
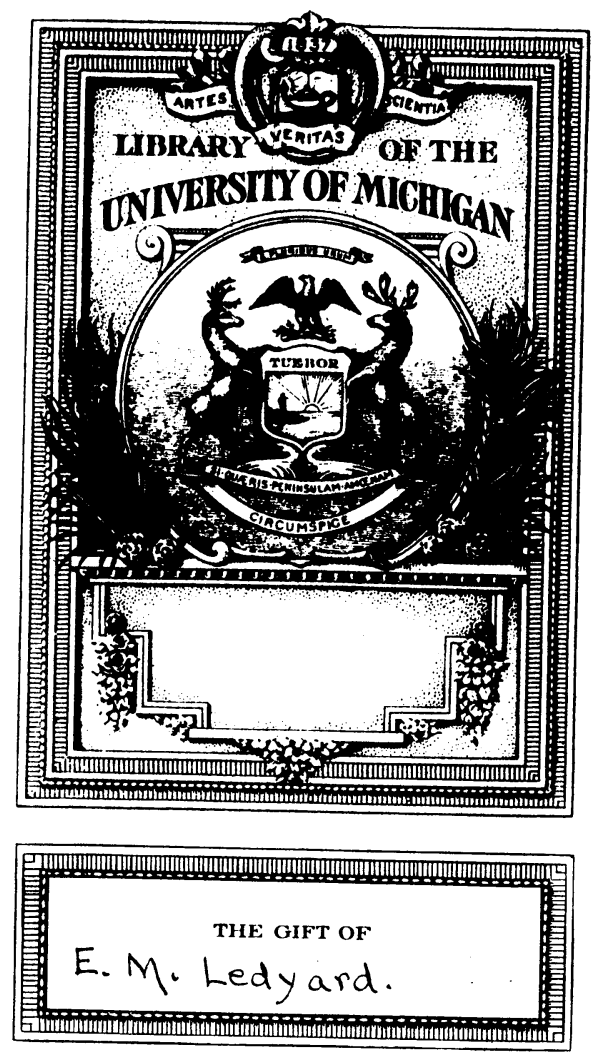
32

, E 
. 


\title{
CATÁLOGO SISTEMÁTICO
}

DE: TOLA I.A

\section{FAUNA DE FILIPINAS \\ CONOCIDA HASTA EL PRESENTE, \\ Y Á LA VIZZ}

EL DE LA COLECCIÓN ZOOLÓGICA

DEL MUSEO DE PP. DOMINICOS DEL

\section{COLEGIO-UNIVERSIDAD DE STO. TOMÁS DE MANILA,}

ESCRITO CON MOTIVO

DE LA EXPOSICIÓN REGIONAL FILIPINA

POR EL

R. $\rho, F_{R}$. CASTO DE ELERA,

PROFESOR DE AMPLIACIÓN DE HISTORIA NATURAL

Y DIREC TOR DEI. MENCIONADO MËSEO

VOLUMEN I.

\section{VERTEBRADOS}

\author{
MANILA \\ IMPRENTA DEL COLEGIO DE SANTO TOMAS \\ 1895
}


Es propiedad.

- 


\section{AL LECTOR.}

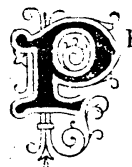

REsentar, en lo que cabe, de un solo golpe de vista, científicamente y por primera vez toda la Fauna de Filipinas conocida hasta el presente; ofrecer en compendio y metódicamente reunido, todo lo que sobre el Reino animal de este Archipiélago se halla esparcido en centenares de libros, folletos, memorias y revistas, publicado en diferentes idiomas por distinguidos Naturalistas; consignar algunas especies y variedades, ó desconocidas como existentes en esta Fauna, ó completamente nuevas, descubiertas por los colectores de este Museo de Santo Tomás, que años ha vienen recorriendo los diversos puntos del Archipiélago; formar como el croquis general de la obra que describa por extenso la Fauna Filipina, la cual, Dios mediante, saldrá á luz lo antes posible con las descripciones y láminas correspondientes; vulgarizar hasta cierto punto esta clase de conocimientos entre los habitantes de este pais, consignando los nombres vulgares, con que son conocidos aquí los distintos animales, á fin cle que por la clasificación científica puedan aquellos apreciar debidamente lo mismo que quizá conocen de una manera irreflexiva; estimular á todos al estudio de esta abundante é instructiva fase de la riqueza natural de Filipinas, y facilitársele mediante la reunión de la sinonimia de todas sus especies, salvando así una de las mayores dificultades que ofrece el trabajo de clasificación; manifestar, en fin, al propio tiempo lo bien surtido de ejemplares zoológicos que se halla el Museo de este Centro científico, el más completo acaso, no sólo de estas regiones océanicas, do:ade los asuntos mercantiles 
más que los científicos suelen llamar la atención, sino aun quizá más rico y variado que muchos Nuseos de Europa: tal es el objeto del presente Catálogo, que el Director del Museo del Colegio-Universidad de Santo 'Tomás de Manila presenta á la Exposición Regional Filipina.

Ninguna ocasión, al parecer, más oportuna para dar a luz este modesto trabajo que la presente, en que todo el país se esfuerza generosamente en cooperar á la más brillante manifestación de la riqueza natural, agrrícola, industrial, mercantil y hasta cientifica y literaria que encierra esta bella porción de los dominios españoles; y ya que por la premura del tiempo y dificultades insuperables de impresión, no haya sido posible presentar la Fauna completa y detallada, sirva el presente Catálogo como resumen suficiente para formarse idea de su grande riqueza y variedad.

Hállanse colocadas las Filipinas por lo que respecta á la geografía zoológica en la subregión Oriental, llamada Indo-Malaya, pero constituyendo al propio tiempo por sí solas un grupo particular, que sirve de lazo de unión a dicha Región con la Austro-Malaya, puesto que participa, aunque en diferente proporción, de las formas zoológicas de las dos Regiones, la Oriental y la Australiana.

Patentiza esta afirmación la mera lectura de este Catálogo. En él se comprueba fácilmente que Filipinas se halla respecto á la Fauna Malaya en la misma relación que lo están Madagascar con el continente Africano, las Antillas con la América del Sur, y Nueva Zelandia con Australia, esto es, que se distingue por la ausencia de casi todas las familias y géneros que caracterizan el resto de la Región Malaya á que pertenece, y al propio tiempo posee multitud de especies y hasta géneros propios y exclusivos de que carecen los demás paises incluidos en la misma Región. De modo que, aún zoológicamente estudiadas estas Islas, son con todo rigor insulares, mientras que Borneo, Java y Sumatra que forman parte de la misma subregión que Filipinas, son verdaderamente continentales en casi todas las formas zoológicas de su respectiva Fauna, y esto mismo sucede con los paises que se agrupan formando parte de la Región Australiana, pues todos ellos contienen las mismas especies que caracterizan á dicha Región Australiana. 
Esta especialidad de la Fauna Filipina, unicla á la menor profundidad de sus mares del Súr relativamente. a la de sus mares (Otientales, demostrada por múl. tiples y minuciosos sondeos, ha movido á algunos sabios á pensar que estas Islas en unión con Borneo, Java y Sumatra no sólo formaron parte en tiempos anteriores relativamente recientes del continente Asiático, lo que ya habian sospechado nuestros primeros historiadores, sino que posteriormente y en época más reciente aún, Filipinas, después de haber permanecido unidas á alguna-de esas Islas, se separaron definitivamente; con la cual hipótesis más ó menos probable, quieren explicar la causa de por qué Filipinas, careciendo de las formas zoológicas características de su Región, contienen sin embargo especies total y exclusivamente peculiares, que suponen haberse formado en el tiempo trascurrido desde su separación hasta el presente. Explicación gratuita es esta que no nos detendrémos en refitar, por no separarios de nuestro objeto, derivada sin duda de la hipótesis no menos gratuita de la mu. tación de especies en sentido trasformista. Baste decir lo que todos sabemos, que cada animal, como cada planta, tiene su área, estación y habitación determinadas según su organización particular, formada por el conjunto de causas físicas y agentes que los rodean. Difícil sería á esos sabios demostrarnos que las formas zoológicas peculiares de Filipinas se formaron después de su separación del continente ó de esas Islas a las que se supone estuvieron unidas, y que no existían en ellas en los tiempos de su unión al continente. Especies peculiares contiene Mindoro, por ejemplo, que no se encuentran en Luzón, y sería ridículo afirmar que esas especies se hayan formado en Mindoro, después de haberse separado de Luzón. En una misma Isla se encuentran especies al Norte que no se hallan en la misma al Súr: si suponemos que esa Isla se divide en dos partes por un agente geológico cualquiera, cada parte seguirá conteniendo las propias especies que contenia antes, sin recibir más trasformación que las modificaciones debidas á la acción de los agentes físicos que les rodeen.

También por este Catálogo podrá apreciarse el inmenso valer de la Fauna Filipina, no menos rica y variada que su Flora, contra lo que hasta ahora se ha 
renido creyendo por algunos, sino más abundante y variada que ella, con ser tan selecta y copiosa. Más de cliez mil especies y variedades zoológicas de Filipinas contiene el presente trabajo, dos mil cinto doce de Vertebrados en este volúmen, y las restantes en el segundo; mientras que las especies botánicas hasta el presente estudiadas no pasan de cinco mil cuatrocientas once. Se incluyen además unas setecientas especies exóticas que forman parte de la colección del Museo de Santo Tomás, y unas ciento veinte pertenecientes á Carolinas y Marianas, que se consignan aquí por formar parte de estas posesiones españolas, aunque zoológicamente consideradas corresponden á la Régión Austro-Malaya.

Si se ha llamado á Filipinas el paraíso de los muluscos por la abundancia y hermosa variedad de sus especies, no es menos rico y hermoso en las otras ramificaciones zoológicas. Aun en Mammalogía, en la cual algunos Naturalistas han escrito que este país se distinguia por la pobreza de especies, el presente $\mathrm{Ca}$ tálogo demuestra que es tan abundante y múltiple como la de cualquier otro país ó región del globo. Ciento setenta y seis especies ó variedades de mamíferos se consignan en este Catálogo, y aun cuando, como verémos al publicarse la Fauna detallada, y hacer la crítica de todas ellas, disminuirán probablemente las especies de Cérvidos y de alguna otra familia, siempre quedará esta clase abundantemente representada. No vacilamos en decirlo; ni por su Flora, ni por su Fauna tiene que envidiar Filipinas á ninguna otra región del mundo.

Estudio tan agradable é instructivo ha tenido en tock tiempo sus cultivadores. Ya descle los primeros años de la anexión á España, nuestros Misioneros, á la ve\% que difundían nuestra santa Religión entre los incligenas, y les enseñaban á vivir civil y cristianamente, no se descuidaron en estudiar este país y sus productos naturales, y en múltiples y apreciables escritos consignaron descripciones de algunos animales peculiares, las cuales, si bien es verdad que en la actualidad no están en armonía con el método científico, no dejan de ser interesantes, por ser los primeros datos sobre esta Fauna. Posteriormente distinguidos Vaturalistas así españoles como extranjeros, que sería prolijo enumerar, hánse dedicado, muy en particular de algunos años á esta parte, al estudio de la Fana Filipina. Comisiona- 
dos por sus Gobiernos respectivos, ó por los Centros científicos de Europa y América, han recorrido casi todas las provincias del Archipiélago, recogiendo en sus excursiones científicas colecciones abundantes que han dado por resultado un conocimiento si no completo de esta tan rica Fauna, al menos más que suficiente para poder comprender su interés é importancia.

Entre los primeros, además de los Sres. Gilly, Baranda, Montero, Fungairiño, Domec, Busto, Mazarredo, y Pérez Maeso, quienes visitando localidades antes poco conocidas y estudiadas, reunieron importantes colecciones, que contribuyeron en gran manera á dar á conocer muchas de las especies características del Archipiélago, merecen citarse los Sres. D. Ramón Jordana y Morera, ingeniero de Montes que en su Bosquejo geográfico $\dot{c}$ histórico natural del Archipiélago, único trabajo de conjunto que se ha publicado en español, reunió casi todo lo relativo á la Fauna Filipina que entonces se conocía, posteriormente D. José Gogorza publicó en 1888 sus Datos para. la Fauna Filipina, en donde consigna quinientas ochenta especies de Vertebrados filipinos, el distinguido conchólogo D. José Cuadras que en I 887 publicó sus importantes Investigaciones conchológicas de Filipinas, y el año pasado el Catálogo di Moluscos de esta Inspección de Montes, y el no menos célebre conchólogo Dr. D. J. F. Hidalgo que ha publicado un interesante Catálogo casi completo de los moluscos observados hasta hoy en las Islas, y por último los Sres. D. Hipólito Fernández, incansable colector, D. Domingo Sánchez, zob́logo auxiliar de esta Inspección de Montes, inteligente, activo y entusiasta de esta Fauna, que además de haber formado una importante colección, en I 890 publicó una bien escrita $M i$ moria sobre el Xylotrechus sp. insecto destructor de los cafetos que tanto daño ha ocasionado en algunas de estas provincias, y el laborioso D. Regino García, auxiliar botánico de la misma Inspección, y no menos entusiasta colector zoológico, á quien se debe el conocimiento de muchos nombres vulgares de los peces de Filipinas.

Entre los extranjeros que más han enriquecido la Fauna Filipina, además de los sabios colaboradores de los Catálogos del Mıseo Británico, de donde se han tomado muchas de las sinonimias de este Catáloga, 
merecen particular mención los distinguidos Naturalistas franceses Sonnerat, Mallat, P. de la Gironiere, Souleyet, Marche y el P. Heude S. J.; los ingleses Cuming, Sharpe, Günther, Boulenger; Swnihoe, Lord Walden, Everetti, y el Marqués de 'Tweeddale; los alemanes Meyer, Semper y Jagor; el italiano Salvadori; y los norte-americanos Dr. Steere, Franck S. Bourns, y Worcester, y otros muchísimos más, todos los cuales en sus diferentes y utilísimos escritos han tratado distintos puntos de esta Fauna, formando un nucleo importantísimo de estudio que demuestra bien claramente su riqueza y variedad.

Con el deseo de contribuir por nuestra parte á que la Fauna de Filipinas sea mejor conocida y apreciada que hasta el presente, unimos á todos los escritos de esos distinguidos Naturalistas este pequeño trabajo, que esperamos sea acogido con benevolencia por el público ilustrado.

Manila 23 de Enero de 1895.

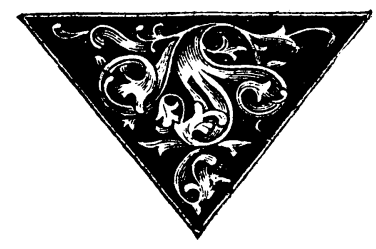




\section{ADVERTENCIA.}

Para mejor inteligencia del presente Catálogo, debemos advertir que después de ordenarle metódicamente consignando el tipo, la clase, familia y género, antes del nombre específico se consigna sólo la inicial del género, á fin de evitar repeticiones.innecesárias; á continuación de la especie y en la misma línea se ponen los signos $t$, macho, $q$ hembra, las iniciales $N$. $v$. que signifi(an Nombre vulgar, y después se añaden las iniciales (M.S.T.) que se leen Museo de Santo Tomás, para indicar que dicha esperie figura en la colección de este Museo. Después sigue la sinonimia ó sea los diferentes nombres que han dado á aquella especie los distintos Naturalistas en sus escritos respectiros que allí se citan. Continúa la palabra Hab. que se lee habitat, y sirve para manifestar los diversos puntos donde dicha especie se encuentra. Para conocer si una especie es ó no peculiar y exclusiva de Filipinas, basta tener presente que siempre que sea especie común á otros paises, después de consignar todos los puntos de las Islas, donde hasta el presente se ha encontrado, st: expresa á continuación algún nombre de los otros paises, y si sólo figuran nombres de puntos de Filipinas es señal de que dicha especie es peculiar y exclusivamente Filipina. Si por el contrario en el habitat, no aparece ningún nombre de las Islas, indicase que la especie es exótica, y forma sin embargo parte dela colección Zoológica del Museo de Santo Tomás. Finalmente, las especies que á continuación de su nombre científico no lleren ias iniciales de algún Naturalista, son nuevas y hasta el presente no clasificadas, $y$ las que en el Índice lleven el asterísco * son especies por primera vez conocidas como existentes en Filipinas. De este modo sencillo y breve se comprenderá más fácilmente el presente Catálogo. 


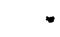




\title{
Tipo 1. VERTEBRadOS.
}

Clase i $^{a}$ MAMIFEROS.

Subclase I $^{\mathrm{a}}$ MONODELFOS.

Orden $1 .{ }^{\circ}$ CUADRUMANOS (Simios.)

\author{
Fam. I. ${ }^{\text {a }}$ SÍMIDOS. \\ Subfam. I. SIMINOS. \\ I. Género SIMLi, Iin.
}

I. S. satyrus, Lin. varr. bicolor. \& N. v. Orang-outan. Museo Sto. Tomás.)

S. abelii, Fischer; Reich., 49h, tîte: gigantica, Pearson.

s. wurmbii, Owen; Reich., 450, 499;:? mias-pappan, Van Kessel.

S. bicolor, Is. Geoff.; Gero., Mamm. I, pl. I; Reich., 497, tète.

S. morio, Ozeen;? mias-rambi, Van Kessei; Reich., 4.58, 500.

S. wallichii, Blainv.,? mias-rassar, Van Kessel; Reich., 498, tête. Habita en Borneo, Sumatra.

Subfam. 2." HILOBATINOS.

2 Gén. HiYlobates, Mhi.ig.

2. H. funereus, Is. Genff. ${ }^{\circ}$ N. v. G. wou-wou.

(M. S. T.)

H. funereus, Is. Geoff. Arch. du Mits. v. p. 532, t. 26; Reich. 475. Hab. en la Isla de Joló y Mindanao.

\section{Fam. 2. ${ }^{\mathrm{a}}$ CERCOPITÉCIDOS.}

Subfam. SEMNOPITECINOS.

3 Gén. Seninoptects, Cuv.

3. S entellus, F. Cu\% $\sigma^{\top}:$ N. v. Mandi. (II S. T.)

S. entellus, Dufrêne; F. Cuv.; Audeb. 4, 2, pl. 2; Bennet, Gard. and Menag., I, fig.; Reich., 227-230.

Hab. en Bombay, Bengala, Nepal. 
4 8. dussumieri, Dism. o N. v. Houneman.

(M. S. T.)

S. dussumicri, Is. Gioff., Arch. Mus., 2. $187_{1}$, pl. 1; Reich., 234, p. 9. Jobnii, Grav 23, Giri., pl. t; «Entelle vieux», $F$. Cur., M.. (nec Fischir, nec Martin).

s. hypoleucus, Blyth; Keich., 231, 232.

s. thersites, Elliof. Proc. As. Suc. Being., figr.

Hab. en Cilan.

5. S. leucoprymnus, Desm. ؟ N. r. id.

Cercopithecus? leucoprymnus, Ott, Nov. Acta Acad. Nat. Citr. xii. p. 505. Sepnopithecu? leucoprymnus, Desm. S. Nestor, Bennett, P. Z. S. 183,3, p. 67, B. M.! S. fulvogriseus, Desmoul. S. dentatus, Shro. Cercopithecus latibarbatus, Geoff. S. porphyrops, limk. Presbytis cephalopterus, Ciray, List $M . B$. MI. 4.' ('cropithecus cephalopterus, Zimm.

Hab. Ceilan.

6. S. albipes, I. Gioff. N. v. id.

S. pallipes, Elliot; Blyth, An. et Mag. N. H. 1844 P. 312.

s. albipes, I. Geoff. Cat. 14; Arch. du Mus. v. p. 576.

Hab. en la isla de Joló, Madrás.

4 Gén. NaSAlis, Georf.

7. N. nasica, Schrob. $q$ N. v. Kahau.

(M. S. T.)

N. nasica, Audeb., 4, 2, pl. 1; Schreber, pl. го, г; Buff., Supp. 7, pl. I 1, г 2. Dahlbom, pl. 4; Müll. et Schl., Verh., pl. 12, juv.: Reich., 317-320.

N. larvatus, E. Geoff. ex Wurmb.; rostrata, Blum. Abb., pl. I3.

N. recurvus, Vig. et Horsf., juv.; Gerv., p. $5^{8}$, tête.

Hab. Burneo.

5 Gén Cynanolgus, Reich.

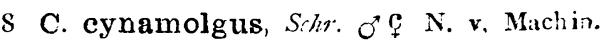

(M. S. T.

Macaque í aigrette, Buff. H.N. XIV, t. $20,2 \mathrm{r}$.

Macacus aygula, Aud, 4, 2 pl. 3.

Simia cynomolyus, Lin. Sy'st. Nat.

Semnopithecus fascicularis, Raffles; Mart. (apued Schlegel) Reich 222. Hab. en tolas las Filipinas, India, Sumatra, Borneo, Jara, Malaca, Siam.

9. C. philippinensis, I. Giaff. var. albint. N. v. Chongo.

Macacus philippinensis, I. Geoff. Arch. Mus. 2, pl. 5; Reich. 370; Jerv. p. 88.

Hab. Luzón, Cávite, Pangasinan. 
ro C palpebrosus, I. Giaff. O N. r. id.

Macacus palpebrosus, I. Geoff. Cat. p. 92: Arch. Mus. r. p. 5t2. Hab. Luzón, Laguna, Albay.

I I. C fur, Siack. $\varepsilon^{\prime} a r$. N. v. id.

Macacus fur, Slack. Proc. Nat. Si?. Ph\%, s's pl. 1. Hab. Luzón, Islis de Nicobar.

12. C. $\pi(a r . c u m i n s i i, G r a v$. C? N. v id.

Macacus cynamolgus, var. cumingii. Gray.

Hab. Luzón.

\section{Gén. M.lCACLS, I, ACrep}

13. M. speciosus, $F \cdot C u v \cdot$ o N. v. Bruh.

M. speciosus, F. Guv. M.tmm. Lith.; Roich. 35.5. 366.

Innuus speciosis, Tem. Fann. Jap. pl. I, 2.

In. fuscatus, Sclat, 1876 : speciosius. Sclat 1875, P. Z. S. pl. 47. Hab. Joló y Japón.

14 M. mauras, F. Cuv. O

Hab. la India.

I 5 M. rhesus, Desm. ơ N. v. Bunder.

(M. S. T.)

Macaque à queue courte, Buffon, H. N. Supp. vii. t. I3. Simia Rhesus, Audeb. t. I. S. erytraa Schreb. Macacus erythræus, Is. Geoff. Voy. Bilang. Simia patas, Link. M. rhesus. Desm. ¿M. nipalensis, Hodgson.

Hab. Bombay.

16. M. sinicus, Lir. o N. v. Munga.

(M. S. T.)

Bonnet chinois, Buff, H. N. xiv. t, 3o. Sinia sinica, Linn. Cyno(ephalus sinensis, Latr. Cercopithecus sinicus, Desm. (not Groff.) Macacus radiatus, Geoff. (Mus. Paris). Hab. Bengala.

17. M. neinestrinus, Dum. $\& \mathrm{~N}$ v. Barou.

(M. S. T.

Pig-tailed Monkey, Edw. Glean. t. 214, Maimon, Buffon, $H . N$. xiv. t. 19. Simia nemestrina, Liun. Macacus nemestrinus, Desm. S. carpolegos, Baffles! S. cristata, Fischer. S. platipygos Schreb. S. fusca, Shaw.

Junior? Babouin à longues jambes, Buffon, Supp. vii. t. 8. S. longicruris, $L$ ink. Hab. Bumbay. 
18. M. nemesirinus, a'ar philipfina, o? N. v. Chongo. (M. S. T?) Hab. Iuzón, Indan (Ciavite).

it Gén. CYNopitinects, itss.

19. C. æthiops, Zim. ơ? N. v. Machin

(II. S. T.)

Papio athiops, Zimm.

Cynocephalus niger, Desm.; Bennet, Gray; Spic. Zool. pl. r. fig. 2.; Schleg. Handl. pl. I. 4 .

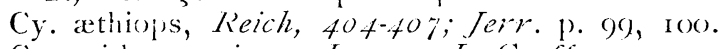

Cynopithecus niger, lesson, $I$. Geoff.

Papio nigrescens, Temm.

Hab. isla de Negros, y Joló. Célebes, Corontalo y Tomini.

\section{Fam. $3 \cdot^{a}$ CEBIDOS.}

8. Gén. CËbls, Erxi.

20. C. chrysopus, F. Cuv. $q$ N. v. Sajou.

(M. S. T.)

¿C. unicolor, Sprx, Bras. t. 4? Cebus chrysoyes, F. Cua. Mamm. lith. t. C. chrysopus, Fischer.

Hab. Colombia.

\section{Orden 2. PROSIMIOS.}

Fam. LEMÚRIDOS.

Subfam. NICTICEBINOS.

I. Gién. NYCTICEBUS, Geofr.

21. N. tardigradus, (Lin.) Fisch. G N. v. Tonger. (M. S. T.)

Nyct. tardigradus, Finch. ex I..; Vosmaer pl. 6.; Aledeb. pl. r. Buff. Suppi. 7, 11. 36; Gero. p. I 57 .

Lemur tarigradus, Lim. Syst. 1. nat.

Loris bengalenss, E. Geoff., sondaicus, Fitz.

Hab. Bohol, Bengala, Malaca, Birmania, Sumatra, Borneo.

Fam. TÁRSIDOS.

2. Gén. TARSIUS, Strorr.

22. T. spectrum, (Pall.) G $\sigma^{\prime}$ N. v. Mago. M. S. T.)

Lemur spectrum, Pall.; Buff. 13 pl. 87: Schleg. Handl. 1857. 11. 1. f. g.; Aud. f. 1. 
Didelphis macrotarsus, Schreb; tarsins, Er.t.'s.

Tarsius daubentonii, I. Geoff.

T. tuscomanus, Fischer; lakis fis. 3; Fischeri, Mesint. bancanus, Sa'.: fuscus. Horsf.

Hah. Samar, Leyte, Bohol, Mindanao; Java, Borneo, Célebes: Sumatrat.

\section{Orden 3." QUIRÓPTEROS.}

\section{Suborden 1. MEGAQUIRÓPTEROS.}

\section{Fam. I. ${ }^{\mathrm{a}}$ TEROPÓDIDOS.}

I. Gén. PTERopus, Briss.

I. Pt phæocephalus, Oiifficld.

Hab. rn las Islas Carolinas.

2. Ft. breviceps, Olficld.

Hab. en las Islas Carolinas.

3 Pt. leucopterus, Tim. $\sigma \rho$ N. v. Paniqui.

(II. S. T. I

Pt. leucopterus, Temminck. Esquiss. Zoolog. sur la côte de Guiné, p. $60,(1853)$.

Spectrum leucopterum et Pt. chinensis, Gray, Catal. Monkeys and Frunteating Bats, pp. 102 \& III (1870).

Hab. Luzón, Cagayan.

4 Pt. edulis, Geoff. $\sigma, 0$ N. v. id.

(M. S. T.)

P't. edulis, Geoffrov, Ann. du Mus. xv. p. 9o (1810); Temmi, Monogr. Mainmal. i. p. I72, ii. p. 58 ; Peters, Mi3. Akad. Berl. I 667, p. 324; Dobson, Journ. Asiat. Soc. Beng. 1873 p. 197, pl. ai?. lig. I (ear); Monogr. Asiat. Chiropt. p. 20 (1876).

Pt. jaranicus, Jesm. Lamm. p. 109 (1820).

Pt. funcreus, Temm. l. c. ii. p. 63 (I835-41).

Hab. Samar, Dinagat, Leyte, Cebú, Malasia, Java, Sumatra, Borneo.

5 Pt. hypomelanus, Tim. $\sigma \rho \mathrm{N}$ v. id.

(M. S. T.)

Pt. hypomelanus, Temm. Esquiss. Zoolog. sur la còte de Guinê, p. 6I (1853); Wagner, Suppl. Schreb. Säuseth. v. p. 599 (1857); Pters, MB. Akad. Bierl. 1867 p. 30.

It. tricolor, Gray, Catal. Monkeys and Fruit-eating Bats, p. 108 (I 8 \%).

Hab. Dinagat, Mindanao, Luzón, Célebes, 'Ternate y Nueva Guinea. 
6 Pt Keraudrenii, Q. it $G$. N. v id.

¿Pt. mariannus, Desm. Mamm. p. $5+7$ (1 822$)$.

P.. keraudren, Quoy et Gaimard, Voyge de l'Uranie, i. p. 51, pl. $3($ i 824$)$.

Pt. keraudrenius, Temm Monggr. Mimmal. i. p. I\$6 (I827); Wagner, Suppl. Schreb. Säugeth. v. p. 6or (1857).

Pt. tonganus et vanicorensis, Quoy et Gaimard, Voyage de l'sstrolabe, i. p. 7 t, pls. 8 \& 9 (1833).

P.. insularis. Hombrom et Jacquinst. Voyage au Pôle Sud, pl. 5; Peters, MB. Akad. Berl. 1860 , p. $37 \mathrm{I}$.

Pt. dussumierii, Geoffroy, Dict. Class. xv. p. 70I.

Pt. reddici, Macgilliwray, Peters, l. c. 1867, p. 326.

Pt. keraudrenii, Peters. l. c. p. $33 \mathrm{I}$.

Pt. flavicollis et greddiei et vitiensis, Gray Catal. Monkeys and. Fruit-eating Bats, pp. I07, I09 (1870).

Hab. las Islas Marianas, y en casi toda la Subregión Polinesiana.

7. Pt. insularis, $I I$. e't $G$. var. N. v id

Pt. insularis, Hombr. et Jacq. Voy. au Pôle Sud. pl. v.; Ptro, MB. Akad. Berl. 1869 , p. $39 \mathrm{I}$.

Hab. en las Islas Carolinas, Jap. y Palaos.

8. Pt. jubatus, Esil. ơ N. v. id.

(M. S. T.)

Pt. caniceps, Gray, Catal. Monkeys and Frut-eating Bats, p. Io7 (i 870$)$.

Pt. affinis, Gray, l. c. p. Io8.

Hab. la Paragua, Mindanao, Joló, Dinagat, Luzón y Batangas.

9. Pt. pbilippinensis, $\sigma \circ \mathrm{N}$ v. id.

Hab. Luzón, Cagayan, Pangasinan, Samar, Borongan, Paragua, P. Princesa.

2 Gén. CYNONYCTERIS, PTrs.

Io Cy. amplexicaudatus, (Geoff.) $\sigma \circ$ N. x. Calapnit. (M. S. T:)

Pt. amplexicaudatus, Geoffroy, $A u d u$ Mus. xv. p. 95 (I 8 ro); Temminck, Monogr. Mammal. i. p. 200 (1827).

Pt. leschenaultii, Desmar. Mammal. p. I IO (1821); Blyth, Catal. Maminal. Mus. Asiat. Soc. Beng. p. 2 I ( 1863 ).

Pt. pyrivorus, Hodgson, Journ. Asiat. Soc. Byns. I835, p. 7o(). Pt. seminudus, Kelaar, Journ. Asint. Sx. B:is. I852, 1. 345. Cy. amplexicaudata, Peters, MB. Akad. Berl. г 37, p. 865; Dosion, Proc. Asiat. Soc. Beng. I872, p. I 54 ; Journ.

Asiat. Soc. Beug. 1873, p. 202, pl. xiv. fig. 8 (ear); Monogr. Asiat. Chiropt. p. 29 (1876). 
Eleutherur. marginata, infumata, philippinensis, Gray, Catal. Monk,'s ant Fruit-eating Bats, pp. II 8 iा9, (1870.)

Cy. infuscatal, Piters, l. c. 1873, p. 487. Hab. Luzón, Laguna, Bataan, Sumatra, Ceilan, Flores, 'Timor.

\section{Gén Cynopterus, F. Cuv.}

I1. Cy. marginatus, Gioff $\sigma \circ$ N. v. Calapnit.

Pt. marginatus, Geoff. Ann. du Mus. xiv. p. 97; Temm. Monogr. Mammal. i. p. 202, pl. xiv.

Cynopterus marginatus, $F$. Cuvier, Dents des Mammif. p. 39) (1825); kelaart, Prodr. Fanna Zeylanica, p. Is (1852); Blyth, ibid. p. 37; Peters, MB. Akad. Berl. I867, p. 866; Jeriton. Mamm. of Inda, p. 2o (1857); Gray, Cat. Monkeys and Fruteating liats, p. 122 (1870); Dobson, J. A. S. B. 1873, p. 20), pl. xiv. fig. 4 (ear); Monggr. Asiat. Chiropt. p. 24.

Pt. tittheacheilus, Temm. l. c. i. p. I98.

Pachy. oma diardii et duvaucellii, Is. Geoffr. Dict. Class. Hist. Nat. xis. p. 705; Temm. l. c. ii. pp. 95, 96.

P. brevicaudatum, Is. Geoff. l. c.; Temininck, l. c. p. 92.

Pt. pyrivorus, Hodsong, P. Z. S. 1836, p. 36 .

Cynopterus horsfieldii, Gray, Cat. Mamm. Brit. Mus. p. $3^{\text {s }}$ (18+3); Horsfield, Cat. Mamm. Mus. E. I. Comp. p. 30 (1851). Pa. luzoniense, Peters, MB. Akad. Berl. 1861, p. 708.

Eleutherura marginata, Gray, P. Z. S. i 866, p. 64. Hab. Luzón, Abra, Paragrua, Java, Sumatra, Borneo, Siam, Ceilan.

12. C. latidens, Dolis. $\sigma \circ$ N. v. Colapnit.

M. S. T.j

Hab. en Luzón, Lagruna, Batangas, y el Archipiélago Malayo.

Subgen. Ptenochirus, Ptrs.

13. Pt. jagorii, Ptrs. N. v id.

Pachysoma (Ptenochirus) jagorii, Ptrs, M. B. Akad. Bevl. 1861 p. 707 .

Cynopterus jagorii, Dols. Monogr. Asiat. Chirop. p. 28 (1876). Hab. Luzón, Ilocos, Pangasinai, Pampanga, Nueva-Écija.

4. Gén. maciroglosits, F. Cuv.

14. M. minimus, Ginff. N. v. Cabag.

(M. S. T.)

Pteropus minimus, Geiffry, Ann. d. Mus. xv. p. 97 (1810). Pt. rostratus, Horsfield, Zool. Research. in Java (1825).

Macroglossus minimus, Temminck, Monogr. Mammal. i. p. I9I, ii. p. 96 (1835-41); Horsfield, Cat. Mammal. Mus. E. I. Comp. p. 29 (1851); Gray. Proc. Zool. Soc. Lond. 1866, p. 64; Peters, Monatsb. Akad. Wissens i, Berl. 1867 , p. 871; Gray, 
Cat. Monke's and. Fruiteating Bats, 1870, 1. 115; Dobson, J. A. S. B. $1 \delta^{2} 73$, p. 205; Monogr. Asiat. Chiropt. pp. 34, 35 , fig.

Hab. Panay, Samar, Cuyo.

\title{
Suborden 2. IMICROQUIRÓPTEROS.
}

\author{
Fam. RINOLÓFIDOS. \\ Subfam. RINOLOFINOS. \\ I Gén. RHINOLOPHUS, Grorr.
}

I. Ph. luctus, Tim. Ó N N. v. Cabag.

(II. S. T.)

Rh. luctus, Temn, Moner. Mammal. ii. p. 24, pl. xxx.; Peters, MB. Akad. Berl. 1871, p. 304; Dobson, Monogr. Asiatic (hiroptera, p. 39 (1876).

Rh. luctus, var rufus, Eydoux et Gervais, Voy. Favor. Zoologie,

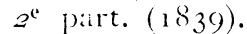

R. morio, Gray, Ann. \& Mag. Nat. Hist. I $8+2$, p. 257.

Rh. perniger, Hougs., Journ. As. Soc. Beng. xii. p. 41 t. xiii. p. $48+$; Horsf., Cat. Mamm. Mus. E. I. Comp. (1851).

Aquias luctus, Gray. P. Z. S. 1847 , p. i 7 ; id 1866, p. ช. Hab. en Luzón, Paragua, Leyte, Mindanao.

2 Rh. philippinensis, Wat. $\sigma 0$ N. v. Cabag. (M.S.T.)

Rhinolophus philippinensis, Waterh. P. Z. S. $18+3$, p. 68; Peters, MB. Akad. Bisrl. 1871, p. 305, Dobson, Monogr. Asiat. Chiropt. p. $43(1876)$.

Phyllotis Philippensis, Gray, P. Z. S. 1866,1 . 81 . Hab. en Luzón, Laguna, Cavite.

3 Fih. rufus, Ptrs. o N, v. Cabag.

(M. S. T.)

Rh. rufus, Peters, MB. Akad. Berl. 1861, p. 7 Io; Dobson. Monogr. Asiat. Ciniropt. p. t+ (1876).

Rh. euryotis, Peters (non Temm.), l. c. 1851, p. 7 ro.

Rh. arcuatus, Peters, l. c. 1871 , p. 305.

Hab. Luzón, Manila, Bataan, Bulacan, N. Eacija, Batangas.

Subfam. FILORRININOS.

2. Gén. PHyLlokHiNi, ibr.

4. Ph. diadema, Groff. U'. N. v. Cabag.

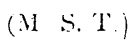

Rhinolophus diadema, Geoff. Ann. Mus. d'Hist. Nat. xx. p. 263.

Rhinolophus nobilis, Horsfeld, Zool. Research. in Java. 


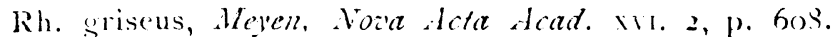

Rh. diatema of nohilis, Temm., Monogr. Mammal. ii. p. 10.

Hipposideros nobilis, Cantor, Journ. dstatic. Soc. Beng. xv. i). 182.

Ilip). lankadiva, Kelaart. Prodt. Faun. Zejlan. p. 19.

Pintlorhina diadema, Peters, MB. Akad. Ber. 1871, p. 315; Dobson, Monogr. Asiat. (Miropt. p. 61 $(18-6)$.

Hab. Luzón, Manila, Laguna, Carvite, Indan, Paragua, Mindatrato, Jata, bornco.

5 Phy. pygmæa, Wat. $0^{\circ}$. N. v. Cinguing.

Rhinolophus pygmax, Ilaterh., P. Z. S. 28 t3. p. 67.

'hollorhina pyomat, Gräy, P. Z. S. 1800 , p. sz; Peters, .Vonalsb. Akad. Berl. 1̇jt, p. 317; Dobson, Monogr. Asiat. ('hiropt. p. 63. $(1876)$.

Mab. Luzón, Batangas, Laguna, Los Baños.

6. Fhy larvata, IIr.s: $0^{\circ}: \mathrm{N}$. v. Talibatab.

Rhinolophus larratus, vulgaris, insignis et deformis. Horsf., Zoo. low. Resectrch. in Jav'r (182t.)

Rh. insignis, Temm., Monog. Mammal. ii. p. 74 .

IIip. insiginis, Gray Mag. Zool. \& Bot. ii. p. 492.

Hip. rulgaris, Blyth, Journ. As. Soc. Beng. xiii. p. 488.

Hip. larratus, Biyt, Cat. Mammal. Mus. As. Soc. p. 26.

Speorifera vulgaris, Gray, Proc. Zool. Soc. Lond. 1866, p. 82.

Phyllorhina larvata, Peters, MB. Akad. Berl. 1871, p. 320; Dobson, Monogr. Asiat. Chiropt. p. 68, figs. a, b (nose-leaf) $($ I 876$)$.

Hab. Luzón, Manila, Antipolo, Java, Siam, Bengala.

7. Phy. obscura, Ptrs. N. v. Cuyayapnit.

(M. S. T.)

Phyllorina obscura, Peters, MB. Akad. Berl. 1861 , p. 709, and хsjг, p. 326; Dobson, Monogr. Asat. Chiropt. p. 73 (1876).

Hab. Dinagat, Mindanao.

8. Phy. coronata, Plis. N. v. id.

Phyllorhina coronata, Peters, MB. Akad. Berl. 1871, p. 327; Dobson; Monogr. Asiat. Chiropt. p. 72 (1876).

Hab. Minclanao, Paragua, Samar.

9. I'hy. bicolor, Temm. O. N N. v. Balacbalac. .

(M S. T.)

Rhinolophus bicolor, Temm., Monogr. Mammal. ii. p. i8 (1835-41).

Phyllorhina antricola, Peters, MB. Akad. Berl. 1861 , p. 709.

Phy. bicolor, Peters, l, c. 1871, p. 323; Dobson, Monogr. Asiat. Chiropt. p. $70(18,6)$.

Hab. Cuyo, Calamianes, Paragua, Borneo, Java y Nicobar. 


\section{Fam. NICTÉRIDOS.}

I. Gén. MEGLDERMA, Groff.

I. M. epasma, (Lin.) O*. N. ¿. Talibatab.

(M. S. T)

Vesperitio spasma, Linnaus, Syst. Nat. $175^{8}$, p. 32; Schreher, Sïllychiere, i. p. I 58 , p. 1. xlviii. (1 775 ).

Megaderma spasina, Geoff., dinn. du Mus. p. I95 (1810); Caitor. journ. Asiat. Soc. Beng. 1876 , p. 179; Blyt. Append. Ke'aart's Prodr. Fanna Zeylanica p. 38 (1852); Peers, MB. Akad. Berl. 1872, p. 193; Dobson, Monogr. Asiat. Chiropt. p. 79 (18;6).

Meg. trifolium, Geoff., l. c. p. 193; Wagner, Suppl. Schreb. Süugeth. i. p. 415 (18+t), v. p. $6+2$ i 855 ).

Meg. philippinensis, Waterh., $P$. Z. S. 1843, p. 69.

Meg. horstieldii, Blyth, Catal. Mammal. Mus. Asiat. Soc. Bens. p. 23 (1 863$)$.

Hab. Luzón, Placer, Mindanao, Borneo, Célebes, Java, Singaf.ur.

\section{Fam. VESPERTILIÓNIDOS.}

\section{Gén. Vesperugo, Keys.}

I. Vespərugo pachypus, T(mm) O O N. v. Cabag.

(M. S. T.)

Vespertilio pachypus, Temm., Monogr. Mammal. ii. p. 2i7, pl. 5t. figs. $4-6$.

Vesierus pachypus, Wagner, Suppl. Schreb. Säugeth. v. p. 74I (1855); Dobson, Proc. Asiat. Soc. Beng. 1871 , p. 2 г 2.

Sec tophilus fulvilus, Blyih, Journ. Asiat. Soc. Beng. xxviii, p. 293. Tylonycteris pachypu:, Peters, MB. Akad. Berl. 1872 p. 70.4 .

Ty. meyeri, Peters, l. c. p, 705.

Vesperugo pachypus, Dobson, Monogr. Asiat. Chiropt. p. 7 I 5 $(18,6)$.

Hab. Luzón, Cebú, Negros, Mindanao, Java, Sumatra.

2. Vesp. ncctula, Schr. $\sigma^{\top}$, N. v. Curarapnit.

(M $\quad \mathrm{S} \quad \mathrm{T}$.)

Vespertilio noctula, Schreber, Säugeth. i. p. r66, pl. 52 (I875); Gimel. Limn. Syst. Reg. Auim. i. p. I46 (1788): Desmarest, Mammalogie (1820), p. I 36 ; Temm. Monogr. Mamm. ii. p. I69 (1835-41); Jenynts, Brit. Vertebr. p. 23 ; Bonap. Fanna Ital. fasc. xxi. (1837); Bell's Brit. Quadrup. p. I2, wdet (I837); De Séys-Longch. Fanne Bulge, 1842 .

Vesp. magnus, Berkenhout, S:nop. Nat. Hist. Gt. Brit. \& Irel. p. ii. ( 789 ).

Vesp. altivolans, White, Nat. Hist. Seltorne ( 1780$)$.

Vesp. serctinus, Geof. Ann. an Mus. viii. p. I0) (1805).

Vesp. proterus, Kuhl, Wetter. Ann. iv. p. 4 i (1817).

Vesp. labiata, Hotgson, Jotrn. Asiat. Soc. Beng. iv. p. 700 (1835). scotophilus noctula, Gray, Mag. Zool. \& Bot. ii. p. t97 (1838). 
Vesperugo noctula, Keys. \& B!as. Wiegin. Arih: 18.39 , p. 317 Wirbelth. Europ. p. 46; Wagner, Suppl. Schreb. Süugeth. v. p. 728 (1855); Blasius, Fanna Dentschl. p. 53, wilct. p. 54 (1857); Lilljeborg, Sver. Norges Rygrradsdjur, p. 134 (1874); Dobson, Monogr. Asiat. Chiropt. p. 88. (1876).

Vespertilio macuanus, Peters, Reise wach Mossambique, Säagath. p. 6 I $(1852)$.

Noctulinia noctula, Jerdon, Mamm. of. India, p. 36 (186j).

Hab. Luzón, Manila, Laguna, Borneo, Java, China.

3 Vesp. maurus, Bias. o๐ $\mathrm{N}$ v. Cabag.

(M. S. T.)

Vesperugo maurus, Blasine Wiegm. Archiv, 1853, p. 35; Fanna Dentschl. p. 67, figs. 4344 (1857); Dobson, Monogr. Asiat. Chiropt. p. 99, figs. $a, b .(1876)$.

Scotophilus darwini, Tomes, P. Z. S. 1859 , p. 70.

Vesp. mordax, Peters, MB. Akad. Berl. $1866, \mathrm{p}, 408$.

Vesp. pulveratus, Peiers, P. Z. S. 1870 , p. 6ri7.

Pipistrellus austenianus, Dobson, $P$. A. S. B, 1871, p. 213.

Hab. Luzín, Manila, y toda las regiones Paleartica y Oriental.

4. Vesp. abramus. Temm. $\sigma \circ \mathrm{N}, \mathrm{v}$ id

(M. S. T.)

Vespertilio imbricatus, Temm. (non Horfield) Monngr. Mammal. ii. p. 2 r6, pl. 54; Wagneg, Suppl. Schreb. Saugeth. v. p. $73^{8}$ ( 1855$)$.

V. abramus, Temm. l. c. 232, pl. 58; Wagner, l. c. p. 739.

Scotophilus javanicus, Gray, Mag. Zool. \& Bot. ii. p. 498 (1838).

Sc. coromandra, Gray l. c.

Vesperugro nathusii, Keys. \& Blas. Wiegm. Archiv, I 839, p. 320;

Blasius, Fauna Deutschlands, p. 58, figs. 37, 38 (1857).

Vespertilio coromandelicus, Blytg, J.A.S. B. p. 1.59 (1855).

Vesperugo blythii, Wagner, op. cit. p. 742 (I855).

Scotophilus coromandelicus, Horsfield, A.\& M. N. H. 1855 , p. 5 .

Sc. pumiloides, Tomes, P. Z. S. 1857, p. $5 \mathrm{I}$.

Verpertilio coromandelicus, Tomes, $P$. $Z$. S. $18 S 8$, p. 539.

Pipistrellus coromandelicus, Dobson, J.A.S. B. 1871 , p. 461.

Vesperug() imbricatus, et micropus, (Hutton), Peters, P. Z. S. $1872, \mathrm{pp} .707,708$.

Vesp. abramus, Dobson, Monogr. Asiat. Chiroptera, p. 97 (1876). Hab. en Luzón Manila, Bosobosó, y con toda la región Oriental, China.

\section{Vesp var imetitus, Can'or. ơ o N V id.}

(M. S T.)

Vesperilio irretitus, Cantor, Ann. \& Mag. Nat. Hist. ix. p. $48_{\mathrm{I}}$ $(\mathrm{I} 8+2)$.

V. meyeni, Waterhouse, P. Z. S. 1845 , p. 7 ; Ann. \& Mag. Nat. Hist xvi. p. 53.

Vesperugo irretitus, Dobson, Monogr. Asiat. Chiropt. p. 98 (1876). Hab. en Luzón, Laguna y China. 
6 Vesp tylopus, Dis. o̊ $\mathrm{N}$ v. Cuyayapnit.

Vesperugo (Cilischropus) tylopus, Dobson, P. Z. S. 1 $87.5 \cdot$ P. +73 , Monogr. Asiat. (Kiropt. p. IIt (Isj(o).

Hab. en Paragua, Borneos.

2. Gen. SCOTOPHILLS, I,ACH.

7. Sc temminckii, $I_{0}, s f .0,0$ N. v. id.

PScotophilus kuhlii, Ieach, Trans. Limn. Soc. xiii. p. 7t (1822). Vespertilio temminckii, Horsficlu, Zool. Researches in Jaz'a (18zt).

V. belangeri et noctulinus, Is. Geoff., Be'ang. Voy. aux Intes Orient. $(1834)$ pp. $87 \& 92$, pl. 3.

V. castaneus, Gray, Illustr. Indian Zool.

Scotophilus temminckii, Gray, Mag. Zool. \& Bot. ii. 1). 497; P' $\epsilon^{\prime}$ ters, MB. Akad. Berl. 1866 , p. 679; Dobson, P. Z. S. 1S7.5, p. $370 ;$ Monogr. Asiat. Chiropt. p. i 20, figs. a, $b$.

Nycticejus temminckii, Temm. Monogr. Mammal. Ir. p. Ifo; Hagner, Suppl. Schreb. Säugeth. I. p. 541 $(18+1)$, v. p. it) (1855); Cantor; J. A.S. B, 1876 , xv. p. $185 ;$ Blyth, J. A. S. B. 1851 , 1. 157; Horsfield, Cat. Mamm. Mus. E. I. Comp. p. $37(1851)$; Blyth. Cat. Mamm. Mus. As. Soc. Beng. 1). 31 (1863).

Nycticejus luteus, Blyth.J.A. S. B. xx. p. I57, xxi. p. $3 f^{\text {th; }}$ Wagner, Suppl. Schreb. Süugeth. v. p. 765 (1855).

N. flaveolus, Blyth, Horsfield in Cat. Mamm. Mus. E. I. Comp. p. $37(185 \mathrm{I})$.

Hab. en Dumaguete, Negros, Jara, Sumatra, Borneo.

3. Gén. Vespertíto, Kiss.

8. V. macrotarsus, Whit N. v Cabag.

Vespertilio macrotarsus, Wraterh. Anz. \& Mag. Nat. Hist. vol. xvi. p. $51(18+5)$; Wagner, Suppl. Solureb. Säugeth. v. p. 7 to (1855); Dobson. Monogr. Asiat. Chiropt. p. 130 (1876).

Hab. en Mindoro.

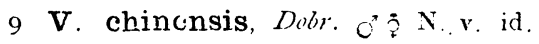

(M. S T.)

Vespertilio chinensis, Tomes, P. Z. S. 1857, P. 52; Dobson, Monogr. Asiat. Chiropt. p. I 39 (1876).

Hab. Luzón, Manila, China.

so $\mathbf{V}$. formosus, $H \omega_{i} d . \mathrm{N}, \mathrm{v}$ id

Vespertilio formosus, Ho:lgson, J. A. S. B. iv. p. 700 (1835); P. Z. S. 18,36, p. $46 ;$ Wagner, Suppl. Schreb. Saugeth. $\because$ p. 736 (1855); Tomes, P. Z. S. 18.58, p. 82, pl. 1x.; Mobson, Monogr. Asiat. Chiropt. p. ifo (1876).

Kerivoula formosa, Gray, List of Mammals in Co!l. Brit. Mus. p. $27(1843)$. 
Vesportilis rufo-pictus, Wryterhouse, Ann. \& et Mag. Nat. Wat. Hist. xri. p. $5+(18+5)$; Tons. P. Z. S. 1858 , p. 85.

Vesp. (Kerivoula) formosa, Horsfielt, Cat. Mamma!. Mus. E. $I$. Comp. 1). ti (1851).

Lerivoula pallida, Blyt, Catal. Mamm. Mus. Asiat. Sx. Bens. p. $3+(1863)$.

Murina formosa, Jerdon, Mammals of India, p. 42 (1867).

Vespertilio auratus, Dobson, J. A. S. B. xl. p. 186, pl. x. (1871). Hab. Luzón, India, Formosa, (hina.

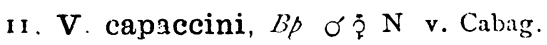

(M. S. T)

Vespertilio capaccinii, Bonap. Fannx Italica, 18,32, fasc. xx.; Temm. Monggr. Mammal. II. p. I39 (1839-11); 1)>bson, MongsrAsiat. Cliropt. p. I $29(\mathrm{I} 876)$.

Verpertilio megapodius, Temm. l. c. p. 189.

Vesp. macrodactylus, Temm. l. c. p. 231 (vide Peters, in $M B$. Akad. Berl. 1866 , p. 679).

Hab. en Luzón, Japón.

12 V. muricua $M u d g_{s}$ N. v. id

Vespertilio arlversus, Temminck (non Horsfield), Mongor. Mammal. ii. p. 221; Petèrs, MB. Akad. Ber-l. 1866, p. 399; 1868, p. 626.

V. tralatitius, Temm. (non Horsf.) l. c. p. 228.

V. muricola, Hodgson, J. A. S. B. x. p. 998; Gray, Cat. Mamm. Brit. Mus. 1873, p. 26; $2 d$. List. of Mammalia and Birds of Nepaul presented by B. H. Hodo:ion, Esq., to the British Musetum, $18+6$; Dobsoin, Monogr. Asiat. Chiropt. p. I34 (1876).

Kerivoula trilatitoides, Gray, Cat. Mamm. Erit. Mus. 1873, p. 27 (nomen mudum) (Vide Pters, MB. Akad. Berl. 1866, p. 399).

V. Caliginosus, Tomes, P. Z. S. 18.56, p. 73 .

V. ater, Bernstein, Peters, l. c. 1856. p. 18.

V. (Pternopterus) lobipes, Pters, l. c. 1867, p. 706.

V. Blanfordi, Dobson, P. A. S. B. 1871 , p. 2 I 4 .

V. moupinensis, Alph. Milne-Edwards, Mammif. du Tibot: p. 255 pl, xxxvii. fig. 2, pl. xxxvii. fig. 4 (1872).

Hab. Joló, Borneo, Célebes.

4. Gén. Kerivoula, Gray.

13. Kerivoula pellicida, Wiat o o N. v. Cuyayapnic (M.S. T)

Vespertilio pellucidus, Witterhouse, P. Z. S. 1845 , p. 6; Wagne:-, Supp!. Schreb. Süugeth. v. p. 742 (1855).

Kerivoula hardwickii, Tomes, P. Z. S. 1858 p 329.

Kcreiroula pellucida, Dobson, Monsgr. Asiat. Chiropt. p. 149 $(1876$.

Hab. en Mindanao, Luzón, Samar.

I4. Kerivoula jazorii, Pirs o $\sigma \mathrm{N}$ v. Balacbalac.

(M S T )

Vespertilis) (Kerivoula) jagorii, Peters, MB. Akxd. Berl. 1866 , 399; Dobson, Monogr. Asiat. Chiropt. p. I85 (1875).p.

Hab. en Samar. 


\section{Gén MINIOPTERUS, Bp.}

15. M scinreibersii, Natt N v. Cabag.

Vespertilio schreibersii, Natterer, in Kuhl, Deutsch. Flederm. Wetteran. Ann. iv. p. 4 I (1819); Desmarest Mammalogie, p. I38 (1820); Temminck, Monogr. Mammal. ii. p. I74 (I835-4I)

V. natalensis, Smith, S. African. Quart. Journ. 1831.

V. fuliginosa, Hodgson, Journ. Asiat. Soc. iv. p. 700 (1835).

Miniopterus ursinii, Bonaparte, Fauna Italica (1837).

V. orsinii, et blepotis, et dasythrix, Temminck, l. c. pp. i79, 2 I 2, 268 (I $835-4 \mathrm{I})$.

'Trilatitus blepotis, Gray. Ann. \& Mag. Nat. Hist. 1842 , p. 258.

V. eschscholtzii, Waterhouse, P.Z.S. 1845 , p. I 2 I.

Miniopteru; schreibersii, Keys, et Blas. Wirbelth. Europ. p. 44.; Blasius. Fauna Deitschl. p. 46, fis. 28, 29 (head and ear) (1857); Dobson, Monogr. Asiat. Chiropt. p. i6o (1876).

M. schreibersii, et blepotis, et tibialis, Tomes, P. Z. S. 1858, p. I 26. Hab. en Luzón, Australia, Java, Ceilan, Italia y España en Sevilfa.

16. M. var pusillus, Doks. N. v id.

M. pusillus, Dobsnn, Monogr. Asiat. Chiropt. p. I62 (1876).

M. australis, Tomes, (in part.) P. Z. S. I858, p. 125.

Hab. en Luzón, Madras, Nicobar.

17. IM tristis. Wat ơ N. v. Calapnit.

(M. S T.)

Vespertilio tristis, Waterhouse, Ann. \& Mag. Nat. Hist. xvi. p. 50 (I845); Wargner, Suppl. Schreb. Säugeth v. p. 743 (I 855). M. tristis, Tomes, P. Z. S. 1858 , p. I 24.

Hab. en Luzón, Cebú, Samar.

\section{Fam. EMBALONÚRIDOS.}

Subfam. EMBALONURINOS.

I. Gén. EMBALlonURA, Tem.

1. Fim. semicaudata, Pcale. N. v. Cabag.

Vespertilio semicaudatus, Peale, United States. Expl. Exp., Mammalia, p. 23, pl. III. fig. 2 ( 1.848 ).

E. semicaudata, Wagner, Suppl. Schreb. Saugeth. v. p. 698 (1855); P'eters, MB. Akad. Berl. 18607 , p. 77.

Emballonura fuliginosa, Tomes, P. Z. S. 1859 , p. 77.

Hab. en Carolinas, Marianas, Subregion Polinesiana.

2. F. monticola, Temm. $\sigma \circ$

(M S. T.)

Eimb. monticula, Temminck, Van der Hoeven, Tijdsch. voor 
Nat. Gesch. v. p. 25, pl. II. figs. I, 2 (1839); Peters. MB. Akad Berl. 1867 , p. 480; Dobson, Monogr. Asiat. Chiropt. p. $165(1876)$.

Vespertilio (Nycticejus) alecto, Eydoux et Gervals. Voy. 'Favorite' 2. part. Zcology, p. 7 (1839).

Emballonura discolor, Peters, MB. Akad. Berl. 1861, p. 7 I I. Hab. Luzón Laguna, Java, Sumatra, Borneo.

\section{Gén taphozous, Georf.}

3. T. melanopogon, Tim. $\sigma \circ$ N. v. id.

(M. S. T.)

Taphozous melanopogon, Temm. Monogr. Mammal il. p. 287

(1835-41); Wagner, Suppl. Schreb. Säugeth. v. p. 687; Cantor, Journ. Asiat. Soc. Beng. xv. p. I8o; Dobson, Proc. Asiat. Soc. Beng. Aug. I872, p. I53; P. Z. S. 1875, p. 549. fig. 2; Monogr. Asiat. Chiropt. p. ${ }_{167}$ (1876).

T. bic olor, Tem. l. c. p. 290.

T. philippinensiș, Waterhouse, P. Z. S. 1845, p. 9.

Hab. Luzón, Manila, Java, Borneo, India, Cochinchina.

\section{Gén. NyCtinomes, Geofr.}

4. N plicatus, Buchrn. $\sigma \circ \mathrm{N}$ v. id.

V. plicatus, Buchanan-Hamitton, Trans. Linn. Soc. v. p. 26, fig. (18c0).

N. bengalensis, Geoffroy, Descr. de l'Egypte, II. p. Izo (1812).

N. tenuis et dilatatus, Horsfeld, Zoolog. Research. in Java (1825); Cantor, J. A. S. B. xv. p. I79.

D. murinus, Gray Illustr. Ind. Zools (figured), 1830.

D. plicatus, Temminck, Monogr. Mammal. i. p. 223 (18354 I.

D. tenuis, Temminck, l. c. p. 228 .

N. plicatus, Jerdon Mammals of India p. 33 (1867); Dobson, J. A. S. B. 1874, p. I43; Monorg. Asiat. Chiropt. (1876); P. Z. S. 1876 , p. 721 .

Hab. en Luzón, Laguna, Java, Sumatra, Borneo, Singapur. 


\title{
Orden $4 .^{\circ}$ INSECTÍVOROS
}

\section{Subord. MESODONTOS.}

\section{Tribu IDERHÓPTEROS.}

\author{
Fam. GALEOPITÉCIDOS. \\ Subfam. GALEOPITECINOS. \\ 1. Gén. GaleopitheCUS, Pall.
}

1. G. volans, (Shaw) $0^{*} \cap$ N. v. Caguan.

(M. S. T.)

La'mur volans, Shaw, Zool. 1, p. $3^{8}$; Stour in Linn. Fdtit. 12. t. 1. p. 455 .

(i. volans, Pall. Act. Petrop. 4 pl. 7. 8: Schreb., Saigrs jö: Aut., Mokis pl. I.

Sonlegel, Handl. pl. i. fog. 12; Gerv. Mamm. 1. p. I80.

Hab. en Bohol, Cebú y toda la Malasia.

2. G. rufus; Gerff. var: $\sigma \Varangle$ N. v. id.

(M. S. T.)

G. rufus, I. Geoff. ct Curv.; G. ternatensis, Geoff.; Seba, Thes. pl. $5^{8} \mathrm{f}$. 2, $3 .$. E Encycl. pl. 22. fig. 1.

Hab. en Samar, Cebú, Siam, Malaca, Ienaserin.

3. G. variegatus, Geoff. var. or $\bigcirc$ N. v. id.

(M. S. T.)

(i. variegatus, E. Geoff. et Cuv., Aud. Makis pl. 2. Schreb, S. 307. D.: Temminckii, Waterh.

Hab. Cebú, Leyte, Samar, Java, Borneo, Sumatra.

4. G. marmoratus, Tem. var. $† ?$ N. v id.

(M. S. T.)

G. marmoratus, Temm. undatus, Wagner; Pall. loc. cit. pl. 7 . Hab. en Basilan, Samar, Banka.

5. G. philippinensis, Wat. var. tf N. v. id.

G. philippinensis, Waterh, P. Z. S. 18.38 p. 119.

Vespertilio admirabilis, Buntius, Hist. $\dot{N}_{a x}$. fig. 1). 69; Griffith

Animal Kingd. I. p. 158. f. 4.

Hab. en Samar, Mindanao, Java y Timor. 


\section{Subord. INSEGTÍVOROS (Verdaderos.)}

Fam. TUPÁYIDOS.

Subfam. TUPAINOS.

I. Gén. TUPAIA, Raffl.

I. T. ferruginea, Raffl. t\& N. v. Visin.

(M. S. T.)

T'. ferruginea, Raffl, Linn. Trans. 13. p. 256; Horsff. Zost. Res. 3. fig.: id Zool. Java fig.: F. Cuv. Mamm II. fig. Press.; $S$. Miull Ver handl. 1. p. 163 pl. $26 \mathrm{f} .3$ pl. 27. fig. 7-10: Reich. Raubth. f. 451 .

Sorex glis, Diard, Duvancel, Asiat. Res. 14. pl. 9.

Hab. Culión, Paragua, Java, Sumatra, Borneo, Singapur, Malaca.

2. T. javanica, Horsf. t\& N. v. id.

(M. S. T.)

'T. javanica, Horsf., Zool. Res. 1824, fg. 3; S. Mïll Verh. 1. p. 165 pl. 26 fig. 4. Reich 451 .

T.. tana. Is. Geoff. Belang. Voy p. I05.

Sorex glis press, F. Cuv. 1121 , Mam. II. fig. Cerps on Banxing. Hab. Calamianes, Culion, Paragua, Borneo, Java, Sumatra.

\section{Fam. ERINACÉIDOS.}

1. Gén. ERINACEUS, L.

I. E. europæus, Lin. むq N. v. Erizo. Españ.x.

(M. S. T.)

\section{Fam. SORÍCIDOS.}

I. Gén, Crocidura, Wagler.

r. C. cærulescens, Shaz». $0^{\pi}$, N. v. Sang̃iô.

(M. S. T.)

Cr. cærulescens, Shaze. Gen. Zool. 1800; Wagner, Schreb. Saug. Suppl. 5. 1855, p. 5.51: Jerd. Mamm. of India, 1867 p. 53. pilorides, Shaw, Mus Lever. 1. p. 31. pl. 8. moschatus, Rob. Assam. p. 96. giganteus, I. Geoff. Dict. Plass. $1827,11$. p. 326.: indicus, p. E. Geoff. Ann. Mus. p. I83.; id. Mem. Mus. 1815; Reich. fig. 500: Blyth. et Auders P. Z. S. 1873 p. $23 \mathrm{I}$.

Hab. Luzón, Manila, India, Bombay.

2. C. murina, (Lin) $\bigcirc \curvearrowright$ N. v. Langam.

Sorex murinus, Lin. Schreb. Saug. $1892 ;$ Cantor, Jour. As. Soc. Beug. 1846; Blyth. J. As. S. B. 1875.

Hab. Luzón, Paragua, Java, Malaca, Cochinchina y China. 
3 C. luzoniensis, Ptrs. $\sigma \rho$ N. v. Bulilit.

('r. luzoniensis, Peters, M. B. Akad. Berlu! 1870, 35 p. 593.

C.r. inurinus, p. et myosurus, p. Auct.

Hab. I,uzón, Minila, Painpanga.

4. C. aibina, Blith. ơ N. v.

(M. S. T.)

Cr. albina, Blyth. J.As. L. Beng. 1 S50, 29 P. 87.; Symun. P. Z. S. 187,3 p. 615 .

Hab. China, Imuy.

5 C. Edwartsiana, Tit. N. v. Langam

('r. edwardsiana, Trt. Le Natural. 1.1880 p. 330. Hab. Joló.

6 C. aranea, Schrel. o* N. v. Musaraña.

(M. S. T.)

(. araneus, Schreb. (nec L.), Saug., 3, p. 379, pl. I60; «Musaraigne de 'T'erre," Daubenton, Mém. de l'Acad. des Sc., 17.56 , 1. 2I I, pl. 5, f. 2; «La Musette,» Buff., Quadr., 8, pl. Io, f. 1; E. Geoff., Ann. Mus., 1811, 17, p. 174, pl. 2, f. 2; 1) 1tzernoy. Mag. Zool., 1842 , p. is, pl. 38; Reich., 478-480; Blasius, Fauna Deutschl., 1858, p. I44, f. 94; E. Brandt, Bull. Nat. Moscou, 1868,2 , pl. 4, dents.

(.. russulus, Hermann, Zimm., Geog. Ges. 2, p. $3^{82}$.

C. musaranea, Cuv., Bp., F. Ital., pl. I9, f. 5, 6.

(. inodorus, (Savi), Sèlys, Micromamm., p. 34, 35.

(. fimbriatus, Wagler, Isis, 1832 , p. 54 (juv.).

( $\quad$ moschata, major, rufa, poliogastra, Wagler, Isis, 1832, p. 275, 1218 (Var.)

C. thoracica, (Savi), Bp., F. Ital., pl. r9, f. 7; Reich., f. 720 ) (Var.); $=965$ a. apud Blasius.

(.) pachyurus, Kuster, Isis, 1835, p. 79 (Var.).

C. priscus, Pomel (Musaraneus), C'at. Vert. foss. de l'Allier, 18.54 , araneus fossitis, $\mathrm{n}^{0}$ i 4 ; Gervais.

C. mauritanicus, Pomel, C. R. Ac. Sc. Paris, 42, 1856, p. 653. Hab. Europa, Francia, España.

Fam. TÁlPIDOS.

I Gén. MYGale, G. Cuv.

I M. pyrenaica, E. Gioff. ơ N. v. Desman.

(M. S. 'T.)

M. pyrenaïca, E. Geoff., Ann. Mus. 17, 1811, p. 193, pl. 4, f. 1-7; id.. Mém. Mus. 1, pl. I 5, f. 10-12, dents; Blainv., Ostéogr. Insect. p. 53, pl. 5, 9; Wran?r. S. S. 2, p. I(r); 5, 1). 568; Gervais, $M$. I. fig. p. 248; Trutat, Catal. Mainm. des Pyrénées (in Bu!l. Soc. H. N. Tou!ouse), p. 95, fig. en photograv. 1.

Hab. Europa, Francia. 


\section{Fam. CENTÉTIDOS.}

I. Gén. CENTETES, It.ı.

I. C. ecaudatus, Sikrcb. ON N. v. Centetes.

(N. S T.)

C. ccaudatus, Schreb., Sang. 3, 1778, p. 584, 11. 164; "Tanrec", Buff., Quadr. 12, p. 438, pl. 56; Gimel., Linn. Syst. Nat., E.d. 12, 1788,1 , p. I I7; Illig., Prodrom. 1811, p. I 24; Wagn., S. S. 2 , p. $55^{2} ; 5$, p. 583 ; Reich., f. $45^{6} ;$ Fitz.. S... t. 80; Gervas, M. I, p. 233 et 234 , go. crâne, deuts; Pollon et Van Dam, Reckerches, 1868,2 2" part., 1. 25; Jentink, Notes, l. c.. p. 144.

C. setosus, Desm. (nec Schreb., Gm.. Blainv. et Auct.), Mamm. p. I6r, n" 25I; Encycl. pl. 37, f. 2.

(. variegatus, Gray (nec E, Geoff.), 1836.

C. arnatus, Is. Geoff., Ann. Sc. Nat. $2^{e}$ Sér., 1837, S, p. 6); id.; Ma3. Zool. 1839, p. 17 et 31, pl. 2; Wagn., S. S. 2, p. $35 ; 5$ p. 584 .

Hab. Madagascar, Isla Mauricio.

\section{Orden $5 \cdot^{\circ}$ ROEDORES.}

\section{Fam. ESCIÚRIDOS.}

Subfam. ESCIURINOS.

I Gén. PTeromys, G. Cuv.

1. Pt. petaurista, Pall. $\sigma \circ$ N. v. Taguan.

(M. S. T.)

Pt. petaurista, Pallas, Miscell. p. 54. Scherb. (part). Saug. 4. p. 8rg pl. 214: G. Cuv. et Auc Jerd. Mamm. of. India 1867 p. $175 ;$ Gerv. M. 2. p. 296.

Taguan, p. Buff. Suppl. 3. p. I50. pl. 21.

Pt. oral, Gick. Calc. G. N.H. 1842 p. 401. pl. I1; Anders. A. aud Z. Res. 1878 p. 279.

Hab. Paragua, Ceilan, Himalaya, China.

2. Pt. philippensis, Elliot. var. $\circlearrowleft, \mathrm{N}$. v. id.

(M. S. T.)

Pt. Philippensis, Elliot, Madras Journ. 1839. p. 217. Hab. Paragua.

3. Pt. innornatus, Niall var. $\sigma \rho \mathrm{N}$. v Tapilac.

Pt. inornatus, Müll et Schleg.; Auder, Rescarch. p. 279. Hab. Paragua y Molucas. 
2 Gén. SCILROPTERUS, F. Cuv.

4 Sc melanotis, Gray. oo N. v: Alalacsin.

(M. S. T)

Sic. melanotis, Gr. P. Z. S. 1836. p. 88: Anders. Res. p. 292. Sc. melanopis, Mottley et Dihv., Cont. to Nat. H. of. Labuan and Borneo, 1855 p. 2.

Hab. Mindanao, Balabac.

5 Sc. pulverulentus, Gth. \& N. v. id.

(M. S. T.)

Sc. pulverulentus, Giinther. P. Z. S. 1873 P. 4I I pl. 38; Auder Res. p. 297.

Hab. Mindanao, Malaca.

3. Gén. AOSCÍURUS, I,at.

6 Ao. affinis, Roffl. ơ N. v. Alalacsin.

(M. S. T )

Sciurus bicolor, Sparr., Gothcb. Wet. Sevensk. Haudl. $1778-1$. p. 7o: Desm. Mam. 1820 p. 336 pl. 75 f. 3; Horef. Zool. Res. 1824 Wagner. S. S. $1843, \mathrm{pl} .2 \mathrm{I} 6$ : Auder, Auct. aud Zool. Rescarches, 1878 p. 215.

S. javensis, Zimm., Schreb. Saug. 1792 p. 781 .

S. affinis, Raffles, Trans. Linn. Soc. 1822,13 p. 259.

Hab. Calamianes, Culion, Singapur, Malaca, Célebes.

4 Gén HETEROSCÍURUS, TrT.

7 Het. prevostii, Dism $\emptyset$ N. v. Ardilla.

(M. S. 'T.)

Hab. Singapur, Bornè̀, Célebes.

8. Het philippinensis, Wat. $\delta ?$ N. v. Alalacsin. (M. S. T)

H. Philippinensis, Waterh. P. Z. S. 1839 P. I I7; Auders. Res. p. 255 .

Hab. Balabac, Mindanao.

9. Het. steerii, Gthr. $t \&$ N. v. Visin

(M. S. T.)

H. Steeri, Gïuther. P. Z. S. 1876 p. 735 pl. 59.

$H a b$. Paragua, Balabac.

10. Het. lateralis, Var. $\delta \hat{\mathrm{N}}$ v. id.

Hab. Samar, Paragua, Bohol, Culión, Calamianes.

5. Gén. nannosciurus, Trt.

1r. No an. exilis, Müll et Schl t. N. v. Vising. (I. S T)

Sciurus exilis, Mäll et Schlec., Tijd. Natur. Ges. $1838-39$, p. 148. Wagner, S. S. 3 p. 208.

Hab. Basilan, Mindanao, Burneo, Sumatra, Malaca. 


\section{Gén. FUNANBULLS, Less.}

12. F. vittatus, Raff. \& \& N. v. id.

(M. S. T.;

F. vittatus, Raffles, Trans. L. S. 1822,13 , p. 259; F. Cuv.,

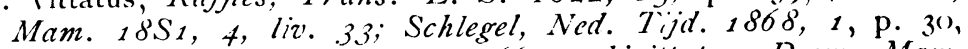
pl. 2, f. 4; Auderson, Res., p. 266. a. bivittatus, Desm., Mam. 1920, Suppl. p. 5+3; «toupaye», Less., Manuel, 1827, p. $23^{8 .}$ Hab. Samar, Borneo, Sumatra.

7. Gén. SCIURUS, Lin.

13. Se. vulgaris, Lin. \& N. v. Ardilla comun. (M. S. T.)

s. vulgaris, L., Syst. Net. éd. 12, p. 86, I; F. Cuv. Mam. 2, liv. 22; Buffon, H. N. 7, pl. 32-35; Blasius, Gauna Deuts. 1857, p. 272 , f. 153,154 .

s. Var. alba, Erxleb., Gerv., M. 1, fig. p. 305.

s. Var. niger, Erxleb., Mam. p. 415.

S. alpinus, F. Cuv., Mam. 2, liv. 24.

s. italicus, Bp., Faun. Ital. fig. 20.

Hab. Europa, España.... etc.

\section{Fam. MIÓXIDOS.}

I. Gén. Eliomys, Wagner.

I E nitela, Pall. $\hat{q}, \mathrm{~N}$ v Moscardina.

(M. S. T.)

E. nitela, Pallas. Nov. Sp. Quaarr. Glir., 1778 , p. 88; Buff., H. N. 8, pl. 24, 25; Schreb., Sä̈g. 3, p. 833, pl. 226; F. Cuv., Mam. liv. 4o, fig., id., Dents des Mam., pl. 58; Gerv., Mam. 1, p. 373, fig. dents.

E. quercinus, L., Sy's. Nat. Ed. 12, p. 84 ; Blasius, Fauna Deuts. p. 289.

Hab. Europa, España....

\section{Fam. MÚRIDOS.}

Subfam. FLEOMINOS.

I. Gén PHL eOMYS, Wat.

1. Fh. cumingii, Wat. $\delta \&$ N. v. Parret.

(M. S. T)

Ph. cumingii, Waterh. P. Z. S. 1839 p. 108; Syd. et Sonley., Voy de la Bonite, 1841 . pl. 7, 8; Gerv. Mam. 1. p. 399.

Hab. Luzón, N. ${ }^{a}$ Ecija, Rosales, Laguna, Mahaihai.

2. $\mathrm{Ph}$. albayensis, $\delta \&$ N. v. Parrot.

(M. S. T)

- Hab. Luzón, Albay, Mindoro. 
2 Gén. Bandicota, Gray.

3 B3. gigantea. Hurdte o o N. v lampong.

(M. S. 'T)

Echimys gigantcus, Harda. Trans. Linn. Soc. 1804 p. 306 pl. I Grav. P. Z. S. 1832 ; Auders. l. c. p. 232 pl. 14.

Ech. baudicota, (Bechst) Blyth. Cat. 1869 p. i i 2: Jord. Mam. India 1867.

Arv. setifer, Horsf. Zool. Res. núm. 8.

:Perchal, Buff. Suppl. p. 276 pl. 69.; F. Cuv. Mam. 61.

Hab. Luzón, Cavite, Alfonso, Cagayan, Pamplona.

3 Gén. EPIMYS, Trt.

4 Ep. decumanus, Pall. Ö N. v Ulag.

(M. S ' I

Mus decumanus, Pall. Non. Sp. Glir 1778 p. 9r: Blas.. Fauna 1)cuts. $18.5 \mathrm{~T} \cdot \mathrm{1}$. 313.

Hab. Europa, Asia, Africa, América, Oceania, ¿Filipinas?

4. Gén. I.EGGADA, Gray.

5 L Everetti Githr.

Mus everitti, Günther, P. Z. S. 1879. p. 75 .

Hab. Mindanao.

5 Gén. MUS, Lin.

6. M. musculus, Lin. is N. v. Bubuit

(M. S. ' $1 ;$

M. musculus, L., Sy'st. Nat. 12, 1766, p. 83; Pall., Glir., p. 45: Buff., H. N., 7, p. 309, pl. 39; Suppl. 3. p. I8 1, pl. 3(); 7 , pl. 40; Schreb. Saüg. 4, pl. 181; Blasius, Fanna 1)cuts. 18.57, p. 320 .

M. mucculus domesticus, Albert Magnus.

M. minor, Klein: Sorex, Brisson.

M. islandicus, Thienem., Nat. Bem. p. 153.

M. incertus, Savi; flavescens, Fischer. mollisimus, Dchne.

M. poschiavinus, Fatio, Fanue de Suisse, 1870. 1. pl. 7 (Var. Melan.)

M. azoricus, Schinz. Synops. Mam. 2, 1875, p. 161 (Var Melan.)

M. algirus, Pomel, C. R. Acad. Sc. Paris, 1856,42, P. 6.5t.

M. Hayi, Waterh., Phil. Mag. 1838, p. 597.

M. Alleni, Waterh., Phil. Mas., l. c., p. 597.

M. Abbottii, Waterh. Phil. Mag., l. c., p. 597.

M. rama, Blyth.

M. adelaidensis, Gray', Gray's Journ. 1. n 86.

M. taitiensis, Fitzang. et Zelebor, Reis. Novara, 1868 .

M. vitiensis, Pcale, U. S. Expl. Exp., 1848,8 , p. 49.

M. brevirostris, Waterh., P. Z. S. 1835 , p. 119. $H a b$. Europa, Asia, Filipinas. 
7. $M$ rattus, Lin $\delta ? ~ N$ v. Dagâ.

(M. S. 'T.)

11. rattus, L... Sys. Nat. 1766, 1, p. 79; Pall., G!ir., p. 93; Buff. H. N. 7, p. 278 , pl. 28t: Schreb. Siär. t, pl. 179; Giebel, Odont. p. 47; Blasius, Fauna Deuts. 1857 , p. 317.

M. caruleus, Lesson, Tabl. Régne An:m., $18+2$.

M. latipes, Bennet, P. Z. S., 1833, p. 89.

M. insularis, Walerh., Voy. Beagle, p. 35.

M. alexandrinus, E. Geoff., Descr. Egypt. pl. 5, f. r; Blasius, Fauna Deuts. p. $316 ; A$. de l" sle, Ann. Sc. Nat. 1865,4 , p. I73; Lache, Explor. Algér. Mam. p. 1 I3, p!. 5, f. I.

M. tectorum, Savi, Nuov. Giornal, 1825; Bonap., Faun. Ital. fasc. 3 et 16, fig.

M. flaviventris, Brants, Muiz. 182$\rceil,$ p. 108.

M. flavigaster, Heuglin, Nov. Act. Sic. Leop. 1861.28 , p. 4, 1). 2 , f. 2 .

M. setosus, Lund, Blik. Bras. Dyr. 3, p. 277.

M. leucogaster, Pictet, Mém. Soc. H. N. Genéve, $184^{1}$, p. 153.

M. Jacobiax, Waterh., Voy. Beagle, p. 35.

M. caledonicus, Wagner, S. S. 4 .

M. nova-zelandia, Buller, Proc. N. Ze:l, Inst. 1871 , 3, p. 1' fig.

M. exulans, Peale, U. S. Expl. Exp., 1878,8 , p. 47. Hab. en todas las Islas.

S. M. peguensis, Blyih. ơ N v. Utot

M. peguensis, Blyth, J. A. S. B. 1859 . p. 295.; C.ut. of. Matm. Burmo, 1875 .

Hab. Luzón, Laguna, Pegou.

6 Gén. MICROMYS, DHNE.

9. M castaneus, $W_{i l^{2}}$. $0^{\circ} \varrho$ N. v. Daguis.

(M. S. T.)

Mus castaneus, Waterh. A. N. H. 1873. p. I34. Hab. Luzón, Manila, Cébú.

ı. IN. minutus, Pall on

(M S. T.)

M. minutus, Pall. Glir., 1778; p. 96, 345, pl. 24 b; Schreb. Sä̈g. 4, p. 660, pl. I83; Gloger, Nov. Acta Leop. 14, p. 358, pl. 23; Blasius, Fauna Deuts., p. 326; Schlegel, Zoogd, Nederl. 1857, pl. 6.

M. campestris, E. Geoff. et F. Cuv., Mam. liv. 64, Ag.

M. messorius, Shaw, Gen. Zool. 2, p. 62.

M. pumilus et messorius, E. Geoff. et F. Cuv., l. c., liv. 64 , fig.

M. soricinus, parzulus et pendulinus, Herm., Obser. Zool., 1, p. $57-62$.

M. pratensis, Ochskay, Nov. Acta Leop., 1831, p. 243.

M. agilis, Dehne, Hoflóssnitz, $18+1,16$. H.x. Europa, Siberia, China. 
Fam. HISTRÍCIDOS.

I. Gén. ACanthion, F. Cuv.

I. A. pumila, Gthv. $\delta \& \mathrm{~N}$ v. Durian

A. pumila, Günther A. N. H., 1879. p. ıо6.

Hab. Paragua, Balabac.

Fam. CÁVIDOS.

I Gén. Cavia, Pall.

I. Cavia cobaya, Murcgv. $d \hat{q}$ N. v. Cui.

(M. S. T.)

Hab. Anérica, Filipinas (introd.) Manila.

Fam. LEPÓRIDOS.

I. Gén LEPUS, Lin.

I. L. variabilis, Pall. ơ N. v. Liebre.

(M. S. T.)

Hab. Europa, Asia, etc.

2. Gén. ORYCTOlaGUS, Lilly.

2 Or. cunículus. $(\operatorname{Lin}) . \delta \& \mathrm{~N}, \mathbf{v}$ Conejo

Hab. Europa, etc. Filipinas (introd. domest.)

\title{
Orden 6. ${ }^{\circ}$ CARNÍVOROS.
}

\section{Subord. FISIfPEDOS.}

\author{
Fam. ÚRSIDOS. \\ I Gén. URSUS, L N.
}

I. U. aretos, Lin. a'ar. Py'cnaicus. t N. v. Oso pardo. (M. S. T)

Ours brun des Alpes, Buffon, H. N. viii. pp. 24, 86, 61; Cuvier, Oss. Foss. iv. t. 22 . f. I, 2.

Ours brun des Pyrénées, Cuv. Oss. Foss. iv. p. 332.

Ours des Asturies, fœem. (U. arctos.) De Blainv. Ostéogr. Ursus,

t. 3 (skeleton), t. 7 (skull $ф$, adlult.)

Ursus arctos, Schreb. t. (from Buffon).

Ursus pyrenaicus, F. Cuv. Mamm. Lithogr. xlv. t. (young).

Ursus arctos pyrenaicus, Gray, P. Z. S. 1864 , p. 683.

Hab. Europa, Asia... España. 


\section{Fam. MUSTÉLIDOS.}

ז. Gén. MELES, Storr,

I. IM. taxus, Bocial. o" N. v. 'Tejon.

(M. S. T.)

Crsus taxus, Blumenb. Handl. p. ro.

U. meles, Linn. Syst. Nat. i. p. 70; Schreb. Sängeth. p. 5 I6.

Meles taxus, Boddaert, Elench. i. p. 80; Schreb. Säugeth. t. I +2;

Blainv. Ostéogr. Subursus, t. 2 (skeleton), 6 (skull), 9 (teeth);

Gray, P. Z. S. 186.5 , p. × 39; 1 868; p. 207.

M. europaus, Desn. N. Dict. Hist. Nat. iii. p. 465.

M. vulgaris, Desm. Mamm. p. 173 .

Taxus vulgaris, Tiedm. Zool. i. p. 376.

Blaireau, Buffon, $H . N$. vii. p. io4, t. 7,8 .

Blaireau d'Europe, Cuvier, $R$. $A$. i. p. I 45.

Common Badger, Penne Brit. Zool. p. 3o; Bell, Brit. Quad. P. I 22.

Hab. Europa, Asia, España.

2. Gén. MYDAUS, F. Cuv.

2. M. meliceps, F. Cuv, $O^{*}, \mathrm{~N}$ N. v. Pantoc.

(M. S. T.)

M. meliceps, F. Cuv. Mamm. Lith. 1825 pl. 159; Horsf., Zosl.

Res. Java. 1824 pl. 3.

Müll, Veraudl. Nat. Ned. Ov. 1835; Gray. P. Z. S. 1865 p. 142.

Mephitis javanensis et javanicus, Desm. Mam. 1820 p. 147.

Hab. Paragua, Java, Sumatra, Malaca.

3. Gén. MUStela, Nils.

3. M. erminea, Lin. O' N. v. Armiño.

(M. S. T.)

Mustela erminea, Linn. S. N. i. p. 68; Nilsson, Skand. Faun. p. I 57; Illum. Fig. t. I 2, Gray, P. $Z$. S. 1855 , p. I I .

Putorius erminea, Owen, Brit. Foss. Mamm. p. iा 6 , f. 4o, $4^{\mathrm{I}}$, 42 , (skuli).

M. ermineum, Pallas, Zoogr. i. p. 90; Buffon, H. N. vii. t. 29 . f. 2, t. 3 I.

Stoat or Eirmine, Penn. B. Zool. i. pp. 89,67, f. i 8. Hab. Europa, Francia.

4. M. vulgaris, Bris., $\sigma$ N. v. Comadreja.

Mustela vulgaris, Briss. R. A. p. 241; Erxl. M. p. 47ı; Gray, P. Z. S. 1865 , p. I I 3 ; Blainv. Ostéogr. Mustela, t. 7 (skull), t. I 5 (teeth).

M. gale, Pallas, Zoogr. p. 194 (albino in winter).

M. nivalis, Linn. Act. Suec. vi. t. $8 ; S . N$. p. 169.

Common Weasel, Penn. B. Z. i. p. 95 I, t. 7. f. I7. Hab. Europa... Francia. 
4. Gén. LUTRA, Erxl.

5. Lutra vulgaris, Erxl. t $\mathrm{N}$ v. Nutria

(M. S. T.)

Lutra vulgaris, Erxl. Mamm. p. 488; Nilsson, Illum. Fig. t. 20; Bell, Brit. Quad. p. 129, f. 4; Blainv. Osteggr. Mustela, t. 8. (skull), t. 5 (skeloton), t. I3 (teeth); Bonap. Icon. t. ; Gray, P. Z. S. 1865 , p. I 26.

Mustela, lutra, Limn. S. N. i. p. 66; Retz. F. p. 18.

Viverra lutra, Linn. F. S. p. г 2; Pall.ss, Zoogr. i. p. 76.

La loutre, $B u f f . H . N$. vii. p. I 34, t. 2 , xiii. p. 325, t. 45 .

Otter, Penn. B. Z. i. p. 92, t. 8. f. I 9.

Hab. Europa...

6 I. sumatrana, Gray. ơ N. v.

(M. S, T.)

L. sumatrana, Gray. P. Z. S. $1865:$ Anders. l. c. 1878 p. 203 pl. Io.

Hab. Paragua, Malaca, Sumatra.

\section{Fam. CÁNIDOS.}

\section{Gén. CANIS, Liv.}

I. Canis japonicus, Tem. $\sigma$ N. v Aso.

(M S. T.) Hab. Japón.

2. Canis molossus, Lin $\sigma$ N. v. Mastin.

Hab. Europa.

3 Canis terræ novæ. ơ N. v. P. de T. $n$.

Hab. en Terra-nova.

4. Canis pollokensis. N. v. Irô.

Hab. Mindanao.

2. Gén. VULPES, Briss.

4. Vulpes vulgaris, Briss. I N. v. Zorra.

(M. S. T.)

V. vulgaris, Briss., Regn. Anim., $175^{\circ}$, p. 239.

V. vulpes, L., S. Nat., 1766,1, p. 59; Blainv., Ost., Canis, pl. 4.

V. alopex, L., l. c., p. 59.

V. crucigera, Briss., R. A., p. 240: Gester, Quadr., fig. p. 9); Alirov., Quadr. Digit., f. p. 222.

V. japonica, Gray, P. Z. S., 1868 , p. 517.

V. hoole, Swinh., P. Z. S., 1870 , p. 631.

V. melanogaster, Bp., Faune Ital., 1835 , fig.

V. lineiventer, Swinh., P.Z.S., 1870, p. $63 \mathrm{I}$. Hab. Europa, Francia, China, Formosa. 


\section{Fam. VIVÉRRIDOS.}

Subfam. ARC'IIC'ININOS.

I Gén. AR'TICls, Tw.

1. Arctictis binturung, Reffe. $0^{*}$ N. v. Mirj.

(N. S. T.)

Viverra binturong, Raffles, Linn. Trans. 12 p. 253.

Arc. binturong, Tem. Monogr. 2. p. 308.; Gray. P. Z. S. 1867

p. 525: Sternd. Jud. Mam. 1894 p. 221.

Arc. peniciliatus, Tem. Monogr. 2. t. 62: Mïll, Zood. iud. Arch. p. 32.

Paradoxurus albifrons, F. Cuv., Mem. Mus. 9. p. 44. pl. 4.; Manm. Sith. pl. 201. Deuts des Mam. p. Io4. pl. 34; Valeuc. Ann. Sc. Nat. 1925, 4 p. 57. pl. 1.

Ictides ater, F. Cuv. Mam. Lith. pl. 202.

Ictides aureus, F. Cuv. Mem. Mus. 9 p. 47. Tem. Mongr. Mam. 2. p. 3o8.

Hab. en Filipinas Java, Sumatra, Malaca.

2. Gén. PARADOXURUS, F. Cuv.

2. P philippensis, ơ $\bigcirc$ N. v. Alamid. Miló.

(M. S. T.)

Martes philippensis, Cam. Phil. Trans. 24 p. 2.204.

Par. zeylanicus, (part) Gray. Cat. Mamm. B. M. p. 55.

Par. philippensis, Temm. Monogr. 2. Esquis Z. p. г 20: Gray.

P. Z. S. 1864 p. 537. Ogilby, Zonl. Journ. 4, p. 300.

Par. aurtus, Wat. Cat. Zool. Soc.

Hab. Luzón, Laguná, 'Tayabas, Cagayan, Ilocos, Leyte.

3. P. dubius, Gray. O $\bigcirc$ var. N. v. Alamid y Musang. (M. S.T.) Hab. en Luzón, Cagayan, Java.

4. P. nigrifrons, Gray. O $0^{3} \mathrm{O}$ var. N. v. Mungao.

(M. S. T.)

P. hermaphrodyta, Pallas, Schreb. Sait., 1777, p. 426; Gray P. Z. S., i332, p. 69 .

P. nigra, Desm., Mam., 1820, p. 208; Buff. Suppl., 3, pl. +7.

P. typus, F. Chov., Mam. Lith., 1821,2 , livr. 24; 7 emm., Monogr. Mam., 2, p. 315; Blanv, Ost., pl. 2.

P. Pallasii, Otto, Noz. Act. Leop., 17, p. Io7, pl, 72, 73.

¿P. prehensilis, Balano, Bull. Soc. Phil., i 816 ; Sclater, P. Z. S., 1877, pl. 71 .

P. felinus, Wagizer, Screb. Saug., 2, p. 349.

P. nubia, F. Cur., Mam., 3. liv. 75.

P. nigrifons, Gray, Ill. Ind. Zoo!., pl.; P. Z. S., 1864, p. 535.

P. macrorlus, Grayy, P. Z. S., $186+$ P. 538, fig. crâne.

P. quadriscriptus, Hodge., Aim. Nat. Hist., 1855, 16, p. 106.

P. strictis, Hoots., lac. cit., p. $10_{5}$.

P. fascita, Desm., Mam., 1820 , p. 209; Gray, P. Z. S., 1864 , p. $53^{6}$; Alst n, P. Z. S., 1879 , p. 666. 
P. musanga, Raffles, Linn. Trans., 13,1812, p. 255; Sterndale, Mam. Ind., 188 , p. 216.

P. typus, orar. sumatranus, Fischer, Synops., 1829, p. I59.

P. Geoffroyi, Fischer, l. c., p. $17 \mathrm{I}$.

P. musanga, var. javanica, Horsfield, Jai'a, pl.; Temm., Monogr., 2, p. 3I7, pl. 53, 54 .

P. "Musang», Marsden, Sumatra, p. I Io, pl. i 2.

P. setosus, Hombr. et Jacq., Voy. Astrol,, Zool., 3, p. 25. pl.

P. Finlaysonii, Gray P. Z. S., 1832, p. 68.

P. Pallasii, Gray (nec Otto), P. Z. S., 1832, p. 67; Ill. Ind. Zool., pl.

P. albifrons, Bennet (nec Cuv.)

P. quinquelineatus et musangoüdes, Gray, Ann. Nat. Hist., 1837 , 1). 579 .

P. dubius, Gray, P. Z. S., 1832, p. 66.

P. crassiceps, Pucheran, Rev. et Mag. Zool., 1855, p. 393. Hab. Luzón, Gagayan, India.

5. P. Crossii, Gray. ơ ${ }_{k}$ var. N. v. Galong, Lampog.

P. Grayi, Bennet, P. Z. S., 1835, p. I8; Gray, P. Z. S., 1831 , p. $95 ; 1864$, p. 541; Amm. Nat. Hist., 187t, 17, P. 24.: Sterndale, Ind. Mam., 1884 , 1). 2 i 7.

P. nipalensis, Hodgs., As. Res. Beng., 1836,19, p. 76.

?. L.urvatus et bondar, p., Femm. Monog. 2, 11,55 , fig. I-t.

P. loniger, Hodgs., Gray, P. Z. S., $186+$, p. $5+2$.

P. leucepus, Orilby', Zool. Journ., 4, p. 303, pl. 35 .

P. Crossii, Gsay, P. Z. S., 1832, p. 67; Ill., Ind. Zool., 2, pl. 7 ; P. Z. S., P. Z. S., 1864 , p. 533, fig. cràne.

P. musanga, var., Esq. Zool. 1853 , p. 120.

P. leucomysiax, Gray, Mag. Nat. Hist., $1 \rho_{37}, 1$, p. 579; P. Z. S., 1864 , p. 540; Temm. Monogr. Mam., 2, p. 325; pl. $6+$, f. 4-6, crâne.

P. auratus, Biainv., Os/. Viverra, pl. 12, deuts.

P. «Amblyodon doré», Jourdan, Amn. Si. Vat., 18.37, 8, p. 372.

P. Jourdanii, Gray, Mag. Nat. Hist., $1837,1,1.579$.

P. Ogilbii, Fraser, Zool. typ., pl.

P. lencocephalus, Gray, Voy. of Samarang; 1850 .

P. 1:hilippensis, p., Schinz, Syzops., p. 357 .

P. Crossii, Gray. Proc. Zool. Soc. 2. p. 67. 1832.; Illustr. Init. Zool.

P. Musanga, var. Tem. Esq. Zool. p. I zi).

Pagkma Crossii, Gray. Cat. Mam. B. M. P. 54. Hab. Luzón, Ilocos Norte, India, Borren.

\section{3. (ín. VIVERRA, IAN.}

6. V. tangalunga, Gray. o. N. v. Musang.

(AI. S. T.)

V. tangalunga, Gray. P. Z. S. 1832 1). 63: $186+11.512$. Cat. Mam. B. M. p. 48. Cantor Mrmm,; Horif. Cat. ilus. Ind. Honse. p. 57.

V. zibetha, Raffles, Lin. Trans. 13 P. 23 i.; F. Cur. Mam. Luthog. 
Mab. Luzón, Cavite, Cagayan, Nueva-kxija, Borneo, Su:a:tra, Célebes.

7. V. zibetha Lin. 0 \% N. v. Mundo.

Vivera zibetha, Linn. S. N. i. p. 65; Gray, Illust. Ind. Zool.

ii. t. 5 ; Proc. Zool. Soc. 1832 , p. 63; Cat. Mamm. B. M.

p. $47 ;$ P. Z. S. 1864 , p. 512; Gerrard, Cat. Ost. B. M.

p. 71 ; Schinz, Sinn. Mamm. i. 362; Horsfield, Cat. Mus. India House, p. 54.

Meles zibethica, Iinn. S. N. 1756 .

Viverra undulata, Gray, Spic. Zool. t. 8.

Vivera civettoides, V. melanurus, V. orientalis, Hodgson, J. Asiatic Snc. Bengal, x. gog.

Zibet, Buffon. ix. 299, t. 3 r. ashtoni, Swinh. P. Z. S. 1870. Hab. Paragua, Negros, Panay, Formosa, China, India.

\section{Gén. VIVERRICULA, HODg.}

8. Viverricula malacensis, Cantor. $\sigma \circ \mathrm{N}$. v Musang. (M S. T.)

Viverra malaccensis, Cantor, C'at. Mamm. Malay. p. 29; Gray, P. Z.S. 1864, p. $5 \mathrm{I} 3$.

V. malaccensis, Gmelin, S. N. p. 92 (from Sonn.); Gray, Cat. Mamm. 13. M. p. 48; Gerraxd, Cat. Ost. B. M. p. 7o.

V. gunda, Hamil!on Bucnaman, Icon.

V. rasse, Horsf. Zool. Java, t.; P. Z. S. ii. (1832) p. 23; Schinz, Sin. Mamm. i. p. 362.

V. indica, Geoff. M. S.: Fischer, Syn. Mamm. p. I7ı; Desm. Mamm. p. 2 Io; Geroais, Mag. Zool. I835, p. Io, t. Iэ; Horst. P. Z. S. ii. $(1832)$ p. 23.

:V. bengalensis, Gray, Illust. Ind. Zool. i. t. 4.

Viverra leveriana, Shaw, Mus. Lever. t. $2 \mathrm{I}$.

Genetta manilensis, Eydoux.

(i. indica, Lesson, Man. I74.

G. rasse, F. Cuvier, Mamm. Lithogr. t.

Civette de Malacca, Sonnerat, Voy. ii. I 44, t. 91.

Viverricula indica, Hodgson, Journ. Asia., Soc. Beng. x. 1. 9:09. Hab. Luzon, Cagayan, Java, Madras, Sumatra.

5. Gén. Herpestes, Illig.

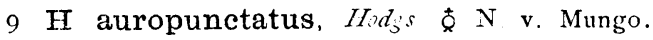

(M. S. T.

H. auropunctatus, Hodgs., J. As. S. Beng., 1836, 5, p. 235; Anderson, Anat. and Zool. Res. Yunnan, 1878 , p. i $72 .$, pl. 11 . fig. 11, 12; Sternd:le, Ind. Mim., 1884 , p. 225.

H. nepalensis, Gray, Mag. Nat. Hist, 1836, p. 578 .

H. javanicus, Blyth, J.As. S., 1852 , p. 349 .

H. microcephatus, Temm., Esq. Zool. côte de Guinèe, 1853 , p. I I 3 .

?H. Edyyarusii, Geoff.

H. persicus, Crray, P. Z. S., 1864 , p. 554; Anderson, l. c., p. I $7+$, pl. 9 .

H. pallipes, Blyth. J. As. S., 1845,14, p. 346. 
H. griseus, Hutton.

Hab. Bengala, Malaca.

\section{Fam. FÉLIDOS.}

1. Gén. CYNailurus, Wagi.er.

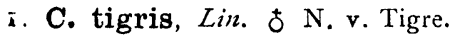

(M. S. 'T.)

F. tigris, L., Syst. Nat., 1766,1 , p. 6r; Schreb. Saug., 3, p. 38 I, pl. 98, 98 a; Elliot, Monogr. Felida, 1883 , pl. 3; Sterndale, Ind. Mamm., 1887 , p. 161.

T. regalis, Gray, P, Z. S., 1867 , p. 263.

'T. sondaïca, Fitzing., Sitc. Ak. Wiss. Wien, 1868,58, p. 34.

T. longipilis, Fitzing., l. c.. p. 35.

Hab. Asia, Java, China, Sumatra, Corea.

2. C macroscelis, Griy. \& N. v. Rinaulahau.

(M. S. T.)

C. Diardi, Desmoul., Dict. Class. H. Nat.. 1823, 3, p. 495; Elliot, Mongrr. Felida, 1883 , pl. 8; Stern., Ind. Mam., 1884 , fig. p. I 86.

C. macroscelis, Gray, P. Z. S., 1867, p. 266. fig. 3, crâne.

C. nebulosa, $H$. Simith.

C. macrosceloïles, Hodgs.

C. brachyurus, Stuinkoe, P. Z. S., 1862, p. 352, pl. 43.

Hab. Asia, China, Malaca, Sumatra, Java, Borneo.

2. Gén. FELIS, I،in.

3. Felis viverrin $\imath$, Binnt $\mathrm{N}$ v. Tarai, wagati

(M. S. T.)

F. viverrina, Bennet, P. Z. S., 1833, p. 68; Elliot, Monagr. Felide, $18^{8} 83$, pl. 22; Sternd., Ind. Mam., 1884 , p. 187, Jig. crane.

F. viverriceps, ilodgson.

F. himalayana, Jardine.

F. celidosaster, Gray (nec Temm.)

F. Bennestii, Gray. P. Z. S., 1867 , p. 268, fig. 5 .

Hab. Chini.l, Formosa, 'Tungquin, Malaca.

4 F. javanensis, Horsf. $\circlearrowleft \circ$ N. v. Singa!on.

(M. S T.

F. javanensis, Horsf. Zool. Res. 1924.; Elliot. Monoso. Fel.de $1883 \mathrm{pl} .28$.

F. sumatrana, Horsf. l. c.

F. undat', (Desm.) Less. Man. de Mam. 1827 p. 188.

F. Diardi, Cuw. (nec Desmoul.), Oss. Foss., 4, p. 437.

F. minuta, Temm., Monogr. Mam., 1. p. i $z^{\circ}$.

F. chinensis, Gray, Mag. N.H., $1837 ;$ P. Z.S., 1857, p. 274, 400 .

F. servalinus, Gray', (nec Ogilby), P. Z. S., 1857 , p. 401.

F. Reeresii, Gray.

F. Smithii et angulifera, Fitzinger. 
F. Herschelii, Grav, Cat. Carn. Mam., 1869 , p. 28.

F. bengalensis, p., Anderson, An. and Zool. Res. Yunnan, 1878, p. 162.

Hab. Panay, Iloilo, Negros, Cápiz, China, Jara, Borneo, Sumatra.

5. F. domesticus, Briss. $\sigma \rho$ N. v. Pusa, Curring.

(M.. S T.)

F. domestica, Briss., Quadrup., p. 191; Regn. Anim., 1756, p. I64; Blasius, Faun. Deuts., 1857, p. 167, f. ro4-105; Elliot, Monogr. Felida, 1883 , pl. 42.

F. maniculata, p., Fitzinger, 1869 .

Hab. Filipinas, Europa, etc. cosmopolita.

6. F. angorensis. Briss. ơ N. v. G de Angola.

(M. S T:)

F. sinensis, Fisch.

Hab. Asia menor, China.

\section{Orden $77^{\circ}$ PINNÍPEDOS.}

Fam. TRIQUÉQUIDOS.

1. Gén. Trichechus, Gray.

1. Trichechus rosmarus, Sihr. (colmillos). $\mathrm{N}$ v. Morsa. Hab. Pacífico, Islas Batanes.

(M. S. T.)

\section{Orden 8.'. SIRENIOS.}

\section{Fam. HALICÓRIDOS.}

I. Gén. Halicore, Illig.

I. Halicore dugong, Lin. N. v. Dugon.

Hab. Oc. Indico, Mindanao.

\section{Orden 9. ${ }^{\circ}$ CETÁCEOS.}

Fam. BALÉNIDOS.

1. Gén. BALAENA, LiN.

1. Balæna australis, Desm. (barbas). N v. Ballena.

(M. S. T.)

Hab. Mindanao, Dinagat, N." Zelandia. 


\title{
Fam. BALENOPTÉRIDOS.
}

1. Gén. balemoptera, Gray.

1. B. Swinhcei, Gray. (cílélra). N. v.

(MI. S ' '.; Hab. Batanes, Joló, Formosa.

2. B. mindorensis.

Hab. Minduro.

\section{Fam. CATODÓNTIDOS.}

1. Gén. Catodon, Gray.

I C. macrocephalus Gray (maxilur inf.)

Hab. Batanes, Marianas, Mindanao, Mares tropicales.

\section{Fam. DELFÍNIDOS.}

I. Gén. StenO, Gray.

I S. chinensis, Osbeck.

Hab. Formosa, China.

2. Gén. Delphinus, Gray.

2. D. longirostris, Gray ơ jov. N. v.?

Hab. Japón, Marianas, Batanes.

\section{Orden IO. $^{\circ}$ ARTIDACTILOS.-(Rumiantes.)}

\section{Subord. GAVIGORNIOS.}

\author{
Fam. BÓVIDOS.
}

I. Gén. BOS, Lin.

r. B. taurus, Gray. $\mathrm{O}^{\star}$ N. v. Toro.

Hab. (introd.) en Filipinas.

2. Gen. Bubalus, H. Smitr.

2. B. carabao. $\sigma$ N. v. Carabao.

$H a b$. en todas las Islas.

3. B. mainitensis, $H$. N. v. Cimarron.

Hab. Mainit, Mindanao. 
4 B. mindorensis. $\sigma \circ \mathrm{N}$ v. Tamarao.

(M. S. T.)

Hab. Mindoro, Célebes.

Fam. ANTILÓPIDOS.

I. Gén. RUPICAPRA.

I R. tragus, Gray. $\sigma$ N. v. Gamuza.

(M. S. T.)

Hab. Alpes Europeos.

Fam. CÁPRIDOS.

I Gén. hircus, Gray.

r. H. ægagrus, Gray. $\sigma: 0$ N. v. Cambing.

(M. S T.)

Hab. (domesticado introd.) en Filipinas.

\section{Fam. ÓVIDOS. \\ I. Gén. OVIS, Gray.}

I. O. aries, Gray. $\sigma \rho \mathbf{N}$ v. Tupa

(M. S T.)

Hab. Filipinas (introd.)

\section{Suborden GAPREOLOS.}

Fam. ÁlCIDOS.

1. Gén. AlCES.

I. A. malkis, Gray. $\sigma \circ$ (cabeza N v. Elk.

(Ad S T.)

Hab. Norte Europa y N. América, Canadá.

Fam. CÉRVIDOS.

1. Gén. Strongyioceros, Gray.

I, S. canadensis, Gray. $\sigma$ (cabeza) N v. Wapiti.

Hab. Norte América, Canadá.

2. Gén. PSEUdixis, Gray.

2. Ps. Tunquinen 3 is, $o^{\prime}$

Hab. 'Tunquin. 
3. Ps. soloensis, Hétute.

El sábio naturalista P. Heude en sus Etudes sur les cerfs des Philippines, fundándose en los caracteres craneales y oflontológicos enumera y clasifica los ciervos de Filipinas de la manera siguiente.

4. Gén. Sikelaphus, i.

4 S. soloensis, $I I$.

Hab. Joló.

3. Gén. USSA, Ir.

5. U. Gorrichant:s, $H$. N. v. Usá.

(M. S. T.

Hab. en Batangan, Luzón.

6. U. Barandanus, $H, \mathrm{~N}, \mathbf{\text { v. iđ。 }}$

(N. S. T.)

Hab. Mindoro.

7. U. crassicorms, $H . \mathrm{N} . \mathrm{v}$. id.

IM. S T.;

Hab. Cebú.

8. U. Francianus, $H . N$. v. id.

(M S. T)

Hab. Mindanao, Mati.

9 U. Nublanus, $H$. N. v. id.

(M S. T

Hab. La Laguna, Luzón.

1o U. Tuasoninus, If. N. v. id.

(M S. T.)

Hab. Batangas, Luzón.

11. U. spatharius, $H_{\bullet}$ む N. v. Usá.

Hab. La Laguna, Luzón.

12. U. Ramosianus, $H$. N. v. id.

Hab. N. ${ }^{a}$ Ecija, Luzón.

13. U. Ambrosianus, $H . N$. v. id.

Hab. N." Ecija, Luzón.

14. U. Macarianus, II. \& N. v. id.

Hab. N. Ecija, Luzón. 
15. U. Elorzanus, $H . \sigma^{*} \mathrm{~N}$. v. id.

Hab. Bataan, Luzón.

6. U. Garcianus, $H . O^{\prime}$ N. v. Usá.

Hab. N. ${ }^{a}$ Ecija, Luzón.

17. U. Guixoteanus, $I H . \sigma N$ N. v. id.

Hab. Batangas, Luzón.

I8. U. Rosarianus, H. N. v. id.

Hab. N. ${ }^{a}$ Ecija.

19 U. nigricans, $H . O^{*}$ N. v. Usá.

Hab. Jala-Jala, Laguna, Luzón.

2o. U Maraisianus, $H . \sigma N$ N. v. id.

Hab. Jala-jala, Laguna, Luzón.

21. U. Dailliardianus, $I T . O^{*}$ N. v. Usí.

Hab. Jala-jala, Laguna.

22. U. Marzaninus, $H . \mathrm{N}$. v. id.

Hab.

23. U. Roxasianus, II. $O^{*}$ N. v. id.

Hab. Batangas, Luzón.

24. U. longicuspis, $H$. N. v. id.

Hab.

25. U. microdontus, $H, \sigma^{\prime} \mathrm{N}$, v, id.

Hab. Batangas.

26. U. Gonzalinus, $H$; N. v. id.

$\mathrm{Hab}$.

27. U. Tolesforiænus, $H$. N. v. id.

Hab.

28. U. brachyceros, $H \cdot G$ N. v. Usá

(M. S. T.)

Hab. Batangas. 
29 U. Corieanus, $I I$. ơ $\mathrm{N}$ v. L̇á.

(M. S. T)

Hab. Bataan, Mariveles.

3o. U. Vorzusanus, $H . \mathrm{N}$. v. id

Hab.

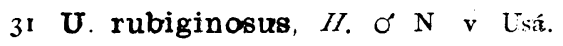

(M. S. T.)

Hab. Bataan, N. 'Ecija.

32 U. Hipolitianus, $H$. of N. v. id.

Hab. Batangas, Laguna.

33 U. chrysotricas, $H_{\circ} \propto \alpha$ N. v. id.

Hab. Laguna, Batangas.

34. U. Guevaranus, $H$. of N. พ. id.

Hab. La Laguna.

35 U. Marianus, $H . \quad \mathrm{N}$ v. $\dot{x}\}$.

Hab.

4 Gén. Meidanaxis, H.

36. M Alfredi, Sclat. $\delta f$ N. v.

Cervus Alfredi, Sclat P. Z. S. 1870 . p. 385.

Hab. Guimarás, Negros, Cebú, Panay.

37. M. Masbatensis, $H$. N. v. Usá.

Hab. Masbate.

38 M. breviceps, $H$.

Hab. Masbate.

39. $\mathbf{M}^{\circ}$ el $\cong g a n s, ~ H$.

Hab. ?

40. M. Basilanensis, $H$.

Hab. Basilan.

5. Gên. HYELAPHU'S, SUNy

41. H. Calamianensis, H. I N. v. Calachao.

Hab. Calamianes, Culion. 
Fam. TRAGÚLIDOS.

I. Gén. TRAGUluS.

I T javanicus, Pall. \& N. v. Pilandoc.

(M. S. T.)

Hab. Balabac, Java, Sumatra.

2. T fulviventer, Graj. t\& \& N. Pilandoc.

Moschus Kanchil, Horsf.

'Tr. affinis, Ciray. Lut of Mam. B. M. 1843. p. 173: P. Z. S. 1861 , p. 138.

M. fulviventer, Gray. P. Z. S. 1836 p. 65.

'Ir. pygmaus, z'ar. 2. Gray. Cat. Vug. B. M. i. p. 250.

'Tr. Kanchil, M. Edzirds. Au. Sc. Nat. 1864 p. 122.

Hab. Balabac, Java, Camboja.

3. T. pygmæus, Gray. of $\mathrm{N}$ v. Pilandoc

(M. S. T.)

Hab. Balabac, Jàra, India.

Fam. SÚIDOS.

I Gén. SUS, LiN.

I S Ecrofa, Lin む N. v Babuy

Hab. Manila, Laguna, Luzón. etc.

2. S. papuensis, Less. $t \hat{q}$ N. v. Babuy.

(M. S. T.)

El laborioso é inteligente P. Heude citado, en su «Etude sur les sangliers des Philipines» enumera los Súidos filipinos y los clasifica del modo siguiente:

$3 \mathbf{S}$ effrenus, $H . \downarrow\{\mathrm{N}$. v. Babuy.

Hab. Laguna, Jala-jala.

4. S. frenatus, $H . \delta$ N. v. id.

(M. S. T.)

Hab. Jala-jala.

5. S. microtis, $H$. \& N. v. id.

$\left(\begin{array}{lll}M & \mathrm{~S} & \mathrm{~T}\end{array}\right)$

Hab. Bataan.

6. S. cebrifons, $H$. $\&$ N. v. Babuy-ihas.

(M. S. T.)

Hab. Cebú. 
7. S minutus, II. N. $\mathrm{v}$ id.

Hab. ?

8 S. arietinus, $H$. N. v. id.

Hab. ?

9. S. bucculutus, $H$. N. v. id.

Hab. ?

10. $\mathbf{S}$ culamianэnsis. $H . \mathrm{N}$ v. Babuytalong.

S. longirontris, Nch.

Hab. Calamianes, Culion.

I. Gén BabIRUSA, Gray.

II B. alfurus, Less. \& N. v. Babirusa (cabezas).

Hab. Molucas.

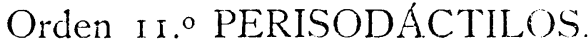

Fam. EQQUIDOS.

I Gén EqUUS, Irx.

I. E caballus, Lin \& N. v. Caballo.

Hab. Filipinas, (import.)

2. Gén. ASINUs, inin.

2. A vulgaris, $\operatorname{Lin} . \mathrm{N} . \mathrm{v} \Lambda$ sno.

Hab. Filipinas, (introduc.)

$$
\begin{aligned}
& \text { Fam. TAFÍRIDOS. } \\
& \text { I. Gén TAPIRUS, Iin. }
\end{aligned}
$$

x. T indicus, Desm, $\delta$ N van'a.

Jlab. Malaca, Sumatra. 


\section{Orden I $^{\circ} .^{\circ}$ PROBOSCIDIOS.}

\section{Fam. ELEFÁNTIIOS.}

I. Gén. Elephas, Lin.

Hab. India, Ceilan, China, Java.

\section{Orden $13 .^{\circ}$ DESDENTADOS.}

\section{Fam. MANÍDIDOS.}

I. Gén. PhOI.IDOTUS, Bkús.

2. P. javanicus, Desm. $\delta \mathrm{N}$. v. Balintong.

P. Manis pentadactyla, Raffles, Linn. Trans. xiii. p. 249.

P. Manis javanica, (partly), Fischer, Syn. p. 400 (not of Sundeoall.)

P. Manis aspera, Sundevall, l. c. p. 253; Rapp, Edent. t. 2." (good), t. 6. f. I, 2 (skull); Desm. Mamm. p. 377; Gerrard, Cat. Bones B. M. p. 285 .

P. Pholidotus javanus, Gray, P. Z. S. 1865 , p. 366. Hab. Paragua, Culion, Chima, Java.

3. P. dalmanii, Sazud.

4 P. culionensis, $t f$ N. v. Halinteng.

P. Manis, Dalmann, Act. Stockh, 1749 , p. 265, t. 6 .

P. Manis, Förster, Act. Berol. 1789 (printed 1793), p. 90, t. 5, 6; Sundevall, l. c. pp. 262, 269.

P. Manis Dalmanii, Sundevall, l. c. p. 256, t. 4 a. f. Io (teobones ), 18'tz; Rapp, Eadnt. p. 17.

P. Pholidotus Dalmannii, Gray, P. P. S. 1865, p. 366.

P. Manis aurita, Flodgson.

P. Manis pentadactyla (partly), Gerrard, Cat. Bones B. M. P. 285 .

P. Manis javanica, Sundeva!l, l. c. p. 25t, t. 4 a. f. I1; Turner, P. Z. S. is.5t, p. 219 ?

P. Pangolin, Bufjon, x. t. 37 .

Hab. Callamianes, Culion. 
Fam. DASIPÓDIDOS.

r. Gén. Tatusia, F. Cuv

I T. novenzincta, Lin. $\mathrm{N}$ v. Armadillo.

(M. S. T.)

Hab. América del Sur.

\section{Sub-clase 2." DIDELFOS.}

\section{Orden MARSUPIALES.}

\section{Fam. PERAMÉLIDOS. \\ I Gén PERAMELES, GEofF.}

2. P nasuta, Geoff. N. v. P. de nariz aguda.

(M S. T.)

P. nasuta, Geoff. Ann. Mus. iv. p. 62, pl. xliv. (animal \& skull) (1804); G. Fisch. Zoogin. iii. p. I3 (1814); G. Cur. R. $A$. i. p. I 77 (18I7); Desm. N. Dict. d'H. N. (2) xxv. p. I83 (1817); id. Mamm. i. p. 265 (1820); Schinz. Cuv. Thierr. i. p. 256 (1821); F. Cuv. Dict. Sci., Nat. xxxviii. p. 4 i6 (1825); Gray, Griff. Cuv. An. K. iii. p. 39, fig. (animal), v. p. 195 (1827); Less. Man. Mamm. p. 2 I6 (1827); id. Dict. Clas, $d^{\prime} H . N$. xiii. p. I99 (1828); Lacép. (Euvr. Buff. xxri. p. 224 (1828); Fisch. Syn. Mamm. p. 273 (1829); Burm. Lehrb. Naturg. p. 548 (1830); Less. B. N. Mamm. (Compl. Buff.) iv. p. 483 (i83o); Grant. Memm. Vern. N. H. Soc. vi. p. I 84 (1832); (anat.) Waterh. Cat. Mamm. Mus. Z. S. p. 65. (1838); Gray, Grey's Austr., App. ii. p. 401 (184I); Waterh. Jard. Nat. Libr., Mamm. xi. p. I 55 pl. xiii. (animal) (18+1); Ozvelt. Tr. Z. S. ii. p. $320(\mathrm{I} 8+1)$; Less. N. Tabl. R. A. Mamm. p. I9I (I842); Gray, List Mamm. B. M. p. 96 (I 843); Wagn. Schr. Säug. Supp. iii. p. 57, pl. clv. A (animal) (1843); v. p. 2I3 (I 855); Schinz, Syn. Mamm. p. 5I5 (I844); Owen, Todd's Cyclop. Anat. iii. p. 260, fig. 84 (skull) (1 $8+5)$; Waterh. N. H. Mamm. i. p. 374 (г 846$)$; Ne m. Dict. Univ. d'H. N. ix. p. 578 (1849); Gould, Mamm. Austr. i. pl. xi. (animal) (1855); Schleg. Dierk. p. I 38 (1857); Gieb. Saïg. p. 72 I (1859); Gerrard, Cat. Bones Mamm. B. M. p. I32 (1862); Krefft, Cat. Mamm. Austr. Mus. p. 33 (1864); id. Austr. Vert. p. I3 (1871); Schleg. Dierent. p. I64 (I872); Bhrem, Thierl. ii. p. 565 (1880); Dobs. J. Anat. Plys. xvii. p. I 52 (anat. foot) (1832); Poulton, P. Z. S. 1883, p. 599 (anat. tongue); id. Q.J. Micr. Soi. xxxiii. p. 69, pl. i. (I883); (anat. tongue); Flow. \& Gars. Citt. Ost. Coll. Surg. ii. p. 738 (1884); Lyd. Cat. Foss. Mamm. B. M. v. p. 255 ( 1887); Jent. Cat. Ost. Leyd. Mus. p. 306 (1887); Coll. Zool. Jahrb. ii. p. 874 (1887). Olfield. T. Cat. of Mars. and Mon. Brit. p. 242 (1888). 


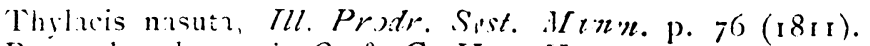

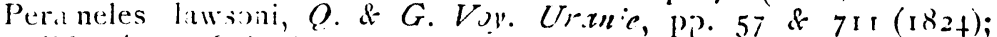
iid. Ann. Si. N.z. id p. tos (1825); Fisch. Syn. Mamm. P. 27t (1'29); Less. N. Tabl. R. A., Mamm. p. IOI (I8+2); Schinz, Syln. Mamm. i. p. 520 ( $18+4)$; Desm. Dict. Unio. d'H. N. ix. p. 579 ( 849$)$.

"Perameles aurita, Mlus. Paris," Waterh, Gray, et al. Hab. Australia.

\section{Fam. DASIÚRIDOS.}

\section{Gén DAsyurus, Geofr.}

I. D. viverrinus. Shato. N. v. Tapoatafa.

(M. S. T.

Spotted Opossuin, Phillip, Voy. Botany Bay, p. I47, pl. xv. ( 7789$)$. laapla tafa, shotted vixriety, White, Journ. Voj' N. S. W. p. 285 , pl. lix. ( 790$)$.

Ditelphis macula a, Kerr, Linn. An. K. p. i99 (1792) (nec Viverra maculata, id. op. cit. p. г7o); G. Cuv. Tabl. E!em. P. I25 (1798); Wiedem. Cuv. Naturg. Thiere, i. p. I79 (I 800$)$; Turton, Limn. Syst. N.zt. i. p. 69 (i 8.06.)

D. viverrinus, Shawe. Gen. Zool. i. pt. ii. p. 49i, pl. cxi. (г 8 (o).

D. viverrinus E. Geoff. Anin. Miss. iii. p. 360 (1804); Seyast. Min. Ac. Péterss. i. p. $4+3$ ( 1807 ); Ill. Prodir. Syst. Manm. p. 77 (г8гі); G. Fisch. Zoggn. ii. p. 585 (г813); G. Cuv. R. $A$.i. p. г 76 (1817); Dssm. N. Dict. d'H. N. (2) ix. p. 139 (18г7); Geoff. Dict. Sci. Nitt. xii. p. 5i г, (18г8); Desin. Mamm. i. p. 263 (1820); Desmoul Dict. Class. d'H. N: v. p. 339 (1824); Gray, Griff. Cuv. An. K. v. p. I93 (1827); Los. Man. Mamm. p. 215 (1827); Temm. Mon. Mamm. i. p. 72 (1827); J. B. Fisch. Syn. Mamm. p. 272 (i 829); Less. H. N. Mamm. (Compl. Buff. v. p. 373. pl. xxv. (I836); Waterh. Cat. Mamm. Mus. Zool. Soc. p. 65 (I838); Gunn. Ann. Mag. N. H. (1) i. p. Iot (I 838$)$; Gould, P. Z. S. $18+0$, p. I 5 ; ; Oven. Tr. Z. S. ii. p. 408, pl. lxx. fig. 2 (cráneo) (i841); Gray. Grey's Austr., App. ii. p. too (18+1); Less. N. Tabl. R. A., Mamm. p. I9: (18+2); Gray, List Mamm. B. M. p. 97 (1843); Wagn. Schr. Saiegg. Supp. iii. p. 33, 1873, v. p. I94 (1855); Waterh. N. H. Mamm. i. p. 442 , pl. xii. fig. I (1 846$)$; Goutd, Mamm. Austr. i. pl. I. (animal) (I85I); Gunn, P. Roy. Soc. Tasm. ii. p. 8I (1852); Gerv. S. N. Mamin. ii. p. 282 (1 855); Schleg. Dierk. p. I35 (1857); Gieb. Saüg. p. 731 (1859); Gerrard, Cat. Bones Mamm. B. M. p. I 34 (1862); Krefft, Cat. Mamm. Austr. Mus. p. 26 (1864); id. Notes Finun. Tasm. p. 4 (1868); id. Austr. Virtp. It (I871); Schleo. Dierent. p. I59 (1872); Brehm, Thierl. ii. p. 549 (1880); Higg. \& Pett. P. Roy. Soc. Tasm. 1883, p. I97; Flowe \& Gurs. Cat. Ost. Coll. Surg. ii. p. $7+2$ (1881); Jent. Cat. Ost. Ley . Mus. p. 3o+ (1837); Thos. Phil. Tituns. clxxvii. p. 46r, pl. xxvii. fig. 5 (dientes) (1887); Lyd. Cat. Foss. Mamm. B. M. v. p. 268 (1887). Olfield. Cint. of Mars. Brit. Mus. p. 265 ( I 888 ).

D. maugei, Geoff. Ann. Mus. iii. p. 35 (1804); T.edem Zool. 
p. +20 (18os), G. Fisch. Zoogn. ii. p. $58+(1813) ;$ Desm. $N$. Dict. d'H. N. (.2) ix. 1. I 38 ( п г z); Geoff. Dict. Sci. Nat. xii. p. 5 I I ( 818$)$; Tesm. Mamm. i. p. 263 ( I820); Coldf. Handb. Zool. ii. p. 449 (1820); Desmonl. I)ict. ('lass. d'H. N. v. p. 336) (rszt; H. Cuto H. N. Mamm. (fol.) iii. livr. xliv. (animal) (182t); Cuoy \& Ga'm. Loy. Lrauie, p. 5t, pl. ir. (182t); Gray, Gr.ff. Cuz. An. K. v. p. 193(1827); Temm. Mon. Mamm. i. p. $7 \mathrm{I}$, pl. vii. figs. 5-8 (1827); Less. Man Mamm. p. $21+$ (1827); J. B. Fisch Syn. Mamm. p. 27 I (1829); Less. H. N. Mamm. (compl. Buff. v. p. 373, pl. xxxvi. (animal) (I836) Binn. Cat. N. H. Austr. MFus. p. 2 (1837); Owen. P. Z. S. 1838, p. 121 et seqq.; Waterh. Cat. Mamm. Mus. Zool. Soc. p. 65 (1838); Gould, P. Z. S. 1840 , p. I1 5 ; Ouen. Tr. $Z$. S. ii. pp. 397, 408, p!. lxx. fiess. 3 (cráneo) (I $8+1$ ); Waterh. Jard. Nat. L:br., Manm. xi. p. i33, pl. vii. (animal) (I $8+1$; Less. N. Tabl. R. Á. Mamm. p. I90 (1 $8+2)$; Wagn. Schrel. Säug. Supp. iii. p. 24, pl clii. B. b. (animal) (1 $8+3)$; Sehinz; Sym. Mamm. i. 1. 49 I (1 $8+4)$; Gieb. Odont. p. 39, pl. xvii. fig. 8 (dientes) (1855).

D. guttatus, Desm. $N$. Dict. d'H. $N^{r} .24$ p. Io (1804).

Hab. N." Gales, Victoria y 'Iasmania.

\section{Fam. DIDÉLFIDOS.}

\section{Gén. Didel.phis, len.}

\section{D. marsupialis, Lin. \& N. v. Zarigiieya.}

(NI. S. T.)

Philander maximus orie..talis, Seba, Thesaurus, i. p. xxxix. (animal) (1734).

Didelplys marsupialis, Linn. Syst. Nat. (13) p. 54 (г760; Mïll. Ltmin. Naturs. i. p. 288 (1773); Erxl. Syst. R. A. p. 73 (1777); Schreb. Säug. iii. p. 536, pl. cxlv. (animal) (I778); Blum. Handb. Naturg. p. 87 (i779); Zimm. Geogr. Gesch. ị. p. 22 I (1780); Bcdd. Elench. Anim. i. p. 76 (1785); Gmel. Lim. S. N. i. p. 105 (1789); Kerr, Linn. Au. K. p. r9i (1792); Donnd. Zool. Beytr. i. p. 34 I (1792); G. Cuv. Tabl. Elum. p. I24 ( т 798); Desm. N. Dict. d'H. N. (2) xxiv. p. 19 ( I 804); Turt. Limn. S. N. i. p. 65 (1806); Blumenh. Abbild. nat. Gegenst. pl. liv. (animal) (1810); Ill. Prodr. S. M. p. 75 (1811); G. Fisch. Zoogn. ii. p. 576 (1813); Ill. Abh. Ak. Berl. 1811, p. Io7 (1815); G. Cuv. R. A. i. p. I73 (1817); Schinz, Cuv. Thierr. i. p. 25I (1821); Wied. Beitr. Nat. Bras. ii. P. 387 (1826); J. B. Fisch. Syn. Mamm. p. 264 (1829); Kaup: Thierr. p. 237 (1835); Less. N. Tabl. R. A., Mamm. p. I86 (1842).

D. karkinophaga, Zimm, Geogr. Gesch. ii. p. 226 (1780).

D. carcinophaga, Boc'd. Elench. Anim. i. p. 77 (1785); G. Cuv. Tabl. Elim. p. I 24 (1798).

D. cancrivora, Gmel. Linn. S. N. i. p. ro8. (1789); Kerr. Linn. An. K. p. 195. (1792); Donnd. Zool. Beytr. i. p. 352 (1792); Siana, Gen. Zool. i. pt. ii. p. 482 ( $18(0)$ ); Bechst. Uebers. Vierf. Thiere, ii. p. 684 (1800); E. Geoff. Cat. Mus. p. I 39 (1803); Desm. N. Dic.. d'H. N. (1.) vi. p. $45^{8}$ (1803); Turt. 
I.nn. S. N. i. p. 6h (1806); Ill. Abh. Als. Berl. es11, p. 107 (1

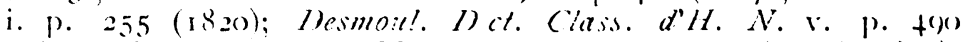

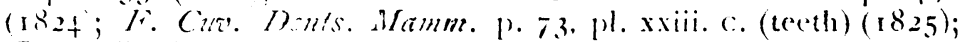

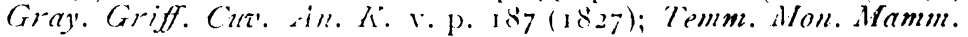
i. p. 32, pl. r. (s'iull \& s'sleton) (1827); less. Man. Mamm.

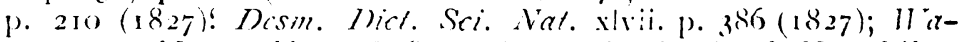
ter. Cat. Mamm Miss. Z. S. p. of (1838): gat. Jard. Nat. Libr.,

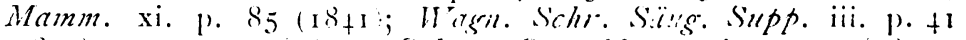

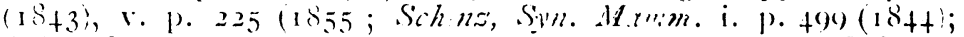

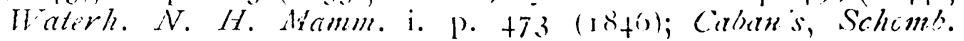

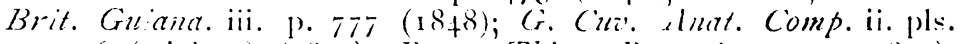
174-176 (mio'og.) (18+9); Burn. Thiere Bras. i. p. I 29) (185t); Gera. H. N. Mamin. i. p. 286 (1855); Burm. Firl. Fanu. Bras. p. 66, pl. iv. (animal), pl. r. fig. 2 \& pl. vi. fig. 2 (1856); Gieb. Säug. p. "7ot' (1859'; Tones, P. Z. S. $1800,1.268 ;$ Gerrard, Cat. Bones Mamm. B. M. p. I 38 (1862); Hens. Abh. Ak. Berl. 1872 , P. 114, pl. i. fig. 2 (dientes); Schleg. Dierent. 1). 160 (1872); Guldiver, P. Z. S. 287.5. P. 491; Alix, Bull. Soc. Philoin. (S) ii. p. 148 (anat.) (1879); Brehin, Therl. ii. !). 56 I (1880'; Natt. Pelź. Bras. Säug., Verh. z.-b. Wien, xxxiii Anh. p. 110 (1883); Floze. \& Gars. Cat. Ost. Coll. Sueg. ii. p. 749 (1884; Jent. Cat. Ost. Leyd. Mus. p. 3oo (1887).

D. virginiana, Kerr. Linn. An. K. p. 193 (1792); G. Cuv. Tabl. Elém. p. 124 (1798); Shaw, Gen. Zool i. pt. ii. p. 473, pl. crii. (animal)(1800); Bechst. Uebers. Vierf. Th. ii. p. 684 (180)); E. Geotf. Cat. Mus. p. I 40 (1803); Desm. N. Dict. d'H. N. (1) xx. p. I47 (1803); G. Fisch. Zoogn. ii. p. 577 (1813); Desm. N. Dict. d'H. N. (2) ix. p. 420 (1817); G. Cuv. R. A. i. p. 172 (1817; Desm. Mamm. i. p. 254 (1820); Desmoul. Dict. Class. d'H. N. N. v. p. 489 (1824); F. Ciuv. Dents Mamm. p. 75 (1825; Gray", Griff. Cuv. An. K. v. p. 186 (1827); Temm. Mon. Mamm. i. p. 27 (1827); Less. Man. Mamm. p. 209 (1827); Desm. Dict. Sci. Nat. xlvii. p. 383 (1827); J. B. Fisch. Syn. Mamm. p. 263 (1829); Burm. Lehrb. Naturg. p. 548 (1830); Waterh. Cat. Mamm. Mils. Z. S. p. 64 (1838); id. Jard. Nat. Libr., Mamm. xi. p. 79 (18+1); Gulliver, P. Z. S. 1842, p. 49; Less. N. Tabl. R. A., Mamm. p. 186 (1842; De Kay, $N$. H. New-York, Znol. i. p. 3, pl. x. v. fig. 2 (animal) (1842); Gray, List Mammt. B. M. p. Ioo (1843); Wagn. Schr. Säug. Supp. iii. p. 37, pl. cxlv.* (animal) (1843); v. p. 219 (1855); Schni, Sym. Mamm. i. p. 497 (1844); Walerh. N. H. Mamm. i. p. $465(18+6)$; Aud. \& Bach. Quadr. N. A. ii. p. IO7, pl. lxvi. (animal) (i 854$)$; Gerv. Casteln. Am. Slid, Mrmm. p. Ioo, pl. xx. fig. 7 (dientes) (1855); id. H. N. Mamm. ii. p. 286 (1855); Gicb. Odontogr. p. 41, pl. xvii. tig. I 2 (dientes) (1855); Burm. Erläut. Faun. Bras. p. 6o, pl. v. fig. I \& pl. vi. fig. 3 (cráneo) (1856); Baird, MAamm. N. A. p. 232 (1859); id. Zoo!. Mex. Bound. Surv. p. 31 (1859); Gieb. Säug. p. 708 (1859); Gerrard, Cat. Bones Mamm. B. M. p. I 37 (1862); Flower, Phil. Trans. 1867 , p. 635 pl. xxx. figs. $3-5$; Coues, P. Ac. Philad. 1871 , p. I5; id. Mem. Bost. Soc. N. H. ii. pp. 41-149, figs. I-35 (1872) (íosteolog. \& miolog.); Wyman, Mem. Bost. 
Soc. N. H. ii. p. I51 (1872); Schler. Diercnt. p. I60 (1872'; Gulliver, P. Z. S. 1875 , p. 49r; Brehm, Thiert. ii. p. S5, (1880); Alst. Biol. Cent.-Am., Mamm. p. 196 (1881); Floa. d' Gars. Cat. Ost. Coll. Surg. ii. .p. $7+7$ I 884 ); Siclenka, Biol. (intralbl. v. p. 29+ (1885); Jent. Cat. Ost. Leyd. Mus. p. 300 (188,. Philander virginianus, Tiedem Zool p. 427 ( 8008$)$.

D. aurita, Wied. Beitr. Nat. Bras. ii p. 395 (1826); Schina, Syn. Mamm. i. p. 5(x) (18+t); Burm. There Bras. i. 1. I. (i $\left.\times_{5+}\right)$; id. Erläut. Fann. Bras. p. 64, pl. iii. (animal), pl. v. fig. 3 \& pl. vi. fig. I (cráneo) (1856); Gieb. Säug. p. 709 (1859); Hens. Zool. Gart. viii. p. 290 (1867); Frantz. Arch. f. Nat. xxxi. p. 355 (1869); Alst. Biol. Cent.-Am., Mamm. p. 197 ( 881$) ;$ Natt. Pelz. Bras. Säug. Verh-z.-b. Wien xxxiii Anh. p. 109 (1883); Jent. Cat. Ost. Leyd. Mus. p. 3o1 (1887).

D. californica, Benn. P. Z. S. 1833 , p. 4o; Less. H. N. Mamm. (Compl. Buff.) v. p. $3^{6}+(1836) ;$ Waterh. Cat. Mamm. Mus. Z. S. p. $64(18 z 8)$; id. Jard. Nat. Libr., Mamm. xi. p. $s_{7}$ (1841); Wagn. Schr. Säug. Sucpp. iii. p. to (1843), v. p. 223

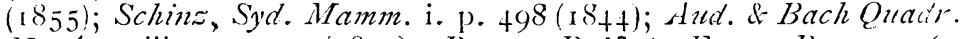
N. Am. iii. p. 335 (1854); Burm. Brläut. Faun. Bras. p. 63 (1856); Baird, Mamm. N. A. p. 233 (1859) id. Zool. Mex. Bound. Surv. p. 32, pl. iii. (animal) (1 859$)$; Gieb. Säug. p. j(x) (1859); Tom s, P. Z. S. 1861, p. 287; Gerrad, Cat. Boncs Mamm. B. M. p. I $38(1862)$.

1). breviceps, Benn. P. Z. S. 1.533, p. to; Less. H. N. Mamm. (Compl. Buff.) v. p. $36+(1836)$; Warterh. Cat. Mamm. Mus. Z. S. p. 64 (1838); id. Jard. Nat. Libr. Mamm. xi. p. $88(18+1)$; Wagn. Schr. Säng. Supp. iii. p. fo (1843), v. p. $22+$ (1855); Schinz, Syn. Mamm. i. p. 498 (1 $\left.{ }_{+4}\right)$; Waterh. N. H. Mamm. i. p. $478(1846)$; Aud. \& Bach. Quadr. N. A. iii. p. 330 (1854; Gieb. Säug. p. 7 (o) (1859); Jent. Cat. Ost. Leyd. Mus. p. zo (188̈7). Micuureus californicus y breviceps, Less. N. Tabl. R. A., Mamm. 1. $186(18+2)$.

D. pruinosa, Wagn. Schr. Säug. Sucpp. iii. p. fo (18+3); Schinz. Syn. Mamm. i. p. 499 (184t).

D. pilossisima, e illinensium, Link, apud Gray'; List Mamm. B. M. p. IOO (18+3), y otros antores.

Philander marsupialis, Gray, List. Mamm. B. M. p. Ior (1 $8+3)$; Gorrad, Cat. Bones Mamm. B. M. p. I 39 (I 862).

Hab. N. América.

\section{Fam. MACROPÓDIDOS.}

\section{r. Gén. MaCROPÚ, Shaw.}

r. M. antilopinus, Gould. $₫ q$ N. v. Canguro antilopino.

(M. S T.)

Osphranter antilopinus, Gould, P. Z. S. 18+1, p. So; Gray, List Mamm. B. M. p. 91 $\left(\mathrm{s}^{8}+3\right)$; Gould, Mon. Macr.pl. xxii. (animal) (1842); id. Mamm. Austr. ii. pls. viii. \& (animal) (1 $\left.s_{5} 8\right)$; Gerrard, Cu'. Bones Mamm. B. M. p. I 27 (1862).

Halmaturus antilopinus, Schinz, Syn. Mamm. i. p. $524(18+4)$; Wagn. Schr. Säug. Supp. v. p. $309\left(18_{55}\right)$. 
N. antilupinus, Waterh. N.H. Mamm. i. p. 95 (1895), pl. v. fig. I5 (incisivos); Giebel, Odontogr). pl. xix. fig. 12 c (1855) (incisivos); Schli's. Dierk. p. It2 (1857); Gieb. Säng. p. 677 (1859); kreft. Mamim. Austr. text. pl. x. p. 2. (ns;i); Gieb Bronn's k\%. u. Odrn. vi. Abth. v. pl. xlvii. fig. 2 I (incisivos) (187(6); Flozi. of Gars. Cat. Ost. Coll. Surg. ii. p. 7 1 (1884); Olfield, Cat. of Mars. Brit Mus. p. 2 I (1888).

Hab. Australia.

2. M. gi£anteus, Shon. $\sigma \circ$ N. v. Canguro gigante.

(II. S. T.)

Kangaroo, Cook, Hazikesz'. Voy. (4to) iii. pp. 157 \& 173 , pl. xx. (animal) (1773); Phillip. Voy. Bot. Bay, pp. io6 of 168 , pls. x. of xxx. (animal y craneo) (1789); Withe, Journ. Voy. N. S. II. P. $272(1 ; 90)$.

Yerboa gigantea, Zimm. Spec. Geogr. p. 526 (1777).

Jaculus giganteus, Erxl. Syst. Regn. An. p. fog (1777); Blumenb, Handb. Naturg. p. 88 (1779).

Didelphys gigantea Schreb. Saitg. iii. p. 552, pl. cliv. (1778); Gmel. Linn. S. N. i. p. Iog (I788); Donnd. Zool. Beytr. i. p. $35+$ (1792); Kerr, Linn. An. K. p. 197 (1792); Meyer, Syst. Uebers. Zool. Ent. p. is (1793); G. Cur. Zlabl. Elém. p. 1:6 (1798); Geoff: Ann. Mus. i. p. 178 (1802); Desm. N. Dict. d'H. N. xii. p. 355 (1803); Blumenb. Man. d'Hist. Nat. i. p. го9 (1803); G. Fisch. Anat. Maki, p. is9, (craneo) (1804); Turt. Linn. S. N. pl. xix. i. p. 67 (1806).

D. kanguru, Zimm. Geogr. Gesch. ii. p. 231 ( 1780$)$.

D. kanguro, Bodd. Elench. An. i. p. 78 (1785).

Macropus giganteus, Shaw'. Nat. Misc. i. pl. xxxiii. (1790); Pinder \& I'Alton, Vergl. Osteol., Beutelth. pls. i. \& vii. (cránees y esqueleto) (1828); Fisch. Syn. Mamm. p. 28 I (1829); F. Cur. H. N. Mamm. (fol.) iii. livr. lv. (1829); Less. H. N. Mamm. (Compl. Buff. v. p. 377 ( 1836 ); Waterh. Cat. Mamm. Mus. Zool. Soc. p. 66 (1838); id. Jard. Nat. Libr., Mamm. xi. p. I92 (I841); Less. N. Tabl. R. A. Mamm. p. $193(18+2) ;$ Waterh. N. H. Mamm. i. p. 62 pl. ini. figs. 3-5, y v. figs. I \& 2 (dientes) (18+6); Desm. Dict. Univ. d'H. N. vii. p. г 7 (1 849 ; Gninn, P. Roy. Soc. Tasm. ii. p. 88 (1852); Gerv. Hist. Nat. Mamm. ii. p. 70 , pl. xlv. (1 855$)$; Giebel, Odontogr. p. 43 pl. xix. fig. It, $a$, b (incisivos), (1855); Schleg. Dierk. p. I42 (I857); Gieb. Säug. p. 672 (1859); id. Thierr. i. p. 235 figs. 397 (o) (1859) Blyth, Cat. Mamm. Mus. As. Soc. p. 183 (1863) Schäfer \& IIilliams, P.Z. S. 1876 , p. I65 (anat.); Giebel, Bronn's Klass. " Ordn. vi. Abth v. pl. xlvii. fig. 2 I, I \& 2 (incisivos) (1876); Brehm, Thierl. ii. p. 591 (1880); Flone'er, Encycl. Brit. (9) xiii. p. 838 (I88I), art. Kangaroo; Chapman, P. Ac. Philad. 1881 , p. 468 pl. xx. (anat.); Flow. of Gars. Cat. Ost. Coll. Surg. ii. p. 707 (1884); Ly'd. Cat. Foss. Mamm. B. M. v. p 225 (1887); Jont. Cat. Ost. Leyd. Mus. p. 3 I7 (1887); Cotl. Zool. Jahrb. ii. p. 816 (1887).

Macropus major, Shaw, Gen. Zool. i. pt. ii. p. 505, pl. cxv. (1800); G. Cuv. R. A. i. p. I83 (1817); F. Cuv. Dict. Sct. Nat. xxiv. p. $3+7$ ( 1822 ); id. Dents Mamm. pl. xliii. (dientes) (1825); Ocien; P. Z. S. 1831 , p. 1591833 , p. I28, \& 1837 , 
pp. 82 \& r 20; Gray, Charlesw. Mag. .V. H. i. p. 582 (1837); Blyth. Cur. An. Kingd. p. Io6 (1840); Owen, 7r. Z. S. ii. pp. 228 \& 388 (1841); Gray, Grey's Austr., App. ii. p. 402 (18+1); Fennel, Nat. Hist. Quadr. p. 275 (1841); Gould, Mon. Macr. pl. i. (animal) (1841); Gray, List Mann. B. M. p. S7 (1843); Gould, Straelcez!'s $N$. S. W. p. 3 I6 (1845); Öw口, Odontogr. Text, Tex. p. 390, Alt. pl. c. fig. 8 (dientes) $(18+5)$; id. Art. Marsupialia, Todd's Cycl. Anat. Ply's. iii. p. 266, fig. 92 (1847), y Art. Teeth, op. cit. iv. p. 993 fig. 594 dientes (1848); G. Cuv. Anat. Comp. ii. pls. clxxxiii.-cciii. (miolog.) (1849); Owen, Ciat. Ost. Mus. Coll. Surg. i. 1). 320 (185.3); Gould, Mamm. Austr. ii. pls. i. \& ii. (animal); (1857); Gerrard, Cat. Bones Mamm. B. M. p. I24 (1862); Krefft, Cat. Mamm. Austr. Mus. p. 47 (1864); Huxl. \& Hazuk. Atl. Comp. Ost. pl. x'. figs. $4 \& 6$ (pelvis y pié) (1864); Flower, Phil. Trans. 1857, p. 633, pl. xxix. figs. I. 2, of 3 Owen, Comp. Anat. Phys. iii. p. 379 , fig. 296 (denticion) (1 868); Krefft, Notes Faun. Tasm. p. 4 (1868); id. Mamm. Austr. pls. x. \& x. bis. (animal, (1870); Owen, Phil. Trans. $187+1$ p). 245 of seqq. p. xx. fig. I5 (maxilar inferior); $F$. G. Waterh. "Harc. S. Austr. p. 284 (1876). Owen, P. Z. S. 1877 , p. 358; Fictcher, P. Linn. Soc. N. S. W. vii. p. 651 (1882), and viii. p. 9 (anat.) (1883); Higg. \& Pett. S. Roy. Soc. Tasm. 1883 , p. 196. Kanguro gigas, Lacép. Mím. Inst. iii. p. 491 (I80i).

Kangurus giganteus, E. Geoff. Cat. Mus. p. I 54 (I803); Tiedem. Zool. i. p. 436 (1808); G. Fisch. Zoogn. iii. p. I9 (1814).

Halmaturus giganteus, Ill. Prodr. Syst. Mamm. p. 8o (I 8I I); Goldfuss, Zool. ii. p. 446 (1820); Schinz, Cuv. Thierr. i. p. 263 (182I); Leuckh. Meck. Arch. Phys. viii. p. 442 (anat), (1823'; - Burm. Lehrb. Natnrs. p. 547 (1830); Kaup, Tierr. i. p. 243 (1835); Wag. Schreb. Sä̈g. Saupp. iii. p. Io8 (I843); v. p. $3^{(1)}$ (1855); Schmz, Syn. Mamm. i. p. 546 (1844).

¿Halmaturus rutilans, Ill. Abh. Ak. Berl. 1811, p. I02 (1815); (nom. nudum).

Kangurus labiatus, Desm. N. Dict. d.H. N. (2) xvii. p. 33 (1817); id. Manm. i. p. 273 , pl. xxi. fig. 4 (animal) (1820); Schinz, Cuv. Thierr. i. p. 263 (1821); F. Cur. Dents. Mamm. p. I 37, pl. xliii. ^. (dientes) (I835); Geoff. D)ict. Class. d'H. N. ix. p. I Iо (1826); Gray. Griff. Cuv. An. Kinged. v. p. 201 (1827).

Halmaturus griseofuscus, labiatus, y griseorufus, Goldfuss, Is:s, 1819, pp. $266,267$.

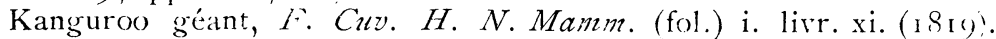
M. labiatus, F. Cuo. Dents Mamm. p. 137, pl. xliii. A (die.tes) (1825); Less. Man. Manm. P. 225. (1127)

M. ocydromus Gould, Ann. Mag. N. H. (1) x. pl. (18+2); Grin', List Mamm. B. M. p. \&7 (18+3); Gilbert, P. Z. S. 18+1, 1). 33 (habits); Gould, Mamm. Autr. ii. pls. iii. \& iv. (animal) I 860, and Introd. i. p. xxix. (1863); Gerrard, Cat. Bones Mamm. B. M. p. 24 (1862); Kreft. Mamm. Austr. text to pl. x. p. I $(287 \mathrm{r})$.

Yerbua kanguru, Fiorst. Descr. Anim. p. 367 (1844).

M. giganteus, zar. typicus, Olfield. Cat. of Mars. of Brit Mus. p. I5 ( 1888 ).

Hab. Australia. 
Fam. FALANGÍSTIDOS.

\section{I, Gén. TRICH()SLRUS, Lrss}

1. T. vulpinus (.Mili) \& $₹$ N. v. F. zorro.

(M. S. T.)

V'ulpine Opossum, Phillips, V'oy. BJ'. B.ry, p. I50, pl. xvi. (animal) $(1-89)$.

Didelphis vulpecula, Korr, Linn. An. K. p. I98 (г792); Turt. L nn. S. N. i. p. 68 (1806).

Didelphis vulpina, Meyer, Uebers. Zool. Entd. Neuholl. p. 23 (1793); Shaw, Geu. Zool. i. pt. ii. 503 (1800); Bechst. Uebers. vierf. Thiere, ii. pl). $34^{8}$ \& $685(1800)$.

Didelphis tapoudru, Mejer, Uebers. Zool. Entd. Neuholl. p. 24 ( 1793$)$.

Didelphis lemurina, Shaw, Gen. Zool. i. pt. ii. p. 487 pl. cx. (animal) (1800)

Ursus nove-hollandix, Bechst. Uebers. vierf. Thiere, ii. pp. 337 \& 683 ( 800$)$.

Phalangista lemurina, G. Cuo. R. A. i. p. I79 (I8I7).

Phalangista rulpina, Desm. N. Dict. $\ddot{a} \cdot H . N$. (2) xxv. p. 475 (1817); id. Mamm. i. p. 267 (1820); Schinz; Civ. Thierr. i. p. 258 (1821); F. Cuv. I).ct. Sci. Nat. xxxix. p. 4I4 (I826); Gray, Graff. Cuv. An. K. iii. p. 4r, pl. (animal); v. p. 197 (1827); Temm. Man. Manm. i. p. 5. (1827); Less. Man. Mamm. p. 218 (1827); J. B. Fisch. S;'ll. Mamm. p. 277 (1829); Less. H. N. Mamm. (Compl. Buff.) iv. p. 475 (I830); Martin, P. Z. S. 1835, p. 2. (anat.); U'aterh. Cat. Mamm. Mus. Z. S. p. 67 (гs38); id. Jard. Nat. Libr., Mamm. xi. p. 265, pl. xx ii. (animal) (I8+1); Gray, Grey's Austr., App. ii. p. 40I (18+1); Less. N. Tabl. R. A., Mamm. p. i 88 (1842; Gray, List Mamm. B. B. p. I86 (1843); Wagn. Schr. Säug. Supp. iii. p. 74 (1 $8+3)$; v. p. 269 (1855;. Schinz, Syn. Mamm. i. p. 526 (1844); Own, O.tontogr. Atl. pl. c. fig. I. (dientes) (I 845); Gieb. Ondontogr. p. 42 , pl. xviii. figs. 8 \& (dientes) (1855); Gould, Mamm. Austr. i. pl. xvi. (animal) (1856); Gieb. Säug. p. 697 (1859); Gerrard, Cat. Mamm. B. M. p. I22 (I862); Kreff, C't. Mamm. Austr. Mus. p. 37 (1864); Gieb. Z. gos. Nat. xxrii p. 392 (1866); Flow. Phil. Trans. 1887, p. 634, pl. xxix. firs. 5 \& 6 Krefft, Notes Faun. Tasm. p. 4 (i 868); id. Mamm. Austr. pl. viii. (aninal) (I87I); Sahleg. Dierent. p. I65 (1872); Tauber, Naturh. Tidsskr. (3) viii. p. 163, pl. xi. fig. 6 (1872); Gulliver, P. Z. S. 1875, P. 491; Brehm, Thierl. ii. p. 577 (1880); Dobs. J. Anat. Phys. xvii. p. I5I (I8\$2); Flow'. \& Gars. Cat. Ost. Coll. Surg. ii. p. 7or (1884); De Vis, P. Linn. Soc. N. S. W'. (2) i. p. I I 29 (1887).

Phalanger de Bougainville, G. Guv. R. A. (ed 2) i. p. 183 (1829). Ph. cooki, F. Ciwo. (no Desm.) H. N. Mam. 3. 45. (1824).

Phalangista bougainvillei, J. B. Fisch. Syn. Mamm. p. 583 ( 1829); Less. N. Tab!. R. A. Mamm. p. I88 (1842); Wagn. Sckr. Säug.

Supp. iii. p. 82 (I 843$)$.

Phalangista ('Trichosurus) vul'ona, Less. Dict. Class. d'Y. N. xiii. 
p. 335 (1823); Waterh. N. H. Mamm. i. p. 28t, pl. ix. fig. I (animal) \& xix. fir. I ( $8+6)$.

Balantia vulpina, Wagl. Syst. Ambh. Säug. p. 25 (1830).

Phalangista xanthopus, p. Os.b. P. Z. S. 1.381 p. I $35 ;$ Waterh. Cat. Mamm. Mus. Z. S. p. 67 (1883); id. Jard. Nat. Libr., Mamm. xi. p. 269 (18+1); Gray, Grej's Austr., App. ii. p. for (I8+1); Wagn. Schr. Säutg. Supp. iii. p. $77(18+3)$; Schinz, Synn. Mamm. i. p. 529 (18+t); Water. h N. H. Mamm. i. p. 294 (1846).

Balantia bouganvillei, Kaup, Thi.rr. i. p. 240 (I 835 .)

Phalangista curieri, Gray, Grey's Austr., App. ii. p. tor (1 $\left.s_{+1}\right)$; Waterh. Jard. Nat. Libr., Mamm. xi. p. 286 (I $8+1)$; Gray, List. Mamm. B. M. p. 86 (1 $8+3)$;

Trichosurus xantophu;, Less. N. Titbl. R. A., M.rmm. p. I 89 $(18+2)$.

Phalangista melanura, Wygn. Schr. Säıg. Supp. iii. p. 8 I (18+3).

'T. vulpinus, Gerv. H. N. Mamm. ii. p. 275 (1855.)

Phalangista hypoleucus, Wign. Schr. Säug. Suppp. r. p. 273, pl. xxii. (animal) (I855).

Phalangista vulpecula, Thass. Ann. M.tg. N. H. (5) iv. p. 397 (1879); id. Encycl. Br.t. (9) xviii. p. 728 (1885); Coll. Z.ol. Jahrb. ii. p. 9 10 (1887).

'T. vulpecula, Je'ut. Notes Levd. Mus. vii. p. 21 (1834); id. Cat. Ost. Leyd. Mus. p. 3it ( I887).

T. vulpeculd, var. typicus, O!field. Catt. of Mars. of Brit. Mits. p. 187 . (I888).

Hab. Australia.

\section{Fam. FASCOLÁRCTIDOS.}

\section{Gén. phascolarctos, bi.}

2. P. cinereus, Schr. O $0 \mathrm{~N}$ v. Coala ceniciento

(M. S. T.)

Koala, G. Cuv. R. A. i. p. I84, iv. pl. i. fig. 5 (ani.nul) (I817); Desm. N. Dict. d'H. N. (2) xvii. p. I IO (I8I7).

Lipurus cinereus, Goldg. Isis, 1819, p. 274; Sihinz, Ciuv. Thier. i. p. 265 (г82 ). Burm. Lehrb. Naturg. p. 547 (1830); Oven, P. Z. S. 1838 , p. I 54 .

Morolac.ylus cinereus, Goldf. Zool. ii. p. 445 (i 820 ).

P. fuscus, Desm. Mamm. i. p. 276 ( I $20 ; 1$ id. Dict. Sci. Nat. xxxix. p. 448 (1826); J. B. Fisch. Syn. Mamm. p. 28+ (1829); Martin P. Z. S. 1836 , p. Io9 (anat); Waterh. Cat. Mamm. Mus Z. S. p. 68 (1838); id. Jard. Nat. Libr., M.mm. xi. p. 295, pl. xxxi. (animal) (I8+1); Less. N. Tabl. R. A., Mamm. p. 192 (1842); Wagn. Schr. Säı. Supp. iii. p. $94($ I $8+3)$, v. p. 28 I (I855); Owen, Odontogr. Atl. pl. c. fig. 6 (dientes) $(18+5)$.

P. koala, Gray, Griff. Cuv, An. K. p. 205 (1827); Cerv. H. N. M:tmm. ii. p. 273, pl. (animal) (1855).

P. flindersi, Less. Man. Mamm. p. 221 (I827).

P. cinereus, J. B. Fisch. Syn. Mamm. p. 285 (1829); Gray, List. Mamm. B. M. p. 87 (1843); Wagn. Schr. Süug. Sıpp. iii. p. 95 (18+3); Schinz, Syri. M.xmin. i. p. 537 (18+t); Waterh. N. H. Mamn. i. p. 259, pl. ix. fig, 2 (animal), y 


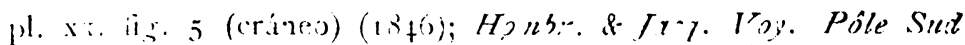
Zon!. p. 3.5. Atl. pls. xivii. of xivi. A. (aninal, crínen y esquelets) (1sij-5.3); Gou!d, Mranm. Alustr. i. pls. xiii. \& xiv. (anim:l) (1854); Grov. H. N. Mamm. ii. p. 273 (1855); Gieb. Sizug. p. 60)3 (1859); Benn. P. Z. S. 1862 , 1. 85; Cierard, Cat. Bones Mamm. B. M.) p. г 23 (1862); Krefft, Cat. Mamm. Austr. lius. p. +t (1864); id Matnm. Austr. pl. vi. (animal) (1871); Schleg. Dierent. 1. 167 (1872); Young, J. Anat. Phys. xiii. p. 305 et seqq. pl. xviii. (anat.) (1879); Sclater, P. Z. S. 1880 , p. 35.5 ; Brehm. Thiorl. ii. p. 579 (1880); Forbes P. Z. S. 1881 , P. I8I; et seqq. fig. I-6 (anat.); Dobs. J. Anat. Phis. xrii. p. I 5 I (1882); Flow. Encycl. Brit. (o) xv. p. $3_{82}$, figs. 25 (cráneo) \& 32 (1883); Flow. \& G.trs. Cat. Ost. Coll. Surg. ii. p. 609 (I884); Thos. P. Z. S. 1897 , p. 338, fig. I (o!l. Zool. Jahrb. ii. p. 935 (1887); Jent. Cat. Ost. Lovd. Mus. p. 307 (1887). Olfield. Cat. of Biritish Mus. p. 210 (1888).

Hab. Australia.

\section{Sub-clase $3 .^{\circ}$ ORNITODELFOS.}

\section{Orden MONOTREMOS.}

\section{Fam. EQUíDNIDOS.}

I. Gén ECHIDNA, G. Cuv.

I. M. aculeata, Shaze. $\delta \hat{f} \mathrm{~N}$. Equidno equinoso.

( $\mathrm{N}$ S. 'T.)

Myrnecophaga aculeata, Shaw, Not. Misc. iii. pl. cix. (ani nal) (I792); id. Gen. Zool. i. pt. i. p. I75, pl. liv. (animal) (18:00); Bechst. Uebers. vierf. Thiere, ii. P. $57 \mathrm{I}$ (1800); Turt. Linn. S. N. i. p. 30 (1806).

Ornithorhynchus hystrix, Home, Phil. Trans. 1802, p. $34^{8}$ et seqq., pls. x.-xii. (animal y anat.)

E. hystrix, E. Gooff. Cat, Mus. p. 224 (1803); id. Bu!l. Soc. Philom. iii. «no. 77,» 226 26), pl. xiv. (animal) (1803); Desm. N. Dict. d'H. N. (1) xxiv., Tabl. Meth. p. 27 (1304); id.op. cit (2) x. p. 53 (1817); G. Cuv. R. A. i. p. 226 (1817); Schinz, Cuz. Thierr. i. p. 339 (1821); Desm. Mamm. ii. p. $378(1822) ; F$. Cur. Dict. Sci. Nat. xxxvi. p. 448 (1825); Gray, Griff. Cuv. An. K. v. p. 284 (1827); J. B. Fisch. Synn. Mamm. p. 40I (1829); Less. H. N. Mamm. (Compl. Buff.) v. 1. 28+ (1827); J. B. Fisch. Syn. Mamm. p. 401 (1829); Less. H. N. Mamm. (Compl. Buff.) v. p. 384 (1836); Waterh. Cat. Manum. Mus. Z. S. p. 68 (1838); id. Jard. Nat. Libr., Mamm. xi. p. 303, pl. xxxiii. (animal) (r8+r); Less. $N$. Tabl, R. A., Mamm. p. 196 (18+2); Gray, List Mamm. B. M. p. 192 (18+3); Ozien, P. Z. S. 1845 , p. 80 Gould, Mamm. Austr: i. pl. ii. (animal) (i 852); Gieb. Süug. p. 399 (1859); Gerr., Cat. Bones Mamm. B. M. p. 289 (1852); Krefft, Cat. Mamm. Aus- 
1r. Mus. p. 55 (1864); $2 d$. Vert. Lower Murray, p. 23 (1865); id. Mamm. Aust. pl. xiv. (animal) (1871); G. F. Benn. 'P. Z. S. 1881 , p. 737 .

E. longiaculeata, Tiedem. Zool. i. p. 592 (1808).

'Tachyglossus aculeatus, Ill. Prodr. Syst. Mamm. p. 114 (1881).

Goldf. Handb. Zool. p. 409 (1820); Wagl. Syst. Amph. Säug. 1. $59(1830)$; Lïtken, P. Z.S. 1885 , p. $15 \%$.

Echinopus hystrix, $G$. Fisch, Zoogn. iii. p. 692 (1814).

E. aculeata, Garnot, $N$. Bull. Soc. Philom 1825, p. 45; Waterh, N. H. Manm. i. p. 41 (1846); Gray, j’. Z. S. 1865 , p. 316 ; id. Handl. Edentates, q. 31 (1873); Floze. \& Gars, Cat. Ost. Coll. Surg. ii. p. 751 (i884; Coll. Zool. Jahrb) ii. p. $937(\mathrm{~s} 887)$.

i.. Australiensis, Less. Man. Mamm. p. 318 (1887).

'Tachyglossus hystrix, Kaup. Therr. i. p. 255 (I 835); Glog. Handb. Naturo. i. p. in (1842); Wagn. Schr. Säus. Supp.' iv. 1). 242 (1844); Schnz, Sy'n. Mamm. ii. p. 329 (1845); Armit, J, Linn. Soc. xiv. p. 41 I $(1878)$.

E. australis, Less. H. N. Mamm. (Compl. Buff.), v. pl. 1 i. $(1836)$.

E. acanthion, Coll. Forh. Vid. Selsk. 1884, no. 13 (1088); id. I'. Z. S. 188.5, p. 150.

E. aculeata thypica, Thos. P. Z. S. 1885, p. 338, pl. xxi i. fig. B. (cráneo). Olfeld. Cat. of Mars. of Brit. Mus. p. 379 (I88I). Hab. Australia.

\section{Fam. ORNITORÍNQUIDOS.}

\section{Gén. ORNITHORHYNCHUS, Blum.}

r. O. anatinus, Saze. \& \& N. v Or paradójico. (M S. T.)

Platypus anatinus, Saw., Nat. Misc. x. pls. $385 \& 386$ (animal, pico \&c.) (1799); id. Gen. Zool. i. pt. p. 229, pls. 66 \& 67 (animal \&c.) (1800); Turt. Linn. S. N. i. p. 30 (1806); Gerrard, Cat. Bones Mamm. B. M. p. 288 (1862); Gray. P. Z. S. 1865, p. 385 ; id. Handl. Edentates, p. 29 (1873).

O. paradoxus, Bhimenb. Voigt's Mag. Naturk. ii. p. 205 (1800); Home, Phil. Trans. 1800 , p. 432. pls. xviii. \& xix. id. op. cit. 1802 , p. 67, pls. ii.-iv. (anat.); Calkoen, Nat. Verh. Bat. Mantsch-Wet. ii. pt. i. p. 177 (I803); Desm. N. Dict. d'H. N. (1) xxiv., Tabl. Méth. p. 97 ( I 804); Blumenb. Abbild. Nat. Gegent. no. 4 I (animal) (1810); G. Fisch. Zoog. iii. p. 689 (1814); G. Cuv. R. A. i. p. 227 (1817,; F. Cuv. Dents Mamm. p. 202, pl. 1xxxiii. (1825); E. Geoff. Ann. Sci. Nat. ix. p. 451 ( 1826); id. Meckel's Arch. f. Nat. x. p. I4 (1827); Gray. Griff. Cur. An. K. iii. p. 265 (or 27); Less. Man. Mamm. p. 319 (1827); J. B. Fisch. Syn. Mamm. p. 402 (1829); Wagl. Syst. Amph. Sâug. p. 59 (1830); Maule, P. Z: S. 1832, p. 145; Benn.P. $Z$. S. 1834 , p. I41; id. Tr. $Z$. S. i. p. 229, pl. xxxiv. (animal) (i834); Knup, Thierr. i. p. 25I (I835); Less. H. N. Mamm. (Compl. B iff.) v. p. 316, pl. liii. (animal) (1836); Waterh. Cat. Mamm. Mus. Z. S. p. (1838); id, Jard. Nat. Libr., 
.Y.tmm. xi. p. 309, pl. xxxir. (animal) $\left(\mathrm{I}^{2}+1\right)$; Glog. Hantb. Natturg. i. p. $115(18+2)$; Less. V. Tabl. R. A., Malmm. p. 196 $(18+2)$; Wagn. Schr. Säug. Supp. iv. p. 262 (1 $8+t)$; Gieb. Od)m-

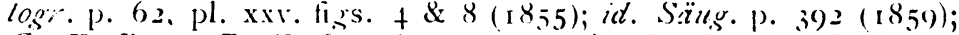
G. F. Benn. P. Z. S. $1 \delta^{7} 77$, p. 161 drmit, J. Limn. So . xiv. p. $413(1878)$.

Dermipus anatinus, Wiedem. Arch. Zosl. i. pt. i. p. $\mid 80$, pl. iii. (animal) is $3: 7)$.

(). rufus et fuscus, Pér. \& Les. Voy. Terres Alustr., dll. pl. xxxir. (animal) (1807); Tiedem. Zool. p. 5 to (1808); Ill. Prodr.

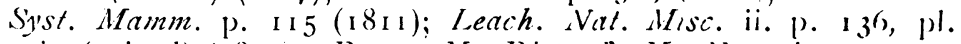
cxi. (animal) (1815); Desm. N. D) ct. d' H. N. xxiv. p. I31 (1818); Goldf. Handb. Zool. p. 4.8 (1820); Sch ibz, Cin' Tilierr. i. pp. 340 \& 34I (1821); Desm. Mamm. ii. p. $380(1822)$; V.an der Hoeven, N. Act. Acad. Leop. xi. p. 3br, pl. xlvi. (animal, pico, \&c.) (1823) F. Cuv. Dict. Sci. Nat. xxxit. p. $4+3$ (1 25 ); Gray, Griff. Curv. An. K. p. $28+\& 285$; Schinz, Syn. MI:nm. ii. p. $327($ I 845$)$.

O. brevirostris, Ogib. P. Z. S. 1831 . p. 150; Schinz, Syn. Mamm. ii. $327(1845)$.

(). crispus et lavis, Macgillivray, Mem. Weris. Soc. vi. pp. 138 \& 132 (1832).

O. anatinus, Gray. List. M.rmm. B. M. p. I9 I (18+3); Waterh. N. H. Mamm. i. p. 25, pl. i. figs, r-6 (pico, piés, \&ce.); Gunn. P. Roy, Soc. Tasm. ii. p. 89 (1852); Gould, Mamm. Austr. i. pl. i. (animal) (1855); Bennett. P. Z. S. 1859 , p. 2г 3, pl. lxxi. (pico); Krefft, Cat. Mamm. Austr. Mus. p. 56 (I864); id. Vert. Zower Murray, p. 22 (1865); id. Mamm. Austr. pl. xv. (animal) (I87r); Flow. \& Gars. Cat. Ost. Coll. Surg. ii. p. 753 (1884); Jent. Cat. Ost. Leid. Mus. p. 325 (1887); Coll. Zool. Jahrb. ii. p. 940 (1837). Olfleld. Cat. of Mars. of Brit. Mus. p. 388 (I 888).

Hab. Australia. 


\title{
Clase 2." A VES.
}

\section{Orden $1 .{ }^{\circ}$ PRENSORAS.}

\author{
Fam. CACATÚIDAS. \\ 1. Gen. Cacatua, Viúili.
}

1. C. hæmaturorygia (S. Miill) $\mathrm{O}^{7} \mathrm{O} \mathrm{N}$ v. Catala, Abucay. ([3. 191). (M.S. T.)

(acatua minor, Brisson, Orn.iv. p. 21 2, no. « « «hilippincs».

Le petit Kakatoës à bec couleur de chair, Bufforn, Hist. Nat. vi. p. 96 (patr. non indic.)

Petit Kakatoës des Philippines, D'Aubcnton Pl. Enl. I9I.

Ps. hæmaturopygius, L. S. Müller, S. N. Suppl. p. 77, no. 5 1 ( 1776) ex Buffon; Walden \& Layard, Ibis, i 872, p. 96; Bonchariat Cat. Av. no 3142 p. Io6, ( 876 .)

Ps. philippinarum, Gm. S. N. I. p. 331, no. 95 (г788) ex Brisson; O. Finsch, Monogr. Papag. I. p. 3Іо; V. Martens, J.f. O. 1866 p. $2 \mathrm{I}$, no. II 2.

Lophochroa minor, (Briss.), O. Finusch. Nederd, Tijdschr. Dierk; Berigten, 1863 , p. xxiii, "Luçon». C. hamaturopyrgia, On birds. Wald. of Philip. Works of Tie. p. 299. (1881).

Hab. Luzón, Bataan, Latguna, Calamba, Batangas, Guimarís, Negros, Valencia, Cebú, Leyte, Amparo, Nipah, Mindanao, Joló, Balabac, Samar, Paragua, P. Princesa, Butuan, Panaon.

2. C. pusilla $\operatorname{Var}$ C $\cap \mathrm{N}, \mathrm{v}$ Alucay, Buqué.

(M S $\mathrm{l}$.

Hab. Luzón, Laguna, Calamba, Bataan, Balabac.

3. C. rosea, $V$. $\&$ N. v.

(M. S. T.)

Eolophus roseicapillus V. Bouch. Cat. An. 107 i 876 . Ps. roseus, Tem. pl. 8I.

Hab. Australia,

4. C. sulphurea, Gm. (B. I4 . \$ N. v.

(M. S. T.)

Kakatoes à huppe jaune.

Psittacus sulphureus, Lin. (B. pl. 14).

C. sulphurea, Vieill; Bouch. Cat. Av. p. Io6, n. ${ }^{\circ} 3146$ (1876). Hab. Molucas.

5. C. nasica, Tem. 6. (T. 33I) N. v. Yacul. (T. $33 \mathbf{I}$ )

(M. S. 'I.)

Licmetis nasicus, (Tem). lam. 331; Cat. Av. p. 107. n." 316 ( Bouch.

Hab. California. 
6. C. moluccensis, (im. (1). 498 ) N. v. Cacalla.

(M. S. T.)

( . moluceensis, (Gm.) S. .V. 1. p. $33 \mathrm{I}, \mathrm{n} .{ }^{\circ} 90$ (1788); Bouch. (ial. An. p. $106, \mathrm{n}^{\circ} 3 \mathrm{I}+3(18 ; 6)$.

Hab. Nolucas, Ceram, Amboina, Joló?

2. Gen. MiCROGLOSSUS, GeògF.

7. IM. aterrimus, (Gm.) of N. v. Rasmalo.

(M. S. T.)

Ps. aterrimus, (Gm.) S. N. 1, p. 330 n. 93 (1788); gigas, lath. griscus, Bech.; goliath, Kuhl.

M. ater, Less. Eda'. B. 310. Lew. Perr. 11-13. Vil. La Creac. 1. 22. 1. $21(1873$.

Hab. N. ${ }^{a}$ Guinea.

Fam. SITACIDAS.

I. Gen. Tanygnathus, Wagler.

I. T. luzonensis, (Briss) ơ N. v. Picoy.

(M. S. T)

Psittacus lucionensis, Brisson, Orn. iv. p. 295, no. +1 , «Lüļon». Limn., S. $N$. i. p. г 66, n. ${ }^{\circ}$ I $($ 1 766), ex Briss.

La Perruche de l'isle de Luçon, Somerat, Voy. Nouv. Guin. p. $80 \mathrm{pl} .44$.

La Perruche aux ailes chamarées, Buffon, Hist. Nat. vi. p. I 51 . Le Vaill, Pers. pl 6o.

Perroquet de l'isle de Luçon, D'Aubent. Pl. Enl. 287 .

Ps. marginatus, L. S. Müller, S. N. Suppl. p. 77, no. 54 (г776), ex Buffon.

Ps. gala, Bodd. Tabl. Pl. Enl. p. I7 (1783) ex D'Aubent.

Ps. pileatus, Scopoli, Del. Fl. Fantn. Insubr. ii. p. (85) 86, no. 2 I (i 786), ex Sonn.

Ps. olivaceus, Gm. S. N. i. p. 326, no. 76 (1 788$)$ en Sonn.

La Perruche aux ailes chamarées, Le Vaillant, Perr. pl. 6o.

Ps. phrygrius, Shaw. Gen. Sool. viii. pt. 2 I p. 42 I (1811). ex Le Vaillani.

Eclectus luconensis (Lim.), O. Finsch, Monogr. Papag. ii. p. 362.

Tanygnathus muelleri, ap. Walden \& Layard, Ibis, 1872 p. 95 , «Negros».

T. luzoniensis, Sharpe. Tr. L. Soc. 2. series 1876. Zool 1 p. $3^{\text {I } 2 .}$ Wald. Tr. Z. S. g. p. I 33 .

Hab. Luzón, Cagayán, Guimarás, Negros, Valencia, Cebú, Leyte, Mindoro, Marinducue, Masbate, \$s. Bernardino, Dumalon, Paragua, P. Princesa, Panay, Mindanao, Zarrboanga, Butuan, Surigao, Basilan, Malanipa, Joló, Cagayán de Joló, Lapac.

2. T. viridis, $O^{*}$ ? Var. N. v. Quilaquil.

(M. S. T )

Hab. Paragua, Carangac, Luzón, Ilocos Norte, Samar, Calbayog, Culión, Calamianes. 
3 T Cyanescens, $O^{*}$, v'ar N. v. Calangag.

Hab. Luzón, Cagayán, Negros.

4. T. torquatus, $0^{*}, 0$ vir N. V. Calangag.

¿Psittacus manillensis; Bech.

¿Palicornis torquatus, (Bodel.) Finsch.

Hab. Sa nar, Paric, Lazín, Cagreyán, Ilocos.

5. T. everetti, Tw. $\sigma^{*} \rho \mathrm{N}$. v. Carang $\iota c$.

(M. S. T.)

T. everetti, Twe. An. et Mag. N. H. ser. 4. vol. 2o. p. 533. (1877).

T. su.natramus, (Rxffles), G. R. Giray, Hand-list. n. ${ }^{0} 82+8$ «Island of Sama.» Sharbe, on Birds. coll. by. Steere; Trans. Lin. Soc. 1878. 2. ser. p. 3I2.

H.zb. Negros, Mindana, Butuan, Samar.

\section{T. burbidgei. Shat ps N. v.}

T. burbilgei, Sharpe, P. Z. S. 1879, p. 313; Guillerm. Voy of the Jacht «Marchesa», 1885, p. 252. Has. Jols.

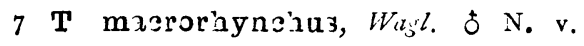

Ps. macrorhynchus, L. (nusutus, Lath. megalorhynchus, Bodd. T. masr. Wigl. lin. 713. Lev. Perr. t. 83. Hab. Molucas, N." Guinea.

2. Gén. Triclaria, Wagi.

8. T. cyanogaster, Shuze. (B. 6I), b N. v.

(M S. T)

Ps. cyanogaster, Vieill.; malachitaceus, Spix. Av. Bras. 28 Bourj. Perr. 57.

Hab. Brasil.

3. Gén. FLITYCERCUS, Vig.

9. Pl. eximins, Shriv. U'? N. v. R s;llı.

(.I. S T.)

Perruche omnicolore, $B u f f$.

Psittacus eximius, Sharv-Leraill. pl. 28-29.

Hab. Singapur, Molucas.

\section{Gen CYCLOPSITTA, H. ET J.}

ió C. lunulata, Scop. $0^{7} \rho \mathrm{N}$. v Bombuctuc.

(il S. T.)

Petite Perruche á collier de l'isle de Luçon, troisieme espece, Sonn.

Voy. Nour. Gunn. p. 77, pl. 39. 
Psittacus lunulatus, Scopoli. Del. Fl. Faun. Insubr. ii. p. 86, no. $24(1-86)$, ex Sonn.

Ps. torquatus, Gm. S. N. i. p. 351, no. 1 $34(1-83)$, ex Sonn.; Lear. Illustr. Psitt. pl. tos

Ps. loxia, Cur. Mus. Paris, Lesson, Tr. p. 204, «Manla», adult (1831); Pucheran, Rev. Mag. Zool. 1853, p. 163; Bourjot, tom. cit. pl. 94 .

Psittacula squamo-torquata, Bourjot, Perr. pl. 97 (1837-8), en Lear.

(yclopsitta loxia, (CuO.), Bp. Rea' et Mag. Zool. i 854 , p. i 54 .

Ps. lunulati et loxias, O. Finsch, Monogr. Papag. ii. pp. I16, 618; v. Martens, $J . f . O .1866$, p. 21, nos. 118, 117; $G$. R. Gray. Hand-list, nos. 8377,8376 .

Psittacula lunulata, Gray, Schlegel, Mus. Pays-Bas, Psittaci p. 72.

Cy. lunulata, IVald. On birds of. Philippines, p. 116, 301. (1875); Sharpe T. Lin So:. 2. ser. 1877 . p. 312, Tw. P. Z. S. 1877 p. 689; id, Works. p. 5I4. I88I.

Hab. Luzón, Cagayán, Pamplona, Manila, San Mateo, Morong, Batang.ıs, Laguna, Samar, Zuinárraga, Mindanao, Butuan, Zamboanga.

11. C. squammotorquata, $\sigma \circ\left(a^{\prime}(a)\right.$. N. v Guringag. (M. S. T.) Hab. Luzón, Ilocos, Cagayán.

32. C. mindanensis, (Steeri). $\mathrm{N}$ v. Quiliquil. (M S T.)

Ifab. Mindanao, Zamboanga.

5 Gén PRIONITURUS, Wagi..

13 P. discurus. (Vicill.) o $\circ \mathrm{N}$ v. Caguit

(M. S. T:)

Psittacus discurus, Vieillot, Gal. des $O$ s. i. p. 7, pl. 26, « Mindanao" (1825),

Ps. spatuliger, Bourjot ( 9$),$ Perr. pl. 53, «Mindanao» (1837-8).

Pionias discurus (V.eill.), O. F nsch, Monogr. Papag. ii. p. for.

Irodiscus discurus (Vie.ll.), G. R. Gray, Mand-list, no. 8047 . «Philippine»Isl.»

Ur. spatuliger (Bourjot), G. R. Gray, ton. cit. no. 8048, «Manila».

Prioniturus discurus, Wald. Brds. inhab. Phil.pp. p. 300. (1881) Tw. P. Z. S. p. 535. (1877) (1878) Sharpe. Tr. L. Soc. p. 31 2: id. P. Z. S. 1879 p. 133 Guill. Voy. of. March. 1885. P. 133 .

Hab. Luzón, Manila, Bulacan, Morong, Laguna, Batangas, Samar, Paranas, Mindor:, San Bernardino, Guimarás, Negros, Mindanao, Zamboanga, Pasananca, Surigan, Placer, Cebú, Panaton, Basilan, Joló, Balabac.

I4 P. cyaniceps, of z'ar. N. v. id.

$\left(\begin{array}{lll}M & S & \mathrm{~T}\end{array}\right)$

Hab. Luzón, Cagayán, Samar, Paríc.

I5.P. cianeus, ơ N v. Quilit.

(M. S T)

Hab. Puragua P. Priacasa, Samır, Borongan. 
16. P. luzonensis, (Stirre) o $0 \mathrm{~N}$. v. id

Hab. Luzón, Mindanao, Marinduquc.

17. P. mindorensis, Stecte. of of N.v. id.

(M. S. T.)

Trab. Mindoro.

6. Gén LORICULUS, Bhyth.

18. T. philippinensis, (Briss). o م N. v. Culasisi.

(M S. T.)

Psittacula philippensis, Briss. Orn. iv. p. 392, no. 87, «Philippines» ( т60); Meyen, Nov. Act. Ac. C. L. C. Nht. Cur. xvi. Suppl. prim. p. 9t, «Manila».

Le coulacissi, Buffon, Hist. Nat. Os. vi. p. i69, ex Brisson.

Perrur:he des Philippines, D'Aubent. Pl. Enl. 520 f. I.

P'sittacus philippensis, L. S. Mïller S. N. S'appl. p. do, no. os (1776); ex Buffon.

Ps. galgulus, var. b Gm. S. N. i. p. 349 , no. 46 , ex Briss.

I'sittacula rubrifrons, Vigors, Phil. Mag. 1831, 1). 1+7, no. 11; Lear, Illustr. Psitl. pl. $4 \mathrm{I}$.

P's. culacissi, Wrager, Mongor. p. 626 (1832); O. Finsch, Monogr. Papag. ii. 1. 705; G. R. Gray. Hand-li;t, no. 81 \&.

Petite Perruche de l'isle de Luçon Sonn. Voy. Nouv. Guin. 1'. 77, pl. 40 fig. sup., $M$.

?Psittacus melanopterus, Scopoli, Del. Fl. Fann. Insubr. ii. p. 86, no. $23, M .(1786)$, ex som.

Ps. ninor ( $1 / 1)$, Gm. tomi. cit p, 351, no. I 35 (1 788$)$, ex Sonn.

loriculus philippensis, Wald. on b.rds of Philipp. 1877 .

Hab. Luzón, Manila, Laguna, Morong, Bulacan, Batangas, Catbalonga, Negros, Marinduque, Joló, Mindanao.

19. I siquijorensis, S«cre. N. v Cusi.

Hab. Siquijor.

20. L. mindorensis, Steere. $\delta$ \& $\mathrm{N}$ Cusi.

(M. S. 'T)

Hab. Mindoro.

2 I L. Worcesteri, Stecr.

(M. S. ' ' )

Hab. Leyte, Samar, Calbayog.

22. L. occipitalis, (Finsch) N. v Gulasisi.

Coryllis occipitalis, Finsch. Pap. 2. p. 7 I0. Ibis. $187+$ p. 2is. Tru. Works. p. 530. (1881) P. Z. S. 1877.1 .757$.

Hab. Mindanao.

23. I. chrysonotus, $S: l, N$. v. Caguit.

L. chrysonotus, Sclater, Ibis, 1872 , p. 323, pl. I I, «Zebu.» Hab. Cebú. 
24. I. hartlaubi, (Finsih,) t N. v. Caguit.

(M. S. 'T.)

Coryllis hartlaubi, O. Finsch, Monogr. Papag. ii. p. 701, «Mindanao» (1868.)

L. melanopterus, (Scop.); G. R. Gray, List. Psitt. Brit. Mus. p. 55, fide $O$. Finsch 1. c.

L. apicacalis, Sunancé, G. R. Gray, tom. cit. p. 56, fide 1. c. nec Souance.

:Petite Perruche de 1'isle de Luçon, Sonn. Voy. Nouv. Guin p. 77, pl. 40, fig. inf. i.

¿Psittacus melanonti rus, Scopoli, Del. Fil. Faun. Insubr. il. p. 86, no. 23, $\pm(178 ;)$, ex Somn.

1.. melanopterus et apicalis, G. R. Gray, Hand-list, nos. $8_{\text {I } 75}, 8$ I 7 6.

L. apicalis, Souance, v. Martens, J. f. O. 1866 , p. 21, no. I I5, nec Sonancé.

L. bonapartei, Son. Rev. et Mag. de Zool. $185^{6}$. p. 222.

L. hartlaubi, Tw. P. Z. S. 1877 . p. 8I9.

Hab. Mindana , Leyte, Bohol, Dumaguete, Bazol, Dinagat, Basilan, Mindanao, Surigao, Placer, Butuan, Joló.

25. L. regulus, Sozv. むf N v. Caguit, Cusi.

(M. S. T)

Loriculus regulus, Sonancé, Rev. et Mag. Zool. 1856, p. 22, "patr. incert;" v. Martens, J. $f . O .1866$, p. 2 1, no. $114 ; G$. R. Gray, List Brit. Mus.

L. panayensis, Tw. Works. p. 462.

Hab. Negros, Panay, Iloilo, Dumaguete, Guimarás, Masbate, Mindanao.

26 T, galgulus, (Lin) (B. 190) † \& N. v. Talisoc.

(M. S. T')

Ps. galgulus, Lin; Alavigula, Bod; pumilio, Scop. pl. igo Edw. B. 293 2. Sonn. Voy. Gininée. 38. Bourj. Perr. 88. Horsf., Tr. Lin. Soc. xiii, p. I 82 (1821); Raffl. ibid. p. 281 (1822); S. Mïll., Verhandl. Lauden Volk. p. Io7, 381 (1839-18+4).

Psittaculus galgulus, Sws. Class. of B. ii. p. 303 (1837); Mottl. $y$ Dilw., Contr. N. H. of Lab. p. 27 ( 1855 ).

Loriculus galgulus, Bp. Consp. i. p. 6 (1850); Rosemb., Journ. f. Orn. 1862 p. 60.; Sclat. P. Z. S. 1863 p. 208 , esp. I $5 .:$ Vall. P. Z. S. 1864 p. 287 , esp. 51; Schleg. Mus. P. B. Psittaci, p. I 3 I (1864); G. R. Gray, Haud List, ii. p. $5_{2}^{2}$ esp. 8169 (1870); Salv. Cat. Ucc. di Borneo, p. 26 (1874).

Coryllis galgulus, Finsch, Die Papag. ii. p. 699, esp. 286 (1868). Hab. Borneo, Sumatra.

\section{Gén. ecclectus, Wagler.}

27. E. grandis. (Gm) (B. 683) む\& N. v.. Ecléctido rojo. (M S. T )

Ps. grandis, (Gin.) Syst. Nat. 1. p. 335, n. 102..(1788.)

ji. grandis, Wagler.

E. ceylonensis, Gr. ex Bodd. pl. 683, Brown, Ill. Zool. t. 6 Levai. Parr. t. I 26- I 28.

Hab. Ternate, Molucas. 
28. E. polychlorus. (Siop) \& N. v. Ecléctids verde. (M. S. T.)

Ps. magnus et sinensis, Gm.; viridis, Lath.; lateralis, $S h: w$. Mascarinus prasinus, Less-Psitacoides magnus, Wagl. Fe. polychloros, Gr. ex Scop.) Pl. 514. Edw'. B. t. 231. I.ev. Perr. 132.

E. polychlorus, Scop. Del. Filor. et Fann. Ins. p. 87. n. 27, (1786.)

Hab. N." Guinea, I. Aru, N." Bretaña...

\section{Gén. MELOPSITTACUS, Goui.D.}

29 M. undulatus, Shute. $t \&$ N. v Eufemia ondulada. (M S T)

Ps. undulatus, Shaw, Nat. Mis. t. 673; Gould Austr. t. 43 Nat. libr. Parr. t. 28; Bourj. Perr. t. 9; Vil. La Creación p. $3^{6}$ f. $29(1873$.

Psittacus, Nanodes, Euphema, Euphemia undulata (auctorum.)

Hab. Australia.

\section{Fam. TRICOGLÓSIDAS.}

I Gén LORILS, Br.

I. L. domicella, Lin. (B. 84.) N. v. Castorie.

Ps. domicella, L.; raja, Shaw.; rex, Bechst.; radhea, Vieill. Pl. 84 y 119. Lev. t. 94, 95. Lear. Parr. t. 37.

Hab. Molucas.

2 L. garrulus, (Lin.) \& $\&$ (B. 216.) N. v.

(M. S. T.)

Ps. ruber, It. Wgoth. 137. Lorius ceramensis, Briss. av. 4. p. 215.n. 13. Ps. garrulus, Lin. Syst. Nat. t. 1.0 p. 333. n.० 25. (1789.)

Hab. Halmahara, Molucas, Gilolo, Batchian.

\section{Gén. Chalcopsittaces, salv.}

3. Ch. ater, $(S \operatorname{sop}$.$) t N$ v.

(M S. T.)

Ps. novæ-guineæ, Gm. ater, Scop. Platicercus ater, Gr. Sonn Voy. 110 Lev. Perr. 109.

Hab. Molucas, N..$^{\text {G Guinea. }}$

3. Gén. Coriphilus, wagl.

4. C. placene, $(T e m$.$) \& N. v.$

(M S. T.)

Hab. N. Guinea. 
4. Gén. Charmosyna, Wagr.

5. Ch. papuensis, $\left(G^{\prime} \prime \prime\right)$ (Prev. 36.) \& N v. Rasmal. (M S. T.)

Perruche lori papoua, Sonil. Voy.

Psitticus pipuensis, Gin. learall. pl. 77 ; javan cus, Gm.; jabon:cus et bontü, Lath; omnicolor, Licht; lchtensteini, Bechit; C.'. papnensis, Wagl; (h. papua, Gr. ex Scop.) Sonn. Voy. t. 111. Hab. N. a Guinea.

5. Gán. Calyptorhynchus, Satr.

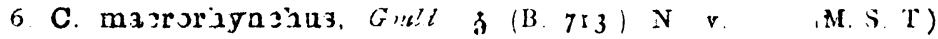

Ps. macrorhyncus, L. nasutus, Lath; megalorhyuchus, Bodt.

'T. macrorhynchus, Wagl. Pl. 71.3. Lev. Perr. \$"3.

Hab. Norte de Australia, ¿Joló?

6 Gén. TRICOGLossus, Wig.

7. T. hæmatr pus. Gm. of N v

$\left(\begin{array}{llll}\text { M } & \mathrm{S} & \mathrm{T}\end{array}\right)$

Perruche des Moluques, Buff. pl. 743 .

Psittarus hamatopus, Gm.-Leva:ll. pl. 24-26.

Hab. Molucis.

\section{Orden $2 .^{\circ}$ RAPACES.}

\section{Sub-orden: HALCONES.}

\section{Fam. I. $^{\text {a }}$ VULTÚRIDAS.}

Subfam. VULTURINAS.

I. Gén GYPS, Sav.

I. G. hizpanioleazis, Shrp. \& N. v. Buitre.

(M $\quad$ S. T.)

Vultur fulvus, Naum. Vög. Deutschl. i. p. 162, Taf: 2, 1822);

Werner, Atlas, Rapaces, pl. 2 (1827); Schl. u. Susem. Vög.

Eur. p. 12, pl. 2 (1839); Dubois, Ols. d' Eur. pls. 2, 3 (1862).

V. chassefiente, Rüpp. N. W. Vög. p. 47 (1835).

V. kolbii. Carst. Naum. ii. Heft i. p. 76 (1852).

?V. fulvus occidentalis, Heugl. Orn. N. O.-Afr. i. p. I (1869).

Gyps hispaniolensis, Sharpe. Cat. Brit. Mus. p. 6. (1874). Hab. España, Europa, Africa. 


\section{Fam. 2.a FALCÓNIDAS.}

\section{Subfam. ACCIPITRINAS. \\ I. Gén. CIRCUS, LACEP.}

1. C. spilorotus, Kaup. \& $\&$ N. v. Balicbalic.

(II S. T.)

Circus spilonotus, Kaup, Contr. Orn. 18.51 , p. 59; Strickl. Oru. Syn. p. I 57 (2855); Swinhoe, Ib1s, 1863, p. 213 v, 1865, p. 230, et I 870, p. 87; Gray, Hand-l B. i. p. 36 (1869); Sizinh. P. Z. S. 1871 , p. 342; Taczan. J. f. O. 1872 , p. 349, Gurwey, Ibis, 1868 ; p. 356. Sharpe. Cat. Bi: Mus. p. $58(1874)$. Wald. on birds of Phitip. 1875. Tw. Works. p. 311: P. Z. S. 1877 p. 686.

Hab. Luzón, Ilocos N., Cagayan, Pampanga; Dauria, China, Península Malayana.

2. C. melancleucus, Forst. $0: 0 \mathrm{~N}$ v. Balicbalic

(M. S. T.)

Falco melanoleucus, Forst Indisch. Zool. p. i1, pl. I 2 (178r).

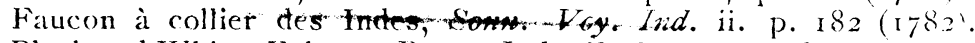
Black-and-White Falcon, Penn. Ind. Zool. p. 33, pl. 2 ( ( $\left.7 g^{\prime}\right)$. lath. Synop. 1. p. 81, n." 65, Ceylon, ex ren.

Tchoug; Levaill. Ois. $a^{\prime}$ Afr. i. p. 133, pl. 32 (1799); Sund. Crit. om. Levaill. p. 27 (1857).

Circus melanoleucus, Vieill. N. Dict. d'Hist. Nat. iv. p. 405 (1816); Less. Traité, p. 87 (1831); Gray, Gen. B. i. p. 32 (1845); Strickl. Orn. Syn. p. I 54 (1855); Jerd. B. Ind. i. p. 98 1862); Schl. Mus. P.-B. Circi, p. \& (1862); Gray, Hand-!, $B$. i. p. 37 (1869); Hume, Rough Notes, ii. p. 307 (1870; Swinh. P. Z. S. 1871 , p. 34; Holdsw. P. Z. S. 1872 , p. 4 rt. Wald.. Tr. Z. S. vol. 9. part. 2, p. 143. Sharpe. Cat. Brit. Mus. p. 6i (I 874$)$.

Strigiceps melanoleucus, Kaup, Mus. Sinck, iii. p. 258 (1845); Bp. Consp. i. p. 35 (1850).

C. cyaneus hudsonius, Sch. M. P.-B., Circi, p. 3. Luzoni, Gurn. Ibis, 1870, p. 4.44 . China.

Hab. Negros, Luzón, Cagayan, Pamplona, Joló; Nepal, India,

3. C. æruginosus, $\operatorname{Lin} \sigma \rho$ N. v. Dumarnguit.

(M. S. T.)

The Meor-Buzzard, Albin, Bird. i. pl. 3. (1731).

Le Busard de Marais, Briss Orn. i, p. 401 (1760).

Le Busard roux, Briss. Orn. i. p. 404 (1760).

Falco aruyinosus, Linn. S. N. i. p. I 30 (1766); Buff. Pl. Enl. i. p. $424(1783)$.

La Harpaye, Buff. Pl. Enl. i. pl. 460 (1783).

Faleo rufus, Gm. S. N. i. p. 266 (1788, ex Buff.); Ten:m. Man. i. p. 69 (1820); Naum. Vog. Deutschl. i. p. 378, Taf. 37, 38. fig. I (1822); Wagner, Atlas, Rapaces, p. 27 (1827); Schl.u. Susen. Vög. Eur. Tat. 36 (1839); Yarr. Brit. B. i. p. go (1840).

F. arundinaceus, Bechst. Orn. Taschenb. p. 23 (1802). 
Circus aruginosus, Savign. Syst. Ois. Egyte, p. 90 (1809); Less. Traite, p. 86 (1831); Macgill. Brit. iii. p. 382 (1840); Graj, Gon. B. i. p. 32 (1845); Bp. Consp. i. p. 34 (1850); Schl. Vos. Nodcrl. pls. 20-22 (1854); Strickl. Oru. Syn. p. 146 (1855; IIcwits. Eggs Brit. B. i. p. 44 (1856); Fritsch, Vog. Eur. tab. 9. fig. 5, tab. Io. figs. 5-7 (x 858$)$; Schl. Mus. P.-B. Circi, p. I I (1862); Jerd. B. Ihid. i. p. 99 (1862); Nezut. Ooth. Wolley. p. I 43 (I864); Bettoni, Ucic. Lomb. i. pls. 31, 3Ia (1865); Suindev. Su. Fogl. p. 222 , pl. xxriii. figs. 4, 5 (1866); Loche, Sci. Alsir. Ois p. 82 (1867); Degl. \& Gerbe, Orn, Europ. i. 1. I05 (1867); Gould, B. Gt. Br. pt. xiii. (r 868); Gray, Han-l. B.. i. ए). $3^{6}$ ( I 869 ); Hengl. Orn. N. O.Afr. p. IO3 (1869); Planf. Geol. \& Zool. Abyss. p. 3or (i 870 ); Hume, Rouch Noies, ii. p. 314 (1870); Ayres, Ibis, 1871, p. 147; Salvadi Fan. Ital. Ucc. p. 24 (1871); Newt. ed Yarr. Brit. B. " p. I 27 (г87 I); Shelley, B. Egypt, p. I8г (1872); Bocage, Jorus Lisb. I872, p. I; Hume, Stray F. i. p. I6o (1873); Schl. M.... P.-B. Revue Accipitr. p. 5o (1873). Sharpe. Cat. Brit. Mus. p. $69(1874)$.

C. rufus, Savign. Syst. Oor. Ois. Esypte; p. 9i (1 809\%); Less. Traité, p. 86 (1831); Gould, B. Eur. pl. 37 (I837).

Accipiter circus, Pall. Zoogr. Rosso-As. i. p. 362 (I 8 I I).

Pygargus rufus, Koch, Sys.: Baier. Zool. i. p. II9 (1816).

Buteo aruginosus, Flem. Brit. An. p. 55 (1828).

C. variegarus, Sylies, $P . Z$. S. 1830 , p. 8 I.

C. arundinaceus, Brehm, Vög. Deutschl. p. 9r (1831).

C. aquaticus, Brhem, Isis, 1832, p. 836 .

C. sykesii, Less. Compl. Buff. vii. p. 16 i (1836).

Buteo rufus, Couch, Corn. Fann. p, i i (i $83^{8}$ ).

Hab. Luzón, Cagayan, Ilocos, Laguna, Paragua; Europa, África, India, China, Formosa.

4. C. cinerescens, $\sigma \circ N . \quad v \cdot T_{a g} z a i$.

(.I. S. T.)

Hab. Luzón, Cagayan, Samar.

5. C. philippinensis? Steere. $\sigma \circ \mathrm{N}$ v.

Hab. Paragua, P. Princesa, Mindanao, Guimarás, Cagayan, Laguna, Luzón.

6. C striatus $\sigma \circ$ N. v. Dumaraguit.

Mab. Borongan, Samar, Paragua.

2. Gén. ASTUR, Lacer.

7. A palumbarius, Lin $\ddagger$ N. v. Azor.

(M. S. T)

The Goshawk, Albin, N. H. Birds. ii. p. 8, pl. 8 (1734); Salrin \& Brcdr. Falconry, p. I03, pls. xviii. xix. (1855).

L'Autour, Briss. Orn. i. p. 317 (1760); Buff. Pl. Enl. i. pl. 418 (1770); Scht. \& Verst. Traité Fauc. pls. x. \& xi. (1844). Le Gros Busard, Liris. Orn. i. p. 398 (1760). 
L? Busaril rarié, Briss. Orn. i. p. 400 (1 760$)$.

Falco palu nbarius, Limn. S. N. i. p. г 30 (1760); Daud. Trats, pls. r, 2 (г 800$)$; Temm. Man. d'Oriz. i. p. 55 (1820); Naum. Vüg. Deutsch. i. p. 249, pls. I7, I8 (1822); Werner, Atlas, Rapaces, pl. 2 I (1827); S'chl. u. Stısem. Vög. Eur. 'Taf. 25 (1839-45); Yarr. Brit. B. i. p. 57 (18+3).

L'Autour sors, i. Buff. Pl. Enl. i. pl. $46 \mathrm{r}$ ( г 770$)$.

Filco marginatus, Lath. Ind. Orn. i. p. 26 (i 790$)$.

F. albescens, Bott. Tikl. Pl. Enl. p. 25 (i 783 ).

F. dubius, Sharrm. Mus. Carls. pl. 27 (1787); Sundev. Crit. p. 6. $\left(185^{8}\right)$.

F. gallinarus, G.n. S. N. i. p. $\mathbf{2}^{6}$ (1788).

F. nxius, $G n . S N$. 1. p. 256 (1788).

F. incertus, Lath. Int. Orit. i. p. 32 (1790).

Dadalion palu nbarius, S.zvigny. Hist. Nat. Ois. d'Egypte, p. ${ }^{26}$ T (18.)8); Loss. Tr rité, p. 65 (1831).

Ac.i jiter astur, P.x!!. Zoorr. Rosso-As. i. p. 357 (18II).

Sparivis palumbarius, Vieull, $N$. Dic. d'Hist. Nat. x. p. 331

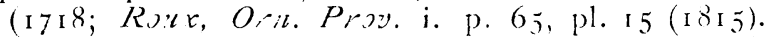

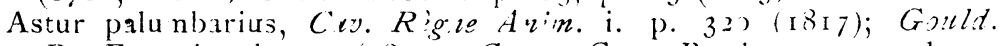

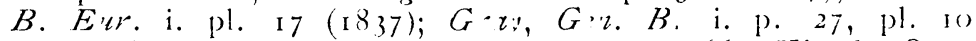
fir. I. (18+9); Kvetp, Cons. Or2. 185 ) p. 65; Kjo-b. Orn. I) ru. Aft. iv. fis. 5 (1851); Morsf. \& Moore, B. Mils. E. I. Co. i. p. 4 I (185t); Sch!. Vog. Ne.terl. pls. I I, I 2 (185t-58); B3. Rev. et M.eg. de Zool 1854, p. 539; Strickl. Orn. Syin. p. II5 (I855); Hewts. Eoss Br. B. i. p. 3t, pl. xi. (1856); Frits=h, Vjg. Eur. tib. i. (1853); S: t. Dier. Nod:r!. Vog. pl. г. figs. 9, го, г) (1951); it $M$ L. P. B. Aisres, p. í (1852); Jert. B. Int. i. p. t5 (1952); $N$ xt. Oxth, Wollej.

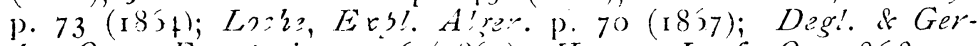
be, Orn. Europ. i. p. $9^{5}$ (18'7); H,mey J. f. O. 1868 , p. 253; Heugl. Ora. N. O.-Afir. i. p. 59 (IS5y); Suztev. Su. Fogl. pl. xxvii. figs. 2, 3 (18j)); Hule, $B$ ) ugh Notss, i. p. I 2 (1859); Graty Hant-l. B. i. p. 29 (1859); Gould, B. Gt.

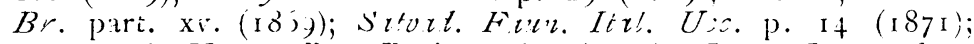
Newt. ed. Yarr. Br. B. i. p. 83 (187I); Tert. Ibs, 1871, p. 243; Sewinh. P. Z. S. 1871 , p. 3+1; 1871, Finsch, Nuc'r. Heugl. 1. xxiv 1871, Shelley, B. Egypt, p. 185 (I772). Shxrpe. Cat. Brit. Mus. p. 95 (1874).

Buteo palumbarius, Flem. Brit. An. i. p. 54 (1828)

Astur gallinarun, B:2, n, Vjr. Dousvihl. p. 83 (г831).

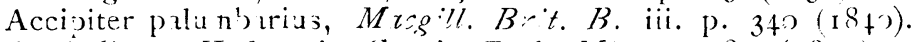

A. iadicus, $H$ ) toss. in Groxy's Zool. $M$ so. p. 8I (18+t).

A. brachyragacha;, Brohn, N.t:n. p. 25 ).

A. piradoxus, Brehm, N.un. p. 259.

Hab. Europa, Corea, Cnina.

8. A trivirgatus, $T_{e: n} \sigma o$ N. v. Ciเııni.

(M. S. T.)

Falco trivirgatus, T?nn. P!. Col. i. pl. 3)3 «Stmatra» (182t).

Nisus trivirgatus, Less. $M$ un. d.Orz. i. p. 93 (1828).

Astur trivirgatus, Cuv. R:gne An. i. p. 332 (1829); Grav, Gen. B. i. p. 27 is 89 ; B. Consp. i. p. 31 (185); St.ictl. Orit, Sy'l. p. 221 (1955); Jer.t. B. In.t. i. p. 47 (1862); Schl. Mus. 
P.-B. Astures, p. 22 (1862); id. Vog. Nederl. Ind. Valko. pp. 18, 57, pl. Io (1866); Vall. Ib.s, 18 6 s', p. 6; Graly, Hand-!. i. p. 30 (1860); Holitsa. P. Z. S. $18 ; 2$, p. +10; Ëchl. Reoule Accipitr. D. 65 (1873). Sharp. Ca'. Brat. Mus. p. 105 (1574); T. Salv. Uccelli di Rorneo. p. 17 (1874).

Dadalion trivirgatum, Leess. Traité, p. 60 (1831).

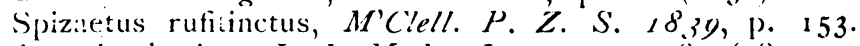

A. palumbarius, Jerd. Madi. Journ. x. p. 85 (1839).

A. cristatus, Gray, Ann. N. 1\%. xi. p. $371(18+3)$;

A. indicus, Hodgs. in Gray's Zool. Misc. p. Si (18+4); Gray, Cat. Accipitr. $18+4$, p. 33.

Lophospiza trivirgata, Kaup, Contr. Orn. 18.50, p. 6.5; Gray, Cat. Hodgs. Coll. 1863 , p. 19; Hume, Rough Noles, i. p. 116 (1869); Swinh. P. Z. S. 1871, p. 3+1. Wilt. on Birds. of Philipp. 1877 llorks. of Tiv. p. 587 (1881).

Hab. Lizón, Cagayan, Paragua, P. Princesa, Calamianes, (Culion, Samar, Leyte, Guimarás, Mindanao, Surigao, Java, Sumatra, Formosa, China, Borneo.

9. A soloensis, Lrth C'. N. v Sicut.

(M S T )

Falco soloensis, Lath. Gen. Hist. i. p. 209 (1821).

1)adalion soloensis, Horsf. Tr. Linn. Soc. xiii. p. 137 (1822).

Falco nisus (pequeno Raff. Tr. L mn. Soc.var.), xiii. p. 278 (1822'.

Accipiter s.luensis, Vig. Zuol. Journ. i. p. 338 (1824); Ciray, Gen. B. i. p. 29 (1849); Striekl. Orn. Syn. p. IO7 (1855); Swinh. P. Z. S. 1863 , p. 315; Scl. P. Z. S. 1853 , p. 207 .

Astur soloensis, Less. M.m. d'Or-n. i. p. $9+$ (1823).

Nisus cuculoides, Less. Man. d'Orn. i. p. $97(1828)$; id. Traite, p. 61 ( 1831$)$.

N. minutus, Less. Traité, p. 60 (1831); Pucher. Rev. Zool. 1850, 1). 210 .

Táchyspiza soloensis, Kaup, Classif. Säug. u. Vög. p. I I7 (1944); id. Contr. Orn. 1850, p. 63; Bp. Res. et Hag. de Zool. P. 538; Kaup, P. Z. S. 1867 , p. 172; Walden, Tr. Z. S. viii. Pp. 34,1 IO $(1872)$.

Micronisus soluensis, Gray, Cat. Accipitr. B. M. p. 75 (1848); . Bp. Consp. i. p. 33 (1850); Horsf. \& Moore, Cat. B. Mus. E. I. Co. i. p. $3^{8}$ (1854); Gray, P. Z. S. 1860, p. 344; Sruinh. P. Z. S. 1863 , p. 261; P'elz. Reis. Novara, Vög. p. 12 (1865); Wall. Ibis. 1868, p. I 2; Gray, Hand-l. B. i. p. 35 (1869); Swinh. P. Z. S. 1871, p. 342. Salv. Cat. di Vecelle di Borneo p. I7 (II74).

M. badius, Swinh. Ibis, 1860 , p. 359.

Accipiter virgatus, Swinh. Ibis, $185 \%$ p. 264.

Nisus soloensis, Schl. Mus. P.-B. Astures, P. 44 (1862); Blyth, Ibis, 1863 , p. 16; Schl. Vog. Nederl. Ind., Valkv. pp. 28, 66, pl. I9. figs. 4-6 (1866); id. Revile Accipitr. p. 97 (1873).

Astur soloensis; Sharp. Cat."Brit. Mus. p. II 4 lam. 4 fig. I (1874.)

Hab. Samar, Borongan, Java, China, Malaca, Sumatra.

10 A. cuculoides, Tim. $\sigma^{\circ}$ N. v. Taui.

(M S. T )

Falco uculcites, Temm. Pi. Col. i. pls. Iro, I39 (1823). 
Nisus cuculoides, Less. Man. dOrn. i. p. 97 (1828); ad Trante, p. 6 I $(183 \mathrm{I})$.

Astur cuculoides, Sharpe. Cat. Brit. Mus. p. I I5 lani. 4 fig. 2 (I 874$)$.

Hab. Luzón, Cagayan, Ilocos N., China, Célebes.

II. A. poliopsides $0^{7} .0$ N. v. Taui.

(M. S. T.)

Hab. Calamiznes, Culion.

3 Gén. ACCIPITER, Briss.

12. A. nisus, (Lina) $O^{*}: \mathrm{N}$ v. Gravilán comúa

(M s. i.)

The Sparrow-Hawh, Albin, Nat. Hist. B. i. pl. 5 (i 73 I).

L'Epervier, Briss. Orn. i. p. 3 rо (1760); Buff. Pi. Ėhl. i. pls. 4 I 2,467 (1783).

L'Epervier tacheté, Briss. Orn. i. p. 314 (1760).

Le petit Epervier, Briss. Orin. i. p. 315, pl. 30. fir. I ( 760 ).

Falco nisus, Limn. S. N. i. p. г зо (г766); Naum. Vör. Deul. i. p. 258, 'Taf. I9, 20 (1822); Yarr. Brit. B. i. p. $62(18+3)$;

Schl. u. Susem. Vög. Eur. Taf. 29 ( I 839$)$.

F. minu us, Limn. S. $N$. i. p. г 3 I ( 766 , ex Briss.)

F. nisus major, Bek., Teutsche Orn. Heft iii. pls. I, 2 (c. г80)).

F. nisus minor, Bek., Teutsche Orn. Heft iii. pls. 3, 4, 5 (c) I $8(0)$ ).

Dadalion fringillarius, Sxvign. Ois. d'Egypte, p. 270 (1803).

Accipiter nisus, Pall. Zoogr. Rosso-As. i. p. 370 (I8I I); Gray, Gen. B. i. p. 29, pl. Io. fig. 4. (1849); Bp, Consp. i. p. 32 (1850); Strickl. Orn. Syn. p. IO4 (1855); Hewits. Esrsi Hirit. B. i. p. 35 .(1856); Fritsch, Vög. Eur. tab. 4. figs. 3, 4, 5 (1858); Jerd. B3. Ind. i. p. 5I (1862); Newt. Ooth. Woúley. p. 79 (1864); Gould, B. Gt. Br. pt. v. (1865); Brodr. Falc. Fav. pl. ii. (1865); Degl. et Gerbe, Orn. Eur. i. p. 99 (1867); Loche, Expl. Sci. Algér. i. p. 72 (1857); Bettoni, Ucc. Lomb. ii. tav. 58 (1869); Gray, Hxud.l. B. i. p. 31 (1860); Hume, Rough Notes, i. p. I24 (1869); Newt. ed. Yarr. Brit. B. i. p. 88 (1871); Salvad. Faun. Ital. Ucc. p. I5 (1871); Swinh. P. Z. S. 1871 , p. 34I; Sharpe \& Dresser, B. Eur. pt. ix. (1871); Shelley. B. Egyp', p. I85 (1872); Huine, Stray F. i. p. I 24 (1873). Sharpe. Cat. Brit. Mus. p. I 32 (1874.)

Ierax fringillarius, Leach Syst. Cat. Mamm. \&c. Brit. Mus. p. Io (1816).

Sparvius nisus, Vieill. N. Dict. x. p. 3 I9 (I8I7).

Ac. fringillarius, Vig. Zool. Journ. i. p. $3^{8}$ (1824); Gould. B. Eur. i. pl. I8 (1837).

Buteo nisus, Flem. Brit. An. i. p. 55 (1828).

Nisus communis, Less. Traité, p. 58 (1831).

N. elegans, Bremh, Vög. Doutschl. p. 83 (i 831 ).

N. fringillarum, Brehm, tom. cit. p. 87 (183I).

N. peregrinus, Brehm, tom. cit. p. 88 (1831).

Falco nisosimilis, Tic., J.A.S. B. ii. p. 57 I (1833).

Astur nisus, Keys. \& B'as. Virb. Eur. p. I 4o (I 8 to); Bailly, Orn. Sav, i. p. 75 (1853); Dubois; Ois. Belg. pls. I 5, I6 (1854); 
Schl. Vog. Nederl, pls. I3, It (185t); Sicrteo. So. Fogl. p. 22i, pl. xxriii. fig. I (1860().

Ac. nisosimilis, Blyth, J. A. S. B. xii. p. 3 I I ( $8+3)$; Streckl. Or-n Syn. p. 105 (1855).

A. subtypicus, Hoilgs. in Gray's Zool. Misc. p. 8I (18+4).

Astur major, Degl. Orn. Eir. i. p, $85(18+7)$.

N. fringillarius, Kanp, Contr. Orn. 1850, p. 6+; Soln!. Mus. P.-B. Astures, p. 28 (1852); H:U?. Orn. N. O.-Atr. p. 65 (1869); Schl. Remue Accipitr. p. 69 (1873).

N. major, Jaub. Mots. Eur. Orn. p. 29 (1851).

N. intercedens, Bremh, Naum. 18.55, p. 269. Hab. Eurupa, Asia, frica, China.

13. A. virgatus, (Reinzv) o o N. v. Lauin Sa .

(M. S. T.)

F.llco virgatus, Temm. Pl. Col. i. p. Io9 (1823, ex Reinw. MS.)

Accipiter virgatus, Vig. Zool. Journ. i. p. $33^{8}$ (182t); Grav. Gen. B. i. p. 29 (1849); Blyth, Cat. B. Mus. A. S. B. p. 22 (1849); id. J. A. S. B. xix. p. 331 (I 85 ); Bp. Consp. i. p. 32 (1850); Horsf. \& Moore, Cat. B. Mus. E. I. Co. i. p. 37 (1854); Moore, P. Z. S. 1854 , p. 260; Strickl. Orn. Syn. p. Io6 (I 855 ); Jerd. B. Ind. i. Gurney, t. c. p. 366 et 1865 , p. 236; Stvinh. t. c. p. I08; Bearw. P. S. S. 1858 , p. 395; Wall. Ihis, 1868 , p. 6; Hume, Hongh Notes i. p. I32 (1869); Gray Hant-l. B. i. p. 33 (1869); Jerd; Ibis, 1871, p. 243; 1872 p. 4II. Sharpo, Cat. B. $M$. 1. p. I $5 \mathrm{I}$.

N. virgatus, Less. Man. d'Orn. i. p. 97 (1828); Cuv. Regn. An. p. 334 (1829). Bp. Rev. et Mag. de Zool. 1852 , p. 538; Schl. Mus. P.-B. Astures, p. 32 (1862); id. Vog. Neiderl. Ind. Valkv. pp. 20, 59, pl. i 2, figs. id. Revue Accipitr. p. 74 (1878).

A. dussumieri (nec Temm.) Sykes, P. Z. S. 1832, p. 79; Hodgs. J. A.S.B. xi. p. $308($ I $8+3)$.

N. manillensis, Meyeu, Beitr. p. 694, 'Taf. ix. (1834)*.

A. besra, Jerd. Medr. Journ. x. p. 23 (1839); Il!. In.t. Orı. pl. 4 (I 847 ).

A. fringillarius, Jerd. Madr. Journ. x. p. $8+$ (1839).

A. minutus, Blyth, J.A.S. B. xi. p. 882 (1 $8+2$ ).

A. affinis, Hodes. in Gray's Zool. Misc. p. 81 (1844).

A. nisoides, Blyth, J. A. S. B. xvi. p. 727 (I847); id. Cat. B. Mus. A. S. B. p. 22 (1849; id. Ibis, 1855 , p. 28 , et I 865 . p. 240 , et i 870, p. i 58 .

Hieraspiza virgatus, Kaup, Contr. Orn. 1850, p. 63.

Astur gularis, Temm. \& Schb. Faun. Jap. Aves, p. 5, pl. 2 ( 1850$)$.

A. gularis, Bp. Rev. et Mrg. de Zool. 1854 , p. 538; Gurney, Ibis, 1865 , p. 547; Swinh. P. Z. S. 1871 , p. 342.

Micronisus badius, Swinh. Ib s, 1850, p. 359, et I851, p. 263.

M. sp, Srvinh. Inis, 1861, p. 25.

M. gularis, Swinh. Ibis, 1863 , p. 2 I 2.

A. gularis, Swinh. Ibis, 1863, p. 89.

M. virgatus, Swinh. Ibis, 1856, p. 403.

'Teraspiza virgata, Kaup, P. Z. S. 1867 , p. 171. Wall. Tr. Z. P. S. 9. p. I4 I 
Ac. viryatus, Sharpe, Cat. Brit. Mhs. p. г jo (18 $8+f)$; Salo. Cat. di Lice. di Bormeo p. I 7 (1874).

llab. Luzón, Pampanga, Negros, Guimarás, Paragua, Borneo, Java, China, Japon, Malica.

12. A. etevengonii, Gremey.

Ac. stcrensoni, Gurmey. Ibis, 1863 [s. $4+7$ Sharp. Cat. Oruit. Mus. p. rog Tiu. Horks. p. G29 Pl. P. Z. S. (1878.) p. 938.

Hab. Minclanas, Zamboanga.

13. A. maculosus, $\delta N$.

(M. S. T.)

Hab. Ilocos $\mathrm{N}$.

Subfam. BUTEONINAS.

1. Gén. BUteo, Cuv.

I. B. vulgaris, Leach. \$ N. v. Buzo vulgar.

(II. S. T.)

The Puttock or Buzzard, Albin, N. H. Birds, i. p. I, pi. I ( 7738 ).

La buse, Briss. Orn. i. p. 406 (1760); Buff. Pl. Enl. i. pl. 419 ( 1770$)$.

Falco buteo, Linn. S. N. i. p. 227 (1766 ex Albin); Naum, V̈̈g. Deuts. i. 'Taf. 32, 33 (1822); Yarr. Brit. B. i. p. 76 (18+3).

?Aquila glaucopis, Merr., Beitr. ii. p. 22, Taf. 7 (1786).

F. glaucopis, Gm. S. N. i. p. 255 ( 7788 ).

?F. versicolor, Gm. S. N. i. p. 272 (r788).

F. albus, Daud. Traité, ii. p. I 55 ( $18(0))$.

Accipiter buteo, Pall. Zoogr. Rosso-Asiat. i. p. 362 (1811).

Buteo vulgaris, Leach, Syts. Cat. Mamm. \& B. Brit. Mus. p. io, (1816); Steph. Gen. Zool. xiii. p. 46 (1826); Gould, B. Eur. pl. I4 (1837); Gray, Gen. B. i. p. I I (I849); Kaup, Contr. Orn. 1850, p. 77; Schl. Vog. Nedert. pls. 35, 36 (I854), Strickl. Orn. Syn. p. 27. (1855); Pelz. Verh. z.-b. Wien, 1862 , p. 144; Jerd. B. Ind. i. p. 87 (1862); Schl. Mus. P.-B. Buteones, p. 3 (1862); Gould, B. Gt. Br. pt. iv. (1863); Nerut. Ooth. Wolley. p. 138 (г 864); Sundev. Su. Fogl. pl. xxviii. fig. 4 (1869); Heugl. Orn. N. O.-Afr. p. 91 (r869); Hume, Rough Notes, ii. p. 261 (1870); Salvad. Faun. Ital. Ucc. p. I I (1871); Newt. et Yarr. Brit. B. i. p. Io9 (1871); Shellcy, B. of Egypt, p. 200 (1872); Schl. Revue Accipitr. p. I05 (1873).

B. fasciatus, Vieill. Faune Franc. p. I7, pl. 8. fig. I (c. I820).

B. mutans, Vieill. Faun. Franc. p. 18, pl 8. fig. 2 (c. I 820 ).

F. mutans, Savi, Nuov. Giorn. Pisk, xxii. p. 64 (1822).

F. fasciatus, Savi, Nuov. Giorn. Pisa, xxi.. p. 66 (1822); Roux, Orn. Prov. pls. 20-22. (1855); Savi. Orn. Tosc. iii. p. 195 (1851).

F. pojana, Savi, Nhov. Giorn. Pisa, xxii. p. 68 (1812); ad. Orn. Tosc. iii. 197. (;831).

B. communis, Less. Traité, p. 78 (183r). 
B. septentrionalis, Brehm, Vög. Deutschl. p. 42 (1831).

B. medius, Brekm, V. D. p. 43 (183I).

B. murum, Brehn, V. D. p. 44 (1831).

B. fuscus, Macgill. Bist. Brit. B. iii. p. I 83 (1840).

F. albidus, De Sely's. Fann. Belg. p. 55 (1842, nec Gm.)

F. variegatus, De Selys, $F$. B. p. 54 (1842, nec Gm.)

B. cinereus, Bp. Consp. i. p. 18 (1850); Loche, Expl. Algér. Ois. p. 4 I (I867).

B. variabilis, Bailly, Orn. Sav. i. p. г 27 ( 1853 ).

B. major, Brchn, Naum. 1855, p. 268.

B. minor Brehm, Na!ım. 1855, p. 268 ,

B. vulgaris, var. obscura, Pelz. Verh.z.-b. Wien, 1862, p. 144.

B. vulgaris, var. etrusca, Pelz. tom. cit. p. i if.

B. minor, Pelz. tom. cit. p. I I 4 (nec Hengl.)

Hab. Europa, Alemania, At

2. B plumipes, Hoitgs, $\sigma$ N., v. Anol.

Circus plumipes, Hodgs. in Gray's Zonl. Misc. p. 81 (1844);

Buteo plumipes, Hodgs. P. Z. S. 1845 , p. 37; Gray, Gen. i. p. II (1849); Kaup, Contr. Orn. 1850, p. 77, Bp. Comp. Av. i. p. 19 (1850); Strickl. Orn. Sinn. p. 38 (1855); Jerd, B. Ind. i. p. 91 (1862); Blyth, Ibis. i 863 , p. 21, et i 866 . p. 245; Gray, Hand-l, B. i. p. 7 (1869); Hume, Rough Notes, ii. p. 285 (1870); Jerd. Ibis, 1871 , p. 340; Blanf. Ibis, 1872, p. 87. Sharp. Brat. Mus. p. r8o lam. 7.j. 1 (1874).

Archibuteo plumipes, Blyth, J. A. S. B. xix. p. 338 (1850).

Falco buteo japonicus, Temm. \& Schl. Fann. Jap. p. 16 (1850).

B. rulgaris japonicus, ïd. ut supra pls. vi. \& vi. B.

B. japonicus, Bp. Comsp. i. p. 18 (1850); Schl. Mus. P.-B. Buteones, p. 7 (1862); Blakist. Ibis, 1862 , p. 314; Gray, Hand-l. \#.-i. p. 6 (1869); Swinh. Ibis, 1870 , p. 87; Jerd. Ibis, 1871 ; p. 337; Schl. Bevue Accipitr. p. 106 (1873).

B. vulgaris, Blyth, Ibis, $186_{3}$, p. 20.

B. asiaticus, Szinh. P. Z. S. 1871, p. 339.

Hab. Cagayan, Pamplona, China, Fo-kien, Japon.

Subfam. AQUILINAS.

I. Gén. NEOFUS, Hodgs.

I. N. malayensis, $\left(T_{e m}\right)$ o N. v. Manoyo.

(M. S. T.)

Falco malayensis, Temm. Pl. Col. i. pl. x 7 (1824, ex Reinze. MS.)

Aquila malayensis, Vig. Zool. Journ. i. p. 337 (I814); Gray, Gen. B. i. p. I4 (1845); Bp. Consp. i. p. I4 (1850); Strickl. Orn. Syn. pp. 6o, 220 (1855); Schl. Mus. P.-B. Áquilæ, p. I I (1862); id. Vog. Nederl. Ind. Valkv. pp. 8, 49, pl, 3. figs. I, 2 (I 866); Gray, Hand-l. i. p. I I (1869) Schl. Revue Accipitr. p. II 7 (1873).

A. malayana, Less. Traité, p. 39 (1831).

A. (Heteropus) pernigra, Hodgs. J. A. S. B. v. p. 222 (1836). 
Hetcropus perniger, Hodgs. J. A. S. B. xii. p. $127(18+3)$.

Ni-aetus ovivorus, Jerd. Madr. Journ. xiii. p. I57 (I $8+t)$.

Ictinatus perniger, Blyth. Amn. $N$. $H$. xiii. p. IIt (i $8+t)$.

Neopus perniger, Hodg. in Gray's Zool. Misc. p. 81 (is+4).

I. malayensis, Blyth.J.A. S. B. хг. p. 7 (18+6). Jerd., Madr. Journ. XIII, 2, p. 117 (1844).-Blyth. Cat. B. Mus. As. Soc. B. p. 28 (18+9).-Lay rd. Ann. Nat. Hist. Ser. 2, XII, p. 99 (1 853).-Irby. Ibis, 1861 , p. $22 \mathrm{I}$.

Onychatetus malayensis, Ka $: p$, Class. der Säug. und. Vög. p. I 20 (i $8+4) .-B p .$, Rev et Mag. de Zool. 1854 , p. 531.-Pelz., Verh. der k.-k. zool. bot. Gesell. "n Wien. 1862 , p. 162. Salv. Uíc. di Borneo p. 4. (I 874.$)$

Aquila malayensis, G. R. Gray, Cat. B. Brit. Muss. 1, p. 7 (18+4).-Id., Gen. C. 1, p. I4 (8+5).-Bp., Consp. 1, p. It (1850).-Id., Rev. Zool. 1850 p. 479.-Harlt., Journ. für. Orn. 1855, p. 331.—"chleg., Mus. c'es P. B. Aquilae, p. I I ( 8 2).-Id., Ois. des Ind. Néerl. Accipitres p. 43, pl. 3, f. I, 2 (1856).-Id., Mus. P. B. Accipitres, p. I 16 (1873).

Neopus malayensis, Gray, Cat. Hodgs. Coll. B. of Nep. p. 42 (1846).-Horsf. and. Moore, Cat. B. Mus. E. I. Comp. 1, p. 38 I (1854).-Jerd., B. of Ind. 1, p. 63 (1862).-Blyth, Ibis, 1863 , p. I9.-Wall., Ib s, 1868 , p. i $3 .-$ Wald., Trans. Zoo! Soc. VIII, 2, p. 34 (1872). Sharp. Cat. Brit. Mus. p. 257 (I87t'.

Heteropus malayensis, G. R. Gray, List of. Gen. and. Sutgen. of. B. p. 3 (1 855).-Gray, Cat. Spec. present. by Hoégs. to the Brit. Mus. p. I8 (1863).-Id., Hand-List; 1, p. II, sp. 96 i869. Beav. P. Z. S. 1868 , Hume, Rough Holdsw. P. Z. S. 1872 , p. 4iा.

Hab. Luzón, Mindoro; Java, Sumatra, Célebes.

\section{Gén. PiZaletus, Vialll}

2. Sp. kieneri Gerv. \&? N. v. Lauin pira.

(M. $S$ 'T.

Astur kieneri, Geru. Mag. Zool. 1835, Ois. pl. 35. Geoffroy St.-Hil. Rev. Zool. 1845 , pl. 25 (1835).

Sp. cristatellus, Jard. et Selb., 3. Or. pl. 66.

Sp. albogularis, Tick. J.A.S. B. xi. 45 ( ( $84+4)$;

Limnaetus kieneri, Strickl. Ann. $N, H$. xiii. p. 33 (18+4); Jerd. B. Ind. i. ए. ( 1862$)$.

Sp. kieneri, Gray', Gen. B. i. p. It $(1845)$; Bp. Consp. i. p. 29 (1850); Strickl. Orn. Syn. p. 7 I (1855); Schl. Mus, P.-B. Astures, p. I I (1862); Wall, Ibis, 1868 , p. I4; Hume, Rough Notes, i. p. 216 (1869); Gray, Itand-l. i. p. 21 3 (1869); Hume, Str. F. i. p. 31 I (1873); Schl. Rivue Acciptr, p. 58 (1873). Salv. Cat. Ucc. di Borneo. p. i6 (1874).

Lophotriorchis kiencri, Sharpe, Cat. Brit. Mus. p. 255 (1874).

Nisactus kieneri, Jerd. Ill. Ind. Oria. p. 5. (1347)

Hab. Marinduque, Mindanao, Panay, Borneo, Calaca, Java.

3 s lanceolatus. $\sigma$ N. v. Lauin pira

(M. S. T )

Spizaetus lanceolatus, Bp. Consp. i. p. 29 (185)); Wall. Ibis, 1868 , p. I3; Gray, Hand-l. B. i. p. I3 (1869). Sharpe. Cat. Brit. Mus. p. 270 (1874). 
S. fasciolatus, Temm. \& Schl. Fann. Jap. p. 8 (1850).

S. cirrhatus, Schl. Vog. Netderl. Ind. Valkv. pl. 7 figs. 2, 3 (1866); id. Rev. Accipitr. p. 52 (1873, pt.)

Limnaetus lanceolatus, IIald. Tr. $Z$. S. viii. p. $3+(1872)$. Hab. Panay, Mindanao, Marinduque, Cagayan, Célebes.

4 S. alboniger, $(B / y / l)$ \& N. v. 1,auin-pira.

(M. S. T.)

Nisaetus alboniger, Blyth, J.A.S. B. xiv. p. $173(\mathrm{I} 8+5)$.

Spizaetus borneonensis, Gray, Cat. Accipitr. $18^{2}+8$, p. 16.

S. alboniger, Blyth. J. A. S. B. xix. p. 335 (1850); Strickl. Orn. Syn. p. 71 (1855) Gould, B. Asia, pt. xr. (1863); Gurn. in Gould's B. of. As.; Wall. Ibis, 1868 , p. 215; Hume, Roush. Notes, i. p. 200 (1869); Salv. Cat. Ucc. di Born. p. I3 (1874); Sharp. Cat. Brit. Mus. p. 27 I (187t); Gray, Hant-l. B. i. p. I3 (1869).

S. nanus, Wall. Ibis, 1868 , p. 14 , pl. $\mathrm{r}$.

Limnatus albiniger, G. R. Gray, Hand-l. i. p. I 3 esp. I I 3 (1859). Sp. cirrhatus, Low. Saraz'ak, p. 409 (1848); Schleg. Mus. P. B. Astures. p. 6. (1862) (en parte); id. Ois des Ind. Necrl. Neerl. Accipitres. p. 54 (en parte) pl. 7. f. 1.; Id. Mus. P. B. Accipitres. p. 52, n. ${ }^{\circ} 43(1873)$.

Hab. Luzón, Ilocos Norte, Malaca, Borneo.

5. Sp. limnaetus, $H_{i}$ if $c 0$ Lauin-pira.

(M. S T.)

Falco limnaetus, Horsf. Tr. Linn. Soc. xiii. p. I 38 (1822); id. Zool. Res. Java, pl. 36 (1824); Temm. Pl. Col. i. pl. I34 (1824.) Bernst., Jouriz. f. Orn. 1860. p. 419.

Morphnus limnaetus, Vig. Zool. Journ. i. p. 325 (1824). Steph. Gen. Zool. xiii. pt. 2 p. 18 (1826); Less. Tr. dOrn. p. 53 (1831).

A. limnaetus, Less. Man. d'Orn. i. p. 94 (1828).

L. horsfieldii. Vig. Mem. Raffl. p. 649 (1830).

L. unicolor, Blyth, Amn. N. H. xiii. p. 114 (1844).

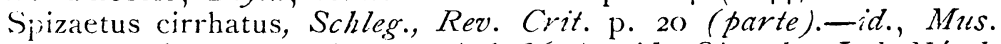
P. B. Astures, p. 6 (parte) (1862).-id., Ois, des Ind. Néerl. Accipitres, p. 53, pl. 8, f. 1 (1866).-Mus. P. B. Accipitres, p. 52 (1873) (parte).-Horsf. \&a Moore, Cat. B. Mus. E. Y. Comp. 1 , p. $32(1854)$-Moore, P. Z. S. 1854 , p. 259.

S. limnaetus, Gray, Gen. B. i. p. I4 (1845); Strickl. Orn. Syn. p. 7 I (1855), Blyth. Cat. Mus. A. B. p. 24 (1849) (en parte.); Sharp. Cat. Brit. Mus. p. 272 (1874) (en parte.)

Limnactus cirrhatus, Kaup, Contr. Orn. 1850 , p. 6r. Hab. Luzón, Laguna, Java, Sumatra, Borneo, Malaca.

6. S philippinensis, Sharpe. \& $\mathrm{N}$ v. Lauin.pira.

(M. S T.)

Sp. philippensis, Gurney in Gould's B. Asia, pt. .xv. 1863 ; Gray, Hand-l. B. i. p. 13 (1869); Sharp. Cat. of the Birds. Brit. Mus. p. 26r. (1874).

Hab. Basilan, Negros, Masbate.

7. Sp caligatus. (Riffl). $\delta \hat{\imath} N . \quad$ Lauin-pira.

$\left(\begin{array}{lll}\mathrm{M} & \mathrm{S} & \mathrm{T}\end{array}\right.$.)

Falco caligaús, Raffl. Linn. Trans. xiii, p. 230 (1822). 
F. niveus, Temm. Pl. Coll. 127 (1823).

Morphnus niveus, Steph., Gen. Zool. xiii, pt. 2, p. 19 (1825):Vig., Zool. Journ. 1, p 235 (1825).-Cuv, Regn. An. ed. 2." t, p. 331 (1829).-Less., Tr. dOrn. p. 53 (1831).

Astur niveus, Less. Man. dorn, i. p. 9+ $(1828)$.

Limatus caligatus, Vig. Raffl Life, p. 649 (1831).-G. R. Gray, Hand-List 1, p. I 3 , esp. $110(1831)$.

Spicuetus niveus, Blyth, Ann. and. Mag. Nat. Hist. xii. p. 91 (1843).

Linnaetus niveus, Strickl. Ann. and. Mag. Nat. Hist. xiii. p. 33 $(1847)$-Jerd., B. of Ind. i. p. $70(1862)$.

S. caligatus, Gray, Cat. Accipitr. 1844 , p. 8; id. Gen. B. i. p. I 4 (18+5); Strickl. Orn. Syn. p. 7o (1855); Schl. Mus. P.$B$. Astures, p. 6 (1862, pt.); $\dot{i d}$. Vog. Nederl. Ind. Valkv. pp. I4, 53, pls. 6, 7. figs. I, 8 (1866); Wall. Ibis, 1868 , p. I 3; Gray, Hand-!. B. i. p. I3 (1869'; Hume, Rough Notes, i. p. Ios (1869). Gurn in Gould's B. Asia. pt. xv (1863).

Spizatus cirriatus, Schleg., Rev. Crit. p. 20 (1844) (parte.)G. R. Gray, List. B. Brit. Mus. i. p. I6 $(1848)$.-Bp., Consp. i, o. $29(1850)$ (parte).-Biyth. J.A.S. B. xix, p. $334(1850)$; Moore, P. Z. S. 1854 , p. 259.-Schleg., Muts. P. B. Astitres, p. $6(1862)$ (parle).-id., Ois. des Ind. Néerl. Accipitres, pp. 14, 63, pl. 6, f, i. 2, 3 y 1. 8, f. 2, 3 (no f. 1, ni pl. 7) (1866)-IVall., Tbis 1868 , p. I3 (parte).-Schleg., Mus. P. B. Accipitres, p. $5^{2}$ (1873) (in parte.) Sharpe. Cat. Brit. Alus. p. 272 (en parte) (1874).

Spizaetus limnatus, Blyth, Cat. B. Mus. A. S. B. p. $24(18+9)$ (parie).

Hab. Luzón, Cagayan, Burneo, Java, Sumatra.

\section{Gón SPILORNiS. Giray.}

8 S. cheela, Lath. $\sigma, 0$ N. v Mamuhac, Banog.

(M S T.)

Cheela Falcon, Lath. Gen. Syn. Suppl. p. 33 (1787).

Falco cheela, Lath. Ind. Orit. i. p. 14 ( 1790$)$.

F. albidus, Temm. Pl. Col. i. pl. 19 (1824, ex Cur.)

Cymindis albidus, Vigors, Zool. Jutrn. i. p. 324 (I824).

Buteo albidus, (az. Régne An. i. p. 337 (1829).

Hximatornis undulatus, Vigors, P.Z. S. 1830-31, p. 171: Gould, Cent. Himal. B. pl. I (1832).

Circaetus nipalensis, Hodgs. As. Research. xviii. pt. 2, p. i 7, pl. 2 (I 833$)$.

Haliaetus bacha, Meycn, Beitr. p. 68 (1834).

Spilornis undulata, Gray, Cat. Accipitr. 1844 , p. 10

(:. undulatus, Gray', Gen. B. i. p. I6 (1845).

H. cheela, Biyth, J.A. S. B. xiv. p. 179 (1845); Horsf. \& Moore, Cat. B. Mus. E. I. Co. i. p. 59 (1 854 ).

C. cheela, Gray, Cat. Accipitr. 1848 , p. 1 I; Schl. Mus. P.-B. Buteones, p. 25 (1862); Gray, Hand-l. B. i. p. It (1869.)

S. cheela, Bp. Consp. i. p. I7 (I850); Strickl. Orn. Syin. p. I7, (1855 pt.); Jerd. B. Ind. i. p. 77 (1862), Blyth, Ibis, 1863 , p. I 2, et 1866, p. 243; Hume, R. N. i. p. 222 (1869; Srvinh. P. Z. S. 1871 , p. 340 .

Sp. orientalis, Gurney, Ibis, 1866243. 
Sp. hoya, Szwinh. Ibis, 1866 , pp. 304, 399; Gurus. t. c. p. 24r. Sharpe. Cat. Brit. Mus. p. 217 (1874).

Hab. Luzón, Cagayan, India, Formosa, China.

9 Sp bacha, (Datud.) o o N. v. Lauin-buhac

(M. S. T.)

Le bacha, Lev. Ois. d'Afr. 1. p. 60 pl. ${ }_{5}$ (1799) Sund. Krit. um Lev. p. 25 (I838).

Falco bacha, Daud. Traité, 11 p. 43 (1800, ex Lev.; Lath, Ind. Orn. Suppl. p. 4; id. Syn. Suppl. 11 p. 22; id., Gen. Hist. i. p. I72: Shazw. Gen. Zool. vii. p. 157, pl. 22 (181 I); Raffl. Linn. Tr. xiii. p. 278 (1822).

F. bido, Horsf. Tr. Lin. Soc. xiii. p. I 37 (1822); Lath. Gen. Hist. i. p. 149.

Cymindis bacha, Vig. Zool. Journ. i. p. 324 (1824); Steph. Gen. Zool xiii. p. 2. p. 24 (1826).

Buteo bacha, Vig. Mem. Raffl. p. 650 (1830); Cuv. Reg. An. P. 324 (1817) P. 337 (1720; Vieill. N.D. iv. p. 467 (1816); id. Enc. Meth. 117. p. I219 (1823); Less. Tr. d.Orn. p. 77 (I83 I).

Hamatornis bacha, Vig. P. Z. S. $1830-31$, p. 270; Horsf. et Moore, Cat. B. Mus. C. L. Co. i. p. 49 (1854).

Spilornis bacha, Gray, List. Gen. B. 1840, p. 3: Bp. Consp. 1. p. 16, (1850); Blyth, Ibis 1863 , p. I 2; Wall. Ibis, 1868 , p. I 5; Hume, Rough Notes, i. p. 230 (1869); Sharpe, Cat. Brit. Mus. p. 290 (1874).

Circaetus bacha, Gray, Gen. B. i. 16 (1845); Schl. Mus. P. B. Buteones, p. 26 (I862); id Vog. Ned. Ind. Val. pp. 36, 71, pl. 22 fig. I-3 (1 866); Gray; Hand-l. B. i. p. I4 (1869).

H. bido, Blyth, Cat. B. Muts. A. S. B. p. I9 (1849).

(. Lascha, Schl. Mus. P.-B. Rev. Accip. p. II 3 (1873). Hab. Luzon, Laguna, Cavite; Java, Borneo, Malaca.

I . S. pallidus, Walı o o N. v. Cananamà.

(M. S. T.)

Spi. cheela, Wall. Ibis. 1868 . p. I 5 .

Sp. pallidus, Wald. Ibis. 1872 p. 363. Sharpe. Ca'. Brit. Mius. p. 290, pl. ix. (I874).

Hab. Luzón, Bulacán, Cavite, Indán, Laguna, Cạlamba, Paragua; Borneo.

11. S. holospilus, Vigors o o N v. Sagueg.

(M S. T.

Buteo holospilus, Vigors, P. Z. S. $1830-31, \mathrm{p}, 96$.

Hæmatornis holospilus, Vigors, t. c. p. I70; Horsf. \& Moore, Cat. B. Mus. E. I. Co. i. p. 50 (1854).

S. holospilus, Gray, Cat. Accipitr. p. Io (I844); Bp. Comp. i. p. I7 (1850); Strickl. Orn. Syn. p. 45 (1855); Blyth, Ibis, 1.866, p. 243, Wall. Ibis, 1868 , p. 16. Sharpe. Cat. Brit. Mus. p. 293 (I 874).

Circaetus holospilus, Gray, \& Mitch. Gen. of. B. i. p. 16, pl. 7 (1845); Schl. Mus. P.-B. Buteones, p. 28 (1862); Gray, Hand-l.

i. p. 14 (1869); B. Schl. Revue Accipitr. p. I14 (1873). Hab. Luzòn, Laguna, Calauan, Cagayán, Tuguegarao, Nueva 
Vizcaya, Cavite, Indán, Marinduque, Mindoro, Samar, Paric, Leyte, Cebú, Paragua, Mindanao.

12. S. parayenses, Strie, on N. v. Sagueg.

Hab. Panay, Negros, Guimarás.

4. Gén BUtastur, Hodgs.

13. B. liventer, $\left(T T^{2} m\right) \sigma \rho \mathrm{N}$ v. Limbas, Sicut.

(M. S T.)

Falco liventer, Temm. Pl. Col. i. pl. 438 (1827).

Buteo liventer, Curv. Rigne An. i. p. 337 (1829); Temm. \& Schl. F. J. Aves, p. 2 I (1850); Schl. Mus. P.-B. Buteones, p. 2 I (1862); id. Vog. Nederl. Ind. Valky, pp. 32, 69, pl. 2 I fig. I (1 866); id. Revule Acciptr. p. I I I (1873).

Buteo pallidus, Less. Traité, p. 82 (1831).

Astur liventer, Gray, Catt. Accipitr. p. 34 (1844).

Poliornis liventer, Kaup, Classif. Säug. u. V̈̈g. p. I22 (1 $8+f$; Gray, Gen. B. i. p. 30 (18+9); Bp. Consp. i. p. 18 (185(); Strickl. Orn. Syn. p. I 26 (1855); Wall. Ibis, 1868 , p. i9; Gray, Hand-l. B. i. p. 16 (1869); Walden, Tr. $Z$. S. viii. p. 37 (1872); Hume, Stray F. i. p. 319 (1873). Salv. Cat. Ucc. do Borneo, p. 9 (1874).

Butastur liventer, Sharpe. Cat. Brit. Mils. p. 296 (1874).

Hab. Luzón, Pampanga, Laguna, Calauan; Siam, Célebes, Java, Timor.

I4 B. indicus, $(G m.) \sigma \circ$ N. v. Sicup, Ticui.

(M. S. T)

Javan Hawk, Lath. Gen. Syn. Suppl. p. $32^{*}$ (1787).

Falco indicus, Gen. S. N. i. p. 264 (1788, ex Lath).

F. javanicus, Lxth. Gen. Hist. i. p. I63 (1821).

F. poliogenys, Temm. Pl. Col. i. pl. 325 (1825).

Astur poliogenys, Gray, Cat. Accipitr. 1844 , p. 34.

Buteo fasciatus, $A$. Hay, Madr. Journ. xiii. p. I 45 (1844).

A. barbatus, Eyton, Anu. N. H. xvi. p. 228 (18+5).

B. pignaus, Blyth, J. A. S. B. xiv. p. I77 (18+5); id. Cat. B. Mus. A. S. B. p. $29(9849)$.

Poliornis indicus, Gray, Cat. Accipitr. 1878 , p. 68; Strick!. Orn. Syn. p. 125 (1855); Walden, Tr. Z. S. viii. p. 37 (1872). Salv. Cat. Uccel. di Borneo. p. 9 (1874).

P. pyrrhogenys, Gray, Gen. B. i. p. $30(18+9)$.

P. poliogenys, Gray, Gen. B. i. p. 30 (1849; Bp. Consp. i, p. 18 (1850); Wall. Ibis, 1868, p. 19; Gray, Hand-l. B. i. p. I6 (1869); Swinh. P. Z.S. 1871 , p. 34 I.

B. pyrrhogenys, Tem. \& Schl. F. J. Aves, p. 2 I pl. 7 в $(1850)$.

B. poliogenys, Schl. Mus. P.-B. Buteones, p. 22 (1862); id. Vox. Nederl. Ind. pp. 33, 70. pl. 2 I figs. 2, 3 (I866); Blyth, Ibis, 1866, p. 247; Schl. Revue Accipitr. p. I I I (1873).

P. barbatus, Gray, Hand-l. B. i. p. 15 (1859).

Butastur indicus, Sharpe. Cat. Brit. Mus. p. 297 (1874). Hab. Samar, Calbayog, Leyte, Parag a, Bısilin, Mindanao, 
Guimarás, Negros, Panay, Cebú, Siquijor, Bshol, Luzín, Ilocos Norte, Cagayan; Japon, China, Malaca, Burneo, Célebes.

\section{HALIATUS, SAv.}

15. H albicillus, (Lin.) N \& $\&$ N. v. Pigargo vulgar, ó Borni. (M. S. T.)

The Golden Eagle, Albin, N. H. Birds, ii. pl. I (1738).

L'Aigle à queue blanche, Briss. Orn. i. p. 427 (1760).

Le Petit Aigle à queue blanche, Briss. t. c. p. 429 (1760).

L. Grand Aigle du mer, Briss. t. c. p. 437 (i 760 ); Buff. Pl. Enl. i. pls. I I 2,4 I 5 .

Vultur albicilla, Linn. S. N. i. p. I 23 ( г766).

Jalco ossifragus, Linn. $S . N$. i. p. I 24 ( 7766 ).

F. albicilla, Gm. S. N. i. p. 253 ( 788$)$; Naum. Vög. Deutsch!. i. p. 224, pls. I 2, I 3 , It (1822); Werner, Atlas, Rapaces, pl. I I (1827); Kittl. Kupf. Vög. pl. 2 fig. 2 (1832); Sch!. u. Susen. Vög. Eur. Taf. 25. 26 (c. I839); Nordm. in. Démid. Voy. p. 98 (1840); Yarr. Br. B. i. p. I8 (184r).

F. melanaetus, $G m$. S. N. i. p. 254 (i 788 ).

F. albicaudus, Gm. S. N. i. p. 258 ( 788 ).

F. hinnularius, Lath. Ind. Orn. i. p. I 5 ( 1790 ).

F. pygargus, Daud. Tratté, ii. p. 62 (1800, cx Briss.)

Haliaetus nisus, Savign. Descr. Egriple, Ois. p. 253 (I 809); Less. Traité, p. 40 , pl. 8 fig. 2 ( $183 \mathrm{r}$ ).

Aquila leucocephala, Meyer in Wolf u. Mey. Taschenb. deutsch. Vogelk. p. 16, pl. 4 (1810).

A. albicilla, Pall. Zoogr. Rosso-As. i. p. 345 (I8I r); Midd. Sibir. Reis. Zool. p. I 25 (1851).

Haliaetus albicilla, Leach, Syst. Cat. Mamm. \&c. Brit. Mus. p. 9 (1816); Cuv. Règne An. i. p. 315 (1817); Gould, B. Eur. pl. Io (1837); Macgill. Brit. B. iii. p. 22 I (1840); Graxy, Ge':. $B$. i. p. I7, pl. 7 fig. 8 (I 845$) ; B p$. Consp. i. p. I 5 (1850); Schl. Vog. Neverl. pls. 28. 29 (1854); Cass. B. Calif. p. in (1855); S'rick!. Orn. Syn. p. 48 (1855); Fritsch, Vjg. Eur. tab. 7 figs. 4, 5, (r858); Cass. 21 Baird's B. N. Am p. 43 (1860); Schrenk, Reis. Amurl. p. 223 (1860); Suntev. Sv. Folg. pl. xxx. figs. 2, 3 (c. I 850); Schl. Mus. P.-B. Aquilix, p. I (1862); Guit'd. B. Gt. Br. pt. 3 (1863); Pelz. Berh. z.b. Wien, 1863, p. 587; Radde, Reis. Sibir. Vög.: p. 95 (1863); Scl. $P$. Z. S. 1853 ; p. 252; Nezet. Ooth. Wolley. p. 345 (1864); Srí. P. Z. S. 1865 , p. 73I; Degl. ct Gerbe, Orn. Eur. i. p. 3') (I857); Heug!. Vög. N. O.-Afr. p. 5I (I869); Gray, Hand-.. B. i. p. 16 (1869); Salvad. Faun. Ital. Ucc. p. 9 (1871); Swinh. P. Z. S. 1871 , p. 339; Nezet. ed. Yarr. Brit. B. i. p. 25 (1781); Shelley, B. Egypt, p. 20+ (1872); Hume, Str. F. i. p. I59 (I873); Schl. Revue Accipitr. p. II7 (1873). albicillus, Sharpe Cat. Brit. Mus. p. 302 (1874.)

F. albicilla borealis, Fabser, Isis, 1827, p. 56 .

H. groenlandicus, Brehm, Naum. 1855, p. 267 . borealis, $B r e h m$. l. c. islandicus, Brehm. l. c. orientalis, Breln. l.c. cinereus, Brehm. l. c. funereus, Brehm. l. c. pelagicus, Hume, Rough Notes, ii. p. 252 (1870, lxpsu). brooksi, H.tm?, Ibis, 1870 , p. $43^{8}$. Hab. Luzón, Leguna, Mindors; Europd, Asia, China, Japón. 
Acquila pelagica, Pall. Zoogr. Rosso.As. i. p. 343, pl. I8 (18ы ); Kittl. Kupt. Vög. p. 3, pl. 2 fig. I (Is32; Midd. Sibir. Relse, Zool. p. 125 (1853).

Falco leucopterus, Temm. Pl. Col. i. pl. 4iog (1824).

F. imperatur, Kittl. Kupf. Vög. p. 3 (1832).

Thalassaetus pelagicus, Kaup, Mus. Senck. iii. p. 261 (1845).

Haliaetus pelagicus, Gray, Gen. B. iii. App. p. I (18+9); Temm. \& Schl. F. J. Aves, p. Ir pl. $4\left(15^{\circ}\right)$; Cass. B. Calif. pp. 31 , IIO, pl. 6 (1855); Strickl. Orn. Syn. 5I (1855); Schrenh, Reis. Amurl. Zool. p. 222 (1859); Cass. in Bard, B.,N. A. p. 42 (1860); Schl. Mus. P.-B. Aquila, p. It (1862); Elliot, B. N. Am. part 6 (1867); Homey. J. $f$. O. 18268 , p. 248; Grav, Hand-1. 1. p. 17 (1869); Sruinh. P. Z. S. 1871, p. 339; Cones. Key N. A. Birds, p. 226 (1872). Sharpe, Cat. B. Mus. p. 3(t) ( 1874$)$

Hab. Luzón, Cagayan; Siberia, China, Jałpón, N. América.

6. Gén. CUNCtMA, Hơgeds.

17. C. leucogaster, (Gm.l.) \& N. v. Lauin, Anol.

(M. S. T)

White-bellied Eagle, Lath. Gen. Sy'n. i. p. 33 (1781)

Falco leucogaster, Gm. S. N. i. p. 257 (i788) ex Lath.); Temm. Pl. Col. i. pl. 49 (1823).

F. maritimus, Gm. S. N. r. p. 260 (1 788 ).

Le Blagre, Lev'aill. Ois. d'Afr. i. pl. 5 (1797); Sund. Krit. om Levaill. p. 23 ( I 5 I).

F. blagrus, Dand. Traité, ii. p. 70 ( 800$)$, ex Levaill).

Haliaetus blagrus, Cuv. Régne An. i. p. 316 (1817); Smith, S.-Afr. Q. Journ. i. p. 108 (1 830); Bp. Cousp. i. p. I 5 (1850).

F. dimidiatus Raffl. Tr. Linn. Soc. xiii. p. 277 (1822).

Pandion blagrus, Bonu. et Veill. Enc. Mèth iii. p. I 200 (1823).

F. oceanica, Less, Voy de la Coq. Zool. I p. 343 (1829).

H. leucogaster, Vig. Zool. Journ. i. p. 336 (1824); Gould. Syn. B. Austr. pt. 3. pl. 37 fig. I (I838); Strickl. Orn. Syn. p. 53 (1855); Schl. Mus. P.-B. Aquila, p. I4 (1862); Jerd. B. Ind. i. p. 85 (1862); Schl. Vog. Nederl. Ind. Valkv. pp. 9, 5(, pl. 4. figs. I, 2 (1866); Finsch. u. Hartl. Fann. Centralpolyn. p. I (I867); Gray, Hand.l. B. i. 1. I7 (1869); Hume, Rough Notes, '. p. 259 (1869); Schl. Mus. P.-B. Revue Acciptr. p. II7 (1873). Sharpe, Cat. Brit. Mus. p 307 (1874).

H. dimidiatus, Vig. Mem. Buff. p. 648 (1830); Gray, Gen. B. i. p. I7 (I845); S'rickl. Orn. Syn. p. 54 (1855).

H. sphenurus, Gould, P. Z. S. 1837, pp. 97, I38; 1d. Syn. 13. Austr. pt. 3, pl. 37 fig. 2 (1 838 ).

Ichthyaetus cultrunguis, Blyth, J.A. S. B. xi. p. I IO (18+2).

I. leucogaster Gray, Cat. Accipitr. 1844 , p. I 3 ; Gould. B. Ausir. i. pl. 3 (1848); Diggles, Oru. Austr. pt. 5 (1866).

Pontoaetus leucogaster, Gray, Gen. B. i. p. 18 (18+5); Pelz. Verh. z.-b. Wien, 186.3 , p. 591.

P. blagrus, Gray, Cien. B3. i. p. I8 (1 845$)$. 


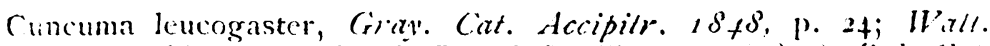
16is, 1868 , p. 15; Hald. Tr. Z. S. viii. p. 35 (1872); Sial. Cat. L'cc. di Bormeo, p. 5 ( I $87+$ ).

Blagrus leucogaster, Blyth, Citt. B. Mus. A. S. B. p. 30 (1849); id. J. A. S. B. xix. p. $3+1$ (1850); $2 d$. Ibis, 1863 , 1. 22 ; Swinh. Ibis, $1870, \mathrm{p} . \mathrm{I} 6$.

I. blagrus, Blyth, J. A. S. B. xi p. I16 (18+9).

P. leucogaster, Gould, Hantb. B. Austr. i. p. I3 (1863).

Hab. Luzón, Cagayan, Pamplona, Manila, Negros, Samar, Mindanao, Paragua; India, Ceilán, Australia, Polinesia, Malaca.

\section{Gén. HaldaAtur, Shliy.}

18. H. Indus, (Budtl.) 3 \& N, v. I.auin.

(iI. S. 'T.)

L'Aigle de Pondichery, Briss. Orn. i. p. 450, pl. nxxv. (1760). Agile des Grandes Inde:, Buff. Pl. Enl. i. pl. 416 (1770).

Falco indus, Bodd. Tabl. Pl. Enl. 2.5 (1783).

F. pondicerianus, Gm. S. N. i. p. 265 (1783). Horsf. Tr. Tin.

Soc. 13.1 . 135 (1821); Raffl. Tr. Lin. Soc. 13, 1). $278(1822)$;

S. Mrull, Verh. Land ea lol pp. $2 \mathrm{I}, 38 \mathrm{I}, 405,406(1839-1844)$. Haliactus pondicerianus, Cut. Régne An. i. p. 310 (1817).

H. garuda, Less. Traité, p. 4 I (I $83 \mathrm{I})$.

Milrus pondicerianus, ferd. Madr. Journ. x. p. 72 (1839).

H. pondicerianus, Selby, Cat. Gen. \& Subg. Types, p. 3 (1840). Dentiger pondicerianus, Hodgs. in Gray's Zool. Misc. p. 81 (1844'. Milvus? rotundicauda, Hodhs. ut suprat p. 8 I (I 844$)$.

Haliastur indus, Gray, Gen. B. i. p. 18 (1845); Blyth, Cat. B. Mus. A. S. B. p. 31 (18+9); Moore P. Z. S. p. 26 I (1854); Sclat. P. Z. S. p. 397 esp. 3 (1863); Pela. Novar. Reic. Vög. pp. 7, I6г (1865); Vall. Ibis, p. I6 (1868); G. R. Gray, Hand. 1. i. 1. I 8 ; esp. 155 (1869). Strickl. Orn. Syn. p. 73 (1855); Jerd. B. Ind. i. p. Ior (1 862); Gray, Hand-l. B. i. p. I8 (1869); Hume, Rough Notes, ii. p. 316 (1870\%; Holdsw. P. Z. S. 1872, p. 414; Hume, Str. F. i6o (1873). Sharpe, Cat. Brit. Mus. p. 3 I 3 (1874).

Ictinaetus indus, Kaup, Arch. f. Naturg. xvi. p. 39 (1850).

H. ponticerianus, $B p$. Consp. i. p. I 5 (1850).

Ictinoaetus pondicerianus Kaup, Contr. Orn. 1850 , p. 73.

Haliaetus indus, Sch. Mus. P.-B. Aquila p. I9 (1862); id. Revue Accipitr. p. I19 (1873).

Hab. Luzón, Manila, Laguna, Cagayan; India, Ceilán.

19. H. intermedius, Gurn. $\delta\{$ N. v. Lauin-bulic.

(M. S. T.)

Haliaetus pondicerianus, Horsf. Tr. Linn. Soc. xiii. p. I 36 (1822); Meyen, Beitr. p. 69 (1834).

Falco pondicerianus, Raffl. Tr. L. S. xiii. p. 278 (I822).

Haliastur intermedius, Gurney, Ibis, 1865, p. 28. Sharpe, Cat. Brit. Mus. Mus. p. 314 sub. esp. a. $(1874)$.

H. indus, Blyth, Ibis, 1868 , p. I6.

H. indus, Schl. Vog. Nederl. Ind. Valkv. pl. 4 fig. 4 (г866, nec fig. 3.

Hab. Luzón, Manila, Mindoro, Marinduque, Bohol, Panay, 
Negros, Siquijor, Samar, Leyte, Guimaras, Mindanao, Joló; Java, Sumatra, Célebes, Borneo.

20. H. girrenera. (Vicill.) $0^{\circ} .0 \mathrm{~N}$. v. Lauin-Culic.

(M. S. T)

Falco ponticerianus, Shaw \& Nodd. Nat. Misc. x. pl. 389 (1799).

White-headed Rufous Eagle, Lath. Gen. Hist. i. p. 218 (1821).

Haliaetus girrenera, Vieill. \& Oted. Gal. Ois. i. pl. x. (1825).

1H. leucosternus, Gould, P. Z. S. 1837 , p. г $38 ; i d$. Syn. B. Austr. iii. pl. 3. fig. ( 1838$)$.

Haliastur leucosternus, Gray, Gen. B. i. 1). I8 ' 845$)$; Gonld. B. Austr. i. pl. 4 (c. I 845); Bp. Consp. I. p. I5, (1850). Strickl. Orn. Syn. p. 75 (1855). Gould. Handb. i. p. I7 (1865); Wadl!. Ibis, 1868 , p. 16; Gray, Hand-b, B. i. p. 18 (1869); Wall. Ibis. p. 16 (1868); Gray Handl. B. i. p. 18 (1869). Diggles, Orn. Austr.: pt. I 7 (1870); Walden, Tr. Z. S. viii. p. 35 (1872). Ictinoaetus leucosternus, Kaup, Contr. Orn. $1850,1.73$.

H. indus, Schl. Vog. Nederl. Ind. Valkv. p. 5i, pl. 4 fig. 3 (1 866). Haliastur girrenera, sub esp. b. Sharp. Cat. Brit. Mus. p. 315. (1874).

Hab. Luzón, Laguna, Cagayan, Pampanga; Australia, Célebes 'Ternate, Amboina.

8. Gén. MrlvUS, Cuv.

21. M. íctinus $S a z$. む N. v. Milano real.

(M. S. T.)

The Kite or Glead, Albin, N. H. Birds. i. pl. 4 ( 738 ).

Le Milan royal, Bris. Orn. i. p. 414, pl. 33 (I860).

Falco milvus, Linn. S. N. i. p. I26 (1766); Naum. Vög. Deuts. i. p. 333, 'Taf. 3I fig. I (1822); Werner, Atlas, Rapaces, pl. 23 (1827); Schl. u. Susem. Vög. Eur. 'Taf. 3 I fig. I (1839); Yar. Brit. B. i. p. 66 (1 843$)$.

Le Milan, Buff. Pl. Enl. i. pl. 422 (1770).

Milius castaneus, Daud, Traité, ii. p. 148(1800).

M. ictinus, Sav. Syst. Oys. d' Egypte, p. 259 (1809); Nez't. Ooth. Wolley. p. I82 (184); id. ed. Yarr. Brit.B. i. p. 92 (1871); Sharpe, Cat. Brit. Mus. p. 319 (1874).

Accipiter milvus, Pall. Zoogr. Rosso-As. i. p. 356 (i 81 I ).

M. regalis, Roux. Orn. Prov. i. p. 44 pls. 26, 27 (1825); Macgill. Brit. B. iii. p. 265 (1840); Graj', Gen. B. i. p. 24 (I845); Bp. Consp. i. p. 21 (1850); Schl. Vog. Nederl. Ind. pl. 3 I (1854); Struckl. Orn. Syn. p. I 32 (1855); Sundev. Sv. Fogl. p. 235, pl. xxix. fig. 4 (1858); Fritsih. Vög. Eur. 'Taf. 6. fig. 7 (I $\left.5^{8}\right)$; Schl. Mus. P.-B. Milvi, p. I (1862); Degl. et Gerbe, Orn. Eur. i. p. 64 (1867); Loche, Expl. Sci. Alger. Ois. i. p. 76 (1867); Gould. B. Gt. Br. pt. xiii. (1 868); Gray, Han-d.l i. p. 26 (1869); Hengl. Orn. N. N. O.-Afr. i. p. 97 (1869); Salvad. Faun. Ital. Ucc. p. I 3 (i 87 I); Shelley, B. Egypt. p. 195 (1872); Schl. Revue Accipitr. p. 126 (1873).

Milvus ruber, Brehm, Vog. Deutschl. p. 5 I (1831).

M. vulgaris, Selby, Br. B. i. p. 74 , pl. 5 (i 833 ); Gould, B. Eur. i, pl. 2 I (1837).

Hab. Furopa, Francia, Alemania. 
9. Gén. ElANís, Saz'.

22. E. hypoleucus, Gould. ơ N. v. Lauin-puti.

(M S T)

Falco melanopterus, Horsf. Tr. Linn. Soc. xiii. p. 137 (1822).

Elanus hypoleucos Gould. P. Z. S. 1859 , p. I 27; id. B. Asia, pt. xii. (1860); Schl. Mus. P. B. p. 7. (1862); Sclat. P. Z. esp. 8. p. 207 (1863). Wall. Ib s, 1868 , p. 17; Grai, Hand-'. i. p. 28 (1869); Wald. Tr. Z. S. viii. p. 36 (1872); Schl. Mus. P.-B. Rucve Accipitr. 1). I 39 (1873); Sharpe, Cat. Brit. Mus. p. $33^{8}$ ( 1874$)$.

Elanus intermedius, Schl. Mus. P. B. Milvi, p. 7 (1862); id. Vog. Nederl. Ind. pp. 31, 68, pl. 24, figs. 2, 3 (1866; Gray, Hana-l. i. p. 28 (1 860$)$.

Hab. Luzón, Cagayan, Pampanga, Mindoro, Cel ú, Guimaras, Negros, Basilan, Mindanao; Borneo, Célebes, Java.

\section{Io. Gén PERNIS, Cuv.}

23. P. ptilonorhynchus $\left(\Gamma_{t^{\prime}}\right)$ \& N. v. $\Lambda$ nol.

(M. S T)

Falco ptilorhynchus, Temm. Pl. Col. i. pl. 44 (1823); Müll et Schl. Verh. Nat. Gesch. Ned. ov. bez. Zool. Aves. 1). 49, t. 7. I $835-44$ ).

Pernis ptilonorhynchus, Steph, Gen. Zool. xiii. Fl. 35 (1826); Holdsw. P. Z. S. 1872 , p. 414. Sharpe, Cat. Brit. Mus. P. $347 \quad(1874)$.

P. cri tati., Cuv. Règne. An. i. p. 335 (1829); Less. Tr. d' Orn. p. 76 (I831 ; Jerd. Madr. Jour. L. S. x. p. 73; Gray, Gen. B. i. p. 24 (I845; Bp. Consp. Av. i. p. 20 (I850); Str.ckl. Orn. Syn. p. I 3 I (I 855); lerd. B. Ind. i. p. IOS (1862); Wall. Ib's, 1868 , p. I7; Gray, Hand-l. B. i. p. 26 (1869); Hume, Rough Notes ii. p. 330 (1870); Schl. Mus. P.-B. Revue Accipir. p. I 32 (I 873 ).

P. torquata; Less. Traite, p. 76 (1831); Gray, Gen. B. i. p. 24 (1845); Des. Murs. Iconogr. Orn. pl. I3 (1845); Pucher. Rev. et Mag. de Zool. $1850,2 \mathrm{I} 2$.

P. ruficollis, Less. l. c. p. 77 (1831); Gray, l.c. p. 24 (1845); Des Murs. l. c. pl. I4 (1845); Pucher. l.c. p. 2 I 2.

P. albigularis, Less. l, c. p. 77 (I831); Gr.ty. l. c. p. 24 (1845); Pucher. l.c. p. 2 I 2.

P. maculosa, Less. in Bèlang. Voy. In!. p. 223 (1834);

P. ellioti, James. Tr. Wern. Soc. vii. p. 493 (1836). Jerd. Madr. Jour. L. S. x. p. 74.

P. bharattensis, Hodgs. in Gray,'s Zool. Misc. p. 81 (1844).

P. apivorus, Temm. \& Schl, Faun. Jap. Aves, p. 24 (1859); Swinh. P. Z. S. 1871 , p. 34 I.

P. ptilorhyncha, Wald. Tr. Zool. Soc. 8. 2. p. 36 (1872); Salv. Cat. Ucc. di Born. p. io (1874).

Hab. Paragua, Mindoro; Borneo, Célebes, Java, India, Sumatra, Banka. 


\section{Subfam. FALCONINAS.}

I. Gén BAZA, IIo:igs.

I. B. magnirostris, Graj. N. v. Ialuin.

Baza magnirostris, Gray, Cat Acciptr. Brit. Mus. p. in (18+f'; Strikl. Orn. Syn. p. 127 (1855); Gray, And, B. i. p. 25 (I869)). Sharpe. Cat. Brit. Mus. p. 356 (1874).

Aviceda magnirostris, Bp. Consp. i. 1). 20 ( $\left.185^{\circ}\right)$. et Rev. et Mag. de $Z$ Zool; 18.54, p. $535^{\circ}$

Pernis madagascariensis, Kaup, Contr. Orn. 1850, p. 77. Hab. Filipinas.

2 Gén. MICROHIERAX, Simirt.

2. M. fringillarius, (IHirsf) \& \& N. v. Lauin-munti.

(M. S. T.

Falco cærulescens, (nec Limn.), Horsf. Tr. Linn. xiii. p. I 35 (1822); id. Zool. Res. Java, pl. 35 (1824); Tem. Pl. Col. i. pl. 97 (1824); Vieill. \& Out. Gal. Ois. i. p. 46, pl. (1825); Kitll. Kupf. Vog. p. 4, pl. iii. fig. 2 (1832); Schl. Mus. P.-13. Falc p. 33 (1862); id. Vog. Nederl. Ind. Valkv. pp. 7, 49, pl. 2 fig. I (1866).

F. fringillarius, Drap. Dict. Class. d'Hist. Nat. vi. p. 41 $2, \mathrm{pl}^{\mathrm{l}}$ v. (I 824$)$;

Hierax cærulescens Vig. Zool. Jouru. i. p. 339 (1824); id. Life Raffl. p. 649 (1830); Blyth. J. A. S. B. xii. p. I80 (1843); Gray. Gen. of. B. i. p. 2 I (1844); Kaup. Contr. Orn. 1850, p. 52; Bp. Consp. i. p. 28 (1850); Bp. Rev. et Mag. de Zool. 1854 , p. 537; Moore, P. Z. S. 1854; p. 258; Strickl. Orn. Syn. p. 102 i $855 \mathrm{Scl}$. P. Z. S. 1863 , p. 206; Wall. Ibis, 1863 , p. 6; Gray Hand-l. B. i. p. 24 (1869): Wald, Ibis, 10 ;2, p. 36z. Salv. Cat. Ucc. di Borneo p. 3 (187t).

H. malayensis, Strickl. Ann. N. H. xiii, p. I33 ( 8444$)$; Gray'. Gen. of B. i. p. 2 I (1844); Strickl. Orn. Sym. p. IO4 (1855); Pelz. Verh. z.-b. Wien, xiii. p. 629 (1863); id. Reis. Noi., Vög. p. 3 (1 865).

H. fringillarius, Blyth, Cat, B. Mus. As. Soc. Beng. p, I 7 (1849); id. Ibis, 1863 , p. 11 ; Wald. Ibis, 1871 , p. 16I.

H. melanoleucus, Horsf. \& Moore, Cat. B. Mus. E. I. Co. i. p I6 (I 854 me Blyth.)

Hab. Mindanao, Samar; Borneo, Java, Sumatra.

3. M. erythrogenys, Vigors. \& \& N.v. Lauin-munti. Guit-guit. (M. S T.)

Hierax erytlirogenys, Vig. P. Z. S. p. 96, 1831; Blyth, J. A. S. B. xi. p. 2 p. 789 , I842; Gray, Gen. B. i. p. 21, I 844 ; Fraser, Zool. Typ. pl. 31, i 849; Kaup, Contr. Orn. p. 52, 1850; Strickl. Orn. Syn. p. I02, I 855; Gray, Hand-l. B. i. p. 24,1869 .

Falco sericeus, Kittl. Kupf. Vög. p. 4. lam. 3. fig. 3, (1832); 
ad. Mem. Acad. St. Petersb. 2 p. I tab. 1, 1835 ; Schl. Mus. P. B. Fialc. p. 24, 1862.

F. girunieri, Ey'd. et Soul. Voy. Bonite, Zool. i. p. 7I lam. I, $(18+1$.

Hitax sericens, Gray, Gen. B. i. p. 21, 1844; Bp. Rea. et Mag. di Zool. p. 536, 1854; Strickl. Orn. Syn..p. 104, 1855; Pelz. Verh. s-b, Vien, 13, p. 630, 1863; Wall. Ibis, p. 6, 1868 ; Gray, Hand-l. B. i. p. 2t, I 869.

Microhierax erythrogenys, Sharp. Cat. B. Mus. i. p. 369, 874 . Hab. Luzón, Laguna, Pampanga, Cagayan, Samar, Paragua, Mindanas.

4. M. latifrons Sharpe. N v. Lauin.

Hab. Cagayan de Misamis, Joló.

3. Gén. FALCO, Li:.

5. F. communis, Gm. $\sigma \rho$ N. v. Limbas, Lagpau.

(M. S. T)

Le Faucon, Briss. Orn. i. p. 321 (1760); Buff. Pl. Enl. i. p. 197, pl. 421, et Hist. Nat. Ois. i. p. 249 (1 770$)$.

Le Faucon pèlerin, Briss. Orn. i. p. 34I (1760).

Le Faucon sors, Buff. Pl. Enl. i. pl. 470. (1770).

Oriental Hawk, Lath. Gen. Sym. Suppl.p. $34^{*}(1877)$.

Behree Falcon, Lath. Gen. Syn. Suppl. p. 35 (1787).

Falco orientalis, Gm. Syst. Nat. i. p. $26+$ ( $\left.77_{1}\right)$, ex Lath.); Lath. Ind. Orn. i. p. 22 (1790); Daud. Traté, ii. p. 76 (1800); Strik!. Orn. Syn. p. I3 (1855); Gray, Hand-l. B. i. p. 19 ( 1869 ).

F. communis, Gm. S. N. i. p. 270 (1788), ex Buff:); Less. Trante, p. I8, pl. I6 fig. I (1831); Schl. Rev. Crit. p. ii. (184t); Temm. \& Schl. F. J. Aves, p. I (1850); Bp. Consp. i. p. z3 (1850); id. Rev. et Mag. de Zool. 1854 , p. 536; Schl. Vog. Nederl. p. 6, pls. I.3 (I 854$)$; id. Mus. P.-B. Falcones, p. I (1862); id. Vog. Nederl. Ind. Falcones, pp. 2, 44, pl. I fig. 2 (1866); Dol. \& Gerbe, Orn. Eur. i. p. 81 (1867); Sundev. Sv. Fogl. p. 206, pl. xxvi. fig. 2 (1867); Heugl. O.m. N. O.-Afr. i. p. 20 (1869); Bocage, Cat. Lish. Mus. p. 42 (I869); Salvad. Faun. Ital. Ucc. p. I7 (1871); Sharpe, Ann. N. H. (4) xi. p. 222 (1873); Schl. Revue Accipitr. p. 32 (1873). Sharpe. Cat. Brit. Mus. p. 376 (1874).

F. hornotinus, Gm. S. N. i. p. 270 (1788, ex Buff.)

F. peregrinus, Gm. S. N. i. p. 272 (178, ex Briss.); Wils. Am. Orn. ix. p. I 20 , pl. lxxvi. (1814); Temm. Man. d'Orn. i. p. 22 (1820); Naum. Vög. Deutschl, i. Taf. 24, 25 (1822); Werner, Atlas, Rapaces, pl. 8 (1527); Sw. \& Rich. Faun. Bor.-Am. p. 23 (I831); Gould. B. Eur. pl. 28 (1837); Audribon, B. N. Ain. i. p. 84, pl. xx. (1839); Magill. Hist. Brit. B. iii. p. 294 (1840); Yarr. Hist. Brit. B. i. p. 32 (1 $\left.8_{43}\right)$; Gray, Gen. B. i. p. 19 (1844); Blyth, Cat. B. Mus. As. Soc. Benr. p. I 3 (1849); Kaup, Contr. Orn. 1850 , p. 55; Horsf: \& Moore, Cat. B. Mus. E. I. Co. i. p. I6 (1854); Strickl. Orin. Sym. I. 89 (1855); Salvin \& Brodr. Falconry, p. 19, 
pls. 1-5 (1855); Fr.tsch, Vog. Eur. tab. x. firs. 8, 9 (1858); Reinh. Ib.s, 1861 , p. 5; Gurney, t. c. p. 331; Jerd. B. Int. i. p. 2 I (I862); Blakist. Ibis, 1862 . p. 314; Gould, B. Gt. Br. pt. I (1862); Pelz. P. Z. S. 1853 , p. 206; Gray, Cat. Brit. B. p. I I (1863); Blyth, Ibis 1866, p. 234; Whitely, Ibis, 1867 , p. 19t; Wall. Ib.s, 1868, p. 5; Hume, Rough Notes, i. p. I9 (1869); Gray, Hand-l. B. i. p. I9, (1869); Jerd. Ibis, 1871 p. 237 Newton. et Yarr. Brit. B. p. 53 (187 I); Swiwhoe, P. Z. S. 1871 , p. 340; Allen, Bull. Harv. Coll. ii. p. 316 (1871); Godmin, Ibis, 1872 , p. 165; Scl. \& S.rlv. Nomencl. p. I 2 I (I 873$)$.

F. calidus, Lath. Ind. Orn. i. p. 4 I (1790); Gray, Hand-l. B. i. p. I9, (1869); Blyth; Ibis, 1863, p. 7 .

F. lunulatus, Daud. Traite, ii. p. 127 (1800, ex Lat's.)

F. abietinus, Bechst. Naturg. Deutschl. ii. p. $759($ I 8.5.$)$

F. gentilis, Wils. Mem. Wern. Soc. ii. p. 587 (1818); Thienem. Abb.ld. Vogeleiern, 'Taf. li. figs. I a.c. (1844).

F. cornicum, Brehm, Vög. Deutschl. p. 62 (1831).

F. griseiventris, Brehm. Is s, 1833 , p. 778 .

F. anatum, B Bp. Comp. List. B. Eur. \& N. Am. p. 4 (1838) ex Audubon); Gusse, B. Jumaica, p. 16 (I8+7); Bp. Consp. p. 23 (1850); Kaup, Contr. Orn. 1850 , p. 56;Bp. R v. et Mag. de Zool. 1854, p. 536; Strick!. Orn. S'nl p. 83 (1855); Cass. B. Cixlif. p. 85 (1855); Newton, Ibis, 1859 , p. 63; S.t. \& S.t. t. c. p. 219; Cass. in B.r red's Ibis, 1857 , pp. 282, 233 ; Cosper, B. Calif. ed Baird, p. 457 (1870).

F. micrurus, Hodgs. in Gray's Zool. Misc. p. 8 I (18+4); Jerd. Ibis, 1871 , p. 238 ; id. tom. cit. 1872 , p. 2 ; Sharpe, Ann. N. H. (4) xi. p. $223,($ I 873$)$.

Le Faucon hagard, Sch! \& Verst. Fanc. pl. i (1853).

Le Tiercelet sors de F.ucon iid. ut suprà, pl. I 2 (1 853 ).

F. ni yriceps, Cass. B. Cillif. p. 87 (1855); i.t. Pr. Phil. Acad. - 1855 , p. 277; id. U. S. Astr. Exp. pl. xiv. (1855); Strick!. Orn. Syn. p. 85 (1855); Cass. in Baird, B. N. Ain. p. 8 (1860); Gurney, Ibis, 1857 , p. 465; Brown, Ibis, 1858, p. 418; Conper, B. Calif. ed. Baird. p. 456 (1870? Allen, Bu'l. Harv. Coll. ii. p. 317 (1871); Sharpe, Ann. N. H. (4) xi. p. 223 (1873).

F. leucogenys, Breh:n. Numinnia, 18.55 p. 268.

F. atriceps, Hume, Ibis, 1859 , p. 356 ; ict. Rough. Notes, i. p. $5^{8}$ (1869); $1 d$. Ibis, 1871 , p. 24; Jerd. tom. cit. p. 237; Rudc!. tom. cit. p. 363 ; Jert. Ibis, 1872 , p. 2.

F. peregrinoides, Vian, Rev. et Mag. de Zool. 1867 , p. I 74 .

F. brookii, Sharp, Ann. N. H. (4) xi. pp. 202221873.

Hab. Luzón, Pampanga, C.tgayan, Negros, Mindlnao; Europa, América, Japón.

6. F. pere zुrinator, Sund. $\sigma \ngtr$ N. v. Cageg.

(M. S T.)

Falco peregrinator, Sundev. Physiogr. Tidsskr. Lund, 1837, p. 177, pl 4. Gray, Gen. of. B. i. p. 19 (18+4); Sundev. Ann. N. H. xviii. p. 454 (1846); Gray, Cat. Manm \& B. Nepal. Hodgs. p. 44 (1846); Bלytí, Citt. B. Mus. As. Soc. Beng. p. I4 (1849); Bp. Cons. i. p. 23 (1850); Kinp. Cuitir. 
Orn. 1850 , p. 55; Gould, B. of, Asua, part iii. (1859); Blith. J. A. S. B. xix p. 321 (1851); Bp. Rev. et Mag. de Zool. 1854 , P. 536; Strickl. Orn. Synn. p. $74(1855)$; Jerit. B. i. p. 55 (1862); Blyth, Ibis, $185_{3}$, p. 8; Gray, Hant-l. B. i. p. I9 (1869); Hume, Rough Motes i. p. 55 (1869); Jerd. Ibis, 1870 p. 237; Sharpe, Ann N.H. (t) xi. p. 223 (1873). id. Cat. Brit. Mus. p. 382 (1874).

F. shaheen, Jerd. Madr. Journ. x. p. 8 I (1839); id. Ill. Iud. Orn. pls. xii. \& xxviii. $(18+7)$; Pelz. Verh. z.-b. Wien, xiii. p. $610(1863)$.

F. sultaneus, Hodgs in Gray's Zool. Misc. p. 81 (184+).

F. ruber, Schl. Mus. P. B. Falc. p. 5. (1862).

Hab. Luzón, Cavite, Indan, Cagayan, Mindoris; India, Himalaya.

7. F. melanogenys; Goult. ơ N v. Sicup.

(M. S. T.1

Falco peregrinus, Vig. \& Horsf. Tr, Linn. Soc. xv. p. ${ }_{1} 5.3$ (1826); Diggles Orn. Austr. part. 20) (1868).

F. melanogenys, Gould. P Z. S. 1837 , p. I39; id. Syn. B. Austr. pt. ii. (1838); Gray, Gen. of B. i. p. I9 (1844); Gould, $B$. Austr. fol. i pl. 8 (18+8); Sturt, Exp. C Austr. ii. App. p. It (I849); Bp. Consp. i. p. 23 (1850); id. Rev. et Mag. de Zool. 1854 , p. 536; Strickl. Orn. Syn. p. 56 (1855); Gould. Handb. B. of Austr. i. p. 26 (1865); Blyth, Ibis, 1856, p. 234; Gray, Hand-l. of B. i. p. 19 (1859); Sharpe, Ann. N. H. (4) xi. p. 224 (1873). id. Cat. Brit. Mus. p. 385 (1874).

F. macropus, Sizunins. Au. in Menxg. p. 34I (1837).

F. communis, Schl. Valkv. Ned. Ind. pp. 2, 4t, pl. 8. fig. 2 ( I 866).

Hab. Luzón, Laguna; Australia, Java, Molucas.

4. Gén. HYPOTRIORCHIS, Boie.

8. H. severus (Horsf) oo N. v. Cageh, Samalinut.

(M. S. 'T.)

Falco severus, Horsf. Tr. Limn. Soc. xii. p. I 35 (1822); Strik!. Orn. Syn. p. 87 (1855); Schl. Mus. P.-B. Falc. p. 23 (1862); Blyth, Ibis, ${ }_{136} 3$, p. 8; Sch!. Vog Nederl. Ind. Valku. pp. 4, 45, 'Taf. 2. figs, 2, 3 (1866); Radel. Ibis, 1871 , p. 366; Sch:. Revule Accipitr. p. 46 (1873); Sharpe. Cat. Brit. Mils. p. 397 (1874).

F. aldrovandii, Temm, Pl: Col. i. pl. 128 (1823); Less. Traite d'Orn. p. 9I (I83i).

F. rufipedoides, Hodgs. Calc. Jonrn. $N . H$. iv. p. 283 (i 844 ).

F. guttata, Gray, Ciat. Accipitr. Brit. Mas. p. 26 (1844).

Hypotriochis severus, Gray, Gen. of B. i. p. 20 (18+4); Blyth, Cat. B. Mus. As. Soc. Beng. p. I 5 (1849); Kaup. Contr, Oru. 1850 , p. 54; Bp. Consp. i. p. 25; (1850); Horsf. \& Moore, Cat. B. Mus. E. I. Co. i. p. 23 (1854); Jerd. B. Int. i. p. $3+$ (1862); Wall. Ibis, 1868 , p. 5; Gray, Hand-l. B. i. p. 20 (1869); Hume, Rough Notes, i. p. 87 (1869); Wald. Tr. Z. S. viii. p. 33 (1872); Salv. Cat. Vcc. di Born. p. 2 (1874).

Hab. Luzón, Cagayan, Negros, Sa:nar, Parazua; Subregión Indo-Malaya, Célebes, Nueva Guinea. 


\section{Gen CERCHNEIS, Bole.}

9 C. tinnuncula, (Lin.) ơ N. v Lagpang.

(M. S. T.)

L'Epervier des Alouettes, Briss. Orn. i. p. 279 ( $17(x)$ ).

La Cresserelle, Briss. Orn. i. p. 393 (1760); Buff. Fl. Enl. i. pls. 402,47 I $\left(177^{\circ}\right)$.

Falco tinnunculus, Linn. S. N. i. p. 127 (г 766); Temm. Man. d'Orn. i. p. 29 ( 1820$)$; Naum. Vog. Deutschl. i. p. 323, Taf. 30 (1822); Werner, Atlas, Rapaces, pl. I2 (1827); Gondd. B. Eur. i. pl. 26, (1837); Schl. u. Susem. Vog. Eiur. 'Taf. I (1839-45); Macgill. Hist. Brit. B. iii. p. 325 (18f(1); Yarr. Brit. B. i. p. $52(18+3)$; Kjarb. Orn. I)an. Afb. iii. fig. 4 (1851); Schl. Vog. Nederl. pls. 9, го (1854); Hartl. Orn. $H^{2}$. Afr. p. 9 (1857); Fritsch, Vog. Eur. tab. 4 figs. 6, 7 (1858); Schl. Mus. P. B. Falc. p. 25 (1862); Dgl. \& Gerle, Orn. Eur. i. 1. 93 (1867); Hengl. Orn. N. O.-Afr. i. p. 40 (1869); Sunder. Siv. Figl. p. 216 , pl. xxvi. figs. 5, 6; Blanf. Geolf. \& Zool. Aliyss. p. 290 (1870); Sharpe \& Dresser, B. Eur. pt. 2 (1871); Newt. ed Yarr. Bit. B. p. 79 (I871); Salvad. Fann. Ital. licc. p. 22 (1871); Shelley, B. Esypt, p. $594(1872)$; Schl. Revue Acciptr. p. 4 I (1873).

F. alaudarius, Gm.S. N. i. p. 279 (1 788 , ex Briss.)

F. fasciatus, Retz. Faun. Succ. p. zo (1800).

F. brunneus, Bechst. Orn. Taschenh. p. 38 (1802).

Cerchneis tinnuncula, Boie, Isis, 1828 , p. 314; Brehm, Vög. Deutsch. p. 73 (1831); Sharpe. Cat. Brit. Mus. p. 425 (1874).

C. murum, Brehm, Vög. Deutsch. p. 70 (1831). media, Brehm.

F. architinnunculus, Bremh, ut supra.

Tinnunculus alaudarius, Gray, Gen. of B. i. p. 2 I (1844); Bp. Consp. i. p. 27 (1850); Kaup. Contr. Orn. $185^{\circ}$, p. 53; Gould, B. Gt. Br. pt. ii. (1862); Jerd. B. Ind. i. p. 38 (1862); Pels. Verh. z.-b. Wien, xiii. p. 623 (186.3); Blyth, Ibis, 1863, p. 9; Nezit. Ooot. Wolley. p. 80 (1864); More, Ibis, 1865, p. 9; Tristr. t. c. p. 259; Hume, Rough Notes, i. p. 96 (1869); Gray, Haud-l. B. i. p. 22 (1869); Finsch, Tr. Z. S. vii. p. 205 (1870) Sharpe, Ibis, 1870 , p. 486 .

Cerchneis tæniura, intercedens accedens, fasciata, guttata, Brehm, Natmania, 1855 , p. 269.

Tinnunculus tinnunculus, Heugl. Peterm. Mitk. 1861 , p. 20. $H a b$. Luzon, Cagayan; Europa, Africa, China, Japon.

\section{Suborden 2.० PANDIONES.}

\section{Fam. PANDIÓNIDAS.}

\section{Gén. PANDION, $\mathbf{S}_{\mathrm{Aw}}$.}

1. P. haliœtus, (Lin.) $\sigma \circ$ N. v. Anol.

(M. S. T.)

The Fishing, Hawk, Catesby N.H. Carol. i. pl. 2 ( I73I).

Le Faucon pescheur, de la Caroline, Briss, Orn. i. p. $362(1760)$. 
. Lirle de Mer, Briss. Oru. i. p. $4+0(1760)$.

Falco haliaetus, Linn. S. N. i, p. г $29(1766)$; Naum. Vög. Deıtschl. i. p. 24I, pl. 16 (1822); Werner, Altas, Rapaces, pl. 19 (1827); Schl. u. Susem. Vög. Ëur. 'Taf. $2+$ (1839).

Le Balbuzard, Buff. Pl. Enl. i. pl. $+1+$.

Falco arundinaceus, Gm.S. N. i. p. 263 (1788).

F. carolinensis, Gm. N. S. i. p. 263 (1788).

F. cayenensis, Gm. S. N. i. p. 263 (1788).

Aquila americana, Vieill. Ois. Am. Sept. i. p. 31 ( 1 Bon).

A. piscatrix, Vieill. Ois. Am. Sept. i. p. 29, pl. $+(18,7)$.

Pandion fluvia!is, Sav gin. Descr. Foypte, Os. p. $272(1807)$.

Aquila haliaetus, Meyer. in Mey. u. Wolf. Taschend. i. p. 23 ( I $80 \mathrm{I})$.

Accipiter haliaetus, Pall. Zoogr. Rosso-As. i. p. 355 (1811).

Triorches fluvialis, Leach, Syst. Cat. Mamm. \&c. B. M. p. $10(1816)$. Aquila balbusardus, Dlumont, Dict. Sci. Nat. i. p. 351 (1816).

Pandion americanus, Vieill, et Oud. Gal. Ois. pl. I1 (1825.)

Balbusardus haliaetus, Fleming, Brit. An. p. 5 I (1828).

Pandion haliaetus, Les. Man. d'Orn. i. p. 86 (1828; Sw. \&. Rich. Fam. Bor.-Am., Birds, p. 20 (1831); Gould, B. Eur. pl. 12 (1837); Gray Gen. B. i. p. 17, pl. 7. fig. 5 (18+5); Bp. Consp. i. p. 16 (1850); Schl. Vog. Nederl.pl. 30 (1854); Strickl. Orn. Syn. p. 63 (1855); Harll. Orn. W.-Afr. p. 7 (1857); Fritsch, Vög. Eug. tab. 9 fig. I (1858); Schrenck, Reis. Amurl., Zoo!. p. 227 (1859); Jerd. B. Ind. i. p. 80 (1862); Schl. Mus. P.-B. Aquil.e, p. 22 (1862); Radde, Reis Sibir., Ois. p. 97 (1863); Nezut. Ooth. Wolley, p. $5^{8}$ (1864); Sunder Su. Fugl. pl. xxix. fig. I (1 866); Degl. \& Gerbe, Orn. Eur. i. p. 47 (1867); Layard, B. S. Afr. p. I6 (1867); Hume, Rough. Notes, i. p. $23+$ (1869); Gould. B. Gt. Br. pt. xvii. (1870); F.nsch. u. Hartl. Vög. Ostafr. p. 40 (1870); Nezut. ed. Yarr. Brit. B. i. p. 3o (1871); Pela. Orn. Bras. p. 4 (1871); Swinh. P. Z. S. 1871 , p. 340; Coues, Key N. Am. B. p. 219 (1872); Holdsw. P. $Z$. S. 1872, p. 412; Salvad. Faun. Ital. Ucc. p. 9, 1872; Sehllley, B. Egypt, p. 203 (1872); Schl. Mus. P.-B. Revue Accipitr. p. I 23 (1873). Sharpe, Cat. Brit. Mus. p. 449 (1874).

P. alticeps, Bremh, Vög. Deutschl. p. 33 (I83r), planiceps, id. id.

P. carolinensis, Andub. B. N. Am. pl. 81, et Orn. Biogr. i. p. 415 (1831); Bp. Comp. List B. Eur. \& N.-Am. p. 3 (1838); id. Consp. i. p. 16 (1850); Cassin. B. Calif. \& Texas, p. I I 2 (1855); Strickl. Orn. Syn. p. 64 (1855); Cass. in Baird's B. N. Am. p. 45 (1860); Dall \& Bann. Tr. Chic. Acad. p. 272 (1869); Gray, Hand-l. B. i. p. I 5 (1869); Cooper, B. Calif. ed. Baird, p. 454 (1870.)

P. indicus, Hodgs. in Gray's Zool. Misc. p. 81 (I844).

Hab. Luzón, Ilocos, Cagayan, Marinduque; Europa, Asia, China, América.

2. P. leucocephalus, Gauld. $\sigma$ N. v. Anol.

Pandion leucocephalus, Gould. Syn. B. Austr. pt. iii. pl. 6 (1838); Gray, Gen. B. i. p. I7 (1845); Gould, B. Austr. i. pl. 6 (1848), Consp. Av. i. p. I6 (1850); Strickl. Orn. Syn. p. 65 (1855); Gould, Handb. B. Austr. i. p. I5 (i869); Schl. Vog. 
Nederl. Ind. pp. I 2, 52, pl. 3. fig. 3 (1866); Wall. Ibis, 1868, p. 15; Gray, Hand-l. B. i. p. I 5 (1 860 ). Sharpe, Cat. Brit. Mus. p. 45I (Sub esp. a) (1874).

P. gouldi, Kaup, Isis, 1847 , p. 270 .

Hab. Luzon, N." Ecija, Paragua, Marinduque; Australia, Molucas.

\section{POLIO(ETLS, KAUP.}

3 P ichthyøtus, (Horsf.) \& $? \mathrm{~N}$ v. Anol.

(M. S. T.)

Falco ichthyaetus, Horsf. Tr. Lin. Soc. xiii, p 136 (1822); id. $Z$. Res. Java, pl. 34 i 824$)$.

Pandion ichthyaetus, Vig. Zool. Journ, i. p, 321 (s824); Gray', Cat. Accipitr. 1848 , p. 23; Bp. Consp. i. p. 16 (1850); Schl. Vog. Nederl. Ind. Valkv. pp. I 3, 62, pl. 5 figs. I, 2 ( I 866); Gray, Hand-l. B. i. p. I 5 (I 869).

Haliaetus ichthyaetus, Cuv. Rig. An. i. p. 327 (1824); Schl. Mus. P.-B. Aquilæ, p. I 8 (1 862 ).

H. unicolor, Gray, \& Hardzo. Ill. Ind. Zool. i. pl, ig (183()).

H. plumbeus, Hodgs. J. A. S. B. vi. p. 367 (1837).

Ichthyaetus bicolor, Gray, List Gen. B. i. p. + (18+1); Strickl. Oru. Syn. p. 66 (1855).

Ich. hosrfieldi, Blyth, J.A. S. B. xi. p. I IO (I $8+2)$.

I. plumbeus, Hodgs. in Gray's Zool Misc. p. 81 (18+4).

I. hucarius, Hodgs. in Gray's Zool. Misc. p. 8I (1844).

Pontoaetus ichthyaetus, Gray, Gen. B. i. P. I 7 ( 1845); Blyth, Cat. B. Mus. A. S. B. p. 30 (1849; id. Ibis, 1863 , p. 20 et 1865, p. 28 .

Polioaetus ichthyaetus, Kaup, Contr. Orn, 1850, p. 73 ; Jerd. 13. Ind. i, p. 81 (1862); Blyth, Ibis, 1866, p. 243; Beav. \& Tytl. Ib s, 1867, p. 316; Wall. Ibis, 1868 , p. It; Hume, Rought $N$. ii. p. I (1870); Jerd. Ibis, 1871 , P. 336; Blyth, Ibis, 1872 , p. 88. Sharpe, Gat. Brit. Mus, p. 452 (1874).

Hab. Luzon, Ilocos, Cagayan, Mindanao; Borneo, Célebes, Java, Sumatra, Malaca, India.

\section{Suborden 3. ${ }^{\circ}$ NOCTURINAS.}

Fam. BUBÓNIDAS.

Subfam. BUBONINAS.

I. Gén. BUBO, Cuv.

1. B. ignavus, Forster, N. v. Gran iluque.

(M. S. T.)

'The Great Horn-Owl or Eagle Owl, Albin, Birds, ii. pl. 9.

Great Horned Owl, from Athens, Edwards, Glean. pl. 227.

Le Grand Duc, Briss. Orn. i. p. 477; Buff. Pl. Enl. i. pl. 435.

Le Grand Duc d'Italie, Briss. Orn. i. p. 482.

Strix bubo, Lin. S. N. i. p. I3 ; Shaw, Mus. Lever. p. I 9, pl. 29; 
Temm. Man. d'Orn. i. p. wor Roux. Orn. Prö. p. 6\% pl. fo;

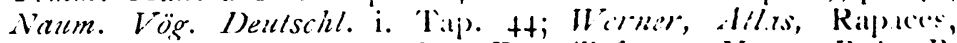
pl. +1; Schil. \& Susem. lög. Eur. Taf. 50; Yar. Bl\% B. i. p. Io7; Kjarb. Orn. I)an. pl. vii. tig. 3 ; Mdt. Sibir. Reis. p. Itr: Sundea. Sir. Fogl. p. Iss, pl. xxir. tig. 4.

Bube microcephalus, Leack, Syst. Cat. Mamm. So. Brit. Mus. p. I I (1816); Steph. Gen. Zool. xiii. pt. 2. p. 55.

B. ignarus, Forster, Sy'n. Cat. Brit. B. p. 3 ; Nezit. ell Yari. Brit. B. i. p. I68; Shellev, B. Egypte, p. 180 ; Dressir, $B$. Eur. part xxii. Sharpe. (iat. Brit. Mus. 2 p. It (1875).

13. maximus, Fileming, Bril. An. p. 57; Gould, B. Eiur. i. pl. 37; Bp. Comp. I. st B. Eur. \& N. Am. p. 6; Wacgill. Brit. B. iii. p. 228 ; Gray, Gen. B. i. p. 37; Kaup, Tr.Z. s. iv. p. 239, et Contr. Orn. 1852 , p. I 6 ; Horsf. \& Moore, Cat. B. Mus. E. I. Co. i. p. 72 ; Strickl. Orn. Syn. p. 212 ; Hewits. Eegs. Br. B. i. p. 5o, pl. xvii. fig. t; Frilsch, Vög. Eur, tab. I2. tig. 5; Jaub. \& Barth. Lapom. Rich. Orn. p. 8o; Schil. Mus. P.-B. ()ti. p. 7 ; Newt. Ooth. Wollyy. p. Itor; I)egl. \& Gerbe, Orn. Eur. i. p. I4I; Loche, Expl. Sci. d'Algér. Ois; p. Ioo; Gray. Hand-l. B. i. p. 43 ; Heugl. Orn. N. O.-Alfr. i. p. I o; Gould, B. Gt. Br. pl. xxx.; Hume, Rough, Notes, ii. p. 374; Bettoni, Ucc. Lomb. i. tav. Io7; Salvad. Faun. Ital. LCC. p. 32 .

B. germanicus, Brehm, Vög. Deutschl. p. 119. septentrionalis, id. id. p. 120 .

B. europaus, Less. Traité, p. I I5, pl. i7. fig. I.

Asio bubo, Swatins. Classif. B, ii. p. 287.

Otus bubo, Schl. Rev. Crit. p. xiii.

B. atheniensis, Bp. Consp. i. p. $48 ;$ Taczan. J. f. O. 1860 , p. 39 .

B. grandis, Brekm, J. f. O. 1853, p. 346 .

B. bubo, Licht. Nomencl. Av. p. 7 .

B. melanotus, Brehm, Natum. 18.55 , p. 270 .

Hab. Europa, Asia, China, Himalaya, Africa.

2. B. philippinensis, S:hl. ơ N. v. Cuago Cul-lalao. M.S.T.)

Scops phil ppensis; Gray, Cat. Accipitr. 1844, p. 45.

Syrnium philippens, Gray, Cat. Accipitr. 1848 , p. $105 ;$ Bp. Consp. i. p. 52; Strickl. Orn. Syn. p. I9I.

Pseudoptynx philippensis, Kaup, Cantr. Orn. 1852, p. I I7; id. Tr. Z. S. iv. p. 24t; Walden, Tr. Z. S. ix. p. 144, pl. xxv. fig. 2.

Bubu philippensis, Schl. Mus. P.-B. Oti, p. I4; id. Dierent. p. Io, fig. 3; Wall. Ibis, 1868 , p. 25; Gray, Hand-l. B. i. p. 45; Sharpe, Cat. Mus. 2 p. 43 (1875.)

Hab. Luzon, Laguna, Pampanga, Cagayan, Bulacan, IlocosNorte.

3. B. gurneyi, Tw. N. v. Cuago.

(M S. T)

Pseudoptynx gurneyi, Tw. Works. p. 632 (1881); P. Z. S. 1878. p. 94I.

Hab. Mindanao, Zamboanga, Paragua. 
2. Gén. SCOPS, SAvig.

4. S. mogalotis, Gray. ơ N. v. Cuago.

(M. S. T.)

Scops megalotis, Gray, Cat. Accipitr. 1844 , p. 45 ; Strickl. Orn. Syn. p. 202; Gray, Hand-l. B. i. p. 46. Sharp. Cat. Brit. Mus. p. 692 (1873).

Ephialtes megalotis, Gray, Cat. Accipitr. 1848, p. 98; Bp. Consp. i. p. 45 .

Ptilopsis megalotis, Bp. Rev. et Mag. de Zool. 1854, p. $5+3$.

Lempijius megalotis, Walden, $\operatorname{Tr} . Z$. $S$. ix. p. I45, pl. xxv. fig. 3 . Hab. Luzon, Manila, Bulacan.

5. S. lempiji, (Horsf.) ơ N. v. Cuago.

(M. S. T.)

Strix lempiji, Horsf. Tr. Lnun Soc. xiii. p. i fo.

St. noctula, Temm. Pl. Col. ii. pl. 99. ex Reinzw.

Scops noctula, Steph. Gen. Zool. xlii. pt. 2, p. 52; Schil. Mus. P.-B. Oti, p. 24 .

Sc. javanicus, Less. Traité, p. I07; Pucher. Rav. et Mag, de Zool. 1849 , p. 26.

Sc. lempiji, Blyth, J. A. S. B. xv. pt. 1; p. i $82 ;$ id. Cat. B. Mus. A. S. B. p. 36; Bp. Consp. i. p. 47; Strickl. Orn. Syn. p. 200; Gray, Hand-l. B. i. p. 46; Schl. Mus. P.-B. Revue Accipitr. p. I 3; Salvad. Ucc. Borneo, p. I9 I874; Sharpe, Cat. Brit. Mus. 2 p. 9i (1875).

Megascops lempiji, Kaup, Contr. Orn. 1852, p. iा 2 ;id. Tr. Z. S. iv. p. 229.

Lempijius indicus, Bp. Rev. et Mag. de Zool. 1854 , p. $5+3$.

L. noctula, Bp. Rev. et $M a g$. de Zool. 1854 , p. 543 .

L. lempiji, G. R. Gray, Hand-l. i. p. 46 esp. 47 I (i869).

Ephialtes lempiji, Gray, Gen. B. i. p. 38 ; Horsf. \& Moore, Cat. B. Mus. E. I. Co. i. p. 7 I Wall. Ibis, 1868 , p. 2+. Hzome, Str. F. ii. p. 469 .

Hab. Samar, Borongan, Paragua; Borneo, Java, Sumatra.

6. S. rufescens, Horsf. $\sigma \mathrm{N}$ v Cuago.

(M. S. T)

Strix rufescens, Horsf. Tr. Linn. Soc. xiii. p. i 40.

Scops rufescens, Sw. Classif. B. ii. p. 2 г7; Blyth, J. A. S. B. xiv. p. I8I; Strickl. Orn. Syı. p. 201; Gray, Hand-l. B. i. p. 46; Schl. Mus. P.-B. Revue Accipitr. p. io; Salvad. Ucc. Born. p. 19 (1874); Sharpe. Cat. Brit. Mus. 2. p. 102 (1875).

Otus mantis, Temm. \& Schl. Faun. Jap. Aves, p. 25.

Ephialtes mantis, Gray, Gen. B. i. p. 38; Wall. bis, is68, p. 24. Scops mantis, Strickl. Orn. Syn. p. 20r; Schl. Mus. P.B. Oti, p. 25; Gray, Hand-l. B. i. p. 46.

Ephialtes rufescens, Horsf. \& Moore, Cat. B. Mus. E. I. Co. i. p. $7 \mathrm{I}$.

Lempijius mantis, Bp. Rev. et Mag. de Zool. 4854, p. $5+3$.

Hab. Samar. Basilan, Mindanao, Joló; Borneo. 
7. S. everetti, Tiu. ơ N. v. Cuajao.

Works, P. 633 (I88I).

Hab. Samar, Basilan, Mindanao, Zamboanga.

8. S. asio, (Lin.) ơ N. v. Peq. duque de la Carolina.

(M. S. T.)

The Little Owl, Catesby, N. H. Carol. i. p. 7, pl. 7 .

Le Petit Inuc. de la Caroline, Briss. Orn. i. p. 497.

Strix asio, Linn. S. $N$. i. p. г $32 ;$ Wils. Am. Orn. vi. 1.1. 42. fig. I; Temm. Pl. Col. ii. pl. 80; Audub. B. Am. pl. xvii.; id. Orn. Biogr. i. p. 486.

Red Owl. Penn. Arctic Zool. ii. p. 23I, pl. xi. fig. I.

Mottled Owul, Penn. t. c. p. 23I, pl. xi. fig. 2.

Strix nævia, Gm. S. N. i. p. 289. (ex Penn.); Wils. Am. Orn. pl. xix. fig. I.

Bubo asio, Vieill. Ois. d' Am. Sept. p. 53, pl. 2 I.

B. striatus, Vieill. t. c. p. 54 .

Otus asio, Steph. Gen. Zool. xiii. pt. 2, p. 57; Schl. Faun. Jap. 1). 25 .

Asio asio, Less. Man. d' Orn. i. p. 187.

()tus navius, Cuv. Regne Aluim. 1829, p. 24 I.

Surnia navia, James. ed. Wils. Am. Orn. i. p. 96.

Ephialtes asio, Gray, Gen. B. i. p. $3^{8}$.

Scops asio, Bp. Consp. i. p. 45; Kaup, Contr. Orn. Is52, p. I 1 2; Strickl. Orn. Syn. p. r99; Cisss. B. N. Am. p. 51; Schl. Mus. P.-B. Oti, p. 27; Gray, Han.t-l. B. i. p. 46; Sch!. Revue Accipitr. p. 9; Ridgw. B. N. Am. iii. p. 49; Cones, B. N. W. p. 3(3. Sharpe, Cat. Brit. Mus. 2. p. IIt (1835).

Megascops asio, Kaup, Tr. Z. S. iv. p. 228.

Asio nævia, Bp. Rev. et Mag. de Zool. 1854 , p. 543.

Hab. Estados Unidos, Canadá.

3. Gén. CARine, Kaup.

9. C. nostua, (Scops.) to Pequeño mochuelo

(M. S. T.)

Little Owl, 'Edwards,, Gleanings, pl. 228.

La Petite Chouette ou La Cherèche, Briss, Orn. i. p. 5 r.

Strix noctua, Scop. Ann. i. p. 22; Naum. Vög. Deutschl. i. p. 493, pl. 48. fig. I; Schl. z. Susem. Vög. Eutr. 'Taf. 47. fig : I, 2 ; Nordm. in Demid. Voy. Russ. Mérid. iii. p. ıo9; Bailly, Orn. Sav. i. p. I94; Keulem. Onze Vogels, pl. I.

La Chevêche, Buff. Pl. Enl. pl. 439.

S. passerina, Gm. S. N. i. p. 296; Temm. Man. d'Orn. i. p. 92; Roux, Orn. Prov. i. p. 78, pl. 53; Werner, Atlas, Rapaces, 1). 39; Yarr. Brit. B. i. p. I 42 .

S. psilodactyla, Nilss. Skand. Faun. Ist. ed. p. 88.

S. nudipes, Nilss. Orn. Suec. i. p. 68.

Athene passerina, Boie, Isis, 1822 , p. 549; Brehm, Vög. Dzutschl. P. IIO.

Carine noctua, Kaup, Natiurl. Syst. p. 29; Newt. ed. Yarr. Brit. 
B. i. p. 178; Irby, B. Gibr. p. 58; Shtrpe, Ibis, 1875, p. 25 S. id. Cat. Brit. Mus. p. I 33 (1875).

Noctuin passerina, Cuv. Régne An. i. p. 345 ; Less. Traile de Orn. 1. 103; Selby, Ill. Brit. Orn. i. p. ro7, pl. 25; Stevenson, B. Norf. i. p. 59.

Surnia Noctua, Bp. Oss. Regn. Anim Cav. p. $4^{8 ;}$ Keys. n. Blas. Wirb. Eur. p. xxxii; Borggr. Vogelf. Norddeutschl. p. 64.

Scotophilus nulipes, Jard. Brit. B. i. p. 274.

Noctua nudipes, Gould, B. Eur. i. pl. 48; Hezvits. Egogs Br. B. i. p. 67, pl. xix. fig. 3 .

Athene noctua, Bp. Comp. List. B. Eur. \& N. Am. p. 6; Gray, Gen. B. i. p. 34; Bp. Consp. i. p. 39; Kaup, Contr. Orn. 1852 , p. I I 5, et Tr. Z. S. iv. p. 2 Io; Sch!. Vog. Nederl. pl. 43; Strickl. Orn. Syn. p. I 39; Fritsch, Vög. Eür. tib. I I. fir. 2; Jaub. et Barth. Lapom. Rich. Orn. p. 77; Newt. Ooth. Wolley. p. I5I; Gould, B. Gt. Br. pl. xxxvii.; Gray, Hand-l. B. i. p. 39; Salvad. Fanun. Ital. Ucc. p. 29; Sharps \& Dressis, B. Eutr. part. iv.

Syrnia psilodactyla, Macgill. Brit. B. iii. p. 4 I7.

Athene psilodactyla, indigen.1, Brehm, Naun. 1855 , p. 270.

Noctua reterum, Schl. Mils. P.-B. Strigcs p. 28; id. Revue Acc:pitr. p. 26,

Noctua minor, Degl. \& Gerbe, Orn. Eur. i. p. 122 Hab. Euroja, Francia, España, Alenania.

4. Gén. NinOX, Hovgs.

เo. N. lugubris, Tich. $\sigma \circ$ N. v. Caruang.

Strix lugubris, Ticksll, J. $A$ S. B. ii. p. 573.

Ninox nipalensis. Hodgs. Madr. Journ. v. p. 24 , pl. I4.

()tus lugubris, Jerd. Madr. Journ. x. p. 7.

Ninox lugubris, Blyth, Ann. N. H. xii. pp. 93, 23I; Sizrpo, Ibis, ${ }_{1875}$, p. 258 . id. Cat. B. 2. p. I 54 (1874).

Ninox jeridius, Hods. in Grays Zool. Misc. p. 82.

Ninox scutellatus, Blyth, J. A.S. B. xvi. p. 518; Jerd. B. Ind.

-i. p. I47; Hume, Rough Notes, ii. p. 420.

Athene hirsuta, Bonap. Consp. i. p. 4I.

Ninox madagascariensis, Bonxp. R:J. et Mxg. de Zosl. 1854, p. 543; Hartl. Faun. Matag. p. 22; Gurnyy, Ibis, i'85g, p. +53.

Noctuit hirsuta, Schl. Mas. P.-B. Striges, p. 25.

Athene lugubris, Gray, H.nnt-l. $\dot{B}$. i. p. 4 I.

Athene Madagascariensis, Gray, Hand-l. B. i. p. 42.

Hab. Luzón, Guinarás, St.nar, Paragua, P. Princesa, B.ısilan, Mindanao; India Himalaya, Malabar.

11. N. paraguauensis, $\sigma$ N. $\mathrm{V}$ G:ague.

Hab. Paragua, Puerto Princesa.

$12 \mathbf{N}$. philippinensis, $\sigma, 0 \mathrm{~N}$. v Caago.

( $\left(\begin{array}{lll}M & \mathrm{~S} & \mathrm{~T}\end{array}\right)$

Ninox philippensis, Bp. C. R. xli. p. 654; W.tlen, Tr. Z. S. p. I 44, pl. xxv. fig. I. Sharpe, Cat. Brit. 2. p. I67 (1875). 
Noctua hirsuta philippinensis, Schl. Mus. P.-B. Striges, p. 20. Athene philippensis, Wall. Ibis, $18^{2} 08$, p. 22; Gray, Hand.l. $B$. i. p. 42 .

Hab. Luzón, Manila, Laguna, Bulacan, Marinduque, Siquijor, Samar, Negros, Guimarás.

I3. N. scutulata. (Raff.) ơ N. v. Balimpasayo.

(.I. S T T.)

Strix scutulata, Raffl. Tr. Linn. Soc. xiii. p. (28(1)).

Strix hirsuta, Temm. Pl. Col. i, pl. 289) (1824).

Noctua hirsuta, Steph. Hen. Zool. xiii. pt. 2, 1). 66.

Surnia hirsuta, Less. Man. d'Orn. i. p. I Io.

Athene malaccensis, Eyton, Ann. N. H. xvi. p. 228.

Athene scutulata, Gray. Gen. B. i. p. 35; Kelaart, Prodr, Fann. Zeyl. p. 28; Horsf. \& Moore, Cat. B. Mus. E. I. Co. i. p. os; Strickl. Orn. Sys. p. I70; Wall. Ibis, 1868 , 1. 23.

Strix hirsuta japonica, Schl. Faun. Jap. Aves, p. 20, pl. 9 13.

Athene hirsuta, Bp. Consp. i. p. 4r; Gray, Hand-l B. i. 1. +1; Pelz. Voy. Naziara, Aves, p. 26.

Athene japonica, Bp. Consp. i. p. 41; Gray, Hand-l. B. i. 1. +2.

A. borneensis, Bp. Consp. i. p. 4 r; Gray, Hand-l. B. i. 1. $42 ;$ Pelz. Voy. Novara, Vög. p. 26, Wall. Ibis, 1868, p. 22.

Ctenoglaux scutellatus, Kaup, Contr. Orn. 1852, p. iog; et Tr. $Z$. S. iv. p. 219.

Ninox japonicus, Bp. Rev. et Mag. de Zool. 1854 , p. 543; Halden, Tr. Z. S. viii. p. 40; Swinh. P. Z. S. 1871,1 . 34.3; Sharpe, Ibis, 1875 , p. 259.

Ninox hirsutus, Bp. Rev. et Mag. de Zool. 1864 , p. 543; Holdsw. P. Z. S. 1873, p 418; Hume, Str. F. 1874 , Pl. I 5I, 469, et 1875, p. $44 \mathrm{I}$.

Ninox bornensis, Bp. Rev. et Mag. de Zool. 1854, p. 5+3; Walden, Ibis, 1872, p. I 8; Salvad. Ucc. Born. p. i 8 .

Noctua hirsuta japonica, Schl, Mus. P.-B.. Striges, p. 24.

N. hirsuta borneensis, Schl. t. c. p. 24.

Athene florensis, Wall. P. Z.S. 1863 , p. 488; id. Ibis, 1868 , p. 23 ; Gray, Hand-l. B. i. p. 48.

Noctua hirsuta minor, Schl. Mus. P.-B. Revue Accipitr. p. 25

Ninox scutulata, Sharpe, Cat. Brit. Mus. 2. p. ${ }_{156}$ (1875).

Hab. Luzón, Paragua, Mindanao, Basilan; India, China, Malaca.

I4 N. spilocephalus, (Stcere) N. v. Bucao.

(M. S. T.)

Hab. Paragua, Basilan, Mindanao.

Subfam. SIRNINAS.

I. Gén. SYRniUm, Sav.

I. S. leptogrammicum, (T'm.) \& N. v. Cuago.

(M. S. T.)

Strix leptogrammica, Temm. Pl. Col. ii. pl. 525 .

Syrnium leptogrammicum, Gray, G»n. B. iii. App. p. 3; Strickl. Orn. Syn. p. 193; Hand-l. B. i. p. 49. Sharpe, Cat. B. Mus. 2 p. 264 (i 875 ). 
Ciccaba lejtogrammica, Bp. Consp. i. p. 4t; Kaup, Tr. Z. S. iv. p. 255; Wall. Ibis, 1868 , p. 26; Salvad. Ucc. Born. p. 21.

Bulaca leptogrammica, Kaup. Contr. Orn. 1852, p. $13 \%$.

Myrtha leptogrammica, Bp. Rev. et Mag. de Zool. 1854,1 . $5+1$. L'lula leptogrammica, Schl. Mus. P.-B. Striges, p. 20, et Reoue Accipitr. p. 2I.

Hab. Paragua; Borneo.

2. S. sinense. (Lath.) \& N. v. Cuago

(M. S T.)

(hina ()wl, Lath. Gen. Syn. Suppl. ii. Add. p. 368.

Strix sinensis, Lath. Ind. Orn. Suppl. p. xvi.

S. orientalis, Shaw, Gen. Zosl. vii. p. 257 (e.t Luth.).

S. seloputo, Horsf. Tr. Linn. Soc. xiii. p. 14ø; Less. Man. i. p. 1og.

Strix pagodarum, Temm. Pl. Col. i. pl. $23 \%$.

Noctua pagodarum, Steph. Gen. Zool. xiii. pt. 2, 1). $6_{5}$.

Ulula pagodarum, Less. Man. d'Orn. i. p. 113 .

Syrnium pagodarum, Cuvier, Régne An. i. p. $3+3$.

Bulaca sinensis, Jerd. Madr. Journ. x. p. 88; Hume, Rough Notes, ii. p. 357 .

Syrnium selopufo, Gray, Gen. B. i. p. 39; Cass. Cat. Strig'de Philad. Mus. p. 6; Horsf. \& Moore, Cat. B. Mus. E. I. Co. i. 1. 84 ; Strickl. Orn. Syn. p. I91; Gray, Hand.l. B. i. p. 49.

Bulaca seloputo, Kanp, Contr. Orn. 1852, p. 122.

Liccaba pagodarum, Bp. Consp. i. p. 44.

Myrtha pagodarum, Bp. Rev. et Mag. de Zool. 18.54, p. 54 .

Ciccaba seloputo, Kanp. Tr. Z. S. iv. p. 254; Wall. Ibis, 1868 , p. 26; Salzad. Ucc. Born. p. 21.

Ulula seloputo, Schl. Mus. P.-S. Striges, p. 22.

Syrnium sinense, Sharpe, Cat. B. Mils. 2, 26I (1875).

Hab. Luzon; Java, Cochinchina.

\section{Fam. EXTRÍGIDAS.}

\section{Gén. STRIX. Lix.}

I S. flammea, Lin. N. v. Cuago, Puec.

(M. S T.)

'The Common Born-Owl or White Ow-1, Albin, N. H. Birds, ii. pl. xi. Le petit Chat-huant, Briss. Orn. i. p. 503.

Strix flammea, Linn. S. $N$. i. p. 133; Meyer u. Wolf. Vög. Deutschl. pl. to p. 185 ; Wils. Am. Orn. vi. p. 51, pl. 58 , fig. 2; Vieill. Faun. Franc. pl. 22. fig. I; Temm. Man. d'Orn. i. p. 91; Max. Reis. Bras. ii. 1821, p. 265; Naum. Vög. Deutschl. i. p. 483, pl. 47. fig. 2; Roux, Orn. Prov. pls. 54, 55; Werner, Atlas, Rapaces, pl. 36; Brehm, Vög. Deutschl. p. 186; Less. Traité, p. п 2 ; Audub. B. Amer. pl. clxxi.; id. Orn. Biogr. ii. p. $4^{8} 3$; Gould, B. Euer. i. pl. 36; Schl. u. Susem. Vög. Eur 'Taf. 46; Macgill. Brit. B. iii. p. 473; Nordm. in Démid. Voy Russie Mérid. iii. p. 118; Pears. J. A. S. B.x. p. 638; Darw. Voy, Beagle, Birds, p. 34; Yarr. Brit. B. i. p. 127; Gray, Gen. B. i. p. 41, pl. 15. fig. 1; Thomps. B. Irel i. p. 92; Blyth, Cat B. Mus. A. S. B. p. 41; Bp. Consp. i. p. 55; Kaup, Contr. Oru. 1852 , p. 18 ; Horsf. \& 
Moore, Cat. B. Mus E. I. Co. i. p. 8r; HarH. Orn. Hintur. p. 21; Schl. Vög. Nederl. pl. ti; Strickl. Orn. Sinll. p. 1-i;; Hezvits. Eggs Brit, B. i. p. 61, pl. xriii. fig. 1; Sunder'. Si. Fogl. p. I3r, pl. lxxi. fig. I; Fritsch, Vög. Eur. tab. ii. lig. +i Jaub. \& Barth.-Lap. Rich. Orn. p. 86; Gould, B. Cit. Bi. i. pl. xxriii; Schl. Mus. P.-B. Strigres, p. 1; Nez\%. Ooth. Wolley. p. I52; Sal Ibis, 1864 , p. 387; Pelz. Reis. Noo. Vï;; p 29; Degl. \& Gerbe, Orn. Eiur. i. p. 13.3; Loche, E.xp!. Sci. Algér. (Ois. i. p. 91; Schl. \& Poll. Firun. Madtur. (lis. p. 48; Gran, Hand-l B. i. p. 52; Heugl. Os"n. N. O.-Alfi. i. p. I 24 ; Allen, Bull. ara. Coll. ii. p. 3tı; Godman, Azores, p. 22; Finsch \& Hartl. Vög. Ostafi. p. In; Bard, ed. (bo. per's B. Calif. p. 4r5; Salvad. Faun. Ital. Ucc. p. 27; Pelz. Orn. Bras. pp. is. t8s; Scl. \& Salv. Nomencl. di'. Niotr. p. I 6 ; Sharpe, ed Layard, B. S. Afr. p. 82; id. Cat. B. M. 2 p. 291 ( 1875$)$.

Strix aiba, Scop. Ann. i. p. 2.

L'Effraye ou La Fresaye, Buff. i. pl. 448.

Strix javanica, Gm. S. N. i. p. 295; Jerd. Madr. Journ. x. p. $85 ;$ Gray, Gen. B. i. p. + r, pl. i 5 ; Blyth, J. A. S. B. xix. p. 513 ; Bp. Consp. i. p. 55; Horsf. \& Moore, Cat. B. Mus. E. I. C'o. i. p. 8z; Strickl. Orn. Sym. p. I88; Jerd. B. Ind. i. 1. I17; Wall. Ibis, 1868, p. 26; Gray, Hand-l. B. i. p. 52 ; Salvad. Ucc. Born. p. 22; Hume, Nests \& Eggs Ind. B. p. 59.

Lechuza, Azara, Apunt. ii. p. 2 ro; Hartl. Iud. Azara, p. 3. Strix perlata, Litcht. Verz. Doubl. p. 59; Max. Beitr. iii. p. 263; D'Orb. Voy. Amér. Mér. Ois. p. i35; Gray, Gen. B. i. p. 4'; Schomb. Reis. Guian. iii. p. 732; Gray, Cat. Accipitr. 1848 , p. Io9; Cass. Cat. Strigide Philad. Mus. p. 1; Bp. Consp. i. p. 55; Kaup, Contr. Orn. I852, p. II9; id. Tr. Z. S. iv. p. 247; Strickl. Orn. Syn. p. I $80:$ Burm. Th. Bras. ii. p. I 37 ; id. Reis. La Plata-St. ii. p. 448; Gray, Band-l. B. i. p. 52.

Aluco flammeus, Fleming, Brit. An. i. p. 157; Newt. ed. Yarr. Brit. B. i. p. 194; Shelley. B. Egypt. p. i 76.

Strix furcata, Temm. Pl. Col. i. pl. 432; D'Orb. in De la Segra's H. N. Cuba, Ois. p. 34; Gundl J. f. O. 1871, p. 377.

S. guttata, Brehm. Vög. Deutschl. p. 106.

Ulula flammea, James, ed Wils. Am. Oru. ii. p. 264.

S. delicatula, Gould, P. Z. S. i836, p. i40; id. Synops. B. Austral. pt. 3, pl. 5o. fig. I; id. B. Austr. i. pl. 31; Sturt, Exp. C. Austr. App. p. I 7 ; Bp. Consp. i. p. 54; Kaup, Contr. Orn. 1852 ; p. I19; id. Tr. Z. S. iv. p. 247; Strickl. Orn. Syn. p. 180 ; Cass. U. S. Expl. Exp. 1858 , p. I05; Gray, B. Trop. Isl. p. 3; Verr. Rev. et Mag. de Zool. 1860, p. 42I; Gould, Handb, B. Austr. i. p. 66; Gray, Haad-l. B. i. p. 52; Finsch \& Hartl. Vög. Cent.-Poly'n. p. I1.

S. pratincola, Bp. Comp. List. B. Eur. \& N. Amer. p. 7; De Kay, Zool. N. Y. Birds, p. 31, pl. r3, fig. 28; Gray, Gen. B. i. p. 41 ; Bp. Consp. i. p. 55; Strickl. Orn. Syn. p. 179; Cass. B. Calif. p. 176; id. B. N. Amer. p. 47; Gray. Hand-l. B. i. p. 52 .

S. punctatissima, Gray in Darw. Zool. Baagle, Birds, p. 34, pl. 4 ; id. Gen. B. i. p. 4 I; B. Consp. i. p. 55; Kaup, Contr. Orn. 1852, p. I 8 ; id. Tr. Z. S. iv. p. 246 ; Strickt. Orn. 
Syn. p. 182; Gray, Hand-l. B. i. p. 52; Scl. \& Satu. Nomencl. p. I 6 .

S. poensis, Fraser, P. Z. S. 1848, p. 189; Gray, Gen. B. i. p. 4I; Strickl. Orn. Syn. p. 182; Hartl. Orn. Westafr. p. 22; Gray, Hand-l. B. i. p. 52; Gurney, ino Anderss. B, Dam. Id. p. 36 .

Stridula flammea, Selys-Longch. Fann. Belge, p. 60.

Strix bakkamma, Forts. Descr. An. p. I 57.

Eustrinx flammea, Webb. \& Berth. Orn. Canar. p. 8.

Strix lulu, Peale, U. S. Expl. Exp. Birds, 1843, p. 74. pl. 2 ; Gray, Hand-l. B. i. p. 52 .

S. pusilla, Blyth, J.A. S. B. xviii. p. tor,

Athene forsteri, Bp. Consp. i. p. 42; Gray, B. Trop. Isl. Pacif. p. 2.

Strix glaucops, Kaup, Contr. Orn. 1852 , p. I 8 ; $i d$. Tr. Z. S. iv. p. 246; Gray. Hand-l. B. i. p. 52; Pelz.J.f. O. 1872 , p. 23.

S. forsteri, Hartl. Arch. Naturg. 1852 , p. 3\%.

S. americana, Brewer, ed Wils. Am. Orm. p. $68_{7}$.

S. africana, Bp. Rev, et Mag. de Zool. 1854, p. 540; Gray, Hand-l B. i. p. 52 .

S. adspersa, margaritata, maculata, splendens, vulgaris, obscura, paradoxa, kirschoffi. Brehm, Naum. 1855 , p. 270 , et 1858, p. 2 \& 5 .

S. indica, Blyth Ibis, 1860 , p. 25I; Huwie, Rough Noles. ii, p. 342; Gould, B. Asia, -pt. nit.; Holdsw. P. Z. S. 1872 . P 415; Hume, Str. F. 1873, p. r63; et 1875, p. 36 .

S. flammea americana, Sckl. Mus. P..B. Striges, p. 4, et Revibe Accipitr. p. 16.

S. rosenbergi, Schl. N. T. D. iii. p. ısı; Wall. Ibis, 1868 , p. 62; Gray, Hand-l. B. i. p. 52; Walden, Tr. $Z$. S. viii. p. 41; Schl. Mus. P.-B. Revue Accipitr. p. 16.

S. affinis, Layard, B. S. Afr. p. 43.

S. insularis, Pelz. J. f. O. $1872, \mathrm{p} .23$.

S. flammea, var. americana, Coues. Key N.Am. S. p. 202; id. B. N.W. p. 298.

S. flammea, var. guatemalæ, var. furcata, var. pratincola, var. javanica, var. delicatula, Ridgw. N.^Am. B. ii. p. I I.

Aluco delicatula, Sharpe, ed Voy. Ereb. \& Terr. Birds, p. 22. Asia. Hab. Luzón, Manila, Bulacán, Pampanga, Cagayan; Europa,

2. S. candida Tick. oo N. v. Manonoctoc.

(M S. T.)

Strix candida, Tickell. J. A. S. B. ii. p. 572; Jerd. Ill. Ind. Orn. pl. 30; Bp. Consp. i. p. 55; Kaup, Contr. Orn. 1852, p. II9; Sir.ckl. Orn. Syn. p. I 8 ; Jerd. B. Ind. i. p. I 8 ; Schl. Mus. P.-B. Striges, p. 6; Gray, Hand-l. B. i. p. 53; Swinh. P. Z. S. 1871 , p. $344 ;$. Gould, B. Asia, part xxiv. Sharpe, Cat. B. 2. p. 308 (1875).

S. longimembris, Jerdon, Madr. Journ. x. p. 86; Gray, Gen. B. i. p. 41 .

Glaux candida, Blyth, J.A. B. S. xix. p. 513; Ball. Str. F. 1874 , p. 381 ; Hume, Nests and Eggs Ind. B. p. 60.

G1. javanica, Blyth, Cat. Mus. A. S. B. p. 42.

Scelostrix candida, Kaup, Tr. Z. k. iv. p. 248; B.lyth, Ibis, 1856 , p. 25I; Hume, Rough Notes, ii. p. 345; Walden, Tr. Z. S. ix. p. 145 . 
S. amauronota, Cab. J. f. 0, \&66, p. 9, et ז872, p. $316 ; G r a y$, Hand-l. B. i. p. 52 .

S. pithecops, Swinh. Ibis, 1866, pP 396, 397; Gray, Hand-l. B. i. p. 53 .

S. walleri, Diggles, Orn. Austr. pl.—; Gray, Hand-l. B. i. p. 52.

Hab. Luzón, Bulacán, Cagayan, Ilocos, Samar; China, Formosa, Australia, India.

\section{Gén. PHODILUS Georf.}

3. Ph. badius, (Harsf.) $\delta \&$ N. v. Cul-laao

Strix badia, Horsf. Zool. Research. Java, pl. 37; Temm. Pl. Col. i. pl. 318 ; Less. Traite, p. I 2.

Phodilus badius, Isid. Geoff. St.-Hil. Ann. Sc. xxi. p. 201; Gray, Gen. B. i. p. 42, pl. I5. fig. I; Blyth, Cat. B. Mus. A.S. B. p. 4 ; Bp. Consp. i. p, 55 Kaup, Contr. Orn. 1852 , p. II 8 ; id. Tr. $Z$. S. iv. p. $245 ;$ Mottl. \& Dillw. N. H. Labuan. p. 8; Horsf. \& Moore, Cat B. Mus. E. I. Co. i. p. 80; Strickl. Orn. Syn. p. I83; Jerd. B. Ind. i. p. I I9; Scl. P. Z. S. 1863 , p. 207 ; Blyth, Ibis, 1866, p. 25 ; Wall. Ibis, 1868 , p. 26; Gray Hand-l. B. i, p. 52; Gould, B. Asia, pl. xxii.; Walden, Ibis, 1872, p. 365; Hume, Rough Notes, ii. p. 346 ; id. Str. F. 1873 , p. 429; Salvad. Ucc. Born. p. 21. Sharpe, Cat. B. Mus. 2. 309 (1875).

Ulula badia, Schl. Mus. P.-B. Striges, p. 23.

¿Phodilus nipalensis, Hume, Str. F. 1875, p. $47^{*}$.

Hab. Luzón, Cagayan, Ilocos; Java, Borneo.

\section{Orden $3 \cdot^{\circ}$ PÁJAROS.}

\section{Grupo: COLIOMORFOS.}

\section{Fam. CÓRVIDOS.}

I. Gén. COLfeUS, Kaup.

I. C. monedula, (Lin). \& N. v. Cuervo.

(M. S. T.)

Le Choucas, Briss. Orn. ii. p. 24.

Le Choucas á collier, Briss. t. c. p. 27.

Le Choucas blanc, Bris. 1. c. p. 27 (var).

Le Choucas noir, Briss. t. c. p. 28; Montb. Pl. Enl. iii. pl. 522. Corvus monedula, Linn. S. N. i. p. I56; Temm. Mann. d'Orn. i. p. I I ; Naum. Vög. Deutschl. ii. Saf. 56. fig. I; Roux. Orn. Prov. pl. I33; Werner, Atlas, Omnivores, pl. vi.; Gould, B. Eur. iii. pl. 223; Macgill. Br. B. i. p. 552; Yarr. Br. B. ii. p IO2; Gray, Gen. B. ii. p. 31 5 ; Schl. u. Susem. Vog. Eur. ii. Taf. iv. fig. I; Nordm. in Démid. Voy. Russie Mérid. iii. p. I 20; Schl. Bier. Nederl, Vog. pl. xiii. fig. 6; id. Vog. Nederl. pl. 140; Kjaeb. Orn. Dan. Taf. xi. fig. I; Schl. Bijdr. 
Dierk. Ansterd. folio, p. I5; Jaub. et B trth. Lupoinn. R'ch. Orn. p. 95; Schrenck, Reis, Amurl. Zool. p. 32t; Surdst. Sv. Fogl. pl. xx. fig. 4; Linderm. Vög. Griechenl. p. 69; Gould, B. Gt. Br. iii. pl. 6r) Filippi, Viagg. Pers. 1) 263 ; Degl. et Gerbe, Orn. Eur. i. p. 202; Ssh!. Mus. P.-B. Coraces, p. 33; Gray, Hand-l. ii. p. 13; Doderl. Avif. Sicil. p. 63; Salva.t. Faun. Itàl. Ucs. p. 173; Heirsl. Orn. N. O., Afr. p. +98 ; Shelley, B. Egypt, p. i6o; Hume, Nests \& Ind. B. p. iI4; Irby. B. Gbr. p. I $28 ;$ Dresser, B. Eur. part. xlvi.; S'cullly, Str. F. ${ }_{1876}$. p. I58; Bianf. East. Persia, p. 263.

La Grolle on Choucas yris, M) mtb. Pl. En!. iii. p. 523.

Corvus sper:nolegus, Vieil!. $N$. DCt. d.Hist. N.t. viii. p. to; Gray, Gen. B. ii. p. 3r5. Sihl. u. Susem. Vög. Eur. ii. Tat. I. fig. 2.

Lycus monedula, Boie, Isis, 1822 , p. $55 \mathrm{r}$; B. . Cons, i. p. 39 t. Coløus monedula, Kaup, Nattiirl. Syst. p. II 4 ; Horsf. \& Moore, Cat. B. Mils. E. I. Co. ii. p. 562; Jerd. B. In.t. ii. p. 3(1); Hume, Lahoreto Yark. p. 239. Sharpe, B. Mus. p. 25 (1877). Monedula turriu.n, Brehm, Vög. Dsutsch. p. 172; Loche. Expl.

Sci. Algé. i. p. II 4.

N. arborea, septentrionalis, Brehm, t. c. p. 173.

M. nigra, $B p$. Consp. i. p. $38+$.

fr. spermolegos, Brehm, Naum. 1855, p. 273. Hab. Europa, España, Francia, Africa.

2. Gén. CORONE, KAUP.

2 philippina, $(B A) \&$.$\& N. v. Uac.uac.$

Corvus philippinus, Bp. C. R. xxxii. p. $830 ;$ Gray, Hand-l. $B$. ii. p. I 2 ; Walden, Tr. $Z$. S. ix. p. 20 .

Corvus solitarius, Kittl. Reis. Russ. Amer. Mkronesien, \&c., ii. p. 43r. $\left(185^{8}\right.$, desc. nullâ).

Corvus brevipennis, Schl. Bijdr. Dierk. Amsterd. fol p. 9, pl. i. fig. 8 ; id. Mus. P.-B. Coraces, p. 22.

Corone philippina, Sharpe, Cat. Brit. Mus. $3 .^{\circ}$ p. 42 (1877). Hab. Luzón, Negros, Cuys, Ca niguin, Iloilo, Z laboliag, Surigao, Mindanao, Dumaguete, Leyte, Samar, Bohol, Bısilan, Paragua, Panay, Cebú, Panzon, Dinagat, Joló.

3. C. pusilla, (Tw) む£ N. v Ugac, Cuare

(M. S. T)

Corrus pusillus, Tw. P. Z. S. 1878 . p. 622; id. Works. p. 6 I 7 (1881).

Hab. Paragua, P. Princesa, Canigaran.

3. Gén CISSA, Bole

4. Cissa chinensis, (Bodll.) \& N. v. Rolièr de China.

(M $\mathrm{S}$ 'T)

Le Rollier de la Chine, Briss. Orn. ii. p. 77, pl. 6. fig. 2 ; Pl. Enl. 620 .

Chinese Roller, Lath. Gen. Sy'n. i. p. 4i4. 
Coratias chinensis, Bodd. Tab!. Pl. Enl. p. $3^{\text {s. }}$

Corrus speciosus, Shari, Gen. Zool. vii. p. 364 .

Pica speciosa, I'agler, Sist. Av'. Pica, sp. $3^{\circ}$.

Corapica beng lensis, Less. Traite, p. 353.

Kitta venatoria, Gray, Ill. Ind. Zool. i. pl. 2; Mc Clell. P. Z. S. 1839, p. I') ; Blyth, J. A. B. xii. p. 938; Bp. Cons;. i. p. 100.

(hloris ma sinensis, Swains. Classif. B. ii p 231.

(issat sinensis, Blyth, J. A. B. S. xiii. p. 93+; Gray, Gen. B. ii. p. 3oó; id. Cat. Mamm. dec. Nepal, Hodtgs. p. 101; Horsf. \& Moore, Cat. B. Mus. E. I. Co. ii. p. 575 ; Jerd. B. Int. ii. p. 312; Schl. Mus. P.-B. Coraces, p. 68;; Blyth. \&- Wald. B. Burm. p. 89 .

Cissa venatoria', Blyth, J.A. S. B. xv. p. 29; id. Cat. B. Mus. A. S. B. p. 92 ; Gould, B. Asia, part. ix.

Kitta speciosa, $B p$. Consp. i. p. 166.

(itta sinensis, Licht. Nomencl. Av. p. 9.

(issa speciosa, Gray', Hand-l. B. ii. p. 6; Hume, Nests \& Egys Ind. B. p. $42 \mathrm{I} ;$ id. Str. F. 1875 , p. 146.

Cissa chinensis, Sharpe, Cat. B. M. $3^{\circ}$ p. 85 (1877).

Hab. Himalaya, Nepal, China, ¿Filipinas?

\section{Gén. XANTHOURA, BP}

5. X. cyanocapilla. (Cah.) \& N. v

(M. S. T.)

:Pica chloronota, Wagler, Is s, 1829 , p. $7+9$.

Cyanocorax cyanocapillus, Cab. in Tschudi, Faun. Peruan. p. 233, note; Scl. \&. Salv. Nom ncl. Av. Neolr. p. 39.

Xanthoura guatemalensis, Bp. Consp. i. p. $3^{80}$.

Xanthocitta cyanocapilla, Cab. Mus. Hem. i. p. 223

Cyanocorax guatemalensis, Scl. \& Salv. Ibis, 1859, p. 22; 1860 , 1. I 13 ; Scl. Cat Amer. B. p. 144.

Cyanurus cyanocapillus, Gray, Hand-l. B. ii. p. 5 .

Xanthoura incas, var. guatemalensis, Baird, Brewer, \& Ridgw. N.-Amer. B. 295 .

Xanthura cyanocapilla, Sharpe, Cat. B. $30^{\circ}$ p. I3 I (1877).

Hab. Guatemala.

\section{Fam. FARADISÉIDOS.}

Subfam. EPIMAQUINOS.

I. Gén. SELEUCIDES, LESS.

I. S. niger, (Shaw.) むo N. v. Paradisea negra.

(M. S. T.)

Le Manucode à douze filets, Audeb. et Vieill. Ois. Dor. ii. p. 29, pl. I 3.

Le Nébouleux, Levaill. Ois. de Parad. i. pls. 16, 17.

Le Promèrops multifil, Levaill. H. N. Promér. et Guép. q1. 17.

Paradisea nigra, Shaw, Gen. Zool. vii. pt. 2, p. 488, pl. 60 (1809.)

P. alba, Blumenb. Abbild. nat. Gegens. pl 96; Schl.J.f. O. 1861 , p. 386 . 
P. nigricans, Shaw, t. $t$. p. 480 .

P. violacea, Bechst. Kurse Uebers. p. I 33.

P. vaillanti, Shaw. \& Nodd. Mat. Misc. xxiv. pl. I025.

White-winged, Paradisedbird, Lath. Gen. Hist. iii. p. 197.

Twelve-wired Paradise-bird, Lath. t. c. p. I99; pl. +8 .

Le Promérops à douze filets, Vieill. Gal. Ois. pl. ${ }_{1} 8$.

Epimachus albus, Wagler, Syst. Av. 1827 , Epinachus, sp. 9; Gray, Gen. B. i. 1. 94; id. P. Z. S. 1861, p. 433 ; Wall. P. Z. S. 1862 , p. 160; Schl. Mus. P.-B. Coraces p. 95; Gray, Hand-l. B. i. p. 105.

Falcinellus resplendens, Less. Traité, p. 32 .

Seleucides acanthylis, Less. H. N. Ois. Parad. pls. 36-38; id. Syn. p. 29.

Nematophora alba, Gray, List. Gen. B. p. I 2.

Seleucides alba, Bp. Consp. i. p. +12; Wallace. Malay Archip. ii. p. 253; Élliot, Monsgr. Parad. pl. xxii., Salvad. Ann. Mits. Civic. Genov. vii. p. 785 ; Baccari, t. c. p. 7 I 3 ; Scl. l. $Z$. S. 1876 , p. 252 .

$\$$ resplendlens, Rosenb J f. O. 1854, p. 123.

S. ignota, Salvad Ann Mils Civic. Genov. ix p. Igr.

S. niger, Sharpe, Cat. B. Mus. 3." p. I59 (1877)

Hab. Nueva Guínea.

\section{Subfam. PARADISEINOS.}

I. Gén. PARAdisea, Lin.

2. P. apoda, Lin. O'. N. v. Gran paradisea.

The Greater Bird of Paradise, Edwards, Birds, iii. pl. I Io.

L'Oiseau de Paradis, Briss. Orn. ii. p. 130, pl. xiii.

Paradisea apoda, Linn. S. N. i. p 165; Wagler, Syst. Av Paradisea sp. I; Gray, Gen B. ii. p. 323; Bp. Consp i p. 41 2; Gray, P. Z. S. 1861 , p. 436, 1862, p. +35; Wall Ibis, 1859 , p. I I I, 1861 , p. 289; Schl. J.f. O 1851 , p $38+$; id. Mus. P.-B. Coraces p. 78; Wall. Malay Arch. ii. p. 238; Rosenb. J. f. 0.1864 , p. 42 ; Gray, Hand-l. B. ii p. i6. Elliot, Monogr. Parad.pl. I; Salvad. Ann. Mrus. Civic. Genov. ix. p. г91. Sharpe, Cat. B. M. $30^{\circ}$ p. 166 (1877).

L'Oiseau de Paradis des Moluques, Buff. Pl. Enl p. I9o; pl. 254.

L'Emeraude, Audeb. et Vieill Ois. Dor. ii. p. 9.

Le Grand oiseau de Paradis émeraude, Levaill. H. N. Ois. Parad. i pls. I-3.

Paradisea major, Shaw, Gen. Zool. vii. p. 480, pl. 58; Less. Ois. Parad. Syn. p. 6; id. H. N. p. I 55, pl. 6.

Paradisea apoda, var, wallaciana, Gray. F. Z. S. 1858 , p. I8I, Hab. Islas Aru.

3. P sanguinea, Shaw. $\sigma \rho$ N. v. Ave roja del Paraiso.

(M. S. 'T.)

L: Paradis rouge, Audeb, \& Vieill. Ois. Dor. ii. p. it, pl. 3. L'Oiseau de Paradis rouge, Leva ll. Ois Parad. i. pl. 6.

Paradisea sanguinea, Shaw, Gen. Zool. vii. p. 487, pl. 59; Gray, 
Hant-l. B. ii. p. เ6; E!!iot, M נngrre. Parrat. pl. iv.; Salvad. Ann. Mus. Civic. Genov. vii. p. 782 . Sharpe, Cat. B. M. 3 p. $170(1877)$.

Paradisea rubra, Vieill. Gal. Ois. i. p. 152, pl. 99; Loss. Voy, Coquille, Zool. p. 660; pl. 27; Wagler, Syst. Av. Paradistia sp. 3: Less. Traité p. 33t, pl. 3; idt. Ois. Parat. Syn. p. í; id. Hist. Nat. p. r69, pls. 7, 8; Gray, Gen. B. ii. 1. 32.3; pl. 79; Bp. Consp. i. p. 4r3; Wall. Ibis, 1859 , 1) 11; 1861, p. 287 ; id. P. Z. S. 1862 , p. roo; Schl. J. f. O. 1851 , 1. $385 ; i d . N$. T. D. ii. p. 249 ; it. Mis. P.-B. Coraces p. St. Uranornis rubra, Salvad. Aın. Miss. Civic. Genov. ix. p. 19)1. Hab. Molucas, Batanta.

\section{Gén. Semiol'tera, Gray.}

4. S. wallacii, Gray ơ $\bigcirc$ N. v. Paradisea de Wallace. (M. S. T)

Paradisea wallacii, Gray, P. Z. S. 1859, p. I30; Schl. Mut. P.B. Coraees, p. 385 .

Semioptera wallacii, Wall. Ibis, 1859, p. 210, note, 1860, p. 61; Sal. Ibis, 1860 , p. 26, pl. 2; Gray, p. Z. S. 1860, p. 355; Gould. B. Austr. Suppl. pl. 3; Rssenh. J. f. O. 1864 , D. 125 ; Wall. Malay Arch. ii. p. I7; Elliot, Monogr. Parad. pl. in; Salvad. Anu. Mus. Civic. Ginov. vii. p. $78_{5}$, ix. p. ig). Sharpe, Cat. B. M. 3 p. 178 (1877).

Epimachus wallacii, Gray, Hand-l. B. i. p. Io5.

Hab. Batchian, Gilolo.

\section{Gén. LOPHORHINA, Vifili..}

5. L. superba, $\left(P_{\iota n n}\right) O^{n} \varrho$ N. v. P. soberbia, ó el Soberbio. (M. S. T.)

L'Oiseau de Paradis de la Nouvelle Guince, dit le Superbe, Briss. Orn. iii. p. I69; Buff. Pl. En!. iii. p. 197.

Paradisea superba, Penn. in Fiorster Ind. Zool. p. 4o; Scop. 1)el. Faun. et Flor. Insubr. ii. p. 83; Gn. S. N. i. p. 4oz; Shaw, Gen. Zool. vii. p. 49t, pls. 63-65; id. \& Nodt. Nit. Misc. xxvi. pl. Ioz ; Wagler, Syst. Av. Paradisea, sp. 5; Wall. Ibis. 1859 , p. I I ; Schl, J. f. O. $13^{61}$, p. $3^{85}$.

(O)iseau de Paradis à gorge violette, Sonn. Voy. Nouv. Guin 1). $157, \mathrm{pl} .96$.

superb Bird of Paradise, Lath. Gen. p. 479.

Paradisea dtra, Bodd. Tabl. Pl. Enl. p. 38.

Lo Superbe, Audeb. et Vieill. Ois. Dor.ii. pl. vii.; Levaill. H. $N$. Ois. Parad. i. pls. I4, I 5.

Paradisea furcata, Bechst. Kurze Uebers. p. I 32.

Lophorhina superba, Vieill. N. Dict. d'Hist. Nat. xviii. p. I 8 t; id. Gal. Ois. p. I 49 , pl. xcriii.; Less. Traite, p. 337; id. Ois, Parad. Syn. p. iz; id. H. N. Ois. Parad. pls. I3, I 4 ; Bp. Consp. i. p. 414; Wall. Ibis, 1861, p. 287, 1852 , p. Io; Salvad. Ann. Mits. Civic. Genov. ix. p. 19o. Sharpe, Cat. B. M. 3 Sharpe, Cat. B. M. 3 p. 179 (1877).

Epimachus ater, Schl. Mics. P.-B. Coraces, p. 95, note.

Lophorhina atra, Wall. Malay Arch. ii. p. 249; Elliot, Mong, 
Parad. pl. I ; Salvad. Ann. Mus. Civic. Genow. vii. p. Tx.; Beccari, t. c. p. 712 ; Schl. Ibis, 1876. 1. 251.

Hab. Nueva Guinea.

\section{PHONYGAMA, Less.}

6. $\mathrm{Fh}$ keraudreni, Less. む† N. v.

(M. S T.)

Phonygama keraudreni, Less. Voy. Coquille, Ois. i. p. 636; id. Man. d'Orn. i. p. I4 I; Gray, Gen. B. ii. p. 3*3; Bp. Consp. i. p. 368 ; Rosenb. J. f. O. 1864 , p. i 23. Sharpe, Cat. B. M. 3 p. $180($ ( 877$)$.

Barita keraudreni, Less. Voy. Coquille, Ois. atlas, pl. I3.

(halybeus cornutus, Cuv. Regn. Anim. i. 1) 354 (1829); Gould in Voy. Rattlisnake, ii. p. 357 .

Phonygama lessonia, Swains. Classif. B. ii. p. 264.

Manucodia keraudreni,, Gray, P. Z. S. 1859 , p. I 58, i 861, p. 436 ; id. Hand-l. B. "ii. p. I 7 ; Elliot, Monogr. Parad. pl. viii. Salvad. Ann. Mus Civic. Genov. vii. p. $78 \mathrm{r} ;$ id. op. cit. ix. 1. I $89 ;$ Scl. Ibis, 1876 , p. 252.

(halybeus keraudreni, Schl. Mus. P.-B. Coraces, p. I 20. Hab. Nueva Guinea.

\section{Fam. ORIÓLIDOS.}

I. Gén. ORIOLlS, LiN

I. O. philippinensis, Gray. N. v. Culiauan.

(M. S. T.)

O. philippensis, J. E. Gray. Zool. Misc. p. 3; Bp. Consp. i. p. 346; Gray, Hand-l. B. i. p. 4. 32 r.; Walden Tr. Z. S. i. p. 186. Hab. Filipinas.

2. O. unicolor, $\sigma, 0$ N. v. Dulao.

Hab. Luzón, Cagayan, Pamplona.

3. O. achrorchynchus, Vigors. Lin. $\sigma, 0$ N. v. Culiauan. (M. S. T.)

Le Loriot de la Cochinchine, Briss. Orn. ii. p. 326, pl. 33 fig. I. Oriolus chinensis, Linn. S. N. i. p. I60 (ex Briss.); Gray, Gen. B. i. p. $232 ; B l y t h, J . A . S . B$. xv. p. 46 ; $2 d$. Cat. B. Mus. A. S. B. p. 215; Horsf. \& Moore, Cat. B. Mus. E. I. Co. i. p. 270; Gray, Hand-l. B. i. p. 292. Sharpe, Cat. B. M. 3 p. 203 ( 1877 ).

Le Couliavan de la Cochinchine, Montb. Pl. Enl. 570.

Oriolus acrorhynchus, Vigors, P. Z. S. 1831, p. 97; Griy \&Mitch. Gen. B. i. p. 232; pl. 58; Bp. Consp. i. p. 348; Schl. Mus. P.-B. Coraces, p. rot, (pt.); Walden \& Layard, Ibis, 1872 , p. Iог; Hume, S. F. 1875 , p. г 32.

Oriolus cochinchinensis, Bp. Consp. i. p. 348.

Broderipus acrorhynchus, Walden, Tr. $Z$. S. ix. p. I 85. Hab. Luzón Guimarás, Negros, Cebú, Bohol, Balabac, 'Minda- 
nao, Panay, Panaon, Leyte, Dinagat, Basilan, Jolo, Lapar, sibutu, Mindoro, San Bernardino, Dumalon.

4. O. steerii, Sharf. \& \& N. v. Tangsit. (M S. T.)

Sharpe, Cat. B. M. 3 p. 2r3. lam. x. (1877).

Hab. Negros, Samar, Basilan, Paragua, P. Princesa.

5. O. assimilis, Tiv. N. v. Tangsit.

(.M. S. T.,

(). assimilis, Tw. Works. p. 53+ (1881).

Hab. Cebú, Masbate.

6. O. xanthonotus, Horsf. \&\& N. v. Tangsit.

(.I. S. T.)

Oriolus xanthonotus, Horsf. Tr. Linn. Soc. xiii. p. 15.3; Timm. Pl. Col. 214; Horsf. Zool. Research. in Java. Birds, pl. 12; Wagl. Syst. Av. Oriolus, sp. 9; Gray, Gen. B. i. p. 232; Blyth, J.A. S. B. xv. p. 49; id. Cat. B. Mus A. S. B. 1. 34t; Horsf. \& Moore, Cat. B. Mns. E. I. Co. i. p. 268; Pelz. Reis. Novara, Vôg. pp. 75, I51; Schl. Mus. P.-B. Coraces, 1). I(x); Gray, Hand-l. B. i. p. 292; Salvad. Ucc. Born. p. 277. Sharpe, Cat. B. M. 3. p. 21 3 (1877).

Oriolus leucogaster, Temm. Pl. Col. 214 (texte, ex Reinzi. MS.)

Oriolus castanopterus, Blyth, J. A. S. B. xi. p: 796; Gray', Gen. B. i. p. 232.

Xanthonotus leucogaster, Bp. C. R. xxxviii. p. $53^{\text {r. }}$ Hab. Paragua.

7. O. suluensis, Sharp. N. v.

O. suluensis, Sharpe, Cat. B. M. 3. p. 205 (1877). Hab. Joló.

8. O. palawanensis, Tw. $0^{0} \mathrm{~N}$. v. Tangsit.

Broderipus palawanensis, Tw, Works. p. 6I I (I88I). Hab. Paragua.

9. O. Samarensis, Steere. $\sigma \circ$ N. v Tangsit.

(M. S. T)

Hab. Samar.

\section{Fam. DICRÚRIDOS.}

I. Gén. DICRURUS Mieill.

r. D. balicassius, Lin. $ఠ \bigcirc$ N. v. Balicasiao.

(M. S. T.)

Le Choucas des Philippines, Briss. Orn. ii. p. 31, pl. 2. fig. I; Montb. Pl. Enl. 603.

Corvus balicassius, Linn. S. N. i. p. 157 (ex Briss.).

Le Balicasse des Philippines, Month. Hist. Nat. Cis. iii. p. 83 . 
Edolius furcatus, Wagl. Syst. Av. p. 322. (ex linn.)

Edolius viridescens, Gould, $P . Z$. S. 1836,1$) .6$.

Dicrurus viridescens, Blyth, Cat. B. Mus. A. S. B. p. 262, AN. xxii.

Balicassius philippensis, Bp.C.R. xxxviii. p. 539 .

Balicassius furcatus, $B p . t . c$. p. 539 .

Dicrurus balicassius, Gray, Hand-l. B. i. p. 284; Wald. T\% Z. S. ix. p. I 80, pl. xxxi. fig. I. Sharpe, Cat. B. $M .3$. p. 236. ( 1877$)$.

Hab. Luzón, Manila, Cagayan, Ilocos, Paragua, Samar, N:rinduque, Mindoro.

2. D. mirabilis, Wald. $\sigma \circ$ N. v. Balicasiao.

(M. S I I.)

D. mirabilis, Wald. et Lay. Ibis, 1872 , p. 103, pl. 5; IIald. Tr. Z. S. ix p. i8I; Sharpe, Cat. B. M. 3. p. 231 (1877). Hab. Negros, Cebú, Panay, Guimarás, Másbate.

3. D. striatus, $T w . \sigma \circ \mathrm{N}$ v. Balicasiao.

(II. S. T.

D. striatus, Twe. Works. p. 469 (1881).

Hab. Panaon, Leyte, Samar, Basilan, Mindanao.

4. D. palawanensis, Tw. $\sigma \circ$ N. v. Pasian-sian.

(N. S. 'T)

Dicrurus palawanensis, Tw. Works. 1, 619) (1881).

Hab. Paragua, P. Princesa.

2. Gén. bucilangis, Iodis.

5. B. cineracea, (Horsf.) $\sigma^{\prime} \rho \mathrm{N}$. v. Tambibiligao.

(N. S. 'l.)

Edolius cineraceus, Horsf. Tr. Linn, Soc. xiii. p. I +5.

Dicrurus leucophæus, Gray, Gen. B. i. p. 287 (nec V.); Bp. Consp. i. p. 352 ; Horsf. \& Moore, Cat. B. Mus. E. I. Co. i. p. 155 .

D. intermedius, Blyth.J.A.S. B. xv. p. 299; id. Cat. B. Mus. A. S. B. p. 203 .

D. cinerasceus, Gray, Gen. B. i. p. 187 .

D. cinaraceus, Bp. Consp. i. p. $35^{2}$; Horsf. \& Moore, Cat. B. Mus. E. I. Co. i. p. I 55.

Buchanga wallacii, Walden. Ann. \& Mag. Nat. Hist. (4) v. p. 220.

B. mouhoti, Walden, Ann. \& Mag. Nat. H st. (4) v. p. 220 ; Swinh. P. Z. S. 1870 , p. 245 ; id. P. Z. S. 1871 , p. 378.

Buchanga cineracea, Sharpe, Cat. B. M. 3. p. 250 (1877). Hab. Paragua, P. Princesa; Java, Lombock, Pegu.

\section{Gén CHibia, Hotjgs.}

6. Ch. pectoralis, (Wall) $0^{t} . \mathrm{N}$ v. Tambibiligao. (M. S. T.)

Dicrurus pectoralis, Wallace, P. Z. S. 1862 , p. 335 y 342; Finsch, Nen-Guinea, p. 170; Gray, Hank-!. B. 1. p. 285. 


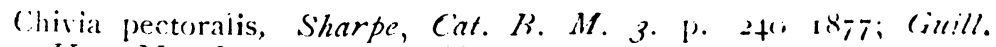

- Voy. Marchesa. p. 259, 1885.

Bh. borneensis, Sharpe, P. Z. S. $18^{\prime} 79,1$. $2 f^{\prime}$.

Hab. Maimbun y Parang, Joló.

\section{Grupo: CICLonorfos.}

Fam. CAMPEFÁGIDOS.

I. Gen. ARTAMIISES, HARTI.

I. A. sumatrensis, (S Mïll.) $\sigma 0$ N. v. Babuy-babuyan. (M. S. T)

Ceblepyris novx-zuinex, S. Muller, Verh. Natuurl. Gesched. Landen Volkenk, p. igo (nec Gm.)

(.) sumatrensis, S. Mïll. t. c. p. I go.

(iraucalus fasciatus, Bp. Consp. i. p. 354 (nec. V.): Jerd. B. Ind. i. p. $418 ;$ p. 218 ; p. 444 ; Sclat. P. Z. S. p. 218. I 863 ; Hartl. J. f. O. 1864, p. 444 .

G. concretus, Hartl. J. f. O. 1864, p. 445; Walden, Ibis, 1872 , p. $37 \mathrm{I}$,

(ampephaga sumatrensis, Gray, Hand-l. B. i. p. 337, n. $5^{077}$.

C. concreta, Gray, t. c. p. 337, n." 5089.

Graucalus sumatrensis, Salvad. Ucc. Born. p. I 5(; Tweedd. Ibis. I877, p. 312; Sharpe, Tr. Linn. Soc. new series, vol. i. 1). 323? Trueedd. P. Z. S. 1878, p. 6I4.

Artamides sumatrensis, Sharpe, Cat. B. Mus. 4. p. 12. (1879). Hab. Paragua, P. Princesa; Región Indo-Malaya, Malaca, Sumatra, Borneo.

\section{A pollens, Salv. N. v.}

Graucalus pollens, Salv. An. Mus. Civ. Genov. 6. p. $75 ;$ id. $P$. Z. S. 1878 p. 87 .

Artamides pollens, Sharpe, Cat. B. Mus. vol. 4. p. i 3. (1879). Hab. Joló, I. Lapac; Molucas.

3. A. striatus, (Bodd) $\sigma 0 \mathrm{~N}$ v. Balabang.

(M S. T.)

Choucas de la Nouvelle Guineé, Daubent, Pl. Enl. iii. pl. 629; Montb. Hisl. Nat. Ois. iii. p. 8o.

New-Guinea Crow, Lath. Gen. Syn. i. pt. 1, p. $38 \mathrm{r}$.

Corvus striatus, Bodd. Tabl. Pl. Enl. p. 38. (ex Daubenton).

C. novæ guineæ, Gm. Syst. Nat. i. p. 37 r.

Coracina fasciata, Vieill. N. Dict. viii. p. 8.

Graucalus novæ guineæ, Cuvier. Regn. Anim. i. p. 341 (1817); Less. Man. d'Orn. i. p. I 44.

Ceblepyris novæ guineæ, Temm. Man. d'Orn. i. p. lxii; Rüpp. Mus. Senckenb. iii. p. I 44.

C. plumbea, Wagler, Syst. Av. art. Corvus, App. sp. 44.

Graucalus lineatus, pt., Lesson, Traité, p. 349.

G. dussumieri, Lesson, tom. cit. p. 349; Jacq. et Pucher. Voy. 
PJ'c siud, Zjo!. iii. p. 65; Pucher. Arch. du Mus. vii. p. 3!3: Biyth, J.A.S, B. xxx. p. 96.

(houcari de Dussumier, Hombr. et Jacq. Voy. Pöle Sud, Atlas, pl. 8 fig. I.

(:impephalga striata, Gray, Gen. B. i. p. 283; id. Haud-l. B. i. p. $337, \mathrm{n}^{\circ} 5^{(3)} 6$.

Gracicalus lagunensis, Bp. C: R. xxxviii. p. $5 f^{\circ}$; Blyt, J. A. S. B. xxx. p. 96; Hartl. J.f. O. 1864 , p. 445.

Campephaga novæ guinex, Gray, P. Z. S. 1858, p. 193; id. Cat. B. Neru. Guinea, p. 58; id. P. Z. S. 1851 , p. 435; Finsch, Veu-Guinea, p. I 7 I.

(. Clussumieri, Gray, Hand-l. B, i. p. 336, no. 5070 .

(. lagunensis, Gray, tom cit. p. 337 , no. 5080 .

Graucalus striatus, Walden, Ibis, 1873 , p. 3 í $2 ; i d, T r . Z$. S. ix. p. I75, pl. xxx. fig; I; Sharpe, Tr. Linu. Soc. new series, i. p. 323; Tweedd. P. Z. S. 1877 , pp. 693, 759, 827, et 1878 , pp. I08, $283,380$.

Corvus papuensis, Von. Kittl., Lïtke, Vog. (Postels), iii. p. 326 (nec Gm., teste Trueeddale)

Artamides striatus, Sharpe. Cat. B. M. vol. 4. p. 18. (1879).

Hab. Luzón, Lzguna, Calauan, Cagayan, Nesros, Cebú, Panaon, San Bernardino, Leyte, Samar, Mindanao, Zamboanga, Paragua, Malamaui, Nipah, Pasananca, B.ssilan.

4. A. mindorenzis, Stzere N. v. Balicasiao.

(M. S. T.)

Hab. Mindoro.

5. A. mindanensis, Steere. If N. v. Balabang.

(M. S. T.)

Hxb. Sumar, Mindanào.

6. A. panayensis, Stiere. N. v.

Hab. Guimaras, Panáy, Masbate.

2. Gen. EDOLIISOMA, Jacr.

7. E. cærulescens, Blyti. \& \& N. v. Babuy-babuyan.

(M. S T.)

Ceblepyris cerulescens, Blyth, J. A. S. B. $184^{2}$, p. $463,184^{6}$, p. 307; Cat. B. Mus. A. S. B. p. I9I.

Campephaga cærulescens, Gray, Gen. B. i. p. 283; Hartl.J. f. 0.1865, p. I 57.

Ceblepyris aterrima, Blith, Ibis, 1866, p. 368.

Campephaga aterrima, Gray, Hand-l. B. i. p. 338, no. 5 w) (descr.. nullä.)

Volvocivora cærulescens, Walden, Tr. Z. S. ix. p. i 78, pl. xrx. fig. 2; Sharpe, Tr. Linn. Soc. new series, vol. i. P. 351; Tweed. P. Z.S. 1877, pp. 693, 759, et 1878, p. 283 .

Edoliisoma cærulescens, Sharpe, Cat. B. Mus. vol. 4. p. 4t. (1879). Hab. Luzón, Manila, Cebú, Negros, Parazua, P. Princesa. 
8 E. mindanensis. Ti. $Y$ r.

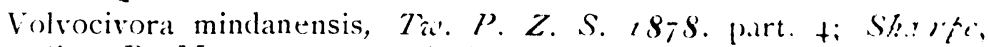
(at. B. 1f. +. p. $+7(0)(18 ; 0)$.

Hab. Mindanao, Basilan.

9. E. panayensis, Stiriv. N.

Hab. Guimaras, Panáy.

3. Gen. PERICROCOTUS, HUI:.

ro. P. igneus, Biyth ơ N. v. Simbag

(II. S. 'T')

Pericrocotus igneus, Blyth, J. A. S. B. xv. p. $3(x)$; $i d$. (iat. B. Mns. A. B. B. p. I93; Bp. Consp. i. p. 356; lerr. Rea. et Mag. de Zool. 1867 , p. I69; Gray, Hand-l. B. i. p. 33.5; Swinh, P. Z. S. 1871, p. 379; Salvad. Ucc. Bern: 1. 14 ; Sharpe, Str. F. 1876 , p. 209; id. Tr. Lann. Soc. new scr. i. p. 324; id. Cat. B. Mus. vol. 4. p. 78 (1879); Hume, Str. F. 1877 , p. I90; 2d. \& Davison, Str. F. 1878, p. 2 гा.

P. minutus, Strickl. Contr. Orn. 1849 , p. 94, pl. 3 r.

P. flagrans, Bp. Consp. i. p. 357 (ex Boie, MS.); Gray, Hand-l. B. i. p. 335 .

Hab. Paragua, P. Princesa, Inagauan; Borneo, Malaca, (hinat.

Ir. P. miniatus, Tem. $\sigma$ N. v. Simbag.

(M. S. T.)

Muscicapa miniata, Temm. Pl. Col. $15^{6}$.

Pericrocotus miniatus, F. Boie, Isis, 1826 , p. 972 ; Gray, Gen. B. i. p. $282 ;$ Bp. Consp. i. p. 357; Cab. Mus. Hein. 'Th. i. p. 6o; Gray. Hand-l. B. i. p. 336; S. Sharpe, Str. F. 1876 , 1). 2ro; id. Cat. B. M. 4. p. 80, I879. Hume, Str. F. 1877 , p. 192 .

Acis miniata, Lesson, Traite, p. 388.

Phrenicornis miniata, Jerd. Ill. Ind. Oru. text to pl. xi. Hab. Samar, Paragua, Java.

12. P. cinereus, Lafiesn. N. v Simbag.

Pericrocotus cinereus, Lafrens. Rev. Zool. viii. p. 94; Bp. Consp.

i. p. 357; Schrenck, Reis. Amurl. p. 381 ; Radde, Reis. Sibir.

ii. p. 273; Gould, B. Asia, pt. ix.; Gray, Hand-l B. i. p. 335. n." 5050; Stinhoe, P. Z. S. 1871 , p. 378; id. Ibis, I 875, p. I I6; Dibowsk. J. f. O. 1875 , p. 249; Tacz. Bull. Zool. Soc France, i. p. 165; Sharpe, Str. F. 18;6, p. 211; Hume, Str. F. 1877 , pp. I74, I75; Sharpe, Ibis, 1877 , p. I9; id. Cat. B. M. p. '3. 1879 David \& Oustal. Ois. Cgine, p. 137; Hume \& Davison, S. F. 1878 , p. $2 \mathrm{II}$.

P. modestus, Sirickl. P. Z. S. 1846 , p. Io2; Bp. Consp. i. p. 357 (ex Boie, MS. in Mus. Iugd.); Gray, Hand-l. B. i. p. 335 . no. 5053 .

Ceblepyris luctuosus, De Filippi, Mus. Mediol. p. 31; Salvad. Atti R. Acead. Torin. $1868, \mathrm{p}, 27 \mathrm{I}$.

Hab. Samar, Borneo, Sumatra, Malaca, China. 
13. P marchesæ, Guill. N. v.

(M S T)

Pericrocotus marchesæ, Guill. Voy. of. the Jacht "Marchesa». lam. I 8 f. I. 1885 .

Hab. Joló, Maimbun.

14. P. Leytensis, Sterre. N. v.

Hab. Leyte.

4. Gen. LALAGE, Bore.

15. L. melanoleuca, Blyth. $\sigma \circ$ N. v. Manlalaņ̃is.

(M. S. T.)

Pseudolalage melanoleuca, Blyth.J.A.S.B. xxx. p. 97; Ilaldeli, Tr. Z. S. p. 1 78, pl. 29. fig. 2; Sharpe, Tr. Linn. Soc. new series, vol. i. p. $35 \mathrm{I}$.

P. melanictera, Sclater, Ibis, 1862, p. 78 lapsu cal.)

Lalage melanoleuca, Hartl. J. f. O. 1865 , p. 163 ; Von Martens, J.f. O. 1866 , p. i 2 ; Sharpe, Cat. B. M. 4. p. 91 (1879!. (ampephaga melanictera, Gray, Hand-l. B. i. p. 339, no. 5129. Hab. Luzón, Mindoro.

16. I. terat, Bood. o o N. v. Dominico.

( $\mathrm{M}$ is T.)

Le Merle des Indes, Briss. Orn. ii. p. 248 , pl. 31. fig. 3.

Le 'Terat-Boulan, Montb. Hist. Nat. des. Ois, iii. p. 397.

Le Merle des Indes orientales, D'Aubent. Pl. Enl. iv. pl. 40. fig. 2. Ash-rumped 'Thrush, Lath. Gen. Syn. ii. p. 74.

'Turdus terat, Bodd. Tabl. Pl. Enl. pl. i 7. (ex Buff. pl. enl. 273 f. 2 ).

T. orientalis, Gm. S. N. i. p. 82r. Lath. Ind. Orn. p. 355. ( 790$)$. Sylvia orientalis, Bonn. \& Vieill. Enc. Meth. ii. p. 487.

Sy. leucophæa, Vieill. ibidev. p. 452. 1823 .

Ceblepyris striga, Horf. Tr. Limn. Soc. xiii. p. I 45.

'Turdus striga, Raffl. t. c. p. 31 . Lath. Gen. Hist. v. p. 87.

Lanius striga, Raff. Tr. Lin. Soc. 13. p. 305 (1822).

Lalage orientalis, Boie, Isis, 1826, p. 973; Blyth. Cat. B. Mus. A. S. B. p. 192; Bp. Consp. i. p. 355;Cab. Mus. Hein. 'Th. i. p. 6o; Horsf. \& Moore, Cat. B. Mus. E. I. Co. i. p. 875; Moore, P. Z. S. 1854 , p. 273; Bernst. J.f. O. 1859 , p. 274; Hartl. J. f. O. 1865, p. 866.

Erucivora orientalis, Swains, Faun. Bor.-Amer., Birds, p. 48.2 ; Pelz Reis. Novara, Vög. p. 8I.

Ceblepyris orientalis, Tem.; S. Müller, Nat. Versch. Land-en Volkenk. p. 190; Less. Echo du Monde Sav. p. 232.

Pycnonotus humeraloides, Less. Descr. Mamm. et Ois. p. $3^{0} 5$.

Campephaga terat, Gray, Hand-l. B. i. p. 339.

Colluricincla maculosa, Peale, Un. St. Expl. Exp. 1. $8_{1} . \mathrm{pl}$. 23 f. I. I848; Hartl., Wiegm. Arch. 1852, p 97.

Lalage terat, Hume, Str. F. 1873 , p. 454, I874; p. 202; Salvad. Ucc. Born. p. 145; Sharpe. P. Z. S. 1875 , 1. 107; id. Cat. B. M. 4. p. 95 (1879). 
Lalage dominica, Halden, Tr. Z. S. ix. p. s; 8 ; Tivecddale, Ibis, 1877 , p. 383; Sharpe, Tr. Linn. Soc. new ser. i. p. 324; Trueed P. Z. S. 1877, pp. 5+5, 759, 827, 1878, Pi. $285,3+2 ;$ id. Ibis, $18^{3} 77$, p. $3^{8} 3$.

Hab. Luzón, Manila, Montalban, S. Mateo, Laguna, Calauan, Marinduque, Mindoro, Panáy, Cebú, Leyte, Bohol, Negros, (iuimaras, Dinagat, Mindanao, Dumalon, Basilan, Malamaui, Joli, Paragua; Borneo, Sumatra, Jara, Malaca, Nicobar.

17. L. aurea, ( $T$ im $) \sigma \emptyset$ N. v. Manlalang̃is

(M. $S$ \%

(eblepyris aureus, Tem. Pl. Col. 2. pl. 382, fig. 2; S. Mäll. Nat. Versch. Land-en Volkenk. p. igo; Rüpp. Mus. Sensch. 3. p. 24; Jacq. et Pucher. Voy. Pöle Sud. 3. Ois. p. 71 ; Hartl. J. F. O. 1857, p. 163.

Echinelleur oranga, H. et Jacq. Voy. Pöle Sud, Atlas, pl. I0. fig. 3 .

Campephaga aurea, Gray, Gen. B. i. p. 283 ; id. Hand-l. B. i. p. 339 .

Lalage aurea, $B p$. Consp. i. p. $335 ; i d . C$. R. xxxviii. p. 5ti; Hartl. S. F. O. 1865 , p. 168; Finsch Nerv. Guinea, 1) $172 ;$ S. F. O. 1866 , p. I 2 ; Walden, Tr. Z. S. viii. p. 7o; Sharpe. Cat. B. M. 4. p. 108 (1879).

Campephaga (Lalage) aurea, Gray, P. Z. S. 1860, p 35.3. Hab. Molucas, Gilolo, 'Ternate. ¿Filipinas?

18. I. insperat 1, Finsch. N. v.

Volvocivora insperata, Finsch, P. Z. S. 1875 , p. 643; id. Journ. Mus. Godeffr. Heft. xii p. 27; id. P. Z. S. 1877, p. 779.

Lalage insperata, Sharpe, Cat. B. M. 4. p. I03 (1879). Hab. Ponapé, Islas Carolinas.

19. L. monacha. (H. et Finsch.) o N. v Malalang̃is. (M. S. T.)

Campephaga monacha, Hartl. et Finsch. P. Z. S. 1772 , p. 99. Volvocivora monacha, Finsch Journ. Mus. God. Hetf. viii. tat. 3. f. 2 y 3 .

Lalagre monacha, Sharpe, Cat. B. Mus. 4. p. 105. 1879. Hab. Ponapé, Carolinas; Palaos.

2o. L. leucopygialis, (Grayj. † N. v. Malalang̃is.

(M. S. 'T.)

Campephaga leucopygialis, Gray Hand-l. B. 1. p. 339. n. ${ }^{\circ}$ I 25. (descr. nulla).

Lalage leucopygialis, Walden, Tr. Z. S. viii. p. 69. n. ${ }^{\circ} 88$ lam. 8, f. 2; Salv. Ann. Mus. Civuc. Genov. vii. p. 658; Sharpe. Cat. B. Mus. 4. p. 98.1879.

Hab. Joló; Célebes.

21. L. minor, Steere.

Hab. Mindanao. 


\title{
Fam. MUSCICÁPIDOS.
}

\author{
1. Gén. ALSEONAX, Cab.
}

I. A. latirostris, Raff. 00 N. v. Pipit.

(M. S. T)

Muscicapa grisola, var. dauurica, Pall. Zoorg. Rossc-Asiat. i. p. 46 r . M. latirostris, Raff. Tr. Linn. Soc. xiii. p. 312; Salvin. Cat. Strickl. Coll. p. 136.

M. poonensis, Sykes, P. Z. S. 1832 , p. 85; Gray, Cat. Manizi. \&.c Nepal. Coll. Hodgs. p. 90.

Hemichelidon latirostris, Gray, Gen. B. i. p. $262 ;$ Blyth, Cat. B. Mus. A. S. B. p. 175; Bp. Consp. i. p. 321 ; Horsf. \& Moure, Cat. B. Mus. E. I. Co. i. p. i 37 ; Moore, P. Z. S. 1854 . P. 270 ; Swinhoe, Ibis, 1866 , p. 57 ; Gray, Hand-l. B. i. p. 324 , n." 4864 .

Butalis terricolor, Blyth, J. A. S. B. xvi. p. 120; id. Cat. B. Mus. A. S. B. p. I75; Bp. Consp. i. p. 318 .

leutalis latirostris, Blyth. J. A. S. B. xvi. p. I21; Layard, Ann. Nat. Hist. 1854 , xiii. p. 1 27; Swinh. P. Z. S. 1871, p. 379; Blyth \& Wald. B. Burm. p. so4; Dybozesk. J. f. O. 1872 , p. 447; Taczan. Bull. Soc. Zool. France, i. p. i68; Davis \&- Oustal. Ois. Chine, p. 123; Tweedd. P. Z.S. 1878 , p. 284.

Muscicapa cinereo-alba, Temm. \& Schl. Faun. Japon, Aves. p. 42, pl. I5, 1850; Schrenk, Reis. Amurl. p. 379; Radde, Reis. Sibir. ii. p. 273.

Alseonax latirostris, Cab. Mus. Hein. Th. i. p. 53; Jerd. B. Ind. i. p. 459; Holsw. P. Z. S. 1872, p. 441; Hume \& Henders. Lahore to Yark. p. 185, pl. ..; Salvad. Ucc. Born. P. I 29; Hume, Str. F. 1875, p. 219 id. Slr. F. 1874 , p. 475; Brooks, Str. F. 1875 , p. 276; et 1876 , p. I 73 ; Fairb. op cit. p. 257; Hume, op cit. p. 396; id. \& Davison. Str. F. 1878 , p. 227. Sharpe, Cat. B. M. 4. p. I 27. (1879).

Alseonax terricolor, Cab. Mus. Hein. 'Th. i. p. 52, note.

Butalis cinereo-alba, Bp. Consp. i. p. 318.

B. pondiceriana, Bp. Consp. i. p. 318 (ex Licht. in Mus. Berol.)

Muscicapa pondiceriana, Middend. Sibir. Reis. Vög. p. 188.

Hemichelidon terricolor, Gray, Hand $/$. B. i. p. 324, n. ${ }^{\circ} 4865$.

Alseonax cinereoalba, Brooks, Str. F. 1875 , p. 276. Chind.

Hab. Luzón, Negros, Borneo, Sumatra, Java, India, Ceilán,

2 Gén. MUSCICAPA, LiN.

\section{M. cæruleocephala, Scop. N. v.}

Le Gole-mouche à tête bleuâtre de l'isle de Laçon, Sonu. Voy. Nouv. Guin, p. 57, pl. 26. n. ${ }^{\circ}$ I.

Muscicapa caruleocephala, Scop. Del. Fann. et Flor. Insubr. p. 95, no. 106 (ex Sonn.); Walden, Tr. Z. S. ix. p. 184.

Muscicapa cyanocephala, Gm. S. N. i. p. $9+3$, n. ${ }^{\circ} 65$ (ex Sonn); v. Martens, J.f. $O .1866$, p. 11 .

Hab. Luzón? 
3. M. luzoriensis, Gm. N. v.

Le Gobe-mouche noir de l'isle de Luçon, Sorn. Voy. No:tv. Guin. p. 59, pl. 27. fig. 2 .

Muscicapa tessacourbe, Scop. Del. Faun. et Flor. Insubr. p. 95, no. 108 (ex Sonn.)

Muscicapa luzoniensis, Gm. S. N. i. p. $942, n^{0} .62$ (ex Sonn.); Walden Tr. Z. S. ix. p. I 84.

Hab. Luzón?

4. M. macrura, Scop. N. v.

Le Gobe-mouche à tête bleue de l'isle de Luçon, Sonn. Voy. Nouv. Guin. p. 58, pl. 27. fig. I.

Muscicapa macroura, Scop. Del. Faun. et Flor. Insubr. p. 95, no. 107.; Walden, Tr. Z. S. ix. p. 184.

Hab. Luzón?

5. M. pbilippinensts, Gm. N. v.

Le Moucherolle des Philippines, Buff., Hist. Nat. Ois. iv. p. 565 . Muscicapa phillippinensis, Gm. S. N. i. p: 943. n. ${ }^{\circ} 63 ;$ v Martens, J. f. $O .1860, \mathrm{p} . \mathrm{I}$, no. 34; Walden, $\operatorname{Tr} . Z . S$, ix p. 184 . Hab. Luzón?

6. M. manillensis, Gm. N. v.

L.e Gobe-mouche à gorge janne de l'isle de Lucon, Sonn. Voy. Nonv. Guin. p. 57, pl. 26. fig. 2.

Muscicapa manillensis, Gm. S. N. i. p. 943, n. 66 (ex Sonn.) Walden, $\operatorname{Tr} . Z$. S. ix. p. 184 .

Hab. Luzón?

7. M. griseisticta, (Sroinh.) $\$ \&$ N. v. Calag. (M, S. T)

Butalis manillensis, Bp. C. R. xxxviii. p. 652 (non Muscicapa manillensis, Gm. ex Sonn.; nec Vieill. N. Dict. xxi. p. $47 \mathrm{~b}$, ex. Sonnerat); Walden, Tr. Z.W. xiii. p. 66, ix. p. 183; Shaxpe, Ir. Linn. Soc. new series, i. p. 326 ; p. Treedd. P. Z. S. 1877, p. 694.

Hemichelidon fuliginosa, Swinh. Ibis, 1860 p. 57 (nec Hondgs.)

H. griseosticta, Swinh. Ibls, 1871 , p. 330 .

Butalis hypogrammica, Wallace, Ibis, 1862 , p. 350; Walden, Tr. $Z$. S. vii. p. 66.

B. griseosticta, Swinh. P. Z. S. 1863 , p. 288, 1866, p. 1 3 1, I 871 , p. 379; David \& Oustal. Ois. Chine, p. i22.

Muscicapa hypogrammica, Gray, Hand-l. B. i. p. 321, n. ${ }_{4}^{814}$.

M. griseisticta, Sharpe, Cat. B. M. $44^{\circ}$ p. 153, (1879).

Hab. Luzón, Montalban, Panay; China, Formosa, Molucas. 
3. Gén, PRATiNCOIA, Koch.

8. P. caprata, Lin. ¿ $\$$ N. v Cipao.

(M. S. 'T.)

L.a 'Traquet de l'isle de Luçon, Briss. Orn. iii. p. 44t, pl. 24. figs. 2, 3; Haubent. Pl. Enl. vi. pl. 235.

Motacilla caprata, Linn, S. Nat. i. p. 335 ; Martens, J. $f$. (). 1866, p. Io.

(Enanthe caprata, Vieill. N. Dict. xxi. p. +33.

Saxicola fruticola, Horsf. Tr. Linn. Soc. xii. p. 157.

\$. bicolor, erythropygia, Sykes, P. Z. S. $1832,19.92$.

Motacilla sylvatica, Tickell, J. A. S. B. ii. p. 575 .

Saxicola caprata, Jerd. Madr. J. x. q. 20.5; Gray. Gen. B. i. p. I79; id. Cat. B. Nepal. coll. Hodgs. p. 71; Finsch, NeuGuinea, 1. 167.

S. meloleuca, Hodgs. Icon. ined. Passeres, pl. 98 (Hodgs. no. 420); id. in Gray's Zool. Misc. p. 83.

Pratincola caprata, Blyth, J. A. S. B. xvi. p. I29; $2 d$. Cat. IS. Mus. A. S. B. p. i69; Bp. Consp. i. p. 305; Cab. Mus. Hein. 'Th. i. p. II Horfs. \& Moore, Cat. B. Mus. E. I. Co. i. p. 284 ; Jerd. B. Ind. ii. p. 123 ; Gran', Hand-l. B. i. 1. 22 , no. 3282 ; Holdsw. P. Z. S. 1872 p. 454 ; Hume, Str. F. 187.3 , p. I82; Adam, t. c. p. 379; Ball, Str. F. 187 , p. 41 3 ; Hume, t. c. p. 477 , et 1875, p. I $34 ;$ id. Nests and Eggs. Ind. B. p. 313 : Brooks, S. F. 1875. p. 238; Blyth of Walden, B. Burm. 1. 102; Walden, Str. Tr. Z. S. viii. p. 63, ix. p. 192; Salvad. Ucc. Born. 1. 252; Sharpe, Tr. Lin. Soc. new series, i. p. $335 ;$ Blandford, East Persia, p. 144; Fairb. I. F. 1876 , p. 259: Batler, S. F. 1877 , p. 229; Trueedd. P. Z. S. 1877 , pp. 696, 761,1878, p. 710; Salvad. P. Z. S. 1877 , p. 393; Hume \& Davison, S. F. 1878 , p. 334; Sharpe, Cat. B. M. 4. p. 19.5. 1879 .

P. atrata, Blyth, J. A. S. B. xx. p. 177 ; Jerd. B. Ind. ii. p. 124 ; Gray, Hand-l. B. i. p. 228 , no. 3281 ; Holdsw. P. Z. S. 1872, p. 454 ; Walden, Tr. $Z$. S. viii. p. 63.

P. bicolor, Hume, Nests and Eggs Ind. B. p. 3 I 4.

Hab. Luzón, Laguna, Panay, Negros, Cebú, Bohol; Célebes, Java, Borneo, India, Flores, 'Timor.

9. P. pampilonensis, $\sigma \circ \mathrm{N}$ v. Taimbabuy.

(M. S. T.)

Hab. Luzón, Cagayan, Pamplona.

\section{Gen Gerygone, Goun.}

ro. G. simplex; $C a b . \sigma \circ \mathrm{N}$. v. Pipit-bulac.

(M. S. T.)

Gerygone modesta, Cab. J. f. O. 1866, p. 10 (nec Pelz.)

Acanthiza, sp., Gray, Hand-l. B. i. p. 220, no. 3I47.

Gerygone simplex, Cab. J. f. O. 1872 , p. 316 ; Walden, Tr. $Z$.

S. ix. p. I94; Sharpe, Tr. Lmm. Soc. n. s. i. p. 352; it. Notes Leyden Mus. i. n. ${ }^{\circ}$ 9, p. 28, id. Cat. B. M. 4. p. 21t, (1879).

Hab. Luzón, Laguna, Calauan. 
11. G. flaveola, Cub O $\curvearrowright$ N. v. l'ipit-bulac.

(M. S. T)

(i. flaveola, Cab.J. f. O. 1873, p. 157; Mever, t. 2. p. foti

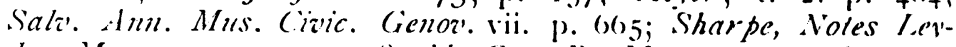
ten Mus. 1. n. 9. p. 28; id. Cat. B. M. 4. p. $21+$ lam. $\therefore$. t. 2. (18т9); Guill. Log. Marchesa, p. 203. (1881).

Hab. Joló; Célcues, Borneo.

\section{Gén. METABOLLS, Br.}

1 2. M. rugensis, II it $\mathcal{F}$. $む N$. v. Pipit-bulac.

IM. S. 'T ,

Drymophile de Rouk, Hombr. et Jacq. Voy. Pole Sud, Atlas, pl. 1.3. Muscicapa rugensis, Hombr. et Jacq. Ann. Sci. (2) xvi. p. 312. Monarcha rugensis, Hartl. Arch. f. Naturg. 1852 , p. I 33 ; id. P. Z. S. I 867 , p. 829; Gray, Hand-l. B. i. p. 321, n. ${ }^{3} 480$. Colluricincla rugensis, Jacq. et Pucher. Voy. Póle Sud, Zool. iii. p. 62.

Metabolus rugensis, Bp. C. R. xxxviii. p. 650. Sharpe, Cat. B. M. 4. p. $238,(1879)$.

Monarcha (Metabolus) rugensis, Gray', Cat. B. Trop. Isl. Pacific Ocean, p. I9.

Hab. Islas Carolinas.

6 Gen XANTHOPYGiA, Blytu.

13. X narcissina, Tem. ơ $\mathrm{N}$ v. Pipit-bulao.

(M. S. T.)

Musicapa narcissina, Temm. Pl. Coll. iii. pl. 577. fig. I; Gray, Gen. B. i. p. 263; Temm. \& Schl. Faun. Japon., Aves, p. 46, pl. 17 C; Gray, Hand-l. B. i. p. 323, n. ${ }^{\circ} 4848$; Sharpe, Cat. B. $M$. 4. p. 250 . (1879).

Xinthopygia chrysophrys, Blyth, J. A.S. $B$. xvi. p. i24.

X. narcissina, Blyth, Cat. B. Mus. A. B. B. p. i 55; Bp. Consp. i. p. 327; Horsf. \& Moore, Cat. B. Mus. E. I. Co. i. p. 283; Swinh. Ibis, 1860 , p. 58; id. P. Z. S. 1871 , p. 380 ; David \& Oustal. Ois. Chine. p. I I9; Sharpe, $7 r$. Limu. Soc. new series, vol. i. 327; Blakiston \& Pryer, Ibis. 1878, p. 234.

Hab. Mindanao, Paragua, China, Japón.

I4. X. cyanomelæna, (T'm.) o, N. v. Pipit-bulac.

(M. S T.)

Muscicapa cyanomelana, Tem. lam. 470; Tem. et Schl. Faun. Jap. p. 47 .

.1. bella, A. Hay, Madr. Journ. XIII. pt. 2. p. $5^{8}$.

Cyanoptila cyanomelanura, Blyth, J.A. S. B. XVI. p. I $25 ; i d$. Cat. B. Mus. A. S. B. p. I74; id. Ibis. 1870 , p. 164.

Niltava cyancmelæna, Gray, Gra'', Gen. B. i. p. 264; Swinh. Ibis, 1860 , p. 398 y i 86 I, p. 4I; Gray, Hand-l B. i. p. 326 n. 4892 . M. melanoleuca, Tem. et Schl. Faun. Jap., Aves, pl. i7 D.

M. gularis, Tem. el Sch. t. c. pl. i6. 8 .

Butalis gularis, Bp. Consp. 1 p. 318 . 
Iypothymis cyanomatena, Bp. id. p. 32i); Ciss. in Perry's E.rp. Jap. p. 239; Swinh. Ibis, 1862 p. 306 .

(yanoptila cyanomalena, Horsf. et Moor. Cat. B. M. E. I. C'C. i. p. 291; Srvinh. P. Z. S. 1871 , p. 386 ; Navd et Onst. Ois. China, p. I16, pl. 8г; Blaksist. et Proy, Ibis, 1878, p. $23+$.

Xanthopygia cyanomelæna, Sarphe, Cat. B. Mus. 4. p. 2ú1. 187\%. Hab. Luzón, Cagayan; Borneo, China, Japoón.

7. Gen. HYPOTIYMIS, BuL.

15. Hy. azurea, (Botla.) $\checkmark, \circ$ v l'ipit azul.

(M. S. I.)

Gube-mouches bleu des Philippines, Daubent. Pl. Enl. 666. fig. \&. Le petit Azur, Montb. Hist. Nat. Ois. iv. p. $53+$.

Muscicapa azurea, Bodd. Tabl. Pl. Enl. p. 4 I (ex Daubent.)

Azure Flycather, Lath. Gen. Syn. ii. pt. i, p. 339.

Muscicapa carulea, Gm. S. N. i. p. 9t3; Jerd. Madr. Journ. xi. p. I 4 .

M. caruleophala, Sykss, P. Z. S. 18,32, p. 85.

Myiagra torquata, Swains. Monogr. Flycatchers, p. 208.

M. carrulea, Swains. Monogr. Flycathers, p. 208; Gray', Cat. Mamm. \&.c Nepal. Coll. Hodgs. p. 93; Blyth, Cat. B. Mus. A. S. B. p. 204 .

M. azurea, Gray. Gen. B. i. p. 26I; Horsf. \& Mcore, Cat. B. Mus. E. I. Co. i. p. I 38 ; Jerd. B. Ind. i. p. 450 ; Pele. Reis. Novara, Vög. pp. 78, I6I (pt.); Gray, Hand-l. B. i. 1. 328, no. 4930; Godw.-Aust. J. A. S. B. xxxix. p. Ioc; Swinh. P. Z. S. 1871 , p. $38 \mathrm{I}$; Ball, Str. F. 1873, p. 68; Hume, Str. F. 1874 , pp. 2 I7, 474; Ball, t. c. p. 40.; Hume, Nests \& Eggs Ind. B. p. 198; id. Str. F. 1875, p. 103; Fairb. Str. F. 1876 , p. 257; Armstr.t. c. p. 322 ; Hume, t. c. p. $395 ;$ David \& Oustal. Ois. Chme, p. I1 4 ; Sharpe; Tr. Linn. Soc. n. s. i. p. 325 .

T'chitrea cæruleocephala, Swinh. Ib:s, 1360 , p. 57.

Hypothymis azurea, Blyth \& Wald. B. Burm. p. I3I; Sharpe, Cat. B. M. 4. p. 274. (1879).

Hab. Luzón, Laguna, Cagayan, Cebú, Mindanao, Malanipa, Bısilan, Paragua; Negros, Guimarás, Mindoro, Marinduque; Borneo, Java, 'Timor, India, China.

16. H. occipitalis (Vigrors.) o o N V. Pipit-azul

(M. S T )

L'Azur à calotte et à collier noir, Levaill. Ois. d'Afr. iv. p. I I, pl. I 53 .

Muscicala carulea, (nec Gm.), Raffl. Tr. Lmm. Soc. xii!. p. j!.; Kittl. Kupf. Vög. p. 7, 'Taf. 9. fig. 8.

Hypothymis cærulea (nec Gnt.) Boie, Isis, 1825 , p. 273 , et 1828 , p. 318 (ex Levaill.); Bp. Consp. i. p. 320; Cab. Mus. He.n. Th. i. p. 56 .

Muscicapa occipitalis, Vigors, P. Z. 18.31. p. 97.

Myiagra azurea, (nec Bodd.), Moore, P. Z. S. 1854, p. 271; Pelz. Reis. Nov., Vög. p. 78 (pt.).

M. carulea, Wallace, P. Z.S. 1863, p. 485. 
M. tytleri, Beavan. Ibis, 1867 , p. 324; Gray, Hand-l. B. i. p. 328, no. 4928; Hume, Str. F. 1874 , p. 217.

Hypothymis azurea, (nec Botd.). Walden, Ibis, 1872, p. Io:; Salvad. Ucc. Born. p. I 33; Walden, Tr. Z. S. ix. p. $83 ;$ Oates, Str. F. 1877 , p. 149; Sharpe, Ibis, 7877 , p. 18; ad. Tr. Linn. Soc. n. s. i. p. 325; Tweeddale; P. Z. S. 1877 , pp. 693, 76o, 825, et 1 878 . p. 284.

Hypotlymis occipitalis, Sharpe, Cat. B. M. 4. p. 275 (1879;.

Hab. Luzón, Laguna, Calauan, Mindoro, Marinduque, Paragua, P. Princesa, Basilan, Joló; Borneo, Java, Sumatra, Formosa.

17. H. superciliaris, $S /$. $\sigma \mathrm{N}$ v. Pipit.

(M. S. T.)

H. superciliaris, Sharpe, Tr. Luu. Soc. n. s. i. p. 326.

Hab. Basilan, Mindanao.

18. H samarensis, Steere. N. v.

Hab. Samar, Leyte.

\section{Gén Cyanomyas, Sharp}

I 9 C. cælestis, 7 $7 \%, \sigma \circ \mathrm{N} . \mathbf{v}$ Piric.

(MI. S. T.)

Hypothymis calestis, Tze. An, et Mag. Nat. H. 20 p. 53\%; id. P. Z. S. 1878 , p. ro9, pl. 7 f. I.

Cyanomyas calestis, Sharpe, Cat. B. M. 4. p. 278.1879.

Hab. Dinagat, Samar, Basilan, Mindanio.

20. C. helenœ, Stecre $\mathrm{N}$ v.

Hab. Samar.

9. Gén. RHIPIDURA, Vigors.

2 I Rh. kubarii, Finsch. N. v.

Rhiphidura kubaryi, Finsch, P. Z. S. 1875 , p. 644 ; id. Journ. Mus. Godeffr. 1876 (Heft xii.) p. 29, pl. 2. fig. 2; id. P. Z. S. 1877 , p. 779; Sharpe, Cat. B. M. 4. p. 314 (1879). Hab. Ponapé, Isl is Carolinas.

22. Rh. lepida, H. et Finsch. N v..

Rhipidura lepida, Hartl. et Finsch, P. Z. S. 1868 , pp. 6, i17; Gray, Hand-l. B. i. p. 331, n. ${ }^{\circ} 4975$; Finsch, Jouru, Mus. Godeffs. viii. p. 21, pl. 4. fig. 23; Sherpe, Cat. B. M. 4. p. 322 ( I 879).

Hab. Ponapé, Carolinas, Palaos.

23. $\mathbf{R h}$ versicolor, $H$. et Finsch. N. v.

Hab. Uap, Carslinas. 
24. Rh. ayauiceps, Cass, $\sigma \circ \mathrm{N}$. v. Pipitgubat.

(M. S. 'l')

Muscipeta cyaniceps, Cas. Pr. Philad. Acad. 1855 , p. $438 ;$ id. U. S. Expl. Exp. p. 145, pl. 9. fig. I.

Rhipidura caniceps (lapsu), Gray, Hand-l. B. i. p. 33 I, n. ${ }^{\circ} 4966$ ). Philentoma cyaniceps, Waldeu, Tr. Z. S. ix. p. 182; Sharpe, Tr. Linn. Soc. new seres, i. p. 325; Tweeddale, P. Z. S. 1877 , p. 693. Sharpe, Cat. B. M. 4. p. 323 (1879).

Hab. Luzon, Montalban, Laguna, Calauan, Mindanao.

25. Rh. albiventris, Sharp. N. v.

Philentoma albiventris, Sharpe, Tr. Linn. new series, i. p. 325; Tweeddale, P. Z. S. 1878 , p. 284.

Rhipidura albiventris, Sharpe, Cat. B. M. 4. p. 324. 1879. Hab. Negros, Guimarás, Panay, Masbate.

26. Rh. nigritorquis. Vigors. oo N. v. Maria-capra. (M. S. T.)

Rhipidura nigritorquis, Vigors, P. Z. S. 1831 , p. 97; Gray, Gen. B. i. p. 259; Bly h.J.A. S. B. xii. p. 936; Sharpe, Tr. Linn. Soc. new series, i. p. 325. Sharpe, Cat. B. M. 4. p. 334 (1879).

Muscicapa bambusæ, Kittl. Kupf. Vög. p. 7; pl. 9. fig. 2. it. Mém. Acad. St. Pétersb. ii. p. 5, pl. 6.

Leucocerca javanica, Blyth, Ibis, 1865, p. 3o (nec Sparm.)

L. nigritorquis, Walden, Tr. $Z$. S. ix. p. i $82 ;$ Tweedd. P. Z. S. 1877, pp. $693,759,826 ;$ id. P. Z. S. 1878, pp. I09, 284 . 709 .

Hab. Luzon, Laguna, Marinduque, Cebú, Mindanao, Paragua, P. Princesa, Basilan, Joló, Bohol, Negros, Guimarás, Dinagat, Panay.

Io. Gén. ZEOCEPHUS, BP.

27. Z. rufus, $B p \quad \sigma, O$ N. v. Pipit canelo.

(M. S. T.)

Tchitrea rufa, Grav, Ann. N. H. xi. p. 37 г; id. \& Mitch. Gen. $B$. i. p. 260 , pl. 64 ; Bp. Consp. i. p. 325 ; id. Han-l. B. i. p. 334, n. $^{\circ} 5025$.

7eucephus rufus, $B p$. Comptes. Rendus, xxxviii. p. 552; Cass. $U$. S. Expl. Exp. p. 144; Walden, Tr. Z. S. ix. p. I83; Sharpe, Tr. Linn. Soc. new series, i. p. 328 ; id. Cat. B. $M$. 4. p. 343 (1879).

Hab. Luzon, Laguna, Mindoro, Marinduque, Negros, Panay.

28. Z. cinnamomeus, Sharp. N. v. ९す Pipit-canelo. (M. S. T.)

Zeocephus cinnamomeus, Sharpe. Tr. Linn. Soc. new series, i. p. 329, pl. 48. fig. I; id. Cat. B. Mus. 4. p. 3431879. Hab. Basilan. 
29. Z. cyan əsceng, Sh. むᄋ N. v. Sarit-sit.

(M. S. 'T.)

Zeocephus cyanescens, Sharpe, Tr. Linn. Soc. new series, i. p. 328 , pl. 48. fig. 2 ; id. Cat. B. M. 4. p. 343 1879.

Hab. Paragua.

11. Gén. PHILENTOMA, Erton.

3o. Ph. velatum. (Tem.) \& \& N. v.

(M. S I.)

Drymophila velata, Temm. Pl. Col. 334.

Monacha velata, Swains. Classif. B. ii. p. 257.

M. cæsia, Less. Rev. Zool. 1839 , p. 167.

Muscicapa pectoralis, Hay, Madr, Journ. xii. pt. 2, p. I6r.

Myiagra pectoralis, Strickl. P. Z. S. 1846 , p. Ior.

Monarcha velata, Gray, Gen. B. i. p. 260; Bp. Consp. i. p. $321 \%$.

Philentoma velatum, Blyth, Cat. B. Mes. A. S. B. p. 2ot; Bp.

Consp. i. p. 324; Horsf. \& Moore, Ciat. B. Mus. E. Cio. i.

P. 392; Moore, P. Z. S. 1854 , p. 270; Jerd. B. Ind. i. p. +49;

Gray, Hano-l. B. i. p. 334, n. ${ }^{\circ}{ }^{(027}$; Walden, Ibis, $18^{\prime} ; 2$,

p. 373; Salvad. Ucc. Bern. p. I 32; Sharpe, Ibis, 1877, p. 19;

Humc \& Davison, Str. F. 1878, p. 224; Sharpe, Cat. B. .l.

4. p. 365.1879 .

P. unicolor, Btyth, I.bis, 1865, p. 46 .

Tehitrea velata, Gray, Hand-l. B. i. p. 334, no. 5027 .

'T. unicolor, Gray, Handl. B. i. p. 334, n. ${ }^{\circ} 5028$.

Hab. Luzon, Laguna, Paragua; Borneo, Sumatra.

12. Gén. RhINOMYAS, Sharp.

31, Rh. ruficauda, Sharp. \& N. v.

(M. S. 'T.)

Setaria ruficauda, Sharpe, Tr. Linn. Soc. new series, vol. i.

p. 327 ; id. Cat. B. M. 4. p. 368 (1879).

Rh. ruficauda, Sharpe, Cat. B. M. 4. p. 367 (1881).

Hab. Basilan.

32. $\mathrm{Rh}$. samarensis, Stecre. N. v.

Hab. Samar, Mindanao.

13. Gén. CUlicicapa, Swinh.

33 C. panayensis, Sharb. N. \& \& v

(M. S. T.)

Xantiolestes panayensis, Sharpe, Tr. Linn. Soc. new series, i. p. 327 ; id. Cat. B. M. 4. p. 37 I (1879).

Hab. Panay, Negros, Paragua.

14. Gén MYIAGRA, Vigors

34 M. pluto, Finsch. N. v.

Myiagra pluto, Finsch, P. Z. S. 1875 , p. 644; id. Journ. Mus.

Godeffr. Heft. xii. p. 29; id. Cat. B. M. 4. p. 380 (1879).

Hab. Ponapé, Carolinas. 
35. M. oceanica, Facy. et Puch. N. v.

Platyrhynche océanien, Hombr. et Jacq. Voy. Pôle Sud, Atlas, pl. 12 bis, figs. I, 2.

Myiagra oceanica, Jacq. et Pucher. Voy. I’ôle Sud, texte, iii. 1. 77; Gray, Cat. Birds Trop. Isl. Pacific. Ocean, p. Is; 1 d. Hand-l. B. i. p. 328 , n." 4929; Sharpe, Cat. B. M. 4. 1. $3^{*} 3$ $(1879)$.

Hab. Carolinas, Marianas.

36. M. erythrops, Hart et Finêch. N. v.

Myiagra erytrops, Hartl. \& Finsch, P. Z. S. 1868, pp. 6, 117, (t) 1872 , p. 97; Finsch, Journ. Mus. Godeffr. Heft. viii. p. 2u; Sharpe, Cat. B. M. 4. p. 383, (1879).

Hab. Marianas, Paraos.

15. Gén MONarcha, Vig. et horsf.

37 M. goגeffroyi. Hartl. \& N. v.

(M. S. T.)

Monarcha godeffroyi, Hartl. P. Z. S. i 867, p. 829, pl. 38; Gray, Hand-l. B. i. p, 321, n. ${ }^{8}$ Io; Sharpe, Cat. B. M. 4. p. 432,1879 .

Hab. Uap, Carolinas.

16. Gén. STOPAROLA, BLYTh

38. S. panayensis, Sharp. O.0 N. v.

(M. S. T.)

Eumyias panayensis, Sharpe, Tr. Linn. Soc. new series, i. p. 326 ; Sharpe, Cat. B. M. 4. p. 440, (1879).

Hab. Panay, Negros.

\author{
17 Gén SIPHIA, Hodgs. \\ 39. S. elegans, $T \cdot m$ o $0 \mathrm{~N} \mathbf{v}$ Ibon-tulog
}

$\left(\begin{array}{lll}M & \mathrm{~S} & \mathrm{~T}\end{array}\right)$

Muscicapa elegans, Temm. Pl. Col. iii. pl. 596. fig. 2.

M. cantatrix \&, Temm. Pl. Col. iii. pl. 226: fig. 2.

Niltava elegans, Gray, Gen. B. i. p. 264; id. Hand-l. B. i. p: 326 , n. ${ }^{\circ} 4805$.

Cyornis elegans, Bp. Consp. i. p. 320; Walden, Ibis, 1872 ; p. 373; Salvad. Ucc. Born.p. I 30; Walden, Ibis, 1877, p. 316 ; Brïggem. Abhandl. nat. Ver. Bremen, v. p. 458.

C. beccariana, Blyth, Ibis, 1870 , p. 165 (nec Salvad.).

C. banyumas, Tweedd. P. Z. S. 1878 , p. 6I5 ( $\$$ ).

Siphia elegans, Sharpe, Cat. B. M. 4. p. 447, (1879). Hab. Paragua; Borneo, Java, Sumatra, Malaca.

40. S. banyumas, Horsf. $\sigma 0 \mathrm{~N}$. v. Ibon tulog.

(M S. T)

Muscicapa banyumas, Horsf. Tr. Linn. Soc. xiii. p. I $4^{6} ; 2 d$. Zos!. Researches in Java' pl. 
M. rufigastra, Raffl. Tr. Linn. Soc. xiii. p. 3 I 2.

M. cantatrix, Temm. Pl. Col. iii. pl. 226 ( $\vartheta^{\circ}$ only).

Niltava banyumas, Gray. Gen. B. i. p. 264; id. Hand-l. B. i. p. 325, n. $^{\circ} 4879 ;$ Brïggem. Abhand-1. Nat. Vereine Breme'l, v. p. 68.

(yornis banyumas, Bp. Consp. i. p. 320; Horsf. \& Moore, Ciat. B. Mus. E. I. Co. i. p. 290; Bernst. J. f. Orn. 1859, p. 265; Pelz. Reis. Novara, Vög. pp. 78, 16ı; Walden, Tr. Z. S. viii. p. 117 ; Salvad. Ucc. Born. p. I 3o; Skarpe, P. Z. S. 1875, p. 107; id. Ibis, 1877 , p. 18; Brüggem. Abhandl. nat. Ver. Bremen. v. p. 458 ; Trueeddale, P. Z. S. 1878, p. 615 (3 only).

C. simplex, Blyth, Ibis, 1870, p. 165 (8).

Siphia banyumas, Sharpe, Cat. B. M. 4. p. 449 (1879).

Hab. Luzon, Marinduque; Java, Borneo, Célebes.

41 S. philippinensis, Sharp. o, N. v. Ibon tulog.

M. S. T)

Cyornis banyumas, Von Martens, J. f, 0.1866, p. 11; Waldei. Tr. Z. S. ix. p. I 82 .

C. philippinensis, Sharpe, Tr. Linn. Soc. new series, i. p. 32.5; Taceeddale, P. Z. S. 1377 , p. 693; et 1878 , pp. 109, 284, 342. Sharpe, Cat. B. M. 4. p. 4501879.

Hab. Panay, Dumaguete, Mindoro, Marinduque, . Cebú, Samar, Basilan, Panay, Luzon, Laguna, Leyte, Negros, Joló.

42 S. lemprieri, Steere. N. v.

Hab. Paragua.

\section{Fam. TÚRUIDOS.}

Subfam. SILVINOS.

I. PHYLLOSCOPUS, BoIE.

I. Ph. borealis, (Blas) $\checkmark \bigcirc$ N. v. Pipit.tulog.

(M. S. T.)

Sylvia (Phyllopneuste) eversmanni, Bp. apud Midd. Sibir. Reise, p. I78, (1851), apud Radde, Reis. Sibir., Vög. p. 263 (1863). Phyllopneuste borealis, Blas. Naum. 1858, p. 313; Szvinh, P. Z. S. 1871 , p. 356; Tacz. Bull. Soc. Zool. France, 1876 , p. I4I; Prjev. Rowley's Orn. Misc. ii. p. I71 (1877); David et Oust. Ois. Chine, p. 27 I (1877).

Phylloscopus sylvicultrix, Szvinh, Ibis, 1860, p. 53; 1866, p. 295.

Sylvia flavescens, Gray, P. Z. S. 1860, p. 349; id. Hand-l. B. i. p. 2 I $5 ;$ n. ${ }^{\circ} 3047$ (I 869 ).

Philloscopus hylebata, Swinh, J. A. S. Beng. xxix p. 265 (1860).

Sylvia (Phyllupneuste) Borealis (Blas.), Naum. Vög. Dentschl. xiii. p. 69, pl. 375. fig. I (i860).

Phylloscopus borealis, (Blas.), Blyth, Ib:s, 1867, p. 28; Seebohm 
\& Harvie Brown, Ibis, 1876 , p. 216 ; Hume, Str. F. is. 1). 290 (1876); Sharpe, Ibis, 1877, p. I6; Seeb, Ibis, 1877, p. 69; Hume, Str. F. vi. p. 352 (1878); Seeb, Ibis, 1879, p. 9; id. Cat. B. Mus. 5. p. $40(\mathrm{1} 88 \mathrm{I})$.

Phyllopneuste kennicotti, Baird, Trans. Chicago Ac. Sc. i. p. 3! 3, pl. xxx. fig. 2 (I 869).

Sylvia sylvicultrix (Swinh), Gray, Hand-l. B. i. p. 216. n. $^{\circ} 305 t$ ( 1869 ).

Sylvia borealis, (Blas.), Gray, Hand-l. B. i. p. 216. no. $3(6) 3$ (i 869).

Phyllopmeuste eversmanni, Bp. apud Salv. Alston \& Harvie Brown, Ibis, 1873 , p. 61.

P. javanica, Bp. apud Salv. Ucc. Born. p. $24+$ (1874).

Phyllopseuste borealis, (Blas.), Meves, Journ. Orn. 1875, p. 429.

Hab. Luzón, Cebú, Leyte, Mindanao, Paragua, Guimarás. Panay, Masbate, Marinduque, Mindoro; Borneo, China Gilolo.

2 Ph. lugubris, Blyth, o' $0^{\circ}$ N. v. Pipit-tulog.

(M. S. T.)

Phyllopneuste lugubris, Blyth. Ann. Nat. Hist. xii. p. 98 (1843); Blyth, \& Wald. B. Burm. p. Io5 (1875).

Phylloscopus lugubris, (Blyth), Blyth, J. A. S. Beng. xii. p. 968 (1843); id. J. A. S. Beng. xiv. p. 591 (1 845 ); id. Cat. B. Mus. As. Soc. p. 185 (1849); Jerd, B. Ind. ii. p. 192 (1863); Blanf. J. A. S. Beng, xli. pt. 2, p. 53 (1872); Seeb, Ibis, 1377 , p. 78; Hume, Str. F. ri. p. 355 (1878); Seeboh. Cat. B. Mus. 5. p. 48 ( 188 I).

Phyllopneuste trochilus, (Linn.), apud Hodgs. MS. Drawings Brit. Mus.) of B. of Nepal Passeres, pl. 57. n. ${ }^{0} 86 \mathrm{1}$, unde Hodgs. Gray's Zool. Misc. p. 82. no. 86ı (1844), apud Gray', Cat. Mamm. \& B. Nepal Coll. Hodgs. p. 65 (1846).

Phylloscopus indicus, (Jerd), apud Blyth, J. A. S. Beng. xiv. P. 593 ( 1845 , parte).

Phyllopneuste magnirostris (Blyth), apud Gray, App. Cat. Mamm. \&c. Nepal Coll. Hodgs. p. 151 (1846).

Regulus lugubris, (Blyth, Gray, Gen. B. i. p. I 75 (1848).

Abrornis lugubris, (Blyth.) Bp. Consp. i. p. 290 (1850).

Sylvia lugubris, (Bly/h), Gray, Hand-l. B. i. p. 215 . no. 3049 ( I 869).

Phyllopseuste lugubris, (Blyth), Giebel, Thes. Orn. iii. p II) (1 377 ).

Phylloscopus lugubris, Seeh. Cat. B. Mus. 5. p. 48. (188I). Hab. Mindanao, Basilan, Samar; Nepal, Calcuta, Bengala.

3. Ph. superciliosus. Gm. o N. v. Pipit

(M. S. T.)

Yellow-browed Warbler, Lath, Gen. Syn. ii. p. 459 ( I 783 ).

Motacilla superciliosa, Gmel. Syst. Nat. i. p. 975 (1788).

Sylvia superciliosa (Gmel.) Lath. Ind. Orn. ii. p. 526 ( I 790);

Sund. Sv. Fogl. pl. 68 fig. 1. (1858); Gray, Hand-l B. i. p. 2 г6 n. ${ }^{\circ} 3067$ (i 869 ).

Regulus modestus, Gould, Hancock, Ann. Nat. Hist. ii. p. 3Io 1839); Yarr. Brit. B. i. p. 316; (1843); Cab, Naum, ii. pt. I. p. 5 (1852); Gaetke, Jonrn. Orn. 1853, p. 91; Naum, 
Vög. Deutsch. xiii. pl. 37s. tigs. 2 \& 3 31 shon: Tristram, Ibis, 1864,1 . 230 .

R. inornatus, Blyth. J. A. S. Beng. xi. p. 2(1) (1842),

Phylloscopus modestus, (Gonli), Blyth, J. A. S. Beng. xii. p. $963(18+3)$.

Phyllopneuste modesta, (Could), Bl;th, Anm. Nat. Hist. xii. p. 98 (18+3; Blyth, Ann. Nat. Hist. xiii. p. 116. (1 $s_{+4}$ ).

Reguloides modestus, (Gould), Blyth, J. A. S. Bellg. xvi. 442 (I 847); Blyth, Cat. B. Mus. As. Soc. P. $18+$ (18+9, parte).

Sylvia (Phyllopneuste) proregulus, (Pall.), Midd. Sib. Reise, p. 183 (1853, parte).

Phyllobasileus superciliosus, (Gmel.), (abaus, Journ. Orn. 185.3, p. 8I; Fritsch, lög. Eiur. pl. 19. tigs. 3 if 4 (1871).

Reguloides proregulus, (Pall.), Horsf. \& Moore, (at. B. Mus. E. I. Co. i. p. 342 (1854); Swinh, Ibis, 1853. p. 307, ct ante; Jerdon, B. Ind. ii. p. 197 (1803); Bp. Consp. i. p. 29)1 (1850).

Ficedula proregulus, (Pall.), Schleg. Vög. Avederl. pp 1 3 \%, $2+1$ (1854).

Phyllopneuste proregulus, (Pal!.) Blass, Naum. viii. p. 31 ( 1858 ); Blasius bis, 1862, p. 66.

Sylvia bifasciata, Gaetke, Naum. viii. p. 4 I9) (1858):

Phyllopneuste (Phyllobasileus) superciliosi, (Gmel.), Schrenck, Reis. Forsch. Ainur-Lande, i. p. 363 (1860).

Sylvia (Phyllopneuste) superciliosa, (Gmel.), Naum. Vög. Dentsch!. xiii. pt. 2, p. 74 (1860); Radde, Reis. Sibir. Vög. p. 264 (1863, parte.)

Reguloides superciliosus, (Gmel.), Blyth, Ibis, 1862, p. 386; Swinh, Ibis, 1863 , p. 307, et subseq.; Tristram, Ibis, 1857 , p. 83; Gould, Ibis, 1859, p. 128; Swinh, P. Z. S. 1871 , p. 357; Harting; Handb. Br. B. p. 107 (1872); Gould, B. Gt. B. ii. pl. 68 (1873); Hume. Nests \& Eggs Ind. B. p. $36+$ (1873; Blyth, \& Wald, B. Burm. q. 106 (1875); Prjer. Rowley's Orn. Misc. ii. p. I72 (1877); David \& Oust. Ois. Chine, p. 273 (1877); Hume \& Davison, Str. F. vi. p. 358 (1878).

Phylloscopus pallasii, Dubois, Ois. Eur. p. 83 (1862)

Phyllopneuste superciliosa, (Gmel.), Bolle, Journ. Orn. 1863 , p. 6o; Tacz, Bul. Soc. Zool. France, 1876 p. 141.

R(gulus superciliosus, (Gmel.), Gray, Cat. Brit. B. p. 54 (1863).

Phylloscopus superciliosus, (Gmel.), Crommelin, Ned. T. D. iii. p. 244 (1866); Newt. ed. Yarr. Br. B. i. p. 443 (1873); Dresier, B. Eur. pt. xxx. (1874); Seebohm, Ibis, 1877, p. I02; id. Ibis, 1879 . p. I2; id. Cat. B. M. 5. p. 68. (1881).

Sylvia inornata, (Blyth), Gray, Hand-l. B. i. p. 2 16. no. 3066 (1869).

Phyllopseuste proregulus, (Pall.) Giebel, Thes. Orn. iii. p. I20 (1877).

Hab. Paragua; China, India. 
2. Gén. ACROCEPHALUS, NaUm.

4. A. orientalis. Tem. $\sigma^{\circ} \mathrm{O}$ N. v.Terat.

(MI. S. T.)

Sylvia turdoides, Meyer apud Kittl. Lütke, Voy. (Postels) iii. p. 327.

Salicaria turdina orientalis, Tem. et Schleg. Faun. Jap. Aves, p. 5 o (1847).

S. turdoides orientalis, T.mm. et Schleg. F،un. Japon, Aves, pl. $\mathrm{xx}$ B. ( 1837 ).

Calamoherpe orientalis, (Temm. et Schleg.), Bp. Consp. i. p. 285 (1850); Swinhoe, Ibis, $186_{3}$, p. 305; id. P. Z. S. 1863 , p. 293 and 1870, p. 427; Tacz. Journ. Orn. 1874. p. 419, et 1875 , p. 245; Tacz. Bull. Soc. Zool. France, 1876, p. 138; Blakiston \& Pryer, Ibis, 1878, p. 237.

Acrocephalus orientalis, (Temm. et Schleg.), Gray, P. Z. S. 1860 , p. 349; Wald. Tr. $Z$. S. viii p. 64 (1872), ix p I95 (1875; Seebohm, Cat. B. M. 5. p. 97 (1881).

A. magnirostris, Swinhoe, Ibis, 1850, p. 5I, and I86r, pp. 32,329 .

Calamodyta orientalis, (Temm. et Schleg.), Gray, Hand-l. B. i. p. 207. $110^{\circ}$ 2919 (1869); Swinhoe, P.Z. S. 1871 , p. 352; Prjez". Rowley's Orn. Misc. ii. p. I69 (1877); David et Oust. Ois. Chine, p. 253 (1877).

Hab. Luzón, Manila, Cebú, Mindanao; Célebes, Borneo, China, Sumatra.

5. A. syrinx, Kittl. N. v.

Sylvia syrinx, Kittl. Mém. l'Acad. St. Pétersb. ii. p. 6, pl. 8 (1 835).

S. oceanica, «Kittl. in Mus. Senckenb,» fide Finsch, Journ. Mus. Godeffr. xii. p. 30 (1876).

Tataré rousseroll:, Haenbr. et Jacq. Atl. Voy. Pôle Sud, pl. 20. fig. 5 (I 853 ).

Tatarè syrinx, (Kittl.), Jacq. et Puch. Voy, Pôle Sud, iii. 1. 92 (1853).

Eparnetes syrinx, (Kittl.), Reichb. Nat. Syst. pl. lviii. (9859); B. Compt. Rend. 1855, p. I I1.

Acrocephalus orientalis, (T. \& S.), apud Pelz. Nov. Reise, pp. 63, 162 (1 865).

Calamodyta syrinx, (Kittl.), Gray, Hand-!. B. i. p. 208. n. ${ }^{\circ} 294^{\prime}$ (1869).

Calamoherpe syrinx, Kittl., Finsch, Journ. Mus. Godeffr. xii. p. 30 (1876); id. P. Z. S. 1877 , p. 778 .

Arocephalus syrinx, Seeboh. Cat. B. Mus. 5. p 100 (1881). Hab. Ponapé, Carolinas.

3. Gén. Locustella, Kaup.

6. I. fasciolata, Gray. $0^{\circ}, 0 \mathrm{~N}$ v. Pipit.

(M. S. T.)

Acrocephalus fasciola'us, Gray, P. Z. S. 1860 , p. 349; Sucbohm, P. Z. S. 1878, p 806; Tweeddale, P. Z. S. 1878, p. 439. 
Acrocephalus insularis, Wallace, Ibis, 1862, p. 350 .

Calamoherpe fumigata, Szwinhoe, P. Z. S. 1863, pp. 91, 29.3.

Locustella fasciolata, (Gray), Blyth, Ibis, 1867 , p. 21; Seebohm. Ibis, 1879 , p. 15. id. (at. B. M. 5. p. $109(1881)$.

L. insularis, (Wallace), Blyth, Ibis, $1867 \mathrm{p.} 21$.

(alamodyta fumigata, (Swinh.), Gray, Hand-l. B. i. p. 207 11.' (2937) 1869.

(.) fasciolata, (Gray), Gray, Hand-l. B. i. p. 207 n. 2924 (1869); Srvinhoe, P. Z.S. 1871 . p. 352; David et Oust, Ois. Chine, p. $253(1877)$.

(.) insularis, (Wall.), Gray, Hand-l. B. i. p. 207. no. 2924 (1869); Swinh, P. Z. C. 1871 p. 352; David et Oust. O.s. Chine, p. $253(1877)$.

Calamoherpe subflavescens, Elliot, P. Z. S. 1870, p. 243.

C. fasci latus, (Gray), Tacz. Journ. Orn. 1872, p. 353; id. Bull. Soc. Zool. France, p. I 78 (1876).

Hab. Luzón, Marinduque; China, Gilolo, Batchian.

7. I. Ochotensis, Middl. $\delta$ N. v. Pip't.

(M. S T:

Sylvia (Locustella) certhiola, (Pall.) apud Midd. Sib. Reis. ii. p. I 84 (1853).

s. (Locustella) ochotensis, Midd. Sib. Reis. ii. p. 185 (1853); Seebohm, Cat. B. M. 5. p. 113. (1881).

Lusciniopsis japonica, Cossin, Proc. Ac. Sc. Ph. 1858, p. 193.

(alamodyta ochotensis, (Midd.), Gray, Hand-l. B. i. p. 2 Io. n.. 2973 (1879).

Locustella subcerthiola, Swinh, Ibis, 1874, p. 154; David et Oust. Ois. Chine, p. 249 (1877).

Arundinax blakistoni, Swinh, Ibis, 1876, p. 332 (bird of the year).

locustella ochotensis, Seeb. Cat. B. Mus. 5. p. I 3 (1881).

Hab. Marinduque; Borneo, Japon. Arch. Malayo.

4. Gèn. Itijciniota, Gray.

8. L. fuscata, Blyth. $\sigma \circ \mathrm{N}$ v. Pipit.

(M S T.)

Phyllopneuste fuscata, Blyth, J. A. S. Beng. xi. p. 113 (1842); Bp. Consp. i. p. 290 (1850); Swinh. P. P. S. 1871, p. 356; Homeyer, Journ. Orn. 1872, p. 202; Blyth. \& Wald, B. Burma, P. IO5 (1875); Tacz. Bull. Soc. Zool. France, 1876, p. I40; Prjev. Rowley's Orn. Misc. ii. p. I7I (1877); David et Oust. Ois. Chine, p. 267 (1877).

Horornis fulviventris, Hodgs. MS. Drawings Brit. Mus. of Birds of Nepal, Passeres, pl. 63. no. 878 (circ. 1844); Hodgs. P. Z. S. 1845 , p. 3r; Gray, Cat. Mamm. \&c. Nepal Coll. Hodgs, j. $6+$ (1846); Bp. Consp. i. p. 290 (1850).

Phylloscopus brunneus, Blyth J.A. S. Beng. xiv. p. 591 (1845); Blyth, Cat. B. Mus. As. Soc. p. 185 (1849); Horsf. \& Moore, Cat. B. Mus. E. I. Co. p. 338 (1854).

Sylvia fuscata, (Blyth), Gray, Gray, Gen. B. i. p. 174 (1848).

Regulus fulviventris, (Hodgs.). Gray, Gen. B. i. p. 175 (1848).

Piylluscopus fuscatus, (Blyth), B!yth, Citt. B. Muss. As. S'oc. p. 
${ }_{185}$ (18+9); Jerd. B. Ind. ii. p. 181 (1863); Blyth, Ibis, 1857 , p. 25 ; Se.?. 16 s, 1877 , p. 85 ; Hume. Stray Fizth. vi. p. 352 (1878); Seeb. Ibis, 1889 , p. II.

Sylvia (Phyllopneuste) sibirica, Midt. Sibr. Reise, p. I8o (1853); Radde, Reis. Sibir., Vög. p. 260 (1863).

S. brunnea, (Blyth), Gray, Hand-l. B. i. p. 2 I6 n." 3057 (1869).

Phyllopneuste brunneus, (Blyth), Blyth, Cat. Mrnim. \& B. of Burma, p. Io5 (I 875$)$.

Phyllopseuste fuscata, (Blyth), Giebel, Thes. Orn. iii. p. 118 (I877).

P. brunea, (Blyth), Giebel, Thes. Orn. iii. p. I 8 (1877).

Lusciniola fuscata, (Blyth), Seebohm, Ibis, $18 \delta 0,277$; id. Cat. B. Mus. 5. p. I 27 (1881).

Hab. Luzón, Cryayan; Formosa, China, Jupon.

\section{Subfam. TÚRDINOS.}

5 Gén. GEOCICHLA, KuHL.

9. G. varia, Pall. $\sigma \circ$ N. v.

(M. S. T.)

'lurdus varius, Pall. Zooorr. Rosso-As. i. p. 4t9 (I8I I); Macg. Brit. B. ii. p. It6 (1839;) Sund. Vet. Ak. Hant-l. 1840, 1). 37; Temm. Man. d'Orn. iv. p. 602 (18to; Werner, Allas in. sectiv. App. pl. 7 (1842); Sund. Is is, 1845 , p. I I9; Homeyer. Rhea, 1849 , p. I44; Kjarb. Dirnm. Filgl. p. I34 (1852); Sund. Svensk, Fogl. pl. Ixvii. fig. I ( 856 ); Radde, Reis. Sibrr., Vög. p. 231 (1863); Gray, Hant-l. B. i. p. 254. n. 3387 (1869; Newt, ed. Yarr. Brit. B. i. p. 25I (1872); Tweedlale, P. Z. S. 1878 , p. 429; Dresser, B. Eur. pt. lxxii. (1878); Seebohm Cat. B. $M$. 5. p. I $5 \mathrm{I}$ ( $188 \mathrm{I})$.

'T. aureus, Holandre, Ann. de Verr. 1825, p. 310; Schinz, Eur. Faun. i. p. 16 I (1840); Dubois, Journ. Orn. 1356, p. 237: Degl. et Gerbe, Orn. Eur. i. p. 420 (1867).

T. squamatus, Boie, Isis, 1835, p. $25 \mathrm{I}$.

'T. whitei, Eyton, Rarer Brit. B. p. 92 (1836); Gould. B. of Eur. ii. pl. 81 (1337); Keys. u. Blas. Wirb. Eur. pp. lii et 179 i $840 ;$ Yarr. Brit. B. ed. i. p. 184 (18+3); Sch. Rev. Crit. pp. xli et 70 (I844); Gray, Gen. B. i. p. 218 (I847); Temm. et Schl. Faun. Japon. p. 67 (1847); Naun. Vög. Deutschl. xiii. pl. 354 (1 360).

Oreocincla whitei, (Eyton), Gould, P. Z. S. 1837, p. 136; Bp. Comp. List B. Eur. \& N. Amer. p. I7 (1838).

O. varia, (Pall.) Gould, P. Z. S. 1837 , p. I36; Cab. Mus. Hein, i. p. 6 (1850); Sund. Journ. Orn. 1857, p. I60; Swinh. P'. Z. S. 1871 , p. 367; Tacz. Bull. Soc. Zool. France, 1876, p. I46; David et Oust. Ois. Chine, p. I 58 (1877); Prjev. Rowl. Orn. Misc. ii. p. 200 ( 1877$)$.

O. aurea, (Hol.), Bp. Cat. Ucc. Eur. p. 34. n." I $5^{6} \quad(18+2)$; id. Consp. i. p. 362 (1850); id. Rey. et Mry. Zos!. 1857 , p. 205; Tomes, Ibis, 1859, p. 307; Jaub. et Barth.-Lxpomm. Rich. Orn. p. 202 (1859); Gould, B. Gt. Br. ii. pl. 39 (1873).

T. lunulatus, Lath. apud Blas. List B. Eur. p. 9 (1362). 
'T. dauma, Lxth. apud Pelz. Verh. k. k. zsol.-ḃot. Gesell. Wien,

Geocichla varia, Seeb. Cat. B. Mus. 5. p. I 5 I (1881). Hab. Luzón.

10. G interpres, Kuhl.

'Turdus interpres, Kuhl, fide Temn. Pl. Coll. ii. n. ${ }^{\circ} 45^{8}$ (1828); Loss. Traité, p. 410 (1831); Gray, Gen. B. i. p. 220 (1847); Gray, Hand.l. B. i. p. 260. n. ${ }^{\circ} 3794$ (i 869).

(Geocichla interpres, (Temm.), Hartl. Syst. Verz. p. 4I (1841); Bp. Consp. i. p. I6 I (1830); Horsf. \& More; Cat. B. Mus. E. I. Co. i. p. igi (1854); Seebohm Cat. B. M. 5. p. i6! (I88I).

Hxb. Basilan, Java, Sunatra, Lombock.

\section{Gén. TURDUS, LiN}

I I. T. musicus, Lin. $0^{*}$ N. v. El músico.

(M. S, T.)

La petite Grive, Orn. ii. p. 205 (i760).

La Grive, Montb. Hist. Nat. Ois. iii. p. 280 ( 1775 ).

La Litorne, Daubent. Pl. Enl. n. ${ }^{\circ} 406$ (i775).

La Calandrotte, Daubent. Pl. Enl. n. ${ }^{\mathrm{a}}$ 49) (1775).

'T. musicus, Linn. Syst, Nat. i. p. 292 ( ( 765$)$; Scop. Ann. i. p. I33 (i759;) Lath, G?n. Syr. Suppl. i. p. 285 (1787); Gmel. Syst. Nat. i. p. 809 (I788); Lath. Ind. Orn. i. p. 327 (I790); Bechst. Naturg. Doutschl. iv. p. 201 (I795); id. Orn. Tacchenb). i. p. I44 (1802); Wolf, Taschenb. i. p. I95 (1810); Pallas Zoogr. Rosso-Asiat. i. p. 454 (I8I I); Temm. Man. d'Orn. p. $8 \mathrm{~s}$ (1815); Koch, Syst. baier. Zool. i. p. I75 (1816); Temm. Man. sern. i. p. I64 (1820); Naum. Vög. Deutschl. ii. p. 262 (I822); Gould, B. Eur. ii. pl. 78 (1837); Keys. u. Blas. Wiro. Eur. pp. I, I77 (1840); Nord. Dend. Voy. Russ. màrid. iii. p. I 25 (1340); Werner. Atlas, Insectiv. pl. I3 (1842); Degl. Orn. Eur. i. p. 465 (1849); Gray, Gen. B. i. p. 2 I 8 (4847); Bp. Consp. i. p. 270 (1850); Cab. Mus. Hein. i. p. 2 (1850); Middend. Reis Sibir. Zool. ii. pt. 2, p. 169 (1858); Licht. Nomencl. Av. p. 25 (1854)? Sch!. Vog. Nederl. p. 21 I (1854); Sundev. Sv. Fogl. p. 50 (1856); Jaub. et Barth.-Lapomm. Rich. Orn. p. 205 (I859); Linderm. Vög, Griechenl. p. 85 (1860); Nut. List B. Eur. Blasius, p. 9(1862); Radde, Reis. Sibir. Vög. p. 234 (I863); Gould, B. Gt. Brit. ii. pl. xxxii. (I 866); Degl. et Gerbe, Orn. Eur. i. p. 422 (18.67); Loche, Expl. Sci. Algér., Ois. p. I86 (1867); Gray, Hand-l. B. i. p. 253. n. ${ }^{\circ} 3677$ (1869); Doderl. Avif. Sicil. p. Io7 (1869); Droste, Vogelw. Borkum, p. 93 ( г 869); Heugl. Orn. N. O.-Afr. p. 3 I8 (1869); Fritsch, Vög. Eur. p. I39. (1870); Salvand. Faun. Ital, Ucc. p. 79 (1871); Sharpe \& Dresser, B. Eur. pt. v. (1871); Newt. ed. Yarr. Br. B. i. p. 264 (1872); Harting, Handb. Br. B. p. I I. (1872); Shelley, $B$. Egypt, p. 66 (1872); Irby, B. Gibr. p. 73 (1875); Blanf. Exst. Pers. ii. p. 156 p. (1876); Tacz. Bull Soc. Zool. France, i. p. $148(1876)$; Bodg, B. Cauc. p. 78 (1879); Seebokm, Cat. B. M. 5. p. r9i (1881). 
Trhostle, Lath. Gen. Syn. ii. pt. I, p. 18 (1783).

T. iliacus, Linn., apud Bodd. Table Pl. Enl. p. 29 (1783).

'T. pilaris, Linn., apud Bodd. Table Pl. Enl. p. 29 (1783).

Sylvia musica, (Lin.), Savi, Orn. Tosc. i. p. 211 (1727).

'T. minor, philomelos, Brehm, Vög. Deutschl. p. 3 I 2 (I831).

Merula musica, (Linn.), Selby, Brit. Orn. i. p. 162 (1833); Rüpp. Sy'st. Uebers, p. 6o (1845).

Hab. Europa, Africa.

12. T. viscivorus, Lin. ON N. v. El drene.

(M. S. T.)

la grosse Grive, Briss. Orn. ii. p. 200 (1760).

'. viscivorus, Linn. Syst. Nat. i. p. 291 (1766); Scop. Ann. i. 1. 132 (1769); Lath. Gen. Syn. Supp. i. p. 285 (1787); Gmet. Syst. Mat. i. p. 806 ( 788 ); Lath. Ind. Orn. i. p. 326 ( $79(1)$; Bechst. Naturg. Deutschl. iv. p. I 8 (1795); in. Orn. Taschenb. i. p. 143 (1802); Wol., Taschenb. i. p. 191 (1810; Pallas. Zoogr. Rosso-Asiat. i. p. 454 (1811); Temm. Man. d'Orn. p. 86 (1815); Koch, Syst. baier. Zool. i. p. 174 (1816); Temm. Man. dOrn. i. p. I61 (1820); Nawm. Vög. Deutschl. ii. p. 248 (1822); Ménétr. Cat. Rais. Cauc. p. 29 (1832); Gould, B. Eur. ii. pl. 77 (1837); Keys. u. Blas. Wirb. Eur. pp. li, I77 (1840); Nordm. Demid. Voy. Ru s. mérid. iii. p. I25 (1840); Werner, Atlas, Insectiv. pl. I I (1842); Gray. Gen. B. i. p. 2 is (1847); Degl. Orn. Eur. i. p. 467 (1849); Blyth, Cat. B. Mus. As. Soc. p. I60 (1849); Bp. Consp. i. p. 269 (1850); Cab. Mus. Hein. i. p. 2 (1850); Licht. Nomencl. Bv. p. 25 (1854) Schl. Vog. Nederl. p. 208 (1854); Horf. \& Moore, Cat. B. Mus. E. I. Co. i. p. 194 (1854); Sundev. Sv. Fogl. p. 49 (1856); Jaub. et Barth.-Lapomm. Rich. Orn. p. 204 (1859); Linderm. Vög. Griechenl. p. 86 (1860); Nzwt. List B. Eur. Blasius; 1. 9 (1862); Degl. et Gerbe, Orn. Eur. i. p. 418 (1867); Loche. Expl. Sci. Algér., Ois. i. p. 183 (1867); Gould, B. Gt. Br. ii. pl. xxxiii. (1869); Heugl. Orn. N. O.-Afr. i. p. 379 (1869); Gray, Hand-l. B. i. p. 253. n. ${ }^{\circ} 667$ (1869); Doderl. Avif. Sicil. p. 106 (1869); Droste, Vogelw. Borkum, p. 93 (1869): Fritsch, Vög. Eur. p. I35 (1870); Salvad. Faun. Ital. Ucc. p. 78 (1871); Sharpe, \& Dresser, B. Eur. pt. vi. (1871); Newton, ed. Yarr. Brit. B. i. p. $25^{8}$ (1872); Harting. Handb. Br B. p. I1 (1872); Shelley. B. Egypt, p. 65 (1872); Severts. Turkest. Jevotn. p. 65 (1873); Dresser, Ibis, 1875 p. 334; Irby, B. Gibr. p. 73 (1875); Blanford, East. Pers. ii. P. 157 (1876); Tacz. Bull. Soc. Zool. France, i. p. 146 (1876); Bogd. B. Cauc. p. 78 (1879); Seebohm, Cat. B. M. 5. p. I94 (1881).

La Draine, Montb. Hist. Nat. Ois. iii. p. 295 (1775).

La Drenne, Daubent. Pl. Enl. n. ${ }^{\circ} 489$ (1775).

Missel Thrush, Lath. Gen. Syn. ii. pt. 1, p. I6 (1783).

Sylvia viscivora, (Linn.), Savi. Orn. Tosc. i. p. 208.(1827).

Ixocossyphus viscivorus, (Linn.), Kaup, Natürl. Syst. p. 145 (1 829 ).

T. major, arboreus, Brehm, Vög. Deutschl, p. 379 (1831).

Merula viscivora, (Linn.), Selby, Brit. Orn. i. p. 158 (1833); Rüpp. Syst. Uebers. p. 6o (1845).

'T. hodgsoni, Homeyer, Rhea, ii. p. I 50 (1849); Bp. Corisp. i. 
p. 269 (185o); Gray, Hand-!. B. i. P. 25.3. n.” 3668 (186:); Hume, Nests and Eggs Ind. B. p. $23^{6}$ (1873).

T. bonapartei, Cab. Journ. Orn. 1860 , p. i 83.

Hab. Europa, Africa, Himalaya.

13. T. mustelinus, Gm ơ N. v.

(.I. S. T.)

Tawny 'Thrush, Lath. Gen. Syn. ii, pt. 1, p. 28 (1 783 , ex Pennant). I. mustelinus, Gmel. Syst. Nat. i. p. 817 (1788, ex Penn. et Lath). Lath. Ind. Orn. i. p. 33 I ( 790$)$; Vicill. Ois. Amér. sept. ii. p. 6, pl. Ixii ( 807$)$; Vienll. N. Dict. d'Hist. Nat. xx. p. 24I (1818); Audub. Orn. Biogr. i. p. 372 (1831); Bp. Comp. List. B. Eur. \& N. Amer. p. I7 (1838); 1)'Orb., La Sagra's Cuba, Ois. p. 49 (1838); Cosse, B. Jamaica, p. 144 (1847); Grab, Gen. B. i. p. $219(1847)$; Bp. Gonsp. i. p. $270(1850)$; Cab. Mus. Hein i. p. 5 (1850); Baird, Gass. \& Laur. B. N. Amer. p. 212 1858); Sclat. P. $Z$. S. 18.59, p. 362 ; id. Amer. B. p. 2 (1862); Baird, Reviez', p. ' 3 (1864); Gray, Hand-l. B. i. p. 254 n. ${ }^{3679}$ (1869), Coues, Key N. Amer. B. p. 72 (1872); Baird, Brewer, \& Ridgw. Hist. N. Amer.'B. i. p. 7 (1874); Salv. \& Godm.. Biol. Centr.-Amer. Zool., Aves, i. p. 9 (1879); Seebohm, Cat B. Mus. 5. p. 196 (1881).

T. melodes, Bartr. Trav. p. 290 bis (1791).

'T. melodus, (Bartr.), Wilson, Amer. Orn. i. p. 29, pl. ii. fig. I ( 8 o 8 ).

'T. melodius, (Bartr.), Bp. Journ. Phil. Acad. iv. p. 33 (1824). Merula mustelinus, (Gmel.), Denny P. Z. S. 1847 , p. 38 .

'Turdus densus, $B p$. Compt. Rend. xxxviii. p. 2 (1854).

T. (Hylocichla) mustelinus, (Gmel.), Couses, Birds Colorado Vall. p. 28 ( ( 878$)$.

Hab. América, Canadá.

14. T. pallasi, Cab. ơ N. v. Culin.

(M. S. T.)

Turdus pallasii, $C a b .^{*}$

T. solitarius, Wilson, Amer. Orn. v. p. 95, nec pl. xliii. fig. 2 (181 2, nec Müll., uec Gmel.); Bp. Comp. List. B. Eur. \& N. Amer. p. I7 (1838); Audub. B. Amer. iii. p. 29, pl. cxlvi. (184I); Gray, Gen. B. i. p. 219 (1847); Bp. Consp. i. p. 270 ( 1850$)$.

'T. minor, Gmel. apud Bp. Journ. Phil. Acad. iv. p. 33 (1824), apud Audub. Orn. Biogr. i. p. 303. pl. lviii. •( I83I)

Merula solitaria, (Wilson), Sw. Faun. Bor.-Amer. Birds, ii. p. I 84 pl. xxxxv. ( $183 \mathrm{I})$.

'I. nanus, Audub. Orn. Biogr. v. p. 201, pl. ccccxix, (I839); id. B. Amer. iii. p. 32, pl. cxlvii. (184r); Gray, Gen. B. i. p. 219 (1847).

'I. guttatus, (Pall.), apud Cab. Tschndi's Faun. Per. p. 187 (1846).

'T. pallasii, Cab. Arch. fïr Naturg. 1847, p. 205; Homeyer, Rhea, ii. p. 147 (1849); Cab. Mus. Hein. i. p. 5 (1850); Gundlach, Journ. Orn. 1855, p. 470; Baird, Review, i. p. I4 (1864); Gray, Hand-l. B. i. p. 254. no. 3684 (1869); Coues, Key $N$. Amer. B. p. 72 (1872); id. B. N. West, p. 2 (1874); Baird, Brewer, \& Ridgre Hist. N. Amer. B. i. p. I8 (1874); Hen- 


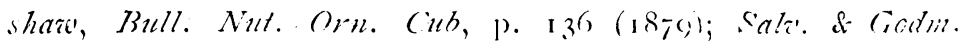
Biol. Centr.-stmer. Zool., Ares, i. p. 13 (18-9); Seebohm, Cat. B. M. 5. p. 199. (1881.

'J. (Hylocichla) pallasi, (Cab.), Cones, B. Col. Vall. p. 20 (1878).

Hab. América, Canarlí, Cuba. California.

15. T. leucauchen, Sclat ơ N. v.

$\left(\begin{array}{lll}\text { M } & S & \mathrm{~T}\end{array}\right.$

'Turdus leucauchen, Sclater, P. Z. S. 1858 , p. 447; Scl. \& Sali'. Ibis, 1859 , p. 6; Sclater, P. Z. S. 1859 , 1. 328; id. (at. Amer. B. p. 3 (1862); Baird. Rev. Am. B. i. p. 24 (1864: Salvin, P. Z. S. 1867 , p. i 32 ; Lazwr.* Ann. L. New York. ix. p. 91 (I 868); Gray', Hand-l. B. i. p. 257. n. 3749 (1 86 (x); Scl. \& Salv. P. Z. S. t87o, p. 838; Seebohm, Cat, B. Mus. 5. p. $212(188 \mathrm{I})$.

Hab. Guatemala.

16. T. grayii, $B p . \sigma \mathrm{N}$. v.

(M. S. T.)

'Turdus helvolus, Licht. Preis-Verz. Mex. Vög. no. 85 (Is $z^{\prime}$, de:cr. mulla); Licht. Nom. Av'. Muts. Zool Berol, p. 26 (185ti; Cab. Journ. Orm. 1868, p. 57.

T. grayii, Bp. P. Z. S. 1837 , p. 118; Gray, Gen. B3. i. p. 211 (1847); Bp. Consp. i. p. 272 (1850); Cab. Mus. Hein. i. p. + (1850); Sclater. P. Z. S. 1859 , p. 330; Scl. \& Salv. Ibis, 1859 , p. 5; Cab. Journ. Orn. 1860 , p. 323; Owen, Ibis, r861 p. 6o; Baird, Kev. Am. B. p. 26 (is64), parte.); Larer. Aun. L. N. H. New York, ix. pp. 91 199 (1868); Sumchrasi. Mem. Bost. Soc. N. H. i. p. 54t (1809); Gray, Hand-l. l'. i. p. 257 no. 3741 (1869) Frantaitis, Journ. Orn. 1869 , 1. 29x: Scl. \& Salo. P. Z. S. 1870,1870 p. 836 Grayson, Pr. Bost. xiv. p. 276 (187 I); Scl. \& Salv. Nomencl. Ala. Neotr. p. I (187.3); Seebohm, Cat. B. M. 5. p. 219 (2881).

Planesticus grayii, (Bp.), Bp. Compt. Rent. xxxviii. p. $4(185+1$. $P$. luriclus, $B p$. Compt. Rend. xxxviii. p. $+(185+)$.

'Turdus tristis, Swains, apud Sclater, P. Z. S. 1856, p. 294.

'T. luridus, (Bp.), Scl. \& Salv. Nomencl. Av. Neotr. p. 2 (1873). Hab. Guatemala.

7 Gén. merula, Leacif.

17. M. merula, Lin. o N. v. El mirlo.

(M. S. T.)

Le Merle, Briss. Orn. ii. p. 227 ( 1760$)$; Montb. Hist. Nat. Ois. iii. p. 330 ( 1775$)$.

Le Merle de France, Daub. Pl. Enl. nos. 2 (d), 5.55 (む) (1775).

'Tû́rdus merula, Linn. Syst. Nat. i. 1. 29.5 (1766); Scop. Anth. i. p. I34 (1769); Lath. 'Gen. Syn. Suppl. i. 1). 285 (1787); Gmel. Syst. Nat. i. p. 831 (1788); lath. Ind. Orn. p. 3t" (1790); Bechst. Naturg. Deutschl. iv. p. 219 (1795); id. Orn. Tachenb. i. p. I49 (1802); Wolf, Tachenb. i, p. I99 (1810); Pallas, Zoogr. Rosso-Asiat. i. p. $449(1 \mathrm{1} 1) ; \mathrm{T}$ ('mm. Man. d'Orn. p. 9I (1815); Koch, Syst. baier. Zool. i. p. I72 (1816); Vieill. Dict. d'Hist. Nat. xx. p. $244(1818) ;$ Timm. Man. d'Orn. i. 
i. p. 168 (1820); Nirm. Vög. Deutschl. ii. p. 326 (1822.) Ménètr. Cat. Rais. Cauc. p. 29 (1832); Keys, u. Blas. Wirb. Eur pp. li, I78 (1840); Nordm. Dèmid. Voy. Russ. mèrid iii. p. I 28 (I840); Werner. Atlas, Insectiv. pl. I6 (I842); Grai, Gen. B. i. p. 218 (1847); Degl. Orn. Eur. i. p. 456 (i849); Bp. Consp. i. p, 274 (1850); Cab. Mus. Hein. i. p. 3 (1850); Licht. Nomencl. Aa. p. 25 (1854); Schl. Vog. Nederl. p. 204 (1854); Sundev. Sv. Fogl. p. 25 (1858); Linderm. Vög Gricchenl. p. 86 (1860); Newt. List. B. Eur. Blasius; p. 9 (1862); Neut. Baring-Gou'd's Iceland, p. 408 (1863); Filippi, Viagg. Pers. p. 347 (1865); Degl. et Gerbe, Orn. Eur. i. p. 399 (1867); Doderl. Avif. Sicil. p. ${ }_{0} 5$ (1869); Droste, Vogelwu. Borkum, p. 92 (I 869); Gray, Hand-l. B. i. p. 255 no. 3697, (1 869); Heugl. Orn. N. O.-Afr. p. 386 (1869); Gogman, Azores, p. 23 (1870); Fritsch, Vög. Eur. p. I47 (1870); Salvad. Faun. Ital. Ucc. p. 76 (1871); Newit. ed Yarr. Br. B. i. p. 280 (1872); Sharpe \& Dresser, B. Eur. pt. x. (1872); Harting, Handb. Br. B. p. I 2 (1872); Shelley, B. Egygt. p. 66 (1872); Sivertz, Turkest Jevotn. p. 64 (1873); Irby, B. Gibr. p. 74 (1875); Dresser, Ibis, 1875 , p. 332; Blanf. East. Pers. ii. p. 157 (1876).

Blackbird, Lath. Gen. Syn. ii. p. 43 (I783);

Merula nigra, Leach, Syst. Cat. Mamm. \&c. Brit. Mus. p. 20 (1816); Forster, Syn. Cat. Br. B. p. 51 (1817).

M. merula, (Linn.), Boie, Isis, 1822, p. 452; Kaup, Natürl. Syst. p. I 37 , I 829$)$.

Sylvia merula, (Linn.), Savi, Orn. Tosc. i. p. 205 (1827).

Merula pinetorum, truncorum, alticeps, carniolica, Brehm, Vög. De utschl. p. 372 ( 1831$)$.

'Turdus merula, var. syriaca, Hempr. et Ehrenb. Symb. Phy's. Aves, fol. bb (r833).

Merula vulgaris, Selby. Brit. Orn. i. p. 167 (1833); Gould, B. Eur. ii. pl. 72 (1837); Rïpp. Syst. Uebers. p. 60. (1845); Jaub. et Barth.-Lapomm. Rich. Orn. p. 214 (1859); Goild, B. Gr. Br. ii. pl. 37 (1862); Loche, Expl. Sci. Alger., Ois. i. p. 192 (1867); Scully, Str. F. 1876, p. 139; Bogd. B. Cauc. p. 8 o (1 879$)$.

M. major, Brehm, Naum. p. 28 I (1855).

'Turdus menegazzianus, Perini, Uccolli Veronesi, p: $5^{6}$ (1858).

'T. dactylopterus, Bp., fide Gray, Hand-l. B. i. p. 255 n. 37 I 4 (i 869).

T. merula, var. montana, Savi fide Salvad., Dresser, B. Eur, pt. x. (1872).

Merula merula, Seebohm, Cat. B. M. 5. p. 235 (288I). Hab. Europa, Francia.

18. M. torquata, Lin. t N. v. Mirlo de collar.

(M. S. T.)

Le Merle à collier, Briss. Orn. ii. p. 235 (1860); Daubent. Pl. Enl. no. 516 (I775).

'Turdus torquatus, Linn. Syst. Nat. i. p. 296 (1766); Scop. Anr. i. p. I 35 ( I769); Lath. Gen. Syn. Suppl. i. p. 285 (1787); Gmel. Syst. Nat. i. p. 832 (I788); Lath. Ind. Orn. p. 343 (1790); Bechst. Naturg. Deutschl. iv. pr 214 (1795); id. Orn. Taschenb. i. p. 148 (1802); Wolf. Tascheub i. p. 198 (1810); 
Pallas, Zovgr. Rosso-Asiat. i. p. 45I ( $18 \mathrm{II})$; Temm. Man. dOrn. p. 90 (1815); Koch, Syst. baier. Zool. i. p. I73 (1816); Temm. Man. a'Orn. i. p. I66 (1820); Naum. I'ög. Deutsch!. ii. p. 318 (1822); Ménétr. Cat. Rais. Cauc. p. 29 (1832); Keys. u. Blas. Wirb. Eur. pp. li, I 77 (1840); Nordin. Démid. Voy. Russ. mérid. ii. p. I 27 (1840); Werner. Atlas, Insectiv. pl. xv. (1842); Gray, Gen. B. i. p. 2 I9 (1847); Degl. Orn. Eur. i. p. $45^{8}$ (1 849); Bp. Consp. i. p. 1274 (850); Cab. Mus. Hein. i. p. 3 (1850); Schl. Vög. Nederl. p. 25 (1854); Licht. Nomencl. Ar. p. 25 (1854); Sundev. Sv. Fo!g. p. 52 (1856); Linderm. Vög. Griechenl. p. 85 (1860;) Neruton, List B. Eur. Blasints, p. 9 (1862); Degl. et Gerbe, Orn. Eurr. i p. 401 (1867); Doderl. Avif. Sicil. p. I06(1 869); Droste, Vogelw. Borkum, p. 92 (1869) Heugi. Orn. N.O.-Afr. i. p. 386 (1869); Gray, Hand-l. B. i. p. 256. n. 3720 (1869); Fritsch, Vog. Eur. p. 146 (1870); Salvad. Faun. Ital. Ucc. p. 77 (187i); Newt. ed. Yarr. Brit. B. i. p. 287 (1872); Harting, Handb. Br. B. p. I 2 (I872); Shelley B. Egypt, D. 67 (1872)? Sharpe \& Dresser, B. Eur. pt. x. (1872); Irby, B. Gibr. p. 74 (1875); Blanf. East. Pers. ii. p. $158(1876)$.

Le Merle à plastron blanc., Montb. Hist. Nat. Ois. iii. p. 349 (I 775).

Ring-Ouzel, Lath. Gen. Syn. ii. p. 146 (1783).

Merula torquata, (Linn.), Boie, Isis, 1822, p. 552; Selby. Brit. Orn. i. p. 169 (1833); Gould, B. Eur, ii. pl. 72 (1837); Jaut. et Barth.-Lapomm. Rich, Orn. p. 2 I6 (I859); Loche, Expl. "c". Algér., Ois. p. I90 (1867); Gould, B. Gt. Br. ii. pl. xxxviii. (1873); Bogd. B. Cauc. p. 8o (1879); Seebohm, Cat. B. M. 5. p. 246 (1881).

Sylvia torquata, (Linn.), Savi, Orn. Tosc. i. p. 206 (1827).

(Copsichus torquatus, (Linn.), Kaup, Natürl. Syst. p. I 57 (1829).

Merula montana, collaris, alpestris, Brehm, Vög. Deutschl. p. 375 $(183 \mathrm{r})$.

'Thoracocincla torquata, (Linn.), Reich. Nat. Syst. pl. xliii. (1850).

Merula vociferans, maculata, Brehm, Naum. 1855, 28I. Hab. Europa, Alemania.

19. M. obscura, Gin. $\delta$ \& N. v Tamsi.

(M. S T.)

Dark Thrush, Lath. Gen. Syn. ii. pt. 1, p. 3r (I783).

'Turdus obscurus, Gmel. Syst. Nat. i. p. 816 (1788, ex Lath.j; Lath. Ind. Orn. i. p. 333 (1790); Gray Gen. B. i. p. 219 (1847); Bp. Consp. i. p. 273 (1850). Middena'. Reis. Sibir., Zool. ii. pt. 2, p. 169 (1853); Jaub et Barth-Lapomm. Rich. Orn. p. 214 (1859); Swinhoe, Ibis, 1863, p. 277; Radde, Reis. Sibir., Vög. p. 235 (1863); Fritsch, Vög. Eur. p. 143 (1870); Swmh. P. Z. S. 1871, p. 367; Tacz. Journ. Orn. 1872, p. 440; Szinhoe, Ibis, 1874. p. 443; Blyth \& Walden, B. Burm. P. 99 (1875); Tacz. Bull. Soc. Zool. France, 1876 , p. I48; David et Oust. Ois. Chine, p. I 53 ( 1877 ); Dresser, B. Eur. pt. lxix. (1878); Hume \& Davison, Stray Feath. vi. p. 25I (1878); Seebohm, Ibis, 1879 , p. 4.

'I. ocragaster, Sparrm. Mus. Carls. iv. pl. 85 (1789).

'I. pallens, Pallas, Zoogr. Rosso-Asiat. i. p. 457 (1811; Teinm. 
et Schl. Faun. Japon., Aves, p. 0.3, pl. xxvii. $(18+7)$; Cab. M/us. Hein. i. p. 2 ( $185(0)$; Swinh)e, Ibis, 1860 , p. 56; Nerot. List. B. Eur. Blasius, p. 9 (1862); Sinliad. Faun. Ital. Ucc. p. B: (1871); Severtz. Turkest. Jevotn. p. 05 (1873; Dresser, Ibis, 1875 , p. 334; Prjev. Rowcley's Orn. Misc. ii. p, 198 (1837).

T. seyffertitzii, Brehm, Lehrb. eur. Vög. ii. p. 97 ₹ (18zt).

'I. rufulus, Drapiez, Dict. Class. d'Hist. Nat. x. p. 443 ( 1826$)$; Gray, Hand-l. B. i. p. 256 n." 3728 (1869).

'T. werneri, Géné, Mem. Ac. R. Tor. xxxvii. p. 296, pl. 2 (183t).

T. pallidus, Gmel. apud Temm. Man. d'Orn. iii. p. 97 (1835), apud Degl. Orn. Eur. i. p. 46r (1849), apud Newol. List. B. Eur. Blasius, p. 9 (1862), apud Degl. et Gerbe, Oru. Eul. i. p. 402 ( 1867$)$.

T. modestus, Eyton, P. Z. B. 1839, p. 103; Gray, Gen. B. i. p. $218 \quad(1847)$.

T. javanicus?, Horsf. apud Blyth. Cat, B. Mus. As. Soc. p. i61 (1849).

Planesticus obscurus, (Gmel.), Bp. Cat. Parzud. p. 5 (18,5).

'Turdus davidianus, Milne-Adw. Nouv. Arch. du Mus. H st. N.t. i. Bull. p. 26 (i 825); Gray, Hand-l. B. i. p. 253. n. ${ }^{\circ}$ (67) ( 1869 ).

T. chrysolaus, Temm., apud Godw.-Aust. J. A. S. Beng. xxxix. pt. 2, p. IOI (I 870$)$.

(Geocichla obscura, (Fmel.), Jerdon, Ibis, 1872 , p. 136.

'Turdulus pallens, (Pall.), Godw.-Alıs!.J.A.S. Bing. xliii. pt. 2, p. 178 (1874).

Merula obscura, Seebohm, 5. p. 273. (1881).

Hab. Luzón, Cagayan, Borneo, Japón, China, Malaca.

20 M chrysolaus, Tem. $\downarrow ? \mathbf{N}$. v. Tams;.

(M. S. T.)

'Turdus crysolaus, Temm. Pl. Col. ii. pl. 537 (1831); Temm. et Schleg. Faun. Japon., Aves, p. 64, pl. xxviii. (1847); Bp. Consp. i. p. 273 (1850); Licht. Nomencl. Av. p. 25 (1854); Swinhoe, Ibis, 1860 , p. 56; Schrenck, Reis. u. Forsch. Amur. i. 1. $35^{2}$ (1860); Sclater, Ibis, 1863 , p. 197; Swinhoe, Ibis, 1863 , p. 276 ; id. P. Z. S. 1863 , p. 280; Gray, Hand-l. i. p. $25^{6}$. n. ${ }^{\circ} 3724$ (1869); Sinh. Ibis, 1870, p. 248; id, P. Z. S. 1871 , p. 367; David et Oust. Ois. Chine, p. I52 (1877); Blakiston \& Pryer, Ibis, 1878 , p. $24 \mathrm{I}$.

'I. chrycolaus, Temm. Pl. Col. ii. text to pl. 537 (1831).

T. chrysolotus, Temm., fide Gray, Gen. B. i. p. 219 (1847).

Merula chrysolaus, Seebohm, 5. p. 275. (I88I). Hab. Luzón, Laguna.

\section{Gén ERITHACUS, Cuv.}

21. E. calliope, (Pall) $\& \&$ N. v. Iolin.

(M. S. T.)

Motacilla calliope, Pall. Reis Russ. Reichs, iii. p. 697 (1776).

Kamtschatka Thrush, Lath. Gen. Syr. ii. p. 28 (1783).

Turdus camtschatkensis, Gmel. Syst. Nat. i. p. 817 (1788, ex Lith.)

1. calliope, (Pall.), Lath. Int. Orn. i. p. 33 I (г790). 
Accentor calliope, (Pall.), Temm. M.tn. dOrn. iii. p. $172(\mathrm{r} s 35)$. (alliope lathani, Gould, B. Eur: ii. pl. In (1837); Byth, J. A. S. Beng. xi. p. 11 2 (1842).

Lusciola calliope, (Pall.), Keys. u. Blas. Wirs. Ë.r. Pi. Wiii. 190 (1840); Temm. et Schleg. Fiuun. Japon. Ayss, p. 57 (18+7).

(alliope camtschatkensis, (Ginel.), Strickl. Ann. Nat. Hist. vi. p. $422(18+1)$; Blyth, J. A. S. Beng. xvi. p. I $3+(18+7)$; id. Cat. B. Mus. As. Soc. p. $149(\mathrm{I} 8+9)$; Bb. Consp. i. p. 29) (1850); Ciab. Mus. Hein. i. p. I (185(3); Horsf. \& Moore, Cal. B. Mus. E. I. Co. i. p. 31 3 (1854); Jaub. et Barlh.-Lapomm. Rich. Orn. p. $236(1859) ;$ D.gel. et Gurbe, Orn. Eur. i. 1). 464 (1867); Swinh. P. Z. S. 1871 , p. 359; Dresser, B. Eilu. pt. xlvi. (1875); Biyth, \& Wald. B. Burm. p. ror (r $\left.8_{75}\right)$; Seebonm, Ibis, 1879 , p. 7 ; Gould. B. Asia, pt. xxxi. pl. 's $(1879)$.

(yanecula calliope, (Pall.), Gray, Gen. B. i. p. I82 (18+8).

lirithacus calliope, ('lall.), Degl. Ora. Eur. i. p. 51+(18fo); Seebohin, Cat. B. M. 5. p. 305. (1881).

Sylvia (Calliope) kamtschitrensis, (Ginel.), Middend. Reis Sicir. Zool. ii. pt. 2, p. $174(1853)$.

(alliope kamtschatcensis, (Gmel.), Hartl. Journ. Orn. $185 \%$, 1. 50; Jerdon, B. Int. ii. p. 1.50 (1863).

Erithacus ignigularis, Dubois, OS. Ëer. pl. 6r (г762).

Melodes calliope, (Pall.), Nezet. List B. Eur. Blasius, p. Io (1852).

Lusciola (Calliope) kuntschat'kensis, (G.nel.), Radus, Reis. Sibir. $V \ddot{g} g$. p. $248(1863)$.

(yanecula camtschatkensis, (Gmel.), Gray, Hant-l. B. i. p. 22 . n. 3202 (1859).

Lusciola (Melodes) calliope, (Pall.), Fritsch, Vüg. Eur.p. lít $(1870)$.

Calliope ka ntschatkensis, (Gimel.), Godw.-Aitst. J. A. S. Bens. xxxix. pt. 2, p. 270 (180); Ta:z. Bull. Soc. Zool. Firance, 1.

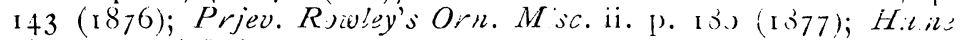
vi. p. $337(1878)$.

Hab. Luzón, Lagun a, Minila, Minlaloyan; Coina, Calcuta, Nepal, India.

9. Gén. MONTICOLA, Boit.

22. M. affinis, Blyth, $\delta \&$ N. v. Solitario.

(M. S. T.)

Petrocincla affinis, Blyth, J. A. S. Beng. xii. p. I $77^{*}(18+3$, p.xrtim). 'Turdus (Mouticola) erythropterus, Gray, P. Z. S. 1850, p. $35^{\circ}$. Petrocossyphus cyaneus, (Linn.)?. apud Jerd. B. Ind. i. p. $51_{2}$ (1862), apud Blyth \& Wald. B. Burm. p. 99 (1875, partion). Monticola affinis, (Blyth), Dauid et Oust. Ois. Chine, p, I62 (1877). Monticola cyanus solitaria, Sesb. Cat. B. M. 5. p. 3 18 (i381). Hab. Luzón, Panaon, Formosa, China, Jaìn.

23. M. soiitaria, Briss. $\sigma \rho$ N. v. Solitario.

(M. S. T)

Le Solitaire do Manille, Briss. Orn. ii. p. 270, pl. xxaiii. fig. 2 (1760). 
Le Solitaire de Philippines, Briss. Orn. ii. p. 272, pl. xxviii. fix. I $(1760)$.

Le: Merle solitaire de Manille, Montb. Hist. Nat. Ois. iii. p. $3^{6,3}$ (1775); Daubent. Pl. Enl. n. 564. fig. 2 (1775;) id. Pl. Enl. n." 636 (1775, \&).

Le Merle solitaire de Philippines, Montb. Hist. Nat. Ois. iii. 1. 364 (1775); Daubent. Pl. Enl. n. ${ }^{\circ} 339$ (1775).

Turdus solitarius, P. L. S. Mïll. S. N., Anhang, p. 142 (1776); Gray, Hand-l. B. i. p. 260. n. 3807 ( 1869 ).

T. philippensis, P. L. S. Müll. S. N., Anhang, p. I45 (1776); Bodd. Tabl. Pl. Enl. p. 21 (1783); Gray, Gen. B. i, p. 221 ( 1847 ); id. Hand-l. B. i. p. 260 n. 3807 (1869).

Pensive Thrush, Lath. Gen. Syn. ii. p. 53 (1783).

Hermit Thrush, Lath. Gen. Syn. ii. p. 54 (1783).

Turdus manilla, Bodd. Tabl. Pl. Enl. p. 39. ( 1783 )

T. manillensis, Gmel. Syst. Nat. i. p. 833 (1788); Lath. Int. 8 Orn. i. p. 345 ( I 790); Vieill. N. Dict. d'Hist. Nat. xx. p. $2 \dot{x} x$ (1818); Gray, Gen. B. i. p. 220 (1847); Temm. et Schleg. Faun. Japon. Aves, p. 6 (1857).

T. eremita, Gmel. Syst. Nat. i. p. 833 (1788); Lath. Ind. Orn. i. p. 346 ( 1790$)$; Vieill. N. Dict. d'Hist. Nat. xx. p. 288 (1818).

Petrocincla manillensis, (Gmel.), Blyth. J. A. S. Beng. xi. p. 460 (1842); id. J. S. Beng. xvi. p. I 5 I (1847); id. Cat. K. Mus. As. Soc. p. 164 (1849); Horsf. \& Moore, Cat. B. Mus. E. I. Co. i. p. I 88 (1854); Cassin, Perry's Exp. to Japan, p. 240 (1856); Swinhoe, Ibis, 1862 , p. 307.

P. eremita, (Gmel.), Bp. Consp. i. p. 297 (I850).

Petrocossyphus manillensis, (Gmel.), Bp. Consp. i. p. 297 (1850); Swinhoe, Ibis, 1860 , p. 56.

Petrocincla violacea, Swinhoe, Zoologist, p. 6228.

P. manilla, (Bodd.) Swinhoe, P. Z. S. 1871 , p. 368.; Tacz. Bull. Soc. Zool. France, 1876 , p. 146.

Manticola solitaria, (Mïll.), Wald. Tr. Z. S. viii. p. 63 (1872); id. Tr. Z. S. ix. p. 162 (1875); Dxvid. et Oust. Ois. Chine, p. I6I (1878); Seebohm, Cat. B. M. 5. p. 319 (I88I).

Cyanocincla solitaria, (Mïll.), Hume \& Davison, Str. F. vi. p. 248 ( 1878 ).

Hab. Luzón, Laguna, Panay, Guimarás, Negros, Panaon, Leyte, Samar, Paragua, Mindanao, Basilan, Balabac.

\section{Gén. SIALIA, Sw.}

24 S. sialis, (Lin.) $\sigma \circ$ N. v. Pipit-tolog.

(M. S. T)

La Rouge-gorge bleue de la Caroline, Briss. Orn. iii. p. 423 ( 1760 ).

Motacilla sialis, Linn. Syst. Nat. i. p. 336 ( 7766 ).

Le Rouge-gorge bleu de l'Amérique sepentrionale, Buff. Hist. Nat. Ois. v. p. 2 I 2 ( 1778 ).

La Gorge-rouge de la Caroline, Daubent. Pl. Enl. n. 395 (1778).

Blue Warbler, Lath. Gen. Syn. ii. p. 446 (I783).

Motacilla scalis, Gmel. Syst. Nat. i. p. 989 (1788), probable mis. print). 
Syivin sialis, (Linn.), Lath. Ind. Orn. ii. p. 522 (1790); Jard. ed. Wilson's Amer. Orn. p. 55 pl. iii. fig. 3 ( 1832 ).

(Enanthe sialisi, (Linn.), Vieill. N. Dict. d'Hist. Nat. Ois. xxi. 1. $417(1818)$.

Saxicola sialis, (Limn.), B. Ann. Lyc. N. York, ii. p. 89 (1 826) Sialia wilsonii, Swains. Zool. Journ. iii. p. 173 (1827); Bp. Comp. List B. Eur. \& N. Amer. p. I6 (1839); Audub. B. Amer. Sro, ii. p. 171, pl cxxxiv. (1839); Gray, Gen. B. i. P. Is+ (18+6); Bp. Cousp. i. p. 298 (1850); Cab. Mus. Hein. i. p. 7 (1850); Scl. Cat. Amer. B. p. Io (1862, partim); Gray, Hand-l. B. i. p. 229. n. ${ }^{\circ} 3312$ (1869).

Anpelis sialis, (Linn.), Nuttall, Man. Orn. i. p. 444 (1832).

Virythaca (Sialia) wilsonii, (Svains), Sw. \& Rich. Faun. Bor.Amer. Birds, p. 210 (1831).

Sialia sialis, (Linn.), Haldemann, Trego's Geogr. Pennsylv. p. 77 ( 1843$)$; Baird, Cass. \& Lawr. B. N. Amer. p. 222 (1858); Baird, Review', i. p. 62 (1874); Coues, Key N.-Amer. B. 1). 76 (1872); Coues; B. N. West, p. I3 (1874, parte); Baird, Brezer, \& Ridgw. Hist. N.-Amer. B. i. p. 62 (1874); Cones, Birds Colorado Wall. p. 77 (1878, parte); Salv. \& Godm. Biol. Centr.-Amer. Zool., Aves, i. p. 45 (1879), parte); Seeboh, Cat. B. M. 5. p. 329 (1883).

Luscinia scialis, (Linn.), Giebel, Reich. Nat. Vög. p. 44 ( ( 860 ). Scialia scialis, (Linn.), Le Moine, Ois. Canad. p. 202 (I86I). Hab. Luzón, Laguna; Cuba, Canadá.

\section{Gén. RUTiCILliA, BreMh.}

25. R. phœnicurus, Lin. o N. v

(M. S T.)

Le Rossignol de muraille cendré, Briss. Orn. iii. p. 406 (i760).

Le Rouge-queue à collier, Briss. Orn. iii. p. 4 I I ( I760).

Le Rossignol de muraille, Briss. Orn. iii. p. 403 ( п766); Buff. Hist. Nat. Ois. v. p. I70 (1778); Daubent. Pl. Enl. n. ${ }^{\circ} 35 \mathrm{I}$ ( 778 ).

Motacilla phonicurus, Linn. Syst. Nat. i. p. 335 (i 766); Gmel. Sy'st. Nat. p. 987 ( 1788 ); Bechst. Naturg. Deutschl. iv. p. 609 ( I 795.)

Redstart, Lath. Gen. Syn. ii. p. 42 I ( 1783 ).

Sylvia phonicurus, (Linn.), Lath. Gen. Syn. Suppl. i. p. 287 ( 1787); Lath. Ind. Orn. ii. p. 5 I I ( 790); Bechst. Orn. Taschenb. i. p. I81 (1802); Wolf, Taschenb. i. p. 244 (1810); Temm. Man. d'Orn. p. I 99 (1815); Vieill. N. Dict. d'Hist. Nat. xi. p. 267 (1817); Temm. Man. d'Orn. i. p. 220 (1830); Ménétr. Cat. Rais. Cauc. p. 35 (1832, partim); Nordm. Démid. Voy. Russ. mérid. iii. p. I 35 (1840); Wern r, Atlas, Insectiv. pl. $52(\mathrm{I} 842)$.

Saxicula phœenicurus, (Linn.), Koch, Syst. baier. Zool. i. p. I 88 $(\mathrm{i} 8 \mathrm{i} 6)$.

Ficedula phønicurus, (Linn.), Boie, Isis, 1822, p. 553; Heitgl. Syst. Uebers. p. $25(1856)$.

Ruticilla sylvestris, Brehm, Vög. Deutschl. p. 363 (1831).*

R. arborea, Brehm, Vög. Deutschl. p. 36.3 ( I $83 \mathrm{I}){ }^{*}$

R. hortensis, Brehm, Vög. Deatschl. p. $3^{6}+$ (1831).* 
Phnenicura muraria, Szains. \& Rich. Faun. Bor.-Am. ii. p. fis) ( $183 \mathrm{I}$ ).

Ficedula ruticilla, Eyton, Cat Brit. B. p. 10 (1836).

Phonicura ruticilla, (Eyton), Gould, B. Eur. ii. pl. 95 (18.37); Swains, Classif. B. ii. p. 240 ( I 837 ).

Ruticilla phonicura, (Linn.), Bp. Comp. List B. Eur. \& N. Amer. p. 15 (1838); Büpp. Syst. Ueber.: p. 57 (1845); Gray', Gen. B. i. p. $180(18+6)$; Bp. Consp. i. p. 296 (1850); Cab. Mus. Hein. i. p. 2 (1850); Moore, P. Z. S. 1854, p. 25; Hartl. Orn. W.-Afr. p. 68 (1857); Jaub. et Barth-Lapomm. Rich. Nrn. p. 230 (1859); Newt. List B. Eur. Btasius, p. Io (1862); Jerd. B. Ind. ii. p. I 36 (1863); Filippi, Viagg. Pers p. 347 (1865); Degl. et Gerbe, Orn. Eur. i. p. $43^{8}$ (1867); Loche, Expl. Sci. Alger., Ois. i. p. 215 (1867); Ciould; B. Gt. Br. ii. pl. li. (r869); Gray, Hand;l. B. i. p. 221. n. ${ }^{\circ} 3153$ (1869); Heugl. Orn. N. O.-Ajr. i. p. 333 (1869); Doderl. Avif. Sicil. 1. I I 5 (1 869); Droste, Vogelw. Borkum, p. 9t (1 869); Harting. Handb. Br. B. p. I 2 (172); Shelley, B. Egoypt, p. 82 (1872); Newt. ed. Yarr. Br. B. i p. 329 (1873); Scvertz. Turkest. Jevotn. p. 65 (1873); Dresser, B. Eur. pt. xxvi. (1864); Irby', B. Gibr. p. 81 (1875); Dresser, Ibis, 1876 , p. 77; Blanf. East. Pers. ii. p. $163($ 1 876$)$; Tacz. Bull. Soc. Zool. France, i. p. 143 (1876); Bogd. B. Canc. p. 95 (1879); Seebohm, Ibis, 1880 , p. 191 id. Ciat. B. 5 p. 336 (1881).

Lusciola phonicurus, (Linn.), Key's. u. Blas. Wirb. Eur. P\}). lviii, 191 (1840; Schl. Rev. Crit. p. xxxi. (1844); Linderm. Vög. Griechenl. p. 105 (1860).

Erithacus phonicurus, (Linn.), Degl. Orn. Eur. i. p. 502 (1849).

Luscinia phoenicurus, (Linn.), Snndtv. Sv. Fogl. p. 59 (1856).

Sylvia (Ruticilla) phænicura, (Linn.), Radde, Rets. Sibir. Vög. p. 254 ( 1863$)$.

Lusciola (Ruticilla) phœnicura, (Linn.), Fritsch, Vög Eur. p. $186($ I 870$)$.

Hab. Europa, Alemania, Africa.

\section{Fam. TIMÉLIDOS.}

Subfam. BRAQUIPODINOS.

\section{Gén. AEGITHinA, Vicil}

I A eg. viridis, (BD.) O 0 N. v. Curibangao.

(M. S. T )

'Turdus scapularis, Raffles, Trans, Linn. Soc. xiii. p. 31і (nec IIorsf.)

Iora viridis, Bp. Consp. i. p. 397 (ex Temm. MSS. in Mus. Latg. $*$ )

1. scrpularis, (nec Hor.s.), Blyth, J. A. S. Beng. xiii. pt. 1, p. $38 \mathrm{I}$; id Cat. B. Mus. As. Soc. p. 214 ; id. Ibis, 1876 , p. so; Salkad. Ucc. Born. p. igo; Sharpe, Ibis, 1877 , p. I4; id. Trans. Linn. Soc. new. series, Zool. i. p. 333; id. P. Z. S. 1879, p. 339. 
Ayithina viridis, Tweedd. Ibis, 1877, p. 304; id. P. Z. S. 1878 , p. 619. Sharpe, Cat. B. M. 6. p. i1. (1881).

A. scapularis, Salvad. Ann. Mus. Civic. Genov. xiv. p, 216. Hab. Paragua, P. Princesa, Borneo, Sumatra.

\section{Gén. CHLOROPSIS, JARd.}

2. Ch. palawanensis, Sharp. 0,0 N. v. Calandinao.

(M. S T)

Phyllornis palawanensis, Sharpe, Trans. Linn, Soc, and series, Zool. vol. i. p. 333, pl. 50. figs. 1, 2 ; Trueeddale, $P . Z$. S. 1878 , p. 619.

(hloropsis palawanensis, Sharpe, Cat, B. M. 6. p, 33. ( $188 \mathrm{I})$.

Hab. Paragua, P. Princesa,

2 Ch. flavipennis, Tw. $\sigma^{\prime}$ N. v. Calindao.

Phyllornis flaoipennis, Tweedd. P. Z. S. 1877, p. 76 I, pl. Ixxvii. fig. 1 .

Chloropsis flavipennis, Sharp. Cat, B. M. 6. p, 34. (1881), Hab. Cebú.

3. Ch. nigricollis, (Vicill.) $0^{\prime} \mathrm{N}$ v.

(M. S. T.)

Le Verdin de la Cochinchina, Daub. Pl. Enl. 643, f. $3 ; B u f f$. H. Nat. Ois. III, p. 392,

Turdus cochinchinensis, Gm. S. N. i. p. 825 (ex Daub); Audeb, et Vieill. Ois. Dor, ii, pls, 77, 78,

Philemon nigricollis, Vieill, Now. Dict. XXVII, p, 432,

Pyllornis cochinchinensis, Tem. Pl, Col. 484, f, 2; Less. Rev. Zool. 1840 , p. I64; Gray, Gen. B. i. p, 124; Bp. Consp. i. p. 396 (pt); Gray, Hand $=l$. B. i. p. 277, n. 4080 ; Wald. Ibis, 1871 , p. 169.

(hloropsis nigricollis, Nichols, Ibis, 1881 , p. 148; Sharpe. Cat. B. Mus. 6. p. 27. (1881).

Hab. Java.

3 Gén. IXOCINCLA, Blyth.

4. I. crassirostris, E. Newt. N. v.

Hypsipetes crassirostris, E. Nezwt, P. Z. S. 1867 , p. 334; id. Ibis, 1867 , p. 334. Gray, Hand-l. B. i. D. 273, n. $3996 ; A$. Newt. in Rowley's Orn. Misc. ii. p. 52, pl. xlii. figs. 4a, $4 b$; Oustal. Bull. Soc. Philom. ; (7) i. p. Ior; Hartl. Vög. Madag. pp. I37, 409; Oustal. Bull. Soc. Philom. 1878, p. 172. Ixocincla crassirostris, Sharpe, Cat. B. M. 6. p. 47. (1881).

Hab. Marianas.

4 Gén. IOle, Blyth.

5. I. olivacea, Blyth. $070 \mathrm{~N}$ v.

(M. S. T.)

Iole olivacea, Blyı h, J. A. S. Beng. xiii. p. 386, xiv. p. 573; 
id. Cat. B. Mus. As. Soc. p. 207; Bp. Corsp. i. p. 261; Horsf. \& Moore, Cat. B. Mus. E. I. Co. i. p. 254; Salvad. Ucc. Born. p. 210; Sharpe, Ibis, 1876, p. 4o; id, Cat. B. M. 6 . p. 55. (1881). Tueedd. Ibis, 1877 p. 307.

('riniger, sp., Gray, Gen. B. i. p. 236, sp. 10.

C. charlotta, Finsch. $J . f . O .1867$, p. 19; Gray, Hand $l . B$. i. p. 274, no. 4033; Giebel, Thes. Orn. i. p. 811.

'Trichophorus brunnescens, S, Mïll. itl Mus, Lugd*; Fïrusch, t. $c$. p. 19; Blyth, Ibis, 1870 , p. 170.

Criniger olivaceus, Gray, Handsl. B. i. p. 275, no. 4048 (nec Srwains.)

Hab. Luzon, Laguna, Bataan, Orion; Borneo, Malaca.

6 I. everetti, Tw. $\sigma \circ \mathrm{N}$. v.

(M. S. T.)

Criniger everetti, Tweedd. Ann. \& Mag. Nat. Hist: (4) xx p. 535; id. P. Z. S. 1877 , p. 827, pl. Ixxxiv., et 1878, p. I10. Sharpe, Cat. B. M. p. 57 . (1881).

Hab. Panaon, Dinagat, Samar, Paranas, Leyte, Surigao, Mindanao.

7. I. rufigularis, Sharpe. $\sigma \mathrm{N}$. v.

Hypsipetes rufigularis, Sharpe, Tr. Lin. Soc. Zool. 1. p. 335; id. Cat. B. M. 6. p. 57. (1881); Tw. P. Z. S. 1877, p. 546, y 1878 , p. 949 .

Hab. Mindanao, Basilan, Malamaui, Pasananca, Zamboanga.

8. 1. philippinensis, Montb. 0 . N. v.

(M. S. T.)

La petite Grive des Philippines, Montb. Hist. Nat. Ois. iii. p. 316. Philippine Thrush, Lath. Gen. Syn. ii. part I, p. 38 .

Turdus philippensis, Gim. Syst. Nat. i. p. 814 (ex Montb.)

Galgulus philippensis, Kittl. Kupf. Vög. p. 8, 'Taf. I2. fig. 2.

Hypsipetes philippensis, Strikl. Ann. Nat. Hist. xiii. p. 41 3; Gray, Gen. B. i. p. $238 ; B p$. Consp. i. p. $26_{1} ;$ vou Martens, $J . f . O$. 1866, p. 13; Walden, Trans. Zool. Soc. ix. p. 172; Sharpe, Trans. Linn. Soc. nexo series, Zool. i. p. 335; Tweedd. $P$. Z. k. 1877, pp. $546,696,76$ I, 827 , et 1878, pp. $285,242,381$.

Philedon gularis, Cuv. Mus. Paris; Pucher. Arch. Mus. vii. p. 344, pl. I 8 .

Microscelis philippensis, Gray, Gen. B. i. p. 235; Bp. Consp. i. p. 263; von Martens, J. f. O. 1866, p. i 3 ; Gray, Hand-l. $B$, i. p. 268 , no. 3917 .

Hypsepetes gularis, Gray, Hand-l. B. i. p. 272, no. 3992.

I. philippensis, Sharpe Cat. B. M. 6. p. 58 (1881).

Hab. Luzon, Lagana, Morong, Cagayan, Guimarás, Negros, Leyte, Samar, Borongan, Panaon, Cebú. Mindanao, Bohol, Marinduque.

8. I. guimarasensis, Steere. N. v.

Hab. Negros, Panay, Guimarás. 
9. I mindorensis, Steere. N. v.

Hab. Mindoro.

io. I. siquijorensis, Gm. N. v.

Hab. Siquijor.

5. Gén. poltolorhus, Sharpe

I P urostictus, Salv. $\sigma \rho$ N. v.

(M. S. T.)

Brachypus urostictus, Salvad. Atti R. Accad. Torino, v. p. 509.

Ixus? urostictus, Wald. Trans. Zool. Soc. ix p. I9I, pl. xxxii, fig. 2. Poliolophus urostictus, Sharpe, Trans. Linn. Scc. neze series, Zool. i. p. 33t; id. Cat. B. M. 6. p. 63(1881); Tweedd. P. Z. S. 1877 , p. 827, I 878 , pp. I1 $10,380,949$.

Hab. Luzon, Laguna, Negros, Panaon, Dinagat, Mindanao; Surigao.

12. P. basilanicus, Steere. N. v.

Hab. Mindanao, Basilan, Java, Sumatra, Borneo.

6. Gén. MICR OPUS, Swain.

13. M. melanocepialus, Gm. $\sigma \circ \mathrm{N}$. v.

(M. S. T.)

Black-headed Shrike, Lath. Gen. Syn. i. pt. i. p. 165, pl. vi.

Lanius melanocephalus, Gm. Syst. Nat. i. p. 309.

'Turdus melanocephalus, Rafl. Trans. Linn. Soc. xiii. p. 3 Iо.

T. atriceps, Temm. Pl. Col. 147.

Ixos atriceps, Temm. Tabl. Méth. Pl. Col. p. 15 .

Brachypodius melanocephalus, Blyth, J. A. S. Beng. xiv. p. 576; id. Cat. B. Mus. As. Soc. p, 211 ; Horfs. \& Moore, Cat. B. Mus. E. I. Co. i. p. 248; Moore, P. Z. S. 1854 , p. 278; Salzad. Ucc. Born. p. 201; Blyth. \& Wald. B. Burm. p. I 36 ; Armstr. S. F. 1876 , p. 324; Tweedd. Ibis, 1877 , p. 307; id. P. Z. S. 1878 , p. 618; Hume, Str. F. 1878, p. 318, 1879, p. 98; Sharpe, P. Z. S. 1879 , p. 339; Salvad. Ann. Mus. Civic. Genov. xiv. p. $22 \mathrm{I}$.

Ixos metallicus, Eyton. Ann. Nat. Hist. xvi. p. 228.

Pycnonotus melanocephalus, Strickl. P. Z. S. 1846 , p. ıо ; Gray, Gen. B. i. p. 237 ; id. Hand-l. B. i. p. 270 , no. 3972.

P. metallicus, Gray, Gen. B. i. p. 237 ; id. Hand-l. B. i. p. 271 , no. 3973 .

Brachypus melanocephalus, $B p$. Consp. i. p. 264.

Prosecusa melanocephala, Cab. Mus. Hein. Th. i. p. IIo.

Ixus melanocephalus, Schl. Handl Dierk. i. p. 272.

Brachypodius immaculatus, Sharpe, Ibis, 1876 , p. 39, I 877 , p. I4.

Micropus melanocephalus, Skarpe, Cat. B. Mus. 6. p. 65 (1881).

Hab. Paragua, P. Princesa; Sumatra, Java, Borneo, Malaca. 
 \\ 7 Gén. Criniger, Tem.}

14. C. frater, Shupp: $\sigma \circ \mathrm{N}$ v. Piroc-piroc.

(M. S. T.)

(riniger frater, Sharpe, Tr. Linn. Soc. 2nd. series, Zjol. vol. i. p. $33 \mathrm{t}$; id. Cat. B. M. 6. p. 79 lam. v. (1881). Trueedd. P. Z. S. 1878 , p. 619 .

Hab. Paragua, P. Princesa, Balabac.

15. C. palawanensis, Tu. $\sigma \rho \mathrm{N}$ v.

(M. S. T.)

C. palawanensis, Tweedl. P. Z. S. 1878, p. 6r8; Sharpe, Cal. B. M. 6. p. 83 lain. 6 fig. 2 (1881).

Hab. Paragua, P. Princesa.

8. Gén. PYCNONOTUS, Bole.

16. Pyc. goiavier, $(S c o p$.$) \& \& N v. Guayabero.$

(M. S. T.)

Petit Goiavier de Manille, Sonn. Voy. Nouv. Guin. p. 59, pl. 28.

Guava Flycatcher, Lath. Gen. Syn. ii. pt. I, 1. 335.

Muscicapa goiavier, Scop. Del. Faun. et Flor. Insubr. ii. p. $9^{6}$ (ex Sonn.)

M. psdii, Gm. Syst. Nat. i. p. 94 I (ex Sonn.)

Pycnonotus goia.ier, Gray, Gen. B. i. p. 237; id. Hand-l. B. i. p. 269. uo. 3942; Sharpe, Cat. B. M. 6. p. I4I (1881).

Ladorusa goiavier, Cab. Mus. Hein. i. p. Iog.

Ixus goiavier, Wald. Tr. Z. S. ix. p. i90; Trueedd. P. Z. S. 1877 , pp. $546,695,8: 7,1878$, pp. 110, 285, 342, 710, 949. Hab. Luzon, Manila, Navotas, San Mateo, Montalban, Laguna, Cal:uan, Calamba, Cagayan, Leyte, Bohol, Negros, Nipah, Amparo, Mindanao, Surigao, Zamboanga, Pasananca, Samar, Basilan. Guimarás, Marinduque.

17. Pyc sinensis, $(G m$ ) to N. v Cul-lac.

(M. S. T.)

Le Gobe-mouches verdâtre de la Chine, Scop. Voy. Ind. Orient. ii p. 197 .

Wreathed Flycatchor, Lath. Gen Syn. ii. part I, p. 336 .

Muscicapa, sinensis, Gm. Syst. Nat. i. p. 942.

'I'urdus occipitalis, Less. Trrité, p. 4 10 (descr. nullâ); Eyd. \& Gervais, Mag. de Zool. 1836, pl. 66; iid. Voy. Favorite, Zool. p. 36, pl. I 4 .

Prenonotus sinensis, Blyth, J. A. S. Beng. xiv. p. 569; id. Cat. B. Mus. As. Soc. p. 210; Gray, Hand-l. B. i. p. 270, no. 3948. Sharpe, Cat. B. M. 6. p. I 49 (1881).

P. occipitalis, Gray, Gen. B. i. p. 237; Horsf. \& Moore, Cat. B. Mus. E. I. Co. i. p. 246.

Ixus stnensis, Bp. Consp. i. p. 266; Cass. in Perry's Exped. Japan, ii. p. 24I; Swinh. Ibis, 1863 , p. 289; id. P. Z. S. 1863 , p. 278; 2., P. Z. S. 1871 , p. 369; Wald. Tr. Z. S. 191; David. \& Oustalet, Ois. Chine, p. r40.

Hab. Luzon, Laguna, Cagayan. China, Formosa. 
18. Pyc. cinaroifrons, Tzu. $\sigma \rho \mathrm{N}$. v.

(II S. T.)

Brachypus cinereifrons, Twzed. P. Z. S. 1879. p. 617.

Pycnonotus cinereifrons, Sharp?, Cat. B. M. 6. p. I53 (1831). Hab. Paragua, P. Princesa.

Subfain. IRENINOS.

I. Gén. IRENA, Horsf.

1). 1. cyanogastra Vigors. $0^{\circ} . \mathrm{N}$. v. Bella irane.

(M. S. T.)

Irena cyanogastra, Vigors, P. Z. S. 1831 , p. 97; Gray \& M tch. Gen. B. i. p. 288, pl. 70; Bp. Consp. i. p. 349; Cass. U.S. Expl. Exp., Aves, p. 143; Gray, Hand-l. B. i. p. 288; W.t!den, Tr. $Z$. S. ix. p. I9o; Sharpe, Cat. B. iii. p. 267; id. Tr. Linn. Soc. nev series, Zsol. i. p. 352 ; id. Cat. B. M. G. p. I75 (188I); Gould, B. Asia, part xxxii.

Hab. Luzon, Laguna, M ınila, Paray.

20. 1. melanocilumys, Sharpe. ot. $\mathrm{N}$. v. id.

(M S. T.)

Irena melanochlamys, Sharpe, Cat. B. iii. p. 255 ; id. Truns. linn. Soc. new series, i. pp. 33t, 352; id. Cat. B. M. 6. p. I7t) (1881); Treed. P. Z. S. 1879, p. $7 \mathrm{I}$.

Hab. Basilan.

21. I. turcosa, Horsf. $0^{\infty} \bigcirc$ N. v. Dalaga-irene.

(M S. T.)

Irena puella, Horsf. in Linn. Trans. xiii. p. r 53; id. Zool. Research. in Java, Birds. pls. I 3, I 4; Steph. G:n. Zoo!. xiii. pt. 2,. p. I42, pl. 48; Gray, Gen. B. i: p. 283; Cab. Mus. Hein. 'Th. i. p. I Io.

Edolius puellus, Temm. Pl. Col. pls. 70, 476, 225.

Irena turcosa, Walden, Ann. N. $H$. (4) v. p. 417; Shzop?, Ibis, 1876, p. 44 ; id. Ca'. B. iii. p. 267; id. Cat. B. M. 6. p. г7i (1881); Nichols. Ibis, 1881 , p. 147.

Hab. Luzon, Laguna, Calauan; Java.

22. I. ellø, Steere. N. v.

Hab. Samar, Leyte.

23. I. twoeddalii, Sharpe. $0^{\prime} \bigcirc \mathrm{N}$. v. Dulipdipan.

(M. S. T)

Irena tweeddalii, Sharpe, Cat. B. iii. p. 258; id. Trans. Linn. Soc. new series, pp. 333, 352; id. Cat. B. M. 6. p. I 78 ( $\left.188_{1}\right)$; Wardlaw-Ramsay, Orn. Memoirs of M.x. 'quis of Tivedllale, p. 657 (1 88x.)

Hab. Balabac, Paragua, P. Princesa.

24. I. paraguanensis, $\sigma \circ$ N. v. Dxlip-dipan.

(M. S. T.)

Hab. Paragua, P. Princesa. 
Subfam. TROCiLODINOS.

1. Gén. CAMpylorhychius, sirix.

25. C. zonatus, Liss. $\sigma$ N. v.

(AI. S ' T.)

Picolaptes zonatus, Less. Cent. Zool. pl. 70; Lafr. Rev. Zool. $184^{6}$, p. 94.

(ampylorhynchus zonatus, Gray, Gen. B. i. p. 159; Bp. Consp. i. p. 223; Cab. Mus. Hein. Th. i. p. 80; Scl. Pr. Philad. Acad. 1856 , p. $364 ; 7 d$. P. Z. S. 1856, p. 290, 1859, p. 363; Scl. \& Salv. Ibis, 1860 , p. 29; Scl. Cat. Amer. B. p. 17; Baird, Review Amer. A. p. ro4; Gray, Hand-l. B. i. p. 192, n." 2636; Lawer. Ann. Lyc. N. Y. ix. p. 92; Sumichr. Mem. Bost. Soc. N. H. ii. p. $545 ; \mathrm{Scl}$. \& Salv. Nomencl. Av. Neotr. 1. 5 ; Lazer. Bull. U. S. Nat. Mus. n. ${ }^{\circ}$ iv. p. 13; Boucard, P. Z. S. 1878, p. 51 ; Salv. \& Godm. Biol. Centr.-Amer., Aves. i. p. 68; Sharpe, Cat. B. M. 6. p. 195 (1881).

(. nigriceps, Sclater, P. Z. S. 1860 , p. $46 \mathrm{r}$; id. Cat. Aver. B. p. I 8; Baird, Review Amer. B. p. Io9; Gray', Hand-l. B. i. p. $193, \mathrm{n}^{\circ}{ }^{\circ} 2654$.

Hab. América, Guatemala.

2 Gén thryothorus, Vicill.

26. Th. maculipectus, $\sigma \circ \mathrm{N} . \mathrm{v}$.

(M. S. T.)

Thryothorus maculipectus, Lafr. Rev. Zool 1845, p. 338; Sclater, P. Z. S. 1856 , p. 290, 1859, pp. 363, 372; Slc. \& Salv. Ibis, 1860 , p. 30; Scl. Cat. Amer. B. p. 21 ; Sel. \& Salv. P. Z. S. 1870, p. 836; iid. Nomencl. Av. Neotr. p. 7; Lawr. Bnll. U. S. Nat. Mus. no. 4, p. i 3; Salv. \& Godm. Biol. Cent.-Amer., Aves, i. p. 92; Sharpe, Cat. B. M. 6. p. 237 (188r).

Pheugopedius maculipectus, Baird; Review Amer. B. p. 1 35; Sumichr. Mem. Bost. Soc. N. H. i. p. 545 .

Troglodytes maculipectus, Gray, Hand-l. ${ }_{B}$. i. p. 189, n. $^{0} 2587$. Hab. América, Guatemala.

\section{Gén HeNiCORHINA, ScL.}

27 H. loucosticta, Licht. $\sigma$ N. v.

(M. S. T.)

Troglodytes leucostictus, Licht. in Mus. Berol., undè.

Cyphorhinus leucostictus, Cab. Arch. f. Naturg. xiii. pt. i. 1847, p. 206; Bp. Consp. i. p. 224; Sclater, P. Z. S. 1858, p. 63; Gray, Hand-l. B. i. p. 193, no. 267 I. Sharpe, Cat. B. M. 6. p. 287 ( I $88 \mathrm{I}$.

Heterorhina leucosticta, (pt.) Baird, Review Amer. B. p. I 7 . Hab. Colombia, Ecuador, Guatemala. 
Subfam. 'TIMELINOS.

1. Gen. Thamnobia, Siwa.

28. T. fulicata, (Lin.) $\sigma \circ$ N. v. Cipao.

(11. S. 'T.

1.e 'Traquet des Philippines, Bris. Orn. iii. p. 4tt, pl. xxxiii. fig. 2 (1760); Buff. Pl. Enl. vi. pl. 185. fig. I.

Motacilla fulicata, Linn. Sys. Nat. i. p. 366 (1766, ex Briss.) Le 'Traquet à queue striée, Levaill. Ois. d'Afr. iv. p. i I1, pl. 188. fig. I* (1805); Sundew. Krit. Framst., Levall. p. 45 (1857).

(Enanthe fulicata, Vieill. N. Dict. d' Hist Nat. xxi. p. 435 (1818).

(E. ptygmatura, Vieill. t. c. p. 436 (1818, ex Levxill.)

Thamnobia rufiventer, Swains. Falun. Bor.-Amer., Bird, p. $4^{8,}$ (1831, ex Levaill.)

Ixos fulicatus, Sykes, P. Z. S. 1832, p. 89.

Tha:nnobia fulicata, Jerd. Madr. Journ. x. p. 264 (1839); Gray', Gen. B. i. p. 185 (1845); Blyth, J. A. S. Beng. xvi. p. 139 (1847); id. Cat. B. Mus. As. Soc. p. 165 (1849); Bp. Consp. i. p. 298 (1850); Layard, Ann. \& Mag. Nat. Hist. (2) xii. p. 266 (1853); Burgess. P. Z.S. 1854 , p. 142; Horsf. \& Moore. Cat. B. E.I. Co. Mus. i. p. 281 (1854); Jerd. B. Ind. ii. l. 121 (1863); Gray, Hand-l. B. i. p. 211. n. 2988 (1869); Holdsw P. Z. S. 1872, p. 454; Hume, Str. F. 1877, p. 307 (1873); Fairb. Str. F. 1876, P. 459; Hume, Str. F. 1877 , p. 406; id. Str. F. 1878 , vol. ii. p. 55; Ball. t. c. p. 216 ; Legge, B. Ceylon, p. 440 (1879); Hume, Str. F. 1879, p. 99. Sharpe, Cat. B. M. 7. p. 54 (1883).

Hab. Luzón, Laguna, India, Ceilán.

2. Gén. COpSychUS, Wagi.

29. C. mindanensis, (Gm.) $\sigma^{\circ} 0 \mathrm{~N}$ v. Maria-capra.

(II. S. T.)

Le Merle de Mindanao, Daubent. Pl. Enl. 627. fig. I; Montb. H. N. Ois. iii. p. 387 .

Turdus mindanensis, Gm. S. N. i. p. 283 (ex Moutb.)

Copsychus mindanensis, Sund. Krit Framst., Levaill. p. 36; $v$. Martens. J. f. O. 1866 , p. 10; Walden \& Layard, Ibis, 1872, p. IO2; Walden. Tr. Z. S. ix. p. 194, pl. xxxiii fig. I (1875); Sharpe, Trans. Linn. Soc. new ser., Zool. i. p. 352 (1877); Tweedd. P. Z. S. 1877 , pp. 695, 761; 828, 1878, pp. 285 , 342, 994, 1879 p. 72; Wardlaze Ramsay', Orn. Works Trueedd. p. 656, (188I); Sharpe, Cat. B. Mus. 7. p. 6o, (1883).

Hab. Luzón, Manila, Montalbán, S. Mateo, Laguna, Calamba, Los Baños, Calauan, Guimaras, Negros, Cebú, Mindanao,
Basilan.

3o. C. seychellarum, $A$ N $N_{\mathrm{c}}$ wt. N. v.

Copsychus seychellarum, $A$. Newton, Ibis, 1865, p. 332, pl. 8; E. Newton, Ibis, 1867 , pp. 337, 352; Grav, Hand-l. B. i. p. 266, n. 3898 (1869); Sharpe, Cat. Afr. B. p. 28 (1871); Hartl. 


\section{$-139-$}

lög Madag. p. 132 (187j); Sarphe, Cat. B. Mus. 7. p. 61 (188.). Hab. Marianas.

31. C. cebuensig, Stiere. N. v.

Hab. Cebú.

\section{Gén Cittocincla, Scrat.}

32. C. nigra, Sharpe. o". N. v. Mananataon.

(M S. 'r.)

Cittocincla nigra, Sharpe, Trans. Linn. Scc. Zool. new series; i. p. 335, pl. lii. (1876); Tweedd. P. Z. S. 1878. p. 619, Wardlaw Ramsay, Orn. Mein. Tuieedd. pp. 614, 657. (1881); Sharpe, Cat. B. Mus. 7. p. 90. (1883).

Hab. Paragua, P. Princesa.

33. C. Luzoniensis, Kittl. $O^{\pi}$ N. v Mananatdon.

(M. S. T)

Turdus luzoniensis, Kittl. Kupf. Vög. p. 7, pl. Ix. fig. 2(1832); id. Mém. Acad. S. Petersb. ii. p. 5, pl. 7 (1833).

Copsychus luzoniensis, $B p$. Consp. i. p. 266 (1850); Sclater. $P$. Z. S. 1861 , p. 186; Gray. Hand-l. B. i. p. 265, no, 3887 (1869).

Cossypha pyrrhopygia, Hartl. Orn. Westafr. p. 78 (1857); id. J. f. O. 1861 , p. I63; Sharpe, Ann. \& Mag. Nat. Hist. (4) xvi. p. $236(1875)$.

Bes-onornis pyrrhopygia, Gray, Hand-l. B. i. p. 265, n. ${ }^{\circ} 3872$ (1 869).

Cittocincla luzoniensis, Waldeu, Trans. Zool. Soc. ix. pp. 193, 250 (1875); Sharpe, Trans. Linn. Soc., Zool. new series, i. p. 352 (1876); Wardlaw Ransay, Orn. Mem. Trveedd. pp. 358, 411, 647; (1881); Sharte, Cat. B. M. 7. p. 91. (1883)

Hab. Luzón, Laguna, Calau. n, Calamba.

4. Gén. PSAMA'IHIA, H. ev F.

34. Ps. annæ; $H$. el $F$ N. v.

Psamathia annæ, Hartl. \& Finsch, P. Z. S. 1868 , pp. 5, 118, pl. ii., 1872, p. 94; Finsch, Journ. Mus. Godeffr. Heft v. p. 22 (1875); Sharpe, Cat. B. M. 7. p. Ior. (1883).

Calamodyta annx, Gray, Hand-l. B. i. p. 208, n. 2949 (1869). Hab. I. Palaos, Marianas.

5 Gén. Bebrornis, Sharp.

35. B seychellensis Oust. N. v.

Ellisia seychellensis, Oustalet, Bull. Soc. Philom, Paris. (7) i. p. 102 (187).

Bebrurnis scychellensis, Sharpe, Cat. B. M. 7. p. 103 (1883). Hab. Marianas. 


\section{Gén. MEGALURUS, Horsf.}

36. M. palustrie, Horsf. $\sigma \circ$ N. v. Sunut-calabao.

(M. S. T.)

Megalurus palustris, Horsf. Trans. Linn. Soc, xiii. p. I 59 ( 1820$)$; Gray, Gen. B. i. p. 169 (1845); Blyth, Cat. B. Mus. As. Soc. p. I 39 (1849); Bp, Consp, i, p. 279 (1850); Cab. Mus. Hein. Th. i. p. 45 (1850); Horsf. \& Moore, Cat. B. E. I. Co. Mus. i. p. 330 (1854); Jerd. B. Ind. ii. p. 70 (1863); Blyth, Ibis, 18655 ; p. 30; Gray, Hand-l. B. i. p. 205, n. 2903 (1869); Blanf. Ibis, 1870, p. 467 ; Hume, Nests \& Eggs Ind. B. p. 276 (1873); id. Str. F. 1874, p. 476 ; id. Oates, Str. F. 1875, p. 124; Walden, Tr. Zool. Soc. ix. pp. 189, 250 (1875); Blyth\& Wald. B. Burm. p. I 8 (1875); Ball. Str. F. 1876, p. 233; Godwin. Austen, J. A. S. Beng. xlv. p. 78 (1876); Sharpe, Trans. Linn. Soc, new series, Zool. i. p. 352 (1876); Oates, Str. F. 1877, p. 156; Twoedd. P. Z. S. 1877 , p. 694; Anderson, Zool. Exped. Yun-nan, Aves, p. 639 '1878); Ball, Str. F. 1878 . vol. ii. p. 214; Hume, Str. F. 1879, p. 97; Inglis, Str. F. 1880 , p. 253; Wardlaw Ramsay. Trveedd. Oru. Works, p. 657 (1881); Oates, B. Brit. Burm. p. 106 (1883). Sharpe, Cat. Is'. M. 7. p. I 23 (1 883 ).

Malurus marginalis, Temm. Pl. Col. iii. pl. lxv. fig.. 2 (1823, ex Reinw. MSS.); Kittl. Voy. Lütke (Postels). Bd. iii. p. 326 .

M. citrinus, Gray \& Mitch. Gen. B. i. p. 169, pl, 48 (1838); Bp. Consp. i. p. 370 (1850); Gray, Hand-l. B. i. p. 206, n. ${ }^{\circ} 2906$ (1869).

Hab. Luzón, Manila, Nabotas, Laguna, Calauan, Samar, Marinduque, Mindoro, Baco.

37 M ruficeps, $T w . \sigma$ N. v. Tulturioc.

(M. S. T.)

Megalurus ruficeps, Treeedd. Ann. \& Mag. Nat. Hist. (4) xx. p. 95 (1877); id. P. Z. S. 1877 , pp. 687, 695. pl. 72; id. P. Z. S. 1877 , pp. $756,76 \mathrm{r} ;$ id. P. Z. S. 1878, p. 285 , 1879, p. 71; Wardlaw Ramsay in Tweedd. Orn. Works. p. 657 (1881); Sharpe, Cat. B. M. 7. p. I 25 (1883).

Hab. Luzón, Cebú, Negros, Valencia, Basilan, Guimaras, Panáy, Bohol, Marinduque, Mindoro, Baco.

7. Gén. SUTORIA, NichoI

38. S sepium, Raff. $\sigma \mathrm{N}, \mathrm{v}$ Pipit-callasan.

(M. S. 'T.) .

Motacilla sepium, Raff. Tr. Linn. Soc. xiii. p. 313 (1820, nec Horsf.).

Edela ruficeps, Less. Cent. Zool. p. 212, pl. 7 I (nec Less. Traite, p. 309).

Orthotomus edela, Temm. Pl. Col. v. pl. 599. fig. 2 (1836); Gray, Gen. B. i. p. $162(1848)$; Blyth, Cat. B. Mus. As. Soc. p. 144 (1849); Bp. Consp. i. p. 282 (1850; Moore, P.Z. S. 1854, p. 79; Horsf. \& Mocre, Cat. B. E. I. Co. Mus i. p. 314 
(1854); Gray, Hand-1. B. i. p. 195, n." 2183 (1869'; Wrt!den. in Blyth's B. Burm. p. 120 (1875); Sharpe, Ihis, 13577. 1. 11. Sutoria edela, Sharpe, Cat, B.M. 7, p. 218 (1883).

Hab. Luzón, Java.

\section{Gèn. ORTHOTOMUS, HORSk.}

39. O. frontalis, Sharpe. ơ N. v. Pipit.

(M. S. T.)

Orthotomus frontalis, Sharpe. Ibis. 1877, p. 112, pl. ii. fig. 1; id. Trans. Linn. Soc. new series, Zool. i. p. 336 (1877); Tzeedd. P. Z. S. 1877 , p. 828, 1878, pp. 11, 710, 950; sharpe, Cat. B. M. 7. p. 220 (1883).

Hab. Bohol, Dinagat, Basilan, Mindanao, Zamboanga,

40. O. cinereiceps, Sharp. N. v Pipit.

(M. S. T.)

Orthotomus cinereiceps, Sharpe, Ibis, 1877 , p. I13, pl. ii. fix. 2; id. Tr. Linn. Soc. new series, Zool. i. pp. 337, 352 (1877); Sharpe, Cat. B. M. 7. p. 222 (1883).

Hab. Basilan, Mindanao.

41. O. nigriceps, $T z w . \sigma \rho$ N. v Pipit.

(M. S. T.)

Orthotomus nigriceps, Trveedd. P. Z. S. 1877, p. 828, pl. 1xxxv.; Wardlaw Ramsay, Orn. Works Treeedd. pp. 554, 658 (1881); Sharpe, Cat. B. M. 7. p. 222 (1883).

Hab. Mindanao.

42. O. castaneiceps, Wald. $\checkmark 0 \mathrm{~N}$. v. Iipit-cauayan.

(M. S. T)

Orthothomus castaneiceps, Walden, Ann. \& Mag. N. H. (4) x. p. 252 (1872); id. Tr. Zool. Soc. ix. p. 195 (1875); Sharpe, Ibis, 1877 , p. I14; id. Tr. Linn. Soc. new series, Zool. i. pp. 337, 342 (1877); Tweend. P. Z. S. 1877 , p. 546, 1878 , p. 286; Sharpe Cat. B. M. 7. p. 223. (1883).

Hab. Panáy, Iloilo, Guimaras, Negros, Valencia.

43. O. derbianus, Moore. $\sigma$ N. v Pipit-cauayan.

(M S. T.)

Orthotomus derbianus, Monre P. Z. S. 1854 , p. 309, pl. Ixxvi.; Gray, Hand-l. B. i. p. 195, n. ${ }^{2} 2690$ (1869); Walden; Tr. $Z$. S. ix. p. 195 (1875); Sharpe, lbis, 1877 , p. 114; Treedd. P. Z. S. 1877 , p. 697; Sharpe, Cat. B. M. 7. p. 224 (1883). Hab. Luzón, Manila.

44. O. ruficeps, Less. $\mathrm{O}^{\prime} \bigcirc \mathrm{N}$. v. Uit-uit.

(M. S. T.)

Edela ruficeps, Lesson, Traité d'Orn. p. 309 (1831, nec Less. Cent. Zool. pl. 71).

Orthotomus sericeus, Temm. Pl. Col. texte to livr. IoI (1836); Gray, Gen. B. i. p. $162(9848)$; Bp. Consp. i. p. 1082 (1850).

o. edela, Blyth, Cat. B. Mus. As. Soc. p. 144 (1849). 
o. ruficeps, Moore, P. Z. S. 1854 , p. 79; Horsf. \& Moore, ('a'. Moore, Cat. B. E. I. Co. Mus. i. p. 316 (1854); Gray, Hant-l. B. i. p. 195, n. 2685 (1869); Salvad. Ucc. Born. p. 248 (1874); Sharpe, Ibis, 1877 , p. I I 4 ; id. Tr. Lisn. Soc. new series, Zool. i. pp. 337, 352 (1877); Truedd. P. Z. S. 1878, p. 619; Hume \& Davison, Stu. F. 1878 , vol. vi. p. 346; Hume, Str. F. 1879 , pp. 94, 108; Sharpe P. Z. S. 1879 , p. 341, 1881, p. 798; id. Cat. B. Mius. 7. p. 224 (1883).

Hab. Paragua, P. Princesa, Borneo, Sumatra, Malaca.

45. O. cineraceus. Blyth. \& N. v Pipit.

(M. S. T.)

(Ohthotomus sepium, Lafr. Mag. de Zool. 1836 , pl. (no Horsf).

Or. cineraceus, Blyth, J. A. S. Beng. xiv. p. 489 (1845); id. Cat. B. Mus. As. Soc. p. I 44 (1849); Bp. Consp. i. p. 282 (I 850$)$; Moore, P. Z. S. 1854 , p. 80; Horsf. et Moore Cat. B. E. 1. Co. Mus. i. p. 315 1854); Scl. P. Z. S. 1863 , p. 215. Gray, Hant-l. B. i. p. I95, n. 2692 (1869); Salv. Ucc. Born. p. 248 (1874); Sharpe, Ibis; 1877 , p. 1 I4; Tzeeed. t. c. p. 312; Hume, Str. F. 1879, p. 64; Sharpe, P.Z.S. 1879, p. 34r; I88I, p. 798; id. Cat. B. Mus. 7. p. 225. I 883.

Or. borneonensis, Salv. Ucc. Born. p. 247 (1874); Sharpe, Ibis, 1876, p. 4I. pl. ii. fig. I; Salv. Ann. Mnis. Civic. Genor, xiv. 1). $235($ I 879$)$.

Hab. Cayayan de Joló; Borneo, Malaca, Sumatra.

46. 0 panayensis, Stesre $\mathrm{N}$ v.

Hab. Panáy.

47. O. Samarensis, Steere. N v.

Hab. Samar.

9. Gén. Cisticola, Kaup.

48. C. cisticola, Vicill \& N. v. Pipit tubigan.

(M. S. T.)

La Fauvette cisticole, Vieill. Faune Franc. p. 27, pl. 102. fig. I ( 1820$)$.

Sylvia cisticola, Temm. Man. d'Orn. i. p. 228 (1820); id. Pl. Col. vi. fig. fig. 3 (1820), id. Man. d'Orn. iii. p. I23; Von der Mühle, Monogr. Europ. Sylv. p. I 22 (1856).

Prinia cursitans, Frankl. P. Z. S. 1831 , p. I I8; Jerd. Madr. Journ. xi. p. 5 (184o); id. Ill. Ind. Orn. pl. vi. (1847).

Gysticola cisticola, Less. Traité p. 4I5 (1831); Licht, Nomencl. p. 31 (1854); Sharpe, Cat. B. M. 7. p. 259 (1883).

Sylvia (Cisticola) typus, Rüpp Neue Wirb. Vög. p. I.13 (1835)-40).

Drymoica cisticola, Swains. Classif. B. ii. p. 242 (1837); Gray', Gen. B. i. p. $164(1848)$.

Salicaria cisticola, Gould. B. Enr. 11. I1 3 (1837); Keys. u. Blas. Wirb. Eur. p. lv. (1840); Schl. Rev. Crit. p. xxx (1844); Linderm. Vög. Griechenl. p. 95 (1869).

(isticola schonicola, Bp. Comp. L st. B. Eur. \& N. Amer. p. 12 
(1838); Blyth. Cat. B. Mus. As. Soc. p. I 45 (18+9); Bp. Cousp. i. p. 286 (1850); Cab. Mus. Hein. Th. i. p. 44 (1850); Hougl. Syst. Ucbers. p. 21 (1856); Tristr. Ibis, 1859, p. I60; Homeyer, J. f. 0.1862 p. 283 ; Jerit. B. Int. ii. p. 174 (1303); Szilinh. Ibis, 1863 , p. 303; Giglioli, Ibis, 180.5, p. 52; Litr:\%, Bull. Soc. Orn. Suisse, i. p. 10 (1865); Blyl/h, Ibis, 1850 . p. 23; Loche, Expl. Sci. Alger, Ois. i. p. 281 (1867); Degl. of Cierbe, Orn. Eur. i. p. 537 (1867); A. C. Smith. Ibis, 1868, p. +tl; Broocks, t. c. p. I 30; Hume, Ib.s, 1870, p. 136; Swinh. t. c. p. 345; Sharpe, t. c. p. 475; Gurney, Ibis, 1871, p. 83; Swinh. P. Z. S. 1871 , p. 352 ; Holdsw. P. Z. S. 1873 , p. 45.5; Shetley, B. Egypt, p. 97; Hume, Nests \& Eggs Ind. B. p. 343 (1873);id. Str. F. 1873 , p. 439; Adan, t. c. p. 382; Tants, t. c. p. 420 ; Hayes Lloyd, Ibis, 1873, p. 412 ; Huine Str. F. 1877, p. 233, 187.5 , p. I 37 ; Butler, t. c. p. $48 \mathrm{r}$; Huise, $t$. c. p. 48I, note; Irby, B. Gibaltrar, p. I I9 (1875); Lifurd, Ibis, 1875 , p. I7; Blyth \& Wald. B. Burm. p. I19 (1875); Wharton, Ibis, 1876 p. 19; Ball. Str. F. 1876 , p. 234.

Drymoica uropygialis, Fraser, P. Z. S. 1843 , p. I7; Allia \& Thomps. Exped. Niger, ii. App. p. 49r (1849)) Fraser, Zool. Typ. pl. 42. fig. 2 (1849); Gray, Gen. B. i. p. I64 (1848); id. Hand-l. B. i. p. I98, n. ${ }^{\circ} 2756$ (1869).

Prinia subhimalayana, Blyth, J, A. S. Beng. xiii. p. 377 (1844.)

P. cisticola, Blyih, t. c. p. 377 (1844).

(isticola subhemalayana, Hodos. Icon. ined. in Brit. Mus., Pas. seres, pl. 5I (n. $\left.{ }^{\circ} 437\right)$; id. in. Gray's Zool. Misc. p. 82 (18+t).

(isticola cursitans, Gray, Cat. M.xmm etc. Nepal pres. Hotys. p. 62 (1846); Blyth, J. A. S. Beng, xvi. p. 457 (1847); Tikel!, op. cit. xvii. p. 3oo (1849); Blyth, Cat. B. Mus. As. Soc. p. 145 (1849); Bp. Consp. i. p. 286 (1 850); Horsf. \& Moore, Cat. B. E. I. Co. Mus. i. p. 324 (1854); Cass. Proc. Phitad. Acizd. 1856, p. 318; Hartl. Orn. W.-Afr. p. 264 (1857); Swinh. Ibis, 1861 , p. 329; Antin. Cat. descr. Ucc. p. 37 (1864); Harll. P. Z. S. 1867, p. 825; Finsch \& Hartl. Vög. Ostaffr. p. 29 (1870); Salvad. Faun. Ital. Ucc. p. I19 (1871); Sharp:, Cal. Afr. B. p. 29 (187 I, pt.); Gurney, in Anderss. B. D.tm. L.t. p. 88 (1872); Walden, Trans. Z. S. viii. p. 64 (1872); Antin. \& Salvad. Viagg. Bogos. p. ror (1873); Sharpe \& Bourier, Bull. Soc. Zool. France, i. p. 305 (1876); Dresser, B. Eur. iii. p. 3, pl. 96 (1877); Huine, Str. F. 1877, p. 90: D.xvidson \& Wenden, Str. F. 1878 , vol. ii. p. 85 ; Ball, t. c. p. 217 ; Cripps, t. c. p. 235; Tweedd. P. Z. S. 1877 , p. 7 I0; Hume \& Davison, Str. F. 1878 , p. $349 ;$ Legre, B. Ceylon, p. 531 (1879); Meyer, Ibis, 1879 , p. 146; Hume, Str. F. 1879, p. 101; Vidal, Str. F. 1880 , p. 67; Bingham, t. c. p. I86; Butier, t. c. p. 407; Wardlaw Ramsay, Orn. Works Twecdd. p. $6_{5}$ (1881); Bocage, Orn. Angola, p. 554 (1881); Reid, Str. F. 1881 , p. 46; Kelham, Ibis, $1881, \mathrm{p} 586$.

Cisticola omalura, Blyth, Cat. B. Mus. As. Sac. p. 145 (18\$9) descr. nulla); id. J. A. S. Beng. xx. p. I76 (1851); B. C.misp. i. p. 286 (1850); Kelaart, Prodr. Cat. p. 120 (1852, pt.); Byytin, Ibis, 1867 , p. 302; Hume, Str. F. 1877 , p. 9).

Salicaria (Cisticolı) brunneiceps Tenm. \& Schleg. Faun., Jap., Aves, p. I 34, pl. $20(1850)$. 
Cisticola brunneiceps, Bp. Consp. i. p. 286 (i $8 \mathbf{5}^{(i)}$ )

Caricicola cisticola, Brchm, Vogelf. p. 237 (1855).

Sylvia arquata, Von Müller, J. f. O. 1856,1$) .224$.

I)rymoca uropygialis, Harll. Orn. W.-Afr. p. $5^{4}$ (1857).

Calamanthella tintinnabulans, Swinh. J.As. S, . V. Chint Bis. 1859 p...

(isticola tintinnabulans, Swinh. I6:s, 1860, pp. 51 \& 131, I851, p. 32.

Schonicola cisticola, Blasius, List. B. Eur. ed. Neivton, p. I (1862); Salvad. Ucc. Sardegn. p. 56 (1864); Bolle, J. f. $O$. 1865 . p. 142; Brooke, Ibis, 1873 , p. 34t.

(isticola fuscicapilla, Wall. P. Z. S. 1863, p. 489 .

( $\therefore$ e uropæa, Hartl. Ibis, 1863, p. 325 .

I) rymoeca cisticola, Heugl. Ibis, 1860 , p. I 32.

Drymoica omalura, Gray, Hand-l. B. i. p. 200, no. 2812 (1864).

I). cursitans, Gray, t. c. p. 200, no. 2806 (1 869).

I). fuscicapilla, Gray, t. c. p. 200, no. 28 ro (1869).

(isticola munipurensis, Godwin-Austen, P. Z. S. 1874, p. 47; id. J. A. S. Beng. xliii. pt. 2, p. I65 pl. ix. fig. 2, $(1 \$ 74)$; Hume, Str. F. 1875, p. 398 , I877, p. 90, 1879, p. IoI.

I.xcustella brunneiceps, Seebohm, Ibis, 1879 , p. 37 .

(isticola cisticola, Oates, B. Brit. Burm. p. II 5(1883); Sharpe, Cat. B. Mus. 7. p. 259 (1883).

Hab. Luzón, Manila, Laguna, Calauan; Europa, China, Japon.

4S. C. exilis, Vig. at Horsf. N. v. Mamansit.

(M. S. T.)

Malurus exilis, Vig. \& Horsf. Trans. Linn. Soc. xv. p. 223 (1827, ex Lath. MSS.)

(isticola lineicapilla, Gonld, P. Z. S. 1347, p. I; id. B. Alustr. fol. iii. pl. 43 (18+8); Bp. Consp. i. p. 287 (1850); Goult, Handb. B. Austr. i. p. 35I (1865); Ramsay, Ibis, 1856, p. 328 .

(. ruficeps, Gould, P. Z. S. 1837 , p. 150; id. B. Austr. fol. iii. pl. 45 (1848); Bp. Consp. i. p. 287 (1850); Wallace, P. $Z$. S. 1863 , p. 485; Gould, Handb. B. Austr. i. p. 353 (1855); Ramsay, Ibis, 1868, p. 277 ; id. P. Z. S. 1860 , p. 385, it. P. Z. S. 1875 , p. 589; Sharpe, Journ. Linn. Soc. xiii. pl). 31 5, 494; Ramsay, Proc. Linn. Soc. N. S. W. ii. p. I 85 (1878); iii. pp. 108, 275 (1879); iv. p. 97 (1880); Salvad. Ann. Mus. Civec. Genov. viii. p. 376 (1879); xvi. p. 189 (1880); Layard, Ibis, 1880 , p. 297; Finsch, Ibis, 1881, p. 335; Nichols. Ibis, 1881 , p. 150; Salvad. Orn. Papuasia, ii. p. 433 (1880).

C. isura, Gould, P. Z. S. 1847 , p. 32 ; ict. B. Aust. fol. iii. pl. 44 (1848); Bp. Consp. i. p. 287 (1850); Gould, Handb. B. Austi. i. p. 352 (1865); Ramsay, Ibis, 1866 , p. 326.

(. exilis, Gould. B. Austr. iii. pl. 42 (1848); Bp. Consp. i. p. 287 (1850); Gould. Hand. B. Astr. i. p. 350 (1865); Ramsay, Ibis, 1866, p. 328; Oates B. Brit. Burn. p. 117 (1883); Sharpe, Cat. B. M. 7. p. 269, (1883).

Drymoica ruficeps, Gray, Gen. B. i. p. 164 (18+4); id. Hand-l. $B$. i. p. 200, n. 2817 (1869).

D. exilis, Gray, Gen. B. i. p. I64 (1848); id. Gand-l. B. i. p. 200 , no. 2817 (1869). 
1). lineicapilla, Gray, G.'n. B. i. p. 16+ (18+8); dd. Hand-l. B. i. p. 200 , no. 2808 ( 1869 ).

D) isura, (iray, Gen. B. i. p. Io+ (1 $8+8)$; id. Hantl-l. B. i. p. 200, no. 2816 (1899).

(iiticola erythrocephala, Blyth, J. A. S. Beng. xx. p. 523 (1851); lerd. B. Ind. ii. p. I75 (1863); Hume, Str. F. $1 \delta^{3} 7$, p. (1); id. Str. F. 1879 , p. 101.

( volitans, Swinh. Journ. N. China Branch As. Soc. $18.5 \%$. P. ; id. Ibis, 186,3 p. 304; id. P. Z. S. $18^{3}, 1$, p. 352 ; l)avid. Oust. Ois. Chine, p. 256 (1878).

(. tytleri, Jerd. B. Ind. ii. p. ${ }_{7} 6$ (186.3, ex Blyth MSS.S. ): Blyth, Ibis, 1865. p. 24; Godain-Austen, J. A. S. Beng. xh. 1. $199(1876)$; Hume, Str. F. 1877, pl). 90, 350.

( rustica, Wall. P. Z. S. 186.3, Pp. 19, 25; Finsch, Nin. (iuinea, p. 166 (1865).

(. semirufa, Cab. J. f. O. 1866 , p. го, г872, p. 316 ; Wall. Tr. Z. S. ix. p. 195 (1875); Sharpe, Tr. Linn. Soc. new ser. i. 1. 352 (1877); Wardlaw Ramsay, Orn. Works Tiveedd. p. 0.57 (1 881$)$.

1)rymoica tytleri, Gray, Hand-l. B. i. p. 200 , no. (281 3 1864).

D). volitans, Gray, t. c. p. 200, no. 2815 (1869).

(isticola delicatula, Blyth, Ibis, 1870 , p. I70 (ex. MSS. in .Mus. Lugd.)

(. melanocephala, J. Andersou, P. Z. S. 1871 , p. 212 ; Swinh. I. Z. S. 1871, p. 352; Godvin-Aust. J. A. S. Beng. xiiii. p. 1 165 , pl. x. fig. I (1874); Hume, Str. F. 1877 , p. 90; Allderson, Zool. Exped. Yun-nan, Aves, p. 641 (1878) David it (Justalet Ois. Chine, p. 257.

C. ruficollis, Walden, Ann. \& Mag. Nat. Hist. (4) vii. p. $2+2$ ( I87 I); Godvin-Austen, J. A. S. Beng. xliii. p. 165, pl. ix. fig. 2(1874); Wardlaw Ramsay, Orn. Works Tweed. p. 664 (1881\%.

(. Grayi, Walden Ann. \& Mag. Nat. Hist. (4) ix. p. 400 (1872;; id. Trans. $Z$. S. viii. p. I 77 (1874); Tweedd. P. Z. S. $187 \%$, 1. 828, 1878, p. 215; Wardlaw Ramsay, Orn. Works Tweedd. p. $66+$ (188I).

(.) ruficapilla, Salvad. Ann. Mus. Civic. Genov. vii. p. 376 $(1876)$.

(. sp., Salvad. Ann. Mus. Civic. Genor. ix. p. 35 (1876); id. Orn. Papuasia etc. p. 424 (1881).

C. erythrocephalus, Hume, Str. F. 1879, p. 1ог.

C. mclanocephalus, Hume, Str. F. 1879 , p. ror.

Hab. Luzón, Negros; Australia, Molucas, Formosa.

io Gén Cinclosoma, Vigors

49 C. punctatum, (Lath. $\sigma^{7}$ N. v.

(M. S. 'T.)

Punctated Thrush, Lath. Gen. Syn., Suppl. ii. p. I87 (1801).

Turdus punctatus, Lath. Ind. Orn. Suppl. p. xliv. (1801).

Cinclosoma punctatum, Vig. \& Horsf. Trans. Linn. Soc. xv. 1. 220 (1826); Gray, Gen. B. i. p. 224 (1846); Gould, B. Ausli. fol. iv. pl: 4 (1848); Cab. Mus. Hein. Th. i. p. 85 (1850); Reichenb. Vög. Neucholl, p. 105 (1850); Guuld, Handb. B. Aitst; . i. p. 433 (1865); Gray, Hand-l. B. i. p. 280 , no. 4r24 (1869); 
Ramsay, Proc. Linn. Soc. N. S. W. ii. p. $18 ;$ (1878). Sharpe, Cat. B. M. 7. p. 332. (1883).

(.. punctatus, Bp. Consp. i. p. $278\left(185^{\circ}\right)$.

Hab. Australia.

$$
\text { 11. Gén. TATARE, I.fiss. }
$$

5o. T. syrinx, $\operatorname{Set}$. N. v.

Acrocephalus syrinx (Kittl.), Seebohm, Cat. B. Brit. Mhes. r. p. roo; Tristr. Ibis, $1 \& 83$, p. 44.

Calamoherpe syrinx, Finsch, Ibis, 1891, pl. I 2 , 115.

Tatare syrinx, Sharpe, Cat. B. M. 7. p. 527 (1883).

Hab. Ponapé, Carolinas.

5I. T. Juscinis, Q. et C.N. v.

'Iatare luscinia, Quoy et Gaim. Voy. Astrol., Zool. i. p. 202, pl. 5. fig. 2 (1830); Gray, Gen. B. iii. App. p. \& (18+9); id. Cal. B. Trop. Isl. Pacific Ocean., p. 14 (1859).

T. luscinius, 13p. Const. i. p. 224 (1850); Gray Hand-l. B. i. p. 194, n. ${ }^{\circ} 268$ r (1869).

Hybristes luscinia, Reichenb. Syst. Av. pl. 57. fig. 7 ( $1850(0)$.

Acrocephalus marianna, Tristr. Ibis, 1883, p. 4.5 .

Tatare mariannæ, Sharpe, Cat. B. M. 7. p. 528 (1883).

Hab. Marianas.

12. Gén. TURDINUS, BhYTH.

52. T. rufifrons, Tiv. $\sigma^{3} 0 \mathrm{~N}$ v. Uit-uit.

(M. S. 'T.)

Trichostoma rufifrons, Tiveed P. Z. S. 1878, p. 6rG, pl. xxxviii. C'urdinus rufifrons, Sharpe, Cat. B. M. 7. p. 546 (1883).

Hab. Paragua, P. Princesa.

13. Gén. DASYCROTOPHA, Tw.

53. D. speciosa, ơ Tzu. N. v. Tangsit.

(II. S. I.)

Dasycrotopha speciosa, Tweedd; P.Z. S. $18 ; 8$, p. I 14, pl. ix. p. 28t). Wardlaw Ramsay, Orn. Mem. Trueed. p. G57 (1881); Sharpe, (at. B. M. 7. p. 574. (1883).

Hab. Negros, Bohol.

14 Gen. MIXORNIS, Hodgs.

54. M. woodi, Sharpe. o, N. v. Uit-uit.

(M. S. T.)

Mixornis wodi, Sharpe, Tr. Linn. Soc. new ser. Zool. i. p. 331 ; (1876); id. Cat. B. M. 7. p. 577. (1883), Tweedd. P. Z. S. i878, p. 617; Wardlaw Ramsay, Orn. Works Ta'eedd. 1. 657 (1 $88 \mathrm{r})$.

Hab. Paragua, P. Princesa. 
55. M. cagayanensis, Guill.

Mixornis cagayanensis, Guillermard, On birds coll. voy. of. «Marchesa.» p. 419 (I885).

Hab. Cagayan de Joló.

56. M. capitalis, Tw. $\sigma \rho$ N. v. Bancao-bancao.

(M. S. 'T.)

Mixornis capitalis, Tweed. Ann. \& Mag. Nat. Hist. (4) xx. p. 535; (1877); id. P. Z. S. 2878 , p. 110 pl. vii. fig. 2; id. t. c. pp. 342, 380; id. P. Z. S. 2879 , p. 71 ; Wardlaw Ramsay, Orn. Works Trueedd. p. 657; (1881); Sharpe, Cat. B. M. 7. p. 582 (1883). Hab. Leyte, Samar, Panaon, Dinagat, Basilan.

57. M nigro-capitatus, Stecre. $\sigma$ o N. v. Tangsit.

(M. S. T.)

Hab. Samar, Leyte.

I5. Gién. MACRONUS, JARD. 、

58. M. striaticeps. Sha'pe, $\sigma 0$ N. v. Tangsit. •

Macronus striaticeps, Sharpe, Trans. Linn. Soc. new series, Zool. i. p. 33 I; id. Cat. B. M. 7. p. 584, 1883. Treedd. P. Z. S. 1877 , p. 827 , I878, pp. 110, 949; Wardlaw Ramsay, Orn. Works Tweedd. p. 657 (1881).

Hab. Basilan, Malamaui, Dinagat, Mindanao, Surigao.

59. M. mindanensis, ơ Steere. N. v. Tangsit.

(M. S. T.)

Hab. Mindanao, Samar, Leyte.

6o. M. kettlewelli, Guill. o N. v.

(M S T.)

Macronus kettlewolli, Guillermard, Voy. of the Marchesa, p. 262 ( I 885 ).

Hab. Joló, Lucatlapas.

16. Gén PTILOCICHLA, Sharpe.

6I. Pt. falcata, Sharpe. $\&$ N. v. Inahing.

(M. S. T.)

Ptilocichla falcata, Sharpe, Tr. Lin. Soc., Zool. p. 332 (1876); id. Cat. B. M. 7. p. 586 (1883); Wardl. Ramsay, Orn. Works Tweed. p. 657 (1881).

Hab. Paragua.

62. Pt basilanica, Steere. \& N. v.

(M. S. T.)

Hab. Basilan.

6: Pt. mindanensis, Steere. $\delta \mathrm{N}$ v.

(M. S. T.)

Hab. Mindanao. 
i7. Gén ANUROPS S, Sharpe.

64. A. cintreiceps, (Tre) i \& N. v. Tangsit.

(M. S. T.)

Drymocataphus cinereiceps, Treed $P . Z$, S. 1878, p. 617. Inuropsis cinereiceps, Sharpe, Cat. B, M. 7, p, 590 ( 1883 ),

Hab. Paragua, Puerto Princesa.

18. Gén. DEndrobiastes, Sharpe.

65. D. basilanica, Sharpe o" N. v. Uit-nit.

(M. s. T.)

Dendrobiastes basilanica, Sharpe, Tr. Lin. Soc., i, p. 332, pl. 53. fig. I I 876 ; id. Cat, B. M. 7. p. 630 (1883).

Hab. Basilan.

Fam. PÁRIDOS.

Subfam, PARINOS.

I. Gén PARUS, LiN.

1. P. coruleus, Lin. $\$ \mathrm{~N}$.

(M. S. T.)

Blue 'Titmouse, Genn. Arctic. Zool. p, 427 (785).

Parus caruleus, Linn. S. N. i, p. 34I ( 760$) ;$ Lath. Gen. Syn. ii. p. 543; Beseke, Vög. Kurlands, n. ${ }^{\circ}$ 193; Retzius, Faun. Suec. p. 269; Koch, System. baier. Zool, p. 205; Temm. Man. d'Orn. p. 290; Roux, Orn. Hrov, p. 190; Less. Traité d'Orn. p. 456; Pall, Zoogr. Ross.-As. i. p. 554; Naum. Vög. Deutschl. iv, pl. 95; Nilsson, Skandin, Faun, i. p. 34I. (1835), i. p. 408 (1858); Schinz, Europ. Faun. p. 217; Gould, B. Eur ii. pl, I54; Moquin-Tandon, Orn. Canar, p. I8; Yarrell. Brit. B. i. p, 330; Selys-Longch. Orn, Belge, p. ro4; Blyth, Cat B.M. A. S. B. p, ro4; Bf. C. A. p. 229; Naceyro, Cat, Av. Galizia, Mem. R. Acad. Madrid, p. Io6; (I850); Kjarbölling, Danmarks Fugle, pl. xxviii.; Horsf. \& Moore, Cat. B. M. E. I. Comp. p, 37 ; Schlegel, Vög. Ncderl. pl. г 26; Sunder. Svensk. Fogl. pl, xvi. fig. I; Wright, Finl. Foglra, i. p, I8 8 ; Fritsch, Vög. Eur. pl. I7, fig. 20; D'Orbigny, Ois. d'Eur. pl. xxviii.; Goeld, B. Gr. Brit. vol. 'ii, pl. 24; Keulemans. Onze Vogels, pl. I4; Holland. Wrbelth. Pommerns, p. 6o, Salvad. Faun. Ital. ii. p. 66; Danford \& Brown, Ibis, 1875 , p. 303; Dresser, B. Eur. iii. p, 131, pl, I I3; Mcore, Brit; Birds, ordo n. ${ }^{\circ} 55 ;$ Yarrell, Brit. B. ed Newiton, p. $483 ; G a$ dow, Cat. B. M. 8. p. I 2 (1883).

Cyanistis cæruleus, Kaup. Natürl. Syst. p. 99 (1839); Cab. Mus. Hein. i. p. 90.

Hab. Eiropa, Asia, Francia. 
2. $\mathbf{P}$ amabilis, Sharfi. o 0 N. v. Uit-uit.

(M. S. T.)

larus amabilis, Sharpe, Trans, Linn. Soc. Zool. new ser. i. 1. $33^{8}$, pl. 53. fig. 2 (1876); Gadori', (at. B. M. 8, p. 22 (188,3);

II ardani Ramsay, Orn. Works. Ticeedd. P. 658 ( $188 \mathrm{~s})$.

Hab. Balabac, Paragua, P. Prince'sa.

3 P. elegans, Less. 0. 0 N. v Pipit-maquing.

(M. S. T.)

Parus regans, Lesson, Traité d'Orn. p. 450 (1831); Pucheran, Rez, et Mag. Zool, $185 \mathrm{f}$, p. 68; Bp. C. R. xxxviii. p. 6.3; Blyth, Ibis, 1867, P. 34 (note); Walden, Tr. Z. S. $187.5, \%$. 199; id, P. Z. S $1877, p p .688,696,756, \& 762,1878, p$. 286; Wardlaw Ramsay, Orn. Works Tweedd, p. $65^{8}$ (1881); (iadore, Cat. B. M. 8. p. 22 (1883).

P. quadrịrittatus, Lafresn. Rev. Zoal. 1870, p. г29; Bp. C.A. i. p. 229 .

Hab. Luzón, Manila, Guimarás, Ccebú, Panay, Negros, Masbate, Paragua.

4. P. semilarvatus, Salv. o o N. v. Pipitmotas. (M. S T.)

Melaniparus semilarvatus, Salvad, Atti Soc. Ital. Sc, Nat. viii. p. 375 (1865); id. J. f. O. 1868 , p. 68; id. Ibis, 1879, p. 300 , pl. ix.

Parus sermilarvatus, Gadow, Cat. B, M. $\delta, ~ .38$ (188),

Hab. Luzón; China,

\section{Subfam. REGULINOS.}

I. Gén. Regulus, Kocir.

5. R. ignicapillus, Brehm. 3 N. v.

(M. S. T.)

Souci ou Poule, Buff. Pl. Enl. pl 651. fig. 3.

Sylvia regulus, Bechstein, Ornith. Taschenb. p. I 89 (1802).

s. ignicapilla, Brehm. in Temm. Man. d'Orn. p. 231 (1820); Naum. Vög. Deutsch. iii, pl, 93. figs. 4, 5, 6.

Regulus ignicapillus, Meyer, Tasch:nb. Deutschl Vogelk. iii. p. log (1822); Werner, Atlas, Insectivores, pl. 61; Roux, Orn. Provenc. pl. 235; Temm. Man. d'Orn. iii. p. i58; Gould. B. Eur. pl. I48. fig. 2; Schinz, Europ. Faun. i. p. 207; Keys. \& Blas. Wirb. Eur. p. lv; Schlegel, Revne, p. xliv; Gray, Gen. B. i. p. r75; Bp. C. A. i, p. 291; Cab. Mus. Hein. Th. i, p. 34; Kjarböll. Danmarks Fugle, pl. 54, fig. 5; sch, Vög. Eur. pl. 19. figs. I, 2; Degland \& Gerbe, Ois. Eur. i. p. 555; Gould, B. Gt. Br. ii. pl. 70; Schlegel, Vög. Nederl. pl. I 24; von Droste, Vogelw. Borkums, p. 90; Perini, Ornic Veronese, p. 2r 2; Altum, Forstzoologie, p. г94; Newiton, ed. Yarr. Brit. B. i. p. 456; Dresser, B. Eur. ii. p. 459, pl. 72. fig. I: Gadow. Cat. B. M. 8. p. 85 (1883). 
R. prosephalus, Brehm, Beitr. Ioge'k. ii. p. 136, pl. i. tis. I (1822); id. Lehrbuch, p. 276 ; id. Vör. Ditutschl. p. $t^{8} 3$.

R. mystaceus, Vieill. Fiaun. Franc. p. 231 (1822).

R. nilsonii, Brehm, Vög. Dentschl. p. 482 (1831).

R. brachyrhynchus, Brehm, t. c. p. $4^{8} 3(1831)$.

Hab. Europla, Esıaña, Francia.

Fam. LAANIDOS.

Sulfam. GIMNORRINOS.

I. Gén. GYMNORHINA, GRAY.

1. G tibican, $(L: t / h)$, $\sigma$ N. v.

(M. S. T)

Piping Roller, Iath. Gen. Syn. Suppl. p. 122.

Coracias tibicen, Leth. Ind. Orn. Suppl. p. xxvii.

Barita tibiern, Temm. Man. a'Orn. i. p. li; Lese. Traité, 1). 345 ; Bip. C. A. i. p. 367 .

Cracticus tibicen, Vir. \& Horsf. Tr. Limn. Soc. xv. p. 200 ; Gould, Syn. B. Austr. part i.; Schl. Mus. P.-B. Coraces, 1). 125 .

Gymnorhina tibicen, Gray, List Gen. B. 1843 , p. 50; id. \&Mitch. Gen. B. ii. p. 302, pl. 73; Gould, B. Austr. ii. pl. 46; Cab. Mus. Hein. Th. i. p. 226; Gould, Handb. B. Austr. i. p. 175; Gray, Hand-l. B. ii. p. 2 , n. ${ }^{\circ} 6062$; Ramsay, Proc. linn. Soc. $N$. S. W. ii. p. I8o.

Gymnorrhina tilicen, Reichenb. Handb. Naturg. n. ${ }^{\circ} 214$ (1850). $H a b$. Australia.

Subfam. PAQUICEFALINOS.

1. Gén. PaChycephala, Vig, et Horsf.

I P. philippinensis, Wald. O० 0 N. v. Piroc-piroc. (M S T)

Hyloterpe philippinensis, Walden, Ann. \& Mag. N. H. 1872, vol. x. p. 252 ; id. $\operatorname{Tr} . Z$. S. 1877 , viii. p. I79, pl. xxxi. fig. 2, ix. p. 250; id. P. Z. S. 1879. p. ıo9; Wardlaw Ramsay, in Tweeddalc's Ornith. Works, Append. pp. 656, 666; Could, B. Asia, part. 33 .

Pachycephala philippinensis, Gadow, Cat. B. M. 8. p. 22 I I883. Hab. Luzón, Dinagat, Paragua, Inagauan.

Subfam. LANINOS.

I. Gén. Lanius, Lin.

I L. schach, Lin. $\sigma \mathrm{N}, \mathrm{v}$. Tarat.

(M. S $\mathrm{S}$ T.)

Lanius a-scack, Osbeck, Ostind. Resa, p. 227 (1757).

L. schach, I.inn. S. N. i. p. $94\left(175^{8)}\right.$; Gm. S. N. i. p. 30.3; 
l'reill. Enc. Meth. ii. p. 7.37; Less. Traite d'Orn. p. 37:Blyth. J. A. S. Beng. xu. p. 3or; id. Cat. B. Miss. As. Sil: p) I51; Bp. C. A. i. p. $36+18501 ;$ Horsf. of Moure, ('i'. B. M. E. I. Co, i. p. I03 (185t); Cassin, Narrat. of. Expert. Amer. Squadr. ii. p. 238 (18+6); Swinh. Ibis, 1850, p. t.3. and. 1871, p. 59 ; id. P. Z. S. 185.3, p. 286, and $1871, \mu$. 375; Severtz. Turk. Jevotn. (1873); Walden, Tr. Z. Soc. 187.5 . p. 170; Dresser, Ibis, 187.5 , p. $184 ;$ Dav. \& Oust. Ois. Chine. p. 96, pl. 75 (1877); Schalow. J. $j . O .1878$ p. 139; Ciadori. Cat. B. M. 8. p. 26r. (1883).

(hestnut-backed Shrike, Lath. Gen. Syn. i. p. 159 (1781).

I anius castaneus, Gm. S. N. xiii. p. 297, n. 5 (1788); Lath. Ind. Orn. i. p. 57.

L. macrourus, Cuv. Mus. Par. (teste Pucher. Arch. du .Mus. d'Hist. nat. vii. p. 325, I854).

L. chinensis, Gray, Zool. Misc. p. I (1831).

Collyrio schach, Bp. Rev. et Mag. Zool. 1853, p. 43t; Criat', Hand-l. B. i. p. 392, n. ${ }^{0} 5949$ (1869); id. Fasc. B. China, p. t.

Lanius longicaudatus, Gould, P. Z. S. 1859 , p. i 5 I.

L. schach, var. formosx, Swinh. Ibis, 185.3, p. 270; id. P. $Z$. S. 1863 , p. 335 .

Hab. Luzón, Cagayan; Formosa, China.

2 I nigriceps, Franlo. $\sigma, 0 \mathrm{~N}$, v. Tarat San Diego. IN S. T:

Pie-grièche d'Antigue, Sonner. Voy. Nowz. Guin. p. 114, pl. ( 776 ).

Lanius antiguanus, Gml. S. N. i. p. 301, n. ${ }^{\circ} 29$ (1788, ex Sonnerat).

L. nasutus, Scop. Del. Fl. Faun. Insubr. ii. p. 85, n. 13 (1786, ex Sonn.); Walden, Tr. Z. S. ix. p. I69.

Collurio nigriceps, Frankl. P. Z. S. 1831 , p. iा 7; Bp. Rẽ'. et Mag. Zool, 1853 p. 435; Hume, Nests. \& Eggs. Ind. B. p. 172.

Lanius tricolor, Hodgs. Ind. Rev. i. p. 446 (1837); id. in Gray's. Zool. Misc.p. 81.

L. nigriceps, Jerd. Madr. Journ. x. p. 236; id. Ill. Ind. Orn. pl. 17. fig. I (1830-34); Gray', Gen. B. i. p. 290, pl. 71; Blyth. Cat. B. Mus. As. Soc. p. i5 ; Bp. C. A. i. p. $364(1850)$; Horsf. \& Moore, Cat. B. M. E. I. Co. i. p. 166 (1854); Jerl. B. Ind. i. p. 404 (1863); Blyth, lbis, 1870 , p. I64; Jerd. Ibis, 1872 , p. I I5; Hnme, Str. F. 1874 , p. 473; David \& Oust. Ois. p. 95 (1877); Schalow. J. f. O. 1878 , p. 153; Anters. B. Yun-nan, p. 644 (1878); Scully, Str. F. 1879. p. 26+; Gadow, Cat. B. M. 8. p. 268. (1883).

(ollyrio pyrrhonotus, Gray, Hand-l. B. i. p. 392, n. 5952 (г 869).

(.. nigriceps, Gray, Hand-l. B. i. p. 392, n. ${ }^{\circ} 5955$.

( $\therefore$ nasutus, Gray, Hand-l. B. i. p. 392, n. ${ }^{\circ} 5958$.

Lanius pileatus, Temm. (in. Mus. Ludo.), file Blyth, Ibis, 1870 , p. 164 .

Hab. Luzón, Laguna, Negros, Guimarás, Panay, Cebú, Leyte, Samar, Bohol, Siquijor; Himalaya, Cochinchina. 
3. L. cephalomelss, $B p \cdot \sigma^{*}: \mathrm{N}$ v Tarat.

(II. S. T.)

I anius cephalomelas, Bp. Rev. et Mag. Zool. 18.53, p. +36; (i.ldow, Cat. B. M. 8. p. 269. (I 883$)$.

I. nigriceps (pt.), Walden, Ibis, 1868 , p. 70 ; Schalow, J. f. (). 1878 , p. I 53, et auct. plurim.

Collyrio cephalomelas, Gray, Hand-l. B. i. p. 392, n." 5959.

Hab. Leyte, Sanar, Paragua.

4. L. superciliosus, Lath. O', N v. Tarat.

(M. S. T)

Le Rousseau, Levaill. Ois. d'Afr. 1)l. 66. fig. 2 (1796-1808).

lanius superciliosus, Lath. Ind. Orn., Suppl. 20, n. $^{\circ}$ It (1801): Walden, Ibis, 1867, 1. 218 ; Gray, Hand-l. B. i. p. 393, n." 5971; Swinh. P. Z. S. 1871 , p. 375; id. Ibis, 187.5, p. +5!; Vian, Rev. Zool. 1872 , p. 327; Dab. \& Oustal. Ois. Chin", P. IOO (1877); Legg , B. Cieylon, P. 279, note (1879); Ciadoni, Cat. B. M. 8. p. 273. (1883).

linneoctonus superciliosus, Gray, Gen. i. p. 29 I (1846); Bp. C: A. i. p. $3^{6} 3$ (1850).

()tomela superciliosa, Bp. Rev. Zool. 1853, p. 437; Schalow. J. f. 0.187 .5$, p. 140 .

lanius phonicurus (non Pall.), Schrenck, Reis. Amur. i. P. 38t; (1860), teste Schaloze et Dav. \& Oustal.; Sundev. Phy's. Sills. Tidssk. ${ }^{1837-38}$ p. 6+; id. Ann. Nat. Hist. $18 .+6$, p. 169; Blasius, Nach'r. Naum. Vög. Deutschl. xiii. p. 39; IValden, Ibis, 1867 , pl. 5. fig. 2.

Hab. Luzón, Cagayan; Malaca, Chi ra.

5. L. lucionensis, Lin. O . N v. Cabezote,

(M $\mathrm{S}$ T.)

Lanius lucionensis, Linn. S. N. i. p. I 35 ( I 766 ); Swinh. Ibis, 1860 , p. 59, 1863 , p. 272; Blyth, Ibis, 1867, p.304, 187.5. p. I 6 ; Walden, Ibis, 1867 , p. 215, 1373, p. 309; Gray, Hand-l. B. i. p. 393, n. 5970; Jerdon, Ibis, 1872, p. II5; Swinh. P.Z. S. 1863, pp. 286 \& 335, 1871 p. 376; Hume, Str. F. 1873 p. 434,1874, p. 199; Walden, Tr. Z. S. is. 1875, p. I7I, pl. 29. fig. I; id. P. Z. S. $1877, \mathrm{pp} .692$ i. 758; Sharpe, Ibis, 1877 , p. 19, 1876, p. 43; Dav. \& Oustal. Ois. Chine, p. 99 (1877); Legge, B. Ceylon, p. 378 (1879); Oates, B. Brit. Burm. p. 25I (1883); Gadow', Cat. B. M. s. p. 274 ( ( 883 ).

L. phœnicurus (non Pall.), Meyen, Act. Acad. Cas. Leopold. xvi. p. 74 (1834) (teste Schalow.); id. Reise um. d. Erd, p. rgi
(ex Philippin. ins., teste Schalow).

Otomela schwaneri, Bp. Rev. Zool. 18.53 , p. 437.

Enneoctonus schwaneri, Bp. C. A. i. p. 3.

Lanius jeracopsis, De Fil. Mus. Mediol. p. 31 ; Bp. Rev. Zool. 1853, p. 437, note; Salvad. Atti. Acc. Sc. Torino, 1868 ,
p. 272 .

L. schwaneri, Walden, Ibis, 1867 , p. 223; Gray, Hand-l. 13. i. P. 293, n. 5974 ; Swinh. P. Z. S. 1871, p. 376.

Otomela lucionensis, Schalow, I. f. O. 1875, P. I 37.

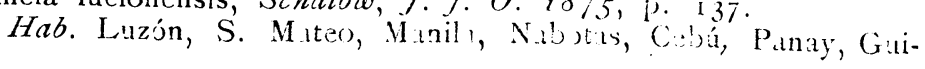


marás, Negros, Basilan, Leyte, Bohol, Patayua, Panaon, Mindanao, Borneo; China.

\section{Fam. VIREÓNIISOS.}

I. Gén. VIré(), Vieil.L.

I. V. gilvus. Vieill. o $\mathrm{N}$ v. .

( $\mathbf{A}$ S. $\%$ )

Muscicapa gilva, Vieill. Ois. Am. Sept. p. 65, pl. 34 (1807).

.1. melodia, Wils. Am. Orn. v. p. 85, pl. 42. tig. 2 (r8r 2 ).

Vireo gilvus, $B p$. Obs. Wils. no ${ }^{\circ} 123(1825)$; id. (: A. i. p. 33:; Audub. Orn. Biog. ii. pl, 118; ia. Birds Am. iv. pl. 241; Baird, Birds N. Am. p. 335; Sclat. P. Z. S. 18.58 , p. 30_; I)resser, Ibis, 1865 , p. 480; Samuels, Birds Neze. Engl. [.. 273; Salv. \& Godm. Biol. Centr-Amer., Aves, i. p. I92; Girdoze. Cat. B. M. 8. p. 296 (1883).

V. bartramii, Swains. Faun. Bor-Am ii. p. 235 (1831).

Vireosylvia gilva, Cassin, Pr. A. N. Sc. Philad. 1851 p. 153; Sclat. P. Z. S. $185^{6}$, p. 298, 1858 , p. 302; Baird. Rev. Aill. B. p. 342; Sumichrast, Mem. Bost. S'sc. $N$. H. i. p. 54; Baird, Brew., \& Ridgze. i. p. 368.

V'ireo swainsoni, Baird, B. N. Ain. 18.58, p. 336; Elliot, Illus/r. Birds N. A. i. pl. 7 .

Vireosylvia swainsoni, Baird, Rev. Am. B. p. 343 (1858).

V. gilva, var. swainsoni, Cooper, Orn. Californ. i. p. I I6 (1870); Baird, Brew., \& Ridgw. N.. Am. B. i. p. 37 i; Scl. \& Sali. Nomencl. p. I 2; Lawr. Bull. U. S. Nat Mus. i. p. 17.

Vireo gilvus swainsoni, Coues. Birds. Colorad. Vall. p. 501. Hab. W. América, Canadá.

2 V. flavoviridis, Cass. o N. v.

Vireosylvia flavoviridis, Cassin, Proc. A. N. Sc. Philad. 1851, ․ p. 1 52, vi. pl. 2; Sclat. P. Z. S. 1856, p. 298, 1859, 1). 375; id. Cat. Am. B. p. 44 (186r); Sclat. \& Salvin, Ibis, $185 \%$. 1. 12; Baird. Rev. Am. B. p. 336; Lawr. Ann. Lyc. N. '. vii. p. 323; Salvin, P. Z. S'. 1857 , p. I37, 1870, p. 184; ''. Frantsius, $J . f$. $O .1869$, p. 509; Baird, Brew., \& Ridgw. $N$. A. B. i. p. 366; Sumichrast, Mem. Bost. Soc. N. H. i. p. 547; Taczanowski, P. Z. S. 1874, p. 509; Merrill, Pros. U. S. Nat. Mus. i. p. r 25 ; Scl. \& Sixlv. Nomencl. p. I I.

Vireo flavoviridis, Baird, Birds N. Am. p. 332 (1858); Coues, 13. Colorado Vall. i. p. 490; Salvin \& Godman, Bioll. Centr.Aner., Aves, p. 189 ; Gadow. Cat. B. M. 8. p. 295 (1883).

Phyllomanes flavoviridis, Cab. J. $f . O .1861$, p. 93.

Vireosylvia agilis flavoviridis, Ridgow. Bull. U. S. Nat. Mus. i. p. I 9. Hab. Bolivia, Guatemala.

$$
\text { 2. Gen. HylophiLes, This. }
$$

3. H dozurtatis, (B), ON $\mathrm{N}$ v.

Sivicula decurtata, Bp. P. Z. S. 1837 , p. I 8 . 
Parhysylvia decurtata, Bp. C. A. i. p. $3(x)$.

Ilylophilus decurtatus, Baird. Rev. Am. B. i. p. 380 (1864); Salv. P. Z. S. 1967, p. 137, 1870 , p. 184 ; id. Ibis. 1969, 1. 313,1872, p. 314; Scl. \& Salv. P. Z. S. 1870, p. 836; lawer, Bull. U.S. Nat. Mus. N. ${ }^{4}$ 4, p. 18 ; Scl. Ibis, $88 \mathrm{~d}$, p. 298; Salz. \& Godm. Biol. Centr.-Amer., Aves, p. zo; Giadow. Cat. B. M. 8. p. 307 (1883).

I1. cinereiceps, Scl. \& Salv. P. Z. S. 1860, p. 299, 1864, p. $384 ;$ id. Ibis, 1060 , p. 397 .

11. pusillus, Lawer. Ann. Lyc. N. Y. vii. p. 323, vii. p. 29, ix. p. 97; Baird, Rev. Am. B. i. p. 381.

Hylophilus plumbiceps, Lawr. Ann. Lyc. N. Y. vii. 1. 323. llielmintheros ? Scl. P. Z. S. 1856, P. 291. Hab. América, Guatemala.

3. Gén. Vireolanius, ibu bus.

4. V. pulchellus, Scl. O N. v

(M. S T.)

Tircolanius pulchellus, Scl.\& Salv. Ibis, 1859 , p. r 2; Salr. Ibis, 1861 , 147; Lawr. Ann. Lyc. N. Y. vii. p. 468 ; Scl. Cas. Am. 13. p. 45; Baird. Rev. Am. B. i. p. 397; Scl. \& Salv. Exotic Ornith. pl. 8; id. Nomencl. p. I3; Salv. Ibis, 1878,$12443 ;$ (iadow, Cat. B. M. 8. p. 315 (1883).

Hab. América, Guatemala.

\section{Grupo: CERTIONORFOS.}

Fam. CÉRTIDOS.

Subfam. CERTINOS.

1. Gén. CERTHIA, LiN.

1. C. mexicana, $G \log$. N. v.

$\left(\begin{array}{lll}M & S & \mathrm{~T} \\ \end{array}\right)$

Certhia mexicana, Gloger, Handb. p. $3^{81}$ note (1834, e.r Licht.); Reichenb. Handb. spec. Orn. Scans. p. 266 pl. 6r 2; Sclater, P. Z. S. 1856 , p. 290; id. ib. 1859 , Pp. 363, 372; Salvin', Ibis, 1866 , p. 190; Gadow, Cat. B. M. 3. p. 326 (1883).

C. americana, Baird, C. N. Amer. p. 373, pl. 83. fig. 2.

C. mexicana, Cooper, Orn. Calif. p. $5^{8}$ (1870).

C. familiaris, var. mexicana, Baird, Brew., \& Ridgw. B. N.Am. p. I 28 (1874).

Hab. Méjico, Guatemala.

2. Gén. Climacteris, Trum.

2. C. mystacalis. ( $7 \mathrm{em}$ ) N. v. Lang̃gaás.

(M. S. T.)

Mcliphaga mystacalis, Temm. Pl. Col. pl. 335. fig. 2.

Climacteris striolata, Kitilits, Kupfer. Vög. pl. 6) fig. 2 (1832). 
(. mystacalis, Gray' ITant-l. B. i. p. 184, n. ${ }^{\circ} 2528$; Gailon', Cat. B. M. 8. p. $339(1883)$.

Rhabdornis mystacalis, Reichenb. Handb. spec. Orn., Scans. n. 640, pt. 566 (185I); Wald. Trans. $Z$. S. ix. 1). 20I. Triedd. IP. Z. S. 1878, PU. II 3,95 I.

Hab. Luzón, Manila, Samar, Paranas, Dinagat, Mindanao, Leyte, Panay.

Subfam. SITINOS.

2 Gén. SITTA, IiN.

3. S. carolinensie, Wils. of N. v. Sita de Carolira.

(M. S. T.)

Sitta europæa, var. $y$, Gmel. S. N. i. p. 440 (1788).

S. canadensis, Lath. Ind. Orn. i. p. 262 ( 7750 ).

S. carolinensis, Wils. Am. Orn. tab. 2. fig. 3 (1808); Nuttall, Man. i. p. $58 \mathrm{I}$; Licht. Verz. Doubl. p. I 7 (1823); Suinins. Faun. Bor. Amer. Intr. p. xxxvii; Aul. Orn. Biog\%. ii. p. 2oy (1834), v. p. 473, pl. I52; id. B. Amer. iv. p. I75, pl. IIt; Swains. Class. B3. p. $318 ;$ Bp. C. A. i. p. 227; Cab. Mus. Hein. i. p. 93; Reichenb. Handb., Scans. p. I53, pl. 513. nos. $3.563,3.564$ (1851); Prinz Wied. J. f. O. 18.58. p. 1o6; Hartl. P. Z. S. $185 \%$, p. 300; Sclat. P. Z. S. 18.59 , pp. 363 \& $373 ;$ Baird, B. N. Amer. p. 374; Sclat. Cat. Am. B. p. i 5; Baird, Rev. Am. B. p. 86; Sclat. Ibis, 186.5 , p. 3 Io Baird. Ibis, 1867 , p. 270; Cones, Key N.Am. B. p. 83; Baird, Brew., \& Ridgre. N. Am B. p. I I 4 ; Nerhling, J. f.O. 1880, p. II 4 ; Gadore, Cat. B. M1. 8. p. 353, (1883).

s. melanocephala, Vieill. N. Dict. xxxi. p. 336 г 889); id. Gal. Ois. tab. 171, p. $280(1825)$.

S. aculeata, Cass. Proc. Phil. Acad. viii. p. 254 (1856); Baird, B. N. Amer. p. 375 ; id. Review. Amer. B. p. 86; id. J. $f$. O. 1866 , p. 257 ; id. Ibis, 1867 , p. 270; Brown, Ibis, $1868^{\circ}$, p. 421 ; Cooper, Orn. Calif. p. 54 (1870).

S. carolinensis, var. aculeata, Cassin, Baird, Brew. \& R. N. Am. B. p. Ii 7 ( 1874$)$.

S. carolinensis aculeata, Coues, B. Colorad. Vall. p. 134 (1878). Hab. América, Canadá.

4. S frontalis, Szo. $\sigma 0$ N. v. I.tlit

(M. S. T.)

()rthorhynchus frontalis, Swxins. Zool. Ill. Ist. ser. pl. 2 (1820-28). Motacilla frontalis, Ruff. Tr. Linn. Soc. xiii. p. I33 (1822).

Sitta relata, Temm. Pl. Col. 72 (1823); Less. 'Traité, d'Orn. 1). 3 r6.

6. frontalis, Horsf. Tr. L. S. xiii. p. 162 (1821); Vig. Men. Raff. p. 67o; Tickell, J. A. S. Beng. ii. p. 579; Mc Clell.:P. Z. S. 1839 , p. ${ }_{16} 6_{5}$; Gray, Gen. B. i. p. 148; id. Cat. Hodgr. Coll. B. Mus. p. 62; Oates, B. Brit. Burm. p. 134; Gadow', Cat. R. M. 8. p. 358 , (1883).

s. corallina, Hodgs. j. A. S B. v. p. 779 (1836); id. Gray's Zool. Misc. P. '2; Gray, Hand-l. B. i. p. $182,{ }_{*}^{8}{ }^{\circ} 2499$. 
Iendrophila frontalis, Suains. Class. B. ii. p. 313 (1837; Jori. Madr. Journ. xi. p. 2 I8; Blyth, J. As. Soc. Beng. xvi. 1. :8o; id. Cat. B. Mus. As. Soc. p. igo; Mason, Burmal. p. $280 ; B p$. C. A. i. p. 220; Cab. Mus. Hein. i. p. 93; Reichenh. Handb., Scans. p. I 54, pl. 514. figs. 3571-72; Kelaart, Prodromus Cat. p. I 20; Layard, Ann. \& Mag. N. M. xii. p. 176; Horsf. \& Moore, Cat. B. M. E. I. Co. ii. p. 722; Bernsten, I. f. O. 1861 , p. I 22; Jerdon, B. Ind. i. p. 388; v. Pelecln, Vovar, Reise, Vög. pp. 59, I61; Beavan, Ibis, 1869 , p. 425; Ierdon, Ibis, 1872 , p. 2r; Holdworth, P. Z. S. 1872, I. 435; Hume, Nests \& Eggs. i. p. I6r; Salvad. Vac. Born. p. itu; legge, Ibis, 1874, p. 16; Kall, F. 1877, p. 397; Bourdillon, Sitr. F. 1876 , p. 393; Fairbank, Str. F. 1877, p. 399; Huth \& Davison, Str. F. 1878, p. 201; Legge, Birds of Cevlon, 1. 560 ; Hume, Str. F. 1879 , p. 459.

1). corallina, Reichenb. Handb., Scans. p. r54 (1851); Hume, Sitr. F. 874 , p. 473, 1875 , p. 89; Sharpe, Str. F. 1870 , 1. 436 ; Anderson, Zool. Exped. Yuman, p. 633.

Hab. Paragua, P. Princesa, Balabac; Java, Borneo, Ceilan.

5 S. œnochlamys, Sharpe. $\sigma \circ \mathrm{N}$. v Pipit-mingan.

(M. S. T.)

1)endrophila cenochlanys, Sharpe, Trans. Linn. Soc. 2nd. ser. Zool. vol. i. p. 338 , pl. liii. fig. 3 (18;6).

Sit:a anochlamys, Gadow, Cat. B. M. 8. p. 359. (1883),

Hab. Guimarás, Panay, Basilan, Cebú.

\section{Gimpo 4. CONIRINGRFOS.}

\section{Fam. NECTARÍNIIOOS. \\ 1. Gén Chalcostetha, ciak.}

1. Ch insignis, (Fmd.) $\circ \circ \mathrm{N}$ v. Saritsit.

(M. S. ' t.)

Nectarinia pectoralis, Tem. pl. 138 fig. 3 (1823 no Horsf); Schl. et Mül!. Natuurl. Gesch. Zool., Aves p. 57, pl. 9. f. (1846).

(ynniris pectoralis, Less. Man. d'Orn. 2. p. 33. (r846).

ec. pectoralis, Drapiez, Dict. Class. i 5 p. 513 (1829).

Nec. cal :ostetha, Jard. Mon. Sun-birds, p. ${ }_{26} 6_{3}$ (1842).

ire. insignis, Jard. t. cit. p. 274 (1842 ex Temm.); Gray, Gen. B. i. p. 98,1847 ; Gould. P. Z. S. 1865 , p. 663; id. B. Asia, p. i 9. pl. 5. (1867).

(y. macklotii, Bp. C. A. I. p. 408, n. 44 ( 8830 ex Temm).

(halcostetha pectoralis, Cab. Ams. Hein. I. p. IO3 ( 1850 ).

(h. macklotii, Reichb. Handb. S ansoria, p. 286, n. 662, 1$)$. 572. figs. $3898-99$ ( 1854$)$.

inthreptes chalcostetha, Licht. Nom. Ar. Mus. Beral. p. 5h (1854).

A rachnechthra chalcostetha, Horsf. et Moor., Cai. B. E. I. Ci'. Mics. 2. p. 746 (i $856-58$ ).

Nec. macklotii, Sclat. P. Z. S. 1863 , p. 220.

Promerops pectoralis, Gray, Hand-l. B. i. p. 110, n. 1254 (1869).

Arachnothera insignis, Grai', tom. cit. P. riz, n. 1384 
Ch. insignis, Wald. Ibis, 1870, p. 44 ; Saln. An. Mus. Civ. Genoz. $\therefore$ p. I77 I874; Hume, Str. F. . . p. 319 (1875; Tr'. $10 \mathrm{~m}$. towe cit. p. 302; id. P. Z. S. 1878 , p. 621; Hume et Nar, Str. F. 6. p. ${ }_{18} 8_{3}(18-8 ;$; Shellev, Mon. Nect. p. \&7, p. 3o; Hume, Srt. F. 1879, p. 89; Gadow, Cat. B. M. 9. p. 12,1884 .

(Ch. inspirata, Hume. Str. F. 187.5, p. $320, n) t a$.

Nec. porphyrolæma, Brïg. Abh. nat. L'er. Bremen, v. p. 73 ( 876 ).

Nec insignis, Brïg. Abh. nat. ver. Bremn, v. p. 465 (i 876 ).

Ch. pectoralis, Oates, B. Br. Burm. i. p. 317. (1883).

Hab. Paragua, P. Princesa, Samar; Célebes, Sumatra, Siam.

2 Gén. ETHOPYGA, Car.

2. At temmiricki, (S. Mïll, $\circlearrowleft, 0 \mathrm{~N}$ v. Aramasit. (M. S. T.)

Nictarinia temmincli, S. Müll. Natumrl. Gesch. Land. en Volkeuh. p. 173 nota (1843); id. et Schl. op. cit. Zool. Aves. p. 56, pl. 8. fig. 2, (1546); Gray, Gen. B. i. p. 98 (184i); Bp. Consp. Av. 1. p. 405, n. 15 (1850).

At. temmincki, Reichb. Handb. Scans. p. 300, n. 702, pl. 594.,

f. 3967 (1854; Wald. Ib:s, 1870 , p. 419; Sharpe, Ibis, 187.3, 1. 419; Salv. An. Mus. Civ. Gen. XIV. p. 2 1 3 (1879); Shellev, Mong. Mect. p. 47, pl. 16. f. 1.; Gad. Cat. B. Mus. \%. p. If (1 884).

Promerops temmincki, Gray, Haud-l. B. i. p. I Io, n. I 347 (1867).

(ynnir is temmincki, Beccari, An. Mus. Civ. Genov. XIII. p. 453 $(18 ; 8)$.

Hab. Paragua, Basilan; Borneo, Sumatra.

3. At. eximia, (Horsf.) $\sigma \circ$ N. v. Saritsit.

(M. S. 'T.)

Nectarinia eximinia, Horsf. Tr. Lin. Soc. XIII, p. I68 (1820); Jard. Monog. Sunbirds, p. 268 (1842); Gray, Gen. B. i. p. 98 (1847);Bp.C.A. i. p. 405 (1 850 ).

Nec. Kuhli, Tem. pl. 376, figs. I, 2, (1825); S. Müll et Schl. Natuur. Gesch. Zoal. Aves. p. 56 (1846); Haud-l. Dierk. p. 256 (1 857 ).

(innyris Kuhli, Less. Man. d'Orn. 2. p. 36 (1828).

C. eximiia, Vig. Mem. Raffl. p. 673 (183o).

Aethopyga eximia, Cab. Mus. Gein. 1. p. I03 (1850); Reech. Handb. Scans. p. 303, pl. 587, fig. 3984-85 (1854); Horsf et Moor. Cat. B. E. I. Cv Mus. p. 736 (1 856); Wrald. Ibis, 1878 , p. 37; Shelley, Monogr. Nect. 1. 27. pl. 9.; Gad. Cat. B. $M$. g. p. 17.1884 .

Anthreptes eximia, Licht. Nom. A2'. p. 56 (1854).

Promerops eximia, Gray, Hand-l. B. 1. p. 109. n. $18_{40}$ (1869)). Hab. Paragua; Jara.

4. Att. magnifica, Slarpe $\sigma 0$ N. v. Aramasit.

(M. S. T.)

Ethopyga magnifica, Sharpe, Nat. Ird. Aug. 1870 , p. 297; id. Tr. Lin. Soc. 2. ser. Zcol. 1. p. $332(1876)$, Tz'. P. Z. S. 


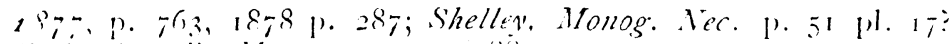
(ind. Cat. Bs. Mass. o p. $24\left(18 s_{t}\right)$.

Hab. Negross, (ébú.

5. Wt. shelleyi, Sharpe, ¿\& N. v. Sacsac.

(M. S. T.)

Aithopyga shellevi, Sharpe, Nal. 3 Aug. 1876, p. 297; id. Tr. linn. Soc. 2. ser. Zool. 1. p. 243; T2k. P. Z. S. $187 s, 1$. 621; Shelleyi, Monog. Nect. p. 75, pl. 24 et 29. fig. 1.; Ciad. (at. B. lius. 9. p. 29 (1884).

/tab. Paragua, P. Princesa, Balabac.

6. Wt. bella, Tiu o o N. v. Suing.

(M. S. T.)

Ait. Wella, Trueddale, An. et Mag. Nat. Hist. xx. p. 3.37 (гs7-); id. P. Z. S. 1877 , P1. 819, 829; Shelley, Mon. Ned. 1. 77. pl. 25 ; liad. (at. B. M. 9. p. 29 (1884).

Mirh. Surigao, Mindanao.

7. Vit. pulcherrima, Shartie, $\sigma, 0$ N. v. Tigbobolan.

(M. S. T.)

Ait. pulcherrima, Sharpe, Nat. 3rd. Aug. 1879, p. 297; Gad. (a). B. 1\%. q. $(188+)$.

Endrepanis pulcherrima, Shelley, Mon. Nect. p. 8.3. pl. 28. Ait dubia, Té. P. Z. S. $18 ; 8$, p. I 2.

End. dubia, Shelley, Monog. Nect. p. 85. pl. 20. fig. 2.

Hab. Dinagat, Samar, Leyte, Basilan.

3. Gén. CINNYRIS, Cuv.

8 C. sperata, (Lin). $\sigma, 0$ N. v. Suing.

(M. S. T.)

Le Grimpereau pourpré des Philippines, Briss. Orn. 3. p. 6.55 , n. 27. pl. 3r. fig. 2, 3. (1860).

Certhia sperata, Linn. S. N. i. p. г86, n. ${ }^{0}$ i7, (1760 ex Briss);

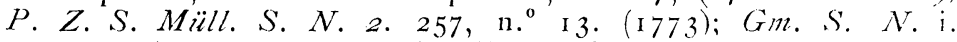
1). 477. n. ${ }^{\circ}$ I , et var. B. (1788); Lath. Ind. Orn. i. p. 28,3, n. ${ }^{\circ}$ 8. et var. g. (1790); Shaze, Gen. Zool. viii. p. 205. (1811).

Grimpereau des Philippines, I)aub. Pl. En!. pl. 2ff. fig. I, 2.

(erthia jugularis, $P$. J. S. Mäll. S. $N$. Anhang. P 98, n." 20) (1776 ex Daub.); Cass. Pr. Acad. Philad. 1864 , p. 244.

'Troisieme grimpereau, de l'islo de Luzón, Somn. Voy'. Nom. Guin. p. 63 , pl. 3o. fig. 9. (1776)

Soui-manga marron pourpré à poitrine rouge, Montb. Hist. Nat. Ois. v. p. $497(1778)$.

Le Grimpereau ou Soui-manga à gorge violette et poitrine rouges, Montb. t. cit. p. 499.

Rcd-Createsd Creeper, var. B. Lath. Gen. Syn. B. p. 708 ( 1781); ex Sonn. pl. 3o f. D.; id. Gen. Syn. B. 2. p. 706 n. ${ }^{\circ} 8$ (1781); id. Gen. Hist. B. v. p. 219 n. ${ }^{\circ} 8(1822)$.

Certhia chalybra, Scop, Flor. et Faun. Insub. 2. p. 91, n. ( 1786 ex Son).

Snui-manga à gorge violette, Audeb. et Vieill. Ois. Dor. 2. pp. 55,56 , pls. 32, 33. (1802). 
C. affinis, Shaw. Gen. Zool. viii. p. 208 (181 r, ex Audeb. et Vieill).

C. pusilla, Bechts, Kurze Vebers, p. i 78 n. 8 (I\& I2).

Cinnyris speratus, Cuv. Regn. An. i. p. $4 \mathrm{r}_{2}\left(\mathrm{I} 8_{17}\right)$; Vieill $N$. Dict. d'Hist. Nat. xxxi. p. 505 (1819); Bonn. et Vieill. Ent. Meth. ii. p. 593 (1823); Less. Man. d'Orn. ii. p. 56 (1828); id. Mraité d'Orn. i. p. 297, n. ${ }^{\circ} 4$ (I831); Shelley. Mon. Nect. p. I 3 I pl. 43 .

Nect. coccinigastra, Temm. Pl. Col. pl. 388, f. 3. (1826); Drapiez, Dict. Class. xv. p. 51 2; Gray Gen. B. i. p. 28 (1847); Von. Mart. J. f. O. 1866 , p. I 2, n. 48 (1866); id Prenss Exped. Ost-Asien, p. I 89.

Cin. coccinigaster, Less. Man. d'Orn. ii. p. 30. (1828).

Nect. sperata, Drapiez, Dict. Class. xv. p. 5 I I (1829).

Nect. philippensis, (part) Meyen, Reise Zool. p. 206 (1834).

C. zeylonica, Meyen. tom. Cit. p. 206 (ns Lin.)

Nect. affinis, Jard. Monog. Sun-birds, pp. 216, 262, pl. (1) ( 1843 ).

Leptocoma sperata, Cab. Mus. Hein. i. p. I05, n. ${ }^{6} 69$ (185()); Bp. Compt. Rend. 1854, p. 265.

Anthreptes expectata, Licht, Nom. Av. Mus. Berol. p. 56 (i 854 ).

An. sperata, Licht. tom. cit. p. 56 ( 1854 ).

Chalcomitra pusilla, Reichb. Handb. Scans. p. 280, n. $^{0} 649, \mathrm{pl}$. 568 figs. $387 \mathrm{I}-72$ (1854).

Nectarophila sperata, Rechb. tom. cit. p. 281. n. 651; Wald., Ibis, 1870 , p. p. 42; id. Tr. Z. S. ix. p. 200 (i 875$)$.

Nectaro aftinis, Reichb. Handb. Scans. p. 28I, n. 651, pls. 35I, 568 , figs. 3879-88, et 4066-67 (1854).

Nectaro coccinigastra, Reich. tom. cit. p. 283, n. 652, pl. 569 fig. 3882 .

Arachnot ra sperata, Gray, Hand-l. B. i. I I 2, n. I 398 ( I 869).

Cinnyris sperata, Gadow. Cat. B. Mus. 3 p. 63 (I884).

- Hab. Luzon, Manila, Laguna, Calamba, Bataan, Orion, Mindoro, Marinduque, Guimarás, Negros, Samar, Dinagat, Masbate, Paragua, P. Princesa.

9. C. juliæ (Tweed.) $\varnothing \circ$ N. v. Suing.

(M. S. T.)

Nectarophila julix, Tweed. P. Z. S. 1877 . pp. 535, 547; 1878 . p. $95 \mathrm{r}$.

Cinnyris julix, Shellev, Monog. Nect. p. I35 pl. 44; Gad. Cat. Mus. 9. p. 64 (1884).

Hab. Malanipa, Mindanao, Joló, Maimbun.

ı. C. aurora, (Tzv.) o\& N. v. Saritsit.

(M. S. T.)

Cyrtostomus aurora, Tweeddole; P. Z. S. 1878, p. 620.

(innyris aurora, Shelley, Monog. Nect. p. 149; Gad. Cat. B. Mus. 9. p, 88 (1884).

Hab. Paragua, P. Princesa, Inagauan.

I I. C. flagrans, (Oust) $O^{T} \rho \mathrm{N}$. v. Suing.

(M. S. T.)

Aethopyga flagrans, Oustalet, Journ. de l'Instit. 1876, p. 108. 
Cinnyris flagrans, Shelloy, $M, m g . N \geq c . p . \quad 1+7$, pl. 47 f.e 2.; Gad. Cat. B. Mies. 9. p. 88 (I8s4).

Hab. Luzon, Bataan, Orion.

2. C. guimaraensis, Stire. O" N. v. Ticuy.

(M. S. T.)

Hab. Guimarás.

13. C. jugularis, (Lin;. $\sigma \circ$ N. v. Suing

(M. s. 'T.)

:Le Grimpereau des Philippines, Briss. Orn. iii. p. 613, n. ${ }^{\circ}$ t, pl. 3o. fig. 2 ( 1760$)$.

Le Petit Grimpereau des Philippines, Briss. tom. cit. p. 616, 11." 7, pl. 32. fig. 5; Daub. Pl. Enl. 576 . fig. 3.

le Grimpereau olive des Philippines, Briss. tom. cit. p. 623, n. ${ }^{\circ}$ io, pl. 32. fig. 4; Daub. Pl. Enl. 576 . fig. 4.

Certhia jugularis, Linn. S. N. i. p. I 87 , n. 2 1 (1766, ex Briss. n. $\left.{ }^{\circ} 6\right)$; P. L. S. Mïll. S. N. ii. p. 256, n. ${ }^{\circ} 7$ (1773); Gill. S. $N$. i. p. 474, n. 7 ( 788$)$.

:C philippina, Linn. S. $N$. i. p. 187, n. ${ }^{\circ} 28$ (1766, ex Briss. n. $\left.{ }^{\circ} 4\right) ; P$. L. S. Müll. S. N. ii. p. 260. n. $^{\circ}$ 2 I (1.773); Latiz. Ind. Orn. i. p. 293, n. $9(\mathrm{r} 790)$.

C. tricollaris, P. L. S. Mïll. S. N., Anhaug, p. 99, 1..$^{\circ}$ it ( I 776) ex Buff. fig. 4).

Premier Grimpereau de l'Isle de Luçon, Sonn. Voy. Now. Ciuin. p. 63, pl. 3o. fig. A (1776).

Second Grimpereau de l'Isle de Luçon, Sonn. l. c. fig. B.

Soui-manga á gorge pourprée, Mont. Hist. Ois. v. p. 506 (1778).

:Philippine Creeper, Lath. Gen. Syn. ii. p. 7 II, n." I2 ( 78 I).

Grey Creeper, Var. a Lath. tom. cit. p. 714, n. ${ }^{\circ}{ }^{2} .^{2}$; id Cien. Hist. B. iv. p. $23 \mathrm{I}$, n. ${ }^{\circ} \mathrm{i} 9$.

C. quadricolor, Scop. Del. Faun. et Flor. Insub. ii. p. 91. n." 69 ( 786 , ex Sonn.)

(. gularis, Sparrm. Mus. Carls. pl. 79. «Martinique» (!) 1780; Lath. Ind. Orn. i. p. 300, n. ${ }^{\circ} 69$ (1790).

(. currucaria, Var. B, Lath. Ind. Orn. i. p. 286, n. ${ }^{\circ}$ i $5,1790^{\circ}$.

Soui-manga á gorge bleue, Aud. et Vieill. Ois. Dor. ii. pp. 51 , 52 , pls. 29, 30 ( 1802$)$.

(. 'currucaria et jugularis, Bechst. Kurze Uebers. p. 180, n." I5 (I 8 I 2 ).

(innyris zeylonicus, Cuv. Régne Anim. i, p. 4 I 2 (1887, ex Daubent. fig. 4); Vieill. N. Dict. d' Hist. Nat. xxxi p. 507 (Isig); id Encycl. Meth. ii. p. 594 (1823).

(.) philippinensis, (part.), Meyen, Reise Th. iii. p. 206 (183t).

(.) sperata $\delta$, Meyen, tom. (it. p. 207.

Nectarinia jugularis, Jard. Monog. Sun-birds, pp. 223, 267, p1. 25. fig. 2 (1842); Gray. Gen. B. i. p. 98 ( I 1 47).

Coreba gularis, Gray, Gen. B. i. p. Iо1, n. ${ }^{\circ}$ (18+7); B3. C: A. i. p. 400, n. $^{\circ} 4\left(185^{\circ}\right)$.

Nectarinia pectoralis, Cab. Mus. Hein. iii. App. p. $33(13+9$, ex Daubent. fig. 4).

Cyrt. jugularis, Cab, Mus. Hein. i. p. 105, n. 570 (185); Reichb. Handb. Scansorio, p. 309, n. ${ }^{\circ} 723$, pl. 573 . is 5.380 (1854). 
Cyrtostonus gularis, Richb. tom. cit. p. 310; n. ${ }^{\circ}$ 712, pl. 519. . tig. 3997 .

Arichnera jugularis, Grav, Hant-l. B. i. p. Ir 2 n. ${ }^{\circ}$ I 389 (1869).

Aracmontira jugularis, Waldon, Ibis, 1870 , p. 27; id. Trans. Zo. : Soc. ix. p. 200 (1875).

(inn is jugulari, Gadou'. Cat: B. Mus. 9. p. 84. ¿.s. Luzon, Mindoro, Marinduyue, Bohol, Dinngat, Negros, Pan, Iloilo, Caniguin, Sinar, Lète, Siquijor, Gainarás, Mi: L.: 1.20, Bısilan, Joló.

I- $p$ pectoralis. (IIorsf) $\sigma \rho$ N. v. Saritsit.

Nectria pectoralis, Horsf. Tr. Linn. Soc. xiii. p. I67 (I820); V..., Mem. Rinffles, p. 673 (1831); Jards. Monogr. Sunbirs, pp. 226,.226, pl. 25. fig. I; Blyth, J. A. S. B. xi, p. 370 (1846); Gray, Gen. B. i. p. 98 (1847!; Blyth, Citt. B. if ss. As. Soc. p. 225. n. ${ }^{\circ} 866$ (18+9); Matley \& Dillreyn, Vat. tist. Labarn, p. I5 (1855); Hartl. J. f. O. 1855 , p. $319 ;$ sclat. P. Z. S. 1863 , p. 220; Wallace, tom. cit. p. 486; Peis:!n, Reise Nazura, Vög. pp. 52, r6r (1865); Tytler \& Beivin, Ibis, 1867 , p. 322 ; Ball, Str. F. 1874, p. 334; G.l. dow. Cat. B. Mus. 9. p. 88 (1884).

Pector:1 Creeper, Lath Gen, Hist. B. ix. p. 266, n. ${ }^{\circ} 75$ (r822'.

Nectarinia eximia, Temm. Pl. Col. 138. figs. I, 2 (1823); S. Mïll. \& Schl. Verh. Nat. Gesch., Zool. Ar. p. 6i, n. ${ }^{\text {io }}$ $(18+6)$; Finsch. Neu-Guinea, p. I63 (1865).

Cinnyris ornatus, Less. Dict. Sc. Nat. i. p. I5 (1827, ex Temm. Pl. Col. 138. figs. I, 2); id. Man. d' Orn. ii. p. 36 (1828).

('in. luteoventer, Less. Trailé d' Orn. i. p. 298, n. ${ }^{\circ} 29$ (1831); Pucheran, Rev. et Mog. Zool. 1853 , p. 488.

( in. pectoralis, Bp.C. A. i. p. 408, n. ${ }^{\circ} 29$ (1850); Bernstein, J. f. O. 1859 , p. 179; Sharpe, P. Z. S. 1875, p. 106; N:cholson, Ibis, 1879 , p. 166; Sharpe, tom. cit. p. 259; id. $P$. $Z$. S. 1879 , p. 341; Shelley, Monog. Nect. p. 165, pl. 53 Gadoze. Cat. B. M. 9. p. 88 (1884).

(Yrtostomus pecturalis, Cab. Mus. Hein. i. p. 105 (1850); Reichb. Handb. Scans. p. 307 , n. ${ }^{\circ} 722$ pl. 570. firs. 3891.92 (1854; Horsf. \& Moore Cat. B. E. Y. Co. Mus. ii. p. 739, n. $^{\circ}$ ro79 (185), Hzume, Ness and Eggs. Ind. B. p. I55 (1873); Sa!'ad. Ann. Mus. Civ. Genova. $\therefore$ p. I7o (1874); id Ann. Mus. Civ. Genova, xiv. p. 212 (1879).

Cy. eximius, Bp. Comptes Rendus, 1854, p. 265.

Arachnothera pectoralis, Gray, Hand-l. B. i. p. I 1 2, n. ${ }^{0}$ r 385 (1869).

Arachnechthra pectoralis, Waldeu, Ibis, 1870 , p. $25 ;$ Ball, Str. F. 1872, p. 64; Hume, Str. F. 1874 , p. 196; Ball, J. $A$. S. B. xxxix. p. 51 (1870); Treedd. Ibis, 18 Fi, p. 302. Hab. Samar, Paranas, Burongan; Java, Sumatra.

4. Gén. ARAChNothera, Trm.

15. A. longirostris, (Lath.) $\sigma \circ$ N. v. Saritsit.

(M, S. 'l:)

Long-belled Creeper, Lrth. Gen. Syn. Supp. i. p. I33 (1787); $\imath$ d. Gen. Hist. iv. p. $25 \mathrm{I}$ (1822). 


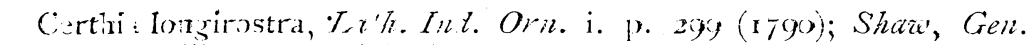
Zool. viii. p. 270 ( $181 \mathrm{I})$.

Sini-manga á lo.ux bec, Auder. ef Vuti. Os. Dor. ii. p. 65

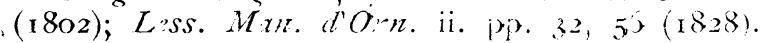

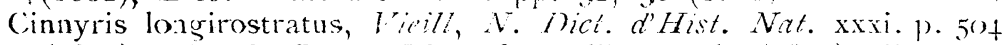
(1819); Horsf. Trans. Linn. Sic. xlii. p. 167 (1820); Bonn. et Vieill. Dnc. Meth. ii. p. 59 I (Isz3).

Cia. Iongirostratu, Loss. Mre dorn. ii. p. 32 (1828.)

Nectarinia longiros rata, Temm. Pl. Col. \& f. fics. I (1823.)

Arachnotera longirustrata, Teinm. Pl. Col. pl. 388 , t x. (1825; Loss. Man. d'Orn. ii. p. 32 (1828); id. Tratéd'Orn. i. p. 229 (183I); Mäll. \& Solulag. Natumel. Gesch. Landen Vo!kcuk. Pp). 69, 71 (1846); Low, Sarawak. p. Ho (17+S); Blyth, Cat. B. Mius. As. Soc. pp. 222, $3+7$ (1849); Bp. C. A. i. p. 409; Salo. Ann. Mui. C. Gen. v. P. 185 (187t); Tivedale, Ibis, 1877 ,

'p. 3os; Brägsem. Abhansl. nat. Ver. Bremen, v. p. 458 (1877); Hume \& Incvison, Str. F. vi. pp. I74, 507 (1878); Hume Str. F. vii. p. 35 (1878; Salu. Anz. Mus. Co. Genova, xiv. p. 214 (1879); Shelley, Monogr. Nect. p. 357, pl. I1 4 ; Hume \& Mavis. Str. F. 18.78 , p. 330; Huine, Str. F. 1879 , p. 300; Oxtes, B. Br. Burm. i. p. 330; Gxdow, Cat. B. Mus. 9. 1). $103(1884)$.

A. inornata, Blyth (nec Temm.), J. A. S. B. xii. p. 982 (1 $8+3)$.

A. longirostris, Blyth, J.A. S. B. xii. p.982, p. $43(1846)$ Schl. Hanol. Derk. 1. 237 (1857); Gray. Gen. B. i. p. 99; Pelzeln, Novar. Reise. Vög. pp. 52, I6I (1865).

A. atfinis, Blvth, (nec Horsf.), J. A. S. B. xv. p. 43 (18+6); Horsf. \& Moore, Citt. B. E. I. Co. Mus. ii. p. $730(18+7)$; Jerd. B. Ind. i. p. 361 (1862); Blyth, Ibis, 1856, p. 364; Hume, Str. F. 1874 , p. 473, 1875 , p. 85; Blyth \& Walden, Cat. Mamm. \& Birds Burma, p. I 40 (1875); Blanford, Ibis, 1870 , p. 467.

A. pusilla, Blyth. Cat. B. Mus. As. Soc. p. 222 (1849).

A. Iongirustris, Reichenb. Hanob. Scansor. P. 3I 5, pl. 592 figs. $4018-19$ (1854).

Arachnothera? Walden. Trans. Zool. Soc. viii. p. 70 (1874). IHab. S.mar, Paranas, Leyte, Paragua; Borneo, J:va, Célebes.

16. Ar. Himbifera, Two $\sigma o$ N. v. Aramasit.

(M. S. T.)

Arachnothera flannifera, Twedtrle, P. Z. S. 1878 . p. $3+3$; Shelleyi. Monog. Nect. p. 361, p. I1 5; Ciadow. Ciat. B. Mus. 9. p. Iot. 1887 .

Hab. Samar, Leyte, Paragua; Borneo, Java.

17. Ar dilutior, Shanpe ơ N. v.

(M. S. T.)

Arachnothera dilutior, Sharpe. Nature, 3 d. Aug. 1876, p. 296; i!l. Tr. Iin. Soc n. ser. Zoo!. i. p. 3+1; Tw. P. Z. S. 1878 , 1. 621; Shalley, Mongg. Nect. 1. 363, pl. 1 16 ; Gadow Cat B. Mus. 9. p. I05. ( 1884 ).

Hab. Paragua, Inagauan. 


\section{Gén ANTHOTHREFTES, GADOw.}

18. A. chilorogaster, G:aduc. $\sigma \circ$ N. v. Saritsit.

(M. S ' T )

Anthothreptes malaccensis, (pt) Salà. Au. Mas. Civ. Gen. vii. p. 661 (1875); id. ix. p. $57(18-6)$; id. Atti R. Acc. Torin. xii. p. $320(1877)$.

Anthreptes chlorigaster, Sharpe, Tr. Lin. Soc. 2d. ser. Zool. i. p. $342(1877)$.

Ant. chlorigastra, Shelley, Monog. Nect. p. 321, pl. 103. fig. I, Ant. rhodolitma, Shelley, Monog. Nect. p. 3 r 3 pl. ior. fig. I. Anthothreptes chlorogaster, Gacter. Cat. B. Mus. g. p. ${ }_{123}$ ( 18 I4).

Hab. Nasbate, Negros, Cebú, Paragua, P. Princesa, Mindanao, Joló, Cagrayan de Joló; Célebes.

19. An griseigularis, $\delta \subsetneq$ N. v. Aramasit

(M. S. T.)

Anthothreptes griseigularis, Treeddale, P. Z. S. 1877, pp. 8I 7 , I.30, 1878 , p. I 3 ; Gadow, Cat. B. Mus. g. p. I 26. (1884).

Hab. Mindanao, Samar, Borongan.

\section{Fam. MELIFÁGIDOS.}

Subfam. MIZOMELINOS.

I. Cién myzomela, Vig. et Horsf.

\section{Mg, rubratra, (Lass.) o' N. v. Uuit-uit.}

(M. S. T.)

Cinnyris rubrater, Less. Voy. Cog., Zool. p. 678 (1826); id. Man. d'Orn.. ii. p. 55. (1828); Kitt. Kupf. Vög. pl. 8. f. 1; id. Denkw. einer Keise, i. pp. 364, 381 (1858).

My. rubratra, Bp. C. R. t. 38 . p. 263 (1854); Hartl. P. Z.S. 1868, p. $5 ;$ id. et Finsch, P. Z. S. 1872, p. 94; Finsch, Journ Godeff. Mus. xii. p. 26; Forbes, P. Z. S. 1879 , p. 270; Gadow. Cat. B. Mus. 9. p. I 29 (1884).

Hab. Islas Carolinas, Palaus.

2. My. chermesina, Giray. N v. Uit-uit.

Myzomela chermesina, Gran, Gen. B. i. pl. 38 (1840); iä. Cat. B. Trop. 3sl. p. гा.; B̈p. C. M. i. p. 394; Gray, P. Z. S. 1858, P: 190; id. Cat. B. New Gu.n. p. 55; id. P. Z. S. 1861 , p. 434; Rosenb. Nat. Tijdschr. Ned. Ind. xxv. 'p. 236; id. J. f. $O .186$, p. I 22; Gray, Hand-l. B. i. p. i 54. n. 1989; Rosenb. Malay. Arch. p. 553; Forbes, P. Z. S. 1878, P. 352, 1879 , p. 273; Salv. Orn. Papuasia, ii. p. 306; Gadow. Cat. B. Mus. o. p. 137. (1884).

Hab. Ponapé, Carolinas. 
Subfam. ZOSTEROPINOS.

2. ZOSTEROPS, Vig. ET HoRsF.

3. Z. everetti, $T w, \sigma \circ$ N. v. Ticuit

(M. S T.)

Znstcrops everetti, Tweeddale. P'. Z. S. 187 . Pp. 762, 829; id. ibid. (г878), pp. I I, 950; Wardlaw Ramsay, Orn. Work: Tw. p. $65^{8}$ (1881); Gadow. Cat. B. Mus. 9. p. i63. (I88+). Hab. Dinagat, Cebú, Mindanao, Joló.

4. Z palpebrosus, (Tim.) $\sigma \rho$ N. v Ticuic.

(M. S. T.)

Sylvia palpebrosa, Tem. Pl. Coll. 293 f. 3. (1824).

Zosterops maderaspatensis, Jerd. Madr. Journ. xi. p. 7. (18+o).

\%. nicobarica, Blyth, J. A. S. Bieng. xiv. p. 563 (1845); Gra:", Gen. B. i. p. I98 (1848); Bp. Consp. i. p. 399 (1850); Griai, Hund.l. B. i. p. 163, n. ${ }^{\circ}$ I 49 (1869).

\%. palpebrosus, $J$. A. S. Beng. xiv. p. 44 (1846); Gray, Grin. B. i. p. $19^{8}$ (1848); Blyth, Cat. B. Mus. As. Soc. p. 2w (1849); Bp. Consp. i. p. 398 (1850); Lelaart, Prodr. (at. $)$ I21 (1852; Layard, Ann. \& Mag. Nat. Hist. 1853, p. 267; Horsf. \& Moore, Cat. B. E. I. Co. Mus. ii. p. 265 (i 854 ); Jeri. B. Ind. ii. p. 265 (1863); Garll.J. F. O. 1865 , p. I4 Blyl., Ibis, 1867 , p. 33 ; Bervan, t. c. p. 328 ; Stoliczka, J. A. S. Beng. xxxvii. p. 5I (1868; Beazan, Ibis, 1868 , p. 82; Tytler, t. c, p. I97; Pelz t. c. p. 3II ; Brooks, Ibs, $186 \%$, p. 5i; Gray, Hand-l. B. i. p. 162, n. ${ }^{\circ} 2122$ (1869); Godwin-Austen, J. A. S. Beng. xxxviii. p. I09 (1870); Holdsw. P. Z. S. 187-, p. 458; Hume, Nest. \& Eggs. Ind. B. p. 356; p. 397 (1873); Cock \& Marsh. Str. F. 1873, p. $35^{6}$; Idam, t. c. p. $3^{8}+$; Hayes Lloyd, Ibis, 1873, p. 412; Legge, Ibls, 1874 , p. 22; Walden, t. c. p. I43; Ball, Str. F. 1874 , p. 4r7; Hume, Sl: F. 1875 , p. 143; Brooks, t. c. p. 252; Hume, t. c. p. 49r; Butler, t. c. p. 49r; Blyth. \& Wald. B. Burm. p. I 1.0 (187.5); Fairb. Str. F. 1876 , p. 260; Hume, t. $\therefore$. p. 463; Fairb. 'S. F. 1877 , p. 407; Davids. \& Wenden, S. It. 1878, p. 8+; Bull, t. c. p. 202; Auders. Zool. Exped. Zuman, p. 63I (1878); Butler, Cat. B. Sind \& c. p. 42 (1879); Legge, B. Ceylon, 1). 582 (1879); Hume, Str. F. (1879), p. I04; Scully, t. c. p. 322; Vidal, Str. F. 1880 , p. 69; Butler, t. e. p. 41 3; Hume, t. c. p. 505; Butler, Cat. B. South. Bomb. Pres. p. 57 (1880); God. Cat. B. M. 9. p. I65 (1884).

Zosterops japonicus, (nec T. \& S.) Sreinh. His, 1861, p. 3.5. Z. simplex, Swinh. Ibls, 1861 , p. 331, 1863, p. 294 ; Harti. J. F. O. 1865 , p. 13; Swinh, Ibis, 1866 , p. 121; Giray, Hand-l. B. i. p. I63, n. ${ }^{\circ} \mathrm{j}_{\mathrm{I}}$ (1869); Sovinh. Hi $s, 1870$, p. 348; Gould. B. Asia, part. xxiii. (1871); Srvinh. P. Z. S. 18;8, p. 34); David \& Austalet, Ois, Chine, p. 85 (1877) Finme, Str. F. 1878 , vol. ii. p. 403 ; id. Str. F. $18 \% \%$, p. so4.

Z. nicobariensis, Hume, Str. F. 1874 , p. 242, 1879 , p. 104.

Z. palpebrosa nicobariensis, Hume, Str. F. 1876 , p. 291. Hab. Luzón, Laguna, Samar, Borongan; China, Ceilán. 
5. z. meyeni, hs ơ N. v. Ticuit.

Dicarum Harum, (nec Hersf), Kittl. Ciatff. lög. pl. 19, li... 2. $(18,321)$ id. Mem. sicat. S. Petersb. $183.5, \mathrm{pl} .3$.

silvia flara, Megen, Acta Acad. Cas.-Leop. Vat. Cur. xvi. Suppl. p. $203(183+)$.

Zosterops., sp. (iray. (ien. B. i. p. $198(1848)$.

7. meyeni, Rp. Consp. i. p. 398 (rs 50$)$; Resch. Hanib. Merop.

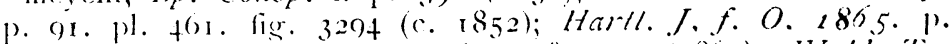
It); Gray, Hand-l. B. i. p. I62, n." 2123 (1869); Hald. T). 2. S. ix. p. 190 (1877); Gadoue. (at. B. .1. g. p. I $80(188+)$. Hab. Luzon, Manila, Cagayán, Mlaalá.

$6 \mathbf{Z}$ semperi. II. it Fïnsch.

Z. scmperi, Hartl. ei Finsch, P. Z. S. 1868 , p. I17; Grai, Hand-l. B. i. P. 163, n. ${ }^{n} 21+$ (1869). Hartl. P. Z. S. 139; p. 95; Finsch, Journ. Mus. Ciodeff. Heft. viii. p. i6, Taf. 4 t. I; id. P. Z. S. 1880, P. 575; id. Ibis. 1881, P. 115; Gadow. Cat. B. M. q. p. I83 (1884).

Hab. Islas Carolinas, Palaos.

7. Z. nigrorum, Tiw. t $\mathrm{N}$. v Suing.

(N. S. T.)

Z. nigrorum, Taecddale, P. Z. S. 187s, p. 286; Wardlaw Ramsai, Orn. Works. Treedd. p. 658; Gadari. 9. p. 186 (1884).

Hab. Negros.

8 Z. hyypolais, Hartl. el Finsch.

Z. hypolais, Hartl. ct Finsch, P. Z. S. 1872 , p. 95; Gadoz'. Cat. B. M. g. p. 186 (1884).

Hab. Carolinas, Uap.

9. Z. oleagina. H. et $F$.

Z. oleagina, Hartl. et Finsch. P. Z. S. 1872 , p. 95; Gad. Cat. B. $M$. g. p. 187 (1884).

Hab. Carolinas, Uap.

Io Z. conspicillata, (Kittl.) $3 \mathrm{~N}$ v.

(M. S. T.)

Dicxum conspicillatum, Kittl. Kupf. Vög. pl. г9. f. I (1832); id. Mem. Acad. S. Petersb. ii. pl. 4. (1835).

Z. conspicillata, Gray, Gen. B. i. P. 398 (1848); Bp. C. Av. i. p. 398 (1850); Reich. Handb. Merop. 1). 92, pl. 46r. f. 3295 (c. 1852); Gray, Cat. B. Trop. Jcl. Parif. Ocean. p. ${ }^{6}$ (1859); id. Hand-l. B. i. p. 163. n." 2141 (1869); Gadon', Cat. B. Mus. 9. p. I 87 (1884).

Hab. Guam, Marianas. 
11. Z. Eemiflava, Niaton.

\%. scniflava, E. Nerion, Ibis, pp. 354, 359, I867; Gray, Handi-l. B. i. 1. 163, n." 2160 (1869); Hartl. Vög. Madag. 1). 1114 1877 ; Oust. Bull. Soc. Phlom. 1878, p. I7o; Gad. B. M. (at. 9. p. 190 (1884).

Hab. Islas Marianas, Scychelles.

12. Z. modesta, Niwlon. ठ N. v.

(M. S. T.)

7. modesta, E. Nereton, 1867 , p. 345, 359; Gray, Hand-l. B. i. p. 163, n." 2159 (1859,; IIartl. Vös. Madag. p. 98, 1877; Oust. Bull. Soc. Phlom. 1878 , p. izo; Gad. Cat. B. M. o. p. I94 (1884).

Hab. Marianas.

13 Z. finschii, (Ha/li). N. v.

'Tephras finschii, Hartl. P. Z. S. 1858 , pp. 6, i17) pl. 3.

Z. tinschii, Gray", Hant-l. B. i. p. 164. n." 2 I7.3 (1869)? Harl!. ct Finsch. P. Z. S. 1862 , p. 96; Gad. Cat. B. M. 9. p. 197 (1884).

11ab. Carolinas, Pakos.

14. Z ponapsngis, Finsih. N. v.

(M. S T)

Z. ponapensis, Finsch, Journ. Mus. Godeff. Heft. xii. p. 27, 'Taf. 2. f. I; id. P. $Z$. S. 1877, p. 778 ; id. Ibis, 1881, p. 11 5 ; Gad. Cat. B. M. g. p. 198 (1884).

Hab. Ponapé, Carolinas.

15. Z. basilanica, Steese. t \& N. v. Mananataon.

(M. S T.)

Hab. Samar, Paric; Basilan.

Subfam. MELIFAGINOS.

1. Gen PHILEMON, Vimilot.

1. $\mathbf{P h}$. philippinensis, Steere. t N. v. Ticuit.

Hab. Samar.

\section{Grupo: FRINGILIFOR MES}

Fam. DICÉIDOS.

I. Gén. DIC RUU, Cưv.

I. D. retrocinctum, Gonld. 0.0 N. v. Pipit.

(M. S. 'T.)

Dicæum retrocinctum, Gould, Ann. \& Mag. Nat. Hist. x. p. 114 (1872); Walden, Trans. Z. S. ix. p. 200 (1875); Gould, 
B. Asia, part. xxxvii. (1875); Salvat. Ann. Mils. Civic. Geno:" viii. p. 309 (1876); Irird!ze Rtmsay, Ora. IVorks Twzed. 1. 658 (1881). Sharpe, Cat. B. M. 10. p. 35 (1885).

Hab. Luzón, Manila, Mindoro, Baco, Cébú.

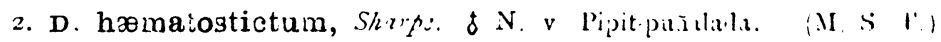

Dicxum hamatostictum, Sharpe, Nature, Aug. 1876, p. 297; it. Transl. Linn. Soc. and ser. Z) o!. vol. i. p. 339 (rg76); it. (i.zt. B. M. 10. p. 35 (1885); Tivedt. P. Z. S. 1878, p. 235; Wardlaw Ransay, Orn. Wories Tiust.t. p. 658 (1331).

Hab. Guimaras, Negros, Pan.ty.

3 D. rubriventer, L:s. $\sigma^{\circ} . \mathrm{N}$ v Pipit p.uñ.lade

(II. S. I.)

I.e Manikor de la Nouvelle Guinée, Driçnn!. P!. Ėı. v. pl. 70j. fig. 2.

I,e Manikor, Buff. Hist. Nett. Ois iv. p. 43 r.

Papuan Manakin, Lath. Gen. Syn. iv. p. 532 (1783).

Pipra papuensis, Gin. Syst. N.ut. i. p. Iont (1733).

Muscicapa papuensis, Temm. M.tn. d'Orn. i. p. Ixvii (1820); it. Pl. Col. Tabl. Méh. p. 25 (1839).

Dicæum rubriventer, Less. Trait', p. 303 (1831); Salvat. Ant. Mus. Civic. Genor. viii. p. 5 I I (1876); Trueedd. P. Z. S. 1877 ,

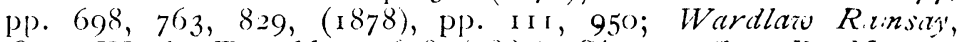
Orn. Wories Tived.t. p. $65^{8}$ (1831); S.i.trp:, Cit.. B. M. 1 J. p. 36 (I 885$)$.

D. schistaceu.n, Trusedd. Ann. \& M tg. Nxt. Hist. (4) xx. p. 537 (1877); id. P. Z. S. 1878 , p. I I ; Ward!.uw Ransay, Oria. Works Tweedd. p. 658 (1881); Sharpe, P. Z. S. 183.3; p. 53j.

D. papuense, Hartl. Rev. Zoul. I8+5, p. 47; Gray, Gen. B. i. p. IOU (1874); Bp. Conss. i. p 403 (1850'; Rsichens. II:aidtb. Scansoria, p. 24), fig. 3782 (1853); Gray, P. Z. S. 1859 , p. 19o, id. Cat. B. New. Guinad, p. 55 (1859); id. P. Z. S. 1861 , p. 434; Finsch, Nen-Guinea, p. 163 (1855); Gray, Hant-l. B. i. p. I14, n. ${ }^{\circ}$ I421 (186́).

D. rubriventris, Pucher. Rev. Zool. 1845, p. I35, note.

D. retrocinctum, Gould. Ann. \& M.tg. Nat. Hist. x. p. II (1872); id. B. Asia, part xxvii. i1875, Wald. Trans Z. S. ix. p. 199(1875). Hab. Luzón, Manila, Laguna, Calanba, Cebá, Dinazat, Mi.1danao, Zamboanga, B.silan, Marinlu.ju, Mindoro.

¿ D. hy poleucum Sharp: \& $\mathrm{N}$ v Uit-uit.

(M. S. T.)

Dicaun hypoleucum, Sharpe, Nature, Aug. 1876, p. 293; id. Trans. Linn. Soc. and ser. Zool. vol. i. p. 339; id. Cat. B. M. 10. p. 37 (1885); Trueedd. P. Z. S. 1879, p. 72; Warlaw Ramsay, Orn. Works Tweedd. p. 658 (1881).

Hab. Basilan, Malamaui.

5. D. mindanense, Tw. $\delta \mathrm{N}$ v. Pipit.

(M. S. 'T.)

Dicreum mindanense, Tzeedd. P. Z. S. 1877 , p. 547; id. Repor l'oy. 'Cinallungu,' Birds. p. 20, pl. 5. fig. I (1881'); Warlta: 
Ramsay, Orn. Works Tiveedd. pp. 471, 638 (1881). Sharpe, (at. B. M. 10. p. 37 (1885).

Mab. Pasananca, Zamboanga, Mindanao.

6. D. trigonostigma, (Scop) $\hat{\imath} \mathrm{N}$ v. Uit-uit.

(M. S. T.)

1.r Grimpereaur sifleur de la Chine, Sonn. l'oy. Ind. Orient. p. 2 Io, pl. 6r. fig. $2(1776-82)$.

(erthia trigonostigm?, Scop. Del. Flor. et Fann. Insubr. ii. p. 91 ( 7786$)$.

()range-breasted Creeper, Lath. Gen. Syn. Suppl. p. г 32 (г787).

(crthia cantillans, Lath. Ind. Orn. i. p. 299 (1790).

J)icaum cantillans, (u:. Reggne An. i. p. 410 (1817; Vieill N. Dict. a'Hist. Nat. ix. p. 409 (1817); Temm. Pl. Col. iv. pl. 47 s. fig. 3 (1829).

1). croceoventre, Vigors, Mem. Raffl. p. 673 (1830); Mottley i Dillw. N. H. Labuan, p. I 7 (1855).

1). trigonostigma, Gray, Gent. B. i. p. IOO (1847); J3lyth, Cat. B. Mus. As. Soc. p. 226 (18+9); Bp. Consp. i. p. $433(1850)$; Cab. Mus. Hein. Th. i. p. 98 (1850); Reichenb. Handb. Scansoria, p. 24I, figs. $3788-89$ (1853); Horsf. \& Moore, Cat. Is. E. I. Co. Ms. ii. P. 748 (1854); Sclater P. Z. S. 1863 , p. 220 ; Wald. P. Z. S. 1866 , p. 545; Beavan, 16.s, 1869 , p. 422; Gray, Hand-l. B. i. p. 114, n. ${ }^{\circ} 416$ (1869; Grod-

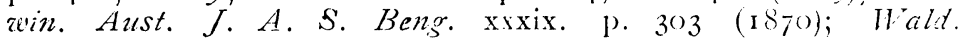
Jbis, 1872, p. $380 ;$ Hume. Sitr. F. 1874 p. 473 ; Satuad. Uic. Born. p. I65 (1874)) Blyth \& Wald. B. Burin. p. It2 (1875!; Walden Ibis, 1876 , p. 349, pl. x. fig. 2; Sharpe, Ibis, 1870 , - p. 42; id. Trans. Linn. Soc. and ser. Zool. i. 1) 340 (1876; Tweet. Ibis, 1877 , p. 302; Hume, of Dxoisoil, Sir. F. 18-8, p. 194; Sharpe, Ibis, 1879 , p. 261; it. P. Z. S. 1879,1 . 343; Hume, Str. F. 1879 , 1. 90; Bingham, Str. I. 188n, 1. 195; Wardlaw Ramsay, Orn. Works Truedd. p. 958 (1881); Sh: pe, P. Z. S. 1881 , P. p. 795; 1d. Cat. B. Mus. 10. p. 38 (1885); Müller, Vög. Insel Salanga, p. 22 (1882); Oate's, $B$. Brit. Burm. i. p. 336 (1883).

Hab. Negros; Burneo, Java, Sumatra.

7. D. dorsale, Sharpi. of N. v Uit-uit.

(M. S. T.)

Dicaum dorsale, Sharpe, Nature, Aug. 1876, p. 298; id. Trans. Linn. Soc. and ser. Zool. i. p. 340 (18;6); id. (at. B. M. 1 (i. p. 40 (r885). Treed. P. Z. S. 1877,1 . 763 ; Conld. B. Asia, part xxx. (1877); Wardlaw Ramsay, Oill. "l'oins Treed. 1). 658 ( I 88r).

Hab. Cebú, Panay.

3 D. xanthopygium, Tre C:O N. v. I'ipit-dilio.

(M. S. T.)

Diceum xanthopygium, Tweedd. Ann. \& Mag. Nat. Hist. (4) xx. p. 95 (1877); id. P. Z. S. 1877 , p. 698, pl.1xxiii. fig. I; Wardlaw Ramsay, Orn. Works Tweedd. p. 658 (I881); Meyer, Vög. Ostind. Areh. p, 39 (1884); Sharpe, Cat. B. MI. 10. P. 40 (1885).

Hab. Luzón, Mindoro, Calapan. 
9 3. cinereigulare, Tiv. $\sigma 0$ N. v. Uit-uit.

(M. S. T.)

1)irxum cinercigulare, Tiweedd. P. Z. S. 1877, p. $829 \& 1878$, P. 950; W.rddla?c Ramsay, Orn. Works Tieedd. p. 658 (r88r) Sharpe, Citt. B. M. 10. p. 40 (1885).

Hab. Mindanao, Placer, Zanboanga, Samar, Paranas, Leyte.

io D. bistj, Stecre.

Hab. Siquijor.

I. D. pvgmæum, (Kittl) $\sigma \rho$ N. v Uit-uit.

(M S. T.)

Nectarinia pygman, Kittl. Mem. pres. Acad. St. Petersb. ii. pts. I, 2, pl. 2 (1833).

Dicaum pygmaun, Gray, Gen. B. i. p. 100 (1847); Bp.Consp. i. p. 403 (1850); Reichenb. Handb. Scans. p. 242 fig. $378 \mathrm{I}$ (1853); Gray, Hand-l. B. i, p. I 5 5, no. I436 (1869); Sharpe, Cat. B. M. 10. p. 43 ( 1885$)$.

Myzanthe pygmaa, Walden, Trans. Z. S. ix p. 200 (1875; Sharpe, Trans. Linn. Soc. and ser. Zool. i. p. 352 1876;; Truedd. P. Z. S. 1877 , p. 698; 1878, pp. 343, 620; Wardlaw Ramsay, Orn. Works Tweedd. p. 658 (188г).

Hab. Luzón, Manila, Montalban, Leyte, Guimarás, Paragua, Negros, Marinduque, Mindoro.

12. D. everetti, Tw. $\sigma \circ$ N. v. Uit-uit.

!)icæum everet i, Truedd. Ann. \& Mag. Nat. Hist. (4) xx. p. 537 (I877); id. P. Z. S. 1878 , p. II I, Wardlaw Ramsay, Orn. Works Tweedd. p. 658; (1881); Sharpe, Cat. B. M. 10. p. 47 ( 1885 ).

D. modestum, Tweedd P. Z. S. 1878. p. 380; Sharpe, Z. S. 1883 , p. 580 .

Myzanthe modestum, Wardlaw Ramsay, Orn. Works, Tweedd. p. 658 (188I).

Hab. Dinagat, Panaon, Samar, Borongan, Loquilocun.

2. Gén, PRIONOCHILUS, StrickL.

13. P. samarensis. Stecre oo N. v. Uit-uit.

(M. S. T.

Hab. Samar, Paric, Borongan.

14. P. percussus, Tim. o 0 N. v. Uit-uit.

Pardalotus percussus, Temm. Pl. Col. iii. pl. 394. fig. 2 (i 826). Prionochilus pèrcussus, Sharpe, P. Z. S. 1883, p. $580 ;$ id. Cat. B. - M. 1o. p. 65 ( 1885 ).

Hab. Paragua, Puerto Princesa; Java. 
15. P quadricolor, Tiu. o N. v. Uit-uit.

Prionochilus quadricolor, Tzecedd. P. Z. S. $187 \%$, p. 762, pl. Ixxvii. figr. 2; Thardlaze Ramsay, Orn. I'orks Taied, p. 65. (1881); Skarpe, Cat. 13. M. 10. 1). 70(1885).

Hab. Panaon, Cichú.

16. $\mathbf{P}$ olivaceus, Ti. \& $\mathrm{N}$ v. Uituit.

(M. S. T.)

Prionochillus olivaceus, Tieedd. Ann. \& Mag. Nat. Hist. (t) xx.

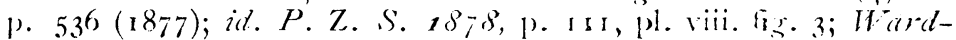

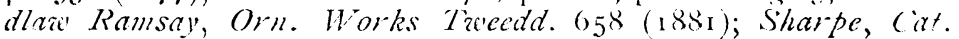
13. M. 10. p. $75(1875)$.

Hab. Dinagat, Samar, Paric.

17. P. xanthofygius, Sat $z$. o, 0 N. v. Uit-uit.

(M. S T.i

Prionochilus xanthopygius, Salvad. Alti R. Accad. Torin. iii. p. 416, cum fig. (1868); Walden, Tbis, 1872, 1). 379; Scla'er, Ibis, 1874 , p. 2; Salvad. Uic. Borm. 1. 162 (1874); Sharpe, Ibis 1877, p. 17; id. Ibis, 1879 , ए. 261 ; id. I. Z. S. 1831 . p. 795 ; id. Cat. B. M. 10 p. 66 , (1885).

Hab. Paragua, P. Princesa; Bornco.

18. P. Joannes, Stcire.

Hab. Paragua.

Fam. HIRUNDÍNIDOS.

I. Gén. CHELtbON, Boie

1. Ch. urkica, (Lin.) $0^{\top} \rho$ X. v. Langay-lngagan. (M $\mathrm{S} \mathrm{T}$ )

La petite Hirondelle oa le Martinet á cul blanc, Briss. Orn. ii. p. 490 ( I 760$)$.

Hirundo urbica, Linn. Sist. Wat. i. p. 3tt (1766); Temm. Man. d'Orn. i. p. 428 (1885); Roux, Orn. Provenc. pl. I44 (1825); Herner, Atlas, (helidones, pls. 2, 3 (1827); Naum. Vög. i)eutchl. vi. p. 75, Taf. $1+5(1823)$; Mén'tir. Cat. ras. (auc. p. 45 (1832); Selby, Brit. B. p. 213 pl. 42 fig. 2 (1833); Gould.

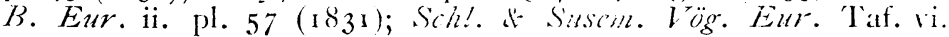
fig. I (1839); Macgill. Brit. B. iii. 1). 573 (1840); Nordm. in

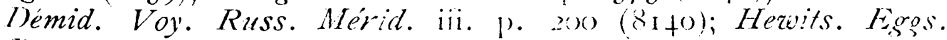
Brit. B. i. p. 216 ; pl. 57 . lig. $3(18+6) ;$ Tlomps. Nat. Hist. Irleand, i. p. 319 (1849); Blylin, (at. B. Mus. As. Soc. p. 198 (1849, pt.); Kjaroh. I anm. Frote, pl. xiv. flg. 5 (1852); Schl. Vog. Nederl. pl. $58(185+)$; Sinnder. Sir. Fogl. pl. xvii. fig. 6

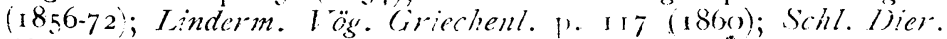
Nederl Vogels, pl. 6. fius. 5, 5." (1861); Keulim, N. T. D. iii. p. 384 ( 1866); Borggr. Vögeif. Vorddeutschl. p. Io0 (1 860); Gray, B. W. Scotl. p. 209 (1871); Harting, Handb. Brit. B. p. 35 (1872); Godm. Ibis, 1872, ए. 171; Keal. Onze, Vogels, ii. pl. 9 (1873); Saxby, B. Shelt. p. 1 f) (1874); Harting, Sum- 
mer. Mhor p. I8t (1875); Fallon, Os. Bels. p. 124 (1875); Seebohm, thrs, 1882 , Pp. 210,372 ; id. Brit. B. iii. p. I7s, pl. I7 (1883); Dixon, Ihis, $185^{\circ} 2$ p. $5^{\text {hr }}$; Homeyer of Taucri, Mitth. orn. I cr. Wicn. Nay. assi, p. I3.

L. Petit Marince, Mambent. Pl. Einl. vii. pl. 542.

L'Hirondelle au croupion blanc ou L'Hirondclle de Fenetre, Month. Hist. Nal. Ois. vi. p. Grt, pl. xxv. fig. 2 (i 7799$)$.

Martin, lath. (irin. Sin. ii. pt. 2; p. $\left.5^{6}\right)_{4}(8783)$.

Hirundo donestica, leach, Syst. (at. Ilamm. \& Brit. Mus. p. is $(1,16)$.

Chelidon urbica, Boie, Isis, 1822 . p. 550; Rü̈pt. Neue. Wirb.

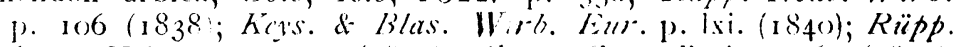
Sist. Uaters, p. $22(\mathrm{r}+4+5)$; (iray, Gen. B. i. p. 6o (r845); id. Cat. Fissir. Brit. Mlis. p. 31 (184s); Rp. Consp. i. p. 34.3 (1850); Cab. Mus. Hein. Th. i. p. 51 (1550); Bolle, J. $f .0$. 1854 , p. 460; Heiugl. Syst. Lebors. p. 17 (1856); Jaub. et Barth-Lapomim. Rich. Orn. p. 340 (1859); Salv. Ibis, 1859 , p. 302; Tristr. t. c. p. 434 ; Heugl. J. f. O. 1861 , p. 419 ; Gould. B. Cil. Br. vol. ii. pl. $6(1862-73)$; Jerd. B. Ind. i. p. 166 (1862); Weation in Barino-Gould's Icrland, p. 408 (1863); Brehm, Res. Habesch. p. 272 (1863); Filippi, Viagg. Pers. P. 346 (1865; Bettoni, lice. nid'f. Lomb. tav. 67 (1865-70); More, Ibis, 1865 , p. I 39; Baird, Ibis. 1867 , p. 28I; Degl. et Gerbe, Orn. Hur. i. P. 592 (1867); loche, Expl. Sci. Algér., Ois. ii. p. 7 I I 867 ; Tristr. Ibis, 1867 , p. 364 ; Drake,t.c. p. 425 ; Saunders, Ibis, 1869 , p. I74; Heugl, Orn. N. O.-Afr. i. p. i68 (1869); Doderl. Avif. Sicil. p. 145 (1869); Droste, Vogelw Bork. 1. 87 (1869); Wiatt, Ibis, 1870, p. г2; Elwes \& Bnckley, $t$. c. p. 200; Blanf. Geol. \& Zool. Abyss, p. 349 (1870); Sharpe, P. Z. S. 1870, 1. 202; Fritsch, Vög. Eur. tab. 23 fig. 5 (c. 1870); Scliad. Fann. Ital., Ucc. p. 5r (1871); Shelley, B. Espt. 1. 125 (1872); Alst. \& Harvie Brown, 1bis, 1873, 1. 59 ; Brooke, t. c. p. 237; Hume, Str. F. 1873 , p. 323; Brooks, S\%. F. 1875, D. 323; Dresser, B. Eur. iii. p. 495 , 1l. I62 (1875); Ir.by, B. Gibr. p. I02 (1875); Wharton, Ibis, $15^{5}$, p. го; Walden, t. c. p. 356; Blanf. East. Persia, ii. p. 216 (1876); Hume, Str. F. 1879 , p. 84; Bogadanoff, $B$. Cancas. P rits (1879); Nezt. ed. I'drr. Brit. B. ii. p. 349 (r880); Butler, Cit. L). S. Bombay Pres. p. 15 (1880); Colietl, Aoreces Finole, i). 286 (188r); Giglioli, Elench. Uic, Ital. p. 6.3 (1881); Biddulph, Ibis, 1881 , p. +7 ; Scully, t. c. p. 428; Shelley, Ibis, 1882 , p. 250; Biddulph, t. c. p. 269; Severtz. Ib s; 1883, p. 70; B. O. U. List Brit. B. p. 44 (1883); Tristr. Faun. \& Fior. Palest, p. 62 (1884); Radde, Orn. Cauc. p. $3^{6}$ (1814). Sharpe, Cat. B. M. 10. p. 87 (I885).

Chrlidon fencstrarum, rupestris, candida, varia, pallida, Naum. lö̈r. Deutsch!. vi. p. 77 (I833). C. L. Brehm, Vög. Dewtschl. l. I 40 ( $183 \mathrm{I})$.

Cheliton tectorum, C. L. Brehm. Naum. 1855, p. 27 I.

Hab. Luropa, Africa, India.

2. Ch. dasypus, $B f \cdot O^{\circ} \bigcirc \mathrm{N}$. v. I,angagy lang̃ayan.

(M. S. T.)

Chelidon dasypus, Bp. Consp. i. p. 343 (1850, ex Temm, MS:); 
Horsj. \& Moore, Cat. B. E. I. Co. Mhts. i. p. $38+(185+)$; Giry', Hand-l. B. i. p. 74, n. ${ }^{\circ} 883$ (1869); Dresser, B. Eur. iii. p. 49. (1875); Sharpe, Cat. B. M. 10. p. 9I (1885).

C. blakistoni, Swinh. P. Z. S. 1862 , p. 320 ; id. Ib:s, $186 . \therefore$ p. 90; Whitely, Ibis, 1867 . p. 196; Gray, Hand-l. B. i. 1) 74 , n. ${ }^{\circ} 882$ (1869); Swinh. Ib s, 1874 , p. I 52 , pl. vii. fig. I; $i d$. Ibis, 187.5, p. 448; Blakist. \& Pryer, Ibis, 1878. p. 231; id. B. Japan, p. $139(\mathrm{I} 882)$.

Delichon (:) dasypus, Salvad. Ucc. Born. p. I27 (I874).

Hirundo dasypus, Seebohm, Hist. Brit. B. iii. p. I79 (1883), Hab. Luzón, Catgayan; Japón, Borneo.

2. Gén. CO'TIIE, BOIE.

3. C. sinensis, Gray. $\sigma_{0}$ N. v. Lang̃aylanğayan.

(M. S. T.)

Hirundo chinensis, J. E. Gray in Hardw. Illustr. Ind. Zoo!. i. pl. 35. fig. 3 (1830-32).

H. brevicaudata, Mc Clell. P. Z. S. 1839 , p. I56; Gray, Gen. $B$. i. p. $58^{\prime}{ }^{\prime} 845^{\prime}$,

H. sinensis, Jerd. Madr. Journ. xi. p. 238 (г94o); Blyth. J. A. S. Beng. xvi. p. 119 (1847); id. Cat. Ba. Mus. As. Soc. p. Iy') (1849).

Cotyle brevicaudata, Boie, Isis, 1844 , p. i 70 .

Hirundo minuta, Hadgs. Icon. ined. in Brit. Mus., Passeres pl. 9. fig. 2 (n. ${ }^{0} 333$ ); id. in Gray, Zool. Misc. p. 82 (1844).

H. subsoccata, Hodg. Icon. ined. in Brit. Mus., Passeres, pl. 9. fig. I n. ${ }^{\circ} 32$; id. in Gray's Zoo!. Misc, p. 82 (18+4).

Cotyle sinensis, (iray, Cat. Fissir, Brit. Mus. p. 30 (1848); Bp. Consp. i. p. 342 (1850); Cass, Cat. Hirman. Philad. Mus. p. 12 (1853); Horst. \& Morre, Cat. B. E. I. Co. Mus. i. p. 96 (i 854 ); Jerd. B. Ind. i. p. 164 (1862); iii. p. 875 (1864); Swinh. Ibis, 1863 , p. 259 , 1866 p. I $34 ;$ Blyth, t. c. p. 338 ; Beavan, Ibis, 1869 , p. 404; Godzvin-Austen, J. A. S. Beng. xxxix. p. 266 (1870); Swinh. P. Z. S. 1871 , p. 347; Adam, Sir. F. 1873 , p. 370; Hume, Str. F, 1874, p. 469, I875, p. 452; Blyth \& Wald. B. Burm. p. I 27 (1875); Fairb. Str. 1876 , p. 254; Batler, Str. F. 1877 , p. 227 ; Dav d \& Oust. Ois. Chine, p. 128 (1877); Anders. Rep. Zool. Exped. Yunnan, Birds, p. 651 (1878); Hume, \& Davison, Sir. F. 1878, p. 45 ; Davids. \& Wend. Str. IF. 1378 , vol. ii. p. 76 ; Ball, t. c. p. 202; Cripps, t. c. p. 257; Hume, Sir. F. 1879, p. 84; Scully, t. c.p. 234; Dorg; t. c. p. 37o; liatl., Cat. B. Sind ete. p. I3 (1879); id. Cat. B. S. Bomb. Pres. p. I4 (1880); Murray, Vertebr. Fian. Sind. p. 103 (1884).

C. subsoccata, Adams, P. Z. S. 1858 , P. 495, I859, p. I $7^{\text {f); }}$ Jerd. B. Ind. i. p. 163 (1862); Blyih, Ibis, 1806 , p. 338; Jerd. Ibis, 1871, p. 353 .

C. riparia, (nec L.) Swinh. Ibis, 1861, p. 328, 1883, p. 89 .

C. sinensis, Gray, Hand-l. B. i. p. 37, n." 865 (r869); Hume, Nests. and Eggs. Ind. B. p. 82; id. Str. F. 1873 , p. Itot; Brit. Burm. i. p. 309 (1883); Sharpe, Cat. B. M. 10. p. Iot $(1885)$. 
( subsoccata, Huine, Nests. and Egrgs. Ind. B. p. 82 (1873).

(. olscurior, Hume, Str. F. 1875, p. 43.

('. obsoleta, Legge, B. Ceylon, p. 599 (is79).

Hab. Luzón, Manila, I aguna, Los Baños, Calamba; Formosa,

( hina, India, Bengala,

3. Gén. hiRLNIJO, Liv.

4. H rustica, Lin. ơ N. v. Langay-lang̃ayan.

(M. S. T.)

L'Hirondelle de Cheminé, Briss. Orn. ii. p. 486 (1760); Daubent. Pl. Enl. vii. pl. 543 fig. I; Montb. Hist. Nat. Ois. vi. p. 591, pl. 25 , fig. I, (1779).

Hirundo rustica, limn. Syst. Nat. i. p. 343 (1766); Temm. Man. d'Orn. i. 1). 427 (1820); Roux. Orn. Provenç. pl. I4I (1825); Werner, Atlas, Chelidones, pl. I (1827); Ménér. Cat. rais. Cauc. P. 45 (1832); Naum. Vög. Deutschl. vi. pl. 145 fig. I (1833); Gould, B. Eur. ii. pl. 54 (1837); Schl. \& Susem. l'ög. Eur. vi. 'Taf. 2. fig. I (1839); Macgill. Brit. Li. iii. p. 55 ' $^{\circ}$ (1840); Key's. u. Blas. Wirb. Eur. p. 196 (1840); Nordm. iue Démid. Voy. Russ. Mérid. iii. p: 201 (1840); Yirrell, Brit. B. ii. p. 213 (1843); Hodgs. Icon. ined. in. Brit. Mhus. Passeres, pl. 8 (n. ${ }^{\circ} 331$ ); id. in Gray's Zool. Misc. p. 82 : 844 ); Gray, Gen. B. i. p. 57 (1845); id. Cat. Fissi. Brit. Mus. p. 22 (1848); Blyth, Cat. B. Mus. As. Soc. p. 197 (1849, pt); Thompson, N. H. Irel. p. $374(1849) ; B$. Consp. i. p. 338 (1850); Cab. Mus. Hein. Th. i. p. 46 (1850); Kjarb. Danm. Fugle, ju. I4. fig. 4 (1852); sichl. Vog. Nederl. pl. 57 (1854); Horst.\& Monre, Cat. B. E. I. Co. Mus. i. p. 91 (1854, pt.) Hewits. Eggs. Brit. B. i. p. 257, pl. 65. figs. 3, 4 (1857); Sundev. Sv. Fogl. pl. I 7 fig. 5 (c. 1856); Grill, Zool, Anleckr. p. 35 (1858); Iaub. et Barth.-Laponm. Rich. Orn. p. 307 (1859); Salv. Ibis, 1859 , p. 334; Lindern Vög. Griechenl. p. 117 (1860); Naum. Vög. Deutschl., Anhang. xiii. 'Taf. 383 . fig. 2 (1860; Schl. Dier. Nederl. Vög. pl. 6 fig. 4 (1861); Hartl. J. f. O. 1861 , p. IO3; Jerd. B. Ind. i. p. I 57 (1862); Gray, Cat. Brit. B. p. 33 (1863); Filippi, Viags. Pers. p. 346 (I865); Bettoni, Ucc. Nidif. Lomb. ii. tav. 30 (1865-70); Deol. et Gerbe, Orn. Eur. i. p. 587 (1867); Loche, Expl. Sci. Algér., Ois, ii. p. 64 (1867); Borggr. Vogelf. Norddeutschl. p. 100 (1869); Doderl. Avif. Sicil. p. I43 (1869); Grav, Hand l. B. i. p. o8, no. 786 (1869); Kenl. Onze Vogels, pl. ro (1869;; Heugl. Orn. N. O.-Afr. i. p. I 50(1869:; Godman Azores, p. 34 I (1870); Godae'Aust.J.A. S'. Beng. xxxiv. p. 94 (1870); Fritsch, Vög. Eur. Taf. 2. fig. 4 (1870); Sharpe, P. Z. S. 1870 , p. 305; id. \& Dresser, t. c. p. 244; Gillett, Ibs, 1870 , p. 306; Blanf. Geol. \& Zool. Abyss. 1. 347 (1870); Finsch \& Hartl. Vög. Ostafr. p. I 34 (1870); R. Graj', B. W. Scotl. p. 205 (1871); Jerd. Ibis, 1871 p. 351; Saluad. Faun. Ital., Ucc. p. 51 (1871); Sharpe, Cat. Air, B. p. 45 (1871); Harting, Handb. Br. B. p. 35 (1872); Holdsw P. $Z$. Z. S. 1872 , p. 418; Gurney, in Anderss. B. Dam. Ld. p. 50 (1872); Shelley, B. Egypt. 1872 , p. I. O; Gocim. Ibis, 1872, 1. 61; Antin \& Saliad. Viagg. Rogos, p. 72 (1873); Hengl. 
Orn. N. O.Afr. iv. App. p. 1. (1873); Hume of Heners. Lahore to Yark. p. I76 (1873); Severtz. Turkest. Je: otn. p. 67 (1873); Gould, B. Gt. Br. ii. pl. 5 (1873); Salvad. An'. Mus. (ivic. Genov. (2) i. p. 120 (1884); Murray, Verteb:" Faun. Sind, p. 102 (1884); Sharpe, Cat. B. M. 10. p. I28 (1885'.

(himney swallow, Laih. Gor. $y$, ii. pt. 2, p. 56r (1783).

llirundo domestica, Pall. Zoogr. Rosso-Asiat. i. p. 528 (pt. i 811 ); Sreinh. Ibs, $1967,1 \mathrm{p} .414,420 ;$ Wratt. Ibis, 1870, 1. Ir; Sivereta. Turkest. Je: o'n. p. $67(1873)$.

('heliclon progne, Forst. Syn. Cat. Br /. B. p. 55 (1817).

(icropis rustica, Bo e, Isis, 1826 , p. 97 I et 1844 , p. 174 , Less. Compl. Buff) viii. p 498 ( 1827$)$.

( Vargorum, stabulorum, C. L. Brehn, lög. Dentschl. p. г 3 S (1831);id. Nanill. 1855, p. 27 I.

Hirunde cahirica, (non Licht.), Hartl. Orn. W.-Afr. p. 26 (1857); Cass. Proc. Acad. N. Sci. Philad. 1859 , p. 32 ; Sa!rad. Ibis, 1870 , p. I 53; Saunders, t. c. p. 299; Elwes \& Buckley, t. c. p. 2oo.

H. boissoneauti (nec Temm.), Linderm. Vog. Griechenl. p. Ir) (1860); Krïper, J.f. O. 1860. p. 281.

H. sivignii (nec Aut.), Tristr. Ibs, $186 \frac{1}{4}$, p. 230.

11. rustica orientalis (alec Schl.), Wright, Ibis, 186.4, p. 57.

H. riocouri (nec Aud.), Gurnzy, jun., Ibis, 1865 , p. 423 .

Cheliton rusticil, Slejn. Pr. $U$. S. Nat. Mus. vi. p. 31 (1832.) Hab. Dumalon, Mindanao; Europa, Africa, China.

5 H. eutturalis, Scop. of N. v. Lang̃ay-lang̃ayan.

(M S. T.)

L'Hirondelle d'Antigue, Sonn. Voy. Nou'. Guin. p. 118, pl. lxxii. ( 1776 ).

L'Hirundelle d'Antigue à gorge couleur de rouille, Montb. Hist. Nat. Ois. vi. p. 607 (1779).

Panayan swallow, Lath. Gen. Syn. ii. pt. 2, p. $565\left({ }^{6} 8_{3}\right)$.

Hirundo gutturalis, Scop. Del. Flor. ct Faun. Insubr. ii. p. 9h (1786); Temm. Man. a'Orn. i. p. 427 (1820); Gray', Gen. B. i. p. $57($ I 845$) ; B l y t h, J$. A. S. Beng. xvi. p. II7 (I 847 ); Gray, Cat. Fissir. Brit. Mus. p. 22 (1848); Cab. Mus. Hein. Th. i. p. 46 (1850); Cass. Cat. Hirmat. Mus. Philad. Acad. p. I (1853); Gray, P. Z. S. 1860,1 . 365 ; Sucint. I6 s, 1860 , pl). $148,429, \& 16 \mathrm{I}$, p. 30 ; Jerd. B. Ind. i. p. 157 (1802, pt.); Swinh. Ibis, $1863, \mathrm{p} .255,1867, \mathrm{p} .4 \mathrm{1} 1, \& 1870, \mathrm{p}$.). (a), 240; Jerd. Ibis, is71, p. 35I; Ha!d. Tr. Z. S. viii. p. 65 (1872); Dybowski, J. f. O. 1872, p. 36r; Saladt. Ucc. Born. p. I 25 (1874); Swinh. Ibis, 1874, p. I5 r; Salnat. Ann. Mas. Civic. Genov. ix. pp. 55, 64 (1876;) Tacz. Bull. Scc. Zool. France, ii. pp. 133 (pt. i 8 ;6); Blakist. Ib s, 18,6 , P. 331; Hume, Ibis, 1876 , p. 286, \& 1877, p. I7; Treecel; P. Z. S. 1877 , pp. 545, 694; Wald. Tr. Z. S. ix. p. Ist (1877); Prjel. in Daweson Rou'er's Orn. Misc. ii. p. I60 (1877'; David. \& Oust. Ois. Chme, p. 124 (1877, pt:); Hume \& I)avis, Str. F. 1878 , p. 4r; Cripts, Sir. F. 1878 , vol. ii. p. 2-6; Salvad. Ann. Mus. Civic. Cenou. xiv. 1) 490 (1879); Mever, Ibis, 1879 , p. 128; Hume, S/r. F. 1879 , pp. 47, 84; Shat-

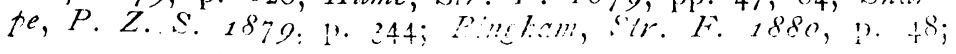


Hume, t. c. p. 24.5 ; Trueld. Report l'oy. "Challengrs, ii. p. to (1881); Sclaler, t. c. p. 1 Io (1831); K:lham, Ib 1 s's 1. 372; Saliad. Oral. Papursia etc. ii. p. 1 (18S1); B!akis!. dPyer, B. Jafan, P. 139 (1882); Sharpe, Cat. B. M. 10. P. $1.34\left(1885^{\prime}\right.$.

H. panayana, Gin. Sist. Net. i. p. wos (1738); Moore, P. Z.S. 18.57, p. 204; Horts \& More. Cat. B. E. I. Co. Mus. i. P. ()) $(1854)$.

H. jewan, Sykes, P. Z.S. 1 832, p. 83; Jerd. Madr. Jouru. xi. 1. $237(1840)$,

11. rustica, (non L.), Meyen, Noina Acta Acad. Coes Losp.-Car. Nat. Cur. Suppl. 'Taf. Io. fig. I (183t); Blvth, Cat. B. MI.s. As. Soc. p. 197 (18+9. pt.); Te:nm. \& Schl. Fa:tn. Tapon., Aves, p. 31 (1850); Swinh. Ibis, 1851 , pp. 25t, 329, \& 1853, p. 89; Scl. P. Z. S. 1853 , p. 217 ; Gray, Hant-l. 13. i. p. 68, n. 786 (i 869, pt.) $^{\circ}$ Holdsio. P. Z. S. 1872, p. 418; Sharpe, Trans. Linn. S-c. 2nd s r. Zool. i. p. 328 (1876); Anderson, Zoo!. Yun-nun Exped., Birds, p. 649 (i878)? Blakist. \& Pryer, .Ib.s, $187 s$, p. 320 ; O.ttes, B. Brit. Burm. i. p. 302 (1883).

Cecropis payana, Less. Compl. Buff. viii. p. 498 (1837.)

( $\therefore$ jewan, Less. t. c. p. 499 (1837); Boie, Isis, 1854, p. 174.

(. rusticoides, panayana, Boie, Isis, $18+4$, p. I 74 (e.x Kuh!, MS.)

Hirundo javanica, (non Sparrm.), Bp. Consp. i. p. $33^{8}$ (185()); Bernst. J. f. O. 1859 , p. 267; Blakist. Ibis, 1862 , pp. 315 , 316; Whitely, Ibis, 1367 , p. 196; Hayes Lloyd, 16is, 187.3 , p. 405 .

H. fretensis, Gould, Handb. B. Austr. i. p. I10 (1865); Gray, Hand-l B. i. p. 70, n. 8 I 5 (1869); Diggles, Orn. Austr. text to pl. 22 (c. 1870$)$.

H. frenat:, Blyth, Ibis, 1866 , p. 336; Gray, Hand-l. B. i. p. 7o, n. ${ }^{\circ} 816(1869)$.

H. andamanerisis, Tytler of Bear. Ibis, 1867, p. 316; Grai, Hand-l. B. i. p. 68, n. ${ }^{\circ} 79$ I (1869); Ball, Str. F. 1873 , 1). 55 ; Hume, Str. F. 1874 , P. 155, \& 1876, p. 286.

H. rustica, var. gutturalis, Seebohm, Hist. Brit. B. ii. p. I7 I (1883). Hab. Luzón, Manila, S. Mateo, Laguna, Samar, Paranas, P.tragua, Mindanao, Guimarás, Panay, Carolinas; Java, Sumatra, Malaca, China.

6. H. javanica, Sparr. Ơ, N. v. Lang̃ay-lang̃ayan. (M. S. T.)

Ilirundo javanica, Sparrm. Mus. Carls. ii. pl. Ioo (1780); Vieill. N. Dict. d'Hist. Nat. xiv. p. 523 (1,817); Temm. Pl. Col. iv. pl. 83. fig. 2 (1823); Gray, Gen. B. i. p. 57 (18+5); Temm. \& Schl. Faun. Jap., Aves, p. 32 (1850); Cab. Mits. Hen. Th. i. p. 46 (1850); Wall. Ibis, 1860 , p. 147; id. P. Z. S. 186.3 , p. 485 ; Gray, Hand-l. B. i. p. 70, no. 81 3 (1869); Finsch. \& Hartl. Vög. Ostafr. p. I 37, uote (1870); Wrlden, Tr. $Z$. S. viii. p. 66 (1872); Swinh. Ibis, 1873, p. 231; Margan, Ibis, 1875, p. 313; Salvad. Ucc. Born. p. I 26 (1874); id. Ann. Mus. Civic. Genno. viii. p. 767 (1875); ix. p. 23 (1876); x. 1. 130 (1877); Sharpe, Ibis, 1876, p. 43; Bourd. Str. IF. 


$$
-1 ; 6-
$$

1876 , p. 374; Farib. Str. F. 1877 , p. 392, Sharpe, Journ. Linn. Soc. Zool. xiii. p. 498 (1877); Tweedd. Ibis, 1877 , p. $316 ;$ id. P. $Z$. S. $1877,760, \& 1878$, pp. ro9, 342, 615, 709; Salvad. Ann. Mus. Civic. Genov. xiv. pp. 492, $6+7$ (1877); id. P. Z. S. 1878 , p. 95; Hume \& Davison, Str. IF. 1878 , p. 43; Ramsay, Proc. Linn. Soc. N. S. W. iii. p. 275 (1879), iv, p. 9i (1879); Meyer, Ibis, 1879 , p. I 28; Hume, Str. F. 1879 , pp. 47, 84; Sharpe, P. Z. S. 1879 , p. 344; Leg.ge, B. Ceylon, p. 597; Hume, Str. F. 1880 , p. I 20; Salz'ad. Reprot Voy. C'hallenger,' 1i. Brids, p. 78 (188I); Davison, Str. F. 1883, F. 345; Sharpe, Cat. B. M. 10. p. 142 (1885). lavan swallow, Lath. Gen. Syn. Sutpl. ii. p. 259 (г8or).

if. frontalis, Quoy et Gaim. Voy. de l'Astrol. Zool. i. p. 204, pl. I2. fig. I (1830); Gray, Gen. B. i. p. 57 (1845); Sil. Journ. Linn. Soc., Zool. ii. p. I55 (I858); Gray, P.' Z. S. 1858 , p. 189. 1859, p. I 54; id. Cat. Mamm. et. N. Gitin, pp. 88, 54 (1859); id. P. ZZ. S. 1861, p. 433; Finsch, NeuGuinea, p. 162 (I 865, pt.); Gray, Hand-l. B. i. p. 70, n." 8 I 2 (г869, pt.); Ramsay, Proc. Linn. Soc..N. S. $W$. ii. p. 179 (1 878 ).

Herse frontalis, javanica, Less. Compl. Buff. viii. p. 497 ' 837 ). Cecropis javanica, frontalis, Boie, Isis, 1844 , p. I 74 .

1 irundo domicola, Jerd. Madr. Journ. xiii. p. I73 (18+4) Blyth, Cat. B. Mus. As. Soc. p. 198 (1849); Kelaart, Prodr. Cat. p. II 8 (1852); Layard. Ann. \& Mag. Nat. Hist. xii. p. 170 (1853); Moore, Cat. B. E. I. Co. Mus. i. p. 384 (1854); Jerd. B. Ind. i. p. 58 (1862); Scl. P. Z. S. 1863 , p. 217; Blyth, Ibis, 1866 , p. 336; Bulger, P. Z. S. 1866 p. 568; Gray, Hand l. B. i. p. 70, n..$^{\circ}$ 814 (1869); Holdsw P.Z. S. 1872 , p. I I8; Elates, Ibis, 1870 , p. 527; Jerd. Ibis, 1871 , p. 35I.

11. pacifica, Motl. \& Dllw. N. H. Labuan, p. ro (i 855 ).

H. neoxena, (non Gould), Gray, P. Z. S. 1858 , p. I 89 I 861 , p. 433; Rosenb. N. T. D. Nederl. Ind. xxv. p. 235 (1 863).

Hypurolepis domicola, Gould, B. Asia, i. pl. 32 (i868); Hume; Ness and Eggs Ind. B. p. (8873); id. Str. F. 1874 , p. 155.

11. javanica, Oates, B. Br. Burm. i. p. 308 (1883).

Itirundo fretensis, Ramsay, Proc. Limn. Soc. N. S. W. ii. p. I7y. ( 1878$)$, nec Gould.

Hab. Mindoro, Baco, Samar, Paric, Negros, Paragua, P. Princesa, Cebú, Leyte, Bohol, Dinagat, Joló; Ceilan, Java, Borneo, Célebes, Molucas.

7. H striolata, (Boie). $\sigma 0$ N. v. Lang̃ay lang̃ayan.

(M. S. T.)

Cecropis striolata, Boie, Isis, 1844 , p. 174 (ex Teinm. MS.); Cass. Cat. Hirund. Mus. Philad. Acad. p. 3 (1853); Wald. in Blyth B. Burm. p. I 27 (1875).

Hirundo striolata, Gray, Gen. B. i. p. 58 (1845); Tem. \& Sichl. Faun. Jap. p. 33 (1850; Bp. Consp. i. p. 340 (1850); Blyth, Ibis, 1866 , p. 337; Gray, Hand-l. B. i. p. 69, no. 801 (r 869); Hume. \& Davison, Str. F. 1878 , p. 44; Seebohm, Ibis, p. I69; Sharpe, Cat. B. M. 10. p. I6I (1885).

Lillia striolata, Hume, Str. F. 1877 , p. 261.

Hab. Luzon, Laguaa, Paragua; Java. 
Fam. MNIOTILTIDOs.

I. Gén. MNIOTILTA, Vieill.

1. Mt. Varia, (Lin.) \& $\mathrm{N}$ v.

(M. S. T.)

The small black and white Creeper, Sloxne, N. H. Jamaica, ii. p. 309, p!. 265. fig. I (I725).

Black and waite Creeper, Elwards, Gleanings, vi. p. igr pl. 300 (1760).

1. Figuier varié de S. Domingue, Bris;. Orn. iii. p. 529, pl. pl. 27. fig. 5 (1760).

Hotacilla varia, Linn. Syst. Nat. i. p. 333; Gm. Syst. Nat. i. p. 979 ( I 788 ).

le Figuier varié, Buff: Mist. Nat. Ois. v. p. 329, (1778).

liniotilta varia, Sharpe, 10 p. $25 \mathrm{I}$ (1885).

$H a b$. Guatemala.

2. Gén. DENDRACA, ShIRPE.

2. D. pennsylvanica, (Lin.) $\sigma$ N. v. D. de pechs rojo. (M S. T.)

The Red-throated Flycatcher, Edw. Glean. ii. p. 193, pl. 30r (1760).

Figuier à teste jaune de Canada, Briss. Orn. iii p. 517, pl. 27, fig. (1760).

F. á teste jaune de Pensilvanie, Br ss. Orn. vi. App. ro5 (1760).

Notacilla pensylvanica, Linn. S. N. i. p. 333 (i 766).

lendrœca pennsylvanica, Sharpe, Cat. B. M. 10. p. 286 (1885). $H a b$. América, Canadá.

3. D. cærulescens, Gm. \& N. v. D. azul.

(M. S. T.

Blue Flycatcher, B.lw. Glean. p. 91, pl. 252, fig. I (1758).

le Petit Figuier cendré de Canada, Bris. Orn. iii. p. 527, pl. 27. fig. 6 (i76o).

Motacilla canadensis, Linn. Syst. Nat. i. p. 336 (1766, nec Linn. $o p$. cit. p. 334).

Figrui $r$ cendré du Canadá, Daub. Pl. Enl. pl. 685, fig. 2.

I. Figuier bleu, Buff. Hist. Nat. Ois. v. p. 304 (1778).

La Fauvette bleuâtre de S. Domingue, Buff. Hist. Nat. Oes. v. p. I64 (1778).

Dendroca carulescens, Sharpe, Cat. B. M. 10. p. 329 (I885). Hab. América, Canadá.

\section{Gén. Geothlypis, can}

4. G. philadelphia, Wils \& $\mathrm{N}$ v.

(M S. T.)

Sylvia philadelphia, Wils. Amer. Orn. ii. p. 1or, pl. 14, fig. 6 (1810); Audub. B. Amer.; id. Orn. Biogr. v. p. 79 (1839).

Gicothlypis philadelphia, Baird, B. N. Amer. p. $243(1858)$ id. 
op. cut. pI. 79 fig. 3 (1850); Lazer. Altz. Iyc. N. Y. vii. p. 322 (1861); Scl. (at. Amer. B. 1. 27 (1862); Sharpe, Cat. B. M. 10 . p. $365+(1835)$.

Hab. Cinnadá.

\author{
4. Gén. SETOPHACit, Sw.
}

5. S. ruticilla. (Ling) \&. v. Aurcra.

(M. S T.)

The Redstart, Catesby, Nat. Hist. Carol. i. tab. 67 (1731).

The Small American Redstart, Edwards, Nut. Hist. B. p. \&o, pl. 80 ( 1747 ).

Yellow-tailed Flycatcher, Edivards, Gleatings, p. ior, pl. 257, (1758)

Le Gobe-mouche d'Amèrique, Bris Orn. ii. P. $38_{3}$ (1760); Daubent. Pl. Enl. v. pl. 566 figs. 1, 2.

Nuscicapa ruticilla, Lmin. Syst. Nat. i. p. 326 (1766); Bodd. Tabl. Pl Enl. p. 33 (I783); Vieill. Ois. Amér. Sept. i. p. (66, pls. 35, 36 (1807); Wils. Amer. Orn. i. p. 103, pl. 6. fig. 6 (1808), v. p. s19, pl. 45 fig. 2 (1812); Audub. B. Amer. pl. 4o; id. Orn. Biogr i. p. 302 (1831), v. p. 428 (1839); B. Amer. i. p. $24 \%$, pl. $68(1839)$.

Figuier noir et jaune de Cayenne, Daubent. Pl. Enl. vi. pl. 391. fig. 2.

Setophaga ruticilla, Swains. Phil. Mag. new series, i. p. 365 (1827); Sharpe, Cat. B. M. 10. p. 41 L (1885).

Hab. Canadá.

\title{
Fam. MOTACÍLIDOS.
}

Gén MOYTACILLA, Lis.

1. M. alba, Lin. Õ.g N. v. Carisayao.

(M. S. T)

1.a Lavandière, Briss. Orn. iii. p. $46 \mathrm{r}$ (1760); Daubent. Pl. Enl. vi. pl. $65_{2}$ fig. I.

La Bergeronette grise, Bris. Orn. iii. p. 465, pl. xxr. fig. I (1760); Buff. Hist. Nat. Ois. vi. p. 261, pl. xlv. (1778); I)aubent. Pl. Enl. vi. pl. 674 fig. I.

Motacilla alba, Linn. Syst. Nat. i. p. 331 (i 766); Gm. Syst. Nat. i. p. 960 (I788); Temm. Man. d'Orn. i. p. 254 (1885); ii. p. 255 (1820); Naum. Vög. Deutschl. iii. p. 803, Taf. 86. figs. 83 (1823); Roux, Orn. Prov. pl. I93 (1825); Werner, Atlas, Insectiv. pl. 73 (1827); Ménètr. Cat. Rais. Cauc. p. 36 (1832); Temm. Mau. d'Orn. iv. p. $7^{8}(1835)$; Rüpp. Noue Wirb., $V \ddot{g}$. p. 84 (1835-40); Gould; B. Eur. ii. pl. 143 (1837); Macgill. Hist. Br. B. ii. p. 221 (1839); Keys. \& Bias. Wirbelth. Eur. p. 174 (1840); Nordme. in Démid. Vöy. Russ. Mérid. iii. p. 153 (1850); Rüpp. Syst. Uebers. p. 59 (1845); Gray, Gen. B. i. p. 203 (1847); Thomps. B. Iret. i. p. 218 (1892); He. Consp. i. p, 250 (1850); Cab. Mus Hein. Th. i. p. 14; (185(2); Kjärb. Dann. Fagle, pl..xix. fig. (1852); Scht: Iög. 
Vederl. p's. 100, 101 (1854); Sundev. Sv. For?. pl. 9: figs. 7 . ' (c. 1856); .Jaub. \& Birlh-lapomm. Rich. Orn. p. 276 (1858); Salvin, Ibis, 1 s59, p. 310 ; Holker; Ibis, 1860, p. 166; Poaeys; t. c. p. 228 ; Simpson, t. c. p. 394; I. inderm. lög. Ciriechenl. P. 79 (1860); Reinh. Ibs, 1soo, p. 6; F. \& P. Ciodman, t. $c$. 1. 82 ; Schl. Dier. Nederl. Vog. pl. 9. figs. 7, 8, 8, a (1861); Reinh. Ibis, 1861 , p. 6; A. Nez't. in Bar-ing-Ciould's Iceland, 1. 406 (1863); Ciould. B. Cit. Br. iii. pl. 2 (c. 1863); Grar. Lisı. Br. K. p. 66 (1863); Radde, Reise Sibir., Vö.s. p. 224 (1863); Baird, Revi@w, p. ${ }_{52}$ (1864); Aldams, Ibis, 1804 , p. 27 ; It right, t. c. p. 62; Nore, Itis, 186,5, 1. 122; Bettori, Ucc. Nidif. L.omb. tav. 37 (186.5-70); l.ilford, Ib.s, 1866, p. 187 ; Tristr. t. c. p. 200; Deg\%. \&. Cierbe, Orn. Fur.

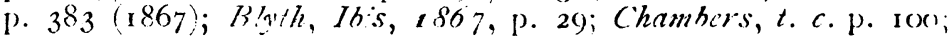
Drake, t. c. 427 ; Joche, Expl. Sic. Alger., Ois. ii. p. 3 (1869);

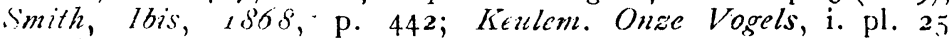
(1869); Gray, Hand-l. B. i. p. 245, no. 3562 (I 860); Borgy. Vogelf. Deutschl. p. 86 (1869); Doderl. Avif. Sicil. p. 100 (1 869); Heugl. Orn. N. O.-Arf. i. p. 316, iv. p. Ixxxviii. (1869-7I; Blanf. Geol. \& Zool. Abyss p. 3 (10 (1870); Finsch, Trans. Zool. Soc vii p. 324 (1870); 4 yatt, bis, 1870 , p. I5; Elzes \& Buckles, $t$ c. p. 195; Fritsch, Vög Eur pl 21 fig. 14 (c) 1870$)$, (iztriey, junr, Ib:s, 1871 , p. 85; Saunders, $t$. c. 215; Shurpe, Cat. Afr. B. p 73 (1871); Saliad. Faun. Ital, Ucc. p. I20 (1871); Gray, B. p. 73 (1871); Salvad. Faun. Ital., licc. p. I 20 (1871); Gray, B. West Scotl. p. I I (1871); Harting, Handb Brit. B. p. 23 (1872'; Shelley, B. Figypt. p. I 26 (1872); Severlz Turkest. Jevotn. p. 66 (1873); Alston \& Harvie Brown, Ibis, 1873, p. 6r; Blanf. t. c. p. 220; Brooke, t. c. p. 244; Hume, Str. F 1873 , pp. 29, 3०; Baird, Brewer, \& Ridgw. Hist. N. Amer. B. i. p. 165 (1874); Durnf. Ibis, 1874, p. 396; Nezuton, ed. Yarr. Br. B. i. p. 541 (1874); Stoliczka, Str. F. 1875, p. 217; Dresser, B. Eur. iii. p. 233, pls. 125, I 26 (1875; Irby, $B$. Gibr. p. 108 (1875); Danf. \& Havvie Brown, Ibis, 1875, p. 309; Dresser, Ibis, 1876, p. 176; Wharton, t. c. p. 22; Seebahm \& Harvie Brown, t. c. p. $125 ;$ Blanf. East. Persia, ii. p. 232 (1876); Scully, Str. F. 1876 , p. 151; Wardlaw Ramsay. Ibis, 1877 , p. 465; Brooks, Str. F. 1877 , p. 472 ; l)anf. Ibis, 1878 , p. 20; Scebohm, t. c. p. 343; Brooks, Str. ff. 1878 , vol. ii. p. 137; Bogáanctf, B. Cauc. p. 166 (1879); Irty, Ibis, 1879, p. 344; Hume, Sitr. F. 1879, p. IO3; Scully, t. c. p. 313; Brooks, t. c. p. 414; Scebohm, Ibis, 1880 , 1). $199 ;$ Vidal, Str. F. 1880 , p. 69; G glioli. Icon. Avif. Ital. 1.1. 163 (1881); Ridgway, Buil. U. S. Nat. Mus. n. ${ }^{\circ}$ I, p. 1.5 (1881); Biddu!ph. Jbis, 1881, p. 61; Scully, t. c. p. 45!; $\therefore$ Swinhoe, Ibis, 1882 , p. 109; Seebohm, t. c. pp. 216; Bitathph, t. c. p. 2го; Seebohm. t. c. pp. 375, 376; id. Ibis, 1883, p. 19; Scrertz. t. c. p. Iо; Irby, $\therefore$ c. p. 184; Sannders, t. c. p. $350 ;$ B. O. U. List. Br. B. p. 29 (1883); Oates, B. Br. Burm. i. p. I56; (1883); Booth, Rough Notes. part. 4 (1883); Seetohm, Hist. Brit. B. vol. iii. p. I99 (1883); Homeyer \& Tancré, Mitth. orn. Ver. Wien, 1883, p. 85; Tristr. Fann. \& Flor. Palest. p. 54 (I884); Radde, Orn. Cauc. P. 
223, Tuf. xii. (1884); Salvad. Ann. Mus. Civ. Gcnov. (2) i. p. 166 (1884); Sharp?, Cat. B. M. 10. p. 464 (1885).

White wagtail, Latk. Gen. Syn. ii. pt. 2, p. 395 (I783).

Cinereous wagtail, Lath. tom. cit. p. 397 (1783).

Motacilla cinerea, Gm. Syst. Nat. i. p. 961 ( 1788).

M. albeola, Pall. Zoogr. Rosso-Asiat. i. p. 516 (1811).

M. septentrionalis, sylvestris, brachyrhynchos, Brehm, Vog. Derkchl. p. 347 (1831).

M. dukhunensis, Sykes, P. Z. S. 1832, p. $91 ;$ Bp. Consp. i. !. 250 (1850); Horsf \& Moore, Cat. B. E. I. Co. Mus. i. p. 319 (1854, pt.); Gould, B. Asia, pl. 62 (1861); Blanf. Ibis, $186 . \therefore$, p. 289; Blyth, Ibis, 1865 , p. 49; Swinh. P. Z. S. 1870 , P. I30; Finsch \& Hartl. Vög. Ostafr. p. 259 (1870); Hume, Sir. F. 1873 , pp. 29 30; Ball. Str. F. 1874 , p. 416; Brooks, I. c. p. 457 ; id. Str. F. 1875 , p. 249; Butler, $t$. c. p. 481); Wald. in Blyth B. Burm. p. 97 (1875); Brooks, Str. F. 1876 , p. 506; Prjev. in Rowley's Orn. Misc. ii. p. 192 (1876); Butler, Str. F. 1877 , pp. 221, 230; Hume \& Davison, Sir. It. 1878 , p. 362; Brooks, Str. F. 1878 , vol. ii. p. 1 37, Huni, Str. F. 179 , p. 104; Butler, Cat, B. Synd etc. p. 40 (1879); id. Cat. B. S. Bomb. Pres. p. 54 (1889); Vidal, Str F. 1880 , p 69; Reid, Str. F. 1181, p. 48; Davidson, Str. F. 188 , p. 3ro; Davison, Str. F. 1883 , p. 395.

Motacilla gularis, Swains. B. W. Afr. ii. p. 38 (1837); Allen \& Thomps. Exped. Niger, ii. p. 310 (1838); Hartl. Orn. W. Afr. p. 72 (1857).

M. cervicalis, Brehm, Vogelf. p. 143 (1853).

M. major, fasciata, Brehm, Naum. 1855, p. 280.

M. luzoniensis, Beavan, Ibis, 1868 , p. 76 .

M. alba $\beta$. dukhunensis, Severtz. Turkest. Jerotn. p. 66 (1879)).

M. dakhanensis, Fairb. Str. F. 1176, p. 260.

Hab. Luzón, Manila, Navotas, Laguna, Orani, Bataan, Samar, Loquilocun, Paragua, P. Princesa; Europa, Asia, Africa, India.

2. M. ocularis Szwinh. O' $\bigcirc$ N. v. Baticulo.

Motacilla ocularis, Swinh. Ibis, $116_{1}$, p. 55; Gray, Hand-l. B'. i. p. 246, n. ${ }^{\circ} 3576$ (1869); Swinh. P. Z. S. 1870 , p. 129, 1871, p. 364; Prejev. in Rowley's Orn. Misc. ii. p. 192 (1877); David \& Oustal. Ois. Chine, p. 299 (1878); Hume, Davison, Str. F. 171, p. 518; Hume, Str. F. 1179. p. I03; Scully, t. c. pp. 312, 315; Hume, t. c. p. 4I3; Oates, B. Brit. Burm. i. p. 158 (1883); Dybowski, Bull. Soc. Zool. France. viii. P. 360 (1883); Nelson, Cruise R. S. «Corwsin,» p. 62, pl. ii. (1883); Ridgw. Pr. U.S. Nat. Mus. iv. p. 414 (1882); Coucs, Key $N$. Amer. B. and ed p. 284 (1884); Sharpe, Cat. B. $M$. 10. p. $47 \mathrm{I}(\mathrm{r} 885)$.

Hab. Luzón, Manila, Montabban, Laguna, Ios Baños, Samar, Paragua, P. Princesa; China.

3. M. melanop?, (Pall.) oo N. v. Baticulo.

(M S. T.)

The yellow water wagtail, Albin, Nat. Hist. B. ii. p. 54, pl. iviii. $\left(173^{8-40)}\right.$ 
The grey water wagtail, Edwards, Glianings, p. ros, pl. 2:") (1758).

La Bergeronette jaune, Briss. Orn. iii. p. 47 , pl. xxiii. fig. 3 (1700).

La Bergeronette de Java, Briss. t. c. p. 474, pl. xxr. fig. 2 ( 1760$)$.

Motacilla flava, (nec L.) Scop. Ann. i. p. 154 (1769); Bodd. Tabl. Pl. Enl. p. 2 (1783).

Bergeronette jaune mâle, Daubent. Pl, Eul. vi, pl. 28. fig. I; Buff. Hist. Nat. Ois. v. p. 268 (1 778).

Motacilla melanope, Pall. Reis. Russ. Reichs, iii. App. p. 6yh (1776); Gm. Syyst. Nat. i. p. 997 (1888); Pall. Zoogr. RossoAsiat. i. p. 500 (1811); Dresser, B. Eur. iii. p. 25I, pl. 128 (1875); id. Ibis, 1876 , p. 177; Dauf. Ib.s, 1877, p. 262; Blakist. \& Prye., Ibis, $\$ 878$, p. 237; Seebohm, t. c. p. 352; Legge, B. Geylon, p. 610 (1879); Seebohm, Ibis, 1879, p. 34; Wardlaw Ramsay,. t. c. p. 448; id. Ibis, 1881, p. 60; Seebohm, t. c. p. I89; Severtz. Ibis, 1883, p. 64; Sharpe, Cat. B. M. 10. p. 497 (1885).

M. Eoarula, Linn. Mant. p. 527 (1771'; Gm. Sysl. Nat. i. p. 997 (1788); Temm. Man. d'Orn. i. p. 257 (1820); Roux. Orn. Provenç. pl. 195 (1825); Werner, Atlas, Insect.v. pl. 74 (1827); Rüpp. Neue Wirb. p. 84 (1835-40); Eyton, Cat. Brit. B. p. I 5 (1 836); Gould, B. Eur. ii. pl. I 47 Bp. Comp. List. B. Eur. \& N. Amer. p. 19 (1838); Macg. Br. B. ii. p. 235 (1835); Keys. u. Blas. Wirb. Fur. p. xlix. (1840'; Nordm. in Démed. Voy. Russ. Mérid. iii. p. 155 (1840); Crespon. Orn. Gard. p. I72 (1840); Jerd. Madr. Journ. xi. p: 10 (1840); Rüpp. Syst. Uebers, p. 59 1 845 ; Heqvits. Eggs. Br. B. p. 1 26, pl. 33. fig. 2. (I846); Gray, Cat. Mamm. etc. Nepal pres. Hodgs. p. 75 (1846); id. Cen. B. i. p. 203 (1847); Blyth Cat. B. Mus. As. Scc. p. 137 (1849); Thomps. B. Irel. i. p. 218 (1861); Temm. \& Schl. Faun. Jap., Aves, p. 59 (1850); Kelaart, Prodr. Cat. p. I 2 I (1852); Kjarb. Orn. Dau. pl. i9 fig. 5 (1852); Layard, Ann. \& Mag. Nat. Hist. xii. p. 268 (1853); Vernon Harcourt, op. cit. 1855, p. 437; Bolle, J.f. O. 185\%, p. 268; Jaubert \& Barth.-Lapomm. Rich Orn, p. 274 (1856); Linderm. Vög. Griechenl. p. 80 (1860); Swinh, Ibis, 1860, p. 55; Powys, t. c. p. 229; Simpson, t. c. p. 394; Swinh. Ibis, 1861 , pp. 35, 383; Irby, t. c. p. 232; Swinh. Ibis, 1862 , p. 260 Blakist, t. c. p. 318 Swinh. Ibis, 1863 , p. 309; Adams, Ibis, 1864 , p. 22; Wright, t. c. p. 62; Salvad. Orn. Sard. p. 57 (1864); Filippi, Viags. Pers. p. 348 (1865); Altum, J. f. O. 1865 , p. 246; Swinh. Ibis, 1866 , p. 138 Pclz. Ibis, 1868 , p. 31 2; Swinh, t. c. p. 442; Gray, Hand-l. B. i. p. 247, n. ${ }^{\circ}$ 3592, (1869); Vorggr. Vogelf. Deutschl. p. 87 (1869; Doderl. Avif. Sicul. p. ro (1869); Salvad. Faun. Ibis, Ucr. p. I21 (1871);B. Gray, B. W. Scoll. p. 112 (1871); Saunders, Ibis, 1871, p. 215; Harting, Handb. Br. B. p. 23 (1872); Blith \& Wald. B. Burm. p. 97 (1875); B. O. U. List Br. B. p. 30 (1883); Seebohm, Ibis, 1883 , p. 30; Jouy, Pr. $U$. S. Nat. Mus. vi. p. 290 (1883); Racide, Orn. Cauc. p. 223 (1884); Blakist. Amended List B. Japan, p. 55 (8884).

Grey wagtail, Iatk. Gen. Syn. ii. pt. 2, p. $308(1,783)$. 
Tschutschi wagtail, Penn. Arctic Zool. ii. p. 397 (1 785 ).

Motacilla tschutschensis, Gm. Syst. Nat. i. p. 962 (1788).

M. sulphurea, Bechst. Naturg. Deutschl. iii. p. 459 (1807); il. Kurze Uebirs, aller bekannt. Vügrl, p. 326 (1811); Naum. lig. Deutschl. iii. p. 824, Taf. 87 (1823); Brehm, Vög. Dentsill. 1. 346; Cab. Mns. Hein. 'Th. i. p. 13 (1850; Midd. Sitir. Reis. p. 168 (1851); Schl. Vög. Nederl. pl. 103 (1854); Sunder. Sv. Fogl. pl. 66 fig. 4 (c. 1856); Schrenck, Amur. Ruis. Zool. p. 344 (1859); Schl. D) er. Nederl. Vög. pl. 9 figs. 11, 12 (1861); Trisir. Ibis, 1862 , p. 279; .1. Neiton, Ib s, 186 , p. 194; Gray's List Br. B. p. 67 (1863; Brehnt, Re:s, Hi:besch, p. 2 I 3 (1863); Radde, Rcis. Sib.r. p. 227 (1863); Moli, Ibis, 1865 , p. iz2; Gocim. Ib:s, i 866, pp. 96, ic6; Tristr. t. c. p. 29 ; C. B. Wharicn, t. c. p. 323; Liljord, t. c. pp. 386, 391; Degl. et Gerbe, Oru. Eurr. i. p. 385 (1867; Heugl. Crn. N. O.-Afr. i. p. 318. (1869); Godmä, Azcrè, 1. 25 (1870); Fritsch, Vög. Eur. Taf. xuii. figs. 12, $14 \%$. (I870); Wyalt, Ibis, 1870 , p. I5; Likes \& Buckley, t. c. p. 195; Blanf. Gcol. \& Zcal. Aly'ss. p. 381 (1870; Godm. Ib:s, 1872 , p. I76; Shelley, B. Egypt, p. I27 (1872); Keulem, Orn. Vogels, ii. pl. 33 (1873); Nea't. ed. Yarr. Br. B. i. p. $5=2$ (1873); Sezerta. Turkisl. Jevotn. p. 67 (1873); Brook, Ib.s, 1873, p. 245; Dauf \& Harvie-Brou'n. Ibis, 1875, p. 3og; Irty, B. Gibr. p. 108 (1875); Blanf. East. Persia, it. p. 23 (1876; Irly, Ibis, $188_{3}, \mathrm{p} . \mathrm{I}_{5} ;$ Bcoth, Rough Notes, pairt 4 (1883); Scebolim, Hist. Brit. B. ii. p. 263 (1883); id. Ib is, 1884 , p. 39; Tristr. Fann. \& Flar. Palist. p. 54 (1884); Salvad. Ann. Mus. Civic Genov. (2) i. p ig6 (I884).

M. cinerea, Leach, Syst. Cat. Mann. eic. Brit. Mus. p. $22(7816)$.

M. bistrigata, Raff. Trans. Linn. Soc. xiii. p. 31 2 (1820); Gran', Hand-l. B. i. p. 248, n. ${ }^{\circ} 3593$ (r 869 ).

Calobates sulphurea, Kaup, Natürl. Syst. p. 33 (1829); Moore, P. Z. S. 1854 , p. 284; Horsf. \& Moore, Cal. B. IE. I. Co. Mus. i. p. 349 (1854); Jerd. B. Ind. p. 220 (1863); Ciould. B. Gt. Br. iii. pls. 6, 7; Holdsw P. Z. S. 1872, P. 415; Hume \& Henders. Lahore to Yark. p. 224. (1873); Butter \& Hume, Str. F. 1875 , p. 419; Butler, Str. F. 1877, p. 230; Bogdan. B. Canc. p. 108 (i879); Homczer \& 7 aniré, $M T$. orn. Ver. Wien, 1883 , p. 86.

Motacilla montium, Brehm, Vög. Deutschl. p. 345 (I83r).

11. xhanthoschista, Hodgs. in Ciray's Zcol. M SC. p. 83 (184t'.

11. lunulata, Dos Murs in Lejebroe, Voy. Alyss., Allas, pl. I (1845).

M. ophthalmica, Des Murs, op. cit., texte Ois. p. 94 (1845).

Pallenura sulphurea, Bp. Consp. i. p. 250 (I850) Brehm, Natm. 1855, p. 280; Loche, Exféd. Sci. Algér., Ois. ii. p. 5 (I86;); Dytcuski, J. f. O. 1873, p. 82, 1874, p. 335; Tacsin. Bu!!. Sec. Zool. Frnce, 1876 , p. I 50; Dyboziski, op. cit. $1883, \mathrm{p} \cdot 3^{60 ?}$ P. javensis, Bp. Consp. i. p. 250 (1850).

Motacilla montium, rivalis, Brehin, Vogelf. p. 143 (i 855 ).

M. lindermayeri, Brehm in Linderm. Vög. Criechenl. p. $82(1800)$.

Budytes boarulus, Tristr. Ibis, 1859, P. 4I; C. B. Wharlon, Ibis, 1876 , p. 22.

3. flava (nec I.), Betton; Ĺce. Nidif. Lomh. tav. 62 (1865-70). 


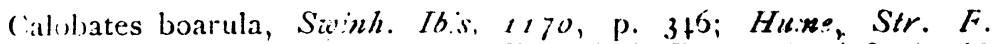
1173 , p. 201; id. Nesis: and Eggs In:l. B. p. 381 (1874); id. Str. F. 1174, p. 237.

Mrtacilla (Calobates) sulphuria, Bl.rnf. Geol. \& Zool. Abyss. p. $38 \mathrm{i} \quad(\mathrm{i} 870)$.

... (Calobates) boarula, Helgr!. Orn. N. O.-Afr. ii. pt. 2, Apl). 1. $\operatorname{lxxxix}(\mathrm{I} 87 \mathrm{I})$.

(alobates melanope, Swinh. P. Z. S. 1871 , p. 364; Brooks, J. A. S. Beng. xli. p. $82(1872)$; id. Str. F. 187.5, p. 250; F.xir.'. Sitr. F. 1876 , p. 260 ; Bourd. t. c p. 401 ; Priev. in D.xwsont Roivley's Orn. Misc. ii. p. 193 (1876); D.vvid \& Oust. O.s. (hine, p. 302 (1877); Walden, Tr. Z. S. ix. p. 196 (1877); Tiweedd. Ibis, 1877,$310 ;$ Hume \& Davison, Sir. F. 1878 , p. $362 ;$ Davison \& wend. Str. F. 1878 , vol. ii. p. 84; Hu :e, Str. F. 1879 , pp. 65, го3, г6г; Scully, t. c. p. 315; Brooks, t. c. p. 484; Butler, Cat. B. Sind \&c. p. 40 (1879); id. Cat. B. S. Bomb. Pres. p. 54 (1880); Vidal, Sir. F. $18^{\prime} 8^{\circ} 0$, p. 69; Reid, Str. F. 1881 , p. 48 ; Salvad. Orn. Papuasia etc. ii. p. 431 ( I88I); Biddulph, Ibis, 1881 , p. 68; Scully, t. c. p. 452; Dai'idson, Str. F. 1882 . p. 3ro; Nichols. Ibis, 1882, p. 62; C. Sivinhoc, t. c. p. Io9; Slater, t. c. p. 433; Nichols. Ib.s, 1883 , p. 253; Davison, Str. F. 1883 , p. 395; Oates, B. Br. Burm. i. p. I59 (I883).

(. bistrigata, Salvad. Ucc. Born. p. 259 (1874).

Budytes novæ guineæ, Meyer, Sitz. Isis Dresden, 18.75, p. 74 ; idt. Mitth. K. Zool. Mus Dresden, i. p. Io (1875); Salvad. Ann. Mus. Ctvic. Genov. xii. p. 345 (1878).

(alobates melanops Ball, Str. F. 1174, p. 446; id. Str. F. 1171, vol. ii. p. 219 .

Hab. Luzón, Manila, Montalban, Paragua, P. Princesa, Mindanao, Pasananca, Zamboanga, Bısilan, Guimarás, Panay, Negros, Samar, Paranıs, Cebú, Leyte; Europa, y Región IndoMalaya, China, Japón, Borneo, Java, Sumatra.

4. M. Hava Lin. O $\bigcirc$ N. v. Pugapug.

(M. S. T)

I a Bergerenotte de printemps, Briss. Orn. iii. p. 468 ( 1760, pt.).

La Bergeronette de Java, Briss. $t$. c. p. 475 , pl. 25, fig. 2 ( 760$)$; Montb. Hist. Nat. Ois. v. p. 266.

Motacilla flıva, Linn. Syst. Nat. i. p. 331 ( 760 ); Horsf. Trans. linn. Soc. xiii. p. I 56 (1820); Temm. Man. d'Orn, i. p. 26) (1820); Naum. Vög. Deutschl. iii. p. 839. Taf. 87 (1823); Roux, Orn. Prov. pl. 196 (1825); Werner, Atlas, Insectivores, pl. 7t (1827); Rüpp. Neue Wirb. p. 84 (1835-4o); Temm. Man. d'Orn. 1835, p. 181, (1840); p. 622; Crespon, Orn. Gard. p. 174 (1840); Keys. \& Blas. Wirb. Eur. p. 175 (1840); Nord. in Démid. loy. Russ. Mérid. iii. p. 156 (1840); Selys-Longch. Faune Belge, p. 88 (1842); Schl. Rev. C'rit. p. xxxviii. (1844); Hewits, Eggs Brit B. i. p., I72, pl. 34. figs. I, 2 (1846); Gray, Gen. B. i. p. 203 (1 847); Middend. Reis. Sibir., Zool. p. 168 (1851); Bjerb. Danm. Fugle, Taf. xix. fig. 3, xix. Suppl. fig. 4 (1852); Sichl. Vog. Nederl. pls. 98, 99 (1854); Sundev. Svensk. Fogl. pl. ix. fig. 4, 5 (c. 1856); Selys-Longch. Naum. 1856 p. 399 (cum var.) Salvin, Ibis, 1859 , p. 30; Tristr. t. c. p. 423; 
Simpson, Ibis, 1860 , p. 393; Linderm. Vög. Grieche'nl. p. 8( (1860); Blasius, in Nachtr. Naum. Vög. Deutschl. p. 120 (1860): Schl. Dier. Nederl., Vog. pl. ix. figs. 9, 10 (1861); $F$. \& P. Godman, Ibls, 1861 , p. 82; Wright, Ibls, 1864 , p. 62: Finsch, Neu-Guinea, p. 167 (1865); Simith, Ibis, 1868, p. 442, Gray, Hand-l. B. i. p. 246, no. 3578 (i869); Kelsl. Onz: Vogels, i. pl. 26 (1869); Baird, Trans. Chicago Acad. i. p. 312, pl. 3o. fig. I (1869); Dall \& Bann. t. c. p. 297 (1869); Heugl. Orn. N, O.-Afr. i. p. 320 (1869); Wyatt, Ibis, 1870. p. 15; Finsch \& Hartl. Vög. Ostafr. p. 268 (1870); R. Gray, $B$. W. Scotl. p. I13 (1871); Swinh. P. Z. S. 1871, p. 364: Harting, Hanab. Brit. B. p. 23 (1872); Pelz. Verh. z.-b. Gesellsch. W.en, viii. p. 428 (1872); Baird, Brever, \& Ridgit'. Hist. N. Amer. B. i. p. 167 (1874); Danford \& Hervie.Brown, Ibis, 1875 , p. 310; Dresser, B. Eur. iii. p. 26r, pl. I29. figs. I, 2 (1875); id. Ibis, 1876, p. 178; Finsch, t. c. p. 62; Seebohm, Ibis, 1878 , p. 351; E. Adams, t. c. p. 423; Coues, B. Color. Vall. p. 192 (1878); Seebohm, Ibis, 1882, p. 216; Dixon, t. c. p. $57 \mathrm{I}$; Seebohm, Ibis, 1883 , p. 20; id. Hist. Brit. B. ii. p. 208 (1883); Shelley, t. c. p. 543; B. O. L'. List Brit. B. P. 31 (1883); Tristr. Fal!n. \& Flor. Palest. p. 55 (1884); Radde, Orn. Ciauc. p. 220 (1884); Sharpe, Cat. B. M. 10. p. 516 , lam. 6. figs. $3-5$. (1885).

Parus luteus, caspicus, S. G. Gmelin, Reis. Russl. iii. p. 1о1, pl. 20. fig. I (I774).

La Bergeronette de l'isle de Timor, Montb. Hist. Eat. Ois $r$. p. 275 .

Motaciila boarula, $\beta$. javensis, Gm. Syst. Nat. i. p. 998 (1788).

M. flaveola, Pall. Zoogr. Rosso-Asiat. i. p. 501 (1811).

M. chrysogastra, Bechst. Kurze Uebers. p. 327 (1811).

M. flavescens, Steph. Gen. Zool.pt, 2, p. 559 (1817); Gray, P. $Z$. S. 1860 , p. 350; Wall. P. Z. S. 1863 , p. 485; Gray, Hand-l. B. i. p. 247, n. 3586 (1 869).

Budytes flava, Cuvier, Règne Anim. p. 37г (1817); Brehm, Vög. Deutschl. p. 344 1831); Ménétr. Cat. Rais. Cauc. p. 36 (1832); Bp. Faun. Ital, Ucc. pl. 31 fig. I $\left(183^{2-44)}\right.$; Maggill. Brit. B. ii. p. 208 (1839); Bp. Consp. i. p. 249 (1850'; Jaub. $\epsilon$ ' Barth.-Lapomm. Rich. Orn. p. 279 (1859); Trist. It s, 1859 , pp. 31, 420; Salvin, t. c. pp. 310, 358; Swinh. Ihis, 1860, p. 51, I861, pp. 36, 333, 4 I I I862, p. 260 I863, p. 208; Brehm. Reis. Habesch, p. 14 (1863); Adams. Ibis, 1864, p. p. 20; Salvad. Ucc. Sard. p. 58; (1864); Sol. P. Z. S. 1864 , p. 108; Altum. J. f. O. 1865 , p. 247; Blytk, Ibis, 1865, p. 50; Giglioli, t. c. p. 55; Lilford, Ib.s, 1866 , pp. 187,386 , 391; Tristr. t. c. p. 290; Chambers, Ibis, 1867 . P. I04; Drake, t. c. p. 427; Malmgr. t. c. p. 230; D.gi. \& Gerbe, Orn. Eur. i. p. 376 (1867); Loche, Expl. Sci. Algér., Ois. p. 7 (1867); Heugl Orn. N. O.-Afr. i. p. 321 (1868; Doderl. Avif. Sicil. p. IOI (1869); Dall. \& Bann. Tr. Chicago Acad. i. p. 312, pl. 3o. fig. I (1869); Wyatt, Ibis, 1870, p. I5; Elwes \& Buckley, t. c. p. 195; Layard. t. c. p. 282; Gould, B. Gr. Brit. iii. pl. 4 (c. 1870 ); Fritsch, Vög. Eur. tab. 17 . 18 (1870); Salvad. Faun. Ital., Ucc. p. 122 (1871); Sissinh. $P$. Z. S. 1871, p. $364 ;$ Ayres, Ibis, $187_{11}$, p. I54; Sambders, t. c. 
p. 215; Shelley \& Buckley, t. c. p. 292; Shurpe, Cat. Afr. B. p. 73 (1871); Gurney in Anterss. B. D.tm. Lt p. I1 2 (1872); Shelley, B. Eyypt, p. г 28 (1872); Coues, Key N. Amer. B. p. 90 (1872); Severtz. Turkest. Jevotn. P. 67 (1873); Bairat. Brewer of Ridgw. Hist. N. Ams. B. p. 167 (187t); B!.znf East. Pers. ii. p. 233 (1876); Tacs. Bull. Soc. Zool. Firanze 1376 p. I50; D.xidd \& Oust. Os, Chine, p. 302 (I877); Brooks, Ibis, 1877 , p. 208; Ayres, t. c. p. 294; Homeyer. $J$. $f . O \quad 1878$, p. I $30 ; B$ B.t. $B$ Cauc. p. 108 (1879); Gigliolt, Ibis, 1881 , p. 200; Kelham, t. c. p. 517; Shelley, P. Z. S. 1881 , p. 573; Ridgw. Bull. U. S. Nat. Mus. n. ${ }^{\circ}$ 1, p. 15 (1811); Coues, Check-list, p. 32 (1882); C. kwinh. Ibis, 1882. p. I го; Irby, Ibis, 1883 , p. г 35 ; Severtz, $t$ c. p. 64; Nelson, Cruise R. S. "Corwin», p. 62 (1883); Dybozuski, Eull. Soc. Zool. France, viii. p. 360 (1833); Homeyer \& Tancre, M. 1. orn. Ver. Wien. T113, p. 86; Sharpe, ed. Lajard's B. S. Afr. p 550 (1884); Saluad. Ann. M.is. Civic. Genso. (2) i. p. $166(188+)$.

Motacilla neglecta, Gould. P. Z. S. 1832 , p. 129; i:t. B. Eur. ii. pl. 146 (1837); E. C. Taylor, Ibis, 1859 . p. 48.

M. flava vulgaris, Sundev. Efv. K. Vet.-Akad. Förh. Stockh. 1840, p. 53 .

Budytes gouldi, Macgill. Man. Brit. B. i. p. I63 (1840).

Motacilla (Budytes) flava, Schrenck, Sibir. Reis. p. 345 (1853); Radde, Reis. Ost-Sibir., Vög. p. 230 (1863).

Budytes pygmaus, A. E. Brehm, J. f. O. 1854, p. 74, note.

B. fasciatus, C. L. Brehm, Vogelf. p. I4 I (1855).

? B. pygmaus, Oscar Brehm, in C. L. Brehm's Vogelf. p. 142 (1855).

Motacilla flava typica, Radd:, Reis. Ost. Sibir., Vög. p. 229 (1863). Budytes viridis, (non auct.), Sclater, P.Z. S. 1853 , p. 2I 4 ; Swinh. Ibis, 1864 , p. 418; Wialden, Trans. Zool. Soc. viii. p. 65 (1872); Salvad. Ucc. Brrn. p. 260 (1874); Tweedt. Ibis, 1877 , p. 22ó; id. P. Z. S. 1878 , p. 82; Meyer, Ibss, 1879 , p. I27; Sharpe, t. c. p. 262; Salvat. Orn. Papuasia etc. ii. p. $43^{\circ}$ (1881).

B. cinereocapillus, Swinh. Ibis, ${ }_{185} 3$, pp. 94, 309; id. P. Z. S. 1871 , p. 364.

Motacilla flava cinereocapilla fasciata, Heugl. Orn. N. O.-Afr. i. p. 32 I (1869).

Budytes leucostriatus, Homeger, $J . f . O .1878$, p. 128.

B. flavescens, Homeyer, $J$. $f$. $O .1878$, p. I 3 I. Hab. Paragua, P. Princesa; Java, Célebes.

5. M. borealis, (Suntev). N. v. Baticulo.

(M. S. T.)

Green wagtail, Brown Zool. pl. 33. f. 2 (I775).

Mutacilla fliva borealis, S.uıdev. O,fo. K. Vet. Akad, Förh. Stockh. $18^{2}+0$, p. 53 .

M. viridis, Gin. S. N. i. p. 962 (1788); Lath. Ind. Orn. ii. p. $505(1783)$.

M. borealis; Sharpe, Cat. B. M. 10. p. 522, lam. 8, fig. I-3. Harb. Luzón, Bataan, Orani, Bohol, Mindando, Joló; Burneo, Cólebes. 
3 Gen. Anthus, Bechis.

\section{A. richardi, Vicill. ơ N. v. Pugapug}

Anthus richardi, Vieill. N. D)ict. d'Hist. Nat. xxvi. p. 49r (1818); Temm. Pl. Co!. iii. pl. Ior (1824); V.g. Zool. Journ. i. p. 41 I (1825); Roux, Orn. Prov. pls. I89, 19) (1825); Goveld. B. Eur. ii. pl. I35 (1837); Macgill. Brit. B. ii. p. I99 (1839); Norm. in Dénid. Voy. Ritss. Mérid. iii. p. I 5 (18fo); Bu. teille, Orn. Dauphine, pl. 29. fig. I. (18+3); Yarr. Brit. B. i. p. 368 (1 $8+3)$; Von der Muh!e, Orn. Giciechntul p. $5^{8}$ (1 $\left.8+4\right)$; Gray, Gen. B. i. p. 205 (18+7); Blyth. Cat. B. Mits. As. Soc. p. 35 (1849); Lryard, Ann. \& Mag. N. H. xii. p. 268 (1853); Schl. Vög. Nederl. pl. 93 (1854); Horsf. \& Moore, Cat. B. E. I. Co. Mus. i. p. 355 (1854); Blasius, in Naum. Vög. Deutschl. Nachtr. xiii. P. 94, pl. 37 I (I860); Swvinh. Ibis, 1860 p.55, 1861; p. 36; B3lasius, Ibis, 1862 , pp. 62, 7 1; Swinh. Ibis, 1863 , p. 311; Gray, Lyst Brit. B. p. 69 (1863); Wright. Ibis, 1864 , p. 6r; Schomb. t. c. p. 241; Dybozeski, J. f. $O$. 1868 , p. 231; Hume, Ibis, 185 g, p. I20; Saunders, t. c. p. p. 392; Borgyr. Vogelf. Norddeutschl. p. 86 (1869); Doderl. Avif. Sicil. p. 97 (1 869); Droste, Vogelw. Bork p. I04 (1 869); Gray. Hand-l. B. i. p. 252, n. 3649 (1869); Swinh. Ibis, 1870 , p. 83; Suudev. Svenk. Fogl. p. 42, pl. 66. fig. 5 (c. 1870); Fritsch. Vög. Eur. pl. 16. fig. 4 (1870); Salvad. Faun. Ital., Ucc. p. I 28 . (1871); Harting, Handb. Brit. B. p. 24 (1872); Gould, B. Gt. Br. iii. pl. 8 (c. I870); Newt. ed. Yarell's Br. B. i. p. $598\left(18_{74}\right)$; Dresser, B. Eur. iii. p. 325 , pl. I38 (1875); Irby, B. Gibr. p. I I I (1875); Cordeaux, Ibis, 1865 , pp. 173, 18r; Danf. \&.Harvie Brown, c. c. p. 3I I Blanf. East. Persia, ii. p. 236 (1876); Seebohm, Ibis, 1877 , p. 343; B. O. U. List Br. B. p. 34 (1883); Sharpe, Cat. B. $M$. 1o. p. 564 (1885).

Corydalla richardi, Vigors, Zool. Journ. ii. p. 397 (I 862); Bp. Consp. i. p. 247 (1850); Kelaart, Prodr. Faun. Zeyl. Cat. p. I2I (1852); Jaub. et Barth-Lapomm. Rch. Orn. p. 287 (1859); Szuinh. Ibis, 1861 , p. 265; Jerd. B. Ind. ii. p. 23 I (1863); Dagl. et Gerbe, Orn. Eur. i. p. 363 (1867); Loche, Expl. Sci. Algér., Ois. ii. p. I I (1867); Beav. Ibis, 1868, p. 79; Hume, Ibis, 1869 , p. 120; Collett, J. f. 0.1869 , 1. 393; Blanf. Ibis, 1870 , p. 469; Swinh. t. c. p. 3i7; id. P. Z. S. 1871 , p. 366; Holdsw. P. Z. S. 1871 , p. 458; Brooks, Str. F. 1870 , p. 358; Hume, Str. F. 1874, p. 239; Ball, t. c. pp. 416, 479, 496; Blyth \& Wald. B. Brim. p. 95 (1875); Armstr. Str. F. 1876 , p. 330; Tacz. Bull. Soc. Zool. France, i, 957 (1876); Prjeb. in Rowley's Orn. Misc. ii. p. 195 (1876); Dxvid \& Oust. Ois. Chine, p. 310 (1877); Anders. Zool. Erp. Yun-nan. p. 365 (1878); Hume \& Davison, Str. F. 1878 , p. $365 ;$ Ball. Str. F. 1878 , vol. ii. p. 220; Cripps, t. c. 288; Legge, B. Ceylon, p. 6or ( I 879); Hume, Str. F. 1879, p. Io3; Scully, t. c. p. 3i6; Oates, B. Brit. Burm. i. p. I66 (1833).

Anthus rupestris (nec Nilss.), Ménétr. Cat. Rais. Cauc. p. 37 (1832). 
1. macronyx, Gloger, Handb. lög. Eur. i. p. 260 (1834).

A. longipes, Holandre, Faune de la Moselle, p. $8+(1836)$.

(ichlops monticolus, Hodgs. Icon. ined. in Brit. Mus., Passeres, pl. 121, 12t fig. I (no. +31); id. in Gray's Zool. Misc. 1. R.3 (i $8+4)$.

(orydalla sinensis, Bp. (onsp. i. p. 247 ( 1850$)$; Dazid of Oust. Ois. Chine, p. 3 п 1 (1877).

Anthus maximus, Blyth in White's Nat. Hist. Selborne, p. 202 ( 1858$)$.

Corydalla chinensis, Szimh. P. Z. S. 1871, p. 366 .

Agrodromas richardi, Saunders, Ibis, 1871, p. 216; Giglioli, Ibis, 1881, p. 206.

Hab. Luzon, China, Ceilan, Molucas.

7. A. rufulus, Vieill. $0^{*}: \mathrm{N}$ v. Pugapug.

Anthus rufulus, Fieill. N. Dict. d'Hist. Nat. xxxri. p. $49+(1818)$; Gray, Gen. B. i. p. 206 (1817); Blyth. J. A. S. Beng, xri. p. 437 (1 $8+7)$; id. Cat. B. Mus. As. Soc. p. $135(1849 ;$ Bp. Consp. i. P. 248 ( 1850$)$; Layard, Aun. \& Mag. Nat. Hist. xii. p. 268 (1853); Horsf \& Moore, Cat. B. E. I. Co. Mus. ii. p. 356 (1854); Schomb. Ibis, 1864 , p. 249; Gray, Haud-l. B. i. p. 252 , n." 3651 (1869): C. Swinh. Ibis, 1882, p. II0; Sharpe, Cat. B. M. 1o. p. $57+(1885)$.

Alauda pratensis, Raffl. Trans. Linn. Soc. xiii. p. 315 (1820). Anthus cinnamomeus, Rüpp. Neue Wirb. p. Io3 (1835); id. Sj'st. Uebers. p. 59 (1845); Gray, Gen. B. i. p. 206 1847, Bp. Consp. i. p. 248 (1850); Heugl. Syst. Uebers. p. 20 (1856); id. Orn. N.O. Afr. i. p. 329 (1869).

A. malayensis, Eyton, P. Z. S. 18.39 , p. I04; Blyth, J. A. S. Beng. xi. pp. 797, 885 (1842); xvi. 436 (1847); Horsf. \& Moore, Cat. B. E. I. Co. Mus. i. p. 357 (1854); Gray, Hand-l. B. i. p. 252, n." 3653 (1869); Hume, Str. F. 1874 , p. 497; Tweedd. Ibis, $187 \%$, p. 3IO; Hume, \& Davison. Str. F. 1874, p. 366; Hume, Str. F. 1879 , pp. 65, гоз.

Agrodroma agilis, Jerd. Madr. Journ. xi. p. 33 (1840).

Cichlops ubiquitarius, Hodgs. Iton. ined. in Brit. Mus. Passeres, pl. 122 figs. I, 2 (n. $\left.{ }^{\circ} 433\right)$; id. in Gray's Zool. Misc. p. 83 ( 1844$)$.

C. fortipes, Hodgs. Icon. ined. in Brit. Mus. Passeres, pl. I 22 a. fig. 2 (n. $\left.{ }^{\circ} 73^{8}\right)$; id. in Gray's Zool. Misc. p. 83 (1844).

Anthus ubiquitarius, Gray, List Mamm. \& B. Nepal. pres. Hodgs. p. $77(1846)$.

A. cafler, Sundev. Efvo. K. Vet.-Akad. Förh. Stockh. I $85^{\circ}$, p. 1oo; Layard, B. S. Afr. p. I 23 (1867); Gray, Hand-l. B. i. p. 249 , n." 3624 (1869); Layard, Ibis, 1869 , p. 368; Tristr. t. c. p. 437 ; Sharpe, Cat. Afr. B. P. 72 (I871); Ayres, Ibis, 1871, p. I56; Gurney in Anderss. B. Lam. Ld. p. II3 (1872); Buckley, Ibis, 1874, p. 384; Feugl. J. f. O. I874, p. 48; Ayres, Ibis, 1880 , p. IO4; Sharp. in Oates's Matabele Land; App. p. 317 (1881); Shelley, Ibis, 1882 , p. 350; Butler, Feilden, \& Reid, Zool. 1882 , p. 335; Schalcz', J. f. O. I 883, p. 367; Boehm, t. c. p. 207; Sharpe, ed. Layard's B. S. Afr. p. 434 (1884).

A. raalteni, Bp. Consp. i. p. $248(1850)$ ex Temm. MSS. in Mus. 


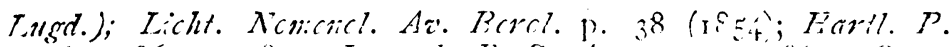
Z. S. 1867 , p. 825; Logard, B. S. Ajr. p. 123 (1867); Gray, Hand-l. S. i. p. 249, n. 3616 (1869); Cab. in Von der Decken's Reis. iii. p. 22 (1869); Finsch \& Hartl. Vög. Ost-Ajr. p. 274 (1870'; Shelley, B. Esypt. p. I 33 (1872); Gurney in Anderss. B. Dam. Ld. P. II 3 (1872); Gadcte, J. f. O. i87o, p. 431; Fischer, J. f. O. 1877 . p. 207; id. J. f. O. 1878 , pp. 268, 269; Cab. t. c. p. 220; Nichols. P. Z. S. 1878 , p. 356 ; Fischer, J. $f$. O. $1879, \mathrm{pP}$ 294, 299; 203; id. \& Reichen. t. c. p. 355 ; Shelley, P. Z. S. 1881, p. 573 .

A. fuonyx, Cab. Mus. Hein. 'Th. i. p. I4 (I850; Gray', Hand-l. B. i. p. 252, n. $^{\circ} 3659$ (i 869 ).

Corydalla rufula, Kelaart, Prodr. Faun. Zcyl. Cat. p. i2 I (1852: Jerd. B. Ind. ii, p. 232 (1863); Blyth, Ibis, 1867, p. 31; Pel:. Ibis, 1168 , p. 31 2; Blanf. Ibis, 1870 , p. 469; Holdsw. P. Z. S. 1872 , p. 458; Hunie, Nests and Eggs Ind. B. p. $38+(1873$; Brooks, Str. F. 1873 , p. 359; Adam, t. c. p. 384 ; Ball, Str. F. 1874 , p. 4 r6; Hume, t. c. p. 497, I875, p. I42; Brooks, t. c. p. 252; Butler \& Hume, t. c. p. 190; Blyth, \& Wald. B. Burm, p. 69 (1875); Fairb. Str. F. 1876 , p. 260; Armstr. t. c. p. 330; Bourd. t. c. p. 401; Hume, t. c. p. 458 ; Fairb. Str. F. 1877, p. 107; Hume, \& Davis. Str. F. 1878, p. 366; Davids. \& Wenden, Str. F. I 878 , vol. ii. p. 84 ; Ball, $t$. c. p. 220; Cripps, t. c. p. 288; Butler, Cat. B. Sind \&c. p. 4 I (1879); Legge, B. Ceylon, p. 625 (1879); Hume, Str. F. 1879, p. Io3; Scully, t. c. p. 317; Butler, t. c. p. 386 ; id. Cat. B. S. Bomb, Pres. p. 56 (1880); Vidal, Str. F. 1880 , p. 69; Reid, Str. F. I880, p 49? Davids. Str. F. 1882, p. 310; Oates, B. Brit. Burm i. p. I68 (1813'; Davison, Str. F. I883, p. 397 .

C. hasselti, rasselti, Brehm, Naum. 1156, p. 463; Salvad. Ucc. Born. p. 262 (I874).

Anthus hasselti, Schl. Handl. Dierk. p. 263 (1857, ex Temm. MSS.) Gray, Hand-l. B. i. p. 252, n. ${ }^{\circ} 3655$ (1869).

A. medius, Wall. P. Z. S. 1863 , p. 488 ; Gray, Hand-l. B. i. p. 252, n. $^{\circ} 3654$ (i 869 ).

A. sp., Antin. \& Salvad. Viagg. Bogos, p. гог (1873).

- Corydalla malayensis, Trueedd. Ibis, 1877 , p. 310; Home \& D. vis. Str: F. 1878, p. 366; Hume, Str. F. 1879, p 65; Kelham, Jbis, 1881 , p. 518 .

C. lugubris, Trueedd. P. Z, S. 1877 , pp. 547 696, 762, 828; id. Z. P. Z. 1878 pp. 286, 380, 710; Wardlaw Ramsay, Orn. Works Treeed. p. 658 (1881).

C. ubiquitaria, Anders. Zool. Yun-nan Exped., Aves, p. $3(x)$ (1 878 ).

Hab. Panay, Hoilo, Mindoro, Leyte, Samar, Cuimarás, Nigros, Dumaguete, Siquijor, Bohol, Luzon, Cebú, Panaon, Mindanao, Borneo, 'limor, Célebes, Molucas.

\section{A. cervinus, Pall. $\mathrm{N}$ v}

Motacilla cervina, Pall. Zoogr. Rosso-Asiat. i. p. 51 1 (1811); Eversm. Bull. Soc. Mosc. xxiii. pt. 2., p. 570 ( ( 850$)$.

Anthus cervinus, Naum. Vög. Deutschl. iii. pl. 85 fig. I ( 823 ; Keys. u. Blas, Wirb. Eur. p. 172 (1840); Gray, Gen. B. i. 
p. $206(18+7)$; Rp. Consp. i. Г. $24 \delta$ (1 $\delta 50)$; Milldend. Sibir. Reis. p. 165 (1851); Svensk. Fogl. pl. 8, fig. 6 (c. 1858 ; Jaub. et Barth.-Lapomm, Rich. Oru. P. Is4, pl. (1859); Blasius, Isis, 1861, P. 261, (1862); p. 71; Swinhoc, Ib.s, $186 \therefore$

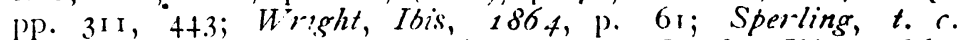
p. 279; Tristr. Ibis, 1856, p. 2co; Blyth, Ibis, 1867 , p. 31; Degl. \& Gerbe, Orn. Enr. i. p. 369 (1867); Lcche, Expl. Sci. Alseir., Os. ii. p. 17 (1867); Beavan. Ibis, 1867 , p. 328; Doderl. Avif. Sicil. p. 99 (1869); Gray, Hand-1. $B$. i. p. 251, n. ${ }^{\circ} 36+6,(1869)$; Gould, $B$. Asia, iv. pl. 6i) (1869); Fritsch, Vög. Eur. pl. 16. fig. 5 (18jo); Swinh, Ibis, 1870, p. 83; Elaves \& Buckley, t. c. p. 195; Hume, Ibis, 18 it, p. $35 ;$ Tristr. t. c. p. 233; Swinh. P. Z. S. 1871, p. $365 ;$ Salvad. Faun. Ital., Ucc. p. 126 (1871); Helugl. Ibis, 1872 , p. 6r; Vian, Rev. et Mag. Zool. (2) xxiii. p. 4t (1872) Harting, Handb. Brit. B. p. Io9 (1872) Shelley, B. Egypt, p. 121 (1872); Alst. \& Harvie Brown, Ibis; 1873 , p. 61; Brooke, t. c. p. 345 ; Ball, Sr. F. 1873, p. 74; Hume, t. c. p. 3IO; Hume Str. 1874, pp. 239, 479; Lilford, Ib:s, 1875, p. 21 ; Cordeaux, t. c. p. I8I; Danf. \& Harvie Braz'n, t. c. p. 310; Stolicaka, Str. F. 1875 , p. 310; Blyth \& Walden, B. Burn: p. 96 (1875); Seebohm \& Harve Braz'n, Ibis, 1876 , p. I22; Dresser, t. c. p. I80; Brooks, t. c. p. soo; Tacz. Bull. Soc. Zool. France, i. p. I 59 (1876); David \& Oust. Ois. Chine, 1 . 306 (1877); 'Finsch, Ibis, 1877 , p. 59; Hume, \& Davison,. St' . F. 1878 , p. 367 ; Danf. Ibis, 1878 , p. 20 ; Scebohm, t. c. 1. 342, I879, p. 34; Gätke, t. c. p. IO3; Finsch, Verh. z.-L. Gesellsch. Wien, 1879 , p. 66; Hume, Sir. F. 1879, p. 103; Seebohm, Ibis, 2880 , p. i 88 ; Biddulph, t. c. p. 70; Gigliol, Ibis, 1881 , p. 300; Ridgw. Bull. U. S. Nat. Mus. vi. p. $5^{6}$ (I88I); Belding, t. c. p. 350; Seebohm, Ibis, 1882, p. 2i6; Bidulph, t. c. p. 280; Seebohw.t. c. p. 376 ; B. O. U. List Brit. B. p. 32 (1883); Seebohm, Hist. Brit. B. ii. p. 229 (1883); Dybowski, Bull. Soc. Zool. France, viii. p. 36I (1883); Hcyer \& Tancré, MT. orn. Ver. Wien, 1883 , p. 86; Sharpe, P. Z. S. 1884 , p. 206; Tristr. Faun. \& Flor. Palest. p. 55 (1884); Radde, Orn. Cauc. p. 219 (1884); Sharp. Cat. B. M. 10. p 585 (i 885$)$.

A. cecilii, Audouin, Deser. Egipte, p. 36o, pl. 5 fig. 6 (1828; Blanf. Geol. \& Zool. Abys. p. 382 (1870).

A. pratensis nubicus, Hempr. \& Ehr. Symb. Phys. fol, dd (1828;

A. rufogularis, Brehm, Vög. Deutschl. p. 340 (I831); Gould, B. Eur. ii. pl. I40 (1837); Bp. Comp. List B. Eur. \& N. Amer. p. 18 (1838); Middend. Sibir. Reis. p. I64 (I851); Licht. Ncmencl. Av. p. $3^{8}$ (1854); Adams, Ibis. 1864 , p. 20? Tristr. Ibis, 1871 , p. 233; Hauf, Verh. z.-b. Gesellsch. Wien, xxii. p. 400 (1873); Tacz. Bull. Soc. Zool. France, i. p. I59 (1876).

A. pratensis, (nec L.), Eversn. Add. Pall. Zoogr. p. I 5 (1835; Nordm. in Demid. Voy. Russ. Mèrid. iii. p. I 59 (1840); Finsch, Trans. Z. S. vii. p. 239 (1870); Nelson, Cruise R. S. C Crwin, p. 62 ( 1883 ).

A. pratensis, rufogularis, Schl. Rev. Crit. p. xxxvi. (1840); Gould, P. Z. S. 1859, p, I5 ; Blyth, Ibis, 1867, p. 3 I.

A. rufosuperciliaris, Blyth, $J$. A. S. Beng. 1860 , p. 105. 
A. thcrmophilus, Swinh. Ibis, 1860 , pp. $55,429,186 \mathrm{I}, \mathrm{pp} \cdot 3^{6,}+\mathrm{I}$.

A. japonicus; Swinh. Ibis, 1861, p. 333,1863, p. 443 .

A. ruficollis, Heugl. Orn. N. O.-Afr. i. p. 323 (1869).

A. cervinus, var. rufogularis, Severtz. Turkest. Jevont. pp. 67. $140(1873)$.

Hab. Mindanao; Basilan, China.

8. A. gustavi, Szinh. N. v.

Anthus arboreus, var., Gray, P. Z. S. 1860 , p. 350; Finsch, Neu-Guin?a, p. I67 (1865).

A. gustavi, Szoinh, P. Z. S. 1863 , p. 90; Gray; Hand-l. B. i. p. $25 \mathrm{I}$, n. ${ }^{\circ} 3^{6} 3^{8}$ (1869); Swinh, Ibis, 1874 , p. 442; Brïggen. Abhandl. nat. Ver. Bremen, v. p. 67 (1876); Sharpe, Trans. Linn. Soc. 2nd ser. Zool. i. p. 338 (1876); Seebohm. Ibis, 1877 , pp. г 29, 258, 1878, p. 341; Tweedd. P. Z. S. 1878, p. 430; Finsch, Reis, West-Sibir. p. 69 (1879); Sharpe, Ibis, 1879 , p. 262 Homeyer \& Tancré, $M T$. orn. Ver. Wien, $1883, \mathrm{p}$. 86 ; Dybozeski, Bull. Soc. Zool. France, 1883 , p. 36r; Sharpe, Cat. P. M. 10. p. 61 3 (1885).

A. (Agrodroma) gustavi, Swinh, P. Z. S. 1863, p. 273.

1. batchianensis, Gray, Hand-l. B. i. p. 25 I, n. ${ }^{\circ} 3642$ ( 1869).

Corydalla gustdvi, Swinh. P. Z. S. 1871 , p. 366; Walden, Trans. $Z$. S. viii. p. II 7 (1872); Meyer, J. f. O. 1873, p. 405; David. \& Oust. Ois. Chine, p. 309 (1877); Saluad. Am. Mus. Civic. Genov. xvi. p. I91 (1880); id. Orn. Papuasia etc. ii. l). 432 (I $88 \mathrm{I}$ ).

Anthus secbohmi, Dresser, B. Eur. iii. p. 295, pl. I 34 (1875); Seebohm \& Harvie Brown, Ibis, 1876 , Pp. I20, I80; Brook, Ibis, 1876 , p. 50 I; Finsch, Ibis, 1877 , Seebohm, t. c. p. I 28 . Hab. Leyte, Guimarás, Basilan, Luzón; Borneo, Timor, Cílebes, Molucas.

9. A. undatus, $\sigma \circ$ N. v.

(M. S. 1.)

Hab. Luzón, Laguna, Calauan.

го. A. maculatus, Hodgs. N. v.

Anthus maculatus, Hodgs. Icon. ined. in Brit. Mus., Passeres, pl. I I 8A (n. $\left.{ }^{\circ} 435\right)$; id. in Gray's Zool. Misc. p. 83 (1844); Cab. Mus. Heln. Th. i. p. I5 (1850); Bp. Consp. i. P. 248 (18 5 ()); Ball, Str. F. 1874 , p. 416 ; Brooks, Str. F. 1876, p. 278; id. Ibis, 1876 , pp. 499, 504; Ball, Str. F. 1877 , p. 417 ; il. Sir. F. 1878 , vol. ii. p. 219; Cripps, t. c. p. 287; Butle: Cat. B. Sind \&c. p. 4I (1879); Seebohm, Ibis, 1879, p. 3t; Hume, Str. F. 1879 , p. 103; Brooks, t. c. p. +84 ; Scebohin, Ibis, 1880 , p. i89; Reid, Str. F. 1881 , p. 48 ; l)anison, Str. F. 1883 , p. 396 ; Blakist. Chrys. ii. p. +25 (1882); id. lom. cit. p. 30 (1883); id. \& Pryer, B. Japan, P. 153 (1882); Jown, Pr. U. S. Nat. Mus. vi. p. 289 (1883); Blakist imended List B. Japan, p. $5^{2}$ (1884); Sharpe, Cat. B). M. 10. p. $5+7$ (I885). Pipastes agilis, Blanf. J. A. S. Beng. 1872, p. 60; Dybowski, Bull. Soc. Zool. France, viii. p. 26 I (1883, nec Sykes).

Hab. Luzon, Japon, China, India. 


\section{Fam. CERÉBIDOS.}

Subfam. CEREBINOS.

Gén. ChLOROPh.INES, Reich.

I. Ch. spiza, Lin. $0^{\circ} \circ$ N. v.

(M. S. T.)

Certhia spiza, Lin. Syst. Nat. i. p. I 86.

Grimpereau à tête noir de Brésil, Daub. Pl. Enl. 578 fiğ. 2 (お). Grimpereau verd de Cayenne, Daub. Pl. Enl. 682 fig. I (.0). Coreba spiza, Max. Beitr. iii. p. $77 \mathrm{I}$.

Dacnis spiza, Cab. Mus. Hein. i. p. 95; Burm. Syst. Ueb. iii. p. I 52 .

Chlorophanes spiza, Cassin, Pr. Ac. Sc. Phil. $186_{4}$, p. 267; Law. Ann. Lyc. N. Y. viii. p. 174; Salvin, Cat. Strickl. Coll. p. 177; 2d. Ibis, 1885 , p. 206, Salvin \& Godman, Biol. C.-A., Aves, i. p. 247; Berlepsch, Ibis, 1881 , p. 242; id. J. f. O. 1884 , p. 286; Sclat. Cat. B. M. 11 . p. 29 (1886).

$H a b$. Guatemala.

\section{Fam. TANÁGRIDOS.}

I. Gén. CALliste, Bore.

r. C. larvata, Du Bus. $\delta$

(M. S. 'T)

Calliste larvata, Du Bus, Esq. Orn. pl. 9; Bp. Consp, i. p. 236; Sclater. Contr. Orn. 1851 , p. 64; id. P. Z. S. 1856 , pp. 19, 26o; id. Mon. Callist. p, 81, pl. 36; id. Syn. Av. Tan. p. 86; id. Cat. A. B. p. 70 ; Scl. et Salv. Ibis, 1859 , p. I6, 1860, p. 33 ; iid. P. Z.S. 1870 , p. 836 , et 1879 , p. 499; iid. Nomeucl. p. 19; Salv. Ibis, 1859 , p. 467 , et 1872, p. 316; Salv. et Godm. Biol. Contr.-Am., Aves, i. p. 274; Scl. Cat. B. M. 11. p. I 24 (1885).

Hab. América, Guatemala.

2. Gén. PYRANGA, Vieiel.

2. P. rubra, (Linn). ó N. v.

(M S. 'T)

'Tanágra nubra, Linn. S. $N$. i. p. 3r 4 ; Gm. S. N. i. p. 889 Lath. Ind. Orn. i. p. 420 ; Wils. Am. Orn. ii. p. 42, t. xi. fig. 3, 4; Aud. Orn. Biogr. i. p. 338, t. cccliv.; Desm. Tang. t. 34 .

'Tangara du Canada, Daub. Pl. Enl. 156. fig. I.

Red Tanager, Lath. G. H. vi. p. 5 .

Pyranga rubra, Sw. North. Zool. ii. p. 273; Jard. Wils. Am. Orn. i. p. 192; Bp. Consp. i. p. 24I; Sclat. Cat. B. M. 11. p. 188 (I 885$)$.

Hab. América, Guatemala. 
3. Gén PHANICOTHRAUPIS, Cab.

3. Ph rubicoides, Lafi. oo N. v.

$\left(\begin{array}{lll}\mathrm{M} & \mathrm{T}\end{array}\right) \quad$,

Saltator rubicoides, Lafr. Rev. Zool. 1874 , p. 4 i; C.zss. Proc. Acat. Sc. Philad. iv. p. 90.

Ihonicothraupis rubicoides, Cab. Mus. Hein. i. p. 24; Sclater. Cat. B. 11. 1. 197 (1885).

Hab. Méjico, Guatemala.

4. Gén. SALTATOR, Vieill.

4. S. magnoides, Lafr. ơ $\mathrm{N}$ v.

(M S 'T.)

Saltator magnoides, Lxfr. Rev. Zool. 1847, p. 41; B. Consp. i. p. 489 ; Sclater, Cat. B. M. 11. p. $28+(188.5)$.

Hab. Méjico, Guatemala.

5. Gén. IITYLIJS, Cuv.

5 P, poliogaster, Du Bus. Ơ N. v.

$\left(\begin{array}{lll}\mathbf{H} & \mathrm{S} & \mathrm{T}\end{array}\right)$

Pitylus poliogaster, Du Bus, Bull, Ac. Brux. xiv. pt. ii. p. Io5; id. Rev. Zool. 1848 , p. 245; id. Esq. Orn. t. 22; Sclater, Cat. B. M. 11. p. 307 (1885).

Hab. Méjico, Guatemala.

\section{Fam. ICTÉRIDOS.}

I. Gén. DOLICHONYX, Sw.

I. D. oryzivorus, Lin. $\$$ N.v.

(M. S. 'T.)

Emberiza oryzivora, Linn. S. N. i. p. $31 \mathrm{I}$; Wils. Am. Orn. ii. p. 48. pl. I 2. f. I, 2.

Passerina oryzivora, Vieill. N. D. xxv. p. 3.

Psarocolius caudacutus, Wagl. Syst. Av. sp. 32.

Dolichonyx oryzivora, Sw. Fa.ın. Bor.-Am. ii. p. $278 ;$ Bp. Consp.

i. p. 437; Sclat. Cat. B. M. 11. p. 331 (1885).

Hab. Anérica, Canadá.

\section{Gén. MOLOThkUS, Sw.}

2. M. pecoris, Gm. $\sigma \rho$ N. v. Irupial de la Carolina. (M S. T.)

Troupiale de la Caroline, Daub. Pl. Enl. 606. fig. I.

Fringilla pecoris, Gm. S. N. i. p. 9 ro.

Emberiza pecoris, Wils. Am. Orn. ii. p. 145, p!. I 8. f. 1, 2, 3. Agelaus pecoris, Szo. Phil. M.tg. 1837; i. p. t35 (Mexiou). 
Wolothrus pecoris, Siu. Faun. Bor.-Am. ii. p. 277; Bp. Consp.

i. p. 436; Cab. Mus. Hein. i. p. 192; Baird. B.. .V. Am. p. .24 ; Slater, Cat. B. M. 11. p. 333 (1885).

Hab. América, Canadá.

3. M. æneus, Wagl. \& N v.

(M. S. T.)

Ps.rocolius teneus, Wald. Isis, 1829, p. $75^{8} ;$ Bp. Consp. i. p. 426.

Molothrus aneus, Cab. Mus. Hein. i. p. 192; Sclater, Cat. b'. M. 11. p. 334 (1885).

Hab. América, Guatemala.

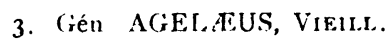

4. A. phœniceus, Lin. \& $\mathrm{N}$ v Trupial de alas rojis. (M. S T)

()riolus phoniceus, Lin. S. N. i. p. 16 r; Gm. S. N. i. p. I86; Lath. Ind. Orn. i. p. I 78 .

Troupiale à ailes rouges de la Louisiane, Daub. Pl. Enl. $\neq 02$. Agelaius phoniceus, Szu. Fann. Bor-Am. ii. p. r80; Bp. Consp. i. p. 430; Scl. Cat. B. M. 11. p. 340 (1885).

$H a b$. América, Canadá.

\section{Fam. FRINGÍL.IDOS.}

I Gén Volatinla, Reich.

I. V. jacarini, (Lin.) \& N. v. Volatin de Cayenna.

(M. S. T.)

The Jacarini, Ewards, Gleanings, ii. p. 202, pl. $306^{\circ}(1760)$.

Lee Tangara noir du Brésil, Briss. Orn. iii. p. 28 (1760).

'lanagra jacarini, Linn. Syst. Nat. i. p. 3 r4 (1760).

l.e Jacarini, Buff. Hist. Nat. Ois. iv. p. 293 (1778); Vieill. Ois. Chant. pl. 33 (1805).

Moineau de Cayenne, Daubent. Pl. Enl. iv. pl. 224. fig: 3 .

Volatinia jacarina, Sharpe, Cat. B. M. 12. p. I52 (1888).

Hab. América, Guatemala.

2. Gén. CARDUELIS, Briss.

2. C. carduelis, (Lin.) $\checkmark \circ$ N. v. El gilguero.

(M. S. 'T.)

L.e Chardonneret, Briss. Orn. iii. p. 53 ( 1760). Daubent, Pl. Enl. iv. pl. 4. fig. I; Montb. Hist. Nat. Ois. iv. p. 187 pl. io ( 778 ).

The goldfinch; Penn. Brit. Zoo!. p. ro8, pl. ro8. figs. r, 2 ( 1766! Fringilla carduelis, Linn. Syst. Nat. i. p. 3 18 (1766), Gm. Syst. Vat. i. p. 903 (I 788 ).

Carduelis carduelis, Boie, Isis, 1122 , p. 554; Licht. Nomencl. Av. p. 46 (1854); Sizarpe, Cat. B. M. 12. p. I85 (1888).

Harb. Europa, Canarias. 
3. Gén. PASSLLR, BRISS.

3. P. mintanus, (Lin) oo $\mathrm{N}$ v. El gorrion.

(M. S. T.)

The Hamburgh Tree-Greeper, Albin, Nat. Hist. 13. iii. p. 23, pl. $24(17+0)$.

The Rod-heal Sparrow, Albin. t. c. p. 6r, pl. 65 (17+o).

Homantain Sisarrow, Albin, t. c. p. 62, pl. 65 (fis. pass.).

M. S. Cuck and Hen, Edwotrds, G'exnungs, ii. p. I24, pl. 269 $(1,70)$.

Le Moineau de Montagne, Briss. Orn. iii. p. 79 ( 1760$)$.

L. M. de Cinasige ou Le Friquet, Briss. t. c. p. 82 (1760).

Le Bouvreuil de Hamburg, Briss. t. c. p. $31+$ (1760).

Fringilla montana, Linn. Syst. Nat. i. p. $32+(9763)$; Tenum. Mam. dOrn. i. p. 35t (1820); Roux, Orn. Prov. pl. 83 (1825); Naum. Vög. Deutschl. iv. pl. i 6 6. figs. I, 2 (1825); Werner, Atlas, Graniwores, pl. 42 (1827); Crespon, Orn. Gard, p. $24^{8}$ (1840); Yarr. Brit. B. i. p. 469 (1843); Kjarb. Danm. Fugle, pl. xxvi. fig. 5 (1852); Suntev. Suensi.. Fugl. pl. v. fig. 7 (1856); Schl. Dier. Nederl., Vög. pl. s6. fig. 3 (1861); Fallon, Ois. Belg. p. 99 (1875).

Le Friquet, Buff. Hist. Nat. Ois ii. p. 489 (r775); Daubent. Pl. Enl. iv. pl. 267. fig. I.

L'Hambouroux, Montb. Hist. Nat. Os. iv. p. $399^{\circ}$ (1778).

Tree Finci, Lath. Gen. Syn. ii. pt, I, p. $25^{2}$ (1783).

Loxia hamburgia, Gin. Syst. Nat. i. p. 854 (1788).

Fringilla campestris, Schrank, Fiauna Boica, p. i81 (1798).

Passer montanina, Pall. Zoogr. Rosso-Asiat. ii. p. 30 (181 I).

P. hamburgensis, Leach, Syst. Cat. Mamm. \&c. Brit. Mus. p. i). $14(1816)$.

P. montanus, Koch, Syst. baier. Zosl. p. 2 I9 (1816); Macgill. Brit. B. i. p. 351 (1837); Keys. u. Blas. Wirb. Eur. p. xxxix (1840); Mïhle, Orn. Griechen!. p. 44 (18+4); Schl. Rev. Crit. 1. Ixiv (18+4); Rüpp. Syst. Uebers. p. $78(18+5)$; Gray, Gen. B. ii. p. $372(18+9)$; Bly!h, Cat. B. Mus. As. Soc. p. г 20 (18+9); Bp. Consp. i. p. 50 (1850); Cab. Mus. Hein. 'Th. i. 1). 156 (1850); Middent. Reis. Sibir., Zool. p. I 48 (1851); Schl. Vög. Nederl. pl. 162, (1854); Horsf. \& Moore, Cat. B. E . Co. Mus. ii. p. $500(1856)$; Hewits. Eggos. Brit. B i. p. I53, pl. 42. figs. I, 2 (1856); Jaub. et Bartí.-Lupsin M. Rich. Orn. 1. I 3 I (I859); Schreack, Reis. Amurl. p. 289 (1859); Tristr. Ibis, 1859 , p. 33; Sruinh. Ibis, 1850, p. 6I; Pozeys. t. c. p. 137; Linderm. Vög. Griechent. p. 56 (1860); Sivinh. Ibis, 1861 , pp. 45, 255; Blyth, t. c. p. 408; Swinh. Ibis, 1862 , p. 258 ; Blakist. t. c. p. 327 ; Gray, List Brit. B. p. Iou (1863); Jerd. B. Ind. ii. p. 366 (186.3); Radde, Reis. Sibir., Vös. p. I81 (1863); Swinh. Ibis, 1863 , p. 378; Wright. Ibij, 185 , p. 53; Schomb. t. c. p. 256; Giglioli, Ibis, 1855 , p. 57; More, t. c. p. I 26; Swinh. Ibis, 1856 , p. 40I; Beavan, t.c. p. 419, 1857, p. 131; Degl. \& Gerbe, Eur. i. p. 245 (2967); Whitely, Ibis, 1867 , p. 202; Beavan, Ibis, 1858 , p. I 74 ; Bul-

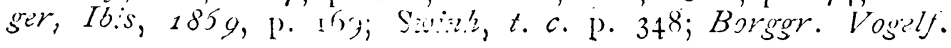




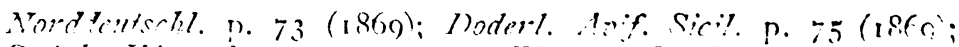

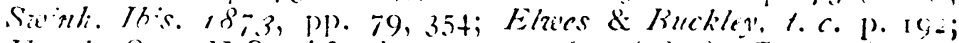

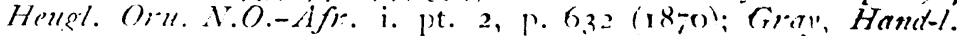
B. ii. P. 86, n.o 725 (18jon); Gould, B. (st. Brit, iii. pl. 3.3

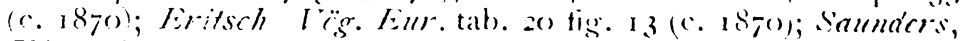

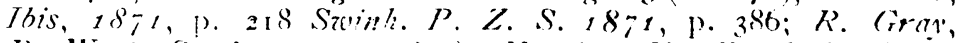
33. West. Scotl. 1. I to (18ju); Harting, Handb. Lrit. B. p.

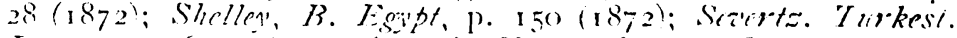

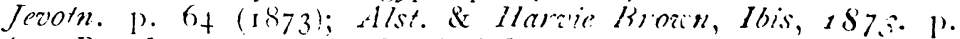
64; Brooks, t. c. p. 2q6; Savinh. t. c. p. 37а; Illume, Nests Figrss. Ind. R. p. foo (1873); id. \& Honders. Lahore to Yark. P. $25+$ (1873); Hume, Str. F. 1874, p. 48r, (1875); p. I75; Joby, B. Citir. P. I I9 (1875); D)resser, B. Eur. iii. p. 597, pl. I78 (1875); Blyth, B. Burm. p. 9t (1875); Sicinh, Ibis. 187.5, P. I20; LYesser, t. c. p. 239; Danf. \& Harvie Brown, t. c. p. 31 2; Nezet. ed Yarr. Brit. B. i. p. 82 (1876); Blaf. East. Persia, ii. p. 255 (1876); Scully', Str. F. $1876,10.165 ;$ Hume, t. c. p. 499; Taez. Bull. Soc. Zool. France, i. p. i 7 s (1876); Secbrhm \& Harvie Brown, Ibis, 1876 , p. In 4 ; Oates, Str. F. 1877 , p. I63; Prjou. in Roneley's Orn. Misc. ii. p. 204 (1874); Darid \& Oust. Ois. Chine, p. 340 (1877); Sreinh. Ibis, 1877 , p. 145; Seebohm, Ibis, 1878 , p. 334; Anders. Zool. Exped. Inn-nan, p. 6or (1878); Hume \& I)avison, Str. F. 1878 , pP. 407, 520; Tirand, Bull, Com. Agric. Cochinchine, i. p. 132 (1879) Finsch, MT. zool-bot. Gesellsch. Wien, 1779 , p. 210 ; Hume, Str. F. 1879,1 p. 67, 107; Scully, t. c. p. 334; Wardlaze-Ramsay, Ibis, 1883 , p. 64; Danf. t. c. p. 94; St. John. t, c. p. 145; Scebohm, t. c. p. I87; Bingham, Str. F. 1880 . p. 193; Barnes, t. c. pp. 218, 456; Kelham, Ibis, 1881 , p. 524; Giglioli, Iconogr. Avif. Ital. pt. Iо (1881); C. Suinh. Ibis, 1882 . p. I I 3 ; Seebohm, t. c. pp. 21 8, 378; Blakist. \& Pryer, B. Jatan, p. I78 (1882); Salvin, Cat. Strickl. Coll. p. 2 10・(1882); Seebohm. Ibis, 1883 , p. 9; Dalgl. t. c. p. 564 ; Oates, B. Brit. Burm. i. p. 348 (1883); B. O. U. List Brit. B. p. 5 I (1883); Homeyer \& Tancró, MT. orn. Ver. Wien, 1883 , p. 89; Clarke, Ibis, 1884 , p. I43; Sannders. t. c. p. .75; C. H. T. Marshall, t. c. p. 419 ; Booth, Rough Notes, pt. vii. (1884); Seebohm, Hist. Erit. B. ii. p. 69 (1884); Radde, Orn. Cauc. p. 477 (1884); Coucs, Key N. Amer. B. 2nd. ed. 1. 345 (1884), Dixon, Ibis, 188.5, p. 82; Gegloll, Avif. Ital. 1. 23 (I 886); Salvad. Elench. Ucc. Ital. p. I69 (1888); Sharpe, Cat. B. M. 12. p. 302 (1885).

Pyruita montana, Cuv. Regn Anim. i. p. 385 (1817); Gould. H3. Eurr. iii. pl. I84 ( $\left.8_{37}\right)$; Bp. Comp. Liss B. Eur. and. $N$. Amer. p. 31 (1838); Selys-Longch. Faun. Belge, p. 80 (1842); Hodgs. Icon. ined, in. Brit. Mus, Passeres, pl. 285. fig. 2, n. ${ }^{\circ} 479$; id. in Gray's Zool. Misc. p. 84 (1844); Gray, Cat. Mamm. etc. Nepal press. Hodgs. p. I07 (18+6); Licht. Nomencl. Av. Berol. p. 47 (1854); Filippi, Viagg. Persia, p. 349 (1865); Bettoni, Ucc. nidif. Lomb. i. pl. 9 (1865); Loche, Exp!. Sci. Algér., Ois. i. p. I 3 (1 867 ).

Pyrgita campestris, septentriona is, Bretsm. t. c. p. 267 (183r). Passer arboreus, Blyth in Rennie's Fid!l Vaturalist, i. p. 467 $(\mathrm{I} 833)$. 
Fringilla rampestris, Nordm. in Memid. Voy. Rliss. Merid. iii. 1). 180 (1840):

Salicipasser montanus, Bogd. B. (auc. p. 6o (1879). Hab. Luzón, Manila, Laguna, Bataan; Eurupa, China.

4. P. flaveolus, Blyth, $\$ \mathrm{~N}$.

(M. S. T.)

Passer flaveolus, Blyth. J. A. S. Beng, xiii. p. 946 (1844); id. Cat. B. Mus. As. Soc. p. 120 (1849); Bp. Consp. i. p. 50 's (1850); Gray, Hand-l. B. i!. p. 86, n. ${ }^{\circ} 267$ (1870); Blanf. t. c. p. 469; Hume, Nest. \& Eggs. Ind. B. p. 4 o $(1873)$; id. Str. F. 1874, p. $48 \mathrm{I}$; id. \& Oates, Str. F. 1875 , p. I 56 ; Blyth \& Walden, B. Burm. p. 94; (875); Anderson, Zool. Exped. Yun-nan, p. 682 (1878) Hume \& Davison, Str. F. 1878 , p. 406; Tiraud, Bull, Com. Agric. Cochinchine, i. p. I I 3 (1879); Hume, Str. F. 1879 , p. 107; Oates, Str. F. 1882, p. 233; id B. Brit. Burm. i. p. 349 (1883); Sharpe, Cat. B. M. 12. p. 330 ( I 885 ).

P. jugiferus, Bp. Consp. i. p. 508 (s 850 , ex Temm. MSS.); Blyth. Ibis, 1870 , p. 172; Gray, Hand-l. B. ii. p. 86, n. 7271 (1870). Hab. Cochinchina, Pegu, ¿Luzón?

4. Gén. POÆCETES, BaIRD.

5 P, gramineus, $(G m.) \ddagger$ N. v.

(M. S T.)

Grass Finch. Lath. Gen. Syn. ii. pt. i, p. 273 (1783); Penn. Arctic. Zool, ii, p. 375 ( $\left.7^{8} 85\right)$.

Fringilla graminea, Gm. Syst. Nat. i. p, 922 ( I 788 ); Audub. B. Amer. pl. 94; id. Orn. Biogr. i. p. 473 (1831), v. p. 502 (1839). loacetes gramineus, Sharpe, Cat. B. M. 12. p. 670 (1889). Hab. Ainérica, Canadá.

\section{Gén. SERINUS, Koch}

6. S. calaria, (Lin.) \& \& N. v. Canario

(M. S. T.)

Canary Birds. Albin. Nat. Hist. B. i. pl. 65 (1738).

Le Serin des Canaries, Briss. Orn. iii. p. I 84 ( I760); Buff. Hist. Nat. Ols, iv. p, I (I 778 ).

Fringilla canaria, Linn. Syst. Nat. i. p. 321 ( I 766$) ;$ Webb, et Berth. Orn. Canar. p. 21 , tab. 2 (1836-44); Gray, Hand-l. $B$. ii. p. 83, n. $7207(1870)$.

Serinus canaria, Cab. Mus. Hein. 'Th. i. p. 163 (185o); Bp. Conp. i. p. 523 (1850); Sharpe, Cat. B. M. 12. p. 370 (1885). Hab. Canarias, Madera, Azores, Tenerife.

6. Gén. LOXIA, LiN.

7. L. curvirostra, ơ Lim. N. v. Pico cruzado.

(M. S. T)

I. curvirostra, Lin, S. N. 1. p. 290 (1885); Sharpe, Cat. B. M. 12 p. 435 (1885).

Le bec-croisè “'Allegmane, Daub. Pl. Enl. pl. 218 . Hab. Europa, Asia, China. 


\title{
Gipo: ESTIRNIFUMIUES.
}

\author{
Fam. ARTAMIIDOS. \\ I. Gén. AR'Payls, Virus.
}

I A. leucogaster, Vale'ni, $\sigma \circ \mathrm{N}$ v. Pagat at.

(M. S. T)

¿La Pie-Grièche de Manille, Briss, Orm, ii. p. ı80, pl. xviii. fig. $2(17(0))$.

'Lanius leuccrhynchus, I, nn, Mantissa, p. $52+$ (1771, ex Briss.'; Cim. S. $\lambda$. i. p. 305 (1 788$)$.

I La Pie-Grièche clominiquaine des Philippines, Sonn. Voy. Nour'. Guin. p. 54 (1776).

? Lanius philippinus, Scop. Del. Filor. et Faun. Insubr. ii. p. 85 (1 785 , ex Sonn.)

: L. dominicanus, Gm. Syst. Nat. i. p. 307 (1788, ex Sonn.)

Ocypterus leucogaster, Valenc. Mêm, Mus. a'Hist. Nal. vi. p. 21, pl. vii. flg. 2 (1820).

Leptopteryx leucorhyrchus (nec L.), Horsf. Trans. I. inn. Soc. xiii. p. 244 ( $182 \mathrm{I})$.

Lanius leucorhynchus (nec L.), Raftl. Trans. Linn. Soc. xiii. p. 386 (1821).

Leptopteryx leucogaster, Wagl. Sy'st. Av., Leptopteryx, sp. 2 (1827).

Ocypterus albiventer (nec Gould), Less. Traite, p, 370 (1831).

O. lt ucorhynchus, Kittl. Kupf. Vög. p. 23, Taf. xxx. fig. I (1832); S. Mïll. Verh. Land en Volkenk. p. 2 I (1839-44).

Artamus leucopygialis, Gould, P. Z. S. 18+2, 1. 17; Gray, Gen. B. i. p. 285 (1845); Gould, $B$, Austr. fol. ii. pl. 33 (1848; Blyth, Cat. B. Mus. As. Soc. p. 199 (1849); Bp. Consp. i. p. $3+4$ (18+o); Reicheb. Vög. Neuholt. p. I7 I (1850); Gray, P. Z. S. 1858 , p. 493; id. Cat. B. New Guinea, p. 58 (1859); Gould, P. Z. S. 1863 , p. 233; id. Andb. B. Austr. i. p. 15. (1865); Finsch, New-Gumea, p. I7 г (1865); Walden, P. $Z$. $\because$. 1866, p. 555; Gray, Hand-l. B. i. p. 289 , n. 4279 (1869; Ball. J. A. S. xli. p. 283 (1872); Cab. \& Reichen, J. f. O. 1876 , p. 322; W. A.FForbes, P. Z. S. 1877. p. 122; Masters, Proc. Linn. Soc. N. S. W. i. p. 48 (1877); Casteln. ¿ Rams. t. c. p. 380 ; Rams. t. c. p. 392 ; idl. op. cit. i. p. I7) (1878), iii. p. 276 (1878), iv. p. 98 (1880); W. A. Forbeo, Rej. Voy. "Challenger.» Zool. ii. p. 85 (1881); Rams. Proc. Lim. Soc. N. S. W. (2) ii. p. 167 (1887).

A. leucorhynchus, Gray, Gen. B. i. p. 285 (1845); Bp. Consp. i. p. 34.3 (1850); Cab. Mus. Hein. 'Th. i. p. 208 (1850); Cass. U. S. Expl. Exped., Birds, p. 140 (1858); Gray, P. Z. S. 1860, p. 354; Wall. Ibis, 1860 , p. Itr; Graj, Hanc':. $B$. i. p. 289, n. ${ }^{\circ 269}$ (1869); Walden, Ibis, 1872, p. 371; id. Trans. 2. S. viii. p. 67 (1872); id. Ibis, 1873. p. 309; Huine, Str. F. 1874 , p. 214 ; id. Nests. \& Eggs Int. B. p. 195 (1875); Salada. Itec. Porr. p. Ito (1875); Slorp, Ib.s, is j6, 
p. 45, 1877, D. 21; p. 147 (1+75); Ticeld. t. c. p. 31: 1878, p $3^{8} 3$; id. P. Z. S. 1877, pp. $544,692,759,826$, (1878) Pl. $283,342,709$ 937; Sharpe, Journ. Linn. Sor. Zosl. xir. p. 688 (1879); id. P. Z. S. 1879 , pp. 315, 3+5; Meyer, $t$. i. p. 146; Nichols. Ibis, 1879 , p. 170; Treedd. Rep. «Challenger» Exped., Zool. ii. p. I6 (1881); Sharpe, Rep. Voy. «Aler,» 1. 21 (1884); Guillim. P. Z. S. 1885, Pp. 25 I, 409, 505, 553.

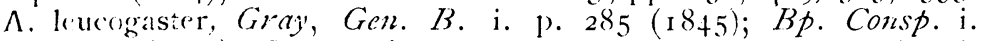
1) $343(1850)$; Horsf. \& Moore, Cat. B. Mus. F.I. Co. i. p. I61 (1854); Bernst. J. f. O. 1859, p. 268; Wall. P. $Z$. S. 1853, pp. 28,$485 ;$ Scl. t. c. p. 217 ; Finsch. Neu-Guinea, p. I7I (186.5); Beavan, Ibls, 1867 , p. 324; Hartl. \& Fincsh. P. Z. S. 1867, p. 117; Gray, Haud-l. B. i. pi 289, n. ${ }^{\circ} 4273$ (1869); Mcyer, Sitz. z.-b. Gesellsch. Wien, lxix. p. $20+(1874)$; Salvad. Ann. Mus. Civic. Genov. vii. pp. 656, 770 (1875); id. op. cit. viii. p. 377 (1876); ix. p. 28 (1876), x. p. 138 (1877); Sharpe in Rowlcy's Orn. Misc. iii. p. I84 (1875); IJ'Albert. \& Salvad. Ann. Mus. Ciric. Genow xiv. p. (1879); Salad. t. c. xv. p. $3^{8}$ (1879); id. Orn. Papuasia etc. ii. p. I67 (1881); Scl. P. Z. S. 1883, pl. 51, 195; H. O. Ferbes, P. Z. S. 1884 , pp. 247, 432; Guillem. P. Z. S. 1885, p. 635; Vorderm. N. T. Nederl. Ind. xlv. p. 878 (1886). Ramsay', Tab. List. Austr. B. p. 3 (1888); Sharpe, Cat. B. M. 13. p. 3. (1890).

A. paupensis, Bp. Consp. i. p. 344 (1850, cx Temm. MBS. in Mus. Lugd.); Scl. Journ. Linn. Soc. ii. 1858, p. I 59; Gray, P. Z. S. 1858 , p. I79, г86 г, p. 435; Finsch, Neu-Guined, p. I7I (1865); Walden, P. Z. S. 1866 , p. 555; Gray, Haud-l. B. i, p. 289 , n. ${ }^{0} 4282$ (1869); Beccari, Ann. Mus. Civic Gevii. p. 709 (1875).

A. leucorhynus, Walden, Trans. Z. S. ix. p. I74 (1875); Sharpe, Trans. Linn. Soc. (2) i. p. 323 (1877); Trueedd. P. $Z$. S. 1877 , p. 544, I 878, pp, 282,342 .

A. leucorhynchus, var. celebensis, Brïgg. Abhandl. nat. Var. Bremen, v. p. 69 (i \& 76 ).

A. celebensis, Brüggem. t. c. p. 7o (1876); Tweedd. Ib:s, 1878 , p. 386 .

A. muschenbroeki, Meyer, Vög. Nester nud. Eer Ostind-Arch. p. 30 (1884); Salvad. P. Z. S. 1884 . P. 578 ; id. Ibis, 1884 , P. 355 .

Hab. Luzón, Manila, Navotas, S. Mateo, Lagruna, Calamba, Negros, Cebú, Amparo, Butuan, Surirao, Bohol, Dumaguete, Basilan, Joló, Mindanao, Guimarás, Panay, Siquijor, Marinduque, Mindoro; Java, Célebes, Borneo, Sumatra.

\section{Fam. ESTÚRNIDOS.}

\section{Gén STLRNIA, Less.}

r. St. violacea, (Bodl). $\sigma^{\prime}$, N. v Culacling puri.

(M. T. S )

Le Grand Traquet des Philippines, Briss. Oru. iii. p. 446, pl. 22. fig. 3 (1760); Daubent. Pl. Enl. iii. pl. 185. fig. 2; Monbt, Hist. Nat. Ois. v. p. 23 ) (1 778 ). 
Motacilla violacea, Bodd. Tabl. Pl. En!. p. i 1 (1783).

Pastor ruficollis, Wagler, Sys'. Ar., P.tstor, sp. 19 (18;2); Gray, Gen. B. ii. p. $33+\left(1 \delta^{\prime}+j\right)$.

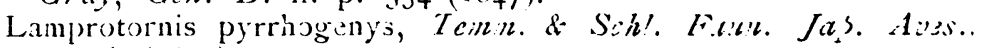
p. $86(1850)$.

L. pyrrhojogon, iid. t. c. pl. to (1850).

Heterornis pyrrogenys, Bp. Consp. i. p. fis (185), c.x M.ï!ter, MSS. ); Swinh. P. Z. S. 1353, p. 202.

H. ruficollis, Bp. Consp. i. p. +18 ( 1850$)$.

Sturnia pyrrhopogon, Gray, P. Z. S. 1850, p. 355.

Calornis albifrons, Byth. (leste Sruinhoe) P. Z.S. $188_{3} 3$, p. 302, note.

'Temenuchus pyrrhogenys, Gray, Hind-l. B. ii. p. 21, n." 63ol ( 870$)$.

I. ruficellis, Gray, c. t. p. 21 n..$^{\circ} 6302(1+70)$.

Acridotheres pyrrhogenys, Giebel, Thes. Orm. i. p. 263 (1872).

St pyrrhogenys, Wald. Trans. Z. S. viii. p. 78 (1872); Swinl . Ibis, 1874 , p. I 59; Blakist. \& Pryer, Ibis, 1878 , p. 233; Meyer, Ibis, 1879 , p. 1.34; Blakist. Amended List B. Jap.n, p. 14 (1884).

St. dominicana, Salvad. Ucc, Born. p. 269 (I874); Verderm. Nat. Tijidschr. Nederl. Ind. xlvi. p. 235 (i $8+7)$.

St. daurica, (nec Pall.), Sharpe, Ibis, 1879, p. 262.

St. violacea, Walden, Trans. Z. S. ix. p. 203 (1877); Ramsay, Ibis, 1886 , p. 172; Blasins, in Madaasz, Zeitschr. 1886 , p. I 20; Sharpe, Cat. B. M. 13. p. 70 (1890).

Hab. Luzón, Manila, Navotas, Lazuna, Calauan, Sinar, Borongan, Paragua, P. Princesa, Negros, Dunalon, Mindanio; Célebes Java, Sumatra.

\section{Gén. ACRIDOTHERES, VieiLL.}

2. A. cristatellus, $(G m)$. $\sigma \circ$ N. v. Murtin langostero.

(M. S. T.)

The Chinese Starling or Bluckbird, Edw.trds, N. H. Birds, i. pl. (I 743$)$.

Le Merle hu;é de la Chine, Briss. Orn. ii. p. 253 (1860); Daubent, Pl. Eul. ix. pl. 5o7; Montb. Hist. Nat. Ois. iii. p. 3's7 (I775).

Crested Grakle, Lath. Gen. Syn. ii. pt. i. p. 464 (I 783$)$.

Gracula cristatella, Gm. Syst. Nat. i. p. 397 (1788).

Acridotheres ater, Bonn. et Vieill. Enc. Méh. ii. p. 692 (1+23); Gray, Hand-l. B. ii. p. 20, n. 6290 (1870).

A. critatellus, Bonn. et Vieill. Enc. Méth. ii. p. 691 (1823); Blyth, J. A.S. Beng. xv. p. 33 (18+6); id. Cat. B. Mus. As: Soc. p. 108 (I849); Cab. Mus. Hein. 'Th. i. p. 205 (1850); Horsf. \& Moore. Ciat. B. Mus. E. I. Co. ii. p. 536. (1856); Swinh. Ibis, 1860 , pp. 60, 429, I861, p. 44, 1862, p. 260; iil. P. Z. S. 1863 , p. 302; id. Ibis, 1863 , p. 382; id Ibis, 1867 , p. 387 ; id. P. Z. S. 1871 , p. 384; Gray, Hand-l. B. ii. p. I 9, n. ${ }^{\circ} 6283$ (1870); Walden, Tr. $Z$. S. ix. p. 202 (1875); Tweedd. P. Z. S. 1877 , p. 698; Darid \& Oust. Ois. Chine, \& p. 365 pl. 86 (1877); Stater, Ibis, 1892, p. 434; Seebohm, Ib:s, 1887, p. 265; Sharp?, Cat. B. M. 83. p. 29 (1890;. 
A. fuliginosus, Blyth, J. A. S. Beng. xiii. p. $3^{62}$ (184t,--jur.). Mrrula philippensis, «Brisson,» Bp. Consp. i. p, $420(185 \%$, nec Brisson).

Acrilotheres philippensis, Bp. Consp. i. p. 420 (1850); Swinh, Ib s, 1870, p. 352; Gray, Hand-l. B. ii. p. 20, n. ${ }^{\circ} 6287$ (1870). Hab. Luzón, Manila, Laguna, Bataan, Cavite, Batangas; China.

\section{Gén SARCOPS, Wald.}

3. L. calvus, Lin. $\delta \varnothing$ N. v. Culing, Gulin, Coleto (M. S. T.)

I. Merle chaure des Philippines, Briss. Orn. ii. p. 280, pl. xxxvi. fig. 2 (I760); Daubent. Pl. Enl. iv. pl. 200.

(iracula calva, Linn. Syst. Nat. i. p. 164 ( 1766); Gin. Syst. Nat. i. p. 396 (1788); Daud. Traité, ii. p. 690; (1800); Kittl. Kupf. Vög. p. 9, pl. xiii. fig. 2 (1832); Gray, Gen. B. ii. p. 33" (1845).

1,: Goulin, Montb. Hist. Nat Ois. iii. p. 420 (1775).

Bald Grakle, Lath. Gen. Synt. ii. pt. 2, p. 457 (1872).

Acridotheres calvus, Bonn. et Vieill. Enc. Méth. ii. p. 6\% (1823).

Gymnops calvus, Cuvier, Règne Anim. i. p. 38I; (1829); Bp. Consp. i. p. 422 (1850); Licht. Nomencl. Av. p. 52 (1854); Walden, \& Layard, Ibis, $1872, \mathrm{p}$ IO3.

(iymnops griseus, Meyen, N. Acta Acad. C. L.-C. G. Nat. Cur. xvi. Supl. prim. p. 78 (teste Walden).

Mino calvus, Blyth, Cat. B. Mus. As. Soc. p. in I (1849).

lulabes tricolor, Gray, Hand-l B. ii. p. 19, n. ${ }^{\circ} 6275$ (1870).

Sarcops calvus, Walden, Trans. Z. S. ix. p. 205 (i 875 ; Sharpe, Trans. Linn. Soc. new. ser. i. p. 344 1876); Trueedd. P. Z. S. 1877 , pp. 699, 764, 839; id. P.Z. S. 1878 , pp. 113, 287, 343; Wardlaw Ramsay, Orn. Mem. Tweedd. p. $75+$ (1881); Gulllem. P. Z. S. 1885 , p. 267; Sharpe, Cat. B. M. 13. p. 97 (1890).

S. lowii, Sharpe, Trans. Linn. Soc., znd. ser. Zool. .i. p. $34+$ (1876); id. P. Z. S. 1870 , p. 315 .

Hab. Luzón, Mindoro, Paragua, Sanar, Cebú, Guimarás, Negros, Leyte, Dinagat, Mindanao, Basilan, Joló, Butuan.

4 Gén. Mainates, Less.

4. M religiosus, (Lin.) 3 \&. v. Tiao.

(M. S. T)

The Minor, Albtn, Nat. Hist. B. ii. pl. 38 (1738).

The Minor or Mino, Edwards, Nat. Hist. B. i. p. 17, pl. 17 ( I 743).

le Grand Mainate, Briss. Orn. ii. p. 308 (1760).

Gracula religiosa Linn. Syst. Nat. i. p. 104 (1766); Jerd. Madr. Journ. xi. p. 24 (1840); A. Hay, Madr. Journ. xiii. pt. 2, p. 156 (1844); Gray, Gen. B. ii. p. 330 (1845, pt.); Blyth, J. A. S. Beng. xv. p. 3 I (1846); id. Cal. B. Mus. As. Soc. p. I09 (1849); Bp. Consp. i. p. 422 (1850); Cab. Mus. Hein. Th. i. p. 207 (1850); Layard. Ann. N. HI. (2) xiii. p. 216 (185t; Sclil. N. T. D. i. p. 3, pl. i. is. I (1843). 
I. Mainate des Indes Orientales, D.xuhen!, Pl. Enl. iv. pl. 268. Minor Grakle, Lath. Gen. Syn. i. pt. 2, p. 455 (1783).

Mainatus religiosus, Less. in Ferussac, Bull. x. p. I $5^{8}$ (1827); Sharpe, Cat. B. M. 13. p. 99. (1890).

Giracula indica Blyth, J. A. S. Beng. xii. p. 1718* (143).

(i. minor, A. Hay, Madr. Journ. xiii. pt. 2, p. I3t (1 $8+t)$.

liulabes religiosa, Horsf. \& More, Cat. B. Mus. E. I. Co. ii. p. 522 (1856); Jerd. B. Ind. ii. p. 337 (1863); Blyth, Ibis. 1867, p. fo; Melliss. Ibis, 1870 , p. ror; Gray, Hand-l. li;. ii. p. I8, n." 6264 (1870); Holdsio P. Z. S. 1872, p. 46.3; Hume, Nest. \&. Egg. Ind. B. p. 435 (18;3); Ball, Sir. 1 . 1874 , p. 420; Hume, Str. F. 1876 , p. 402; Fairb. Str. $t$. 1377 , p. to7; Ball, Str. F. vii. p. 221 (1878); L.egge, 13. Ceylon, P. 682 (1879); Hume, Str. F. 1879 . P. Iob; Davison, Str. Fr. 1883 , p. tor; Taylor, Str. F. 1887 , p. 463 ; Terry. Str. F. 1887 , p. 478 .

Hab. Paragua, Canigaran; Ceilan.

5. M. javanicus, Osbeck. ơ $\rho \mathrm{N}$ v Tiao.

(M. S T I

Corrus javanensis, Osbeck Iter. p. IO2 (1757); id. Voy. (Engl. transl.), p. I 57 (I 77 I).

Gracula religiosa (nec L.), Raffl. Trans. Linn. Soc. xiii. p. 30,3 (1820); Gray, Gien. B. ii. p. 330 (1854).

Eulabe; javanensis, Horsf. Trans. Linn. Soc. xiii. p. 162 (1820).

?Gracula religiosa, Vieill. Gal. Ois. i. p. 144, pl. 95 (1825).

Eulabes javanus, Cuvier, Règne Anim. i. p. 377 (1829).

Mainatus javanus, Less. Traité, p. 357 (I83i).

M. sumatranus, Less. Traité, p. 357 (1831).

Gracula javanensis, Blyth, J. A. S. Beng. xv. p. 31 (1846); id. Cat. B. Mus. As. Soc. p. Io7 (1849); Bp. Consp. i. p. 422 (1850); Motley \& Dillv. Nat. Hist. Labuan, p. 25 (1855); Schl. N. T. D. i. p. 5, pl. i. fig. 3 (1863) Scl. P. Z. 'S'. ${ }_{1863}$, p. 219 ; Salvad. Ucc. Born. p. 274 (1874); Sharpe, $P$. $Z$. S. 1875 , p. Iо7; Tweedd. Ib s, 1877 , p. 319; Brïggem. Abhandl. nat. Ver. Bremen, v. p. 461 (1877(; Sharpe, Ib.s, 1879 , p. 262; id. P. Z. S. 1879, p. 346; Salvad Aun. Mus. Civic. Genov. xiv. p. I 38 (1879); Nichols. Ibis, 1881 , p. I53; Kelham, t. c. p. 520 ; Nichols. Ibis, 1882 , p. 63; I883, p. 25; Oates B. Brit. Burm. i. p. 393 (1883); Snellem. in Veth. Midden-Sumatra Vög. p. 43 (1844); Büttik. Notes Leyden Mus. ix. p. 7 I (1887).

Gracula javana, $A$. Hay, Madr. Journ. xiii. pt. 2, p. I56 (1844); Cab. Mus. Hein. Th. i. p. 207 (1850); Ball. Str. F. 1874, p. 334 .

Eulabes javanensis, Horsf. \& Moore, Cat. B. Mus. E. I. Co. ii. p. 524 (1856); Moore, P. Z. S. 1859 , p. 444; Stoliczka, J. A. S. Beng. xxxix. p. 326 (1870); Gray, Hand-l. B. ii. p. 81, n. ${ }^{\circ} 6267$ (1870); Walden, Ibis, 1871, p. 176; Hume, \& Davison, Str. F. vi. p. $395(1878$, pt.); Hume, Str. F. 1879 , pp. 66, Io6; Sharpe, Cat. B. M. 13. p. 102 (1890).

Hab. Pardgua, P. Princesa; Java, Borneo. 
6. M palawanensis, Sharp. ơ $?$ N. v. Tiao

(iracula javanensis, (nec Osbeck), Sharpe, Trans. Lin. Soc. new. ser, i. p. 344 (1876); Tweedt. P. Z. S. 1878 , p. 622; Shar pe, Ibis, 1884 , p. 32I; Blassins, Ornis, 1888 , p. 4 I 5.

Fulabes javanensis, Sharpe, Ibis, 1888 , p. 202.

11. palawanensis, Sharpe, Cat. B. M. 13 , p. 104 (1890).

Hab. Paragua, P. Princesa, Inagauan, Canigaran.

\section{Gén. MINO, I.ess.}

\section{M. dumonti Less. o 0 N. v. Martin}

Nino dumonti, Less. Voy. Coquille, Zool. i. p. 652, pl. 25 (1826); Kittl. KupJ. Vög. p. 9. 'T'af. xiii. fig. I (1833); Salvad. Ann. Mus. Civic. Genov. vii. 1. 775 (1875), viii. p. 402 (1476), ix. p. 39 (1476), x. p. I50 (1877); Salvad. P. Z. S. 1878 , p. 98; D'Albert. \& Salvad. Ann. Mus. Civ c. Genov. xiv. p. 93 (1879); walvad. op. cit. xvi. p. 195; id. Orn. Papuasia, etc. ii. p. 466 (1881); id. Rep. Voy. H. M. S. «Challenger», ii. Birds, p. 8I (1881); Sharpe, Cat. B. M. 13, p. I I (1890). Mainatus dumonti, Lesson in Férussac, Bull. x. p. I59 (1828). Giymnosp dumonti, Cuvier, Règne. Anim, i. p. 38 I (1829).

Gracula dumonti, Wagler, Ibis, 1829, p. 747; Mïll. \& Scl. Verh. Land-en Volk. p. $22(\mathrm{1} 839-44) ;$ Gray, Gen. B. ii. P. 330 (1845); Bp. Consp. i. p. 422 (1850), Scl. Proc. Linn. Soc. ii. p. $164(1858)$; Gray, $P . Z$. S $1858, \mathrm{pp} .182,194,1859$, p. I58; id. Cat. B. Nere Guinea, pp. 38, 59 (1859); id. P. Z. S. 1861 , p. $43^{6}$; Schl. Nederl. Tijdschr. i. p. 8, pl. i. fig. \& (1863); Rosenb. J. f. O. 1864 , P. I 3 I; Finsch, Neu-Guinea, p. 174 (1865); Gray, Hand-l. B. ii. p. 18, n."6272 (1870); Ramsay, Proc. Linn. Soc. N. S. $W$. i. p. $592(1876)$.

Eulabes dumonti, Sharpe, Journ. Linn. Soc. xiii. p. 82 ( I876), p. 318 (1877), p. 508 (1877), xiv. p. 687 (1879); Ramsay Proc. Linn. Soc. N. S. $W$. iii. pp. 107, 279 (1878), iv. p.' 99 (1879). Hab. Molucas.

6 Gén. Aplonis, Gould.

8 A. pelzelni, Finsch. N. v

(M S. T.)

Aplonis pelzelni, Finsch, P. Z. S. 1875 , p. 6tt; id. Journ. Mus. God:ffr. Heft. xii. p. 32 , pl. ii. fig. $3(1876)$; id. $P . Z$. S. 1877 , p. 779; id. Ibis, 1881 , p. I Iо; Sharpe, Cat. B. M. 13, p. I 36 ( I 890$)$.

Hab. Ponapé, Carolinas.

9 A. Khittlitzi, Finsch.

? Pacific Shrike, Lath. Geu. Syn. i. pt. i. p. 16+ (1871).

?Lanius pacificus, Gm. Syst. Nat. i. p. 306 ( 1788 , ex Lath.).

Turdus columbina (noc ( $\mathrm{mm}$.), Kittl. Küpf. Vög. p. I r, Taf. r 5. fig. 2 (I 833 ). 
Lamprotornis columbinus, (nec lim.), Bp. Consp. i. p. 417 (1850); Hartl. J. f. O. 18.54 , p. 168.

L. opaca, Licht. in. Mus. Beroi.; Cab. Mus. Hein. Th. i. p. 201 (I\&50); Letcht Nonencl. Ar. Neotr. p. $53(1854)$.

Calornis pacifica, Gray, Cat. h. Tropical 1sl. Pacifie Occall, p. 25 (1859); id. Hand-l. b. ii. p. 27, n. 0.390 (is 50$)$.

C. opaca, Gray, Cat. B. Tropical Isl. p. 26 (1851); Gray, Hand-l. B. ii. 1). 27, n." 6391 (18-0).

C. columbina, (net: Gen.), Pela. Rés. Nowara, l\%gg. p. 88 (1881).

C. kittlitzi, Finsch. \& Hartl. Faun. (entrlapolyn. p. Iog) $(1867)$; Hartl. P. Z. S. 1867 , p. 380; id. d- Finsch, P. Z. S. 1868 , pp. 7, 117; ind. P. Z. S. is 72, p. 100; Finsch, Journ. Mus. Godeffr. Heft. viii. p. $23(1875)$; Silurpe, llis, $18 ; 6$, p. 47; Finsch, P. Z. S. 1877 , p. 779. Sharpe Cal. B. M. 13. p. 136. (i $8(x))$.

C. pacificus, Finsch, Journ. Mus. Godeffr. Heft xii. p. 3r (1876); id. P. Z. S. 1880, p. 575 ; id. Ibls, 1881 , p. 194; id. lös. der Südsee, p. 49 (1884).

Hab. Ponapé, Carolinas.

7. Gén. CaLornis, Gray.

ro. C. metallica, Tiv. N. v. Culagling.

(M. S. T.)

Lamprotornis metallica, Temm. Pl. Col. ii. pl. 266 (1824); Lesis. Voy. Coquille, i. p. 343 (1826); Mïll. Verh. Land-en Volkenk. pp. IO2. I IO (1839-44); Bp. Consp. i. p. 417 (1880); Jacq. et Pucher. Voy. Póle Sud, Zool. iii. p. 81 (1851); Ciass. Proc. Philad. Acad. 1862 ; p. 3r 3; Finsch, Neu-Guinea p. I84 (1863); Cab. \& Reichen. J. f. O. 1876, p. 322.

Siourne bronzé, Hombr. et Jacq. 'Voy. Póle Sud, Atlas, pl. i6. fig. $2(1832-35)$.

Aplonis metallica, Gould, B. Austr., Suppl. pl. 33 (1851); Macgiltiuray, Voy. «Rattlesmake,» ii. p. 351 (1852).

Calornis metallica, Gray, Gen. B. ii. p. 327 (1846); Hartl. J. f. O. 1854 , p. I64; Scl. Journ. Linn. Soc. ii. p. I64 (1858); Gray, P. Z. S. 1858 , p. I94; id. Cat. Mamm. etc. New Guinea, p. 59 (I859); Wall. P.Z. S. 1862 , p. 335; Gould, Hand.b. B. Austr. i. 417 (I865); Rosenb. Reis. naar Zuidoostereil p. 8o (1867); Ramsay, P.Z. S. 1868,$385 ;$ Scl. P Z. S. 1859 , 1. 120; Gray, Hand-l. B. ii. p. 26. n. 6376 (1870); Walden, Trans. Z. S. viii. p. 80 (1874); Ramsay, P. Z S ${ }^{2} 875,1$. 593; Salvad. \&. D'Albert. Ann. Mus. Civic. Genov. vii. p. 827 (1875); Sharpe, Ibis, 1876, p. +7; Saluad Ann Mus. Civic. Genov. viii. p. 402 (1876), ix. p. 38 (1876); Brïgg. Abhandl. nat. Ver. Bremen, v. p. 78 (18-6); Masters Proc. Linn. Soc. N. S. $W$. i. p. 54 (1877); Salvad. Ann. Mus. Civic. Genov. x. p. 148 (1877); id. P $Z$. S. 1878, pp 88, 97; Ramsay, Proc. Limn. Soc. $N$ S. $W$ iii. p. io7 (1878), p. $379(1874)$ iv. Pp. 77, $99(1879)$; Rasenb. Malay. Arch. pp. 322, 370 (1879); D'Albert. \& Salvad. Ann. Mus. Civic. Genor. xiv. p. 190 (1879); Salvad. t. c. p. 656 (1879); Tristr. Ibis, 1879. p. 442; L. C. Layard, Ibis, 1880, p. 195; Salvad. Ann. Mus. Civic. Genov. xvi. p. 193 (1881); id. Orn. Paturara, ii. p. 447 (i881); 


\section{$-204-$}

Blasius, J.f. O. 1883 , p. 160; Meyer, Sitz. inat. Gesellscí, Isis, 1884 , Abth. i. p. 48; Finsch, Vög. der Südsee, p. 13 (1884); id. t. c. p. 36. Sharpe, Cat. B. M. 1.3. p. 138 (1890). (. nitida, Gray, P. Z. S. 1858 , p. $18 \mathrm{I}$; id. Cat. Mizmm, eti. New Guinea p. 38 (1859); id. Cat. B. Trop. Isl. Pacific Oceall. p. 26 (1859); id. Hand-l. B. ii. p. 27, n. ${ }^{\circ} 3_{3} 83$ (1870); Walden, Trans. Z. S. viii. p. 80 (1872); Sharpe, Ibis. 1876 , p. 4 ; Scl. P. Z. S. 1877 , p. 104, 1878, p. 28c; Finsch, P. Z. S. 1879 , p. I ; Layard, Ibis, 1880 , p. 295; Pinsch, Ibis, 1881 , p. 537; Tristr, Ibis, 1882 , p. I 37 .

C. viridescens, Gray, P. Z. S. 1851, p. 181 id. P. Z. S. $180 \%$, p. 436; id. Hand-l. B. ii. p. 26, n. ${ }^{\circ} 6378$ (1870); Walden. Trans. Z. S. viii. p. 80 (1874); Sharpe, Ibis, 1876, p. +7: id. Journ. Linn. Soc. Zool. xiii. pp. 318, 501 (1877).

C. amboinensis, Gray, P.Z. S. 1858 , p. 182; $2 d$. Cat. Mamm etc. New Guinea, p. 38 (1859); id. P Z. S. 1860, p. 35.5; Bernst. Nederl. Tijdschr. ii. p. 350 (1865); Gray, Hand-l. B'. ii. p. 27, n. ${ }^{\circ} 6382(1870)$; Walden, Trans. Z. S. viii. p, \& (1874); Sharpe, Ibis, 1876 , p. 48; Rosenb. Malay. Arch. p. $322(1879)$.

C. virescens, Gray, P. Z. S, 1859, p. 158; id. Cat, Mamm. etc. New Guinea, p. 59 (1859).

Lamprotornis virescens, Rosenb. Nat. Tijdschr. Nederl. Ind. xxv. p. 248 (1863); id. J. f. O. 1864, p. г 3 .

Calornis purpurascens, Gray, Hand-l. B. ii. p. 26. n. ${ }^{\circ} 6377$ (1 87 (3): Walden, Trans Z. S. viii. p. 8o (1874); Sharpe, Ibis, 1876 , p. 47 .

Lamprotornis purpurascens, Giebel, Thes. Orn. ii. p. 430 (1 875 . L. metallica, var. nitida, North, Proc. Linn. Soc, N. S. it. (2) ii. p. 443 (1887).

Hab. Luzón, Cagayan, Pamploną; Molucas.

11. C chalybæa, (Horsf.) $\delta \& \mathrm{~N} \quad \mathrm{v}$. Culngling.

(M. S. ' F.)

Turdus chalybeus, Horsf. Trans. Linn. Soc. xiii. p. 148. (1820). L. strigatus, Horsf. Trans. Linn. Soc. xiii p. It8 (1820).

Lanius insidiatur, Raff. t. c. p. 337 (I \&20).

Lamprotornis cantor, Mïll, Verh. Land-en Volkenk, p. I 74 (1839-44); Bp. Consp. i. p. 416 (1850); Licht. Nomerocl p. $53(1864)$.

L. chalybeus, Eyton, $P . Z$. S. $1839, \mathrm{p}$ yo3.

Calornis cantor, Blyth, J.A.S. Beng. xiii. p. 366 (1844), xix. p. 225 (1850); id. Cat. B. Mus. As. Soc. p. III (1849; $\mathrm{Sc}$ P. Z. S. 1863 , p. 21 8; Stoliczk.r, J. A. S. Beng. xxxix. p). 326 (1870); Waldeu, Ibis, 1871 , p. 176 .

C. affinis, A. Hay, J. A. S. Beng. xv. p. 36 (1846); Blyth, t. c. p. 369 ; id. op. cit. xix. p. 255 ( 1850$)$; id. Cat. B. Mus. As. Soc. p. II I (1849); Gray, Hand-l. B. ii. p. 27, n. ${ }^{0} 638$ I (1879), Blyth. \& Wald. B. Burm. p. 91 (1875; Sharpe, Ibis, 18;0, p. 46; Hume, Str. F. 1877 , p. 38.

C. panayensis, pt., Gray, Gen. B. ii. p. 327 (1846); Motley \& Dillw. Nat. Hist. Labnan, p. 56 ( $(1856)$.

Lamproturnis affiuis, Bp. Consp. i. p. 410 ( 1850$)$.

L. insidiator, Cab. Mus. Hein. Th. i. p. 201 (1850). 
Calcrnis chalylea, Horsf. \& Moore, Cat, B. IIis E. I. Co. ii. 1) $5+3$ (1856); Moore, P. Z. S. 1859, p. +45; Gral, Hand.l. B. ii. p. $26 \mathrm{n} .0374$ (1870); Hald. Trans. $Z$. S. viii. p. 80 (1872); Salvad. Lic. Born. D. 2i (1874); Blyth of IVald 1?. Purm. p. 91 (1875); Sharpe, Ibis, 1876, p. 45, 1877, p. 21; Trieed'd. Ibis, 1877 , p. $318 ;$ Hume of Mavison, Str. F. 1878 , p. 39t; Hume, Sir. F. i879, pp. 60, 106; Nichols. Ibis, 1879 , p. I70; Sharpe, P. Z. S. 1879 , p. 345; 2d. Ibis, 1879, p. $262 ;$ Salvat. Ann. Mus. Civic. Genoz. xiv. p. 238 (1879); Kelham, Ibis, 1881 , p. 519 ; Nichols. Ibis, 1882 , p. 62; lorderm. N. T. Nederl. Ind. xliii. p. 189 (1884); Büttik. Notes Leyden Mus. ix. p. 7I (I887); Sharpe, Cat. B. M. 13. p. 143. (1890). C. insidiator, Walden, Ibis, 1872 , p. 38 r.

Aplonis cantor, Snellem. Exped. Midden-Sumalra, Vogels, p. 43 (1884).

C. inwini, Hunie, Str. F. i. p. 481 (1873).

Hab. Luzón, Paragua, Sumatra, Java, Borneo.

12. C panayensis, (Sisp) o 0 N. v. Culagling.

(M. S. T.)

Le Petit Merle ou Muscicien de l'Isle Panay, Sonn. Voy. $N$. Guin. p. I I 5, pl. 73 (1776).

Muscicaja panayensis, Scop. Del. Flor. et Faun. Insubr. ii. p. $96 \quad$ ( I 783$)$.

Le Merle des Columbiers, Montb. Hist. Nat. Ois. iii. p. $3^{8} \mathrm{r}$ $(17 ; 5)$.

Songsier thrush, Lath. Gen. Syn. ii. pt. i. p. 59 (1783).

Pigcon thrush, Lath. t. c. p. 64 (I8I3).

Turdus colun binus, $C \mathrm{~m}$. S. N. i. p. 836 (1088).

T. cantor, Gm. S. N. i. p. 837 (1788); Kittl. Kupf. Vög. ii. p. I I ; pl. 5. fig. I (I 832 ).

Lanprotornis panayensis, Cab. Mus. Hein. Th. i. p. $2 \mathrm{CO}$ (1850).

Calcrnis panayensis, Gray, Hand l. B. ii. p. 26, n. 6373 (1870); Walden \& Layard, Ibis, 1872 , p. 97; Watden, Trans $Z$. S. viii. p. 79 (1872); Sharpe, Ibis, 1876 , p. 46; id. Trans. Linn. Soc. 2nd ser. Zool. i. p. 343 (1876); Trueedd. P. Z. S'. 1877 , pp. 549, 763, 831; id. P. Z. S. 1878 , pp. I I3, 287, 343, 622, 710, 951; id. P. Z. S. 1879, p. 72; Sharpe, Ibis, 1884 , p. 321 ; Wardlaw Ramsay, Ibis, 1886. p. 162; Sharpe, Ib.s, 1888 , p. 202; Blassius, Ornis, 1888, p. 315; Sharpe, Cat. B. M. 13. p. $148(18 \mathrm{go})$.

C. columlina, Gray, Hand-l. B. ii. p. 27, n. $^{\circ} 6384$ (1870).

Mrab. Luzón, Cebú, Negros, Dumaguete, Panay, Amparo, Leyte, Camiguin, Bohol, Surigao, Zamboanga, Basilan, Paragua, Dinagat, Mindanäo.

\section{Gén. STREPTOCITTA, Br.}

:4. St. torquata, (Temm.) oo N. v Martin

(M. S T.)

Girrula torquata, Temm. Pl. Col. ii. pl. 444 (1828).

Pica tcrquata, Gray, Flard-l. B. ii. p. I I, n. 6180 (1870).

Streptocitta torquata, Walden, Trans. Z. S. viit. p. i6 (I87t;

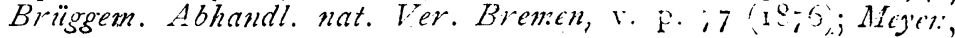


Ibıs, 1879, pp. 133. 146; id. Abbild. Vögel-Skel. pl. «хх. (1882); Blasius Zeitschr. ges Orn. ii. p. 296, Taf. xiii. tig. : (1885); Sharphe, Cat. B. M. I3. I8ço, p. I 52.

Hab. Paragua;? Célebes.

\section{Gén ENODES, Trm.}

15 E. erythrophrys, (Ten.) ơ N. v. Piroc.

I amprotornis erythrophrys, Temm. Pl. Col. ii pl. $267(1824)$. kn des erythrophrys, Temm. Pl. Col. i. Tabl. Méth. p. in (1838); Blyth, Cat. B. Mus. As. Soc. p. III (1849); Bp. Consp.i. p. 4I7 (1850); Wallace, Ibis, 1860 , p. I4r; Walden, Traus. Z. S. viii. p. 78 (1874); Brïggem. Abhandl. nat. Ver. Bremen, v. p. 77 (1876); Meyer, Ibis, 1879 , p. i34; Sharpe', Cat. B. M. 13. 1890, p. 192.

Calornis erythrophrys, Gray, Gen. B. ii. p. 327 (1846); id. Hant-l. B. ii. p. 27, n. $^{\circ} 6394$ (1879).

Hab. Paragua;? Célebes.

\section{Fam. PILOCÉIDOS.}

$\cdot$

I. Gén. MUNIA, Hodg.

I. M. oryzivora, (Lin.) o $\bigcirc$ N. v. Mayang costa.

(M. S. T.)

The Cock Padda or Rice bird, Edwards, Nat. Hist. B. i. p. 4I, pl. 4I (I 743).

Le Gros-bec cendré de la Chine, Briss. Orn. iii. p. 244, pl. xi. fig. 4 ( 1766$)$.

Loxia oryzivora, Linn. Syst. Nat. i. p. 302 ( г766); Vieitl. O.S. Chant. p. 94, pi. 1xi. (1805); Raffl. Trans. Linn. Soc. xiii. p. 314 (1820); Hahn \& Küster, Vög. ans Asien etc. Lief. x. 'Taf. 3 (1821); Schl. \& Poll. Faun. Madag, Ots. p. I54 (1868). Le pedda ou l'Oiseau de riz, Buff. Hist. Nat. Ois. iii. p. to 3 (I775).

Le Calfat, Buff. Hist. Nat. Ois. iv. p. 37 I (г778).

Grosbec de la Chine, Daubent. Pt. Enl iv. p1. I52. fig. I.

Java Grosbeak, Lat. Gen. Syn. ii. pt. i. p. I 29 (1783).

Red-eyed Bunting, Lath. t. c. p. 210 ( 1783 ).

Emberiza calfat, Gm. Syst. Nat. i. p. 887 ( 1788).

Loxia javensis, Gm. Syst. Nat. i. p. $85^{\circ}$ (1788); Sparm. Mus. Carls. fasc. iv. pl. 89 (1789).

Coccothraustes oryzivora, Vieill. N. Dict. d'Hist. Nat. xiii. ए). 545 ( 18 I 7 ).

Fringilla oryzivora, Horsf. Trans. Linn. Soc. xiii. p. 861 (1820); Swains. Zool. Illustr. iii. pl. I $5^{6}$ (i $8822-23$ ).

Amadina oryzivora, Strickl. P. Z. S. 1842, p. I67; Blyth J. A. S. B. xv. p. $285(1846)$; id. Cat. B. Mus. As. Soc. p. I 8 (1849); Gray, Gen. B. ii. p. 369 (1849); id. Hand-l. B3. ii. p. 55, n. ${ }^{\circ} 6776$ (1870); Hume, Slr. F. viii. pp. 67, I1) (1879).

Munia oryzivora, Bp. Consp. Av. i. p. 451 (1850); Szunh. Ibis, 
$1 S 61$, p. 45; E. Verifon, t. c. p. $115 ;$ Scl. P. Z. S. 186,3 , 1. 219; Hartl. P. Z. S. 1867. p. 826; id. Vög. Madah. p. for (1877); Shelley, Ibis, 18'30, p. 312; Bartlett, Monogr. Weaver.B. pt. i. (1888); Sharpe, Cat. B. M. 13. 1890, p. $32 \mathrm{~S}$.

()ryzornis oryzivora, Cab. Mus. Hein. i. p. I74 1850, id. ia I'on der Decken's Reis. iii. p. 30 (1869); Frinsch \& Harii. l"̈̈g. Ostafr. p. 433 (1870); Sharpe, Cat. Afr. B. p. 63 (1871).

Paldla oryzivora, Horsf. \& Moor, Cat. B. E. I. Co. Mue. ii. P. $5^{\circ}+(1856)$ Moore, P. Z. S. 18.59, p. 443; Reichenb. Sing\%. p. 42, pl. xv. figs. 135-139 (1861); Wall. P. $Z$. S. 186,3 , p. 486; Melliss. Ibis, 1870 . p. Ioo; Suinh. P. Z. S. 187s, p. 385 ; Wald. Tr. Z. S. viii p. (1872); Hume, Nests. \& Eggs:. Ind. B. p. 454 (1873); Stölker, J.f. O. 1874 , p. I r Salvad. L'cc. Born p. 263 (1874); Walden, Tr. Z. S. ix. p. 207 (1875); Tweedd. Ibis. 1877 p. 317; David \& Oustal. Ois. Chine, p. 334 (1877); Legge, Birds of Ceylon, p. 646 (1879); M'son, Ibis, yer. Ibis, 1879 , p. I 32 ; Sharpe, P. Z. S. 1874 , p. 344; Nicholson, Ibis, 1881 , p. I 53; Vorderm. Nat. Tijdschr. Nederl. Ind. xliv. p. 222 ( I885), xlvi. p. 87 (1887).

Padda verecunda, Reichenb. Singy p. 4I, 'Taf. xv. fig. I53 (186I). (Oryzivora leucotis, Jerd. B. Ind. ii. p. 359 (1863).

Spermestes oryzivora, Russ, Stubenz. p. I 36 , 'Taf. viii. fig. I 33 ( I 879).

Hab. Luzón, Manila, Mindanao.

2. M. jagorii, Cab. $\sigma \circ \mathrm{N} . \mathrm{v}$ Mayang paquing.

(N. S. T.)

Munia (Dermophrys) jagori, Cab. in MIus. Berol.; Martens. J. f. $O .1866$, p. 14 .

Dermophrys jagori, Cab. J.f. O. 1872, p. 3 I 6.

Amadina jagori, Gray, Hand-l. B. ii, p. 55, n. ${ }^{\circ} 67.66$ (1870).

A. minuta, Gray, t. c. p. 55, n. ${ }^{\circ} 6768$ (i 870 ).

Munia jagori, Walden, Trans. Z. S. ix. p. 207 (1875); Salvad. Ann. Mus. Genov. vii. p. 667 (1875); Sharpe, Trans. Linn. Soc, and ser. i. p. 353 (1876); 7reedd. P. Z. S. 1877 , pp. 549. 699, 764; id. P. Z. S. 1878, pp. 288, 343, 710. 95I; id Rep. Voy. "Challenger,» Zool. ii. p. 22 (1880); Salvad. Ann. Mus. Genov. xvi. p. 192 (1880); id. Orn. Papuasia etc. ii. p. 347 (г881); Wa'dlaw Ramsay, Treedale Mem. p. 654 (1881); Sharpe, Cat. B. M. 13. p. 337 (1890).

Munia atricapilla, (nec V.), Sharpe, Ibis, 1888, p. 202.

Hab. Luzón, S. Mateo, Cebú, Leyte, Bohol, Valencia, Negro;, Dumaguete, Butuan, Placer, Zamboanga, Mindanao, Paragua, 'Taguso.

3. M. minuta, Mejer. $\mathrm{N}$ v. Maya.

(M. S 'T )

Fringilla minuta, Meyer, N. Acta Acad. Cos. Lesp.-Carol. Nat. Cur. xvi. Suppl. p. 86, pl. xxii. fig. 2 (I834); M.trtens, J. f. O. 1866, p. 14 .

Munia minuta, Walden, Trans. Z. S. ix. p. 208 (1877); Sharpe, Zat. B. M. 13. p. 337 (1890).

Hab. Manila, Luzón. 
4 M. nisoria, ( $\left.T_{e \prime \prime}\right)$ N. v. Maya.

Le: Grosbec tacheté de Java, Briss. Orn. iii. p. 239, pl. xiii. fig. 2 (1760).

loxia undulata, (nec Müll.), Lath. Ind. Orn. i. p. 387 (1790). Fringilia punctularia, (nec Gm.), Horsf. Trans. Linn. Soc. xiii. p. 161 (I820).

F. nisoria, Temm. Pl. Col. iii. pl. 500 fig. 2 ( 1830$)$.

Amadina punctularia, (nec Gm.), Hay, J.A.S. B. xiv. p. 554, ncte (1845).

(Oxycerc เ nisoria, Gray, Gen. B. iii. App. p. Io (1849).

Munia punctularia, (nec Gm.) Blytk, Cat. B. Mus. Ac. Soc. p.

I 7 (1849); Bp. Consp. Av. i. p. 452 (1850); Horsf. \& Moo-

re, Cat. B. Mus. E. I. Co. ii. p. $665(1856)$.

Amadina undulata, Gray, Hand-l. B. ii. p. 56, n." 6778 (1870).

A. nisoria, Hume, Str. F. viii. p. 66 (1876).

Lonchura undulata, Vorderm. Nat. Tijdschr. Nederl. Ind. xiiv. p. $224(1885)$.

M. nisoria, Sharpe, Cat. B. M. 13. p. $35^{2}$ (1890). Hab. Luzón, Java.

5. M. cabanisi, Sharp. N. v. Mayang bulic.

(M. S. T.)

Uroloncha jagori, (nec Munia jagori, Cab.), Cab.J.f. O. 1877 , p. I 4 .

Oxycerra jagori, Cab.J.f. O. 1872 , p. 3r7; Walden, Trans.

Z. S. ix. p. 208 (1875); Tweedd. P. Z. S. 1877 , p. 699.

M. cabanisi, Sharpe, Cat. B. M. 13. p. 353 (1890).

Hab. Luzón, S. Mateo, Montalban.

2. Gén. UROLONCHA, Cab.

6. U. everetti, (Tw.) N. v. Mayang bulic.

(M. S. T )

Oxycerca everetti, Tweedd. Ann. \& Mag. Nat. Hist. (4) xx. p. 96 (1877); id. P. Z. S. 1877 , pp. 699, 764, pl. lxxiii. fig. 2. id, P. Z. S. 1878, p. 622; Sharpe, Ibis, 1888, p. 202.

U. everetti, Sharpe, Cat. B. M. 83. p. 363 (1 890 ).

Hab. Luzón S. Mateo, Montalban, Cebú, Paragua, P. Princesa.

7. U. leucogastra, Blyth. N. v. Maya.

(M. S. T.)

Amadina leucogastra, Blyth, J. A. S. Beng. xv. p. 286 (1846; Gray, Gen. B. ii. p. 370 (1849); Hume, Str. F. 1879 , p1. 66, 107, 161; Oates, Handb. B. Brit. Burm. i. p. 367 (1883).

?Munia melanictera, Blylh, Cat. B. Mus. As. Soc. p. I I 7 (I849).

Munia leocogastra, Horsf. \& Moore, Cat. B. E. I. Co. Mus. ii. p. $510(1856)$; Jerd. B. Ind. ii. p. 359 (1863); Salvad. Ucc. Born. p. 267 (1874); Hume, Str. F. 1875 , p. 323; D.tv.son, Str. F. 1877 , p. 460 ; Hume, \& Divison, Str. F. iv. p. 46.2 (1878); Vordorm. Nat. T.jdschr. Neder!. Int. xlvi. p. 234 (1887). 
N. chrysura, Bly!h. Ibis, 1870 , p. 172 (ex MSS. Miss. Liıg.t.).

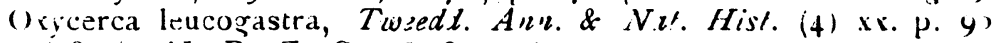
(1877); id. P. Z. S. 1878, p. 622.

l. leucogastra, Sharpe, Cat. B. M. 13. p. 362 (1890)).

Hab. Paragua; Borneo.

\section{Gén. ERYThruka, SPakr.}

8. E. prasina, (Sparr.) oo N. v. Maya.

(M. \$ 1.1

le Verdier de Java, Briss. Orn. iii. p. 198, pl. vii. fig. 4 (17001).

L.e Quadricolor, But. Hist. Nut. Ois. iii. p. 467 (1775).

l.e '́oupet bleu, MIJntb. Hist. Nat. Ois. is. p. 179 (1778).

Ciros-bec de Java, Daubent. Pl. Enl. iv. pl. Ior tig. 2.

ked-ru uped Bunting, Lath. Gen. Syn. ii. pt. i. p. $20 x$ (1783).

Biue-faced Bunting, Luth. Gen. Sun. ii. pt. i. p. $2(199$ ( 1783$)$.

Juxia prasin.ı, Sparrn. Mus. Carli. ii. pls. 72, 73 (1788); Raftles, Trans. Linn. Soc. xiii. p. $3^{1+}+(1820)$; Sundev. Crit. om. Sparrm. 1. $12(1857)$.

Kimberiza cyanopis, Gmi. Syst. Nat. p. 886 (1788).

1:. quadricolor, Gm. Syst. Nat. p. 886 (1788).

Coccothraustes prasina, Vieill. N. Dict. d'Hist. Nat. xiii. $537(1817)$.

Fringilla prasina, Horsf. Trans. Linn. Soc. xiii. p. 161 (1820).

ji. sphecura, Temm. Pl. Col. iii. pl. 96. figs. 1-3 (1824).

J.snciura quadricolor, Sykes, P. Z. S. 1832, p. 94 .

Eirythrura viridis, Swains. Class. $B$. ii. p. 280 (1837).

IV. prasina, Blyth, Cat. B. Mus. As. Syc. p. 118 (18+9).

Ainadina prasina, Gray, Gen. B. ii. p. 370 (1849); id. Hand-l. $B$. ii. p. 58, n. $^{\circ} 6305(1870)$.

Erythrura prasina, Bp. Consp. Av. p. 457 (1850); Horsf. \& Moore, Cat. K. E. I. Co. Mus. ii. P. 503 (1856); Hartl. P. Z. S. 1858 , p. 46 г; Reichenb. Sin๗ve. p. 3t, Taf. xi. fi ss. 99-101 (1861); Salvad. Ucc. Born. p. 2 ) I (1874); Hume, Str. F. 1875 , p. 323; Sharpe, Jbis, 1875. p. 5\%; Hume, \& Davison. Str. F. vi. p. $405(1878)$; Nicholsne, Ibis 1879, p. 170; Sharpe, t. c. p. 252; Hun?, Str. F. viii. pp. 67, 107, ix. p. 121 (1881); Scl. Ibis, 1881 , p. 545; Nicholson, Ibis, 1882, p. 63; Vordern. Nut. Tijdschr. Nederl. Ind. xliii. p. $106(1884)$, xivi. p. 254 (1887); Shtrpe, Ibis, 1889, p. 435. id. Cat. B. M. 13. p. 381 . (1890).

Hab. Luzún, Panpanga; Borneo, Java, Sumatra.

\section{E. trichroa, Kiltl. N. v.}

Fringilla trichroa, Kittl. Mem. Acad. St. Petersb. id. p. 8, pl. 10 (1835).

Estrelda trichura, Gray, Gen. B. ii. p. 369 (1849).

Errythrura trichura, Bb. Consp. Av. i. p. 457 (1850); Hartl. P. Z. S. 1858, p. 461 ; Reichenb. Singu. 0. 33, pl. xi. fige. 98 (1861); Scl. P. Z. S. 1870 , p. 158; Meyer, Sitz. k. Akad. Wien, lxx. p. 127 (1874); Säbvad. Ann. Mus. Civ. Genov. xivi. p. 192 (1880); Finsch, P. Z. S. 1880 , p. 576; id. J. f. $O$. 1880, p. 290; id. Ibis, 1881, pp. 108, 115; Sc!. Ibis, 1881, 
p. 545, Salvad. Orn. Pap. ii. p. 442 (1881); id. Ibis, $188+$,

p. 354; Guillem. P. Z. S. 1885 , p. 573; Graut, P. Z. S. 1889 , p. I97; Sharpe, Cat. B. M. 13. p. 385 ( 1890).

E. kittlitzi, Bp. Consp. Av. i. p. 457 ; Gray, Hand-l. B. ii. p. $58, \mathrm{n}^{\circ} 6084$ ( 1870$)$.

Fistrelda trichroa, Gray, Cat. B. Trop. Isl. p. 27 (1859).

Hirythrura modesta, Wall. P. Z. S. 1862 , p. 35I.

A.nadina modesta, Finsch. Neu-Guinea, p. i 76 (1865).

Erythrura phanicura, Bernst. Nederl. Tydschr. Dierk. ii. p. 331 i 865$)$.

Amadina trichroa, Gray, Hand-l. B. ii. p. $5^{8}$ n. 0806 (1870); Rosenb. Reistocht. Geelbinteb. p. 7 (1875); id. Malay. Archip. p. 406 (1879).

Erythrura glauca, Finsch. Journ. Mus. Goddeffr. xii. p. 36 (1876).

E. sp. inc., Finsch. P. Z. S. 1877 , p. 779.

Hab. Carolinas; Molucas.

\section{Gén. Ploceus, Cuv.}

10 P. baya, Blyth. N. v. Maya.

(M S T.)

Le Grosbec des Philippines, Briss. Orn. iii. 1760, p. 232, pl. xii. fig. (б), pl. xviii. figs. I, 2 Daubent. Pl. Einl. iv. pl. I35. fig. 2.

Loxia philippina, Linn. Syst. Nat. i. p. 305 (1766).

Le Toucnam-courvi, Buff. Hist. Nat. Ois. iii. p. 465 (1775).

Philippine Grosbeak, Lath. Gen. Syn. ii. pt. i. p. i 29 (1783).

Ploceus philippinus, Cuv. Règne An. i. p. 363 (I817); Sykes, P. Z. S. 1832 , p. 94; Gray, Gen. B. ii. p. 252 (1849); Blyth, Cat. B. p. II 5 (1849); Layard, Ann. \& Mag. N. H. (2) xiii. p. 256 (1854); Reid, Str. F. v. p. 54; Davidson, t. c. p. 31 2; Davison, t. c. p. 402; Taylor, t. c. p. 462; Ball, Str. F. vii. p. 22; Legge, B. Ceylon, p. 641 (1879); Hume, Str. F. 1879 , p. Io6; Butler, Cat. B. Sind \&c. p. 45 (1879; id. Cat. B. S. Bomb. Pres. p. 60 (1880); Vidal, Str. F. ix. p. 71 (1880); Davidson, \& Wenden, t. c. p. 415; Swinh. \& Barnes, Ibis, 188.5 , p. I 28; Vidal, Str. F. ix. p. 71 (1880); Reid, Str. F. x. p. 54 (1881); Davidson \& Wenden, t. c. p. 312; (1882); Davison, t. c. p. 402; Taylor, t. c. p. 462 .

Euplectes philippinus, Jerd. Madr. Journ. xi. p. 25 (1840); Blyth, J. A. S. Beng. xi. pp. 87 I, 889 (1842).

Plocens baya, Blyth, J. A. S. Beng. xiii. p. 945 (1844);Bp. Consp. i. p. 442 (1850); Horsf. \& Moore, Cat. B. Mus. E. I. Co. ii. p. 5 I 5 (1856); Jerd. B. Ind. ii. p. 343 (1863). Stoliczha, J. A. S. Beng. xli. p. 246 (1875); King, J. A. S. Beng. xxxvii. p. 2 I 5 (I868); Holdsw. P. Z. S. 1872 , p. 46.3; Huine, Nests \& Eggs Ind. B. p. 346 (1873); Adam, Str. F. 1873, p. 387; Hume, Str. F. 1874 , p. 481; Ball, t. c. p. 420; Butter \& Hume, Str. F. 1875 , Butler, Str. F. 1876. p. 39; Farrb. t. c. p. 260; Hume, Str. F. 1877, p. 323; Walden, Tr. Z. S. ix. p. 209 (1875); Scully, Str. F. 1879 , p. 331; Sharpe, Cat. B. 13. p. "488, (I890).

P. bengalensis, (nec L.), Sundev. Ann. \& Mag. Nat. Hist. xviii. p. $260(1846)$. 
Euplectes baya, Jerd. Madr. Joarn. xiii. p. I3t (1 $8+4$ '.

Nelicurvius fuscicollis, Reichenb. Singv. p. 75, 'Taf. xxxiii. figs. $263-265$ (1801).

Ploceus fuscicollis, Reichen, Zool. Jahrb. i. p. I 56 (2886). Hab. Luzón, Abra, Cagayan, Pamplona; Ceilan.

II. P. atrigula, Hodgs. N. v. Maya.

(M. S T.)

Ploceus atrigula, Hodgs. Icon. ined. in Brit. Mus. Passeres, pl. 278. figs. 1, 2 (n. ${ }^{\circ} 743$ ); id. in Gray's Zool. Misc. p. 8+ (18+4; Sharpe, Cat. B. M. 13. p. 491 (1890).

P. passerinus, Hodgs. Icon. ined. in Bibl. Zool. Soc. Passeres, pl. 277. fig. 3 (n. ${ }^{\circ}$ (0); Reichen. Zool. Jahrb. i. p. I 56 (1886); Nelicurvius baya, Reichcnb. Singo. p. 76, 'Taf. xxxiv. figs. 260268 (г 86 I).

P. baya, (nec. Blyth), Horsf. \& Moore, Cat. B. Mus. E. I. Co. ii. p. 5 I 5 (1856, pt.); Godzin-Austen, J. A. S. B. xxxix. p. 810 (1870); Blanf. op. cit. xli. p. I67 (1872); Hume, St. F. 1875, p. 153; Blyth \& Wald. B. Burm. p. 92 (1875); Anders. Rep. Zool. Exped. Yunnan, Aves, p. 597 (1878); Hume, Str. F. 1878 , p. Io6; id. Str. F. ix. p. 257 (1880); Oates, Handb. B. Br. B. i. p. $35^{8}$ (1883); Vorderm. Nat. Tijdschr. Nederl. Ind. xlii. p. 84 (1883).

Ploceus philippinus (nec Linn.), Hume \& Davison. Str. F. vii. p. 399 ( 1878 ).

P. megarhynchus, Hume, Str. F. 1875, p. 406, 1878, p. 400, I 879 , p. 106.

Hab. Luzón, Bataan, Hermosa, Pampanga, S. Fernando, Arayat; Java, Sumatra.

Gén. Ploceella, Oates.

I2. P. javanensis, (Less.) N. v. Maya

(M. S. 'T.)

Fringilla philippina, Horsf. Trans. Linn. Soc. xiii. p. I60 (1820). Loxia philippina, Raff. Trans. Linn. Soc. xiii. p. 313 (1820).

L. javanensis, Less. Traité, p. 446 (1831).

Ploceus philippinus, (nec L.), Blyth, J. A. S. Beng. xiii. p. 945 ( 1843 ); id. Ibis. 1867 , p. 40 .

P. hypoxanthus, Blyth, Cat. B. Mus. As. Soc. p. I I 4 (I849) Bp. Consp. Av. i. p. 443 (1850); Cab. Mus. Hein. Th. i. p. I80 (1850); Horsf \& Moore, Cat. B. Mus. E. I. Co. ii. p. 5 I 3 (I 856); Gray, Hand-l. B. ii. p. 44, n. ${ }^{\circ} 66$ I 3 (1870); Hume, Nests \& Eggs Ind. B. p. 442 (1873); id. Str. F. iii. p. I 5 t (1875); Vorderm. Nat. Tijdschr. Nederl. Ind. xlii. p. 85 (1883); Reichen. Zool. Jahrb. i. p. I6I (i886).

Pliceolus hypoxanthus, Reichenb. Singv. p. 77, Taf. xxxv. figs. 277,278 (186I).

Ploceella javanensis, Oates in Hume's Nests \& Eggs Ind. B. p. 443 (1873); id. Str. F. v. p. I60 (1877); id. ibid. x. p. 23 I (1887); Hume, $F$. viii. p. Io7 (1879); Oates, Handb. B. Brit. Burm. i. p. 362 (1883); Sharpe, Cat. B. M. 13. p. 474. (1890).

Ploceus javanensis, Wald. Tr. $Z$. $S$. ix. p. 2 io note (1875); Blyth \& hald. B. Burm. p. 9i (1875). 
P. chrysauus, Hume, Str. F. vi. p. 399, note $(1878)$.

Hab. Luzón, Cavite, Indan; Java.

\section{Fam. ALÁUDIDOS.}

1. Gén. MELANOCORYPHA, Boje.

1. M. Calandra, ( lint.) \& N. v. Calandria.

(M. S. T.)

Alauda calandra, Lın. S. N. 1. p. 288; Sharpe, Cat. B. M. 1.3. p. 55 t ( 1890$)$.

La calandre, Jaub. Pl. Col. pl. 363, f. 2. Hab. Europa, España.

\section{Gen aliauda, LiN.}

2. A. gulgula, Frankt. $\sigma \rho$ N. v. Laclac bunga

(M. S. '?.)

Alauda gulgula, Frankl P. Z. S. 1838 , p. ira; Jerd. Madr. Journ. xi. p. 30 (1840); Blyth, J. A. S. Beng. xiii. p. 962 (I84t; id. Cat. B. Mus. As. Soc. p. 132 (1849, pt.); Bp. Const. i. p. 245; Jerd. B. Ind. ii. p. 434 (1863); Stoliczka, J. A. S. Beng. xxxvii. p. 64 (1868); King. t. c. p. 216; Beav. Ibis, 1868 , p. 181; Brooks, Ibis, 1869 , p. 59; Grav, Hand-l. B. ii. p. 117, n. ${ }^{\circ} 746$ (1870; Holdsw. P. Z. S. 1872, p. $465 ;$ Huine \& Henders. Lahore to Yark. p. 269, pl. 29 (1873; itume Nest \& Eggs Ind. B. p. 486 (1873); id. Str. F. i. pl). 39, 420 (1873); Adain t. c. p. 389 ; Brooks, t. c. p. $485 ;$ Bly $y^{\prime} /$. \& Wald. B. Burin. p. 95 (1875); Brooks, Ibis, 1876 , p. 49r); Butler, Str. F. iv. pp. 2, 39 (1876); Arms'. t. C. p. 337; Godwin-Aust. J. A. S. Beng. xlv. p. 83 (1876); Butler. Str. F. v. p. 185 (1877); Ball, t. c. p. 223; Hume \& Davison, Str. $F$. vi. p. 409; (1878); Davids. \& Wenden, Str. F. rii. p. kis (1878); Legge, B. Cevlon, p. 630 (1879); Hume, Str. F. viii. p. 108 (1879); Scully, t. c. p. 330; Vidal, Str. F. ix. 1.73 (1880); Wardlaw Ramsay, Ibis, 1880 , p. 67; C. Swinh. Ibis, 1882, p. 116; Reid, Str. F. x. p. 58 (1883); Dav'son, t. c. p. 406; Vidal, t. c. p. 424; Oates, Handb. B. Br. Burm. i. p. 373 (I 583); Murray, Vertebr. Faun. Sind, p. 197 (I 884 ); Swinh. \& Barnes, Ibis, 188.5 , p. s30; Terry, Str. F. x. p. 478 (1887); Hume, Str. F. xi. p. $289(1888)$; Sharpe, Cat. $B$. M. 13, p. 575 (1890).

A. triborhyncha, Hodgs. Icon. ined. in Brit. Mus., Passeres, pl. 293. fig. 2 (n. ${ }^{0}$ 739); id. in Gray's Zool. Misc. 1. 84 (1844); Gray, Cat. Manm. \&c. Nepal pres. Hodgs. p. Ion (1846): Horsf. \& Maore, (at. B. Mus. E. I. Co. ii. p. $467(1856)$ pt.); Gray, Hand-l. B. ii. p. I17, n." 7745 (1870).

A. gracilis, Blyth, J. A. S. Reng. xi. p. 201 (1842).

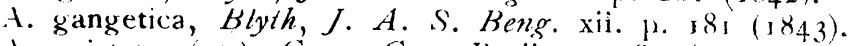

A. cristata, (pt.); Gray, Gen. B. ii. p. 380 (1 844$)$.

A. malabarica, (nec Sykes), Horst. \& Moore, (at. B. Mus. L. I. Co. ii. p. $467(1856)$.

1. coelivox, Swinn. Zool. 1839, p. 6723; ia. Ibis, 1.86o, pp. 52, 
$132, \quad 186 \mathrm{~s}$, p. $+6,1863$, p. 3ij; id. P. Z. S. 1863. pp. 81), 272 ; id. Ib s, $18 ; 0$, p. 83; Grai, Hand-!. B. ii. p. I 8 , n." 7753 (1870); Swinh. P. Z.S. is 1 , p. 399; David \& Ous:. Ois. Chine P. $314(18,7)$.

1. sala, Swinh. Ibs, 1870, p. 355. id. P. Z. S. 1871, p. $3^{\text {(o); }}$ David of Oust. Ois. (hine, p. $31+(1871)$.

A. wattersi, Szinh. P. Z. S. is; 1 , p. 389; David \& Ou:t. Ois. Chine, p. 3rt (1877); Taieed. P. Z. S. I $\delta ; \delta$, p. Tw; IVardlaze Ramsa', Ibis, $1880^{\circ}$. p. 160.

A. australis, Brooks, Str. F. 187,3, p. $4^{8}+$; Hume, Str. IN 1579, p. 108; Brooks, t. c. p. 489; Terry, Str. F. x. p. $+79(1887)$.

A. peguensis, Oates, Ntr. F. 187,5, p. 343 .

Hab. Luzón, Manila, Bohol; Formosa.

\section{Cién. MIRIIFRA, Hor:s.}

3. M javanica, (Horsf) \& N v Pugaput.

(M. S. T)

M. javanica, Horsf. Tr. Lin. Soc. xiii. p. 159 (1820); Gray, Gen. B. ii. p. $3^{8} 3$ (1844); Bp. Consp. i. p. 243 (1850; B Blytl, J. A. S. Beng. xiv D. 255 (1855; Horsf. et Moore, Cat. 13. Mus. B. I. Co. li. I856, p. 474; Gray, Hand-l. B. ii. I8jo, p. г 2 г, n." 7796; Salí. Ucc. Bern. 1874, p. 262; Nich. Ibis, $1 \& \& 1$, p. 1 54; Vord. Nat. Tijds. Ned. Ind. xliii. I884, p. 107, xlvi. I 887 , p. 234 ; Sharpe, Cat. B. M. 13. p. 602 (1890).

Alauda mirafra, Tem. Pl. Col. iii. fl. j05, f. 2 (1824).

Geocoraphus javanicus, Cab. Arch. f. Natur. $8_{77}$, p. 328; id. Mus. Hem. i. p. I 26 (1 850 ).

M. Borneensis, Stuinh. An. et Mag. N. Hist. viii, 1871, p. 257; Salv. Ucc. Born. 1874 , p. 263; Vord. Nat. Tijds. Ned. Ind. xlvi. I $887,234$.

Hab. Samar, Borongan, Paric; Java, Borneo.

4. M. philippinensis, Wiral. $\bigcirc \bigcirc \mathrm{N}$. v. Pugapug.

(M. S. T)

Mirafra philippinensis, Wardlaw Ramsay, Ibis, 1886, p. 160; Sharpe, Cat. B. M. 13, p. 605 (1890).

Hab. Luzón, Manila, Laguna.

\section{Fam. TIRÁNIDOS.}

I. Gén. TODIROSTRUM, Lliss.

1. T. sehistaceips, $S c l$. $\sigma$ N. v.

(M. S. T)

T. schitaceiceps, Sclater, Ibis, 1859 , p. 44+; id. Cat. B. M. 14. p. 208; id. Cat. B. M. 14. p. it (1888).

Hab. América, Guatemala.

\section{Gén. LEGA'TUS, Si H.}

2. I albicollis, (Vicill.; $\sigma \mathrm{N}$. v.

(II. S T.)

Barhichon de Cayenne, o, Daub. Pl. Enl. 83. fig. 2. 
L. albicollis, Sclater, P. Z. S. 1859 , p. $+6 ; 1 d$. Cat. B. M. $1+$. p. 155 (I 888).

Hab. América, Guatemala.

3. Gén. MYODINASTES, Br.

3. M. Iuteiventris, $B p . \sigma \mathrm{N}, \mathrm{v}$.

(M S. T)

'Tyrannus audax, Sclater, P. Z. S. 1856, p. 297.

My. luteiventer, Bp. Compt. Reud. xxriii. p. 659; Sclater, Cat. B. $M .14$ p. 183 (1888).

Hab. América, Guatemala.

4. Gén. megarhynchus, Thumb.

4. M. pitangua, Lin. N. v.

(M. S. T.)

Lanius pitangua, Lin. S. $N$. i. p. I 36 .

M. pitangua, Hein. J. f. O. 1859 p. $3+3$; Sclater, Cat. B. M. 14. p. 190. (1 888).

Hab. América, Guatemala.

5. Gén. Myobius, Gray.

5. M. sulphureipygius, Scl. $\sigma$ N. v.

(M. S T.)

Tyrannula sulphureipygia, Scl. P. Z. S. 1856, p. 296.

M. sulphureipygius, Scl. Cat. B. M. 14. p. 200. (1888).

Hab. América, Guatemala.

6. Gén. MYARCHUS, Сав.

6. M. lawrencii, Gir. $\sigma$ N. v.

Muscicapa lawrencii, Girad., B. of. Texas. 1841 , pl. 2. f. I. My. lawrencii, Scl. Cat. B. M. 14. p. 256 (1888).

Hab. América, Guatemala.

7. Gén. TYRANNUS, Cuv.

7. T. pipiri, Vieill. \& N. v. Tirano intrépido.

(M S. T.)

Lanius tyrannus, Lin. S. N. i. p. I 36 , (parte) var carolinensis, Gm. S. N. i. p. 302 .

'T. pipiri, Vieill, Ois. Am. Sept. i. p. 73 pl. $44 ;$ Scl. Cat. B'. M. 14. p. 267 (I 888 ).

Hab. América, Canadá.

Fam. PÍPRIDOS.

r. Gén. CHIRoXiphia, Вав.

1. Ch. caudata, Shaw. t N. v.

Pipra candata, Ch. Shaw, Nat. Mis. v. pl. I53 (r794). 
Ch. candatu, C.xb. Wieg.n. Arch. $18+7$, i. p. 235; Bp. Consp

1. p. I7I; Scl. Cat. B. M. 14. p. 318 (1888).

Hab. América, Canadá.

2. Gén. CHIROMACH.ERIS; CaB.

2. Ch. candæi, Pars. \& \& N. v.

(M. S. T.)

Pipra candai, Parzud. Rev. Zool. 1841, p. 306.

('h. candxi, Cab. et Heine, Mus. Hein. ii. p. 97; Scl. Cat. B. M. 14. p. 3I 4 (1888).

Hab. América, Guatemala.

\section{Fam. COTÍNGIDOS.}

I. Gén. Pachyrhamphus, Gray.

I. P. major, Sclat, $\delta$ \& $\mathrm{N}$.

(M. S. T.)

P. major, Scl. Cat. B. M. 14. p. 346 (1 888 ).

Hab. América, Guatemala.

2. Gén. Lipaugus, Bore.

2. I. holerythrus, む Scl. N. v.

(M. S. T.)

L. holerythrus, Scl. et Salv. P. Z. S. 1860 p. 300; Scl. Cat. B. $M$. 14. p. 357 (1888).

Hab. América, Vera-Paz.

3. Gén. RUPICOLA, Bris.

3. R. peruviana (Lath.) む N. v. Gallo de peñasco.

(M. S. T.)

Pipra rupicola, var. Gm. S. N. i. p. 998.

R. peruviana, Sct. Cat. B. M. 14. p. 370. (1888).

Hab. América, Perú.

4. Gen. XiPhOlÆNA, Glog.

4. X. pompadora, (Lin.) \& N. v. Cotinga purpurea. (M. S. T.)

Ampelis pompadora, Lin. S. N. i. p. 218.

Cotinga pourpre de Cayenne, Daub. Pl. Enl. 279.

X. pompadura, Scl. Cat. B. M. 14. p. 387 (I888).

$H a b$. América, Cayena. 


\section{Fam. PÍTIDOS.}

I. Gén. PitTa, Vifill.

1. Fitta cyaroptera. Tem. 0.0 N. v. Uac.uac.

Mirle des Moluques, Daub. Pl. Enl. 257.

T'urdus moluccensis, P. L. S. Mïll. Natursyst., Suppl. p. 1 tt $(1 ; ; 6)$.

'. macrourus fasciatus, Bodd. Table d. Pl. Enl. p. I6 (1783).

( rrus brachyurus, var. d. madagascariensis, Gm. S'. N. i. p. 376 . ( 1785 ).

Myiothera brachyura, Raff. Trans. Minn. Soc. xiii. p. 300 (1822). l'itta cyanoptera, Temm. Pl. Coll. $218(1823) ; \cdot V i g$. Mem. Ruffl. 1. 659, (1830); Less. Tr. d'Orn. p. 394 (1831), Mïll. et Schl. Verh. Zool. Pitta, pp. 7. 17; G. R. Gray, Gell. B. i. 1. 21 3: low', Sarawak, p. $410 ;$ Blyth. Cat. Mus. A. S. 1. 157; Moore, P. Z. S. 1854, p. 274; Horsf. et Moore, Cat. B. Mus. IE. I. C. i. p. 183; Mottl. et Dillw. Contr. Nat. Hist. Labuan, p. 22, Schlegel, Vog. Ned. ind. Pitta, pp. 9, 32, pl. 4 fig. 1 (1863'; id. Mus. de Pays, Bas, Pitta, p. 9. (1863). et p. 15 (1874); Wallee, Ibis, 1864, pi). 105, I10; Walden, P. Z. S. 1866, p. 545; Beavan Ibis, 1870 , p. 322 ; Salvad. Ib.s, 1871 , 1. 248 ; id. Att. R. A. Sc. Tor. ix. p. 631;id. Ucc. d. Born.o, p. 235; Sharpe, 1bis, p. 262 Nicholson, Ib.s, 1883, p. 88, Sicl Cat. B. M. 14. p. 420 (1888).

(oloburis cyanoptera, Cab. et Hein. Mus. Hein. v. i. p. 4.

l'itta malaccensis, Blyth, J. A. S. B. xii. p. $950(1843)$.

Jirachyurus malaccensis, $B p$. Consp. i. p. $25+$.

(Coloburis malaccensis, Cab. et. Hein. Mus. Hein. ii. p. 4.

Brachyurus cyanopterus, Blyth, $J$. A. S. B. xvi. p. 153. $(18+7)$; Bp. Consp. i. p. 254; id. Consp. Vol. Anisod. p. 7; Elliot, Mon. Pitt, pl. iv.

l'itta nimpha, Swinh, Ibis, 1861, Pp. 412, 414; id. P. Z. S. 1863, p. 277.

P. moluccensis, Swinh. P. Z. S. 1871 , p. 374; Oates, Str. Feath. ․ p. 149 ; id. B. Brit. Burm. i. p. 415 , Hume et Dav. Str. Teath. vi. p. p. 240; Hume, Str. Feath, viri. p. 94; Kelhain, Ibis, 1881, p. 509; David et Oast O.s. d. Chine, p. 144.

Brachyurus moluccensis, Elliot. Ibis, 1880, p. 413; Hume, Str. Feath. iii. p. 106; Blyth. et Walden, B. Burina, p. 98: Sicurpe, Ibis, 1877 , p. 10 .

Hab. Samar, Borongan; Borneo, China.

2. Pitta erythrogastra, Tem. o. L Liocn.

(M. S. T.)

Pitta erythrogastra, Temm. Pl. Col. 212 (1823); Less. Tr. $d$. Orn. p. 394; Mull. \& Schl. Verh. Zool. Pitta. p. 18; Blyth. Cat. B. Mus. A. S. B. p. I 57; Gray, Gen. B. i. p. 213; Schleg. Mus. P. B. Pitta, p. 8 (1863); et p. 12 (1874); Ibis, 1864 , p. 106; Wardl. Ramsay, Ibis, $189+$. 1. 33t; Sal. Cat. B. $M .14$. p. 432 (1888). 
Brachyurus erythrogaster, Bp. Consp. i. p. 255; Ellıol. Mols. Pllt. pl. xvi; id. Ibis, 1870 , p. 417 .

( ) huris erythrogaster, Cah. ef Hein, Mus. Hein. ii. p. 5.

lirithopitta erythrogastra, Bp. Consp. Vol. An sod. P. i; Walden, Ir.zns. Zool. Soc. ix. p. ix. p. 187 ; id. P. \%. S. 1877. p. 5 k5, 1878, p. 948, et 1879, p. 71 ; Wordl. Rams:14, Tiüedt. Mem. App. p. 657.

Hab. Luzón, L guna, Mindoro, Marinduque, Ciuimaris, Zambounga, Mind mao, Basilan.

\section{P. propinqua, Sharpe oo N. v. Uastuas.}

Brachyurus propinquus, Sharpe, Trans. linn. Soc. ser. 2. Zool. i. p. 330 .

P. propinqua, Sclat. Cat. B. M. 17. p. 433 (1888).

Hab. Sa nar, Dumalon, Mindana(o.

4. P. Kochi, Brug. O० 0 N. v. Lioco.

(M S. 'T)

P'ittakochi, Brüggem, Abhandl. nxt. Vor. Bremen, r. p. 65, pl. iii. f. 6; Oust. Bull. Soc. Phil. ser. 7. vi. p. 256; Gould, $K$. Asia, v. pl. 7 I; Scl. Cat. B. M. 14. p. 433 (1888).

Erythropitta kochi, 7\%. P. Z. S. 187ı, p. +3\%, pi xxvi.

Hab. Luzón, Cagayan, Alcalá, Bataan, Orión.

\section{P. makloti, Tem. t N v.}

Pitta Macloti, Temm. Pl. Col. 547. (1834); Mïll. \& Schleg, Verh. Zool. Pitta, pp. 12, 18; Gray, Gen. B. i. p. 213, id. P. Z. S. 1858 , p. $175,-1859$. p. I 551861 . p. 434 ; id. Cat. $B$. New. Guinea, p. 26; Sclat. Proc. Linn. Soc. ii. p. 158; id. P. Z. S. 1877 . p. 99. Schleg. V.ög. Ned. Ind. Pitta, p. 15. pl. 4 fig. 3 ; id. Mus. P.-B. P.tta, p. 7. (1863) et p. Io. 1874 ; id. Ned. Tydschr. iii. pp. 188, 189; Wallace, Ibis, 1864 , pp. 102, 105, го6; Krefft, P. Z. S. 1867 , p. 31, (Cape York); Ciould, Suppl. B. Austria. pl. 29, Salvad. Ann. Mus. Civ. Genov. vii. p. 778 , x. p. I 28 , xiv. p. 656, x i, p. I 85 ; id. Ibis, 1879 . p. 32t; id. Orn. Pap. ii. p. 395; Ramsay, Proc. Linn. Soc. $N$. S. $W$. ii. p. 187 , iii. p. 277 , iv. p. 98; Guillemard, P. Z. S. 1885 , p. 643; Nehrkorn, J.f. O. 1885 , p. 33; Sclat. Cat. B. M. 14. p. 436 (1888).

Brachyurus mackloti, Bp. Cons. i. p. 255; Wallace, Ann. N. H. ser. 2, xx. p. 477; Rosenb. Nat. Tydsehr. xxv. p. 232; id. J. f. O. 1864, p. II9; Elliot, Ibis, 1870, p. 415; Scl. Cat. B. M. 14. p. 436 (1888).

lirytropitta mackloti, Bp. Consp. Vol. Anisod. p. 7; Gray, Hand-l. i. p. 296.

Brachyurus (Erythropitta) mackloti, Elliot. Mon. Pitti. pl. 2 I.

Pitta digglesi, Krefft, Ibis, $186 \mathrm{~g}$, p. $35^{\circ}$.

P. strenua, Gou!d, M. S.; Elliot, Ibis, 1870 , p. 4 Io.

P. novae-hiberniae, Ramsay, Proc. Linn. Soc. N. S. W. iii. p. 73 (cf. Salvad. Ibis, 1879, p. 324.)

Hab. Australia, Nueva-Bretaña. 
6 P atric .pillz. Bris. N. v. ợo Uas.urs.

(M. S. T.)

Merula viridis atricapilla moluceensis, Briss. Orn. ii. p. 319. (引).

Merle des Philippincs, Daub. Pl. Enl. 89?

Breve des Pinilippines, Bub. Hist. Nat. OS. iii. p. fiz (!).

'Turdus sordidus, P. L. S. Müller, Natursyst. Suppt. p. I +3. n. ${ }^{\circ}$ I $(1776$, ?

Turdus brevicauda, Bodd. Ticll. d. Pl. Enl. p. 6 (1783):

Curvus brachyurus, var. B. philippensis, Cim. S. N. i. p. 375 (!).

Citta melanocephala, Walg. Sijst. sh. Corrus, n." it (1827).

Pitta atricapilla, Less. Tr. d'Orn. p. $39+\left(18_{31}\right)$; id. Compl. d. Buffon. p. 5on (1848); Tellm. Pl. Col. Tab. Meth. p. 16 (1832). Gray, Gen. B. i. P. 214; Gould, B. Asia. v. pl. 76; Scl. Ciat. $B$. $M$. 14. p. $43^{8}$ (1888).

Brachyurus atricapillus, Bp. Consp. i. p. 256 ; Elliot, Mon. Pitt. pl. xxv.

Melanopitta atricapilla, Bp. Consp. Vol. Anisod. p. 7.

Coloburis brevicauda, Cab. et Hein. ii. p. 5 .

Melanopitta sordida, 7iveed. P. Z. S. 1878 , 1. 949; id. Trans. Zool. Soc. ix. pp. 187, 250; Wardl. Ramsay, Trueedd, Mem. App. p. 657 .

Brachyurus sordidus, Sharpe, Trans. Linn. Soc. ser. 2. Zool. i. p. 331; Elliot. Ibis, 1870 , p. 419 (part.)

Pitta sordida, Sharpe, Ibis, 1884 , p. 321.

P. philippensis, Müll. \& Schl. Verh. Zool. Pitta, p. 19; Schl. Mus. P.-B. Pitta, p. $2(1863)$, et p. $5(1874)$.

P. macrorhyncha, Gray, Zool. Misc. p. 3 (1831).

P. leucoptera, Elliot. Proc. Ac. Sc. Phil. 1861, p. 133 (?) Hab. Luzón Negros, Camiguin, Siquijor, Marinduque, B.ssilan, Mindanao, Paragua, P. Princesa, Mindoru.

7. P. mudleri, $B p$. o $\rho$ N. v. Uar-uac

(M S. T )

Pitta atricapilla, Müll, \& Schl. Zool. Pr'fa, pp. 8, 19; Müll, Verh. Ethn. p. 405; Horsf. et. Moore, Cat. B. Mus. E. I. Co. 1. p. 399; Schl. Hand-l. Dierk. i. p. 254; id. Vög. Ned. Ind. Pitta p. 6, pl. ii. figs. 2, 3; id. Mus. P.-B. Pitta, p. 3 (1863), et p. 5 (s 844$) ;$ Pelz. Novara Reise, Vög. pp. 70, i6r; Gould, B. Asia, v. pl. 76 .

Brachyurus muelleri, Bp. Consp. i. p. 256 ; Mon. Pitt. pl. xxvi; Sharpy, P. Z. S. 1875 , p. 164; id. Ibis, 1877 , p. 10.

Pitta muelleri, Scl. P. Z. S. 1863, p. 215 ; Wallace, Ibis, 1864 , p. Io6; Wald $n$, Trans. Zool. Soc. viii. p. 62; Salvad. Ucc. Born. p. 240; Sharpe, Ib s, 1870 , p. 263; id. P. Z. S. 1879 , p. 346, et 1881 , p. 798 ; Nichols. Ibis, 1882, p. 63, et 1883 , p. 88; Guillemard, P. Z. S. 1885, P. 413; Sclat. Cat. B. M. 14. p. 439 (1888).

Coloburis muelleri, Cab. et Hein. Mus. Hein. ii. 1. 4.

Melanopitta muelleri, Bp. Consp. Vol. Anisod. p. 7; Walden. Ibis, 1872 , p. 374 .

Brachyurus sordidus, Elliot, Ibis, 1870 , p. 4 I 9 (part.)

l'itta malaccensis, Mull. \& Sichl. Verh. Zool. Pitta, p. 19. Hab. Paragua, P. Prinesa; Borneo, Sumatra. 
8. P. steerii, Sharh. ơ N. v. Uas-uac.

(M. S. T )

Brachyurus steerii, Sharpe, Vature, xiv. 1. $297(18 ; 6)$; id. Trans. linn. Soc. ser. 2. Zool. i. p. 329, t. xlix.

Melanopitta steerii, Treedt. P. $Z$. S. $18 ; 8$, p. 949.

Pitta sterii, Gould, B. Asia, v. pl. 74; Sil. Cat. B. M. 14. p. $4+2$ (1888).

Cyanopitta steerii, Gould. Mon. Pilla, pl. s.

Hab. Dumalon, Zamboanga, Mindanac, Luzón, Paragua, P. Princesa, Samar.

9. P. Iuzonica, $\sigma \circ$ N. v. I.ioco.

Hab. Luzon, Laguna, Calauan.

ro. P. guttata, ơ N. v. Uas-uac.

Hab. Paragua, P. Princesa.

II. P. maxima, o 0 N. v. Pitta gigantea.

(M S. 'T.)

Pitta maxima, Müll, et Schleg Verh. Zool. Putta, p. I4; Gray, Gen. B. i. p. 273; id. P. Z. S. $185 \mathrm{c}$, p. 351; Westerman, Bijdr. t. d. Dierk. i. p. 45, Pitta, pl. I; Schelg. Hant-l. t. d. Dierk. i. p. 253, Aves, pl. 2. fig. $2 \mathrm{r} ;$ id. Vog. Net. Int. Pita, p. 4 pl. i. fig. 4 ; id. Mus. P.-B. Pitta, p. 2 (1863), et p. 4 1874; id. Ned. Tijschr. Dierk. iii. p. I89; Walluce, Ibis, 1859 , p. I I 2, I860, p. 197, 1864, pp. 803, I05, I08; Bernst. Nat. Tijdschr. Dierk. ii. p. 373; Salvad. Ann. Mus. Civ. Genov. vii. p. I84; id. Orn. pap. ii. p. 378; Gould, B. New. Guin. pt. ii. pl. 5. Scl. Cat. B. M. 14, p. 419 (1888).

Brachyurus maximus, Bp. Consp. i. p. 253 ; Elliot, Mon. Pitt. pl. xii.; id. Ibis, 1870 , p. 4 I 3 .

Gigantipitta maxima, Bp. Consp. Vol. Anisod p. 7.

Leucopitta maxima, Gould, Mon. Pitta, pl. 7 . Hab. Gilolo.

12. P. nympba, Tem et Schl. $\sigma \mathrm{N}$ v. Iloco.

(M. S. 'T.)

Pitta nympha, Temm. et Schleg. Frun. Jap., Aves, Suppl. t. A; Wallace Ibis, 185.7 p. IO5; Swinhoc, P. Z. S. 1873, p. 730; Scl. Cat. B. M. 14. p. 425 (1888).

Brachyurus nympha, Bp.Cmsp. i. p. 25t; id. Consp. Vol. Anisod. p. 7; Elliut, Mon. Pitt. pl. viii.; id. Ibis, 1870 , p. 4I5. Pitta oreas, Swnh. Ib.s, $186_{4}$, p. 428 (Formosa); id. P. Z. S. 1865 , p. 678 1871. p. 375 ; Elliot, Ibis, 1878 , p. 408; Gould, B. Asia v. pl. 65; David et Oust. Ois. d. Chine, p. I53.

Brachyurus oreas, Elliot, Ib s, 1870, p. 4r 5 , pl. xiii. flg. I. Hab. Luzon; Fornosa, Borneo. 


\section{Fam. EURILÉMIDOS.}

\section{Gén. SARCOPHANOPS, Sharpe.}

1. 8. steerii, Sharpe. $\delta q$ N. v. Tueat.

(M. S T )

Eurylaemus steerii, Sharpe, Nature, vol. xiv. p. 297 (Aug. i $8 j$ th. Sharcophanops steerii, Sharpe, Trans. Linn. Soc. ser 2 , vol. i. Zool. p. 344, pl. 53; Gould, B. Asia, i. pl. 65; Tweddale, 'P. Z. S. 1878 , p. 110, et 1879 , p. 70, Wardlaw Ramsay, Twedd. Mem., App. p. 658; Sclat. Cat. B. M. 14. p. 462 (1888).

$H a b$. Dinagat, Basilan, Mindanao; Java, Borneo, Sumatra.

2. S. samarensis, Steere. N. v.

Hab. Samar.

2. Gèn. EURYLaimuS, Sclat.

3. E. javanicus, Hors. $\$ \&$ N. v. 'Jucat.

(M. S. 'T'

Eurylæmus javanicus, Horf. Trans Linn. Soc. xiii. p. r7o; 1d. Zool. Res. pl. 54; Vigors, Mem. Raffles, p. 65.3; Less. Man. d. Orn. i. p. 175, id. Trait. d. Orn. p. $26 \mathrm{I}$; Blyth, Cat. B. A. S. p. 195; id. B. Burm. p. 125; Bp. Consp. i. p. I68; Moore, Cat. B. Mus. E. I. Co. p. 116; id. P. Z. S. $18.5 \mathrm{t}$. p. 267; Cab. et Heine, Mus. Hein. ii. p. 121; Walden, Ibis, 1872 , p. 369; Gauld, B. Asia, i. pl. 57; Salvad, Ucc. Born. p. 107; Davison, Str. F. v. p. 456; Hume \& Dav. Str. F. vi. pp. 89, 489; Hume, Str. F. ii. p. 470, viii. pp. 50, 86; Birgham, Str. F. ix. p. I j7; Sharpe, Ibis, 1876 , p. 48, I877, 1. 22, et 1879 , p. 263; Ramsay P.Z. S. 1880 , p. 14; Nicholson, Ibis, 1882 , p. 63, et 1883 , p. 88; Oatas, B. Brit. Burm. i. p. 427; Guillemard, P. Z. S. 1885 , p. 407; Scl. Cat. B. M. 14. p. 463 (1881).

Eurylaimus horsfieldi, Tem. Pl. Col. г zo, г z I (1823); Size. Flycatch. p. 240. pl. 30; Wagl. Syst. Av. Euryl. esp. 3.

Platyrhynchos horsficldi, Vieill, Gal. Ois. i. p. 200, t. cxxv.

Javan tody, Lath. G. H. iv. p. 96.

Hab. ¿Paragua; Java, Borneo, Sumatra.

\section{Fam. DENDROCOLÁPTIDOS.}

1. Gén. SYNallaXis, Vieill.

i. S. erythrothorax, $S c l$. $\delta$ N. v.

(M. S. T.)

S. erythrothorax, Sclater. Cat. B. M. 15, p. 55 (1888).

Hab. América, Guatemala. 
2. Gén AUtomolds, Reick.

2 A. cervinigularis, $S c l$, $\mathrm{N}$ v.

(MI S. ' l.)

A, cervinigularis, Scl. Cat. B. M. 1.5, p. 91 (1888).

Hab, América, Guatemala.

3. Cién. DENDRORNIS, Eyton.

3. D. eburneirostris, (Less) $\delta \mathrm{N} \mathbf{v}$.

(M. S. T.)

Dryocopus eburneirostris, L.ess. Scho. cl. M. S. 187,3 .

Dendrornis eburneirostris, Scl. Cat. B. M. 15, p. 130 (1888). Hab. América, Guatemala.

4. D. erythropygia, $S c l$. \& $\mathrm{N}$.

D. erythropygia, Scl. Cat. B. M. 15, p. 131 (1888).

Hab, A mérica, Guatemala.

Fam. FORMICÁRIDOS.

1. Gén. THAMNOl'Hilus, Vikiti.

1. Th. doliatus, (Lin.) Q 0 N. v.

(M. S T)

Lanius doliatus, Lin. S. N. i. p. 136; Lin. S. N. i. p. 309.

Th. doliatus, Scl. Cat. B. M. 15, p. 207 (1888).

Hab. América, Venezuela.

2. Gén. RhAMphOCGNUS, Vieilit

2. Rh. ruflventris, $B p . \delta \mathrm{N} . \mathrm{v}$.

(M. S. T.)

Scolopacinus rufiventris, $B p . P . Z$. S. 1837, p. I19.

Rh. rufiventris, Scl. Cat. B. M. 15, p. 26 I (1888.)

Hab. América, Guatemala.

3. Gén. CERComaCra, Sci.

3 C. tyrannina, $S c l$. o $\circ$ N. v.

(M. S. T.)

Cercomacra tyrannina, Sclater, Cat. B. M. 15, p. 265 (1888).

Hab. Méjico, Vera Paz. 


\section{Orden $44^{\circ}$ PICARIOS.}

\section{Suborden: UPUPOS.}

\section{Fam. UrÚPIDOS.}

1. Gén LPUPA. Lis.

1. U. epops, (Lin.) ơ N. v Abubilla.

Ia Huppe on Puput, Briss. Orn. ii. p. +55 . lam. 43 ( 760() . Upupa epops, Lin. Syst. Nat. i. p. I83 (г766); Gin. S. N. i. p. 466 (1788); Bp. Consp. I p. 88 (1850); Osbert, Cat. B. M. 16. p. $5(1892)$.

Ja huppe, Daub. Pl. Enl. vi. p. 52.

Hab. Europa, España, Africa, India, Japón.

\section{Suborden: TROQUILOS.}

\section{Fam. TROQUÍLIDOS.}

I. Gén. EUL $\Lambda$ MPIS, Bore.

1. E. jugularis, (Lin.) o N. v. El granate.

Le grenat, Buff. Hist. Nat. Ois. vi. p. $48 ;$ Aut. et Vieill. Ois. Dor. i. p. 2o, pl. 4.

Colibri violet de Cayenne, Buff. Pl. Eul. 6oo. f. 2.

Trochilus jugularis, Lin. Syst. Nat. i. p. r 9o; Gn. S. N. i. p. 479.

Eulampis jugularis, Reich. Anfz de Col. p. I ; Bp. Consp. i. p. 72 ; Osbert. Cat. B. M. 16, p. 102 (1893).

Hab. Guadalupe, Dominica, Martinica.

2. Gén. FLORISUGA, BP.

2. F. mellivora, (Lin.) $\sigma 0$ N. v. Colibri de garganta azul.

(M. S. T.)

'Trochilus mellivorus, Lin. S. $N$. i. p. r93; Gn. S. N. i. p. 499, Buf. Pl. Enl. 640, f. 3; Osbert Cat. B. M. 16. p. 329, 1893 .

Hab. América, Guatemala.

3. Gén. AMAZII.IA, Reich.

3. A. riefferi, (Bourc.) $\& \hat{q}$ N. v. Colibri.

(M. S. T.)

'Trochilus riefferi, Bourc. Ann. Soc. Phys et Nat. Lyon: Osbert Ca!. B. M. 16. 1893, p. 216.

Hab. América, Cuatemala. 


\title{
Suborden: CORAGIOS.
}

\author{
Fam. CIISÉLIDOS.
}

Subfam. CHETLRINOS.

(ién CHOElURi, STEPH.

I. Ch. gigantea, (Tim.) ơ $\mathrm{N}$ v. Balintagus.

(MI S. T.)

(ypselus giganteus, Tem. Pl. Col. $364(1825)$ Java.

Acanthylis caudacuta, part, Blyth, Cat. B. Mus. As. Soc. p. It $(18+9)$.

A. giganteus, Bp. Consp. i. p. 64 (1850).

('hoetura gigantea, Scl. P. Z. S. 1805, p. 608; id. J. f. O. 1867 , p. 129; Hume, Str. F. iv. p. 223 (1876), viii. p. ti. (1879); Sharpe, P. Z. S. 1879, p. 335; id. Ibis, 1888 ', p. 198; Hartert, Cat. B. M. I6. p. 475 (1892).

Hirundinapus giganteus, Salv. Uc. Born. P. 124 (1874); Oxtes, B. Burmah, ii. p. 5 (1883); Büttik. Notes Loyden Mus. ix. 1. 40 1887; Hart. J. f. O. 1889 , p. 400; Sharpe, Ibis, 1890 , 1). 24 ; Steere, List. B. et Mamm. Philippin. p. 12 (18g0).

Hab. Paragua, P. Princess, Inagaua; B.rneo, Singapore, Malaca.

3. COLlocalia, Gray.

2 Collocalia lowi. (Sharpi). o o N. v. Salangana. (M. S T.)

(ypselus lowi, Sharpe, P. Z. S. 1870, p. 333 .

(y. labuanensis, Ibis, 1879 , p. 116 .

Collocallia lowi, Hartert. Cat. B. M. 16. p. 498 (1892).

Hab. Paragua, P. Princesa; Borneo, Sumatra.

3 C fuciphaga, (Thumh), \&? N. v. Salangana.

(M. s. '1.)

Hirundo fuciphaga, Thumb. Act. Holm. xxxiii. p. 15I, pl. 4. I772; Stepk. Gen. Zool. x. p. III (1817).

lisculent swallow, Lath. Gen. Syn. Suppl. ii. p. 257, pl. I35 $(1802)$ :

Hirundo esculenta, (nec Lin.) Horsf. Tr. Lin. Soc. xiii. 1. 142; Raffl. ibid. p. 315 (1822).

Cypselus fuciphaga, S. Mïll. Verh. Land-en. Volkenk, p. $45^{6}$ (1839-44).

Ilirundo unicolor, Jord. Madr. Journ. 1840, p. $23^{8}$.

(ypselus concolor, Blyth, Journ. As. Soc. Bens. xi. p. 886 (1842).

(y. unicolor, Jerd. Journ. As. Soc. Beng. xiii. pt. i. p. 173, pt. ii. p. It+ (I844).

(Sllocalia unicolor, Blyth, Journ. As. Soc. Beng. xix. pp. 209, 212 (1845); Hitne. Stray Fuath. iv. p. 37+ (1876); Scully ibid. viii. p. 235 (1979); Butle, Stray Fexth. ix. p. 380 (1850); 
Davison, Stray Feath. x. p. $3+4$ (1832); Oxtes, id. H:ume Nests. et Egg Int. B. iii. p. 28 (189.)).

(. nidifica, Gray, Gen. B. i. p. $55(18+3)$; Blyth, Cat. B. M. As. Soc. p. 86 (1849); Bp. Consp. i. p. $3+3$ (1850); Horsf. et Moore. Cat. B. Mus. E. I. Comp. i. p. 98 (Ió54); id. Cat. B. New Guinea pp. 17, 54; Bernst. J. f. O. 1859, p. I18; Jerd. B. India. p. $182(1862)$; Rosenb. N.t. Tij.tschr. Veder!. Ind. xxv. p. $234(1863) ;$ Scl. P. Z. S. 1863, p. 21 2; id. J. O. 1864 , p. I 20; Finsch, Neu-Guinea, p. I62 (1865); Peli. Reis. Nozara, Vög. p. 39, (1865); Gray, Ann. \& Mag. Nat. Hist. 1866 , xvii. p. 118; id. Hand-l. i. p. 65, 746 (1869); l.egoe, Ibis, 1874, p. I 3; Rey, J. f. O. 1875, p. 285; Brooks, Stray Feath. iii. p. 232 (1875); Green, Jouril. Physiology, 1895 , 1. 40; Varigny, Nature, 1885, p. 186; B.att.k?f. P. Z. S. 1885 , p. 6ro; Pryer, Zoologist, 1885 , p. 296; Mitrray, $t . c$. 1. 147 .

Iemiprocne salangana, Streubel, Isis, 1848, p. 368.

(ollocalia fuciphaga, $B p$. Compt. Rind. xli. p. 977 (1 855$)$ i id. Rev. et Mag. Zool. 1855, p. 581 ; Wallace, P. Z. S. 185,3 , lp. 384,484 , Scl. P. Z. S. 1855 , p. 616; id. J. f. O. 186 ; p. I 39; Holdsw. P. Z. S. 1872 , p. 420; Wald. Trans. Zool. Soc. viii. p. 46 (1872); Salvad. Ucc. Bornes, p. I 20)1874); id. Ann. Mus. Civ. 1879, p. 6+7; id. Orn. Papuas. i. p. $5+t$ (1880); id. Agg. Orn. Pap. i. p. 63 (1889); Tristr. Ib.s, 1880 , 1) 246; Sharpe, Journ. Linn. Soc., Zool. xvi. p. 430 (183.); 1. B. Meyer, Abb.lal. Vogelsk. pl. 46. fis. 2 (1883); id. Zeitschr. ges. Orn. 1884, p. 278; W. Blas. f. O. 1884, p. 217; Kutter, J. f. O. 1885, p. 346; Finsch. \& Myer, Zeitschr. ges. Orn. 1885 , p. I 4 ; Hartert, J. f. O. 1839; Tristr. 1bis, 1989 ; p. 556; Sharpe, Ibis, 1890, p. 23; Hartert, Cat. 13. Mus. 16, i892, p. 498.

llirundo vanikorensis, Quyy et Gaim. Voy. Astrolabe, Zsol. i. 1. 206, pl. xii. fig. $3(1830)$.

(Cotyle vanicorensis, Boie, Isis, 1844, p. I70.

collocalia vanicorensis, Hartl. \& Finsch, P. Z. S. 1868 , pp. ${ }_{4}$ 116 ; Finsch \& Harll. Arn. Centralpol. p. 47, part. (1 $\$ 67) ;$ Finsck, Journ. Mus. Godeffr. viii. p. I 5 (1875); id. ib d. xii. p. 23 (1876); Layard, P. Z. S. $187^{5}$, p. 53; id. ibs, $187^{6}$, 1. 391; Finsch, P. Z. S. 1877 , P. 778; id. J. f. O. 1880 , p. $285 ;$ id. P. Z. S. 1880,575 ; 2 . J. f. O. 1880 , fi. 298 ; id. Ibis, $1881, \mathrm{pp}$. 105, 108, I. I 5,536 .

(ypselus inquietus, Kittl. Denkz. Reise, ii. p. 26 (1858); Finsch, Ibis, 1881 , p. 105.

Collocalia francica, Tweedd. P. Z. S. 1878 , p. 283 ; Steere. List. C. sp. inc., Scl. P. Z. S. 1879 , p. 447 .

C. linchi, Tristr. Ibis, $1880, \mathrm{pp} .223,298$.

(.) cinera, Lxyard, Ibis, 1881, pp. 223, 298; id. Ibis, $1882,1.503$. Hab. Paragua, Ihuaging; Borneo, Sumatra.

4. C. troglodytes. Gray. $3 \&$ N. v. Salangana. (M. S. 'I')

C. troglodytes, Gray, Gen. B. pl. I9 (18+5); id. Hand-l. i. p. 65 (1869); Wall. P. Z. S. 1853 , p. 384 ; Martens, J. F. O. 1856 , 1. I8; Bps. Compt. Rent. xli. p: 977 ; Pela. Reise Novara, 
Vog. p. 40 (1865); I'alden, Trans. Zool. Soc. ix. p. 158 (8175); 7iveedd. P. Z. S. 1877, p. 823; Sharpe, Trans. Liun. ioc. i. p. 320 (1877); Hume, Stray', Feath. viii. p. 47 (1879); Sharpe, Ibis, 1888, p. 198; Whilely, Ibis, 1800, p. 46; Steere, List B. \& Mamm. Philippines, p. 12 (1800); H.z\%. Cat. B. M. 16, p. 507 (1892).

Hab. Paragua, P. Princesa, Mindanao, Placer.

5 C marginata Sali: $0^{\circ} \rho \mathrm{N}$ v. Balinsayao.

(M. S. T.)

Collocalia fuciphaga. Treedd. P. Z. S. 1878, p. 429.

( marginata, Salvad. Atti R. Ac. Sc. Tor. xvii. p. $44^{8}$ (1882). Reichen \& Schalwo J. f. O. 1884, p. 382; Hartert, Cat. $K$. M. 16,1892, p. 508 .

Collocalia cebuensis, Kutter, J. F. O. 1882, p. 171; Reichen to Schalow, J. F. O. $1884, \mathrm{p} .3^{82}$.

Hab. Cebú, Samar, Paranas, ¿Luzón: :Manila?

6. C. linchi, If. et Moore. of N. v. Balinsayao.

(M. S T.)

Hirundo fuciphaga, Horsf. (nec Thunb.) Trans, Linn. Soc. xiii. p. I 43 (I82I).

Collocalia fuciphaga, G. R. Gray, Gen. B. i. p. 55 (1845); Blyth. Journ. As. Soc. Beng. xiv. p. $548(18+5)$, xv. p. 32 (184(1); id. Cat. B. Mus. As. Soc. Beng. p. 86 (1849); Walden, Ib/s. 1874, p. I 33 .

(ollocalia linchi, Horsf. \& Moore, Cat. B. Mius. C. Y. Comp. i. p. 100 ( 1845$)$; Bp. Compt. Rend. xli. p. 548 ( 1855); Berust. J. F. O. 1859 , p. I19; Wall. P. Z. S. 1863, p. 548; Scl. P. Z. S. 1865 , p. 616; Pelz. Reis. Novara. Vög. p. 3" pl. ii. fig. 2, \& pl. vi. fig. 2 (1865); Gray, Ann. \& Mag. Nat. Hist. 1866 , xvii. p. I I ; Gray, Hand-l. i. p. 65 (1869); Hartert. Cat. B. Mus 16, 1892 p. 508; Ball, Stray Feath. i. 1. 55, (1873); Hume, ibid. ii. p. I 57 (1874), vi. p. 48 (1878), viii. 0.47 (1879); Salvad. Ucc. Borneo, p. I2I (1874); Sharpe, Ibis, 1879 , p. 250; Tristr. Ibis, 1879, p. 438; Nicholson, Ibis, 1881, p. 143; Kelham, Ibis, 1881, p. 374; Oates, $B$. Burmah, ii. p. Io (1883); Vordem. Nat. Tydschr. Nederl. Ind. p. 193 (1883); Kutter. J. F. O. 1883 , p. 302; id. J. F. O. 1885 , p. 344; W. Blas, J. F. O. 1884 , p. 227; Grabowsky, Urnis, 1885 , p. I57; Sharpe, Ibis, 189o, p. 23; Oates, ed. Hum's Nests. \& Eggs. Ind. B. iii. p. 33 (1891); Hartert. Cat. B. $M .16 .1892$, p. 508 .

C. atfinis, Tytler \& Beavan, Ibis, 1867 , p. 38 ; Blyth, Ibis, 1868 , p. I 31 ; Walden, Ibis, 1873 , p. 302; Salvad. Ibis, 1880 , p. 130.

Hab. Samar, Borongan; Borneo, Java. 


\section{Subfam. MACROPTERIGINOS.}

1. Gén. MACROPTERYX, Siwaiss

7. M. comata, $\left(T_{e m}\right) O^{\prime} \rho$ N. v. Layanliyan.

(M. S. T.)

(ypsclus comatus, Tenm. Pl. Col. $268(1824)$.

Dentrochelidon comatus, Boie, Isis, 1847 p. I66; Bp. Consp. i. 1). 66 (185()); Gould, B. Asia, i. 1). 25 (1859). (ab. . He ne, Mus. Hein. iii. p. 82 (1860); Scl. P. Z. S. 1863,1 .. hi7; id. J. f. O. 1867 , p. I41; Gray, Hand-l. i. 65 (1869); Sialvad. Ucc. Borneo, p. I 23 (1874); Hume, Stray Feath. iii. p. 318 (1875); id. ibid. viii. p. 47 (1879); Sharpe, Ibis, 1878 , 1. 414 ; id. ibid. 1879 , p. 250; Hume \& Davison, Stray Feath. i. p. 5 I. (1878); Sharpe, P. Z. S. 1879 , p. 334; Kellam, Ibis, 1881 ; p. 376 .

Macropteryx comatus, Stwains. Class. B. ii. p. 340 ( 1837$)$; Blyth, (at. B. Mus. As. Soc. p. $87(1849)$; Walden, Trans. Żon. Soc. ix. p. 158 (1875); Sharpe, Ibis, 1876 , p. 53; id. Trans. linn. Soc. ser. 2, i. p. 319 (1877); Tweedd. P. Z. S. 1877 . 1. 823; Salz. Cat. Strickl. Coll. p. 373 (1882); Oates, B. Burmah, ii. p. I4 (1883); Hartert, J. t. O. 1889 , p. 40I; id. J. f. O. 1891, 1. 295; id. Cat. B. M. 16.1892, p. 517.

Hab. Luzón, Manila, Laguna, Los Biños, Calamba, Cebú, Negros, Mindanao, Placer; Java?

\section{Fam. CAPRIMÚL.GIDOS.}

Subfam. CAPRIMULGiNOS.

I Gén. CAPRimulgus, Iis.

I. Caprimulgus europæus, Lin \& N. v. Papavientos. (M. S. T)

Caprimulgus europeus, Linn. Syst. Nat. el. x. p. 193 (1758); ed. 12, i. P. 346 (1866); Gm. Syst. Nat. ii. p. 1027 1 788 ; Hart. Cat. B. M. 16. p. 526 (1892).

La téte de Chévre cu Crapaud volant, Briss. Orn. ii. p. 47 (', pl. $4+(1 ; 60)$.

L'lingoulevent, Buf. Hist. Nat. Ois. vi. p 512 (1779),

Crapaud volant, Daubent. Pl. Enl. i 93 ( 1783 ).

Hirundo caprimulgus, Tunst. Orn. Brit. p. 2 (гі7г); Pall. Zoogr. Rosso-Asiat. i. p. 542 (I8I I).

(aprimulgus punctatus, Meyer \& Wolf. Taschenb. p. 284 (1810); Brehm, Vög. Deuntschl. p. 1 30 (1831); id. Vagelf. p. 44 (1855).

C. vulgaris, Vieill. Faune Franc. i. p. I40, "pl. 61. fig. 2 (1828); Ronx, Orn. Provenc. p. 240, pl. I47 (1825).

(. maculatus, Brehm, Vög. Deutsckl. p. I31 (1831); Vogslf. 1. $4+(1855)$.

Nictichelidon europæus maculatus, punctatus, punctorum, foliorum, y peregrinus, A. E. Bremh. Verz Samml. C. L. Brehm, p. 3 (1866); teste Dresser, I. c. 
Caprimulgus smithii, Bp. Consp. i. p. 50 (1850); Cass. (ait. Capr. Ac. Phil. p. 2 (1851); Harll. Ibis, 1002 , p. 143 Ger:ney, Ibis, 18 sos, p. 151 Gray, Handt i. p. 56 (1869): Cab. J.J. f. O. $18 ; 8$. p. 236; Reichen. J. f. O. id8 8 , p. 61.

C. capensis, Vers. M. S. leste Cat. Capr. Atc. Phil. p. 2 (1851). Hab. Europa, España, Africa, China, :Luzón, Cagayan?

2 C macrurus, Hor $\sigma^{\circ} . \mathrm{N}$. v. Paturtur.

(M. S. T)

Javan goatsucker, Lath. Gen. H. B. vii. p. 346 (1823).

C. macrourus, Horsf. Tr. Lin. Soc. xiii. p. It2 (1821); Jercl. Ill. Ind. Orn. n." $2+(18+7) ; B l y t h$, Cat. B. M. As. Soc. p. si (1849); id. Ibis, 1860, p. 341, Bp. Consp. i. p. Oo, (1850); Horsf. et Moore, Cat. B. M. E. I. Co. i. p. I I 2 (1854); Gral', P. Z. S. 1850 , p. I 54; Cab. et Helne, Mr. Hein. iii. p. Xis (1860); Jerd. B. Ind. p. I95 (1862); Wall. P. Z. S. 180, Gould, B. Austr. ii. pl. ix.; id. Handb. B. Austr. i. p. i(u), (1865); Koening-Warth. J. f. O. 1868, p. 378; Gray, Hand-l. i. p. 57 (1869); Beavan. Ibis, 1869, p. 406; Walden, Ibis, 1874 , p. I 31 ; Hume, Stray F. ii. p. 469 (1874); id. Stray F. iii. p. 46 (1875); xiii. p. 48 (1879); xi. p. 39 (1888); Sali. Ucc. Born. p. I $77,(1874)$; id. Orn. Papuas. i. p. 528 (1880); A. B. Meyer, Sitzb. K. Ak. Wissensch. Wien. 1874 , pp. (",

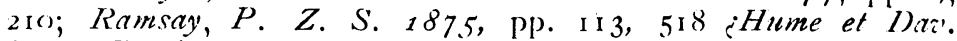
Stray F. vi. p. 58, (1 878$)$; Cripps, Stray F. vii. p. $25^{8}(1878)$; Sharpe, Journ. Lin. Soc. xiii. p. 493 (1878); id. Ibis, $187 \%$, p. 250; id. P. Z. S. 1879 , p. 332; id. Ibis, 1888, p. 298, I 890 , p. 22. Bingh. Stray, F. ix. p. i 50 (1880); Layard, Ibis, 1880, p. 298; Kelam, Ibis, 1881, p. 376; Aug. Müll. J.f. O. $1882,1.403$; Vorderm. Nat. Tijdschr. Nederl. Indie, 1883, p. 57 ; Oxtes, B. Burm, ii. p. 20, $188.3 ;$ id. ed. Humes Nests. et E.ggs. Ind. B. iii. p. 45. (1890); A. B. Meyer, Zeitschr. ges. Orn. 1884 , p. 197; Hart. J. f. O. 1889, p. 424; Witch. Ibis, $1890, \mathrm{p} 46 ;$ Steere, List. B. et Mamm. Exp. Philipp. p. I 2 ; Hart. Cat. B. M. 16 p. 537 ; (1892).

$\therefore$ schlegelii, Gray, Hand-l. i. p. 57, I869; Aru, New. Guin.

C. Salvadorii, Sharpe, P. Z. S. 1875, p. 99, pl. xxii. f. I; id. Ibis, 1877 , p. 4 .

Hab. Paragua, P. Princesa, Inagauan; Australia, Borneo, Java, Sumatra.

3. C. afỉnis, Horsf $t$ N. v. Paturtur.

(M. S ' ' ' )

C. affinis, Horsf Tr. Lin. Soc. xiii., I $82 \mathrm{I}$ p. I42; G. R. Gray, Gen. B. i. 1847 , p. 48 ; Blyth, Ciut. B. M. As. Soc. 1849 p. $8_{4}$; Bp. Consp. i. p. 60 (1850); Cass. Cat. Capr. M. Philud. esp. I7 (185I); Horsf. et Moore, Cat. B. M. E. 1 Comp. i. p. I 44 (1854); Scl. P. Z. S. 1863 , p. 312; Gray, Hand-l. i. p. 57" (1869); Wald. Tr. Zool. Sioc. viii. I872, p. I I 4; Salv. Ucc. Borneo, 1874 p. I 5 ; Vorderm. Nat. Tijds. Ned. Int. 1.883, p. 54; Hart., Ibis, 1892 , p. 280; $2 d$. Cat. B. M. 16. p. 549 (I 892).

C. curopæus, Raf, Tr. Linl. Sof. xiii. p. 315, (1822). 
(huppa goatsucker, lath. Gen. H. B. vli. p. 359 (1823).

lingoulevent des roscaux, $H$. et Jacq. Voy. Pole Ind. pl. 21 fig. 28 ( 1846$)$.

('. bisignatus, Boie, M. L. teste Cass. Cat. Capr. M. Phil, (1851); Koenig-Warth. J. f. O. 1868 p. 373.

( . arundinaceus, Bp. Consp, i, p. 6o (1850); Jacq. et Puch. Vov au Pole Ind., Ois. p. $93(1853)$; Hartl. J. f. O. 1854, p. I64; Wald. Tr. Zool. Soc. viii. p. 1151872 ; Salv. Ucc. Borneo, 1874, p. 116.

(' faberi. Meyer, Sitzeng. Ges, Isis, 1884 , i. p. 20.

Hab. Paragua, P. Princesa, Joló, Samar, Borongan; Borneo, ( élebes, Java, Sumatra,

4. C. manillensis, Gray. N. v. Candarapa.

(M. S. T.)

( manillensis, G. R. Gray, List. Fissirostres Brit. Mus. p. TA 11. ${ }^{\circ}$ I, «Manilla», descr. nulla! (1848); id. Hand-l. p. 57, n." 637 (1869).

$\because$ (. macrurus, Martens, J. f. 0,1866, p. 19.

('. manillensis, Walden, Trans. Zool. Soc. ix. pp. 160, 410 (1875); Trueedd. P. Z. S. 1878 , p. 635; id. P. Z. S. 1879 , p. 60; Whiteh. Ibis; 1890 , p. 46; Steere, List B. \& Mamm. Exped. Philipp. p. I 2 (1890); Hart. Cat. B. M. 16, p. 544 (1892). $H a b$. Luzon, Manila, Nayotas, Mindanao, Zamboanga, Basilan; Célebes.

5. C. griseatus, Gray. N. v. Indarapa.

(M. S. T.)

(. griseatus, G. R. Gray, Hand-l. i, p, 57, n. 629; Walden, Trans. Zool. Soc, ix. p. 159 (1875); Tweedd. P. Z. S. 2877 , p. 691 Mutter, J. f. $O .{ }_{1} 882$, p. 175 ; Hart. Cat. B. M. 16. p. 550, lam. xi. ( 1892 ).

Hab. Luzon, S. Mateo, Bulacan, Lolomboy, Mindoro, Baco.

6. C. phalæna. Hart. ¿ N. v.

(M. S. T.)

( . phalæna, Hartl. \& Finsch, P. Z. S. 1872, p. 91; Finsch, Journ. Mus. Godeffr. 1875 , p. I3, pl. ii. figs. 1, 2; Hartert. Cat. B. M. 46. p. $545(1892)$.

Hab. Carolinas, Palaos.

\section{Gén. LYNCORNIS, Gould.}

7. I. macrotis, o 0 N. v. Dapolac, Sacsaculap.

(M. S. 实)

(aprimulgus macrotis, Vig. P. Z. S. 1838, p. 97.

Lycornis macretis, Gould, Icon. Av. pt. 2 (1883); Bp. Consp. i. p. 62 (1850); Gray, Hand-l. i. p. 62 (1869). Yrecedd. Zool. Soc. ix. p. 159 (i877); Wardl. Ramsay, Ibis, 1886 , p. I 57; Harter. Cat. B. M. 16,1892 p. 605.

Hab. Luzon, Manila, Cagayan, Ilocos, Mindoro, Baco. 
8. I mindanensis, Tiredt. \& ? N. v.

(M. S. T.)

Lyncornis mindanensis, 7iw. P. Z. S. $1 s^{2} 78^{8}$, p. 944; Hart. Cat. B. M. 16. p, 605 (1892).

Hab. Mindanao, Zambranga.

9. I temmincki, \& \& Goull. N. v. Parturtur.

(M. S. T.)

L. temminckii, Gould, Icon. Av. pt, $2\left({ }_{18} 8\right)$; Bp. Consp. i. p. 62 (1850); Gray, Handel, i. p. 62 (1869); Wald. 16is, 1872, p. 369; Salvad. Ucc. Borneo, p. 114 (1874); Tweedd. Ibis, 1877 , p. 298; Hume, Stray F. viii, p. 48; Nicholson, Ibis, 1882, P. 58; Grabowsky, Ornis, 1885 , p. 187; Everelt, Journ Straits Branch Asiat Soc. 1889 . Hart. Cat. B. M. 16. p. 606 (1892). Hab. Paragua, P. Princesa; Borneo, Java.

10. I. paraguanensis, $0.0 \mathrm{~N}$. v.

(M. S. T.)

Hab. Paragua, Inagaua.

Fam. PODARGIDOS.

1 Gén batkachostomls, Govip.

I. Bat auritus, Gr. $\delta \mathrm{N}$ v. Ijao. (M. $S \mathrm{~T}$.)

Podargus auritus, Gray in Griffith, id. Cuver's An. Kingd. ii. p. II4, cum tab. (ex Vig. M. S.) (1829); Vig. App. Mem. Raffí p. 658 .

Batrachostomus auritus, Gould, Icon. Av. ii. I4, pl. vii. (1838); Gray, List. Spec. B. Brit. Mus. ii. pt. i. p. 3 (1848); Bp. Consp. i. p. 57 (1850); Cass. Cat. Caprim. Mus. Phlad. p. \& (1851); Horsf. \& Moore. Cat. B. Mus. E. I. Co. i. p. 387 (1854); Cab. et Heine Mus. Hein. ii. p. 123, (1860); Gray, Hand-l. i. p. 54 (1869); Sharp:, P. Z. S. 1875, p. 99, 187y, p. 332; Tweedd. Ibis, 1877, p. 388 ; id. P. Z. S. 1867, p. 434; Sharpe, Ibis, 1879 , p. 249; Hume, Stray F. viii. p. 48; Hartl. Ciat. M. 16. 1892, p. 637 .

Bombycistomas fullertonii, Hay, Jouru. A. S. Beng. 18+1, p. 574, zoodcut.

Pordargus fullertoni, Blyth, Journ. A. S. Beng. 1842, p. 798. Hab. P. Princesa, Paragua; Borneo, Sumatra.
2. Bat septimus, Tw. $\sigma \circ$ N. v. Paturtur.
(M. S. T)

Batr. septimus, Tw. P. Z. S. 1877 . p. 542, 1878 , p. 944; id. Voy. Challengir; p. I3, pl. ii. 1880 ; Harter. Cat. B. $M$. 16,1891, p. 638 .

Hab. Mindanao, Zamboanga, Paragua, P. Princesa. 
3. Bt. javan€nsis, Horsf. \& $\mathrm{N}$ v. Ijao.

(M. S. T.)

Pulargus javanensis, Horsf. Trans. Linn. Soc. xiii. p. I 4 I (I821); Bart. Cat. B. M. 16. 1892, p. 640.

Pulargus cornutus, Temm. Pl. Cal. p. i 59; Schleg.J.f. O. 1856 , p. 460; id. Nederl. Tydschr. Dierk iii. p. 3+1, part. (1866); P. javanensis, Horsf. Zool. Res. Javx, pl. 37 (1824); Blyth. Cat. 13. Mus. As. Soc. Beng. p. 8I (1 849); Schleg. Handl. Dierk. i. p. $224(1857)$.

Battrachostomus javanensis, Gray, Gin. B. i. p. 45 (1846); id. List. B. Brtt. Mus. ii. pt. i. p. $3($ I $8+8)$; id. Handl. i. p. $5+$ ( 1869$)$; Bp. Consp. i. p. 57 (1850); id. Cass. Cat. Caprim. Mils. Philad. p. 7 (1851); Horsf. \& Moore, Cat. B. Mus. E. I. Co. i. p. 41 2 (1854); Cab. \& Hein'; Mus. Hein. ii. p. I 23 (1860); Bernst. J. f. O. 1850 , p. 428 ; Scl. P. Z. S. 1863 , 1. 21 ; Salvad. Ucc. Bormeo, P. 102 (1874); Sharpe, P. Z. S. 1875, p. 99,1879 , p. 332; Twoedd. Ibis, 1877, p. $3^{89}$; 1d. P. Z. S. 1877 , p. +35; Hume, Stray Feath. vii. P. I+3, note (1878; Hart. Ciat. B3. M. 16 p. 640 (1892).

Batrachostomus cornutus, Gray, Gen. B. i p. 45 (i $8+6)$; Pels. Reise Navara, Vog. p 34; Tweedd. Ibis, 1877, pp, 293, 388 ; id P. Z. S. 1877 , p. 432 , pl. xlvi; $W$. Blas. J f. O. $188+$, 1) 217 ; Kut'er, J.f O. 1885 , p. $3+1$; pl. ix. Gr.zbowiky, $O=$ nis, 1855 , p. I57; Sharpe Ibis, 1888 , p ig8; Which Ibis, $1890, \mathrm{p} .46$.

13 stellatus, (?) Büggem. Abh. naturw. Ver. Brem n, v. p, 35\% $(1876)$.

B. alspersus, Bruggem. Ann. \& Mag. Nat. Hist. 1877 , xx. 1. 17\$; id. Abh naturw. Ver. Bremen, v. p. 528.

Hab. Paraya, P Princesa; Sunatra, B rneo

\section{Fam. CORÁCIDOS.}

I. Gén. EURYSYOMUS, VIELLL.

I. E. orientalis, (Lin). $\sigma \circ \mathrm{N}$. v. Uac.uacan. Sagagsac.

(M. S. T.)

Le Bollier des Indes, Briss. Orn. ii. p. 75, pl. 7. fig. 2 ( 760 ); D.ubent, Pl. Enl. iii. pl. 6I9; Mintb. Nat. Hist. Ois. iii. p. I 47 (I 775 ).

Coracias orientalis, Linn. S. N. i. p. I59 (1766); Gm. S. N. i. p. 379 (1788); Raffl. Trails. Linn. Soc. xiii. p. 302 (1820). Oriental roller, Lath. Gen. Syn. i. p. 4I I (I78I).

Le rolle á gorge bleue, Levaill. Ois. de Parad. Rolliers, p. I 83 , pl. 36 (1806).

Eurystomus orientalis, Steph. in Shaws Gen. Zool. vii. p. 403 (1809); Swains. An. in Menag. 326 (1837); Gray, Gen. B. i. p. 62 (1845); Bp. Consp. i. p. I68 (1850); Reichenb. Handb. Merop. p. 54, Taf. 437. figs. 3195, 3196, 3167 (1852); Moore, P. Z. S. 1854 , p. 267; Horsf. \& Moore, Cat. B. Mus. E. I. Co. i. p. I21 (1854); Cab. \& Heine, Mus. Hein. Th. ii. p. 120 (185)); P.la. R.:'S. Novara, Vög. p. 42 (1865); Schl. 
Mus. Pay's.Bas, Coraces, p. 139 (1867, pt); Gray, Hasd-i. B. i. p 76, n." 906 (1809) Blanford, Ibis, 1870, p. 465: Elliot. Ibis, 1871, p. $20+(\mathrm{pt})$; Walden \& Lajard, Ibis, $18 ; 2$, p. I(X); Walden. Ibis, $1 \$^{3} 7.3$, p. Io8; Ball, Str. F. i. p. $5 i$ (1873); Hume, Str. I. ii. p. itot (1874); Valden, Trans. 弚. S. ix. p. 152 (1875); Saluad. Ucc. Born. p. 105 (187t); Blwtl: \& Wald. B. Burn. p. 72 (1875); Sharpe, Ibis, I $877,1.7 ;$ Ilardlaw Ramsay, t. c. p. 457; Hume, \& Inavison, Str. I. ri.

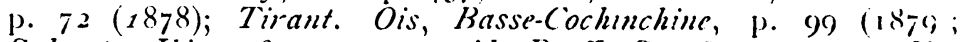
Saharpe, Ibis, 1879 , p. 24(); id. P. Z. S. 1879 , p. 332; Hume, Str. F. p. 85 (1879); Bingham, t. c. p. 193. id. op. cit. ix. p. 153 (1880); Saliad. Orn. Papuasia i. p. 508 (1880)!; Kelham, Ibis, 1881 , p. 373; Sharpe, P. Z. S. $188^{\prime} 1$, p. 793; Nichols. Ibis, 1882 , p. 67; Oa'es Handb. B. Brit. Burm. ii. p. 70 (1883) Suellem, in Veth's Midden-Sumatra, Vïg. p. 3(1885); Büttik. Notes Leyden Mus. ix. p. +1 (1887) Huni. Str. F. xi. p. 43 (1888); Sharpe, Ibis, 1888 , p. I97; Blasius, Ornis, 1888 , p. 579; Sharpe, Ibis, 1889 , p. 21 ; Whitehead, t, c. p. 45; Salvad. Agg. Orn. Papuasia, p. 60.(1889) Shtrpe, Ibis, 1890 , p. 21 ; id. P. Z. S. 1890 , p. 550; Stuere, Linst. B. \& Mamm. Philipp. p. 9. (1890); Oates, cd. Humc's Nets \& Eggs Ind. B. iii. p. 57 (1890, pt.); Sharpe, Cat. B. M. 17 , 1892, p. 33, lam. 2, fig. I.

(o) aris orientali:, Cuv. Regne Anim. i, p. 4or (18I7); Horsf. Trans. Lialn. Soc. xiii. P. 162 (1820).

Eurystomus cyanicollis, Vieill. t. c. p 425 (1819); id. Gal. Ois. i. p. 176, pl. iii. (1825); Lp. Consp. i. p. 188 (1850).

E. fuscicapillus, Vieill. N. Dict. d'Hist. Nat. xxix. p. $426(1810)$.

Colaris cyanicollis, Wagl. Syst. Av. Colaris, sp. 4. (1827).

(. garula, (nec L.), Guérin-Ménev. Iconogrr. Régne, Anim. pl. 22. fig. 2 (1829-38).

Eurystomus pacificus, (nec Lath.), Motl. \& Diliw. N. H. I.abuan, p. I I ( 855$)$.

Hab. Luzón, Carite, Indan, Laguna, Calamba, Bataan, Orion, (angayan, Manila, San Mateo, Mariquina, Mindoro, Baco, Matrincluque, Samar, Paranas, Borongan, Negros, Valencia, Guimarás, Dinagat, Cuyo, Paragua, P. Princesa, Leyte, Amparo, Cébú, Mindanao, Panaon; Dumalon, Zamboanga, Butuan, Basilàn, Joló; Borneo, Java, Sumatra, Célebes.

2. E calonyx,? Hodgs o o N. v. Uac-uacan.

(M. S. T.)

liurystomus calonys, Hodgs. Icon. ined. in Brit. Mus., Passeres, pls. IO, I I (n. ${ }^{\circ}$ 247); id. in Gray's Zool. Misc. p. 82 (1844); Sharpe, P. Z. S. 1890, p. 55I; Styan, Ibis, 1891, 1. 317, Sharpe, Cat. B. M. 17. I892, p. 38, lam. 2, f. 2.

E. orientalis, (nec L.), Hogs. Icon. pls. Io, I I (n. $\left.{ }^{\circ} 248\right)_{\text {; }}$ id. in Gray's Zool. Misc. p. 82 (1844); Gray, Cat. Mamm. Ac. Nepal. press. Hodgs. p. $55(1846)$; id. List. Fissir. Brit. Mus. p. 3I (1848); Blyth, Cat. B. Mus. As. Soc. p. 5I (i 849); Swinh. 1bis, 1860 , p. 48, 1861, p. 31; Radde, Reis. Sibir. p. II 4, pl. ii. fig. 2 (186I); Jerd. B. Ind. i. p. 2 I9 (1862); Swinh. Ibls, 1866 , p. I 29; Bulger, Ibis, 1869 , p. I 55; Swinh. P. Z. S. 1871, P. 347; Jerd. Ibis, 1872 , p. 3; Pelz. Ib:s, 
1873, p. 108; Huine, Nests \& Eggs Ind. B. p. 105; (18731\% Tacz. Bull. Soc. Zool. France, i. p. 135 (1876); Dybowski, J. f. O. 1876, p. 192; David \& Oust. Ois. Chine, p. 72 (187-); Hume, Str. F. viii. p. 49 (1879); Blakist. Amend. List. K. Japan, p. 24 (1884); Styan, Ibis, 1887, p. 229; Oxtes, ed Hilme's Nests \& Eggs, iil. p. 57 (1890, pt.)

Hab. Samar, Paranas, Paragua, Negros, Mindanao, Lsusín, Cagayan; China, Borneo.

\section{Fam. MEROPÓDIDOS.}

1. Gến. MEROPS, Irn.

I. M bicolor, Budd. oo N. v. Puraojurao

(M S. T.)

le Guepier de l'isle de France, Briss. Orn. ir. 5+3, pl. 44 tig. 2 (1860); Daubent. Pl. Finl. vi. p. 253.

J.e Petit Guepier des Philippines, Briss, t. c. p. 555, pl. +3 . tig. 2 (1760).

Je: Guépier marron et blue, Montb. Hist. Nat. Ois. vi. p. 493 ( 779 )'.

Merops americanus, $P$. Z. S. Müll. Syst. Nat. Suppl. p. 95 (1776); Cass. Proc. Philad. Acad. 1364, p. 243.

(hestnut Bee-cater, Lath, Gen. Syn. i. pt. 2, p. 677 (1782).

Merop bicolor, Bodd. Tabl. Pl. Enl. p. I5 (1783); Wald. Trans. Zool. Soc. ix. p. 150, pl. xxvi. fig. I (1875); Trueedd. P. Z. S. 1877 , pp. $540,690,757,822 ;$ id. P. Z. S. 1878 , pp. 282 , 340; Dreser, Monogr. Merop, P. 27, pl. 7 (1884); Steere, last. B. \& Mamm. Philipp. p. 9 (1890); Sharpe, Cat. B. M. 17. 1892, p. 60 .

M. badius, Gm. Syst. Nat. i. p. 462 (1788); Gray Gen. B. i. p. 86 (1846); Reichenb. Handb., Merop. p. 75, 'Taf. 449 fig. $3244(1852)$.

Merops casteneus, Lath. Ind. Orn. i. p. 273 (1790); Vieill. $N$. Dict. d'Hist. Nat. xiv. p. I 8 (1817).

Le Guépier marron et bleu, ou Le Guépier Latreille mále, Levaill. Hist. Nat. Guépiers, iii. p 45, pl. 12 (1807).

Merops hypoglaucus, Reichenb. Handb., Merop. p. H. Taf. 449. Fig. 3245 (1852).

Melittophas badius, Reichenb. t. c. p. 82 (1852).

M. hypoglaucus, Reichenb. t. c. p $82\left(185^{2}\right)$.

Merops ornatus, (nec Lath.) Von Marteus, J. F. O. 1866 p. 17. Hab. Luzon, Bulacan, Lolomboy, Manila, Montalban, San Mateo, Cavite, Indan, Cagayan, Mindoro, Bacó, Marinduque, Cebù, Leyte, Sanar, Paranas, Guimarás, Panay, Negros, Valevcia, Mindanao, Placer, Butuan, Zamboanga.

2. M. sumatranus, Ruffl. \&\& N. v Piliopilioan.

(M S. T)

Merops sumatranus, Raffl. Trans. Linn. Soc. xii. p 29+(1821); Gray, Gen. B. i. p. 86 (1846); Blyth, Cat. B. Mus. As. Soc. p. 53 (1849); Gould, P. Z. S. 1850 , p. I5 I; Walden, Trans. $Z$. S. ix. p. I5 I, pl. xxvi. Fig. 2 (1875); Twsedd. Ib s, 1877, pl. 7, 297; Sharpe, Ib.s, 1879 , p. 248; Hit:ze, Stir. F. viii. 
p. $4^{8}$ (1879); Tirant, Bu!l. Soc. Comm. Agrie. Cochmehine, (3) i. P. 9\$ (1879); Shtrpe, P. Z. S. 1879, p. 329, 188t, p. 79.3; Ncholson, Ibis, 1882, p. 56, 1883. p. 243; Dresser. Moniggr. Merop. p. 23, pl. 6(188t); Sharpe, Cat. B. M. i7. p. 61 (1892). M. cyanopy yius, Less. Tratè, p. $23^{8}(18+1)$.

M. badius, (n:C Gin.), Bp. Consp. i. p. 162 (1850); Horsf. \&. Moore, Ca!. B. Mus. E. I. Co. i. p. 87 (1854); Modre, P. Z. S. 1854 , p. 264; Motloy \& Dillew. Nat. Hist. Labilżt, p. It $(1855)$; SCl. P. Z. S. 185,3 , p. 213 ; Schl. Mus. Pays-Bas, Mirops, p. 3 (1863); Kelhum, Ibis, 1881, p. 377.

M. bicelor, (nec Botll), Gray, Hantl. B. i. p. 99, fig. I dis (1869'; Salvad. Ucc. Born. p. 9: (1874); Sharpe. P: Z. S.

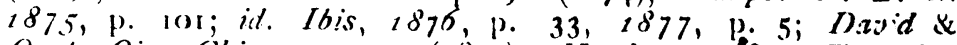
Oust $O$ s. Chine, p. 73 ( 1877$)$; Vorderm. Nat. Tijdschr. Nederl. Ind. xliv. p. 191 (1885), xlvi. p. 69 (1887).

M. rochechouardi, Herude. Anz. Sci. Nat. 187.3 (teste Dresser). Hab. S.tmar, Mindors, Parigua; Borneo, Sumatra, Java.

3. M. apiaster, Li:a. o $\supset \mathrm{N}$ v Abejaruco comun.

(M S. T)

The Bec-eater, A!bin, Nat Hist. B. ii. p. 40, pl. $44\left({ }^{7} 3^{8}\right)$; Yar, Brt. B ii. P) $200\left(\mathrm{I}_{43}\right)$.

Le Guespier, Brison, Orn. iv. p. 532 (1760); Daubent, Pl. En'. vi. p. 938; Montb. Hist. Nat. Os. vi. p. 480 , pl. 23 (1779).

Le Guespier á teste jaune, Br.ss. Orm. iv. p. 537 (176ci).

Mero;s apiaster, Limn. Syist. Nat. i. p. 182 (1766), Gm. Syst. Nat. i. p. 460 (1788); Sharpe, C't. B. M. 17, 1892, p. 63.

Hab. Europa, España, Francia, Asia, Africa.

4 M. philippinus Lin. $00 \mathrm{~N}$ v Pirie-bundoc.

(M. S. T.)

Le Grand Guespier des Philip ines, Briss. Orn. iv. p. 560, 43. Fig. I. ( 760$)$; Dutubent. P!. Enl. vi. pl. 57.

Marops philippinus, Limn. Syst. Nat. I 3 th. edit., ${ }^{i}$, p. ${ }_{1} 8_{3}$ (1787, teste Witden, Tr. $Z$. S. vii. p. 145); Gin. Sysi. Nat. i. p. 463 (1788); Vieill. N. D.ct. d'Hist. Nat. xiv. p. 72 (1817); . Frankl. P. Z. S. 1831 , p. I15; Strickl P. Z S. 1842, p. 167; Gray, Gen. B. i. p. 86 (1846); id. Citt. Mamm. \& Nepal. press. Hodgs p. $57\left(18_{16}{ }^{\prime}\right.$; id. List. Fistr. Brit. Mus p. 69 (1848); Horsf. \& Moore. Cat. B. Mus. E. I. Co. i. p. 86 (1854); Moore, P. $Z$. S. 1854, p. 263; Goutd, B. Asia, i. pl. 36 (1855); Moore, P.Z. S. 1857, p. 87 ; Cab \& Heine, Mus. Hein, Th. ii p. I39 (1860); Irby, Ibis, 1861, p. 228; Scl. P. Z. S. 1863, p. 213; Schl. Mus. Pays-Bas, Merops, p. 2 (1863); Blyth, Ibis, 1865 , p. 30,1866 , p. 344; Beavan, Ibis, 1867, p. 318; Gray, Hand-l. B. i. p. 99, n. ${ }^{\circ} 207$ (1869); Wald. Ta. Z S. vii. p. I45 (1872); Hume Nests \& Eggs Ind. B. p. ror (1873); Ball, Str. F. i. p. 57 (1873); ii. p. 386 (1874); Holdew. Ibis, 1874 , p. 125; Ball, Str. F. iii. p. 289; Salvat. Ucc. Born. p. 89 (1874); Blyth \& Wald. B. Burm. p. 72 (1875); Hums, t. c. p. 326; Butler, t. c. p. 456; Fairb. Str. iv. p. 25 , (1876); Hume, t.c. p. 326 ; Butlem t. c. p. 456; Hitns; Str. F. '. p. 18 (1377); F.xirb. t. c. p. 394; B.tll. t. c. p. 4r3; 
Davin \& Oust. Ois. Chine, p. $72(187 j)$; Treedd, P. Z. S. 1877, PP. 549, 690; 1878, Pp. 107, 282, 340, 709; Hume \& Davis, Str. F. vi. pp. 67, 498; Ball, Str. F. vii. p. 203 (1877); Cripps. t. c. p. 258; lesge, B. Ceylon, p. 306 (187(1); Hume, Str. F. viii. pp. 48,85 (1879); Bingham, t.c. p. 192; lloig, t. c. P. 37o; Tirant, Bull. Comm. Soc. Agric. Cochinchine, p. 78 (1879); Meyer, Ihis, 1879 , p. 57; Sharpe, t. $c$. p. 248; Vidal, Str. $F$. ix. p. 49 (188(a); Bingham, t. $c .1$. 152; Doig, t. c. p. 278; Nichnls. Ibis, 1881 , p. 143; Kelham, Ibis, 1881, p. 378; Reid, Str. Li. x. p. 21 (1881); Davidson, t. c. p. 295 (1882); Davison, t.c. p. 350 (1883); Oates, Handb. B. Br. Burm. ii. p. 66 (1883); Dresser, Monogr. Merop. 1. 55, pl. 15 (1874); Vorderm. Nat. Tijdschr. Nederl. Ind. xliv. p. 191 (1882); Salvad. Ann. Mus. Civic. Genov. (2) iv. p. $5{ }^{\circ} 3$ (1887); Hume, Str. F. xi. P. 42 (1888); Steere, List. B. $\$$ Mamm. Philipp. p. 9 (1890; Oates, ed. Hume's Nests \& Eggs Ind. B. iii. p. 63 (1890); Sharpe, Cat. B. M. 17. p. 71 (1892).

L.t Guépier vert á queue d' azur, Mont. Hist. Nat. Ois. vi. p. 5 of $^{\circ}$ ( 779 ).

Philippine Bec-eater, Lath. Gen. Syn. i. pt. 2, p. $07+(1782)$.

L.' Guépier á queue d' azur, ou Le Guépier, Daudin, Levaill. Hist. Nat. Guépiers, p. 49, pl. 14 (1807).

Mesops javanicus, Horsf. Trans. Linn. Soc. xiii. p. 171 (1820); Eyton, P. Z. S. 1839 , p. rox; Gray. Gen. B. i. p. 86 (1846; Bp. Consp. i. p. 162 (1850); Reichemb. Hand-l. Merop. p. 60, 'raf. 444 Figs. 3227-28 (1852); Molley, P. Z. S. 1863 , p. 2 I3.

M. philippensis, Steph. in Law's Gen. Zool. xiii. pt. 2, p. 75 (1826); Jerd. B. Ind. i. p. 207 (1862); Swinth. Ibis, 186.5, 1. 230; Beavan, t. c. p. 407; Hancock, Cat. B. Northumb. p. 28 (1874); Anderson, Kep. Zool. Exped. Yun-nan, p. 5 I (1878).

M. daudini, (uvier, Régne Anim. i. p. $422(1429)$ Gray, Hand-l. B. i. p. 99, n." г 208 (1879); Swinh. P. Z. S. 1871, p. 349; Hume, Str. H. ii. Pp. 16z, $469(187+)$; iii. p. $49(1873)$.

M. typicus, Hogs. Icon. ined. in Brit. Mus. Passeres, pl. 23 fig. 1, 2 (n. $\left.{ }^{\circ} 746\right)$; id. in Gray's Zool. Misc. p. 82 (1844).

Blepharomerops javanicus, Reichenb. Handb., Merop. p. 72 (1852).

H. phitippinus, Reichenb. t. c. p. 82 (1852).

H. savigni, (nec Audouin), Reichenb. t. c. p. 82 (1852).

M(rops philippinus, war. celebensis, Blasius, Zeitschr. ges. Orn. ii. p. $239(1885)$.

Hab. Luzon, Manila, Mariquina, Montalban, S. Mateo), Latguna, Bulacan, Pampanga, Pangasinau, Cebú, Negros, Valencia, Panay, Bohol, Mindoro, Siquijor, Guimatás, Samar, Mindanao, Zamboanga, Basol, Batsilan; Java, Célebes, Sumatra, Singapore. 


\section{Suborden: HALCIONES.}

\section{Fam. ALCEDINIDOS.}

Subfam. ALCEDININOS.

I. Gén PEIARGOPSIS, Gi.ogke.

I P melanorhyncha, $\left(T_{t m}\right)$ is N. v. Salacsac.

(M. S. T.)

Alcedo melanorhyncha, Temm. Pl. Col. iv. pl. 391 (1826); Schl. Mus. Pays. Bas., Alced. p. 15 (1863), id. Vög. Nederl. Int. Pp. 10, I6, pl. 2 fig. I (1864); 2d. Mus. Pays-Bas, Alced, Revue, p. (1874).

Halcyon melanorhyncha, Gray Gen. B. i. p 79 (1846); Bp. Consp. i. p. 155 (1850); Cass. Cat. Halc. Philad. Wus. p. io (1852); Wall. His, 1860 , p. $142 ;$ id. P. Z. S. 1862, pp. 335, 338; Gray, Hand-l. B. i. p. 92, n. ${ }^{\circ} 202$ (1869); Pelz. u. Lorenz. Alun. k. Nat. Cofm. Wien, i. p. 258 (1886).

Hylcaon melanorhyncha, Reichenb. Hand-b., Alced. p. 18 Tat. 399 , fig. $307+$ (185r).

Rhamphalcyon-melonorhyncha, Bp. Consp. Volucr. Anis. p. Io (1854).

Pelargopsis melanorhyncha, Sharpe. P. Z. S. 1870, p. 62; id. Monogr. Alced p. 94, pl. 29 (1870); Walden, Tr. Z. S. viii. p. 95 (1872); Meyer Ibis, 1879, p. 64; Guillem. P. Z. S. 1833, p. 547, Blasius, Zeitschr ges. Orn. iii. p. 92 (1886); Sharpe, Cat. B. M. 17. p. 97 (1892).

Hab. Célebes, ¿Filipinas?

2. P leucocephala, (Gm.) on. N. v. Salalu.

(M. S. T.)

Martin-Pecheur de Java, Daubent. Pl. Enl. viii. pl. 757.

Le Martin-pêcyeur à tête et cou couleur de paille, Buff. Hist. Nat. Ois. vii. p. 190 ( 780$)$.

White-headed kingfisher, i. 1.t. 2, p. 6i7 (i782).

Aledo javana, Bodd. Tabl. Pl. Enl. p. 47 (1783).

A. leucocephala, Gm. Syst. Nat. i. p. 456 (1788); Shaw. \& Nodd. Nat. Misc. xix. pl. 793 (1807); Schl. Mus. Pays-Bas, Alced. p. 13 (1863. pt.); id. Vög. Nederl. Ind., Alied. pl. 2. (1864); Wald n, P. Z. S. 1866 , p. 553; Schl. Mus. Pay's-Bas, Alced., Revule, p. 7 (1874).

1. jaranica, Shaw, Gen. Zool. viil. p. 67 (1812).

Halcyon leucocephala, Steph. in Shave's Gen. Zool. xiii. pt. 2, p. I 00 (1826); Gray, List. Fissir Brit. Mus. p. 55 (1848, pt.) Bp. Consp. i. p. I54 (1850); Horsf. \& Moore, Cat. B. Mus. E. I. Co. i. p I 23 (1854, pt.); Scl. P. Z. S. 1863, p. 213.

Halcyon javana, Gray, Gen. B. i. p. 79 (1846); id. Hand-l. B. i. p. 92, no. IIO4 (1869).

Rhamphalcyon gurial, (nec Pears.), Reichenb. Handb., Alced. 'Taf. 399 fig. $3075: 185 \mathrm{I}$, nec descr.) 
Palargopsis leucocephala, Sharpe, P. Z. S. Is;o, 1. 64, it. Monogr. Alced. pr 99, pl. 31 (1870); Walden, Ibis, 1872, 1. 368; Salvad. Ucc. Born. p. 95 (1874); Sharpe, Trans. Linn. Soc. n. s. i. p. $317(1876)$; idt. Ibis, 1876, p. 33, 1879 , ए. 249, id. P. Z. S. 1870, p. 329; id. P. Z. S. 1881 , p. 793; Guillem. P, Z, S. 1855 , p. 407; Blastus, Ormis, i, p. 156 ( 785$)$; Everett, Journ. Straits. Branch. R. As. Soc. $18 \delta^{\circ}$, 1. 159, Sharpe, Ibis, 1890 , pp. 19, 283; id. Cat. B. MI. 17. p. $98(1892)$.

Hab. Paragua, Mindoro, Baco; Borneo.

3. P, gouldi, Sharpe. $\sigma \circ \mathrm{N}$ v. Picaul.

(M. S. T.)

Pelargopsis gouldi, Sharpe, Ibis, 1870, p. 63; id. Manogr. Alced. p. Iо1, pl. 32 (1870); Blasius Ibis, 1888, p. 373; id. Oinis, iv. P, 307 (1888); Everett. P. Z. S. 1889, P. 225; id. Journ. Straits, Branch. R. As. Soc. 1889 , p, 159; Sharpe, Cat. B. M. 17, p, $100(1892)$.

P. leucocephala (nec Gm,), Sharpe, Ibis, 1884 , p. 318 (err. ; id. Ibis, 1888 , p. 197 .

Hab. Luzón, Laguna, Cagayan, Abra, Ilocos Norte, Panay Iloilo, Paragua, P. Princesa.

4. P. gigantea, Wald $\sigma \circ$ N. v. Salulu.

(II. S. T.)

Pelargopsis gigantea, Walden, Amm. \&. Mag. Nat. Hist. (4.) xiii. p. I23 (1874); Meyer, t. c. pp. 401, 475; Salvad. UCC. Born. p, 95 (1874), Sharpe. Trans. Linn. Soc. n. s. i. p, 317 (1876); Trueedd P. Z. S. 1877, pp. 541, 822. 1871, pp. I08, 34I, 943, 1879, p. 69; id. Rep. Exped. Challenger, i. Birds, p. 12, (1801); Guillem. P. Z. S. 1885, p 266; Sharpe, Cat. B. M. 17. p. 100 (1892).

Hab. Samar, Borongan, Paric, Panay, Leyte, Amparo, Guimarás, Masbate, Dinagat, Mindanao, Malanipa, Butuan, Zairboanga, Paragua, Inagauan, Joló, Rasilan, Lapac.

5. P. mindorensig, $\sigma \circ$ N. v. Salasac,

(M. S. T.)

Hab. Mindoro, Bacó.

2. Gén. AlCEdo, LiN.

6. A. ispida, Lin. $\sigma \rho$ N. v. Casay-casay.

(M S. T.)

The Little Indian kingfisher, Edwards, Nat. Hist. B. i. p. I, pl. II (I 743).

Le Martin pescheur, Bris. Orn. iv. p. 471 (1760).

Le Martin pescheur de Bengale, Briss, Orn. iv. p. $47 \times$ ( $176(3)$. Alcedo ispida, Linn. Syst. Nat. i. p. 179 (1766); Gm. Syst. Nat. i. pp. 448 (1788); Pall. Zagr. Rosso-stsiat. i. p. 435 (1811); Vieill. N. Dict. d'Hist. Nat. xix. p. 396 (1818); Temm. Man. d'Orn, i. p. 423 (1820); Naum. Vög. Deutschl. v. p. 480, Tatt. 144 (1826); Werner, Atlos, Alcyons, pls 2, 3 (1827); Brehm, Vog. Dentschl p. 148 (18:1); Menétr. Cat. Rais. Cauc. p. $4^{6}$ 


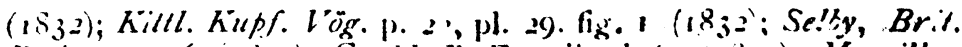
B. i p. 136 (1 333 ; Gould, B. Eut ii. pl. bi (is 37 ); Macg.ll :. Br. B. iii. p. 671 (1840); Crespon, Orn. Gard, p. 294 (1840;

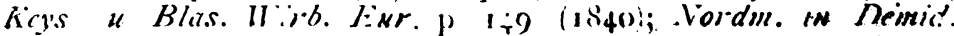
Voy. Russ. Mer-d iii p. 207 ( I 8 +o); Selys-Songrh. Janne Belgc, p. Ior (1842'; Rüpp. Sist licbers p. 23 (18+4); Sichl. Res'. (rit. p. lii. (1itt); Muhle, Beit). Orn. Griechens. p. 31 (18+4); Gray, Gen. B. i. p. $\mathrm{X}_{1}\left(1_{47}\right)$; id. List Fissir. Brat. Mus. p. 62 (I $8+8)$; Thomps. B. Irel. i. p. 369 (1849); Blith, Cat. B. Mus. As. Soc. p. t9 (1s+9); Bp. Consp. i. p. 158 (1850; Cassin, Cat. Halyon. Philad. Mtus. p. I (1852); Kjarrb. Orn. I)an. pl. xiii. fig. 3 (1852); Schl. Toy. Nederl. pl. '13+(185t'; Bp. Const. laluer. Inis p. 10 (1854); Herwits. Eggs. Br. B. i. p. 25.5 pl. it. fig. I $(1856)$; Surzdev. Sa. Fingl. pl. Ixx. fig. 2 (1856-72); jout'. it Barth-Lapomm. Rich. Orn. p. 326 (1859); E. C: Taylor, Ibis, 1859 , p. 47; Wright, Finl. Fog:. p. 287 (1859); Cab. \& He ne, Mus. Heill. Th. ii. p. 143 (1860); Parys, Ib:s, 1860 , p. 236; Lunderm. Vög. Gricchon!. p. +3 (1860); Schl. D)ier. Wederl. Vög. pl. it fig. 2 (1801); Tristr. Ibis, 1862 , p. 278; Schl. Mus. Pay's-Bas, Alcedimes, p. 6 (1863; Gray, L.st Brr. B. 1. \$6 (1863); Radde, Reis. Sib.r., Vöy. p. 143 (1863); Adams, Ib.s, 1864, p. 15; Wright. t. $c$. p. 73 ; Hllen. t. c. p. 237 ; Sperling, t. c. p. 28., Morc, Ibis, 1865 , p. I 38 ; Triste. Ibis, 1866 , p. 84; Lilford. $t$. c. p. 178; Degl. \&- Cirbe, Orn. Eur. i. p. 173 (1867); Tajlor, Ibis, $186 \%$, p. 56; Drake, t. c. p +25; Loche, Expl. Sci. Alger, Ois. ii. p. 9+ (1867); Beltoni, Ucc. ind'f. Loml. ii., Alced. pls. I, 1. a (1868); Smith, Ibis, 1868, p. 849; Cillett, Norges, Fugle, p. 147 (1869); Gray, Hand-l. B. i. p. 95, n. ${ }^{\circ}$ I15 (1869); Kenlen. Onze. Vög. i. pl. 45 (1.869); Borggr. Vogelf. Nordents hl. p. 68 (1869); Suzunders, Ib.s, 1869 , p. 172; Daderl. Avif. Sicil. p 55 (1869); Heugl: Orn. N. O.-Afi. i. p I77 (1869); Droste, Vög. Bork. p. 84 (1869); Wyatt, Ibis, 1870 , p. I2; Eizues \& Buckley t. c. p. I 89; Gould, B. Gt. Br. ii. pl. 10 (1870'; Sharpe, Monogr. Alced. p. I, (187 pl. i. (1870); Fritsch, Vög. Eur. pl. xiv. fig. I (1871); Salvad. Faun. Ital., Ucc. p. 45 (I87I); Saunders, Ibis, 1871, p. 67; R. Gray, B. W. Scotl. p. 204 (1871); Harting, Handb. Br. B. p. 34 (1772); Godman, Ibis, 1872, 1. 169; Shelley, B. Egypt. p. 168 (1872; Brooke, Ibis, 1873 , p. 236; Severtz. Turkest. Jevotn. p. 68 (1873); Hume, Str. F. i. p. 168 (1773); Wright, Ibis, 1874, p. 257; Silford, Ibis, 187.5 , p. 16; Cordeaux, t. c. p. 184 ; Daly. \& Havie-Brown, l. $\therefore$ p. 300; Dresser, B. Eir. v. p. I13, pl, 290 (1775); Irby, B. Gibr. p. 68 (1875); Stoliczka, Sir. F. iii. p. 219 (1775); Fallon, Ois. Belg. p. 122 (1775); Whartou, Ibis, 1870, p. I9; Dresser, t. c. p. 320 ; Blanf. Last. Persia, ii. p. I 2 I (1776); Butler, Str. F. v. p. 207 (1777); D.nnf. Ibis, 1877, p. 262, (1878), p. 7; Bogd. B. Cauc. p. 114 (1879); Finsch. Verh. zool-bot. Ges. Wien. xxix, p. I73 (1779); Butler, Cat. B. Sind. etc. p. I7 (1879); Hume, Str. F. viii. p. 86 (1879); l)anf. Ibis, 1880, p. I3; Seeb. t. c. p. 293; Nezut. ed Yarell's Br. B. ii. p. 443 (188I); C. Swinth. Ibis, 1882, p. 102; Seeb. t. c. p. $289 ; B . O$. L. List. Br. B. p. 80 (183; 
Liebe, J. f. O. 1883 , pp. 286-291; Secb. Ib.s, 188.3 , I. 2т; Irby, t. c. p. 179; Booth, Rough. Notex, ii. pl. It ist-si, Gigl. Yconogr. Avif. Ital. sp. 59 (1882); Horreyer \& Tancie', MT. orn. Ver. Wien, $1883, \mathrm{p} .83$; Capm. I6 s, $1884, \mathrm{p}$. $9 ;$ Saunders. t. c. 379; Seeb Hist. Br. B. ii. p. 341 (18st); 7 ristr. Faun. \& Flor. Palest. p. 86 (1884); Radde, Or.n. Cauc. p. 323 (1884); Murray, Vertebr. Fann Sint, p. וा (1884); Whitehead, Ibis, 1885 , p. 27; Reid, t. c. p. 247; $G g^{\prime}$. Ital. p. 283 (1886); Sharpe, Ibis, 1895, p. i66; Salvad. Elench. Ucc. Ital. p. 76 (1887); Backh. Ibis, 1887 , p. I2; Tait. $1 . c$. p. 305; Lorenz, Beitr. Or. Kauc. p. 43 (1887); ielford, (ol. Fig. Brit. B. part. viii. (1888); St. John, Ibis, 1889, p. 157; Lelford, t. c. p. I 3 I; Gigl. Avif. It.rl., lst Resoc. p. 3.5' (1889); Seeb. B. Japan. Emp. p. I 75 (1890); Sharpe, Cat. B. "ir. 17. p. 14r (1892).

Martin-pecheur, Daubent. Pl. Enl. viii. pl. 77.

Le Martin-pecheur, ou l'Alcyon, Buff. Hist. Nat. Ois. vii. p. ift (1780).

Le Martin-pecheur, Buff.t.c. p. 201; (1790).

Common Kingfisher, Lath. Gen. Syn. i. pt. 2, p. 626 (1782); Yarrell, Hist. Brit. ii. p. 206 (1 843 ).

Indian Kingfisher, Lath. t. c. p. 631 (i 782).

Alcedo bengalensis, Gm. Syst. Nat. i. p. 450 (1788); Vieill. $N$. Dict. a' Hist. Nat. xiv. p. 400 (1811); Sykes, P.Z. S. 1832 , p. 84; Kittl. Kiipf. Vög. p. 22 pl. 29, fig. 2 (1842); Me Clell. P. Z. S. 1839 p. 156 ; Jerd. Madr. Journ. xi. p. 231 (1840); Hodgs. Icon. ined. in Brit. Mus., Passeres, pl. i 7. fig. 2 (6o6); id. in Gray's Zool. Misc. p. 82 (1 844 ; Blyth, F. A. S. B. xv. p. iा. (1846); Gray Cat. Mamm. etc. Nepal pres. Hodgs. p. 57 (1846; id. Gen. B. i. p. 81 (1847); id. Cat. Fiss. Brit. Mus. p. 68 (1848); Blyth, Cat. B. Mus. As. Soc. p. 49 (18+9); Bp. Consp. i. P. I5 (1850'; Cass. Cat. Halc. Philad. Mus. p. I (1852); Layard, Ann. \& Mag. $N$. H. (2) xii. p. I72 (1853); Moore, P. Z. S. (I854), p. 269; Bp. Consp. Vol. Anis. p. (185t;) Horfs. \& Moore, Cat. B. Mus. E. Y. Co. i. p. I 29 (1854); Adams, P. Z. S'. 1858 , p. 474 ; Gould, P. Z. S. 1859 , pp. I50, I5 I Adams, t. c. p. I 74 Swinh, Ibis, 1860 , p. 49, I86I; pp. 3 I, 328; Cab. \& Hene, Mus. Hein. Th. ii. p. I 44 (1860); Gould, B. Asia, i. pl. 53 (1862); Jerd. B. Ind. i. p. 230 (1862); Blakist. Ibis, 1862 , p. 325; Schomb. Ibis, 1864 , p. 247; Blyth, Ibis, 1866 , p. 348; Whitely, Ibis, 1867 , p. I96; Swinh. t. c. p. to8; $7 y$ tler, Ibis, 1868 , p. I97; Bulger, Ibis, 1869 p. $15^{\circ}$; Beazan, t. c. p. 409; Gray, Hand-l. B. i. 95, n. ${ }^{\circ}$ I 52 (1869); Godw-Aust. J. A. S. Beng. xxxix. p. 95 (1870. Stol. t. c. p. 297; Swinh, Ibis, 1870 , p. 92; Blanf. t. c. p. 465 ; Sharpe, Monoggr. Aleed. p. i I, pl. 2 (1870); Swinh. P. Z. S. 1871 , p. 347; Blanf J. A. S. Beng. xl. p. 27 I (1871 ; (1871); Shelley, B. Egypt, p. I66 (1872); Holdsw. P. Z. S. 1872 , p. 424; Stoliezka, J. A. S. Beng. xli. p. 232 (1872); Hume \& Henders. Lahore to Yark. p. I78 (1873); Walden, Ibis, 1873 , p. 202; Hayes, Lloyd, t. c. p. 407; Hume Nests. \& Eggs. Ind. B. p. 107 (1873); id. Str. F. i. pp. 168, 305, $45^{\circ}$ (1873); Adam t. c. p. 372; Rainey, t. c. p. 496; Hume, 
S\%. F. ii. pp. 173, t70 (1874); Ball, t. c. p. 387; Salv.rd. Licc. Borll. p. $92(1874)$; Hume, Str. F. iii. p. 72 (1875): Blylh. \& I'ald. B. Burm. p. 71 (1875); Butler, t. c. 456; Fairb. Str. F. iv. p. 254 (1876); Armstr. t. c. p. .307; Drcsiser, Ibis, 1876 , p. 320; Sharpe, Ibis, 1877 , p. 6; 'T2ueed. I'. Z. S. 1877 , pp. 690, 757; id. Ibis, 1877, p. 297; Wud.'. Ramsay, t. c. p. 457 ; Dazid \& Oust. Ois. Chive, p. 74 (1877): Tieedd. P. Z. S. 1878 , pp. 341 709, 9+3; Anders. Rep Zoo!. Exped. I'unnan, p. 580 (1878); Blakist. \& Pryer. Ibis, 1875 ', p. 229; Hume \& Inavison. Str. F. vi. p. 77 (1878); Ball, Str. F. vii. p. 204 (18;8); Cripps, t. c. p. 26o; Segge, B. Ceylon, i. p. 292 (1878); Meyer, Ibis, 1879 , p. 64; Hicme, Sir. 1. viii. pp. +9, 86 (1879); Salvad. Ann. Mus. Civ. Genov. xiv. p. 193. (1879); Scully, t. c. p. 238; Doig, t. c. p. 370; Brosks. t. c. p. 468; Sharpe, P. Z. S. 1879 , p. 329; Butl:!', Cat. B. Sind of p. 16 (18791; id. Cat. B. S. Boinb. Pres. p. 20 (1880); Salvad. Orn. Papuasia e delle Molucch, i. p. 407 (1880); Vidal, Str. F. ix. p. 50 (1880); Binsham, t. c. p. I55; Butler. t. c. p. 383 ; Boluzw. J. f. O. $1 \delta^{8} s^{\circ}$, p. I15, 1881, p. 53; Kelham, Ib.s, 1881, p. 382; H. H. Slater, Ibis, 1882 , p. 432; C. Szwink. Ib s, 1882, p. 102; Joul', Proc. U. S. Nat. Mus. vi. p. 310 (1883); Homejer \& Tancré M. 1: orn. Ver. Wien, 188 3, p. 83; Parker, Ibus, 1883 , p. 193; Oates, Handb. B. Brit. Burm. ii. p. 72 (188.3'; Baklist. Amended. List. B. Japan, p. 13 (1884); Murray, Vertebr. Faun. Sind., p. I I ( I884); Seeb. Ibis, 1884, pp. 261, +28; Sharpe, t. c. p. 318; C. H. T. Marshall, t. c. p. 40y; Guillem. P. Z. S. 1885 , pp. 255, 407, 504, 547; C. Sivinh. \& Barnes, Ibis, 1885 , p. 6I; A. Mull. J. F. O. 1885, p. 150; Voderm. N. T. Nederl. Ind. xivi. p. I I (1886); Gigl. \& S'ilvad. P. Z. S. 1887 , p. 581 ; Sharpe, Ibis, 1886 , p. 483; Reid, Str. F. x. p. 22 (1887); Oates, t. c. p. I87; Davilson, t. c. p. 295; Davidson, t. c. p. $35 \mathrm{I}$; Taylor, t. c. p. 457; Teerry, t. c. p. $47 \mathrm{I}$; Styan, Ibis, 1887 , p. 230; Salvad. Ann. Mils. Gensi. (2) iv. p. 584 (1887); Darries, J. f. O. 1888 , p. 65; Vorderm. N. T. Nederl. Ind. xlix. p. 393 (1889); Hartert, J. f. O. 1889 , p. 365 ; St. John. Ibis, 1889 , p. 157; Everell, P. Z. S. 1889 , p. 225; id. Journ. Straits Branch R. As. Soc. 1889 , p. 158; Sharpe, Ibis, 1890 , pp. 18, 283; Whitehead, t. c. p. 45; Oates ed Hume, Nests. \& Eggs. Ind. B. iii. p. I $(1890)$.

Alcedo ispida bengalensis, Temm. \& Schl. Fann. Jap., Aves, p. 76, pl. 38 (1830); Seebohm, bis, 1882, pp. 248, 424; id. Ibis, 1887 , p. 176 .

A. ispida, var. bengalensis, Raffl. Trans. Linn. Soc. xiii. p. 293 (1822); Schrenck, Reis, Amurl. p. 265 (1859); Radde, Sibir. Reis., Vög. p. 143 (1863); Severtz. Turkest. Jevatn. p. 6r (I873).

A. hispida, Less. Traité, p. 243 (1831); Filippi, Viagg Persia, p. 346 (1865).

A. subispida, Brehem. Vög. Deutschl. p. 149 (1831).

A. advena, Brehm, t. c. p. I 50 , Taf. xi. fig. 2 (1835).

A. pallasi, Reichenb. Handb., Alced. 1. 3, 1'af. 393 figs. $3^{0}+3-14$, 'Taf. 393 . figs. $30+5-46(1858)$. 
A. bengalensis, oar. sondaica, Reichnb. t. c. P. 3 (1851).

A. japonica, Bp. Consp. Vol (Anis. p. Io $(185+)$.

A. brachyrhychus, Brekm, Vogelf. P. 51 (1 855$)$.

A. pallidu, Brehm, $t$ c. p. 5 .

A. sondaica, Cab. \& Heine, Mus. Hein. 'Th. ii. p. Itt $(18600)$ : Gray, Hand-l. B. i. p. 95, n. ${ }^{\circ}$ I 57 ( 869 ).

A. minor, Schl. Mus. Pays-Bas, Alced. p. 7 (1863); id. Vös. Nederl. Ind., Alced. pp. 4, 43, pl. i. fig. 3 (1864).

A. ispida minor, Heugl. Orn. N. O.-Afr. i. p. 178 (1869).

Ispida bengalensis, Tacz. J. f. O. 1875, p. 25t; id. Bull. Soc. Zool. France, i. p. $135(1876)$.

A. sindiana, Hume, Str.f. i. p. 168 (1873).

Jacelo minor, Snellem, in Vetk's Mid.len-Suinatra, Vög. 1). 36 (1887). Hab. Luzon, Manila, San Mateo, Montallin, Panpanga, Mindoro, Baco, Negros, Cebú, Bohol, Leyte, Sa:nar, Masbate, Siquijor, Guimaràs, Basilan, Zamboanga, Minlınao, Paragua, B.1labac, Joló, Mainbun; Chins, Fornosa, Europa, Asi i.

7. A. merinting, Horsf. $\delta ?$ N. v. Bintis.

(M S. T)

Alcedo meninting, Horsf. Trans. Linn. Soc. xiii. p. I 72 (1821).

Temm. Pl. Col. ii. p. 239 fig. 2 (1823); Blyth, J. A. S. Beng. xv. p. I 2 (1846); Gray, Gen. B3. i. p. 81 (1847); Kanp, Fam. Alced. p. 16 (1848); Blith, Cat. B. Mus. As. Sö. p. 49 (1849): Bp. Consp. i. p. I 58 (1850); Moore, P. Z. S. 1854 , P. 26); Horsf. \& Moore, Cat. B. Mus. E. I. Co. i. p. I $3^{\circ}(185 t)$; Reichenb. Handb., Alced. p. 4, 'Taf. 394. fig 3050.51 (1851); Cass. Cat. Halc. Philad Mus. p. 3 (1852); Bp. Consp. Voluer. Anis. p. 10 (1854); Cab. \& Heine, Mus. Hein. Th. ii. I'. I 44 (1860); Wall. P. Z. S. 1863 , p. 484; Schl. Mus. Pay'sBas. Alced. p. 9 (1863); id. Vög. Nederl. Ind., Alced. pp. 0 , 44, pl. 3 (i 864); Gray, Hand-l. B. i. p. 95, no. i 154 (1 869); Salvad. Ucc. Born. p. 93 (1874); Sharpe P. Z. S. 1875, p. Iог; Salvad. Ann. Mus. Civ.c. Genov. vii. p. 652 (1875); Tweed. Ibis, 1877 , p. 397; Hume \& Davison. Str. F. vi. p. 8, (1878); Sharpe, P. Z. S. 1879, p. 329; id. Ibis, 1879, 1). Hume, Str. F. viii. pp. 50, 16 (1879); Salvad Ann. Mus. Civ. Genov. xiv. p. 193 (1879); Vorderm. Nat. Tijdschr. Nederl. Ind. xlv. p. 185 (1881); Kelham, Ibis. 1881 p. $381 ; A$. Müll J.f. O. 1882, p. 397; Blasines, J.f. O. 1883 , Pp. 213 , 215 Nichols. Ibis, 1883 , p. 83; Biasius, Zeitschr. ges. Orn. iii: p. 94 (1886), Vorderm. Nat. Tijschr. Nederl. Ind. xlvi. p. 88 (1886); xlix. p. 393 (1889); Blasius Ornis, 1888, p. 307 ; Sharpe, Ibis, 1890, pp. 18, 283; Sharpe, Cat. B. M. 17.1892 p. 157.

Alcedo asiática, Swins. Zool. Illustr. lst ser. i. pl. 50 (1821); Sharpe, Ibis, 1869 , p. 218 ; id. Monggr. Alced. p. 25, pl. 5 (1870); Waln. Ibis, 1872 , p. 368; Hume Str. F. i. p. $45^{\circ}$ (1873); Meyer, Ibis, 1879, p. 64; Guillem. P. Z. S. 1885 , p. 255; Sharpe, Ibis, 1888 , p. I97; Everett, P. Z. S. 1889 , p. 226; id. Journ. Straits Branch R. As. Soc. 1889 , p. 159 ;

Whitehead, Ibis, 1880 , p. 45.

Ceyx meninting, Cnvier, Régne Anim. i. p. H+ (1829).

Alcedo bengalensis, (nec Gn.) Less. Trati, p. $2+3$ (1821, pt.) 
A. verreauxi. D. In Berg^, Rez. Zool. 1851 , p. 305, pl. 9; Reichenh. Handb., Alced. p. 5, 'Taf. 403 fig. 3173 (1851); Rlvth, Ibis, 1866, p. 348; Gray, Hand-l. B. i. p. 95, n.' is.5 (1869).

Hab. Paragua, P. Princesa, Joló, Maiıbun; Borneo, Sumatra, Java. Célebes.

\section{Subfam, DACELONINOS.}

\section{Gén. CEYX, I,acḱr.}

\section{C. tridactyla. (Pall.) \& \& N. v. Susulbot.}

(II. S T:)

Alcedo tridactyla, Pallas, Spic. Zool. vii, p، to, tals, 2 figu, 1 (1769); Linn. Mant. Plant. p. $52+$ ( 77 I), Scop. Del. Fantl. et Flor. Insubr. ii. p. 90 (1795); Gin. List. N.t. i. p. 459 $(\mathrm{I} 788)$.

Martin-pècheur de l' isle de Luçon, Sonn. Vög، Now, Guirı. p. 6), pl. 32 (1776).

Martin-pêcheur de Pondicherry, D.ubent. Pl. Enl. vii. pl. 79 s. tig. 2.

1,: Martin-pècheur á trois doigts, Buff. Hist. Nat. Ois، vi. $).$ $203(1780)$.

Alcedo rubra, Bold. Tabl. Pl. Enl. p. 49 (1783).

A. purpurea, Gm. Syst. Nat. i. p. 449 (1788).

A. erythaca, Gm. Syist. Nat. i. p. 449 (1788).

(Ceyx luzoniensis, Steph. in Shaze's Gen. Zool. xiii. p. Ior (1825).

(. tridactyla, Sykes, P. Z. S. 1832 , p. 84 ; Strickl. P. Z. S. 1846, p. 69; Gray, Gen. B. i. p. Io (1847); id. Cat. Fissir. Brit. Mus. p. 59 (1848); Jerd. Ill. Int. Orn. pl. 25 (1847); Bp. Consp. i. pp. 157, I58 (1858); Cass. Cat. Halcyon. Philad. Mus. p. 13 (1852); Bp. Consp. Volucr. Anis. p. 9 (1854); Hartl. J. f. O. 1854, p. 413; Moore, P. Z. S. 1854, p. 269; Jerd. B. Ind. i. p. 229 (1862); Gray, Cat. Mamm. \& Nepal. ,res. Hodgs. p. 24 (1863); Sharpe, P. Z. S. 1868, p. 271: id. Monogr. Alced. p. I 19, pl. 4o (1868); Gray, Hand-l. B. i. p. 94, n. ${ }^{1}{ }_{42}$ (1869); Stlvad. Atti R. Acead. Sci. Torino, iv. p. 45.5 (1 886); Stoliczka, J. A. S. Beng. xxxix. p. 395 (1870: Prov. Vellesley.); Holdsw. P.Z. S. 1872 , p. 424 Hume, Str. F. ii. p. I74 (i874: Butler, t. c. p. 453 Walden Ibis, 1874, p. 137 Hume, Str. F. iii p. 51 (1875: Blyth, B. Burm. 1. $7_{1}(1875)$; Hume, Str. F. iv. p. 287 (1876: id. Str. F. v. 1. I 9 (1877: id. \& Davison, Str. F. vi. pp. 80, 499 (1878): legge, B. Ceylon, p. 303 ( 1879$)$; Hume, Str. F. viii. p. 80, ( 879 ; Bingham, t. c. p. 193 id. Str. F. ix. p. I 55 (1880); Butler, Cat. B. B. Somb. Pres. p. I9 (1880; Oates, Handb. B'. Br. Burm. ii. p. 80 (1883); Salvad. Ann. Mus. Civ. Genon. (2) iv. p. 537 (1887); vii. p. 333 (1889); Hume, Str. F. xi. p. 45 ( 1888); Oates, ed. Hume's Nests \& Eggs Ind. B. iii. p. I.3 (1890); Sharpe, Cat. B. M. 17.1862. p. 174.

(. purpurea, Less. Traite, p. $3^{8}$ (1831); Reichenb. Handb. Alced. 9, Taf. 398. fig. 3071 (1851); Bp. Consp. Vol. Anis. 1. 9 (1 854$)$; Hartl. J. f. O. 1855, p. 423. 
C. microsoma, Burton, P. Z. S. 1837 , p. 89.

(.) erythaca, Blyth, Cat. B. Mits. As. Soc. p. 5o (1849).

llacelo rubra, Schl. Mus. Pays-Bas, Alced. p. 49 ( I 863$)$; id. Vög. Nederl. Ind. Alced. pp. 40, 68, pl. 16. fig. 2 (1864).

Hab. Luzon, Laguna, Calauan, Los Raños.

9. C. rufldorsa, Sharpe, $\varnothing, 0 \mathrm{~N}$ v. Bintis.

(M. S. T)

Ceyx rufidorsa, (nec Strickl.), Monogr. Al ed. p. I21, pl. +1 (1869); id. Ibis, 1877 , p. 6 Trceedd. Ibis, 1877, p. 297 (SF.) Kelham, Ibis, 1881 , p. 318 Salvin, Cat. Strickl. Coll. p. +18 (1882); Nichols. Ibis, 1883, p. 243; Sharpe, Ibis, 1884 , p. 318; Buttik Notes. Leyden. Mus. ix p. 37 1887: W. Whitehead, Ibis; 1890. p. 45 Everett, Journ, Straits Branch R. Asiat. Soc. 1889 , p. I59; Steere, List Birds \& Mamm. Steere Eixped. p. $10(1890)$.

(C. innominata (nec Salvad.), Sharpe, P. Z. S. 1875, p. 101.

C. dillwynni (nec Sharpe 1868), Sharpe, Ibis, 1879 , p. 249 id. P. 7. S. 1881 , p. 793

Ijacelo rufidorsa (nec Strickl), Schl. Mus. Pays Bas, Alced. p. t9 (1863); id. Vög Neder-l. Ind. Alced. pl. 40, 67, pl. 16. fis. 1 (1864); Snellem. in Veth's Midden-Sumatra, Vog. 1). 36 ( I88+).

C. euerythra, Sharpe, Cat. B. M. 17. $1892,1.179$.

Hab. Mindoro, Calapan, Paragua, P. Princesa Igualvig; Borneo, Sumatra, Malaca.

Io. C. melanura. Koup. O $0^{0}$ N. v. ( asaycasay.

(M. S. T.)

Ceyx melanura, Kaup, Fam. Eisv. p. 15 (1848); Gray, Cat. Fissir. Brit. Mus. p. 59 (1848); id. Gen. B. iii App. p. $5\left(18_{49}\right) ; B p$. Consp. i. p. ${ }_{158}$ (1850); Reichenb. Handb. Alced. p. 9 (1851); Cass. Cat. Halcyon Philad. Mus. p. 13 (1852; Amborna); Cab. \& Heine, Mus. Hein. 'Th. ii. p. 151 (1860); Sharpe, P. Z. S. 1868, p. 27ı; id. Monogr. Alced. 1. I17, pl. 39 (1868); Gray, Haud-l. B. i. p. 94, n. 144 (1869); Salvad. Atti R. Accad. Torino, iv. p. 453 (1869); Sharpe, P. Z. S. 1869, p. 511; Treedd. Trans. Z. S. ix. p. 152 (1875); Wardlaw Ramsay, Ibis, 1884 , p. 332; (Manila), 1886, p. 1 56; Steere, List Bircts * Mamm. Exped. Philipp. 1. 9 (1890). Sharpe, Cat. B. M. 17. 1892, p. 180 .

Dacelo melanura, Schlegd, Mus. Pays-Bas, Alced. p. 49 (1863).

Ceyx samarensis, Steere, List Birds \& Mamm. Steere Exped. Philipp. p. 40 (1890).

?. platenx, Blasius, J.f. $O_{i} 1890$, p. 148 .

Hab. Luzón, Manila, Bulacan, Laguna.

11. C. basilanica. Stiere. $\delta$ N. v. Bintis.

(M. S. T.)

Ceyx basilanica, Steere, List. Birds et Mamm. Steere Exp. Philipp. p. Io (1890). Sharpe, Cat. B. M. 17. 1812, p. 181.

Hab. Basilan. 
12.C. mindanensis, Stiere. N. v.

Cryx mindanensis, Steere, List. Birds. Exped. Philipp. p. 10 (189); Shaipe, Cat. B. 17, (1892); p. 181 .

Hab. Mindanao.

13. C. lepida, $T_{i} m$. $\delta \&$ N. v.

(M. $S$ T )

('eyx lepida, Temm. Pl. Col. iv. pl. 595 fig. I (1836); Less. Compl. Buff., Oss. p. 649 (1838); Gray', Cat. Fissir. Brit. Mus. p. 59 (18+9); Bp. Consp. i. p. 158 (1850); Reichenb. Handb., Alced. p. 10 Taf. 398 . ig. 3066 (1851); Cass. Cat. Halcyon. Philad. Mus. p. It (1852); Bp. Consp. Vol. Anss. p. 9 (1854); Harll. J.f. O. 1854, P. 414; Gray, P. Z. S. 1858, p. 190; id. Cat. B. Nero Guinea, p. 55 (1859); id. P. Z. S. 1860 , p. 348,1861, p. 433; Wall. P. Z. S. 1862, pp. 335, 338 (pt.); id. P. Z. S. 1873, p. 25 ; Rosenb. Nat. Tijdschr. Nederl. Ind. xxv. p. 251 (1863); id. J.f. O. 1864, p. 118; Finsch, Neu-Guinea, p. I6r (1865); Sharpe, $P$ Z. S. 2868 , pp. 27 I 596; id. Monogr. Alced. p. I31, pl. 46 (1868); Gray, Hand.l. B. i. p. 94, n. ${ }^{\circ}$ I 45 (1869); Salvad. Atti R. Accad. Sei. Torino, iv. p. 471 (1869); Sharpe, P. Z. S. 1869 , p. 509; Salvad. Ann. Mus. Civ. Genov. x. p. 30 (1877); id. P. Z. S. $187 \delta$, p. 8I; id. Orn. Papuasia, i. p. 4 I 7 (1880); id. Rep. Zool. H. M. S. «Challenger,» ii. p. 62 (1881); Blasius \& Nehrk. Verh. z.-b. Ges. Wien. xxxii. p. $418(1882)$; Blasius, P. Z. S 1882, p. 704; Pleske, Bull. Acad. St. Petersb. xxix. p. 525 (1884); Guillem. P. Z. S. 2885, p. 567 ; Salvad. Agg. Orn. Papuasia, i. p. 53 (189); Sharte, Cat. B. M. 17. 1892 , p. 883.

Alcyone lepida, Gray, Gen. B. i. p. 82 (1847).

Ceyx uropygialis, Gray, P. Z. S. 1860 , p. 348; Finsch, NeuGuinea, p. 161 (1865); Sharpe, P. Z. S. 1868, pp. 271, 596; id. Monogr. Alced. p. 133, pl. 47 ( 1869); Salvad. Atti R. Accad. Sci. Torino, iv. p. 474 (1869); Gray, Hand-l. B. i. p. 94, n. I 48 (1869); Salvad. Ann. Mus. Civ. Genov. v. p. 764 (1875).

Dacelo lepida, Schl. Mus. Pays-Bas, Alced. p. 48 (1863); id. Vög. Nederl. Ind. Alced. pp. 39, 66, pl. I6. figs. 4, 5 (186); id. Mus. Pays-Bas, Alced. Rovue, p. 34 (1874).

Alcedo lepida, Rosenb. Reis. naar Geelvinkb. p. 6 (1875).

Hab. ¿Filipinas? Molucas.

4. C. malımaui, Steere. $\mathrm{N} v$

Coyx malanaui, Steere List. Mam. et Birds. Exp. Philipp. 1. I (1890); Shrrpe, Cat. B. M. 17 , p. I84, (1892).

C. suluensis, Blasius, J. f. O. 1890, p. I 4 r.

Hab. Malamaui, Basilan, Joló.

I5 C bournsi. Steere. N. v.

Ceyx bournsi, Steere, List. Exped. Philipp. p. Io (1890); 17 p. 185 (1892). Sharpe Cat. B. M. 17. 1892, p. 185 .

C. margar, Blasius, J. f. O. 1890 p. 140.

Hiab. Basilan, Joló. 
16. C. cyanipectus, Lafr. $0^{\circ} \circ$ N. v. Susulbat.

(M. S.T.)

Ceyx cyanopectus, Lafr. Rev. Zool, 1840 , p. 33; Ciray, Gen. B. ii. App. p. 5 (1841); Wardlaw Ramsay, Ibis, 1884, p. 332, pl. ix; id. Ibis, 1886 , p. 156; Stcere, List Birds \& Metmm. Steere, Exped. Philipp. p. 10 (pt. 1890); Sharpe, Cat. B. .I. $17, \quad \mathrm{p}, 184$ (1892).

Mcyone cyanopectus, Jord. Coctr. Orn. 18.50, p. 82; Bp. Consp. i p 158 (1850); Reichent. Handb, Alced P 71, Taf 3(2). fig, $3060(1851) ; B p$ Consp. Voluer Anis. p $10(1854) ;$ Hartl. $J f O$ 1854, p lxiv; Sharpe, P.Z S. t86 9, p 353; it. Monogr. Alced. p 55, pl. 17 (1869); Walden, Trans $Z$. ix. pp. 1 53, 249 (1875); Wardlaw-Kainsay, App. Tiveedd. Mem. 1) 655 ( 1 $88 \mathrm{r})$.

A. cincta, Jard. Contr. Orn. 1850, p 82, pl. only.

Alcedo cyanipectus, Schl. Miss. Pays-Bas Alced. p. 18 (1803:; Gray, Hand-l B. i. p. 95, n ${ }^{\circ}$ ir6r (1869).

('eyx philippinensis, Gould, P. Z. S. 1868 , p. 404; Sharpe. Monogr. Abced. p. I I 3 , pl. 37 (1869;) Walden, Trrns. Z, S'. ix. pp, 153, 249 (1875); Wardlaw Ramsay, Truedd. Mem. App. p. 654 (188 r).

Hab. Luzón, Manila, Marinduque, Mindoro, Calapan.

17. C. sterii, Shurpe. $\delta \oint$ N. v, Casay+casay.

(I. S. T.)

('ryx philippinensis, Steere, List. Bird. Exped. Philipp. p, IN (1890); Sharpe, Cat. B. M. 17. p. 187 (192).

$H a b$, Mindoro, Baco, Naujan,

18. C. argentata, Tw. $\bigcirc \subsetneq \mathrm{N}$, v. Sibit.

(M. S. T.)

(eyx argentata, Tzweeddale, An. el Mag. N. H. xx. p. 533 (1877). id. P. Z. S. 1877 , p. 822 ; id. Ibid. 1878 . p. 108 pl. vi.; Steere, List. Exped. Philipp. p. 10, 1890; Sharpe, Cat. B3. M. 17. P. I87 (1892).

Hab. Samar, Paranas, Paric, Borongan, Calbayog, Dinayat, Mindanao, Placer, Zamboanga.

19. c. fluminicola, Tw. \&? N. v Sibit.

(M. S. T, ;

(ryx argentata, Tw. P. Z. S. 1878 . p. 341.

( flumenicola, Steere, List. Exped. Philipp. p. $101890 ;$ Sharpe, Cat. B. M. 17. p. $187(1892)$.

Hab. Samar, Borongan, Leyte.

4. Gèn. dacelo, heach.

20. D gizas. (Betl). t N. v.

(M. S. T.)

Grand Martin-pecheur de la Nouvelle Guinee, Sonz. Voy. Now. Guin. i. p. 171, Taf. Io6 (1766).

Martin-Pècheur de de la Nouvelle Guineé, Daubent, Pl. Enl. viii. pl. $66_{3}$. 
Le flus grand Martin-pecheur, Buff. Hisi. Nat. O.s. vii. p. IRt i $1 ; 80$ ).

Dacelo gigas, Gray, Gell. R. i. p. 78 1184h; Sharpe, Cat. K. H. 17.1892 1. 204.

Hab. Australia.

\section{Gèn HAICTON, SWANS.}

21. H. coromendus, (Lath.) $\checkmark \circ \mathrm{N}$. v. Salasac.

(M. S. T.)

Ie Martin Pècheur videt des Indes, Sonn. Vay. Ind. Orienl. ii. p. $212(1-82)$.

le Murtin Pécheur violet de la còte de Coromandel, Sonn. t. c. pl. $118(1782)$.

Alcedo coromanda, Lath. Ind, Orn. i. p. 252 (1790; Horsf. Trans. Linn. Soc. xiii. p. I74 (1820).

Halcyun ccromanda, Sttph. in Shaw's Cien. Zool. xiii. pt. 2, p. 100 (1826); Cass. Cat. Halc. Philad. Mus. p. 7 (1852); Sharpi, Monogr. Alced. p. 155, pl. 57 (1870; Ball. J. A. S. Beng. xli. p. 277 (1872); id. Str. F. i. p. $5^{8}$ (1873; Hume, Sti.

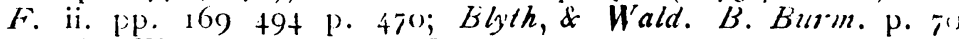
(1875); Wardlw Ramsay, Ibis, 1870 , p. 456 Hume it Davis. Str. F. xi. p, 75 1878; Sharfe, Ibis, 1879, p. 249 id. H. $\because$. S. 1879 , p. 3.: ; Hume, Sir. F. viii. Pp. 49, 85 (1879; Sharte P. Z. S. 1881 , p. 793; Jenig, Proc. U. S. Niat. Iifu. . vi; p. 310 (1883); Oates, Handb. B. Br. Brirm. ii. p. 81 (1882); Guillem. P. Z. S. 1885, pp. 407, 5+8; Blakst. Anent. Lisi. B. Japan, p. It (1884); Secb. Ib.s, 1887, p. 176; Stejn. Proc. U. S. Nat. Mus. x. p. 403 (1887); Hume, Str. F. xi. p. 44 (1888); Tacz. P. Z. S. $188^{3} 8$, p. 154; Sharpc, Ibis, $1888, \mu$. 197 ; Styan, Ib.s, 1889 , p. 446; Evereth, P. L. S. 1889, p. 226; id. Journ. Strqits Branch. R. Asiat. Soc. 1889 , p. I60; Sharpe, Ibıs, 1590, pp. 20, 283; Whitehead, t. c. p. 46; Secbohn, B. Japan, Emp. p. I73. (I8go); Steere, List. Birds. \& Mamm. Steere. Exped. Philipp. p. 11 (1890); Sharpe, Cat. B. M. 17, 1892, p. 208.

Halcyon coroinaidelicus, Vigors, App. Mem. Rafft. p. 654 (18,50; Stoliczka, J. A.S. Beng. xxxix. p. 296 (1870); Walden, Ibis, 1 's $71, \mathrm{p} .165$.

Dacelo coromanda, Less. Traite, p. 247 (1831); Schl. Mus. PaysBas, Aicud. Revue, p. 16 (1874;.

Halcyon lilacina, Szalns. Classif. B. ii. p. 335 (1837); Bp. Consp. i. p. ${ }_{156}$ (1850); Motley \& Dilize. Nat. Hist. Labuan, p. 1.3 (1855); Sic. P. Z. S. 1863, p. 213 .

Alcyun calipyga, Liodgs. Ic ch. ired. in Brit. Mus., Passere., pl. 16. n." 769; idt. in Gray's Zool. Miss. p. 82 (1844).

Halcyon coro...andra, Graj, Cat. Mamm. etc. Nepal pres. Hodsg. p. $56($ i $8+6)$.

H. coromanceliana, Gray, Gen. B. i. p. 79 (1846); id. List. Fisssir. Brit. Mus. p. 55 (18+8); Horsf. \& Moore, Cat. B. Mus. E. I. Co. i. .p. 126 (1854); Jera. B. Ind. i. p. 227 (1862); Swinh. Ibis, $186_{3}$, p. 259; id. P. Z. S. 1863 , p. 333; id'. P. Z. S. 1871, p. 347; Gray, Hand-l. B. i. p. 91, n. ${ }^{\circ}$ log9 (1869); Blakist. \& Pryer, Ibis, 1878, p. 230; Seebohm, Ibis, 1879, p. 24. 
II. coromanda major, Temm. \& Schl. Faun. Japon. p. 75, pl. $39(1842)$.

11. coromanda minor, Temm. \& Schl. Faun. Japon. p. 76 (18+2).

H. coromander, Blyth, Cat. B. Mus. As. Soc. p. 47 (18+9).

H. schlegeli, $B p . C u s p$. i. p. 156 (1 850$)$.

Callialcy on coromanda, Reichenb. Handb. Alced. p. 15, Taf. to5. fig. 3092 (185I); Bp. Consp. Vol. Ans. p. 9 (185t); Wald. Ibis, ${ }_{1873}$, p. 302; Saluad. Ucc. Born. p. 793 (1874); Walit. Trans. Z. S. ix. p. I55 (1875); P. Z. S. 1878 , p. 944 David \& Oist. Ois Chine, p. 75 (1877); Muller, J. f. O. 1882,1 . 398; Vorderm. Nat. Tijdschr. Neverl. Ind. xlvi. p. I I (I886).

C. lilacina, Bp. Consp. Vol. Anis. p. 9 (I854).

(. schlegeli, Bp.t.c. p. 9 (I 854$)$.

Dacelo coromandeliana, Schl. Mus. Pays-Bas, Alced. p. 25 (1863, pt.); id. Vög. Neterl. Ind. pp. 24, 56, pl. 8. fig. (1864).

D. cora :andeliana major, Schl. Mus. Pays-Bas, Alced. p. 25 (1863).

Halcyon rufa, Wall. P. Z. S. 1862 , p. $33^{8}$.

H. coromanda, var. rufa, Brüggem. Abhandl. nat. Ver. Bremen, v. p. 54 (1876).

Callialcyon rufa, Wald. Trans. $Z$. S. viii. p. 44 (1872); Salvad. Ann. Mus. Civ. Genov. vii. p. 653 p. (1877); Meyer, lbis, 1879 , p. 62; Blasius, Zeitschr. ges. Orn. iii. p. 90 (1886); id. Ornis, iv. p. 572 (1 888 ).

Halcyun coromanda rufa, Stejn. Proc. U. S. Nat. Mus. x. p. 403 ( 1887 ).

Hab. Luzón, Manila, Laguna, Cavite, Cagayan, Guimaras, Mindanao, Zamboanga; Jara, Sumatra, Formosa, Borneo, Célebes.

22. H smyrnensis, (Lin.) $\sigma \circ \mathrm{N}$ v Salacsac.

(M. S T.)

The smirna Kingfisher, Albin, Nat. Hist. B. iii. p. 27, pl. 27 ( 1740 ).

T. Large Kingfisher from Bengale, Albin, t. c. p. 27, pl 28 (1740).

'T'. Great Kingfisher from the River Gambia, Edwards, N. Hist. $B$. i. p. 8, pl. 8 (1 743$)$.

Le Martin-pescheur de Smyrne, Bris. Orn. iv. p. 499 (1760).

L. Grand Martin pescheur de Bengale, Bris. t. c. p. 50r (1760).

Alcedo smyrnensis, Linn. Syst. Nat. i. p. 18г; Gm. Syst. Nat. i. p. $456(1788)$; Eyton, P. Z. S. 1839 , p. гог.

Martin-pêcheur de la côte de Malabar, Daubent. Pl. Enl. riii. pl. 894 .

Smyrna Kingfisher, Lath. Gen. Syn. i. pt. 2, p. 6r 5 (1782).

Great Gambian Kingfisher, var. A, Lxth. t. c. p. 616 (1782).

G. Bengal Kingfisher, var. B. Lath. t. c. p. 616 (i782).

Alcedo fusca, Bodd. Tabl. Pl. Enl: p. 54 (i 783 ).

Dacelo smyrnensis, Less. Traité, ii. p. 246 (I 831 ).

Halcyon smyrnensis, Steph. in Shaze's Gen. Zosl. xiii. p. 99 (1826); Sykes, P.Z. S. 1832, p. 84; Mc Clell. P. Z. S. 1839, p. I56; Jerd. Madr. Jouru. xi. p. 230 (1840); Strickl. Ann. \& Mag. Nat. Hist. ix. p. 441 (1842); Gray, Cat. Mamm. etc. Nepal. pres. Hodgs. p. 57 (1846); id. Gen. B. i. p. 79 (I846); id. List. Fissir. Brit. Mus. p. $55(1848)$; Blyth, Cat. B. Mus. 
As. Boc. P. 47 (1849); Bp. Consp. i. p. 155 (1850), Crss. (at. Halc. Philal. Mus. p. 6 (1852); Moore, P. Z. S. 18.5t, p. 288; Horsf. \& Moore, Cat. B. Mits. E. I. Co. i. p. I2: (1854); Sainh, Ihis, 1860 , p. 48, 1861, p. 21; Irby, Ibis, 186i; p. 227; 7rist). Ibis, 1856 , p. 86; Blyth, t. c. p. 348; Grav. Hand-l. B. i. p. 91, n..$^{\circ} 1095$ (1869); Sharpe, Mor.ggr. Alceit: p. I6I, pl. 59 (18;0); Siinh. Ibis, 1870, p. 93; Blanf. t. c. P. 465 (Pegu); Swenh. P. Z. S. 1871 , p. 3+7; Blanf. I. A. S. Beng. xl. p. 37 I (1871); Holdsw. P. Z. S. 1872, p. 424; Stsliczk.x, J. A. S. Beng. xli. p. $232(1872)$; Hayes Llayd. Ibis, 1873 p. 407; Huine, Nests \& Eggs. Ind. B. p. 105 (1873); id. Str. F. i. Pp. IO3, I1 5, I68 (1873); Adame, t. c. p. 372; B.z!!. Str. F. ii. p. 387 (1874); Hume, t. c. p. 470 ; Blyth. \& Wald. B. Burm p. 70 (1875); Hume, Str. F. iii. p. 5i (1875: Pegu); Butler, t. c. p. 456; id. Str. F. iv. p. 34 (1876); Fuirb. t.c. p. 254; Armstr. t. c. p. 306; Hume, t. c. p. $3^{8} 3$; Blanf. Ens'. Persia, ii. p. I 2 I (1876); Hume, Str. F. v. p. 19 (1877); Oxtes, t. c. p. 143 ; Fairb. t. c. p. 394; Hume, \& l)avison, Str. F. vi. p. 74 (1873); Hume, Str. F. p. 54 (1878); Davidson \& Wenden, t. c. p. 77; Murray,t.c. p. II9; Ball, t. c. p 2ot; Cripps, t. c. p. 260; Anderson, Zool. Exped. Y'un.un.n, p. 579 (1878); Hume, Str. F. viii. pp. 49, 85 Scully, t. c. p. 238; Doig, $t$ c. p. 377; Legge, B. Ceylon, p. 298 (1879), Butler, Cat. B. Sind etc p. 16 (1879), id. Cat. B. S. Brnt. Pres. p. 19 (1880); Vidal, Str. F. ix. p. 49 (188(s); B.ngh.rm, $t$ c. p. 154; Danf Ibis, 1880, p 83; Bu-Str. F. ix. p. 382 (1881); Kelham, Ibis, 1881 , p. 380 ; Reid, Str. F x. p 21 (1881); Davidson, Str F. x. p. 2961882 ; Dizvison t c. p $35 \mathrm{r}$ I883; Oates, Handb. B. Br. Burm. ii. p. 82 (1883); Tristr. Fiann. \& Flor. Palest p. 87 (1884); Murray, Vertebr. Finun. Sint., 1) 110 (1884); C. H. T. Marshall, Ibis, 1884, p. 409; Swinh. \& Barnes, Ibis, 1885 , p. 61 Radde, Ihis, 1885, p 237; Shar. pe, Ibis, 1886 , p. 488 (Fao); Taylor, Str. F. x p 457 (1887); Teery, t. c. p 47 I (Palanis); Styan, Ibis, 1887 , p. 230; Gmi$11: m$. Ibis, 1888 , p. I02; Hume, Str. F. xi. p. 441888 ; St. John, Ibis, 1889, p. 157; Oates, ed. Hume's Nests. \& Eggs. iii. p. 15 (1890); Sharpe, Cat. B. M. 17 1892 p. 222.

Alcyon smyrnensis, Hodgs. Icon. ined. Brit Mus., Passeres, pl. I 7 fig. I (no. 590); id. in Gray's Zool. Misc. p. 82 (1844).

Halcyon fusca, Gray, Gen. B. i. p. 79 (18+6); id. List Fissir. Brit. Mus. p. 6 (1852); Horsf, \& Moore, Cat. B. Mus. E. 1. Co i. p. I 25 (185t); Jerd. B. Ind. i. p. 22+ (1862); Beavan, Ibis, 1865 , p. 408; Blyth, Ibis, 1868 , p. 348; Walden, Ibsis, 1871 , p. ${ }_{165}$; Cordeaux, Ibis, 1888 , p. 225, (Dras).

Entorrothera smyrnensis, Reichenb. Handb.; Alced. p. I 3 ( 1851 ). E. fusea, Reichenb. $t$. $c$ p. 12, Taf. 404. figs. $3088-89$ (1851). Entomobia smyrnensis, Cab. \&. Heine, Mus. Hein. Th. ii p. I55, note (1860); David \& Oust. O s. Chine, p. 76 (1877); Salvad. Ann. Mus. Genov. iv. p. 857 (1 886, Blamo).

I. fusca, Cab. \& Heine, t. c. p. I 55 (1860).

Dacelo fusea, Schl. Mus. Pays-Bas, Alced. p. 28 (I863); id. Rez'lue, p. I8 (1874); Heugl. Orn. N. O.-Afr. p. I88 (i 869).

Hab. Luzón, Cavite, Indan; China. 
23. H. gularis, (Kشhl; $\sigma .0 \mathrm{~N}$. Sulacsac.

I.: Martin-pescheur bleu de Madagascar, Briss. Orn. iv. p. 490 , pl. xxxviii. fig. $2(r ; 60)$.

Ciran Martin-Pécheur de Madagascar, Daubent. Pl. Enl. viii. pl. 232.

Alcedo smyrnensis, var $\beta, G m$. Syst. Nat. i. p. 456 (1 788 .

A. gularis, Kuhl, Buffon. \& Daubent: Fig. Av. Col. Nom. Syst. p. 4 (1820).

A. rufigirostris, Kittl. Kupf. Vög. p. Io, 'Taf. It. fig. 2 (1832); Meyen, Beitr. p. 94 (Ir 34 ).

A. melanoptera (nec Horsf.), Temm. Tabl. Méth. p. 75 (18z6): Halcyon ruticollis, Swains. Class. B. ii. p. 335 (1837).

Alcyon smyrnensis, var. albogularis, Blyth. J. A. S. Beng. xii. p. 99 (I 843 ), xiii. p. 394 (1 884 ).

11. gularis, Grav, Gen. B. i. p 79 (18+6); Kaup, Fam. Es:s: p. 8 (1848); Bp. Consp i p I55 (1850); Gould, B. Asia. i pl. (1861!; (jr.zy, Hant-l B. i. p 91, n. 1095 (1869); Sharo:, Monogr Alced. p. 165 , pl. 6o (187o); Wald. \& Layard, Ib s, 1872 , pp. 96, 97; Sharpi, Trans Linn. Soc. (2) i p. 317 (1876); Steere, List Birds \& Mamm Steere Exped Philipp. P) II (1890; Shurpe, Cat. B. M. 17, 1802. p. 227.

Entomothera gularis, Reichenb. Handb., Alced. i. p. I3, Taf. for. fig. 3082 ( $186 \mathrm{r})$.

linti. obia gularis, Cab. Mits Hein. Th. ii. p. I55 (1860); Wald Tr Z.S. ix p. I54 (1875); Treedd. P. Z. S 1877 . p $53^{8}$ (Mindanao), p. $54 \mathrm{I}$ p 690 p. $75^{8}$ p. 823; id. P. $Z$. S. 1878, p 282 p 34 p. 379 p. 943.

Alecdo fusa, (nec Bodd.) Martens J.f. O. 1866, p. 16; id. Preuss. Exped. Ost.-Assien, p 189 (1867)

1)acelo gularis, Schl. Mus. Pays-lsas, Revue. Alced. p y9 (1867). $H a b$. Luzón, Manila, S. Mateo, Montalban, Cavite, Indán, Bataan, Orani, Bulacán, Lólonboy, Lngona, Los Baños, Cagayán, Pamplona, Mindoro, Baco, Cebú, Negros, Sibulan, S. Bernardins, Valencia, Panáy, Iloilo, Guimarás, Samar, Mas!ate, Marinduque, Mindanao, Zamboanga, Panaon, Surigao.

24. H pileatus, (B)id ) $\sigma 0$ N. v. Salacjac.

(M. S. T.

Martin-pecheur de la Chine, D.xubent. Pl. Enl. vii. pl. 673.

J.e Martin-pêcheur á coiffe noir, Ruff. Hist. Nat. O:s. vii. p. I I9 ( 780 ).

Illack-capped Kingfisher, Luth. Gen. Syn. i. pt. 2, p. $62+(\mathrm{I} 782)$.

Alcedo pileat ı, Bodd. Tabl. Pl. Enl. p. 41 (1783).

A. atricapilla, Gm. Syst. Nat. i. p. 453 ( 1780 ); Lath. Ind. Orn. i. p. 251 , (1790); Raffl. Trans. Lrnn. Soc. xiii. p. 293 (1820).

Halcyon atricapilla, Vig. Mam. Raffl. p. 655 (1830); Blyth, Cat. B. Mus. As. Soc. p. 47 (1849); Bp. Consp. i. p. I55 (1850); id. Consp. Veluer. Anis. p. 9 (1854); Horsf. \& More, Cat. B. Mus. E. I. Co. i. p. I 24 (1854); lerd. B. Ind. i p. 226 (1852); Steliezkx, J. A. S. Beng. xxxix. p. 296 (1870); Hum, Ib s, 1870 , p. $43^{8}$ Blanf. t. c. p. 465 Ga!l!d, B. Asia, i. pl. 45 (1850); Pels. R.s. Novira, Vög. p. 9t (18'5); B.t. Str. IF. 
i. p. 58 (1873); IIume Str. F. ii. p. 167 (1874); ad. Str. F. iv. p. $(1876)$.

Alcelo brama, less. (ant. Zool. pl. 8 (1830).

Dacelo atricapilla, Less Tratte, p. $246(1831)$.

Alcedo puella, Lion Kreling, teste Recchenb J. f. O. 2854, p. 149.

Ilalcron pileata, Gray, Gen. B. i. p. $79(18+6)$; id. List. Fissir. p. $54(18+8)$; Cass. Cat. Halc. Phil. Mfus. p. 5. (1852); Sharpe, Monogr. Alced. p. $105 ;$ pl. 62 (1868); Gray, Hand-1. B. i. p. 91, n." 1097 (1809); IVald. Ibis, 18 j1, p. 165; Swini. Ibis, 1870, p. 93; id. P. Z. S. 1871 , p. 3+7; Holdsw. P. Z. S. $18^{2} 72$, p. +24; Hume, Str. F. ii. p. 470 (1874); iii. p. 54 (1875); Armstr. Str. F. ir. p. zo6; Sharpe, P. Z. S. p. 101; Blyth. \& Wald. B. Burn. p. 70 1875; Sharpe, Ibis, 1876, 1. 33; Tweedd. Ibis, 1877 , p. 296; Wardlaw Ramsay, t. c. p. 45b; Hume, \& Dawison Str. F. vi. pp. 499 (187\%); Ball. Str. F. vii. 1. 235 (1878); Sharpe, P. Z. S. 1879, P. 331; itt. Ibis, 1879 , p. 249; Legge, B. Céglon, i. p. 301 (1879); 11ume, Str. F. viii. p. to p. 85; Bingham, t. c. p. r93; Vidal, Str. F. ix. 49 (1880); Bingham, t. c. p. 154; Butler, Cat. B. S. Bomb. Pres. p. I9 (1880); id. Str. F, ix p. 382 (1881); Kelham, Ibis, 1881 , p. 380 ; Oates, Handb. B. Brit. Burn. ii. p. 83 (1883); Nichols. Ibis, 1883 , p. 243; Seeb. Ibis, 1884, 1. 26 I (Kiang R.); Guillem. P. Z. S. 1885 , p. 548; 7acz. P. Z. S. 1887 , P. G(0); Styan, Ibis, 1887 , p. 237 ; Taca. P. Z. S. 1888 , p. 46.2; Sharpe, Ibis, 1888 , p. 197; Whitehead, Ibis, 1800 , p. 45; Steere, List. Birds \& Mamm. Steere Exped. Philipp. p. I1 (1890); Sharpe, Cat. B. M. 17. 1892 p. 229.

lintomothera pileata, Reichenb. Handb., Alced. p. I 5, Taf. 401, figs. $3080-80$ ( 1851 ).

Eintomobia pileata, Cab. \& Heine, Mus. Hein. 'Th. ii. p. 155 (1860); Salvad. Ucc. Born. 1. I02 (1874); Wald. Trans. Zool. Soc. ix. p. ${ }^{1} 54$ (1875); David \& Oust. Ois. Chine, p. 75 (1877); Meyer, Ibis, 1879, p. 61; Tacz.J. f. O. 1881 , p. 180; Mïller, J. f. O. 1882 , p. 398 ; Vorite m. Nat. Tijdschr. Nederl. Ind. xlvi. p. I I (1885); Blasius, Ornis. iv. p. 307 (1888).

Incelo pileata, Schl. Mus. Pays-Bas, Alced. p. 27 (1863); id. Vög. Nederl. Ind., Alced. pp. 22, 54, pl. ix. fig. 2 (1864); id. Mus. Pays-Bas, Rev. Alced. 1. is (1874).

Hab. Balabac, Cuyo, Calamianes, Basilan; China, Sumatra, Java.

25. H. albicillus, (Cuv.) N. v.

(M. S. T.)

Alcedo albicilla, Cuvier, M. S. S.; Dumont, Dict. Sc. Nat. xxix. 1. 273 (1823); Pucher. Rev. Zool. 1853. p. 388.

Sauropates albicilla, Salv. Oru. Papuasia, i. p. 470 (1880).

Halcyon albicillus, Sharpe, Cat. B. M. I7. p. 249 (1892).

Hab. Islas Marianas.

26. H. wirchelli, Sharpe, ơ N. v. Bintis.

(M. S. ')

Halcyon winchelli, Sharpe, Tr. Lin. Soc. i. p. 318, pl. 47 (1876); id. Cat. B. M. 17. p. 255, (1892); Tweedd. P. Z. S. 1878, p. $94+$; Steere, List. Exped. Philipp. 1890, p. I I.

H. alfredi, Oustalet, Le Nat. 1890, p. 62.

Hab. Samar, Borongan, Mindanao, Zamboanga, Basilan, Joló. 
27. H. cinnamominus, Swnins. N. v.

(M. S. T.)

Ilalcyon cinnamomina, Szwaison, Zool. Illustr. ser. i. rol. ii. pl. 67 (1821); Vig. \& Horsf. Tranzs. Linn. Soc. xt. p. 206 (1827); Gould, Syn. B. Austr. part. iii. (1838); Gray, Gen. B. i. p. 97 (1846); id. B. Trop. Tsl. Pacific Ocean, p. 5 (1859); id. Hand-l. B. i. p. 93, n" 123 (1869); Sharpe, Monog. Alced. p. 21 3, pl. 80 (1870;; Finsch, Jowrn. Mus. Godeffr. Heft. xii. p. $20(1876)$; Sharpe, Cat. B. M. 17, 1). 259 (1892).

'Jodirhamphus cinnamominus, $B p$. Consp. i. p. 157 (1850); Reicheub. Handb., Alced, p. 32, Taf. 420, fig. 3140 (1851); Cass. Cat. Halc. Philad. Mus. p. $12(1852)$; id. U. S. Expl. Exped., Birds, p. $226\left(185^{8}\right)$.

Sauropatis cinnomamina, Cab. \& Heine, Mus. Hein. Th. ii. p. $159(1860)$.

Dacelo cinnamomina, Schl. Mus. Pays-Bas, Alced. p. 39 (1860); id. Rewe, Alced. P. 29 (1874).

Nlcedo ruficeps, Cuvier, MSS. in Mus. Paris; Dumont, Dict. Sci. Nat. xxix. p. 273 (1823); Pucher. Rev. et Mag. de Zool. 1853 , p. 367; Hartl. J. f. O. 1855, p. 423 .

Dacelo ruficeps, Lesson, Traité d'Orn. p. 247 (1831.

Halcyon ruficeps, Gray, Hand-l. B. i. p. 93, n." II 22 (1869). Hab. Islas Marianas.

\section{H. mediocris, Sharpe, N. v.}

11. cinnamomina (no Vig.), Finsch, J.f. O. 1880, p. 285 .

11. mediocris, Sharpe, Cat. B. M. 17, p. 260 (1892).

Hab. Ponapé, Carolinas.

29. H. chloris, (Boct.) o 0 N. v. Casaycasay.

(M. S. T.)

Slartin-pecheur á collitr blanc des Philippines, Sonn. Voy. Now. Guin. p. 67, pl. $33(1776)$.

Martin-pècheur á tete verte du Cap de Bonne Esperance, Daulent, Pl. Enl. viii. pl. 783 . fig. 2.

L. Martin-pecheur á tete verte, Buff. Hast. Nat. Ois. vii. p. igo $(1780)$.

i.: Martin-pechror á collier blanc, Buff. t. c. 192 (1780).

Girecn-headed Kingtisher, Lath. Gen. Syn. i. pt. 2, p. 620 (1782).

Aleedo chloris, Bodd. Tabl. Pl. Enl. p. 49 (i 783 ).

A. collaris, Scop. Del. Flor. et Fann. Insubr. ii. p. 90 (1786); lath. Ind. Orn. i. p. $25^{\circ}$ (1790); Kittl. Kupf. Vög. i. pl. 14. tig. I (1833); S. Müll. Verh. Land-en Volkenk. p. 2 I (183944); Rosenb. Malay. Arch. p. 364 (1878-79).

Alcedo sacra, var. e, Gm. Syst. Nat. i. p. 453 (1788, ex Sonn.)

A. clor cephala, Gm. S. N. i. P. 454 ( 1788 , ex Daub.); Lath. Ind. Orn. i. p. 240 (1790); Shau, Gen. Zool. viii. p. 77 (1811); Cuvier, Regne Anim. i. p. 417 (18.7); Vicill. Nowv. Dict. d'Hist. Nat. xix. 1, 417 (1818); Horsf. Tr. Linn. Soc. xiii. v. 174 (1821); Ramfft. t. c. pt. 2, p. 293 (1821); Reichenb. J. f. O. 1854, D. 150 . 
Haliyon collaris, Szuxims. Zool. Illuslr. i. p. 27 (1820); S. Mï'l. Verh. Land-en Volkenk. pp. I74, 175 (1839-44); Gray, Gen. B. i. p. $79(1846)$; id List Fissir. B) rit. Mus. p. $56(1848)$; Horsf. \& Moore, Cat. B. Mus. E. I. Co. i. p. I27 (1854'; Gray, P.Z. S. 1858, Pp. 171, 190; id. Cat. B. New Guin. Pp. 19, 55 (1859); id. P. Z. S. 1860 , p. 311; id. P. Z. S. 1861 , p. 433 ; Scl. P. Z. S. 1863, p. 213 ; Wall. t. c. pp. 23484.

H. sacra, var. F. Bonn. et Vieill. Fnc. Meth. i. p. 295 (1823).

H. chlorocephalus, Steph. in Shav's Gen. Zool. xiii. p. Ion (1826); Swains. Classif. B. ii. p. 335 (1837); Hart. Ibis, 1854 , p. 339 .

Dacelo chlorocephalus, Less. Traitè p. 246 (1831).

Halcyon chloris, Gray, Gen. B. i. p. 79(1846); Motley \& Dillu. Nat. Hist. Labuan, p. I 2 (1855); Pelz. Reis. Novara, p. $4 t$ (1865); Gray, Hanctlist B. i. p. 92, n. ${ }^{\circ}$ I 16 (1869); Sharpe, Monogr. Alced. p. 229, pl. 87 (1870); Walden \& LaIard, Ib s, 1878 , p. 96; Hume, Str. F. i. p. 45I (1873); Buller, Ibis, 1874 , p. II 4 ; Bruggem. Abhandl. nat. Ver. Bremen, $\therefore$ ' p. 73 (1876); Pelz. Verh. z-b. Ges. Wien, xxiv. p. $718(1876)$; Sharpe, Trans. Linn. Soc. (2) i. p. 319 (1876; Sharpe, P.Z. S. 1879 , p. 332; i.t. P. Z. S. 1881 , p. 793; Nichols Ibis, 1881 , p. 142 Java 1883 , p. 89; Sharpe, Ibis, 1888 , p. 197; Everett, $P$. Z. S. 1880 , p. 225; Whitehead, Ibis, 18 90, P. 45; Sharpe, t. c. pp. 90, 283; Steere, List. Birds \& Mamm. Exped. Philipp. p. I I (1890); Sharpe, Cat. B. M. 17. p. 27.3, (1892).

Todirhamphus collaris, Blyt, Cat. B. Mus. As. Soc. p. 48 (1849); Bp. Consp. i. p. I 56 (1 850$)$; Reichenb. Handb., Alced. p. 30, Taf. 4r7. fig. 3129, Taf. 403. B. fig. 3390 (1851); Bp. Consp. Volucr. Anis. p. 9 (1854); P. Z. S. 1862 , p. 338; id. P. Z. S. I 863 , p. 23 Bourn.

'Todirhamphus chlorocephalus, Bp. Consp. i. p. I56 (185); Reichenb. Handb, Alced. p. 3I, 'Taf. 417. fig. 3128 (1851); Bernst. J. f. O. 18859 , p. 189.

T. chloris, Cass. Cat. Halc. Philad. Mus. p. Io (1852); id. U. S. Expl. Exped. p. 203 (1858); Pelz. Reis. Novara, Vög. p. 44 (1865); Meyer, Ibis, 1879, p. 6r.

Dacelo chloris, Schl. Handl. Dierk. p. 32 (1857); id. Mus. PaysBas, Alced. p. 32 (1863); id. Vög. Nederl. Ind. pp. 26, 58, pl. x. figs. 3, 4 (1864); Finsch, Nou-Gun? p. I6I (I 865$)$; Schl. Mus. P.-B. Alced. Rewe, p. 2 I (1874); Buttik. Notes Leyden Mus. viii. p. 62 (1886).

Sauropatis chloris, Cab. \& Heine, Mus. Hein. 'Th. ii. p. I60 (1860); Salvad. Ucc. Born. p. I()3 (1874); Walden, Trans. ZZ. S. ix. p. I 55 (1875); Salvad. Ann. Mu:. Genov. vii. p. 6.53 (1875); viii. p. 374, ix. p. 53 (1876); x. pp. 126305 (1877); Tweedd. P. Z. S. 1877 , p. 690; id. Ibis, 1877 , p. 296; id'. P. $Z$. S. 1878 , p. ro8, p. 282, p. 34, p. 709; $i d . P$ P. $Z$. S. 1879 , p. 69; Salvad. Ann. Mus. Genov. xix. p. 646 (1879; id. Orn. Papuasia etc. i. p. 470 ( 1880 ) Tweedd. Rep. Zool. Coll. H. M. S. Challenger, ii. p. I3 (1881); Salvad. $t$. c. p. 76; Meyer, Verh. z.-b. Ges. Wien. xxxi. p. 763 (г881); p. 770. p. $772 ;$ Blas. u. Nehrk. Verh. z.-3. Ges. Wien, xxxii. p. 121; (1883); Kutter, J.f. O. 1883,1 , 301; Sel P. Z. S. 
1883 , p. 200; Meyer, Zeitschr. Ges. Orn. i. ए. 276 (1884; id. Sitz. u. Abhandl. Ges. Isis Dresden, 1884 , p. I9; Fo:P. Z. S. 1884, p. 432; Pleske, Bull. Acad. St. Petersb. xxxix. p. 256 (1884). Guillem. P. Z. S. 1885 , p. 216, p. 502, 1. 547, p. 568; Voderm. Tijdschr. Nederl, Ind. xliv. p. I (1886); p. II; Blasius, Zeitschr. ges. Orn. iii. p. 90 ( 1886); id Ornis, iv. p. 307 p. 573 (1888); Saliad. Aggr. Orn. Papuasia, i. 1). 57 (1889); Vorderm. Nat. Tijdsçhr. Nederl. Indie, I, Aff. t, p. 439 (1890).

Hab. Luzon, Manila, Mariquina, Montalban, Navotas, Laguna, Los Raños, Cavite, Indan, Mintoro, Baco, Marinduque, Panay, Iloilo, Guimarás Negros, Valencia, Cebú, Dumaguete, Leyte, Amparo, Samar, Paric, Bohol, Dinagat, Paragua, Mindanao, Surigao, Butuan, Camiguin, Basilan, Joló, Cagayan de: Joló; Java, Sumatra, Gilolo, Borneo.

30. H. humii, Sharpe, ơ N. v. Casaycasay.

(M. S T.)

Alcyon chloris, Sharpe, Monogr. Alced, 1870 , pt p. 229; Hume, Str. F. viii. p. 49 (1879); Kelham, Ibis, 1881, p. $3_{81 .}$

Todirhamphus collaris, Moore, P. Z. S. 1854 , p. 269.

II. humii, Sharpe, Cat. B. M. 17, p. 281 , pl. viii. 1892. Hab. Mindoro Baco; Sumatra.

3r. H. variegatus, (F. et Puch.) OD:O N. v. Bintis.

(M. S. T.)

Actenoide variée, Hombr. \& Jacq. Vey. Pde. Sud. Pl. 23. fin. $2(1846)$.

A. variegata (nec Bonn.), Jacq. \& Pucher. Vog. Pde. Sud, Zool. iii. p. 18 I ( 1853$)$.

1. hombroni, Bp. Consp. i. p. I57 (1850); Reichenb. Handb., Alced. p 36, Taf. 422. fig. 3147 (1851); Bp. Consp, Voluer. Anis.. P. 9 (1854); Walden, Tr. $Z$. S. ix. P. I55 (1875); Gould, B. Asia, i. pl. 40 (1877); Tweedd. P. Z. S. 1878, p. 994; Steere, List. B. \&. Mamm. Steere. Exped. Philipp. p. I I (i 890$)$.

Ilalcyon hombroni, Gray, Hand-t. B. i. p. 93, n." I 36 (I 869); Sharpe, Monogr. Alced. p. 221, pl. 84 (1871); id. Trans. Linn. Soc. 2nd. ser, Zool. i. p. 318 (1876); Sharpe, Cat. B. M. 17. p. 284 (1892).

Hab. Mindanao, Zamboanga, Samar, Paric.

2. H. lindsayi, (Vig.) o o N. v. Casay-casay.

(M. S. T)

Dacelo lindsayi, Vig. P. Z. S. 1831 , p. 97; Eyd. \& Souleyct, Voy. Bonite, Zool. p. 100 (1841; Schl. Mus. Pays.-Bas, Alced' p. 23 (1 863 )

D. lessoni, Vig. P. Z. S. 1831 , p. 97.

Halcyon lindsayi, Gray, Gen. B. i. p. 79, pl. 27 (1846); id. Sist. Fissir. Brit. Mus. p. 52 (1848); Bp. Consp, i. p. I 53 (1 850 ; Gray, Handl. B. i. p. 93, n." II 35 (1869; Sharpe, Monogr." Alced. p. 217, pl. 82 (1871); id. Cat. B. M. 17. p. 286 (1892).

Paralcyon lindsayi, Reichenb. Handb., Alced. p. 36, 'Taf. $42 \mathrm{I}$. figs. 3143-44 (1851); Bp. Consp. Veluer. Anis. p. 9. (1854). 
Acienoides lessoni, Hartl. J. f. O. Isit, p. 64 .

Astacophilus lindsayi, Cab. \& Heine, Mias. Hein. Th. ii. p. 10? $1860)$ !.

Alcedo lindsayi, Martens, J. f. O. 1 sof, p. 18 ; id. Preuss. E.xp. Ost. Asen, p. $\left.189\left(1 \mathrm{~s}^{2}\right) 5\right)$.

Actenoides lindsayi, Walden, Tr. Z. S. ix. p. 156, (1875); Gould. 13. Asia i. pl. +1 (187j); S'erre, list B. \& Mamm. Steere Exped. Philipp. p. i (1890).

Hab. Luzón, Manila, Malabon, S. Mateo, Montalban, Laguma, Los Baños, Calauan.

33. H. meseleri Sharpe o N. v. Bintis.

(M. S. T.)

Actenoides moseleyi, Steere, List. Exped. Philipp. 1sqo, p. 1. H. moseleyi, Sharpe, Cat. B. M. 17. p. 288 (1893).

Hab. Isla de Negros.

6 Gén. TANYSIPIERA, Vigors.

:4. T. dea, (Lin) ơ N.v.

(M. S. T.)

? Martin-pècheur á long brins, Valentyn, dmboina, p. zor, 'Taf. iii.

Le Martin-pescheur de Ternate, Briss. Orn. iv. p. 525, pl. 4o. fig. 2 ( I 760$)$.

Alcedo dea, Linn. Syst. Nat. i. p. I 8 r (1766); Gm. Sist. Nat. i. p. $45^{8}$ ( 1788$)$.

Martin pècheur de Ternate, Daubent. Pl. Enl. pl. iı6.

Tanysiptera dea, Vibors, Tr. Linn. Soc. xir p. 433 (1825, pt); Gray, Gn. B. i. p. $78(18+5) ; B p$. Consp. i. p. $157\left(185^{\circ}\right.$, pt.); Reichens Handb., Alced p. 42 Taf. 4a5. tig. 34 ğ (i851). Hab. Molucas.

Fam, MOMÓTIDOS.

1. Gén. hylomanes, licir.

I Hy. momotula, Litht. $\sigma$ N. v.

(M. S. 'T')

H. momotula, Licht., Sharpe, Cat. B. M. 171892, p. 332.

Hab. Guatemala.

\section{Suborden: BUCEROTES.}

\section{Fam. BUCERÓTIDOS.}

1. Gén. HLDROCORiX, Briss.

I. H. hydrocorax. (Lin.) $\sigma \circ$ N. v. Calao, Calo.

(M. S. T.)

Le Calao, Briss. Orn. iv. p. 566, pl. xlv. (1760).

Le Calao des Moluques, Daubent. Pl. Enl. n." 283 ; Buff. Hist. Nat. Ois, vii. p. 147 ( 1780$)$. 
Philippine Hornbill, var. A, Lath. Gen. Syn. i. p. 345 [adill'| (178I).

Indian Hornbill, Lath. Gen. Syn. i. p. 35 [ [immaturc] (г78̈).

Le Calco roux, Levaill, Hist. Nat. d'Ois. l'Amer. i. p. I 3, pl. vi. (I 80 I).

Le Calao á casque plat, Levaill. Hist. Nait. O.s. d'Afr. i. p. 127 , pl. 240 (1806).

Buceros hydrocorax, Linn. Syst. Nat. i. p. I53 (1766); Forst. Ind. Zool. p. 40 ( $178 \mathrm{r}) ; G \mathrm{Gm}$. S. N. i. p. 359 (1788); Latí. Ind. Orn. i. p. I4t (I790); Shaw, Gen. Zool. viii. p. 3 I (18г1); Vieill. N. Dict. d'Hist. Nat. iv. p. 597 (1816); Temm. Pl. Col. ii. p. 73, pl. 283 (1824); Wagl. Syst. Av. Buceros, n. 7 (1827); Less. Traté Orn. p. 254 (1831); Gray, Gen. B. ii. p. 399 (I847); Blyth, Cat. Birds. Mus. As. Soc. p. +3 (1849); Bonap. Consp. Gen. Av. i. p. 89 (1850); Schl. Mus. Rais-Bas, i. Bucces p. 2 (1862); Von Mart. J. f. O. p. is (i 866); Walden, Trans. Z. S. ix. p. I64 (1873); Tweed. I'. Z. S. r 877 . pp. $543 \& \cdot 692$; Steere, List Birds \& Mamm. Philippines, P. I 3 (I890); Hartet, Cat. Mus. Senckeilb. Frankf. p. I 40 (1891); Ogilvie, Cat. B. M. 17. p. 358, (1892).

Platyceros hydrocorax, Cab. \& Heine, Mus. Hein ii. p. I7t (1850).

Buceros hydrocorax, Gray, Hand-l. B. pt. ii. p. I28, sp. $788+$ ( 870 ); Dubois, Bull Mus. Relo. iii. p. 195 (1884).

Buceros bicornis, var., Shaw, Gen. Zool. viii. p. I7 (I8I I).

Buceros planicornis, Merrem, Erseh. Gruber, Encycl. xiii. p. 287 (1 824 ).

Hydrocorax planicornis, Bonap. Consp. Vol. An sod. p. 2 (1854; Elliot, Monogr. Bucerot. pl. vii. [text] (1877).

Buceros platyrhynchus, Pears. J. As. Soc. Beng. p. 652 (1841). Hab. Luzón, Montalban, Cavitə, Indán, Cagayán, Abra, Ilocos, Mindoro, Calapán, Marinduque, Samar, Paranas, Calbayog.

2. H. mindanensis. Twued. $\sigma$ N. v. Tulasi Calaoa.

(M. S. T.)

Buceros mindanensis, Trueeddale, P. Z. S. 1878, pp. 543 \& 823; ibid. 1878, p. 278, figs. I \& 2, \& p. 946, \& 1879, p. 70; Sclater, Chall. p. I5, pl. iii. (1880); Steere, L.st Birds \& Mamm. Philippines, p. I 3 (1 890$)$.

Hydrocorax mindanensis, Elliot, Monsgr. Bucerot. pl. viii. [text] ( 1880); Ogilvie, Cat. B. M. 17. p. 359, (18)2).

Buceros hydrocorax, var. mindanensis, Dubsis, Bull. Mis. Belg. iii. p. I 96 ( 1884 ).

Hab. Bas lan, Mindanao, Surigao, Zamboanga, Butuan.

3. H. semigalealus, (Tresed.) $07.0 \mathrm{~N}$ v. Calao.

(M. S T.)

Buceros semigaleatus, Tweeddale, P. Z. S. I 878 , p. 279, fixs. 3 \& 3 4, pp. 34I, 379; Steere, List. Birds \& Mamm. Philippines, p I 3 (I 890).

Hydrocorax semigaleatus, Elliot, Monogr. Bucerot. pl. ix. [text] (1882); Ogilvie, Cat. B. M. 17. p. 360, (1892).

Buceros hydrocorax, var. semigaleatus, Dubois Bull. Mus. Belg. iii. p. 196 (1884). 
Hab. Samar, Borongan, Loquilocum, Calbayog, Leyte, Anparo, Panaon.

\section{Gén ANTHRACOCEROS, Rfich.}

4. A montani, \& $N \vee$ Talusi.

Buceros montani, Oust. Bull. Hebd. Assoc. Sc Fr. 1880, p. 204.

Limnophalus montani, Elliot, Moul Buc. 1882 pl. 25.

B. montani, Dubois, Bull. Mus. Belg: iii. 188 f. p. 199.

Anthracoceros montani, Ogilvie, Cat. B. M. 17,1892 p. 370.

Hab. Joló.

\section{Gén GYMNOL.Gilus, Ogilv.}

5 Gy. marchii, (Oust.) $6 \Omega \mathrm{N}$ v. Taluchi, Talisi.

(M. S. T.)

Anthracoceros marihii, Oustalet, Le Nat. 1885 , p. 108.

A. lemprieri, Sharpe, P.Z. S. 1885, p. 446 , pl. xxvi.; id. Ibis, 1888, p. 198, i 890, p. 45.

Gymnolomus marchii, Oglvie, Cat. B. M. 171892, p. 370.

Hab. Paragua, P. Princesa, Inagauan, Calamianes, Cuyo.

\section{Gén. PENELOPIDES, Reich.}

6. P. panini.: (Budd.) o $\&$ N. v. Tarigtig, Calo.

(M. S. T).

Le Calao de l'Isle Panay Daubent. Pl. Enl. n. 780 [ร], n." 78 ; Buff. Hist. Nat. Ois. vii. p. I 45 ( 780$)$.

Le Calao mâle á bec cis lé de l'Isle Panáy pl. 82, et Le Calao femelle \& pl. 83; Sonn Voy. Nouv. G.lin p. I 22 (1776).

Panayan Hornbill, Lath Gen. Syn. i. p 353 (188I).

Le Calao á bec ciselé, Levaill, Hist. Nat. d'Ois. l'Amer. i. p. 35, pls xvi \& xvii. (1801)

Buceros panini, Bodd. Tab. Pl. Enl. p. $4^{8}$ (1783); Gray, Gen.

B. ii. p. 400 [part] (18+7); Eyton, Osteol. Av. p. 63 (1867).

Penelopides panini, Cab. \& Heine, Mus. He.n. ii. p. I68 [part] (1860); Walden, Tr. $Z$. S. ix. p. i66, pl. xxviii. fig. I \&, fig. 2 \& (I 875); Elliot, Monsgr. Bucerot. pl. 2 I [text] (1877); Steere, List Birds \& Mamm. Philippines, p. I 3 (1 890); Ogilue, Ciat. B. $M .17$. p. 372 (1892).

Buceros panini, Von Martens, J.f. O. p. I8 (I866); Gray, Hand-l. B. ii. p. I 29, p. 7892 (1870).

B. panayensis, Scop. Del. Faun. et Flor. Insubr. ii. p. 87 ( 1780 ; Gm. S N. i. p. 360 (1788); Lxth. Ind. Orn. i. p. Itt (179); Shaw, Gen. Zool. viii. p. 33 (г81 i); Vieill. N. Dict. d'Hist. Nat. iv. p. 594 (1816); Temm. Pl. Col. sp. I 2 (1824); M:rrem, Ersch u. Gruber, Encycl. p. 287 (1824).

Penolopides panayensis, Bonap. Consp. Vel. Ansad. p. 3 (1854). Buceros insculptus, Dumont, Dict. Sc. Nat vi. p. 209 (1817).

Buceros sulcirotris, Wogl. Syst. Av. Buceros, n." i 3 [0"0] (1827). Tockus sulcirostris, Bonap. Consp. Gen. Av. i. p. 9 r (1850).

Penelopides sulcirostris, Bonap. Consp. Vol. Anisod p. 3 (1854). Buceros (Penelopides) manillx, var. panini, Dubois, Bull. Mus. Belg. iii. p. 211 (1884). 
Hab. Mindoro, Baco, Guimarás, Valencia, Panxy, Negros, Masbate, Luzón, Catgayan.

7. P, manillø, (Bo.ll) $\sigma \circ \mathrm{N}$. v. Tarigtig.

d: Calao de Manilla, Daubent. Pl. En!. n." $891 ;$ Buff. Hist. Nat. Ois. wii P. It+ $(1-80)$.

Manilla Hornl ill, Lath. Geth. Syn. i. p. 354 ( I $\left.78_{1}\right)$.

le Calao á b c' ciselé, le vaill Hist. Nat. d'Ois. l'Amer. i. 1. 35 pl. xriii. (180I).

Buceros manilla, Bodd. Tabl. Pl. Enl. p. 54 (1782);

P'enelopides manilla, Walden, Tr. Z. S. ix. p. I68 (1875); id. P. Z. S. 1871 , p. 692; Elliol, Monogr. Bücerot. pl. 20 $\mid$ text $\mid$ (1878); Steere, List Birds \& Mamm Phitippines, p. I3 (1890); Ogilvie, Cirt. B.. M. 17. p. 373, (1892).

Buceros (Penelopides) manilla, Dubors, Bull. Mus. Belg. iii. 1). $210 \quad(1884)$.

B. manillensis, Gm S. N. i. p. 36 г ( 788$)$; Lath. Ind. Orn, i. p. I 45 ( 1790); Vieill. N. Dict. d'Hist. Nat. iv. 1). 546 (1816); I)umont, Dist. Sc. Nat. vi. p. 208 (1817); Meyen. Nov. Act. Ac. C. L. C. Nat Cuv. Suppl. prim. p. 91, pl. xiii. Lo a!l. (1834); Schl. Mus. Pays-Bas, Buceros, p. I I ( I 8's).

B. sulcirostris, Wagl. Syst. Av. Buceros, n. ${ }^{\circ}$ i (1827).

B. panini, Gray, Gen. B ii. p. 400 [part.] (i847); v. Martens, $J$ f. O. 1866, p. i 8 .

P'enelopides panini, Cab. \& Heine, Mus. Hein ii. p. i68 [part.] (1860). Huceros panayensis, Blyth. (nec Scop.), J. As. Soc. Beng. xvi. 1). 997 ( 1847$)$.

Hab. Luzón, Manila, Montalban, Morong, Laguna, Los B.ıños, Calamba, Marinduque, Negros.

8. P. mindorensis, Steere, $0.0 \mathrm{~N}$. v. Talictic.

(H. S T )

P. mindorensis, Steere, List. Exped. Philipp. 1890 , p. I 3 ; Hartert Cat. Mus. Senkenb. Frankf. p. $25 \mathrm{I}$; Ogilvie, Cat. B. M. . 17. p. 374 (1892).

P. affinis schmackeri, Hart. Cat. Mus. Senck. fr. 1891 , 1). 139.

Hab. Mindoro Baco.

9. P. affinis, $T w . \& \mathrm{~N}$ v. Talusi.

(M. S. T.)

P. affinis, Tweedd. An. Mag. N. H. xx. 1877, p. 53t; id. P. Z. S 1877 , p. 824, fig. 2; 1878 , ii. 947; Elliot, Mon. Bucer. pl. 22 (1881); Ogilvie, Cat. B. M. 17. 1892, 1) 375 .

Buceros (Penelopides) manilla, var. affinis, Dubois, Bull. 'Mus. Belg. iii. I 884 , p. 2 I I.

Hab. Mindanao, Surigao, Butuan, Paragua.

ro. P. basilanica, Stcere, N. v.

P. affinis, Tw. P. Z. S. 1879 , p. 70 .

P. basilanica, Steere, List. Exped. Philipp. 1 s9o, 1. 1.3; Ogilv. Cat. B. M. 17. 1892, p. 375 .

Hab. Basilan. 
11. P. samarensis, Steert, ơ N. v. Talusi.

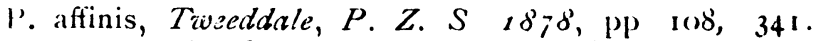

l'. samarensis, Steere, List. Exped. Pällipp. $18^{2} \mathrm{~g} \cdot \mathrm{d}, \mathrm{p} .13 ;$ Oslv. (it. B. M. 17. 1892 p. 376 .

IIab. Samar, Loquilocu.n, Zuinárraga, Borongan, Calbajog, L.yte, Amparo, Dinayat.

5. Gén. CRANORRIINUS, C. ET HEINE.

12. C. cassidix, \& \& $\mathrm{N}$.

(M. S. 'T.)

Buceros cassidix, Temm. Pl. Col. ii pl. zro (1823); Vagl. Sy'st. Av. Buceros, n. 3 (1827); Griff. An m. K̈ing. ii p. $43+$, pl. (1829); Less. Traite Orn. p. 253 (1831); Müll. \& Schl. Verh. Gis. Ned. Ind. p. 24 (1839-tt); Griy, Gen. Birds, ii. p. 3\%) ( 1847$)$; Schl. Mus. Pays-Bas, $i$. Buceros, p. 9 (1862).

Cal.ı (Cassidix) cassidix, Bon xp. Consp. Gen. Av. i. p. 90 (1850).

Rhyticeros (Cassidix) cassidix, Bonap. Consp. Vol. Anisod. p. 3 (1854).

Cranorrhinus cassidix, Cab. \& Heine, Bus. Hein. ii. p. I 73 (1860); Müll \& Schl. Verh. Ges. Ned. Ind., Aves, p. 24, pl. 4 bis [4] (1839-44); Wallace, Malay. Arch. i. p. 364 (1869); Wabt. Tr. Z. S. viii. p. 47, figs. I-4 (1872); Lenz, J. f. O. 1877 , p. 370; Elliot, Mon. Bucerot. pl. I6 [text] (1877); Meyer, Ibis, 1879 , p. 65; Guillemard, P. Z. S. 1885 , p. 54; 2d. Marchesa, ii. p. 188 (1886); Ogilvie, Cat. B. M. 17. p. 377, (1892).

Buceros (Cranorrhinus) cassidix, Gray, Hand-l. B. ii. p. I 29) (1870); Dubois. Bull Mus. Belg. iii. p. I92 (1884).

Hab. Célebes, Gilolo.

13. C. lou socephalus, (Virill.) to N. v. Aguiec.

(M. S. T.)

Buceros leucocephalus, Vieill. N. Dict. d'Hist. Nat. iv. p. 592 (1816).

Cranorrhinus leucocephalus, Wald. Tr. $Z$. S. p. I65, pl. xxvii. (1875); id. P. Z. S. 1877 ; p. 824; Elliot, Mon. Bucerot. p. I1 [text] (1880); Ogilvie, Cat. B. M. 17. p. 378, (1892).

C. leucocephalus, Steere, List Birds \& Mamm. Philippines, 1). I 3 (I 890).

Buceros (Cranorrhinus) leucocephalus, Dubois, Bull. Mus. Belg. iii. p. 193 (1884).

B. sulcatus, Temm. Pl. Col. ii. p. 89, pl. 69 (1823); Wagl. Syst. Av. Buceros, n. ${ }^{\circ}$ (1827); Less. Tranté Orn. p. 254 (1831); Gray', Gen. Birds, ii. p. $400(1847)$; Schl. Mus. Pays-Bas, i. Buceros, p. IO (I 862 .

Tockus sulcatus, Bonap. Consp. Gen. Av. i. p. 9i (1850).

Calao sulcatus, Bonap. Consp. Vd. Anisod. p. 3 (1854).

Buceros (Penelopides) sulcatus, Von. Mart.J. f. O. 1866 , p. 18.

B. (Cranorrhinus) sulcatus, Gray, Hand-l. B. ii. p. I 29 (1 870$)$.

Hab. Mindanao, Surigao, Butuan, Camiguin, Marianas. 
14. C. Waldeni, Sharpe, \& N. v.

(. Waldeni, Sharpe, Tr. Lin. Soc. i. pp. 322, 351, (1879); Elliot, Mon. Bucer. 1877 , pl. 18; Steere, List. Exped. Philipp. 1890 , p. 13; Ogilvie, C'at. B M. 17, 1392, p. 380.

Hab. Panay, Guimarás, Concepción.

6. Gén. ANORRHINUS, Reich.

15. A. galeritus, ( $T_{e m}$.) \& N. v.

(M S. T.)

Buceros galeritus, Temm. Pl. Col ii. p. 78, p. $520(1824)$; Müll. et Schl. Verh. Ges. Ned. Ind. pp. 23 21 (1839-44), Gray, Gen. B. ii. p. 399 (1847); Low, Sarawak, p. 488 (1841); Blyth, Cat. B. Mus. As. Soc. p. 44 (1849); Schl. Mus. PaysBas, i. Buceros, p. I (1862).

Ilydrocissa galeritus, Bonap. Consp. Gen. Av. i. p. 90 (1850). H. (Anthracoceros) galesitus, Bonap. Consp. Vol. Anisod. p. 2 (1854).

Anorrhinus galeritus, Re.ch. Syst. Av. pl. xlix (1850); Horsf. \& Moore, Cat. B. Mus. E. Ind. Co. ii. p. 594 (1856-58); Moore, P. Z. S. 1859 , p. 450; Cab. \& Heine, Mus. Hein. ii. p. 169 (I860); Salvad. Ucc. Rorneo, p. 79 (1874); Hume, Ibis, 1875 . p. 318; Trueeddale, Ibis, 1877 , p. 292; Hume, \& Dav. Str. F. vi. 109 (1871); Sharpe, Ibis, 1879, p. 246; Hume, Str. F. ix. 1. 16 (1880); Elliot, Mon. Bucerot. pl. 42 [text.] (1880); Sharpe, P. Z. S. 1881 , p. 793; 1d. Ybs, 1890 , p. 16; Elliot, Auk. p. 9 (1891); Ogilvie, Cat. B. M. 17.1892 p. 391.

Buceros (Anorrhinus) galeritus, Gray, Hand-l. B. ii. p. 128, $s p$. 7177 (1870).

B. carinatus, Blyth, J. As. Soc. Beng. xiv. p. 187 (1835), \& xiv. j. 996 ( 1847$)$.

Hab. Borneo, Sumatra, ¿Filipinas?

\section{Suborden: TROGONES.}

\section{Fam. TROGÓNIDOS.}

1. Gén. HaRpaC'TES, Swairs.

1. H. ardens, (Tem.) oo N. v.

(M. S. T.)

Trugon ardens, Temm. Pl. Col. n. 404 (1826); Gould, Mon. Trog. ed. i. pl. 35 [text.] ( 1835$)$.

Harpactes ardens, Gray, Gen. B. i. p. 71 (1845); Gray, List. Fissir. Brit. Mus. p. $44(1848)$; Bonap. Consp. Gen. Av. i. p. 51 (1850); Cassin, U. S. Expl. Exp., Mamm. \& Orn. p. 229 (1858); Gould, B. Asia, pl. 76 [text.] (1862); Gould, Mon. Trog. ed. ii. pl. 39 [text.] (1869); Gray, Hand-l. B. i. p. 14 (186.9); Wald. Tr. P. Z. ix. p. 149 (1875); Tweedd. P.Z.S. 
$1 S 77$, p. 540, 1878, p. 107, 943; Steere, List Birds \& Mamm. Philippines, p. 9 (1890); Ogilvie, Cat. B. M. 17.1892, p. 487. Pyrotrogon ardens, Bonap. Coisp. Vol. Zjg. p. 14 (1854); Cab. \& Heine, Mus. Hein. Th. iv. p. 159 (1863).

Harpactes rhodiosternus, Peale, U. S. Expl. Exp. viii. p. I66, pl. 4I (1878); Hartl. Wieg. Arch. xviii. p. 112 (1852).

Hab. Luzon, Manila, Abra, Bucay, Dinagat, Samar, Paric, Mindanao, Marinduque, Zamboanga, Basilan.

\title{
Suborden TREPADORES.
}

\author{
Fam. PICIDOS. \\ Subfam. PICINOS. \\ 1. Gén. GeCinUS Buit.
}

1. G. viridis, (Lin.) o N. v. Pico-verde.

(M. S. T)

Le Pic verd, Briss. Orn iv. p. 9 ( 1760 ; Buff. Hist. Nat. Oss. vii. p. 355 (1783); Daub. Pl. Enl. 879 (U)).

Le Pic rerd, du Mexique, Briss. Orn. iv. p. 16. (1760).*

Le Pic jaune de Perse, Briss. Orn. iv. p. 20. (1760); Salerne, Hist. Nat. Ois. p. 108 ( 1767$)$.

Picus viridis, Linn. Syst. Nat. i. p. 175 (1766); Tiunst. Orn. Brit. p. 2 (1771); Gm. Syst. Nat. i. p. 433 (1788); Hart. Cat. B. M. 17. p. 86 (1890).

Hab. Europa, Alemania.

2. G. puniceus, (Horsf.) $\sigma$ N. v. Pico puniceo.

(M. S. T.)

Picus punic us, Horst. Trans. Linn. Soc. xiii. pt. I, p. 176 (1821); Raff. tom. cit. pt. 2, p. 289 (1822); Lath. Hist. B. iii. p. 352 (1822); Temm. Pl. Col. ccccxiii. (1827); Wagl. (pt.) Syst. Av. Picus, sp. 96 (1827); id. Isis, 1129, p. 518; Vig. Mm. Raffl. p. 668 (1830); Less. Traité, i. p. 222 (1831); Sundev. Consp. Av. Piein. p. 58 (1866).

Picus gularis, Wagl. Syst. Av. Add. Picus, s. n. 6 (nec n. ${ }^{\circ} 89$ ), On (1827).

Brachylophus puniceus, Strickl, P. Z. S. 1841, p. $31 ; B p$. Consp. Voluer. Zygod. p. 10 (1854).

Gecinus puniceus, Gray, Gen. B. ii. p. 139 (I846); Blyth, Cat. B. Mus. As. Soc. p. 59, n." 27o (1849); Wall. Ann. \& Mag. Nat. Hist. xv. p. 96 (1855); Gray, Sist. Picid. Brit. Mus. P. 77 (1868); id. Hand-l. B. ii. 192, n. 8687 (1870); Harg. Ibis, 1888 , p. 176; id. Cat. B. M. 18 . p. 64, (1890).

Venilia puniceus, Bp. Consp. Gen. Av. i. p. 128 (1850).

V. punicea, Reichenb. Scans. Picina, p. $35^{8}$, n. $^{\circ}$ i 76, pl. dexxviii. figs. 4176-77, ơ ad. (1854); Horsf. \& Moore. Cat. B. Mus. E. I. Co. ii. p. 664, n. ${ }^{\circ} 968\left(185^{\left.6-5^{8}\right)}\right.$; Moore, P.Z. S. 1859, p. $45^{6}$. 
(hornpicus puniceus, Malh. Picida; ii. p. Ino, pl. lxxir. figs. $5,6$, o 0 ( ( 862$)$.

('hrysophlegma puniceus, Jerd. B. Ind. i. p. 29г. (1862); Blyth. \& Wald. B. Burm. p. 77 (1875); Oates, B. Brit. Burm. ii. p. $44(1883)$.

(allolophus puniceus, Salvad. Ucc. Born. p. $49(1874)$; Sharpe, P. Z S. 1875, p. IO3; id. Ibis, 1876, p. 36 ; Hume \& I)avison, Str. F. vi. p. r39 (1878); Hume, (O). cit. I879, 1. 88; Sharpe, Ibis, 1879 , p. 242; id. P. Z. S. 1851, p. 792; Guillemard, op. cit. 1885, p. 405; Büttikofrr, Votes, Seyd. Mus. ix. p. 23 ( 1887 )

Chrysophlegma puniceum, Salvad. Ann. Mus. Civ. Stor. Nut. Genova, ser. 2, iv. pp. 519, 529 (I887).

$H a b$. Sumatra.

2 Gén. CHLORONERPES. Swa.

3. Ch. yucatanensis (Cabot) $\sigma \circ$ N. v. P de Yucatan. (M. S. T.)

Picus yucatensis, Cabot, Bost. Journ. Nat. Hist. 1845, p. 92.

P. yucatacensis, Gray, Gen. B. iii. App. p. 2 I (18+9).

(hrysontilus canipileus, (non D'Orb.), Sclat. P. Z. S. 1855, p. I6 I

(hloronerpes yucatanensis, Moore, P. Z. S. 1859 , p. 60; Sclat. \& Ibis, 1859 , p. 1 36 ; iid. op. cit. 1860, p. 44; Sclat. Cat. Amer. B. p. 339, n. ${ }^{\circ} 2025$ (1862); Cab. J. f. O. 1862 , ए. p. 321 ; Cab. \& Heine, Mus. Hein. iv. p. I6r (1863), pt; Gray, Sist. Piid. Brit. Mus. p. 104 (1868), pt.; id. Hand-!. B. ii. p I98, ${ }^{\circ} 8777$ (i870); Sclat. \& Salv. Nomencl. Aa'. Neotr. p. Ioo (1873); Salv. Cat. Struckl. Coll. p. 396, n. ${ }^{\circ}$ ig 36 (1882); Zeled. Cat. Av. Costa Rica, 1882 , p. 23, n. ${ }^{\circ}$ tix; $^{\circ}$ Bancard, P. Z. S. 1883 , p. 452; Zeled. Proc. U. S. Nat. Mus. 1885 , p. I I I Hargitt, Cat. B. M. 18.1890 p. 84.

$H a b$. América, Guatemala.

3. Gén. CHRYSOPHLEGMA, Gould.

4. Ch. mentale, (Tem.) ơ N. v.

(M. S. T.)

Picus mentalis, Temm. Pl. Col. 384 , \& (1826).

P. gularis, Temm. M. S.; \& Wagl. Syst. Av. Picus, sp. \&o (1827), nec o.

P. squamicollis.*, Less. Traité, i. p. 229 (1831).

Gecinus mentalis (pt.), Gray, Gen. B. ii. p. $439^{\circ}(\mathrm{r} 846)$, \& iii. App. p. 21 (1849); id. List. Picid. Brit. Mus. p. 77 (1868); id. Hand-l. $B$. ii. p. 192, n. ${ }^{\circ} 8686$ (1870).

Venilia mentalis, Bp. Consp. Gen. Av. i. p. I 28 (1850).

Brachylophus mentalis, Strickl. P. Z. S. 1846 , p. 31; Bp. Const. Volucr. Zjgod. p. io (1854).

Venilia gularis, Reichenb. Scans. Picina, p. 358, n. ${ }^{\circ} 827$, pl. dexxviii. figs. 4I 80,4 I 8 I (1 854 ).

Picus mentalis (pt.), Sundev. Consp. Ac. Picin. p. 58 (1866). 
(hloropicus mentalis (pt.), Malh. Picida, ii. p. 12, pl. lxxv. figs. t, $5(1860)$.

Chrysophlegma mentale, Hargitt, Ibis, 1886, P. 267; id. Cat. B. i. 28 is 80, p. 125.

Hab. Java, China.

\section{Gen. MELANERPES, Sw.}

5. M. pucherani. (Malh.) $\sigma \circ \mathrm{N}$. v.

Picus carolinus, zar. d, Lath. Ind. Orn. i. p. 231, sp. I8 (1890). P. alurifrons (non $I T$ agl), Less. Kev. Zool. 1839 , p. 102; Wagn. Arch. fïr. Naturg. $18+1$, p. Ioo.

Zebrapicus pucherani, Malh. Rev. Zool. 1849 , p. 542; Bp. Consp. Volucr. Zigod, p. I I, n. ${ }^{\circ} 224$ (1854); Malh. Picida, ii. p. 227, pl. ciii. figs. I, 2, ơ (1862),

Centurus pucherani, Bp. Consp. Gen. Av. i. p. 120, n. 9 (1850); Reichenb. Scans. Picina, P. 4 I I, n. ${ }^{\circ} 966$ (1854); Sclat. \& Salv. Ibis, 1859 , p. 136; Moore, P. Z. S. 1859 , p. 60; Sclat. \& Salv. Ibis, 1860, p. 43; Sclat. P. Z. S. 1860 , pp. 286, 296; Lawer. Ann. Lic. N. I. 1861, p. 229; Cab. J. f. O. 1862 , p. 328; Sclat. Cat. Amer. -13. p. 342, n. 2044 (1862); id. \& Salv. P. Z. S. 1864 , p. 367 ; iid. op. cit. 1867, p. 230; iid. op. cit. 1879 , p. 387 ; Salv. Ibis, p. 320 ; Sclat. \& Salv. Nomencl. Av. Neotr. p. I0I (1873); Boucard, P. Z. S. 1878, p. 49, Nutting, Proc. U. S. Nat. Mus. vi. p. 406 (1883).

Zebrapicus gerini, Malh. Picide ii. p. 23 I (1862).

Picus gerini, Sundev. Consp. Av. Picin. p. 55 (1866).

Melanerpes pucherani, Hargitt, Cat. B. M. 18. p. 164 (I89o). $H a b$. América, Guatemala.

\section{Gén. DENDROCOPUS, Косн.}

6. D. mixtus, (Bodd.) $\checkmark \circ \mathrm{N}$ v.

(M. S. T.)

L. Epeiche au Pic. variè de la Encenada, Buff. Hist. Nat. Ois. vii. p. 409 ( I 783 ).

Pic. mâle de le Encenada, Daubent. Pl. Enl. 748. fig. I.

Picus mixtus, Bodd. Tabl. Pl. Enl. p. 47 (I783); Gray, Gen. $B$. iii. Arp. p. xxi. (1849); Sclat. Cat. Amer. B. p. 334, n. 1996 (1862); Sundev. Consp. Av. Picin. p. 20 (1866); Gray, Syst. Picid. Brit. Mus. p. 48 (1868); Sclat. \& Salv. P. Z. S. 1868 , pp. 138. 143; Hudson, op. cit. 1370, p. 158; Gray, Hand-l. B. ii. p. I85, n. 8608 (1870); Pelz. Orn. Bxas. p. 443 (1870); Sclat. \& Salv. Nomencl. Av. Neortr. p. 99 (1873); Durnf. Ibis, 1878 , p. 62; White, P. Z. S. 1882, p. 6i7, 1883 , p. 40; Barrow's, Auk, 1884, p. 25; Withington, Ibis, 1888 , p. 467 ; Sclat. \& Huds. Arg. Orn. ii. p. 191889.

Dendrocof us mixtus, Hargitt, Cat. B. M. 18 . (1890) p. 259.

P. bicolor, Gm. Syst. Nat. i. p. 438 (1788); Bp. Consp. Gen. Av. Hab. América, Brasil. 


\section{Gén SPHYROFICUS, BaIR.}

7 sh. varius, (Lin.) $\sigma \Omega$ N. v.

(M. S. T.)

'The Yellow belly'd Woodpecker, Catesby, Nit. Hist. Carol. i. p. 2i, pl. xxi., \& ad. (i73i).

Pic varié de la Cardine, Briss. Orn. iv. p. 62 ( 1760); Daubent. Pl. Enl. 785, o ad.

Picus varius, Linn. Syst. Nat. i. p. i76 (i766); Penn. Arct. Zool. ii. p. 275 (1785); Gm. Syst. Nat. i. p. +38 ( 788$)$.

Sphyropicus varius, Hargitt, Cat. B. M. 18.1890 p. 188. Hab. América, Canadá.

\section{Gen IYNGIPICUS, BP.}

8. I. maculatus, (S॰op) $\sigma \circ$ N. v. Anloague, Calpintero. (M S. '1')

Petit Pic d'Antigue, Sonn. Voy. Now Gunn. p. i I 8, pl. 77 (1776).

Le petit Epeiche (pt.), Buff. Hist. Nat. Ois. vii. p. 64 (1780, ex Sonn.)

Picus maculatus, Scop. Del. Faun. et Flor. Insubr. p. 89 (1786, ex Sonn.); Jerd. B. Ind. i. p. 279 (1863).

P. minor, var. b, Lath. Ind. Orn. i. p. 230 (1790).

P. moluccensis, Less. Traité, i. p. 221 (1831, non Gin.); Malherb:, Pacida, i. p. I 43, pl. xxxii. (186r).

P. nanus, Blyth, J. A. S. Beng. xiv. p. 197 (1846, non Vigors).

P. validirostris, Blyth, Cat. B. Mus. As. Soc. p. 6+ (1849); id. J. A. S. Beng. xviii. p. 805 (18+9); Reichenb. Scans. Picinx, p. 373 (1854); Sundev. Consp. Av. Picin. p. 29 (1866); Gr.xy, Syst. Picid. Brit. Mus. p. 43 (1868); id. Hand-l. B. ii. p. $184, \mathrm{n}^{\circ}$ I 582 (i 870 ).

P. flavinotus, Malh. Picida, i. p. 144 (18861, ex spec. in Mus. Brit.)

Bæopipo validirostris, Cab. \& Heine, Mus. Hein. Th. iv. p. 6) (1 863).

Picus (Yungipicus) validirostris, Martens, J. f. O. 1866, p. 20.

Yungipicus maculatus, Walden, $\operatorname{Tr}$. $Z$. S. ix. p. I48 (1875); Sharpe, Tr. Linn. Soc. 1877 , Zool. i. p. 350; Kütter, J. f. O. 1883 , p. 295 .

Iyngipicus maculatus Hargitt, Ibis, 1882 , p. 26; id. Cat. B. M. 18. I890 p. 332; Gould, B. Asia, vi. pl. xxx., o" $0^{*}(1883)$.

Hab. Luzón, Manila, Montalban, Laguna, Panay, Guimarás, Marinduque.

9. I. fulvifasciatus, Harg. N. v.

Iyngipicus fulvifasciatus, Hargitt, Ibis, 1881, p. $59^{9}$; id. op. cit. 1882 , p. 28 ; id. Cat. B. M. 18 . p. 333 (1790). Gould, B. Asia, vi. pl. xxxi., ơ (1883).

Hab. Mindanao, Zamboanga, Basilan.

10. I. basilanicus, Steere. $\mathrm{N}$.

Hab. Basilan. 
11. I. leytensis, Stecre. N. v

Hab. Leyte.

12 1. ramsayi, Harg. N. v

Iyngipicus ramsayi, Hargitt, Ibis, 1881 , p. 598; id. op. ctt. 1882, p. 49; id. Cat. B. 18. p. 334 (1890); Gould, B. Asia, vi. pl. xxiv, $\sigma \sigma$ (1882); Guillem. P. Z. S. 1885, p. 254.

Hab. Joló.

\section{Gén Tiga, Kaup.}

13. T. everetti, Tew \& \& N. V Malalantuc.

(M. S T )

'Iiga javanensis (non Ljung), Sharpe, Trans. Linn. Soc. 2nd ser. i. p. 315 (1877); Tweedd. Mem. ed. W.-Ramsay, pp. 607, 655, $670(1881)$.

T. everetti, Tweedd. P. Z. S. 1878, p. 61 2, pl. xxxvii. \& ?; Sharpe, Ibis, 1884, p. 317, 1888, p. 197; Hargitt, Cat. B. M. 18. p. 418 (1890).

Hab. Paragua, P. Princesa.

14 T. javanensis. (Ljung.) $0^{\circ}, 0$ N. v. Malalantoc.

(M. S. T.)

Picus javanensis, Ljung, Mém. Acad. Roy. Stockh. 1797, p. I3t, pl. vi. O; Sundev. Consp. Av. Picin. p. 83 (1866).

P. tiga, Horsf. Trans. Linn. Soc. xiii. p. 177 (1822); Raffl. $t$. c. p. 290 (I822); Horst. Zool. Research. Java (Gen. Cat.) p. 4 (1824; W.gl. Syst. Av. Picus; sp. 100 (1827); Gray, Hardze. Ill. Ind. Zool. pl. 3o. figs. $2 \sigma^{\circ}, 2$ a, h ad of 8 (183032); Vig. App. Mem. Raffl. p. 669 (1831).

Chrysonotus tridactylus, Swains. Classif. B. ii. p. 309 (1837); Jerd. Ibis, 1872 , p. Io.

Chrysono us tiga, Eyton, P. Z. S. 1839 , p. ro6; Horsf. \& Moore, Cat. B. Mus. E. I. Co. ii. p. 657 (1856-58).

'Tiga tridactyla, Blyth, J.A. S. B. 1845 , p. 193; Strickl. $P$. Z. S. 1846 , p. 104; Gray, Gen. B. ii. p. 441, pl. cix. fig. 7 (1846); Blyth, Cat. B. Mus. As. Soc. p. 56 (1849); Bp. Consp. Gen. Av. i. p. I 20 (1850); Reichenb. Scans. Picina, p. 396, pl. dclii. figs. 4344-4485, o o (1854); Gould, P. Z. S. 1859 , pp. I50, 151; Sclat. op. cit. 1863, p. $210 ;$ Vord. Over. Nat. Tijds. Nederl. Ind. xlii. p. I7 (1882).

Picus intermedius, Bly h, J.A. S. B. 1845, p. 193.

P. rubropygialis, Malh. Rev. de Zool. 1845, p. 400.

Chloropicoides tiga, Malh. N. Classif., Mém. Acad. Metz, 1848, 49, p. 347; id. Picida, ii. p. 97, pl. lxxi. figs. 5 \$. 6 8. (1862).

'Tiga intermedia, Blyth, Cat. B. Mus. As. Soc. p. 56 (1849); Reichenb. Scans. Picina, p. 396, n. ${ }^{\circ} 918$ b (1854); Schomb. Ibis, 1864 , p. 257; Blyth, op. cit. 1865 , p. 30; Gray, List Picid. Brit. Mus. p. 97 (1868); id. Hand-l. B. ii. p. 196, n. ${ }^{0}$ 8752 (1870); Armstr. Str. F. 1879, p. 31.; Salvad. Ann, Mus. Civ. Stor. Nat. Genova, 1887 , p. 5801888, p. 567. 
Tiga rubropygialis, $B p$. Consp. Gen. Av. i. p. I20 (1850); $u$. Consp. Volucr. Zygod. p. I0, n. ${ }^{\circ}{ }_{3}{ }_{3}$ (1854); Reichenb. Sians. Picina, p. 397, n. 92 I (1854); Grav, List Pic d. Brit. Mus. p. 97 (1868); id. Hand-l. B. ii. p. 196, n. ${ }^{\circ}$ г753 (1870); Hume, Str. F. 1879, p. 88, n. $^{\circ} 185$.

Crysonotus intermedius, Horsf.e \& Mosre, Citt. B. Mils. E. I. Co. ii. p. 657 (1856-58); Moore, P. Z. S. 18.59 , p. 456 ; Jerd. B. Ind. i. p. 299 (1862); Cab. \& Heine, Mies. Hein. iv. p. 872 (1863); Beavan, P. Z. S. 1866, p. 4; id. Ibis, 1859, p. 415; Hume \& Oates, Str. F. 1875, p. 14.

Chloropicoides intermedia, Malh. Picide, ii. p. 99 ( 1862 ).

Chrysonotus rnbropygialis, Jerd. B. Ind. i. p. 299 (1862); Hume, Str. F. 1876 , p. 390 .

Chloropicoides rubropygialis, Malh. Picide, ii. p. Ioo, pl. lxx. fig. 5, juv. (1862).

Chrysonotus javanensis, Cab. \& Heine, Mus. Heir. iv. p. 172 (1863); Mïlll. Orn. Ins. Salanga, p. 64 (1882).

(.) erythropygius, Cab. \& Heine, Mus. Hein. iv. p. I73 (1863).

Picus javanensis, var. B, Sundev. Cunsp. Av. Pacin. p. 83 (iso6).

P. javanensis, var. C, Sundev. Consp. Av. Picin. p. 83 (1866,

Tiga javanensis, Gray, Lyst Picid. Brit. Mus. p. 97 (1868); id. Hand-l. B. il. p. I96, n. ${ }^{\circ} 875$ I (1870); Salvad. Ucc. Born. p. 54 (1874); Blyt. \&c. Wald. B. Burm. p. 75 (1875); Hume \& Davison, Str. F. vi. pp. I46, 50 (1878); Oates, op. cit. $187 \mathrm{y}$, p. I65; Nicholson, Ibis, 1879 , p. I64; Tirant, Ois. B.-Cochinchine, p. 90 (1879); Salvad Ann. Mus. Civ. Stor. Nat. Genova, 1879 , p. 183; Sharpe, Ibis, 1879, p. 243; Hume, Str. F. 1879 , pp. 53, 88, n. ${ }^{\circ}{ }_{1} 84 ;$ Bingh. op. cit. 1880, p. 164 ; Tweed. Mem ed. W.-Ramsay, p. 479, (r881; Kelh Ibis, $188 \mathrm{~s}^{\circ}$, p. 388, Sharpe, P. Z. S. 1881 , p. 792; Oates, Str. F. x. 1882 , p. 192; Davison, t. c. p. 357 (1883); Oxtes, B. Brit. Bur B. ii. p. 55 (1883); Vorg. Over. Nat. Tijds. Nederl. Ind. lxiv. p. I9I (1884); Guzllem. P. Z. S. 1885 , p. 405; Salvad. Ann. Mus. Civ. Stor. Nat. Genova, 1887 , p. 518 ; Hargoitt, Cat. B. M. 18 . p. 4I 2 i 890.

Tiga rufa, Stolic. J. A. S. B. 39. p. 289 (1870). Hab. Culion, Calamianes.

\section{Gén CHRYSOCOLAPTES, Blyth.}

15. Ch. erythrocephalus, Sharpe o' 0 N. v. Balitutu. (M. S T'

Chrysocolaptes erythrocephalus, Sharpe, Trans. Linn. Soc. 1877 , xxx. pp. 315, 350, pl. xlvi. fig. I; Tweedd. P. Z. S. 1878 , p. 61 2; Wardl-Rams. Tweed. Mem. pp. 607, 655 (1881); Sharpe, Ibis, 1884, p. 317, 1888, p. 197; Hargitt, Citt. R. M. 18. 1. $45^{2}$ (I 890 ).

Hab. Culión, Calamianes, Paragua, P. Princesa.

16. Ch. hæmatribon, (Wagl.) ơ $\mathrm{N}$ v. Manunuctoc.

(M. S. T.)

Picus hæmatribon, Wagl. Syst. Av. Picus, sp. 95, \& (1827); Sundev. Consp. Av. Picin. p. 80 (1866). 
P. spilolophus, Vig. P. Z. S. 1831, p. 98, o; Less. Compl. Buff. ix. p. 3 10, $\&$ (1837).

P. philippinarum (non Laht.), Less. Traité, i. p. 222, 9 (1831).

Brachipternus hæmatribon, Strickl. P. Z. S. 1841, p. 31 .

Chrysocolaptes hamatribon, Blyth, $J$. A. S. B. 1843 , p. ron4; Gray, Gen. B. ii. p. 436 (1845); Blyth, Cat. B. Mus. As. Soc. p. 55 (1849); Bp. Consp. Gen. Av. i. p. 121 (1850); id. Consp. Voluer. Zygod. p. Io, n. ${ }^{\circ} 75$ (1854); Reichenb. Seans Picina, p. 400, n." 930, pl. dclv. figs. 4357-58, $\rho$ (1 854); Jerd. $B$. Ind. i. p. 283 (1862); Cab. \& Heine, Mus. Hein. iv. p. 169 (1863); Gray, List. Picid. Brit. Mus. p. 63 (1868); id. Hand-l. $B$. ii. p. I89, n. 8646 (1870); Wald. P. Z. S. 1873, p. 526; Twetd. op. cit. 1877 , p. 689; Wardl.-Rams. Tweeld. Mém. pp. 299, 314, 410, 515,655 (1881); Hargitt, Cat. B. M. 18. p. 454 ( 1890$)$.

Indopicus hamatribon, Malk. Picida, ii. p. 84, pl. Ixviii, figs. I $\sigma 2$ o jicv., 3 o (186a).

Hab. Luzón, Cagayán, Ilocos, Manila, Montalban, Batanes, Calayan, Marinduque.

17. Ch. Iucidus, ! $S c o p$.) ơ 0 N. v. Manunuctuc.

(M. S. T.)

Pic grivelé ou Grand Pic de I'isle de Luçon, Sonn. Voy. Nouzv. Guin. 1776, p. 73, pl. xxxvii.

Le Palalaca on Pic vert tacheté des Philippines, Buff. Hist. Nat. Ois. vii. p. 2 I ( 1780 !.

Pic verd des Philippinés, Dauben. Pl. Enl. 69r.

Bengal Woodpecker, var. B. Lath. Gen. Syu. ii. p. 581 (1782).

Pi.us aurantius (non Linn.), Bodd. Tabl. Pl. Enl. p. 43 (1783).

P. lucidus, Scop. Del. Fl. Faun. Insubr. ii. p. 89 (1 786$)$.

P. bengalensis, var. \&, Gm. Syst. Nat. i. p. 433 (1788).

P. philippinarum, Lath. Ind. Orn. i. p. 236 (1790); Steph. Gen. Zool. ix. p. 190 (1815); Vieill. N. Dict. d'Hist. Nat. xxvi. p. 15 (1818) id. \& Bonn. Enc. Meth. iii. p. 1314 (1823); Valenc. Dict. Sc. Nat. xl. p. 175 (1826); Sundev. Consp. Av. Picinp. 80 (1 866).

P. palalaca, Wagl. Syst. Av. Picus, sp. 92 (1827).

P. squamosus, Less. Traité, i. p. 23o (18.31).

Brachypternus philippinarum, Strickl. P.Z. S. 1851 , p. 31 .

Chrysocolaptes philippinarum Gray, Gen. B. ii. p. 436 (1845); $B p$. Consp. Voluer. Zygod. p. Io, n. ${ }^{\circ}$ 16 (1 854); Gray, List. Picid. Brit. Mus. p. 63 (1 868).

C. aurantius, Gray, Gen. B. iii. App. p. xxi. (1849); Cab. \& Heine, Mus. Hein. iv. p. 168 (1863); Gray, Hand-l. B. ii. p. 189, no. 8648 (1870).

C. palalaca, Bp. Consp. Gen. Av. i. p. I 2 I (1850); Reichenb. Scans. Picina, p. 400, no. 932, pl. dclv. fig. 4360 ( 1854$)$.

Indopicus philippinarum, Malh. Picide, ii. p. 85, pl. Ixvi. figs. 3 3,4 \& (1862).

Chrisocolaptes lucidus, Tweedd. P. Z. S. 1877, pp. 537, 539, 822, 1878, p. 942; Wardl.-Rams. Tweedd. Mem. pp. 314, 410, $461,462,463,548,633,655$ (1881); Kütter, $J . f .0 .1883$, p. 295; Hargitt, Cat. B. M. 18. p. 455, (1890). 
C. maculiceps, Sharpe, Trans. Linn. Soc. 1877, xxx. pp. 314, 35 (), pl. xlvi. fig. 2, \&.

Hab. Butuan, Mindanao, Zamboanga, Basilan, Negros, Sibulan, Luzon.

18. Ch. rufopunctatus, Harg. o o Palalaca.

(M. S T.)

(. lucidus, (non Scop.), Tweedid. P. Z. S. 1878, p. 379; Wardl.Ramsay. ed. Treedd. Mem. p. 603 (1881).

(. rufopunctatus, Harg. Ibis, 1889 , P. 23.1; id. Cat. B. 18. p. $457(1890)$.

Hab. Panaon, Samar, Paranas.

19. Ch. xanthocephalus, Wald. ơ N. v. Palalaca.

(M S. T.)

Crysocolaptes xanthocephalus, Wald. \& Layard, Ibis, 1872, p. 99, pl. iv.; Wald. P. Z. S. 1873 , p. 526; Sharpe, Trans. Linn. Soc. 1877 , xxx. p. 3.14 ; Gould. B. As. vi. pl. xxxvii. o 0 (1877); II ald.-Rams, Tweedd. Mem. pp. I $8,299,314,410,655,664$ (1881); Harg. Cat. B. M. 18. p. 457, (1890).

Hab. Negros, Guimarás, Panay, Masbate.

\section{Gén. microstictus, harg.}

20. M. fuliginosus, $T w$. $\sigma, 0$ N. v. Palalaca.

(M. S. T)

Mulleripicus fuliginosus, Tzveedd. Ann. \& Mag. Nat. Hist. 1877 ; xx. p. 534; id. P. Z. S. 1877 , pp. 817,821 , pl. lxxxiii. o. Uardl.-Rams. Tweed. Mem. pp. 543, 548, 562, App. 665, 667 ( $188 \mathrm{I}$ ). Microstictus fuliginosus, Hargitt, Cat. B. M. 18. p. 492 ( 1890$)$.

Hab. Butuan, Surigao; Mindanao, Samar, Paranas, Borongan.

2 I M funebris, (Valkn.) $ه 0$ Malagayang.

(M. S. T.)

Picus funebris, Valenc. Dict. Sc. Nat. xl. p. 179 ९ (1826); Drap. Dict. Class. d'Hist. Nat. xiii. p. 497 (1828); Sundev. Consp. Av. Picin. p. 10 (1866).

P. lichtensteini, Wagl. Syst. Av. Picus, sp. 31, \& (1827).

P. modestus,' Vig. P. Z. S. 1831 . p. 98; Less. Compl. Buff. ix. p. 310, ó (1837).

P. punctatus, Less. Traite, i. p. 230, \& (1831).

Hemilophus lichtensteini, Gray, Men. B. ii. p. 439 (1846); BP. Consp. Gen. Av. i. p. I3I (1850); Reachenb. Scans. Picina,

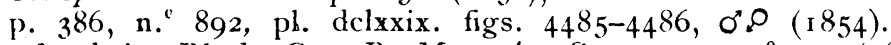

H. funebris, Blyth, Cat. B. Mus. As. Soc. p. 55, n. ${ }^{\circ} 247$ (1849); Gray, List. Picid. Brit. Mrus. p. 84 (1868).

Lichtensteinipicus modestus, Bp. Consp. Volucr. Zygod. p. 7, n. ${ }^{\circ} 6$ (1854).

L. linhensteini, Gray, List Gen. 1855, p. 93, ex Bp.

Dryopicus funebris, Malh. Picide, i. p. $54, \mathrm{pl}$. xv. figs. I $\mathrm{T}$, 2 \% (1861).

Alophonerpes funebris, Cab. \& Heine, Mus. Hein. iv. p. I()] (1863). 
Campethera funebris, Gray, Hand-l. B. ii. p. 193, n. ${ }^{\circ} 215^{8}(1870)$. Mulleripicus funebris, Treedd. Trans. Zool. Soc. $187.5, \mathrm{pl}$. 146, 249; Sharpe, Trans. Linn. Soc. 1877, p. 350, tilh. n. 29; Treeedd. Mem. ed. Wardl.-Ra'nsay, pp. 31.3, 410, -1pp. 65.5 (1881). Microstictus funebris, Harg. Cat. B. M. $1 \delta^{2}$. p. +92, (8890).

Hab. Luzon, Cagayan, Manila, Calay.u.

Ir. Gén. Hevil,ophus, Siv.

22. H pulverulentus, ( $T_{e m}$ (Im.) $\sigma$ Balalatug.

(MS S. T)

Picut javansis 8, Horsf. Trains. Linn. Soc. xiii. p. 176, o (1822); Lath. Hist. B. iii. p. 353, क $(1822)$; Steph. Gien. Zool. xiv. p. I 59, \& (1826).

P. pulverulentus, Temm. Pl. Col. $38^{\circ} 9,0^{\circ}(1826)$; Vig. Mem. Raffl. p. 669 (1830); Less. Traité, i. p. 222, ช (1831); Sun. dev. Consp. Av. Picin. p. ro (1866).

P. gutturalis, Valenc. Dict. Sc. Nat. xl. p. i 78 (1826).

P. wackloti, Wagl. Syst. Av. Picus, sp. 4 o add., sp. 1 o ad., \& imm. (1827); id. Isis, 1829, p. 508 .

P. horsfieldii, \& Wagl. Syst. Av. Picius, sp. 5, o (1827).

Hemilophus pulverulentus, Swains. Class'f. B. ii. p. 309 (1837); Blyth, Cat. B. Mus. As. Soc. p. 54, n. ${ }^{\circ} 24+(1849) ; i d$. I. A. k. B. 1854 , p. 21 5 ; Blanf. Ibis, 1870 , p. 464; Harg. Cat. B. M. 13. p. 494, (8894).

H. mackloti, Gray, Gen. B. ii. p. 439 (18+6); Bp. Consp. Gen. Av. i. p. I 3 I (1850); Reichenb. Scans. Picida, p. 385, n. 889 , pl. dexliv. figs. $43000 \mathrm{OI}$, \& \& (1854).

H. müllet i, Bp. Consp. Gen. Av. i. p. I 31 (1 $\left.85^{\circ}\right)$.

Mulleripicus pulverulentus, Bp. Consp. Volucr. Zygot. p. 7, n." I 5 (I 854 ); Gray, Cat. Gen. B. Brit. Mies. 1855, p. 93, n. ${ }^{\circ} 576$; Horsf. \& Moore, Cat. B. Mus. E. I. Co. ii. p. 651 (1856-58); Moore, P. Z. S. 1859 , p. 455; Jerd. B. Ind. i. p. 284 (1862); Blyth, Ibis, 1866 , p. 355 ; Jerd. op. cit. 1872 , p. 9; Godw-Aust. J. A. S. B. 1873 , p. 155; Hume \& Davison, Str. F. vi. p. I 33 (г878); Hume, op. cit. 1879 , pp. 52. 87, n. ${ }^{\circ}$ I68; Tiraut, Ois. B.-Cochinchine, p. 89 (1879); Bingh, Str. F. 1880 , pp. I4I, 162, 472 ; Kelh. Ibis, 1881; p. 388 ; Oates, Str. F. x. p. 196 (1882); Sharpe, Ibis, 1888, p. 197;

Dryopicus gutturalis, Malh. Picida, i. p. 5I, pl. xv. figs. 4 o ad; 5 o ad., 6 juv. (I86r).

Alophonerpes gutturalis, Cab. \& Hine, Mus. Hein. iv. p. 106 (1863); Blyth. \& Wald. B. Burm. p. 75 (1875).

Herrilophus gutturalis, Gray, List. Picid. Brit. Mus. p. 83 (1863).

Campathera gutturalis, Gray, Hand-l. B. ii. p. 193; n. ${ }^{\circ} 8704$ ( 870$)$.

Mulleripicus gutiuralis, Hume, Str. F. 1874, p. 47I; id \& Oates, op. cit. 1875 , pp. I $2,66$.

Alophonerpes pulverulentus, Salvad. Ucc. Born. p. 5 I (i874); Wardl.-Rams. Ibis, 1877 , p. 457; Sharpe, op. cit. 1879. 1. 242 ; id. P. Z. S. 1879 , p. 326, 1881, p. 792; Oates, B. Brit. Burm. ii. p. 29 (1883); Vorderm. Over. Nat. Tijds. Nederl. In.t. xliv. p. ig $8(1884)$. 
Horrilophus fischeri, Brïgg. Abhaudl. nat. Ver. Brom. 1877, p. 454 (juv.).

Hab. Paragua, P. Princesa, Mindanao; Borno, Java.

12. Gén Thriponax, Cab. et Heine.

23. Th. javensis, (Horsf.) $ఠ 0 \mathrm{~N}$ v. Manugtug.

(M. S. T.)

Picus jaranensis, Morsf. Trans. Linn. Soc. xiii. p. 175 (1822), o; Steph. Gen. Zool. xiv. p. 159 (1826), o; Vig. Mem. Raffl. p. $668(1830)$.

P. leucrugaster, Valenc, Dict. Sc. Nat. xl. p. i 78 (1826), \&; Wagl. Syst. Av, Picus, sp. 7, '0 (1827); id. Isis, 1829, p. 109, o. Temm. Pl. Col. iv. pl. 501, ¿ (1838); Snndev. Consp. Av. Picin. p. 9 (1 866).

P horsfieldii, Wagl. Syst. Av. Picus, sp. 5, ? (1827).

Dryocopus javanensis, Boie, Isis, 1828 , p. 326.

D. leucogaster, Boie, Isis, 1828 , p. 326 .

Picus fulvigaster, Drap. Dict. Class. d'Hist. Nat. xiii. p. 503, (1828).*

J'. maximus malayanensis, Bland, J, A. S. B. vi. p. 952 (1837). Ilemilophus leucogaster, Gray, Gen. B. ii. p. $439(18+6)$, ?.

H. javensis, Gray, Gen. B. ii. p. 439 (1846), o'; Blyth, Cat. B. Mus. As. Soc. p. 55 (1849); Bp. Consp. Gen. Av. i, p. 13 г (1850; id. Consp. Volucr. Zygod. p. 7 (1854); Reichenb. Scans. Pic.na, p. 386, n. ${ }^{0} 894$, pl, dexlv. figs, 4306-4307, ơ (1854); Motley \& Dullw. Contr. Nat. Hist. Labuan, p. 29 (1855); S.lat. P. Z. S. 1863, p. 211; Gray, List. Picid. Brit. Mus, p. 8.5 (1868); Stoliczka, J. A, S. B. $187 a$, p. 290; Brïgg. Abhandi. nat. Ver. Bremen, 1878, p. $53 \mathrm{I}$.

Dryopicus leucogaster, Malh. Mem. Acad. Metz, $1848-49$, p. 322. Mulleripicus javensis, Horsf. \& Moore, Cat. B. Mus. E. Y. Co. ii. p. 652, n. $^{\circ} 946(1856-58)$; Jerd. B. Ind. i, p. 285 (1862).

Dryopicus leucogaster, Malh. Picida, i, p. 47, pl. xiii. figs. 4, 5 , \& i ( I $86 \mathrm{I})$.

'Thriponax javensis, Cab. \& Helne, Mus. Hcin, iv. p. 105 (1863), Salvad. Ucc. Born. p. 52 (1874); Sharpe, P. Z. S. 1875, p. 103; Hume, Str. F. 1875 , p. 319; Trueedd. Ibis, 1877 , P. 288. id. P. Z. S. 1877 , pp. 689, 821; Hume \& Davison, Str. F. vi. p. 135 (1878); Hume, op. cit, 1879, pp. 52, 87; Sharpe, Ibis, 1879 , pp. 52, 87; Sharpe, Ibis, 1870, 2. 243; Tweedd. P. Z. S. 1879 , p. 69; Sharpe, tom. cit. p. 326; Dicholson, Ibis, 1881 , p. I41; Kelham, toin. cit. p. 388 ; Sharpe, P. Z. S. 1881 , p. 792; Mïll. Orn. Ins. Salanga, p. 72 (1882); Nicholson, Ibis, 1882, p. 5t; id. op. cit. 1883 , p. 89; Oates, B. Brit. Burm. ii. p. 27 (1883); Kütter, J. f. $O .1883$, p. 295; Blas, Verhandl, zool-bot. Gesellsch. Wien, 1883, p. 30; Vorderm. List. B. Java, n. ${ }^{\circ}$ 6 I (1884); Hargitt. Ibis, 1885 , p. 145; id. Cat, B. M. 18. 1890, P. 498.

Campethera javensis, Gray, Hand-l. B. ii. p. 193, n. 8707 (1870). Hab. Luzón, Negros, Mindoro, Paragua, P. Princesa, Célebes, Basilan, Java. 
$24 \mathrm{Th}$ pectoralis, Tw. o $0 \mathrm{~N}$. v. Ialalaea.

(M. S. T)

Thriponax pectoralis, Tüeedt. P. Z. S. 1878 , pp. 340, 379, II ardl.-Ramsay, Trieedd. Mém. p. 596, \& App. pp. 655, 670 (1881 ; Harg. Ibis, $188^{2} 5$, p. I+3; id. Cat. B. M. 18.1890. p. 5oo, lam. xiii.

Hab. Leyte, Panaon, Samar, Borongan, Paranas.

25. Th. hargitti, Sharpe. $0^{\circ} .0 \mathrm{~N}$ v. Balalaling.

(M. S T.)

'Thiponax jarensis (pt.), Sharpe, Trans. Linn. Soc. and. ser. Zool. i. p. $31+(1876)$.

Th. hargitti, Sharpe, Ibis, 1884 , p. 317 , pl. viii.; Harg. op. ctt. 1885 , p. I15; id. Cat. B. M. 18.1890 p. 505. Sharpe, op. cit. 1888 , p. 197 .

Hab. Luzón, Manila, Paragua, P. Princesa.

20 Th. philippinensis, Stecre. N. v.

Hab. Guimarás, Masbate.

27. Th. mindorensis, Slecr. $\diamond \varnothing \mathrm{N} . \mathrm{v}$.

Hab. Mindoro.

\section{Fam. CAPITÓNIDOS.}

1. Cién. XANTHOL ENA, BP.

1. X. hœmatocephala (S. Nüll.) $\checkmark$ o Tubantug, Aso ni pusong (M. S. T.)

Le barbu des Philippines, Bris . Orn. iv. p. 99 (s760); Daubent. Pl. Eal. vii. p. pl. 33 r.

Bucco hømacephalus, $P$. L. S. Mïll. S. N. Anhang. p. 88 ( 176 ex Daubent); Cass. Proc. Acad. Phil. 1864 , p. 242.

Barbu á gorge jaune, Buff. Hist. Nat. Ois. viii. p. 102 pl. 5 (1 780$)$.

!Le Petit Barbu, Buff. Hist. Nat. Ois. vii. p. 105 (1780), Senegal! ¿Barbu au Senegal, Daubent. Pl. Enl. vii. p. 746 fig. 2.

Yellow-throated Barbet, Lath. Gen. Syn. i. pt. 2. p. 500 (1782).

Litlle Barbet, Lath. Gen. Syn. i. pt. 2. p. 503 (1 782 ).

Buff-faced Barbet, Lath. Gen. Syn. i. pt. 2. p. 504 pl. xxii. (1782).

Bucco flavigula, Bodd. Tabl. Pl. Enl. p. 20 (1783); ex Daubent.)

¿B. nanus, Bodd. Tabl. Pl. Enl. p. 47 ( 1783 ex Daubent).

Indian Barbet, Lath. Gen. Syn. Suppl. i. p. 97. (1787).

Bucco philippinensis, Gm. Syst. Nat. ii. p. 407 (1788 ex Briss.); Rafs. Trans. Linn. Soc. xiii. p. 283 (1820); Less. Traite, p. 163 (1831).

?B. parvus, Gm. Syst. Nat. i. p. 407 (1788, ex Buff.)

B. lathami, Gm. Syst. Nat. ii. p. 408 ( 1788 ).

B. ind:cus, Lath. Ind. Orn. i. p. 205 (1790). 
Le Barbu á collier rouge, Levaill. Hist. Na!. Barbus, ii. p. 7 ' pl. 33 (1806).

Capito lathanni, Vienll. $N$. Dict. iv. p. 499 (1816).

C. flavicollis, Bonn. et Vieill. Enc. Meth. p. It $2+$ (1823).

Bucco rubricollis, (uv. Régne Anim. i. p. +57 (1892 ex Levaill.)

B. luteus, Less, p. 163 (1831) var; Des Murs, Icon. Orn. pl. 21.

B. rafflesius (nec Less.), Boie Brief. Ost. Ind. n. ${ }^{\circ}{ }_{1} 5$ (1832).

Megalaima philippinsis, Gray, Gen. B. ii. p. +29 (18+6; Blyth. Cat. B. Mus. As. Soc. p. $68(18+9) ;$ Bp. Coisp. Av. i. p. $844(1850)$

?M. parva, Gray, Gen. B. ii. p. $43^{\circ}$ (1846).

M. lathami, Gray, Gen. B. ii. p. $+29(18+6)$.

M. raffiesius, Bp. Consp. Av. i. p. $1441850 ;$ Gray, Hand-l. B. ii. p. $175 \mathrm{n}^{\circ} 845^{2}$ (1870).

Xantholæma philippensis, Bp. Consp. Volucr. Zygod. p. I 2 I 854 .

$\mathrm{X}$. flavigula, $B p$. Consp. Volucr. Zygod. p. 12 I 854.

X. indica, Horsf. \& Moore, Ca'. B. Mus. E. I. Co. ii. p. 6+t (1856); Gould, P. Z. S. 1859, p. 15I; Jerd. B. Int. i. p. 315 (1862); Be.van, Ibis, 1865 , p. 4I 2; Blyth, Ibis, 1866 , p. 359; Beavan, Ibis, 186 g, p. 4 Io; Godiv. Aust. J. f. S. Beng. xxxix. p. 267 (1870; Stol. t. c. p. 289 (1870); Wald. Ibis, 1871 , p. 862; Holdsw. P. Z. S. 1872 , p. 430; Jerit. Ibis, 1872, p. 12; Hume, Nests \& Eggs Ind. Bp.1131 (1873).

Megalaima in lica, Moore, P. Z. S. 1859 , p. 454 .

Megalæma philippensis, Irby, Ibis, 1861, p. 229; Schoinb. Ib.s, 1864 , p. 258 ; Von. Marlens, J. f. O. 1866, p. 20.

Megalaima flavigula, Goffin, Mus. Pays-Bas, Buccones, p. $2+$ (1863); Snelleman in Velh's Midden-Sumatra, Vög p. 35 i 885.

M. rubrifrons, Gray, List. Capit. Brit. Mus. p. II I868; id. Hand-l. B. ii. p. 175; n. ${ }^{\circ} 8453$ (1870).

M. hæmatocephala, Gray, Hand-l. B. ii. p. 175 n. $8+48$ (1870).

Xantholæ.na hxmatocephali, Macshull, Moncgr. Capit. p. Ior pl. 42 (1871) 1891, p. 89.

X. hæmatocephala, Hayes. Lloyd, Ibis, 1873, p. 407; Blyth. \& Wald. B. Burm p. 74 (1875); Wald, Tr. Z. S. ix. P. 156 (1877); Tivced. Ibis, 2877, p. 299; id. P. Z. S. 1877, pp. $69,0,823$; id. P. Z. S. 1878 , p. 944; Ball. Str. F. vii. p. 206 (1878); Cripps, t. c. p. 264; Legge, B. Ceylon. i. p. 2 18 (1879); Hume, Str. F. 1879 , pp. 53, 38; Scully, t. c. p. 253; Salvad. Ann. Mus. (iv. Genov. xiv. p. 180 (1879); Vidal. Str. F. ix. p. 54 (1880); Biughan. t. c. p. 166; Butler. t. c. p. 387; Wardlaw Ramsay, Tweedd. Mem. p. 656 (1881); Keiham, Ibis, 1881, p. 390; Nicholson, I6.s, 1883 , p. 2+3; Oatc's, Handb. 13. Brit. Burm. ii. p. 136 (1883); Murray, Vertebr. Faun. Siud. p. 116 (1884); Swinh. \& Barnes, Ibis. 1885; p. 63; Buttilc Notes Leyden. Mus. ix. p. 16 (1886); Reid, Cá. Lucknon, Mus. p. 31 (1886; id. Str. F. x. p. 26 (1887); Davison, t. c. pp. 299, 358; Salvad. Ann. Mus. Civ. Genov. (2) iv. p. 577 (1887), v. p. 564 (1888); Shelley, Cat. B. M. 19. 1891, p. 89.

X. rafflessi, Traud, Bull. Soc. Agric. Coch. Ch. 1879, p. 91. $H a b$. Luzón, Manila, Montalban, Abra, Ilocos, Cagayan, Leyte, Mindoro, Mindanao, Placer, Surigao, Zamboanga. 
2 X. ro:ea, (Dum) of N. v. Tubantug.

Le Barbu rose-gorge, Levaill. Hist. N.at. Ois. Parad:s, ii. p. $76,33(1806)$.

Bucco roseus, Dumont. Dict. Sci. Nat. (Ist. ed) iv. p. 52 (18w) cx Levaill). Cuv. Régne An. i. p. +28 (1817).

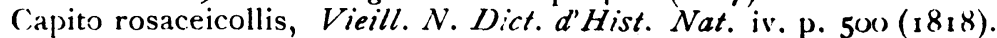
Bucco philippensis (nec Gm.), Horsf. Trans. Linn. Soc. xiii. p. 181 (1820).

B. roseicollis, Vigors, Mem. Raffl. p. 667 (1830).

Megalaima rosea, Gray, Gen. B. ii. p. 429 (1846); Bp. Cousp. Av. i. p. 14 (1850); Goffin. Mus. P. B. Buccones, p. 27 (1863); Gray, Hand-l. B. ii. p. 174 n. ${ }^{\circ} 8+45$ (1870).

Xantholøema rosea, Horsf. \& Moore, Cat. B. Mus. E. I. Co. ii. p. (1856); Marshall. Monogr. Capit. p. Io7, pl. 43 1871; Wa!den, Tr. $Z$. S. ix. p. I 57 (1875); id. Ibis, 1877 , p. $299 N$ : cholson, 1bis, 1881, p. 142; Vorderm Nat. Tijdschr. Nederle, Ind. xli. p. 187 (1882) id. op. cit. xlvi. p. 71 (1887); Shelley, Cat. B. M. 19. p. 96 (1191).

Hab. Negros, Luzón, Abra, Bucay; Sumatra, Java.

$3 \mathbf{X}$. intermedia, (Shell.) $\subset \circ$ N. v. Tubantug.

(M. S. T.

Xantholœma rosea, (nec Dum.) Walden Ibis, 1872, p. Ioo; id. Tr. $Z$. S. ix. pp. 131, I57, 250, (1875); Tweeddale, $P . Z$. S. 1877, p. 758,1878, p. 283 ; Shelley, Cat. B. M. 16. 1891 p. 97 .

Hab. Cebú, Negros, Valencia, Guimarás.

2. Gen. CHOTORHEA, Br.

4 C. versicolor. Raffl. $\sigma 0 \mathrm{~N}$. v.

(M. S. T.)

Bucco versiccolor, Raffl. Trans. linn. Soc. xiii. p. 284 (1820);

Temm. pl. Col. iii. pl. 309 (1825); Blyth, J. A. S. Beng. xv. p. I 3 (1846).

B. rafflesii, Less. Rev. Zool. 1839 , p. 137.

.Megalaima versiccolor, Gray, Gen. B. ii. p. 429 (1846); Bp. Consp. i. p. 143 (1850).

Chotorhea versicolor, Shelley, Cat. B. M. 19. 1891, p. 59. $H a b$. Sumatra.

\section{Fam. RAMFÁSTIDOS.}

I. Gén. RHAMPHASTOS, LiN.

I. Rh dicolorus, Lin. $\sigma \mathrm{N}$ v. Tucan de vientre rojo.
(M. S. T.)

Ramphastos dicolorus, Linn. S. N. i. p. I52; Gm. S. N. i. p. 356;

Shelley, Cat. B. M. 19. I891, p. I 33.

Lepetit 'Toucan á ventre ronge, Levill. Ois. de Par. ii. p. 22 pl. 8. Hab. Brasil. 


\section{Fam. GALBÚLIDOS.}

I. Gén. Galblla, Briss.

I. G. melanogenia, Scl. $\sigma$ N. v.

(M. S. T.)

Galbula melanogenia, Scl. Contr. Orn. 1852, p. 61, pl. 90; Shelley, Cat. B. M. 19. I891, p. 166.

Hab. América, Guatemala.

\section{Suborden: GOGGIGEOS.}

\section{Fam. CÚCULIDOS.}

\section{Subfan. CUCULINOS.}

\section{COCCYSTES, Glog.}

\section{C. coromandus, (Lin) $\delta \&$ N. v Sabucut.}

Le Coucou hupè de Coromandel, Briss. Orn. iv. p. I 47 , pl. xi. A. fig. I (i 760$)$.

Cuculus coromandus, Linn. Syst. Nat. i. p. 17 I ( 766 ).

Coucou huppè de Coronandel, Pl. Enl. vi. pl. $27+$ ex Briss.

Le coucou huppé á collier, Montb. Hist. Nat. Ois. v. p. 389 ( I 779).

Collared cuckow, Lath. Gen. Syn. i. pt. 2, p. 529 (1782).

Le coucou á collier blanc, Levaill. Ois. d'Afr. v. pl. 213 (1806); Sundev. Krit. orn. Levaill. p. 47 (1858).

Cuculus collaris, Vieill N. Dict. Hist. Nat. viii. p. 299 (1817); Borm. et Vielll. Enc. Meth. p. I333 (1823).

C. coromandelicus, S. Müll. Naturl. Geseh. Land-en Volkenk. p. 234 , note $(1839-44)$.

Oxylophus coromandus, Jerd. Madr. Journ. xi. p. 272 ( $184(1)$; Blyth, J. A. S. Beng. xi. p. 920 (1842); Gen. B. ii. p. 464 (1847); Blyth, Cat. B. Mus. As. Soc. p. 74 (1849); Bp. Consp. i. p. 102 (1850); Licht. Nomencl. Av. Berol. p. 79 (1854); Motley \& Dillw. Labuan. N. H. p. 55 (1855); Irby, Ibis, 1861, p. 230 Sclat. P. Z. S. 1863, p. 209.

Oxylophus coromandus vel rubrarmus, Hodgs. Icon. ined en Brit. Mus. Passeres, pl. 345 figs. I, 2 (no. 611); id. in. Gray's Zool. Misc. p. 85 (1844).

Coccystes coromandus, Horsf. \& Moore, Cat. B. Mus. E. I. Co. ii. p. 693 (1854); Moore, P. Z. S. 1859 , p. 459; Jerd. B. Ind. i. p. 341 (1862); Cab. \& Heine, Mus. Heine, iv. p. 45 (1862); Schl. Mus, Pays-Bas, Cuculi, p. 43 (1863); Blyth, Ibis, 1866, p. 364; Swinh Ibis, 1867, p. 227; Bulger, Ib s, 1869, p. 169; Gray, Hand-l. B. ii. p. 229, no. 906 (1870); Swinh, P. Z. S. 1871 , p. 394; Holdsw. P. Z. S. 1872, p. 432; Hinme, Nests, \& Eggs. Ind. B. p. I 38 (1873); Salvad. Ucc. Born. p. 67 (1874); Blyth \& Walden, B. Burm. p. 81 (1875); Hume, 
Str. F. 1871 ; 1. 82; Brïggem Abhandl. nat. Ver. Bremen, i. p. 405 (1876); David \& Olust. Ors. Chine P. 71 (1877); Hume \& Davis, Str. F. 1878 , P. I62; Sharpe, Ib's, 1878, p. 414 ; Tweedd. P. Z. S. 1878 , p. 946; Legge B. Cevlon, p. 24") (1879); Hume, Str. F. 1870 , p. 89; Seully t. c. 1. 257; Wardlazu-Ramsay, App. Trueedd. Mem. p. 650 (1881); Simsnn. Ibis, 1882, p. 87; Silvin, Cat. Strickl. Coll. p. 437 (1882); Müller, J. F. O. 1882 , p. 406; Blasius, J. F. O. 1883, p. 155; Oates B. Brit. Burm. ii. p. I17 (1883); Guillem. P. Z. S. 188.5, p. 406; Büttlk, Notes, Leyd. Mus. ix. p. 29 (1886'; Vorderm. Nat. Tijdschr. Nederl. Ind. xlvi. p. I0 ( I 846); No'-man, Ibis, $1888^{\circ}$, p. too; Sharpe, Ibis, 1890, p. I 2 ; Shelley, Cat. B. M. 19, p. 214 ( 1891 ).

Hab. Mindanao, Zamboanga; Borneo, Sumatra, Java, Célebes.

\section{Gén. SURNICULUS, I.ks.}

2. S. Lugubris. (Horsf.) $\sigma \circ$ N. v. Meisaquit.

(M. S. T.)

Cuculus lugubris, Horsf. Tr. Linn. Soc. xiii. p. I79 (1820); id. Zool. Res. Java, pl. $5^{8}$ (1824); S. Mïll. Verh. Lant-en Volk. p 178 (1839-44;) Less. Traité. Orn. p. I5I (1831); id. in. Belang. Voy. Ind. Orient. p. 238 (1834); Blyth, J. $A$ S. Beng. 1872 , p. 916; Bp. Consp. i p. I05 (1850); Schl. Mus. Pays. Bas, Cuculi,. p 28 (1864); Pelz. Novara Reis., Vög. p. 104 (1865); Gray, Hand-l. B. ii. p. 219 , n. ${ }^{\circ} 9052$ (1870).

Cuculus albopunctatus, Drap. Dict. Class. Hist. Nat. iv, p. 570 (1823).

C. albopunctatus, Horsf. Zool. Res. Java, pl. (1824).

Pseudornis dicrur ides, Hodgs. Icon ined. in Brit. Mus., Passeres, pl. 353 no. 502; id. J. A. S. Beng. 1839 , p. I36; 1d. in Gray's Zool. Misc. p. 85 (18+4); Gray, Cat. Mamm. \& Nepal. pres. Hodgs. p. i i $9 .(1846)$.

P. lugubris, Hodgs. J. A. S. Beng. 1839 , p. 137.

Cuculus dicruroides, Jerd. Madras, Journ. x. p. 22 I (I840); Blyth, J. A. S. Beng. 1842 , p. 91 5 (1843); p. 244; kelaart., Cat. Prodr. App. p. 6o (I 853); Lryard, Ann. \& Mag. N. H. xiii. p. 453 (I854); Gray, Haud-l. B. ii. p. 219, no. 905I ( 1870$)$.

Polyphasia lugubris, Blyth, J. A. S. Beng. xii. I843, p. 244.

Surniculus dicruroides, Blyth, Cat. B. Mus. As. Soc. 1849, p. 72; Horst. \& Moore, Cat. B. Mus. E. I. Co. ii. p. $696(1856)$; Jerd. B. India, i. p. 336 (1862); Swinh. P. Z. S. 1871 , p. 394; Holdsworth, P. Z. S. 1872, P. 431; Hume, Str. F. 1874 , p. 472 ; David \& Oust. Ois. Chine, p. 6r (1877).

S. lugubris, Blyth, Cat. B. Mus. As. Soc. p. 72 (1849); Horsf. \& Moore, Cat. B. Mus. E. I. Co. ii. p. 695 (1856); Sclat. P. Z. S. 186 g, p. 209, Walden, Ibis, 1873 , p. 368; Salvad. Ucc. di Borneo, p. 63 (1874); Sharpe, Ibis, 1877, p. 8; Tweeddale, t. c. p. 287 ; id. P. Z. S. 1878 , p. 6r 3 ; Hume. \& Dason, Str. F. vi. pp. 159, 502 (1878); Ball, Str. F. vii. p. 207 (1878); Salvad. Ann. Mus. Civ. Gen. 1879, p. 184; Sharpe, Ibis, 1879 , p. 244; id. P. Z. S. 1879 , p. 328; Hume, Str. $F$. viii. pp. 54, I9 (1879); Legge, Legge, $B$. Ceylon, p. 243 
(1850); Hume, Str. F. ix. p. 24r (1880); Waldlaw Ramsay, Trueeddale's Orn. Works, App. p. 656 (1881); Sharpe P.Z. S. 1881, p. 792; Oates, Handb. B. Br. Burm. ii. p. I I 2 I883, Blasius, Verh, zool-bot. Ges. Wieu, xxxiii. p. 30 (1883); Buttik, Notes Leyden Mus, 1886, p. 27; Sharpe, Ibis, 1888 , p. 394,1890 , p. 10; Whttehead. t. c. p. 46 ; Shelley, Cat. B. M. $19,1891, \mathrm{p} .227$.

Calangelus lugubris, Cab. \& Heine, Mus. Hein. iv. p. iz (1862); A. Mill. J.f. O. 1882 p. 404.

$\therefore$ dicruroides, Cab. \& Heine, Mus. Hein. iv. p. 18, note (1862). Surniculus velutinus, (nec Sharpe,) Treeddale, P. Z. S. 1879 , 1. 69.

Hab. Paragua, P. Princesa; Java, Sumatra.

3. S. velutinus, Sharpe. $0^{n} .0$ N. v. Misaquit.

(M. S. T.)

Surniculus velutinus, Sharpe, Tr. Linn. Soc. ser. 2, Zool. i. p. 320 (1877); Trueeddale P. Z. S. 1877 , p. 823, 1878, p. 945; Wardlaze Ramsay, Trueeddale's Orn. Works, App. p. 659 (1881); Shelley, Cat. B. M. 19, 1891 p. 230.

Hab. Luzón, Manila, Cavite, Indan, Butuan, Zamboanga, Basilan, Mind..nao, Malamaui.

\section{Gén hierocoCCyx, S. Mula.}

4. H. sparverioides, ( $V_{i j}$ ) o $\bigcirc \mathrm{N}, \mathrm{v}$ Sicosocop.

M. S. T.)

? Coucou á ventre rayé de l'Isle de Panay, Sonn, Voy. Now. Givin. i. p.' 20 pl. I 79 (1776).

¿coucou brun et j.une á ventre rayé, Buff. Hist. Nat. Oss. vi. p. 379 (1779).

(Cuculus flaviven ris, Scop. Del Flor et Fann. Insubr. ii. p. 89 (cx Sonn. 1786); Strickl. J. A. S. Beng. 1844, p. 390, note.

( . radiatus, Gm. S. N. i. p. 420 (ex Sonn. I 788 '.

C. sparverioides, Vig. P. Z. S. 1831 , p. 173; Gould, Cent. Himal. B. pl. 53 (1832); Blyth, J. A. S. Beng. xi. p. $898(1842)$, xii. p. 240 (1843'; Hodgs. in Gray's Zool. Miss. p. 85; Gray, Cat. Mamm. \& B. Netal pres Hodgs. p. I20 (1846); Blyth, Cat. B. Mus. As. Soc. p. 7o (1849); Schl. Mus. Pays-Bas, Cuculi, p. 15 (pt., 1864); David. \& Oust. Ois. Chine, p. 63 (1877); Blakiston, Ibis, 1878, p. 228.

Hierococcyx sparverioides, Bp. Consp. i. p. 104 (1850); Horsf. \& Moore, Cat. B. Mus. E. I. Co. ii. p. 699 (1856); Jerd. B. Ind. i. p. $33^{1}$ (1862); Blyth, Ibis, 1866, p. 362; Jerd. Ibis, 1872 , p. I4; Hume, Neste \& Eggs. Ind. B. p. ${ }^{3} 35$ (1873); id. Str. F. rii. p. 80 (1875); Armstrong, Str. F. iv. p. 3 I I (1876); Tacz J. F. O. 1876 , p. 201; Hume \& Davisson, Str. F. vi. p. 157 (1878); Ball. Str. F. vii. p. 207 (1878); Hume, Str. F. vi i. pp. 54, 89, (1879); Scully, t. c. p. 256; Binghaam, Str. F. ix. p. 167 (1880); Hume, t. c. p. 248 ; Davison, Sir. F. x. p. 359; Oates, Handb. B. Br. Burm. ii. p. $108(1880)$; Marshall, Ibis, $18 \delta 4$, p. 41 ; A. Müll. J. f. O. $1885,1$. 157; Salvad. Ann. Mus. Civ. Gen. 1887 , P. 581 ; Shope, 
Ibis, 1890, p. 11; Oales, et Hume's Nests of Eggs. Ind. $B$. ii. p. $38+$ (1 890); Shelley, (at. B. M. 19. $18^{2} y 1$, p. 232.

Cuculus strenuus, Gould, P. Z. S. 18.56, p. 96; id. B. Asir, vi. p. t $^{2}\left(185^{6}\right)$; Blyth. Ibis, 1860 , p. 362.

Hierococcyx sparverinus, Cab. \& Heine, Mus. Hein. iv. p. $2 x$ note $(1862)$.

H. strenuus, Cab. \&. Heime, Mus. Hein. iv. 28 note (1862); Hume, Pr. Z. S. Beng. $1 \delta^{\prime} z 2$, p. $7 \mathrm{r}$; Ilalden, Tr. Z. S. ix. p. I61 (1875); Sharpe, P. Z.S 1879, P. 327; Blas us, Ormi,

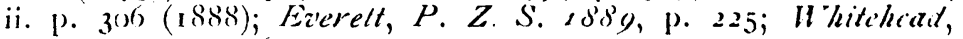
Ibis, $1890, \mathrm{p} .+6$.

Hab. Paratgua, Tagburus, Luzón, Manila, Cagayan, Pamplona; China, Borneo.

5. H. fugax, (HH, sf.) ơ N. v. Sabucot.

(M. S. T.)

Cuculus fugax, Horsf. Tr. Limn. Soc. xiii. p. 178 (1821); Vis. Mem. Raftl. p. 67 I (1830); Sykes, P. Z. S. 1832 , p. 98; Jerd. Mad. F. xi. p. 2 I9 (18f(o); S. Mäll. Verh. Land-en Volk. p. 233 (1839-4t); Blyth, J. A S. Beng. xi. p. gor) (1842), xii. p. 240, (18+3), xiv. pp. 20+, $943($ (1845); id. Inu. \& Mag. Nat. Hist. 18+6, p. 385; Gray, Cat. Mamm. \& B. Nepal pres. Hodgs. P. I $20(1846)$.

C. lathami, J. E. Gray, Ill. Zook. p. 34 fig. 2 (1834).

C. vagaus, S. Müll. Verh. Land-en Volk. p. 233 note (1839-44).

C. nisicolor, Hodgs. Icon. ined. in Brit. Mus., Passercs, pl. 357. fig. I (n." 872); Blyth. J. A. S. Beng. 1843, p. 18.

C. flaviventris, (nec Scop.), Strickl. J. A. S. Beng. 1873, 1. $390^{\circ}$; note.

Hi rococyx flaviventris, Blyth, J. A. S. Beng. p. 390, note 1844 ; id. Ibis, 1866 , p. 362 .

H. fugax, Bp. Consp. i. p. I04 (1850); Cab.\& Heine, Mus. Hein. iv. p. 28, note (1862); Salvad. Ann. Mus. Civ. Gen. v. p. 65 (1874); Trucedd. Ibis, 1877 , p. 7; Salvad. Ann. Mus. Civ. Gen. xiv. p. 185 (1879); Sharpe, Ibis, 1879, p: 245; Hume, Str. F. viii. p. $5^{2}$ (1879); Kelham, Ibis, 1881, p. 391; Wardlawo. Ramsay, Ibis, 1884 , p. 333; A. Miüll. J. f. O. 1885, p. 157; Butiih. Notes Leyd. Mus. 1886 , p. 28; Sharpe, P. Z. S. 1887 , p. 442; id. Ibis, 1890 , p. 10; Shelley, Cab. B. M. 19. 1891, p. 236 .

H. vagans, Bp. Consp. i. p. I04 (1850); Cab. \& Heine, Mas. Hein. iv. p. 3o, note (1862).

Cuculus hyperythrus, Gould. P. Z. S. 1856, p. 96; id. B. Asic vi. pl. 431 (1856) Swinh. P. Z. S. 1871 , p. 395; David \& Oust. Ois. Chine p. 64 (1874); Layard. Ibis, 1878, p. 327; Seebhm, Ibis, 1884 , p. 36.

H. pectoralis, Cab. \& Heine, Mus. Hein. iv. p. 27 (1862); Walden, Tr. Z. S. ix. p. 161 (1875); Tweedd. P. Z. S. 1878 , p. 946.

H. hyperythrus, Cab. \& Heine, Mus. Hein. iv. p. 3o, no e (1867); Walden, Tr. Z. S. ix. p. 161 (1875); Wardlare Rainsay, Ibis, 1886 , p. I 57, Tweed. P. Z. S. 1878 , p. 946.

H. sparveroides (nec Vig.), Schrenck, Reis. im Amurlande, Vogr. p. 257 , pl. Io ( $1859-67)$.

H. nisicolor, Jerd. B. Ind. i. p. 330 (1862); Blyth. P. A. S. 
Beng. xxx. p. 93 (1861); id. Ibis, 1866, p. 361; Jerd. Jbis, 1872 , p. 14; Hume, Str. F. vi. p. 157, (1870); Jerd. Str. $F$. vii. p. $371(1878)$; Hume, Str. F. viii. pp. 53, 80 (1879); Oates, Handb. B. Bs Burm. ii p, 109 I 883 ; id. ed Humés Nests. \& Eggs. Ind. B. ii. p. 383 (1890).

Hab. Luzón, Manila, S. Mateo, Zamboanga, Mindanao; Borneo, Java, Sumatra.

4 Gén. CUCUL US, Lin.

6. C. canorus, Lin. $\mathrm{m} \mathrm{N}$ v. El Cuclillo.

(M. S. T.)

Cuculus canorus, Linn. Syst. Nat. ip. i68 ( 1766); Horsf. Trans. Linn. S. xiii. p. 179 (1821); Naum, Vög. Deutschl. pls. 127; 1 28, 129, (1822-53); Temm. Mam. d'Orn. i. p. 381 (1820); Werner, Atlas, Zygod. pls. I, 2 (1828); Sykes. P. Z. S. 1832, p. 98; Gould, B. Eur. iii. p. 240 (1837); Blyth. J. A. S. Beng. xi. p. 901 (1842); Rupp. Syst Uebers p. 96 (1845\% Blyth, Cat. B. Mus. As. Soc. p. 71 (1849); Karb. Orn. Dan. pl. ro, \& Suppl. pl. 4 (1851); Schl. Vög. Nederl. pls. 5.5, 50 (1854-58); Hartl. Orn. W. Afr. p. 266 × 857 , Horsf. \& Moore, Cat. B. Mus. E. I. Co. ii. p. 702 (1858); Irby, Ibis, 1861, p. 229; Sundew, Svensk. Fogl. pl; 21. figs. 4, 5 (1862); Blakiston, Ibis, 1862 p. 325; Cab \& Heine, Mus. Hein. iv. p. 32 ( 1862); Jerd. B. Ind. i. p. 322 (1862); Swinh. Ibi, 1863 , p. 396; Schl. Mus. Pays-Bas, Cuculi, p. 4 (1864); Betteni, Ucc. Lumb. ii. pl. 92 (1865-70); Blyth, Ibis 1866 , p. 359; Tytler Ibis 1868 p. 202; Blan Geol \& Zool, Abyss. p. 352 (1870:; Fritsch, Vög. Eur. pl. iv. figs. 8, 9 (1870); Gray, Hand-1. B. ii p. 215, n. ${ }^{\circ} 8985$; Swinh. P. Z. S. 1871, p. 395, Heugi. Orn. N. O. Afr. p. 779 (1871); Shelly, B. Egypt, p. I62 (1872); Gurney in. Anderss B. Damara Ld. p. 227 (1872:, Jerd. ibis, 1872 p. 12; Cab. J. f. O. 1872 . p. 235; Gould, B. Gt. Britt. iii. pls. 67,68 (1873); Hume, Nests. \& Eggs.. Ind. B. p. 133 (1873); Alston. \& Harvie-Brown, Ibis, 1875 , p. 59; Brooke, t. c. p. 236; Cock. \& Marshall, Str. F. i. p. 35I (1873); Adams, t. c. p. 373; Steineger, J. f. $O$ 1873. p. 305; Ussher, Ibis 1874 , p. 52; Durnford, t. c. p. 395; Ball, Str. F. ii. p. 393 (1874); Swinh, Ibis, 1875 , p. I 25; Danford, t. c. p. 299; Krüper, J. f. O. 1875, p. 279; Sachse, t. c. p. 424; Hume, Str. F. iii. p. 78 (1875); Bro.ks, t. c. p. 232; Bygrave Wharton, Ibis, 1876 , p. 26; Seebohm. \& Harvie-Brown. Ibis 1876 . p. II ; Barratt, t. c. p. I99; I)resser t. c. p. 320 ; Scully, Str. F. iv. p. 134 (1876); Hume, t. c p. 28nं; Bchalow, J. f. O. 1876 , p. 186; Bocage, Orn. Angola. p. I $3^{8}$ (1877); David \& Oust. Ois. Chine, p. 64 ( 1877); Ayres, Ibis, 1877, p. 342; Wardlaw Ramsay, t. c. p. 458; Hume, Str. F. v. p. 27 (1887); Schalow J. f. O. 1877 , p. I96; Dresser, B. Eur. v. p. 199, pl. 299. (1878); Dauford, Ibls, 1878, p. 7; Blakiston \& Pryer t. c. p. 227; Seebohm, t. c. p. 326; Hume', Str. F. vi. p. I $56(1878)$; Ball, t. c. p. 2o6; Finsch, P. Z. S. 1879 p. 12 ; Hume, Str. F. viii, p. 88 (1879); Scully t. c. p. 253;A. Mïll J. f. O. 1879 , p. 387 ; Legge, B. Cevl"n i. p. 221 (1880); Wardlau Ramsay, Ibis, $188^{\circ} 0$; p. 50 Biddulph, 
Sir F. ix. p. 314 (1889); Butler, t. c. p. $3^{8} 7$; Barnes, t. c. p. 453; Biddulph, Ibis, 1881 p. 49; Siully, t. c. p. 430; Reid. Str. F. x. p. 26 (188I); Scully t. c. p. 102 Davison, t. c. p. 299; Swith Ibis 18\&2, p. IO3; Seebohm, t. c. pp. 309, 37j; Shclly t. c. p. 309, Oates, Gandb. B. Brit. Burmah, ii. 1. 103, (1883); Seebohm, Ibis, 1883 , p. 22; Severts. t. c. p. 71, Isby. t. c. p. 179; Sharpe, ed Layarid's B. S. Afr. pp. 147; 889 (1875-84); Tristr. Faun \& Hor. Palest ne, p. 89 (1884); Seebohm, Hist. Brit. B. ii. p. 378 , pl. i9 (1884); Agres, Ib S, I 884, p. 223; Saunders, t. c. p. 380 Marshall, t. c. p. 410; Fisch. Zeitsehr. ges. Orn. $188+$ p. 365; Swinh. \& Barnes, Ibis, 1885 p. 63; Dixon, t. c. p. 257; Guillemard, P. Z. S. 188.5 p. 551 Fisch. J. f. O. 1885 , p. 1 24; Deditius, t. c. p. 203 ; Dubois, Bull. Mus. Belg. 1885, p 181; Schalow, Zeitschr. ges. Orn. 1885 p. 86; Booth, Rough. Notes, part. xiii pl Sharpe Ibis, 1866 , pp. 489, 498; Hartert, J. f. O. 1886 p. 597; Stynn, Ibis. 1887 , p. 230; Tait. t. c. p. 306; Bocage, Forn. Lisb. 1887, p. 5; Leverk. J. f. O. 1887 , pp. 79, 191; W. Müll. t. c. p. I69; Schalow, t. c. p. I88; Hartert, t. c. p. 253; Eckstein, t. c. p. 286; St. Pohn, Ibis, 1889 p. I 59; A. Miüll. t. c. p. 219 ; Lilford, t. c. p. 330; oates, t. c. p. 355; Clarke, t. c. p. 549; Oates, ed Hume's Nests. \& Eggs. ii. p. 379 (1890); Shelley, Cat. B. M. 19.1891. p. 245.

Le coucou, Montb. Hist. Nat. Ois. vi. p. 305 (1779); Daubent. Pl. Enl. vi..pl. 8i I.

Coucou du Cap de Bonne Esperance, Month. pl. Enl. vi. pl. 390.

Cuculus capensis, P. Z. S. Mäll. Syst. Nat. Suppl. p. 90 (1776); Gm. Syst. Nat. i. p. 4 Io (1788; ; Steph. Gen. Z. ix p. 85 (1 825); Cab. \& Heine, Mus. Hen. iv. p. 42 (pt., 1862); Cass. Proc. Philad. \& 1864, p. 243.

C. hepaticus, Sparrm. Mus. Carls. iii. p. 55 (1789); Lath. Ind. Orn. i. p. 215 (1790); Sundev. Krit. Orn. Sparrm. p. 10 ( 1857 ).

C. rufus, Bechst. Nat. Hist. Deutschl. ii. p. II42 (1792); id. Oru. Taschenb. i. p. 84 (1802).

C. tristis, Hermann, Observ. Zool. p. I 31 (1804).

Coucou vulgaire d' Europe, Levaill. Ois. a' Afr. v. p, 26, pl. $202,203$ ( 1806$)$.

Cuculus borealis, Pall. Zoogr Rosso-As. i. p. 442 (1811).

?C. tenuirostris, S. Müll. Verh. Nat. Gesch. Land-en Volk. p. 235, note (1839-44); Bp. Consp. Av. i. p. I03 (1850); Ciib. \& Heine Mus. Hein. iv. p. 39 (1862).

C. Iongipennis, Brehm, Naumannia, 1855, p. 271.

C. striatus (nec Drap), Swinh. Ibis, 1861 , p. 259.

C. telephonus, Heinc, J. f. O. 1863, p. $35^{2}$.

C. libanuticus, Tristr. P. Z.S. 1864, p. 432.

C. canorus telephonus, Stejn. Bull. U. S. Nat. Mus. n. ${ }^{\circ}$ 29, p. 224 (185); Trazanowski, P. Z. S. 1887 , p. 607, I888, p. 466 .

C. canorus borealis, Stejn. Bull. U. S. Nat. Mus. n. ${ }^{0}$ 29, p. 225 i 885.

C. peninsulæ, Stejn. t. c. p. 227.

Hab. ¿Luzon; Europa, y casi cosmopolita. 
7. C. intermedius, Vahl. o o N. v. Sabucot. Mesayuit. (M. \$. T.)

C. intermedius, Vahl. Skriz. af. Natuur. Selzk. iv. p. $5^{8}$ (1797); Oates, ed. Hume's Nests. \& Eggs. Ind. B. ii. p. 3ri (1 Squ); Seeb Birds: Japan Emp. p. 169 (1890); Shelley, Cat. B. M. 19. 1891, p. 252.

C. striatus, Drap. Dict. Class. Hist. Nat. p. 570 (1823); id. Dict. Class. Sc. iii. p. Itt $\left.183^{8}\right)$; Horsf. \& Moore, Cat. B. Mus. E. . Co. ii. p. 703 , pt. $(1856-58)$; Swillh. Ib 18,1861 , pl 259, 340; Cab. \& Heine, Mus. Hein. iv. p. 37 (1862); Schl. Mus. Pays-Bas, Cuculi, p. 7 1864); Bly'h, Ibis, 1866 , p. 359; Swinh. P. Z. S. 1875 , p. 395; Humn, Str. F. i. p. 422 (1873); id. Nests \& Egos. Int. B. p. I3t (1873); id. Str. F. ii. pp. 19o; Rosenb. Reits naar Geslbinkb. p. 6 (1875; Hume, Str. F. vi. p. 156 (1878), viii. pp. 53, 88 (1879); Scully, t. c. p. 254; Davison t. c. p. 359 ; A. Mïll. J. $f . \quad O$. 1882 , p. 306; Oates, Handb. B3. Brit. Burm. ii. p. I05 (1883); id. Ib s, 1889, p. 357 .

C. himalayanus, Vis. P. Z. S. 1831 , p. 172; Blyth. Cat. B. Mus. As. Soc. p. 71 (1849); Horsf. \& Moore, Cat. B. Mus. E. I. Co. ii. p. $704\left(185^{8}\right)$; Jerd. B. Illd. i. p. 323 (1862); Tytler, Ibis, 1868 , p. 202; Jerd. Ibis, 1872 , p. 82; Seebohm. Ibis, 1878 , p. 326; Sharpe, P. Z. S. 1879 , p. 327; Biddulph, Str. F. ix. p. $314(1880)$; id. Ib.s, 1881 , p. 50; Scully, t. c. p. 431, id. Str. F. x. p. 103 (1881); Seebohm. Hist. Brit. B. ii. p. 379 I 88 t, p. 36; Marshall, t. c. $1.4+10$.

C. canoroides, S. Mäll. Verh. Nat. Gesch, Land-en Volk. p. 235, note (1839-44); Bp. Consp. Av. i. p. 103 (1850); Gray, P. Z. S. 1860, p. 359; Wallace, P. Z. S. 1863, p. $48+$; Finsch, Nen-Guinea, p. I 59 (1865); Gould, B. Australia, p. 6I4 (1865); Blyst, Ibis, 1865, p. 31; Gray, Hand-l. B. ii. p. 2 I6, n." 9006 (170); Blyst, Ibis, Cat. B. Burmah, p. 79 (1875); Wardlaw Ramsay, P. Z. S. 1875 , p. 600; Saliad. Ann. Mas. Civ. Genov. x. p. I 24 ( 1877 ), xiii. p. 457 (1 878 ); D' Albertis \& Salvad. Ann. Mus. Civ. Genov. xiv. p. 42 (1879; Ramsay, Pr. Linn. Soc. N. S. Walcs, iv. p. 96 (1879); Salvad. Orn. Papuasia, i. p. 328 (1880); App. op. cit. p. 48 (1883); Blits. u. Nehek. Verh Gesellsch. Wien 1882. . 417; Finsulh, Vög. der Sudsee, p. 16 (1884); Plcske, Bull. Act. Petersb. xxix. p. 524 (1884); Guillemard, P. Z. S. 1885, pp. 565, 624; Blasints. Ornis, v. p. 306; Whiteliead, Ib.s, $1890, \mathrm{p} .+6$.

C. saturatus, Horsf. J. A. S. Beng. xii. p. $9+2$ (1 $8+3)$; Blyth, J. A. S. Bang. xi. p. 18 (1846; Cab. \& Heine, Mils. He.n. iv. p. 37 , note (1862).

C. optatus, Gould, P. Z. S. 1845, p. 18; Gray, Gen B. ii. p. 463 (1847); Gould, B. Australia, iv. pl. 48; id. Introd. B. Australia p. 67 (1848); Rchb. Vög. Neuholl. ii. pp. I13, 309 (1851); Bp. Consp. Av. i. p 103 (1850); Cab. \& Heine, Mus. Hein. iv. p. 34, note I 862 ).

C. canorus indicus, B.yth, J. A. S Beng. xr. (1846 p. i9; Stcjin. Bull. U. S. Nat. Mus. n." 29, p. 228 (1885).

Nicoclarius optatus, Bp. Consp. Vol. Zygod. p. 6, sp. 169 (1854); Gray', Hand-l. ii. p. 216 n. 9007 ( 1870$)$. 
Nicoclarius cannroides, Bf. Consp. Fol. Zygod. p. 6 sp. I69 (185t); Gray', Iland-!. 13. ii. 1). $216(18 ; 0)$.

Cuculus horsfiedi, Moore, Cat. B. Mus. E. I. Co ii. p. 703 $(1858)$.

C. fucitus, Peale, $U^{r}$. S. Exped. p. I 36, pl. 37. fig. a (18+8); Cab. \& Hsine, Mus. He 1 . iv. p. 40, note, (1862); Gray', Hand-!. B. ii. p. 216 n. 8997 (18jo).

(. tenuir.sstris, (nec Less.), Cass. U. S. Expl. Exped. 1858, p. 2tt pl. 21 fig. 1 .

C. indicus, Cab. \& Heine, Mus, Hen. iv. 1. 3t (1.862); Taez J. f. O. $18 ; 6$, p. 200 ; id. Bull. Soc. Z. France, 1882, p. 395; Dy'b. op. cit. 1883, p. 368; Lorenz, Beilr. Orn. Kank. 1. $43(1887)$.

C. cantor, Ill. fide Cab. \& Heine, Mus. Hein. iv. p. 3+ (1862).

C. canorinus, Cab. \& Heine, Mus Hen. iv. p. 35 (1862); Guies. Thes, Orn. i. P. 834 (1872); Tassan, J. f. O. 1873 , pp. 9', 1 18; Salvad. Ucc. Borneo, p. 67 (1874); Swinh. Ibis, 1575 , p. 25t; Stejn. Pr. U. S. Nat. Mus. vi. p. 182 (1883).

C. swenhoii, Cab. \& Heine, Mus. Hein. iv. p. 37, note (1062).

C. kelungensis, Swinh. Ib.s, $186_{3}$, p. 394; Gray', Hand-l. B. ii. p. 216, n. ${ }^{\circ} 9000$ (I 070$)$.

C. mon syllabicus, Szwinh Ibis, 1865 , p. 545; Gray, Hant-l. B. ii. p. $216, n{ }^{\circ} 8998(1870)$.

Hab. Luzon, Laguna, Cilauan, Paragua, P. Princesa; Chinat, Célebes, Ausțralia.

8. C poliocephalus, Lath. O'. N. v. Sabucot. Meisaquit (M. S. T.)

Cuculus poliocephalus, Lath. Ind. Orn. i. p 2I4 (1790); Gould. Cent. B. Himalayas, pl. 54 (1832); Blyth, J.A. S. Beng, xi. p. $904(18+2)$; Gray, Cat. Mamm. \& Nepal, pres Holtgs. p. I 9 (1846); Blyth, Cat. B. Mus. As. Soc. p. 7 (1849); Horsf. \& Moore, Cat. B. Mus. E. I. Co ii. p. $704(1856-58)$; Cab. \& Heine, Mus, Hein. iv. p. $3^{\mathrm{r}}$, note (1862); Jerd B. Int. i. 1) 324 (1862); Schl. Mus. Pay's-Bas, Ciucuti, p. I2 (1864); lerd. Ib.s, 1872 , p. I 3; Gray, Hand-l. B. ii. p. 21 5, n. $8990 ;$ Hume, Nests. \& Eggs. Ind. B. p. I 35 (1873); Milne-Edw. \& Grand. Hist. Madag. Ois; Atlas, i. $\mathrm{pl} 66$ (1876); fuirbank, Str. F. iv. p. 255 (1876); David \& Oust. Ols. Ching, p. 66 (1877); Davidson \& Wenden, Str. F. vii. p. 7 I (1878); Huine, Str. F. viii. p. 88 (1879; 'Sharpe, Ibis, 1879, p. 394; Legge, B. Ceylon, p. 23 I (1880); Vidal, Str. F. ix. p. 54 (1880); Butler, t. c. p. 385 ; Davison, Str. F. x. p. 299 (1881); Sharpe, Ibis, 1888 , p. 39t; Oates, Ibis, 1889 , p. 357; Sharpe, Ibis, 1 S9o, p. 11; Shelley, Cat. B. M. 19. 1891 . p. 355.

C. himalayanus vel. bharovus, Hodgs. Zool. Misc. p. 85 (1831).

C. canorus (nec Linn.), Desjard. P. Z. S. 1832 , p. 11 ; Hartl, Ann. \& Mas.-Nat. Hist. ii. p. 391 (i 848 ); id. J. f. O. 1860 . p. I Io; Vinson, Voy. Madag. p. 88 (I865); Verr. Ann. B., Voy. Madag. Vinson, p. 3 (1865).

Le Pigeon ramier gris et verdráte, Sganzin, Mem. Soc. Mus. Hist. Nat. Strasbourg, $1840, \mathrm{p} .40$.

Hierococyx pcliocephalus, Bp. Consp. Av. i. p. $10+(1850)$. 
Cuculus bartlettii, Layard, Ann. \& Mag. Nat. Hist. 2nd. scr. xiii. p. $45^{2}$ (1854).

Nicoclarius temicrostris (nec Less.), Bp. Cousp. Vol. Zygod. p. 6 (1854).

Cuculus rochii, Hartl. P. Z. S. 1862 , p. 224; Roch. \& Newton. Ibis, $186_{3}$, p. I66; E. Newton, t. c. p. 453; Poll. Ned. Tijdschr. v. d. Dierh. p. 300 (1863); Schl. P. Z. S. 1866 , p. 424 ; Schl.\& Poll. Faun. Madag. ii. Ois. p. 53 (1863); Gray, Hand-l. B. ii. p 215, n. ${ }^{\circ}$ 1992; Sharpe, P. Z. S. $18^{\prime} 70$, P. 399, 1873, p. 582; Hartl. Vög. Madag. p. 253 (1877); Bartlett. P. Z. S. 1879, p. $77 \mathrm{I}$.

C. clamosus (nec Lath.), Coquerel, Bull. Soc. Accl. Réunion ii. Io (I 864).

(C. tamsuicus, Swinh. Ibis, 1865, p. 108.

(.) himalayanus (nec Vig.), Graud. Rev. et Mag. Zool. 1867 , p. 418; Poll. Fanı. Madag., Relat. Voy. i. p. 198 (1877).

C. sp. inc., Blakiston \& Pryer, Ibis, 1878 , p. 227.

(.) poliocephalus, var. rochii, Milne-Edw. \& Graud. Hist. Madag. Ois. i. p. 176 (1879).

(.) leptodetus (nec Cab.), Dubois, Bull, Mus. Hist. Nat. Belg. iv. p. 147 (1886).

C. stornsi, Dubots, Bull. Mus. Hist. Nat. Belg. v. p. 3, pl. 2 ( 1817$)$.

Hab. Luzón, Laguna, Calauan; Java, Burneo.

5 Gén. CaComantis, S. Mul.t.

9. C. merulinus, (Scop.) o o N. v. Mesaquit, Pasiay. (M. S. T.)

Le Petit coucou de l' Isle Panay, Sonn. Voy. Noze. Guin. i. p. I 22 p. 8I (I776).

Cuculus merulinus, Scop. Del. Flor. et Faun. Insubr. ii. p. $8 y$ (1786); Strickl. J. A. S. Beng. 1844, p. 391; Blyth, Cat. B. M. As. Soc. p. 7 I (1864); Schl. Mus. Pays-Bas, Cuculi, p. 2 I (I 864); Gray, Hand-l. B. li. p. 2 I 7 n. 90 I6 (I870).

C. flavus, Gm. S. N. i. p. 42 I (1878); Steph. Gen. Zool. ix. p. 107 (1815); Horsf. Trons. Linn. Soc. xiii. p. I79 (1822); Raffl. t. c. p. 285; Vig. Mem. Raff. p. 671 (1830); Less. in Belang. Voy. Ind. Orient. p. 23I (1834); S. Mïll. Verh. Nat. Gesch. Land-en Volk. p. 177 (1839-44); Blyth, J. A. S'. Beng. 1842, p. 91 2, I843, p. 24I; Low, Sararvak, p. +1 1 (1848); Waldeu, Ibis, 1869 , p. 337 .

Surniculus flavus, Less. Traité, p. I5I (1831).

Coucou á téte grisse de Java, Less. in Belang. Voy. Zool. $\mu$. 237 (1 834).

Cuculus sepulcralis, S. Mïll, Verh. Nat. Gesch. Laud-en Volk. p. 177, note, (1839-44); Gray, Hand-l. B. ii. p. 217 , n. ${ }^{\circ} 9022$ (1870).

C. lanceslatus, S. Müll. Verh. Nat. Gesch. Laud-en Volk. 1". I 78 ( $1839-44)$.

C. abruptus, Burm. Verz. Mus. Halle, p. 54 (1850).

Cacomantis flavus, $B p$. Consp. i. p. IO3 (I850).

Cacomantis sepulcralis, Bp. Consp. i. p. Io4 (1850); Cab. \& Heine, Mus. Hein. iv. p. 20 (1862); Sclat. P. Z. S. 1863, p. 
209; Walden, Trans. Z. S. viii. p. 54 (1872); Salvad. Ucc. Borneo, P. 65 (1874); Tweedd. P. Z. S. 1878, p. 945; Wardlaw Ramsay, App. Tweedd. Orn. Works, p. 656 (1881); Gumllemard, P. Z. S. 1885, p. 550; Beddard, P. Z. S. 1885 , p. 175 .

Cuculus lineatus, Plucher, Rei'. et Mag. Zool. 1853, p. 70.

Cacomantis borneensis, Bp. Consp. Vol. Zygod. P. o (185t); Wald. Tr. $Z$. S. viii. p. 54 (1872).

Polyphasia merulina, Horsf, \& Moore, Cat, B. Mus. E. I. Co. ii. p. $697(1856-58)$.

Cocomantis threnodes, Cab. \& Heine, Mus. Hein. iv. p. 19 (1862'; Watd. Tr. Z. S. viii. p. 5t (1872); Hume, \& Davis : vi. p. 158; Ball. op. cit. p. 207 (1878); Cripps, t. c. p. 265; Hume, op. cit. viii. pp. 54, 89 (1879); Bingham, op. cit. ix. p. 167 (1880); Kelham, Ibis, 1881, p. 391; A. Mïll. J. f. O. 1882 , p. 405; Oates, Handb. B. Brit. Burmah, ii. p. In ( 1883$)$; A. Mïll. J. f. O. 1885 , p. г 57 ; Salvad. Ann. Mus. C.v. Gen. 1888, p. 568.

(iuculus rufoviridis, Cab. \& Heine, Mus. Hein. iv. p. 20 (1862).

Cacomantis merulinus, Cab. \&. Heine, Mus. Hein. iv. p. 21 ; Wald. Tr. $Z$. S. viii. p. I56 (1872); Salvad. Ucc. Borneo, p. 64 (1874); Wald. Tr. Z. S. ix. p. 160 (1875); Sharpe, Ibis, 1876, p. 34, 1877 , p. 7; id. Trans. Linn. Soc. ser. 2, i. Zool. p. 320 (1877); Treeedd. P. Z. S. 1877, p. 691, 1878, pp. 61 3 , 709; Salvad. Ann. Mus. Civ. Gen. xiv. p. 185 (1879); Sharpe, Ibis, 1879 , p. 244, Hume, Str. F. viii. p. 54 (1879); Tiveedd. P. Z. S. 1879 , p. 69; Sharpe, t. c. 1879 , p. 328, 1881, p. 792; Wardlw Ramsay, App. Trueedd. Orn. Works, p. 656 (I88I); Büttik Notes Leyden Mus. ix. p. 28 (1886); Salvad. Ann. Mus. Civ. Gen. 1887, p. 532; Sharpe, Ibis, 1888, p. 198, I89o, p. Io; Whitehead, t. c. p. 46; Everett, P. Z. S'. 1889, p. 225; Shelley, Cat. B. M. 19. 1891 , p. 268.

Polyphasia tenuirostris, (nec S. Müll.), Jerd. B. Ind. i. p. 335 (pt.) (1 862).

Cacomantis dysonomus, Heine, J. f. $O .186_{3}$, p. 352.

(C. querulus, pt., Heine, J. $f$. O. 1863, p. $35^{2}$.

Polyphasia rufiventris, Jerd. Ibis, 1876 , p. 15.

Cacomantis passerinus, (nec Vahl), Blyth. \& Walden, B. Burmah, p. 80 (i 875$)$.

Ololygon tenuirostris, Hume, Str. F. iii. p. 8o (1875).

Cacomantis rufiventris, Armstrong, Str. F. ix. p. 31 2 (г876): Wardlaw Ramsay, Ibis, 1877 , p. 458; Anders, Zool. Exped. Yunnan, p. 587 ( 1878$)$.

C. tenuirostris, (nec Bp.), David \& Oust. Ois (hine p. 62 (1877).

Cuculus threnodes, Gray, Hand-l. B. ii, p. 2i 7 , n. ${ }^{0} 9017$.

Hab. Luzón, Manila, Montalban, S. Mateo, Laguna, Calauan, Panay, Negros, Masbate, Cebú, Sanar, Leyte, Bohol, Zamboanga, Mindanao, B.silan, Paragua, P. Princesa, Malanaui; Java, Sumatra, Borneo, I'ernate. 


\section{Gén. CHAL,CococcyX, C^A.}

10. Ch. xanthorhynchus, (Itorsf.) \& \& N. v. Sabucot. (M. S. T.)

(uculus xanthorhynchus, Horsf. Tr. Linn. Soc. xiii. p. 879 (1822); id. Zool. Res. Java, pl. 59 (1824); Vig. Mem. Raffl. p. 67 (1830); S. Miull. Verh. Nat. Nat. Gesch. Land"n Volk. p. 234 (1839-44); Blyth, J. A. S. Beng. xi. p. 919 (1842); Schl. Mus. Pays-Bas, Cuculi p. 32 ( 1864 ; Gray, Hand-l. B. ii. p. 219, n." 9040 (1870); Sharpe, Ibis, 1879 , p. 244.

lampromorpha anethystina, Vig. P. Z. S. 1831, p. $9^{8}$.

Chrysococcyx xanthorhynchus, Blyth, I. A. S. Beng. xi. p. 9rg (1842), xii. p. 245 (1843); id. Cat. B. Mus. As. Soc. p. 73 (1849); Bp. Consp. i. p. $106\left(185^{\circ}\right.$; Horsf. \& Moore, Cat. 13. Mus. E. I. Co. ii. p. 706 (1856-58); Scl. P. Z. S. 1863 , p. 209; Salvad. LCc. Borneo, p 62 (1874); Wald. Ibis, 1874, p. 137 ; Hume, Str. F. iii. p. 472 (1874); Blyth, \& Wald. B. Burma, p. \&o (1875); Tweedd. Ibis, 1877, p. 287; Wardlaw Ramsay, Ibis, 1877 , P. 458; Hume, Str. F. vi. p. $506(1878)$, viii. pp. 54; 89 (1879); Sharpe, P. Z S. 1879 r p. 327; Hume, Str. F. ix. p. 248 (1880); Sharpe, P. Z. S. 1881 , p. 792; Oates, Handb B. Br. Burm. ii. p. I I4 (1883); Büttik notes Leyden Mus. 1886 , p. 21; Sharpe, Ibis, $188^{8} 8$, p. 198, 189(), p. 9; Whitchead t. c. p. 46.

lam.promorpha xanthorhynchus, Bp. Consp. Vol. Zygod. p. 7 (1854).

Chalcucocyx xanthorynchus, Cab. \& Heine, Mus. Hein. iv. p. 15 (1862); Hume, Str. F. ii. p. I91 (1874); iii. p. 81 (1875); Hiume \& Davis Str. F. vi. pp. 161, 506 (1878); Shelley, Cat. B. M. 19, 1891, p. 289 .

(. annethystinus, Wald. Tr. Z. S, ix. pp. I60, 250 (1875).

(halcites xanthorhynchus, Gould., B.Asia vi. pl. 47 (1877).

(hry sococcyx limburgi, Trueedd. P. Z. S. 1877 , p. 366; id. Str. -F. vii. p. $319(1878)$; Hume, Str. F. viii. p. 89 (1879); Burgliam, Stos. F. ix. p. 168 (1880); Oates, Handb. B. Br. Burm. ii. p. II6 ( 1783$)$.

Hab. Mindoro, Calapan, Naujan, Luzon, Bataan, Orion; Java, Sumatra, Borneo.

11. Cb basalis, (Horsf) $\Varangle ?$ N. v. Sabucot.

(M. S. T.)

(uculus basalis, Horsf. Tr. Linn. Soc. xiii. p. 879 (1821); Vig. Mem. Raffl. p. 672 (1830); Gray, Gen. B. ii. p. 463 I 547 , id. Hand-l. $B$. p. 218 no. $9038(1870)$.

Chrysococcyx chalcites (nec Ill.), Blyth, J. A.S. Beng. xi. p. 919 ( 1842 ).

(. lucidus (nec Gm.), Gould, B. Austr. iv. pl. 86, pt. fig. \& et juv. (1848).

C. Lasalis, Blyth, Cat. B. Mus. As. Soc. p. 73 (1849); Horsf. \& Moore, Cat. B. Mus. E. I. Co. ii. p. 107 (1856-58).

Ch..lcites basalis, Bp. Consp. Vol. Zygod. p. 7 (1854); Hume Str. F. iii. p. 319 (1875); Ramsay, Pr. Linn. Soc. N. S. Wiales, (2) ii. p. I 70 (1 887$)$. 
Limprococcyx basalis, Cab. \& Heine, Mus. Hein. iv. p. I 2 (1862); Salvad. Ann. Mus. Cí. Gen xiii p. 459 (1878); id. Orn. Papuasia, i. p. $3+9$ (1880); App. p. 49 (1889); Shelley, Cat. B. W. 10, p. $29+(1+9) 1)$.

Cuculus lucidus, Schl. Mlus. Pays-Bas, Cuculi, p. 3.3 (pt., 186q).

? ( uculus neglectus, Schl. Mus. Pays-Bas, (uculi, p. 35 (1864); Gray, Hand-l. B. ii p. 219 n. ${ }^{\circ} 045(1870)$.

Hab. Negrus, Mindanao; Java, Australia.

12. Ch. lucidus. (G:n.) \&? N v. Sabucot.

(M. S. T.)

Shining cuckoo, Lath. Gen. Syn. ii. p. 528, pl. 23 (1782).

Cuculus lucidus, Gin. Syst. Nat. i. p. 421 (1788); Vig. \& Horsf. Trans. Linn. Soc. xi p. 30 (1826); Temm. Pl. Col. iii. pl. IO2 fig. I I824; id. Tabl. Meth. p. 52 (1838); Gray, Hand-l. B. ii. p. 218, no. 9042 (1870); Shelley, Cat. B. M. 19, 1191 p. 295 .

Variable Warbler, Lath. Gen. Syn. Suppl. ii. p. 250 (1801).

Sylvia versicolor, Lath. Ind. Orn. ii. Suppl. p. 56 (1801).

Cuculus metalicus, Vig. \& Horsf. Tr. Linn. Soc. xv. p. 3o2 (1827).

(halcites lucidus, Less. Traitè, p. 153 (1831); Bp. C:nsp. Vol. Zygod. p. 7 (1854).

(uculus nitens, Forst. Descr. Anim. p. $5_{5}$ (1844.)

Cuculus versicolor, Gray, Gen. B. iii. p. 463 (1847).

C. chalcites, Ill. en Mus. Berol. Temm. Tabl. Meth. p. $5^{2}$ (1 838 ).

Chrysococcyx lucidus, Bp. Consp. Av. i. p. I05 (1850); Horst. \& Moore, Cat. B. Mus. E. I. Co. ii. p. 706 (1856-58); Finsch. Neu Guin. p. I59 (1865); Buller, B. New Zeland, p. 77 (1873); 2nd id. i. p. I32, pl. . 5 (1888).

C. chalcites, Licht. Nomencl. Av. p, 78 (1854).

Limprococcyx lucidus, Cab. \& Heine, Mus. Hein. iv. p. 14 (1862). Hab. Australia.

13. Ch malayanus, (Kinffl.) $\delta \&$ N. v Bahao.

(M. S. T.)

Cuculus malayanus, Raffl. Trans. Linn. Soc. xiii. p. 286 (1822).

C. chalcites (nec Ill.), Blyth, J. A. S. Beng. xi. p. 919 (I842), xii. p. 944 (1843); S. Müll. Verh. Nat. Gesch. Land-en Vilk. p. 234, note (1839-44).

C. lucidus (nec Gm.), Blyth, J. A. S. Beng. xii. p. 944

Chrysococcyx basalis, ? Blyth, J. A S. Beny xv. p. 54 (1846); ? Horsf. \& Moore, Cat. B. Mus. E. I. Co. ii. p. 707 iscri58); Salvad. Ucc. de Borneo p. 62 (1874); Sharpe, Trail. Linn. Soc. 2nd ser. i. Zool. p. 320 (1879); id. Ibis, 18-8, p 416; Wardlaw Ramsay, App. to Tueedd. Orn. Works. p. $656(\mathrm{I} 88 \mathrm{I})$.

C. chalcites (nec Ill.), Blyth, Cat. B. Mus. As. Soc. p. 73 (18.49); Bp. Consp. i. p. 106 (1850); Sclat. P. Z. S. 1163, p. 209; Wall. t. c. p. 484.

C. minutillus, Gould, P. Z. S. 1159 , p. I 28; id. B. Austr. Suppl. pl. 56 (1859).

C. nislaynus, Horsf. \& Moore, Cat. B. Mus. E. I. Co. ii. p. 
706 (1856.58); Walden, P. Z. S. 1878 , p. 945, Shelley, Cat. B. $M .191191$. p. 298.

Lamprococcyx minutillus; Cab. \& Heine, Mus. Hein iv. p. 15, note (1862); Gould, Handb. B. Austr. i. p. 625 (1865).

Cuculus minutillus, Gray, Hand-l. B. ii. p. 218, no. 9043 (1870). Lamprococcyx malayanus, Hume, \& Davison Str. F. vi. p. 503 $(1878)$.

L. basalis, Grant, P. Z, S. 1887 , p. 191.

Hab. Mindanao, Zamboanga; Java, Borneo Maiaca.

7. Gén. EUDYNAMIS, Vig

14 E mindanensis (Linn.) $\mathrm{O}^{*} . \mathrm{O}$ N. v. Bahao, Tarucay, Carajao. (M.S T.)

Caucou tachetè de Mindanao, Briss. Orn. iv. p. Izo (1760).

(uc lus mindanensis, Linn. Syst. Nat i. p. 169, (1766); e.x Briss. Vieill. Enc. Meth. p, 1337 (1823); Wald. Ibis, 1869, p. 327 .

(oucou tachetè de l'Isle de Panay, Sonn. Voy. Nouv. Guin. p. i2) pl. $78(1776)$.

Cuculus variegatus, Scop. Del Fl. et Faun. Insub. ii. p. 8) (1703).

C. panilyanus, Gm. Syst. Nat. i, p. I 43 (1788); Enc. Meth. p. 1336 (1823); Vald. Ibis, 1869 , p. 336.

Eudynamis mindanensis, Cab. \& Heine, Mus. Hein. iv. p. 52; Wald. Ibis, 186 g, p. 340.(pt.); Gray, Handal. B. ii p. $22 \mathrm{I}$ n." 9070; Wald. Trans. Z. S. ix. p. I62 (1875); Sharpe; Trans. Linn Soc. Zool. 4877 , pp. 320, 351; Trueedd. P. Z. S. 1877 , pp. 536, 543, 66 1, 823, г 878, p. 946, 1870, p. 7a; Wardlaiv Ramsay, App. 7weedd. Orn. Works. p. 656 (1881); Meyer Sitz. Ab. Handl. Ges. Isis, p. 17 (1884); Blasius, Ornis, 1888 , p. 306; Everett. P. Z. S. 1889, p. 225; Whitehead, 1890, p. 46; Shelley, Cat. B. M. 19. 1891, p. 321 .

1.. niger, (nec Limn), Salvad. Ann, Mus. Civ. Gen. ix. p. 5.3 (18;6); Treeedd. P. Z. S. 18;8, p. 6r 3 .

Hab. Luzon, Cavite, Indan, Montalban, Laguna, Guimarás, Mactan, Negros, Panay, Zamboanga, Butuan, Marinluque, M.tlanipa, Taguso, Paragua, Samar, Mindoro, Basilan; Sanghir.

15. E. honorata, (Lin.) $\sigma 0 \mathrm{~N} . \mathrm{v}$ Bahao, Tuao.

(M. S. T.)

Black indian cuckoo, Edwards, Nat. Hist. B. ii. pl. 58 (1747). Brown and spotted indian cuckoo, Edwards, t. c. pl. 59 (i747). Coucou tachetè de Bengale, Briss. Orn. iv. p. I32 (1760, ex Edw. pl. 50).

C. tachetè de Malabar, Briss. t. c. p. I 36 , pl. I I fig. 2 ; Daubent. pl. 294.

C. noir de Bengale, Briss. t. c. p. 141 (ex Edw. pl. 58); 1)aubent. pl. Enl. pl. 516 .

?C. tachetè de la Chine, Buff. Pl. Enl. p. 764.

Le Coucou á gros. bec, Levaill. Ois. Afr. v. p. 50, pl. 214 ( 1806$)$.

Le Coucou tacheron, Levaill. t. c. p. 62, pl. 216.

Cuculus honoratus, Limn. Syst. Nat. i. p. 169 (1766 ex Briss. 
pl. I1 lig. 2); Vieill. Enc. Meth. p. $13.32(182.3) ; B l y t h, /$. A. S. Beng. xi. p. 913 (18f2); II allien, Ibis, 136q, p. 327. C. scolopaceus, Linn. Sist. Nat. i. p. 170 (1766, ex Eitio. pl. $50)$; Il ald Ibis, 1869 , p. 327 .

(. niger, Linn. Syst. Nat. i. p. 170 (1766, ex Eatue. pl. 58; Wald 16.5, 1859 pp. 327. 33.5.

c. maculatus, Gin. Siyst. Vat. i. p. +15 (171s, e.t D.ubent. pl. $76+$ ); Wald. Ibis, $180^{\circ} 9$, p. $33^{6}$.

C. indicus, Lath. Ind. Orn. i. p. 211 (1790).

C. orientalis, e'ar, y, lath. t. c. p. 21 r.

(. crassirosiris, Sleph. Gon. Zool. iv. p. 86 (isis e.x Leìnill pl. 215); Vieill. Enc. Meth. p. 1333 (18:3).

(. orientalis, (nec L.mn.), Horsf. Trans. Linn. Soc xiii. p. 178 (1822); Blyth. J. A.S. Beng. xi. p. 913 (18+2); S'trickl. Aun. \& Mag. Nat. Hist. $18+6$, p. foo.

Elyenamis orientalis, Vig. Mem. Raffl. p. 671 (1830); Sykes, P. Z. S. 1832. p. 97 ; Jerd. Madras Journ. xi. F. 220 (1840); Pearsou, J. A. S. Beng. x p. 657 (1841); Blyth I. A.S. Bing. 1843 , p. 245, i847, p. 468; Layard, Ann. \& Mag. Nal. Hist. xii. p. 45 I (1854; Moll. \& Dillw. Conir. N.ut. Mis: Labuan, i. p. 55 (1855); Horsf. \& Moore Cat. B. Mus. E. I. Co. ii. p. 708 (1856-58); Swinh, Ib.s, 1861 , p. 46; Irby t. c. p. 230 ; lerd. B. Ind. i. p. 342 (1862); Blyth, Ibis, 186. , p. 363 ; Gray Hand $B$. ii. p. $22 \mathrm{I}$, n. $^{\circ}$ c072 (1870); Jer!?.

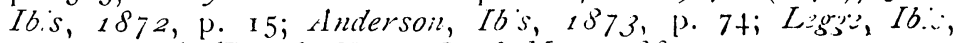
1874 , p. I6; Butlik Notes Ley'd Mus. 1883 p. 29.

E. honorata, Blyth. J. A. S. B. 1842 , p. 912; Gray Gell. B. ii. p. $464(1847)$; Wald. Ibis, I869, p. 338; Gray, Hand-?. B. ii. 220 , n. ${ }^{\circ} 968$ (1870); Holdsworth, P. Z. S. 1872, p. 432; Hume, Nests. \& Egg. Ina. B. p. I 39 (1873); Adams, t. c. p. 373; Ball, Str. F. ii. p. 394 (I874); Anders. Ibis, 1875 , p. I 42 ; Farbank, Str. F. iv. p. $225(1876)$; Himne, t. c. p. 463 ; Fa.rbank, Str. v. p. 257; Davison \& Wendon Str. F. vii. p. 79 (1878); Butler, t. c. p. 182; Ball, t.c. p. 207; Crifps t. c. p. 266; Hume, Str. F. viii. p. 89 (1879); Scul'ly, t. c. p. 257; Legge. B. Ceylon, i. p. 25I (1880); Vidal, Str. F. ix. p. 55 (1880); Butler, t. c. p. 385 ; Reid, Str. F. x. 1?. 27 1881; Davison, t. c. pp. 299, 360; Swinh \& Barnes, 16.s, 1885, p. 63; Shelley, Cat. B. M. 19. 1891. p. 316.

E. maculata, Gray, Gen. B. ii. p. $46+$ (18+7); David \& Oust. Ois. Chine p. 6o (1877).

E. indicus; Gray, Gen. B. ii. p. 464 (1847).

A. australis, (nec Swatins), Bp. Consp. A\%. i. p. 181 (1850).

E. nigra, Cab. \& Heine, Mus. Hein. iv. p. 491862.

E. cylunensis, Cab. \& Heine, Mus. Hein. iv. p. 51, ncte (1862).

E. chinensis, Cab. \& Heine, Mus. Hein. iv. p. 52 note (1862); Blyth; Cat. Mamm. \& B. Burmah, p. 8 I (1875).

Eudynamis malayana, Cab. \& Hein Mus. Hein iv. p. 52 (1862); Wald. Ibis, 1369 , p. 339; Hand-l. B. ii. p. 220, n. ${ }^{\circ} 9069$, (1870); Wald. Ibis, I873; p. 303; Salvad. Ucc. Borneo p. 68 (1874); Hume, Str. F. ii. pp. I92, 472 (1874), iii. p. 82 (1875); Sharpe, Ibis, 1876 , p. 34; David. \& Oust. Ois. Chine, p. I I (1877); Hume, Str. F. iv. p. 162 (1878), viii. pp. 54, 89 (1879; Sharpe, It:s, 1879 , p. 215 ; id. P. Z. S. 1879, p. 328; 
Bingham, Str. F. ix. p. 163 (188)); Huine, t. c. p. 248; K:! ham, Ibis, 1881 , p. 392; A. Mïll. J. f. O. 1882, p. fo'; Ooates, Handb. B. Brit. Burmah, ii. p. 119 (1883; Giallermard, P. Z. S. 188.5, p. 406; Mïll. J. f. O. 1885, p. 15;; Salvad. Ann. Mus. Civ. Gen. $1885,581,1887$, p. 569; Sharpe, Ibis, 1888 , p. 198; Everelt, P. Z. S. 1389, p. 226; Sharpe, Ibis, 1890, p. 12.

Hab. Luzón, Cavite, Alfonso, Mindoro, Baco, Samar, Paric; China.

16 f rufiventer, (Less.) $\sigma \mathrm{N} \quad \mathrm{v}$ Sabucot.

(II. S T.)

Coucou aacheté des Indes Orientales, D.rub. Pl. En!. vi. pl. 771 $\left(\mathrm{I}_{7} 83\right)$.

Cuculus rufiventer, Less. Voy. Coq., Zosl. i. p. 623 (1838); id. Traité, p. $150(183 \mathrm{I})$.

Eudyamis rufiventer, Less. Compl. Butr., O.s. p. 625 ( 833 ); Gray, Gen. B. ii. p. $46+(18+7)$; W.xt.t. Ibs, 1859 , p. 3+t; Gray, Hand-l. B. ii. p 221, 11." 907t; Wald. Trans. Z. .'. ix. p. 162 (1875); Salvad. Ann. Mus. C.v. Gen. xiii p. 401 (1878); id Atti R. Accad. Torino, xiii. p. 3 I8 (1873); itt. Or.⿲. Papuasia, p. 368 (1880); Sharpe, Journ. Linn. Soc. xvi. p. 428. 1882, Guillem. P. Z. S. 1885, p. 625; Salvad. Orn. Papuasia App. p. 51 (1889); Shelley. Cat. B. WT. 19. 1891, p. 325 .

E. picatus, S. Mïll. Verh. Land-en Volk. p. 876, note (1839-4t'; Bp. Consp. Av. i. p. Ior (1850); Cab. \& Heine, Mas. Heill. iv. p. 55, note (1862); Cab. \& Reichen. J. f. 0.1876 , p. 324 .

E. punctatus (nec Linn.), pt., Bp. Consp. Av. i. p ror $\left(1850^{\circ}\right.$; Gray, Cat. B. New Guin. p. 40 (1859); id. P. Z. S. 1354 , p. I 59, I861, p. 437 .

E. rufiventer, Sclat. Journ. Linn. Soc. Zool. ii. p. 166 (1858).

E. orientalis, pt., Finsch, Neu-Guin. p. I59 (1865).

E. cyanocep!rala (nec Lath.), Salval. Anil. Mas. Civ. Gen. iv. p. $17 \quad 1876$.

Hab. Nueva Guinea.

8. Gen. COCCYZUS, V1ELL.

17. C erythrophthalmus, Wils. o N v.

(M. S. T.)

Cucuius erythrophthalmus, Wils. Ann. Orn. iv. p. i6, pl. 25 , fig. 2. (1811); Steph. Gın. Zool. xiv. p. 208 (1826); Harting. Handb. Brit. B. p. I 24 (1072).

Coccyzus dominicus (nec L.), Nutl. Man Orn. i. p. 556 (1832).

C. erythrophthalmus, Bp. Journ. Acad. Philad. iii. p. 307, pl. 2 (1824); Audubon, Orn. Biogr. i. p. I70 (1831, v. p. 523 (1839); Shelley, Cat. B. M. 19.1891, p. 311.

Hab. América, Canadá. 
9. Gen SCY IHROPS, I.ATIt

18. S novæ-hollerdiæ L,th. ó N. v. C. anómalo.

Psttaceous Hornbill, Phrll. Foy. Botrn: B.xy, p. 165, pl. (1789). Anomalous Hornbill, J. While, Jou'n. Voy. N. S. Wales, p. I $+2, \mathrm{I}^{\prime} \cdot(1 ; 90)$.

Scythrops nora-hollandia, Lath. Ind. Orn. i. p. 14 I (1790).

S. novit-hollandia, $v w$. prassagus, Brïgg. Abhandl. nat. Ver. Brem. v. 1. 56 (1S76); Shelley, Citt 13. M. 19. 1891, p. 330. Hab. Australia.

\section{Subfam. CENTROPODINOS.}

Io Gén. CENTROPUS, Il.t.

19 C mindorensis (Steere.) o. N. v Sabucot-talahilban (M. S T)

(entrococcyx mindor nsis, Steere, List. Rirds. \& Mam. Exped. Philipp. p. I 2 ( 1890$)$.

Centropus mindorensis, Hartert. Katt. Vög. Mus. Senckenb., App. p. 253 (1891); Sholley, Cat B. M. 19. 1891. p. 339.

Hab. Mindoro, Calapan, Baco, Naujan.

20 C sinensis, (Stepr.) ơ N. v. Bab but.

Polophilus sinensis, Steph. Gen. Zool. ix. p. 5 r (1815).

(entropus bubutus, Horsf. Trans. Linn. Soc. xiii. p. I89 (1821); id. Zool. Research Java, pl. 6o (1824); Bp. Consp. Vol. Zy'god. P. 5 (1854).

Chestnut Coucal, Lath. Gen. Hist. B. iii. p. 243, pl. 54 (1822).

(entropus philippensis, var. javanica, Horsf. Zool. Research, Java. text to pl. 6o (1824).

C. castanopterus, Steph. Gen. Zool. xiv. p. 215 (1826); Helfec. J. A. S. Beng. 1838 , p. 862; Swinh, op. cit. 1871, p. 658 .

(. philippensis (nec Cuv.), Sykes. P.Z. S. 1832, p. 98; Me. Clelland, P. Z. S. 1839 , p. 166; Blyth, J. A. S. Beng. 1842 , I099, I 843, p. 246, I844, p. 945, г845, p. 202; S. Mïll. Verh. land-en Volh. p. 405 (1839-44); Blyth, Ann. \& Mag. Nat. Hist. 1847 , p. $3^{85}$; id. Cat. B. Mus. As. Soc. p. 78 (1849); Layard, Ann. \& Mag. Nat. Hist. 1854 , xiii. p. 450; Motley. \& Dillweyn, Contr. Nat. Hist. Labuan, p. 54 (1855); Irby, Ibis, 1851, p. $230 ;$ Scl. P. Z. S. 1863 , p. 209.

Cuculus philippensis, Sundev. Physiogr. Sällsk. Tijdskr. 1837, p. I67; Strickl. Ann. \& Mag. Nat. Hist. 1846 , p. 4or.

Centropus pyrrhopterus, Jerd. Madras. Journ. xi. p. 224 (1840).

Cuculus fasciatus, Swinh. J. A. S. Beng. 1841 , p. 659.

Centropus sinensis, Blyth, F. A. S. Beng xii. p. 247 (1843), xiv. p. 202 (1845); id. App. Cat. B. Mus. As. Soc. p. xix. (1852); Swinh. Ibis, 1861 , p. 49; Gray Hand-l. B. ii. p. 2 I 4 n. ${ }^{\circ}$ I 965 (1870); Swinh. P. Z. S. 1371, p. 393; David \& Oust. Ois. Chine p. $5^{8}$ (1877); Styan, Ibis, 1887, p. 230; Shelley, Cat. B. M. 19. 1891, p. 343 . 
Centropus eurycercus, Hay, J. A. S. Beng. xir. p. 55i (1 $8+5)$; Blyth. Cat. B. Mus As. Soc. p. 78 (1849); B). Consp. Vol. Zygod. p. 4 (i 84 ); Horsf. \& Mosre, Cat. B. MIs. E. I. Co. ii. p. 685 (1856-58); Schl. Mus. Pays Bas, Cuculi p. 66 (1 85t!; Gray, Hand-l. B. ii. p. 21 2, n." 1963 (1870); Blyth, \& Halden Cat. Manm. \& B. Rurma, p. 82. (1875); Trueedd. Ihis, 1877 , p. 288; Hume, Str. Fi. v. p. 27 ( 1877 ); Nicholson, Ibis, 1882 , p. 54; Snelleman, in Veth's Midden Sumatra, Vög. P. 34 (1884); Bïttik. Notes Ley'den, Mus. 1886, p. 32.

Centropus rufipennis (nec Ill.) Blyth, Ann. \& Mag. Nat. Hist. 1843 , p. 95; id. Cat. B. Mus. As. Soc. pp. $32 \mathrm{I}$, xix, (1849); Horsf. \& Moore, Cat. B. Mus. E. I. Co. ii. p. 681 (1856-58; Jerd. B. Ind. i. p. 248 (г 862); Schl. Mus. Pays-Bas, Cuculi. p. 68 (1864); Gray, Hant-l. B. ii. p. 2r3 n." 8951 (1870); Jerd. Ibis, 1872 , p. I 5 ; Hume, Nests. \& Eg,s. Ind. B. p. It 2 (1873); id. Str. F. i. p. I73 (1873); Adams. t. c. p. 374; Morgan 1bis, 1875 , p. 315; Blyth \& Walden, B. Burma, p. Bi (1875; Fairbank, Sir. F. vi. p. 255 (1876); Legge, B. Ceylon, p. 260 (1880); Marshall, Ibis, 1884 , p. 4 I I.

C. viridis (nec Scop), Swinh. P. Z. S. 1863, p. 266; id. 1bis, $1870, \mathrm{p} .235$.

C. borneensis, Bp. Consp. Vol. Zygot. p. 5 (1854); Gray, Hand-l. B. ii. p. 2 I 3, n. $^{\circ} 8962$ (1870).

Centrococcyx rufipennis, Cab. \& Heine, Mus. Hein iv. p. II 5 (1862; Ball. Str. F. iii. p. 394 (1874); Fairbank, op cit. vi. p. 297 (1877); Davidson \& Wenden, op cit. vii. p. $79(1878)$; Ball. t. c. p. 207; Hume, op. cit. viii. p. 89 (1879); Vidal, op. cit. ix. p. 56 (1880); Bu'ler, t. c. p. 389; 1)avison, op. c.t. x. pp. 299, 361 (1881);A. Mïll. J.f. O. 1882, p. 411 ; Swinh \& Barns, Ibis, 1885 , p. 64; A. Mïll. J. f. O. 1885 , p. 157 .

C. sinensis, Cab. \& Heine, Mus. Hein. iv. p. I 9 (1862).

G. eurycercus, Cab. \& Heine, Mas. Hein. iv. p. I16, note (1862); Wald. Ib:s, 1872 , p. 395; Hume, Str. F. i. p. 453 (1873); Salvad. Ucc. Borneo, p. 78 (1874); Hitme, Str. F. ii. pp. 193, 473 (1874); iii. p. 83 (1875); Armstrong, op. cit. iv. p. 3 I 2 (1876); Sharpe, 1bis, 1377, p. 8; Tweedd. P. Z. S. 1878, p. 6I4; Sharpe, P. Z. S. 1879, p. 328; Saluad Ann. Mus. Civ. Gen. xiv. p. 188 (1879); Hume, Str. F. viii. p. 55 (1879); Kelham, Ibis, 1888, p. 39t; Sharp", P. Z. S. 1888, p. 793; Wardlaw. Ramsay, App. Tweedd. Orn. Works. p. 656 (I881); Blasius, J. f. O. 1882, p. 247; A. Mull. Orn. Ins. Sxlanga, 1. 60 (1812); Nicholson, Ibis, 1883 p 2+1; Blasius, Verh, Zool.-bnt. Ges. Wien xxxiii. p. 39 (1883); Gilillemard P. Z. S. 1885 , p. 406; Salvad. Ann. Mus. Civ. Geu. iv. p. 533 (1887); Sharpe, Ibis, 1888 , p. 198; Everett, P. Z. S. 1889 226; Hartert. J. f. O. 1889 , p. 372; Sharpe, Ibis, 1890 , p. I3; Whitehead. t. c. p. 46 ; Everett, Journ. Straits. Branch. As. Soc. 1889 , p. 175.

C. validus, Heine, J. f. $O .1863$, p. 357 .

C. intermedius, Hume, Str. F. i. p. 454 (1873); id.\& Davison, op. cit. vi. p. 168 (1878); Cripps., op. cit. vii. p. 266 (1878); Hume, op. cat. viii. pp. 89, I55 (1879); Bingham. op. cit. ix. p. 169, (г 88); Reid. op. cit. x. p. 28 (1881); O.ttes. t. c. 
ј. I96; id. Handb. B. Brit. Burmah, ii. p. I26 (1883); Sabwad. Anu. Mus. Civ. Gen. (2) iv. p. $5^{83}$ (1887); id. op. cut. v. j. 569 ( 1888$)$.

C. maximus, Hume, Str. F. i. p. 454 (1873).

('entropus intermedius, Oates, Str. F. v. p. 145 (1877).

('entrisoccyx acheenensis, Hume, Str. F. vi. p. 17 I $(1878)$. Java.

Hab. Paragua, P. Princesa, Ningpo, Joló; Borneo, China,

21. C. viridis, (Scop.) $\sigma \circ$ N. v. Sagucsuc, Tarucug.

(.M S. T)

Coucou vert d'Antig, e, Sonn. Voy. Nowv Guin. p. 131, pl. Bu ( 1876$)$.

Cuculus viridis, Scop. Del. Fy. Faun. Iısub. ii. p. 89. (1786).

(:. xgyptius, Gm. Syst. Nat. i. p. 420 , vars $3 / .(1788)$.

Coucou des Philippines, Buff. Hist. Nat. Ois. vi. p. 369 (1779); I)aubent. P!. Enl. $82+(1783)$.

Polophilus viridis, Steph. Gen. Zoxl. ix. p. 55 (1815).

Centropus rufipen:nis, Ill. Abhandl. Berl. Acad. 1818, p. 224.

Cuculus philippensis, Cuv. Regne, $A$ n. i. p. 436 (1817).

Corydonix viridis, Vieill, Nouv. Dict. xxxiv. p. 299 (1818); id. Enc. Meth, iii. p. I 335 (1823).

C. pyrropterus, Vieill. Enc. Meth. iii. p I 353 (1823).

Centropus viridis, Blyth, $J$. $A$. S. Beng. vi. p. I IO2 (1842); Gray, Gen. B. ii. p. 455 ( 1846$)$; id. Hand-l. B. ii. p. 213 , n." 8956 (1870); Shelley, Cat. B. M. 19.1898, p. 349.

Centrococcyx viridis, Cab. \& Heine, Mus. Hein. iv. p. I Io, note ( I862); Walden Trans. $Z$. S. vii. p. 58 (1872); ix. p. I63 (1875); id. P. Z. S. 1877, pp. 543, 691, 758, 823; Sharpe Trans. Linn. Soc. 1877 , p. 322; Trueedd. P. Z. S. (1878) pp. 213, 34r, 709, 1879, p. 946; id. Rep. Voy Challenger, ii. p. 15 (I8Iо); Wardlaw Ramsay App. Tzeeddale's Orn. Works. 1). 658 (1880); Kutter J. f. O. 1881 p. I85; Guillemard, P. $Z$. S. 1885 , p. 257; Steere, List. Birds. \& Mamm. Exped. Philipp. 1). I $2(1890)$.

Hab. Luzon, Manila, Montalban, S. Mateo, Laguna, Calamba, Liss Baños, Cigayan, Pamplona, Ilocos, Cebú, Sianar, Calbayog, Paranas, Borongan, Panay, Iloilo, Negros, Valencia, Guimarás, Surigao, Zamboanga, Mindanao, Mactan, Leyte, Masbate, Marinduque, Basilan, Bohol, Jólo.

22. C javanicus. (Dum.) oo N. v. 'Tarucug.

(M. S. T.). .

Cuculus javanicus, Dumont, Dict. Sc. Nat. xi p. I44 (1818).

(entropus tolu, (nec Gm.) Raffles, Trans. Linn. Soc. xiii. $28_{5}$ ( 1822$)$.

C. lepidus, Horsf. Trans. Linn. Soc. xiii. p. I80 (1822); Steph. Gen. Zool. xiv. p. 2 I4 (1826);. Berust. J. f. O. 1859, p. 817; Gray. Haint-l. B. ii. p. 2 I 3 n. ${ }^{0} 8959$ (1870).

C. affinis, Horsf. Trans. Linn. Soc. xiii p. 810 (1822); Vig. Mem. Raff. p. 670 (1830); Blyth, J.A. S. Beng. xi. p. I IO5 (1842); Bp. Consp. i. p. 108 (1850); Bernst. J.f. O. 1859 , p. ${ }^{8} 851860$, p. 269 ; Scl. P. Z. S. 1863 , p. 209 Finsch, Neu-Guin. p. 160 (1855); Grxy Hand-l. B. i. p. 213, n." 
ry6u (1870); Blasints, J. f. O. 1883, p. 1321888, p. 374 ; Sharpe, Ibis, 1888 p. 198.

( . pumilus, Iess. Traite, p. 136 (1831); Pucher, Rev. et Mag. Zool. 1883 p. 66.

(C. molkenboeri, Bp. Consp. i. p. Io8 ( 850$)$; Gray, Hand-l. B. ii. 1). 214 n. 8964 (1870).

(. medius, 1Rp. Consp. i. p. Ios (185); ex Müller, MSS. in Mus. Lutgd; Pucher, Rev. et Mag. Zool. 1852, p. 475, note, 13p. Consp. Vol. Zygod. p. 86 (1854); Gray, P. Z. S. 1860 1. 359 ; Wallace, op cit. 1863, p. 23; Finsch, Neu-Guin. p. $59(1865)$.

C(ntrococcyx lepidus, Cab. \& Heine, Mus. Heine iv. p. Io9 (1862); Hartert, J. $f . O .1889$, p. 372 .

( $\therefore$ affinis, Cab. \& Hcine, t. c. p. I Io; Wald. Trans, Z. S. viii. p). 56 58, 112 (1872); Meyer, Ibis, 1879, p. 70; id. Sitr. nat. Ges. Isis, p. 18 (1884); Guillemard P. Z. S. 1885 , pp. 504, 55I; Whatehcad, Ibis, 1890,1 . 47.

Centropus moluccensis, Cab. \& Hene. t. c. ex Bernst. MSS. in Mus. Berol; Gray, Hand-l. B ii. p. 214 n ${ }^{\circ} 8967$ (1870).

(entrococcyx moluccensis, Cab. \& Heine, Mus. Hein. iv. p. I I 3 (1862); Heine J. f. O. 1863, p. 357, Walden, Trans. Z. S. viii. pp. 50, 60 (1872).

(entropus rectunguis, pt., Schl. Mus. Pays-Bas, Cuculi p. 69 ( 1864$)$.

(entrococcyx javanensis, Walden, Trans. $Z$. S. viii. pp. 5860 (1872); id. Ibis, 18872 p. 367; Salvad. Ucc. Born. p. 76 (1874); id. Ann. Mus. Civ. Genl. vii. p. 651 (1875) Sharpe, Ibis, 1876 1). 34; Salvad. Ann. Mus. Civ. Gen. xiv, p. 188 (1879); Nicholson, Ibis, $1 \& \delta 1$, p. 141, $1 \& \delta_{3}$, p. 241 Blasius Zeitschr. ges. Orn. ii. p. 263 (1885); Everett, P. Z. S. 1889 , p. 226; Sharpe, Ibis, 1890 , p. I 4 ; Everett; Journ. Straits Branct. As. Soc. 1889, p. 175 .

( . medius, Walden, Trans. Z. S. viii. Pp. 57, 50 (1872); Salvad. Aun. Mus. Civ. Gen. vii. p. 762 (1875); xiii. p. 463, (1878); id. Orn. Papuasia, i. p. 375 (1880).

(.) bengalensis, var. javanica, Brïgs. Abhandl. nat. Ver. Brem. r. p. 6r $(18 ; 6)$.

C. jaranensis, Sharpe, Ibis, 1870 , p. 946; id. P. Z. S. 1879 , 1. 328; Shclley, Cat. B. M. 12,1891, p. 354.

Hab. Nindoro, Calapan, Panay, Concepción, S. Antonio, Nesros, Leyte, B hol, Paragua, P. Princesa; Sumatra, Java, Borneo, Célebes, Molucas.

23. C. melanops, (Les.) ơ O Obon-obon.

(M S T)

Centropus melanops, Lees. Traité, p. г 37 (1831); Gray, Gen. B. ii. p. $455,(1846)$; id. Hand-l. B. ii. p. 214 , n." 8980 (1870); Shelley, Cat. B. M. 19. $180 ?$, p. 365.

C. nigrifrons, Peale, U. S. Expli. Exped. irt. ed. p. 137 pl. 38 fig. I (1848); Hartl. Arch. f. Nat. xviii. p. 137, pl. 30 iI852); Cass. U. S. Expl. Exped. 1855 , p. 249, Atlas, pl. 22 (1855); Gray, Hand-l. B. ii. p. 214 , n. 8979 (1870).

Nesocentor nigrifions, Cab. \& Heine, Mus. Hein. iv. p. 19 , nute (1862). 
N. melanops, Cab. \& Heine, Mus Hein. iv. P. 119 note (1862). Pyrrhocentor melanops, Walden, Tr. Z. S. ix. pp. 164, 331; Trueeddale P. Z. S. 1877, p. 823, Sharpe, Trans. Ianth. Soc. ser, 2, Zool. i. p. 322 (1877); Trueedd. Orll. Horks. p. 656 (1881); Killer, J. f. 0.1883, p. 303 .

Hab. Butuan, Surigac, Basilan, Nipah, Zanboanga, Mindanato, Samar, Paranas, Loquilocuin, Calbayog, Paric, Borongan, Leyte, Malamaui.

24. C. unirufus, Cab. A Heine. $\checkmark \circ$ N. v. Siggucu.

(M. S. T.)

Pyrrhoceritor unirufus, Cab. \& Heine. Mus. Hein. iv. p. \$ı, note, (1862); Walden, Trans. Z. S. viii. p. $4^{6}$ (1872); p. 164 $(1875)$.

Ceutropus unirufu , Gray', Hand-l. B. ii. p. 214, n. ${ }^{\circ} 8973$; Shelley, Cat. B. $M$ 19. 1891, p. 367.

Hab. Luzón, Cayayan, Pamplona, Manila, Ilocos Norte.

25. C. var albina, $\sigma \circ$ N. v. Siggucu.

(M. S. T)

Hab. Luzón, Cagayan, Pamplona.

\section{Subfim. FENICOFAINOS.}

I. Gén. PIAYA, Less.

I. $\mathbf{P}$ cayana (Lin.) O $\mathrm{N}$ v. El Tingaru. (M. S. T.)

Cuculus cayanus, Linn. Syst. Nat. i. p. I7o (1766); Wald. Ibis, 1869, p. 335 .

C. rilibunlus, Gm. Syst. Nat. i. p. 414 (1788); Wald. Ibis, 1869, p. 336.

Mingaru, Lzara, Apunt. Hist. Nat. Pax. (1) ii. p. 265 (1805); Hartl. Syst. Ind. Azara, p. I7 (1847).

Piaya cayana, Less. Traité, p. I40 (1831); Shelley, Cat. B. M. 19. 1891, p. 373 .

Hab. América, Guatemala.

2. P. melanogastra, $(U) O^{\prime}$ N. v.

(M. S T.)

Cuculus cayanus, var. y, Gm. Syst. Nat. i. p. I47 (1788); Wald. Ibis, 1869 , p. 337 .

C. melanogaster, Vieill. Nouv. Dict. viii. p. 236; id. Enc. Meth. p. 1337 ( 1823$)$.

Piaya melanogaster, Sehl. Mus. Pays-Bas, Cuculi p. 59 (1864); Shelley, Cat. B. M. 19. 7891 , p. 337 .

Hab. América, Guatemala.

2. Gén. rhopodytes, Cab. ẽt heine.

3. Rh. tristis, (Liss.) of N. v.

(M. S. T)

Melias tristis, Less. Traile Orn. 1831, p. I32; id. in Belang Voy. Ind. Oricut. Zool. 1834 p. 23r, pl. i. 
Rhopodytes tristis, Cab. \& Heine, Mus. Hein. iv. p. 67 (1862); Siluclley, Cat B. M. 19. 1891, p. 386. $H a b$. China.

3. Gén. RHINORTHA, Vig.

4. Rh. chlorophœa, (Raffl.) o N. v.

(M. S. T.)

Rhinortha chlorophœa, Vig. App. Mem. Raffl. p. 671 (1830); Shclley, B. M. 19. 1891 , p. 393. Hab. China.

4. Gén. DRYOCOCCYX, Sharpe.

5. D. harringtoni, Sharpe, oo N. v. Bubbut.

(II. S. T.)

J)ryococcyx harringtoni, Sharpe, Trans. Linn. Soc. (2) Zool. i. pp. 321, 351 (1877) id. Ibis, 1888, p. 198; Whitehead Ibis, 1890 , p. 46; Shelley, Cat. B. M. 19. 1891 , p. 400.

Phomcophaes harring toni, Wald. P. Z. S. 1878, p. 198; War. dlaw Ramsay, App. Tweedd. Orn. Works, p. 656 (1881). Hab. Paragua, P. Princesa, Balabac.

5. Gén. Dasylophus, Swa.

6. D superciliosus, (Cuv.) ơ N. v. Sabucot-palongan. (M. S T.)

Phonicophaus superciliosus, Cuv. Dect. Hist. Nat. x. p. 55 (1826; Less, Man. Orn. ii, p. $127(1828$; Blyth, J. A. S. Beng. xi. p. 925 (1842); Schl. Mus. Pays-Bas Cuculi, p. 5 I (1864).

Melias superciliosus, Less. Traité, p. I 33 (1831).

Malcoha superciliosus, Guer. Icon. Regne An. i. pl. 33. fig. I (1 $864-38)$.

1)asylophus superciliosus, Swains. Class. ii. p. 324 pl. 286, fig. a (1837); Gray, Gen. B. ii. p. 456, pl. I I6 (1845); Blyth, J. A. S. Beng. xiv. p. 199 (1845); id. Cat. B. Mus. As. Soc. p. 75 (1849); Bp. Consp. Vol. Zygod. p. 5 (1834); Cab. \& Heine, Mus. Hein iv. p. 69 (1862); Gray, Hand-l. B. ii. p. 206. n. ${ }^{\circ} 887$ (1870); Sharpe, P. Z. S. 1873, p. 603 fig. 7, head; Wald. Trans. Z. S. ix. p. I62 )1875); Gould, B. Asia, vi. pl. 44 (1876); Treeedd. P. Z S. 1877 p. 691; Sharpe, Trans. Linn. Soc. (2) Zool. i. p. 321 ( 1877 ; Kutter, J. f. O. 1882, p. 175; Shelley, Cat. B. M. 19. 1891, p) 404.

Phœnicophœus ornatus, Blyth, J. A. S. Beng. xi. p. 925 (1842). Hab. Luzón, Manila, Montalban, Bataan, Laguna, Calauan, Marinduque.

6. Gén. Lepidogramius, Reich.

7. L. eumingi, Frasser ơ N. v.

(M. S. 'T.)

Ihœnicophaus cumingi, Fraser. P. Z. S. 1839 , p. 112; Blyth, J. A. S. Beng. xi. 925 (1845); Fraser, Zcol Typ. pl. 53 (1848-49); Schl. Mus. Pays-Bas, Cuculi, p. 51 (1864). 
P. barrotii, Eydoux \& Souleyet, Vay. Brit. Zool. p. 89 Allas, pl. $6(18+1)$.

Dasylophus cumingii, Gray, Gen. B. ii. p. 439 (1845); Blyth, J. A. S. Beng. xiv. p. 199 (18+5); Gray, Hand-l. B. ii. p. 206, n. ${ }^{\circ} 8871 \quad(1870)$.

Lepillogrammus cumingi, Bp. Consp. Vol. Zygod p. 5 (1854); Cab. \& Heine, Mus. Hein. iv. p. $7^{\circ}(1862)$; Sharpe, P.Z. S. 1873 , p. 602 fig 6 cabeza; Wald. Trans. $Z$. S. ix. p. 163 (1875); Gould, B. Asia vii. pl. 45 (1876); Trueedd. P. Z. S. 1877 , p. 691; Shelley, Cat. B. M. 19. 1891, p 404.

Cuculus decorus, «Gravenhorst» Bp. Consp. Vol. Zygat p. 51854. Hab. Luzón, Manila, Montalban, Bataan, Laguna, Calamba.

\section{Subfam. CROTOFAGINOS.}

1. Gén, CROTOPHAGA, Lix.

1. C. sulcirostris, St\%. $\sigma^{\circ} \mathrm{N}$. v.

(M. S. 'T.)

Crotophaga sulcirostris, Swains, Philos. Mag. (n. s.) i. p. 44o (1827); Shelley, Cat. B. M. 19. 1891, p. 432.

Hab. América, Guatemala.

\section{Orden: PALOMAS.}

\section{Fam. TRERÓNIDAS.}

1. Gén. TRERon, Vieili.

1. T. nipalensis. (Hodgs). $\delta \succcurlyeq$ N. v. Curiasiao.

(M. S. 'I')

Columba curvirostra, Raffl. Tr. Lin. Soc. xiii. 2. 311 (1822).

'Toria nipalensis, Hodgs. As. Res. xix. p. ${ }_{1} 64$, ix. fig. (1836; G. R. Gr. Hand-list, ii. p. 222, n. ${ }^{\circ} 9086$ (1870); Blyth, $B$. Burm. p. I 43 (I875).

Columba aromatica, S. Mïll. (nec Gm.) Verh. Landen Volkenk. p. 396 (1 839-44).

Treron aromatica, part., G. R. Gr. Gen. B. ii. p. 467, n. ${ }^{\circ}$ I (1844); id. (nec Gm.) Cat. Hodgs. Coll. B. M. p. I2I (1846); Gieb. Thes. Orn. iii. p. 469 (1877).

T. nipalensis, Blyth. J. A. S. B. xiv. 2, p. 847 (1845); id. Ann. \& Mag. N. H. xix p. 44 (1847);G. R. Gr. Gen. B. App. p. 23 (1849); Blyth, Cat. B. Mus. A. S. B. p. 228 (1849); Bp. Consp. Av. ii. p. II (1854); id. Comp.. Rend. xxxix, p. 873 (1854); xl. p. 286, n. ${ }^{\circ}$ 14 (1855); id. Coup. d'ail. fig. pp. 5, 54, n. ${ }^{\circ}$ I $(1855) ; G$. P. Gr. List B. Brit. Mus. Columbo, p. 10 (1856); Moore, P. Z. S. 1859 , p. 404; Rchnb. Tauben, i. p. I09 (1862); Gray. Cat. Hodgs. Coll. B. Mus. and ed. p. 66 (1863), Jerd. B. of Ind. iii. 445 (1864); Godw.-Aust. J. A. S. B. xliii. p. I I (1874); Hume, Str. 
Feath. iii. p. 160 (1875); Wald. B. Burm. p. 143 (18 5); Hume \& Davis. Sir. Ficath. vi. p. tro (1878); Hume, Str. Feath. vii. p. 109, n. ${ }^{\circ} 771$ (1070); Binch Str. Feath. ix. p. 193 (1880); Pelham, Ibis, 1 s81, p. 225; Sulo. Cat. B. Strickl. Coll. p. 557 (1882); Oates, Sir. Feath. x. p. 235 (1882); id. B. Brit. Burm. ii. p. 306 (1883); Anters. Journ. Linn. Soc., Zool. xxi. p. I25 (1887); Grau, Ann. \& Mag. N. H. (6) ii. p. $35^{2}$ (1888); Hume, Sir. Feath. xi. p. 289 1888); Saluat. Anu. Mus. Civ. Gen. (2) v. p. 619 (1883), vii. p. 423 (1889; Hartert, $J . f . O .1889$, p. 405; id. Kat. Vogelsainl. p. 19I (1891); Salv. Cat. B3. Mus. 21 , p. 34 (1893).

T. aromaticun, Low (nec Gim.), Silraxuxie, p. 4 I I (10+8).

T. nepalensis, Wall. Ibis, 1865,1 . 376; id. J. f. O. 1866 , p. 277; Blyth, Ibis, 1857 , p. I 47 ; G. R. Gr. Hand-list, ii. 322 , n. $908 \mathrm{r}$ (1870); Treedd. Ib.s, 1877 , p. 321; Salvat. Ann. Mus. Civ. Gen. xiv. p. 2to (1879); Tiraut, Bull. Com. Agr. et Find. Cochinch. (3) i. p. I+1, n. 250 (1879); Tweed.l. Orn. Work. p. 502 (1881).

'T. nasica, Sharpe (nec Schleg.), Tr. Linn. Soc., Zcol. i. p. 346 (1876); Treedd. P. Z. S. 1878 , p. 623; id. Orn. Works. p. 659 (188I); Sharpe, I6.s, $183^{2} 8$, p. 202; Gr.unt, Alun. \& Mag. N. H. $(6$ ii. p. 352 (1888); Blïs. Orns, iv. p. 3 г6 (1888); Everett, P. Z. S. 1889 , p. 226; id. Journ. Sir. Br. R. As. Soc. 1889 , p. Ig6; Vorderm. N. T. N. I. xlix. p. 4 I 2 (1889); i. p. 499 (1890); Whitel. Ij.s, 1890, p. 56; Steere, List, p. 24 (1890); Hartert. J. f. O. 1891 , p. 301.

T. nasica, Oust. (Schleg.) Nouz. Arch. Mus. (2) ii. p. 29) (1879).

Rhamphotreron nipalensis, Heine of Rclunw. Nomencl. Mus. Hein.

Orn. p. 279 (1890).

Hab. Paragua, Inagauan, Bınguey; Burneo, Sumatra, Malaca.

2. Gén. OSMOTRERON, Br.

2. Os. axiliaris, Gray $\mathrm{N}$ v. Punay, Pu:ıe.

(M. S. T.)

Treron axillaris, G. R. Gray, MS.; Bp. Compt. Reu.t. xxxix. p. 875 (8854); xl. p. 286, 11. 85 (8855; id. Coilp. d'cail Ordr. Pig pp. 6, 54, n. ${ }^{\circ} 6$ (1855); iat. Compt. Keant. xliii) p. 833 (1856); Wall), Ib.s, 8863, p. 319; id. Ibis, 1955, Pi. 374 , 356; d. J.f. O. 1866 , p. 277; saiv. Cai. B. Strichl. Coll. p. 557-(1882); Tristr. Cut. Coll. B. p. +5 (1889).

? Os. axillaris, Bp. Consp. Av. ii. p. 13 (1 $35+$ ).

Treron aromatica, G: R. Gr. (nec Cin.) Lisi. B. Brit. Mus. Columbe, p. I0 (1856); Bp. Compl. Reial. xliii. p. 833 (1856; Id. Icon. Pig. pl. 7 (1857); Schleg. N. T. D. i. p. 6+(1853); iii. p. 346 (1866); “. Mart. J. f. O. 1865, p. 23 ; Schieg. Mits. P. B. Columba, p. 52 (1873); Gieb. Thes. Orn. iii. p. 6+9) (1877).

'I. amboinensis, part, G. R. Gr. Hand-list, ii. p. 222, n. ${ }^{0} 9079$ (1870).

Osmotreron axillaris, $I$ ald. Tr. Zoal. Soc. ix. p. 21 I, 2.1 2 (1875; Sharpe, Tr. Linn. Soc. Zool. ii. pp. 346, $353(1876)$; Tiveed! P. Z. S. 1877 , pp. 549, 619, 764, 832) 1878. pp. II 3,287 ;

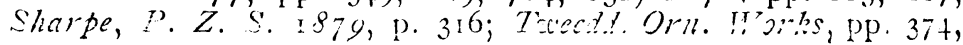


$375,+12,4^{60},+62,+73,525,538,55^{\circ}, 572,593(1831) ;$ Wardl. Rams. Tweedt. Orn. Whis, P. 659 (i831); Kitter, I. f. $O$. 1883 , p. 31t; Grillem. P. Z. S. 1885 , p. 268. Sleere, List. B. \&. M. Philpp. Exp. p. 24 (1890); Salo. Cat. B. M. 21 p. 48 , lan. 4 . ( 1893$)$.

Hab. Luzón, Manila, M intalban, Laçuna, Iloilo, Panáy N-gros, Valencia, Du naguete, Simar, Paric, Borongan, Cebú, Guimarás, Mindanao, Butuan, Zamboanga, Joló.

3 Os. vernans, (Lin.) $\delta$ \& N. v. Punay.

(M. S. T )

Columba viridis philippensis, Briss. Orn. ip. 143, t. I1. f. I. (1760).

C. vernans, Linn. Mant. p. 526 (177I); (ex Brisson); Gm. S. $N$. i. 2, p. 789, n. $^{\circ} 69$ (1788); Lath. Ind Orn. ii. p. 559, n. 22 (1790); Bonn. Enc. Méth. i. p. 239 (179); Tenm. Hist. Nat. Pig. i. pp. 70, 443 (1813); Vieill. N. D. xxvi. p. 392 (1818; Horsf. Tr. Linn. Soc. xiii. i. p. 182 (1821); Raft. Tr. Linn. Soc. xiii. 2, p. 318 (1822); D.sm. Dict. Hist. Nat. xi. p. 369 (1 826); Wagl. Syst. Av. Columb.x, sp. 9 (1897); Rosenb. Versl. Eil. Nias. p. 20 (1 8?).

Pigeon vert des Philippines, D'Aubent. Pl. Eıl. I $38 ;$ Ruff. Hist. Nat. Ois. ii. p. 527 ( $178 \mathrm{r})$.

Columba viridis,. L. S. Mïll. (nec Linn.) S. N. Supp!. p 132 (1776); (ex Daubenton). Scop. Del. Flor et Futun. Insubr. ii. p. 94 n. ${ }^{\circ} 95$ (1786); (ex Sonnerat.) Cais. Pr. Ac. Philad. I 864, p. 248.

Pigeon verd de l'Is'e de Luçon, et d'Antigue Sonn. Voy. Nouv. Guin. p. I Io, pls. 64, 65 (1 776).

Purple Pigeon, Bronvon, Illistr. p. 42, pl. 18 (1776); Lith. Syn. ii. 2, p. 628, n. ${ }^{\circ} 8(1783)$; id. Gen. Hist. viii. p. 76 (1823).

Parrot Pigeon, Lath. Syn. ii. 2, p. 629, n. ${ }^{\circ}$ 20 (1783); id. Gen. Hist. viii. p. $75(1823)$.

Columba puripurea, Gm. $S$. i. 2, p. $78_{4}$, n. ${ }^{\circ} 6$ r (1788); Ltth. Ind. Orn. ii. p. 599, n. ${ }^{0} 20($ I 79$) ;$ Bonn. Ens. Miti. i. p. 24 , 1). $7^{8}$ f. i. 179)); Vicill. N. D. xxvi. p. 359 ( 1899); Desm. Dict. Sc. Nat. xi. p. $37+(1826)$; Resenb. Versl. Eil. Nias, p 2i) i $8-?$

Vinago vernans, Cuv. Regn. An. i. p. 457 (1817); Steph. Gen. Zool. vi. p. Ir 3 (r8I9); Cuv. Rign, An. and. ed i. p. $49^{2}$ (1829); Eyt. P. Z. S. 1839 , p. Io7.

Treron vernans, Steph. Gen. Zool. xiv. i. p. $274(1826) ; G . R$. Gr. Gen. B. ii. p. 467, n. $^{\circ} 7$ (18+4); Strickl. Ant. \& Mag. N. H. xiii. p. 38 (1844); Blyth, J.A.S. B. xir. 2, p. $85 \mathrm{I}$ (1845); R:hnb. Syn. Av. ff. I 34)-41 (18+7); Motl. \& Dilov. Contr. Nat. Hist. Lab. p. 30 (1855); Cass. Narr. Exped. Amer. Squadr. \&. p. 243 (1855); id. J. f O. 1858 , p. 45\%; G. R. Gr. P. Z. S. 1850, p. $350 ;$ Wall. Ibis, 1863 , p. 320; Sclat. P. Z. S. 1853, p. 220; Schleg. N. T. D. i. p. 70 (1863); id. de Dercitit. p. 2 I I (1864); Pelz. Novara Reise, Vög. p. 105 (1865); Schleg. Mus. P-B. Columba, p. 49 (1873); Salvad. Ucc. Born. p 286 (is)4); Sharpe, P. Z. S. 1875, p. 110; Brïggem. Abh. nat, Ver. Brein. v. p. 79 (1876); 
pp. 461; 529 (1877); Gieb. Thes, Orn. iii. p. 652 (1877); Rosenb. Malay. Archip. p. 68 (1876); Sharpe, P. Z. S. 1879 , p. 348; Blas. Jarh. Ver. Nat. Braunscchw. 881 , p. 59; Sharpe, P. Z. S. 1881 , p. 799; Vordem. N. T. N. I. xliii. p. 89 (1882); Salv. Cat. B. Strickl. Coll. p. 556 (1882); Snellem. Sum-Exp. Vogels, p. 47 (1884); Gulllem. P. Z. S. 1885, p. 509; Vorderm. N. T. N. I. xlv. p. 397 (1886); Buttık. Nat. Leyd. Mus. ro p. 75 (1880); Vorderm. N. T. $N$. I. xlvi. p. 235; Blas. Ornis, iv. p. 316 (1888); Everet, P. Z. S. 1889 , p. 225; id. Journ. Str. Br. R. A. Soc. 1889 . p. 196; Eristr. Cat. Coll. B. p. 46 (1889); Vorderm. N. T. N. I. i. p. 501 (1890); Sharpe, Ibis, 1890, pp. I 34, 283.

Columba vernans, var., Less. Tr. d'Orn. p. 426 (1831).

'Treron viridis, Blyth. Cat. B. Mus. A. S. B. p. 229, n. ${ }^{\circ} 1887$ (1849); G. R. Gr. List. B. Brit. B. Mus. Columba, p. I 2 (1856); ?Gould, P.Z. S. 1859 p. I5 I; Wall. Ibis, 1854 , p. 374; id.J. F. O. 1866 , p. 277 ; Mart.J. F. O. 1886 , p. 23; Rosenl. Malay. Archip. p. 220 ( 1878); Sclat. List. Vert. An. 8 th. ed. P. 445 (1883).

Os notreron vernans, $B p$. Consp. Av. ii. p. 12 (1854); id. Compt. Rend. xxxix. p. 874 (1854), xl. p. 216 ; n. 23 (1855); id. Coup. d'cit. Ordr. Pig. pp. 6, 54, n. ${ }^{\circ} 2$ (1855); ld. Icon. des Pig. pl. xiii. (1857); Rchnb, Tauben. i. p. I05, t. 24 I. ff. I $340-41$ (I862); ii. p. I80 (1862); Wald. Tr. Zool. Soc. viii. 2, pp. 81. I13 (1872); Sousa, Mus. Nac. Lisb. Columba, p. 7 (1873); Wald. Tr. Zool. Soc. ix. ps. 2 ro, 25I (1875); Sharpe, Tr. Linn. Soc. Zool. p. 346 (1876); Tweedd. Ib s, 1847, p. 321 ; id. P. Z. S. 1877 , p. 704, I878, p. 623, 1878 . p. 951; Hume. \& Davis. Str. Feath. vi. p. 4I I (1878); Hume, t. c. p. 414 (1878); id. Str. Fealh. viii. pp. 67, 109 (1879); Twsedt. P. Z. S. 1879 , p. 73; Sharpe P. Z. S. 1879 , p. 315; Tirant. Bull. Com. Ahr. et Ind. Cochinch. (3) i p. r 40, n. ${ }^{\circ} 247$ (1879); Salvad. Ann. Mus. Civ. Gen. xiv. p. 242 (1879); Tweedd Oril. Works. pp. 182, 211, 374, 412, 503, 537,618, 623, 642, 650 (1881); Wardl. Rams. Tweedd. Orn. Works, App. p. 659 (1881); Kelh. Ibis, 1881 , p. 526; Salvad. Orn. Pap. c. Mol. iii. p. 3 (1882); Nichols. Ibis, 1882, p. 65, Blas. J. F. $O$. 1883 , p. 138, O.xtes, B. Brit. Bruin. ii. p 309 (1883); Gullem. P. Z. S. 1885 , pp. 268, 416, 509; Blas. Zeltsckr. f. ges. Orn 1886 , p. I27; Anders. Journ. Linn. Soc. Zool. xxi. p. I 25 (1887); Salvad. Ann. Mus. Civ. Gen. (2) iv. Pp 523, 557, Sharpe, Ibis, 1888 , p. 202; Vordern. $N . T . N . I$. xlix. p. 412 (1889); Hartert, J. F. O. 1889 , p. 376; Whitchead, Ibis, $1890 ;$ p. 56; Steere, List. B. \& M. Steere Exp. p. 24 1890, Hortert. Kat. Vogdsamwl. p. i91 (1891); Salv. Cat. B. M. 21. p. 60 (1893).

Osmotreron viridis, Moore. P. Z. S. 1859 , p. 465, G. R. Gr. Hand-list, ii. p. 223, n. ${ }^{\circ}$ 9a95 (1870); Hume, Str. Feath., p. p. 461 (1873), iii. p. I62 (1875); Blyth. B. Burm. p. IIt. (1875); A. Mïller, J. F. O. 1882, p. 431, I885, p. I59; Hein. \& Rchnw. Nomen.l. Mus. Hem. Orn. p. 279 (1890).

Treron griseicapilla, Schleg. N. T. D. i. p. 70 (1863); Salvad. Ucc. Born. p. 288 (1874).

'. vernalis, Pelz. Novira Reise, Vög. p. I76 (1865). 
'T, vernans, Stoliczka, J. A. S. B. xxxix. 2, p. 330 (1870); Oust. Bull. Soc. Philom. (8) iv. p. 117 (1892).

1. chlorops, Salvad. U c. Born. p. 288 (1874).

Hab. Luzon, Manili, Laguna, Calamba, Bataan, Orani, Panay, Guimarás, Bohol, Cebú, Samar, Paric, Paragua, Cuyo, Catla ..ianes, Mindoro, Calapan, Mactan, Zamboanga, Mindanao, B:1silan, Joló; Célebes, Java, Borneo.

\section{Gén. PHABOtRERON, Bp.}

4. Ph. amelhystina. $B p$. $\sigma o$ N. v. Bato batrng-tulug.

(M. S. T.:

Phapitreron amethystina, $B p$. Consp. $A v$. ii. p. 28 (1854). id. Comp. Rend xl. pp. 214, 217, n. ${ }^{\circ} 64$ (1855); id. Coup. d'reii. Ordre. Pig. pp. 52. 55; n. 64 (1855); Rchnb Tauben, i. 1. 91 (1062); G. R. Gr. Hand-list, ii. p. 246; n. 9422 (1870); Wald. Tr. Zool. Soc. ix. p. 25I ) 1875); Elliot, P. Z.. S. 1878 , p. 505; Tweedd. Orn. Works, pp. 378, 412, pl. xxxir. fig. 2 (I $88 \mathrm{I}$ ).

Phaps amethystina, G. R. Gr. List. B. Brit. Mus. Columba, p. 62 (1856); Gieb. Thes. Orn. iii. p. 89 (1877).

Ptilonopus amethystina, Wall. Ibis, 1865 , pp. 382, 397; id. J. F. O. 1866, p. 279.

Phaps (Phapitreron) amethystina, Marı. J. f. $O \quad 1866$, p. 24.

('hlorænas amethystina, Wald. Tr. Zool. Soc. ix. p. 214, $\mathrm{pl}$. xxxiv. f. 2 (1875); Sharpe, Tr. Linn. Soc. Zool. i. p. 353 (1876); Tweedd. P. Z. S. 1871, p. 832,1878 , p. I 13, p. $38 \mathrm{I}$; id Orn. Works, pp. 378, 409, 558, 572, 604 (1881); Vardl. Rams. ibid. p. 659 (1881); Steere, List. Philipp. Birds, p. 24 (1890); Salvad. Cat. Brit. Mus. 21. 1893. p. 66.

Hab. Luzon, Bataan, Orion, Samar, Borongan, Calbayoy, Leyte, Dinagat, Panaon, Butuan, Mindanao, Mindoro, Baco.

5. $\mathbf{P h}$ cinerciceps, Frank et Worc.

Prelim. Notes Menage, Exp. Philipp. 1894, p. 8. $H a b$. Tawi-tawi.

6. Ph. brunneiceps, Fr. et Worc.

Prel. Not. Men., Exp. Philipp. 1894, p. 9. Hab. Basilan.

7. Ph. maculipectus, Fr. et Worc.

Prel. Not. Men., Exp. Philipp. 1894, p. 10.

8. $\mathrm{Pb}$. frontalis, Fr. et Worc.

Prel. Not. Men., Exped. Philipp. 1894, p. ro.

Hab. Cebú.

$9 \mathrm{Ph}$ leucotis, $(T: n) \sigma, 0$ N. v. Bato-batong-tulug.

(M. S. T.)

Columba leucotis, Temm. Pl. Coll. 189, Desm. Dict. Sc. Nat. xI. p. 338 (1826); Wagl. Syst. Av. Columbr, sp. 6o (1827); 
Less Tr. dOrn. I. $47 \mathrm{I}(\mathrm{8} 3 \mathrm{I}) ; G . R$ Gr. Gen. B. ii. I". 470, n. 28 (1844); Rchnb. Syn Av. Columbaria, f. 1259 (1884); Hartl. J. F. O $1854, B$. lxv.

Goura? leucutis, Steph. Gen. Zool. xiv. p. 295 (1826).

Phaps leucotis, G. K. Gr. Gén. B. ii. P. 477, n. ${ }^{\circ} 5(18+5)$; id. List B. Br.t. Mus. Columber, 1. 6r (1856); Gieb. Thes. Orn. iii. p. qo (1877).

Pristera (Oreopeleja) leucotis, Rchnb. Av. Syst. Wat. p. xxv. (1852).

Phapitreron leucotis, Bp. Consp. Av. ii. sp. 28 (1854); id. (ompt. Rend. xxxix. p. 879 (1854; xl. p. 217 no. 65 (1855); id. Coup. d'eil Ordre Pig. pp. II, 55, no. 65 (1855); Rchub. Tauben, i. p. 91, t. 224 f. 1259 (1862);G.R. Gr. Hant-list, ii. p. 246 , no. 9432 (s870); Wald. Tr. Zoot. Soc. ix. p. 25 s (1875); Takeedd. Orn. Works, p. 4 s (1881); Hein. u. Rchnw. Nomeucl. Mus. Hein. Orn. p. 286 ( 890$)$; Hartert. Kat. Vogelsamml." p. 19" (1891); id. J. F. O. 1891 , p. 301.

I:ilonopus leucotis, Wall. Ibis, 1865 , pp. 382,397 ; id. J.F.O. 1866, p. 279 .

Phaps leucotis, Mart. J. F. O. 1866, p. 24.

Phapiscus leucotis, Sund. Meth. Nat. Av. dsp. Tent. p. 99 (1872).

(hloronas leucotis, Schleg. Mus. P. B. Columba, p. 79 (1873).

lhabotreron leucotis, $H^{\prime}$ ald. Tr. Zool. Soc. ix. p. 2l4 (1875); Sharpe, Tr. Linn. Soc., Zool. i. p. 353 (1876); Treeedd. P. Z. S. 1877 , p. 699; id. Orn. Works, pp. 378, 525. 538, 559, 643 (1881); Wardl. Rams. ibid. p. 659 (1881); Salv. Cat. $B$. Strickl. Coll. p. 552 (1882); Guillem. P. Z. S. 1885, p. 270; Tristr. Cat. Coll. B. p. 4I (1889); Steere, List Philipp. Birds, p. 24 (1890); Salv. Cat. B. M. 21, p. 67 (1893).

Hab. Luzon, Manila, Montalban, San Mateo, Laguna, Calauan, Bataan, Orion, Cagayan, Alcalá, Ilocos, Pampanga, Mindoro, Baco.

10. Fh. occipitalis. Tze. \& $\&$ v. Punay.

(M. S. 'T)

Ph. brevirostris, Tweed. P.Z. S. 1879, p. 73; id. Orn. Works p. 650; Steere, List. Philipp. p. 24; Salv. Cat. B. M. 21, p. 68 ( 1893 ).

Hab. Basilan.

11. Ph. nigrorum, Sharpe, \&? N. V limuquin.

(M. ล. ' $)$

I'hapitreron leucotis, Wald et Layard. (no Temm.) Ibis, 1872 . p. io4; 7 wed. Orn. Works, p. 121 (1881).

P. nigrorum, Sharpe, Tr. Lin. Soc. Zool. 1. pp. 3+6, 353; T2u. P. Z. S. 1877 , p. 764 ; id. Orn. Works, p. 538 (1881); Hardl. Rams, Twedd. Orn. Works, p. 659 ( $188 \mathrm{I}$ ); Trist. C'at. Coll. p. 4I, (1889); Steere, List. Philipp. Birds, p. 24 (189o); Salv. Cat. B. M. 21. 1893 , p. 68.

Hab. Negros, Guimarás, Cebú, Panay, San Bernardino.

12. Ph brevirostris, Tze. C $Q$ K. v. Dimucuyo, Limucun. (M. S. T.)

Phaps leucotis, part. G. R. Gr. Iist. B. Brit. Mus. Columba, 1. $61 ;$ specim. c. $(1856)$. 
Phabotreron brevirostris, Treedd. P. Z. S. 1877, pp. 549, 8,2, 1878, pp. I1 3, 344, 952; id. Orn. Works, pp. 461, 473, 55\%, $572,599,643,650$ (1881); Wardlazi Rams. ibiat. pp. 659, 66a (1881;; Tweedd. Voy'. "(hallenger, B Birds, p. 22, pl vi. (1881); Kietter, J. F. O. 2883, p. 314 ; Guillem. P. Z. S. 1885.1 . 209; Tristr. Cat. Coll. B. p. +1 (1849); Sleere, List Philipp. B.rds, p. 24 (1890; Sali. Cat. B. 11. 21, p. 69 (1893).

Hab. Dinagat, Leyte, Samar, Borongan, Bohol, Mindanao, Surigao, Butuan, Pasananca, Zamboanga, Sibulan, Placer, Jolí.

\section{Fam. PTILÓPIDAS.}

1. Gién LEUCOIRlikon, Br.

I. L occipitalis, Gr. N. v Punay.

(M S. T)

Ptilonopus occipitalis, G. R. Gray, List. B. Br. Mus. Ciallinu, p. I (1844); Gr. \& Mitch. Gen. B. ii. p. 467, no. 25, pl. I1 8 (1844) G. R. Gr. List B. Brit. Mus. Columba, p. 7, specim. a, b. (1856); Wallace, Ibis, 1865, p. 378; id. J. Fi. O. 1866, p. 278; Garrod, P. Z. S. 1174 , p. 250; Salv. Cat. B. M. 21, p. $72($ ( 893$)$.

Ramphiculus occipitalis, Bp. Consp. Av ii. p. I7 (1854); id. Compt. Rend. xxxix. p. 878 (1854); xl. p. 216, no. 34; id. Coup. d'xil Ordr. Pig. pp. Io, 54, no. 34 (1855); id. Icon. Pig. pl. 14 (1857); G. R. Gr. Hand-list, ii. p. 227, no. 914) (1870); Sousa, Mus. Nac. Lisb. Columba, p. 7 (1873); Wala'. Tr. Zool. Soc. ix. pp. 214, 251 (1875); Sharpe, Tr. Linn. Soc. (2) i. p. 353, no. $202(1876)$; Treedd. P. Z. S. 1877 , p. 764, ए. 832,1378, p. 952, 1879, p. 73; id. Orn. Works, PI. $378,412,538,558,643,653$ (1881); Wardl. Rams. Tweedd. (irn. Works, p. 659 (188I).

On eutreron batilda, Bp. Consp. Ar. i. p. 27 (1854); id. Compt. Reud. xxxix. p. 878 (1854), xl. p. 217, n. ${ }^{\circ} 61$; $2 d$. Comp. a'ail. Ordr. fig. pp. Io, 55, n. ${ }^{\circ}$ 6r (1855).

Columba occipitaiis, Schleg. Handleid. i. p. 4 II (1857).

Lam rotreron porphyrea, $B p$. (nec Tenm.) Icon. fig. pl. xv.

Ionotreron occipitalis, Kchnb. Tauben, p. Ior, t. 259 f. I 33 I (1862). Ptilonopus batilda, Wall. Ibis, 1865 , p. 382 ; id.J.f. O. 1866 , p. 279.

Ptilopus (Phamphiculus) occipitalis, Mart. J. f. O. 1866, p. 23; n." 124.

P. (Omeotreron), batilda, v. Mart. J. f. O. 1886, p. 23, n. ${ }^{0} 2.5$.

P. uccipitalis, Schleg. Mus. P.-B. Columber, p. 35 (1873); Gieb. Thes. Oru. iii. p. 366 (1877); Elliot, P. Z. S. 1878 , p. 569; Tristr. Cat. Coll. B. p. 44 (1889); Steere, List. Philipp. Birds;, p. 24 (1890).

P. incognita, Tweedd. Ann. \& Mag. N. H. (4) x. p. 538 (1877); id. Orn. Works, p. 565 (1881); Wardl. Rams' Tweedd. Orn. Wolks, pp. 654, 659 (1881).

Leucotreron incognita, Tweed:l. P. Z. S. 1877 , p. 8 Í́.

Xenotreron incognita, Tweedd. P. Z. S. 1877, p. 832.

$H a b$. Luzón, Manila, Calaocan, Bataan, Orani, Laguna, Cagayán, Cebú, Leyte, Mindanao, Butuan, Zamboanga, Basilan. 
2 I marchoi, Oust, $\sigma \circ \mathrm{N}$. v Punay.

(M. S. T.)

Trilopus (Rhamphiculus) marchei, Oust. Le Nat. 1880 , p. 324 ; Rcmhuw et Schel. J. f. O. 1881 , p. 26; Oust. Now Arch. du Mus. d'H. N. viii. p, 304, pl. xiii. (1884); Salvad. Cat. $R$. M. 21, p. 75 (1893).

Hab. Luzón, Bataan, Orion.

3. L. leclanderi, $B p \cdot \sigma \rho$ N. v. Innalatatad

(M. S. T.)

Irerolama leclanderi, $B p$. Compt. Rend. xli. p. 247 (1855); xliii, p. 833 (1856); id. Icon, fig. pl. 16 (1857); G. R. Gr. Ibis, 1862 , p. 342 ; id. Hand-list, ii. p. 230, n." 9201.

Ptile nopus occipitalis, part., G. R. Gr. List. B. Brit. Mus. Colunita, p. 7, sperim. c. (1856).

(arpophaga leclancheri, $G$. R. Gr, List. B. Brit. Mus. Columba, p. 21 (1856); id. P. Z. S. $185^{8}$, p. 196, 1861, p. 437; Resenb. $N . T . N$ I. xxv. p. 249. n. ${ }^{0} 225$ (1863); id. J.f. $O .1864$, p. $132, \mathrm{n}^{\circ} 225$.

Jeucotreron gironieri, Verr. \& Des, Murs, Ibis, 1862, p. 342, pl. xii. G. $R$ Gr. Ibis, 1862 , p. 344; Schleg. Ibis, 186,3 , p. I 20; Wald. Tr. Zool. Soc. ix. pp. 21 $3,25 \mathrm{I}, \mathrm{sp.}$ 1 $37, \mathrm{pl}$; xxxiv. f. I (1875); Sharpe, Tr. Linn. Soc. (2) i. p. 353, 11." 201 $(1876)$; Tweedd. P. Z. S. 1878 , p. 287 ; id. Orn Works, pp. $277,412,593$ (188I).

Ptilopus geversi (errore), Schleg. N, T. D. i. p. 6o (1863),

P. hugonianus, Schleg. op. cit. pl. 3 f. 2 (1863); Wall. Ib.s, 1865 , p. 378, n. ${ }^{\circ}$ 20; Schleg. Mus. P.-B. Columba, p. 36 (187.3; Gieb. Thes. Orn. iii. p. 364 (1877).

Trerolæma gironieri, G. R. Gr. Hand-list, ii. p. 230, no 9292 (1890).

Ptilopus leclancheri, Salvad. Ann. Mus. Civ. Gen. ix. p. 199 n." 32 (1876); Gieb. Thes. Orn. iii. p. 365 (1877); Elliot P. Z. $\therefore 1878$, p. 568; Wardl. Rans., Treecdl. Orn. Works, Pp. h54, 659 (188I); Eberett, P. Z. S. 1889 , p. 225; Steere, List. of. Philipp. Birds, p. 24 (1890).

?. gironieri (errore), Gicb. Thes. Orn. iii. p. 364 (1877),

l.fucotreron leclancheri, Salvad. Atti R. Ac. Sc, Tor. xiii. p. $427,878)$; Blas. Ornis, 1888, p. 316; Meyer, J. f. O. 1891 , . 73; Salv. Cat B. M. 21. p. 79 (1893).

Frinpus leclancheri, (sic), Tr.str. (at. Coll. B. p. 44 (1889). Hab. Luzón, Laguna, Bataan, Guimarás, Valencia, Negros, Celú, Panay, Paragua, P. Princesa,

4 L. roseicollis, Gr. $0^{*}, 0$ N. v. Punay.

(M. S. T.)

Columba porphyrea, Reinw. in Temm. Pl. Col. 106 (1824) nec C. porphyracea, Forst.; Desm. Dict. Sc. Nat. xl. p. $3 j 6$ (1826); Less. Tr. d' Orn. p. 472 (1831); Knip, \& Prev. Pig. ii. pl. $4(183-1843)$.

Ptilinopus porphyreus, Sze. Zool. Journ. i. p. 473 (1825); Steph. Gen. Zool. xiv. p. 277, pl. 33 (1826); Less. Compl. de Buff. viii. p. 37 (1837), 2nd ed p, 279 (1838). 
('o'umba roseicollis, Wagl. Syst. Av. Columba, n. 27 (1827); id. Is $s, 1820$. p. 741 .

Ptilonopus erythrocephalus, Sw. Class. B. ii. p. $347 \cdot(1836)$.

P. porphyreus, Hartl. Syst. Verz. Brem. p. 97 (1844).

P. ros-icallis, G. R. Gr. Gen. B. ii. p. 467, n. 2 I (I844); id. Sist. Gallina B.M. p. I ( 844$)$; id. List. Spec. B. Br-it. Mus. Columba, p. 8 (1856); Pelz. \& Lor. Ann. Naturh. Hofmus. p. 47 ; Salv. Cat. B. M. 21. 18893, p. 75.

Kurukuru roseicollis, Des Murs \& Prev. Voy. Venius, p. 269 (1855)?

Lamprotreron porphyrea, $B p$. Consp. Av. ii. p. is (1854); id. Compt. Rend. xxxix. p. $876(1854)$; xl. p. 216 , n. ${ }^{\circ} 37$; id Coup. d' ail Ordr. Pig. pp. 8, 54, n. 37 (1855); Rchnb. Tauben, p. 97 , t. 237, ff. I 32223 (1862); Sousa, Mus. Nac. Lisb. Columbae, p. 7 ( 1873$)$.

Ptilopus porphyreus, Schleg. De Dierent. p. 210 (1864); id. Mus. P.-B. Columba, p. 33. (1873); Gieb. Thes. Orn. iii. p. 367 (1877); Elliot P. Z. S. 1878, p. 553; Saluad P. Z. S. 1879, p. 64; Nichols. Ibis, 1881 , p. 154; Vorderm. N. T. N. Y. xlv. p 395 (1885).

Pito opus porphyreus, Wall. Ibis, 1865, p. $380 ;$ id. J. f. $O$. 1866, p. 279.

Lamprotreron roseicollis, G. R. Gr. Hand-list, ii. p. 227, n." $9160 \quad 1870$.

Ptilopus roseicollis, Salvad Ann. Mus. Civ. Gen. xiii. p. 454 (note) (1878) id. P. Z. S. 1879 , p. 64; id Anil. Mus. Cir'. Gen. xiv. p. 245 (1879); Vorderm. N. T. N. I. xlix. p. 412 ( 889 , li. p. 400 (1 892 ).

Lamprotreron porphyreus, Hein. \& Rchnw. Nomencl. Mus. Hein. Orn. p. $28 \mathrm{I}$ (1 890$)$.

Hab. Paragua, P. Princesa, Samar, Paric; Java, Sumatra.

5. I. gularis, $Q$. et $G$. $\sigma$ N. v Punay.

(M. S. 'T.)

Columba gularis, Quoy et Gaim. Voy. Astrol. Zool. i. p. 247, pl. 29, I830; Kn.p. et Prov. Pig. pl. xi. I838-4I.

Ptilinopus gularis, Less. Compl. de Buff. viii. Ois. p. 39 (1837); Brïg. Abh. naturw. Ver. Bren. v. p. 82 (1876).

Carpophaga gularis, Gr. $R$ Gray, Gen. B. ii, p. 469 n. 25 (1844).

Leucotreron gularis, Bp. Consp. Av. ii. p. 151854 ; id. Compt. Rend 39 I854 p. 876; id. Coup. d' ceil Ordr. Pig. pp. 8, 54 n." 31 (1855); Wald, Tr. Zool. Soc. viii. p. 83 (1872) Sousa, Mus. Nac. Lisb, Columbe p. 7 (1873); Salvad. Ann. Mus. Civ. Gen. ix. p. 670 (1875); Meyer, Ibis, 1879. p. I35; Blas. J.f. O. 1883 , p. I 38; id. Zeitschx. f. ges. Orn. iii. p. I 3 I (1886).

Trerolæma gularïs, Bp. Compt. Rend. xll. p. 24 (1855); G. R. Gr. Hand-list, ii. p. 230, n. ${ }^{\circ} 9203$ (1870) Schneid. Cat. p. 22 ( 1878 ).

Laryngogramma gularis Rchnh. Tauben, p. I02, t. 233 f. I 297, ii. p. 203 , t. i. f. 44 (1862); Hein. \& Rchnw Nomencl. Mus. Hein. Orn. p. 282 (1890).

Ptilopus gularis, Schleg. De Dierent. p. 2 10 (1864); id. Mus. P.-B. Columba, p. 37 (1873); Gieb. Thes. Orn. iii. p. 364 (1877); Elliot, P. Z. S. 1878 , p. 570; Salv. Cat. B. M. 21 . p. 78 (1 893). 
Ptilonopus gularis, Wall. Ibis, 1865, p. 377 ; id. Journ. f. Orn. 1866 , p. 278.

Hab. Samar, Borongan, Calamianes, Culion; Célebes.

6. L. sp $\sigma 0 \mathrm{~N}, \mathrm{v}$ Lare.

(M. S T.)

Hab. Culion, Calamianes.

7. L. jambu (Gm.) đo N. v Bato bató. Laré.

(M S. T.)

Pooni jamboo, Marsd, Sumatra, p. 84.

Jamboo-pigeon, Lath. Syn. ii. 2. p. 627, n. ${ }^{0}$ i6 (178.3).

Columba jambu, Gm. S. N. ii. 2, p 784, n. ${ }^{\circ} 63$ (1788); Temm. \& Knip, fig. pls. 27, 28 (1808-1 1); Raff Tr. Linn. Soc. xiii. p. 316 (1822); Wagl. Syst. Av. Columba, n. ${ }^{\circ} 28$ (1027); Eyion, P.Z. S. 1839 , p. I07; Schleg. Handl. Dierk. i. p. 4I I $(1857)$.

C. jambos, Lath. Ind. Orn. ii. p. 598, n. ${ }^{\circ}$ i 8 (1790); Bonn. Enc. Meth. p. 252 (1790'; Teinm. Pig. A. Gatl. i. pp 257, 46o (1813'; Vieill. $N$ D. xxvi p. 349 (1818); Desm. Dict. Sc. Nat. xl. p. 333 (1826); Less. Tr. d'Orn. p. 472 (1813).

C. jambon, Steph. Gen. Zool. xi. I, p. 49 (18r9); xiv I, p. 284 ( I I 26 !)

Ptilirnpus jambu, Less Comple de Buff. Ois. viii. p. 27 (1837), and ed. p. 276 (1 838), Hartl. Syst. Verz. Brem. p. 97 (i $8+4$; Blyth, Cal. B. Mus. A. S. B. p. 230, n. ${ }^{\circ}$ I95 (1849); Brïss. Abh. naturw. Ver. Brem. v. p. 5.36 (1878).

Pt. jambu, G. R. Gr. Gen. B. ii. p. 467, n. ${ }^{\circ}{ }_{1} 6$ (1 844$)$; id. Lis'. of Gallina B. M. p. 2 (1844'; id. List. B. Brit. Mus. Calumba, p. 7 (1856); Sclat. P. Z. S. 1863 , p. 221 , Wall. Ibis, 1865 , p. 378 ; id. J. f. O. 1866 , p. 278; Salvad. Ucc. Born. p. 289 (1874); Sharpe, Ibis, 1877, p. 23; Hume, Str. Feath. viii. p. 67 (18;8); Vorderm. N. T. N.I. 1.p. jo3 (1896)).

Ramphiculus jambu, Bp. Consp. ii. p. I7 (is54); id. Compt. Rend. xxxix. p. 878 (1854), xl. p. 215 , n." 35 ; id Coup. d'all Ord. Pig. pp. 10, 54, n." 35 (1855), Moore, P. Z. S. 1859 , p. 465; G. R. Gr. Hand-list, ii. p. 227 , n. 9 1.50 (1870; S'ousa, Mus. Nac. Lisb. Columber p. 7 1873.

R. jambu, Rchnb. Tauben, p. 99, t. 230. ff, 1334-35. t. 244 , ff. 3488-89 (1862); A. Mïll. J. f. $O \quad 1882,43$ I.

Ptilophus jambu, Schleg. De Dierent. p. 210 1 $864 ;$ id. Mis. P.-B. Columba, p. 36 (1873); Gieb. Thes. Orn. iii. p. 365 (1877; Elliot, P. Z. S. 1878 , p 554; Beccari. Ann. Mus. Cv. Genl. xiv. p. 244 (1879); Sharfe, P. Z. S. 1879 , p. 348, 1881 , P. 299, Salv. Cat. B. Strickl. Coll. p. 555 (1882); Sclat. List. Vert. An. 8th ed. p. 444 (1838); Sharpe, P. Z. S. 1887 , 1. 432; Snellem. Sum.-Exped. i. Vög. p. 48 (1887); G.gl. d" Sitivad. Mem. R. Ac. Sc. 7 or. (2) xxxix. p. I Io (1888; Evere't. Journ. Str Br. R. As. Soc. 1889 , p. I 37 ; Vordcrm. V. T. F. I. xlix. pp. 64, 412 (1889); Tristr. Cat. Coll. B. p. tt (1889); Sharpe, Ibs, 1890, p. 4I3; Salv. Cat. B. M. 21. p. 80) (1893).

Ptilinopus jambu, Stoliczka, J.A. S. B. xxxix. 2, p. 330 (1870). Phassa ja.rbu, Heine \& Rchnw. Nomencl. Mus. Hein. Orn. P. 281 (1890).

Hab. Luzón, Paragua, Calamianes, Culion; Borneo, Sumatra. 
2. Gé.s. PTILOPUS, Sikicki.

8. Pt. posapэnsis. Finsiil of N. v. Imunatad.

(M S. M.)

Ptilonopus fasciatus: Finsch, Journ. Mus. Godeffr. Heft. xii. p. $37(1870)$.

Pt. ponapensis, Finsch, P. Z. S. 1877 , p. 779; Schmeltz. Ethnogr. Abth. Mus. Gudeffr. p. 353 (1881).

Ptilopus fasciatus, Elliot, $P Z$. $S .1 \delta^{\prime} 78, \mu p .535,536$; Trist. Cat. Coll. B. p. 4t. $(1889)$.

Pt. ponapensis, Schmeltz, Verh. Ver Hainb. 1877, (1879), pp. 178, 179; Finsch, J. t. O. 1880, p. 291; id. P. Z. S. 1880, p. 576 ; id. Ibis, 1881 , pp. I 1 3, I 1 $5 ;$ Wiglesw. Ibis, 1891, p. 583 ; id. Aves Polynes. p. 50, n." 261 (1191); Salv. Ciat. B. $M$. 21. p. 93 (1803).

Hab. Ponapé, Carolinas.

9 Pt hernsheimi. Fin,eh $\mathrm{N}$ v.

Pt. herusheimi, Finsch, J. f. O. 1880, p. 303; id. P. Z. S. 1880 , p. 577; id Ibis, 1881 , pp. 106-108; Rchmo et Sihcl. J. f. O. 1888, p. 75; Wiglesw. Ibis, 1891 , p. 583 ; id. Aves Polynes. p. 51 1 $10^{\circ} 262$ (1891); Salv. Cat. B. M. 21. p. 94; (1893).

Hab. Ualan, Carolinas.

Io Pt roseicapillus, (Less.) N. v. Curucuru.

Kurukuru, Quoy et Gacin. Vög. Uvanie, Zool. p. 34, I824.

Columba roseicapilla, Less. Tr. d'Orn. p. 472 (183r).

(. purpurata, Kit l. Kupfertaf. Heft. iii. p. 25, t. xxxiii. f. 2 (1833).

Kurukuru des Marianes, Less. Compl. de Buff. viii p. 33 (1837); i.t. op. cit. and eat p. 278 ( $183^{8}$ ).

Ptilonopus swainsoni, G. R. Gr. Gen. B. ii. p. 466, n. 6 (1844).

P. purpureocinctus, G. R. Gr. P. Z. S. 1853 , p. 48, pl. lv.; id. Ann. \& Marg. N. H. (2) xv. p. I 59 (1855); ia. List. B. Brit. Mus. Columber, p. 4 (1856).

Kurukuru roseicapilla, Des Murs \& Prev. Voy Vénus, Ois. pp. 259,269 (1855?).

Ptilopus roseicapillus, Bp. Consp. Av ii. p. 2 I (1 854); id. Compt. Rend. xxxix. p. 877 (I 854 ), xl. p. 246, n" 46 (1855), xliii. p. 833 (1856); id. Rev. et Mag. do Zool. $185^{6}$, p. 536; id. Icon. Pig. pl. xxiii. (1857); Schlig. Mus. P.-B. Columbar, p 8 (1873); Sousa, Mus. Nac. Lisb. Columbe, p. 8 (1873); Gieb. Thes. $O, n$. iii. p. 368 (1877); Elllot, P. Z. S. 1878 , pp. 5i6, 537; Aust. Le Natural 1889, p. 261; Wiglesw. Ibis, 1891 , p. 584; id. Aves Polynes. p. 48, n. ${ }^{\circ} 259$ (1891); Salvad. Cat. B. $M$. vi. p. 108 (I 893 ).

Ptilonopus roseicapillas, G. R. Gr. List B. Trop. Isl. p. 37 1859; Rchnb. Tauben, p. 96 (1862); Finsch. P. Z. S. 1874 , p. 94 .

P. roseicapillus, Rchnb. Tauben, ii. p. I77 (1862); Finsch \& 
Hartl. Orn. Centralpol. p. I 27 ( 1867 ); G. R. Gr. Hand-list, ii. p. $225, n .^{\circ} 9119(1870)$.

P. purpuratus, Hartert, Kat. Vogelsamml. p. 190, n. 2866 (1891). Hab. Islas Marianas, Guahan.

3. Gén. I.AMPROTRERON, Br.

I1. L. temmincki, Des Murs. ơ N. v. Bato-bato.

(M. S. T.

Columba superba, Knip. et Prev. Pig. ii. pl. $42(1838+43)$.

Ptilonopus superbus, part., G. R. Gr. Gen. B. ii. p. 467, n. ${ }^{\circ}$ i (I 844 ).

Kurukuru temminkii, Des Murs \& Prev. Voy. Vémus, Zool. pp. 236, 268 (ex Prevost, l. c.) (1855?)

Ptilonopus formosus, G. R. Gr. P. Z. S. 1860 , p. 360; Wall. Ibis, 1865, p. 379, n. $^{0} 28$; id. J. f. $O .1866$, p. 278.

Ptilopus superbus, Schleg. N. T. D. iii. p. 209 (1866); id. Mus. P.-B. Columber, p. 3o (1863); Gieb. Thes. Orn. iii. p. 368 ( 1877 ).

Jamprotreron formosus, G. R. Gr. Hand-list, ii. p. 277, n." 9I 59 I 870 .

Ptilonopus (?) celebensis, Wall. fide G. R. Gr. Hand-list, l. c. (I870).

Lamprotreron formosa, Wall. Tr. Zool. Soc. viii. p. 82 (1872); I.enz, J. f. O. 1877 , p. 377; Meyer, Ibis, 1879 , p. I35; Rlas. J. f. $O .1883$, p. 138 .

Ptilonopus formosus, Brïgg. Abh. naturw. Ver. Brem. r. p. 79 ( 1876$)$.

Ptilopus temmincki, Elliot, P. Z. S. 1879 , p. 62; Blas. Zeitschr. f. ges. Orn. 1885, p. 302, t. xiv. ff. I, 2 ; Salv. Cat. B. M. 21. P. II 5 (I 893 ).

P. formosus, Guillem. P. Z. S. 1885, pp. $269,556$.

?Lamprotreron temmincki, Heine \& Rchnu. Nomencl. Mus. Hein. Orn. p. Orn. p. 28 I ( 1890$)$.

Hab. Joló; Célebes.

4. Gén. SPILOTRERON, Salv.

12. Sp bangueyensis, Meyer, $0^{\circ}$, N. v. Punay.

Ptilopus melanocephalus, Tweed. P. Z. S. 1878, p. 951, 1879 , p. 73; Sharpe, P. Z. S. 1879 , p. 316; Wardl. Rams. Twsedd. Orn Works, p.659 (1881); Sharpe, Ibis, 1864, p. 322; Gullem. P. Z. S. 1815 , p. 269; Everett, Ibis, 1886, p. 524; Sharpe, Ibis, 1888 , p. 202; Everett, ibid. p. 282; Blasins, Ornis, 1888 , p. 315; Everett, P. Z. S. 1889 , p. 225; id. Joninn. of the Str. Branch R. As. Soc. 1889 , p. 197; Whiteh. Ibis, 1890 , p. 56; Steere, List B.\& M. Phtipp. Steere Exp. p. 24 (1890); Salv. Cat. B. M. 21. 1893 , p. 143.

P. bangueyensis, Meyer, J.f. O. 1891, p. 70.

P. purpureinucha, Meyer, $J . f . O .1891$, p. 7 !. Hab. Mindanao, Zamboanga, Banguey, Basilan, Paragua, P. Princesa, Joló. 
5. Gén. ALECTRAENiS, GikaY.

13. A. pulcherrima, (Scop.). N. v.

l'igeun violet a tète rouge d'Antigue, Son. Foy. Nour'. Ciumn. p. I I 2 , pl. 67 ( I 766).

Columba pulcherrima, Scop. Del. Flor. et Faun. Jnsubr. ii. p. 94, n. ${ }^{\circ} 98$ (1 776$)$.

Red-orowned Pigeon, Lath. Syn. ii. 2, p. 618, n. $0^{0}\left(t_{7} \delta_{3}\right)$.

Columba rubricapilla, Gm. S. $N$. i. 2 , p. 784, n. 62 (i 788 ); Lath. Ind. Orn. ii. p. 599, n. ${ }^{\circ} 19$ (1790); Temm. \& K'nip. Pig. i. pl 20 (1808-1811)i Temm. Pig. et Gallin. i. pp 233. 464 (1813); Vieill. N. D xxvi. p 363 (1818); Step. Genl. Zool. xi. I, p. I I (1819); Desm. Dict. Sc. Nat. xl p. 32y (1826); Wagl. Syst. Av. Columba, sp. 24 (1827); Less. Tr. d'Orn. p. 470 (1831); id. Compl. de Buff. Ois. viii. p. 14 (1837); id. op. cit. and ed. p. 295 (1838).

C. rubrocapillata, Bonn. Enc. Méth. i. p. 334, pl. 77. f. 4 (1790).

Ptilinopus pulcherrimus, G. R. Gr. Lisi. B. Brit. Mus. Gallina, p. I (1 844); id. Gen. B. ii. p. 467, n. ${ }^{\circ} 2$ (1844); id. lis/. B. Brit. Mus. Columber, p. 9 (1856).

Carpophaga rubricapilla, Rchnb. Tauben. Novitia a. vi. p. 1, 1, 230 b. f. 3361 .

Janthonas pulcherrima, Rchitb. Syst. As. p. xxv. (1852).

Ptilopus rubricapillus, Licht. Nomencl. p. 82 (1852).

Erythroena pulcherrima, Bp. Consp. Av. ii. p. 30 (1854); Rchnb. Tauben, i. p. I31 (I862).

F. pulcherrima, Bp. Compt. Rend. xxxix p. 860 (1854), xl. p. 217, n. 70 (1855); id. Coup dacil. Ordr. Pig. pp. i 2, 55, no. 70 (1855); Hartl. J. f. O. 1860, p. 11 2; id. Orn. Bestr. Faun. Madag. p. 65 1861; Pollen, N. T. D. i. p. 318 (1863); E. Newt. Ibis, 1867 , pp. 337, 345, 356, 359, 360; Oust. Bull. Soc. Philom. (7); p. IOI (1 877$)$.

:Alectrœnas nitidissima, E. Neret. Ibis, 1862, p. 196; Rchnb. Tauben, ii. p. 186 (1862).

Ptilopus pulcherrimus, Schleg. De Dierent. p. 2 I I (1864); id Mus. P.-B. Columba, p. 44 (1873); Geb. Thes. Orn. iii. p. 367 (1877).

Erythronas pulcherrima, Sclat. P. Z.S. 1865 , p. $318 ;$ E. Newt. P. Z. S. 1867 , p. 344; Garrod, P. Z. S. 1875 , p. 368; Sclat. List. Vert. An. 8th ed. p. 444 (1883).

F. pulcherrima, G. R. Gr. Hand-list, ii. p. 227, n. ${ }^{\circ} 9163$ (187o).

Alectronas pulcherrima, Hartl. Vög. Madag. p. 264 (1877); Shell. Ibis, 1883 , p. 260; Salv. Cat. B. M. 21. 1863 , p. 161.

Funingus pulcherrimus, Oust. Bull. Soc. Philom. (7) ii. p. i7' ( 1878 ).

Alectrcenas pulcherrima, A. Nezet. P. Z. S. 1879 , p. 4.

A. pulcherrimus, Tristr. Cat. Coll. B. p. 43 (1889).

Hab. Marianas, Panay. 


\section{Fam. CARPOFAGIDOS.}

1. Gén. GL,OBIBERA, BP.

1. G. Oceanica, (Less.) N. v.

Cislumba oceanica, Loss. Voy. Coq. Zool. i. p. 432, Atlas, pl. 41 (1826); 1)csm. Dict. Sc. Nat. xl. p. 3:6 (1826); Less. op. cit. i. 2, p. 708 (1828); id. Min. d'Orn. ii. p. 166 (18.8); W.igl. Jsis, 1829 , p. 739; Less. Tr. d'Orn. p. 468 (1831); Kittl. Kupfert. p. 25, t. 33. f. I (1833); Préu. \& Knip, Pig. ii. pl. 24 (1838-43); Kittl. Reise, i. p. 377 , ii. pp. 39.62 (1858).

('arpophaga oceanica, G. R. Gr. Gen. ii. p. 468, n." 2 (1844); Peale, Un. St. Expl. Exped., Birds, n. ${ }^{\circ} 19$ (1848); Hartl. \|iegm. Arch. f. $N$ :turg. 1852, pp. 115,$135 ;$ id. I. $f .0$. 1854, p. 168; Cass. Pr. Ac. Nat. Sc. Philad. 1854, p. 229; Hartl. P. Z. S. 1867 , p. 830; Harll. \& Finsch, P. $Z$. S. 1072, p. 101; Schleg. Mus. P.-B., Columba, p. 87 (1873); Nillsch, Vög. der Palau Gruspe, p. 26; id. P. Z. S. 1877 , 1. $726, / 75,780$; Salvad. Monogr. sottogen. Globicera, p. \& (1878); id. 16.s, 1879 , p. 364; Nehrk. J.f. O. 1879, p. 407; Finsch, J.f. O. 1880, pp. 292, 304; id. P. $Z$. S. $1880, p$. 576 ; $a d$. Ibis, 1880 , pp. 220, 331, 332, 1881, pp. 108, 113; "chmeltz, Ethn. Abth. Mus. Godeffr. 1881, 1pp. 298, 33(, 353; Finsch. Mith. orn. Ver. Wien, 1884, p. $125 ;$ Tristr. iat. Coll. B. p. 42 (1889); Hartert, Kat. Vogelsanml. p. 130 (1891); Wiglesw. Aves polynes. p. 52,, n. 266 (1891).

Cilobicera oceanica, Bp. Consp. Av. ii. p. 31 (1854); id. Compt. kend. xxxix. p. 1072 (1854); xi. p. 217, n. 73 (1855); id.

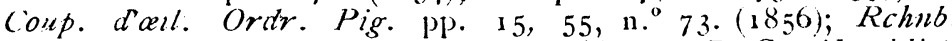
Tauben, i. p. 120 , t. 130 ff. $1283-84$ (1662); (.. R. Gr. Hand-list, ii. p. 229, n. 9179 (1670); Salv. Cat. B. M 21. 1893, p. 176.

( arpophaga (Globicera) oceanica, G. R. Gr. Cat. B. Trop Ist. p. 41 (1859).

(C) pacifica, part., Hartl. \& Finsch, Orn. Centialpolyn. P 142 (1867); ia. P. Z. S. 1868 , PI). 7, I18.

Hab. Punapé, Carolinas, Palaos.

\section{Gén. CARPOPHAGA, Shlix.}

2. C. nuchalis, Call. \& N. v. Punay.

(M. S. I.)

(. paulinà, part. Schleg. N.T. D. iii. PP 200, 201, (1866); Hald. Tr. Zool. Soc. viii. p. $13(1872)$; Schleg. Mus. P.-B. Columba, p. 85 (1873); Wald. Tr. Zool. Soc. ix. p. 215 (1875); iwcedi. Orn. Works. p. 379; Kutter, J. f. O. 1882, p. 176; : Hartert, Kat. Vogelsamml. 1. 181 (1891).

! Ducula paulina, Sousa. Mus. Nac. Lisb. Columba, p. $10(1873)$. Carpuphaya nuchalis, Cab. J. F. O. 1882 , p. 126; Kutter, J. F. O. 1882, p. 877; Rcknw \& Schal. J. F. O. 1883 , p. 403; Hartert, Kat. Vogelsamml. p. 189 (1891); Salv. Cat. B. M. 1,1893 , p. 190 .

Hai. Luzon, Bataan, Mindoro, Mindanao. 
Palumbus moluccensis, Bris. Orn. i. p. 148, pl. xii. f. $2(1760)$; Less. Compl de Buff., Ois. viii. p. 81 (1837); id. op. cit. $2 n 1$ ed. p. 291 ( 1838$)$.

Columba xnea, Linn. S. N. i. p. 283, n. $22(1766)$; Gm. S. N. i. 2, p. 780, n. 22 (1788); Lath. Ind. Orn. ii. p. 6ri2, n." 33 (1790); Bonn. Enc. Méth. i. p. 233 (179:); Temm. \& Knip. Pig. i. fam. sec. pl. 3 (1808-1 1); Temm. Pig. et Gallin. i. 1.. 86, 446 (1813; Vieill. N. D. xxvi. p. 363 (1818); Steph. Gèn. Zool. xi. p. 2r (1819; Horsf. Tr. Linn. Soc. xiii.p 184 (1821); Raffl. Tr. Lann. Soc. xiii. pt. 2, p. 316 (1822); Desm. Dic. Sc. Nat. xl. p. 315 (1826); Wrgl. Syyst Av. Columba, sp. 15 (1827); Less. 7ir. d'Or11. p. 468 (1831).

Pigc ,n Ramicr des Moluques, $D^{\prime} A u b e n t$. Pl. Enl. I64 (17-?); Buff. Hist. Nat., Ois. ii. p. 538 (1771).

Columba moluccensis, Müll. S. N. Suppl. p. 133, n. 35 (177h); Cass. Pr. Ac. Nat. Sc. Philad. 1854 , p. 248.

Nutmeg Pigcon, Lath. Syn. ii. 2, p. 636, n. ${ }^{\circ}$ 30 (1783).

Columba sylvatica, Tickcll,J. A. S. B. ii. p. 58i (1833).

Carphophaga anea, Selby, Nat. Libr., Pigeons, p. I 8 (1834; Sw. Class. B. ii. p. $34^{8}$ (1837); Jerd. Madr. Journ. xii. p. II (1840); G. R. Gr. Gen. B. ii. p. 468, n. ${ }^{\circ}$ I (1844); Harll. Syst. Verz. p. 98 (1844); Peale, U. S. Expl. Exped., Zool. p. 198 (1848); Rchnb. Syn. Av. Novit. Columbaria, t. 23! b. f. 3359 (I85I); Hartl. Arch.f. Naturg. 1852, p. I1 5; Casi. Pr. Ac. Nat. Sc. Philad. 1854 , p. 227 ; Bp. Consp. Av. ii. p. 32 (1854); id. Compt. Rend. xxxix. p. 8074 (8854); xl. p. 287, n. ${ }^{\circ} 80$ (1855); id. Coup, a'xil Ordre Pig. pp. 84, 45, n. ${ }^{\circ} 80$ (1855); Gould. P. Z. S. 1859 , p. 150; G. R. Gr. P. Z. S. p. 36I; Rchnb. Tauben, i. p. I 19, t. 2306 f. 3359 (1862); Sclat. P. Z. S. 1863 , p. $221 ;$ Wall. P. Z. S. 1863 . p. 486; Pelz. Novara Ris., Vög. p. 105 (1865); Wall. Ibis, 1865 , pp. 383, 397 ; id. J. F. O. 1866 , p. 280 ; Schleg. N. T. D. iii. p. 197 (1866); G. R. Gr. Hand-list, ii. p. 228, n. ${ }^{\circ} 9$ r 70 (1870); $G$ eb. Thes. Orn. i. p. $583(1872)$; Wald. Ibis, 1873 , p. 31 4 ; Schleg. Mus. P.-B. Columba, p. 85 ( 1873 ); Sousa, Mus. Nac. Lisb. Columbe, p 9 (1873); Garrod. P. Z. S. 1873, pp. 468, 639, I874, p. 256; Hume, Str. Feath. ii. p. 260; 424 (1874); Salvad. Ucc. di Born. p. 290 (1874); Blyth. \& Wald. B. of Burin. p. 144 (I875); Walt. Tr. Zoot. Soc. ix. pp. 215, $25 \mathrm{I}$ (1875); Hume, Nests \& Eggs. iii. p. 496 (i873); id. Sitr. Feath. iii. p. 163 (1875); Legge, Ibis, 1875, p. 399; Sharte, Ibis, 1875, p. 51; Brïggem. Abh. Ver. Brem. v. p. (1876); Ball. Str. Feath. iv. p. 235 (1876; Armstr. b:b. p. 337 (1876); Sharpe, Tr. Linn. Soc. Zool. i. pp 346, 353 (1876); Inglis, Str. Feath. v. p. 39 (1877); Ball, ibid. p. 418 (1877); Tweedd. P. Z. S. 1877 , pp. $264,832,1878$, pp. I13, 288, 344, 623, 752; Rosenb, Malay, Archip. p. 108 (1878); Hume, \& Davis, Str. Feth. vi. p 416 (1878); Ball. Str. Feath. vii. p. 224 (1878); Hume, Str. Feath. viii. pp. 67. 109, n." 780 (1879); Tweedd. P. Z. S. 1879. p. 73; Sharpe, P. Z. S. 1879, 348; Tirant. Bull. Com. Agr. el Ind. Cochinch. (3) i. 
p. ItI n." 252 (1879); Bingh. Str. Feat. ix. p. 194 (1881: Parker, ibid. p. $48 \mathrm{I}$ (1880); Wardl. Rams. P. Z. S. $18 \delta^{\prime} 0$, p. 15; Sharpe, P. Z. S. 1881 , p. 799; Tweedd. Orn. Works, pp. 245, 378, 379, 380, 41 2, 538, 559, 573, 594, 599, 6ris, 643, 650, (1801); Wardl. Rmis. Tweedd. Orn. Works, App. p. 652(1881); Kelham. Ibis, 18'8'1, p. 527; Blas. Nchrk. Jahresh. ler. Naturw. zerr Braunschw 1881, p. 59; Nichols. Ibis, 1882 , p. 65; Oates, Str. Feath. x. p. 235 (1882); Cab. J. $f$. O. 1882 , pp. 125, 126; Kutter. J. f. O. 1882 , p. 177; Sali. (at. B. Strickl. Coll. p. 553 (1882); Nichols. Ibis, 1883, p. 524; Kutter. J. f. O. 1883 , p. 318 ; Sclat. List. Vert. An. ed. p. 433 (1883); Blas. Verh. z.-b. Ges. Wien, xxxiii. p. bа (1883); Oates, B. Brit. Burm. ii. p. 301 (1883); Meyer, Abbud. Vogel-Skel. t. lviii. (1884); Sharpe, Ibis, $188+$, p. 322; Snellem. Sumatra-Exp., Vogels, p, 48 (1884); Vorderm. N. T. .V. J. Ixiv. p. 226 (1884); Guillem. P. Z, S. 1885, p. $27($, Redd. P. Z. S. 1885 , p. 844 ; Vorder:n. N. T. N. I. xlvi. pp. 25, 236 (1886); Buttik. Not Leyd. Mus. ix. p. 76 (1886); Anders. Journ. Linn Soc., Zool. xxi. p. 152 (1887); Sharpe, Ibis, 1888 , p. 302; Hume, Str. Feath. xi. p. 294 (1888); Eierett, fourn. Str. Br. R. As. Soc. 1889, p. 194; id. P. Z'. S. 1889 , p. 225; Tristr. Cat. Coll B. p. 42 (1889; Vorderm. V. T, $N$. I. xlix. p. 4 I 2 (1889), xl. p. 505 (1890); Whiteh, Ibis, 1890 , p. 56; Sharpe, 1bis, 1890, pl'. 134, 283; Steere, list of. Philipp. Birds, p. 23 (1890); Heln, \& Rehnw. Nomencl. Mus. Hein. Orn. p. 281 (1890); Hartert, Kat. Vogelsamml. p. 189 (1891); id. J. f. O. 1891, p. 300; Salv. Cat. B. $M$. 21. p. 191 (1893).

Hab. Samar, Bora igan, Tablas, Siquijor, Panay, Sibuyan, Ca'a. mianes, Culión, Leyte Negros, Luzon, Paragua, P. Princesa, Mindanao, Mindoro, Baco, Joló, Tawi-tawi.

4. C. chalybura, $B p$. $\sigma \circ \mathrm{N}$ v Balot

(M. S. 'T.)

C. chalybura, Bp. Consp. A2. ii. p. 32 (185t); id. Compt. Rend. xxxix. p. I074, xl. p. 217, n. ${ }^{0} 81$ (1855); id. Coup. d'xil Ordr. Pig. pp. 14, 55, n. ${ }^{\circ}$ 8 I (1855); G. R. Cir. Lisi b̈. Brit. Mus. Columba, p. I7 (1856); Bp. Icon. pl. xlii. (1857); Wall. Ibis, 1861 , p. 286; Rchnb. Iauben, i. p. 119 (1862); Wall. Ibis, 1865 , pp. 383,396 ; id. J. f. O. 1866 , p. 280; Mart. J. f. O. 1866 , p. 24; G. R. Gr. Hand-list, ii. p. 288 , n. ${ }^{\circ} 9169$ (1870); Sousa, Mus. Hein. Orn. p. 281 (1890).

Muscadivora xnea, Schleg. Dierent. p. 2 ro (1864); Salv. Cat. B. M. 21. 1893 p. 192.

Hab. Luzon, Manila, Montalban, Negros, Dumaguete, Cebú, Amparo, Leyte, Dinagat, Surigao, Samar, Butuan, Zamboanga, Basilan, Mindanao, Calamianes, Cuyo.

5. C palawanensis, Blas. ơ $\& \mathrm{~N} v$ Bałut.

(M. S T.)

C. anea palawanensis, Blas. Orn. ix. p. 316 (1888); id. Ibis, 1888 p. 374; Salv. Cat. B. M. 21 p. 192 (1893).

Hab. Banguey, Paragua, P. Princesa. 
Columba perspicillata, Temm. Pl. Col. 246 (tior. 42, 1825); Stephí. Gen. Zool. xiv. I, p 286 (1826); Desm. Dic'. Sc. Nat. xl. p. 31 (1 826); Wagl. Sy'st. Av. Columba, sp. 1,3 (1827);? Less. 7ir. d' Orn. p. 469 (1831); id. Compl. de Buff. Ois. viii. p. X6 ( 1837 ); id. op. cit. and ed p 293 ( 1838 ).

Carpophaga perspicillata, G. R. Gr. Gen. B. ii. p. 468, n. ${ }^{\circ} 14$ (1844); Blyth, Cat. B. Mus. A. S. B. p. 232 n. 1403 (1849); Bp. Consp. ii. p. $33(1854)$; id. Compt. Rend. xxxix. p. 1074 1854), xl. p. 217 , n. 84 (1855); id. Conp. d' ail Ordr. Pig. pp. 14, 55, n. 84 (1855); Cass. Pr. Ac. Nat. Sc. Philad. 1854 , p. 230; Bp. Compt. Rend. xliii. p. 835 (1856); id. Icon. Pig. pl. 45 (1857); G. R. Gr. P. Z. S. 1860 , p. 360 (parl.) 186 I, p. 437; Wall. Ibis, 186s, p. 290; Rchnb. Tauben, i. p. I89, t. 231 ff. 1291-92 (1862); Finsch, Nen-Guin. p. 177 (1865); Schleg. N. T. D. iii. pp. 194, 344 (1865); G. R. Gr. Hand-list, ii. p. 228, n. $^{\circ} 9197$ (170; Gieb. Thes. Orn. i. 1. 587 (1872); Schleg. Mus. P.-B. Columba, p. 89 (1873); Sausa, Mus. Nac. Lisb. Columbee p. ro (1873); Wald. Tr. Zool. Sor. ix. p. 216 (1875); Salvad. Ann. Mus. Civ. Gen. vii. p. 78 (r) (1875), viii. p $3^{82}(1876)$ ix. p. 201, n. ${ }^{\circ} 45$ (1876); Pelz. Verh z.-b. Ges. Wien, 1876, p. 719; Roseub. Malay. Archip. p. 407 (1878-79); Saland. Orn. Pap. e Mol. iii. p. 91 (1882); Pleske, Bull. Acad. Petersb. xxix p. 535 (1884) (Ternate); Tristr. Cat. Coll. B. p. 42 (1889); Heine \& Rchnze. Nomencl. Mus. Hein. Orn. p. 281 (1890) (New Guinea!); Salvad. Mem. R. Ac. Tor. (2) xlii. p. 56 (Agg. Orn. Pap. e Mol. F. I82) (1891); Salv. Cat. B. M. p. 199 (1893).

C: perspicillata, var., Wall. P.Z.S. 1863, p. 33.

C. temminckii, Wall. Ibis, ${ }_{186} 8$, p. $384,{ }^{1}{ }^{\circ} 54 ; i d . J . f . O$. ${ }_{1} 856$, p. $280, \mathrm{n}^{\circ} 54 ; G$. R. Gr. Hand-list, ii. p. 228, n." $9173(1870)$.

Hab. Mindoro, Baco, Paragua, P. Princesa; Batchian.

7. C. pickeringi, Cass. $\sigma \mathrm{N}$ v Balut.

(M. S. T)

C. anea, Peale. U. S. Expl. Exped., Zool. p. 198 (1848); Hartl. Archiv. f. Naturg. 1852 , p. II 5.

C. p ckeringii, Cass. Pr. Ac. Nat. Sc. Philad. vii. p. 228 (1854); Bp. Compt. Rend. xliii. p. o35 (1856); Cass. Un. St. Expl. Exp. and ed. p. 267, pl. xxvii. (1858); Rchnb. Tauben, ii. p. 184; Novit. Suppl. $t$. vi. f. 72 (i862); Wald. Tr. Zool. Soc, ix. p. 215 (1875); Sharpe, Tr. Linn. Soc., Zool. i. p. p. 353 (1876); id. P. Z. S. 1885, pp. 248, 270, 418, 420; Everett, Ibis, 1888, p. 282; id, Jour. Str. Br. R. As. Soc. 1889, p. г95; Blas. J. f. O. 1890, p. 138; Salv. Cat. B. M. 21. p. 201 (1893).

C. chalybura, part., G. R. Hand-list, ii. p. 228, n. ${ }^{0} 9$ rg6 (1870).

C. znea, part., Gieb. Thes. Orn. i. p. $5^{83}$ (1872).

C. everetti, Grant. Ann. \& Mag. N. H. (6) ii. p. $35 \mathrm{I}$ (1888); id. Ibis, 1889 , p. 582 .

Hab. Cagayan de Joló, Mantanani, Joló. 
3. Gén. Ptilocalpa, Br.

8. Pt. griseipectus, $B p \quad$ N. v. Balut-isla.

(M. $s$ r)

Carpophaga pectoralis, G. R. Gr. List. B. Brit. Mus. iii. Gallinar, p. 7 (1844; Hartl. J. F. O. $1854, B$. xlv. 1855, p. 9 \&.

C. griseopectus, G. R. Gr. Mus. Brit. 1854 ; id. List. B. Brit. Mus. Columba, p. 22 (1856); Wall. Ibis, 1865, pp. $38 \equiv$, $398 ;$ d. J. F. O. 1866 , p. $281 ; G$ eb. Thes. Orn. i. p. $5^{\times} 5$ (1872); Schleg. Mus. P.-B. Columba, p. 88 (1073); Tristr. Cat. Coll. B. p. 42 (1889); Salv. Cat. B. M. 21 p. 265 (1893).

Ptilocolpa griseipectus, Bp. Consp. Av. ii. p. 34, n. ${ }^{0}$ (1851); id. Compt. Rend. xxxix. p. 1075 (1854), xl. p. 2i 7, n. 88 (1855); id. Coup. d'cil. Ordr. P.g. pp. I 5, 55, n. 88 (1855); id. Icon. Pig. pl. 5 I (I857); G. R. Gr. Hand-l.s', ii. p. 23 I, n. ${ }^{\circ} 9225$ (1870); Wald. Tr. Zool. Soc. ix. p). 216,251 (187.5); Sharp!, Tr. Linn. Soc., and ser. Zool. i. p. 353 (i 876 ); Trecedd. P'. Z. S. 1878, pp. 937, 952; id. Oril. Works, p. 380, 412 62?, 643 (1881); Wardl. Rims. Tweedd. id. Orn. Works, p. 659 (r88r); Kutter, J. f. O. 1883, p. 315; Wardl. Rams. Ib.s, 1886 , p. 160 ; Steere, List. Philipp. B. p. $2+$ (1890); He.n. \& Rchuw. Nomencl. Mus. Hein. Orn. p. 283 (1890); Hartert. J. f. O. 1191, p. 301.

Pt. griseipectus, Rchnb. Tauben, i. p. 91 (1862), ii. p. 176, Novit. Suppl. t. iii. ff. 30, 3 I (1862).

(arpophaga griseipectus, Mart. J. f. O. 1866, p. 24.

C, sp. Trueedd. P. Z. S. 1878 , p. 937.

Hab. Luzon, Manila, Mindoro, Mindanao, Zarboanga.

9. Pt. carola, $(B p) \sigma \Omega$ N. v Carmaso.

(M. S T.)

Carpol haga carola, Bp. in litt, id. Consp. Av. ii. p. $34(1854)$; G. R. Gr. Lis B. Brit. Mus. Colunba, p. 22 (1856); Wall. Ibis, 1868 , pp. 385, 398; d. J.'f. O. 1866, p. 281; Salì. Cat. B. M. 21. p. 206 (1893).

Ptilocolpa carola, Bp. Consp. Av. ii. p. 34 , n. (1 154); id. Compt. Rend. xxxix. p. 1075 (1854), xl. p. 217, 1. 87 (1855); $d$. Coup. d'ail Ordre Pig. pp. 15, 55, n. ${ }^{\circ} 87$ (is 55); id. Compt. Rend. xli. p. 658 (1855), xliii. p. 8,35 (1856); G. R. Gr. Handlist, ii. p. 231, $1^{\circ} 9227$ (1870); Sousa, Mus. Nac. Lisb. Columbar, p 10 (1873).

Pt. carola, Rchnb. Tauben, p. 91 (1862).

Carpophaga carola, Mart. I. f. O. 1856 , p. 24.

Ptilocalpa griseipectus, Wald. Tr. Zool. Soc. ix. pl). $216,25 \mathrm{r}$ (1875); Trueedd. Orn. Works, pp. 380, 412 (1881). Wirt!. Rams. Tzeedd. Orn. Works, p. 659 (1881).

Hab. Luzón, Manila.

4. Gen ZONOPHAPS, SAl.v.

ro Z. poliocephalue, (Gray) ơo N. v. Balutro.

(M. S. T)

Carpophaga poliocephala, Gr. List. B. Brit. Mus. iii. Rallina, I. 6 (I844; G. R. Gr. \& Mitch. Gen. B. ii. p. $4^{6} 9$, n. ${ }^{\circ}$, pll. 
189 (1844); Rihnb. Syn. Av. Columba, f. 1298 (1847); Harll. J. f. $O{ }_{1854}, B$. lxv. 1855, p. 97 ; G. R. Gr. Lis B. Brit. Mis. Columba, p. 22 (1856); Wall. Ibis, 1865, pp. 387, 398; id. J. f. O. 1866 , p. 281 ; Gieb. Thes. Orn. i. p. 587 (1872); Schleg. Mus. P. B. Columba, p. 92 (1873; Tristr. Cat. Coll. B. p. 42 (1889); Hein. \& Rchnb. Nomencl. Mus. Hein. Orn. 1. 283 (1890); Salv. Cat. B. M. 92. p. 209 (1890).

Zuncenas poliocephala, Rchnb. Av. Syst. Nat. p. xxri. (1852); id. Tauten, i. p. 112, t. 233, f. 1298 (1862).

Hemiphaya poliocephala, Bp. Consp. Av. ii. 39 (1854); id. Compt. Rend. xxxix. p. 1077 (1854); xl. p. 217, n. ${ }^{\circ} 105$ (1855); id. Coup. d'ceil Ordr. Pig. pp. 17, 55, n. ${ }^{\circ} 105$ 1855; id. Coimpt. Rend. xli. p. 658 (1855), xliii. p. 836 (1856); G. R. Gr. Hand-list, ii. p. 231, n. ${ }^{\circ 223}$ (1870); Sousa, Mus. Nic. Lisb. Columba, p. I1 (1873); Wall. Tr. Zool. Soc. ix. Pp. 217,253 (1875); Sharpe; 7r. Linn. Soc. (2), Zool. i. pp. 347, 353 (1876), Tweedd. P. Z. S. 1878 , pp. 113, 952; id. Orn, Works, pp. $381,412,573,643$ (1881); Wardl. R tms. 7 weedd. Orn. Works, 1. 659 (1881); Stcere, List. Philipp. B. p. 24. (1890).

(arpophaga forsterii, port., Cass. Pr. Ac. Nat. Sc. Philad. 1854, p. 231 .

(. (Hemiphaga) poliocephala, Mart. J. f. O. 1867, p. 24.

Hab. Luzón, Laguna, Negros, Dinagat, Panay, Cebú, Samar, Borongan, Masbate, Mindanao, Zamboanga, Basilan.

\section{Gén. MYRIS'TICIVORA, R'HUB.}

\section{M. bicolor, (Joß̣) $\sigma \circ \mathrm{N}$ v. Camsso.}

Pigcon ou Ramier blanc mangeur de muscade de la Nouv. Ciuinere, Sonn. Voy Nouv. Guin. p. 169, pl. 103 (1776).

White Nutmeg Pigcon, Lath Syn. ii. 2, p. 538, n." 31 (1783). Columba bicolor, Scop. Del. Filor. ct Faun. Insubr. ii. p. 94, n. ${ }^{\circ} 97$ (1786).

(.) alba, Gm. S. N. ii. p. 780, n. $^{\circ} 53$ (1788) Lath. Int. Orn. ii. p. 602, n. ${ }^{0} 34$ (1790); Bonn. Enc. Méth. i. p $2_{4} 6$ (i790); Vieill, N. D. xxii p. 362 (г818); Wagl. Syst. Av. Columba, sp. 20 (1827); id. Isis, 1829, p. 741; Less. Cormpl. de Buff. Ois. viii. p. 90 ( 1837 ); id. op. cit. and ed. p. 293 (1838); Harll. Syst. Verz. p. 98 (18+4).

(. littoratis, Temm. \& Kup. Pig. i. pl. 7 (1808-1 r); Temm. Pig. A. Ciallin. pp. 99, 448 (1813); Steph. Gen. Zool. xi. 1, p. 23 (1819); Horsf. Tr. Linn. Soc. xiii. p. 182 (1821); Steph. Gen. Zool. xiv. I, p. 291 (1826); Desm. Dict. Sc. Nat. xl. p. 314, (1826).

Carpophaga myristicivora, G. R. Gr. List Gallina B. M. p. + (1844); id. Gen. B. ii. p. 468, n. $^{\circ} 3$ (1844); Blyth. J. A. S. B. xx. p. 371 (1846); Beav. Ibis, 1867 , pp. 332, 334 .

C. casta, Peale, Un. St. Expl. Exp., Zool. p. 204 (1848); Hartl. Arch. f. Naturg. xviii. I, p. 116 (1852; Bp. Compl. Rend. xliii. p. 836 ( 1856$)$.

C. bicolor, G. R. Gr. Gen. B. App. p. 23 (1849); Blyth, Cat. B. Mus. A. S. B. p. 232, n. ${ }^{\circ} 436$ (1849); Cass. Pr. Ac. Npt. Si Hhilad. 1854, p. 232; G. R. Gr. List. B. Brit. 
Mus. Colnmba, p. 19 (1856); Cass. Un. St. Expl. Exp. and ed. Orn. p. 265, pl. 28 (1858). Sclat, Journ. Pr. Linn. Soc. ii. p. 167, n. ${ }^{\circ} 139\left(185^{8}\right) ; G$. R. Gr. P. Z. S 1858, p. 196; id. Cat. B. New Guin. pp. 47, 61 1859; id. P. Z. S. 1861 , p. 447; Blyth. Mouat's App. p. 362 (1863); Finsch, Nen-Guin. p. 177 (1865); Pelz. Novara Reis., Vög. p. 107 (1865); Wall. Ibis, 1865 , p. 386, n. ${ }^{\circ} 68$; id. J. f. O. 1866 , p. 281; Schleg. N. T. D. iii. pp. 203, 205, 343 (1866); Blyth \& Beav. Ibis, 1867 , p. 332; Ball, J. A. S. B. xxxix. 2, p. 32 (1870); Gieb. Thes. Orn. i. p. $584(1872)$; Ball. Str. Feath. i. p. 79 (1873); Schleg. Mus. P.-B. Columbe, p. 38 (1873); Hume, Nests. \& Eggs, iii. p. 896 (1873); id. Str. Feath. ii. p. 264 (1874); Salvad. Cat. Ucc. Born. p. 292 (1874); Hume; Str. Feath. ii. pp. 80, 84, 96, 103, I 14, I19, 264 (1874). Sharpe, $P * Z$. S. 1875 , p. ro8; Blyth, B. Burm. p. I45 (1875); Koch, Verz. Samml. Vogelbälg. p. 2; Brügs. Abh. Naturw. Ves. Brem. v. p. 85 (1876); Hume, Str. Feath, vi. p. 418 (1878); Kelh. Ibis, p. 527 ; Vorderm. N. T. N. I. xlii. p. 227 ( 832$) ; O a-$ tes, B. Brit. Burm. ii. p. 303 (1883); Sclat. List Vert. An. 8 th ed. p. 443 (1883); Vurderm. N. T. N. I. xliv. p. $23^{6}$ (1886); Büttik. Not. Leyd. Mus. viii. p. 66 (I886); Sharpe, Ibis, 1888 , p. 202; Tristr. Cat. Coll. B. pp. 42, 269 (1889; Vorderm. N. T. N. I. xlix. p. 4I 2 (1889); Everett, Journ. Str. Br. R. As. Soc. 1889 , p. 195; id. P. Z. S. $1889 ;$ p. 225; Whiteh. Ibis, 1890 , p. 56; Sharpe, Ibis, 1890, pp. 135 , 284; Vorderm. N. T. N. I. 1. p. 507 (1890).

Myristicivora littoralis, Rchnb. Av. Syst. Nat. p. xxvi. (1852); Meyer, J. f. O. 1873 , p. 405.

M. bicolor, Bp. Consp. Av. ii. p. 36 (1854); id. Compt. Rend. xxxix. p. ${ }_{1078}$ (1854), xl. p. 217, n. ${ }^{\circ} 98$ (1855); id. Coup. d'ail Ordre Pig. pp. I8, 55. n. ${ }^{\circ} 98$ (1855); Moore, P. Z. S. 1859 , p. 466; Rchnb, Tauben, i. p. I16, t. 229: ff. I 278-79 (1862), ii. p. I82, t. vi. ff. 64-65 (1862); Rosenb. $N . T . N$. I. xxv. p. 249, n. ${ }^{\circ} 228$ (1863); id. J. f. $O$. 1864 , p. 132, n. $^{\circ} 28$; G. R. Gr. Hand-list, ii. p. 229, n. ${ }^{\circ} 9187$ (I 875 ); Gieb. Thes. Orn. i. p. 584 (1872); Wald. Tr. Zool. Soc. viii. p. 84 (1872); Sousa, Mus. Nac. Lisb. Columba, p. IO (1873); Wald. Tr. Zool. Soc. ix. p. 217 (1875); Salvad. Ann. Mus. Civ. Gen. ix. p. 62, p. 202, n. ${ }^{\circ} 54$, p. 270 , f. 3, p. 276 (1876); Sharpe, Tr. Linn. Soc;.(2) Zool. i. pp. 347, 353 (1876); Lenz. J. f. O. 1877 , p. 319; Tweedd. P. Z. S. 1877 , p. 35\%, I878, pp. I 1 3, 952; Saluad. P. Z. S. 1878 , p. 39; id. Ann. Mus. Civ. Gen. xiv. p. 66 I (1879); Meyer, Ibis, 1879, p. I 36 ; Sharpe, P. Z. S. 1881, p. 799; Tzueedd. Voy. Challeng., Birds, p. 23; 1881; Salvad. ibid. p. 71 (1881); Vardl. Rams. Tweedd. Orn. Works, pp. I84, 380, 4 I 2, 460, 462, 474, 573, 643 (188r); Wardl. Rams. Tweadd. Orn. Works, App. 1. 659 (188I); Salvad. Orn. Pap. Mol. iii. p. I07 (1882); Siclat. P. Z. S. 1883 , pp. 51, 195, 200; Kutter, $f$. f. O. 1883 , p. 315; Forbes, P. Z. S. 1884. P. 433; Meyer, Sitzb. u. Abh. Ges. Isis, 1884 . Abh. i. p. 6; Gullem P. Z. S. 1885 , p. 27 I, p. 416 , pp. 558, 575; Blas. Zcitschr. f. d. ges. Orn. 1886, p. 197; Salvad. Anu. Mus. Civ. Gen. (2) iv. p. 559; (1887); B!as. Orn!s, ir. p. 617 (1883); Pels. \& Lorenz. Anth. 
Anin. Niturh. Hofmus. iii. p. 47 (1888); Hein \& Rchuie. Nomencl. Mus. Hein. Orn. p. 291 (1890); Steere, List Philipp. B. p. 24 (I 890); Hartert, Kat. Vogelsamml. p. Igo (I89r); Salvad. Mem. R. As. Sc. Tor. (2) xlii. p. 58; id. Cat. B. M. 21. p. 228 (i 893 ).

Myristivora luctuosa, Bp. Consp. Av. ii. p. 37 (1854); id. Compt. Rend. xxxix, p. 1078 (1854); xl. p. 217, no. 99 (1855); id. (out. d'anl Ordre Pig. pp. 18, 55. no. 99 (1855).

(arpophaga luctuosa, Moll. \& Dilat. Contr. Nat. Hist. of Lab. 1. 5 I (1855); G. R. Gr. List. B. Brit. Mus. Columba, p. 20 (1856); Rosenb. N. T. N. I. xxr. p. 241, no. 224 (1863); $d$. J. F. O. 1864 , p. I32, no. 224 ; Gieb. Thes. Orn. i. p. ${ }^{806}$ (s872); Rosenu. Malay. Archip. p. 220 (I878).

(arpophaga littoralis, Hartl. J. F. O. 1855, p. 319; Finsçh, Neu-Guin. P. 178 (1865); Rosenb. Versl. Eil. Nias, p. 20, id. Malay. Archip. p. 108 ( 878$)$.

Carpophaga littoralis, G. R. Gr. P. Z. S. 1860, p. 361 .

(arpophaga alba, G. R. Gr. P. Z. S. 1860 , p. 36r.

Muscadivora bicolor, Schleg. Dierent. p 2 io (1864).

Myristicirora spilorrhoa, Whitely, in Gurn. Ibis, 1891, p. 275.

'Myristicivora melanura, part., Blas. I'. Z. S. 1882, p. 709; Bernst. Dagboek, pp. I 27, i60 (1883); Nehrk. J. F. O. 1885. p. 34 ; Saliad. Tbis, 1886 , p. I 53 .

Hab. Luzon, Cagayan, Abra, Ilocos, Mindoro, Calapan, Masbate, Negros, Paracrua, P. Princesa, Calamianes, Cuyo, Malanipa, Nipab, Zamboanga, Mindanao, Tawi-tawi; Java.

\section{Fam. COLÚMBIDAS.}

1. Gén. COllimba, Lin.

\section{C. livia, Briss. $0^{0}$ N. v. Palcma doméstica.}

Columba domestica, Gm. S. $N$. i. p. n. ${ }^{\circ}$.

Biset, 'D' Aubent. Pl. Enl. 5 10.

Hab. Cosmopolita.

2. C. griseigularis, Wald $\sigma^{-} \mathrm{O}$ N. v. Balut-muntí.

(M. S. T.)

Carpophaga metallica, pat. Schleg. N.T. D. iii. pp. 202, 203 (i 866). lanthænas griseogularis, Wald. \& Layard, Ibis, 1872 , p. Io4, pl. vi; Sclat. Ibis, 1874 , p. 455; Wald. Tr. Zool. Soc. ix. pp. 218, 25I (1875); Sharpe, Tr. Linn. Soc., Zool. i. p. 353 (1876); Tweedd. P. Z. S. 1878, p. 288, 1879, p. 73; ?Sharpe, P. Z. S. 1879 , p. 316; Tweedd. Orn. Works, pp. II7, I 2 I, 381, 4і2, 594, 651 (1881); Wardl. Rams. Tweedd. Orn.' Works, pp. 659, 666 (I88I); Guilbem. P. Z. S. 1885, p. 27I; Everett. Ibis, 1887,363 ; id. Journ. Str. B. R. As. Soc. 1889, . 1ог 5 ; Stcere, List. Philipp. Birds, p. 24 (1890); Slas. $J$. $f$. O. 1890, p. 145 .

Jantænas luzoniensis, Schleg. Mus. P.-B. Columba, 75 (1873); Sclat. Ibis, 1874 , p. 455 .

Carpophaga griseigularis, Tristr. Cat. Coll. B. p. 42 (1889). 
Columba griseigularis, Salv. Cat. B. M. 21. p. 313 (1893).

Hab. Luzón, Guinarás, Vegrros, Valencia, Basilan, Mindanao, Joló; Borneo.

3. c. cinerea, siop.

Tourterclle cendré de l'isle de Luzon, Sonn. Ioy. Non. Guin. 1. 52 pl. 22 (1776); Wald. Tr. Zool, Soc. 9 p. 220 (1875).

i,uzonian turtle, Lath. Syn. ii. 2. p. 646, var. $\subset(1783)$.

columba cintrea, Scopoli, 1sel. Fl. et Fonun. Insubr. ii. 1 ;66 P. 94 n. 93 .

(: turtur, d. T. Inzoniensis, Gm. S. N. i. 2 1788 p. 786.

Tourterelle: de l'Isle de Luçon, Bonn. Tabl. Enc. Orin. p. 248 , pl. 8 i f. 4 17.90.

( . turtur, d. Lath. Ind. Orn. ii. p. 6006 (1790); Steph. Gen. Zool. 11, p. 73,1819 .

( . turtur, var. d. Tem. Pig. et Gall. i. p. 479 (18г3).

'Tótiterelle grise de l'isle de Luçon, Vieill. N. D) 26, p. 381 , $(18,8)$.

(C turtur, var. d. Wagl. Syst. Av. Columba sp. dubia $9(1827)$.

(.) phrnicorhyncha, Wagl. Ibis, 1829, p. 745 (1829).

T. cincrea, part. Bp. Consp. Av. ii. p. 61 (1854); Wall. Ibis, 1865 , p. 392 ; id. J. F. O. 1866, p. 213.

T. cinereus, Rechnb. Tauben, 1, p. 70 part. 1862, ii. p. 17t; Novit. Suppl. t. iii. f. 25 (1862); v. Mart. J. F. O. 1850 , p. 24 ; G. R. Gr. Hand-l. ii. p. 238, n." 9312 part. (1 870$)$; (iieb. Thes. Orn. iii. 1. 726 (1877); Sali' Cat. B3. 11. 21, p. 6zi (1893).

liab. ¿Luzon.

\section{Fam. MACROPÍGIDAS.}

I. Gién. IURACANA, Bp.

I. T. modesta? $0^{\top}: 0$ N. v. Imamatat.

(I) S. T.)

Columba modesta, Tem. Pl. Coll. 552, (1835).

Turacana modesta, Bp. Consp. Av. ii. p. 59, (1854); Salv. Cat. 13. M. 21. p. 334. (1893).

Hab. Paragua.

2. Gén. MACROPYGia, Sw.

2. M. tenuirostris, Gr. $0 \%$ N. v. Batig-lauin

(II. S. T.)

Columbá phasianella, Tem. Pl. Coll. 100; Steph. Gen. Zool. xiv. (1826); Desm. Dict. Sc. Nat. xl. p. 357 (1826); Wagl. Syst. Av. Columba, sp. 36 (s827); Less. Fi. dorn. p. 473 ( 1831 ); Knip \& Prev. fig. ii. pl. 52 (1843).

Macropygia phasianella, Sw. Class. B. ii. p. $349(1837)$.

Turtur amboinensis, part., Less. Compl. de Buff., Ois. viii. p. 67 (1837); id. op. cit. and. ed. p. $287\left(183^{8}\right)$.

Macropygia amboinens's, part., G. R. Gr. List. Gen. B. P. $3^{8}$ 
(i 840$)$; id. Gen. B. ii. 1. ti1, n.” I (184t); Rchnh. Syn. Aí. f. $1394(18+7)$.

M. tenuirostris, G. R. Cir. MSS.; Bp. Consp. Ã. ii. p. 57 (1854); id. Compt. Rend. xxxix. p. 111 (185t), xl. P. 219, n." inI (1855); id. Coup. d'ail. Ordt. lig. Hu. 27,57, n. 161 (1855'; (i. R. Gr. List. B. Brit. Mils. Columber, p. 39 (1856); Rchnth. Tauben, i. p. 87, t. 251. f. $139+(1802)$; II all. Ib:s, 180.5. p. $390 ;$ id. J. .. O. 1866 , p. 282, M.r. J. f. O. 1866, p. $24 ;$ G. "R. Gr. Haug-list, ii. p. 237, n." $920+13870)$; Wald. Ihis, 1873 , p. 315 ; Schleg. Mus. P.-B. Columbia, P. $106(1873$; Sousa, Mus. Vace Lisb. Columbe, p. 15 (1873); Gieb. Thes. Orn. ii. p. $518(1875)$; Wrild. Tr. Zool. Soc. ix. p. pp. 2 s', +12 (1875; Sharpe. T). Linn. Soc., zat. ser. Zool. i. pl. 347, 253 (1876); 7reedd. P. Z. S. 1879, p. 288; Siarfe, P. Z. S. 1879, ․ 317; Treeitd. Orn. Works, pp. 247, 382, +1259+ (1881); Wrat. Rams. Tiveedt. Orn. Works, App. p. 659 1881; Salì. ('at. B. Strickl. Coll. p. 546 (1882; Ciuillem. P. Z.S. 1885, p. 27 ; Sharpe, lbis, 1888, p. 203; Blass. Ornis, 1888 , p. 317; Tritr. Cat. Coll. B. p. 39 (1889); Everett, Journ. Str. Br. R. As. Soc. 1889 , p. 193; Whiteh. I6s, 1890, p. 57; Wardl. Rams. Ibis, 1890, p. 221; Steere, List. p. 24; PHar.ert, Kat. Vogels. p. 185 (1891); Salv. Cat. 13. M. 21. ए. 346 (1893).

M. eurycerea, Tweedd. P. Z. S. 1878, p. 288, p. 952, 1879, p. 73 ; id. Orn. Works, pp. 586, 594, 643, 651 (1881'; Ward!. Rams. Tweedd. Orn. Works, App. pp. 659, 667 (1881); Steere, List. p. 24 ( 1890$)$.

Hab. Luzón, Manila, Cagayan, Mincloro, Baco, Negros, Valencia, Paniqui, P. Princesa, Calamimes, Culion, Samar, Mindoro, Bacó, Mindanao, Zamboanga, Basilan, Joló, 'Tawi-tawi.

\section{Fam. PERISTÉRIDAS.}

\section{Subfam. TURTURINAS.}

I Gén. Homoplelia, SaLv.

I. H. rostrata, (Bp.) $\mathrm{N}$ v.

(M. S. 'T.)

Columba picturata, var. Prév. fig ii. p 66 pl. 3.5 (1838-43).

Turtur rostratus, $B p$. Consp. Av. ii. I' 62 (185t); id. Rend. xl.

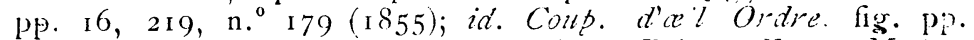
30, 57, n. ${ }^{\circ}$ I79 (1855); Hartert. Om. Beiter. Faun. Madaf. p. 67 (186r); Rchnb. Tauben, i. p. it (1862); E. Vequt. P. Z. S. $1867, \mathrm{p} 344$; id. Ibis, 1867 , pp. 336, 337, 354, f. wing and Load, pp. 35.5, 359; A. Vez Ib 5,1859 , p. 47i; G. R. Gr. Hand-list, ii. p. 238, n. ${ }^{\circ} 9320$ (1870); Schleg. Mus. P.-B. Columber, p. I 30 (1873); Oust. Bull. Soc. Philom. 1871 , p. I80; Shell. Ibis, 1883, p. 320 ; Tristr. Cat. Coll. B. p. 38 (1889); Salv. Cat. B. H. 21. 1893. p. 413.

T. picturatus, part., Schleg. P. Z. S. 1866 , p. 424; Schlig. Thes. Orn. iii. p. 727 (1877), \& Poll. Rech. Faun. Madag. Ols. p. I I 3 (1868); r. Gieb. Thes. Orn. iii. p. 727, (parie) (1877). Hab. Marianas, Seychelles. 


\section{H. picturata, (Tim)}

Columba picturata, Temm. Pig. et Gallin. i. pp. 315, 480 (181,3); Vieill. N. D. xxri. p. 357 (1818); Steph. Gen. Zool. xi. ". 85 (1819); Vieill. Enc. Meth. i. p. 383 (1823); Temm. Pl. Col. 242 (livr. 41, 1823); Steph. Gen. Zool. xiv. p. 280 (1826); Desm. Dict. Sc. Nat. xl. p. 352 (1826); Vagl. Syst. Av. (i) lumba, sp. 61 (1827); Less. Compl. de Buff., Ois. viii. p. 5n (1837); id. op. cit. and ed. p. 282 (1838); Knip. \& Prev. P. ii. pl. $35(1838-43))$ G. R. Gr. Gen. B. ii. p. 470, n." (1 $8+4)$; Rchnb. Syn. Av. f. I 260 (1 847$)$.

Columba dufresnii, Leach, fide Steph. Gen. Zool. xi. p. i7, pl. 3 ( 18 í).

Peristera picturata, Boie, Isis, 1828, p. $32 \%$.

Columba picta, part., Less. Tr. a' Orn. p. 473 1831).

'Turtur versicolor, Kitt. (alec Columba versicolor, Kittl.j fide Rchinis. Syn. Av. f. 2 (1847), et Bp. Consp. Av. ii. p. 6r (1854).

T. picturatus, Blyth, Cat. B. Mus. A. S. B. p. 236, n. ${ }^{\circ} 4.33$ (1849); Bp. Consp. Av. ii. p. 6I (1854); id. Compt. Rend.

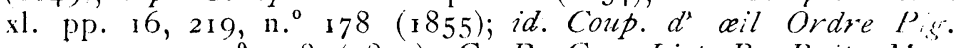
pp. 3o, 57, n. ${ }^{\circ}$ г 78 (1855); G. R. Gr. List B. Brit. Mus. Columber, p. 42 (1856); Hartl.J.f. O. 1860 , p. $16 \mathrm{r}$; id. Orit. Beitr. Faun. Madag. p. 66 (1861); Rchnb. Tauben, i. p. jo, t. 224. f. г 260 (1862); Maill. Net. sur l' ile Réun. p. i6,3; (1862; Sclat. P. Z. S. 1863 , p. 164; Roch \& E. Ncwt. Ibis, 1863 , p. I67; E. Newt. Ibis, 1863 , p. 454; Pallen, N. T. D. i. p. 319 (1863; Coquerel, Bull. Soc. Acclim. Réun. 1. 19 (1864); Schleg. P. Z. S. 1866 , p. 424; E. Newt. Ió: $1867, \mathrm{pP} .346,354,355$ 359; Grand. Rev. et. Mag. de Zoo! 1867, p. 418 ; Schleg. \& Poll. Rech. Fann. Madag. Ois. p. 113 (part.) (1868); A. Newt. Ibis, 1868 , p. 477; G. R. Gr. Hanctlist, ii. p. 238, n. 9314 (1870); Sclat. P. Z. S. 1871, p. h93; Schleg. Mus. P-B. Columba, p. 129 (1873); ? Miln-Edii. Ann. Sc. Nat. (5) xix. p. 14, pl. xii. f. 2. (1874); Baril. P. Z. S. 2875 , p. 67; Hartl Vög. Madag. p. 268 (1877); Ghieb. Thes. Orn. iii. p. 727 (part.) (1877); Oust. Bull. Soc. Philoin. 1878 , p. 178; Shell. Ibis, 1883, P 317 ; Sclat. List. Vert. An. 8th ed. p. $45^{2}$ (1883); Milne-Eaw. \& Grand. Hist. Nat. Madag. xii. Ois p. 460, pls. 185, 186, 187 (1885); Tristr. Cat. Cobl. B. p. $3^{8}$ (1889); Heine \& Rchuw. Nomencl. Mus. Hein. Orn. p. 77 (1890); Sibree, Ibis, 1891, p. 564; Salv. Cat. B. M. 21, 1893, p. 409 .

'Turtur prevostianns, Bp. Consp. Av. ii. p. 62 (1854) (-C. picta, Mus. Paris, Qucy \& Gaimard); id. Compt. Reud. xl. pp. 16, 259, n. $^{\circ} 80$ (1855); id Coup. d'ceil Ordre Pig. pp. 30, 57 n. ${ }^{\circ} 80(1855 ; G . R$. Gr. List B. Trop. Isl. p. 43 (1859); Hart. Orn. Beitr. Faun. Madag. p. 67 (186I); Rchnb. Tauten, i. p. 7 I (1862); G. R. Gir. Hand-list, ii. p. 238, n. ${ }^{\circ} 9361$ (1870); Gieb. Thes. Orn. iii. p. 227 (1877); Oust. Bull. Sicc. Philom. 1878 , p. 179; Wigleszi. Aics Polynes. p. 54 (18911). 1. pictus, Mus. Paris, fide Oust. Bull. Soc. Philom. 1878 p. 179.

'1. picturatus, var., Tristr. Cat. Coll. B. p. 38 (1889). hab. Marianais; Mauricio, Madagascar. 
2 Gén. STREPTOPELIA, BP.

3. St dussumieri. (Tcm) of.0 N. w. Batobatong de collar. (M.S T.)

Cinlumba dussumieri, Tem. Pl. Col. 1 \&\& (18z3; Steph. Gen. Zool. xiv. p. 285 (1826,; Desm. Dict. Si. Vat. xl. p. 348 (1826); Wargl. Sist. Ai. Columba, sp. 991827 ; Less. Tr. dOOrn. p. 473 (1831); id. Compl. de Buff. Ois. viii. P. 54 (1837); id. op. cit. 2n!l. ed. p. 283 (1 838$)$; Prév. \& Kinp, Pig. ii. pl. 20 ( $1838-43)$.

Colombe clussumier, Quoy \& Gam. Vog. Wran., Zool. i. p. 3.5 $(182+)$.

Piristera dussumieri, Boie, Isis. 1828, p. 327.

'lurtur dussumieri, G. R. Gi. Gen. B. ii. p. 472, n." I 2 (18+4); Rchnb. Syn. Av. f. 1360 (18+7); (i. R. Gr. List. B. Br.t. Mus. Columbar, p. $45(1856)$; Rchnb. Tauben. i. p. $7 \mathrm{I}$, t. 246. f. I 360 (1862); Wall. Ibis, 186.5 , p. 392; id. J. F. O. 1866 , p. 283: G. R. Gr. Hand-list, ii p. 239, n. ${ }^{\circ} 9331$ 187u); Schleg. Mus. P.-B. Columbe p. 120 (1873); Wald. Trans. Zoo!. Soc. ix. pp. 218, 251 (1875). Sharp?, Trans, Linn. Soc. (2) i. pp. $347,353(1876)$; Gieb. Thes. Orn. iii. p. $726(1877$; Trieed. P. Z. S. 1877, pl. 700, $765,833,1878$, p. 288, 1. 354, p. 711, p. 952; Tweedd. Orn. Works. Pp. 382, 383, 412, $525,538,559,2594,600,623,628,6+3$ (1881); WFardl. Rams. Treedd. Orn. Work: p. 659 (1881). Sharpe P. Z. S. 1881, p. 799; Guillem. P. Z. S. 1885 , p. 22, Lilas. Ibis, $188 \%$ ", p. 374; id. Ornis. 1888 , p. 317 ; Ewrelt, Jomrn. Str. Br. K. As. Soc. 1889 , p. 193, Tristr. (at.. Coll. B. 1). 38 (1889); Whiteh. Ibis, 1890 , p. 16 ; Stecre, List. p. 24 (1890); Hartert. Kat. Vogelsamml. p. I 8 (1891); id. J. f. O. 1891, p. 300 Wiglesw. Ares Polyn. p. 54 (1891); Sali. Cat. B. M. 21, p. $4 \mathbf{2}_{3}$ (1893).

Streptopelia dussumieri, Bp. Consp. As. ii. p. 65 (1854); id. Compt. Rend. xl. pp. i 8, 2 го, n. ${ }^{\circ}$ I9 $($ i 855$)$ 1d. Coup. a'ail Ordr. Plg. pp. 31, 57, n. ${ }^{\circ}$ 191 (1855); G. R. Gr. Hand-list, ii. p. 269, n. ${ }^{\circ} 933$ I (1870); Sousa, Mus, Nac. Lisb. Columba, p. I7 (1873).

S. gaimardi, Bp Consp. Av. ii. p. 66. (185t; id. Conspt. Rend. xl. pp. 18, 2 I9, n. ${ }^{\circ}$ I92 (1855; id. Coup. d'ail Ordre Prg. pp. 31, 57, n. 192 (1855); Rchnb. Taub?n, i. p. 76 (1862); G. R. Gr. Hand-list, ii. p. 239, n. 9333 (1870).

Turtur gai, ardi, G. R. Gr. List. B. Trop. Isl. p. 43 (1859).

'T. dussumieri, v. Mart. J. F. O. 1811 , p. 24.

T gaimardi, Wald. Trans. Zool. Soc. ix. p. 219 (1875); Tzueedd. Orn. Works, p. 219 (188I).

Hab. Luzon, Manila, Montalban, S. Mateo, Laguna, 'Tayabas, Batangas, Bulacan, Cagayan, Ilocos, Mindoro, Marinduque, Bobol, Romblon, Tablas, Sibuyan, Calamianes, Negros, Siquijor, Masbate, Cebú, Panay, Guimarás, Samar, Leyte, Amparo, Dımaguete, Valencia, Mindanao, Surigao, Basilan, 'Tawi-taẉi, Buruan, Joló, Marianas. 
4 St. humilis, $(T e m$.$) t \$$ N. v. Batobito

(II. S T.)

Columba humilis, (Tem. Pl. Col. $2.59(8) \mathrm{m}$. lam. $259 \mathrm{~g} \mathrm{Q}_{24}$; Steph. Gen. Zool. xiv. p. 280 (1826); Desm. Dict. Sc. Nat. xl. 1. 350 (1826); Walg. Syst Av. Columba, sp. 95 (1827); Knip. \& Prei. Pig. ii. pl. (1838-43); Homey, J. F. O. 1370, р. г70.

Peristera humilis, Boie, Is is, 1828, p. 327.

Turtur humilis, $G$. R. Gr. (Fen. B. ii. p. $472,10.7$ (1 I 44 ); Rcnb. Syn. As. f. I $370(\mathrm{I} 8+7)$; Swinh. Ibs, $1850, \mathrm{p} .63$. 1861 , p. 49,1862 p. 261,1863, p. 397; al. P.Z. S. 1963, p. 306; Bly'h. Monat's App. p. 362 (1863); Jerd. B. of. Lill. iii. p. 482 (1864); Swlinh. Ibis, 1855 , p). $355,54 \mathrm{I}, 542$; Wal!. Ihis, 1865 , pp. 392, 399; id. I. f. O. 1860 , p. 283 ; Blth, Ibis, 1867, p. i5 ; ? Beav. Ibis, 1867, p. 332; Slat. P.Z. S. 1809 , p. 627; Swinh. Ibis; 1870 , pl. 80, 356, id. Ibs, $1871, \mathrm{p} .397$; Schleg. Mus. P.-B. Columba, p. I $25(1873)$; Hume, Str. Feath. ii. p. 269 (1874); Swinh. Ibis, 1875 , p. 125; Hume, Vests and Eggs, p. 507 (1875); Waid. Trans. Zool. Soc. ix. p. 219 (1875) id. in. Bly'h's B. Burm. p. I45 (1875); OHame, Str Featí. iii. p. 165 (1875); iv. p. 292 (1876); Armsir. \& Hune, Sti. Feath. iv. p. $33^{8}$ ( 876$)$; Tarz Bull. Soc. Zool. Fr. i. p. 2t (1876); Sharpe, Tr. Linn. Soc. (2) Zool. i. pl). 347, 353 ( 1876); Gieb. Thes. Orn. iii. p. $272(1877)$; 1)avd. \& Oust. Os. Chine, p. 388 (1877); Hume \& Dav:ds. Str. Feath. ix. p. +23 (1878; Hume. Str. Fealh. viii. p. 2 10 (ritg9; Rringh Sti. Feath. ix. p. 194 (188s); Trueedd. Orn. Works, p. 383 i 838 ;); Wardl. Rams. ibid. p. 659 (188r'; Tacs. J. F. O. 1381 , p. 187 ; Oates, str. Feath. x. p. 235 ( 1882$)$; id. B.rds. of. Birt. Burm. ii. p. $294\left({ }_{188}\right)$; Blakist. Ament. I,st. B. Jab. p. +3 (1884); Seebh. 'Ibis, 1884 , p. I79; Marsh. Ibis, 1884, I'. +21; Stejn. Pr. U. S Nat. Mus. 1887 , p. +28 ; Hune, Sir. Feath. xi. p. 229 (1888); Tristr. Cat. Coll. B. p. 38 (1889); Salvad. Ann. Mus. Civ. Gen. (2) vii. p. 42t (is8(1); Stere, List. p. 24 ( 890$)$; Hartl. Abh. Nart. Ver. Brem. x. p. 406; Seebh. B. Jap. Einp. p. ${ }_{1} 62$ (г 890$) ;$ Styan, Ib s, 1891 , pi). 328, 489; Hartert, Kat. Vogd'samml. p 188 ( $189 \mathrm{I}$ ); id. J. $\mathrm{f}$. O. 1891 , p. 300; Salv. Cat. B. M. 21. p. 43+ (1893).

Columba (?) terrestris, Temm. fide Rchnb. Sym. As. Columburia, p. $3(1847)$.

Streptopelia humilis, Bp. Consp. Ar. ii. p. 66 (1854); id. Compt. Rend. xl. pp. I8, 219 , n. ${ }^{\circ} 193$ (1855); id. (Ous. d'ce!l Ortip Pig. pp. 32, 57, n. ${ }^{\circ} 93$ (1855); id. (Oompt. Rend. xli. p. 65) (1855); Rchnb. Taubent, i. p. 76, t. 247. f. 1370. (1862); (i). R. Gr. Fiand-list. ii. p. 239, n. ${ }^{\circ} 9331$ (187o).

Turtur terrestris, Mercatorum, fide Bp. Conspt. Rend. xl. p. is ( 855$)$; id. Coup. d'ail Ordre Pig. p. 32 (1855).

Columba humilis, Schrenck, Vög. d. Amur-L. p. $28+$ (1860).

Turtur humilis, v. Mart. J. F. O. $1860^{\circ}$, ए. 24.

T. humilior, Hume, Proc. As. Soc. Beng. $1874, \mathrm{p} 24 \mathrm{I}$; id. S6r. Feath. iii. pp. 279, 280 (1875).

T. tranquebaricus, Blyth. B. Burm. p. I 45 (1875).

T. sp., Blakist. \& Pryer, Tr. As. Soc. Jap. x. p. I 29 (1882). Hab. Luzón, Manila, Bulacan, Pampanga, Bataan, Cagayan, Mindoro, Marinduque, Samar; Cochinchina, Formosa. 
3. Gèn. SPII OPLIIA, Sevinev.

5. Sp. chinensis. (Siof.) $\sigma 0 \curvearrowright$ v.

(M. S. T.)

'Tourtelle grise de la Chine, Somn. loy'. Ind. ii. p. 172, pl. w $\left(1 ; x_{2}\right)$.

Chinese grey 'Turtle, lath. Syn. ii. 2, p. 6+9, n." $+2(1 ; 83)$.

Columba chinensis, Scop. Del. Flor. ef Faun. Insubr. P. 94, n." go ( 1786$)$.

C. risoria, i'a). B. (im. S. N. i. p. 787 (1788); Lath. Ind. Orn. ii. p. 608 (1790).

C. tigrina, part., Temm. Pig. et Gallin. i. pp. 317, $4^{81}(1818)$; Steph. Gen. Zosl. xi. p. 78 (1819); Desm. Diet. Sc. Nat. xl. p. 3+7 (1826); Wagl. Syst. A2. Columba, sp. 96 (1827); Less. Compl. de Buff., O.s. viii. p. 5 I (1837); id. op. cil. 2nd ed. p. $2 \mathrm{I} 3$ (I 838$)$.

'Iurtur chinensis, G. R. Gr. Gen. B. ii. p. 472, n. ${ }^{\circ} 9(1844)$; Blyth, J. A. S. B. xir. p. $875(\mathrm{r} 8+5)$; id. Ann. \& Mag. N. H. xix. p. $185($ I $8+7)$; id. Cat. B. Mus. A. S. B. p. 23', n. ${ }^{\circ} 8_{4+}$ (1849); Bp. Consp. A\%. ii. 63 (1854); id. Compt. Ren?. xl. p. I7, p. 19, n. ${ }^{0} 183(1855)$; id. Coup. d'acil Ordre Pig. pp. 30, 57, n. ${ }^{\circ}$ I83 (1855); Blyth. I. A. S. B. xviv. p. 263, p. 480 (1855); Cass. Vurr. Perry's Exped. ii. p. 244 (1856: Sivinh. Ibis, 1860, p. 62, 1861, p. 49, 1862, p. 269; Rchnb; Tauben, i. p. 72 (1862); ii. p. 87t, Novit. Suppl. t. iii. f. 26 (1862); Sreinh. P.Z. S. 1863, p. 306; id. Ibis; 1863, p. 397 . 1865 , p. 54c; Wall. Ibis, 1865 , p. 391; id. J. f. O. 1860 , p. 283 ; Sicinh. Ib s, 1866 , p. 292, 1867, p. 396, 187o, p. 356; G. R. Gr. Hand-list ii. p. 238, n." 9386 (1870); Szvinh. P. $Z$. S. $188^{3} 1$, P. 397; Schlcg. Mus. P.-B. Columber, p. I27 (1873); Garr. P. Z. S. 1874, p. 256 ; D.roid \& Oust. Ois. Chine, p. $386 ;(1877)$; Gieb. Thes. Orn. iii. p. 276 (1877); Finsch, Ibis, 1880 , 1. 78 ; Kelh, Ibis, 1881 , p. 52i; Sclater, Ibis, 1882 , 1. 435; Müll. J. f. O. 1882 , 1. +32; Sclat. List Virt. An. s'th ed. p. 453 (1883); Seebh. Ibis, 1884 , p. 266; Tristr. Cat. Coll. B. p. 38 (1889); Hartl. Abh. nat. Ver. Brem. xi. P. 406 (1889); Styam, Ibis, 1891 , pp. 328, 498; Salv. Cat. B. M. 21 P. 439 (1893).

Turtur tigrinus, Hartl. Syst. Verz. p. 99 (1844).

T. pinensis, Blyth, J. A. S. B. xxiv. p. 480 (1885).

Spilopelia chinensis, Sundev. Meth. nat. Av. disp. p. Ior (г872). Hab. Luzón, Ilocos, Cagayan; Forinosa.

б. Sp. tigrina (Tem.) $\sigma 0$ Tancurur.

$\left(\begin{array}{lll}M & \text { S. T }\end{array}\right)$

Columba tigrinı, Tem. et Knip, Pig i. pl. 43 (1809-8r); Teinm. P'g. et Gallin. i. pџ). 387,48 I (1813); Vieil. $N$. D. xxvi. p. 380 (1818); Steph. Gen. Zool. xi. p. 78 (1819); Horsf. Trans. linn. Soc. xiii. p. 183 (1821); Desm. Dict. Sc. Nat. xl. p. 347 (1826); Wagl. Syst. Av. Columba, sp. 96 (1827); Less. Tr. dOrn. p. 473 (183r); id. Compl. de Buff., Ois. viii. p. 51 (1837); ad. op. cit. and ed. p. 283 ( I 838$) ;$ S. Müll. Verh. Lind-en lolkenk. p. 109 (1839-44; Bchleg. Hand-!. Dierk. i. p. 404 
(1857); Rosenb. Reist. naar Geslvinkb. p. 8 (1875); Gulliv. P. Z. S. 1875 , p. 489 .

(i. turtur, var., Raff. Tr. Linn. Soc. xiii. p. 388 (1822).

Peristera suratensis, Boie, Isis, 1828 , p. 327.

Columbina inornata, G. R. Gr. List Gallina B. M. p. $13(18+t)$.

'Turtur chinensis, G. R. Gr. B. ii. p. 472 , n." 9 $(18+4)$; Rchnb. Syn. Av. ff. 136162) 1847); Bp. Conip. Av. ii. p 63 (1854); id. Compt. Rend xl. pp. 17, 219, n. ${ }^{\circ} 8_{3}$ (1855); id. Coup. d'ail Ordre P'ig. pp. 30, 57, n. ${ }^{\circ} 83$ (1855); G. R. Gr. List. B. Brit. Mus. Columb.e, p. 42 (1856); Wall Ibis, 1800 , p. 147; Pelz. Novar. Reis., Vog. p. 108 (1865); G. R. Gr. Handlist, ii. p. 238, n. ${ }^{\circ} 9316$ (1870); Sousa, Mus. Nac. Lisb. Columber, p. 16 ( 1873 ).

(columba chinensis, Thienem, Fortpfl. p. 59, t. xi. f. 6 (18 +6).

'Turtur suratensis, Blyth, J. A. S. B. X1. p. $37^{2}(\mathrm{1} 8+6)$; id. Aln. \& Mag. N. H. xix. p. $18+(18+7)$; Cionld, P. Z. S. $188 \%$, pl. 150, I51; G. R. Gr. P.Z. S. 1860, p. 361 ; Schomb. Ibis, 1864, p. 252 .

'T. tigrinus, Blyth, J. A. S. B. xxiv. pp. 263, 410 (1855); Moore, P. Z. S. 1859 , p. 467 ; Rchnb. Tauben. i. p. 72, t. 246 . if. $1861-62$ (1862), ii. p. 174 (1862); Sclat. P. Z. S. 183 3, 1. $22 \mathrm{I}$; Blyth, Moulat's Sspp. p. 362 (1863); Wall. J. f. O. 1865 , p. 283; Swinh. Ibis, 1866, p. 292; Blyth; Ibis, 185 ;, 1. 150; Beav. Ibis, 1868 , p. 376; Blanf. Ibis, 1870 , p. 469 ; Blyth, J. A. S. B. xxxix. pt. 2, p. 332 (i 870$)$; Ball. Str. Feath. i. p. 80 (1872); Schleg. Mus. P.-B. Columba, p. 127 (1873); Blyth. \& Wald. B. of. Burm. p. $1+5$ (1875); Brïggem. Abh. naturw. Ver. Brem. v. p. 87 (1876); Pelz. Verh. s.h. Ges. Wien, 1876 , p. 720 ; Gieb. Tues. Orn. iii. p. 728 (1877; Oates, Str. Feath. v p. I6+ (1877); Anders Jurn. Exped. 1). 665 (1878); Hume, \& Davis Str. Feath. vi. p. 422 (1878); Hume, Str. Feath. viii. p. 110 (1879); Rosenb. Malay. Arohit. p. to7 (1879); Bingh. Str. Feath. ix. p. 194 (1880); Keth. Ibis, 1881 , p. 528; Bock, Ibis, 1882 , p. 475; Mïll. J.f. O. 1882 , pp. 431, 432; Salv. Cat. B. Strickl. Coll. p. 545 (1882); Oates, Str. Featk. x. p. 235 (1882); id. B. Burm. ii. p. 290 (1883); Snellem. Sum.-Exp., Vogels, p. 48 (1884); Vorderm. $N . T . N . J$ xliv. p. 228 (1844), xlv. p. 404 (1885); Müll.J. f. O. 1885 . p. 160; Hume, Str. Feath. xi. p. 299 (1888); Tristr. Cat. Coll. B. p. 39 (1889); Hartirt, J. f. O. 1889, p. 376; Salv. Cat. B. M. 11. p. 442 (1893).

Metriopelia in rnata, $B p$. Consp $A r^{2}$. ii. p. 76 (1854); id. 'Compt. Rend. xl. pp. 24, 220, n. $^{\circ} 25$ (1855); id. Coup d'ail Ordre Pig. pp. 40, 58, n. ${ }^{0} 225$ (1855); Burm. Syst. Ueb. Tr. Bras., Vög. ii. p. 301 $(1856)$.

Melopelia inornata, Bp. Compt. Rend. xliii. Pp. 945, 949 (1850). Peristera inornata, G. R. Gr. List B. Brit. Mus. Columba, 1). 53 (1856); Pelz. Orn. Bras. pp. $\$ 278,451$ (1856).

Metropelia inornata, Rchnb. Tauben, i. p. I8 (1862);G. R. (ir. Hand-list, ii. p. $242, \mathrm{n} .^{\circ} 9371 \cdot(1870)$.

lurtur tigrina, Wall. P. Z. S. 1863 , p. 486; id. Ibis, 186.5 . p. 391; Finsch. Neu-Guin. p. 178 (1865!; Wald. Tr. Zoo!. Soc. vii. p. 85 (1872); id. Ibis," 1872 p. 391; Hume, Str. Feath. i. p. $461(1873)$; id. Nests \& Eggs, p. $506(1873)$; id. 
Str. Feath. ii. pp. 269, 481 (1874), iii. pp. It, 164 (1875); Armstr. Str. Feath. iv. p. 337 (1876); Hitme, Str. Feath. vi. p. 424 (1876); Anders. Junn. Exped. p. 665 (1878); Meyer, Ibis, 1879 , p. 137 ; liraud. Bull. Com. Agr. et Ind. Cochinch. (3) i. p. 142, n. 255 (1879); Sharpe, Ibis, 1888, 1. 211.3; Whiteh. Ibis, 1890, p. 57 .

?'I. suratensis, Ibis, 1864 , p. 252; Swinh. Ibis, 1866, p. 24y Spilopelia tigrina, Sundev. Meth. nat. Av. disp Tent. p. I(x) (1872); Salvad. Cat. Ucc. Born. p. 296 (1874); id. Ann. Mfirs. (iv. Gen. vii. p. 673 (1875), ix. p. 205, n. 70 (1876); Tzeeedit. Ibis, 1877. p. 322; Salvad. Ann. Mus. Civ. Gen. xiv. p. $2+7$ (1879); Sharpe, P. Z. S. 1881, p. 799; Meyer, Verh. z.-6. Ges. Wien, 1881, pp. 706, 769, 772; Salvad. Orn. Pap. c. Mol. iii. p I51 (1882); Blas J. f. O. $188^{\prime} 3$, p. I 39; Mever. Zeitschr. f. ges. Orn. i. pp. I15, 215 (1884); Guillem. P. Z. S. 1885 , p. 5 Io, p. 558; Büttik. Not. Leyd. Mtus. ix. p. 76 (1886); Salvad. Ann. Mus. Civ. Gen. (2) ix. p. 490 (1890); Vorderm. N. T. N. I. xlix. p. 413 (1889), l. p. 508 (1890); Salvad. Mem. R. Ac. Sc. Tor. (2) xlii. p. 63 (1891); V'orderm. N. T. N. I. li. pp. 401, 402 (1802).

(hamæelia inornata, Gieb. Thes. Orn. i. p. 634 (1872).

'Turtur striatus, Schleg. Mus. P.-B. Columba, p. I 27 (1873).

Hab. Paragua, Puerto Princesa, Calamianes, Cuyo; Ć́lebes, Molucas.

Subfam. GEOPELINAS.

4 Gén. GeOpelia, Sw.

7. G. striata, (Lin.) $\&$ N. v. Bato-batong camumu.

(M. S. 'T.)

Transverse striped or barred dove, Edw. Bird., i. pl. i6 (I750).

Dove from China, Alb. Orn. iii. p. 43, t. 46.

Turtur sinensis striatus, Bris.. Orn. i. p. ro7 (r760).

'. indicus striatus, Bris. Orn. i. p. 109 (i 760 ).

Columba striata, Linn. S. N. i p. 282, n. ${ }^{\circ} 8$ (1766); Jacq. Beitr. p. 34 n." 28 , t. 15 (1784); Gm. S. $N$. i. 2, p. 775, n. ${ }^{\circ}{ }_{18}$ (1788); Lath. Ind. Orn. ii. p. 608, n. 53 (1790); Bonn. Enc. Méth. p. 243, pl. 74 f. 2 (1790); Vieill. N. D. xxvi. p. $3^{8}+$ (1818); Wagl. Syst. Av. Columba, sp. 106 (1837); Thiehem. Fortpfl. p. 6o, t. xi. f. ro (1846); Schleg. Hand-l. Dierk. i. p. $404(1857)$.

Columba sinica, Linn. S. N. i. p. $284,{ }^{\circ} 28$ (1760); Gm. S. N. i. p. 78.3, n. $^{\circ} 8$ (г788); Lath. Ind. Orn. ii. p. 6o8, n. 52 (1790); Tem. Pig. et Gallin. i. pp. 373, 472 (1813); Vieill. N. D. xxvi.p. 384 (1818); Steph. Gen. Zool. xi. xi. p. 68 (1819); Desm. Dict. Sc. Nat. xl. p. 377 (1826).

Tourterelle rayée des Indes, Buff. Hist. Nat. Ois. ii. p. 557 ( 177 I )

T. rayèe de la Chine, Buff. op. cit.

Petite tourterelle de Quéda, Sonn. Voy. aux Ind. et á la China, ii. p. 177 (1776). 
Striated twetle, Lath, Syn. ii. 2, p. 650, n." +3 (1783).

Bawed turtle, Lath. op. cit. n." 44 .

Malaca turtle, Lath. op. cit. p. 66ı, n." 60.

Columba malaccensis, Gm. $S$ N. ii. p. 788 , n. 68 (1788); Lath. Ind. Orn. ii. p. 612, n. ${ }^{\circ} 69$ (1790); Bonn. Enc. Mèth. p. 523 ( 790 ); Temm. \& Knip. Pig. i. pl. 47 (1803-11); Temm. Tem. Pig. et Girllin. i. pp. 339, 4st (1873i; Vieill. $N$. D. xxvi. p. $313(1818)$; Steph. Gen. Zool. xi. 1, p. 88 (1819); Dict. Sc. Nat. xl. p $353($ 1826); S. Mäll. Verh. L.xnd-en Volkenk. p. 1o9 ( $\left.8_{39} 44\right)$; Schleg. Handl. Dierk. i. 1). 404 (1857).

(olumba bantanensis, Sparrm. Mus. Carls. fasc. iii. t. 57 (i788); Lath. Int. Orn. ii. p. 6r5, n." 77 (1790); Bonn. Enc. Méth. p. 256 (1790); Vieill. N. D). xxvi. p. 375 (1818); Horst. Trans. Zool. Suc. xiii. p. I83 (1821); Raffl. Trans. Zool. Soc. xiii. 2, p. 319 (1822); Less. Compl. de Buff., Ois. viii. p. 57 ('1837); id. op. cit. and ed. p. 284 ( 1838$)$.

(olumba lunulata, Bonn. Euc. Méth. p. 25 s (1790).

Columba fowat, Bonn. op. cit. p. 251 (1790).

l'eristera malaccensis, Boie, Isis, 1828, p. 327.

Columba moluccensis, Less. Tr. d'Orn. p. $47+(1831)$.

(ieopelia lineata, Sw. Class. B. ii. p. 348 (1837).

(ieopelia striata, G. R. Gr. List. Gen. B. p. $5^{8}$ (18qo); id. Gen. 13. ii. p. $47 \mathrm{I}, \mathrm{n} .^{\circ} 2$ (1844); App. p. 23 (1849); Blyth. (at. B. Mus. A. S. B. p. 235, n. ${ }^{\circ}{ }_{2} 8$ (1849); Bp. Consp. Av. ii. p. 94 (1 845); id. Compt. Rend. xl. pl). $210,2 \mathrm{II}, 22 \mathrm{I}, \mathrm{n} . "$ 284 ; id. Coup d'ail Ordre Pig. pp. 48, 49, 59, n." $28+$ (1855); G. R. Gr. List. B. Brit. Mus. Columbe, p. 36 I 856 Moore, P. Z. S. 1859 , p. 466; Hartl. J. F. O. 1860, p. 162; $2 d$. Orn. Beitr. Faun. Madag. Not. Ile Réure. p. 163 (1862); Blyth, Ibis, 1862 , p. 92; Wall. P. Z. S. 1863 , p. 486; Pollen, N. T. D. i. p. 319 (1863); Jerd. B. of. Ind. iii. p. $486(1864)$; Sclat. P. Z. S. 1864 , p. 839; Finsch, Neu-Guin. p. 179 (1865); Wall. Ibis, 1865, p. 394, n. ${ }^{\circ}$ I 5 ; id. J. F. O. 1806, p. 284 ; Mart. J. F. O. ${ }_{1866}$, pp. 24, 284; Schleg. P. Z. S. 1866 , p. 224; E. Newt. Ibis, 1867 , pp. 342, 359; Sclat. I. Z. S. 1869 , p. 227; G. R. Gr. Hand-list, ii. p. 236, n. ${ }^{0} 9286$ (1870); Wald. Ibis, 1871 , p. 177; id. Tr. Zool. Soc. viii. p. 86 (1872); Sundev. Méth. Nat. Av. disp. Tent. p. 100 (1872); Schleg. Mus. P.-B. Columba, p. I 3 I (1873); Sousa, Mus. Nac. Lisb. Columba, p. 23 (1873); Pelz, Ibis, 1873 , p. 34; Garr. P. Z. S. 1873 , pp. $468,639,1874$, p. 256 ; Saliad. Cat. Ucc. Born. p. 298, n. 314 (1874); Gieb. Thes. Orn. ii. p. 250 (1875); Wald. Tr. Zool. Soc. ix. p. 223 (1875); Hume, Str. Feath. iii. p. $3^{23}$ (1875); Sharpe, Tr. Linn. Soc. (2) Zool i. p. 353 (1876); Salvad. Ann Mus. Civ. Gen. ix. p. 205, n. ${ }^{\circ} 73$ (1876); Hartl. Vög. Madag. p. 404 (1877); Tweedd. Ib.s, 1877, p. 322; Hume \& Davis Str. Feath. vi. p. 423, 123 (1878); Becc. Ann. Mus. Civ. Gen. viii. p. 454 (1874); Salvad. ibid. xiv. p. 247 (1879); Hume, Str. Feath. viii. p. 110 (1879); Meyer, Ib:s, 1879 , p. 138; Tweedd. Orn. Works, pp. I 1 2, 186, 386, 412, 503, 513, 525 (1881); Wardl. Rams. ibid. p. 659 1 1881 ); Meyer, Verh. z.-b. Ges. Wien. 1881 , p. 772 ; Kelih. Ibis, 1881, p. 528; Salvad. Orn. Pap. c. Mol. iii. P 155 (1882); Oates, B. of. Brit. Burm. ii. p. 298 (1883); Sclat. Lis:. Vert. Au. Sth 
ed. p. 450 (1883); Shell. Ibis, 1883 , p. 330; Snellem. Surn. Exp., Vogels, p. 49 (1884); Vorderm. .V. T. N. I. xliv. p. 229 (1884); xiv. p. 404 (1885); xlvi. pp. 89, 236 (1886); Bültik. Not. Leyd. Mus. ix. p. 77 (i886); Vorderm. N. T. N. 1. xlix. p. 413 (1889); Tristr. Cat. Coll. R. p. 41 (1889); Stecre. lit Mamm. \& B. p. 24 (a Bgo); Salvad. Mem. R. Ac. Sic. For. (2) xlii. p. 63 (189r); Hartert. Kat. Vogelsamml. p. iss.5 (1891); Vorderm. N. T. N. I. li. P. 422 (1892); Salv. Cat. B. M. 1893 p. $45^{8}$.

Geopelia striata, Rchenb. Av. Syst. Nat. xxv. (1862); id. Taub'n. i. p. 26, t. 250 ff. $1385-86$ (1862), ii. p. 164 (1862).

G. malaccensis, Hom. /. F. O. 18.59 , p. 361 .

'Turtur s'riatus. Schleo. De Dierent. p 207 (1864); Schleg. \& Poll. Rech. Faun. Madag. p. 162 (1868).

I. malaccensis, Schleg \& Poll. Rech. Fanun. Madag p. 155 (1868).

Hab. Luzon, Manila, Navotas, Mcntalban, San Mateo, Laguna, Bataan, Cagayan, Ilocos, Abra; Célebes.

\section{Subfam. FABINAS.}

\section{Gén. Chat.cophaps, Goui.d.}

8 Ch. indica, (Lin.) $\checkmark \circ \mathrm{N}$. $\mathrm{v}$. Bato-batong chile. Sipagan. (M S. T)

Green-winged dove, Edw. Birds, i. pl. I4 I741; Lath. Syn. ii. 2. p. 625, n. $^{\circ}$ I 4 I 783 .

Palumbus amboinensis, Briss. Orn. i. p. i 50, t. i 5 f. I (i760).

Colunba indica, Linn. S. N. i. p. 284 , n. 29 (176); Penn. Faun. Ind. in Forst. Zool, Ind. p. 4 I ( 1781); Jacq. Beyter. p. 35, n. $^{\circ} 29$ (1784); Gm. S. N. i. p. 785 , n. 29 (1788); Lath. Ind. Orn. ii. p. 598, n. ${ }^{\circ} 6$ (1790); Hayes, Portr. of rave \& cur. B. ii. pl. 79 (1799); Vieill. N. D. xxvi. p. 362 ( 18 I 8 ).

Tourterelle de Java, D'Aubent. Pl. Enl. 177.

Turvert. n: $:^{\circ}$, Buff. Hist. Nat. Ols. ii. p. 556 ( 177 I).

l'igeon verd à tete grise d'Antigue, Sonner. Voy. Nouv. Guin. p. I I $2,11.66$ (1766).

Columba javanensis, Müll. S. N. Suppl. p. г 33, n. 35 (г776); Cass. Pr. Ac. Philad. I 864, p. 248.

Bluc-crowned turtle, Lath. Syn. Av. ii. 2, p. 655, n. ${ }^{2}$ (1783).

Columba turtur viridis, Bodd. Tabl. Pl. Enl. p. I I (i783).

C. pileata, Scop. Del. Flor. et Faun. Insubr. ii. p. 94, n. ${ }^{\circ} 96$ ( 1786 ).

C. albicapilla, Gm. S. N. i. p. i 775, n. ${ }^{\circ} 8$ (г788); Lath. Ind. Orn. ii. p. 597, n. ${ }^{\circ}$ I I (1790); Vieill. $N$. D. xxvi. p. 360 ( 1818$)$.

C. javanica, Gm. S. N. i. p. 781, n. ${ }^{\circ} 55$ ( 788$)$; Lath. Ind. Orn. ii. p. 610, n. ${ }^{\circ}$ 6o (i790); Bonn. Tabl. Enc. Méth. p. 237 (1790); Temm. Pig. et Gallin. pp. 254, 468 (1813); Vieill. N. D. xxvi. p. 381 (1818); Steph. Gen. Zool. xi. p. 47 (1819); Horsf. Tr. Linn. Soc. xiii. I, p. 183 (1821); Rafl. Tr. Linn. Soc. xiii. 2, p. 317 (1822); Desm. Dict. Sc. Nat. xl. p. $33^{2}$ 
(1826); Less. Tr. dOrn. p. 471 (1831); id. Compl. de Buff. Ois. viii p. 43 (1 837$)$; id. op. cit. 2nd ed. p. $280(\mathrm{I} 838) ; E ; t$. P. Z. S. 1839, p. 107; S. Müll. Verh. Land-en Volkenk. p. 109 (1839-44); Temm. Tabl. pl. Enl. p. 18 (1840).

( . cyanocephala, Gm. S. N., i. p. $78 \mathrm{r}$, n." 56 (1788).

C. caruleocephala, Lath. Ind. Orn. ii. p. 618, n. ${ }^{\circ}$ 6r (1790'; Vieill. $N$. D. xxvi. p. 389 (1818).

(C) cyaneopileata, Bonn, Tabl. Enc. Méth. p. 238 (1790).

C. griseocap:llata, Bonn. Tabl. Enc. Méth. p. 238 , pl. 77. f. 2 (i790).

(C. aurata, Bonn. Tabl. Enc. Méth, p. 252 (1790).

(: superciliaris, Wagl. Syst. Av. Columba, sp. 80 (1827).

Monormis pelpulchra, Hodgs. Gray's Zool. Misc. p. 85 (1844).

Ptilinopus superciliaris, Hartl. Syst. Verz. p. 97 (1844).

(halcophaps indica, G. R. Gr. List Gallina Brit. Mus. p. is (1 844); id. Gen. B. ii. p. 477, n. ${ }^{\circ}$ I (1 844); Blyth. J. A. S, $B$. xiv. 2, p. 859 (1845); id. Ann. \& Mag. N. H. xix. p. 99 (1847)? Strickl. 2bid. p. 101 (1847); Rchnb. Av, Syst. Nfit. p. xxv (1852); Bp. Consp. Av. ii. p. 91 1854); id. Compl. Rend. xl. pp. 208, 209, 222, n. ${ }^{\circ} 273(1855)$; Bp. Coup. d'a.l. Ordre Pig. pp. 46, 47, 59, n. ${ }^{\circ} 273$ (1855); G. R. Gr. List R. Brit. Mus. Columbar, p. 59 (1856); Moore, P. Z. S. $185 \%$, p. 467; Rchnb. Tauben, i. p. 46, t. 260 ff. 144I-42 (1862), ii. p. 166 (1862); Sclat. P. Z. S. 1861 , p. 365, 1862, p. 321, 1864, p. 139; Pelz. Novar, Reis., Vög. pp. I09, 102 (1865; Schleg. $N . T$. D. iii. p. 265, 266, 245 (1866); Blyth, Ib.., 1867, p. 151; G. R, Gr Hand-list, ii. p. 244, n." 9415 (1870); Swinh. Ibis, 1870 , p. 356; Elwes, Ibis, 1870 , p. 528; Swnh. P. Z, S. 1871 , p. 397; Wald. Tr. Zool. Soc. viii, pp. 86, 1 i 4 (1872); Holdsw. P. Z. S. 1872 , p. 467; Elices, P. Z. . 1873, p. 655; Hume Nests \& Eggs, p. 509 (1873); Schicg. Mus, P.-B. Columbar, p. I 45 (1873'; Sousa, Mus. Nac. Lisó. Columbe, p. 23 (1873); Salvad. Ucc. Born. p. 299 (1874); Hume, Str. Feath, ii, p, 481 (1874); Wald. Trans. Zool Sac. ix. p. 22 I (1875); Hume, Str. Feath. iii. p. 165 (1875); iv. p 404 (1876); Salv. Ann. Mus. Civ. Gen. viii. p. 303 : 1866); Priüg. Abh. Nat. Ver. Brem. v. p. 87 (1876); Siharpe, 7r. Linn. Soc. Zool. i. pp. 348, 353 (1876); Saliad. Ann. Mus. Civ. Gen. ix. p. 206 , n. $77(1876)$; Rowl. Orn. Misc. i. p. 119, pl. 57 (1877); Oust. \& David, Ois. Chine, p. 384 (1877); Rosinb. Malav. Archip. pp. 275, 407 (1878); Anders. Yunn. Exped. p. 667 (1878); \& L)avids, Str. Feath. vi. p. $424(1878$; Hume, Str, Feath. viii. p. 110 (1875); Tirant, Büll. Com. Agr. A. Ind. Cochinch. (3) i. p. 142, 254 (1879); Meyer, Ibis, 1879 , p. I 38 ; Legge, B. of Ceyl. p. 714 (1880); Kelh, Ibis, 1881 , p. 528; Oates, Str. Feall. x. p. 235 (1882); Vorderm. N. T. N. I. xlii. p. 228 (1882); Salvad. Orn. Pap. $55^{2}$ (1882); Oates, B. Brit. Burm. p. 297 (1883); Sclat. List. Vert. An. 8th. ed. p. 4.59 (1883); Pleske Büll. Acad. Petersb. xxix. p. 5.35 (1884); Vorderm. N. T. N. I. xlvi. p. 236 (I886); Sneliem. Sum.Exp. i. Vög. p. 48 (1887); salrad. Ann. Mus. Civ. Gen. (2) iv. p. 561 (1887), vii. P. 424 (1889); Veraerm. N. T. N. I. xlix, p. ${ }^{11} 3$ (1889); Anders. Journ. Linn. Soc., Zcol. xxi. p. ${ }^{52}$ (1889); Brisay, Bull. Soc. Acclim. 1889, 
r. 57: Tristr. Cat. Coll. B. pp. 41, 269 (1889); Steere, List, 1. 24 (1890); Vorderm. N. T. N. I. 1. p. 508 (1890); Hein. \& Rchnov. Nomencl. Mus. Hein. Frn. p. 286 (1890); Hartet. Kat. Vogelsamml p. 188 (1891); Salvad. Mem. R. Ac. Sc. Tor. (2) xlii. p. 64 (1891); Vorderm. N. T. N. I. Sharpe, Ibis, 1892, p. 442. Salv. Cat. B. M. 21. 1893 p. 514.

C. indicus, Tick. in Blyth. J. A. S. B. xlv. 2, p. 848 (1854); id. Ann. \& Mag. N. H. xix p. I(x) $(18+7)$; Blyth. J. A.S. B. xv. p. $47 \mathrm{I}(1846)$; id. Cat. B. Mus. A. S. B. p. 237, n. ${ }^{\circ} 1440$ (1849); Gould. P. Z. S. 1859, p. 149; Blith, Monat's App. P. 362 (1863); Jerd. B. of Ind. iii. p. $484(1864)$; Bear'. Ibis, 1867 , p. 332; Blyth, Ibis, e868, p. $133 ;$ Ball. J. A. S. B. xli. pt. 2, p. 2, p. $287(1872)$; id. Str. Fealh. i. p. $\delta(1)$ ( 1873$)$; Vald. Ib s, $1873, \mathrm{p}$ 315; Hume, Str. Feath. ii. pp. $70,75,80,269(1874)$; Ball, Str. Feath. ii. F. 425 (1874; Blyth. \& Wald. B. Burm. p. 147 (1875).

C. javanica, Bp. Consp. Av. ii. p. 91 (1854); id. Compt. Rend. xl. pp. 208, 209, 222, n. 274 (1855); id. Coup. d'ceil Ordr: Pig. pp. 46, 47, 59, n. ${ }^{\circ} 274$ (1855); Rchnb. Tauben, i. p. 40 (1862), ii. p. 166 (1862); Wall. P. Z. S. 1863 , p. 486; id. Jbis, 186.5 , p. 393; 1d. J. f. O. 1869 p. 484 ; Sousa. Mus. Nac, Lisb. Columba, p. $23(1873)$.

R. augusta, Bp. Consp. Av. ii. p. 92 (1854); id. Compt. Rend. xl. pp. 209, 221, n. 275 (1855); id. Coup a'ail Ord. Pis. pp. 47, 59, n. ${ }^{\circ} 275$ (1855); Schleg. N. T. S. iii. pp. 265, $26 \%$ (I866); Blyth. Ibis, 1868 , p. I33 G. R. Gr. Hand-list, it. p. 244, n." 9419 (1870); Ball, Str. Feath, i. p. 81 (1873).

Peristera bornensis, $S$ Müll. Mus. Lugd. Bp Consp Av. ii. p. (1854); id. Compt. Rend. xl. p. 208 (1855); id. 'Coup. d' ail Ordre Pig. p. 47 (1855).

Chalcophaps bornensis, Bp. Compt. Rend. xliii. pp. 947, 948, (1856); Wall. Ibis 1865 , p. 393; id. J. f. O, 1866 , p. 284.

C. javanica, a bornensis, Bp. Comp. Rend. xliii. p. 949 (1856).

C. moluccensis, G. R. Gr. P. Z. S. 1860 , p. $36 \mathrm{r}$; Wall. P. Z. S. 1862 , pp. $335,345,186_{3}$, p. $34 ;$ id. Ibis, 1865, p. 393 id. J. f. O. 1866,284 Schleg. N. T. D. iii. pp. 265, 266 (1866); G. R. Gr. Hand-list. ii. p. 265, n. 9420 (1870); Wald. Tr. Zool. Soc. ix. p. 222 (1875); Hein. \& Rchnw. Nomencl. Mus. Hern. Orn. p. 286 (18go).

C. albicapilla, Rchnb, Tauben, i, p. 48 (1862), ii. p. 166, x. i. f. Io (1 862$)$.

Goura javanica, Schleg. Dierent. p. 208 (1864).

Peristera moluccensis, Finsck, Neu-Guin. p. 179 (1865).

Chilcophaps formosana, Swinh. Ibis, $1865, \mathrm{pp}$. 357,$540 ; G$. R. Gr. Hand-list, ii. p. 245, n. ${ }^{\circ} 9424$ (1870); Swinh. P. Z. S. 1871 , p. 397; Elwes. P. Z. S. 1873, p. 667.

Chalcopaps, sp. Mart. J. f. $O .1866$, p. 24, sp. 137.

C. pileata, G. R. Gr. Hand-list, ii. p. 245 n. 9422 (1870).

C. javanensis, G. R. Gr. Hand-list, ii. p. 245, n. ${ }^{\circ} 9416$ (1870); Hein. \& Rchnw. Nomencl. Mus. Hein. Orn. p. 286 (1890).

Phaps albicapilla, Gieb. Thes. Orn. i. p. 630 (1872), iii. p. 88 ( 1877 ).

P. augustæ, javanica, formosæ, indica, moluccensis, Gieb. op. cit. i. p. I 30 . 
Chalsphaps marix, «Bp.» Blyth, Biros of Burm. P. It7 (1875).

C. javanus, Rosenb. Reis naar Zuidoostereil p 8 (1875).

C. sp., Becc. Ann. Mus. Civ. Gen. vii. p. 716 (1 $8755^{\circ}$.

Hab. Luzón, Manila, S. Mateo, Montalban, Bataan, Orani, Hermosa, Laguna, Calamba, Negros Valencia, Cebú, Surigao, Zamboanga, Basilan; Célebes, Formosa.

6. Gén. OCYi'HAPS, Gould.

9 O. lophotes, $\left(T^{\prime} / m.\right) \sigma^{*} \mathrm{~N} . \mathrm{v}$.

Columba lophotes, Tem. Pl. Col. $1.52(1823)$.

Ocyphaps lophotes, Gould, P. Z. S. 1842 , p. 20; Salv. Cat. $B$. M. 21 p. 535 (1893).

Hab. Australia.

\section{Subfam. GEOTRIGONINAS.}

7. Gén. I'HL,ugANAS, RсHмB.

Io $\mathrm{Ph}$ luzonica, (Siop) ơ o N. v. Bato punalar'a

(M. S T)

Touterel'e greie ensanglentee de l'isb. Luçon, Sonn. Voy Vou. Guin. p. 52 pl. 21 (1776).

Red.-treasted Turtle, Lath. Sy'n. ii. 2, p. 657, n. ${ }^{0} 56$. (1786).

Columba luzonica, Scop. Del flor. A. Fann. Insubr. p. 94, n." 92 ( 1786 .

Columba cruenta, Gm. S. N. i. 2. p. 785, n. 66 1 7889 ; Lath. Ind. Orn. ii. p. 61 г, n. ${ }^{0} 6$ (г7g(); Bonn. Tabl. Enc. Méth. p. 250 (1790'; Temm, \& Knip, Rig. i. fam. trois. p. i6, pl. 8 (1808-і I); Tem. Pig. et Gallin. p. 494 (1813); Viell. N. D. xxvi. p. 403 (1818; Wagl. Syst. Av. Columb.r, sp. 73 (1827; Less. Tr. d'Orn p. 474 (1831).

C. cruenta, «Lath.» Temm. Pig. i. fam, trois p. 16 (1808-1 I'; id. Pig, et Gallin. i. p. 407 (1813); Desm. Dict. Sc. Nat. xl. p. $306(1826)$; Less, Compl. de Bufff., Ois. viii. p. I5 (1837); id. op. cit. 2nd. ed. p. 128 (1819).

Goura cruenta, Steph. Gen. Zool. xi. p. 128 (1819).

Red. br asted Pigeon, Lath. Gen. Hist. viii. p. 90 (1823).

Columba luzonica, Scop. Del. Flor. A. Fuzm. Insubr. p. 9t, n." $92(1789)$.

C. cruenta, Gm. S. N. i. 2, p. 785, n. ${ }^{\circ} 66$ ( 1788 ); Lath. Ind. Orn. ii. p: 6II, n. ${ }^{\circ} 6$ (I790); Bonn Tabl. Enc. Méth. p. 250 (1790); Temm. \& Knip, fig. i. fam. trois. p. i6, pl. 8 (180-1 I); Tem. $P$ g. et Gallin. p. $49+$ (1813); Vieill. N. l). xxvi. p. 403 (1818), Wagl. Syst. Av. Co'umba, sp. 72 (1827); Less. Tr. d'Orn. p. 474 (1831).

C. cruenta, «Lath.» fig. i. fam. trois. p. 15 (1808-1r); id. Pig. et Gallin. i. p. 407 (1813); Desin. Dict. Sc. Nat. xl. p. 306 (1826); Less. Compl. der Buff., Ois. viii. p. I5 (1837); id. op. cit. 2nd ed. p. I 28 (1819).

Goura cruenta, Steph. Gen. Zool, xi. p. 28 (1819).

Red breasted Pigeon, Lath. Gen. Hist viii. p. 90 (1823). 
Calienas cruenta, Strickl. P. Z. S. 1842, p. 168; Cab. fide Bp. Consp. Av. ii. p. 88 .

C. luzcnica, G. R. Gr. List. G.allina Brit. Mus. p. I8 (1844); id. Gen. B. ii. p. 478, n. $3(18+5)$.

Phleganas luzonica, Rchnb. Syn. Av. Columbaria, Navit. t. 227; f. $2+79$ (1851); Wall. Ib.s, 186.5 , pp. 392, 399; id. J. f. $O$. (1866); p. 283 , n." rot; G. R. Gr. Hand-list, ii. p. 246, n." $9+t^{2}$ (1870); Schleg. Mus. P.-B. Columba, p. 157 (1873); Tristr. Cat. Coll. B. p. 4 I (1889).

Ph. cruenta, Rchnb. Av. Syst. Nat. xxv. (1852); Bp. Consp. Rend. xl. pp. 206, 221 , n. ${ }^{\circ} 262$ (1855); id. Coup. d'ail Ordre Pig. pp. 45, 59, n. ${ }^{\circ} 262$ (I 85.5); Rchnb. Tauben, i. p. 40 (I 862).

Ph. cruenta, Bp. Consp. Av. ii. p. 88 (1854); Sousa, Mus. Nac. Lisb. Columba, p. 22 ( 1873$)$.

Calænas luzunica, G. R. Gr. List. B. Brit. Mus. Columba, p. 63 ( 1856$)$.

Phlogænas cruenta, Sclat. P. Z. S. 1861 , p. 366,1863, p. 377 ; Gould, B.Asia, vi, pl. $5 \mathrm{I}$ (1855); Sund?v. Tentamen. p. Io. (1872); Hartert, Kal. Vogelsamml. p. 189 (1891).

Calienas cruenta, Mart. J. f. O. 1866, p. 25.

Phlogarnas luz̄onica, Wald. Tr. Zool. Soc. viii. p. 29 (187.2), ix. pp. 221, 251 (1875); Sharpe, Tr. Linn. Soc., Zool. i. p. 34h (1876); Tweedd. P. Z. S. 1877, p. 700; id. Orn. Works, p. 659 (1881); Salv. Cat. B. Srickl. Coll. p. $55^{2}$ (1882); Steere, List. p. 24, n. ${ }^{\circ}$ 300 (1890); Salv. Cat. B. M. 21. p. 585 (1893).

Ph. cruenta, Garrod, P. Z. S. 1873, pp. 468, 639, 1874, p. 250; Vagenfïhr, J. f. O. 1876, p. 334; Sclat List. Vert. An. 8th ed. p. 46. (1883); Hein. \& Rchibw, Nomencl. Mus. Hein. Orn. p. 286 (1890).

Ph. cruentatus, Ciab. J. f. O. $1875 .$, p. 126.

Phaps cruenta, Geb. Thes. Orn. iii. p. 89 (1877).

Tourterelle blanche eusanglentée, de L'isle de Luçon, Sonner. Voy. Nouv. Guin. p. 5I, pl. 20 (1776).

Columba nivea, Scop Del. Flor. et Faun. Insubr. p. 94, n. $91(1786)$.

Sanguine Turtle, Lath. Syn. ii. 2, p. 657, n. ${ }^{\circ} 57$ (1873).

Colunba sanyuinea, Gm. S. N. i. 2, p. $785,{ }^{\circ}{ }^{\circ} 65$ ( 1788$)$; Lath. Illd. Orn. ii. p. 611, n. ${ }^{\circ} 66$ (1790); Bonn. Tabl. Enc. Méth. p. 250 (1790); Vieill. N. D. xxvi. p. 400 (188I).

C. cruenta, var., Tem. \& Kn p, Pig. $i$. fam. trois. p. I 7 , pl. 9 (I808-1 I); Temm. Pig. A. Gallin. i. p. 494 (1813); Wagl. Syst. Av. Columba, sp. 73 (1827); Rchnb. Syn. Av. f I 265 (1847).

C. cruenta, var., Temm. Pig. et Gallin. i. p. 410 (1813).

Goura cruenta, var. B. Steph. Gen. Zool. xi p. I28 (I819). Hab. Luzón, Manila, en todas las provincias, Calamianes.

r. Ph. luzonica, var. albina. N. v. Bató bató puñalado.

Hab. Id. id.

12 Ph. samarensis, of. N. v. Amomoquit

(M. S. T.)

Hab. Samar, Borongan, Calbayog, Paris, Paranas.

13. Ph mindorensis. $\sigma^{\circ} .0 \mathrm{~N}$. v. Ala-lauan.

(M. S. T.)

Hab. Mindoro, Bacó, Calapan, Naujan. 
14. Ph. crinigera, (Het Facq.) $\sigma \circ \mathrm{N}$. v. Bato-puñalada.

(M. S. T.)

Peristera crinigere, Homb. et Jacq. Voy. Pole Sud. Atlas, pl. 27. f. 2 ( 1845$)$.

Calænas, G. R. Gr. Gen. B., App. p. 24 (1849).

Peristera crinigera, part., Rchnb. Syn. Av. Columbaria, Novit. t. 259. f. 2597 ( $185 \mathrm{r}$ ).

Pampusana criniger, Pucher. \& Jacq. Voy. Pole Sud, iii. Ois. p. I1 8 (1853); Hartl. J. f. $O$ 1854, p. I66.

Phlegænas criniger, Bp. Consp. Av. ii. p. 88 (1854).

Ph. criniger, $B p$. Compt. Reud. xl. pp. 206, 221, n. $0^{\circ} 2+$ ( 1855 ); id. Coup. d'ail Ordre Pig. pp. 45, 59, n. '26) (1855); Rchnb. Tauben,; p. 40, t. 249. f. 2597 (1862); Wall. Ibis, 1865, pp. 392, 400; G. R. Gr. Hand-list, ii. P 246 . n. ${ }^{\circ} 9+43$ (1870).

Phlogenas bartletti, Sclat. P.Z.S. 1863 , p. 377 , pl. xxxir., 1865 p. 238.

Phlogenas crinigera, Sclat. P. Z. S. 1855, p. 239; Gould, B. of. As. vi. pl. 52 (1865); Sclat. P. Z. S. 1869 , p. 628; Grarrod. P. Z. S. 1874 , p. 250; Tzveedd. P. Z. S. 1877 , pp. 817, 833, 1878; p. 952; id. Orn. Works pp. 544, 559, 643 (1881); Wardl. Rams. Tweedd. Oirn. Works, p. 659, n. ${ }^{289}$ ( 881); Sclat. List Vert. An. 8th ed. p. $46 \mathrm{r}$ (1883); Steere, List, p. 24 (1890); Salv. Cat. B. M. 21. p. 587 (1893).

Phleganas crinigera, Wall. J. F. O. 1866, p. $284, \mathrm{n}^{0} \mathrm{Ios}_{5}$; Tristr. Cat Coll. B. p. 41 (1879).

Phap. crinigera, Gieb. Thes. Orn. iii. p. 89 (1877).

Hab. Samar, Borongan, Mindanao, Placer, Zamboanga, Basilan, Joló.

15. Ph. platenæ, Blas. ơ $\mathrm{N}$ v.

(M. S. T.)

Phlegenas criniger, Hart. J. f. O. 1891, pp. 30\%, 302.

$\mathrm{Ph}$. platenæ, Blas in lits. Hart. J. f. O. $1891, \mathrm{p} .302$.

Phlogenas platenæ, Salv. Cat. B. M. 81. p. 588 (1893).

Hab. Mindoro, Baco.

16. Ph. yapensis, $H$, ct Funch.

Phlegrenas yapensis, Hartl. et Finsch. P. Z. S. 1872, 1. 102; Sch. meltz, Ethn. Abth. Mus. Godeffr. 1881, p. 398; Wiglesw. Aves Pocyn. p. 57, n. ${ }^{\circ} 293$ (1891).

Ph. napensis, Sharpe, Zool. Rec. ix. 1872) p. 53 (1874).

Phaps yapensis, Gieb. Thes. Orn. iii. p. 91 (1877).

Phlogænas yapensis, Salv. Cat. B. M. 21. p. 593 (1893).

Hab. Carolinas, Uap.

\section{Ph. Kubaryi, Finsch}

Phlegenas eryptroptera, pat. Bp. Consp. Av. ii. p. 89 (185t). Phlegænas erythroptera, part., Rchnb. Tauben, i. p. 4I (I862).

P. sp., Finsch, Journ. Mus. Godeff. viii. p. I31 (1875), xii. pp. 16, 17 (1876).

P. crythroptera?, Finsch, P. Z. S. 1877 , p. 780, 1880, p. 576; 
id. Ibis, 1.88, 1,1. 112, 115; 7) is/r. (inl. Coll. B. p. +1 (1889).

P. Kubaryi, Finsch, J. F. O. $188^{\circ} 0$, p. 292: Rchnu. \& Schal. I. F. O. 1881 , p. 75 i Wiglesi Ais. Polvn. p. 5.5, n." 26t; Sala. Cat. B. M. 21. p. 599. (I89,3).

Hab. Carolinas.

17. Ph. menagei. Fi. it $\| \mathrm{i} \%$

Ph. menagri, Frank el Horcester. Pielim. Not. Menage E.xped. Ihilipp. 1s97, 1. 10.

Hab. Tawi-tawi.

1S. Ph. pampusan, Q. et (i)

Columbe rousseau, Tem. Pl. Col. 190 (foì) $1+23$.

Columba xanthonura, Cur. Tem. Pl. Coll. 190; Siteph. Cien. Zool. xiv. p. 2 I ( (126); Less. Compl. de Buff., Ois. viii. p. t+ $(1837) ;$ id. op. cit. and. ed. p. 281 (1838; G. R. Gr. Genl. B. ii. p. 470, n. ${ }^{\circ}$ 30 (1 845$)$; Rehnb. Syn. Az'. Df. 1 269-70 (1847).

colombe crythroptére á gorge blanche, Quoy \& Gaim. Iroy. Tran., Zool. p. 35 (1824).

(.) de couleur rousse, Quoy \& Gaim. Iroy. Iran.. Zool. p. 3.5 (I824).

(columba pampusan, Quoy \& Gaim. Loy., Zool. p. 121, pl. 3" (1824); Desm. Dict. Sc. Nat. xl. p. 345 (1826); Wagl. Syst. A'. Columba, sp. 82 (1827); Less. Tr. d'Orn. p. 47 I (1838); id. Compl. de Buff., Ois. viii. p. $42(1837)$ i id. op cit. 2nd. ed. p. 280 (1838); Hartl. J. F. O. $18+5$, p. 167 .

C. xanthura, Cuv. Régn. Ad. znd. ed. i. p. 491 (1829); Temm. Tabl. Méth. Pl. Col. p. 8 г (1839); Prer. \& Kiup, Pig. ii. p. 45, pl. $23(1838-43)$; Schleg. N. T. D. iv. p. 29 (1871).

Pampusana xanthura, Bp. Consp. A\%. ii. p. 89 (1854); id. Compl. Rend. xl. pp. 207, 221, n. ${ }^{\circ} 267$ (1855); id. Coup. d'ceil. Ordre. Pig. pp. 45, 59, n. ${ }^{\circ} 267$ (1855); Rchnb. Tauben, i. p. 39, t. 226. fig. I 270 (1862).

Pampusana rousseau, Bp. Consp. Av. ii. p. 89. (1854); id. Comp. Rend. xl. pp. 207, 222, n. ${ }^{\circ} 268$ (1855), id. Coup. d'ail, Ordre. Pig. pp. 46, 59 n. ${ }^{\circ} 268$ (1855); Rchnb. Tauben, i. p. 39, t. 226 f. 1269 (1862).

P. erythroptera, part., Bp. Compt. Rend. xliii. p. 947 (1857).

Columba erythroptera, Hartl. J. F. O. 1854, p. 167.

Peristera erythroptera, part., Cass. Un. St. Expl. Exped., Birds. 1). $277(1858)$.

Calanas erythroptera, war., G. R. Gr. Cat. B. Trop. Isl. p. t+ (1859): id. Hand-list, ii pp. 246. n. ${ }^{\circ} 9446$, (1870).

(. xanthura, G. R. Gr. Cat. B. Trop. Isl. p. 45. (1859).

(. ruusseau, G. R. Gr. Cat. B. Trop. Isl. p. 45 (1859).

Phleganas erythroptera, parl., Rcnub. Tauben, i. p. 4i (1862); Oilsl. Le Natural 1869, p. $26 \mathrm{I}$.

Pampusana xanthura, G. R. Gr. Hand-list, ii. p. 247, n. $945^{\prime \prime}$ $($ I 870$)$.

Columba rousseau, Schleg. N. T. D. iv. p. 29 (1.871).

leptoptila pampusan, Schleg. N. T. D. iv. p. 30 (187r); id. Mus. I. - S. Columha, p. $162(1873)$. 
Phaps erythroptera, part., Gieb. Thes. Orn. iii. p. 89 (1877). l'hap xanthura, Gieb. Thes. Orn. iii. p. 91 (1877).

Phiegranas pampusan, Wiglesw. Av. Polyzes. p. 55, n." 283 (1841).

Salv. Cat. B. M. 21. p. 602 (1892).

Hab. Marianas, Guam, Marquesas.

Subfam. CALENADINAS.

8. Gén. Caloenas, Giray.

19. C. nicobarica, (Lin.) $\sigma^{\prime}, 0$ N.v. Dundunay.

(M. S T.)

The Nincombar Pigeon, Abb. Nat. Hist. Birds. iii. p. 4t, pls. 47, 48 (1740); Edw. Glean. iii. p. 27 I, pl. 339 (1761); Lath. iyn. ii. 2, p. 642, n. 38 ( 1783$)$.

columba nicombariensis, Briss. Orn. i. p. I 54 ( 1760$)$.

Columba nicobarica, Linn. S. N. i. p. 283, n." 27 (1760); Cim. S. N. i. 2 p. 783 ; n. 27 (1788); Lath. Ind. Orn. ii. p. 6o5, n." 44 (1790); Bonn. Tabl Enc. Mèth. p. 246, pl. 8o. f. \& (1790); Hayes, Portr. of: rare and curions Birds, ii. pl. 91 (1799); Temm. \& Knip. Pig. i. fam. trois. p. 5, pl. 2 (1808-1 1); Temm. Pig. et Gallin. i. pp. 385,445 (1813); Cuv. Regn. An. i. p. 456 (I817); Vieill. N. 1). xxvi. p. 395, pl. G. 38 (II 8); Desm. Dict. Sc. Nat. xl. p. 303 (1826); Less. Voy. Coq., Zool. i. I, p. 342 (1826); id. Tr. d'Orn. p. 475 (1831); id. Compl. de Buff., Ois. viii. p. 12 (1837); id. op. cit. 2 nd. (d. p. 272 (1838); Temm. Pl. Col. i. Tabl. Métk. p. 79 (1839); Bcnnett. Wauderings, ii. 1). 65 ( 1834); Thienem. Fortpfl. p. 56; (1846); Gulliv. P. Z. S. 1879, p. 419.

Pigcon de Nincombar, D'Aubent. Pl. Eul. 491; Buff. Hist. Nat. Ois. ii. p. 54 r $(167 \mathrm{r})$.

(olumba Nicombar, Penn. Faunula Ind. in Forst. Zool. Ind. 1. 41 ( 1781$)$.

columbi-galline á camail, Levaill. Ois. d'Afr. vi. p. roz, pl. 279 (1808); Sundev. K. Sv. Vet.-Ah. Hand-l. 18.57, 1). 55, $11 . " 279$.

Goura nicobarica, Sóeph. Gen. Zool. xi. pt. 2, p. 122 (1819); Rans. Elem. Zool. iii. pt. I, p. 217 (1821, p. 55, n." 279.

columba gallus, Wagl. Syst. Av. Columba, sp. I 3 (1827).

( gouldia, Gray \& Hardw. Ill. Ind. Zool. ii. pl. 57 ( 1834 ); Bp. Compt. Rend. xxxix. p. 87 I (1854); 2 d. Coup. d'acil Ordie Pig. p. 3 (1855); Bartlett, P. Z. S. 1866 , p. 79.

Gcophilus nicobaricus, Selby, Nat. Libr., Pigeons, p. 22i, p. =9 (1835); Blyth, Calc. Journ. N.H. i. p. 605 (1841); Peale. In. St. Expl. Exped., Birds, p. I25 (1848; Hartl. Arch. f. Natnrg. 18.52. i. ए, i 7 , Rams. Pr. linn. Soc. N. S. $W$. i. p. $370(1876)$.

Calanas nicobarica, G. R. G. List Gen. B. P. 59 (1840); Biylh. J. A. S. B. xv. p. 37 I (18460; Margill. Narr. Voy. "Rattlesnake," i. p. 244 (1852); Harlt.J.f. O. 1854, p. 368, n." 257 ; Bp. Consp. Av. ii. p. 95 (1854); 1 d. Compt. Rend. xi. p. 221 , n." 206 (1855); Hartl. J. f. O. 185.5. p. 319; C. R. Gr lisi. B. Brit. Mus. Columber, p. 63 (1856; Sclieg. Hazdl. 
Dierk. i. p. 408 (1 858$) ; G$. R. Gr. P. Z. S. 1858 , p. 196; id. Cat. B. Trop. Isl. p. 44 (1859); Blyth, Ibis. 1859, p. to5; Flower, P. Z. S. 1860 , pp. 333, 334. pl. I66. ff. 3, 4; G. R. Gr. P. Z. S. 1861 , p. 438 ; Bennett. 1863 , p. 372 ; Blyth in Mouat's dident. \&- p. 362 (1863); Rosenb. N. T. $A$. I. xxr. p. 250, n. $243 \quad$ (1863 ; id. J. f. $O .1867$, p. 13.3, 11. 243 ; Sclat. I'. Z. S. 1865 . p. 139; Finsch, Nest-Guits. p. 179 (1865); Pela. Vovara Reis., lö̈. p. I10 (1865); Wall. lois, 186.5, pp. 370, $39+400 ;$ id. I. f. O. 1866 , p. 284 ; Mart. I. f. $O .{ }^{1} 860$ p 25; Bartlett, P. Z. S. 1866, p. 78 ; Rosenb. Reis. natr Zuidoostereil. pp. $81 ; 82(1867)$; S'clat. $P$. Z. S. 1809 , p. 628; (G. R. Gr. Hand-lis/, ii. p 246, n." 9434 (1870); Ball. J. A.S B. xxxix. 2, p. 32 (1870); Gieb. Thes. Orn. i. 1. 545 (1872); Wald. Trans. Zool. Soc. viii. p. 80, n." I 33 (1872); Pelv. Ibis, 1873 , p. 35; Ball. Str. Feath. i. p. 8I (1873); Ciarrod. P. Z. S. 1873, pp. 468, 639; Elaces, P. Z. S. i873, 1. 663; Sousa, Mus. Nac. Lisb. Columba, 1.. 24, n." 144 (1873); Garrod. P. Z. S. 1874, pp. 250, 257; Hume, Str. Feath. ii. pp. I 33, 27I, 481 (1874); Wald. Tr. Zool. Soc. ix. p. 222, n." I5 I (1875; Sharpe, P. Z. S. 1875, p. Iіо; Rosenb. Reist. naar Geelvinkb. pp. 8, 4, I I7 (1875); Saliad. Ann. Mus. Civ. Gen. ix. p. 208, n. ${ }^{0}$ 90 $(\mathrm{i} 876)$, x. p. I62 (1877); Sclat. P. Z. S. 1877 , p. I 2 , 1878 , p. 289; Rams. Pr. Linn. Soc. N. S. W. iii. p. 295 (1878), iv. p. ioi, no. 879 (1879); Finsch. P. Z. S. 1879 , p. I4; Rosent. Malay, Archip. pp. 323, 372, 396, 407, 56r (1879); Salvad. Ann. Mus. Civ. Gen. xiv. p. 66+ (1879); Tiraut, Bull. Com. Agr. et Ind. Cochinch. (3) i. p. I43, n1. ${ }^{\circ} 28$ (1879). D)'Alb. Nouva Guin. p. 582 (1880); Finsch, Ib s, 1881 , p. 538; Saluad. Orn. Pap. e Mol iii. p'. 209 (1882; Sharpe, Journ. Linn. Soc., Zenl. xvi. p. 446 (1882); Sclat. List. Vert. An. Sthad p. 462 (1883); Meyer, Siizh. u. Abh Ges. Isis, 18st, Abh. i. p. 52; Guillem. P. Z. S. 1885 , pp. 576, 663; Grant. P. Z. S. 1887 , p. 332; Woodf. P. Z. S. 1888 , p. 248; Trislr. Ibis; 1889 , p. 558; id. Cat. Coll. B. p. 4 I (1889); Stecre, List, p. 24, n. ${ }^{303}$ (1890); He:n. \& Rchnze. p. 300; Saliad. Mem. R. Ac. Tor. (2) xlii. p. 67 (1891); Tristr. Ib s, 1892, p. 297.

Calanas nicobarica, G. R. Gr. Gen. B. ii. p. 478, n. ${ }^{\circ}$ I ( $\left.8+5\right)$; Blyth, J. A. S. B. xv. p. 37 I (1846); id. Cat. B. Mhts. 1 . S. B. p. 238 , n. ${ }^{\circ}+44$ (1849); "clat. Joum. Pr. Litms. Sirt. ii. p. I67, $n^{\circ}$ г $3^{\circ}(\mathrm{r} 858)$; Cass. Un. St. Expl. Exped., Birds, 1) $276\left(185^{8}\right) ;$ G. R. Gr. Cat. B. Nezu Guin.: p. 49, 6r (1859); id. P. Z. S. 1860 , p. 362 ; Le Vis, Anm. Rep. Brit. Neni Guin. for the years $1890-91$, p.-; id. An Queensl. Mits. 11. ${ }^{\circ}$ 2, p. 8 (1892); Salo. Cat. B. M. 21.1893, p. 6r 5.

Calanas gouldie, $G$. R. Gr. Gen. B. ii. p. 478, n. ${ }^{\circ} 2(18+5)$. Grophilus nicobarica, Peale, Un. St. Expl. Exped. viii. p. 207 $($ I $8+8)$.

Calrenas gouldia, Rchnb. Syn. An. t. 244 f. 2490 (1851); Harll. Arch. f. Naturg. 1852,1 , p. I 34 ; id. J. f. 0.1854 , p. I65.

Callenas nicobarica, Rchnb. Av. Syst. Nat. p. xxv. (1852); id. Tauben, i. p. 48, t. 263. ff. I $462-68$ (ad.) t. 244. f. 2490 (1862!; Schleg. Mus. P.-B. Columba, p. 170 (1873), Vorderm. N. T. N. I. xlviii. p. I 46 (I889), r. p. 509 (1890). 
Nicobar Pigeon, Bartl. P. Z. S. 1860 , p. 90; Wall. Malay. Archip. ii, p. 65 (1 869).

Calanas nicobaricus, Jerd. B. of Ind. iii. p. $480(1864) ; B e a r$. Ibis, 1867 , p. 332; Hume, Str. Feath. ii. p. 70 (1884); Blyth, $B$. of Burm. p. 147, n. ${ }^{\circ} 524$ (1875).

( $\because$ gouldii, G. R. Gr. Hand.bust, ii. p. 256, n." 9440 (1870).

(alliænas nicobarica, Sund. Meth. nat. Av. disp. Tent. p. 99 (i 809 ).

C. nicobarica, Rams. Pr. Linn. Soc. N. S. W. vi. p. 38 (1882).

Hab. Luzón, Laguna, Paquil, Mindorr. Calapan, Negros, Siquijor, Joló, Tawi-tawi,

\section{Fam. GÚRIDAS.}

I. Gén. GOURA, STEPh.

I. G. coronata, (Lix.) \& N. v. La coronada

(M. S. T)

Columba coronata, Lin. S. $N$. i. p. 282, n." "7 (1766); Gm. S, $N$. i. p. 774, n. ${ }^{\circ}$ i 7 ( 7788$)$.

Faisan couronnè des Indes, D'Aubent. Pl. Enl. 118 .

Goura coronata, Salv. Cat. B. M. 21: p. 620, (189.3).

Hab. N. ${ }^{a}$ Guinea.

2. G. victoria, Fraser. む N. v.

(M. S. T.)

G. victoria, Cat. B. M, 21, p. 624 (889.3).

Hah. N. a Guinea.

\section{Orden: GALLINAS.}

\section{Fam. FASIÁNIDAS.}

1. Gén. Caccabis, Kaur.

1. C. rufa, (Lin) ơ N. v. Perdiz roja.

Tetrao rufus, Lin. S. N. i. p. $276(1766)$; Gm. S. $N$. i. pt ii, p. 756 (1 788$)$.

La perdrix rouge d' Europa, D'Aubent. Pl. Enl. ii, pl. 44.

C. rufa, Ogilvie, Cat. B. M. 22, p. II 8 (1893).

Hab. Europa, España, Francia, Africa.

2. Gén. PERDIX, Briss.

2. P. perdix, (Lin). o N. v. Perdiz gris.

Tetrao perdix, Lin. S. N. i. p. $276(1766) ;$ Gm. S. N. i. pt. p. 757 ( 1788$)$.

I. perdix grise, Buff. Hist. Nat. Ois. ii. p. for (1771); l)' . Aubent. Pl. Enl. ii. pl. $4 \mathrm{I}$ n. ${ }^{\circ} 27$.

P. cinerea, Lath, Ind. Orn. ii. p. 645 ( 1 790). 
3. perdix, Hart. Kat. Mus. Senkeub. p. 194 (1891); Ogil. Cat. B. $M$. 22, p. 185 (1893).

Hab. Europa, Francia, Asia.

\section{Gén. ARBORICOLA, Hod.is.}

3. A. gingica, (Gm.) $\sigma \Omega$ N. v. Pugong.buquit.

(M. S. T.)

La perdrix de Gingi, Sonn. Voy. Ind. Or. euts ii. p. 167 (1782). Gingi partridge, Lath. Gen. Syn. ii. p. 773 (1783); id. Gen. Hist. viii. p. 278 (1823).

'Tetrao gingicus. Gmel, S. N. i. p. 760 (1788).

Perdix gingica, Lath. Ind. Orn. ii. p. 648 (1790); Bonn. Tabl. Ency. Meth. i. p. 212 (1791); Tem. Pig. et Gall. iii. pp. 4 IO y 733 (1815); Vieill. N. Dict. d' Hist. Nat. xxv. p. 197 (1817); Steph. in Shaw. Gen. Zool. xi. p. 351 (1819).

Gorget partridge, Lath. Gen. Hist. viii. p. 302 (1823).

Perdix scutata, Gray, in Griff. ed. Cuv. iii. p. 54 (1829).

Arboricola gingica, Blyth, Ib1s, 1870 , p. I74; Grant, Ibis, 1892, p. 395, pl. ix; Ogilv. (at. B. M. 22, p. 213 (189.3).

Hab. Luzon, Manila, Calaocan.

4. Gén. ROLLULUS, Bonn.

4. R. roulroul, (Scop.) $\sigma_{0}$ N. v. Pugo.

(M. S. T)

Roulroul de Malacca, Sonn. Iyy. Ind. Or. ii. p. 174, pl. 100, ( 782.$)$

Green partridge, Lath. Gen. Syn. iv. p. 777, pl. 67 (I783).

Phasianus roulroul, Scop. Del. Flor. et Falun. Insubr. ii. p. 93 ( 1786$)$.

P. cristatus, Sparrm. Mus. Carls. fasc. iii. pl. 64 (i 788 ).

Columb ! cristata, Gm. S. N. i. pt. ii. p. 774 (1788); Lath. Ind. Orn. ii. p. 596 (1790).

Tetrao viridis, Gm. S. N. i. pt. ii. p. $7^{6}$ I (1788); Ramfft. Tr. Linn. Soc. xiii. p. 322 (1822).

Perdix viridis, Bonn. Tabl. Encycl. Mèth. i. p. 219, pl. 95. fig. 4 I 79 I.

Coturnix viridis, Bonn. Tabl. Enc. Mèth. i. p. 219, pl. 95, fig. 4 ( I 79r)

Tetrao porphyrio, Shaw. \& Nodd. Nat. Misc. iii. pl. 84.

Perdix coronata, Lath. Suppl. Ind. Orn. p. 62 (1801).

Crowned partridge, Lath. Gen. Syn. Suppl. ii. p. 278 (1802); id. Gen. Hist. viii. p. 331, pls. cxxx., cxxxi. (1823).

Cryptonyx coronatus, Temm. Pig. et Gall. iip. pp. 526, 745 (I815); Temm. Pl. Col. v. pls. $43 \& 44$ (1855); Less. Traité d'Orn, p. 499, pl. 86. fig. I (1831); Müll. Verhantl. Land-en Volkenk, p. 395 1839-44); Schinz, Nat. Vogel, p. I54, pl. 75 (1853); Schl. Handl. Dierk. i. p. 395 (1857).

Cryptonyx cristatus, Vig. Zool. Journ. iv. p. 348 (1829); Cuv. Rig. Anim., Ois. pl. 62. fig. 2 (1836-46).

C. coronatus, Schinz, Nat. Abbild., Vög. p. 267 (1833); Jard. Nat. Lib., Orn. iv. p. 187, pl. xxvi. (1834). 
Lipnnyx cristata, Vieill. Gall. Ois. p. 35 , pl. 2 10 18;t; Fin ger, Atl. Iög. fig. 239 (1864).

Rollulus cristatus, Gray. List Gen. B. 8) (i $9_{1} \mathrm{r}$ ); Blyth, Cat. B. Mus. As. Soc. p. 253 (1849); Bonap. C. R. xlii. p. Bifh (1856); Mamm. \& B. Burma, p. г ร1 (1875).

Rollulus roulroul, Gray, List Grall. B. iii. p. $42(18+4) ; i d$. Cien. B. p. 507 (1845); id. Hand-l. B. ii. p. 269 , n. ${ }^{\circ} 9726$ (1870); Wald. Ibis, 1872 , p. 382 ; Saluad. Ucc. Borizeo, p. 308 (187ti; Hume, Str. F. iii. p. 324 (1875); Sharpe, Ibis, 1876 , p. 51; Treedd. Ibis, 1877 , p. 322; Hume \& Davison, Str. F. vi. p. 448 (1878); Becc. Ann. Mus. Civ. Genov. xiii. p. 455 (1878); Salvad. t. c. xiv. p. 252 (1879); Sharpe, Ibis, 1879, p. 270; Hume, Str. F. viii. p. pp. 69, i I I (1879); Hume \& Marsch. Game B. ii. p. Io3, pl. 1879; Sharpe, P. Z. S. 1881, p. 8oo; Kelham, Ibis, 1882 , p. 4; Nicholson, Ibis, 18 \% 2, p. 65; \& 1883 , 1. 90; Blas. Verh. Ges. Wien, xxxiii p. 60 (1883); Oates. H. Burmah, ii. p. 330 (1883); Meyer, Vög-Skelet. pl. lxxxr. (1885); Büttikofer, $N$. Leyd. Mus. ix. p. 78 (1887); Everct, List B. Borneo, p. 200 (1889); Sharpe, Ibis, 1890 pp. I1 $10,28+$; Hos., Ibis, 1893 , p. 423; Osilv. Cat. B. M. 22. p. 225. (1893).

Hemipodius nigrifons, Drapiez, Dict. Sci. Nat. x. p. 562 , pl. 45. fig. 2 (I 845$)$.

Rollulus coronatus, Jerd. B. Ind. ii. p. 580 (1864); ve. Pels. Ibis,

R. rulrul, Brüggem. Abh. nat. Ver. Brem. v. p. 463 (1877).

(ryptonix roulroul, Snelleman, in Veth's Midden-Smeatra, iv, p. 46 (1887).

Rullulus roulroul, Elliot, $A u k$, viii. P I 5 (I 89 I).

Hab. Paragua, ?Borneo, Java, Sumatra.

\section{Gen. COIURNix, Monhing.}

5. C. coturnix, (Lin.) $\varangle \circ$ N. v. Codomiz.

(M. S T.)

La caille, Briss. Orn. i. p. 247 (1860); Buff. Hist. Nat. Ois. ii. p. 449, pl. xvi. (177 I); D.'Aubent. Pl. Enl. ii. pl. 49, n. ${ }^{\circ}$ I 7 o. 'Tetrao coturnix, Lin. S. N. i. p. 278 (1766); Gin. S. N. i. p)t. ii. p. 765 ( 1788$)$.

C. coturnix, Ogilo. Cat. B. M. 22 p. 231 (1893).

Hab. Europa, España, Asia, China.

\section{Gen EXCAILACTORLA, B?.}

6. chinensis, (Lin.) $\sigma^{\circ} \rho \mathrm{N}$ v. Pugong-tico.

The chinese quail, Edw. Glean. Nat. Hist. v. p. $77, \mathrm{pl} .247$ (1758); Lath. Gen. Syn. ii. pt. ii. p. $78_{3}$ (1783); id. Gen. Hish. viii. p. 318 (1823).

Tetrao chinensis, Linn. S. N. i. p. 277 (1766); Gm. S. N. i. pt. ii. p. 765 (1788).

Perdix chinensis, Lath. Fud. Orn. ii. p. 652 (I790); Vieil!. V. Dict. d'Hist. Nat. xxv. p. 260 (1817).

Coturnix chinensis, Bonn. Tabl. Encycl. Mét. i. p. 223, pl. $9^{\text {f). }}$ fig. 3 (i 79r). 
C. rxcalfactoria, Temm. Piy. et Gall. iii. pp. 516, 742 (1815); Steph. in Shazu's Gen. Zool. xi. p. 371 (1819)..

(. flavipes, Blyth, J. As. Soc. Beng. xi. p. 808 (1842); Gray, (ien. B. iii. p. $507(18+0)$.

( . chinensis, Gray. List of B. pt. iii. p. 39 (1844); id. Cat. Hodgs. ed. i. p. 128 (1846); id. Gen. B. iii. p. 507 (1846); layard, Ann. Mag. L. H. (2) xiv. p. 107 (1854); Swinkse, Ibis, $18^{\prime} 61$, p. 50; Legge, B. Cejlon, iii. p. 755 (1880); Parker, Ihis, 1886, p. 187 .

('. philippensis, Hodgs. in Gray's Zool. Misc. p. 85 (r844); id. lcon. ined. in Brit. Wus. nos. 119, 120.

Excaltactoria chinensis, Bonap. C. R. xlii. p. I 88 (1856); Maillard, Votes Réunion, p. I63 (1862); Jerd. B. Ind. iii. p. 591 (1863); Sicunhoe, Ibis, 1863 , p. 398; id. P. Z. S. 1863, p. 308; Gould, B. Asia, vii. pl. 6 (1867); Beavan, Ibis, 1868, p. 386; Swinh. Ibis, 1870, p. 360; id. I'. Z. S. 1871 , p. 401; Holdsw'. I'. Z. S. 1872 , p. 470 ; Hume, Nesis \& E. Ind. B. p. 553 (1873); Ball, Str. F. ii. p. 428 (1874); Legge, Ibis, 1874, 1. 26; Hume, Str F. iii. p 323; (1875); Oates, Str. F. iii. 1. 34.5 (1875); Blyth \& Walden, Cet. Mamm. \& B. Burma, p. I51 (1875; Davd \& Oitstal. Ois Chine, p. 397 (1877); Ball. Str. F. vii. p. $226(1878)$; Hume, Str. F. viii. p. Oy (1879); Oates, Str. F. viii. p. 167 (1879); Bidie, Str. F. ix. p. 209 (1880); Hume of Inglis, Str. F. ix. p. 258 (1880); Butl!n, str. F. ix. p. 423 (1880); Reid. Str. F. x p. 63 (1881); Weuden, Str. Fr. x. P. I65 (I88r); Oates, Str. F. x. p. 236 (1882); Danson, Str. F. x. p. 412 (1883); Kelham, Ibis, $188_{2}$, 1. 3; Uates, B. Burmah, ii. p. 334 (1883); Mäll.J.f. O. 1885, p. I60; Hume, Str. F. xi. p. 310 (1888; Hartert, J. F. (). 2889,1 . 434; Oates ed. Hume's Nests \& E. Ind. B. iii. p. Hir (1890); Ogilv. Cat. B. M. 22. p. 250 (1893).

Exiallactoria minima, Gould, l'. Z. S. 1859 , p. 128; id. B. Asia, vii. pl. $T$ 1867; Walden, T\%. Z. S. viii. p. 87 (1872); ix. p. 224 (1875); Saluad. Ann. Mus. Civ. Genov. vii. p. 675 (1875); x+iii. p. 9 (1882); id Orn. Pap. iii. p. 255 (1882); Pleske, biull. Acad. Pétersb. xxix. p. 536 (1884); Salvad. Agg. Orn. Pap. pt. iii. p. 195 (1891).

Synacus sinensis, Newton, Ibs, 1961 , pp. I $6,275$.

(oiurnix caineana, Swinh. Ibis, $1865, \mathrm{pp}$. 351, 542; id. Ibis, $1870, \mathrm{P} .360$.

C. (Excalfatoria) chinensis, Gray, List Gallina, Brit. Mus. p. ${ }^{3} 3$ (1867); id. Hand-l. B. ii. p. 269 (1870).

C. (Excalfactoria) minima, Gray, List Gallıne, Brit. Mus. p. o3 (i 867 ); id. Hand l. B. ii. p. 269 (1870).

Excalfactoria sinensis, Hume \& Druson, Stz. F. vi. p. $t+7$ (1878); Bingham, Str. F. ix. p. i $9^{5}$ (1880).

¿Excilfactoria sp., Saliad. Orn. Pap. iii. p. 257 (1882).

Hab. Luzon, Laguna, Jolö; Borneo, Formosa, China, Célebes.

7. Ex. lineata, (S:op.) of: O N. v. Fugong-tico.

(M. S. T.

Coturnix philippensis, Briss. Orn. i. p. $25+$ pl. xxv. fig. I ( 1760$)$.

La caille des Philippines, $D^{4} 1 u b u t$. Pl. Ent. ii. 1H. 5 I fig. 2. 
La petite caille de l'Isle de Luçon, Sonnerat. l'oj. N. Gumn. p. 54, pl. 24 (1776).

Manilla Quail, Lath. Gen. Syn. ii. pt. ii. p. 790 ( 1783$)$; id. Gen. Hist. viii. p. 321 (1823).

Oriolus lineatus, Scop. Del. Flor. et Faun. Insubr- ii. p. $87(1-80)$.

Tetrao manillensis, Gmel. S. N. i. pt. ii. p. $j^{6}+(1488)$.

Perdix manillensis, Lath. Ind. Orn. ii. p. 055 (1790).

Coturnix manilensis, Bonn. 7 ab. Encycl. Méth. i. p. 221, pl. 97 fig. 4 ( $179 \mathrm{r}$ ).

Tetrao chinensi, Hayes, Osterl, Menag. pl. 50 (1794).

T. sinensis, Raffl. Tr. Linn. Soc. xiii. p. 324 ( 1822 ).

Chestnut-bellied-partridge, Undulated Partridge, Lath. Gen. Hist. viii. p. 305 ( 1823$)$.

(oturnix nana, Schinz, Nat. Abbild. Vög. p. 275 (1 133).

Synoicus? chinensis, Gould, B. Austr. v. pl. 92 (1848); l)iggles, B. Austr. ii. pt. xx. pl. 96 (1860).

Coturnix chinensis, Sclat. P.Z. S. $186_{3}$, p. 221.

lixcalfactorid chimeńsis? Walden \& Layard, Ibls, $1872, \mathrm{P} .006$ Salvat. Ucc. Borneo, p. 3 I I (1874); Walden, Tr. Z. S. ix. 224 (1875); Sharpe, Ibis, 1878 , p. 419 id. P. Z. S. 1879, p. 350 Nichols. Ibis, 1881 , p. 155, I883, p. 255. Kutier, J. F. O. 1883, pi). 293, 316; Wardlaz-Ramsxy, Ibis, 1886 , p. 160; Everrett. P. Z. S. 1889 , p. 225. Whitehead, Ibis, 1890 p. 57; Sharpe, Ibis, 1890, pp. I 40, 284; Ogilv. Cat. B. M. 22 p. 253 (1893).

Excalfactoria australis, Gould. Handb. B. Austr. ii. p. I97 (1865); Ramsay, Ibis, 1868 , p. 279 id. P. Z. S. 1876 , p. I19 id. Tab. List. Aust. B. p. I9 (1888); North. N. \& E. B. Alustr. p. 29I (I 889).

Coturnix (Excalfactoria) australis, Gray, List Gallina. Brit. Mus. p. 63 (1867); id. Hand-l. B. ii. p. 269 (r87o).

C. sinensis, Jwinh. Ibis, 1869 , p. 348 .

Synoicus (Excalfactoria) sinensis, Ramsay, P. Linn. Soc. N. S. $W$. i. p. I 86 ( 1876 ).

Hab. Luzon, Manila, Navotas, Bulacan, Lolomboy, Lagúna, Calauan, Ilocos Norte, Abra, Panay, Cebú, Masbate, Calamianes, Culión, Paragua, P. Princesa, Taguso. Joló; Borneo, Java, Sumatra, Australia.

7. Gén. LOphURA, Fleming.

8. L. rufa, Raffl. $\sigma^{\rightarrow}$ N. v. Fassan de Sumatra

(M. S. T.)

Phasianus ignitus, Raff. 7r. Lm. Soc.; id. rufus, ibid. p. 23 r 1022 .

J. rufa, Ogilo. Cat. B. M. 22. p. 286 (1893).

Hab. Sumatra, Malaca, Borneo

8. Gén. TRAGOPAN, Cur.

9. T. satyra, (Lin) $0^{7} \bigcirc$ N. v. Faisan cornudu.

(II. S. T.)

Meleagris satyra, Lin. S. $N$. i. p. $229(1-96)$.

Tragop n satyrus, Cur. Regr. An. i. P. 479 (1629); Tem. Pl. Cal. 1. p) $13,1+\left(\mathrm{n} .^{\circ} 5+3,544\right)$ 
1. satyra, Ogilv. Cat. B. M. 22. p. 271 (1893).

Hab. India, Nepal.

10. T. temminck1, Gray. $\sigma$ N. v. Faisan de Temminkci. (M. S. T.)

Satyra temmincki, J E. Gray, Ill: Ind. Zool. i. pl. 50 (1830-3a).

1. timmincki, Ogilv. Cat. B. M. 22. p. 275 (1793).

Hab. China.

\section{Gen GENNÆUS, WAGL}

tr. G. nyctemerus, (Lin.) \& N. v. Facian plateado. (M. S. T.)

Phasianus nycthemerus, Lin. S. N. i. p. 272 (1766); Gm. S. N. i. pt. ii. p. $743(1788)$.

Fasian noire et blane de. la Chine, $D^{\prime}$ Aubent. $P l$. Einl. pls. 123 , 134.

(i. nycthemerus, Ogilv. Cat. B. M. 22. p. $3^{\circ 7}$ (1893).

Hab. China, Fokien.

1o. Gén. PHASIANUS, Iis.

12. Ph. colchicus, Lin. oo N. v. Faisan comun. (M s $\mathrm{T}$.,

le Fasian, Briss. Orn. i. p. 262 (г760); D'Aubent. Pl. Eul. pls. $121, \quad 122$.

Phasianus colchicus, Lin. S. N. i. p. 27 I (1766); Gm. S. N. i. - pt. ii. p. 741 (1788); Ogilv. Cat. B. M. 22. p. 330 (1893).

Hab. Inglaterra, Alemania.

I 3 Ph. torquatus, Gm. oo N. v. Faisan de collar. (M. S. T )

Ph. torquatus, Gm. S. N. i. pt. ii. p. 742 (1788); Ogilv. Cat. B. M. 22. p. 331 (1893).

Hab. China, Canton, Sanghai.

II. Gén. Chrysolophus, Gray.

14. Ch pictus, (Lin) $\sigma \circ$ N. v. Faisan dorado.

(M. S. T.)

Phasianus pictus, Lin. S. N. i. p. 272 (1866); Gm. S. N. i. pt. ii. p. 745 ( 1788$)$.

Le faisan dorè, $D^{\prime}$ Aubent, $P l$. Enl. pl. 2 r 7 .

Chrysolophus pictus, J. E. Gray, Ill. Ind. Zool. ii. pl. 4I. fig. 2. (1834); Ogilv. Cat. B. M. 22. p. 339 (1893).

Hab. China.

12. Gén. GalluS, Lin.

15. G. stramineicollis, Sharpe. N. v. Labuyo.

G. stramineicollis, Sharpe, P. Z. S. 4879, p. 317; Ogilv. Cat. B. $M .22 . \mathrm{p} 343$ (1 893).

Hab. Joló. 
16. G. g.llus, Lin. o $\bigcirc$ N. v. Labuyo.

(M. S. T.)

Phasianus gallus, Lin. S. N. i. p. $270(1766)$; Raffl. 7r. Lint. Soc. xiii. p. $319(1822)$.

Grande Caille de la Chine, Sonn. Voy. Ind. Orient. ii. P. I7t $(1782)$.

Hackled Partridge, Latk. Gen. Syn. ii. p. 766 , pl. 6n $\left(1 ; x_{3}\right)$; id. Gen. Hist. viii. p. 307, pl. 129 (1823).

Tetras ferrugineus, Gm. S. $N$ i. pt. 768 (1788).

Perdix ferruginea, Lath. Ind. Orn. ii. p. 651 (1790).

Coturnix spadnea, Bonnat. Tabl. Eineycl. Méth. i. p). 228 (179s). Ciallus bankiva, Temm. Pig. et Gall. ii. p. 87 (I812); iii. p. 054 (1815); Steph. in Shaw's Gen. Zool. xi. p. 198 (1819); Horst. 'r. Linn. Soc. xiii. p. 185 (1822); Vieill. 7abl. Encycl. Méth. i. 1. $3^{6}+(1823)$; Griff.ed. (uv. iii p. 20 (1829); Guérin, Icon. Reg. Anim., Ois. p. 26, pl. 42. figs. 2, $2 a$ (18 29-38); $J$. E. (iray, Ill. Ind. Zool. i. pl. 43 fig. 3 (1830-32) Less. Traile "'Orn. p. 49I (1831); Schinz, Nat. Abbild. Vög. p. 342, pl. 9t (1833); Jard. Wat. Lib. Orn. iv. p. 175, pl. (1834); Hodgs. in Gray's Zool. Misc. p. 85 (1844); Gray, List. of. B. pt. iii. Gall. p. $27(1844)$; id. Gen. B. iii. p. 499 187.5. id. Cat. Hodgs. ed. i. p. I 25 (1846); Schinz, Nat. Vög. p. 145, pl. ba ( 853 ); Adams, P. Z. S $85^{8}$, p. 498; Wallace, P. Z. S. 1863 , p. 486; Sclat. List. of. Phas. p. 10 (1863); Blyth, Ibis, 1867 , p. 156; Melliss, Ibis, 1870 , p. 103; Wald. Tr. Zool. Soc. viii. pt. 2, p. 86 (1872); Hartl. \& Finsch, P. Z, S. 1872 , p. 89. ro3; Trueedd. P. Z. S. 1878 , p. 953; 1879, p. 73;. Meyer, Ibis, 1879 , p. г 38 ; Grillem. P. Z. S. 1885, p. 272; Blas. Ibis, 1888, p. 374; Shufeldt. J. Comp. Med. \& Surg. Oct. $1888,3+$ pp. 3o figs.; Blas. Ornis, 1888 , p. 317 ; Everett, list. B. Born. p. 199 (1889); Sharpe, Ibis, 1890 , p. 57 .

(i. ferrugineus, Blyth, Ann. Mag. N. H. xx. p. $387($ I $8+7)$; i. 1. 455 (1848); id. Cat. Mus. As. Soc. p. 242 (1849); Adamus. I. Z. S. 1859 , p. 185; Irby, Ibis, 1861 , p. 234. Jerd. B. Ind. iii. p. 536 (1863); Gray, List Gallina Brit. Mus. p. 37 (1867); Blyth; 1bis, 1867 , p. 154; Beaban, Ibis, 1868 , 1. 381; Brooks. Ibis, 1869, p. 60, Bulger, Ibis, 1809 , p. I70; Swinhoe, Ibis, 1 $\$ 70$, p. 357. Gray', Hand-l. B. ii. p. 26I (1870); Swinh. P. Z. S. 1871, p. 399; Elliot, Mon. Phas. ii. pl. 32 ( 1872$)$; Hume. V. \& E. Ind. B. p. $528(1873)$; Ball. S/r. t. iii. p. $+26(1874$; Hume, Str. f. ii. P. $4^{82}(\mathrm{i} 874)$; Blyth. \& Walden, Cat. Mam. \& B. Burma, p. 149 (1875); Hume, Str. f. iii. p. I7 (1875); Armstr. Str. f. iv. p. 331 (1876); Finsch, P. Z. S. 1877 , p. 780 ; Hume \& Inglis, Str. f. v. 44 (1877); Oates, Str. it. v. p. I64 (1877); Wardl. Rams. Ibis, 1877 , P. 468; David\& Onstal. Ois. Chine, p. 420 (1877); Marsh. B. Nest. Int. p. 59 (1877); Hume, \& Marsch. Game B. Ind. i. P. 217, pl. $(1878)$; Auders. B. W. Yuman, D. $669(1878)$; Hume \& Da¿'son, Str. t. vi pp. 442 \& 52 I (1878); Ball, Str. f. vii. P. 225 (1878); Hume, Str. F. viii. p. 68 1879; Scully, Str. F. viii. p. 348 (1779); Salvad. Ann. Mus. Civ. Gen. xiv. p. $25:$ (1879); Binghani, Str. F. ix. p. 895 (1880): Fasson, Sitr. F. i.. p. $205(1880)$; Mäll. J. F. O. 1882 , 1) +32; Keltam, Ibls, 
1882 , p. 8; Vichols. IBis, $18^{2} d^{2} 2$, p. 65; O.tes, Str. F. x. p. 2361882 . id. B. Burmah, ii. p. 322 (1883); Vichols, Jhis, 1883; p. 255; Marsh. Ihis, I $8 \delta_{+}$, p. 423 ; Büttiknt. $N$. deyd. Mus. ix. p. $78(1887)$; Tavlor, Sir. F. x. p. 5.38 (1887); Hame, Sitr F. xi p. $30+(1888) ;$ Hartert. J. A. O. 1989 , pp. 279, 434 ; Tegetm. Ibi, i89o, p. 304; Oates, ed. Hume's Vests. \& liggs, iii. p. 417 (1890).

(i. gallus, Ogito. Cat. B. M. 32. p. 34t. 189.3 .

Hab. Luzon, Manila, Cavite, Laguna, Pampanga Hataan, (agayan, Ilocos, Abra, Guimarás, Cebú, Mindoro, Baco, Paragua, P. Princesa, Calamianes, Cuyo, Balabu, Samar. Borongran, Masbate, Romblon, Sibuyan, I'ablas, Tawi-tawi, Neyros, Panay, Paranas, Basilan, Mindanas, Surigao, Butuan, Zamboanga, Joló; Java, Célebes, Sumatra.

17. G. varius? Shaze. 0 . N. v. Cadlaganon.

(M. S. T.)

Phasianus varius, Shaw. et Nodd. Nat. Misc. x. pl. 35.3.

G. varius, Griff. ed. Cuv. iii. p. 21 (1820); Less. Traite d'Orn. p. 492 (1831); Gray, List. of. B. pt. iii. Gall. p. 27 (1844); id. Gen. B. iii. p. 499 (1845); Sclat. List. of Phas. p. in (1863); Blyth, Ibis, 1867, pp. 307, 308; Gray, List Galline Brit. Mus. p. 40 (1867); id. Hand-l. B. ii. p. 26I (1870); Elliot, Mon. Phas. ii. pl. 35 (1872); Kelham, Ibis, 1881, p. 3; Hume, Str. F. x. p. 68 (1887); Ogilv. Cat. B. M. 22, p. 352 (1897).

Gallus furcatus, Temm. Pig. et Gall. ii. p. 26r (1813), iii. p. 662 (1815); Steph. in Shaw's Gen. Zool. xi. p. 215 (1819); Vieill. Tabl. Encycl. Méth. i. p. 364 (1823); Temsn. Pl. Col. v. pl. 4 (1829); Gray, Ill. Ind. Zool. i. pl. 43, fig. 2(1830-32); Schinz, Nat. Abbild. Vög, p. 242 ( 1833 ); Jard. Nat. Lib. Orn. iv. p. 184, pl. x. (1834); Schinz. Nat. Vög. p. 145 (185.3); Wallace, P. Z. S. 1863 , p. 486.

Gallus javanicus, Horsf. Tr. Linn. Soc. xiii. p. 185 (I 822 ).

Hab. Paragua, Inagauan, Mindoro, Baco; Java.

18. G. domesticus, (Lin) $\sigma \rho$ N. v. Gallo comun.

(M. S. T.)

Hab. Todas las islas.

19. G. d. patavinus, (Lin.) o. N. v. G. de moño. (M S. T.)

Hab. China, Japón.

20. G. d. cochinchinensis, o\& N. v. G. Cochinchina. (M. S. T)

Hab. Cochinchina.

2I. G. sonnerati, $\left(T_{e} e m\right.$. $)$ o o N. v. Manoc de Joló. (M. S. T.)

Coq. et Poule sauvage des Indes, Sonn. Voy. Ind. Orient. ii. p. I 48, pls. 94, 95 (1782).

Wil Cock, Lath. Gen. Syn. ii. p. 698 (i783).

Phasianus gallus, Scop. (nec Linn.) Del. Flor. et Fann. Insubr. pt. ii. p. 93 (1786); Gm. S. N. i. pt. ii. p. 737 (1788); Lath. 
Ind. Orn. ii. p. 625 (1790); Bonnat. Tabl. Encycl. Meth. i. p. 180 , pl. 86, flgs. 4,5 (1791).

Sonnsrat's Wild Cock, Lath. Gen. Hist. viii. p. 181 (1823).

Gallus sonneratii, Temm. Pig et Gall. ii. p. 246 (1813) p. P. 659.(1815); Steph. iu Shaw's Gen. Zool. xv. p. 200, lii. 12 (1819); Temm. Pl. Cot. v. pls. I \& 2 [nos 232, 233] (1823); Vieill. Gal. Ois. ii. p. 26, (1825); Griff. ed. C'uv. iii. p. I9 (1829); Less. Traité, d'Orn. p. 492 (1831); Sykes, P. Z. S'. 1832, p. 151 [W. Ghauts]; Schinz, Nat. Abbild. Vög. p. 24.3, pl. 94 ( 1833 ); Jard. Nat. Lib, Orn. p. 186, pls, xv. \& xii. (1834); Gray, List of B. pt. iii. Gall. p. 27 (1844); id. Genl. B. iii. p. 499 (1845); Blyth, Ann. Mag. N. H. xx. p. 388 (1 847 ), i. p. 455 (1848); id. Cat. Mus. As. Soc. p. 243 (1849'; Schinz, Nat. Vög. p. I45, pl. 69 (1853); Burgess, P. Z. S. 1855 , p. 29; Sacc, Rev. Zool. (2) xiv p. I I, pl. 3 (1862); Jert'. $B$. Ind. iii. p. 539 (1863); Sclat. List of Phas. p, I0 (186.3; Bulger, P. Z. S. 1866 , p. 57 I [Nilghiris]; Gray, Lst. (iallina Brit. Mus. p. 39 (1867); Blyth, Ibis, 1867, pp. 164, 31,; Gray, Hand-l, B. ii. p. 26I (1870); Elwes, Ibis, 1870 , p. 52.4 Elliot, Mon. Phas. ii. pl. 34 (1872); Lloyd, Ibis, 187.3, p. 4\%); Hume, N. \& E. Ind. B. p. 531 (1873); Butl. Str. F. iv. 1. 5 (1876); Fairb. Str, $F$. iv. p. 262 (1876); Hume, Str. F. iv. p. 404 (1876); Butl. Str. F. v. p. 222 (1877); Fairb. Si. F. v, p. 409 (1877); Marsh. B. Nest. Ind, p. 59 (1877); Gould, B. Asia, vii, pl. 56 (1877); Hume, \& Marsh Gome. B. Int. i. p. 23I, pl. (1878); Davidson \& Wend. Sr. F. vii. p. Bt, (1878); Butl. Cat. B. Sind, p. 53 (1879) Mc. Iroy, Str. I. viii. p. 493 (1879); Vidal, Str. F. ix. p. 76 (1880 ; [S'. Konkan); Btler, Str. F. ix.p. 205 (1880); [Belgaum], p. $42 \mathrm{I}$ ( I880); Davidso", Str. F. x. p. 3 I6 (i 88a); Davison, Str. F. x. p. 409 (188.;'; Swinh. \& Barnes, Ibis, 1885 , p. Taylor, Str. F. x. p. 464 (1887); Terry, Str. F. x. p. 479 (1887); Oates, ed. Hume's Nests \& Eggs, iii. p. 420 (1890).

Phasianus indicus, Leach, Zool. Misc. ii. p. 6, pl. 6r (1815); Ogilv. Cat. B. M. 22, p. $35^{\circ}$ (1893).

Hab. Joló.

13 Gén. POLYPLECTRON, TFm.

23. P. emphanes, $S c l$ O .0 N. v. Tandican.

(M. S. 'T.)

P. emphanes, Sclat, Ib s, 1878 , p. 386; Tuv. P. Z. S. 1878 , p 6.23.

P. napoleonis, Tuv, P. Z. S. 1878 , p. 792; Sharpe, Ibis; 1888 , p. 203; Blasius, Ov. 1888 , p. 317 ; Fiveret. List. B. Rorn. p. 198 (189); Witehead, Ibis, 1890, p. 57.

P. nehrkornæ, Blas. Mitth. orn. Ver. Wien. 1891, p. 1.; id. J. f. O. 1891 , p. 10; Ogilv. Cat. B. M. 22. p. 360 (1891). Hab. Paragua, Puerto Princesa, Balabac.

2. P. napoleonis, Le'ss. ơ N. v. 'Tandican.

(M. S. T.)

P. napoleonis, Less. Traité d'Orn. pp. 487, 650, (183) 1 ; id. 
Coup. de Buff. viii. p. 323 (1836); Gray, Gen. B. iii. p. 495 (1845); id. List. Goll. Br. Mus. p. 25 (1867); Ogilu. Cat. B. M. 22, p. 361 (1893).

P. emphanum, Tem. Pl. Col. v. pl. 18 n. 540 (1831); fard. Nat. Lib. Orn. iv. p. 159, pl. 5 (1834); Gray, Hand-l. B. 1. 257 (1870); Elliot, Mon. Phas. i. pl. 9. (1872); Salv. Ucc. Born. p. $3 \circ 4$ (1874); Ocut. Bull, Soc. Phil. vi. p. 259 (1882).

Eimphania napeleonis, Bp. C. R. xlii. p. 878 (1856).

P. emphanes, Scl. List. of Phas. p. 12 (186.3).

Hab. Ralabac, Paragua, Luzón.

14 Gen. ARGUSIANUS, RaF.

24. Ar. argus, Lin. $\sigma \rho$ N. v. Faisan argos.

(M S. T.)

Phasianus argus, Lin. S. N. i. p. 272 (1766); Gm. S. N. 1. pt. ii. p. 742 (1788).

Argusianus argus, Titze. Ibis, 1877, p. 322; Ogilv. Cat. B. M. 22. p. 363 ( 1893 ).

Hab. Sumatra, Malaca.

is Gén. PAVO, Lis.

25. P. cristatus, Lin $\sigma \circ$ N. v. Pavo real.

(M. S. 'T.)

P. cristatus, Lin. S. $N$. i. p. 267 (1766); Gm. S. $N$. i. pt. ii. p. 729 ( 788 ); Ogilv. Cat. B. M. 22. p. 368 (I 893).

Le Paon, D'Aub. Pl. Enl. pls. 433, 434.

Hab. India, Ceilan.

26. P. muticus, Lin. ơ N. v. P. especifero.

(M. S. T.)

Hab. Java.

16 Gién. MELEAGRIS, I.IN.

27. M. gallo-pavo, Lin. $\sigma \circ$ N. v. Pavo comun.

(M. S. T.)

Hab. Filipinas, (introd.)

17. Gén. NUMIDA, Iis.

28. N. meleagris, Lin. ơ 0 N. v. La pintada.

(M S. 'T.)

N. meleagris, Lin. S. N. i. p. 273 (1766); Gm. S. N. i. pt. ii. p. 744 ( I788); Ogilv. Cat. B. M. 22. p. 375 (I 803).

Le Peintade, D'Aub. Pl. Lnl. ii. pl. 8o, n. ${ }^{\circ}$ 108.

Hab. Filipinas (introd.)

29. IN id var, alba, ơ N, v.

Hab. Luzón, Manila. 
18. Gén. L.OPHORTYX, Br.

30. I. californicus, Shaze. ơ N. v

(M. S. T.

Tetrao californicus, Shaze et Nodd. Nat. Mus. ix. pl. 345 (1797).

L. californicus, Ogilv. Cat. B. M. 22 p. 400 (1893).

Hab. California.

Fam. MEGAPÓDIDAS.

I. Gén. MEGAPODIUS, Q E'T Gaim.

1. M. cumingi, Dillze. $\sigma \rho$ N. v. Tavon, Talun, Taliuac-uac, (M. S. M.)

Megapodius rubripes, Wagler, Isis, 1829 , p. 737 :

M. rufipes, Gray, List of $B$. Gall. p. 2 I (1844).

M. cumingii, Dillwyn, P. Z. S. 1851 , p. I 18, pl. 39; Motley of Dillwyn, Contr. N. H. Labuan, p. 32, pl. 7 (1855); Gray, P. Z. S. 1861, p. 29:, I064, p. 43; v. Martens, J. f. O. 1866, p. 26; Schl. Ned. Tidjdschr. Dierk. iii. p. 263 (1866); Gray, List Gallina Brit. Mus. p. 21 (1867); id. Hand-l. B. ii. p. 255 (1870); Walden, Trans. Zool. Soc. ix. pp. 225, 25I (1875); Tweedd. P. Z. S. 1878 , p. 624; Sharpe, Ibis, 1879, p. 267; id. P. Z. S. 1879 , p. 349, p. 322; Oustal. Ann. Sci. Nat. xi. p. I I (188I); Whitehead, Ibis, $18 \delta 8$, p. 4I I; Sharpe, Icis, 1888 , p. 203; Blasins, Ornis, 1888 , p. 217 ; Everett, P. Z. S. 1889 , p. 225; id. J. As. Soc. Str. Br. p. 197 (1889); Sharpe, Ibis, 1890 , p. I4I; Ogilv. Cat. B. M. 22. p. 449 (1893). M. gilbertii, Gray, P. Z. S. 1861 , p. 289; 1864, p. 43; id. Hand-l. B. ii. p. 255 (1870); Walden, Trans. Zool. Soc. viii. p. 87 (1872); Meyer, Ibis, 1879 , p. I 38 ; Oustal. Ann. Sci. xi. p. 107 (1881); Guillem. P. Z. S. 1885, p. 559.

M. lowi, Sharpe, P. Z. S. 1885, p. I I I.

M. pusillus, Treeedd. P. Z. S. 1877 , p. 765 , pl. lxxviii.

M. dillwini, Tweedd. P. Z. S. 1877, p. 766, I 878, p. 953, I879, p. 73; Oustal. Ann. Sci. Nat. xi. p. I I8 i I8 (1881).

M. dillwini, Steere, List Berds. \& Mamm. Philippines, p. 25 (I 890$)$.

Hab. Luzon, Laguna, Cagayan, Ilocos, Tablas, Romblon, Sibuyan, Labuan, Mindoro, Cuyo, Balabac, Dumalon, Cebú, Basilan, Joló, 'Tawi-tawi, Mindanao, Zamboanga, Paragua, P. Princesa, Samar, Paranas, Masbate, Marinduque; Borneo, Célebes.

2 M. laperousii, (Tem.) \& N. v. Tavon.

Megapodius la Perouse, Quoy. et Gaim. Voy Uranie. p. i 27, pl. 33. (1824).

M. laperousii, Tem. Pl. Col. v. livr. 69, p. 3 (1826); Wagler, Isis. 1829 , p. 736; Less. Traité d'Orn. p. 478 (1831); id. Compl. de Buff., Ois. p. $255 \mathrm{t}$ pl. 9. fig. 2 (1840); Gray, Cat. B. Trop. Is. p. 46 (1859); id. P. Z. S. 1861, p. 289, 1864, 
p. +2; Reichenb. Tauben, pp. 5, 190, pl. 276. fig. 1532 (1862); Schl, Mus. Pays-Bas, viii. p. 65 (1880); Oustal. Aun. Sct. Nat. xi. p. г 38 (1881); id. le .iat. 1889 , p. 261; id. Ans. Sai Nat. Nat. xi. p. 196 (1891); Ogilv. Cat. B. M. 22. 1. $+60(1893)$.

M. la peyrouse, Less. Man. dOrn. ii. p. 221 (1828).

M. senex, Hartl. P. Z. S. 1867 , p. 830; Hartl. \& Finsch, P. Z. S. 1858, pp. 7, 118, 1872, pp. 89. го3; iid. Cat. Mus. Godeff. v. p. II (187t); Finsch, J. Mus. Gudoff. iv pt. viii. p. 29 , pl. v. figs. 2. \& 3 (1875); Schl. Mus Pays-Bas, viii. p. 65 (1880); Oustal. Anu. Sci. Nat. xi. p. 1 40 (1881); Schmeltz. Ethn. Abth. M. G. p. 407 (1801); Oustal. de Vat. 1880 , p. 261; Wiglesz. Abh. Mus. Dresd. 1890-01, n.0 6, p. $53(1802)$. Megapodius perousii, Wiglesi'. Abh. Mus. Dresd. 1890-91, n." 6, p. 58 (1892).

Hab. Marianas, Palaos.

\section{Orden: HEMIPODIOS.}

\section{Fam. TURNÍCIDOS.}

I. Gen. TURNIX, Boxsat.

I. T. pugnax, ( $\left(e_{e} m\right)$ N v. Pugong.gubat.

(M. S. '1.)

Hemipodius pugnax, Tem. Pig. et Gall. iii. Pp. 6r2, 794 (179r); Schime, Nat. Abb.ld. Vög. p. 268 (1833); id. Nat. Vögel. 1). I65, pl. 89 (1853).

Ortygis luzoniensis, Horsf. Trans. Linn. Soc. xiii. p. 185 (1822). Tetrao luzoniensis, Raffl. Trans. Limn. Soc. xiii. p. 33+ (1822). Jighting Quail, Lath. Gen. Hist. viii. 1. 34 I (1823).

'Turnix pugnax, Blyth, Ib:s, 1867, p. 309; Nibholson, Ibis, 1881 , P. I 55, Grant, Ibis, 1889 , pp. 449, 450, $45^{8}$ (1893); Ogili'. Cat. B. M. 22. p. 534.

'T. taigoor, Holdsw. P.Z. S. 1872 , p. 470; Legge, Ibis, 1874 , p. 26, 1875, p. 400; 2 d. B. Ceylon, iii. p. 761 (1880).

Hab. Luzón, Manila, Narotis, Laguna, Calauan, Cagayan, Ilocos, Mindoro.

2 T. fasciata, (Tem) $\delta$ N. v. P'ugong gubat.

(M. S. T.)

Hemipodius fasciatus, Tem. Pig. et Gall. iii. pp. 6r+, 757 (1815).

'T. fasciata, Gray', Hand.l. B. ii. p. 270, n." 9740 (1870); Treedd. P. Z. S. 1877, p. 701; Ramsay, Orn. Works. Tweedd., App. p. 659 (188I); Grant, Ibis, 1889 , Pp. 449, 450, 459. Ogilv. Cat. B. M. 22. p. 535 ( 1893 ).

T. nigreesens, Tweedd. P. Z. S. 1877, p. 765; Ramsay, Orn. Works. Tweedd., App. p. 659 (1881); Sharpe, Ibis, 1989, p. 203; Whitehead, Ibis, 1890, p. 58 .

'T. haynaldi, Blasius, Ornis, iv. p. 317 ( 1888 ).

Hab. Luzón, Manila, Narotas, Laguna, Calamba, Cebú, Ne- 
gros, S. Antonio, Masbate, Panay, Sibuyan, Calamianes, Culion, Dumaguete, Paragua, P. Princesa.

3 T. ocellata, (Scop.) ơ $\bigcirc$ N. v. Pugang-daan.

(il. s. ' ' )

(aille de l'Isle de Luçón, Sonn. N. Guinea, p. 5t, pl. 23 (1770).

Oriolus ocellatus, Scopoli, Del. Flor. Faun. Insubr. pt. ii. p. 88 ( 1786$)$.

'Tetrao luzoniensis, Gm. S. N. i. pt. ii. p. 767 (1788).

Perdix luzoniensis, Lath Ind. Orn. ii. p. 656 (1790).

Hemipodius, thoracicus, Temm. Plg. et Gall. iii. pp. 622, 755 (1815).

'Turnix rufus, Vieillot. and. ed. Nouv. Dict. dHist. Nat. xxivi. p. $48(1823)$.

Ortygis ocellata, Meyer, Nov. Act. Acad. C. L.-C. Nat. Curios. xii. Suppl. Primum, p. 101, pl. I7 (1834).

'Turnix ocellatus, Gray, Gen. B. iii p. 511 (1847).

Hemipodius ocellatus, $v$. Martens, $J . f . O . \quad 1866$, p. 26.

'Turnix ocellate, Gray, Hand-t. B. ii p. 271 . n. ${ }^{\circ} 9750$ (1870); ¿Walden, Ibis, 1872, p. 107; id Tr. $Z$. S ix. pp. 224, 25i (1875); 7weedd. P.Z. S. 1877 , p. 7oo; Ramsay, Orn. Works. Tweedd., App. p. 659 (1881); Grant, Ibis, 1889 , pp. +52, 469; Ogilv. Cat. B. M. 22. p. 536 (1893).

Hab. Luzón, Manila, Montalban, Laguna, Calauan, Cavite, Sta. Cruz, Naic, Batangas.

\section{Orden: CORREDORAS.}

\section{Fam. CASUÁRIDAS.}

I Gén. CASUarius, Lin.

I C. emeu, Lath. P. E. 313 N. v. Casuario de casco. (M. S T',

Hab. Ceram.

2. C. bicarunculatus, Sclat. Ð N. v. C. de carúncuculas. (M. S. T)

C. Sclat. Tr. Z. S. iv. pl. 37.

Hab. N." Guinea.

\section{Orden: ZANCUDAS.}

Fam. OTÍDIDAS.

I. Gên. OTIS, LıN.

I. Otis tarda, $L . \sigma^{\prime}$ N. v. Avutarda.

(ii. S. '1')

Hab. Europa, Africa. 


\section{Fam. EDICNÉMIDAS.}

\section{Gén. ORTHORAMPHUS, SAI.}

I. O. magnirostris. (Gerff) $\sigma_{0}$ N. v Tilin-tilin, Tubalabang. (M. S. T.)

()elicnemus magnirostris, Geoff. in Vieill, N. D) xxii, p. 231 (1818); Tem. Pl. Col. 387 (1826); S. Müll., Verhandl. Nal. (jesch. Nederl. Oiverz. Ber. Land-en Volkenle. p. 33 (1839-41); Schleg. Mus. P. B. Cursores, p. 23 (1865).

Charadrius magnirostris, Wag!. Syst. Ao. Gen. Charadrius, esp. 3 $(1827)$.

Ch. griganteus, Licht. in Wagl. Isis, $1829, \mathrm{p} .647$.

Esacus magnirostris, G. R. Gray. List Gén. of. B. 2 ed. p. $8_{3}$ $(18+1)$; d. Cat. B. Neq. Cintu. p. 50 (1859); id. Hand-l. iii. p. io espe. $994^{8}$ (1875); Gould. B. Aust. vi. pl. 6. id. Hands-Austr. ii. p. 213 (1865); Bp Compt. Rend. xliii. p. 4 it ('s). 29 (1856); Sclat. Pr. Lin Soc. ii. p. 169 (1858); Wald. Tr. Zool. Soc. viii. a p. 91 (1872).

Orthoramphus magnirostris, Salo. Cat. Vic. di Borneo, p. 312 $(1874)$.

Hzb. Paragua, P. Princesa, Samar, Borongan, Paric, Joló; Célebes, Java.

\section{Fam. CHARÁDRIDAS.}

I. Gén. SQuaterola, Cuv.

I Sq. helveticz. (Briss.) $\mathrm{N}$ i Tuhec

(M. S. ' $\mathrm{i}$ )

Vanellus helveticus, Briss Av. 5. p. $106 \mathrm{n}^{\circ} 4, \mathrm{t}$ io, f. 1.

Tringa helvetica, L.in. S. N. p. 250 (1766); Gm. S. N. ii. p. 67 (9.

Vanellus melanogaster, Bechst. Horsf. Tr. Lin. Soc. xiii. p. 186 ( $182 \mathrm{I})$.

Vanneau de Suisse, Buff. Hist. Nat. Ois. 8. p. 6o; Pl. Enl. n." 853 .

Tringa subtridactyla, Hassel. Iter Palestinum, p. $397, \mathbf{n}^{\circ} 28$.

Sq. helvetica, Sav., Mottl. et Dilliv. Contr. Nat. Hist. of Labuan, p. $5^{5}$ (1855); Sivis, sandpiper, Arct. Zosl. 2 p. 478, n. ${ }^{\circ} 396$; Lath. Sym. iii. p. I67, n." Io; G. R. Gray, Hand-l. iii. p. 13, esp. 9980 (1871); Wald. "et Layard, Ibis, 187a, p. 105; Salo. Cat. Ucc. Borneo, p. $3^{1} 3$ (1874); Brehm, Vid.t de los animales, 4. p. 347 ( I 88 I

Hab. Luzon, Manila, Navotas, Samar, Paric, Negros, Siquijor, Paragua, P. Princesa, Calamianes, Cuyo, Mindanao; Borneo, Java, China.

2 Sq. grisea, (Briss) o $00 \mathrm{~N}$ v Tarinting

(M. S. T.)

Vanellus griseus, Briss. Av. 5, p. roo, n. ${ }^{0}$ 2. t f. 1 .

Pluvialis cinerea, Bell. Av. 62, Aldr. Orn. 3. p. 333.

Vanneau plurier, Buff. H. Nat. des Oiss. 
V. gris; Buff. Pl. Enl. n. 854 .

'Iringa squaterola, Gm. S. N. 2. p. 628, esp. 23 (1889).

Hab. Luzon, Manila, Samar, Borongan, Paragua, P. Princesa, Europa.

3. 8q. varia, (Briss.) o o N. v. Matang-vaca.

(M. S T.)

Yanellus varius, Briss. Av. 5. p. 103, n. $^{\circ}$ 3. t. 9 f. $z$.

Vanneau varié, Buff. Pl. Enl. n. ${ }^{\circ} 923$.

Tringa varia, Gm. var. b. S. N. 2. p. 628 (1789); Brehm. I. An. 4. p. 347 .

Hab. Luzon, Manila, Navotas, Laguna, Paragua, P. Princesa, Samar, Paranas; Europa.

\section{Gén. iblenopterus, keiche.}

4 B. cayennensis, Gm. (B. 836) ๖ N. r. Avefria de Cayena (M. S. T.) Hab. Cayena. Brasil.

3. Gén. Chakadrius, itiv.

5. Ch. fulvus, Gm. $\sigma \circ$ N. v. Natang-vaca.

(M. S. T.)

Fulvous pluvier, Lath., Gén. Syn. iii. p. 21 I (1785) n." i7.

Charadrius fulvus, Gm. S. N. i. p. 68.7 (1788); Lath., Ind. Orn. ii. p. 147 (1790); Schel. Mus. Pays-Bas, Cursores, p. zo; Hartl. \& Finsch, Beitr. Faun. Centralpolyn, p. 188 (1867); Swinh, P. Z. S. 1871 , p. 403; G. R. Gray, Hand-list, iii. p. 14; sp. 9985 (1875); Salv. Ucc. di Borneo, 1874, p. 313; Wald. Trans. Zool. Soc. IX. 1875 , p. 226.

( pluvialis, Horsf. (nec Limn.), Trans. linn. Soc. xiii. p. 187 ( 1821 ).

(. xanthocheilus, Wagl., Syst. Av. Gen. Charadrits, sp. 37 ( 1827); Gauld, B. Auslr. vi. pl. г $3 ; G$. R. Graj', Mand-list, iii. p. 14, sp. 9986 (18 7 i).

( $\therefore$ taitensis, Less. Man. d'Orn. ii. p. 321 (1829).

( $\therefore$ virginianus, Jard. \& Selb., iii. Orm. ii. pl. 81 ( $88_{3}(3)$.

( $\therefore$ glaucopus, Forst., Diser. An. p. 170 ( 1844).

( virginicus, Blyth (nec Barkhawsen), Cat. B. Mus. A. S. B. p. 262 (1849);. Mottl. \& Dillw., Con!'r. Nal. Hist. Lab. p. 57 (1855); Swinh., Ibis, 1860 , p. 358 èt. loc. al.

C. longipes, Temm. apud Bp., Rev. Crit. p. I80 (1850); Sclat, P. Z. S. 1863 , p: 222; Swinh., P. Z. S. 1863, p. 309 : Jerd., B. F. Ind. iii. p. 636 (1864); G. R. Gray, Hand-list, iii. p. 14, sp. 9984 (1871).

C. auratus orientalis, Temm. \& Schleg. Fann. Japon. Ares, p. Io4 t. 62 (I 850$)$.

Pluvialis longipes, Bp., Compt. Rend. x. L. iii. p. 4I7, sp. 37 (1856).

Pluvialis taitensis, $B p$. ibid. sp. 38 .

P. Fulvis, Bp., ibid. sp. 39. Schleg., Mus. P. B. Cursores, p. (0) $(1865)$.

Charalrius auratus, Schrenck; Reis, Ammrl. Vogel, 1. fro (1860). 
C. nrientalis, Gould, Hand. C. B. Austr. ii, p. 225 (1865).

Hab. Luzon, Manila, Navotas, Malabon, Bulacan, Angat, Paragua, P. Princesa, Calamianes, Culion, Basilan, Samar, Paranas, Negros, Cebú, I,eyte, Bohol, Sibuyan, Masbate, Mindanao, Siquijor, Balabac, Carolinas; Borneo, Sumatra, Célebes, China.

\section{Ch pluvialis: Lin. \& N v. Matang-vaca. (M.S. T)}

Pluviaiis viridis, Mars. Danub. 5. t. 2.5.

P. aurea, Briss. Av. 5. p. 43. n. ${ }^{\circ}$, t. h. f. 1.

Golden pluver, Brit. Zool. n. ${ }^{\circ} 208$ t. 72, Arct. Zool. s. p. 483. n. ${ }^{\circ}$ 399; Lath. Syn. 111. i p. 193, n. ${ }^{\circ}$ I.

Pluvier dore, Buff. H. Nat. Ois. 8. p. 8I. pl. Einl. 904:

Ch. pluvialis, Lin. Gen. l. N. 2. p. 688. n. ${ }^{\circ}$ 7. Chenu. 5. p. 190 (1875); Wood. Nat. Hist. p. 666. f. Brethm. cit. p. 34t, f. 119.

Hab. Luzon, Manila, Navotas, Laguna, Calamba; Europa, Alemania.

\section{Gén. AEGIALITES, Boie.}

7. Ae. peronii, (Tem) o. N. v. Tarin-ting, Candurang. (M. S. T.)

Charadrius peronii, Tem. in Mus. Lugd.; Bp. Compt. Rend. xliii. p. 417 es p. 68 (1856) Schleg. Aitus. P. B. Cursores, p. 33 ( 865$)$; Blyith, Ibis, y 870 p. I 75.

Ch. alexandrinus, Mottl. et Dillaw. Contr. Nat. Hist, Lab. p. 47 (18I5).

Ch. philippinus, Pelz. Navas. Reis. Vög. pp. I16, I62 (1865).

Aegialites peronii, Swinh. P. Z. S. 1870 1. r 39; G. R. Gray, Hand-l. 111. p. 16. up. 10005 (1871); Wal. Tr. Zool. Soc. viii. 2. p. 96. pl. ıо. f. 2 (1872); Sali'. Ucc. Borneo, p. 315 ( 1874$)$.

Hab. Luzon, Manila, Navotas, Lreyte, Bohol, Samar, Borongan, Negros, Siquijor, Mindanao; Borneo, Java, Célebes.

8. F. dubius. $(S \operatorname{scop}) \delta \&$ N. v. Tarinting.

(M. S T )

Charadrius dubius, Scop. Del Flor. et Faun. Insubr. ii. p. 93 . (1786); (ex Sonn., Voy. Nouv. Guin, p. 84, pl. 36).

:(:. corunicus, Bescke, Schr. der berl. Natur, F. Ges. vii. p. 4 (63 (1 787 ). Gm, S. N. ii. p. 692 (1888). Mart., Journ. F. Orn. 1860, p. 26.

C. alex.ndrius, var. d Gm. S. N. i. p. 684 (1788) (ex Sonn.)

C. philippinus, Lath., Ind. Orn. ii. p. $7+5$ (1790). (ex. Sonn., $\iota$. c..) Scheleg, Mus. P. B. Cursores, p. 28 . (1865).

Eigialites philippinus, Sclat, $P Z Z$. S. 1863, p. 222. G. R. Gray, Hand-List, iii. p. I5, sp. 10000 . (parte) (1871).

A. dubius, Wald., Trans. Zool. Soc. viii, 2. p. 89 (I872); Sali. Ucc. di Borneo, 1874 , p. 316; Wald. Trans. Zool. Soc. IX. 1865, p. 227.

Hab. Luzon, Manila, Navotas, Bulacan, Laguna, Calamba, Bataan, Orion, Guimarás, Negros, Leyte, Bohol, Mindanao, Paragua; Borneo, Java, Célebes. 
(haradriu's mongolicus, Pall. Reis. $111,[\%, 700: 1876$ :.

(: mongolicus, Pall., Zoogr. Ross. As. ii. P. 1.36 (1811-1 $\left.{ }^{3} 3 \mathrm{r}\right)$.

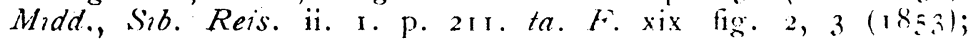
Schrenck Reis. Amurl. Vög. p. 4ri. Ithoo; Racide, Reis. S. O. Sib, ii. p. 324 (1863); Schleg., Mns. P. B. (ursores. i). 81, (1865), Gerl. \& Degl., Orn. Eur. ii, 1) 141 (1867). Blyth., Ibis. 1867 , p. 164 .

(. cantianus, Hors, F. (nec Lath.) Trans. Linn, Soc. xiii. P. 107, (1821).

C. cirrhepidesmos, Wagl., Syst. Av. Gen. Charadrius, sp. ix ( 1887$)$.

(.. gularis, Wagl., op. cit. sp. to. $^{\top}$.

C. sanguineus, Less., Man. a'Orn. ii. p. 330. (1828); id. Tr. d'Orn. p. 544. (183i).

(. pyrihothorax, Temm, apud, Gould, B. of Eur. p)l. 299. (I837; 13p. Comp. List. Birds Eur. and. N. Am. p. $4511838 ; 7 \mathrm{~cm}$. Man. 2. ${ }^{a} e d$ iv p. 355 (1840); Schleg. Rcv. (irt. [it. ii. p. (55, (1844; Bree, B. Eiur iv, p. 18. (1862; Tristr., P. Z. S., 1864, p. 45 a. id., Ibs, 1868, p. 323 .

Egia itis pyrrhotorax, Keys. \& Blas., Wiróltk. Eur. p. 70 ( 8 qu); Jerd., B. of. India, iii. p. 639 (1864); Beavan, Ibis, 1867. p. 332. Pilz., Ibis, 1858 p. $32 \mathrm{I}$.

Charadrius rufinellus, Blyth, Ann, and. Mag. Nat Hist. xii, 1). I 69 ( 1844$)$,

Hiaticala leschenaultii, Blyth, (nec Less); J. A. S. B. xii. p. I $8 \times$, (1843); id. Cat, B. Mus. A. S. B. p. 263. (1849).

Charadrius ruficollis, Cur. \& Temm., apud. Pucher., Rev. Zool. 1851, p. 282, Hengl,, S;rst. Velers. Vög. N. O. Atr. 1). $5^{6}$ $(1856)$.

C. subrufinus, Hodos.. Zool, Misc, p. $86(18+4)$.

Hiaticula inconspicua, Licht., Nomencl. A2. p. 94 (185t); (descr. mully).

H. inornata, Gould, B, Austr. vi, pl. I9

Pluviorhynchus mongolus, $B p$. Compt. Bend. x. L. iii, sp. t I (1856); Id., Cat. Ois. d' Eur. p. It $(3856)$.

Cirrhepidesmus pyrrothorax, Bp. Compt. Rend, t. c. sp. $5^{\circ} . \mathbf{G}$. R. Gray, Hand, List, iii, p. I8 sp, 9995. (I87 I).

Charadrius inornatus, $C_{i}$. R. Gray, Cat, B. N. Guin. p. 5 r. ( 8 89).

(. leschenzultii, Adams. (nec Less.) P. Z. S. 1859, p. isk; id. Wand. Nast. India, p. 283 (1867).

()chthodromus inornatus, Gont. Handb. B. Alustr. ii. p. $237(1805)$.

Aegialitis mongolus, il reting, Ibis, $1870, \mathrm{p} .384$.

Cyrrheptdesmus mongolus, $G$. R. Gr. Hand.-List, iii, p. is.sp. 9994 ( 1871 ).

Aegialitis rengolus, Sainh., P. Z. S. 1871. p. 404.

A. mongolicus, Salv. Ucc. ti borneo, 187t. p. 3i6; Wald. Trans. Zool. Soc. IX. 187.5, p. 227 .

Hab. Leyte, Bohol, Mindoro, Baco, Negros, Panay, Paragual, P. Princesa; Europa, Borneo, Jala, Borneo, China, Australi,. 
.10. Ae. geoffroyi, (IIisl) of N v. Candurng.

Charadrius asiaticus, Horsf. Tr. Lill. Soc. xiii, p. $187(182 \mathrm{r})$; (sec Pall j.; Tristo., P. Z. S. 1864 , p. 450; Id. Ibis, 1867, p. 93. (h. geofliovi, Ilay\%, Syst. Ai. Cien. Charadrius, sp. 19(1827); Kltl, kup. log. p. 26, pl. 34, f 2. (1833); Blyth, J.A.S. B. xii, p. $180(18+3)$; Heugl., Ibis, 1859 . p. 345, Nert. Ibis, 186.3, p. +55; id., Ibis, 1866, p. 343; Blyth, ibis, 2865, p. 34; Schelg, Mus. P. B. (iursores, p. 59 (i865).

('h. leschenaulti, Less. Man. d'an. ii. p. 322 (1828); Swinh., J: A. S. B. xxix. p. 259 (г86r); Wallace, P. Z. S. 1863, P. t88; lajart. B. S. Afi. p. $299(1867)$.

(h. griseus, Less. Tr. a'Orn. p. 544 (1831).

Ch. fuscus, Cu:, in Puch., Rev. et Mag. Zool. 1851, p. 282.

Ch. rufinus, Blyth, Ann. and. Mag. N. H. xii. p. I69 (1843'; Houlgs. Zool. Misc. P. $86(1844)$.

Hiaticul geoffroyi, Rupp., Syst. Veb. p. I18 (1845); Blyth, Cat. B. Mus. A. S. B. P. 262 (1849).

H. columbina, Licht., Nomencl. p. 94 ( 1854 ).

Cirrhepides !ns genfroyi, Bp. Compt. Rend. xliii, p. 4r7, sp. t9 (1856;; G. R. Gray', Hand-List, iii. p. 14. sp. 9993 (1871).

Aegialites geoffroyi, Heusl., Sy'st. Veb. Vog. N. O. Afr. p. it (1856); Sclat., P. Z. S. 1803 , p. 222 ; Sivinh., Ibis, $1863,1$. 403; Jerd. R. of, ind. iii, p. 638 (1864); Harting, Jbs, 187, p. 378, pl. xi; Szunh., P.Z. S. 1871 , p. 404; Salv. Uar. de Borneo, $1 \delta^{2} 74$, p. 3 I8; Wald. Trans. Zool. Soc. 9 p. 227 (1875).

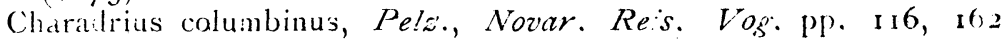
( 1865$)$.

Hab. I,uzón, Manila, Navotas, Pampanga, Leyte, Bohol, Negros, Paragua, Puerto Princesa; Borneo, Java, Sumatra, China, Formosa.

11: Ae. cantianus, (Lath.) $\sigma \circ \mathrm{N}$ v. Tarinting

(M. S. T.)

Charadrius cantianus, Lath. Sectl. Ind. Orn. p. lxvi. (1801).

Ch. littoralis, albifrons, y trochilus, Brehm. t. cit. p. $35 \mathrm{I}$.

Af cantiana, albifrons, ruticeps, dealbata, $y$ albigularis, Breh. id. Hinticula cantiana, y elegans, Brehm. l. c.

Aegi lophilus cantianus, Brehm. l. cit.

Hab. Bohol, Panay, Paragua, P. Princesa, Mindanao.

\section{Fam. GLAREÓLIDAS.}

\section{Gèn. GLAREOLA, Less.}

- $\mathrm{Cl}$ orientalis, Leach. $\sigma^{\circ} \mathrm{O}$ N. v Cananay-buquit.

(M. S. M)

Oriental pratincola, Lath, Gen. Hist. ix, p. 365 .

Pratincola orientalis.

Glareola orientalis, Leach. (nec Jerdon). Trans. Linn. Soc. xiii. 2. 1 32, pl. I5, f. I. 2 (1821), (1); Horsf, ibid. p. 187 (1821); 
Gould, B. Austr. vi. pl. 23; Blyth, Cat. B. Mus. A. S. R. P. 259 (1849); Bp. Compt. Rend. xliii. p. 4 I9 sp. 117 (1856); Schleg. Handl. Dierk i, pp. 445, 48I, t. 7. f. 79 (1857-58); Jerd., $B$. of. India, iii. p. 631 (1864), Schleg. Mus. $P$. $B$. Cursores, p. 16 (1865); Gould, Handb. B. Austr. ii. p. 245 (1865); G. R. Gray', Hand-list, iii p. I8, sp. 10031 (1871); Swinh, P. Z. S. 1871 , p. 403; Salv. Ucc. de Borneo, 187 t, p. 318 .

Hirundo, y trachelia pratincola, Brehm. $V$. An. 4. 1881, p. 3561121 .

Glareola torquata, Jerd. Cat. n. 370.

Hab. Luzón. Laguna, Calamba, Pampanga, San Fernanto, Tarlac, Pangasinan, Negros; Borneo, Java, China, Australia.

2. G, isabella, Vicill $0^{*} \bigcirc \mathrm{N}$. v. Cananay.

Glareola isabella, Vieill. Nouv. Dict. xiii. 1817. p. 221 ; id. Enc. Méth. 1823 , p. 1048; 1d. Gal. des Ois. 11. 1834, p. 159, pl. 263; Sclat., Pr. Lin. Soc. ii. p. $160\left(185^{8}\right)$; G. R. Gray, Cat. B. New. Guin. 1859 , p. 50; Salv. Ucc. di Bornen, 1874 , p. 320 .

(i. grallaria, Temm. Man. d'Orn. ii. I820, p. 503; S. Müll. Verh. Nat. Ges. Nederl. Overz. Bez. Land-en Volkenk, 183944, p. 23; Gould, B. Austr. vi. pl. 22; Schleg. Handl. Dierk, i. 1857 , p. 445; id. Mus. P. B. Cursores, 1855, p. Is; Gould, Handb. B. Austr. ii. I865, p. 243; Wald. Trans. Zool. Soc. viii, 2. I 872 , p. I 7 .

(i. australis, Leach, Trans. Lin. Soc. xiii, p. 132 lam. I 4 f. I, 2 (1821).

Stiltia grallaria, Bp. Compt. Rend. xliii, p. 419, esp. I I 3 (1856); G. R. Gray, Hand-l, iii. esp. I0032, I87I. p. I17.

Hab. Luzón, Manila, Bulacan, Pampanga, Bataan, Orion; Borneo, Java, Célebes.

\section{Fam. HEMATOPÓDIDAS.}

4. Gén. Strepsilas, Illig.

I. St. interpres. (Lin.) $\subset \circ \mathrm{N}$. v. Taringting.

'Tringa interpres, Lin., S. N. I. p. 248 (г766).

Strepsilas interpres, Leach. Cat. B. Mus. p. 29 (1816); Gould, B. Austr. vi. pl. 39; Sclat., R. Linn. Soc. ii. p. 169 (185-); G. R. Gray, Cat. B. New. Guinea, p. 51 (1859); Jerd. B. of. India, ii p. 56 (1864); Schleg. Mus. P. B. Cursores, 1. +3 (1865); Hartl. \& Finsch, Beitr. Faun. Centralpolyn. p. v. iii. 2, p. 91 (1872); Salv. Ucc. di Borneo, 1874, p. 320; Wald. P. Z. S. 1878, p. 7 II.

St. borcalis, littoralis, y minor, Brehm. V. An. 4. p. $5^{8}$ (1881),

f. 122

St. collaris, Mull. Verhandl. Land-en Volkenk, pp. 90, 110 (1 839-1 844).

'Tringa interpres, morinella, y oahuensis, Brehm. l. cit. p. $35^{8}$. 
(inclus interpres, G. R. Gray, Hand-lisl. iii, p. 22. op. 10068 ( i 87 I)

Hab. Paragua, P. Princesa, Tagburus, Bohol, Siquijor, Masbate; Java, Célebes, Borneo, Europa, China.

\section{2. 'Gén HaimatopUS, IiN.}

2. H osculans, Swinh, $\sigma \circ$ N. v, Candurung dagat.

(M. S. 'T.)

Hacmantopus ostralegus, Szvinh. (nec Lin.) Ibis, 1860, p. 63; I 861; pp. 26I, 342; Schleg., Mus. P. B. Cursores, p. 70 (parte) (1865).

Harmatopus longirostris, Swinh. (nec Vieill), Ibis, 1863, p. 406; id. P. Z. S. 186,3, p. 310.

H. ostraleugus, hypoleucus, balthicus, orientalis y longirostris, Brehm. V. An. 4. p. 359. f. I 23.

H. sp., Gray, Hand-list, iii, p. 21, sp. 100060 (187 r).

H. osculans, Swinh., P. Z. S. 1871 , p. 405; Salv. Ucc. di Borneo, 1874 .

IAab. Luzón, Laguna, Calamba; China, Borneo.

\section{Fam. ESCOLOPÁCIDAS.}

- i. Gén. himantopes, Briss.

4. H. leucocephalus, Gould. $\delta \&$ N. v. Ticas-tica.

(M. S. T.)

Himantopus leucocephalus, Gould, P. Z. S. 1837, p. 26; S. Mäll. Verhandl. Land-en. Valkenk. p. I53 (1839-1839); Gould. B. Austr. vi. pl. 24 (1848); Sclat., Pr. Linn. Soc. ii. p. 169 (1858); G. R. Gray, Cat. B. Nere. Guinea, p. 52 (1859); Schleg. Miis. P. B. Scolopaces, p. 106 (1864); Blyth; Ibis, $1865 ; \mathrm{p}$. $35 ;$ G. R. Gray, Hand-list, iii. p. 48 op. 10295 (1871); Wald. Trans. Zool. Soc. viii. 2, p. 91 (1872); Salv. Ucc. di Borneo, 1871 , p. $32 \mathrm{I}$.

H. melanopterus, S. Mäll. (nec Temm.) Verh. Nat. Gesch. Nederl. overz. Bez. Land-en Volkenk. p. 32 (1839-1844).

? H. intermedius, Blyth, J. A. S. B. xviii; ?id., Cat. B.'Mus. A. S. B. p. 265 (1849); G. R. Gray, Hand-list, ili; p. 47. sp. IO2 93 (187 I).

Hab. Mindanao, Luzón, Manila.

2. H. autummalis, Has $0^{\prime}, 0$ N. v. Candurung-dagat.

(M. S. 'T.)

(haradrius autummalis, Hassely. Iter Palestinum, p. 253, n." 29 (1757); Reise nac Palästina, (Gadeb, Germ Tr), p. 308, n. 29, $(1762)$.

Himantopus rufipes, Bechts. v. Martem.J.f. $0.1866,28$, n." 160 . $H a b$. Luzón, Laguna.

3. H. minor, Nat. ơ N. v. Ticas-tica.

(M. S. T.)

Hab. Mindoro; Baco. 
2 Gen. RECLRYiROSTRA, lis.

4. R. avocetta, Lin. \& \& N $\mathbf{N}$ Candurung.

(M. S. T.)

Sccoping avosetta, Brit. Zosl. 2. n." 223 :: 8. A.ct. Zoll. 2. p. 503., B. Lath. Syn. III. i. p. 293 , n." 1.

Avocetta, Buff. H. N. O.s. 8. p. 45's. t. 38. Pl. En!. n. 353.

Recurvirostra arocetta, Lin. S. $N$. 2. p. 693, n." 1; Brehm. "l. An. 1881,4 . p. 392, 1. 126.

Hab. Paragua, P. Princesa.

\section{Gen. CALIDRIS, I,LGRR.}

5 C. arenaria Lin. $\$$ N. v. Canduru

(N. is T.)

'Tringa arenaria, Lin. S. N. i. p. 25 I (1765); ferd. B. of. India. III, p. 964 (186+); Schl:g., Mus. P.-13. Scolopaces. p. 55 (180+); Sze nh, P. Z. S. 1871, p. 4o8; G. R. Gray Hand-List III, p. 51, sp. 10324 (1871); Brehm. 4. p. 371.

Calitris arenaria, Salv. Ucc. de Borneo, 1874,1$) .322$.

Hab. Luzón, Liguna; China, Formosa, Java, Borneo?

\section{Gen. LIMICOLA, Koch}

6 L. platyrhyncha, (Tem) $\&$ \& N. v. Candurung dagat. (M S. T.)

Numenius pigmaus, Lath. Ind. Orn. ii. p 713 (1890).

Limicola pigniaea, Baier. Zool., p. 315 (1816); (nec N. pygmaus, Lath? ): Brehm. V. Anim. 4. 1881 , p. 370.

'Tringa platyrhyncha, Temm; Blyth. Cat. B. Mus. A. S. B. 1. 269 (1849); Schleg, Mus. P.-B. Scolupaces, p. 46 (1864); Jeril. $B$ of. India III, p. 692 (1864); Svinh. P. Z. S. 1871 , p. for; G. R. Gray, Hand-List. III, p. 49, sp. 10305 (1871).

Limicala platyrhyncha, Salv. Ucc. di Borneo, 1874 , p. 322.

Hab. Luzon, Manila, Narotas, Paragua, P. Princesa, Calamianes, Culion, Bohol, Negros; Europa, China, Java, Borneo.

5. Gen. PLLIIINA, Cuv.

7. P. subarquata, (Gulden) t\& N. v.

(M. S. 'T.)

Scolopax subarquata, Gitldenst. Noz. Comm. Petrop. xix, 1) tit ( 1775 ).

Tringa subarquata, Temm. Man. II, p. 6óg (1820); Horsf., Treans. Linn. Soc. xiii. p. p. 193 (1821); S. Müll, Verhandi. Land-en Volkenk. p I10 (1839-1844); Blyth. Cat. B. Mus. A. S. L. p. 269 (1849); Jerd. B. of. Iind. III, p. 689 (1864); Schles. Mus. P.-B. Scolopaces, p. 8 I (1864).

Schoniclus subarquatus (!) G. R. Gray List Stec. B. Brit. Mus. III, Gralla, p. I05 (18+4), Gould, B. A\%str. vi. pl. $32(18+\%)$ A.cylucheilus subarquatus, (!) $B p$. Connt. Rema. XIIII, p. 5gto. 
sp. 213 (1850); Gould. And6. B. Austr. 11, p. $256(1865)$; Brehm. F. An. 4. 1881 , p. $\$ 72$.

A. subarquata, G. R. Gr. Hand-Llist. III., p. 50. sp. 10319 (187I). 'Tringa subarquata (!) Swinh, P. Z. S. 1871, p. 409.

Pelidna subarquata, Salv. Ucc. di Borneo, 1874 , p. 322.

Hab. Luzón, Manila, Malabon, Bataan, Orion, Negros, Paragua, P. Princesa, Calamianes, Culion, Samar, Borongan, Loquilocum; Borneo, Java, China, Europa, Asia.

8. P. alpina, (Lin.) $0^{\circ}, 0$ N. v. Tarniting.

(M. S. ' '.

Tringa alpina, Linn. S. I. 1. p. 294, sp. 11 (1766); Brehm. V. $A n$. 4. p. 372.

'1. cinclus., Linn. ibid. p. 251, sp. 18 (1766); Blyth, Cat. B. Mus. A. S. B. p. 269 (1849); Jerd. B. of. Ind. iii. p. 690 (1864); Schleg. Mus. P. B. Scolopaces, 2 (1864).

'T. chinensis, Gray. Zool. Misc. p. 2.

'T. cinclus, var. chinensis, Swinh., P. Z. S. 1871, p. 408.

Schoeniclus cinclus, $G$. R. Gray, Hand-list, iii. p. 49, sp. IO316 (1871).

Pelidna alpina, Salz. Licc. di Borneo, 1874, p. 323.

Hab. Luzón, Manila, Bulacan, Paragua, P. Princesa; Borneon, Java, Europa.

9. P. acuminata, (Hor f $)$ o $N$ v. Taringting. (M. S T.)

'Totanus acuminatus, Horsf. Trans. Linn. Soc. xiii. p. 192 (1831).

'lringa australis, Jard \& Selby, iii. Orn. ii. pl. 91.

Schoenciclus australis, G. R. Gr.y List. B. Brit. Mus. iii. p. 10.5 (1844); Gould. B. Austr. vi. pl. 80 (1844).

:Tringa rutescens, Midd. (nec Vieill.) Sib. Reis. ii. p. 22 (1853).

'T. acuminata, Szvinhoe, Ibis, 1863 , p. 412 ; Schleg. Mus. P.-B'.

Scolopaces, 38 (1864); Swinh. P. Z. S. 1871 , p. 409.

limnocinclus acuminatus, Gould. Handb. B. Austr. ii. p. 254 ( 1865 ; G. R. Gr. Hand-list. iii. p. 49, sp. 10,304 (1871).

Pelidna acuminata, Salv. Ulcc. di Borneo, 1874 , p. 323.

Hab. Luzón, Manila, Malabon, Laguna, Calamba, Paragua, Inagauan; Java, Borneo, Australia.

\section{Gén. ACTODROMAS, Kavp.}

Io. A. albescens, ( $T e m$.) $0^{\circ} \circ$ N. v. Taguinting, Canduru. (M. S. T.)

Tringa albescens, Tem. Pl. Col. 41. f. 2. (1824); G. R. Gray, Cat. B. N. Guin. p. 52 (1859); Rosenb, Sourn. f. ornith. 1864, p. $135 ;$ Sharpe, \& Dress. B. of. Eur. nel. testo, della T. minuta, pp. 45 1871 (1873).

Calidris australis, Cuv. Mus. Paris.

'Tringa australis, Less. Tr. d' Orn. p. 558 (1831).

'T. pusilla, Mull, Verh. Nat. Gesch. Nederl. Overz. Bes. Land-en. Volkenk. p. 23 (1839-1834).

Schøiniclus, albescens, Gould. B. vi. pl. 3 r (1848); Sclat. Pr. Linn. Soc. ii. p. I 70 (1858).

Actodromus albescens, Bp. Compt. Rend. xliii. p. 596 sp. 220 (1856); Sala'. Ticc; di Borneo. $18.74 \%$ p. 323 . 
Tringa minuta, Schleg. Mus. P.-B. Scolopaces, p. 43 (parle) (1864); Wald Trans, Zool. Soc. viii, 2 p. 97 (1872).

Actodromas australis, Gould. Handb. B. Austr. ii. p. 257 (1865). A. albescens, G. $R$ Gray, Hand-l'st, iii. p. 50. sp. so31 2 (1871). Tringa salina, Swinh.P.Z.S. 1871 , p. 409. (parte).

Hab Luzón, Manila, Navotas, Laguna, Paragua, Tagburus, Calamianes, Culion; Borneo, Java, Célebes.

$$
\text { 11. f.c temminckii, (Leisl) o o N. v. Taguinting. }
$$

Tringa pusilla, Lath. Ind. Orn. p. 737 (189o, nec Linn).

'T. temminckii, Leisl. Nachtragi. zu. Bechst. Naturg Deuslchl. i. p. 75 (I8I I); Blyth. Cat. B. Mus. A. S. B. p. 270 (1849); Jerd. B. of. Ind. iii. p. 62 r (1864); Schleg. Mus. P. B. Scolopaces, p. 47 (1864); Swinh, P. Z. S. 1871. P. 409; Brehm. 4. p. 374 .

Pelidna temminckii, Bore, Isis, 1826 , p. 979.

Limoneites temminckii, Kaup, Naturl. Syst. p. 38 (1829); G. R. Gray, Hand-list, iii. p. 50, sp. Io315 (1871).

Actodromus temminckii, Kaup, Bp. Compt. Rend. xliii. p. 396. sp. 219 (1856); Salv. Ucc. di Borneo, 1874 , p. 324.

Hab. Luzon, Manila, Navotas; Bormeo, China, Europa.

12. Ac salina, (Pall) $\sigma \rho$ N. v. Canduru.

(M. S. T.)

'Tringa salina, Pall, Itin, iii. App. p. 700 ( 1776$)$; Ind. Zoogr. Rosso-As. ii. p. I9I (1810-3I); Dybowsky, Journ. 1868 , p. 337; Hartl. \& Finsch, Vög. O. Afr. p. 766 (nota) (1870); Sharpe \& Dress. B. of. Eur. testo de ella T. minuta, Pl'. $4,5(1871-72)$.

T. ruficollis, Gm. S. N. I. p. 688 (1788); Lath. Ind. Orn. p. 736 (1790); Vieill, Enc. Meth. p. 1088 (1823).

Totanus damacensis, Horsf, Trans. Linn. Soc. xiii. p. 192 (1821).

Tringa brevirostris, Kuhl, litt. et Temm. (ex parte).

'T. subminuta, Midd. Sib. Reis. p. 222 t. xix. f. 6 (1858); Szinh. Ibis, 1862, p. 255 ; $186 \mathrm{r}$, p. 37 .

Actodromus minutus, a. brevirostris, Bp. Compt. Rend. xliii. p. $596(1856)$.

Tringa damacensis, Swmh, Ibis, 1863, p. 418; id. P. Z. S. P. Z. S. 1863 , p. 316; Schleg. Mus. P. B. Scolopaces, 1. 48 (1864); Swinh, P. Z. S. 1871, p. 409; Wald. Trans. Zool. Soc. viii. 2, p. $97(1872)$.

Actodromas salina, G. R. Gray, Hand-list. iii. p. 50. sp. 10313 (1871); Salv. Ucc. Borneo, 1874 , p. 324.

Hab. Luzuil, Manila, Malabon, Cebú, Negros, Paragua, P. Princesa; Borneo, Formosa, Célebes.

\section{Gén. TRINGA, IIIN.}

13. T. crassirostris, Tem. ct Schl o o N. v. Candurung-bato (M.S.T)

Tringa crassirostris, Tem. et Schleg. Hann. Japon. Aves, pl. 64 (1846), p. I07 (1850); Midd, Sibir Reis. ii. 2, p. 219 (1855); Schrenck, Reis, Amum. Vogel, p. 420 (1860); Sch!eg. Mus. 
P-B. Scolopaces, p. 28 (1864); Blyth, Ibis, 1865, p. 34; Salv. Ucc. de Borneo. 1874, p. 325.

Schoeniculus magnus, Gould, P. Z. S. 1848, p. 39 Id. B. Austr. vi. pl. 33 (1848).

Tringa magna, $B p$. Compt. Rend. xliii. p. 596, sp. 211 (1856). T. tenuirostris, Swinh, P. Z. S. 86,3, p. 315; Gould, Handb. B. Austr. ii. p. 260 (1865); P. R. Ciray, Hand-list. iii. p. 49, sp. rozor (1871); Sivinh, P. Z. S. 1871 , p. 408.

Hab. Luzon, Manila, Malabon, Negros; Borneo, Java, China, Japon.

14. T. canutus? (Lath) oo N. v. Canclurung.

(M. S. T.)

Hab. Luzon, Manila, Malabon; Europa.

8. Gén. TRINGOIDES, Br.

15. Tr hypoleucus, (Lin). $\sigma \circ \mathrm{N}$. v. Tica-ticang munti. (M. S. T.)

Tringa hypoleucus, Lin. S. N. i. p. 250 (1766).

Actitis hypoleucus, iii, Prodr. Syst. Mamm. et. Av. p. 262 (1811); F. Boie, Is s, 1822 , p. 649; Blyth. Cat. B. Mus. A. S. B. p. 267 (I 849 ); Bp. Compt. Rend. x iii. p. 597, sp. 250 (1856); Schleg. Mus. P. B. Scolopaces, p. $80(1864) ;$ Jerd. B. of. Ind. iii. p. 699 (1864); Rosenb. Journ. F. Orn. 1864, p. 1 35; Gould, Handb. B. Austr. ii. p. 263 (1865); Wald. Trans. Zool. Soc. viii. 2, p. 96 (1872); Brehm. V. All. 4. 1881. p. 381; Tweed. P. Z. S. 1877 , p. 703 .

Tringa leucoptera, Pall. Zoogr. Rosso-As. p. 196 (1810-31).

Totanus hypoleucos, Temm. Man. p. 424 (1815); Horsf. Trans. Linn. Soc. xiii. p. 192 (1821); S. Müll., Verhandl. Land-en Volkenk. p. 22 (1839-44); Wall. P. Z. S. 1863 , p. 487.

Totanus guinetta, leach, Syst. Cat. M. and. B. Brit. Mus. p. $30(1816)$.

Actitis stagnalis, Brehm. Handb. Nat. Vög. Deutschl. p. 649 (1831).

Guinetta hypoleuca, G. R. Gray List. Gen. B. p. 68 (184).

'Tringoides hypoleuca, G. R. Gray, List. Gen. B. p. 88 (i 848); Mottl. \& Dillw., Contr. Nat. Hist. of. Lab. p. 6o (1855); Sclat. P. Z. S. 1863 , p. 222 ; G. R. Gray, Hand-list, iii. p. 46. sp. I0279 (1871); Szeinh, P. Z. S. 1871, p. 406; Salz'. Ucc. di Borneo, 1874 , p. 326.

Actit s empusa, Gould, P. Z. S. 1845 , p. 222; $2 d$. B. Austr. vi. pl. 35 (1848); Bp. Conpt. Rend. xlviii. p. 597. sp. 259 (1856).

Actitis schlegelii, Bp. I. C. sp. $25^{2}$.

Tringoides empusa, Sclat. Pr. Lin. Soc. ii. p. I7o (1858).

'Totanus empusa, G. R. Gray, Cat. B. New. Guin. p. 52 (1859). Hab. Bohol, Paragua, P. Princesa, Masbate, Calamianes, Culion, Samar, Burongan, 'lawi-tawi; Formosa, Java, Sumatra.

\section{Gén. TO'TANUS, Bechst.}

16. T. glareola, (Lin). o o N. v. Tica-tica.

(M. S. T.)

Tringa glareola, Lin. S. N. i. p. 250 ( 766 ).

Totanus glareolas, Temm. p. 42 I (18r5); Schleg. Mus. P.-B. 
Seolopaces, p. 71 (i864); Pelz. Novar, Reis. pp. 131, 1 tiz (1865); Swinh., P. Z. S. 1871, p. 406; Salv. Ucc. di Bornee, 1874, p. 327; Brehm, V. An. 4. p. 387; Blas. Ornis. 1888 , p. 320.

'Totanus aftinis, Horsf. Trans. Linn. Soc. xiii. p. I91 (1821); Gray \& Hardw. iii. Ind. Zool. t. 5 I, f. 2; Strickl., Aun. and. Mag. Nat. Hist. xiv, p. 120 (1844); Sclat. P. Z. S. 186.3, P. 222; P. Z. S. 1863 . p. $3 \mathrm{II}$.

T. grallatorius, Steph, in Shaw, Gen. Zool. xii. p. $148(1824)$. T. glareoloidos, Hodgs.

Phyacophilus glareola, Kaup, Nat. Syst. p. Ito (1829); (․ R. Gray, Hand-list, iii. p. 44, sp. 10260 (I 871 ).

Tringa littorea, Pall. Zoogr. Rosso-As, p. 195 (1810-31).

'lotanns sylvestris, Brehm, Handb. Nat. Vög. Deutschl. p. 6.3. (18 8 I).

T. palus ris, Brehm, op. cit. p. 639.

Actitis glareola, Blyth, Cat. B. Mus. A. S. B. p. 267 (1 849$)$; Jerd. B. of. India iii. p. 697 (1864); Wald, Trans, Zool. Soc: viii. 2 , p. 96 (1872).

Hab. Luzon, Manila, Negros, Panay, Guimarás, Pragua Basilan, Cebú, Siquijor, Calamianes, Culion; Borneo, Java, Célebes, liuropa.

16. T. ochropus, Lin. $\$$ N. v. Tarinting.

(M. ล. T)

Hab̆. Paragua, P. Princesa.

' 7. T. calidris, (Gm.) o $\odot$ N. v. Tica-tica.

(M. S. 'T.)

Wotanus striatus, Briss. Orn v. p, 196, n." 5, t, 12, f. 1 (1760). 'T. naevius, Briss. op. cit. p. 230, n. ${ }^{\circ} 6$.

Scolopax calidris, Gm., S. $N$, I. p. 664. (1788).

Tringa ga ribetta, Gm., op. cit. p. 671, n. ${ }^{\circ} 3$.

T. striata, Gm., op, cit. 672, n. ${ }^{\circ} 5$.

lotanus calidris, Bechst., Orn. Traschenb. Deulschl. ii, p. ${ }_{2} 8_{4}$ (1803); Blyth, Cat B. Mus. A. S. B. p. 266, (1849); Schleg., Mfus. P. B. Scolopaces, p. 65 (1864); Jerd., of. India, ii, p. 702 (1864).-Swinh., P. Z. S. 1871, p. 406. G. R. Gral. Hand-List, iii, p, 45, sp. I02 72 (1871) - Wald., Trans Zoo'. Soc. viii. 2, P 97 (1872); id. ibiden, IX. 1875, p. 234; Sal7. licc. di Borneo, 187 , p. 328; Brehm, l?. Anim. 4, 1881, 1. 386

Ciambetta calidris, B $p$. , Compt. Rend. xliii, p. 597 (1856).

Hab. Negros, Siquijur, Cebú Paragera, P. Princesa, Basilan; Europa.

18. T. stagnalis. Beh.t. $\sigma \sigma \mathrm{N}$ v Tica ticang-munti.

(M. S. T)

Totanus stagnalis, Bchs. Orn. Tiuschenb. Deutschl. ii, p. 202 (1803); Gould, B. Austr. vi, pl. 37 (1848); Blyth, Cat. B. Mus A. S. B, P. 266 (1849); Jerd. B. of Ind. iii, p. Tor (1864); Sckleg., Mus. P. B. Scolopaces, p. 68 (1864); Swinh. J. Z. S. 1871 , p. 405; Salv. Ucc. di Bormeo, 1874, p. 328; Brehon. $V . A u, 4,1889$, p. $3^{8} 4$. 
Tringa guinetta, Pallas, Zoogr. Rosso-As. ii p. 195 (1816-18,31). Totanus tenuirostris, Horsf., Trans. L.12n. Soc. xiii. p. $192(1821)$.

'T. horsfieldii, Sykes, Cat. p. 196.

T. lathami, Gray, \& Hardw., iii. Ind Zool. pl. 5 I f. 3.

T. stagnalis, G. R. Gray, Hand-list, iii. p. 44, sp 10266 (1871). Hab. Luzon, Manila, Malabon, Navotas, Laguna, Calamba;

Borneo, Java, China, Europa.

19. T glottis, (Lin.) $\checkmark \supset$ N. v. Tica-tica

(M. S. T.)

Scolopax glottis, Lin. S. $N$. i. p. 245, sp. 10 (1766).

S. totanus, Linn., ibid. sp. I3.

S. canescens, Gm., S. N. i. p. 668 (1788)

Totanus glottis, Bechst., Orn. Traschenb. Deutschl. ii. p. 287 (1803; Horsf., Trans. Lin. Soc. xiii. p. 192 (1821); Blyth, (at. B. Mus. A. S. B. p. 265 (1864); Szeinh., P. Z. S. 1871 , p. 405; Wald., Trans, Zool. Soc, viii., 2. p. 96 (1872); Salv'. Vec. di Borneo, 1874 , p. 328 ; Bremh. V. Ani. 4. p. 384 .

Glottis nivigula, Hodgs., Gray, Zool, Misc. ii. p. 36 (1831).

Totanus glottoidos, Vig., P. Z. S. 1831, p. 173; Gould., Cent. Him. B. pl. 76 .

Limosa glottoides, Sykes, P. Z. S. $18_{32}$, p. 163.

(ilottis vigorsii, G. R. Gray, List. Spec. B. Brit, Mus. iii. Grallae. p. 39 (i 844$)$.

Glottis horsfieldii, G. R. Gray, ibid.

Totanus horsfieldii, G. R. Gray, Gén. B. iii. p. 573 (1846); Sclat., P. Z. S. 1863 , p. 222.

Glottis glottoides, Goul, B. Austr. vi. pl. 36 (1848); id., Handl. B. Austr. ii p. $265(1865)$.

Totanus canescens, Adams. P. Z. S. 1859, p. r69; Sharpe \& Dress. B. Eur. pl. $42(1871-1872)$.

Glottis glottis, G. R. Gray, Hand-list, iii. p. 45, sp. 10276 ( 1871 ).

Hab. Luzon, Manila, Malabon, Negros; Borneo, Java, Célebes, China, Europa.

19. T. incanus, $(G m) \sigma o$ N. v. Tugil, Tarinting.

(M. S T.)

Scolopax incanus, Gm. S. N. i. p. 658 (I788); ex.l, ath, Gm. Syn. iii. p. I54; Lath., Ind. Orn. p. 724 (1790).

'Totanus incanus, Vie ll., N.D. iv. p. 4 ro (1816); id. Enc. Meth. p. 1098 (1823); G. R. Gray, $B$. isl. p. 50 (1859); Sclat. P. Z. S. 1864 , p. 9. Schleg. Misc. P. B. Scolopaces, p. 74 (1 864); Swinh, P. Z. S. 1871 , p. 406; Salv. Ucc. di Borneo, 1874, p. 329 .

'I'. brevipes, Vieill, N. D. iv. p. 410 (1816); id. Enc., Meth. p. IIO6 (I823); Puch., Rev. et Mag. de Zool. 1851, pp. 370, 570 ; Cass., Pr. Ac. Ph lad. viii. p. 40 (1856); id., Pr. Ac., Philad. 1861 , p. 321 ; id. Un. Sl. Expl. Exp. Orn. p. 319 (1858);G. R. Gray, B. Trop. isl. p. 5 r (1859); Swinh; P. Z. S. 1863 , p. 312; Pelz., Novar. Reis. Vög. p. 129 (1865).

'Iringa glareola, Pall. (nec. auct)., Zoogr. Rosso-As. ii. 194, t. 60 (1810-3I).

'Yotanus pedestris, Less., Tr. d'Orn. p. 553 (1881). 
'T. fuliginnsus, Gould, Voy. Beagle, Birds, p. 130 (1841).

Scolopax nululata, Forst., Dcscr. An. p. 173 (1844).

S. pacilica, Forts., op. cit. p. 174.

S. oedicnemus, Licht., Mus. Berol.

Totanus pulverulentus, S. Mïll., Verh. Nat. Gesch. Nederl. overz.

Bez. Lint.en. Volkenk. p. I53 (1839-1844); Temm. \& Schleg., Faun. Japon. Aves, p. Io9, t. 65 (1850); Mid., Sibir Reis. Zool. Vög. p. 24 (1853); Rad.te, Reis. O. Sib. ii. p. 326 (1863).

'Totanus oceanicus, Less., Descr. Mamm. et Ois. p. 224 (1847); Bpp., Compt. Rend. xli. p. I I 2 (i855); Ciss., Un. St. Expl. Exp. Orn. p. 318 (1858).

T. griseopygius, Gould, P. Z. S. 1848 , p. 39; id., B. Austr. vi. pl. $3^{8}(18+8) ; G$. R. Gray, P. Z. S. 1860, p. 364.

T. solitarius, Bloxh.; Byr. Vey. p. 244.

'T. polynesia, Peale, Un. St. Expl. Exp. Birds, p. 237, pl. 05, f. I (1 $8+1)$; Hartl. Wiegm. Arch. 1852, p. I20; id., op. cit. p. I 34 .

Actitis brevipes, Blyth, Cat. B. Mus, A. S. B. p. 267 (18+9). 'Totanus scolopaceus, Licht. ap. Mid. Sibir. Reis, Zool, Vög. p. 214 (1853).

Actitis brevipes, Blyth. Cat. B. Mus. A. S. B. p. 267, (1849).

'Iotanus scolopaceus, Licht, ap. Midd. Reis. Zool. Vög. p. 214 ( 1853$)$.

Actitis pulverulentus, Licht, Nomencl. p. 92. (1854).

Crambetta fuliginosa, Bp. Compt. Rend. xliii, p. 597, sp. 234 (1856).

(i. Oceanica, pulverulenta, grisenpygia, brevipes, Bp. op. cit. sp. $235,236,237,238$.

Hrteroscelus crevipes, Baird, B. N. Am. p. 734 (1858); id. Attas, pl. 88. G. R. Gray, Hand-List, iii. p. 45, sp. IO27 I (1871).

'Totanus undulatus, forst. ap. verr. Revet. Mag. Zool. 1860 , p. 437 .

Gambetta pulverulentus, Gould. Handb. B. Austr. ii, p. 268 (1 865).

Actitis incanus, Harll, \& Finsch., Faun. Centralpolin. p. 182 ( 1867 ).

Heterosrelus incanues, G. R. Gray, Hand-List, iii. p. 45, sp. I $8270(\mathrm{I} 87 \mathrm{I})$.

Hab. Bohol, Samar, Calbayoc, Negros, Siquijor, Mindanao, Paragua, P. Princesa; Borneo, China.

\section{Io. Gén TEREKIA, BP.}

21. T. cinerea, (Guldı) 000 N. v Tarinting.

(M. S. T)

Scolopax cinerea, Guldenst, N. Comm. Pe'rop. xix, p. 473, t. xix (1774). Gm. S. N. i. p. 657. (1788)

Terek snipe, Lath., Gen. Syu. v. p. I55 (I785).

Scolop $\mathrm{x}$ terek, Lath, ind. Orn. ii, p. 724 ( 1790 ).

Totanus javanicus, Horsf., Trans. Linn. Soc. xiii, p. 193. (1828)

Scolopax sumatrana, Raffi, Trans. Linn. Soc. xiii, p. 327 (1822).

Numenius cinereus, Vieill. Enc. Meth. p. II 57 (1823). 
Fedoa terekensis, Steph, Gen. Zool. xii, i, p. 83 (18.24).

Nenus cinereus, Kaup. Naturl. Syst. p. I15 (182); $G$ R. Giray, List. spec. B. Bril. Mus. iii. Grallae, p. 96, (1844); Blas, list. B. Eur. p. 18 (1862); Finsch, 7 rans. Zool. Soc. vii. p. 328 (1870); Brehm. v. An. 4. 1881 p. 383.

Limicula (err.) indiana, Less., Tr. d'Orn. p. 554 (1831).

Limosa recurvirostra, Pall. Zoogr. Rosso As. ii. p. I8, (1810) 31; Schleg, Rev (rit. p. Ixxxvii. (18+4).

Terekia javanica, Bp. Comp. List. B. Eur. and. N. Am. p. $5^{2}$ (1838); Swinh., P. Z. S. 1862 , p. 319.

Limosa terek, Temin. Man. d' Orn. iv, p. 426 (1840); Gould, B. Eur. iv, pl. 307 .

L. (Simorhynchus) cinerea, Keys. \& Blas, Wirbelth. Eur. pp. lxxir, 2 I 2 ( 840$)$.

Tenekia cinerea, G. R. Gray, List. Gen. B. p. 88 (184o); Gould. Austr. vi. pl. 34 (1848); Blyt, Cat. B. Mus. A. S. B. p. 267 (1849); Bp. Compt. Rend. xliii, p. 597, sp. 267. (1856); Swinh. Ibis, 1863 , p. 97; id. P. Z. S. 14 3. p. 3 1 2; Jerd, Bof. Ind. iii. p. 682 (1864); Gould. Handb. B. Aus r. ii, p. 261 (1865); Saliad, Ibis, 1870 , p. i $54 ; G$. R. Gray, Hand. List, iii, p. t4. sp. 10255 (187r); Salvad., Faun d'Ital. Liccelli, p. $22 \mathrm{I}$ (1870-71); Swinh., P.Z. S. (1871), p. 406; Sharpe \& Dress, B. of. Eur. pl. Ixxx (1871-72); Sali'. Ucc. di Bormeo, 1874, 1. 330; Trueed: P. Z. S. $187 \delta$, p. 711.

Terekia guttifera, Nordm. apud. Bp. Compt. Rend. xliii. p. 597; sp. 268 ( 1856$)$.

Limosa cinerea, Blas \& Naum, Vög. Deutschl. xiii, pt. 2 p. $4^{8}$, t. $386 ;$ f.3 (I 860$)$.

lotamus cinereus, Schleg., Mus. P. B. Scolopaces. p. 77 (1864). Hab. Paragua, Tagburus, Bohol, Negros, Masbate; Borneo, Siumatra, China.

I I. Gén. IIMOSA, Briss.

22. L. melanuroides, Gruld ơ $\bigcirc$ N. v. Tica-tica.

(M. S. T )

Limosa melanuroides, Gould. P. Z. S. 1846 , p. 84 ; id. B. Austr. vi, pl. 28 (I848); Id. Handb. B. Austr. ii, p. 25 I (I865); G. R. Gray, Hand, List, iii, p. 43, sp. 10260 (1871); Sharpe \& Dress, B. of. Eur. nel testo della L. aegocephala, p. 3 (1872); Sali. Uic. di Borneo, 1874, p. 331 .

L. melanura, Temm. \& Schleg, Faun. Sap. Aves, p. I1 3 (1850); Sivinh., P. Z. S. 186.3 , p. 313; id. Ib.s, 1868 . p. 58 .

L. Irevipes, Scheleg, (nec Gray,) Mus. P.-B. Scolopaces, p. 2 r (1864); Swinh. P. Z. S. 1871 , p. 405.

Hal. Luzon, Manila, Narotas, Bulacan; Borneo, China.

23. I baueri, Naum. oo N. v. Tica-tica.

(M. S. T.)

I.imosa baueri, Naum, Vög. Deutschl. viii, p. $429(1836)$; Buller. B. New. Zealand, p. I98 (1872); Salv. Ucc. di Borneo, 1874 p. $33 \mathrm{I}$.

J. lapponica, var. novae zealandiae, Gray, Voy, Ereb. and. Tirror, Birds, p. 13, 1844 . 
I. australasiana, Gray, List B. Brit. M. III, Grallad, 184t, p. 96.

L. novae zealandiae, Gray, Gen. B. iii. p. $570(18+7)$; $1 d$. HandList. iii, p. 43, sp. 10252 (1871); Sharpe \& D)ress., B. of: Eur. nel testo della $L$. aegocephala, p. 3 (1872).

1.. uropygialis, Gould. P. Z. S. 1848 , p. 38 ; id. B. Austr. vi. pl. 29 (1848); Schleg., Mus. P.-B. Scolopaces, p. 25 (1864); Handl. B. Austr. ii. p. 252 (1863); Harll. \& Finsch., Finun. Centralpolyn. p. 177 ( 867$)$; G. R. Gray, Hand.-List., iii, p. 43, sp. $10261,(1871)$; Szevinh., P. Z. S. 1871, p. 406; Wald., Trans, Zool. Soc. viii. 2. p. 97 (1872); Sharpe \& Dress., B. of. Eur. nel testo della L. aeyocephala, 1). 3 ( 1872 ).

1.. foxi, Peale, V. S. Expl. Exp. Birds, p. 231, pl. $65(18+9)$.

1. rufa, Temm. \& Schleg. Faun. Jap. Aves, p. II 4 (i $\left.85^{\circ}\right)$.

Gallinago punctata, Ellman, Zool 1851 , p. 7470. Java.

Hab. Bshol, Negr ss, Luzon, Manila, Calaocan; Célebes, China,

24. L. gocephala, ชo N. v. Tica-tica.

(M. S. T.)

Scolopax xgocephala, Lin. S. $N$. 2. p. 67 n." i6.

limosa barge rouse, Buff. H. N. Ois. 7. p. 505 Pl. Enl. 876 , 916.

J. agocephala, islandica, y jodraca, Brehm. $V$. An. t. 1861 , p. 389 ; Wood. Nat. Hist. 1864 , p. 694.

Scopax limosa, belgica y egocephala, Brehm. l. c.

Hab. Luzón, Manila, Malabon, Bulacan, Negros; Europa, Asia

25. L. brevipes, Gray. $\mathrm{N}$ v. Tica-tica.

(M. S. '.)

(i. R. Gray, List. B. M. Grella, p. 93 (8844).

Hab. Luzón, Manila, Malabon, Mindoro Bacó; Borneo, Java, China.

12. Gén. PSEUdOSCOLOPAX, Bivry

26. Po. semipalmatus, ( $\mathcal{F}_{i r}$ ) $\sigma \circ \mathrm{N}$. v. Tica-tica.

(M. S. T)

Macrorhamphus semipalmatus, Jerd, in. Blyth, J. A. S. B. xvii. p. 252 (1848); Blyth, Cat. B. Mus. A. S. B. p. 27 (1849): Jerd. B. of. India, iii. p. 679 (I874).

M. guiseus semipalmatus, Bp., Compt. Rend. xliii. p. 579, sp. 201. var. a. $(1856)$.

Pseudoscolopax semipalmatus, Blyth, (1859) Szvinh., P. Z. S. 1863, pp. 181, 313; G. R. Gray, Hanl-list. iii. p. 52, si). 10327 (1871); Swinh., P. Z. S. 3871 , p. 407; Sali. UCC. d! Borneo, 1875 , p. 332 .

Micropalama tacksanawskia, J. Verr, Rez'. et Mag. de Zool. 1860 , p. 206, pl. xiv.

limosa semipal nata, Schleg., Mus. P. B. Scolopaces, p. 26 (186.t). Hab. Luzón, Manila, Navotas; Borneo, China. 
13. Gén. NUMENIL'S, BRIS.

27. N. arquata, (Lin.) oo $\mathrm{N} v$. Balancauitan, Canumiut.

Siolopax arquata, Lin. S. N. i. p. $2+2$ (1766).

Numenius arquatus, Lath, Ind. Orn. is, p. 710 (1790); Jerd., Cat. n. ${ }^{\circ} 357$; Blyth. Cat. B. Mlus. A. S. B. p. 268 (1849); Jerd. B. of. Int. iii. p. 683 (1867); Schleg, Mits. P. B. Sicolopaces, p. 85 (1864); Salo. Lice. di Borneo, 18.54, 1. 3.3. Brehm. I. An. 4. p. 394. f. 127.

Hab. Paragua, P. Princesa; Borneo, Jara, Sumatra,

28. N. lineatus, Stere, N, v.

Hab. Negros.

29. N. major, Tim it S.hleg. oo N. v. Balancauitan, Canunuat. (M. S T.)

Numenius major, Temm. \& Schleg., Finun. Jap. Aves, p. I10, pl. Ixvi. I 850 ; Schleg., Mus. P. B. Scolopaces, p. 80 (165.5): Sirla. Ucc di Borneo, 1847 , p. 333.

Hab. Paragua, P. Princesa; Borneo, Java, Sumatra.

3o. N. australis, Gould o o N. v. Balancauitan.

(M s. 'T)

: Scolopax tahitiensis, Gm. N. i. p. 056 (I888); (ext Lath. Sir'l. iii, i, p. I $22, n .^{\circ} 4$.)

: Numenius tahitiensis, Lath., Ind. Orn. ii, p. 7 I r (1890); Szrinh., P. Z. S. 1871, p. 4 IO.

: N. cyanopus, l'ieill., N. D. viii, P. 306 (r8I7); Gould, Handh. B. Austr. ii, p. 277 (1865).

N. austra!is, Gould, P. Z. S. 1837 , p. 155; idt., B. Austr. ri. 1l. 42 (1888); Schrenck, Reis. Amurl. Vog. p. 426 (I860); Schleg., Mus. P. B. Scolopaces, p. 9r (1864); Salv. Ucc. di Borneo, 1874 , p. 332.

N. major, Temm. \& Schleg., Faun. Jap. Aves, p. ıı, t. Ixvi. ( 1850$)$, (parte).

N. rufescens, Gould, P. Z. S. 1862, p. 286 id., B. of Asia, pt. xvi, pl. i3 (1 864).

Hab. Luzón, Manila, Malabon, Bulacan; Formosa, Japon, Australia.

3I. N. phæopus, (Lin.) $\sigma \circ \mathrm{N}$, v Balangcauitan.

(M. S. T.i

Scolopax phaeopus, Linn., S. N. i. p. 245 (187r); Gm. S. $N$. ii. p. 657 .

Corlieu, Buff. H. N. Ois. 8. p. 27, Pl. Enl. n. ${ }^{0} 84^{2}$.

Numenius phaeopus, Lath, Ind. Orn. ii. p. 7 I 1 (1790); Horsf., Trans. Linn. Soc. xiii, p. 19г (1821); S. Mull, Verh. Nat. Gesch. Nederl. Overz. Bez. Land-en Volkenk. p. 22 (1839-44); Schleg., Mus. P. B. Scolopaces, p. 97 (1864); Swinh. P. Z. S. 1871 , p. 4 ro; Wald., Trans. Zool. Soc. viii. 2. p. 96 (1872); Salo. Ucc. de Borneo, 1874 , p. 332 ; Brehm. T. Ani. 7. p. 394. 
: N. uropygialis, Gould, P. Z. S. 1840, p. г75; id., B. Austr. vi. pl. $43(1848)$.

Hab. Panay, Negros, Cebú, Siquijor, Calamianes, Cuyo; Borneo, Java, Sumatra, Célebes.

32. N. minutus, Gould. ơ $\bigcirc$ N. v. Candurung-dagat.

(M. S. 'T.)

Numenius minutus, Gould. P. Z. S. 1840 , p. 176 ; Scheleg., Mits. P. B. Scolopaces, p. Io1 (1864); id. Ned. Tijdschr. Dierk. 1866 , p. 348; Swinh, P. Z. S. 1871 , p. 469; Wald, Trans. Zool. Soc. viii. 2, p. 96 (1872); Salv. Ucc. di Borneo, 1871 , P. 356 .

N. minor, S. Mull. (nec Briss.), Verh. Nat. Gesch. Nederl. Overz. Bez. Land-en Volkenk. p. 110 (1839-44); Temm. \& Schleg., Faun. Jap. Aves, p. in , pl. 67 (1850); Gould, Handb. B. Austr. ii. p. 28o ( 1865$)$; G. R. Gray', Hand-list, iii. 1). 53. sp. $10254\left(18_{73}\right)$.

N. uropygialis, G. R. Gray, Cat. B. New. Guin. p. 51 (1859) (parte).

Hab. Luzón, Manila, Calaocan, Laguna; China, Japon, Australia.

33. N. Iuzoniensis, (Gm.) O $O^{0}$ N. v. Candurung, Canunuat (M. S T.)

Scolopax luzuniensis, Gm. S. N. ii. 656 .

(ourlis tachatè de l' isle de Luçon, Sonn.; Buff. H. N. Ois. 8. p. 32 .

Hab. Luzón, Manila, Malabon.

14. Gén. Gallinago, Steph

34. G. megala, Szinh. O", N. v. Candurung buquit.

(M. S. T.)

(iallinago solitaria, Swinh. (nec Hodgs), Ibis, 1860 , p. 60.

(i. megala, Stuinh.; Ibıs, 1861 , p. 343; Schleg. Mus. P. B. Scolopaces, p. 12 (1864); Swinh, P. Z. S. 1871, p. 407; Salv. Ucc. de Bormeo, 1874, p. 334; Wald. Trans. Zool. Soc. ix. 1875 , p. 235 .

(i. stenura, Radde (nec Kabl.), Reise S.-O. Sibis. p. 33t. 'Taf. yiii. f. $1-3 \quad(1863)$.

Spilura megala, $\vec{G}$. R. Gray, Hand-list, iii. p. 53, sp. 103+5 (1871); Wald. Trans. Zool. Soc. viii. 2, p. 98 (1872).

Hab. Panay, Negros, Siquijor, Leyte, Samar, Paric, Masbate, Sibuyan, Calamianes, Basilan, Tawi-tawi, Zamboanga, Mindanao; China, Célebes.

35. G. scolopacina, $B p \cdot O^{*} \odot \mathrm{N}$. v. Candurung.

(II. S. T:)

Scolopax gallinago, Limn. S. $N$. i. p. 244 n. ${ }^{\circ} 7$ ( 766$)$.

Becassine, Buff. H. N. Ois. Pl. Enl. n." 883 .

Gallinago scolopacina, Bp. Compt. Rend. p. 579, Wald. 1875, 1. 397. Orn. Rend. Phrlip.

Hab. Luzón, Manila, Bohol, Leyte; Europa. 


\section{I5 Gén. PaVONCELLA, Leach.}

36 P. pugnax, (Lin) ó. O N. v. Pavon de mar.

(M. S. T.)

Hab. Australia.

16. Gén SPLURA, Be.

37. S. stenura, (Kuthl) ơ N. v. Taringting.

(M. $S$ T)

Scolopax gallinago, Horsf. (nec; Linn), Trans. Linn. Soc. xiii. p. I9I (1821); Raffl., ibid. p. 327 (1822); Jerd., Cat. p. 337 (artimp).

S. stenura, Kuhl. in Bp., Ann. di Stor. Nat. iii. fasc. x. 1830 ; Isis, $1833, \mathrm{p}$. 1077.

S. stenoptera; Temm. (ubi:).

S. horsfieldii, G. R. Gray, iii. ind. Zool. pl. 54, f. 2 (1834); Wall., P. Z. S. 1863, p. 487 .

S. strenua, G. R. Gray, (1834).

S. biclavata, Hodgs. P. Z. S. 1837, p. $49 \mathrm{I}$.

S. heterura, Hodgs; Eyt., P. Z. S. $z 839$, p. 107.

S. pectiniciuda, Peale, U. S. Expl. Exp. Bryds, p. $227(18+8)$.

Gallinago stemura, Blyth, Cat. B. Mus. A. S. B p. 272 (I 849 ; Cass. U. S. Exp. Orn. p. 313. (1 858$)$; Jerd, B. of. India, iii. p. 674 (1864); Schleg, Mus. P.-B. Scolofaces, p. I2 (1864); Salv. Ucc. di Borneo, 1874 , p. $33+$.

Scolopax indica, Licht. (nec Hodgs), Nomencl. p. 93 (1854).

Spilura hosfieldii, Bp. Compt. Rend. xliii. p. p. 579, sp. 198 (1856); G. R. Gray, Hand-list, iii. p. 53 sp. I034t (I87 I).

Scolopax (G.lllinago) heterura, Cab., n. ${ }^{\circ}$ sp.» Mart. Journ. f. Orn. 1866, p. 28.

Gallinago horsfieldii, Swinh. P. Z. S. 1861, p. 407.

G. heterocerca, Cab., fide Swinh., P. Z. S. 1871, p. 407. Hab. Luzón, Manila, Bulacan, Mindsro, Baco; Borneo, Sumatra, China.

17. Gén RHYNCHÆAt, Cuv.

38 Rh. bengalen sis. (Lin.) ơ N. v. Pacobo.

Rallus bengalensis, Lin. S. N. i. p. 263 (i 866 ).

Scolopax capensis, Linn; Raffi, Trans. Linn. Soc. xiii. p. 327 (1822). Rhynchaea orientalis, Horsf., Trans. Linn. Soc. xiii. p. 193 (1821). Rh. variegata, Vieill., Gal. Ois. ii. p. I09, pl. 240 (1824); Schleg. Mus. P.-B. Scolopaces, p. 16 (1864); Brehm, V. An. 4. p. 447. p. 147 .

Rh. australis, Gould, P. Z. S. 1837 , p. I35; id. B.'Austr. vi. pl. 4. (I 848 ).

Rh. bengalensis, G. R. Gr. Jerd, B. of. India, xiii. p. 677 (1864); Sw inh. P. Z. S. 2871 , p. 408; G. R. Gr. Hand-l. iii. p. 55 . sp. I0357; Salv. Ucc. Borneo, 1874, p. 335; Wald. Trans, $Z$ ool. Soc. ix. 1875 , p. 235 .

Hab. Zambanga, Mindanao, Luzon, Manila, Malabon, Laguna, Calamba, Siquijor, Panay; Java, Sumatra, China. 
18. Gèn. SCOLOPAX, Iin.

39. Sc. rusticola, Lin. \& N. v. Becada comnn.

(M. S. T.)

Hah. Europa.

\section{Fam. RALIDAS.}

I. Gén. HYPOT enidis, Rchi

I. H. striata, (Lin). ơ N. v. Ticling-gubat.

(M. S. 'T.)

Rallus philippensis striatus, Briss. Orn. v. p. $167,$. n. ${ }^{\circ}$ 5. t. It, f. 2. .

R. striatus, Lin. S. N. i. p. 262 (1766); ex Bris. Gm. S. N. i. p. 7I4 (I788); Less. Tr. d' Orn. p. 536 (1831); Blyth. Cat. B. Mus. A. S. B. p. 285 (1849); Jerd. B. of India, iii. 1). 726 ( I 864 ).

Rallus gularis, Horsf. Trans. Linn. Soc. xiii. p. 196 (1821); Raffles, ibid. p. 328 (1822); Bernst. Journ. f. Orn. 1861, p. $190 ;$ Sclat. P. Z. S. 1863 , p. 223.

R. philippinus, Blylh. (nec R. philippensis, Linn).

R. indicus, Verr. ap. Rchb, Syn. Av. iii. Rassores, t. 201, f. $2575-76$.

Hypotaeniclia striata, Bp. Compt. Rend. xliii. p. 599, sp. 3.34 (1856); Schleg. Mus. P. B. Ralli, p. 24 (1865'; G. R. Grai', Hand-list. iii. p. 57, sp. 10378 (1871); Swinh, P. Z. S. 187i, P. 4I5; Wald, Trans. Zool. Soc. viii. 2, p. 95 (1872); Ib.s, 1872 , p. 382; Pelz. Ibis, 1873 , p. 40; Salv. Ulcc. di Borneo, 1874 , p. 336; Sharpe, Cat. B. M. 23, 1894, p. 33.

Fulabeornis celebensis, Pelz. (nec Auct.), Novar. Reis, Vög. p|). 134,162 (1865).

Hab. Luzon, Manila, Caloocan, Laguna, Cagayan, Hlocos, Mindoro, Samar, Negros, Panay, Guimarás, Mindanao, Siquijor, Calamianes; Java, Borneo, Célebes.

2. H. torquata, (briss.) o o N. v. Ticling-tae, Baintanac.

(M. S. 'T.)

Rallus philippensis torquatus, Briss. Orn. v. p, izo, n. 6 (1760); Gim. S. N. ii, 7I4, n. ${ }^{0} 6$.

R. torquatus, Lin. S. N. ed. 12, i. p. 262 (1766); ex Brisson. R. lineatus, Cuv. Mus. Paris, fide Puch. Rev. et Mag, Zool. 1851, p. 276 ; Lesson. Tr. p. 536, n. $^{0}$ i I (1831).

R. torquatus, Briss Meyer, Nov. Act. Ac. C. L. C. Nat, Cui'. Suppl. prim. p. Io8, pl 19; Lessou. 1. c. n." I 2.

Hypotanidia torquata, Wall. On the Birds. Philipp. 187.5, p. 39); Sharpe Cat. B. M. 23, I894 p. 43.

Hab. Luzón, Manila, Navotas, Laguna, Calamba, Bataan, Orion, Marinduque, Mindoro, Romblon, Siquijor, Masbate, Panay, Samar, Mindanao. 
3 H. philippinensis, (Briss) $\delta \&$ N. ․ Ticling.

(M. S. 'T)

Rallus philippinensis, Briss, Orı. r. p. 163. n. ${ }^{\circ}$ : Lin. S. 1 . i. p. 263, n. ${ }^{\circ} 7.1766$, ex Brisson; Schlegel, Mus. Pays-Bas, Ralli, p. 23; Wald. T,. Z. S. viii. p. 95; Buller. Birds . 1 . Zooland, part. iii. pl. 6.

Rale rayé des philippines, D'Aubent. Pl. Enl. 774 .

Hypotænidia philippinensis, Wald, On Bird, Philipp. p. 394, 187.5. Hab. Luzón, Manila, Mindoro, Calapan.

2. AMALRORNIS, REICII.

4. A. olivacea. (Meyer.) $\delta \&$ N. v. Quijao.

(M. S. T.)

Gallinula olivacea, Meyer, Nov. Act. Ac. C. L. C. Cuv. xiv. Supl. prim. p. 109, pl. 20 (1834).

Amaurornis olivacea, Reich. Natürl. Syst.: Wald. On Birds Philip. 1875, p. 392 lar 33 f. 2; Sharpe, Cat. B. 11. 23, 189': p. 156.

Hab. Luzón, Manila, Siquijor, Leyte, Samar, Bronoan, Panay, Cebú.

3. Gén. RALlina, Rснв.

5. R. fasciata, (Raff.) $\& \&$ N. v. Ticling paule.

(M. S. '1.)

Rallus fasciatus, Raff, Trans. Linn. Soc. xiii. p. 328 (1822).

Gallinula euryzona, Temm. Pl. Coll. 417 (1826).

Crex fasciati, Blyth, 7. A. S. B. xi. p. 797 (1842).

Coretrura fasciata, $G$. R. Gray, Gen. B. p. 595 (1846).

Porzana fasciata, Blyth, Cat. B. Mus. A. S. B. p. 285 (1849'; Jerd, B. of. Ind. iii. p. 275 (1864).

Rallina euryzona, Rchb, Syn iii. Rasores, t. cxx (206), f. I 186. Hypotaenidia euryzona, Rchb, Av. Syst. Nat. p. xxiii. (1852).

Rallina fasciata, G. R. Gray, Gen. and. Subgen. of. B. p. i 2!) (1855); Schleg, Mus. P. B. Ralli, p. 19 (1865); id. Ned. Tijdschr. Dierk, iii. p. 349 (1866); Hartl. \& Finsch. P. Z. S. $186 \%$, p. 7; G. R. Gray, Hant-list, p. 68, sp. ro390 (187r); Salt'. Ucc. di Borneo, 1874 , p. 337.

Euryzona fasciata, Bp. Compt. Rend. xliii. p. 599, sp. $34^{2}$ (1856). Hab. Luzon, Manila, San Mateo, Montalban, Bulacan, Lolomboy; Java, Borneo, Sumatra.

6. R. fusca, (Limn). \& \& N. y Ticling-gubat.

(M. S. T.)

Rallus philippinensis fuscus, Briss., Orn. $U$, p. 173, t. 15, f. 2. R. fuscus Linn. S. N. i, p. 262 (1766); (ex Brisson). Gm. S. N. ii. p. $7 \mathrm{I} 3, \mathrm{n}^{\circ}{ }^{\circ}$

Rale brune des Philippines, D'Aubent. Pl. Enl. 773.

Gallinula rubiginosa, Temm., Pl. Coll. 357 (1825).

Porzana fusca, Blyth, Cat. A. S. B. p. 285 (1849); Jerd., B. of India, iii, p. 724 (1864).

Euryzona rubiginosa $B p$, Compt, Rend, xliii. p. 599, sp. 344 (1856). 
Rallina fusca, Schleg.. Mus. P.-B. Ralli, p. 20 (1863); G. R. Gray, Hand-lis, iii. p. $5^{8}$, sp. 10391 (parte) (1871); Sali'. Ucc. di Borneo, 18 ;4, p. 338.

Limnobrenus rubiginosus, Sund., Meth: Nat. Av. dispon. Tentamen, p. r.3r (1873).

Hab. Luzón, Mani a, Navotas, Cagayan, Alcalá, Abulungs, Ilocos, Mindanao.

7. R. rufigenis, $\delta$ N. v. (Wall.) Ticling-tubigan.

(M. S. 'T.)

Porzana rufigenys, Wall., P. Z. S. 186.5 , p. 480 ; New't. Zool. Rec. 1865, p. 128 .

Rallina rufigenis, G. R. Gray, Hand-list, iii. $5^{8}$, sp. 1036h (I 87 I).

Hab. Luzón, Manila, Navotas, Bulacan; Java, Borneo, Sumatra.

8. R. euryzonoides, (Lafres.) $\sigma \circ$ N. v. Ticling.

(M. S. T.)

Gallinula curyzonoides, Lafr. Rev. Zool. 1875 , p. 368.

Rallina euryzonoides, 77w. P. Z. S. 1877 , P. 767; 1878 , PI. 288,345 ; Sharpe, Cat. B. M. 23, 1894, p. 78, lam. viii.

Hab. Luzón, Mindoro, Baco, Panay, Negros, Leyte, Crbú, Joló.

4. Gén. ORTYGometra, i.in.

9. O. cinэrez. (Vieill) $\$$ N. v. Tica. Matang-bayani

(M. S T.)

Porphyrio cinereus, Vieill, (nec Rallus cinereus, Vieill.), $N$. I). xxviii. p. 29 (1819); id. Enc. Méth. iii. p. Io49 (1823); P’uch., Rev. et. Mag. d: Zool. 1854, p. 563 .

Rallus quadristrigatus Horsf., Trans. Limn. Soc. xiii. p. Iof (is21). Ciallinula superciliosa, Temm. (nec Szes).

G. leucosoma, Sws, Two Cent. ant. a Duxrt. p. 348.

Rallus tannensis, Forst., Descr. An. p. 275 (1844).

R. quadristriatus, Temm. (fide Licht).

(irex quadristriata, Licht., in. Forst., Descr. An. p. 275 (nota) (i 844 ).

Rallus nigrolineatus, Hodgs. (M. S.), G. R. Gray, List, Spec. B. Brit. Mus. iii. Grallae, p. I 17 (1844).

R. superciliaris, Eyt. Ann. and. Mag. N. H. 18.75, p. 230.

Gallinula mystacina, Mus. Paris. (fide Bonaparte).

Zapornia nigrolineata, Hodgs., J. A. S. B. 1845, p. 281 . n." 765 ; Gray, Cat. Hodss. Coll. B. Mus. p. 143 (1840).

Porzana leucophrys, Gould, P. Z. S. 1847 , p. 33; id. B. Ausir. vi. pl. 81 (1848).

P. quadristrigata, Blyth, Cat. B. Mus. A.S. B. p. $28+($ I $8+9)$; Wall. P. Z. S. 1863 , p. 487 .

Erythra leucophrys, Bp., Compt. Rend. xliii. p. 600, sp. to 5 (1 856$)$.

E. cinerea, $B p$. , op. cit. sp. foh.

E. cinerea, var. media, Bp. ibid. (ex Borneo).

li. cinerea var. minima, $B p$. ibul. (ex Vova Guinea).

Ortygometra tannensis, G. R. Gray, Cat. B. Trop. Isl. p. 52 (1859).

Porzana (Zapornia) leucophrys, Verr., Rev. et. Mag. de Zool. 1860, p. 437. 
Ortygometra superciliaris, G. R. Gr., Ca'. Hodgs. Coll. B. Miss.

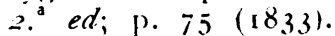

Porzana cinerea, Schleg, Mus. P.-B. Rolli. p. 32 (1865).

Ervthra quadristrigata, Gould, Haudb. B. Austr. ii p. 343 (1865). Ortigometra quadristrigati, Hartl. \& Finsch, Finun. Centralpolin. p. $16+(1867)$; idt., P. Z. S. 1868 , p. 8.

Zajornia quadristrigat:a, (i. R. Grav, Hand-list, iii. p. 68 sp). $10+6+(18,1)$.

Ortygometra cinerea, Wald., Trans. Zool. Soc. viii. p. 2, p. $9+$ (i872); Salv. Ucc. de Bormeo, 1874 , p. 339.

Hab. Luzon, Manila, Laguna, Calauan, Los Baños, Guimarás, Cebú, Naga, Siquijor, I, eyte, Marinduque, Mindoro, Naujan, Panay, Negros, Paragua, P. Princesa, Basilan; Borneo, Célebes, Java.

5 Gén PORZANA. V.

10. P erythrothorax, Sterie. N. v.

Hab. Siquijor.

II. P. palykuli, Stecte. N. v.

Hab. Basilan.

12. P. bailloni tieill. $\&$ N. v. Ticling.

(M. S. T.)

Crax pygmaa, Naum, Schlegl, Mus, Pays-Bas, Ralli. p. 30 1820. Porzana pyomat, Wald. Birds. Philipp. 187.5, p. 392.

Hab. Luzon, Manila, Navotas.

\section{Gén GALLICREX, BıYTH}

12 G. cristata, (Lath) $\sigma \bigcirc$ N. v. Mang̃ungubut.

(M. S. T)

Crested gallinule, Lath, Syn. iii. i. p. 267 n. ${ }^{0} 22$.

¿ Fulica cinerea, Gm., S. N. i. 702 ( 1788 ); (ex Lath.)

Gallinula cristata, Lath, Ind. Orn. ii. p. 773 (1890); Schleg. Mus, I’. B. Ralli p. 39 (1865).

G. cinerea, Vieill. N. D. xii. p. fo+ (1817); Mart., Journ. $f$. Orn. 1866 , p. 29; Sh.trpe, Cat. B. M. 23, I894, p. 183.

G. plumbea, Vieill. ibid.

C. gularis, Horsf.. Trans. Linn. Soc. xiii. p. I95 (1821) ( $)$ ).

(:. lugubris, Horsf., ibid. (t).

C. natevia, Less. Tr. d'Orn. p. 534 (1831).

G. porphyrioides, Less., ibid.

G. rufescens, Jerd., Madr. Journ. xii. p. 205 (I840).

Gallicrex cristatus, Blyth, Cat. B. Mus. A. S. B. p. 283 (18+9); Bp. Compt. Rend. xliii. p. 600, sp. 400 (1865); Jerd. B. of. India, iii. p. 716 (г864); Holsw. P. Z. S. 1872 , p. 475.

Crcx lugubris, Rchb., Syn. Av. iii. Rasores, t. 201 f. $2572-73$.

Hypnodes lugubris, Rchb., Av. Syst. Nat. p. xxiii. (1852).

Giallinulopha cristata, $B p$. (1854?).

Ciallicrex cristatal, Sclat, P. Z S. 1863 , p. 223; G. R. Gray, 
Hand-list, iii. p. 67, sp. 1051 (1871); Swinh. P. Z. S. 1871, p. 404; Salv. Ucc. di Borneo, 1874 , p. 340.

$H a b$. Luzon, Laguna, Cagayan, Ilocos, Mindoro, Calapan, Marinduque, Panay, Masbate, Cebú, Mindanao, Joló; Borneo, Java, China, Formosa.

\section{Gén. ERY THRA, RC.His.}

14. E. phœnicura, (Penn.) ơ م N. v. Taliuacuac.

(M. S. T.)

Kallus phonicurus, Penn. Ind. Zool. p. 19, pl. 9 (1781);Gm. S. N.I. p. 715 ( I 788$)$.

Fulica chinensis, Bodd, Tabl. Pl. Enl. p. 54 (1783) (ex Buff. Pl. Enl. 895.)

(iallinula phœnicura, Lath., Iud Orn. ii. p. 770 (1790); Less., Tr. d. Orn. p. 534; (1831); Mottl. \& Dillw., Contr. Nat. Hist. Lab. p 6o (1855); Jerd. B. of. India, iii. p. 720 (186+); Schleg., Mus. P. B. Ralli, p. 4I (1865); Pelz., Norar. Reis. Vög. Pр. 133, I62 (1865); Suinh., P. Z. S. 1871, p. 414.

(iallinula erythrina, Bechst., fide Schlegel, t. $c$.

J'orphyrio phœnicurus, Vieill., N. D. xxriii p. 29 (1819).

(iallinula javanica, Horsf. Linn. Trans xiii. p. $13^{6}$ (1821); id. Zool. Kes. in Jav'a, Gen. Ind. (1824).

:Rallus sumatranus, Raffl., Linn. Trans. xiii. p. 324 (1822).

Porzana phœnicura, Blyth, Cat. B. Mus, A. S. B. P. 284 (1849); Sclat., P. Z. S. 1863 ; p. 223.

Erythra phœnicura Rchb., Av. Syst. Nat. p. xxi (1852); Bp. Compt. Rend. xliii. p. 600, sp 403 (1856); G. R. Gray, Handlist, iii. p. 67, sp. 10508 (1871); Wald., Trans. Zool. Soc., viii. 2, p. 94 (1872); Salv. Vcc. di Borneo, 1874 , p. 340.

'Porzana?, Sclat., P. Z.S. 1863 , p. 223.

Hab. Luzon, Laguna, Marinduque, Mindoro, Baco, Calamianes, Siquijor, Mindanao, Paragua, P. Princesa, Joló, Basilan, 'Tawi-tawi; Borneo, Jara, Célebes, Sumatra.

\section{Gén. Gatllinula, Bris.}

13. G. orientalis, Horsf. $\sigma \rho$ N. v Uluc, Mang̃ugobog. (M S. T)

Ciallinula orientalis, Horsf., Trans. Linn. Soc. xiii. p. 195 (1821); Raff., ibid. p. 329 (1822); Wald., Trans. Zool. Soc. viii. 2, p. 94 (1872); Sali. Lcc. di Borneo, 1874, p. 342.

(i. ardosiaca, Vienll., Gal. p. I73, pl. 268 (1825).

(;. chloropus, Schleg., Mus. P. B. Ralli, p. 45 (parte), (1865); G. R. Gray, Hand-list, iii. p. 66, sp. Io495 (parte), (1871); Wood. Nat. H. 2, 1. 7r7 fig.; Sharpe, Cit. B. M. 23, 1894, p. 169.

Stagnicola chloropus, minor, parrifrons, brachyptera, Brehm. Ir. An. 4, p. 457 .

Hab. Luzon, Manila, Navotas, Laguna, Biñan, Santa Rosa, Cagayan, Pamplona, Mindero, Naujan, Panay, Ciuimarás; Borneo, Célebes, Sumatra. 
16. G. $s p . \delta$ N. v. Lluc dagat.

(M. S. 'T)

Hab. Luzon, Laguna, Calauan.

9. Gén. PORPHYRIO, Briss.

17 P. pulverulentus, Tim. of N. v. Agbac

(M. S T)

Porphyrio pulverulentus, Tem. Pl. Col. 405 1826; v. Martens. J. F. O. 1866 , p. 29, n. ${ }^{\circ}$ 176; Wald. Or. Birds. Philipp. p. 391, 1875 ; Sharphe. Cat. B. 23, 1894, p. 507.

P. poliocephalus, Lath, apud Schlegel, Mus. Pays-Bas, Ralli, I. n." Luth. Comp. Walden, Tr. Z. S. viii. p. 92.

Hah. Luzon, Laguna, Calamba, Mindoro, Calapan.

Io Gén. PORPHYRIO, Briss

I8. P. indicus, Forsf. 0.0 N. v. Agbac

(M. S. T)

Porphyrio indicus, Horsf. Tr. Lin. Soc. xiii. p. 194 (1821); Cass. Un. St. Expl. Exp. Orn. p. 308 (1858); G. R. Gray, K. Trop. Isl. p. 53 (1859); Schleg., Mus, P. B. Ralli, p. 55 (1865); Finsch \& Hartlaub, Faun. Centralpolyn, p. 170, pi. xii. f. 2 (1867); G. R. Gray, Hand-list, iii. p. 64, sp. ro48, (I871); Wald., Trans. Zool. Soc. viii. 2, p. 92 (1872); Salt. Vcc. di Borneo, 1874 , p. 342 .

Porphyrio smaragdinus, Temm., Pl. Col. 42 I (1827 (ex Java.) less. Man. dOrn. ii. p. 297 (1828).

P. melanopterus, Temm., Mus, Lugd. (ex Geram); G. R. Gr., op. cit. p. 65 sp. 10483 (187 I).

Parphirio samoensis, Peale, U. S. Expl. Birds, p. 220, pi. 62, f. I ( 848 ); Hartl., Wiegm. Arch. f. Naturg. 1852, p. I 18 ; (r. R. Gray, op. cit. p. 65, sp. 10482 , (1871).

Hab. Luzon, Manila, Bulacan, Cavite, Santa Cruz, Laguna; Célebes, Java, Sumatra.

11 Gén FUliCA, LiN..

19. F. atra, Lin. $\&$ N. v.

(M. S. T)

Hab. Europa.

Fam. PÁRRIDAS.

I. Gen. hydrophasiands, Wagl.

I H. chirurgus, Siop.) o 0 N v. Gaoc.

(M. S. T)

Le Chirurgien d'isle de Luçon, Soun. Voy. Nouv. Guın. p. 82, pl. 45 .

Tringa chirurgus, Scop., Del For. et. Faun. Insubr. p. 92 ( 1786) (e.r. Son). 


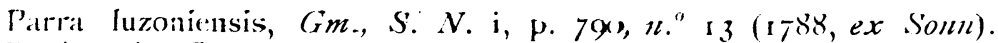

P. sinensis, Gm., ibid. n." 15, ex L,ath. Schles., Mus. P. B. R.t!!!, p. 7 I (1865); Mart., Journ. f. Orn. 1896 , p. 29.

Hidrophasianus sinensis, Wagl., Isis, 1832, p. 279; Gonsld, 13 . As. pt. vii, pl. 3 (1855); Wood. Not. Hist. $1852,2$. p. 7 r(s.

Parra phoenicura, Hodgs. in Gray's Zool. Misc. p. 86.

Hitrophasianus chirurgus, chirurgus, Blyth, Cat. B. Mits. A. S. B. p. 27.3 (18+9); Jerd., B. of. Sirt. iii, p. 70, sp. 10.5 70 , (1871); Sivinh, P. Z. S. 1871 , p. 4it; Holdsw., P. Z. S. $2 d^{\prime} 72$, p. 475; Salo Ucc. di Bornec, 1877 , p. 343.

Hah. Luzon, Manila, S. Mateo, Montalban, Loguna, Calamba, Los Baños, N." Ecija, Rosales, Cagayan, Alcalí, Ilocos, Mindoro; Java, China, Formosa, Ceilan.

\section{Fam. GRÚIDAS.}

1. Gén. AnTigone, Keach.

1 M. torquata, (Wieill) ơ @ N. v. Tipol.

(M. S. T.)

Ardea antigone, Lin. S. N. ii. p. 622.

Grus orieutalis indica, Briss. Av. 5. p. 378. n." 7 .

Greater indian crane, Edw. Glean. Av. t. 45.

Indian crane, Lath, Syn. III. i. p. 38 esp. 4.

Grus tcrqueta, Gm. var. C. S. N. 11. p.622; Brehm. V. An. 4. 1881 p. 435 .

Gruce á collier, Buff. Ois. vii. p. 307. pl. enl. 865.

Ardea torbueta, Vieill. Gal. Ois. t. 256 ; Reich. Syst. Av. t. 214 , f. 425 et t. 18 t. i 239 ad. ex Vieill. 1240.

Autigone torqueta, Bp. Comp. Av. 2. p. 100. (1854).

Hab. Luzon, N. ${ }^{a}$ Ecija, Pangasinan, Villani, S. Nicoles; Australia, Asia.

\section{Fam. ARDÉIDAS.}

1. Gén. ARDEA, LiN.

I. A sumatrana, Retift. $\sigma^{\circ}, 0$ N. v. Arandungao.

(M S. T)

Ardea sumatrana, Raffl., Linn. Soc. xiii p. 325 (182:); Blyth, Cat. B. Mus. A. S. B. p. 278 (1849); Jerd., B. of Ind. iii. p. 740 (5864); Gould, Handb. B. Austr. ii. p. 296 (1865); Wald., Trans. Zool Soc. viii. 2, p. 98 (1872); Salv. Ucc. di Borneo, 1874 , p. 344 .

A. typhun, Temin., Pl. Col. 475 (1829); G. R. Gray, P. Z. S. 1860, p. 363; Schleg., Mus. P. B. Ardeae, p. 3 (1863); Wall. P. Z. S. 1863 , p. 287; Schleg., Ned, Tijdsch. Dierk. iii. p. 348 (1 866).

A. rectirostris, Gould, P. Z. S. 1842, p. 22; id. B. Austr. vi. pl. 54 (1848).

A. fusca, Blyth, Ann. and. Mag. N. H. xiii. p. I 76 (is.t.) (jüs).

A. insirnis, Hodgs. Gras's Zool. M.sc. p. ¿6. 
A. robusta, S. Mïller, in litt.

Typhon temminckii, Rchb Syst. Ao. Nat. p. xvi. (1852).

Ardeomega nobilis, Blyth, apud Bp. Consp. ii. p. Ioy (nec. Blyth), (1855); Blyth, Ibis, 1865, p. 36.

Ardea goliath, Temm. ex Celebes, apud Bp. op. cit. p. 10 (185.5).

'Yyphon robusta, Bp. op. cit. p. 100 (1855).

Ty. sumatrana, Bp. op. cit. p. II3 (1855); Gray, Hand-list, iii. p. $27, \mathrm{sp}$. IOIO5 (187i).

Ty. rectirostris, $B p$. op. cit. p. 1 Io $(1855)$.

Ha.b. Paragua, P. Princesa, Masbate; Célebes, Sumatra, Australia, Gilola.

2. A cinerca, Lin. $\sigma_{0}$ N. v. Candangeo na puti.

(M. s. T.)

Ardea cincrea, Lin. S. N. i. p. 236 (1766); Horsf. Tr. Lin. Soc. xiii. p. 188 (1821); Schleg. Mus. P. B. Asdea, p. 5 (1863); Jerd. B. of India, iii. p. 742 (1864); Gould, Handb. B. Austr. ii. p. 295 (1865); Szelinh. P. Z. S. 1871, P. 411; G. R. Gray, Hand-l. iii. p. 26 esp. I0099 (1871); Saly. Ucc. di Borneo, 1874 , p. 344 ; Bremh, 4. p. 422.

A. brag. Z. Geoffa, Bélang. Voy. pl. 8.

A. leoccophara, Gould, P. Z. S. 1848, p. 58 ; id. B. Austr. ri. pl. 55 ( 1848$)$.

Hab. Guimarás, Luzón, Maniła, Malabon; Java, Chin, Europa.

3. A purpuraa. Lin. 00 N. v. Candang̃ahuc, Candagapi (M. S T I

Ardea purpurea, Lin. S. N. i. p. 236 (1766); Horsf. Tr. Lin. Soc. xiii. p. I 88 (182I); Mottl. et Dellze. Contr. $N$. Mist. of Salv. p. 34 (1855); Schleg. M. P B. Ardece, p. 8 (1863); Sclat. P. Z. S. 1863 , p. 222 ; Pela. Nov Reis. Vög. 1P. iis, I62 (I865); G. R. Gray, Hand-l iai p. 27 esp. Ioroz (1871; Salv. Ucc. di Borneo, 1874 , p. 945; Bremh, 4. 1881. p. 422; Wald. Trans. Zool. Soc. ix. 1875 , p. 338.

Hab. Luzón, Manila, Malabon, Bulacan, Laguna, Sta. Rosa, Panay, Leyte, Negros, Marinduque, Mindoro, 'Tablas, Calaniznes, Tawi-tawi, Paragua, P. Princesa; Burneo, Java, China, Iuropa.

\section{Gen DEMIEGKETTA, IH.YTH}

4 D sacra, $(G m$.$) o \sigma$ N v Candanğioc.

(M S. T.)

Ardea sacra, Gm. S. N. i. p. 646 ( 1765$)$ ex Lath; Lath., Ind, Orn. ii. p. 696 (г790; Vieill, N. D. xiv, p. 423 (I8I7); id. Enc. Meth. I22 I (1823); Hartl. \& Finsch, Faun. Centralpolyn. p. 201 (г86); Buller: B. Nex'. Zeal. 228, p1. (1872).

A. matook, Vieill. $N$. D. xiv. p. 416 (18г7); id. Enc. Meth. p. I I 8 (I827); G. R. Gray, Ib.s, 1862, p. 235 .

A. jugularis, Forst, Icon. Ined.; Wagl. Syst. Av. Gen. Ardea, sp. I8 (1827); Less., Tr. d'Orn. p. 574 (1831) (excl. syn.); forst., Descr. An. p. I72 (1844); Less. Descr. Mamm. et. Ois. p. 34 I (1847); Mottl. \& Dillw., Contr. Nat. Hist. Lat. 
p. 58 (1855); Cass. V. S. Expl. Exp. Orn. p. 206 (1858); Schleg. Mus. P. B.-Ardeae, p, 25 (1863); Pelz,, Novar. Reis. Vög. p. I 8 (1865); Schleg., Ned. Tijdschr. Dierk. iii. p. 348 ( 1866$)$.

A. gularis, Less. (nec Bosc.), Tr. d'Orn. p. 574 (1831).

? A, asha, Sykes, P. Z. S. 1832, p. 157.

Herodias matook, G. R. Gray, App. Dieff. $N, Z$. ii, p. 106 (1843); id., Voy, Ereb. et Terr. Burds. p, I3 (1845).

Herodias grey, G. R, Gray, List Spec. B. Brit. Mus. iii. Grallae, p. 80 (1844); Gould. B. Austr. vi. pl. 61 1848; Bp., Consp. ii, p. 122 (1855); Sclat., P. Z. S, 1862, p. 223.

$\mathrm{H}$, jugularis, $G, R$, Gray, op. cit, ibid.

Demiegretta concolor, Blyth, J. A. S. B. xv. p. 376 (1846).

Herodias pannosus, Gould, P. Z. S. 1847 , p. 221 ; id., B. Austr. vi, pl, 59 (1848); Bp., Consp. ii. p. I 29 (1855).

H. asha, Blyth, Cat. B. Mus. A. S. B. p. 280 (1849); Bp., Consp. ii, p. Iao (1855); Gould, Handb, B. Austr. ii, p. 3'5 ( 1865$)$.

H, jugularls, Blyth, Cat. B. Mus. A, S. B. p. 34 I (1849); Gould, B. Austr. vi, pl. 60 (1848); Wall, $P, Z$, S. 1863, p. 487 ; Sclat., P, Z, S. 1864 , p. 9.

Ardea atra, Cuv.-Pucher., Rev. et Mag. de Zool. 1851, p. 375. Herodias atra, $B p$. , Cousp. ii. p. I 21 (1845).

$\mathrm{H}$, sacra, Bp., Consp. ii. p. I 21 (1855); id., Compt. Rend. xl. p. 1212, sp. 53 (1855).

$H$, concolor, $B p$, Consp. ii, p. 1 аг (1855).

? Ardea aruensis, G. R, Gray, P. Z.S. 1861, p. 458 ; id., $P$. Z. S. 1861 , p. 438; Schleg., Ned Tijdschr. Dierk. iii, p. $34^{8}$ (1866); G. R. Gray, Hand-list, iii. p. 28, sp. IoII9 (1871).

A. albulineata, G. R. Gray, P. Z. S. 1859 , p. I66; Id. B. Trop, Isl. . 48 (1859'; Verr. et Desm., Rex. et Mag. de Zool. 1860 , p. 438; Schleg., Mus, P. B. Ardeae, p. 27 (1863).

A. cinerea, Ellm., Zool. 1861, p. 7469.

Demiegretta asha, erd., $B$ of India, iii, p. 746 ( 1864 ).

D, jugularis, Gould. Handb. B. Austr. ii. p. 307 ( 1865).

Demiegretta greyi, Gould, op. cit. p. 309 (1 865).

D. sacra, $G, R$. Gray, Hand-list, iii. p. 28 , sp. Ior 20 (187)'; Wald, Trans, Zool. Soc. viii. 2, p. Ioo (1872); Salv. Ucc. di Borneo, 1874 p. 246; P. Z. S. 1877 , p. 55I; Sharpe, Ibis, 1894, p. 244; Twed.

Hab. Mindoro, Naujan, Panay, Masbate, Siquijor, Samar, Borongan, Luzón, Bataan, Orión, Cagayan, Pamplona, Panaon, Joló, Marianas, Cebú; Borneo, Java, Sumatra, Célebes, China,

\section{Gén. Hekodias, Boie.}

3. H. torra, Buch-Hamilt.) ơ N. v. Tagac.

(M. S. T.

Ardea torra, Buch. Hamilt. ap. Franklin, P. Z. S. 1830-3i, p. 123.

A. modesta, Gray, Zool. Misc. p. 19; Gray and Hardze., iii.

Ind. Zool. ii, pl. 49, f, I (1 834 .)

A. nigrirostris, Gray \& Hardw., ibid, f. 2. 
Hcrodias ffavirostris, G. R. Gray, Vög. Ereb. et Terr. p. 13 $(18+3)$; Id., Ibis, 1862, p. 235 .

H. modesta, G. R. Gray, List Spec. B. Brit. Mus. iii. Grallae, p. $77(1844)$.

H. torra, Salv. Ucc. di Borneo, 1874 , p. 347.

H. syrmatophora, Could. B. Austr. vi. pl. 56 (1848).

H. alba, Blyth (nec Linn. ', Cat. B. Mus. A. S. B. p. 279 (1849); Jerd., B. of India, iii. p. 744 (1864); Gonld, Handb. B. Austr. ii. p. 3or (1865); Haldsw., P. Z. S. 1872 , p. 477.

Ardea alba, Temm. \& Schleg., Faun. Japon. Aves, p. I 4 (1850); Gray, P. Z. S. 1860 , p. 363 ; Hartl. \& Finsch, Orn. O. Afi. p. 683 (1 870$)$; (parte).

Egretta modesta, Bp., Consp. ii. p. 117 (1855); Swinh., P. Z. S. 1871, p. 412 .

E. nigrirostris, $B p$., op. cit. p. 117 (1855).

li. syrmatophora, $B p$. , op. cit. p. 115 (1855).

Herodias egretta, Blyth, Ib:s, 1860 , p. 64; i861, p. 51; Wald., Trans. Zool. Soc. viii. 2, p. 99 (1872).

H. syrmatophorus, Blyth, Ibis, 1865, p. 36 (=alba).

Ardea egretta, Schleg., Mus. P. B. Ardeal, p. I7 (1863); (partim), Id., Ned. Tijdsch. Dierk. iii. p. 349 (1866).

Egr. tta modesta, Swinh, P. Z. S. 1871, p. 412. Hab. Paragua, P. Princesa, Calamianes, Culion; Borneo, Java, Célebes.

6. H. intermedia, (Hasself) ơ N. v. Tagao, Cobo.

(M. S. T )

Ardea intermedia, Hasself. in litt. Wagl., Isis, 1829, p. 659; Schleg, Mus. P. B. Ardeac, p. 19 (1863); id., Ned Tijdschr. Dierk. iii. p. 348 (1866); Hartl. \& Fins.h. Vög. O. Afr. p. 686 (1870).

A. melanopus, Wagl., Isis, 1829 , p. 659.

A. putea, Buch, Hamilt., ap. Frankl., P. Z. S. $1830-31$, p. 123.

A. nigrirostris, Gray \& Hardw., iii. Ind. Zool. pl. 492 (1834).

A. egrettoides, Temm. (nec Gm.), Man. d'Orn. iv. p. 314 (1840); Temm. \& Schleg., Faun. Jap. Aves, p. 155. t. 69 (1850).

Herodias plumifera, Gould, P. Z. S. 1847 , p. 221 ; id., B. Austr. vi. pl. 57 (1848).

H. intermedia, Blyth, Cat. B. Mus. A. S. B. p. 279 (1879); Wald. Trans. Zool. Soc. ix. 1875, p. 273.

Ardea nivea, Cuv. (nec Gm.) Puch., Rev. et Mag. de Zool. 1851, p. 576.

Egretta plumifera, $B p$. Consp. ii. p. I 1 5, n. ${ }^{0}$ (1855).

E. egrettoides, $B p .$, ibid, n. ${ }^{\circ} 5$.

E. melanopus, $B p$., ibid, p. 116, n. ${ }^{0} 7$.

E. intermedia, $B p$. , ibid, p. 116, n. ${ }^{\circ} 8$.

Heredias egrettoides, Swinh., Ibis, 1861 , p. 261; Jerd., B. of Ind. iii. p. 745, (1864); Gould, Handb. B. Ausir. ii, p. 393 (1865).

Herodias intermedia, Szeinh., Ibis, 1861 , p. 344 ; Id. P. Z. S. 1863 , p. $3{ }^{1} 3 ; i d .$, P. Z. S. 1870 , p. 427 ; G. R. Gray, Handlist, iii. p. 28, sp. 101 10 (1871); Swinh., P.Z.S. 1871, p. 412; Wald. Tr. Zool. Soc. ix. 1875, p. 237.

Hab. Mindoro, Baco, Mindanao, Zamboanga, Bohol, Samar, Borongan, Paragua, P. Princesa, Java, Borneo, China. 
7. H. garzetta. (Iin) ơ N v Tagac

(M. S T.)

Aigrette, Buff. H. N. O:s. 7, p. 372, t. 20 Pl. Enl. gor.

Ardea garzetta, Lin. I. N. 2, p. 628; Wald. Trans. Zool. Soc. ix. p. $237(1875)$.

Hab. Panay, Siquijor; Borneo, Java, Célebes.

4. Cién GAKZETTA. Br

8 G nigripes. (Tem.) o̊ $\mathrm{N}$ v. Bacao-itim, Cabó.

(M. S. T)

Ardea nigripes, Tem. Man. d'Orn. 2." ed. iii. P. 377 (1 840$)$.

Herodias inmaculata, Goul, B. Austr. vi. pl. $5^{8}$ (1848).

Garzetta nigripes, Bp., Consp. ii. p. I19, n. ${ }^{\circ} 3$ (1855).

Garzetta inmaculata, $B p$., ib.d n." 4 (1855).

Ardea garzetta; Mottl. \& Dillze, Contr. Nat. Hist. of. Lab. p. 35 (1855); Scleg. Mus. P. B. Ardeae. p. I 2 (parte), (1863.

Herodias melanopus, Gould., (neg Wagl.) Handb. B. Austr. ii. p. 304 (1865); Brehm, V. Ann. 4 p. 424, f. 139.

Herodias nigripes, Wald, Trans. Zool. Soc. viii. 2. p. 99 (1472); Salz. Ucc. di Borneo, 1874 , p. 349.

Hab. Luzon, Bataan, Orion, Liguna, Paric, Samar, Borongan, Leyte, Bohol; Java, Célebes.

5. Gén. Bubllcus, Br.

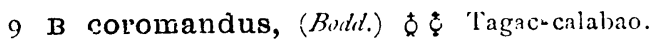

(M. S. T.)

Cancroma coromanda, Bodd. Tabl. Pl. Enl. p. 54 (1783) ex Ruff. Pl. Enl. g10; Schleg. Mus. P. B. Ardeae, p. 30 (1863).

Ardea coromandeliensis, Steph., Gen. Zool. xi, p. 577 (1819) e, xiv. p. 324 (1826).

A. affinis, Horsf. Trans. Linn. Soc. xiii, p. 189. (1829).

A. caboga, Frankl., P. Z. S. 1830-31, p. 124.

A. roussata, Temm., Man. d'Orn. 11, p. 566 (partim) (i 820$)$.

A. russuta. Wagl., Syst. Av. Gen. Ardea, sp. 12. (1827) (partim). Gray, P. Z. S. 1860 , p. 363.

Herodias bubulcus, Blyth, Cat. B. Mus. A. S. B. p. 280 (184); (partim).

Bubulcus coromandelensis, IBp. Consp. ii, p. I 25 (1855).

Herodias coromanda, Wall., $P$. Z. S. 1863 , p. 487.

Buphus coromandus, Jerd., B. of Iridia, III, p. 749 (I864).

Bubulcus coromandus, $G$. R. Gray, Hand-list, iii, p. 3o, sp. 10133 (1871); Swinh., P. Z. S. 1871, p. 412; Sali. Ucc. di Borneo, 1874 , p. 350; Hald. Trans. Zool. Soc. ix. 1875, p. 337 .

Hab. Luzen, Manila, Bulacain, Laguna, Bataan, Negros, Trablas, Masbate, Cebú, Panay, Guimarás, Mindanao, Paragua, Balabac, Bohol; Borneo, Java, China. 
6. Gin. AkDiELA, Buik.

10. A. speciosa, (Hiisf.) ơ N. v. Bacao.

(M. S. 'T.)

Artea speciosa, Horsf. Tr. Lin. Soc. xiii, p. Isy (18́21); Iat., Zool. Res. in Javia, pl. 62 (1824).

Ardeila siseciosa, G. R. Gray, List. Gen. B. p. 80 (1 $8+1)$; Iat. Hand-liät, iii. p, 30, sp. $10135^{2}$ (1871; Wiald., Tritns. Zoul. Sic. viii, 2. p. 97 (1872); Sirlv. Uic. di B.mnes, $18^{2} 7 \mathrm{t}, \mathrm{P} .35 \mathrm{I}$. Buphus sijeciosus, Bp. Consp. ii, p. $127\left(18_{55}\right)$.

Ardea leucoptera speciosa, Schleg., Mus. P. B. Aritial, p. It (1863).

Hab. Paragua, P. Princesa; Burneo, Java, Célebes.

7. Gén. BUTORLDES, BLYTH.

I. B. javanica, (Herff.) $\sigma \circ \mathrm{N}$, Bacao-al, uhin (M. S. T.

Ardea javanica, Horsf. Lin. Tr. Soc. xiii. p. 190. (182 I); R.tfth. ibid. p. 326 (1822); Less, M.tn. d'Orn. ii. p. 2 40 (1821); Schleg. Mus. P. B. Ardeae, p. 43 (1863); Id., Nederl. Tydschr. Deerk. iii, p. 348 ( 1866); Hartl. \& Finsch, Fatul. Ceiniralpelyia. p. 20I ( 867 ); PArlea choriceps, Hodgs.

Ardetta stagnatilis, Gould, P. Z. S. 18+7, p. 221, In., B. Aiøstr. vi. pl. 67. (1848):

?Buturides javanica, Blyth, Cat. B. Mas. A. S. B. p. 281 (18+9); Bip., Consp. ii, p. I 29 (1855); Sclat., P. Z. S. 1853, p. 223 ; Wall., ibid. p. 487 ; Jeril., B. of. Int. iii. p. 752 (186+); Gould, Handb. B. Austr. ii, p. 317 ( 1865$)$; Suvinh., P: $Z$. S. 1871 , p. 4r3; G. R. Gray, Hant-List; iii. p. 3r, sp. 10157 (1871); Haidvw. P. Z. S. 1872 , p. 478 ; Wald. Trans. Zool. Soc. viii. 2 p. 100 (1872); Sialv. Ucc. di Borneo, 1874 p. 351; Willd. Trans Zool. Soc. ix. 1875, p: 237.

?Ardietia thalassina, «Swos.» Kelaart, Prodr. Finun. Ceyl. p. I 33 (1852).

Ardea stagnalis, Cass., U. S. Expl. Exp. Orn. p. 297 (1858); G. R. Gray, Cat. B. Trop. Ijl. p. 49 (1859); Pelz; Novar. Reis. Vög. p. I2t (I 865 ).

Ardea (Butorides) viridiceps, Gray, P.Z. S. 1860, p. 364.

A. (Butcrides) virescens var. scapularis et var. amurensis, Schrenk. Vög. Amurl. p. 437, (1860).

Hab. Luzon, Layuna, Calauan, Mindoro, Naujan, 'Tablas, Sibuyan, Negros, Leyte, Amparo, Dinagat, Paragua, P. Princesa, Mindanao, Guinaras, Panay, Cebú, Samar, Marinduque, Masbate, Siquijor, Calamianes, 'Tavi-tavi, Joló; Borneo, Java, China, Cèlebes.

12. B. macrohyncha. (Gould) $\sigma \rho$ N. v. Bacao-benge.

(M. S. 'T.)

Ardetta macrorhyncha, Gould, P. Z S. 1878, p. 39; Id., B. Anstr. vi. pl. 66 (1848).

Arlea scapularis, Temm. \& Schlog. (nec III), Finun. Jap. Aves, i. II (i) (IBj). 
Butorides macrorhyncha, $B p$. Consp. ii. p. 131 (1855); Gould, Handb. B. Austr. ii. p. 316 (1865); Swinh. P. Z. S. 1811, p. 413; Salv. Ucc. di Borneo, 1874 , p. 353.

Ardea javanica, Mettl. \& Dillw. (nec Horsf.), Contr. Nat. Hist. of Labuan, p. 59 (1855).

A. macrorhyncha, Schleg., Mus. P. B. Ardeae, p. 44 (1863).

Butorides javanicus, Swinh. (nec Bp.), Ibis, 1863 , p. 413.

Hab. Mindoro, Baco; Borneo, China, Java.

\section{Gén. ARDEIRALLA, Verr.}

13. A. flavicollis, (Lath) of $0 \mathrm{~N}$ v. Cobo. Bacao-lati

(M. S. 'T.)

Ardea flavicollis, Lath. (nec Gould.), Ind. Orn. ii. p. 701 (1790); Horsf. Trans. Linn. Soc. xiii. p. I89; (1821); Wagl., Sy.st. Av. Gen. Ardea, sp. 16 (1827); Hardw. \& Gray, iii, Ina. Zool. i. pl. 66, f. 2. (1830-34); Jerd., III. Ind. Orn. pl. Io (1843); Schleg., Mus. P. B. Ardeae, p. 45 (1863); Id., Ned. Tydschr. Dierk. iii, p. 348 ( 1 866).

A. nigra, Vieill., $N$. D. xiv, p. 417 (1817).

A. picta, Raff., Trans. Linn. Soc. xiii. p. 323 (1822).

A. bilineata, Cuv., Mus. Par. Less., Tr. d'Orn. p. $57+$ (1831); Pucher., Rev. et Mag. de Zool. 1851, p. 375.

Ardetta flavicollis, Blyth. Cat. B. Mus. A. S. B. p. 272, (1849); Bp. Consp. ii. p. 13 I (1855); Wall., P. Z. S. 1863, p. 35; Jerd., B. of. Ind. iii. p. 853 (1864); Swinh., P. Z. S. 1871 , p. 4 I $3 ; G$. R. Gray, Hand-List, iii. p. 30, sp. 10144 (1871); Holdsw., P. Z. S. 1872, p. 478; Wald, Trans. Zool. Soc. ix. p. 236 . (1875).

Botaurus flavicollis, Rchb., Syst. Av. Nat. p. xvi, (1852).

Ardetta bilineata, $B p .$, Consp. ii, p. I 32 (1855).

Ardea (Ardetta) flavicollis, Gray, P.Z. S. 1860, p. 364.

Ardiralla flaviacollis, Salv. Ucc. di Borneo, 1874, p. 353.

Hab. Luzon, Bulacan, Malolos, Samar, Paric, Mindoro, Naujan, Cebú, Mindanao; Borneo, Java, Sumatra.

9. Gén. Ardetta, G. R. Gray.

14. A. sirensis, (Gm.) ơ N. v. Bacao beng̃e.

(M S. T.)

Ardea sinensis, Gm. S. N. i. p. 642 (1788); Gray, \& Hardw., iii. Ind. Zool. i. pl. 66. f. 2 (1830-34); Schleg., Mus. P. B. Ardeae, p. 40 (1863).

A. lepida, Horsf., Trans. Linn. Soc. xiii. p. I9o (1821); Wagl. Syst. Av. Gen. Ardea, sp. $3^{8}$ (1827).

A. melanophis, Cuv., apud Less., 7i. d'Orn. p. 573 (errore) (1831).

A. melanotis, Cuv., apud G. R. Gray, Gen. B. iii. App. p. 25 (errore) (1.849).

Ardetta sinensis, Blyth. Cat. B. Mus. A. S. p 282 (1849); Jerit. $B$. of Ind. iii p. 755 (1864); Swinh., P. Z. S. 1871, p. 414; G. R. Gray, Hand-list, iii. p. 31, sp. 10147 (1871); Wald., Trans. Zool. Soc. viii. 2. p. 99 (1872); Salv. Ucc. di Borneo, 1874 , p. 354; Wald. Trans. Zool. Soc. ix. 2875 , p. 237. 
Ardea melanoptera, Cuv., in Mus. Paris.-Pucher., Rev. et Mag. de Zool. 1851 , p. 375 .

Ardeola sinensis, Bp. Consp. ii. p. I33 (1855); Wall., P. Z. S. 1863 , p. 487 .

Hab. Luzún, Laguna, Calamba, Cagayan, Alcalá, Ilocos, Rattac, Panay, Mindoro, Baco, Calapan, Joló, Tablas; Borneo, J:va, Célebes, China.

15. A. paraguanensis, o" N. $\checkmark$ Cabo.

(M. S. T.)

Hab. Paragua, P. Princesa.

I6 A. cinnamomea, $(\mathrm{Gm}$.$) o$. N. v Hacao canela

(M. S. T.)

Ardea cinnamomea, Gm. S. N. i. p. $6+3$ (1788); Raff., Trans, Linn. Soc. xiii. p. 326 (1822); Wagl., Syst. Av. Gen. Ardea, sp. 39 (1827); Gray, \& Hardw., Ill. Ind. Zool pl. 6\%. f. I. (1830-34); Schleg., Mus. P. B. Ardeae, p. 40 (1863); Mart. Journ. f. Orn. 1866 , p. 28.

A. nebulosa, Horsf., Trans. Linn. Soc. xiii. p. 190 (1821).

Ardetta cinnamomea, Blyth. Cat. B. Mus. A. S. B. p، 282 (1849); Jerd., B. of Ind. iii. p. 755 (1864); Sclat. P. Z. S. 1871, p. 413; G. R. Gray, Hand-list, iii. p. 31, sp. I0146 (1871); Holdsw., P. Z. S. 1872 , p. 478; Salv. Ucc. di Borneo, 187t, p. 354; Wald. Trans. Soc. ix. p. 237 (1875).

Ardeola cinnamomea, $B p$., Consp., ii. p. I 32 (1855).

$H a b$ : Luzón, Manila, Malabon, Laguna, Calamba, Bataan, Orion, Cagayan, Pamplona, Ilocos, Batac, Mindoro, Baco, Marinduque, Panay, Amparo, Leyte, Cebú, 'Tablas, Guimarás, Mindanao, Zamboanga, Tawi-tawi; Java, Borneo, China.

17. A. sp. $\sigma^{\prime}, 0$ N. v. Bacao-tulic.

(M. S. T.)

Hab. Cagayan, Pamplona, Ilocos, Batac.

10. BOTAURUS, StEPh.

18. B. limnophylax, (Gen.) o 0 N. v. Bacao.

(M. S. T.)

Nycticorax limnophilax, Tem $P l$. Col. 581 (juv.) (1835).

Botaurus limnicola, Rchb., Syn. Av. ii. Grallatores, t. i48, f. $5 \mathrm{I} 0$, 'Tigrisoma limnicola, Rchb., Syst. Av. Nat. p. xvi. (1852).

Botaurus limnophilax, Bp., Consp. ii. p. 136 (1855); G. R. Gray, Hand-list, iii. p. 32, sp. Ior64 (1871); Salv. Ucc. di Borneo, 1874 , p. 355 .

Ardea limnophylax, Schleg., Mus. P. B. Ardeae, p. 55 (i 863).

Hab. Luzón, Bataan, Orion, Mindoro, Baco; Java, Bangka.

19. B. stellaris, Lin. $\sigma^{\prime}\left(\begin{array}{ll}\text { B } & 289\end{array}\right)$ N. v. Alcaravan.

(M. S. 'T.)

Hab. Europa, Alemania. 
11. Gén. BUPHUS, Bore.

20. B. malaccensis, (Gm.) N. v. Bacao.

(M. S. T )

Ardea malaccensis, Gm. S. N. 2. p. 643 esp. 73 .

Crabrier blanc et brun, Buff. Hist. Nat. des Ois. 7. p. 394.

C. de Malac, Buff. Pl. Enl. n. ${ }^{\circ} 91 \mathrm{r}$; Hardw. Ill. Ind. Zool. 1. t. 48 .

Malacca Heron, Lath. Syn. III. i. p. 78 n. $^{\circ} 44$.

Buphus malaccensis, Cab.; Reich Syst. Av. t. I50. f. 500, 7 Buff.

t. 50 f. 2397; Bp. Comp. Av. 2. p. 126 (1857).

Hab. Luzón, Manila, Malabon, Bulacan, Ilocos, Batac.

12. Gén. ZEBRILUS, Bp.

21. Z. radiolatus, $B p$. $\&$ N. v. Bacaobbulic.

(M. S. T.)

Ardea philippensis et undulata, Gm. S. N. i. p. 670.

Cacrophagus philippensis, $B r . O r$. v. p. 274 t. 33 f. 2.

Butaurus undulatus, $\mathrm{Cab}$.

'Tigrisoma undulatum, Gr. Reich $S$. Av, t. I 50 f. 506 .

Zebrilu; radiolatus, $B p$. Consp. Av. 2. p. I 38 (1857).

(rabier des Philippines, Buff. Pl. Enl. 898 ad et 769 jov. Hab. Luzón, Lagund, Mindoro, Baco; Cayena.

13. Gén. GORSAChiUs, Pucg.

22. G. melanolophus, (Raffl) $\delta \&$ N. v. Bacao-pulu.

(M. S. T)

Ardea melanolopha, Raffl. 7r. Lin. Soc. xiii. p. 326 (1822); Blyth, Ibis, 1865 , p. 38 .

Nycticorax goisagi, Temm., Pl. Col. 582 (1836); Hartl. \& Finsch, P. Z. S. 1868, p. 8.

Ardea goisagi, Temm. \& Schleg., Faun. Jap. Aves, p. i 16, pl. 7o (1850); Swinh., Ibis, 1861 , p. 344; Schleg., Mus. P. B. Ardeae, p. 54 (1863); Srumh., Ibis, 1865 , p. $35^{8}$.

Tigrisoma goisagi, Rchb., Syn. Av. ii. Gailatores, t. i 46 , f. 501 . Botaurus goisagi, Rchb., op. cit. t. 149, f. 2566-2567; id., Syst. Av. Nat. p. xvi. (1852).

Gorsachius typus, Pucher.

G. goisaki, Bp., Consp..ii. p. 138 (1855).

Nycticordx melanolophus, Swinh., P.Z.S. 1863, p. 320.

Gorsachius melanolophus, Blyth., Ibis, 1865 , p. 38; id., Ibis, 1867 , p. 173 (nota); Salv. Ucc. di Borneo, 1874, p. 355; Wald. Trans. Zool. Soc. ix. 1875, p. 238.

Ardea melanocephala, Swinh (errore), Ibis, 1865 , p. 359.

Gorsochius goisagi, Swinh., Ibis, 1866, pp. 122, 1 23403.

Goisakius melanolophos, G. R. Gray, Hand-list, iii. p. 23, sp. IOI 77 (I 87 I).

Goisachius melanolophus, Swinh., P. Z. S. 1871 , p. 41 3; Holdsw., P. Z. S. 1871, p. 478 .

$H a b$. Luzón, Laguna, Calamba, Cebú, Masbate, Siquijor, Guimarás, Mindanao; Sumatra, Japon, Malaca. 
14. Gén. NYCTICOR.AX, STErh.

23. N. griseus, (Lin.) o o N. v. Bacao taling.

(M. S. T.)

Ardea nycticorax, Lin. S. N. i. p. 275 (1 166(; Horsf., Trans, Linn. Soc. xiii, p. 189 (1821); Wagl., Syst. Av. Gen. Ardea, sp. 31 (1827); Temm. \& Schleg., Faun. Jap. Aves, p. 116 (1850); Schleg., Mus. P. B. Ardeae, p. 56 (1863).

A. grisea, Linn., loc. cit.

Nycticorax europaus, Steph., Gen. Zool. xi, p. 609 (1819).

N. griseus, Strickl.-Blyth, Cat. B. Mus. A. S. B. p. 281 (1849); Bp. Consp. ii. p. 140 (1855), Sclat., P. Z. S. 1863, p. 223; Jerd. B. of Ind. iii, p. $75^{8}(1864)$; Wald., Trans. Zool. Soc. viii. 2, p. $100($ I872); Holdsw., P. Z. S. 1872, p. 478; Sali'. Ucc. di Borneo, 1674 , p: 356 .

N. europara, Szus. Class. B. ii. p. 355 (1836).

N. nycticorax, G. R. Gray, Hand-list, iii. p. 33, sp. 10171 (1871); Swinh. P. Z. S. 1871, p. 413.

Hab. Luzón, Manila, Malabon, Bataan, Orion, Laguna, Calamba, Mindanao; Java, Borneo, Célebes, China.

24. N. manillensis, Vig. $\sigma \circ$ N. v. Bacaodagat.

(M. S. T)

N. manillensis; Vigor, P. 2. S. 1831, p. o8; Trans. Zool. Typice, pl. 66; Bp. Consp. 11. p. 140; V Marten, J. f. O. 1866 , p. 28; Schlegel, Mus. Pays.-Bas, Ardea p. 60; G. R. Gray, Handl n. ${ }^{\circ}$ roi $73 ;$ Wald. Ov. Rinb. Philipp. p. 400, 1875 .

Hab. Luzón, Manila, Mindanao, Siquijor, Marinduque, Samar, Paric, Basilan, Amparo, Leyte, Cebú, Negros, 'Tablas, Sibuyan, Panay, Masbate, Siquijor, Tawi-tawi.

\section{Fam. CICÓNIDAS.}

\section{Gén. LEPTOPTILOS, Less.}

I. L. dubius, (Gm.) $\sigma 0$ N. v. Ciguieña marabú.

Ardea dubia, Gm. S. N. i. p. 624 (1788) ex Lath.

A. argala, Lath. Ind. Orn. ii. p. 676 (1790).

Cicoonia marabeu, Temm., Pl. Col. 300 (1824).-Wagl., Syst. Av. Gen. Ciconia, sp. i. (1827).

Hab. Sumatra, Java, Borneo.

2. Gén. MELAN OPELARGUS, Rchi.

2. M. episcopus, (Bodd.) $\checkmark \circ$ N. v. Camabuy, Ligay. (M. S. T.)

Ardea episcopus, Bodd. Tabl. Pl. Enl. p. 54 (1783) (ex Buff., Pl. Enl. go6).

A. leucocephala, Gm. S. N. I. p. 642 (1788).

Ciconia leucocephala, Horsf., Trans. Linn. Soc. xiii. 1. 188 
(1821); Lykes, P. Z. S. 1832, p. I50; Blyth, Cat. B. Mus. A. S. B. p. 277 (1849); Jerd., B. of Ind. iii. p. 737 (1864); Schleg., Mus P. B. Ciconsiae, p. 9 (1864).

C. arrebellatta, Wagl., Syst. Av. Gen. Cicoma, sp. 11 (1827).

C. biclavata, Hodos., Gray's Zool. Misc. (1831).

C. bicaudata, 7ickell, J.A. S. B. xvii, p. 304 ( 1848$)$.

C. episcopus, G. R. Gray, Gen. B. $5^{6}+(1848)$; Hartl. \& Finsch. $V \dot{g}$. $O . A f r$. p. I 22 (1 870 ),

C. microscelis, G. R. Gray, op cit. pl. I 5 I (1848); Rchb., Syan. Av. ii. Grallatores. t. I44, f. 2388.

sphenorhinchus leucocephalus, Rchb., op. cit.; t. 165, f. $45^{6}$; Id., Syst. Av. Nat. p. xvi. (1852).

Melanopelargus leucocephalus, Bp., Consp. Av. ii. p. Io5 (185.5).

Ciconia pruyssenaerii, Heugl., Journ. f: Orn. 1853 , p. 28; Id., Ibis, 1864 , p. 430 ; Id. Journ. f. Orin. 1864 , p. 275.

Melanopelargus episcopus, Blyth, Ibis, 1867 , p. i7 2; G. R. Griy', Hand-List, iii. p. 35, sp. Ior 87 (1871); Wald. Trans. Zool. Soc. viii, 2. p. IoI (1872); Salv. Ucc. di Borneo. $1874 \%$ 356; Tw. P. Z. S. 1878 p. 953 .

Dissoura leucocephala, Cab., v. d. Decken's Reisen, iii. p. to ( 869 ).

Diplura leucocephala, Blyth. (Jerd., B. of Ind. iii. p. 73.7),

Diplocercus leucocephalus, Blyth (Jerd., loc. cit.)

Hab. Luzón, Cagayan, Abra, Ilocos, Laguna, Calamba, Mindoro, Calapan, Marinluque, Bohol, S mar, Borongan, Calbayoc, Paranas, Masbate, Panay, Mindanao, Zambonga; Borneo Java, Sumatra, Célebes.

\section{Fam. TANTÁLIDAS.}

I. Gén. FAl CINELLUS, Bechst.

I. F. igneus, $\left(G m\right.$.) $\sigma^{\circ}$ N. v.

(M. S. 'T.)

I antalus falcinellus, Limn., S. $N^{\star}$. I. p. 241 (1766).

vumenius igneus, S. G. Gm., Nov. Comm. Petrop, xv. p. 4tin, t. 18 (177 I).

v. viridis, S. G. Gr:., op. cit. p. 46,2. t. 19.

rantalus castaneus, P. L. S. Mïll. Sist. Av. et. Mamm. p. 2 tio (1811); Vieill., N. D. xvi. p. 23 (1817); Wagl., Sist. Ao. Gen. Ibis, sp. I (1827); Schleg., Mus. P. B. Ibis, p. 2 (1 86.3); Swinh, P. Z. S. 1871, p. 411 .

Plegadis falcinellus, Kaup. Nat. Sist. p. 82 (1829).

'Tantalides falcinellus, Wagler. Isis, 1832 , p. 1231 .

Falcinellus igneus, $G$. R. Gray, List Gen. B. p. 87 (1841); Blyth, Cat. B. Mus. A. S. B. p. 274 (1849); Bp., Consp. ii. p. $15^{8}$ (18.55); Jerd., B. of Ind. iii. P. 770 (1864); Resenb., Journ. f. Orn. 1864 , p. 173; Gould, Handb. B. Austr. ii. p. 286 (1867); Salv. Ucc. di Borneo, 1874 p. 360 .

Falcinellus bengalensis (Licht.) Bp., Consp. ii. p. I 58 (1855).

F. peregrinus, $B p$., Consp. ii. I 59 (1855).

F. falcinellus, G. R. Gray, Hand-list, iii. p. 39, sp. 10214 ( I87 I). Hab. Luzón, Laguna; Borneo, Java, Sumatra, Célebes. 


\section{Fam. PLATALÉIDAS.}

\section{Gén platalea, lin.}

I P. leucorodia, Lin. ¿ N. v. Espátula blanca.

(M. S. T.)

Hab. Europa.

2. Gén. LEUCORODIUS, Rrich.

2. L. luzoniensis, $(\operatorname{Scop}$.) \& $\mathrm{N}$ v. Tagac-cuchara, Canutsut. (M. S. T.)

La spatule blanche de l'isle de Luçon, Somn. Voy. Nouv. Guin. p. 89 pl. $5 \mathrm{I}$.

La spatule huppéc de l'isle de Luçon, Sonn. $t$. c. p. 95, pl. 52. Plataleå cristata, Scop. t. cit. p. $92, \mathrm{n} .{ }^{0} 76$ ex Sonn.

Pl. leucorodia, var. g. Gm. l. c. ex Sonn.

Pl. tenuirostris, Tem. Man. de`Orn. 820 , ex Sonn. pl. 51, 52; Hand boek der. Eur. Vög. p. I 34 (1824), ex Somn; Finsch. et H. parte.

Pl. luzoniensis, $B p$. Consp. ii, p. 148 n. 61857 , ex Samm. pls. 51, 52; v. Martens J. f. $O$. 1866, p. 27, n. ${ }^{\circ} 159$.

Hab. Luzón, Manila, Malabon, Laguna, Calamba.

\section{Orden: NADADORAS.}

Fam. ANÁTIDAS.

1. Gén. Querquedula, Steph.

1. Q. multicolor, (Scop.) o 0 N. v. Pato sa bundoc.

$\left(\begin{array}{lll}\mathrm{M} & \mathrm{S} & \mathrm{T}\end{array}\right)$

La petite Sarcelle de l'isle de Luçón, Sonn. Voy, Nouv. Guin. p. 9.1. pl. 55 .

Sterna multicolor, Scopoli, Del. Fl. Faun. Insubr. ii. p. 92, n. ${ }^{\circ} 74$ (i 766), ex Sonn.

Anas manillensis, Gm. S. N. i. p. 523, n. ${ }^{\circ}$ 9i (1788), ex Sonn: Eyton, Monogr. Aroatide p. I 25.

Querquedula multicolor, Wald. On Birds Philipp. Arch. in Tw. Works, p. 404 (1876).

Hab. Luzón, Manila, Malabon, Bulacan, Laguna.

2. Q. circia, (Lin) o o N. v. Pato sa bundoc.

(M. S T.)

Anas querquedula, Lin. S. N. i. p. 203 (1766); Schleg. Mus. P.-B. Anseres, p. 49 (1863).

A. circia, Lin. l. c. p. 204.

Querqu dula circia, Steph. in Shaw, Gen. Zool. xiii. 2. p. 143 (1824): Blyth. Cat. B. Mus. A. S. B. p. 305 (1849): Jerd. $B$. of Ind, iii. p. 807 (1864): Swinh. P. Z. S. 1871 , p. 418; 
G. R. Gray, Hand-l. iii. p. 83 esp. 10656 (1871); Wald. Tr.

Zool. Soc. viii. 2. p. 102 (1872); Salv. Ucc. Born. p. 361 (1874). Cyanopterus circia, Eyton, Mon. Anat. p. I 30 (I838).

Anas (Querquedula) humeralis, Müller. Venhandl. Land. en Volkenk. p. 159 (1839-44; Brehm. V. An. 4. 1881 , p. 490.

Pterocyanea querquedula, $B p$. Compt. Rend. xliii. p. 65\%. esp. Querquedula humeralis, $B p$. ibid. esp. 98.

Hab. Luzón, Manila, Malabon; Célebes, Java, China.

\section{Gén. ANAS, Lin.}

3. A. Iuzonica, Fras. $\delta\{$ N. v. Itic, Ngaga, Patiqui.

(M. S. 'T.)

Anas luzonica, Fraser, P. Z. S. 1839, p. I1 3; Zool. Typica, pl. 67; v. Martens, J. f. O. 1866, p. 30. n. 189 ; Walden, On Birds Philiptutrch. in Tweed. Works. p. 404 (1875).

Hab. Luzón, Laguna, Calamba, Cagayan, Alcalá, Ilocos, Batac, Guimarás, Marinduque, Siquijor, Panay, Masbate, Paragua, P. Princesa.

4. A. superciliosa, Gm. \& N. v. Itic.

(M. S. T.)

A. superciliosa, Gm. S. N. 2. p. 537. n. ${ }^{\circ}$ II I . Hab. Luzón, Manila, Malabon.

5. A. boschas, Lin. N. v. Pato sa bundoc.

Hab. Luzon, Manila.

6. A paraguanensis, $\sigma$ N. v Araquit-raquit.

Hab. Paragua, 'Tagburus.

7. A. pusilla, N. v. Patiqui.

Hab. Luzon, Laguna, Calamba.

8. A. cinerescens, $\delta ?$ N. v. Araquit.

(M. S. T.)

Hab. Paragua, P. Princesa.

3. Gén. DEndrocygna, Swains.

9. D. vagans, Eyton. $\sigma \circ$ N. v. Ng̃aga balliri, Pato bundoc, Papan. (M. S T.)

Dendrocygua vagans, Eyton, M. S.; Fraser Zool. Typica, pl. 68, I849; Walden, Zool. Soc. viii. p. Iо2; $2 d$. On birds Philipp. in Works, of 7 w. p. 404 (1875); v. Martens, J. f. O. 1866 , p. 30. n. ${ }^{\circ}$ 90; v. Pelz. Reise Nov. Vög. p. 137.

Hab. Luzón, Manila, Malabon, Bulacan, Laguna, Calamba, Samar, Mindanao, Borongan, Loquilocum, Mindoro, Naujan, Panay, Masbate, Siquijor, Guimarás, Basilan, Cebú, Dinagat, Leyte, Amparo, Mindanao, Zamboanga, Bohol; Australia, Jara, Célebęs. 
10. D. arquata, (Cur.) oo N. v. Papan.

(M. S. T.)

Anas arcuata, Cuv. Mus. Paris; Horsf. Zool. Res. Java, pl. 65 $(1824)$.

A. jaranica, var. b. Horsf. Tr. Lin. Soc. xiii. p. $200(1821)$.

A. sp. Raffl. Tr. Lin. Soc. xiii. p. 331 (1822).

Mareca awsuree, Sykes, $P$. Z. S. 1832, p. 168.

Dendronessa arcuata, Walg Isis, 1832 , p. 282.

Dendrocygna arcuata, Sw. Class. B. ii. p. 365 (1876); Eyton, Moins. Anat. p. Io8 (parte) (1838); Blyth. Cat. B. Mus. A. S. B. p. 301 (1848); Siclat. P. Z. S. 1863, 224; id. P. Z. S. 1864 , p. 300; Blyth, Ibis, 186 5, 39; Schleg. Mus. P.-B. Anseres, 1. 89 (1866); Blyth, Ib:s, 1867 , p. 175; G. R. Gray, Hand-l. iii. 1871 , esp. 10608; Salv. Ucc. Borneo, 1874 , p. 362.

D. arcuata, var. minor, Bp. Compt. Rend. xliii. p. 649 (1856).

D awsuree, Jerd. B. of Ind. iii. 1864, p. 789.

D. javanica, Holdsw. P. Z. S. 1872 , p. 479.

Hab. Luzón, Manila, Bulacan, Laguna, Calamba, Siquijor, Guimarás, Basilan, Bohol, Leyte, Amparo, Samar, Cebú; Borneo, Sumatra, Java, Ceilan, Africa.

II. D. guttata, (Forst) ơ N. v. Fapan, Ng̃aga.

(M S. T.)

Dendrocygna guttata, Forst. in Mus. Lugd.; Schleg. Mus. P.-B. Auseres, 1866, p. $85 ;$ G. R. Gray, Hand-l. p. 79 esp. Io6 3 ( $187 \mathrm{I})$.

D. guttulata, Tem, Wall. P. Z.S. $186_{3}$, p. 32; Müll, M. S., Sclat. P. Z. S. 1864 , p. 300.

D. guttata, Salv. Prodr. Orn. Pap. e, Moln. p. 40 ; G. R. Gray, Hand-l. p. 72 . esp. Io6 г 2 ( 187 г).

Hab. Luzón, Manila, Tinajeros; Célebes, Ternate, Gilolo.

4. Gén. NetTapus, Brand.

I'. N. coromandelianus, (Gm.) o o N. v. Papan.

(M. S. T.)

La sarcelle de Coromandel, Buff. H. Nat. Oes. ix. p. 274, Cote de Coromandel, D'Aubent. Pl. Enl. 549, 850.

Anas coromandeliana, Gm. S. N. i. p. 522 n. ${ }^{\circ} 90$ (1788); ex Bnff., v. Pelz. Reis. Novara, Vögel, p. i 36.

Nettapus coromandelianus, Wald. On. Birds. Philipp. Arch. in Tweed. Worh. p. 404 (1875).

Hab. Luzon, Laguna, Calamba, Bataan, Orion; Java, India.

5. Gén. Spatula, Boie.

I3 S. clypeata, Lin. $\sigma$ o N. v. Papan bundoc.

(M. S. T)

Anas clypeata, Lin. S. N. 2. p. 5 1 8 n. ${ }^{\circ}$ ig. Briss. Av. 6, p. 329 , n. 6 t. 32 f. I; Naum, Vög. t. 306; Gould. B. E. p. 360. A. virescens, Mars. Dan. 5 p. I 20 , t. $5^{8}$.

Souchet., Buff. H. N. Ois. 9, p. г9г; Pl. Enl. n. ${ }^{\circ} 97$ I, 972. 
Clypéata pomorina, macrorhynchos, pla'yrhynchos, y brachyrhytchos, Brehm.V.An. 4, 188 I p. 495 f. 158.

Hab. Luzon, Manila, Malabon, Laguna, Calamba; Europa, China, Australia.

6. Gén. ANSER, LiN.

14. A. segetum, Gm, 0 .. N. v.

(M S. T.)

Bean goose, Brit. Zool. 2, n. ${ }^{\circ}{ }^{267}$; Arct. Zool. a p. 546; n. ${ }^{\circ} 472$; Lath. Syn. iii. 2 p. 464, n. $^{\circ} 23$.

Anas segetum, Gm. S. N. 2, p. 512, n. ${ }^{\circ} 68 ; N a u m, V o ̈ g . ~ t . ~ 287$; Gould. B. E. pl. 348; Brehm, V. An. 4, I881, p. 474.

Hab. Luzon, Manila, Malabon; Europa.

15. A. cinereus, Meyer. $\$ \$$ N. v Oca cenicienta.

(M. S. T.)

Hab. Europa.

7. Gén OLOR, Wagler.

16. O musicus, (Lin.) \$ N. v. Cisne cantor.

(M S. T )

8. Gén, AIX, Bore.

17. A galericulata, Lin. $\$$ \&. v. Pato de abanico.

(M. S. T.)

Hab. China.

8. Gén. DAFILA, Leach.

18. D acuta, Lin. $\varnothing$ N. v. Itig.

(M. S. T.)

Canard á lonque queue, Buff. H. N. Ois. 2, p. i99. t. i3. Pl. Enl. n. ${ }^{\circ} 954$.

Sea pheasant, or Cracker, Raj. Av. p. i 47. A. 5; Will. Orn. p. 376 ; Alb. Av. 2. t. 94,95 .

Pin-tail, Brit. Zool. 2, n. ${ }^{\circ}$ 282. Arct. Zool. a. p. 566. n. ${ }^{\circ} 500$; Lath. Syn. III. 2. p. 526.

Anas acuta, Lin. Gm. S. N. 2. p. 529. n. ${ }^{\circ} 28$.

Dafila acuta, Gould. B. E. pl. $365 ;$ Brehm. V. An. 4. p. 494.

Hab. Luzon, Manila, Malabon, Laguna, Calamba; Formosa, Europa.

9. Gén. FUliX, Sundev

19. F cristata, Lin. $\delta \&$ N. v. Pamituin.

(M. S. T.)

Fuligula cristata, Brehen. V. Am. 4. 1881. p. 502.

Anas fuligula, Gesn. Av. 107. Aldr. Orn. 3. p. $221 ; G m$. S. N. 2. p. $543 \cdot$ n. $^{\circ} 45$.

A. cristata, Pas. Av. 142.

Morillon, Buff, H. Nat. Ois. 9. p. 227, 231. t. i 5 Pl. Enl. n. ${ }^{\circ}$ roor.

Hab. Luzon, Manila, Malabon, Pampanga, Basilan. 


\section{Fam. PELECÁNIDAS.}

\section{Gén PELECANUS, I,inn.}

I P. roseus, Gm• $\sigma \circ \mathrm{N}$ v. Pagala.

(M. S ' )

Pelecanus roseus, Gm.; S. N. I. p. 570. sp. $q$ ( 1788$)$; (ex Sonn.) Salv. Ucc. de Borneo, 1874, p. 363.

P. manilensis, Gm. op. cit. p. 57ı, sp. i1. (jui.) ex Sont. ?Raffl., Trans. Linn. Soc. xiii. p. 330, (1822).

P. philippensis, Gm., op. cit. p. 571, sp. 12, (1788); ex Briss. Horsf., Trans. Linn. Soc. xiii, p. 330, (1822); Schleg., Mus. P. P. Pelec.mi, p. 33, (1863), (parte); Jerd., B. of. Ina. iii, p. 858 (1864); Sclat. P. Z. S. 1868 , p. 268. Swinh., P. Z. S. 1871, p. 420, Id., P. Z. S. 1871, p. 633.

P. rutescens, LCht., Abhandl. Akad. Wiss. Bert. $1838 \mathrm{p} .+39$, (parle); Elliot, P. Z. S. 1869 , p. 583 (parte); G. R. Gray, Hand-List, iii, p. I 28, sp. 10115 (1871); (parte).

Hab. Luzón, Cavite, Naic, Laguna, Calanba; China, Java.

2. P. javanicus, Horsf. $0^{\circ} .0$ N. v. Pagala.

(N. S. T)

Pelecanus javanicus, Horsf., Trans. Lin. Soc. xiii. p. I97 (I821); Jerd., B., of. Ind. iii. p. 857 (i864); Blyth, Ibis, 1857 , p. 179; Sclat., P. Z. S. 1868 Elliot; P. Z. S. 1869 , p. $58 \mathrm{r}$, G. R. Gray, Hand-List, iii, p. r3o, sp. 111.54 (I871), Salá. Ucc. di Borneo, 1874 ; p. 363 .

P. minor, Rüpt., Mus Senckens. ii. p. I 85 (I837); Id., Vög. N.O.Afr. p. I 40, t. $49(18+5)$; Elliot. P. Z. S. 1869 , p. $5^{80}$. P. mitratus, Licht., Abhandl. Akad. Wiss. Berl. $183^{8}$, p. 436, t. iii, f. 2; Blyth, Ibls, 1857 , p. 178; Sclat., P. Z. S. $186 \%$ p. 266; Swinh., P.Z.S. 1871 , p. 420 ; Sclat., P. Z. S. 1871 , p. 633 .

P. brevirostris, Hodgs. (parte).

Hab. Luzón, Crgayan, Panplona; China, Java, Sumatra.

2. Gén. FREGATA, Ill.

3. F aquila, (Linn.) $\sigma \circ \mathrm{N} . \mathrm{v}$

(M. S. T)

Pelecanus aquilus, Linn., S. N. I. p. 2 i6 (i766).

Fregate, Buff. H. N. Ois. 8. p. 381. Pl. Enl. n. ${ }^{\circ} 95 \mathrm{r}$.

P. leucocephalus, Gn., S. N. I. p. 572 (1788); (jov.) Raff. Trans Linn. Soc. xiii, p. 330 (1822).

Fregatia aquila, Ill. Prodr. p. 279 (I8Ir). Sch!go., Mis. $P . B$. Pelecani, p. 2. (1863); Salv. Ucc. di Bornes, 1874, p. 364.

'Trachypetes aquilus, Vieill. Gould., Handb. B. Austr. ii. p. 659 (1865); Hartl. \& F nsch. Faun. Centratolyn. p. 265 (i 867); Brehm. V. An. 4. p. 555 .

Attagen aquilus, Gray. Jerd. B. of Ind. iii. p. 853 (i 864); $G$. R. Gray. Hand. List, iii. p. I 3I, sp. 11161 (I87I).

Hab. Luzón, Manila, Cebú, Carolinas; N. Guinea, Sunatra. 
4. F. minor, $(G m)$ do N. v.

(M. S. T.)

Fregata minor, Briss. Orn. vi, p. 509 (1760); Schleg., Mus. P. B. Pelecani, p. 3 (1863); Salv. Ucc. di Borneo, 1874 p. 364; W'itteh. Ibis, $1890, \mathrm{p} .6 \mathrm{I}$.

Pelecanus minor, Gm. S. N. I. p. 572 (1788) (ex Briss).

Atagen ariel, Gould, Ms. G. R. Gray, Gen B. p. 669, pl. ist (1815); Gould, B. Austr. vii. pl. $72(1848)$.

'Trachypetes minor, Bp. Consp. ii. p. 107. (1855); Gould, Handb. B. Austr. ii. p. 499 (1865); Swinh., Tbis, $18^{2} 68$, p. 56.

Attagen minor, G. R. Gray, Hand-List, iii. p. I $3, s p .11162$ (1871); Swinh., P. Z. S. 1871, p. 423.

Hab. Luzon, Manila, Negros, Mindanao; l.abuan, Molucas, China.

\section{Gén. GRÁCULUS, LiNא,}

5. G. carbo, (Linn.) \& N. v. Cormoran.

Cormoran, Buff. H. N. Ois. 8 p. 3ro. t. 26. Pl. Enl. 972.

l'elecanus carbo, Linn, S. N. I. p. 216 (i766) (spec. major).

Phalacrocorax medius, Nilss, Skand. Fant. Fogl. ii, p) 478 (spec. minor.) (1835).

Jelecanus sinensis, Shaw, Nat. Misc. pl. 529.

('arbo filamentosus, Temm. \& Schleg., Faun. Jap. Aves, p. I20) ( 1850$)$.

(. capillatus, Temm. \& Schleg., op. cit. t. 83, 8t.

Giraculus carbo, Gray, Schleg. Mus. P. B. Pebecani, p. 6 (1863); Jerd. B. of Ind. iii, p. 861 (1864); Salv. Ucc. di Borneo, 1874 p. 364 .

Phalacrocorax carbo, Dum. Bp., Consp. ii, p. 168 (1855); Sainh. P. Z. S. 1871 , p. 420 .

P. novae Hollandiae, Steph., Gen. Zool. xiii. 1. p. 93 (1825); Gould. Handb. B. Austr. ii, p. 488 (1865); Buller, Birds. Nezi'. Zeal. p. 325 (1873).

P. carboides, Gould, P. Z. S. 1837 , p. I56. Id., B. Austr. vii. pl. 68 (1848).

Giraculus carbo, Baird., B. N. Am. p. 876. (1858).

P. macrorhynchus, Cuv., Puch., Rev. Zool. 1850. P. 557.

Hab. Samar, Borongan; Australia, Sumatra, China.

4. Gén. MIROCARBO, BP.

6. M. sulcirostris, (Brandt.) \& N. v.

(M. S. T.)

Garbo graculus, Temm. (nec Linn.), Man. d'Orn. ii. p. 897 (1835), é iv, p. 565 (1840).

Phalacrocorax sulcirostris, Brandt, Bull. Sc. Acad. Imp. Petersto. iii, p. $56($ I838); G. R. Gray. List. B. Brit. Mus. iii, Anseres, 1. I 35 (1843); Gould. B. Austr. vii. pl. 67 (1848).

Graculus sulcirostris, G. R. Gray, Gen. B. p. 667 (1845); Schleg., Mus. P. M. Pelecani, p. I 3 (i 863).

Haliaeus sulcirustris, Bp., Consp. ii. p. I 78 ( I 855$)$. 
H. stictocephalus, $B p$., seu:

Microcarbo stictocephalus, $B p$., Consp. ii. p. ${ }_{17} 8(1855) ; G . R$. Gray, Hand-List, iii. p. 129, sp. 11150 (1871).

Carbo sulcirostris, Temm., Mus. Lugd. ex Borneo (Bp. I. c.)

Phalacrocorax stigtocephalus, Gould, Handb. B. Austr. ii. p. 495 (i 865 ).

Microcarbo sulcirostris, G. R. Gr., Hand; List, iii. p. 129 , sp. IIIt9 (I87 I); Saly. Ucc. di Borneo, 1874 , p. 365 .

Hab. Luzón, Cagayan; Australia, Borneo, Java.

7. M. pygmaeus, (Pall.) ơ N. v.

(II. S. T)

Pelecanus pygmacus, Pall., Itim. Atl. t. 9; Id., Zoogr. RossoAs. t. 74 , f. I ( $1810-183 \mathrm{I})$.

Hydrocorax niger, Wieilt., N. D. viii. p. $88(2817)$.

Carbo javanicus, Horsf. Trans. Linn. Soc. xiii. p. 197 (1821).

C. africanus, Horsf. (nec Gm.), Cat. Jav. B. in Zool. Res. in Java, p. ult. (I 824$)$.

C. melanognathos, Brandt, Bull. Acad. Sc. Petersb. 1837, p. 6.

C. raptensis, Hodgs.

Graculus pygmaeus, G. R. Gray, Gen. B. p. 667 (1845); Blyth, Cat. B. Mus. A. S. B. p. 298 (18+9); Schleg., Mus. P. B. Pelecani, p. I3 (1863); Brehn. 4. p. 559.

Graculus pygmaeus indicus, Rchb., Syn. Av. I, Natatores, t. $5^{8}$, f. 2304 .

G. pygmaeus algeriensis, Rchb., op. cit. t. 6, f. 2305-2306.

Phalacrocorax puinilio, Licht., Nomencl. p. IO3 (I 854 ).

Carbo oriepcii, Malh. Faun. Orn. de l'Alg. p. 38 (I 855 ).

Haliaeus pygmaeus, Bp., Consp. ii. p. 179 (1855).

H. pygmaeus, a. algeriensis, melanognathus, javanicus, niger, $B p$. op. cit. p. I 80 .

Microcarbo pygmas, Bp. Cat., Parzud. p. Io, (1856); G.R. Gray. Hand-List, iii. p. I 29, sp. I I It3 (187 I); Salv. Ucc. di Borneo, 1174 p. 366.

Graculus javanicus, Jerd., B. of Ind. iii. p. 863 (I863); Holdsa'., P. Z. S. 1872 , p. 483 .

Microcarbo melanognathos, G. R. Gray, Hand-List. iii, p. I 29, Sp. I I I 44 ( I 87 I ).

M. niger, G. R. Gray, op. cit. sp. i I 48 (I87 I).

Hab. Paragua, P. Princesa; Borneo, Jaxa.

5. Gén. SUla, Briss.

8. S. cyanops, Suntl. N. v.

(M $\quad S$ T.)

?Sula dactylatra, Less. Voy. Coq. Zool. ii. p. 494 (1823); Id., Tr. d'Orn. p. 601 (1831); Less. Compl. Buff. ii. p. 707 (1838); G. R. Gray, Gen. B. iii. p. 666 (18+5); Pucher., Rev. et Mag. de Zool. 1850 , p. 626; Bp., Consp. ii. p. 165 (1855); Hartl., Journ. f. Orn. 1855 , p. 420 ; Bryant, Journ. f. Orn. 1855 p. 420 ; Bryant, Journ.f. Orn. 1861 , pp. 57, 58; G. R. Gray, Hand-List, iii. p. i 26 , sp. I I 106 (I 87 I).

?S. nigrodactyla, Less. (G. R. Gray, Hand-List, iii. p. 126, in synon $S$. dactylatra). 
S. cyanops, Sundev. Physiogr. Sällsk. Tädskrift, 1837, 1. 218, t. 5; Id:, Isis, 1842 , p, 858; G. R. Gray', Gen. B. iii. ". 666 (parte) (1845); Bp. Consp. ii. p. I65) (1855; Id., Consp. Gav. Syst. sp, I7 (1856); Schleg., Mus. P. B. Pelecani, 1. 3') (1863); Gould, Handb. B. Austr. ii. 1). 506 (I865); G. R. Grav', Hand-List, iii, I 26, sp. I I I07 ( 1871 ); Shelley, B3. of Egypt, 1). 294 (1872); Heugl., Vög. N. O. Afr. p. It8o (1873); Saz'. Ucc. di Borneo 1874, p. 367.

S. personata, Gould, P. Z. S, 1846, P, 2 I; id. B. Austr. vii. pl. 77 (1848); Rchb́., Neuentd. Vög. Neu-Hell. n். ${ }^{0}$ 3(t; $^{\circ}$ Id., Syn. Av. I, Natatores, t. 54. f. $229 \mathrm{I}$ (nec f. 2289-go); Cass U. S. Expl. Exp. Orn. p. 368 (1858); G. R. Gray, B. Trop. Isl. p. 6i (1859); Id., Cat. B. New. Guin. p. 63 ( I 859),

S. piscator, Peale (nec Linn.), U. S. Expl, Exp. Birds. 1. $273($ I 848$)$.

S. melanops, Hart., Ibis, 1859 , p. 35I, pl. x. f. 2 e3; Hetgl., Faun. Roth. Meer. n. ${ }^{\circ} 33$ et p. 32 ; Brehm, Habesch, 1). 230; Hengl., Peterm. Geogr. Mitth. 186 \%, 1). 418.

Sula bossana, Thomps. (nec Linn.), Allen Exped. Niger, ii. p. 175 .

Dysporus cyanops, Licht., Nomencl. p. I03 (1854); Hartl. \& Finsch, Faun. Centrapolyn. p. 252 (1867); Hartl. \& Finsch, Vög. O. Afr. p. $843(1870)$.

Hab. Filipinas; Australia.

9. S piscatrix, (Limn.) $0^{\circ} \bigcirc \mathrm{N} . \mathrm{v}$.

(M. S ' T)

Sula candida, Briss. Orn. vi. p. 501 (1760); Steph., Gen. Zool. p. $103(1826)$.

Fou blanc, Buff. H, N. Ois. 8, p. 37 I.

Pelecanus piscitur, Linn., S. N. I. p. 217 (I766); Forst., Deser. An. p. 244 (1844).

Dysporus piscator, Licht, Doubl. Verz. p. 83 (1823); Sunden., Physiogr. Sällsk Tidskrift, 1837, p. 220; Hartl. \& Finisch, Orn. Centrapolyn. p. 255 (1867).

Sula erythrorhyncha, Less., Tr. d'Orn, p. 6o1 (1831).

S. rubripes, Gould, P. Z. S. 1837 , p. I56; Gray, List Spec. B. Brit. Mus. iii. Anseres p. 183 (18+4).

S. piscator, G. R. Gray, Gen. B. p. 666 (18+5); Blyth, Cat. B. Mus. A. S B. p. 397 (1849); Gould, B'. Austr. Gonld. B. Austr. vii. pl. 79 (1848); Rchb., Nenentd. Vög. Neu Hell. n." 306 ; id., Syn. Av., i. Natatores, t. 53 (xxix), f. 855 ; Ciss., U. S. Expl Exp. Orid. p. 365 ( 1858 ); Hartl., Orn. Beitr. W. Afr. p. 268 (1857); G. R. Gray, Cat. B. Trop. Isl. p. 6i (1859); Hartl., Orn. Beitr. Faun. Malag. p. 87 (1861); (i. R: Gray, 1bis, 1862, p. 250; Salvin, Ibis, 1864, p. 37); Jerd, B. of Ind. iii. p. 852 (1864); Gould., Handb. B' Ausli. ii. p. $5(9)(1865)$

S. rubripeda, Peale, U. S, Expl. Exp. Birds, p. 274, pl. 83 (1848); Hartl., Wiegm. Arch. 1852 , p. 125.

S. piscatrix, Rchb.; Syn. Av. i, Natatores, t. 55 (cclxxvii.), f. 2294-95; Schles., Mas. P. B. Pelecani, p. 40 (1863); Salv. Ucc. di Borneo, 1874 , p. 368. 
Piscatrix candida, Rchb., Ar'. Syst. Nat. p. vi. (1852); Bp., Consp. ii. p. I66 (1 855$)$; id., Consp. Gav. Sj'st. p. 20, sp. 18 (1856). P. piscator, G. R. Gray, Hand-list, iii. p. I26, sp. I I 108 (1871). Hab. Luzon, Mania, Mindanao, Zamboanga; Java, China, Australia.

ro. $\mathrm{S}$ fiber, (Linn) o $\bigcirc \mathrm{N} . \mathrm{v}$.

Sula fusca, Briss., Orn. vi. p. 499, t. 43 , f. I ( 1760$)$.

Petit fou brun, Buff. H. N. Ois. 8, p. 374. Pl. Enl. 974.

Pelecanus sula, Linn., S. N. I. p. 218 , sp. 7 (1866); Rafl., Trans. Linn. Soc. xiii. p. 330 (1822).

P. fiber, Linn., op cit. sp. 8.

Pelecanus parvus, Gm., S. N. I. p. 579 (г788).

Pelecanus leocogaster, Bodd., Tabl. Pl. Enl. p. 57 (1788); (it Buff., Pl. Enl. 973).

Disporus sula, Licht., Doubl. Verz. p. 87 (1823); Bp., Consp. ii. p. I6+ (1855); Hartl. \& Pinsch, Fann. Centrapolyn. p. 260 ( 767$)$; Wald., Trans. Zool. Soc. viii. 2. p. Io6 (1872).

Sula brasiliensis, Spix, Av. Bras. ii. p. 83 t. 107 (av. jun.) (1825).

S. australis, Steph., Gen. Zool. xiii. p. 104 (1826).

S. fiber, Steph., op. cit. p. I05; Blyth, Cat. B. Mus. A. S. B. p. 296 (1849); Schleg., Mus. P. B. Pelecani, p. 41 (1863); Jerd., B. of Ind. iii. p. 85 I, 1864; Gould, Handb. B. Austr. ii. p. 507 (1865); G. R. Gray, Hand-list, iii. p. I 26, sp. I I I09 (1871); Swinh., P. Ż. S. 1871 , p. 420; Salo. Ucc. di Borneo, 187 t, p. 369 .

S. fulica, Less., Tr. d'Orn. p. 60 i (1 831 ).

Pelecanus plotus, Forst., Deser. An. p. 278 (1844).

Phalacrocorax flavirostris, Gould?, apud Licht., in Forst., Deser. An. p. 278 (I 844$)$.

Sula sinicadvena, Swinh., Ibis, 1865 , p. I09; G. R. Gray, Handlist, iii p. I26, sp. IIIII (1871).

S. leucogastra, G. R. Gray, op. cit. sp i i iо (187i).

Hab. Luzon, Manila, Laguna, Mindanao, Carolinas; Australia.

\section{Fam. PLÓTIDAS.}

I. Gén. PLOTUS, LiNs.

I. P melanogaster, (Penn.) Ơ O N. v Pato casili, Casili. (M. S. T)

Anhinga melanogaster, Penn. Ind. Zool. p. 22, pl. 12 (1781).

Anhinga de Cayenne, Buff. Pl. Enl. n. ${ }^{\circ} 959$, et 107.

Plotus melanogaster, Gm. S. N. I. p. 580 (1 788); Lath., Ind. Orn. ii. p. 865 (1790); Forsf., Trans. Linn. Soc. xiii. p. 198 (1821); Bp., Consp. ii. p. 181 (1855); Schleg., Mus. P. B. Pelecani, p. 26 (I 863); Jerd., B. of Ind. iii. p. 86 I (1864); Mant., Journ. f. Orn. 1866, p. 30; G. R. Gray, Hand-List, iii. p. I25, sp. I I 100 (1871); Wald., Trans. Zool. Soc. viii. 2, p. I06 (1872); Salv. Ucc. di Borneo, 1874 p. 367 . Brehm, t. p. 555 f. I 73 . 
P. nova-hol ndia, Gould, P. Z. S. 1877 , p. 3t; Gray, G. $n$. B. pl. 184 (1848); Id., B. Austr. vii pl. 75 (1848); Bp., Consp. ii. p. 181 (1855); Gould, Handb. B. Austr. ii, p. 496 (1865); G. R. Gray, Hand-List, iii, p. I 25 , sp. I102 (1 871$)$.

Hab. Luzón, Manila, Bulacán, Laguna, Calamba, Cavite, Naic, Cagayan, Alc.ilá, Panplona, Abulus, Mindoro, Calapan, Naujan; Borneo, Java, Célebes.

Fam. FETÓNIDAS.

I. Gén. PhaEton, Linn.

1. Ph. rubricauda, Bcdd. $\sigma$ N. v.

Phaeton rubricauda, Bodd., Tabl. Pl. Enl. d'Aub. p. 57 (1783); Schleg., Mus. P. B. Pelecani, p. 44 (1863); Jerd., B. of Ind. iii. p. 849 ( 1864); Hartl. \& Finsch., Faun. Centraltolyn. p. 248 (1867); Heldsw., P. Z. S. 1872, p. 482 ; Salv. Ucc. di Borne?, 1874 , p. 37 \%.

Paille en queue de l'ile de France, Buff., Pl. Enl. 979; id. H. N. Ois. 8, p. 357.

Phaton phơnicurus, Gm., S. N. ii. p. 583 (1788; Gould, $B$. Austr. vii. pl. 73 (1848); id, Handb. B. Austr. ii. p. $50 \mathrm{r}$ ( 1865$)$.

P. xthereus, Bloxh. (nec Linn.), Voy. Blond, App. p. 251 (1826); Blyth, Cat. B. Mus. A. S. B. p. 296 (1849).

P. erubescens, Brandt.

Phoenicurus rubricauda, $B p_{\text {, }}$ Consp. ii. p. 183 (1855); G. R. Gray, Hand-list, iii. p. i25, sp. 11098 (1871).

Phæton rubricandata, Hartl. \& Finsch, Vög. O. Afr. p. 839 ( 1870$)$.

Hab. Mindoro, Baco, Luzón, Laguna, Bataan, Orion; Ceilan, Australia.

2. Ph. candidus, (Briss.)

Orn. vi. pl. xlii f 2, (1760).

Hab. Islas Carolinas.

\section{Fam. LÁRIDAS.}

I. Gén. LARUS, Linn.

1. L. fritzei, (Bruch). $\sigma \circ$ N. v. Cananay.

(M. S. T)

Larus fuscus, Fritze, in Mus. W.estad; Brehm. V. Ani. 4. p. 526.

Dominicanus fritzei, Bruch., Journ. $f$. Orn. 1855 , p. 280 , taf. iv. f. 4 ; $B p$., Consp. ii. p. $214(1856) ; G$. R. Gray, HandList, iii. p. 112, sp. 10957 (1871).

Larus dominicanus, Schleg., Mus. P. B. Lari, p. 12 (parte) ( 1863$)$. 
Larus fritzei, Blasius, Journ. f. Orn. 2865 , p. 378; Salv. Ucc. di Borneo, 1874 , p. 370.

Hab. Samar, Borongan; Borneo, Java.

2. L. ridibundus, Lin. $\sigma$ N. v. Cananay.

(M. S. T.)

L. cinereus, Scop. Ann. 1. n. ${ }^{\circ}$ I05, Raf. Av. p. 128.

Monette rieuse à patres rouges, Buff. H. Nat. Ois. 8. p. 433, Pl. Enl. 970 .

Larus ridibundus, Lin. S. N. 2. p. Gor; Brehm. V. An. 4. p. 527 f. 162.

Hab. Luzón, Manila; Asia.

3. L. herdyi, $B p$. o $\mathrm{N}$ v. Cananay.

(M. S. T)

L. hardyi, Bp. Consp. 2. p. 2 10 (1854); Compt. Rend. Ac. Sc. 1856 .

L. parasiticus, Boie.

Hab. Luzón, Manila; Sandwich.

\section{Fam. ES TÉRNIDAS.}

I. Gén. GELOCHELIDON, Brehм.

1. G. anglica, (Mont.) \& N, v. Cananay.

(M. S. T)

Sterna anglica, Mont., Orn. Dict. Suppl. (1823); S. Müll., Verhandl. Land-en Volkenk. p. I 25 (1839-1844); Schleg., Mus. P. B. Sternae, p. 34 (1863).

S. aranea, Wils., Am. Orn. viii. p. 143. pl. Ixxii. (1814).

s. affinis, Horsf. (nec Büpp.), Trans. Linn. Soc. xiii. p. 199 (182I).

Viralva? affinis, Steph, Gen. Zool. xiii, 1. p. 175 (1826).

?. Sterna macrotarsa, Gould, P. Z. S. 1837 , p. 26.

Gelochelidon anglicus, Blyth, Cat. B. Mus. A. S. B. p. 290 (1849); Jerd., p. of Ind. iii. p. 839 (I864).

G. anglica, $B p$., Compt. $R$ nd. xlii. p. 772 , sp. Ioo (18 $\left.8^{\circ}\right)$; Sclat., P. Z. S. 1863 , p. 224; Blas., Journ. f. Orn. 1866, p. 82; Sclat. \& Salv., P. Z. S. 1871, p. 572; Salv. Ucc. di Borneo, 1874 , p. 371; Brehm. V. Anim. 4. 1881, p. 518.

G. nilotica, G. R. Gray, Hand-List, iii. p. I 19, sp. I 1040 (187 I). Hab. Bálabac; Borneo, Java.

\section{Gèn HYDROCHELIDON, BorE.}

2. H. nigra, (Linn.) \& N. v. Cananay.

(M. S. T.)

Sterna nigra, Linn., S. N. I. p. 227 (1766); Schleg., Mü. P. B. Sternae p. 3I (I863); Gm S. N. 2. p. 608. n. ${ }^{\circ}$.

S. leucoptera, Meisn. \& Schinz, Vög. der Schzveiz, p. 264 (181 5).

Hydrochelidon nigra, G. R. Gray. Jerd., B. of Ind. iii. p. 875 (1864); G. R. Gray, Hand-List, iii. p. I 21, sp. I 1070 (187 I); Szeinh., P. Z. S. 1871 , p. $42 \mathrm{I}$; Wald., Trans. Zool. Soc. 
viii, 2. p. 103 (1872); Holdsw., P. Z. S. 1872 , p. 487 ; Salv. Ucc. di Borneo, 1874 , p. 372; Brehm. V. Anim. 4. 1881 , p. 519 .

H. javanica, Swinh. (nec Horsf.), Ibis, 1860 , p. 68, 186 1 p. 3+5, Hab. Samar, Calbayoc, Minclanao; Célebes, China.

3 H. hybrida, (Pall.) \& N. v. Cananay.

(M. S. T.)

Sterna hybrida, Pall., Zoogr. Rosso-As. ii. p. $33^{8}$ (1810-1831); Schleg. Mus. P. B. Sternae, p. 32 (1863)

S. leucopareja, Natt., in Temm., Man. d'Orn. 2. ${ }^{2}$ ed. ii. p. $7 t^{\prime}$ ) (1820); S. Mïll., Verhandl. Land-en Volk?nk p I'25 (1839-1844).

S. grisea, Horsf., Trans. Linn. Soc. xiii. p. I 9.9 (1821).

? S. cantiaca, Raffl. (nec Gm.), Trans. Linn. Soc. xiii, p. 320 (1822).

S. d-lamotei, Vielll., Enc. Meth. p. $35^{\circ}$ (1823).

Viralva indica, Step., Gen. Zool. i. pl. 70 f. 2 (1832).

Sterna semilis, J. E. Gray; iii. Ind. Zool. i. pl. 70 f. $2(1832)$.

Hydrochelidon fluviatilis, Gould, (nec Naum.), P. Z. S. 1842 , p. 140; id., B. Austr. vii. pl. 3 I (1848).

H. indica, Blyth, Cat. B. Mus. A. S. B. p. 290 (1849); Jerd. B. of Ind. iii. p. 837 (1864).

H. hybrida, Bp., Compt. Rend. xlii. p. 773, sp. 150 (1856); Swinh., P.Z. S. 1871 , p. $42 \mathrm{I}$; Salv. Ucc. di Borneo, 1874, p. 372; Brehm. $V$. Anim. 4. 1881, p. 519.

H. leucopareja, Gould, Handb., B. Austr. ii. p. 400 (1855'; Wald., Trans. Zool. Soc. viii. 2, p. p. 103 (1872).

Pelodes hybrida, G. R. Gray, Hand-List, iii. p. I 2 I sp. I Io7 I (187i).

P. indica, G. R. Gray, op. cit. sp. 11072.

P. fluviatilis, $G$. R. Grayy, op. cit p. $122 \mathrm{sp}$. 11075.

P. delalandei, G. R. Gray, op. cit. sp I 1076.

Hab. Luzón, Manila, Samar, Borongan, Sibuyan, Panay, Ronblon, Guimarás, Negros, Masbate, Cebú, Siquijor, Tablas, Cialamianes, Tawi-tiwi, Mindanao, Joló; Borneo, For nosa, Célebes, Java.

\section{Gén. ONYCHOPRION, WAGL.}

4. O. fuliginosus, $(G m.) \succsim \mathrm{N}$ v. Canaulay.

(M. S. T.)

Hirondelle demer á grande envergure, Buff. H. N. Ois. 8. p. 345.

Sterna fugilinosa, Gm. S. N. I. p. 605 (1788); Temm. \& Schleg.,

Faun. Jap. Aces, p. 13.3, t. 89 (1850); Schleg., Mus. P. B.. Sternae, p. 25 (1863); Hartl. \& Finsch, Finu. Centropolyn. p. 225 ( 867); Brehm. V. Anim. 4. p. 517 (1887).

Onychoprion serratus, Wagl., Isis, 1832 , p. 277.

Planẹtes guttatus, Wagl. op. cit. p. i 222.

Haliplana fuliginosa, Wagl., op. cit. p. r224; Bp., Compt. Rent. xlii. p. 772, sp. I6 (1856); Couss, Pr. Ac. Phil. 1862, p. 557; Blas., Joarn. f. Orn. 1865. p. 81; G. R. Gray, HandList, iii, p. I 22, sp. I 1279 (1 87 I).

Sterna serrata, Forst., Descr. An. p. 276 (1344); Pelz. Novar. Reis. Vög. p. 154 (1865). 
S. guttata, Forst., op. cit. p. 211.

(nnychoprion fuliginosus, Gould, B. Austr. vii. pl. 32 (1848); Sclat. P. Z. S. 1871 , p. 572 ; Salv. Ucc. di Bormeo, 1874, p. 373 .

An wus l'herminieri, Less., Descr. de Mamm. et d'Ois. p. 255 (1848). Sterna gouldii, Rchb., Syn. Av. i. Natatores, t. 37, f. 2267-68. (Baychoprion fuliginosa, Newt., Ibis, 18.55 , p. 371 .

Sterna luctuosa, Phil. \& Lund., W'iegm. Arch. 1866, pt. I, p. i 26. Mab. Luzón, Bataan, Hermosa; Japon, Borneo, Su.natra.

8. O. anasthetus, (Siop.) $\sigma \circ \mathrm{N}$ v Cánanay.

(M. S T.)

Sterna anathetus, Scop., Del. Flor. et Faun. Insubr. ii. p. 92, n. ${ }^{\circ} 2$ (1786), (ex Sonn., Voy', Now. Guin. pl. 84).

S. panayensis, Gm., S. N. I. p. 1607 (1788); ex Sonn., I. C. Schleg., Mus. P.-B. Slernae, p. 26 (1863).

S. panaya, Lath., Ind. Orn. ii. p 808 (1790);-Hartl. \& Finsch, Faun. Centrapolyn. p. 228 (1867).

S. oahuensis, Bloxh., Voy. Blonde, p. 251 (1826).

S. melanoptera Broxh., Voy. Blonde, p. 25 I (1826).

S. melanoptera, Sws., S. W. Afr. ii. p: 249 (1843); Hartl., Syst. Orn. W. Afr. p. 255 (1857).

S. fuligula, Licht. in Furst., Descr. An. p. 276 (nota) (1844).

S. antartica, Cuv. (nec Forst.) in Mus. Paris.

(Onychoprion panaya, Gould, B. Austr. vii. p. 33 (i $8+8)$.

(). anasthatus, Blyth, Cat. Mus. A. S. B. p. 293 (1 849); Jerd., B. of. Ind., iii. p. 844 (1864); Salv. Ucc. di Borneo, 1874 , 1. 374 .

Haliplana panayensis, Bp., Compt. Rend. xlii. p. 772 , sp. 98 (1856); Blas., Journ. fur Orn. 1866, p. 80.

11. panaya, Coues, Ibis, 1864, p 39I.

H. discolor, Cones, Ibis, 1864 , p. 392; Lawe., Ann. Syc. N. I. viii. p. IO4 (1864); Elliot, B. $N$. Am. ii. pl. 57 (1869).

Onychoprion panayensis, Gould, Handb. B. Austr. ii. p. 4I I (1865); Sclat. \& Salv. P. Z. S. 1871 , p. 373.

Sterna nubilosa, Sund., Ofv. of $K$. V. A. Forh. 1869, p. 589. Haliplana anasthaetus, G. R. Gray, Hand-list, iii. p. I 22, sp. I IO80 (I 87 I ).

H. anatheta, Swinh., P. Z. S. 1871 , p. 422.

Onychoprion anaesthetus, Holdsw., P. Z. S. 1872 , p. 482; Wald., Trans. Zool. Soc. viii. 2, p. 103 (1872).

Hab. Luzon, Manila, Navotas, Laguna, los Baños, Biñan, Samar, Paranas, Borongan, Paragua, P. Princesa, Calamianes, Culion, Mindoro, Baco; Borneo, Célebes, Java.

9. O. sumatrunus, (Raffl) $\delta$ N. v. Cananay.

(M. S. T)

Sterna sumatrana, Raff., Trans. Linn. Soc. xiii. p. 329 (1822). S. melanauchen, Temm., Pl. Col. 427 (1827); Less. Man. d'Orn. ii. p. 382 (1829); Id., Tr. d'Orn. p. 622 (1831); Mïll., Verhandl. Land-en Volkenk. p. I 25 (1839-44); Gould, B. Austr. vii. pl. 28 (1848); Rchb., Neicentd. Vög. Neu-Holl. n. ${ }^{\circ}$ 295. Id.. Syn. Av. i, Natatores, t. 32 (xx), f. 282 . et t. 35 (cclxx)', f. 2255-56; Schleg., Di Dierent. fig. p. 291; Id., Haild!. Dierk'. 
t. 8, 102 (1857); Sclat. Journ. Pr. Linn. Soc. 1858, p. 170; G. R. Gray, P. Z. S. 1859 p. $116 ;$ Id., B. Trop. Isl p. 59 (1859); Id., Cat. B. New Guin ii. 53 (1859); Verr. Rev. et Mag. de Zool. 860 , p. 442; Schleg., Mus. P.-B. Sterna, p. 28 (1863); Rosenb., Journ. f. Orn. 1864 , p. 138; Gould. Handb. B. Austr. ii. p. 400 (1865); Pelz., Novar. Rels Vög. p. I 54 (1865); Finsch \& Harll., Faun. Centralpolyn, p. 224 (1867); Swinh., Ibis, 1867 , p. 230; Chao. 3. p. 669.

S. marginata, Blyth, J. A. S. B. xv. P. 373 (nota) (1846). (Inychoprion melanauchen, Blyth, Cat. B. Mus. A. S. B. 293 (1849); Jerd., B. of Ind. iii. p. 844 (1864); Blyth, Ibis, 1865 , p. 39; Wald., P. Z. S. 1866 , p. 556; Swinh., P. Z. S. 1871 , p. 422; Wald. Trans. Zool. Soc. viii. 2, p. 104 (1872).

Sternula melanauchen, $B p .$, Compt. Rend. xlii. p. 773 , sp. 142 (1856); Blas., Journ. f. Orn. 1866, p. 74; G. R. Gray, Hand-list, iii. p. I2I, sp. 11053 (I8II).

Sterna minuta? Swinh. (nec Linn.), Ibis, 1860, p. 429.

liygis sp., Hartl. Ibis, 1864 , p. 232.

i. decorata, Hartl., Hartl. Godeffr. Cat. i. p. 5 (1864).

()nychoprion sumatranus, Salv. Ucc. di Borneo, 1874 , p. 374.

Hab. Mindoro Calapan; Borneo, Célebes.

\section{Gén. GYGIS, Wagi.}

1o. G. alba. (Sparrm) む N. v Cananay.

(M. S T.)

Stern alba, Sparrm., Mus. Carls. n. ${ }^{\circ}$ xi. (1786); Schleg., Mus. P.-B. Sterna, p. 35 (1863).

S. candida, Gm. S. N. p. 607 n. ${ }^{\circ}$ ig (1788); Lath., Ind. Orn. ii. 807 (1790); Forst., Drecr. An. p. 179 (1844).

Gygis candida, Wagl., Isis, 1832, p. 1224; Gould, B. Austr. vii. pl. $30(1848)$; Bp., Compt. Rend. xlii. p. 772, sp. I16 (1856); G. R. Gray, B. Trop. Isl. p. 59 (1859); Pelz., Novar. Reis. Vög. p. 155 (1865).

G. alba, Bp., Compt. Rend: xlii. p. 772 , sp. 115 (1756); Cass., U. S. Expl. Exp. Orn. p. 389 (1858); Gould, Handb. B. Austr. ii. p. 495 (1865); Blas., Journ. f. Orn. 1866, p. 73; Hartl. \& Finsch, Faun. Centralpolyn. p. 232 (1867); Swinh., P. $Z$. S. 1871, p. 422; G. R; Gray, Hand-list, iii. p. 122, sp. ${ }_{11083}(187 \mathrm{I})$; Salv. Ucc. di Borneo, 1874 . p. 375; Brehm. $V$. Anim. 4. 1881 , p. 520.

Gygis nap.leonis, Bp., Compt. Rend. xlii. p. 772 , sp. I 77 (1856). Hab. Mindoro, Baco, Paranas, Samar, Carolinas, Palaos; China.

\section{Gén. Sterna, Lins.}

Subgén. PELECANOpUS, Wagl.

11. St. cristata, Steph. $\sigma 0$ N. v. Taratara.

(M. S T )

Sterna cristata, Steph (nec Sws.), Gen. Zool. xiii. p. 146 (1826); Mottl. \& Dillw., Contr. Nat. Hist. of Lab. p. 61 (1855); 
Szinh., Ibıs, 1863 p. 430; G. R. Gray, Hand-List, iii. p. I 20, sp. 11058 ( $187 \mathrm{r})$.

St. pelecanoides, King, Surv. Intertrop. Austr. II, App. Aves, p. 422 (1827); Schles., Mus. P. B. Sterna, P.9 (1863); Id., Ned. Tijdschr. Dierk. iii. p. 350 (1866).

Pelecanopus pelecanoides, Wagl., Isis, 1832, pp. 277, $1225 ; G$. R. Gr., List B. Brit. Mus. iii. Anseres, p. $180(1844)$; Bp., Compt. Rend. xlii, p. 772 , sp. 90 (1856); Swinh., P. Z. S. 1871, p. 422.

Sterna velox, S. Mïller (nec Rüpp.), Verhandl. Land., en Volkenk. p. I 25 (1839-1814); Sclat. Journ. Pr. Linn. Soc. 1858, p. I70; Swinh., Ibis, 1860 , p. 429; 1861 , p. $345 ; 1866$, p. 134. St. ressa, S. Müller.

Thalasseus pelecanoides, Gould, B. Austr. vii. pl 23 (1848); Rosenb., Journ. f. Orn. 1864 , p. I 38; Swinh., P. Z. S. $18^{2} 71$, p. 422. T. cristatus, Blyth, Cat. B. Mus. A. S. B. p. 291 (1849). Swinh., P. Z. S. 1863 , p. 329; Jerd., B. of Ind. iii. p. 842 (1 864); Gould, Handb. B. Austr. ii. p. 394 (1865); Holdsw., P. Z. S. 1871, p. $48 \mathrm{r}$.

Sterna Bergii. Finsch \& Hartl., Faun. Centrapolyn. p. 216 (partim) (I867); Brehm. V. Anin. 4, 1887, p. 514.

Pelecanopus cristatus, Wald., Trans. Zool. Soc. viii. 2, p. 105 (1872); Salv. Ucc. di Borneo, 1874 p. 376.

Hab. Luzon, Manila, Siquijor, Cebú, Masbate, Negros, Guimarás, Panay, Sibuyan, Romblon, 'Tablas, Calamianes, Samar, 'Tawi-tawi, Joló, Paragua, P. Princesa, Canigaran, Mindanao, Bohol, Leyte; Formosa, Célebes.

12. St. media, Horsf. $\sigma 0$ N. v. Cananay.

(M. S T.)

Sterna media, Horsf. (nec Forst.), Trans. Linn. Soc. xiii. p. 195 (1821); Hartl. \& Finsch, Vög. O. Afr. p. 830 (1870).

S. affinis, Rüpp (nec Horsf.) Atlas, p. 23, tab. 44 (1826); Temm. Mon. iv. p. 454 (1840); S. Miull., Verhandl. Land-en Volkenk. p. I52 (1839-1844); Schleg., Mus. P. B. Sterna, p. 6 (1863).

S. bengalensis, Cuv. Mus. Paris,-Less., Tr. dOrn. p. 624 (1831); Pucher., Rev. et Mag. de Zool. $285^{\circ}$, p. 512.

S. torresii, Gould. P. Z. S. 1842 , p. 140.

Thalasseus torresii, Gonld, B. Austr. vii, pl. 25 (I848); Blyth, Ibis, 1865 , p. 39 .

Sylochelidon affinis, Gray, List. B. Brit. Mus. iii, Anseres, p. I 77 (1844).

Thalesseus maxuriensis, Licht., Nomencl. p. 98 (1854).

'T. bengalensis, Jerd. iii, p. 843 (1864); Gould, Handb. B. Austr. ii, p. 396 (1865); Blyth, Ibis, 1867, p. I77.

T. affinis, Blas., Journ. f. Orn. 1866 . p. 82.

Artochelidon media, G. R. Gray, Hand-List, iii, p. I19, sp. 11047 (1871).

Thalasseus medius, Holdsw., P. Z. S. 1872 , p. 482.

Pelecanophus medius, Wald., Trans. Zool. Soc. viii. 2; p. 104 (1872); Salv. Ucc. de Borneo, 1874 , p. 377.

Hab. Luzón, Manila, Malabon; Java, Célebes, Sumatra. 
7. Subgen. STERNA, LINV.

13. St. melanogastra, Temm. $\sigma \circ$ N. v. Cananay.

(M. S. T.)

?Sterna javanica, Horsf., Trans. Linn. Soc. xiii. p. 199 (juv.) (1821); Blyth, Cat. B. Mus. A. S. B p. 293 (1849); Jerd., $B$. of Ind. iii. p. 840 (1864).

\$. melanogaster, 7emm., Pl. Col. 434, (I827); Horsf., General Catalogue of Jav. Biras, nelle Zool. Res. in Java, p. ult. (I824); Burg., P. Z. S. 1855 , p. i 84 ; Gould., B. As. pt. xix. pl. if (1867); Holsu., P. Z. S. 1872 , p. $48 \mathrm{r}$.

S. acuticanda, Hardw. \& Gray, Ill. Ind. Zool. pl 7o, f. 3 (1 830-34.) Hydrochelidon mélanogaster, G. R. Gray, Gen. B. p. 660 (18461; Bp., Compt. Rend. xlii. p. 777, sp. I 54 ( 1856 ).

Sterna melanogastra, Schleg., Mus. P.. B. Sterna, p. 21 (186.3'; Salv. Ucc. di Borneo, 1874 . p. 377.

Sternula melanogastra, Blas., Jeurn. f. Orn. 1866, p. 74.

Pelodes javanica, G. R. Gray, Hand-list, iii. p. 122, sp. I I073( 1871 ). Hab. Luzon, Manila, Paragua, P. Princesa, Masbate; Ceilán, Java.

14. St. sinensis, Gm. t N. v Cananay.

(M. S. 'T)

St. sinensis, Gm. S. N. 2, p. 608; Whiteh. Ibis, 1890, p. 6r. $H a b$. Mindoro, Calapan.

\section{Gén. STERnUl.A, Boif}

15. St. minuta, (Linn.) $\sigma \circ$ N. v. Canalay.

(M. S T )

Sterna miuuta, Lnn., S. $N . I$. p. 228 (1766); Gm. S. $N$. 2, p. 6o8; Horsf., Trans. Linn. Soc. xiii. p. I98 (1821). ?S. Müll., Verhaudl. Land-en Volkenk. p. I25 (1839-1844); Schleg., Mits. P. B. Sternae, p. 21 (1863); Blas., Journ. f. Orn. 1856, p. 7 t. Petite hirondelle de mar, Buff. H. N. Ois. 8, p. 377; Pl. Enl. 996 .

Sterna netopoleucos, Gm., S. N. ii. p. 6.8 (1788).

Sternula minuta, Boie, Isis, 1822 , p. 564 ; Blyth, Cirt. B Mus. A. S. B. p. 292 (1849); Sclat., P. Z. S. 1863, p. 224; Jerd., B. of Ind. iii. 8+I (186+); G. R. Gray, Hand-l:st, iii. p. I 21, sp. I I062 (187I); Swinh, $P . Z$. S. 1871, p. 422; Wald. Trans. Zool. Soc. viii, 2. p. Ji8 (1872); Salv. Ucc. di Borneo, 1874, p. 378; Brehm. V. Anim. 4, 1881, p. 517.

?Sternula nereis, Gould, $P . Z$. S. 1842 , p. 142; id., B. Austr. vii. pl. 29 (1848); id., Handb. B. Austr. ii. p. 402 (1865).

?Sterna nereis, Rosenb., Journ. f. Orn. 1864 , p. 138.

Hab. Mindanao, Zamboanga; Borneo, China.

9. Gén. ANOUS, Leach.

16. A. stolidus, (Linn.), oo N. v. Cananay.

(M. S. T.)

Sterna stolida, Linn., Amoen. Ac. iv. p. $240 ; I d .$, S. N. I. p. 
227 (г766); Gm. S. N. p. $605(1 ; 88)$; Schleg., Mhus. P. R. Sterna, p. 36 (1863).

Monuette brune, Buff. P!. Enl. n. ${ }^{\circ}$ 997. H. N. Ois. 8. p. 46 r. Gavia fusca, Briss., Orn. vi. p. 199, t. 18, f. 2 (1760).

Sterna pileata, Scop., Del. Flor. et Faun. Insubr. p. 92, n." 73 (1786); ex Sonner., Voy à la Nouv. Guim. p. 125, t. 85. St. philippina, Lath., Ind. Orn. ii. p. 805 (1790).

Anous niger, Steph. Gen. Zool. xiii. p. 110 (1826).

Megalopterus stclidus, Boie,-Gould, Voy. Beagle, p. 145 (1 $8+1)$ Sterna leucoceps, Szv.

S. unicolor, Erman, Verz. von Thier. 14. Pflanz. 1835, p. 17. Anous stolidus G. R. Gray, List, Gen. B. p. Ino (1 841 ); Goull. B. Austr. vii. pl. 34 (1848); Blyth, Cat. B. Mus. A. S. B. p. 293 (I 849); Cass., U. S. Expl. Exp. Orn. p. 391 (1858); Jerd., B. of. Ind. iii, p. $8+5$ (1864); Coness, Ib s, $186+$, p. 392; Gould. Handb. B. Austr. ii, p. 413 1865; Pelz., Nozar. Reis. Vög. p. I 55 (1864); Blas., Journ. f. Orn. 1865, p. 8.3; Hartl. \& Finsch, Faun. Centralpolyn. p. 238 (1867); Sclat. \& Salv., P. Z. S. 1871 , p. 566; G. R. Gray, Hand-List, iii. p. I23, sp. I Io84 (187I'; Salv. Ucc. di Borneo, 1874 \%. 379; Brehm. V. Ani. 4. 1881 p. 52 I Wood. Nat. Hist. a '). 755 fig.

A. pileatus, G. R. Gray, Gen. B. p. 66 I (1846); Pelz., Novar. Reis Vög. p. I 55 (1855).

A. unicolor, G. R. Gray, op. cit. ibd.

A. rousseaui, Hartl., Orn. Beitr. Madag. p. 86 (1861'.

A. frater, Cones, Pr. Ac. Philad. 1962 , p. 558; Id., Ib.s, 186 t, p. 393 .

Hab. Luzón, Manila, Laguna; Borneo, Sumatra, Java, Formosa.

17. A. me'anozenys, Gr, N. v.

Hab. Carolinas.

\section{Fam. PODICÍPIDAS.}

I. Gén. SylveOCYClus, BP.

I. S. philippine̊nsis, (Bun) $\sigma \circ \mathrm{N}$. v. Suliasip.

(M. S. T.)

Le castagneux des Philippines, Buff. Hist. Nat. Ois. viii. p. 246; D'Aubent. Pl. Enl. 946; Tem, Man. a'Orn. ii. p. 729 .

Colymbus minor, var. B. Gm. S. N. i. p. 59i, n. ${ }^{\circ}$ o ex Buffon. C. philippensis, Bonnaterre, Ency. i. p. $5^{8}$, pl. 46 f. 3. (1823); ex Buffon; v. Martens, J. f. $O .1866$, p. $3 \mathrm{I}$ n. ${ }^{\circ}$ i 92.

Podicips philippensis, Wald, On Birds Philip. 1875 p. 406; Brehm. Vida de los animales 4. p. 572.

Hab. Luzón, Laguna, Calauan, Guimarás.

2. S. var. $\sigma 0$ N. v. Suliasip,

Hab. Luzón, Pampanga, Bataan, Orion. 
2. Gén. PODICEPS, I,ATH.

3. 8. australis, Gould o 0 N. v. Calapini.

(M. S. T.)

Hab. Luzón, Laguna, Calamba; Australia.

3 Gén PEdeaithyia, Kaup.

4 S. rubricollis, Lath. \& (B. 93I) N. v.

(M. S T.)

Hab. Europa, Alemania.

Fam. COLIMBIDAS.

1. Gén. C.OLYMBUS, LiN.

1. C. arcticus, Lin. (Buff. 952) N. v.

(M. S. T.)

Hab. Norte de Europa, Alemania.

\section{Fam. PROCELÁRIDAS}

I. Gén. DIOMEDEA, LiN.

I D. exulans, Lin. o o N. v. Pájaro carnero, Albatros aullador (M: S. T.)

Hab. Pacífico, Australia, Africa.

2. Gén. PUFFINUS, BRISS.

2. P. loucomelas, (Tcm.) \& N. v.

(M. S. T.)

Procellaria leucomelas, Tm. Pl. Coll. 587, «Japan» (i836); Tem. et Schl. Faun. Jap. Aves, p. I3I, pl. i5; G. R. Gray, List. Br. Mus. Anseres, p. I6o, «Cataguan» (1844).

Hab. Luzón, Manila; Japon.

Fam. ÁlCIDAS.

1. Gén. CHENISCUS, Moehr.

I. Ch. cirrhatus, $(G m$.) \& (Buff. 76I) N. v.

(M. S. T.)

Hab. América.

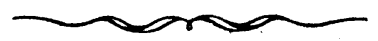


(

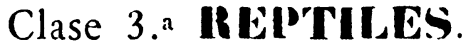

\section{Orden: QUELONIOS.}

\section{Suborden: ATECOS.}

\section{Fam. ESFȦRGIDOS.}

1. Gén. DERMOCHELYYS, Bl.inv.

1. D. Coriacea,? (Lin.) o 0 N. v. Paguican.

'lestudo coriacea, Linn. S. N. i. p. 350 (1766); Schoepff, Test. p. I 23, pl. xxix. (1792); Daud. Rept. ii. p. 62, pl. xviii. fig. I $(1802)$.

T. arcuata, Catesby, Nat. Hist. Carol. ii. p. 4o (1771).

Chelon a coriacea, Schweigg. Prodr. p. (1814).

Sphargis mercurialis, Merr. Tent. p. I9 (1820); Schleg. Faun. Japon., Rept. p. 6, pls. i-iii. (1838).

Corindo coriacea, Harlan, Journ. Ac. Philad. vi. p. 37 (1827). Sphargis tuberculata, Gravenh. Delic. Mus. Uratisl. p. 9 (1829). Dermatochelys porcata, Wagl. Syst. Amph. pl. i. figs I $23\left(\mathrm{r} 83^{\circ}\right)$. Sphargis coriacea, Gray, Syn. Rept. i. p. 5 I (1831); Dum. \& Bibr. ii. p. 560, pl. xxiv. fig. 2 (1835); Bell, Brit. p. I1, tig. (1831); Bonap. Icon. Faun. Ital. pl.-(1841); Holbr. N. Am. Herp. ii. p. 45, pl. vi. (1842); Gray, Cat. Tort. p 51 (1844); et Sh. Rept. i. p. 7 r (1855); A gassiz, Contr. N. H. U. S. i. p. 373 (1857); Gray, Suppl. p. II9 (1870); Gervais, N. Arch. Mus. viii. p. 199, pls. 1-ix. (1872); Schreib. Herp. Eur. p. 509 (I875); German, Bull. U. S. Nat. Mus. n. ${ }^{0} 25$, p. 3(1) (1884); M'Goy, Prodr. Zool. Vict. pls. xlii. \& xliii. (1885); Wood, Nat. Hist. 3. p. 2o. f.

Dermatochelys coriacca, Strauch, Chelon. Stud. p. i 80 (1862), and Verth. Sckildkr. p. 133 (1865); Gïnth. Rept. Brit. Ind. p. 55 (1864); Boulanger, Cat. B. M. p. Io (1889); Brehm. V. An. p. 52 f. 19; Vilan. Creac. 5. p. 22 f. 16.

Hab. Luzón, B. de Manilà, Malabon; Océano pacífico.

\section{Suborder: TEGÓFOROS.}

\section{Fam. PLATISTÉRNIDOS.}

I. Gén. Platysternum, Gray.

I. Pl. megacephalum, Gray. in N. v.

(M. S M.)

Platysternon megacephalum, Gray, l. c. p. 107, et Ill. Ind. Zool. i. pl. Ixii. (1834); Dum. \& Bibr. p. 344, pl. fig. 2; Griv', 
Cat. Tort. p. 35, et Sh. Rept. i. p. 49; Gïnth. Rept. Brit. Int. p. 43 (1854); Theob. Journ. Linn. Soc. x. P. 17 (1868); Bouleng. Ann. \& Mag. N. H. (5) xix. p. 46r, pls. xvi. \& xrii. (1887); id. Cat. B. M. 1889 , p. 46.

linys megacephala, Schleg. Faun. Japon., Rept. p. $4,\left(183^{8}\right)$. P!atysternon peguense, Gray, Suppl. Cat. Sh. Rept. i. 1. 70 (18j(1). Hab. Mindoro; China.

\section{Fam. TESTUdínIDOS.}

I Gén. Callagur, Gray.

I. C. picta, Gray, \& $\mathrm{N}$ v.

Jinys trivittata, (non D. \& B.). Cantor, Cat. Mal. Rept. P. + $(18+7)$.

l'etraunyx affiais, part., Cantor, l. c. p. 6.

litagur picta; Gray, Proc. Zool. Soc. 1862 , 1. 204.

3. affinis, Günth. Repi. Brit. Ind. p. 4o, pl. iii. fig. C. (1864); Theob. Cat. Rept. Brit. Ind. p. 19 (1876).

Clennys grayi, Sirauch, Verth. Schildkr. p. 83 (1865).

Kachuga aifinis, Gray, Proc. Zool. Soc. 1369, p. 203.

Gallagur picta, Griy, Suppl. Cat. Sh. Rept. i. p. 53 (1870); Boul. Cat. B. M. 1889, p. 60.

Cantorella affinis, Gray, l.c. p. $5^{8}$.

Kachuga major, Griy, Ain. \& M.xg. N. H. (4) xi. p. 3oo ( I 873 ). 'letraonyx pictus, 7 heob. $l$. $c$.

Hab. Paragua, Balabac; Borneo.

\section{Gén. OCADIA, Gray.}

2. O. sinensis, (Gray') \& N. v.

(M. S. T)

limys sinensis, Gray, Proc. Zool. Soc. 1834,1 . 53, et Cat. Tort. p. 17 (1844), et Sh. Rept. i. p. 20, pl. vii. (1855); Günth. Rept. Brit. Ind. p. 27 (1864).

l. bermettii, Gray, Cat. Tort. p. 21, and Sh. Rept. i. p. 22, 1)l. $x$.

Clemmys sinensis, Strauch, Chelon. Stud. p. 32 (1862).

C. bennettii, Strauch, l. c., et Verth. Schlldkr. p. 72 (1865).

Ocadia sinensis, Gray, Suppl. Cat. Sh. Rept. i. 1) 35 (18-0); Boul. Cat. B. M. 1889 , p. 85 .

Hab. Vigan, Ilocos; Formosa, China.

3. Gén. DAMONIA, Gray.

3. D. reevesii, Gray. N. v.

(M. S. T.)

Emys reevesii, Gray, Syn. Rept. p. 73 (1831); Dum. \& Bibr. ii. 1). 313 (1835); Gray, Cat. Tort. p. 18 (18+4); Günth. Rept. Brit. Ind. p. 29 (1864); Rütimeyer, Verh. Naturf. Ges. B.tsel, vi. p. 48 (1875).

li. vulgaris picta, Schleg. Abbild. p. 127, pl. xlii. $(18+4)$. 
E. japonica, A. Dum. Cat. Meth. Rept. p. 8 (185I), et Arch. Mius. vi. p. 220 (1852).

Geoclemmys reevesii, Gray, Cat. Sh. Rept. i p: 18, pl. v. (1855). Clemmysmyèii, Strauch, Chelon. Stud. p. 104 (1862).

Damonta vesii, Gray, Proc. Zool. Soc. 1869 , p. 194, et Suppl. Cat. Sh. Rept. i. p. H. (1870), et Ann. \& Mag. N. H. (4) xi. p. 148 (1873); Boul. Cat. B. M. 1889, p. 95.

Hab. Luzón, Cavite; (hina.

\section{Gén. BELlia, Gray.}

4. B. crassicollis, (Gray.) ơ

(M. S. T.)

Emys crassicollis, Gray, Syni. Rept. p. 2i (1831), et Ill. Ind. Zool. i. pl. Ixxvi. fig. 2 (1832); Dlim. \& Bibr. ii. p. 325 (1835); Gray, Cat. Tort. p. 16 (18+4); Cantor, Cat. Mal. Rept. p. 3 (1847); Gray, Cat. Sh. Rept. i. p. 20 (1855); Gïnth. Rept. Brit. Ind. p. 28, pl. iv. fig. E (1864).

E. spengleri, part., Schleg. Faun. Japon., Répt. p. 49 (1833).

E. nigra, Blyih, Journ. As. Soc. Beng. xxiv. 1855, p. $713(1856)$; et $\mathrm{xxxii} . \mathrm{I} 86_{3}$, p. $8 \mathrm{I}$.

Clemmys crassicollis, Strauch, Chelon. Stud. p. 32 (1862); and Verth. Schildkr. p. 69 (1865).

Bellia crassicollis, Gray, Procc. Zool. Soc. 1869 , p. 197, et Suppl. Cat. Sh. Rept. i. p. 40 (1870); Boul. Cat. B. M. 1884 , p. 98.

B. crassilabris, Theob. Cat. Rept. Brit. Ind. p. Io (1876).

Hab. Cuyo, Calamianes; Borneo, Sumatra.

5 Gén. Nicoria, Gray.

5. N. spengleri, Gray, $\sigma$ N. v.

(M S. T.)

Walbaum, Schr. ges. Naturf. Freunde. vi. p. 122, pl. iii. ( 1785 ).

T'estudo spengleri, Gmel. S. N. i. p. 1043 (1789); Daud. Rept. ii. p. 103 (1802).

'I. serrata, Shaw, Zool. iii. p. 51, pl. ix. fig. 2 (1802).

T. tricarinata, Bory de St. Vinc. Voy. dans les quatre princ. Iles des Mers d'Afrique, ii. p. 308, pl. xxxvii. fig. I (1804).

Eimys spengleri, Schweigg. Prodr. p. 4I (1814); Dum. \& Bibr. ii. p. 307 (I 835).

Geomyda spengleri, Gray, Proc. Zool. Soc. 1834, p. Ioo, et Cat. Tort. p. 14 (1844).

Nicoria spengleri, Gray, Cat. Sh. Rept. i. p. I7 (1855); Boul. Cat. B. M. 1889 , p. I 20.

Clemmys spengleri, Strauch, Chelon. Stud. p. 32 (1862); et Verth. Schildkr. p. 63 (1865).

Hab. Balabac, Paragua; China, Borneo.

6. Cén. CyClemys, Bell

6 C. trifasciata, (Gray.) $\sigma$ N. v. Paguican.

(M S. T.)

Sternothœrus trifasciatus, Bell, Zool. Journ. ii. p. 305, pl. xiii. (1825). 
(istudo trifasciata, Gray, Syn. Rept. p. 19 (1831); and IIl. Ind. Zool. ii. pl. Ixi. (1834); Dum. \& Bibr. ii. P. 219 (1835); (iray, Cat. Tort. p. 31 (1844).

Cuora trifasciata, Gray, Cat. Sh. Rept. i. p. $+2\left(1855^{\text {jy }}\right.$ Güntk Rept. Brit. Ind. p. it (1864).

'Terrapene trifasciata, Strauch, Chelon. Stud. p. 27 (1862).

( ) trifasciata, Bonl. Cat. B. M. 1889, p. 133. Hab. Batanes; China:

7. C. amboinensis, Daud. o o N. v. Paguican.

(M. S. T.)

'I'estudo amboinensis, Daud. Rept. ii. p. 369 (1802).

'Testudo melanocephala, Daud. l. c. p. 128.

limys amboinensis, Schrueigg. Prodr. p. 45 (1814).

E. couro, Schweigg. l.c. p. $46 ;$ Schleg. Faun. Japon., Rept. p. 63 ( 1843 ).

Terrapene amboinensis, Merr. Tent. p. 28 (1820); Strauch. Chelon. Stud. P. 99 (1862); et Verth. Schildkr. p. 47 (1865); Sowerby \& Lear, Tort. pl. xxiii. (1872).

Kinosternon amboinense, Bell, Zool. Journ. ii. p. 305 (1825).

'Terrapene bicolor, Bell, l. c. p. 484 , pl. xvi.

T. couro, Filzing. $N$. Class. Rept. p. 45 (1826).

(istudo amboinensis, Gray, Syn. Rept. p I9 (1831); and IIl. Ind. Zool. i. pl. lxxvii. fig. 2 (1832); Dum. \& Bibr. ii. p. 215, pl. xv. fig. 2 (1835); Gray, Cat. Tort. p. 3o (1844); Giebel, Zeitschr. f. ges Naturw. xxvii. p. 11 (1866).

Cuora amboinensis, Gray, Cat Sh. Rept. i, p. 41 (1855); Günth. Rept. Brit. Ind. p. 12, pl. iv. figs. A, B. (1864); Gray, Suppl. Cat. Sh. Rept. i. p. 21 (1870); et Append. p. IO (1872); Tneob. Cat. Rept. Brit. Ind. p. 7 (1876).

Cyclemys amboinensis, Boul. Cat. B. M. 1889 , p. I33.

Hab. Luzon, Manila, Cavite, Laguna de Bay, Negros, Dinagat; Siam.

8. C. flavomarginata, (Gray.) N. v. Bayuyuco.

(M. S. T.)

Cuora trifasciata, part., Gray, Cat. Sh. Rept. i. p. 42 (1855).

(istoclemmys flavomarglnata, Gray, Proc. Zool. 1863 , p. 175, et Ann. \& Mag. N.H. (3) xiii. p. roz (1864), et Suppl. Cat. Sh. Rept. i. p. 20 (1870).

Cuora flavomarginata, Günth. Rept. Brit. Ind. p. 13, pl. v. fig. A. 1864 .

Terrapene flavomarginata, Strauch, Verth. Scihildkr. p. 48 (1865).

Cyclemys flavomarginata, Boul. Cat. B. M. 1889 , p. 135.

Hab. Luzón, Pangasinan, Lingayen; Formosa.

9. C. platy nota, (Gray.) $\sigma \circ$ N. v. Bayuyuco.

(M. S. T.)

Emys. platynota, Gray, Proc. Zool. Soc. 1834, p. 54 et III. Ind. Zool. ii. pl. I I I f. I ( 1834); et Cat. Tort. p. I6, I 844 ; Strauch, Verth. Sichildkv. 1865, p. 57.

Cyclemys platynota, Gray, Cat. Sh. Rept. i. 1855 p. 43. Bouleng. Cat. Ch. B. M. 1889 , p. 130.

Notochelys platynota, Gray, Proc. Zool. Soc. 1863, 1' i77; Githr. 
Rept. B. India, 1864 , p. 17; Gray, Supp. Cat. Sh. Rept. i. 1870, p. 21.

(. dentata (non Gray) Giebel, Zeitschr. J. ges. Natu. xxvii, P. I5, pl. iii. (1866).

Geoemyda granlis, part. Craj, Suppl. Cat. Sh. Repet. i. p. 26. (. Giebelii, Hubrecht, Notes Lej'd. Miss. iii. (188ı); p. 45.

Hab. Paragua, P. Princesa; Borneo, Sumatra.

\section{Fam. QUELÓNIDOS.}

\section{Gén. Chelone, strauch.}

I Ch mydas, (Lin). \& \& N. v. Paguican.

(M. S. T.)

'Testudo mydas, Linn. S. N. i. p. 350 (1768); Schoepff, Testud. p. 73, pl. xvii. fig. 2 (1792); Daud. Rept. ii. p. Io, pl. xvi. fig. I ( 1802 ).

T. macropus, Walbaum, Chelonogr. p. 112 (1782).

I. viridis, Schnetd. Schildkr. p. 299, pl.- (1783); Brehm. I'. An. 5. p. 47. fig.

'T. japonica, Thunberg, Vetensk. Acad. Handl. viii. p. 178, pl. vii. fig. I ( I 787$)$.

T. cepediana, Daud. l. c. p. $5 \%$.

Chelonia japonica, Schweigg. Prodr. p. 21 (1814).

C. virgati, Schreeigg. l. c.; Dum. \& Bibr. ii. p. 54I (1835); Cocteau, in R. de la Sagra, Hist. Cuba, Rept. p. 26, pl. iii. (1843); Gray, Cat. Tort p. 54 (1844); Cantor, Cat. Mal. Rept. p. II (18+7); Gray, Sh. Rept. i. p. 74 (1855); Agass. Contr. N. H. U. S. i. p. 379 (1857); Günth. Rept. Brit. Ind. p. 53 (1864).

C. mydas, Schrueigg. l. c. p. 22; Gray, Syn. Rept. p. 52 (1831); Dum. \& Bibr. ii. p. 538; Holbr. N. Am. Herp. ii. p. 25, pl. iii. (1842); Agass. Contr. i, p. 378; Sowerby \& Lear, Tort. pls. lix. \& lx. ( 1872$)$; Peters, Reiss n. Mossamb. iii. p. 18 (1882); Vilan. Creac. 5. p. I9, fig. I4.

Carretta cepedii, esculenta, nasicornis, thurbergii, Merr. Tent. p. 18 (I 820$)$.

Chelonia maculosa, lachrymata, Cuv. R. A. 2. ed. ii. p. I3 (1829); Dum. \& Bibr. ii. p. 544 .

C. viridis, Temm. \& Schleg. Faun. Japon., Rept. p. i 8, pls. iv. \& vi. (1843); Cocteau, l. c. p. I9; Gray, Cat. Tort. p. 54, et Sh. Rept. i. p. 75; Girard, U. S. Explor. Exped. Herp. p. $45_{2}$ (1858); Gray, Proc. Zool. Soc. 1873, p. 402; Wood. Nat. Ibis, t. 3. p. 21.

C. bicarinata, Lesson, in Balang. Voy. Ind. Or., Zoxl. p. 301 (1834).

C. marmorata, Dum. \& Bibr, ii. p. 546, pl. xxiii. fig. I; Grard, l. c. p. $455, \mathrm{pl}$ xxxi. figs. 5-7.

Euchelys macropus, Girard, l. c. p. 448, pl. xxxi. figs. 9-11.

Chelonia formosa, Girard, l. c. p. 456 , pl. xxxi. figs. 1-4.

C. tenuis, Girard, l. c. p. 459 , pl. xxxi. fig. 8.

Chelone macropus, virgata, viridis, Strauch, Chelon. Stud. p. 6r (1862); p. 185, Verth. Schildkr. p. 141 (1865); Schreib. Herp. Eur. p. 5 I 8 (I 875$)$. 
Chelone maculosa, marmorata, Strauch, Chelon. Stud. I. is6.

Mydas viridis, Gray, Suppl. Cat. Sh. Rept. i. p. I I9 (1870).

Chelonia agassizii, Bocourt, Miss. Sc. Mex., Rept. p. 26, pl. iv.

(1870); Garman, Bull. Mus. Compt. Zool. vi. p. 126 ( $188(1)$.

C. depressa, Garman, b. c. p. I 34 .

C. lata, Philippi, Sool. Gart. xxviii. p. 84 (1887).

('helone mydas, Boul. Cat. B. M. 1889 , p. 180 ,

Hab. Mindanao, Mangsi; Formosa, Australia.

2 Ch. imbricata, Strauck. o". N N. Paguican

(M S. 'T, )

'Testudo imbricata, Linn. S. N. i. p. 350 (2766); Schneid. Schildkr. p. 309 ( 1783 ); Schoepff, Testud. p. 72 , pl. xviii. (I792).

'Y. caretta, Daud. Rept. ii. p. 39, pl. xvii. fig. 2 (1802).

Chelonia imbricata, Schweigg. Prodr. p. 2 I (1814); Gray, Sy'n. Rept. p. 51 (1831); Temm. \& Schleg. Faun. Japon., Rept. p. 13, pl. v. (1833); Dhım. \& Bibr. ii. p. 548, pls. ii. \& xxv. ficr. 2 (1835); Bell, Brit. Rept. p. I (1839); Holbr. N. Am. Herp. ii. p. 39, pl. v. (1842); Sowerby \& Lear, Tort. pls. lviii. (1872); Vilan. Creac. 5. p. I9. f. I 5.

Caretta imbricata, Merr. Tent. p. 19 (1820); Gray, Cot. Fort. p. 53 (1844), et Sh. Rept, i. p. 74 (1855); Girard, U. S. Explor. Exped., Herp. p. 440 (1857); Gray, Proc. Zool. Soc. 1.873, p. 397; Wood. Nat. His. 3. p. 20 fig.

Chelonia pseudo-mydas, Lessan, in Bélang. Vay. Ind. Or., Zoot. p. 299 ( 1834$)$.

C. pseudo-caretta, Lesson. l. c. p. 302.

Caretta bissa, Rüpp. N. Wirbelth. Abyss., Amph. p. 4, pl. ii. (1835).

Eretmochelys imbricata, Agass. Contr. N. H. U. S. i. p. $38 \mathrm{r}$ (1857).

E. squamata, Agass. l. c. p. 302 .

Caretta squamosa, Girard., l. c. p. 443 , pl. xxx. figs. 1-7.

C. rostrata, Girard, l. c. 446 , pl. xxx. figs. 8-1 3 .

Chelone imbricata, Strauch, Chelon. Stud. p. I8I (1862); and Verth. Schildkr. p. I 36 (1865); Schreib. Herp. Eur. p. 522 (1875); Boul. Cat. B. M. 1889 , p. I83; Brehm. V. An. 5 , p. 51 fig. I 8.

Caretta squamata, Günth. Rept. Brit. Ind. p. $5+$ (1864).

Onychochelys kraussi, Gray, Proc. Zool. Soc 1873. p. 398, figs. Hab. Batanes, Joló, Paragua, P. Princesa; Formosa, Célebes.

\section{Gén. THALASSOCHELYS, Fit'zing.}

3. Th. caretta. (Lin.) む M v. Cala.

(M. S. T.)

'Testudo caretta, Linn. S. N. i. p. 35 (1768); Walbaum, Chelonogr. p. 95 (1782); Schapff. Testud. p. 67, pls. xvi. \& xrii. fig. 3 (1792).

Testudo cephalo, Schneid. Schildkr. p. $3 \circ 3$ (1783).

'T. caouana, Daud, Rept. ii. p. 55, pl. xvi. fig. 2 (I 802 ).

Chelonia caouana, Schweigg. Prodr. p. 2 (1814); Wagl. syst. Amph. pl. i. figs. ixxvi. (1830); Dum. \& Bibr. ii. p. 552 (1835).

Caretta atra, Merr. Tent. p. I7 (1820).

C. cephalo, Merr. l. c. 
Chelonia multiscutata, Kuhl, Beitr. Zool, p. $7^{8}$ (1820).

C. olivacea, Eschscholtz, Zool. Atl. p. 3, pl. iii. (1829); Cantor Cat. Mal. Rept. p. $3(18+7)$.

(. cephalo, Temm. \& Schleg. Faun. Japon., Rept. p. 23, pls. iv. * vi. (1833); Cocteau, in $R$. de la Sagra, Hist. Cuba, Rept. p. $35(18+3)$.

Chelonia pelasgorum, Val. in Bory. Expéd. Morêe Zool. pl. vi. (1 833).

C. dussumieri, Dum. \&. Bibr. p. 557, pl. xxiv. fig̀. . .

Careta olivacea, Rüpp. N. W'irbelth. Abyss., Amph. p. 7, pl. iiii. (I 835).

Chelonia caretta, Bonap. Faun. Ital. (1835); Hotbr. N. Am. Herp. ii. p: 33 , pl. iv. $(1842)$.

Caouana caretta, Gray, Cat. Tort. p. 52 (1844), et SA. Rept. i. p. 72 (1855), et Proc. Zool. Soc. 1873 , p. 404 .

C. elongata, Gray, Cat. Tort. p. 53, et Sh. Rept. i. p. 73.

C. olivacea, Gray, Cat. Tort. p. 53, et Sh. Rept. i. p. 73; Gïnth. Rept. Brit Ind. p. 52 (1864).

Thalassochelys caouana, Agassiz, Contr. N. H. U. S. i. p. 384, pl. ri. figs. $13-32$ (1857).

T. corticata, Girard, U. S. Explor. Exped., Herp. p. 43. I, pl. xxix. (1858); Strauch, Chelon. Stud. p. 187 (1862), et Verth. Schildkr. p. 146 (1865); Schreib. Herp. Eur. p. 513 (1875).

Lepidochelys olivacea, Girard, l. c. p. 435; Gray', Proc. Zool. Soc. 1873 , p. 407 .

L. dussumieri, Grard, l. c. p. 437.

"Thalass chelys olivacea, Strauch, Chelon. Stud. p. 63.

Cephalochelys oceanica, Gray, Proc. Zool. Soc. 1873, p. 408. Eremonia olongata, Gray, l. c. fig.

Thalassochelys tarapacona, Philippi, Zool. Gart. xxviii. p. $8_{4}$ ( 1887 ).

Th. caretta, Boul. Cat. B. M. 1889 , p. 184.

Hab. Luzón, B. de Manila; Borneo, Célebes.

\section{Fam. TRIONICIDOS.}

\section{Gén. TRIONYX, Gray.}

1. T. subplanus, Geoffr. ठ N. v.

(M S. T)

Trionyx subplanus, Geoff. Ann. Mus. xiv. p. i I, pl. v. fig. 2 (1809); Gray, Ill. Ind. Zool. i. pl. lxxix (1832); Günth. Rept. Brit. Ind. p. 49.(1864); Strauch, Verth. Schildkr. p. I 30 (1865); Boul. Cat. B. M. 1889 , p. 246.

Gymnopus subplanus, Dum. \& Bibr. ii. p. 496 (I835).

Dogania subplana, Gray, Cat. Tort. p. 49 (1844), et Sh. Rept. i. p. 69, pl. xxxiii (1855), et Proc. Zool. Soc. 1862 , p. 265, ot Suppl. Cat. Sh. Rept. i. p. 106 (1870), et Proc. Zool. Soc. 1873 , p. 57, fig.

Trionyx frenatus, Gray, Cat. Sh. Rept. i. p. 67.

Dogania güntheri, Gray, Proc. Zool. Sac. 1862 , p. 265, et Suppl. Cat. Sh. Rept. i. p. 107, et Proc. Zool. Soc. 1873, p. 58 . 
Trionyx güntheri, Gïnth. Rept. Brit. Ind. p. 49, pl. vi. fig. A. Potamochelyx? frenatus, Gray, Proc. Zool. Soc. $186 \mathrm{f}, \mathrm{p} .87$.

Sarbieria frenata, Gray, Proc. Zool. Soc. 1869 , p. 220, et Suppl. Cat. Sh. Rept. i. p. Ioo.

Flatypeltis ferox, part., Gray, Proc. Zool. Soc 1873, p. 58.

Trionyx? dilwinii, Gray, Ann.\& Mag. N. H. (4) xi. p. 306 (1873). Hab. Mindanao, Paragua; Sumatra, Borneo.

2. T. sinensis, Wiegm. $\delta$ N. v.

(M. S. T.)

Trionyx (Aspidonectes) sinensis, Wiegm. Nov. Act. Ac. Leop.Caral. xvii. p. I 89 ( 1834$)$.

T. stellatus, var, japonica, Schleg. Faun. Japon.,' Rept. p. 33, pl. vii. ( 1838 ).

T. japonicus, Schleg. Abbild. p. ıо8, pl. xxxi. (г840).

'. tuberculatus, Cantor, Ann. \& Mag. N. H. ix. p. $482(18+2)$; Gray, Proc. Zool. Soc. 1861, p. 42.

Tyrse perocellata, Gray, Cat. Tort. p. 48 (I844).

'Trionyx perocellatus, Gray, Cat. Sh. Rept. i. p. 65, pl. xxxi. ( I 865 ).

T. maackii, Braudt, Bull. Ac. St. Pétersb. xvi. p. I Io (1857).

I. schlegelii, Braudt, l. $c$.

'T. sinensis, Strauch, Chelon. Stud. p. I77 (1862); Günth. Rept. Brit. Ind. p. 46 (1864); Strauch, Verth. Schildkr. p. 128 (1865), et Voy. Przewalski, Rept. p. 5, pl. i. (1876): Boul. Cat. B. M. 1889 , p. 256.

Potamochelys? perocellatus, Gray. Proc. Zool. Soc. 1864 , p. 86. P. tuberculatus, Gray, l. c. p. 87 , et Suppl. Cat. Sh. Rept. i. p. $105(1870)$.

Landemania irrorata, Gray, Proc. Zool. Soc. 1869 , p. 216 , fig., and Suppl. Cat. Sh. Rept. i. p. 96. and Ann.\& Mag. N. H. (4) xii. p. I60 (1873).

L. perocellata, Gray, Proc. Zool. Soc. 1869 , p. 2 16, et Suppl. Cat. Sh. Rept. i. p. 96, et Proc. Zool. Soc. 1873, p. 53, figs., et Ann. \& Mag. N. H. (4) xii. p. I 59 (1873).

Psilognathus lævis, Heude, Mem. Hist. Nat. Emp. Chin. i. p. 24, pl ii. (1880).

Temnognanthus mordax, Heude, l. c. p. 26, pl. iii.

Gomphopelta officinæ, Heude, l. c. p. 27, pl. iv.

Bælogathus novem-costatus, Heude, l. c. p. 09, pl. v.

Tortisternum novem-costatum, Heude, l. c. p. 3 I, pl. vi.

Ceramopelta lairostris, Heude, $l$. c. p. 33, pl. vii.

Coptopelta septem-costata, Heude, l. c. p. 35, pl. viii.

Cinctisternum bicinctum, feude, l. c. p. 37 , pl. ix. Hab. Batanes; Formosa, China.

2. Gén. Pelochelys, Gray.

3 P. cantoris, Gray. ơ $\bigcirc$ N. v.

(M. S. T.)

Chitra indica, part., Griry, Cat. Tort. p. 49 (1844); et Sh. Rept. i. p. 70 (1855); Günth. Rept. Brit. Ind. p. 50, pl. vi. fig. C (I 864).

Gymnopus indicus Cantor, Cat. Mal. Rept. p. io (1847). 
Pelochelys cantorii, Gray, Proc. Zool. Soc. 186 \&, p. 9o, figs., et Suppl. Cat. Sh. Rept. i. p. 90 (1870); Theob: Cat. Rept. Brit. Ind. p. 28 (1876); Boul. Cat. B. M. 1889, p. 263.

P. cumingii, Gray, ll. cc. pp. 90, 9r.

P. bibronii, Gray', ll. $c c$.

Hab. Balabac; Borneo.

\section{Gén. ChITrA, Gray.}

4 Ch indica. (G'ay') T N. v.

'Irionyx indicus, Gray, Syn. Rept. p. 47 (1831).

T. xgytiaeus, var. indicus, Gray. Ill. Ind. Zool. i. pl. Ixxx. (1832). Gymnopus lineatus, Dum. \& Bibr. ii. p. 49r (I\$35).

Chitra indica, part., Gray, Cat. Tort. p. 49 (18+4); and Sh. Rept. i. p. 70, pl. xlii. (1855); Gün'h. Rept. Brit. Ind. p. 5' (1764); Boul. Cat. B. M. 1889 , p. 264.

C. indica, Gray, Proc. Zool. Soc. 1864 , p. 91, figs., and Suppl. Cat. Sh. Rept. i. p. 89 (1870); Theob. Cat. Rept. Brit. Ind. p. 27 ( 876$)$.

Hab. Paragua; India.

\section{Orden: EMIDOSAURIOS.}

\section{Fam. CROCODÍlIDOS.}

I Gén. CROCODILUs, Cuv.

I. C. porosus, Sthneid. $\sigma 0$ N. v. Buaya.

Crocidolus porosus, Schneid. Hist. Amph. ii. p. 159. (1801); Gray Cat. Tort. \& p. 58 (1844); Günth. Rept. Brit. Ind. p. 62, (1864); Theob. Cat. Rept. Brit. Ind. p. 36 (1876); Boul. Cat. B. M. 1889, p. 284.

C. oopholis, Schneid. l. c. p. 165 .

Crocodilus biporcatus, Cuv. Ann. Mus. x. p. 48, pls. i. \& ii. (I807); Tiedem., Opp.; \& Lib. Nat. Amph. p. 72, pl. ix. (1817); Lesson, in Belang. Voy. Ind. Or., Zool. p. 303 (1834); Dum. \& Bibr. iii. p. II5 (1836); Schleg. Abbild. p. 3, pl. i. (1837); Huxley, Journ. Linn. Soc. iv. p. I I (1860); Strauch. Syn. Crocod. pp. 52 \& 99 (1866); Brehm. V. An. 5. p. 63. f. 24; Vilan. Creac. 5. p. 3 I.

C. biporcatus raninus, Schleg. \& Mïll. in Temminck, Verh. Nat. Gesch. Nederl. Ind., Rept. p. 28, pl. iii. figs. 7 \& 8 (i 844 ).

()opholis porosus, Gray, Ann. \& Mag. N. H. (3) x. p. 267 (1862), et Trans. Zool. Soc. vi. p. i 38 (i867), et Cat. Sh. Rept. ii. p. 8 (1872).

O. pondicherianus, Gray, ll. cc. pp. 268, 139, 9.

Crocodilus pondicerianus, Gunth. Rept. Brit. Ind. p. 62, pl. vii.; Stoliezka, Journ. As. Soc. Beng. xlii. p. I13 (1873); Theob. l. c. Hab. Luzón, L. de Bay, Pampanga, Cagayan, Mindoro, Mindanao. 
2. C palustriso, Less. N. v. Buaya

Crocodilus palustris, Lesson in Belang. Voy. Ind. Or.; Zool. p. 305 (1834); Kelaart, Prodr. Faun. Zeyl. p. 183 (1852); Günth. Rept. Brit. Ind. p. 61, pl. vii. fig. A. (1864); Strauch, Syn. Crocod. pp. 48 \& 97 (1866); Theob. Cat. Rept. Brit. Ind. p. 36 (1876); Boul. Cat. B. M. 1889 p. 285.

C. vulgaris, var. B. Dum.\& Bibr, iii. p. 108 (1836); Cantor, Cat. Mal. Rept. p. I 5 (1847).

(.) palustris, part., Gray, Cat. Tort.'\& p. 62 (1844).

(.. trigonops, Gray, l. c.

C. bombifrons, Gray, Cat. Tort. p. 59 (1844); Huxley, Journ. Linn. Soc. iv. p. I 3 (1 860).

Bombifrons trigonops, Gray, Ann. \& Mag. N. H. (3) x. p. 269 ( 1862 ).

B. indicus, Gray, Trans. Zool. Soc. vi. p. I4o, figs., pl. xxxi. figs. 1-3 (1867), et Cat. Sh. Rept. ii. p. 9 (1872).

Hab. Mindanao, Placer.

\section{Orden $3 .:$ SAURIOS.}

\section{Subord. $1 .^{\circ}$ LAGARTOS.}

\section{Fam. GECÓNIDOS.}

s. Gén. Gymnodactylus, Six.

I. G. marmoratus, Dum. \& Bilir. 0 . N. v. Butiqui. (M. S. T.)

Cyrtodactylus marmoratus, part, Gray, Cat. p. I73.

Phyllurus marmoratus, (Kuhl), Fitzing. N. Class. Rept. p. 47.

Gonyodactylus marmoratus, (Kuhl), Gray, Griff. A. K. ix. Syn. p. 51; Girard. U. S. Explor. Exped., Herp. p. 304; Boul. Cat. B. M. 1. p. 44 (1885).

Gymnodactylus marmoratus, Dum. \& Bibr. iii. p. 426, pl. xxxiv. fig. I; Schleg. Abbild. p. 8, pl. ii.; Steindachn. Novara. Rept. p. 17 .

Hab. Luzón, Cavite, Indan; Célebes, Sumatra, Java.

2 G. philippinicus, Steindackn. $\sigma^{\circ} \circ \mathrm{N}$. v Butiqui.

(M. S. T)

Cyrtodactylus marmoratus, part., Gray, Cat. p. 173.

Gymnodactylus philippinicus, Steindachn. Novara, Rept. p. 17, pl. ii. fig. I; Boul. Cat. Liz. B. M. 1885, p. 46.

Hab. Luzón, Laguna, Calamba, Los Baños, Dinagat, Albay.

3, G. consobrinus, Peters. Ot: N. v. Bútiqui.

(M. S. T.)

Gymnodactylus consobrinus, Peters, Mon. Berl. Ac. 1871, p. 569, and Ann. Mus. Genov. iii. 1872, pl. ii. fig. I; Boul. Cat. 1. p. 47 .

Hab. Paragua, P. Princesa, Samar, Borongan; Borneo. 
4. G. platurus, While, os N. v. Butiqui.

(M. S. T.)

Phyllurus platurus, Gray, Cat. p. 176 :

P. inermis, Gray, l. $c$.

Jacerta platura, White fourin. N. S. Wales, p. 246, pl:- tig.

s Shaze, Gen. Zool. iii. p. 247.

Sitellio plyyllurus, Schneid. Amph. Phys. ii. p. 31.

s. platurus, Dand. Rept. iv. p. 24.

Agama platyura, Mérr. Syst. Amph. p. 51

A. discosura, Merr. l. $c$.

Phyllurus platurus, Fitzing. N. Class. Rept. p. 47; Guérin, Icon. R. A., Rept. pl. xiv. Sig. I; Gray, Zool. Ereb، \& Terror, pl. rvii. fig. 3; Girard, $U$. S. Explor. Exped.y Herp. p، 303.

P. cuvieri, Bory de St. Vinc. Dict. Hist. Nat. vii. p. $183,{ }^{1}{ }^{1}$. -tig. 2.

(iymnodactylus platyurus, Wagl. Syst. Amph. p. It+; Boul: Cat. 1. P. 49.

Gyrtodactylus platura, Gray, Griff. A. K. ix. Syn. p. 52،

Cymnodactylus phyllurus, Dum. \& Bibr. iii. p، 428 .

Cinyodactylus (Phyllurus) platurus, Fitz Syst. Rept. p. 92.

Phyllurus inermis, Gray, Zool, Ereb. \& Ternor, pl. xvii. fig, I. Hab. Paragua, Ynagauan; Australia.

\section{Gén Hemidaciylus; Cuc.}

5 H. frenatus, Gray. o๐ $\mathrm{N}$ v. Butiqui.

(M. s. T.)

Hemidactylus frenatus, Gray, Cat. p. 155.

11. vittatus, Gray, l. c.

H. frenatus (Schleg.), Dum. \& Bibr. iii. p. 366; Kelaart, Prodr. Faun. Zeyl. p. I8ı; Gïnth. Rept. Brit. Ind. p. го8; Stoliczka, Journ. As. Soc. Beng. xxxix. i870, p. I64, \& xii. I872, p. 96; Anders. Zool. W. Yunnan, p. 801.

H. (Pnoepus) javanicus, Fitzing. Syst. Rept. p. ıо6; Boul. l. cit. 1). I 20 .

: H. punctatus, Hallow. Jerdon, Journ. As. Soc. Beng. xxii. I 854 , p. 467 .

H. inornatus, Hallow. Proc. Ac. Philad. 1860, p. 492.

H. pumilus, Hallow. l. c. p. 502 .

(iecko chaus, Tytler, Journ. As. Soc. Beng. xxxiii. 1864, p. 547.

(i. caracal, Tytler, l. $c$.

Hemidactylus vittatus, Gray; Zool. Erebus \& Terror, pl. xv. fig. 5 .

H. Iongriceps, Cope, Prac. As. Philad. 1868 , p. 320.

Hab. Luzón, Manila, Cebú, Ticao, Paragua, Puerto Princesa; Borneo, Java.

6. H. maculatus Grdy. \& N. v.

(M. S. 'T.)

Hemidacty'us maculatus, part., Gray, Cat. p. I 53.

H. maculatus, part., Dum. \& Bibr. iii. p. 358; Bort. Cat. 1 . p. I 32 . 
I1. sykesii, (Gray), Günth. Rept. Brit. Ind. p. ro8, pl. xii. fig. (.. Hab. Mindoro, Samar, Paric, Borongan; India.

7. H. garnotii. Dum. B/ib. N. v. \& Butiquts.

(M. S. T.)

Doryura garnotii, Gray, Cat. p. 157 .

Hemidactylus garnotii, Dum. \& Bibr. iii. p. 368; Bouleng. Proc. Zool. Soc. 883, p. 18 , pl. xxii. fig. ; id. Cat. Liz. $B$. Mus. p. I 4 I.

Hoplopodion (Onychopus) garnotii, Fitz. Syst. Rept. p. sut.

Doryura vulpecula, Girard, Proc. Ac. Philad. 1857, p. 197; and $U$. S. Explor. Exped., Herp. p. 286, pl. xxiv. figs. $17-24$.

Hemidactylus indekingii, Bleek. Nat. Tijds. Ned. Ind. xvi. 1859, p. 27.

Doryura gaudama; Theob. Journ. Linn. Soc. x. 1868, p. 3().

Hemidactylus (Doryura) mandellianus, Stoliczka, Joutrn. As. Soc. Beng. xli. I 872 , p. IOI, pi. iii. figs. I, 2 .

Hab. Mindoro, Naujan; N." Caledonia, Birma.

8. H. platyurus, Lack. $\sigma 0$ N. v. Butiqui

(M. S. 'T.)

Platyurus schneiderianus, Gray, Cat. p. 197.

Stellio platyurus, Schneid. Amph. Phys. ii. p. 3', et Denkschr. Ak. Münch. 1811, p. 62, pl. i. fig. 3.

Lacerta schneideriana, Shaw, Zool. iii. p. 278.

Gecko platyurus, Merr. Syst. Amph. p. 4r.

(i. marginatus, Cuv, R. A. ii. p 54.

Hemidactylus platurus, Wiegm. Nova. Acta. Ad. Leop. Carol. xvii. i. 1835, p. 288; Cantor Catal. Mal. Rept. p. 24; Boul. Cat. $B . M$. p. 143 .

H. marginatus, Gray, Griff. A. K. ix. Syn. p. 51; Dum. \& Bibr. iii. p. 370, pl. xxx. fig. 2 .

Hoplopodion (Cosymbotus) platyurum, Fitz. Sysl. Rept. p. 104.

Crossurus platyurus, Hirard, $U$. S. Explor. Exped., Herp. p. $28 \mathrm{I}$.

Nycteridium schneideri, Günth. Rept. Brit. Ing. p. II.

N. himalayanum, Anderg. Journ. As. Soc. Beng. xl. ${ }_{1} 8_{71}$, P. I 5 .

N. platyurus, Steliczka, Journ. As. Soc. Beng. xli. i 872, p. 103, Hab. Luzón, Manila, Dinagat; China, Java, Borneo.

\section{Gén GEHYRA, Grax:}

9. G. mutilata, Wiegre. 0.0 N. v.

Peripia peronii, Gray, Cat. p. 159.

Peropus mutilatus, Gray, $l$. $c$.

Hemidactylus (Peropus) mutilatus, Wiegm. Nova. Acta. Ac, Leop. Carol. xvii. p. 238.

P. mutilatus, Dum. \& Birdsi ii. p. 354.

P. peronii, Dum. \& Babr. iii. P 352 , pl. $\mathrm{xxx}$ fig.; Jacqun. Voy. Pole Sud, Saur. pl. i. fig. 2; Cantor, Cat. Mal. Rept. p. 2 i Kelaart, Prodr, Faun. Zeyl. p. $18_{7}$. 
Perophus (Dactyluperus) peronii, Filz. Syst. Rept. p. 103.

P. mutilatus, Fitz. l. c.; Girard, U. S. Explor. Exped, Herp. p. 277.

Peripia peronii, Günth. Rept. Brit. Ind. p. 110; Stolicska, Jowrn. As. Soc. Bellg. xxxix. $2 \delta_{j} o$, p. 163, \& xli. 1872, p. 103.

Gecko pardus tytler, Journ. As. Soc. Beng. xxxiii. 186 f. p. 547.

¿Gecko harrieti, Tyller, l. c. p. 548 .

Hemidactylus (Peripia) mutilatus, Peters, Mon. Berl. Ac. $1866_{\mathrm{i}}$ p. 14; Anders. Zool. W. Yunnan, i. p. 799.

Peropus packardii, Cope, Proc. Ac. Philad. 1868, p. 319,

Peripia mutilata, Günth. Proc. Pool. Soc. 1873, p. 168; Peters \& Doria, Ans. Mus. Genov. xiii. 1878, p. 370 .

Gehyra mutilata, Boul. Cat. Lis. B. M. p. 148 . Hab. Negros, Luzón, Manila, Tayabas, Dolores, Mindanao; Ceilan, Penang, Méjico.

4. Gén. PERoChIRUS, Blik.

s. P. ateles, Dum. $\$$ N. v.

Hemidactylus ateles, A. Dum. Arch. Mus. viii. p. 426, pl. viii. fig. 9. Boul. Cat. Lis. B. M. p. 154.

Hab. Zamboanga, Mindanao.

I. P. depresus, Fisck.

Hemidactilus ateles, var. depressus, Fischer. Arch. f. Nat. 1882, p. 300, pl. xvii. figs. 31-36: Boul. Cat. 1. p. I55. Hab. Carolinas.

I2. P. scutellatus, Fisch

Hemidactylus ateles, var. scutellatus, Fischer. Arch.f. Nat. 1882, p. 299 pl. xvii. figs. 26-30; Boul. Cat. 1. p. 156.

$H a b$. Carolinas.

13. P. var articulatus, Fisck.

Boul. Cat. I. p. I 56.

Hab. Carolinas.

\section{Gén Spathoscaloba'tes, Gthr.}

14. Sp. mutilatus, Glkr.

Spathodactylus mutilatus, Gînth. Proc. Zool. Soc. 1872, p. 594; Boul. (at. p. 156, lam. 13, fig. I.

Hab. Ticao; Archipiélago Indico.

\section{Gén LePidodactytus, Fitzing.}

15. I. lugubris, $D . B$. $\&$ N. v.

(M S. T)

Amydosaurus lugubris, Gray, Cal. p. 162. 
Platydactylus lugubris, Dum. \& Bibr. iii, p. 304; Jacquin. Vo:'. Pole Sud, Saur. pl. i. fig. I; Cantor. Cat. Mal, Rept. p. it). Lepidodactylus lugubris, Fitzing. Syst. Rept. p. 98 ; Bouleng. Proc. Zool. Soc. 1883 , p. 120 , pl. xxii. fig, 3 ; Boul. Cat. 1, p. If5. Hemidactylus meyeri, Bleek. Nat. Tijds. Nederl Ind. xvi, 1859, p. 47 .

Peripia cantoris, Günth. Rept. Brit. Ind. p. I Io; Stohezka, Journ. As. Soc. Beng. xli. 1872, p. IO3.

(iecko mostus, Peters. Mon. Berl. Ac. I867, p. I3.

Gymnodactylus candeloti, Baoay, Rept. $N$. Caled. p. I 3.

Peripia meyeri, Gïnth. Proc. Zool. Soc. 1872, p. 594.

P. mysorensis, Meyer, Mon. Berl. Ac. 1874, p. 129.

P. lugubris, Peters, \& Doria, An. Mus. Genov. xiii, 1878, p. 371.

Platydactylus (Lepidodactylus) crepuscularis, (non Bao.), Salvage, Bull. Soc. Philom. (7) iii, i 878, p. 69.

Hab. Carolinas; Amboina,

16. L. labialis, Pirs. $\delta$ N. v.

(M. S ' 'P)

Cecko labialis, Peters, Mon. Berl, Ac. 1867 , p. I4; Baul. Cat. p. 166 .

Hab. Mindanao:

\section{Gén. LUperosaurus, Gray.}

17. L. curninzii, Gray o̊ $\mathrm{N}$ v. Calasayan.

(M. S. 'I.)

Luperosaurus cumingii, Gray, Cat Boul. Cat. p. 18 I 1. I 5 f, 2. Hab, Luzon, Laguna, Calamba, Cabuyao, Manila, S. Mateo.

Gén. GECKO, LAUR.

18. G. verticillatus, Law;. N. v. Chacon.

( $\mathrm{N}$ S $\mathrm{S}$ T.

Gecko verus, Gray, Cat. p. i6o.

Gecko reevesii, Gray, l. c. p. i6.

Lacerta geeko, Linn. S. N. i. p. 165 .

Gecko verticillatus, Laur. Syst. Rept. p. 44; Boul. Cat. p. 1833.

G. teres, Laur. l. c.

Stellio gecko guttatus, Daud, iv. p. I22, pl. xlix.; Günth. Rept. Brit. Ind. P. IO2.

S. verus, Merrem, Tent. p. $4 z$.

S. annulatus, Kuhl, Beitr. Zool, Vergl, Allat. p. I32.

s. reevesii, Gray, Griff. A. K. ix. Syne p. 4 8; Richards. Zoo:. Herald, p. I 51, pl. xxvii.

Platydactylus guttatus, Guérin, Icon. R. A, Rept. pl. xiii.; Dum. \& Bibr. iil. p. 32 I. pl. xxviii. fig. 4 ; Vilan. Creac. 5. p. 6.2. f. 4 I.

P. reevesii, Fitzing. Syst. Rept. p. гол.

Gekko indicus, Girard, $U$. S. Expet., Herp. p. zqre, pl. xri. fig. 9-16.

Hab. Panay, Iloilo, Negros, Jcló, Luzón, Manila, Lagun:, Los Baños, Batangas, Albay, Gubat, Tayabas, Dolores, Camarines, Mindcro, Mindanno; Siem, Java, China. 
19. G. vittatus Griay N. v.

Ciecko vittatus, Gray, Cat. p. 162.

G. vittatus, Houtfuyn. Vorh. Genotsch. Vlissing. ix. 1782, p. 325, pl.-. fig. 2; Brongn. Bull. Scc. Philcm. ii. $18 \mathrm{e}, \mathrm{p}, \mathrm{pl}$. vi. fig. 3; Daud. Rept. iv. p. I 36, pl. $1 . ;$ Detas \& Doria, dun. Mus. Genor, xiii. $18 ; 8$; p. j68; Boul. Cat. 1. p. 185.

Lacerta unistriata, Shaqi, Matur. Miscell iii. pl. Ixxxix.

Platydactylus vittatus, Dum. \& Bitr. iii. p. 331; Duvernoy, R. A. Rept. pl. xx. fig. 1 .

P. (Scelotretus) vittatus, Fitzing. Syst. Rept. p. 101.

Hab. Marianas, Molucas.

20. G. var: bivittatus, o $\circ$ N. v. Toco

(M. S. T.)

Gecko bivittatus, Gray, Cat. p. 162.

Platydactylus bivittatus, Dum. \& Bibr. iii. p: 334.

P. (Scelotretus) bivittatus, Fitzing. Syst. Rept. p. Ior.

Gecko trachyloemus, Peters. Mon. Berl. Ac. 1872, p. 774.

Hab. Luzón, Manila, Mindanao, Davao; Palaos, N." Guinea.

zI G. monarcbus, Cray $\delta \mathrm{N}$ v. Chacon.

(M. S T.)

Gecko monarchus, Gray, Cat. p. 161; Boul. Cat. Г. 187.

Platydactylus monarchus, (Schleg.), Dum. \& Bibr. iii. p. 33.5; Cantor, Cat. Mal. Rept. p. ig.

P. (Scelotretus) monarchus, Fitzing. Syst. Rept. p. Ior.

Gecko monarchus, Girard, U. S. Explor. Exped., Herp. p. 2yz; Gïnth. Rept. Brit. Ind. p. Io3.

Hab. Mindanao, Placer, Dinagat; Borneo, Célebes.

Fam. AGÁMIDOS.

I. Gén. DRACO, LUN.

I. D. volans, Gray. $\sigma$ o N. v Huniang̃uo.

(M. S. T.)

Draco volans, Gray. Cat. p. 233.

D. volans, Linn. S. N. i. p. 358; Latr. Rept. ii. p. 3 ; Cantor, Cat. Mal. Rept.- p. 37; Günth. Rept. Brit. Ind. 1. I 24; Stcliczka, Journ. As. Soc. Beng. xxxix. 1870 , p. $182 ;$ Boul. Cat. 1. p. 253; Vilan, Creac. 5. p. 50 f. 35; Wcod, Nat. Hist. 3. p. 85 fig.

D. propos, Linn. l, $c$.

D. major, Laur. Syn. Rept. p. 50.

D. minor, Laur. l. c. P. $5 \mathrm{I}$.

D. viridis, Daud. Rept. iii. p. 3or, pl. xli.; Kuhl, Beitr. Zool.

u. Vergl. Anat. p. I02; Schleg. Abbild. p. 89, pl. xxiv. fig. I.

D. fuscus, Daud. l. c. p. $307 ; K u h l, l$. c.

D. daudinii, Dum. \& Bibr. iv, p. 451 . Hab. Samar, Borongan; Borneo, Java. 
2. D. reticulatus, G/hr N. v. id.

(M. S. T.)

Rept. B. India, p. 125; Ptrs et Doria, An. Mus. Genov. xiii. 1878, p. 374; Boul. Cat. 1. p. 127. lam. xxi.

Hab. Luzón, Albay, 'Tayabas, Atimonan; Sanghir.

3 D. id v. cyanopterus, Plis.

Hab. Mindanao.

4. D. guentheri, $B l g r . \sigma o$ N. v.

Boulenger, Cat. p. 257. lam. 20. f. 2.

Hab. Mindanao, Samar, Borongan.

5 D. everetti. $B / g r . \mathrm{N}$. v

Bouleng r, Cat. B. M. 1885 , p. 258. 1. 20. f. 3 .

Hab. Mindanao, Placer, Dinagat.

6. D. ornatus, Günthr. $\sigma 0$ N. v.

(II. S. 'T.)

Dracunculus ornatus, Gray, Cat. p. 235.

Draco ornatus, Gïnth. Proc. Zosl. Soc. 1873 , p. $167 ;$ Boul. Cat. p. 259 .

Hab. Luzón, Laguna, Albay, Daraga, Dinagat, Negros.

7. D. spilopterus, Dum. \& Bibr. ơ N. v.

(M. S. T.)

Dracunculus spilopterus, Gray, l. c. p. 236.

D. spilopterus, Wiegm. Nova Acta Ac. Cas.-Leop. xvii. i. 1835, p. 216, pl. xv.

Draco spilopterus, Dum. \& Bibr. iv. p. 461; Gerv. in Eyd. Voy. Favorite Zool. pl. xxvii.; Schleg. Abbild. p. 92; Günth. Rept. Brit. Ind. p. I $24 ;$ Boul. Cat. p. 260.

Dracontoides personatus, Fitz Syst. Rept. p. $5 \mathrm{I}$.

Hab. Luzón, Cavite, Silan, Albay, Tayabas, Atimonan, Negros, Dumaguete; Java.

8 D bimaculatus, Gthr. ơo N. v.

(M. S. T.)

Draco bimaculatus, Günth. Rept. Brit. Ind. p. I27; Boul. Cat. p. 263, 1. 20. f. 6 .

Hab. Dinagat, Samar, Borongan.

9. D. spilonotus, Gthr. \& N. v.

(M, S. 'T )

Draco spilonotus, Gïnth. Proc. Zool. Soc. 1872, p. 592, pl. xxxv. fig. B. \& 1873 p. 167 ; Boul. Cat. 1. p. 265.

Hab. Paragua, Tagburus; Célebes. 
to D dussumieri, Dum. \& Bibr. of N. v.

Dracocella dussumieri, Gray, Cat. p. 234.

Draco dussumieri, Dum. \& Bibr. iv. p. 456; Jerdon, Journ. As. Soc. Beng. xxii. 1853, p. $+7+$; Gunth. Rept. Brit. Ind. p. 125, pl. xiii. fig. D; Anders. Proc. Zool. Soc. 1871, p. 164 ; Boul. Cat. 1. p. 268.

Draco (Pterosaurus) dussumieri, Fitz. Syst. R:pl. p. 51.

Hab. Luzón, Manila; India, Madras.

I1. D. quinquefasciatus, Gray. $\sigma \in$ N. v.

Dracunculus quinquefasciatus, Gray, Cat. p. 235.

Draco quinquefasciatus, Gray, Zool. Journ. iii. 1827, p. 219, et Zool.; Dum. \& Bibr. ii. p. 455; Günth. Rept. Brit. Ind. p. I 26; Stoliezka, Journ. As. Soc. Beng. xlii. 1873. p. I18; Boul. Cat. 1. p. 269 lam. 20 f. 8, cabeza.

Hab. Samar, Paranas, Calbayog; Penang.

2 Gén. Gonyocephalus, Kaup.

I2. G. sophiæ, (Gray.) ơ $\mathrm{N}$ v Humag̃no.

(M. S. T.)

Tiaris bellii, (non D. \& B.) Gray, Cat. p. 239.

T. sophix Gray, l. c. p. $24 \%$.

T. petersii, Günth Zool, Rec. iv. p. 136.

'I. sophia, Günth. Proc. Zool. Soc. 1872 , p. 593, pl. xxxvii. f. C; Boul. Cat. p. 288.

Hab. Mindanao, Luzón, Albay, Bulusan, Tayabas, Dolores.

13. G. semperi, Ptis. む N. v.

(M. S ' ' ')

Lophyrus ('Tiaris) semperi, Peters, Mon. Berl. Ac. 1867 , p. i6; Boul. Cat. p. 289.

Hab. Luzón, Cagayan, Alcalá.

14. G. interiuptus $B l_{3} r$. N. v.

(M. S T.)

Bcul. Cat. p. 290, 1. 2 1; J. G. Fisch, Jahrb. d. wiss. Aust. Hamb. 2, 1885 , p. 80. (Tiaris subcristata).

Hab. Mindanao.

3. Gén. Calotes, Cuv.

15. C. cristatellus, (Kuthl) o 0 N. v. Layagan.

(M. S. T.)

Bronchocela cristatella, Gray, Cat. p. 241.

Agama cristatella, Kuhl, Beitr. Zool. Vergl. Anat. p. 108.

A. gutturosa, Merr. Tent. p. $5 \mathrm{I}$.

Calotes cristatellus, Fitz. N. Class. Rept. p. 49; Boul. Cat. p. 316.

Agama moluccana, Lesson, Coquille, Rept. pl. i. fig. 2.

(alotes gutturosa, Guerin, Icon. R. A., Rept. pl. vii. fig. 3.

A ama vultuosa, Gray. Griff. A. K. ix. Syn. p. 56. 
Hronchocela cristatella, Kaup, Isis. 1827, H. 6ig; Ditm. \& Bibr. IV. p. 395; Cantor. Cat. Mal. Rept. p. 30 ; Girard, U. S. Ex. ped., Herp. p. 41 ; Gïnth. Rept. Brit. Ind. p. г 38 ; Peters, Mon. Berl. Ac. 1867, p. 17; Steind. Novara, Rept. 1. 27; Stoliezka, Journ. As. Soc. Beng. xxxix. 1870, p. 178.

13. (Lopodeira) crist.tella, Fitz. Syst. Rept. 1. 46 .

13. moluccana, Peters, Mon. Berl. Ac. 1867 , p. 17; Stoliezka, l. c. p. I 79 .

13. intermedia, Peters, \& Doria, Ann. Mus. Genov. xiii. 1878 , P. 375 .

13. burmana, Blanf. Journ. As. Soc. Beng. xlviii. 1878 , p. 127. Hab. Paragua, Inagauan, P. Princesa, Dinagyat, S.znar, Min(lanao, Luzón, Albay, Singalon, Daraga, 'Tayabas, Dolures; Célebes, Borneo.

16. C. marmoratus Gray. ơ N. v. Layagan

(M. S T $)$

Hronchocela marmorata, Gray, Cat. p. 242.

?Hombr. \& Jacq. Voy. Pôle Sud, Rept..pl. iii.

? Lophyrus spinosus, A. Dum. Cat. Méth. Rept. p. 9 I.

Calotes (Bronchocela) philippinus, Peters, Mon. Berl. Ac. 1867 , 1). 16 .

I3ronchocela marmorata, Günth. Proc. Zool. Soc. 1873, p. 168; Boul. Cat. p. 318.

Hab. Luzón, Mąila, Albay, Daraga, Mindanao, Davao.

17. C. ophiomachus, Gray. o N. v. Layagan.

(M. S. T )

Calotes ophiomachus, Gray, Cat. p. $2+3$; Vilan. Creac. 5. p. to.

l,acerta calotes, Linn. S. N. i. p. 367.

Iguana calotes, Laur. Sym. Rept. p. 49.

Igama calotes, Daud. Rept. iii. p. $36 \mathrm{r}$. pl. xlii.

T. ophiomachus, Merr. Tent. p. 51 .

T. lineata, Kuhl, Beitr. Zool. Vergl. An.tt. p. 108.

Calotes ophiomachus, Kaup, Isis, 1827 , p. 621; Duin, \& Bibr. iv. p. 403; Kelaart, Prodr. Faun. Zeyl. p. I69; Günth. Rept. Brit. Iud. p. 142; Boul. Cat. 1. p. 327.

Hab. Luzón, Cavite, Indan; Ceilan.

4. Gén. lophura, Gray.

18. L. amboinensis, Gray oo N. v. Layagan.

(M. S. T')

Lophura amboinensis, Gray, Cat. p. 247; Boul. Cat. p. 402.

L. shawii, Gray, l. c.

Laterta amboinensis, Schlosser, Epist.; Hornst. Abh. Acad. Slockh. iv. 1785 , p. I 30, pl. v. fig. I.

Iguana amboinensis, Latr. Rept. i. p. 271.

I3asiliscus amboinensis, Daud. Rept. iii.p. 322.

Lophura amboinensis, Gray, Phil. Mag. ii. p. 55; Wagl. Icon. Amph. pl. xxviii.; Günth. Proc. Zosl. Sx. 1873, p. 168; Piters \& Doria, Ann. Mus. Gen. xiii. 1878, p. 383 .

Hydrosaurus amboinensis, Kaup, Isis $182 s$, p. II 47 .

Istiurus amboinensis, (uj. R. A. 2n. et. ii. p. $4 \mathrm{I} ; 1): 2 \%$. \& 
B.br. iv. p. $380 ;$ Dutern. R. A., Rept. pl. xr. fig. т; Brehm. V. An 3. p. 122, f. 48 ; Vilan. Creac. 5 p: 4 S.

Histiurus pustulosus, Eschsch. Zosl. Atlas, pl. vii.; Lichtenst. Vomencl. Rept. Mus. Betrol. p. io.

L:phura pustulusa, Fitz, Sy'st. Rept. p. 49.

Istiurus microlophus, Bleek. Natuurk Tijdschr. Ned. Ind. sxii, $1500, \mathrm{p}$. 80.

1.j)hura celebensis, Peters, Mon. Berl, Ac. 1872, p. $5^{8 \mathrm{r}}$.

Hab. Luzón, Manila, Ticao, Negros, (ebú, Dinagat, Mindatnao, Placer, Surigdo; (élebes, Java, Molucas.

\section{Fam. VARÁNIDOS.}

I. Gén VARANUS', MERk

I V. grayi, $G t h v$.) $\&$ N. v Bayauac. (M. S \%॰)

Varanus ornatus, (non. 1)aud) Gray, Cat. p. 10; Boul. 2. Cat. p. 3 I 2 .

Hab. Luzón, Laguna.

2. V. rudicollis, Gray. \& $\mathrm{N}$ v. Bayauac. (M S. T.)

Varanus rudicollis, Gray, Cat. p. Io; Conl. Cat. 2. p. 3 r 3.

Hab. Samar, Borongan; Borneo.

3. V salvator, Laur. ơ N. v. Banias, Bayauat.

(M. S. '.)

Hydrosaurus salvator, Gray, Cat. p. 13.

Stellio salvator, Laur, Syn. Rept. p. 56.

'Tupinambis bivittatus, Kuhl, Beitr. z. Zool. p. 125

Monitor elegans, Gray, Zool. Journ. iii. 1828 , p. 225.

Varanus vittatus, Lesson in Belang. Voy. Ind. Or., Rept. f. 307.

Hydrosaurus bivittatus, Wagl. Syst. Amph. p. I64; Vil. Creac. 5. p. 37 .

H. marmoratus, Wiegm. Nova. Acta Ac. Leop.-Card. xvii. 1835, i. p. 196, pl. xiv.

Varanus bivittatus, Dum. \& Bibr. iii. p. 487 .

V. salvator, Cantor, Cat. Mal. Rept. j. z9; Boul. Cat. 2. p. $31+$; Brehm. V. An. 5. p. 9o. f.

Monitor bivittatus, Schleg. Abbild. p. 76, pls. xxi. \& xxii. figs. I, 2; Müll. \& Schleg. Verh. Naturl. Gesch. Nederl. ov. Bezitt. Rept. p. 38.

Hydrosaurus salvator, Günth. Rept. Brit. Ind. 67, pl. ix. fig. E. Hab. Luzón, Manila, Laguna, Calaguan; Borneo, Java, Célebes.

4. V. nuchalis, Gthr. o $0 \mathrm{~N}$ v. Bayauat.

(M. S. T )

Hydrosaurus nuchalis, Gïnth. Proc. Zool. Soc. 1872, p. 145. pl. viii., et 1873 p. I65; Boul. Cat. 315. 2. p. 215 .

H. marmoratus, (non Wiegm). Gïnth. l. c. 1873 , p. 165.

Hab. Luzón, Albay, Manila, Batangas, Bulacán, Laguna, Calamba, Negros. 
5. V. cumingii, Wart. $\delta$ N. v.

(M. S. T.)

Varanus cumingii, Martin. Proc. Zool. Soc. 1838, p. 69; Boul. C'at. 2, p. 316.

Hydrosaurus cumingii, Günth, Proc. Zool. Soc. 1872 p. $1+5$ il. vii.

Hab. Mindanao, Zamboanga, Placer, Butuan.

Fam. LACÉR CIDOS.

.. 1. Gén. LACERTA, LiN.

I. L. viridis. (Laur). N v. Lagarto verde.

(M. S. T.)

Hab. Europa, Francia.

Fam. ESCÍNCIDOS.

I. Gén. EGERnia, Gray.

I. E. cunninghami, Gray', t N. v. Bubuli.

(M S. T )

Egernia cunninghami, Gray, Cat. p. 105; Boul. Cat. 3, p. 139. 'Tiliqua cunninghami, Gray, Proc. Zool. Soc. 1832 , p. 40.

Egernia cunninghami, Gray, Ann. N. H. ii. 1838, p. 288, et in Stokes, Diocov. in Austral. i. p. 489 pl. ii.

Tropidolepisma cunninghami, A. Dum. Cat. Méth. Rept. p. I77. Egernia krefftii, Peters, Mon. Berl. Ac. 1871, p. 3o.

Hab. Paragua, P. Princesa; Australia.

2. Gén. MABUIA, Fitr.

2. M muiticarinata, (Gray). O' 0 N. v.

(M. S. T )

Tiliqua multicarinata, Gray, Cat. p. ıo9; Boul. Cirt. 3. p. 185 lam. I 2 f. 2.

Hab. Dinagat, Luzón, Bulacan, Angat.

3. M. multifasciata, Kuhl. $\mathrm{N}$.

(M. S. T.)

'Tiliqua rufescens, part. Gr. Cat. p. Iog.

Scincus carinatus, part. Schneid. Hist. Amph. ii. p. 183.

Lacerta rufescens, part. Shaw. Zool. iii. p. 285.

Scincus multifasciatus, Kuhl. Beitr. Zool. u. Vergl. Anat. p. 26.

Mabuya multifasciata, Fitz. N. Class. Rept. p. 52; Boul. Cat. 3. p. 186.

Tiliqua rubriventris, Gray, Ind. Zool.

Euprepes sebæ, part. D. et B. p. 692.

Euprepis rufescens, Cantor; Cat. Mal. Rept. p. 46.

Eup. carinatus, Gravenh. l. c. p. 338; Bocourt. Mus. Sc. Mex. Rept. pl. xxii. c. f. 5 . 
Plestiodon sikkimensci, Gray, An. et Mag. N. H. 12. 185.3, p. 388 .

'Tiliqua rufescens, Girard, V. S. Exped. Herp. p. 227.

Euprepes rufescens, part. Gthr. Rept. B. Ind. p. 79.

'Tiligua carinata, part. Stollia. Journ. As. Soc. Beng. xxxix. 1870 , p. 169.

Euprepes carinatus, part. Theob. Cat. Rept. B. Ind. p. 49.

Eup. ocellatus, Bocourt. An. Sc. Nat. vii. 1878 , art. i6 pl. xxii 18.

Hab. Negros, Mindanao, Zamboanga, Talibon.

\section{Gén lygosoma, Gray.}

4. L. fasciatum, Gray, $\sigma \circ$ N. v. Bubuli.

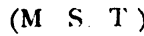

Hinulia fasciata, Gray, Cat. p. 75; Boul. Cat. 3, p. 237.

Lygosoma (Hinulia) fasciatum, Peters, Mon. Berl. Ac. $18 \overline{7} 2$, p. 683 .

Hab. Luzón, Manila, Albay, Daraga, Tayabas, Dolores, Camarines Sur, Negros, Mindanao, Surigao, Mindoro, Boac.

5. I jagorii. Ptrs. $ఠ \supset$ N. v. Bubuli.

(M S. T.)

Lygosoma (Hinulia) jagorii, Peters, Mon. Berl. Ac. 1864 , p. 54; Boul. Cat. 3. p. 240.

Hinulia variegata, (non Peters), Günth. Proc. Zool. Soc. 1873. p. 165 , pl. xvii. fig. B.

Hab. Leyte, Samar, Tacloban, Borongan, Dinagat, Negros, Mindanao.

6. I. variegatum Ptrs. \& N. v, Bubuli.

(M. S. T )

Lygosoma melanopogon, part., Dum. \& Bibr. v. p. 723.

L. (Hinulia) variegatum, Peters, Mon. Berl. Ac. 1867, p. 20.

L. (H.) nævium, Peters \& Doria, Ann. Mus. Genova, xiii. $187 \delta$, p. 340; Boul. Cat. 3, p. 246.

Hab. Dinagat, Mindanao; Borneo, Molucas.

7. L. acutum, Ptrs. $\sigma \circ$ N. v. Bubuli.

(M. S. T)

L. (Hinulia) acutum, Peters, Mon. Berl. Ac. 1864 , p. 54; Boul. Cat. 3, p. 248.

Hab. Samar, Loquilocum, Dinagat.

8. I. cumingii, Gray. N. v.

Otosaurus cumingii, Gray, Cat. p. 93; Boul. Cat. 3, p. 249.

Euprepes otus, Peters, Mon. Berl. Ac. 1867 , p. 20.

Hab. Dinagat, Mindanao; Davao.

9. L. chalcides, (Lin) ơ N N. v. Bubuli.

Lacerta chalcides, Lin. S. N. i. p. 369.

Anguis quadrupes, Lin. l. c. p. 390. 
Lac. serpens, Bloch, Besch. Ges. Natur. Freutude, ii. 17\%0, 1. 28 , pl. .ii.

Scincus brachypus, Schneid. Hist. Amph. ii, p, I92.

Seps pentadactylus, Daud. Rept ix. p. 32.5.

Mabuya serpens, Fitz. $N$. Class. Rept. p. 53.

Lygosoma serpens, Gray, Zool. Journ. iii. 1828 , p. 228.

L. aurata, Gr. Griff. A.K. ix. Syn. p. 72.

L. abdominalis, Gray, An. N. H. ii. $18+9$ p. 332 .

L. brachypoda, D. et B. x. p. $72 \mathrm{I}$.

L. chalcides, Cant. Cat. Mal. Rept. p. t9; Bout. Cat. 3. 1. 3 t'. Eumeces chalcides, Gthr. Rept. B. Ind. p. go.

Podlophis chalcides, Gray, Cat. p. 88.

Hab. Luzón, Cagayan, Pamplona; Jara, China.

ı. L. quaḍrivittatum, Itrs. N. v

Lygosoma (Cophoscincus) quadrivittatum, Peters, Mon. Berl. Ale. 1867 , p. 19 et 1872, p. 583 ; Boul. Cat. Liz. B. M. 3, 1. 2.4. Hab. Mindanao, Davao, Gorontalo.

I I smaragdinum, Less. O' $0^{\prime} \mathrm{N}$. v. Buliuli.

Kenenxia smaragdina, Gray, Cat. p. 79.

Scincus smaragdinus, Eesson, Voy Coquille, Zool. ii. 1) 43, 1'. iii. fig. I; Schleg. Abbild. p. 33, pl. xi.

S. viridipunctus, Lessan, l. c. p. 44 pl. iv. fig. I.

S. crestinus, Guér. Uon. R. A. Rept. pl. xv. fig. 2.

Lygosoma smaragdina, Dum. et Bibr. x. p. 738 ; Boul. Cat. 3. p. 25.

L. (Hinulia) smaragdinum, var. viridifuscum, Peters. Mon. Beil. Ae. 1872 , p. 776 .

L. (Kenenxia) smaragdinum, Peters \& Doria, Ann. Mus. Ciencva, xiii. 1878, p. 345 .

Hab. Luzón, Negros, Cebú, Dinagat, Carolinas, Mlndanaci.

I2 L olivaceum, $G r, \mathrm{~N}$ v.

Dasia olivacea, Gray, Cat. p. Io\&.

'Tiliqua grisea, Gray, l. c. p. I Io.

Dasia olivacea, Gray, Ann. N. H. ii. is 38 , p. 331; Boul. Caí. 3. p. $25 \mathrm{I}$.

Euprepes ernestii, Dum. \& Bibr. v. p. 696; Cantor, Cat. Mal. Rept.p. 47.

E. olivaceus, Günth Rept. Brit. Ind. p. So, pl. x. fig. D; Theot. Cat. Rept. Brit. Ind. p. 5I.

E. (Tiliqua) semicinctus, Peters, Mon. Berl. Ac. $1867, \mathrm{p} .21$.

Tiliqua olivacea, Stoliczka, Journ. As. Soc. Ben. xxxix. i\&ja, p. I72.

Hab. Mindanao; Borneo, Java, Sumatra.

13. L semperi, Ptrs. N. v.

L. (Lipinia) semperi, Peters, Mon. Berl. Ac. 1867,1 . 18; Boul. Cat. p. 254.

Hab. Camiguin, Mindanao. 
14. L. pulchellum, $G r$. $\sigma \rho$ N. v.

(M. S. T)

Lipinia pulchella, Gray, Cat. p. 8t; Roul. Cat. 3. p. 254.

Euprepes pulchelus, Steindachn. Lovara, Répt. p. $4^{8}$.

Hab. Luzón, Manila, Laguna, Calamba.

15. L vulcanium, Cii. \& N. v.

(N. S. T)

Lipinia rulcania, Girard, Proc. Ac. Philad. 18.57, p. 196, it U. S. Explor. Exped., Herp. p. 254 ; Bout. Cat. 3. p. 2.5, Hab. Caldera, Mindanao, Samar, Borongan.

16. I atrocostatum, (Liss.) \& N. v.

(II $\quad$ S $\mathrm{T}$ )

Mocoa cumingii, Gray, Cat. p. 8r.

Mabouya atrocostatus, Gray', l. c. p. 95.

Scincus atrocostatus, Lesson, Voy. Coquille, Zool. ii. p. 50, pl. iv. fig. 3 .

Eumeces freycinetii, Dum \& Bibr. v. p. 648.

Euprepes bitæniatus, Peters, Mon. Berl. Ac. 186 , p. 53.

E. (Mabuya) cumin ii, Peters, Mon. Berl. Ac. 1867, p. 20.

E. (Mabuia) m crestictus, Peters, Mon. Rerl. Ac. 1874, p. 37.3. ? Mabcuia marmorata, Maclecy, Proc. Linn. Soc. N. S. $W$. ii. I 877, p. 65 .

? M. irrorata, Macleay, l. c. p. 66.

Euprepes (Mabuya) atrocostatus, Peters \& Doria, Ann. Mus. Genova, xiii. I878, p. 358 ; Boul. Cat. 3. p. 295.

Eumeces serratus, Fincher, Abh. Natura'. Ver. Hamburg, xi. I 886, p.-, pl. ii. fig. 3 .

Hab. Dinagat, Luzón, Paracale, Samar, Paranas, Catbalogan, Carolinds; N. ${ }^{\mathrm{a}}$ Guinea.

17. I nigrum, H. et facq.

Eumeces niger, Hombr. \& Jacq. Voy. au Pôle Sud (Astrolabe et Zélée), Rept. p. i I. pi. iv. fig. 2; Boul. Cat. 3. p. 597.

Emva nigrita, Girard, U. S. Explor. Exped., Herp. p. 268.

Euprepes (Mabuya) niger, Peters, Mon. Berl. Ac. 1868 , p. 449.

? E. longicaudis, Macleay, Proc. Linn. Soc. N. S. W. ii. 1877, p. 68. Hab. Carolinas.

4. Gén. TROPIDOPHORUS, D B.

18. T. leucospilus, (Pts.) of O N. v.

(M. S. T.)

Lygosoma (Hinulia) leucospilos, Peters, Mon. Berl. Ac. 1872, p. 684; Boul. Cat. 3. p. 360.

Hab. Luzón, Cavite, Indan.

19. T. grayi, Gthr. O" N N. v.

(M.S T.)

Tropidophorus cocincinencis, (non D. \& B.), Gray, Cat. p. Iог. (p. 364). 
'T. grayi, Giüth. Proc. Zool. Soc. 1861 , p. 189; Boul. Cat. 3. Cat. B. M. 3. p. 364, 1887 . lam. 36 t. 2.

Enoplosaurus insignis, Sauvage. Bull. Soc. Philom. (7) iii. 1879 p. 211.

Hab. Luzón, Manila, Laguna, Los Baños.

5. Gén. BRACHYMELES, D B.

2c. B. schandenbergii, (Fucher.) N. v.

Brachymeles, Dum \& Bıbr. v. p. 776; Gray', Cat. p. $9^{8}$.

Senira, Gray, l. c.; Boul. Cat. 3. p. 386. Hab. Mindanao.

21. B. gracilis, Fuch $\mathrm{N}$ v.

Senira bicolor, part., Gray, Cat. p. $9^{8}$.

Eumeces (Riopa) gracilis, Fischer, Fahrb. Wiss. Ans.) Hamb. ii. I 885, p. 85 pl. iii. fig. r.; Boul. Cat. 3. 387 .

Hab. Negros, Mindanao.

22. B. bicolor, Gray. N. v.

(M. S. T )

Senira bicolor, part., Gray, Cat. p. 98; Bonl. Cat. 3. p. 388.

1. $3 \mathrm{I}$.

Hab. Luzón, Manila, Dilao.

23. B. bonitæ, D. B. O 0 N. v.

(M. S. T.)

Brachymeles bonitæ, Gray, Cat. p. 98 .

B. bonita, Dum. \& Bibr. v. p. 777; Boul. Cat. 3. p. $3^{88 .}$

Hab. Luzón, Manila.

\section{Suborden: RIPTOGLOSOS.}

\section{Fam. CAMELEÓNTIDOS.}

I. Gén. CHAMaleon, laur.

1. Ch. vulgaris, Dawd \& N. v. Camaleon.

(M. S. T.)

Hab. Europa, España. 


\section{Orden $44^{\circ}:$ OFII)IOS.}

\section{Fam. TIFLÓPIDOS.}

I. Gén. TYPHLOPS, D. B.

I. T. braminus, (Daucl.) o $\checkmark$ N. v

(M. S. T.)

Eryx braminus, Daud. Hist. Rept t. 7. p. 279.

'Typhlops rondo talooloo, Cuv. Reg. Ani. t. 2. p. 63.

'T. russelii, Merr. Tent. Syst. Tenph. p. 84 ; Schleg. Abbild. Ampl. p. 39 .

'T. braminus, Cuv. Mus. Par.; Fitz. Neuc. Clas. Rept. p. 53; Griff. An. Kingd. Cuv. vol. 9. p. 241; Gray. Synopsis Rept. in Griff. D. et B. 6. p. 309 .

Hab. Luzón, Manila, Tayabas, Dolores, Daraga, Paracale, Mindlanao, Marianas, Guam; China, Molucas.

2. T philippinus, (.Müll). N. v

Trev. Zeits. J. Physiol. 4. p. 349; D. et B. 7. P. I 54 (185t). Hab. ¿Filipinas?

3. T. jagorii, Plrs.

Peters, Mon. Ber. Berlin. Akad. 1861 , p. 684.

Hab. Luzón, Monte Isarog, Camarines.

4. T. petersi, Steind. N. v.

Steindeenher, Verh. Zool. Bot. Gess. Wien 1867, p. 515. lam.

13, fig. 7-9.

Hab. Davao, Mindanao.

5. T. ruficaudus, (Gray.) $\sigma 0$ N. v.

(M. S. T.)

Gray, Cat. Lizards Brit. Mus. 1845, p 136 (Anilios); Ptrs, Mon. Ber. Berlin Aka.t. 1861, p. 684.

Hab. Luzón, Paracale, Daraga.

6. T. $\mathbf{s p .}$

Hab. Davao.

2. Gén. ONYCHOCEPHaLlł, D. B.

7. O. cumingii, (Gray.) $\sigma \circ$ N. v.

(M. S. T.)

Gray, l. c. p. I 33. (Onychophis).

Hab. Luzón, Manila, Malabon. 
8. o. olivaceus, Gray. ơ م. v.

(M. S. T.)

Gray l. c. p. I 33. (Onychophis); Ptrs, Mon. Ber. Berlin Akat. 1881, p. 684 .

Hab. Samar, Paranas, Loquilocum y Borongan.

Fam. UROPÉLTIDOS.

1. Gén. UROPELTis, D. B.

I. U. philippinus, (Cuv.) N. v.

Cuvier, Regn. ani. D. et B. 7. p. i61, (1854).

Hab. Filipinas.

2. Gén. RHINOI'HIS.

I. Rh philippinus, Cuv. N. v.

Jan, Icon. Oph. lam. 5o.

Hab. Filipinas; Ceilan.

\section{Fam. CALAMÁRIDOS.}

I. Gên CALAMARIA, Boie.

1. C. bitorques, Pts. N. v.

Peters, Mon Ber. Berlin. Akad. 1872, p. $5^{8} 5$.

Hab. Filipinas.

2. C gervasii, $D$ B. $\sigma \circ \mathrm{N}$ v

(M. s. 'T.)

Calamaria virgulata, Gervais, Mag. Zool. Guerin, Rept. pl. 16, f. 7-10, et Voy. au monde de la Favorite, Zool. ii. p. 75 .

(. gervasii, Dum. et Bibr. Erp. gen. t. 7. p. 76; Gthr, Cat. Col. snake, Brit. Mus. 1858 , p. 4. et Proc. Zool. Soc. 1879 , p. 77; Peters, Mon. Berl. Akad. 1861 , p. 684; Jan, Icon. d. Ophid. Lief. 10, 1865, lam. 2, f. nn; F.

Mïller, III. Nachtr. Cat. Herp. Sam. Basal Ann. 1883, p. 12 ; Fischer, Jahrb. Harburg. 2, I885, p. 80.

Hab. Luzón, Camariues Sur, Bula, Paracale, Daraga, Tayabas, Dolores, Angat, Bulacian, Bataan, Orion, Negros, Samar, Mindanao; Java, Sumatra.

3. C. grayi, Gthr. N. v

Günther, Colubr. Sn. Brit. Mus. 1858, p. 6. Hab. Filipinas. 
4. C. lumbricoidea, Buiz. \& N. v.

(M. S. T.)

Boie, Isis, 2827 , p. 540; Güntlier, I. c. p. 5; W'agl. Syst. p. $192 ;$ Schleg. Ess. pl. 1: f. 14-16; Cantor, Cat. p. 61; I)mm. et Bibr. p. 89 t. 7.

(.) virgulati, (joven) Bore. l. c. (no. Gervais).

Hab. Samar, Paranas, Borongan; Java, Asia.

5. G. philippinica, Stzind. $\delta$ N. v

(M. S. T.)

Steindachner, Ver. Zool. Bot. Gess. It'ien Bd. 17, 1867; 1. 5it. lam. I 3, f. 4-6.

Hab. Samar, Borongan.

5. C. vermiformis, $D . B . \$ \mathrm{~N}$. v.

(II. S. I)

C. vermiformis, D. B. Erp. gen. t. 7 , p. 85.

Hab. Samar, Paric.

7. C. temminckii, o D. et B. N. v.

(Mi. S. T.)

C. temminckii, Dum et Bibr. vii. p. 87 ; Cithr. Cat. Br. Mrus. 1858, p. 5 .

Hab. Paragua, P. Princesa.

2. Gén. OXYCALAMUS, Gruk.

8. O. oxycephalus, Gthr. \& N. v.

Rabdosoma oxycephalus, Cat. Colubr. Sn. Brit. Mus. 1848,1 . $2+2$ y Proc. Zool. Soc. London 1863, p. 168, f. 9.

Hab. Luzón, Bataan, N. ${ }^{\text {E Ecija. }}$

3. Gén TYPhlogkephus; GThr.

9. T. brevis, Gthr. N. r.

Günther, Proc. Zool. Soc. London 1879, p. 77. Hab. Dinagat, Mindanao.

4. Gén. GEOPHIS, Wagi.

10. G. schadenbergi, Fisoh. N. v.

G. sch. J. G. Fischer, Jahrb. d. wis. Aust. Hamburg. Bd. 2, 1885 , p. 80 y 93, lam. 3 ; fig. 4 .

Hab. Mindanao.

I. G. brevirostris, Ptrs. N. v.

Stenognathus brevirostris, Peters, Mon, Ber. Berlin. Akad. 1872,

p. 586.

Hab. Filipinas. 
12. G. modestus, (D. B.) o 0 N. v.

(M. S. 'T.)

Stenognathus modestus, D. et B. Erp. gen. t. 7 , $1854 ;$ p. 504 . Khabdosoma leporinum, Gthr. Cat. Colubr. Sn. Brit. Mus. i $85 \delta^{\prime}$, p. 12. Proc. Zool. Soc. London 1873, p. 169, y' I879 p. 7.7. St. modestus, Peters. Mon. Ber. Berlin. Akad. 1861, p. 684. Rabdosoma leporinum, F. Müll, III. Nachtr. Cat. Herp. Samml. Mus. 1883 , p. I 2.

Hab. Luzón, Samar, Tubig, Dinagat, Mindanao; Java.

5 Gén. RABDiON, B B.

13. R. torquatum, B. B. $\sigma \circ \mathrm{N}$. v

Rabdion torquatum, Dumeril et Bibron, Erp. gen. t. 7. 1854, p. I 19; Ptrs. Mon. Ber. Berlin. A. Kad. 1861, p. 684.

Ca amaria conica, Mus. de Leyde. 1845.

Pseudorhabdion torquatum, Jan.

Hab. Luzón, Daraga, Davao, Mindanao; Malaca.

6. Gén. ASPIDURA, Wai.ger.

14. A. brachyorrhos, Gth. \&\& N.v.

(M. S. T.)

Scytale brachyorrhos, Boie, Isis, 1827 , p. 517. pl. 25.

As. scytale, Wagler, Nat. Syst. Amph. p. igr. D. et. B. t. 7 . p. 128; (1854).

Calamaria scytale, Schleg. Ess. 2. p. 42. n. ${ }^{0}$ i 2.

As. brachyorrhos, Gthr. Cat. Colub. Sn. Brit. Mus. 1852 , p. I 4. Hab. Luzón, Bulacan, Angat; Ceilan, India.

\section{Fam. OLIGODÓNTIDOS.}

I. Gén. OLIGODON, Boie.

I, O. modestus, Gthr.

O. modestus, Rept. Brit. India, 1864 , p. 2 ro; Proc. Zool Soc. London. 1879 , p. 77.

Hab. Negros.

2. O. notospilus, Gthr.

O. notospilus, Gthr, Proc. Zool. Soc. London 1873 p. I69. fig. A. Hab. Mindanao.

3. O. sublineatus, Gthr. ơ N. v.

O. sublineatum, D. A. B. loc. cit. p. 57.

O. sublineatus, Gthr. Cat. Col. Sn. Brit. Mus. 1852, p. 2 1. Hab. Samar, Paranas; Ceilan. 
2. Gén. ADl,ABES, D. B.

4. A. collaris, (Gray.) $\bigcirc \circ$ N. v.

(.M. s. T.)

Psammophis collaris, Gray, An. et Mag. Nat. Hist. \& $\$ 5.5,1.3(x)$. Adllabes collaris, Gthr. Cat. B. M. p. 28. (18.58).

Hab. Luzón, Laguna, Calamba.

3. Gén SLMOTES, D. B.

5. S. ancoralis. $f_{a n} 0^{\prime} .0 \mathrm{~N}$. v.

(M. S T)

Jan, Elenco sist. d. ofidi, Milano, 1863 p. 45, Prodr. Icon. Gen. d. Ofidi, II.

Coronellidæ, Modena 1863 p. 23, et Icon. a. Ophid. Lief. 11, p. I865. lam. 6, fig. 2; Steind. Reise, d. Novara, Rept. Wien. 1869 p. 6o; F. Müll, III.

Nachtr, Cat. Herp. Samml. Basel. Mus. 1883 , p. 14.

Hab. Luzon, Laguna, Calauan, Camarines Sur, N. Vizcaya, Bayombong.

6. S. aphanospilus, Cope.

Cope, Proc. Acad. Nat. Sci. Philadelpia 1860 p. 245. Hab. Filipinas.

7. S. phænochalinus, Cope. 0 N. v.

(M. S. T.)

Cope, l. c. p. 244; F. G. Fischer, Jahrb, d'wiss. Aust. Hamb. $B d$. 2, 1885, p. 80 .

Hab. Luzón, Manila, Mindanao.

8. S. purpurascens, (Schleg.) \& N. v.

(M. S. T.)

Xenodon purpurascens, Schleg. Ess. pl. 3. f. 13, 14. et Abbildg. pl. I 4 ; $D$. et B. p. 753 ; Cantor. Cat. p. 67.

Coronella albocinct?, Cantor. Proc. Zool. Soc. 1839, p. 50.

Simotes albocincta, $D$. et $B$. p. 633 lam. 32 f. 1 .

S. purpurascens, Gthr. Cat. Colubr. Sn. Brit. Mus. $185^{8}$ p. 25. var. C.; Peters. Mon. Ber. Berlin. A. Kad, 1861 p. 684. Os Kar. Cat. Philip. Rept. 1886, p. 10.

Hab. Luzón, Daraga.

9. S russelli, fan. \& N. v.

Elenco sist, de Offid.; Milano $186_{3}$, p. 45, et Icon. d. Ophi, 11. 1865 , lam. 6. f. 1. B.

Hab. Luzón, Manila, Laguna, Los Baños. 


\section{Fam. $4^{\text {a }}$ COLÚBRIDOS. \\ Subfam. COLUBRINOS. \\ I. Gén. ELAl'HiS, Airr}

\section{E. subradiatus, (Schleg.)}

Coluber subradiatus, Schleg, Ess. ii. p. i 36, et Abbildg. t. 28 f. $7,8, t .29$.

Compsosoma subradiatum, $D$. et $B$. vii. p. 297 .

Elaphis subradiatus, Gthr. Cat. Col. Sn. B. M. $185^{8}$ p. 95; (is Kar. Cat. Rep. Rulip. p. Io7.

Hab. Filipinas; 'Timor.

2. Gén. CORYPHODON, D, I:

2. C. Korros, (Reinw.) O', O N. v

(M. S. 'T.)

Cọlụber korros, Reinw,; Schleg. Ess. ii. p. I 39, et Abbildg. t. 27, t. $2 \dot{\delta}, f .1-6$.

C. cancellatus, Oppel, Mus. Paris; Boie, Isis, 1827 p. 537.

Coryphodon korros, D. et B. vii. p. I86; Gthr. Cat. Colue, Sin. B. $M .1858$ p. I Io.

Hab. Luzón, Manila; Hong-kong, China.

3. C. hexanotus, (Cantor) o N. v.

(M. S. T.)

Coluber hexanotus, Cantor, Cat. of Malay. Rept. p. 74.

Coryphodon hexanotus, Gthr. Cat. B. M. Snakes, $185^{8}$ p. IIo. Hab. Calamianes, Culion; Borneo, Singapor.

4 C. mucosus, (Lin.) $\sigma^{\prime} \rho$ N. x.

(M. S. T)

Coluber"mucosus, Lin. Mus. Ad. Fried. t. $13 \mathrm{f}$. 2. t. $23 \mathrm{f.}$. 2; Boie, Isis, 1827 , p. 536; Russell. i. t. 34 , ii. t. 18 f. 2 ; Lacep. Quadr. Ovip. ii. pl. 34; Latr. Rept. iv. p. 156; Daud. Rept. vi. p. 355 .

C. blumembachii, Merr. Tent. p. I Ig; Schleg Ess. pl. 5. f. 7 r.

C. dhumua, Cantor, Proc. Zool. Soc. 1839. y Cat. of. Malay'. Rept. p. 74 .

Coryphodon blumenbachii, Dum. et Bibr. vii. p. I ${ }_{4}$; Gthr. Cat. B. $M .1858$. p. I I I.

Hab. Luzón, Bataan, (Orion; China, Ceilan.

5. C. fuscus,? Gther. $\sigma$ N. v.

C. fuscus, Gthr. Cat. B. M. Snakes 1858 p. I 2.

Hab. Samar, Borongan; Borneo. 
3 Gén. COMPSOSOMA, D. B.

6. C melanurum, (Sihligr.) N. v.

(M. S. T.)

Schleg, Ess. p. I+1, ii. lam. 5. fig. I , I 2, et Abbild. lams. 5. (Coluber); Peier, Mon. Ber. Berlin. Akad. \& 86 \& p. 685, 684, var. Plegiodon erythrums, Jan. Eleuco sist. d. Ofiidi Milano 1863 p. 6r $e^{\prime}$ Icon. de Ofliti Lief. 211867 , lam. 4, fig: 2 , (arar.-var. Manillensis); Gthr, Proc. Zool. Soc. London 187.;, p. 169, (var.) et 1879 p. 78 (Spilot's); F. Müll, III. Nachitr. Cat Herp. Samml. Basel, Mlies. $188^{2} 3$ p. It (iar.); F. G. Fucher, fadhrb. d. wiss. Aust. Hamburg. Bd. 2. 1885 , p. Sin et 101 (var.); Oskar. Cat. Rept. Phil.pp. p. 108 (1886).

Compsosoma melanurum, D. et B. vii. p. 299.

Hab. Luzón, Manila, Batangas, Lian, Nasugbú, Mindoro, Daraga, Negros, Leyte, Samar, Loquilocum, Paragua, P. Princesa, Mindanao; Borneo, China.

7. C. var. erythrura, D. B. N. v.

Hab. Samar, Paric, Loquilocum, Mindanao.

4. Gén. SPilotes, Wagl.

8. sp. samarensis, Ptrs. $\varnothing \mathrm{N}$ v.

(M. S. T)

Peters, Mon. Ber. Berlin. Akad. 1861 ; p. 685; Oşkar. l. cit. p. Io8.

Hab. Samar, Cubo-cubo.

Fam. DRIADÍNIDOS.

I. Gén. ZAOCYS, Cope.

I. Z. luzonensis, Gthr. $\oint$ N. v. Duhul. $\quad$ (M. S. T.)

Gthr. Proc. Zool. Soc. London, 1893, p. I69; Oskar, loco cit. p. 108 .

Hab. Luzon, Cavite, Indan.

\section{Fam. NATRÍCidOS.}

I. Gén. TROPIDONOTUS, KUHL.

I. T auriculatus, Gthr. $\varnothing \mathrm{N} . \mathbf{v}$.

(M. S. T)

Gthr. Cat. Colubr. Sn. Brit. Mus. 1858, p. 80; Petérs, Mon. Ber. Berlin, Akad. 1861 , p. 697; F. Müll, iii. Nachtr. Cat. Herp. Samml. Basel. Mus. 1883 , p. I5; J. G. Fischer, Jahrb. d. wisc. Aust. Hamburg. Bd. 2. 1885, p. 80; Oskard Cat. Rept. Philipp. 1886 , p. Io8.

Hab. Samar, Loquilocum, Mindanao. 
2. T. chrisargus, Boie. N. v.

(II. S. T )

Boie, Isis, 1827 p. 534; Schleg. Ess. t. 1 2, f. 6, 7; Gthr. Cat. Col. B. Mus. 1858, p. 70 ; Oskar. l. cit. p. 108.

Steriophis chrysargus, Fitz, Syst. Reft. 27.

Amphiesma chrysargum, $D$. et $B$. vii. p. 739 .

Hab. Paragua, P. Princesa; Borneo, Java, Sumatra.

3. T. deudrophiops, Gthr. N. v.

Gthr. An. Mag. Nat. Hist. (5) Bd. 11. 1883, p. I36; Oskar, Cat. Rept. Philip. p. Io9 (I 886).

Hab. Mindanao, Zamboanga.

4. T lineatus, Ptrs $\delta$ N. v.

(M. S. T )

Ptrs. Mon. Ber. Berlin. Akad. 1861, p. 686; Oskar, l. cit.

p. Iog.

$H a b$. Samar, Loquilocum.

5. T. spilogaster, Boie. $\sigma 0$ N. v.

(M. S T.)

'Tr. spilogaster, Boie. Isis, 1828 , p. 559; D. et B. vii. p. 598; Gthr. Cat. Colubr. Sn. Br. Mus. 1858 p. 66; Peter. Mon. Ber. Berlin. Akad. 1861 , p. 687; Jan. Elenco sist. d. Ophi. Milano 1863 , p. 72 et Icon. d. Ophid. Lief. 27, 1868, lam. 2 fig. I; J. G. Fischer, Arch. t. Naturg. Bd. 481, 1882, p. 282 $y$ Jahrb. d. wiss. Aust. Hamburg. Bd. 21885 p. 82; F. Müll. iii. Nachtr. Cat. Herp. Samml. Basel Mus. 1883, p. 15; Oskar Cat. Rept. Philipp. p. 109 I 886.

Tr. quincunciatus, var. Schleg. Ess. ii. p. 309; Eydoux et Gerv. Voy de la Favorita v. pl. 28.

Hab. Luzon, Manila, Laguna, Los Baños, Morong, Bosoboso, Bulacan, Tayabas, Dolores, Bayombong, Nueva-Vizcaya, Batangas, Nasugbú, Daraga, Tabaco, Albay, Camarines Sur, Bataan, Orani, Mindanao.

6. Tr. sp. $\bigcirc \circ \mathrm{N} . \mathrm{v}$.

(M S. T.)

Hab. Luzon, Batangas, Manila.

7. Tr. sp. N. v.

Hab. Mindanao.

8 Tr. stolatus, (Lin.) ơ N. v.

(M. S. T)

Coluber stolatus, Lin. Mus. Ad. Fried. t. 22 f. I et. S. N. i. p. 379; Laur. Syn. Rept. p. 95; Shaw, Gen. Zool. iii. p. 542; Pts. Mon. Ber. B. Akad. i 861 , p. 686.

Coronella cervina, Laur. l. c. p. 88.

Coluber cervinus, Gm. Syst. Nat. i. p. I I I 4 ; Lacép. Hist. Nat. des Serp. ii. p. го7; Latr. Rept. iv. p. 80; Daud. Rept. vii. 


\title{
$-431-$
}

p. I6I; Russell, Ind. Serp. i. t. Io, ii. 19 et ii. t. I5 $B$. jov.

Natrix stolata, Merr. Tent. p. 123.

'Tropidonotus stolatus, Boie, Isis, 1827, p. 535; Schleg. Ess. ii. p. $317 ;$ Gthr. Cat. Sn. B. M. 1858, p. 68.

Amphiesma stolatum, D. et B. vii. p. 727 .

Hab. Luzon, Bulacan, Calumpit; Ceilán, Hong-kong, China.

9. Tr. aff. dorsalis, Gthr ơ N. v.

(M. S T.)

F. Müll, 1. Nachtr. Cat. Herp. Samml. Basel. Mus. 1880, p. 31 .

Hab. Luzon, Manila.

Io $\operatorname{Tr}$ aff hypomelas, Gthr

F. Müll, iii. Nachtr. Cat. Herp. Samml. Basel. Mus. 1883 , p. 15 .

Hab. Mindanao.

1I. Tr. schistosus, (Daud) ơ N. v.

(M. S. T.)

Coluber schistosus, Daud. Rept. 7, p. 132.

Tropidonotus schistosus, Schleg. Phys. Sarp. 1. n. ${ }^{\circ}$ I 3 p. I68;

t. 2 p. 3 18; Det. B. Exp. Gen. 7, p. 596.

Hab. Mindlanao, Mindoro, Luzon, 'Tayabas, Laguna, Calamba;

China, Hong kong.

\section{Fam. HOMALPÓSIDOS.}

\author{
I. Gén. Cerberus, Cuv. \\ I. C. rhynchops, (Schneid) $\sigma \circ \mathrm{N} \cdot \mathrm{v}$. \\ (M. S. T.;
}

Hydrus rhynchops, Schn. H. Amph. Bd. 1, p 246.

Cerberus boxformis, Peters, Mon. Ber. Berlin Akad, 1861 , p. 687.

Homalopsis boxformis, Jan, Elenco List. d. Ojid. Milano, 1863 ,

p. 77; Gthr. Rept. B. India 1864, p. 279 et Proc. Zool. Soc. London, 1879 , p. 78.

C. cinereus, Gray, Wood. p. I.I 8 .

C. obtusatus, Cuv.

Elaps boæformis, Schneid.

Homalopsis rhynchops, Cant.

H. schneideri, Schleg.

Hurria schneideriana, Daud.

Hab. Luzon, Manila, Batangas, Lobo, Camarines Sur, Albay,

Boac, Mindoro, Daraga, Buti, Negros, Mindanao, Placer, Davao, Paragua, P. Princesa. 
2. Gén. CAMPYLODON, i). I3.

2. C. prevostianum, D. B. $\sigma^{*} \mathrm{~N}$. v.

(M) S. T)

1)umeril et Bibron, Erp. gen. t. 7, p. 964, i 854 ; Jan. İon d. Ophid. 3o, 1868, taf. 6. f. 3 .

Coluber prevostianus, Eyd. et Gerv.

I Iomalopsis plumbea, Eyd. et Gerv.

Hab. Luzon, Manila, Laguna, Calamba, Bataan, Orion.

\section{Fam. PSAMMÓFIDOS.}

I. Gén. PSAMmodynastes, Giru.

I. Ps. pulverulentus, (Boie.) o 0 N. v.

(M S. I.)

Psammophis pulverrulentus, Boie, Tsis, p. 5+6, (1827); D. et B. p. 895; Schleg. Ess. 18 , p. (I 7 I I); Gthr. Rept. B. India. 1864 , p. 292; A. Proc. Zool. Soc. London 1879 p. 78 ; F. Müll, III. Nachtr. Cat. Herp. Samml. B. Mus. 1883 p. iz; J. G. Fitcher, Jahrb. d. wiss. Amt. Hamburg. 2, 1885 , p. 81; Oskarb. Cat. Rept. Philipp. 1886 , p. I Iо.

Dipsas ferruginec, Cant.

Hab. Luzón, Isarog, Leyte, Samar, Loquilocum, Dinayat, Mindanao, Placer; Borneo, Java, Célebes, Sumatra.

\section{Fam. DENDRÓFIDOS.}

I. Gén. GONYOSOMA, Wagl.

I. G. oxycephalum, (Boie.) $\sigma \circ$ N. v.

(M, S. T.)

Coluber oxycephalus, Boie, Isis, 1827, p. 537; Ptrs Mon. Ber. Berlin. Akad. 1871 p. 688; Gthr, $\bullet$ Rept. B. India 1864 , p. 294 ; id. Cat. Sn. B. M. 1858 , p. 122.

- Aepidea robusta, Hallow.

Coluber cærulescens, Lin. Mus. Ad. Frind, t. 20, f. 2; Daud. Rept. VI. p. 320; Shaw. Gen. Zool. iii. p. 5I4; Merr. Tent. p. I I 2 .

Alopecophis chalybæus, Gray.

Gonyosoma oxycephalum, D. et B. Erp. Gen. VII. p. 2 I3.

(i. viride, Wagl. Icon. t. 9. et Nat. Syst. p. 185 .

Herpetodryas oxycephalus, Schleg. Ess. t. 7, f. 8-10, et Abbildg. t. 44, f. I-9; Cantor, Cat. of Malay. Rept. p. 80.

Hab. Luzón, Manila, Bulacan, Angat, Camarines Sur, Mindanao, Surigao, Legaspi; Borneo, Java, Sumatra, Célebes.

2. G. frenatum, (Gray) $\sigma \circ$ N. v.

(M. S. T.

Herpetodryas frenatus, J. E. Gray. An. et Mag. Nat. Hist. 1853 , p. $39^{\circ}$. 
(3. frenatum, Gthr. Cat. B. M. 1858 p. 123.

Hab. Luzón, Manila, Malabon.

2 Gén, DEXINUPHIS. Buk.

3 D. pictus. (Gim) $\sigma \circ$ N. v. Remorana (M $\therefore$ \%)

('oluber pictus, (ion. S. V. I p. 1116; D). el B. Erp. gen. T, $18.5+$, 1. 197; Ptrs. mon Ber. Berlin. Akat 1861, p. o8;; litkr. Rep. B. Intia $180^{\circ}+1$ ). $297 ; J$. G. Fischer, Jahrh. at. zisiss. Aust. Hamburg. 2, p. $8(3,1885$.

thetullat belli, Gray, Ill. Ind. Zool,; Cant. Cat, p. 79.

(. ceruleus, Bonn. Ophiol. p. 30.

(. Hecorus, Shaw, Gen. Zool. p. 538; Merr. Tent. p. 128.

Dendrophis chairecacos, Boie, Isis, 1827,4 . 5tr; Erpet Javia. t. 34 ; Wagl. $N$. Sy'st. p. 183.

loptophis mumar, Bell. Zool. Journ. ii. p. 324.

D) p.n. picta, Schleg Ess. t. 9. f. 5-7; 1). et B. VII. p. 197; Gthr. Cit. Sn. B. Mus. 1858, p. ${ }_{1+8 ;}$ Brehm. V. An. 5, p. $23,3, f$. var $B$.

Hab. Luz'on, Manila, Laguna, Los Baños, Bulacan; Angat, Morong, Antipolo, Albay, Daraga, Camarines, Sa:nar, Loquilocum, Mindanao, Davao.

\section{D. punctulatus, (Gray).}

Leptophis punctulatus, J. E. Gray in King's Australia, ii. p. +32, y in Grey. West. Australia, ii. p. $+3 \mathrm{r}$.

Dendrophis (Ahatulla) olivacea, Gray, Zool. Misc. p. 54.

1). lineatus, D. et B. VII. p. 200; Voy au Pôle Sud. sur les: Corvettes l'Astrol. et la Zelee, Ophid. pl. 2.

1). punctulata, Gthr. Cat. Col. Sic B. Mus. 1858 , p. It9; Pa. rente et Picaglia, Atti Soc. Nat. Modena Mem. Orig. 5, 1886 , p. 50 .

Dendrophis arnensis, Doria. D. calligastra, Gthr. D. lineolata, I). B. D. olivacea, Gray. D. striolatus, Pts.

Hab. Ticao.

5. D. terrificus, Pts. $\sigma \varnothing$ N. v,

(M. S. T. )

Peters. Mon Ber. Berlin Alead 1851, p. 688; (caudolineatus, nor

Gray) y 1872 , p. 583; Gthr. Proc. Zool. Soc. London 1879, p. 78 (Philippinensis); Parenti et Picaglia, l. c. p. 50; (octolineata.)

Hab. Luzón, Bulacan, Tayabas, Dolores, 'Tiaon, Batangas, Lian, Albay Trao, Sanar, Borongan, Loquilocun, Paragui, P. Princesa, Mindanao; Célebes.

3 Gén CHRYSOPELEA, BoIE.

6 Ch ornata, (Shuzw). $\sigma \rho \mathrm{N} \mathrm{v}$

(M. S T.)

Coluber ornatus, Shaw. Zool. 3, p. 477; Cope, Pros. Ac. Nat. So: Phl. i85) p. 556; o.tr. A. de Gthr.; Ptrs. Mon. Berlin 
Akad 1\$61, p. 687; Gthr.; Rept. B. India, 1864, p. 298; Stenida, Reise d. Novara, Rept. Wicn 8869 , p. $71 ; J . G$. Fischer, Jahrb. d. wis. Hamb. 2, p. \&o, 1885.

Natrix ornata, Merr. Tent. P. 109.

Chrysopelea paradisei, Boie, Isis, 1827, pp. 546, 547; Wagl. Amph. 1. 188.

Tyria ornata, Kit. 2, None Class. Rept. p. Go.

Dendrophis ornata, Schleg. Ess. pl. 9 f. 8-u; Fil ppi Cat. Serp. Mus. Pav.

Coluber ibiboca, Latr. Rept. iv. p. 131.

D. paradisei, Moll. et Dillw.

Chry sopelea ornata, D. et B. VII. p. 342.

Leptophis ornatus, Cant.

Hab. Luzón, Manila, Laguna, Los Baños, Leyte, Samar, Lauang, Mindanao, Balabac; Borneo, Java, Célebes, Sumatra.

7. Chr. rubsscens, (Gray). ơ $0 \mathrm{~N}$ v.

Dipsas rubescens, Gray, Ind. Zool. pl. tig. 2, Gthr. l. c. p. 299; Steind. l. c. p. $7 \mathrm{I}$.

Dendrophis sumatrana, Bleck.

Leptophis rubescens, Blyth.

Chrysopelea rubescens, Gthr. Cat. B. Mus. 1858, p. 145; Oskar. Cat. p. 112 (1886).

Hab. Luzón, Manila, Cavite, Alfonso.

4. Gén Loptophrs, BenL.

$\& \mathbf{L}$ vertebralis, $D \quad B \quad \& \mathbf{N} . \mathbf{v}$.

(M. S. T.)

J). et B. Erp. Gen. 7, p. 543 \854.

'Tamnosophis vertebralis, Jan.

Hab. Luzón, Manila.

Fam. DRIÓFIDOS.

1. Gén. TRAGiOPS, Wagl.

1. T prasinus, (Reinzer) of $0 \mathrm{~N}$ v Dabunpalay.

(M. S. T.)

Dryophis prasina, Reinwartt. Tijdschritt voor Natiur, Boie, Isis, 20. p. 545, 1827; Schleg Eśs. pl. 10. t. 9-13; et Abbildg. t. 8. f. 1-6; Gthr. Cat. C. Sn. B. Mus. 1858 , p. 159; col Rept. B. India 1864 , p. 303; y Proc. Zool. Soc. London 1879, p. 78 ; Cope, Proc. Ac. Nat. Sc. Phil. 8600 , p. 554; var. lata. Ptrs. Mon. Berlin, Akad 1867, 4. p. 688; F. I. Fischer Jahrb. d. wiss. Aust. Hamb. 2. 1885 p. 80.

Drynus nasutus, Bell. Zool. Jour 11, p. 327.

Tragops nasutus, Wagl.. Syst. Amph. p. 184 .

'T. prasinus, D. B. vii. p. 824 ; Cantor.

Hab. Luzón, Manila, Laguna, Calamba, Los Baños, Daragan, Albay, Samar, Catbalogan, Mindanao, Dıvao; Célebes, Sumatra. 
2. T. var. læta, Cap. \&\& N. v.

Hab. Haguasa, Mindanao.

3. L xanthozonius, oar. hive $\delta \&$ N. v. Dahunpalay. (I. S. T.)

Russell, Ind. Serp. ii. pl. 24.

Dryiophis xanthozonia, (Kuhl.) Boie, Isis, 1827 , p. 545; Githr. Cat. Sn. B. M. $160,\left(185^{8}\right)$.

Tragops xanthozonius, Wagl. Syst. Amph. p. $18_{4} ;$ D. et B. vii. p. 826 .

Hab. Luzón, Bulacan, Lolomboy.

2. Gén passerita, Gray.

4. P. mycterizans, (Lin.) \& \& N. v Dahunpalay.

(M S T T

Coluber nycterizans, Lin. Mus. Ad. Fried. p. 28; Lacep. Quadr. Ovip. ii. pl. 4. f. 2; Bom Ophr. Enc. Meth. pl. 30. f. 62; Russell, Ind. Serp. t. I2, I3; Daud H. Rep. vii. pl. 87.

Drynus nasutus, Merr. Tent. p. 196.

Passerita mycterizans, Gray, Ax. Phil. x. p. 208; Gthr. Cat. Sn. B. Mus. 1858 , p. 160 .

Drynus oxyrhynchus, Bell. Zool. Journ. 1821, p. 325.

Dryiophis nasutus et D. pavoninus, Boie, Isis, 1827, pp. 520, 543; Cuv. Anim.; Guer. Icon. Rept. t. 22. f. 2.

'Tragops nasutus et 'T. pavoninus, Wagl. Sys. der Amph. p. 184 , Schleg. Ess t. IO. f. I-5.

Herpetotragus nasutus, Fitz. Syst. Rept. p. 27; D. et B. vii. p. 809 .

Hab. Luzón, Manila, Bulacan, Lolomboy.

Fam. DIPSẢDIDOS.

I. Gén. DYPSAS, Bore.

r. D. (Dipsadomorphus), angulata, Pts.

Peters, Mon. Ber. Berl. Akad 1861, p. 668.

Hab. Leyte.

2. D. $s p$. ¿\& N. v.

(M S. T)

Hab. Luzón, Bataan, Orion.

3 D. (Eudipsas) cynodon, Cuv. o N. v.

(M. S. T.)

Cuv. Reg. Anim; Boie, Isis, 1627 , p. 559; Guerin, Icononogr. pl. 21. f. 2; Shleg. Phys. di Serp. 2. p. 268 lam. 11. f. Io, 1 ; Cant. Cat. p. 77; Gthr. Rept. India, 1864 , p. 308, y Proc. Zool. London 1879, 1879 , p. 71 . 
Eudipsas cynodon, Fitzingr. Syst. der Rept. p. 27; Wood, Nat. Hist. 139. fig.

Ospetiodon cynndon, $D$. et $B$. vii. p. 207.

Pareas waandersi, Bleck.

Hab. Mindanao, Paragua, Puerto Princesa; Rorneo, Java, Snmatra,

4. D. $s p . \sigma o \mathrm{~N} . \mathbf{v}$.

Hab. Luzón, Cavite, Alfonso.

5. D. (Dipsas) dendrophila, $W_{\text {oggl }} \sigma \circ \mathrm{N}$ r. $\quad$ (M $\mathrm{S}$ T.)

Wagler, Syst. Amph. p. 181, é Icon. lam. 8; Peters Mon. Ber. Berlin. Akad, 1861 , p. 688 (genmicincta); Gthr. Rept. R. India 1864 , p. 310 ; et Proc. Zool. Soc. London, 1879 ; p. $7 x$; J. G. Fischer, Jahrb. d. wiss. Aust. Hamburg. 2. p. 81, I885.

Dy. dentrophila, Reinv; Boie, Isis, 1827 , p. 549; Schleg. Ess.

t. If. f. I-3; Cant. Cat. p. 76; Brehm. V. Anti. 5. p. 2ot. f. 93 .

Triglyphodon dendrophilum et gemmicinctum, D. B. vii. p. Ios, y. 1086.

Hab. Samar, Paragua, Puerto Princesa, Rutuan, Mindanac, Davao.

6. D. drapieai, (Boie.) $\mathrm{N} v$.

D. Arapiezii, Boie, Isis, 182\%, p. 559; Sichleg. Ess. pl. I I. f. 8, 9, et Abbilg. t. I 5.

'Triglyphodon drapiezii, $D$. et $B$. viii. p. 1097.

Hab. Mindanao.

7. D (Eudipsas) guiraonis, Stcind. $\mathrm{N}$ v.

Steindachner, Reise der Novara, Rept. Wien. 1869, p. 75; F. Miüller. III. Nachtr. Cat, Herp. Snmml. Basel Mus. 188,3 , p. I 8, J. G. Fischer, l. c. p. 8ı.

Hab. Mindanao.

8. D. fusca. (Gray ) o $\circ \mathrm{N}$ v.

(M S r.)

Dendrophis fusca, (Gray.) Zool. Misc. 1842, p. 54; Gthr. Cat. B. $M .1858$, p. I 7 I.

Triglyphodon flavescens, $D$. et $B$. vii. p. ro 8 o.

Hab. Luzón, Laguna, Mindoro, Calapan.

9. D philippina, Pts. $\sigma \circ$ N. v.

(M S. T)

Peters Mon. Ber. Berlin, Akad. 1867, p. 27.

Hab. Luzón, Manila, N." Vizcaya, Quiangan,

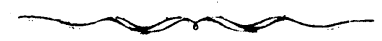




\section{Fam. LICODÓNTIDOS.}

1. I.YCOD(IN, BOtE.

1. L. aulicus, (Lin.) ð.० N. v.

(M. S, T.)

Coluber aulicus, S. N. i. p. 38r ; Cope, Proc. Acat. Nat. Sc. Philadel. 1867, p $262(v i r$. D. B.); Pste:s, Mon. Ber. Be::lin Akad 1861, p. 688; Gthr. Rep. B. Ind a 1864, p. 916; et Proc. Zool. Soc. Kondon 1879, p $78 ; J$. G. Fischer, Jahrt. di wiss, Aust. Hamb. 2. 2885, p. 81 var. g; D. et B. vii. p. 969; Vil. Creac. 5. p. I07.

Lycodon hebe, Schleg.

Hab. Luzon, Manila, Tayabas, Dolores, Batangas, Nasugbu, Camarines Sur, Paracale, Mindoro, Boac, Butuan, Mindanao.'

2. I capucinus, Boie. var. $D$ liwssel. N. v

(M S. T.)

L.y. capucinus, Boie, Isis, 1826 , p. $98 \mathrm{r} ;$ Gthr. Cat. B. M. $185^{\circ}$, p. 203.

'Iy, atro-purpureus, Cont. Cat p. 68.

Hab. Luzón, Laguna, Calamba.

3. L. bairdi, $S$ end.

Steind, Reise a'Nov. Rept. Wien. 1869, p. qo. Hab. Filipinias.

4. I modestum. N. v.

Nom. Rept. M. Zool. B. Berl. 1869, p. 27.

Hab. Manila.

5. L. tesselatus, Fan. $\sigma^{\prime} \rho \mathrm{N}$. v.

(M. S. T.)

Jan. Elenco sist. d. Ofid. Milano 1863 , p. 96; et Icon. di Ophid. 1. 36. I870 lam. 4 f. 2; Mïll. III. Nachtr. Cat. Herp. Samml. Basel. Mus. 1833 , p. 17.

Hab. Luzón, Manila.

6. I cucullatum, N. v,

Nom. Rept. et Amph. Mus. Zool. B. Berl. 1856, p. 27. Hab. Luzón, Manila.

2. Gén. ODONTOMUS, D. B.

7. O. mülleri (D. B.) \& N. v.

(M. S. T)

Lycodon mülleri, Dumeril et Bibron, Erp. gen. 7. 1854, p. $382 ;$ Gthr, Cat. Col. Sn. B. Mus. 1858 , p. 203; et Proc.Zool. 
Soc. London 1879, p. 78; Peters Mon. Ber. Berlin. Akad 1861, p. 688.

Hab. Luzón, Daraga, Iriga, Balabac, Mindanao, Surigao.

$$
\text { 3. Gén CyClocorls, D. B. }
$$

8 C. lineatus, (Reinh.) of $\circ$ N. v.

Lycodon lineatus, Reinw. l. cit. t. I. f 7-9.

Cyclocorus lineatus, D. et B. p. $386 ;$ Gthr. Cat. Col. Sn. B. Mus. 1858, p. 208; Peters Mon. Ber. Berl. Akad. 1861. p. 688; Jam. Icon. d. Ophid. 30, 1870 , lam. 6. 1. 2.; F. Mïll. III. Nachtr, Cat. Herp. Samml Basel. Mus. 1883 , p. 17.

Hab. Luzón, Manila, Tayabas, Dolores, Albay, Mayon, Daraga, Minłanao, Surigao, Samar, Paric.

\section{Fam. ESCITÁLIDOS.}

1. Gén. hologerrhum, Grthr.

I H. philippinum, Gthr. o N. v.

(M. S T )

Gïnther, Cat. Colubr. Sn. B. Mus. 1858 p. 186; id. Proc. Zool. Soc. London. 1873 p. I 7 I, lam. 18 fig. B. et 1879 p. 78 .

Cyclochorus maculatus, Jan. Icon. d. Ophid. 361870 lam. 6 fig. 3 .

C. lineatus, var. maculata, J. G. Fischer, Jahrb. d. wiss. Aust. Hamb. 2. 1885 p. $8 \mathrm{I}$.

Hab. Mindoro, Baco, Mindanao, Placer.

\section{Fam. AMBLICEFÁLIDOS.}

I. Gén. AMblycephalus, Kuhl.

I. A. boa, Bcie. N. v.

(M. S. T)

Boie, Isis 1828 p. 1035; Gthr. Rept. B. India 1864 p. 325; J. G. Fischer l. c. p. $8 \mathrm{I}$.

Dipsas boa, Schleg. Ess. t. i . f. 29, 30; Cant. Cat. p. 78 , pl. 40. f. 3 .

Aplopeltura boa, D. et Bibr. vii. p. 444 .

Hab. Mindanao; Borneo, Java.

\section{Fam. BÓIDOS.}

\section{Gén Piesigaster, Shonne.}

I. P. boettgeri, Seoane. $\mathrm{N}$ v.

Seoane, Abhandi. Senckenbg. Nat. Gen. I2, 1880 p. 219. 1. I. Hab. Panay, Mindanao, Pollock. 
Fam. PITÓNIDOS.

I. Gén. PYIHON, Daud.

I. Py. reticulatus, (Schncid.) ơ N. v. Saua.

(M. S. 'T )

Schneid, Hist. Amph. p. $26+$ (Boa); Petrs Mon. Ber. Berl. Akad. 1861 p. 689; Gthr. Rept. B. India 1864 p. 330; v. Martens, Prenss Exp. O. Asien Zool. 1. 1875 p. 197; Brehm. V. $A n$. 5. p. I 94 .

Python shneideri, Mesr. Tent. Syst. Anph. p. 89.

Hab. Luzón, Manila, Laguna, Calauan, Bulacán, L lomboy.

\section{Fam. ACROCÓRDIDOS.}

I. Gén. Chersidrús, Cuv.

I Ch. granulatus, (silneid.) oم N. v.

(M. S. T.)

Hydrus granulatus, Schneid. l. c. p. $2+3$; Peters, Mon. Ber. Berlin. Akad. 1861 p. 684, et Prenss. Exped. P. Asien. Zno!. 1. p. 382 1876; Gthr, l. c.t. p. 336; Steind. Reise d. Nov. Rept. Wien. 1869 p. 58.

Acrochordus fasciatus, Shawe; Aer. granulatus, Cantor; Ch. ann ulatus, Gray; Ch. fasciatus, Cuv.

Hab. Luzón, Manila; Laguna, Los Baños, Mindoro, Boac, Mindanao; Célebes, Timor, Sumatra.

Fam. ELÁPIDOS.

I Gén NAJA, Lauk.

I. N. tripudians, Merr. N. $\mathbf{v}$.

Merrem, Tent. p. 147; Peters. Mon. Ber. Berlin Akat 185, p. 690 (var.); Gthr. Rept. B. India 1854 p. 338 et Proc. Zool. Soc. London 1879 , p: 78; id. Cat. Sn. B. Mus. p. 223. 1858 , Wood. Nat. Hist. 3. p. 141. fig.; J. G. Fischer, Jahrb. d. wiss. Aust. Hamburg. 2. p. 81, $1885 ;$ D. B. p. 1293.

Coluber naja, Lin. Mus. Ad. Fried. i. t. 25. f. 1. et f. Nat. i. p. 382 ; Lacep. ii. pp. I02. I04; Lxur. Syn. p. 91; Shaz, Zool. iii. p. 409; Rusiell, Int. Serp. i. t. 5, 6 ii. t. 1.

Naja atra, Cantor; kaonthia, Less; larvata, Cant; Brehm. Gl. Am. 5, p. 25I. f. lutescens, Laur.

Hab. Leyte, Mindanao, Davao.

2. N. sp. N. v.

Hab. Mindanao. 
3. N. var. semarensis, Pts. \& N. v.

Jeters, l. cit. p. 6gor.

Hab, Samar, Loquilocum, Leyte.

4. N. sputatrix. (R:inw. Schleg. N. v.

Schleg, Ess. Phys. Serp. 2. 1837 p. 469 (Tripudians, var.); Gthr. Rep. B. India 1864 p. 339; F. Mïll. iii. Nochtr. Cat. Herp. Samml. Basel. Mus. 1883 p. I 8.

N. tripudians, var. nigra.

Hab. Mindanao.

2. Gén. OPHIOFHAGLis, Gink.

5. O. elaps, (Sihles). N. v.

Naja elaps, Schleg. l. c. p. 4, 76; Gthr. l. c. p. 3+1; et Cat. Su. B. M. 1858, p. 319; J. G. Fischer, Jahrb. d. w. ss. Aust. Hamb. 2, 1885 ; p. 81, (Hamadryas); Brehm. V. An. 5, 1). 263 , f. 99 .

Hamadryas bungarus, Schleg. Ess. ii. p. 476 .

I1. ophiophagus, Cant. Cat. p. 116.

Iraja bungaru:, Schleg.

N. vittata, Ell. Madr. Journ. Lit. et Sc. xi. pl. I.

'Irimeresurus ophiophagus, D. B. p. I 245.

Hab. Mindanas; Burneo, Java, Sumatra.

6. O. fasciatus, (Pts.) \& N. v.

(M S. T.)

llamadryas fasciatus, Peters, Mon. Ber. Berl. Akad. 1861 1. 689. ('ph. fasciatus, Oscar Cat. Ript. Philipp. 1886,1 . 117.

Hab. Samar, Lxquilocum.

3 Gén. HEMLBUNGakUs, Prks.

7. H. calligaster, (Wiegm.) o o $\mathrm{N}$ v. Camamalo. (M. S. T.)

Elaps calligaster, Wiegman, $N$. Ant. 1 835, t. 25, f. 2; D. et $B$. p. 1226 ; Ptrs. Mon. Ber. Berlin Akad. 1861 , p. 689; Jan. Icon. d. Opkid. 43, 1873, 1. 2, fig. 2; F. Müll, III Nachtr.

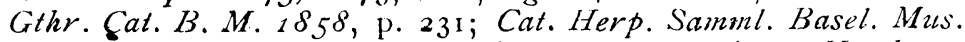
$188_{3}$, p. $11 ; J . G$. Fischer, Jahrb. de wiss. Aust. Hamb. 2, I 885, p. 81 .

Ii. collaris, (Boie) Schleg. Ess. ii. p. +48, et Abbildg. t. +o, fig. I $0,1 \mathrm{I}$.

Hab. Luzón, Bataan, Manila, Camırines Sur, Dirara, Mindanao, Paragua, Bulabac.

8. H. collaris, (Sihleg) $\sigma \circ \mathrm{N}$ v.

(M. S. T)

Elaps collaris, Schleg. Ess. Phys. Sero. 2, 1837, P. +48; Jan. 
Elenco sist. de Ofidi. Milano 1863 , p. 11t et Icon. d. Ophid. 73, $18 ; 3 ; 1.1$ i f. 1 .

Elaps gastrodelus, $B . B$.

Hemibungarus collaris, Oskard. Catt. Rept. Philip. 1836, p. 117. Hab. Luzón, Manila.

9. H. gemmiannulis, Ftrs. N. v.

Peters, Mon. Ber. Berlin. Akad. 18;2, p. 58;; F. Mäll. IIl. Vachtr. Cat. Herp. Samml. Basel Mus. 1853, 1. I8; Oskard. Cat. Rept. Philip p. 117, 1886.

Hab. Mindanao.

4. Gén. Catilophis, Gikay.

Io. C. biineatus, Plrs. of N. v.

(M. S. 'I)

Pelers, Sitz. Ber. Gess. Nat. Freunde Berlin, 1881, p. 109; Oskar, Cat. B. M. 1886 , p. I I 7 .

Hab. Paragua, P. Princesa, Balabac.

5. Gén. Adeniophis, prirs.

II. A. philippinus, (Gthr.) N. v.

(allophis intestinalis, var. Gthr. Proc. Zool. Soc. London 18.59, p. 82, lam. 16 fig. A. et Rept. B. Indic. 1864, p. $348 ; A$. B. Meyer. Sitz. Ber. Berlin. Prens. Akad. Wiss. Berlin, 36, I 886, p. 6i 2 .

Hab: Mindanao.

6 Gen. ELAPS, SCHNEID.

I2. $\mathbf{E}$ intestínalis, $(S e b a) \succsim \mathrm{N}$ v.

(M. S. T.)

E. intestinalis, Seba, ii. pl. I9; Gthr. Cat. Sn. B. M. 1858 1. 230, var.

Aspis intestinalis, Laur. Syn. Amph. p. го6.

Coluber intestinalis, $G m$. S. N. p. Io85. $_{5}$

Elaps furcatus, Schneid. Hist. Amph. p. 303; Schleg. Ess. pl. i6 f. I 2, 13 et Abbildg. t. 46, f. I-8; Cant. Proc. Zool. Soc. 1839, p. 34 et Cat. p. I07; D. et B. p. 1228.

Vipera furcata, Daud. Rept. vi. p. 22.

Hab. S Imar, Paranas.

13. E. gracilis. (Gray.) N. v.

(M S. T.)

Calliophis gracilis, Gray, Ind. Zool. pl. f. 1-2; Schleg. Ess. ii. p. $45 \mathrm{I}$.

lilaps nigromaculatus, Cantor, Cat. pl. to f. 7.

Elaps gracilis, Gthr. Cat. B. M. 1868 , p. 230.

Hab. Mindoro, Baco. 


\section{Fam. HIDRÓFIDOS.}

1. Gén. Platurus, iatr.

I Pl Iaticaudatus, (Lin.) $\sigma \circ$ N. v

(M. S. 'T.)

Pl. fasciatus, D. et B. p. I 321 , Fischer, Secschtangen, p. 28.

Hydrus laticaurlatus, Schneid. Hist. Amph. p. 238 ; Cithr. Cat. $35^{6}$ (scutauns); Ptrs. Mon. Ber. Berlm. Akad. 1861 p. Ggr (fasciatus) 1872 p. 860 et 1877 p. ti; Brehin. V. An. 5 . p. 267 , f. 301 .

Coluber laticaudatus, Lin.

Hydrophis colubrinus, Schleg.

l.aticauda scutata, Laner.

Hab. Samar, Nipa-nipa, Luzón, Legaspi, Joló.

2. P1. var. colubrina, Schneid. N. v.

Hab. Samar, Joló.

\section{Gén. HYDROPHIS, IDACErs}

3 H. abreviatus, Fan. $\sigma \rho$ N. v.

(M. S T)

Jan. Elenco sist. d. Ofidi, Milano $186_{3}, \mathrm{p}$. Iog, et Icon. $d$. Oph!d. 40, I 870 1. 4 , f. 2.

Hab. Luzón, Manila.

4. H. brevis, Fan. I N. v.

Jan. l. cit. p. 109 et 4o, lam. 5, f. 2. Hab. Luzón, Manila.

5. H nigrocinctus, $D . B$.

I). et Bibr. Erp. gen. t. 7, I 854 p. I $35 \mathrm{r}$. Hab. Manila.

6 H fasciatus. Schneid $0 \times \mathrm{N}$ v. Calabicab.

(M. S. T.)

Hydrus fasciatus, Schneid. Hist. Amph. 1, г799 p. 240; Pts.

Mon. Ber. Berlin Akad. 1872, p. 849, lam. I f. I.

Hydrophis gracilis, Schleg.

$\mathrm{Hy}$. chloris, Fayr.

Hab. Luzón, B. de Manila.

7 H loreata, (Gray) o 0 N. v. Dalig-asan.

(M. S T)

Lapemis loreata, Gray, An. Mag. N. Hist. $18+3$, ii. p. 46; Gthr. R:p. B. India, 1864 , p. 386; Steind. Resie d. Nov. Rept. Wien. 1869 , p. 85 (annulata); J. G. Fischer, Jahrb, d. wiss. Aust. Hamburg. 2, p. 81, 1882. 
Hydrophis pelamidoides, a'ar. annulata, Fud.

lapemis hardwicki zar. Grav.

Hab. Luzon, Manila, Mindanao, Joló; Borneo, Cèlebes.

8. H. semperi, Girm \& $\mathrm{N}$ v.

(M. S. r)

Garman, Bull. Mus. Compt. Zool. 8, p. 85, 1885.

Hab. Luzon, Taal, Batanyas.

9. H. spiralis, (Shaw.) \& N. v.

(M. S. T.)

Hydrus spiralis, Shazo, Zool. 3, p. 564, lam. r25; Crthr. I. c. p. 366 lan. 25 f. D. Jan. Ícon. d. Ophid. $t_{1}, 1872$ 1. 2 f. 2 (nigromictus).

Hy:drophis melanurus, Wagl.

1I. spiralis, Oskar, Cat. Rep. Pkilipp. p. I9 (1886).

Hab. Luzon, Manila.

10. H. westermanni, fan. ơ $\mathrm{N}$ v.

(M. S. T)

Jan. Icon d. Ophid. 39 I872, 1. 5 f. I. Oskar. Cat. Rept. Philipp. 1886 , p. I 91 I.

Hab. Luzon, Bahía de Manila.

3. Gén. PElamis, Daud.

1 I. P. bicolor, (Schneid) o 0 N. v.

(M. S. T)

Hydrus bicolor, Schneid. Hist. Amph. p. 2t2; Gthr. Rept. B. India, 1864 , p. 382 ; Ptrs. Prens. Exped. O. Asien Zool. 1, 1876 p. 382 .

Hy. pelamis, Schleg.

H. variegata, Schleg.

P. bicolor, Dum et Bibr. ix. p. I 335; Brehm. V. An. 5, p. 268, f. 103 .

Pelamis ornata, Gray.

Hab. Luzon, B. de Manila; Borneo, Célebes.

\section{Fam. CROTẢILDOS.}

I Gẻn. TRIMERESURUS, Githr.

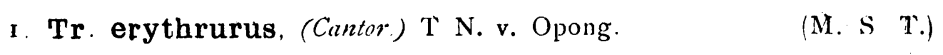

Trigonocephalus erythrurus, Cant. Proc. Zool. Soc. London, 1839 , p. $3 \mathrm{I}$.

'Tr. viridis, Schleg. Ptrs. Mon. Ber. Berlin Akad. 1861 , p. I69; Gthr. Rept. B. India 1864 , p. $386 ; J$. G. Fischer, Jahrb. $d$. wiss. Aust. Hamb. 2, 1885 p. 87.

Trimeresurus albolabris, Gray.

Tr. erythrurus; Oskar. Cat. Rept. Philipp. 1886 , p. I9.

Bothrops erythrurus, Brehm. V. An. 5, p. 315, f. ri6.

Hab. Luzon, Menila, Paracale, Mindanao; China, Java. 
2. T. flavomaculatus, (Gray')

Megzra flavomaculata, Gray, Zool. Aus. 1842 , p. 49.

Parias flavomaculata, Gray, Cat. Vip. Sn. Br. Aus. $18+9$, p. I I; Gthr. Proc, Zool, toc. London, 1879, p. 79.

'Tr. gramineus, F. Mïll. iii, Nachtr. Cat. Herp. Samml. Basel. Mus. 1883 , p. 19.

Megæra ornata et variegata, Gray.

Parias ornata et variegata, Gray.

Hab. Mindanao, Placer, Luzon, Albay.

3. T. hombroni, (Ginsh) N. v. Alimurain

$(\mathrm{M} \quad \mathrm{S} \quad \mathrm{i})$

'Iropidolømus hombroni, Guichenot, Voy Póle Sud et Oceania, Part. Zool 1848 , p. 23 l, 2 f. 2. D. ct B. Exp. gen. 7, 1854 p. I527; Ptrs, Mon. Ber. Berlin, Akad, 1867 , p. 29.

Hab. Mindanao, Zamboanga, Joló.

4 T. philippinensis, (Gray.) \& N. v.

(II. S. T)

Gray, Zool. Misc. 1842 , p. 48 et. Cat. Vip. Sn. B. Mus. p. Io. 'Tropidolœmus philippinensis, Ptrs. Mon. Ker. B. Akad 1861, p. 69 I.

Hab. Samar, Calbigan.

5 T. sp. $0^{7} \Omega \mathrm{N}$ v.

(M. S. T)

Hab. Luzon, Cavite, Alfonso.

6 Tr. schadembergi, Fisch.

J. G. Fischer, Jahrb. d. wiss. Aust. Hanb. 2. 1884 P. 'i et I 16 .

Hab. Mindanao,

7. Tr. wagleri, Sichles. OT, $\mathrm{N}$ v Dupong, Dipong.

(M. S. M.)

Trigonocephalus wagleri, Schleg. Ess. Phys. Serp. 2, p. 542, 1. 19 f. I6-I8.

'Trimesurus maculatus, Gray, Zool. Mis. p. $t^{8}$ et Viper. Sh. p. 8.

Tropidolœmus scheli, Bleeker.

Tropidolamus subannulatus et maculatus, Ptrs, Mon. Berlin Akad 1861 , p. 691; Gthr. Rept. B. India 1864, p. 388 et Proc. Zool. Soc. London 1879 , p. 78 ; F. Müll. III. Nachtr. Cat. Herp. Samml. Rasel. Mus. 1883. p. 19; J. F. Fischer, l. c. p. 8ı; var. Gray, Vip. Sn. p. 9; Motl. et Dilla'. N. H. Labuan, p. 44; var.

'Trigonocephalus sumatranus, Cant. Mal. Rept. p. $12 \mathrm{I}$.

'Trimeresurus formosus, Gray, sumatranus, Gray, Vip. Sn. p. 10. Hab. Luzon, Albay, Daraga, Mindanao, Butuan; Borneo, Java. 
S. Tr bypnale, D. it $B . \times \mathrm{v}$ Alimurain-alupon.

(M. S T.)

Trigonocephalus hypnale, Dum et Bibr. ix. p. I 498 ; Cith. Catr. B. $11.1858, \mathrm{p} .267$.

Hab. Luzon, Manila, Bulacan, Angat.

9. T. viridis, $D$ it $B .00 \mathrm{~N}$.

(M. S. T)

Bothrops viridis, Dum et Bibr. ix. P. I5I3.

'Trimeresurus viridis, Gthr. Cat. B. M. 1858, p. 266.

Hab. Luzon, Ilocos; China, Hong-kong.

\section{Clase $4 \cdot^{\mathrm{a}}$ : A NFIIBIOS (Batracios).}

\section{Orden I. $^{\circ}$ : ANUROS:}

\section{Subord. FANEROGLOSOS.}

Fam. RÁNIDOS.

1. Gén. OXYGLossus, Tschuni.

1. Ox. lævis, Gthr. $\sigma \bigcirc$ N. v. Palacá.

(M. S. T.)

Oxyglossus lævis, Gunth. Cat. p. 7 pl. i. f. A.

Phrynoglossus lævis, Peters. Mon. Berl. Ac. 1867 , p. 29.

Oxyglossus lævis, Stolicka, Proc. Ac. Soc. 1870, p. 273, et 1872 p. 1 I ; Boul. Cat. Batr. Sal. B. Mus. p. 6. 1882.

Microdiscopus sumatranus, Peters, Mon. Perl. Ac. 1877 , pp. 422,682 .

Hab. Luzón, Laguna de Bay, Bulacan, Sibul, Tayabas, Dolores, Samar; Sumatra.

2. O. 11ma, Gthr. ơ o N. v Palacá.

(M. S. T.)

Oxyglosssus lima, Gunth. Cat. p. 6.

O. lima, Tschudi, Batr. p. 85; Dum. \& Bibr. p. 334, pl. 86. f. 4, Gunth, Rept. Brit. Ind. p. 401; Stoliczka, As. Soc. 1070, p. 272, and 1872, p. Ior; Boul. Cat. Batr. Sal. B. Mus. 1882, p. 5

Hab. Luzón, Abra, Cagayan; Java.

$$
\text { 2. Gén RANA, Lin. }
$$

3. R. mindanensis, (Giv.) N. v.

Hylarana mindanensis, Girard. V. S. Expl. Exp. Herp. p. 42; Boul. Cat. B. Sal. of Br. Mus. p. 6. i 882. Hab. Mindanao, Caldera. 
4 R. corrugata, Ptrs. $\varnothing^{\circ} \odot$ N. v.

(.I. S. T.)

Rana kuhlii, var. B. Gïnth. Cat. p. 8.

R.' corrugata, Peters. Mon. Berl. Ac. $1853, \mathrm{p} .+12 ;$ Boul. Cat. B. Sal. Mus. p. ig (1882).

R. kuhlii, Günth. Rept. Brit. Ind. p. 4o 4 pl. 26. f. B. Hab. Mindoro, Naujan, Ilocos; Ceilan, China.

5. R. kuhlii, Günth. $\rho^{\circ} \mathrm{N}$. v.

(MI S. T )

Rana kuhlii, var. A. et C. Gïnth. Cat. p. 8.

R. kuhlii, (Schleg). Dum. \& Bibr. p. $384 ;$ Anders. Anat. Zool. Res. Yunnan, p. 838; Boul. Cat. B. of B. Mus. p. $20,1882$. R. kuhlii, part., Günth. Rept. Brit. Ind. p. 404 pl. 26. f. A. R. conspicillata, Günth. Proc. Zool. Soc. 1872 , p. 595 pl. 40. f. A; id. Zool. Record, ix. p. 97.

Hab. Paragua, Samar, Buronsan; Borneo, Célebes.

6. R. macrodon, Gihr. O* : N v. Palacá.

Rana macrodon, Günth. Cat. p. 8; Boul. Cat. B. Mus. p. 24. lam. I. f. 4 .

R. macrodon, (Kuhl.) Tschudi Batr. p. 80; Dum. \& Bibr. p. 382 ; Pet.rs, Mon. Berl. Ac. 1871 , p. 652; Blanf. Proc. Zool. Soc. 1881 , p. 225 pl. xxi. f. 4 .

R. fusca, Blyth, Journ. As. Soc. xxxiv. p. 719 ; Anders, Proc. Pool. Soc. 1871, p 197; Stolicaka Journ. As. Soc. 1873. p. I $15 ;$ Anders. Anat. Zool. Res. Yunnan, p. 837.

Ixalus aurifasciatus, Peters, Mon. Berl. Ac. 1863, p. 455.

Hab. Luzón, Manila, Laguna de Bay, Dinagat, Negros, Mindanao, Zamboanga; Java, Singapore.

7. R. tigrina, Gthr. $\sigma \circ$ N. v.

(M $\quad \mathrm{S} \quad \mathrm{T}$.)

Rana tigrina, Giöth. Cat. p. 9.

R. tigrina, Daud. Rani. p. 64 pl. 20, et Rept. vii. p. 125, Merr. Tent. p. r74; Cuv. R. A; Dum. \& Bıbr. p. 375; Kelaart, Prodr. Faun. Zeyl. p. 192; Peters. Mon. Berl. Ac. 1863 , p. 77; Gunth, Rept. Brit. Ind. p. 407; Steindachn. Novara, Anat. Zool. Res. Yunnan, p. 837; Bou!. Cat. Batr. Scl. of B. Mus. p. 26 (1882).

R. cancrivora, (Boie) Gravenh. Delic. p. 4I; Tschudi, Batr. p. 79.

R. brama, Lesson in Belang. Voy. Ind. Or., Rept. p. 329 pl. 6.

R. vittigera, Wiegm. Nova, Acta Ac. Leop. 1835 , p. 329. t. 21 . f. I.

R. regulosa, Wiegm. l. c. p. 258, pl. 21 . f. 2.

R. crassa, Jerdon. Journ. As. Soc. 1853 , p. $58 \mathrm{I}$; Theobald. Cat. Rept. As. Soc. Mus p. 79; Anders. Proc. Zool. Soc. 1871 , p. 199.

Hydrostentor pantherinus, Fitzing. Sitz. Ac. Wien, xlii. p. 414.

Pyxicephalus fodiens, Peters. Mon. Berl. Ae. 1860 , pl. 186.

Hoplobatrachus ceylanicus, Peters, Mon. Berl. Ac. 186.3, p. 449; Gïnth. Rept. Brit. Ind. p. 410. 
Rana latrans; David, Noui. Arch. Mus. vii. i872, Bull. p. 76. Hab. Luzón, Manila, Laguna, Los Baños, Tayabas, Dolores, Samar, Basilan, Mindanao, Surigao; China, Borneo.

8 R. gracilis, Wiesm \& N v.

(M. S.'T.)

Rana vittigera, Gïnth. Cat. p. 9.

R. gracilis, Wiegm. Nova. Acta Ac. Lrop. 1835, p. 257; Peters, Mon. Berl. Ac. 1853 , p. 78 ; Gïnth. Rept. Brit. Ind. p. to9; Steindachn. Novara, Amph. p. i 8; Stoliczkr, Journ. As. Soc. 1870 , p. I 2 ; Anders. Proc. Zool. Soc. 1871, p. 200, Günth. Proc. Zool. Soc. 1875, p. 567; Anders. Anat. Zool. Res. Yunnan. p. 84o; Boul. Cat. Batr. Scl. of B. Mus. p. 28 (I 882 ).

R. nilagirica, agricola, Jerdon. Journ. As. Soc. 1854, p. 531.

R. brevipalmata, Peters. Mon. Berl. Ac. 1871 , p. $6+6$.

R. lymnocharis, (Boie) Stoliezka, Proc. As. Soc. 1872 , p. I(1), et Journ. As. Soc. 1873 p. I 6 .

Hab. Samar, Villareal; China, Formosa.

9. R. guentheri, Boul. $\mathrm{N}$ v.

(M $\quad \mathrm{S} \quad \mathrm{T}$ )

Boul. Cat. Batr. Salt. of B. Mus. 1882 p. 48 . pl. ix. f. 2. Hab. Paragua, P. Princesa, Mindoro, Naujan; China.

ı. R. macrodactyla, Gthr. \& N. v.

(M. S. T)

Hylarana macrodactyla, Günth. Rept. Brit. Ind. p. 424 ; Stoliezka Proc. As. Soc. 1872 . p. 104; Boul. Cat. B. Mus. 1882 p. $5 t$. Hab. Luzón, Laguna, Cagayan, Dinagat, Negros, Zamboanga, Mindanao.

II. $\mathbf{R}$ erythræa, Gthr. \& N. v.

(M. $\mathrm{S}$ T)

Hylarana erythræa, Günth. Cat. p. 73.

Hyla erythræa, Schleg. Abbild. pl. 9 f. 3.

Hylarana erythræa, Tschudi, Batr. p. 78 ; Gïnth. Rept. Brit. Ind. p. 425; Stoliezka. Proc. As. Soc. 1872 , p. 104; Anders. Anat. Zool. Res. Yunnan. p. 846; Boul. Cat. B. Mus. 1882 p. 65 .

Limnoclytes erytræus, Dum. \&. Bıbr. p. 5Ir, pl. 88. f. I; Cantor, Mal. Rept. p. I4I.

Hylorana subcærulea, Cope, Proc. Ac. Philad. 1868 , p. I 39. Hab. Mindanao, Samar; Borneo, Sumatra.

I2. R. chalconata, Gthr. $\sigma, 0$ N. v.

(M $\quad$ S. T.)

Hylarana chalconata, Günth. Cat. p. 73.

Hyla chalconata, Schleg. Abbild. pl. 9. f. г.; Boul. Cat. B. M. p. 66. (1 882).

Polypedales chalconotus, Tschudi. Batr. p. 76 .

Limnodytes chalconotus, Dum. \& Bibr. p. 5r3.

Hab. Paragua, Inagauan, Bulacán; Borneo, Java, 
I3 R. jerboa, Gthr. \& N. v.

Hylorana jerboa, Gthr. Proc. Zool. Soc. 1872, P. 599 pl. qu; Boul. Cat. B. Ann. I 882 p. 67.

Hab. Samar, Loquilocum; Borneo.

14. R. luctuosa, (Ptrs) N. v.

Limnoclytes luctuosus, Peters. Mon. Berl. Ac. 1871, p. 579, et Ann. Mus. Civ. Genov. 1872, p. +3 pl. 9. f. I.

Hab. Mindanao, Butuan.

15. R. natatrix, Gthr. $\sigma \circ \mathrm{N}$ v.

(M. S. T.)

Ixalus natator, Gïnth. Cat. p. 75 pl. iv. f. C.

I. guttatus, Gïnth. l. c. p. 76. pl. iv. f. D.

Staurois natator, Cope. Nai. Hist. Rev. 1865 p. 117.

St. guttatus, Cope. l. c.

Rana natatrix, Boul. Cat. B. M. 1882 p. $7 \mathrm{I}$.

Polypedates, guttatus, Gïnth. Proc. Zool. Soc. 1873 , p. Gool. Hab. Dinagat; Borneo.

I6 R. similis, Gthr. o 0 N. v

(M. S. T.)

Polypedates similis, Gthr. Proc. Zool. Soc. 1873 p. I7 I.

Rana similis, Boul. Cat. B. M. 1882 p. 72.

Hab. Luzón, Manila, Laguna de Bay, Los Baños; Borneo.

I7. R. sp N. v.

Hab. Luzón, Bulacán, S. Miguel de Mayumo.

18. R everetti, Boul. o 0 N v. Palacang-saguing. (M S. T.)

Rana everetti, Boul. Cat. B. M. 1882 p. 72 . lam. 6.

Hab. Mindanao, Zamboanga, Cagayan de Misamis.

19. R. glandulosa, Boul. $\$$ N, v.

$\left(\begin{array}{lll}M & \mathrm{~S} & \mathrm{~T}\end{array}\right)$

Rana glandulosa, Boul. Cat. B. Mus. I 882 p. 73 lam. 7 .

Hab. Paragua, P. Princesa; Borneo.

3. Gén RHACOPhORUS, KuhL.

20. R. hecticus plrs. $\sigma \circ$ N. v.

II. S T)

Polypedates hecticus, Peters. Mon. Berl. Ac. 1863 , p. t57; Boul. Cat. B. M. 1882 p. 78.

Hab. Samar, Loquilocum. 
21. R. surdus, Plrs, $\sigma \rho \mathrm{N}$. v.

(M. S. '1.)

Polypedates surdus, Peters. Mon. Berl. Ac. 1863. p. 459 ; Borl. Cat. B. Mus. 1883. p. 79.

Hab. Luzún, Manila, Mindanao.

2: R. maculatus Gthr. $\sigma \circ \mathrm{N}, \mathrm{v}$.

(M. S. 'I.)

Polypedates maculatus, Gïnth. Cat. p. 78 .

Hyla maculata, Gray. Ind. Zool.

H. leucomystax, Gravenh. Delic. p. 26.

Polypedates leucomystax, Tschudi, Batr. p. 75; 1)um. \& Bibr.

p. 5I9; Kelaart, Prodr. Faun. Zeyl. p. 193.

Bürgerid maculata, Techandi, $l$. $c$.

Polypedates rugosus, Dum. \& Bibr. p. 520 .

P. cruciger, Blyth, in Kelaart, Prodr. App. p. 48.

5'. megacephalus, Hallow. Proc. Ac. Philad. 1860, p. 48.

1'. maculatus, Günth. Rept. Brit. Ind. p. 428 ; Blanford, Journ. As. Soc. 1870 p. 376; Anders. Proc. Zool. Soc. $18^{\prime} 71$, p. 307 ; Stoliezka, Proc. As. Soc. 1872 , p. I06; Boul. Cat. B. M. 1882, p. 83.

P. biscutiger, Peters. Mon. Berl. Ac. 1871, p. 649.

Hab. Luzón, Manila, Batangas, Ticao; Borneo, China.

23. R. var. quadrilineata, Wiegm N. v.

Polypedates quadrilineatus, Günth. Cat. p. 79.

Hyla quadrilineata, Wiegm. Acta. Ac. Caes. Leop-Carol. 1835 , p. 260 , t. 22 f. 1 ; Boul. Cat. B. M. 1882 , p. 84 .

Limnodytes celebensis, Fitzing. Sitzb. Ac. Wien. xlii. p. +13. Polypedates quadriineatus, Günth. Rept. Brit. Ind. p. 429; Steindachn. Verh. zool-Cot. Ges. Wien. 1864 , p. 253 pl. Io t. 2; Anders, Proc. Zool Soc. 1871, p. 207.

Hab. Luzon, Manila; Formosa, Java.

24. R. appendiculatus, Gthr \& $\mathrm{N}$ v.

(M S. T.)

Polypedates appendiculatus, Gthr. Cat. p. 79; Boul. Cat. B. M. 1882 , p. 86, lam. 8 fig. 4.

Hab. Dinagat, Mindanao, Samar, Villareal.

25. $\mathbf{R}$ pardalis, Gthr む $\mathrm{N} \mathbf{v}$

(M. S. T.)

Rhacophorus pardalis, Gïnth. Cat. p. 83.

R. reinwarltii, Eyd. \& Soul. Voy. Bonite, pl. ı, f. 1 ; Boul. Cat. B. M. 1882 , p. 91.

Hab. Dinagat, Mindanao; Borneo.

$$
\text { 4. Gén IXALUS, D. ET B. }
$$

26 I. acutirostris, Peters. $\sigma \circ$ N. v. Palaca.

(M, S. T.)

Ixalus acutirostris, Peters, Mon. Berl. Ac. 1867, p. 32; Boul. Cat. B. M. 1882 , p. 99.

Hab. Paragua, P. Princssa, Mindanao. 
27. I pictus, Pitirs T $\mathbf{N}$ v.

Ixalus pictus, Peters. Mon. Berl. Ac. 1871 , p. 5 \%o; et Ann. Mus. Civ. Gent. iii. 1872 , p. 44, pl. 6 f. 3; Boul. Cat. B. M. 1882 , p. 99.

Hab. Paragua; Borneo.

28 I ? bimaculatus, Ptrs. $\sigma \circ \mathrm{N}$. v

(M. S T)

Leptomantis bimaculatus, Peters. Mon. Berl. Ac. 1867, p. 32. I.? bimaculatus, Boul. Cat. B. M. 1882 , p. 116.

Hab. Paragua, P. Princesa, Calamianes, Cuyo, Mindanao.

\section{Gén CORnufer, Tschudt.}

29. C. guentheri, (Buul.) N $\mathbf{v}$ Palaca.

Boul. Cat. B. M. $188_{2}$, p. 108 lam. I f. 3 .

Hab. Dinagat, Mindanao.

3o. C. meyeri, Gth, $\sigma \rho \mathrm{N} . \mathbf{v}$

(M. S T.)

Platymantis meyeri, Günth. Proc. Zool. Soc. 1873, p. 171; Boul. Cat. B. M. 1882, p. 108 lam. I1 fig. 4 .

Hab. Luzon, Manila, Laguna de Bay.

$3 \mathbf{r}$ C. jagorii. Pirs o o N. v.

Halophila jagorii, Peters, Mon. Berl. Ac. 1863. p. +56 ; Boul. Cat. B. M. 1882 , p. 109.

Hab. Luzon, Manila, Samar, Paranas, Mindanao.

32. C. corrugatus, Du\%; Co N. v

Platymantis plicifera, Günth. Cat. p. 95 pl. viii. f. 13.

Hylides corrugatus, A. Dum. Ann. Sc. Nat. (3) xix. p. i76.

Platymantis corrugata, Peters, Mon. Berl. Ac 1873 , p. 6ir; Petcrs. \& Doria, Ann. Mus. Civ. Gen. xiii. p. +2o; Boul. Cat. B. M. 1882 , p. 110.

Pi, plac.fera, Gïnth. Proc. Zool. Soc. 1917 , p. 132.

Hab. Luzon, Layuna, Negros; N.a Guinea.

\section{. Fam. ENGISTO}

I Gén. My'crohylat, Tschud.

1. M. achatina, Gthr.

Micrhyla achatina, Günt. Cat. p. I 21;Boul. Cat. B. M. 1882, p. 166.

Hyplesia achatina, Boie, Isis, 18.27, p. 294. 
Microhyla achatina, Tsckudi, Batr. p. ir; Dum. of Bibr. p. 6r4; Peters. \& Doria Ann. Mus. Genov. xiii. p. +28.

Diplopelma disciferum, Peiers. Mon. Berl. Ac. 1867, p. 36. Hab. Mindanao; Java, Molucas.

2. Gén CailuUi,a, Gr.

2 C picta, Gth, o๐ N. v.

(M. S. T)

Kaloula picta, part., Giunth. Cat. p. 12.3.

Plectropus pictus, (Bibr.) Iiyd \& Soul. Voy. Bonte Rept. pl. 9

f. 2; Dum. \& Bibr. p. 737; Peters, Mon. Berl. Ac. 18263 , p. 455 .

Callula picta, Günth. Repi. Brit. Ind. p. +36 ; Boul. Cat. 1882 , p. 168 .

Hab. Luzon, Manila, Laguna de Bay, Negros, Cebú.

3. C. conjuncta. Pirs. $\sigma \circ \mathrm{N}$. v. IM S T.।

Hylædactylus (Holonectes) conjunctus, Peters, Mon. Berl. Ac. 1863 ,

p. 455; Steindachn. Verh. zool-bot. Ges. Wien, 1864, p. 256, pl. I I f. 5 .

Callula conjuncta, Cope, Journ. Ac. Philad. (2) vi. 1867, p. 192;

Boul. Cat. B. M. 1882 , p. 168.

Hab. Lużon, Manila, Mindanao.

4. C. baleata, Miall ơ $\mathrm{N}$ v.

(M. S T)

Kaloula baleata, Günth. Cat. p. 122; Boul. Cat. 1882, p. 169. Bombinator baleatus, Müll. Verhandel. Batav. Genvotsch. 18,36, p. 96.

Hylædactylus baleatus, Tschudi. Batr. p. 85; Dum. \& Bibr. p. 734 .

Kaloula baleata, Bteindachn. Novara. Amph. p. 68

Hab. Luzon, Laguna de Bay; Célebes, Java.

\section{Fam. BUFÓNIDOS.}

I. Gén NECTOPHRYNE, Buch.

I. N. guentheri (Boul.)

Bufo leptopus, Günth. Proc. Zool. Soc. 1872, p. 598; Boul. Cat. B. M. 1982, p. 280 . Hab. Mindanao; Borneo.

2. N. P sundana, Pirs N. v.

Calohyla sundana, Peters. Mon. Berl. Ac. 1867, p. $35 ;$ Boul. Cat. B. M. 1882 , p. $28 \mathrm{I}$.

Hab. Mindanao; Borneo. 
2. Gén. BUfo, Laur.

\section{B brevipes Ptrs. ơ N. v. Cacapsuy.}

Hylaplesia brevipes, Peters. Mon. Berl. Ac. 1867 , p. 34 Boul. Cat. B. M. 1882, p. 287.

Hab. Mindanao, Zamboanga.

4. B. melanostictus, Gthr. $\sigma \Omega$ N. v. Palacang-bato

(M. S T.)

Bufo melanostictus, Gïnth. Cat.p. 61; Boul. Cat. B. M. 1882 , p. 306.

B. isos, Günth. l. c. p. 62.

B. melanostictus, Schneid. Hist. Amph. p. 216; Gravenh, Delic. p. 57; Cantor, Cat. Mal. Rept. p. I42; Girard. V. S. Expl. Exped:, Herp. p. 92, pl. 5 f. I0-I4; Gïnth. Rept. Brit. Ind. p. 422; Steindachn. Novara, Amph. p. 42; Stoliczka, Proc. As. Soc 1870 , p. I 55.

B. scaber, Dand, Rain. p. 94 pl. 34. f. I et Fept. viii. \%. 194; Tschndi, Batr. p. 98; Dum. \& Birbr. p. 699; Schleg. Abbild, p. 64, pl. 20. f. 2.

B. bengalensis, Daud. Rain. p. 96. pl. 35 f. 1. et Rept. viii. p. 197; Lesson, in Bélang. Voy. Ind. Or. Rept. p. 334.

B. isos, Lesson, l. c. p. 333, pl. 7; Dum. \& Bibr. p. 702.

B. dubia, (Shaze.) Gray, Ind. Zool.

B. carinatus, Gray, l. c.

B. gymnauchen, Bleeker, Nat. Tijdschr. Nederl. Ind. 1858, xvi, p. 46.

Phrynoidis melanostictus, Cope, Proc. Ac. Philad. 1863, p. 357. Bufo spinipes, (Fitzing), Steindachn. l. c. p. 43, pl. 5. f. 6. Hab. Luzón, Bulacan, Lolomboy; Borneo, Java, China.

5. B. biporcatus, ơ Gthr. Palacang bató.

Bufo biporcatus, Günth. Cat. p. 62 .

B. biporcàtus, (Schleh.) Tschudi, Batr. p. 88; Gravenh. Delic. p. 53; Dum. \& Bibr. p. 7 I4; Boul. Cat. B. M. 1889. p. 31 1. Chilophryne biporcata, Cope, Proc. Ac. Philad. 1862 , p. 357. Hab. Luzón, Manila, Batangas, S. Pablo.

6. B. panayensis. Seocan.

Hab, Luzón, Manila, Batangas, S. Pablo.

\section{Fam. HílidoS.}

1. Gén HYLA, LAUK.

1. H. chinensis, Gthr. o o Palacang-saguing.

(M. S. T)

Hyla arborea, var. chinensis, Günth. Cat. p. Io8. pl. ix. f. C. 
H. chinensis, Gïnth. Rept. Brit. Ind. p. 436 ; Cameramo, Att. Acc. Torin. xiv. 1879 p. 894; Boul. Cat. B. M. 1882, p. $3^{81}$.

H. arborea, var. chinensis, Steindachn. Novara. Amph. p. 59.

Hab. Luzón, Basilan; China, Formosa.

\section{Fam. HILEDACTÍLIDOS.}

I. Gén. HYLEDACT YIUS, Tschun.

I. H. pictus, Bibr. \& N. v. Palacá.

(M S. 'T.)

Kalooula, Gray.

H. pictus, Bibr.; Voy. Bonite, Rept. lam. 9.

Hab. Luzón, Bulacan.

\section{Fan. PELOBÁTIDOS.}

I. Gén MEGALOPHRYS, KUHr.

I. M. montana, Gthr. $\sigma \circ$ N. v.

(M. S. T.)

Megalophrys montana, Günth. Cat. p. 36.

M. montana, (Kuhl). Wagl. Syst. Amph. p. 204; Tschudi, Batr. p. 82; Dum. \& Bibr. p. 458; Cantor, Cat. Mal. Rept. p. 140; Günth. Repi. Brit. Ind. p. 4r3, et Ann. \& Mig. N. H. (4) xi. p. 4r9; Boul. Cat. $B$ M. 1882 . p. 442.

Ceratophrys montana, Schleg. Abbild. t. x. f. 3 . Hab. Dinagat, Negros, Mindanao.

\section{Ord. URODELOS.}

\section{Fam. SALAMÁNDRIDOS.}

I Gén. MOLGE, Merv.

1. M sinensis, Boul $\delta$ N. v.

Cinops chinensis, Gray. Proc. Zool. Sor. 1859 , p. 229 pl. 19. f I; Gthr. Rept. Brit. Ind. p. 439.

Triton chinensis, Strauch. Salam. p. 5I; Boul. Cat. B. M. 1882, p. 20.

Hab. Luzón, Cagayan, Pampluna; China. 


\section{Orden ÁPODOS.}

Fam. CECÍLIDOS.

I. Gén. ICHThyop'His, Fitz.

I Ich monochrous. Pirs. I $\mathrm{N}$ v

I. glutinosus, var. Cant. Cal. Mal. Rept. p. I37.

Epicrium monochroum, Blkr. Nixt. Tydschr. Ned. Ind. xvi. I85", p. I88; Gthr. Rept. Brit. Ind. p. 443 .

I. monochrous, Peters, Mon. Berl. Ac. 1879 , p. 932; Boul. Cat. 13. M. 1882 . p. 91 .

Hab. Paragua, P. Princesa, Samar, Calbayoc; Borneo, Java.

\section{Clase 5.a PECWS. \\ Subclase $I^{\text {a }}$ TELEOSTEOS. \\ Orden: ACANTOPTERIGIOS.}

\section{Fam. BERÍCIDOS.}

I. Gén. MONOCENTRIS, Schneid.

I. M. japonicus, Houtt. N. v.

(M S. T.)

Gasterosteus japonicus, Houttuyn, Act. Soc. Harl. xx. pt. 2 p. 329.

Sciæna cataphracta, Thunberg, Nov. Act Scient. Suec. xi. p. I02 lam. 3 .

Monocentris carinata, Bl. Schn. p. Ioo, lam. 24.

Lepisacanthus japonicus, Lacép iii. p. 321 .

Monocentris japonicus, Cuv. \& Val. id. p. 46 I lam. 97; Faun. Japon, p. 50 lam. 22 f. 1; Gthr. Cat. B. M. 1.1859, p. 9.

Hab. Luzón, Manila, Bataan; China, Japon.

2. Gén, MYRI'RISTRIS, Cuv.

2. M. pralinius, Cuv. $\mathrm{N}$ v

(M. S. T )

Myripristris pralinius, Cuv. \& Val. iii. p. 170 et vii. p. 486 ; Gthr. Cat. B. M. 1. 1859 , p. 20.

M. seychellensis, Cuv. \& Val. iii. p. 172.

Hab. Luzón, Samar, Paragua; Célebes, Oc. Índico y Pacífico. 
3. M. murdjan, Forsk. $\mathrm{N}$ v.

(M S. T)

Sciana murdjan, Forskal, p. to.

Pered murdjan, Schneider, p. 86; Lacép iv. pp. 396, +18 Russell, t. I05.

Myripristis murdjan, Cuz. \& Val. iii. p. 177, vii. p. 495; Rüppell, Atl. p. 36, t. 23 f. 2, e! Fische des Rothen Meers, $\mu$. I5; Bleeker Amboin: , iii. p. 19; Gthr. Cat. B. M. 1. $185 \%$, 1. 21 .

Hab. Luzón, Cavite, Santa Cruz, Batangas, Nasugbú; Amboina.

4 M japonicus, Cuv. N. v.

(M. S. T.)

Myripristis japonicus, Cuv. \& Val. iii. p. 173 l:tn. $5^{8 ;}$ F.xun. Jatpon, p. 23 lam. 9; Richardson Schth. China, p. 222; G!hr. C.zt. B. M. 1. 1859, p. 25.

? M. pralinius, Richardson, l c. p. 223.

Hab. Luzón, Currimao, Ilocos; China, Japon.

\section{Gén. holocentruil, akteid.}

5. H. rubrum, (Fiorsk.) $\mathrm{N}$.

(M. S. T.)

Seba, iii. 27. I

Sciana rubra, Forsh. p. 48 (no. Bl. Sch 1.$)$

Kenard, Poiss. t. 29. f. I59; Valentyn, Ind. iii. p. 390. f. 137.

Perca rubra, Bl. Schn. p. 90.

P. praslin, Lacép. iv. p. 418.

Holocentrum alborubrum, Lacép. iv. p. 372; Richardson, Ichth. China, p. 223.

H. orientale, Cuv. \& Val. iii p. 497; Bleeker, Perc. p. 53.

Holocentrus ruber, Rüpp. Atl. p. 83. t. $22 \mathrm{f}$, et $N$. W. Fische, p. 96.

H. rubrum, Gthr. Cat. B. Mus. 1. 1859 , p. 35.

Holocentrum marginatum, Cuv. \& Val. iii. p. 216.

H. spinosissimum, Richardson, l. c. p. 223 (no Fauna Japon.) Hab. Luzón, Manila, Pangasinan, Samar, Paragua, Cebú; Amboina, China.

6. H. punctatissimum, Cuv. N. v.

(M. S. T.)

Holocentrum punctatissimum, Cuv. \& Val. iii. p. 2r 3 ; Less. \& Garn. Voy. Cog. Zool. ii. p. 2 г9; Bleeker, Sumatra, ii. p. 6; Gthr. Cat. B. M. 1. 1859 , p. 38.

? H. lacteoguttatum, Cuv. \& Val. iii. p. 2 I4. Hab. Cebú, Carolinas, Marianas; Sumatra.

7. H. stercus-muscarum, C. V. N. v.

Cuv. \& Val. vii. p. 503; Gthr. Cat. B. MT. 1. 1859, p. 39. $H_{a} b$. Carolinas, Guam, Marianas. 
8. H. spiniferum, $C . V$. N. v.

(M. S. T.)

Sciana spinifera, Forsk. p. 49.

Perca spinifera, Bl. Schn. p. 86; Lacép. iv. p. 4 I8.

Holocentrum leo, Cuv. \& Val. iii. p. 204; Less. Voy. Coq. ii. p. 222; Cuv. Regne Anim. Ill. lam. 14. f. I; Bleeker, Kokos Islands, p. 355; Voy. Astrol. Poiss p. 678. lam 14. f. 3; Gthr. Cat. B. M. 1. 1859 , p. 39.

Holocentrus spinifer, Rïpp $N$. W. Fische, pp. 96, 97. t. 25. f. I. (no Atlas); Vilan. Creac. 5. p. 220.

Hab. Luzón, Cavite, Naic, Batangas, Nasugbú, Mindoro.

9. H. binotatum, $Q$ et $G . \mathrm{N}$ v.

Quoy \& Gaim, Voy. Astrol. Poiss p. 679. lam. 14. f. + (no Bleek); Gthr, Cat. B. M. 1. 1859, p. 4().

Hab. Guam, Marianıs; N. a Guinea.

10. H. spinosissimuim, Tem. et Sih. N. v.

(M. S. T)

Temm. \& Schleg. Faun.' Japon p. 22 lam. viii. et Richardson Ichthyol. China, p. 223; Gthr. Cat. B. M. 1. $1859, \mathrm{p} .+1$. Hab. Luzón, Currimao, Ilocos; China, Japon.

II. H. diadema, Lacep. N. v

(M. S. T.)

Holocentrus diadema, Lacép iv. pp. 372, 374 iii. lam. 32. f. 3; Rüpp. Atl. Fische, p. 84 t. 22 f. 2.; Vil. Creac.; 5. p. $23^{\circ}$.

Perca pulchella, Benn. Zool. Journ. iii. p. 377. lam. f. 3.

1 Lolocentrum diadema, Cuv. \& Val. iii. p. 2 1 3; Less. Voy. Duperr. Zool. iii. p. 220 p. 25. f. 2; Bleeker, Amboina \& Ceram, p. 259; Gthr. Cat. B. M. 1. 1859 , p. 42.

Hab. Mindoro, Cebú, Samar, Paragua; Borneo, China.

12. H. melanotopterum, Blkr. N. v.

(M. S. T.)

Bleeker, Cèlebes viii. p. 302; Gthr. Cat. B. M. 1. 1859, p. 43. Hab. Mindoro, Culion, Calamianes, Paragua, P. Princesa; Célebes.

13 H. cornutum, Blkr. N. v.

(M. S. T.)

Blceker, Ceram 3 p. 24\%; Gthr. Cat. B. M. 1.1859 p. 45. Hab. C. de Samar, Borongan; Amboina.

14. H. violaccum, Blkr. N. v.

(M. S. T.)

Bleeker, Amboina id. p. 335; Gthr. Cat. B. M. 1. 1859, p. 4t. Hab. Iloilo, Panay, Cebú; Amboina.

15. H. sammara, (Forsk.) N. v.

(M. S. T.)

Sciæna sammara, Forskal., p. 48; Lacep iv. p. 3 I 4 . 
Perca sammara, Bl. Schn. p. 89.

labrus angulosus, Lacép iii. p. 43 p lam. f. I .

Holocentrum sammara, Rïpp. Atl. Fische, t. 22. f. 3; Ciuv. \& Val. iii. p. 216; Bleeker, Perc. p. 54. et Amboina ii. p. 555; Githr. Cat B. M. 1. 1859, p 46.

H. christianum, (Ehrenberg); Ciıv. \& Val. iii. p. 219; cfr. Küpp. Atl. p. 85.

Hab. Samar, Cebú; Amboina, Mar Rojo.

4. Gén. RHYNCHICHTHYS, C. V.

16. Rh. pelamides? C. V. N. v.

(M. S. T.)

(uv. et Val. vii. p. 504, lam. 208; Gthr. Cat. B. M. 1. 1859, p. 50 .

Hab. Luzón, Cavite, Sta. Cruz; Amboina.

Fam. PÉRCIDOS.

I. Gén. PERCichithys, Girard.

I. P. ciliata, Gthr. N. v.

(M. S. 'T.)

'Perca ciliata, (范hl \& v. Hasset), Cuv. \& Val. ii, p. 52.

Percichtys ciliata, Gthr. Cat. B. M. 1859 , p. 62.

Hab. Panay, Iloilo, Paragua, Culion; Java.

2. Gén LATES, Cuv.

2 L. calcarifer, (Floek.) N. v.

(M. S. T.)

Holocentrus calcarifer, Block, t. 344.

Russell, ii. t. I 3 I.

Holocentrus heptadactylus, Lacep iv. pp. 344, 39 r.

Coius vacti, Ham. Buch. Ganges, Fishes, t. I6, f. 28.

Lates nobilis, Cuv. \& Val. ii. p. 96, t. I 3; Richardson, Ichth. China, p. 222; Bleeker, Perc. p. 27; Cantor, Catal. p. I; Hageman in Natuurk. Tydschr. Nederl. Ind. 1851, p. 348.

1. calcarifer, Gthr. Cat. B. M. 1. i 859, p. 68.

Hab. Mindoro, Calapan; China, India.

\section{Gén CNIDON, M et Trosch.}

3. C. chinensis. Müll. N. v.

(M. S. T.)

Müll. \& Trosch Hov. Ichth p. 21; Gthr. Cat.. B. M. 1. I859, p. 68 .

Hab. Luzón, Manila, Lingayen, Pangasinan, Cebú; China.

4. Gén. PミAMMOPERCA, Rich.

4. P. Waigiensis, $C$ et $V$ N. v.

(M. S. T )

Labrax waigiennsis, Cuv. \& Val. ii. p. 83; Bleeker, Natuurk. Tydschr, Nederl. Lied. ii. p. 479; Sesson, Voy. Co7. Zool. 237. 
Psammoperca datnioides, Richardson, Voy. Erebus \& Terror Fisches, p. I 6 . lam. 57 f. 1, 2.

Ps. waigiensis, Bleeker, l. c. v. p. 177;Gthr. l. c. 1. p. 69. $H a b$. Luzón, Cagayan, Marinduque, Samar; China, Austratia.

5. Gén. PERCAIABRAX. T'Ku.

5. P. japonicus, $C$ et $V$.

Labrax japonicus, Caco. \& Vat. ii. p. 85.

Percalabrax japonicus, Faun. Japon, p. 2 pl. 2. f. 1; Gthr. l. cit. 1. p. $7 \mathrm{I}$.

Lateolabrax japonicus, Bleek. l. c. p. 177.

Hab Luzón, Bataan, Orion; China, India.

6. Gén. ETELIS, C. V.

6. E. carbunculus, $C$. $V$.

Cuv. et Val. ii. p. 127; Gthr. Cat. B. M. 1. p. $79.185^{8}$. Hab. Carolinas; Seychelles.

\section{Fam. SERRÁNIDOS.}

I. Gén APRION, C. V.

I A virescens, $C . V . \mathbf{N} . \mathbf{v}$.

(M. S. T )

Cuv. et Valenc, 6. p. 544, lam. 168, Gthr. Cat. Brit. Mus. p. 8 .. I. 1859 .

Hab. Paragua, P. Princesa, Samar, Paric; Seycheles.

2. Gên AN'THIAS, BlK.

2. A. cichlops, Blk. N. v.

Serranus cichlops, Bleeker, Sumatra; i. p. 3, Gthr. l. cit. I. p. 95 .

Hab. Samar, Borongan.

3. A. rasor, (Richard). N. v.

Serranus rasor, Richardson, P. Z. S. 1839 p. 95, y Trans, Z. Soc. 1849 , p. 73, lam. 4. f. I. Gthr. l. c. 1. p. 93.

Hab. Samar, Viilareal; Australia.

4. A. schelegelìi, Gthr. N. v

Caprodon, Tem. et Schleg. Faun. Jap. Pois. p. 64, lam. 3o; Richards. Ichth. China, p. 235; Gthr. l. cit. 1. p. 93.

Hab. Mindoro, llocos, Vigan; China, Japon. 


\section{Cén. anyperodon, Gthr.}

5. A. leucogrammicus Gthr.

Renard, Poiss. A. s. f. 6. Valent iii. p. 476 f. 409.

Serranus leucogrammicus, (Reinu'). Cuv. \& V'al. ii. p. 347; Bleeker, Perc. p. 33 .

Anyperodon leucogrammicus, Gthr. l. cht. 1. p. 96. Hab. Marianas; Molucas.

\section{Gén SERRANUS Cóv}

(. S brunneus. Gthr. N. v Lapolapa

(M S. T.)

Epinephelus brunneus, Bloch, t. 328 f. 2 ; B!. Schn. p. zoo.

Serranus kawamebari, Richardson, I.chth. China, p. 234 ("No Faun. Japon.)

S. brunneus, Gthr. Cat. B. M. 1. p. 107 (1859). Hab. Luzón, Cagayan; China.

7. S. albofuscus Gthr. N. v. Lapo-lapo.

(M. S. T.)

Holocentrus maculatus, Bl. id. p. 96. t. 242, f. 3 ; Bl Schn. p. 3 I 5 .

H. albofuscus, Lacep iv. pp. $341,384,385$; Gthr. l. cit. p. 188. Serranus maculatus, Bleeker, Boeroe, p. 398 .

Hab. Paragua; Borneo.

8. \& oceanicus, $C \quad V$. N. v. I.apo-lapo.

(M. S. T.)

?Perca fasciata, Forskal, p. 40.

Holocentrus oceanicus, Lacepp. iv. p. 377. t. 377 t. 7. f. 3.

H. forskulii Lacép. iv. p. 377.

Serranus oceanicus, Cuv. \& Val. ii. p. 302; Gthr. l. cit. p. 109. S. marginalis var., Bleeker, Perc. p. 34.

Hab. Cebú, Samar; Isla de Francia, Mar Rojo.

9. S. louti, Forsk $\mathrm{N}$ v Lapo-lapo.

(M S. T.

Seba, iii. 27. 7; Renard. t. 4I f. 207, t. 21. f. Ioo; Valentyn p. 412 . f. 205 .

Perca louti, Forskat. 1. 40.

Labrus punctatus, Lacép. iii. p. 377 lam. i 7. f. 2.

Bodianus louti, Bl. Schn. p. 332; Lacép, iv. p. 286 . ।

Serranus punctatus, Cuv. \& Val. ii. p. 376 ix. p. 435 ; Bleek.

Linn. i. p. 570; Quכy. \& Gaim. Voy. Astrol. Poiss. p. 654.

lam. 3. f. 2. louti, Rïpp. Atl. p. io6. lam. 26 f. 2. (no Val.);

Gthr. loco cit. 1. p. IOI.

Hab. Paragua, Mindanao; Sumatra, Molucas.

ro. S. trimaculatus, $C . V$. N. v. Lapolapo. (M S. T.)

Epinephelus japonicus, Krusenst. Reisse, lam. 64. f. 2 . 
Serranus trimaculatus, Cuv. \& Val. ii. p. $53 \mathrm{I}$; Faun. Japon. Poiss, p. 8; Richardson, Icht. China, p. 232; Gthr. l. cit. 1. p. 109 . Hab. Samar; China, Japon.

11. S. flavimarginatus. Rüpp. N. v. $(\mathrm{M} S \mathrm{~S} \cdot \mathrm{T})$

Rüpp. Atlas, Fische, p. 109; Gthr. Cat. Brit. Mus. i. p. I1,.3. Hab. Luzon, Manila, Mindanao; Mar Rojo.

12. S diacanthus Cuv. N. v.

Serranus diacanthus, Cuv. \& Val. ii. p. 3 19; Gthr. l. c. 1. p. I Io. s. nebulosus, Richards. (no Cuv. \& Val., ni Bleek.), Ichth. China, p. 232.

S. schihpan, Richards. l. c. p $23 \mathrm{I}$.

Hab. Luzon, Currimao, Ilocos; China, Hong-kong.

13. S stigmapomus. Rich $\mathrm{N}$ v.

(M. S. T.)

Richardson, 1chth. China, p. 232; Gthr. l. c. 1. p. III.

'Hab. Luzo, Cavite, Sta. Cruz; China, Australia.

14. S tigrinus, $C . V$. N. v. Lapo-lapo.

Seba iii 27.5 .

Crochilus, sp. 4, Klein. Miss. p.

Holocentrus tigrinus, Bloch. t. 237; Bl. Schn. p. 3 r4.

Serranus tigrinus, Cuv. \& Val. ii. p. 314; Gthr. l. c. 1. p. 1 it. Hab. Samar, Zumárraga, Borongan; Indias Orientales.

15. S. zananella, Blh. N. v. Lapo-lapo

Serranus zananella, Bleeker. Perc. p. 32.

? Labrus guasa, Lacep. iii. p. 501. lam. 27 f. 1 .

? Serranus zananella, Cuz. \& Val. ii. p. 3o4; Gthr. l. c. p. 1 í. Hab. Mindoro, Cebú, Luzon, Cavite; Java.

16 S micropion, Blk. N. v.

Bleeker, Ambona, ii. p. 552; Gthr. l. c. 1. p. IIt. Hab. Samar, Villareal, Panay; China.

$17 \mathrm{~S}$ cyagnostigma. $C: V \mathrm{~N} \quad \mathrm{v}$

Renard,, Poiss. Mol. t. 28 f. I 53; Valent. iii. p. 392.

Serranus cyanostigma, (Kuhl. \& v. Hass.) (uv. ii. p. 359 ef Regne, Anim. Ill. pl. 8 f. 2; Blecker, Perc, p. 32; Githr. I. c. p. II7.

- Hab. Paragua; Java. 
IS. S aurantius, $C \quad V$. N. v.

(M. S. T)

Cui'. \& Val. ii. p. 305 Bleek. Sum. i. p. 5ir; Gthr. l. c. I. p. I1邓. Hab. Cebú; Sumatra.

19 S guttatus $B i$ N v L.ạo.lapo.

(M. S. T)

Percx miniata, var., B. Forsk. p. 4 I.

Renard, i. pl. 22 f. 3, lam. 30 f. 162; Valent. iii. p. $35^{8}$ f. 37. Bodi nus guttatus, Bl. t. $224 ; B l$. Schn. p. 330 .

Serranus myriaster, Cui'. \& Val. ii. p. 365; Rüppell. Atlas. p. ${ }^{107}$ t. 27 f. I; Richards. Ichthyol China, p. 233 Quoy \& Gaim. Voy. Astrol. lam. 3 f. I; Less. Voy Caquille, pl. 37; Blcek. Natura'k. Tydschr. Nederl. Indie, vi. p. 192; Quoy \& Gain. Voy. Astrol. Poiss. p. 653 lam. 3 f. I.

S. guttatus, Gthr. Cat. B. M. i. p. 119.

Hab. Luzon, Manila, Cavite, Santa Cruz, Batangas; China, Polinesia, Australia.

2o. S. urodelus, $C$. V.

Perca urodela, Forst. Descr. Anim. Cur. Lichtenst. p. $22 \mathrm{r}$.

Serranus urodelus, Cuv. \& Val. ii. p. 306, vi. p. 513 ; Bleck Kokoseilanden, p. 39; Gthr. l. c. 1. p. 122.

$H a b$. Islas Carolinas; Amboina.

21. S. lumbatus, $C$. V. N. v.

Cuv. \& Val. ii. p. 307; Gthr. l. c. 1. p. 125.

Hab. Guam, Marianas.

22. S. suillus, $C . V$. N. v. Lapo-lapo.

(M. S. T)

Russell, pl. I 27 .

Bola coioides, Buchanan Hamilton, pp. 82, 369.

Serranus suillus, Cuv. \& Val. ii. p. 335, Bleeker, Verhand. Bat. Geenootsch xxii. p. 9; Gthr. l. c. 1. p. I 27.

S. coioides, Cant. Catal. p. ii.

Hab. Cebú;. Java.

23. S. altivelioides, Blh N. v.

Bleeker, Perc. p. 38; Gthr. M. B. 1, p. 127

Hab. Samar; Batavia.

24 S. marginalis, $C U$. N. v.

$(\mathrm{M}$ S. T $)$

Epinephelus marginalis, Bloch, t. 328 f. r.

Hol:centrus rosmarus, Lacép. id. pp. 389,392 t. 7 . f. 2.

Serranus marginalis, Cuv. \& Val. ii. p. 301; Richards. Ichth.

China, p. 233; Bleeker, Perc. p. 34 ; Gthr. l. c. 1. p. I35.

$H a b$. Mindoro, Calapan, Panay, Iloilo; China. 
25 S. seba, Blk. N. v.

(II. S. T)

Bleeker, Amboina v. p. 488 ; Gthr. l. c. 1. p. 137.

Hab. Luzón, Manila; China, Amboina.

26. 8. celebicus, Blk. N. v.

(M. S. 'T.)

Bleeker, Célebes i. p. 2 г 7 ; Gthr. l. c. 1. p. I39.

$H a b$. Samar, Cebú, Nasugbú; Célebes.

27. S. sp. N v. Lapo-lapo

Hab. Luzon, B. de Manila, C. de Ilucos.

2S. S. hexagonatus Gthr. N. v.

(M. S. T.)

Gthr. l. c. p. I40.

Hab. Luzón, B. Manila; China, Amboina.

29. S. var. hexigonatı, Iirst. N, v.

(M. S. T.)

Perca hexagronata, Forster, Descr. Anim et Licht. p. 189.

Holocentrus hexagonatus, Bl. Schn. p. 323.

Serranus hexagonatus, Cuv. \& Val. ii. p. 330 vi. p. 516; Guerin, Jcon Poiss, lam. 4. f. I; Richardsora, Voy. Sulph. p. 82 lam. 38 f. I; Cantor, Catal. p. 7; Bleek. natumrk. Tydschr. Nederl., Indie, vi. p. I91; Gay Chile, Zool. ii. p. I5\%; Gthr. var. A.l. c. p. I4I.

S. foveatus, Cuv. \& Val. ii. p. 329.

S. stellans, Richardson, Ann. Nat. Hist. 1842 vol. ix. p. 23. Hab. Luzón, Batangas, Nasugbú; China.

3o. S. var merra C. V. N. v.

Seba, iii. 27-7; Klein, Hist. Pisc. v. p. 43. t. 8. f. 3.

Perca tauvina, Forsk. p. 39.

Epinephelus merra, Bl. t. 329; Bl. Schn. p. 300 .

Holocentrus tauvinus, Bl. Schn. p. 32 r; Lxcép id. p. 338.

H. merra, Lacép. id p. 329.

Serranus merra, Cuv. \& Val. ii. p. 325 ; Gthr. l. c. 1. p. 141. Hab. Carite, Naic, Nasugbú; China, Singapur.

31. S. punctatissimus G.hr N v.

$(\mathrm{M}$ S. T)

S. punctatissimus, Gthr. Cat. B. M. 1. p. I44 (1859) lam.x. f. t. ;Hab. Luzón, Manila; China.

32. S. gilberti, Richard. $\mathrm{N}$ v.

(M. S T )

Percis n. ${ }^{\circ}$ 1 5 , Klein, Hist. Pisc. v. p. 43. t. 8 f. 3 .

Serranus gilberti, Richardson, Ann. Nat. Hist. 1842, p. 19; et Ichth. China, p. 230; Gthr. l. c. 1. p. 148.

S. megachir, Richardson, Ichth. China, p. 230. 
S. pardalis, Blecker, Perc. p. 37 .

Hab. Paragua, 1'. Princesa; China.

33. S. awoara, Sihlig. N. v

(M. S. T.)

Tem. et Schleg. Faun. Japon, p. 9 1. 3 f. 2. Richards Ichth.

China, p. 231; Gthr. l. cit. 1. p 150 .

Hab. Luzón, Cavite, Sta. Cruz; China, Jipon.

34. S. chlorostigma, Githr. N. v.

(il s. 'T.)

Cuv. \& Val. ii. p. 352 ; Gthr. Cat. B. M. i. p. 151 (1859).

Hab. Luzón, Manila; Seychelles.

25. S. altivelis. C. V. N. v

(M. S. T.)

Cuv. \& Val. ii p. 324, lam. 25; Rcharis. Ichth. Chint, p. 23); Bleeker, Perc. p. 33.

Cantor, Catal. p. Io; Gthr. Cat. B. M. i. p. 152 (1859).

Hab. Luzon, B. de Manila, Cavite, St.ı. Crus, Nasugbú, Batangas; China, Cébú, Japon.

36. S. formosus, (Shuw.! N. v

(M. S. T.)

Russel, t. I 29.

Sciæna formosa, Shaw, Zool. Misc. p. 23 t. 1007.

Serranus formosus, Cuv. \& Val. ii. p. 311; Richards. Ichth. China; p. 233; Bleeker, Perc. p. 31; Gthr. l. c. 1. p. 154. Hab. Luzón, C..vite, Santa Cruz; China, Batavia.

37 S. lineatus, $C . V$. N. v.

(M. S. T.)

Cuv. \& Val. ii. p. 31 2; Gthr. Cat. B. M. i. p. I56 (1859). Hab. Luzón, Cavite, Santa Cruz; China.

5. Gén. PLECIROPOMA, Cuv.

38. P oligacanthus, Blkr. N. v.

(M. S. T.)

Bleeker, Java ii. I854, p. 422; Gthr. Cat. B. M. 1, p. 157 ( I 859 ).

Hab. Lúzon, Cavite, Santa Cruz; Batavia.

39. P. susuki, C. V. N. v.

(M. S. T.)

Cuv. \& Val. ii. p. 404; Faun. Japon, p. 11. pl. 4; Richardson Ichthyol. China, p. 230; Gthr. Cat. B. M. 1, p. I60 (I859). Hab. Luzon, Currimao; China, Japon. 


\section{MISSING PAGE}




\section{MISSING PAGE}


M. immaculatus, Cav. \& Val. ii. p. 474 ; Bleek. Perc. p. 45 .

M. gembra, Bleek. Sumatra, ii. p. 2+6; Gthr. l. cit. p. 193. Hab. Luzón, C. Ilocos, Camarines, Cagayan, Samar, Borongan.

49. M. bohar, Forsk. N. v

(M. S. P.)

Sciana bohar, Forsk. p. 46 .

Sparus lepisurus, Lacep. iii. lam. 15. t. 2.

Lutjanus bohar, Bl. Schn. p. 325.

Jiacope bohar, Cuv. \& Val. ii. p. 43.3 ; Rüpp. Atl. Fische p. 73 , et $N$. $W$. Fische, p. Io3.

Mesoprion bohar, Gthr. l. cit. p. 190.

Diacope quadriguttata, Cuv. \& Val. ii. p. 427, vi. p. 533.

Hab. Luzón, B. Manila, Carite, Santa Cruz, C. Mindanao, Zamboanga.

50. M. johnii, Blo.h N. v.

(M. S. T.)

Renard, Porss i. t. 31. f. 172; Russel, t. $9^{6 .}$

Anhias johnii Bloch, t. 318 .

Sparus tranquebaricus, Shaze, Zool. iv. p. $47 \mathrm{~s}$.

Coius catus, Bu:h. Ham. Gaug Fish. p. 90 lam. 38 f. $3^{\circ}$.

Nesoprion unimaculatus, Quoy \& Gam Zool. Frege p. 304; Cuv. \& Val. ii. p. 441; Bleeker, Perc. P. 42; Richards Ichth, China, p. 229; Quoy \& Gaim, Voy de l'Astrol. Poiss. p. 665 lam. 5. f. 3 .

M. johnii, Cuv. \& Val. ii. p. 443; Cantor, Catal. p. i3; Gthr. l. cit. 1 . p. 200.

Hab. Luzón, Camarines Sur, Pası́ıao; China, Australia.

5I. M fuscescens, $C . V$.

(M S T )

Mesoprion fuscescens, Cuv. \& Val. vi. p. $53^{8}$; Bleeker, Batjan ii. p. 197; Gthr. t. cit. p. 201.

M. hotsen, Richards. Ichthyol, China, p. 229.

Hab. Cebú; China, Célebses.

52. M. quinquelineatus, $C \quad V . \mathrm{N} . \mathrm{v}$.

(M. S. T.)

Rissell, ii. lam. I ro.

Mesoprion quinquelineatus, Cuv. \& Val. ii. p. $4+5$; Gthr. l. c. p. 209.

:Diacope caruleolineata, Rüpp. N. W: Fische, p. 93. t. 24 f. 3 . Hab. Cebú; Java.

53. M. fulviflamma, (Forsk). N. v.

(M. S. T.)

Scitna fulviflanma, Forsk. p. 45.

Perca fulviflamma, Bl. Schneid. p. 28.

Centropomus hober, Lacép. iv. p. 255.

Diacope fulviflamma, Cuv. Régne. Anım.; Rüpp. Atl. Fische, p. 72 t. 19. f. 2, et $N$. W. Fische, p. 94; Cuv. \& Val. ii. p. 423 . 
Mesoprion monostigma, Cuv. \& Val. li. p. +66 (no Bleek.)

M. fulviflamma, Bleek. Amb. ii. p. 553. Gthr. l. c. p. 201. Hab. Luzón, B. Manila, C. Cebú; China, Java.

54. M. annularis. C. V. N. v.

(M. S T )

Mesoprion annularis, Cuv. \& Val. ii. p. 484 ; iii. p. 497 , Richardson, Ichth. China, p. 229; Bleeker, Perc. p. +7 ; Cantor, Catal. p. I4. Gth. l. c. p. 200.

Diarope annularis, Rüpp. Atl. Fische, p. 7t. et N. H'. Fisch. p. 91, t. 24 f. 2; Quoy, \& Gaim. Voy. Astrol. Poiss, p. 666 pl. 5. f. 4 .

D. metallicus, Bleek. Topogr. Batav.

Mesoprion sanguineus, Rleek. Perc. p. $4^{\mathrm{s}}$.

Hab. C. Cebú; China, Australia.

55. M. malabaricus Bl. N. v.

(M. S. T.)

?Sparus nalabaricus, Bl. Schn. p. 278 .

¿Mesoprion malabaricus, Cuv. \&. Val. ii. p. $4^{80}$, Gthr. l. c. p. 204.

M. malabaricus, Bleek. Sumatra, iii. p. 3.

Hab. Luzón, Manila; Sumatra.

56 M. decussatus, $C, V$.

(M. S. T.)

Cuv. \& Val. ii. p. 487 ; Bleeker, Perc. p. 43; Gthr. Cat. B. M. 1. p. 210, 1859 .

Hab. Luzón, Camarines Sur, Pasacao, C. Mindanao, Zamboanga; Amboina.

57. M. vitta, Q. et $G$. N. v.

(M S. T.)

Serranus vitta, Quoy, \& Gaim. Voy. de Freyc. Zool. Polss. lam. 58. f. 3. p. 315; Richardson, Ichth. China, p. 234.

?Serranus vitta, Cuv. \& Val. ii. p. 239, vi. p. 505.

Diacope vitta, Schleg, \& Temm. Faun. Japon. p. I3. pl. 6. f. I. ?Mesoprion vitta, Bleeker, Perc. p. 43; Gthr. l. c. 1. p. 2 I4. Hab. Luzón, Cavite, Sta. Cruz; China, Australia, Java.

\section{Fam. PRIACÁNTIDOS.}

1 Gén. PRIACANTHUS, C. V.

I. P. japonicus, C. V. N. v.

(M. S. T.)

Priacanthus japonicus, (Langsdor). Cuv. \& Val. iii. p. io6. lam. 50, Fain. Japon. p. 20. (no Bleek.); Gthr. l. c. 1. p. 217. Hab. Luzón, Cavite, Sta. Cruz; China, Japón.

2. P. carolinus, $C, V$ :

Cur. \& Val. iii. p. го5; Less. Voy. Coq. Zool. Poiss. p. 224; 
Bleeker. Banda i. p. 235; Gthr. Cat. B. M. 1. p. $219(165(1)$. Hab. Carolinas, Ualan.

3. $\mathbf{P}$ holocentrum, Blkr $\mathrm{N}$ v.

Bleeker, Perc. p. 48; Gthr. Cat. B. M. 1. p. 220 (1859). Hab. Luzón, Cavite, Sta. Cruz; Paragua, P. Princesa.

4. P. tayenus, Richard N. v.

(M. S. 'T',

Priacanthus tayenus, Richard, Ichth. of the Seas of China sp. 237; Gthr. Cat. B. M. I. p. 221 (1859).

Hab. Luzón, Ilocos; Vigan, Marinduque, Mindoro, Calapan; China.

\section{Fam. APOGÓNIDOS.}

I Gén. AMbassis, C. U.

I. A. rabustas, G/hr N. v.

(N. S. T.)

A. robustus, Gthr. Cat. B. M. i, p. $222(1859)$.

Hab. Samar, Cebú; Borneo.

2. A interruptus, $B{ }^{\prime} k$. N. v.

(M. S. T.))

Bleeker, Ceram, 2. p. 696; Gthr. Cat. Brit. Mus. t. ${ }^{\circ}$ p. 22t. $H a b$, Luzón, Manila, R. Pasig; Java, Australia.

3. A. safgha, Forsk N. v.

(M. S. 'T.)

Scicena safgha, Forsk. Descr. Anim. p. 53.

Centropomus anbasis, Lacép. ix. p. 273.

Lutjanus gymnocephalus, Lacép. ix. p. 2 r6, iii. lam. 33. f. 2.

Ambassis commersonii, Cuv. \& Val. ii. p. г76. lam. 25; Rüppt. N. W. Fische, p. 89; Gthr. l. c. p. 223.

A. nalua, Bleek. Perc. p. 3o; Batav. p. 453.

Hab. Luzón, Manila; Java, Australia.

4. A urotænia. Blk. N. v. Langaray.

(M. S ' ' ')

Bleeker, Amb. \& Cer. p. 257; Gthr. Cat. B. M. 1. p. $22+(1859)$. Hab. Luzón, Manila, R. Pasig; Amboina.

5. A apogonoides, Blk. N. v.

(M. S. T.j

Bleeker, Borneo, ix. p. 200; Gthr. 1. Cat B. M. 1. p. $224($ I859). Hab. Rio de Catbalonga, Samar; Borneo.

6. A. dussumieri, $C . V . \mathrm{N}$. v.

(M. s. T.)

Ambassis dussumieri, Cuv. \& Val. ii. p. 18 I, vi. p. 5(3), ix. p. 
431; Blecker, Perc. p. 3o; Quoy \& Gaim. l'oy. Astrol. Po:ss, 650 lam. I. f. 3; Gthr. l. cit. p. 225.

(handla dussumieri, Cantor, Catal. p. 6. Hab. Cebú; China, Célebes.

7. A. buruensis, Blik. $\mathrm{N} v$

Bleeker, Boeroe, p. 396; Gthr. Cat. B. M. t. p. 226 (1859)

Hab. Luzón, Pasig, Manila; Burneo.

8. $\Lambda$ wolffü, Blk N. v.

Bleeker, Borneo, i. p. 9; Cat. B. M. 1. p. 226 (1859).

Hab. Samar, Burongan; Borneo.

9. A. batjanensis, $B / k$ N. v.

(M. S. T)

Bleeker, Batjan, ii. p. 196; Gthr. Cat. B. M. 1. p. 225.

Hab. R. Pasig, Manila; Amboina.

2 Gén. APOGON, Lacél'.

ro. A. tæniatug, $C . V . \mathrm{N} . \mathrm{v}$.

(M. S. T.)

Apogon tani: tus (Ehrenberg) Cuv. \& Val. ii. p. 159, Ruppell. Atlas, Fische, p. 48, and. N. W. Fische, p. 87; Cithr. Cat. B. M. 1. p. 234 ( 1859 ).

Hab. Luzón, Manila; Mar Rojo.

II. A. hyalosoma, Blkr.

Apogon thermalis, Bleeker, Perc. p. 27. (no Cuv.); Gthr. l. c. p. $23 \mathrm{I}$.

A. hylosoma, Bleeker, Singapore, p. 63 et Ambogna, iv. p. 329. Hab. Cebú; Sumatra, Batavia.

12. A. nigripinnis, $C . V$. N. v.

(M. S. T.)

Cuv. \& Val. ii. p. 152 Faun. Japon. Poiss. p. 3.

Hab. Luzón Cavite, Sta. Cruz; China.

13. A pæcilopterus, $C$. $V$. N. v.

(M S. T)

Apogon pæcilopierus, Kuhl. \& van Hasselt. Cuv. et Val. ii. p.

154; Cantor, Catal. p. 2; Gthr. l. cit. p. $23^{2}$.

Háb. Cebú; Java.

14. A multitieniata $C . V$. N. v.

(M. S. T.)

Cuv. et Val. l. cit.; Rüpp. Atl. p. 47; Gthr. l. cit. p. 229. Hab. Cebú; Mar Rojo. 
15. A. monochrous Bliekev. N. v.

$\left(\begin{array}{lll}M & S & T\end{array}\right)$

Bleeker, Act. Soc. Nederl. i. Manado en Macassar p. 34; Gthr. loc. cit. p. 236.

Hab. B. de Manila, C. de Cebú; Amboina.

16. A. bifasciatus, Kü̈ppell. N. v.

(M. $\mathrm{S}$ T.)

Apogon bifasciatus, Rüppell, N.W. Fische, p. 86, t. 22, f. 2; Gihr. loc. cit. p. $23^{8}$.

A. trimaculatus, (no Cuv.) Richards. Ichthyol. China p. 221.

Hab. Luzón, Manila, Cavite, Sta. $\mathrm{Cr} z$; China, Mar Rujo.

17. $\Lambda$. anrularis Rüpt. N. v.

(M. S. T.)

Rüppell, Atlas, p. 48; and. N. W. Fische, p. 85;-Gthr. l. cit. p. 239.

Hab. C. de Cébú; China, Amboina.

I8 $\Lambda$. quadrifasciatus, Val N. v.

(M. S. I )

Valenciennes, ii. p.'I53; Bleeker, Perc. p. 28; Cantor, Catal.p.

3; Peters. Wiegm.; Arch. 1855, p. 234; Gthr. l. cit. p. 239.

Hab. Luzón, B. de Manila; China, Australia.

19. A. frenatus, $\operatorname{Val} \mathrm{N}, \mathrm{v}$.

Apogon frenatus, Valenc. Nouv. Ann. Mus. Hist. Nat. 1832 , p. 57, lam. 4 f. 4; Bleeker, Act. Soc. Nederli., Amboinx, p. 25 Gthr. loc. cit. p. $24 \mathrm{I}$.

A. vittiger, Bennett. in Proc. Zool. Soc. 1833, p. 32.

A. melanorhynchos, Bleeker, Amb. \& Cer. p. 255, et l. c. p. 26.

Hab. Marianas, Guam; Mauricio.

2c. A. fasciatus, White. $\mathrm{N}$ v.

(M. S. 'T.)

Mullus fasciatus, White, New. South. Wales, p. 268 f. I.

Apogon novem-fasciatus, Cuv. \& Val. ii. p. I54; Bleeker, Timon, i. p. I63; Peters, Wwiegm. Arch. 1855 , p. 234.

A. fasciatus, Quoy, \& Gam Voy. Freyc. Zool. p. 334; Gthr. l. c. p. 241 .

A. balinensis, Bleeker, Perc. p. 28 et Verhand. Batav. Gnootsch. xxii., Bali p. 5 .

A. endeka-tænia, Bleeker, Banka, p. +49.

Hab. C. de Cebú; India, Molucas.

21. A guamonsis, Val $\mathbf{N}$.

Nouv. Ann. Mus. H. Nat. 1832 , p. 54; Gthr. l. c. p. 229.

Hab. Marianas, Guam. 
22 A cyanotænia, Blecker $\mathbf{N}$ v.

(M. S. T )

Bleeker Solor, p. 71; Gthr. Cat. B. M. 1. p. $2+2$ (1859).

Hab. C. de Cébú.

23 A fuscus, $Q$, $G$. N. v.

Quoy et Gaim Voy. Friye Zool. p. 3+5; Gthr. Ciat. f. 229. Hab. Guam, Marianas.

3. Gén. APOGONICHTHYs, BLKk.

24. A. glaga, Blkr. N. v.

(M. S. T.)

Apogon glaga, Bleeker, Perc. p. 29.

Apogonichthys glaga, Bleek. Verh Batıv. Genustech. xxvi. p. 57; Gthr. Cat. p. 2+7.

Hab. Luzón, Cavite, Sta. Cruz; Java, Singapor, Japon.

25 A. carinatus, $C . V$

Apogon carinatus, Cuv. \& Val. ii. p. r57; Faun. Japon Poiss p. 3 .

Apoyonichthys carinatus, Bleeker, Floris, p. 321, et Verh. Batav. Genootsch xxvi. p. 56, lam I. f. 3; Gthr. l. c. p. 247. Hab. Luzón; China.

26. A. polystigma, Blk. N. v.

(M. S T )

Apogon punctulatus, Bleeker, Nat. Tydschr. Nederl. Indie, iii. p. 616 (no Rüpp.)

Apogonichthys polystigma, Bleeker, l. c. vi. p. $48+$, Gthr. l, c. p. 246.

Hab. Cebú; Sumatra.

4. Gén. Chilodipterrus, Lacép.

27. Ch quinquelineatus, $C \quad V$.

Cheilodipterus quinquelineatus, Cuy. \& Val. ii. p. 167; Rüpp. N. W. Fische, p. 89; Lesson, Zool. Voy. Duperr. ii. p. I67; Bleeker, Amb. \& Cer. p. 252; Gthr. l. cit. p. 248.

Hab. Cebú; Amboina.

\section{Fam. GRÍSTIDOS.}

I. Gén. DULE:, C. V.

1. D. tæniurus, C. $V$. N. v.

Cuv. \& Val. iii. p. II 4 ; Bleeker, Perc. p. 49; Gthr. Cat. B. M. 1. p. 267 (I859). p. 267.

Hab. Luzón, B. de Manila; China, Java.• 
2. D. guamensis, $C . V$.

Ciuv. \& Val. vii. p. 474; Dumont d'Urville Voy. Pole Sud. Porss p. 42 lam. 3. f. I; Gthr. Cat. B. M. 1. p. 269 (1859).

Hab. Guam, Marianas.

\section{Fam. PRISTIPOMÁTIDOS.}

I. Gén Therapon, Cuv.

1. T. theraps, Cuv. N. v

(M. S. T )

'Therapon theraps, Cuv. \& Val. iii. p. 129, lam. 53; Bleeker, Perc. p. 50; Richards Ann. \& Mag. Nat Hist. 1842, vol. ix. 1 26; Rüpp. Neuc. Wirb. Fische, p. 95; Gthr. B. M. 1. p. I7t (1859).

Hab. Luzón, Manila, C. de Cebú; China.

2. T. servus, Block. N. v. Bagaong.

(M. S. T.)

Sciæna jarbua, Forskal, Descr. Anim. p. 50; Shaw, Zool. vi. p. $54 \mathrm{I}$.

Holocentrus servus, Block, t. 238. f. I.

H. jarbua, Lacép. iv. pp. 348,355 .

Grammistes servus, Bl. Schn. p. 185 .

Therapon timoriensis, Quoy \& Gaim Voy Freyc. Poiss p. 3+1.

Th. servus, Cuv. \& Val. iii. p. I25, vii. p. 479; Bleeker Perc.

p. 30; Richardson, Ann. \& Mag. Nat. Hist. 1842 , vol. ix. p. I 26; Rüpp. N. Wirb. Fische, p. 95; Gthr. l. b. p. 278.

l'terapon trivittatus, Gray. Ind Zool. pl. f. I.

Hab. Luzón, B. de Manila, C. de Samar, C. de Mindanao;

China.

3. T. elipticus, Rich. N. v.

(M. S. T)

Datnia alleptica, Rich. Voy Ereb. et Terr. Fisches p. I18 1. 52 f. 4 8; Gthn. Cat. Brit. Mus. 1. p. 276.

Hab. Laguna de Bay; Australia.

4. T. trivittatus, (Buch.) N. v.

(M. T. S )

Russel, ii. pl. I 26.

Coius trivittatus, Buch. Ham. pp. 92, 370.

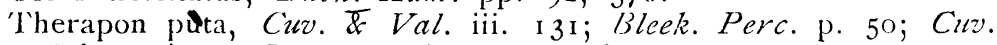 Régne Anim. Ill. Poiss. lam. I 2. f. 2.

'. trivittatus, Cant. Catal. p. i 9; Gthr. l. c. p. 280.

Hab. Luzón, B. de Manila; Ceilan, Singapore.

5. T. oxirbynchus, N. v.

(M. S. T)

Temm. \& Schleg. Faun. Japon p. I6 lan. 6. f. 3; Richards. 
Ichthyol. China, p. 239; Bleek. Verhand. Batav. Genootsch. xxvi. p. 6+; Gthr. loc. cit. p. $28 \mathrm{r}$.

Hab. Luzón, Cavite, Sta. Cruz; China.

6. T. quadrilineatus, (Bloch.) N. v.

Holocentrus quadrilineatus, Bloch, t. 238. f. 2.

Therapon quadrilineatus, Cuv. \& Val. ili. p: 134; Bleeker, Peri. p. 5I; Richards. Ichth. China, p. 239; Gihr. l. c. p. 282.

T. xanthurus, Chvv. \& Val. iii. p. 135.

Hab. Luzón, B. de Manila, C. de Cebú; China, Java.

7. T. argenteus, C. V. $\mathrm{N}$ v.

(M. s. T.)

Datnia argentea, Cuv. \& Val. iii. r 39. lam. 5t; Bbeeker, Perc. p. 52 .

Mesopristis macracanthus, Bleeker, Topogr. Batav.

Therapon argenteus, Gïnther, Cat. B. M. 1. p. 283.

Datnia cancellatoides, Bleek. Sumatra, i. p. 5 .

Hab. Luzón, Camarines Sur, Pasacao, Manila, Laguna de B.ty.

2. Gén. PRistipoma, Cur.

8. P. nigrum, C. V. N. v.

(M. S. T.)

Pristipoma nigrum, (Mertens) Cuv. \& Val. v. p. 258; Ciant. Catal. p. 74; Gthr. Cat. B. M. 1. p. 289 (1859).

Hab. Luzón, Manila; China.

9 P. hasta, (Bloch). N. v.

Luthanus hasta, Bloch, lam. 246. f. 1; Bl. Schn. p. 324; Lacep iv. p. 229 .

Labrus commersoni, Lacép iii. pp. 43I, 477 lam. 23 f. I; Shaw, Zool. iv. p. 493.

Lutjanus microstoma, Lacép iii. lam. 34. f. 2. iv. pp. I8r, 2 I6. Pristipoma Kahaan, Cuv. \& Val. v. p. 244; Rüpp N.W. Fische, p. I 23 t. 20. f. I; Richardson, Ichth. China, p. 227; Bleek. Verh. Bat. Genbotsch. xxii. p. 3.

P. conmersonii, Cuv. \& v. p. 252 ; Cant. Catal. p. 72.

P. hasta, Cuv. \& Val. v. p. 247 , cop. in Belanger, Voy Iud. Orient. p. 362 ; Bleek. l. c. xxiii. Scian. p. 20; Gthr. l. cit. p. 289.

P. crysobalion, (Kuhl. \& v. Hasselt.) Cuv. \& Val. v. p. $24^{8}$. Hab. Luzón, B. de Manila, Batangas, Nasugbu, Cebú; Chin:t, Mar Rojo.

I0. P. ləucurum. C. et $V^{r}$. N. v.

P. leucurum, Cuv. et Val. iv. p. +88 ; Gthr. Cat. B. M. 1. p. 286.1859 .

Hab. Marianas, Guam. 
11. P. argyreum Bleek. N v.

(M S. T.)

Pristipoma argyreum, Bleek. Verh. Bat. Gen. xxiii. Scian. p. 22. Pristipona argyreum, Cuv. \& Val. iv. p. 485 ; Gthr. l.c. p. 292. Hab. Luzón, B. de Manila; Sumatra, Bataria.

32. P. maculatam, (Bl) N. v.

(M. S. T.)

Inthias maculatus, Bl. Tab. 326 f. 2; Bl. Schn. p. 366; Gthr. l. c. p. 293.

Lutjanus maculatus, Lacép. ir. p. 239 .

(aripe, Russell, pl. 124.

Pristipoma caripa, Cuv. \& Val. v. p. 261; Caut. Catal. p. 75; Rüpp. N. W. Fische. p. I 24; Bleek. l. c. p. 2 เ.

Hab. Luzon, B. de Manila; Amboina.

13. P. japonicum, C. V. N. v.

(M. S T.)

Cus. \& Val. v. p. 288; Faun. japon, Poiss. p. 60 lam. 26 f. 2, Richards. Ichth. China, p. 228; Gthr. Cat. B. M. 1, p. 3(3 ( 1859$)$.

Hab. Luzon, Cavite, Santa Cruz; China, Japon.

3 Gén hapalogknys. Richards.

14. H. nigripinnis, (Tem. Schl.) N. v. (M. S. T.)

Pogonias nigripinnis, Temm. \& Schleh. Faun. Japon. Poiss. p. 59 pl. 25 .

Hapalogenys nitens, Richards. Ann. \& Mag. Nat. Hist. 1847, xiii. p. 463, et Ichthyol, Sulph. p. 84 lam. 43 f. I, 2.

? Hapalogenys maculatus, Ruhards. Ichth. China, 1). 235. Hab. Luzon, Cavite, Santa Cruz; Ci.ina.

15. H. mucronatus, (Ey'd. ct Soul.) N. v.

(M. S T.)

Pristipoma mucronatus, Eydoux \& Souleyet, Voy, de la Bonite, Zool. p. I6I lam. 2 f. I (is 84 ).

Hapalogenys analis, Ruchards. l. c. \& Ichth. Sulph. p. 85 lam. 43 f. 2. Hab. Luzon, Manila; China.

4. Gén. Diagramma, Cuv.

16. D. gibbosum, $H$. et $\mathcal{F} a q q$. N. v.

Diagramma gilbosus, Hambr. \& Jacq. Givchen Voy Pole Sud, Poiss. p. 46 lam. 5 f. 2; Gthr. Cat. B. M. 1, p. 319 (1859). Hab. Marianas, Guam.

17. D affine, Gthr N. v.

(M. S. T)

Dityramma crassispinum, Bleek. Werh. Batar. Gensotseh. xiii. 
Scran. p. 26 (no Rüpp); Gthr. Cat. B. M. 1, p. 319 lam. xix. f. A. ( 1859$)$.

Hab. Luzón, B. de Manila; Australia, China.

18. D. lineatum, Lin. N. v.

(M. S. T.)

Seba, iii. 27 18; Benn. Ceyl. Fish. p. 13 f. 13.

Perca diagramma, L. Gm. p. rз 39.

P. lineata, L. Gm. p. I319.

Sciann lineata, L. Mus. Ad. Frid. t. 3i f. 4.

S. abumgaterin, Forsk. p. $5 \mathrm{I}$.

Grammistes lineatus, Bl. Schn. p. 186.

Diagramma lineatum, Cur. \& Val. v. p. 3o9; Bleek. Amboina iii. p. 22; Wood, Nat. Hist. 3, p. 237, fig.

$H a b$. H. de Manila, Cebú; Amboina.

19 D. pardalis, $V . C^{\prime} \mathrm{N}$. v.

(M. $\mathrm{S}$ T)

Diagramma pardalis, (Kuhl. \& v. Hasselt. Cuv. \& Val. v. p. 3ou; Gthr. Cat. B. M. 1, p. 323 (1859).

Hab. Luzón, Manila; Java.

20 D punctatum, $C V \mathrm{~N}$ v.

(M. S. T.)

Diagramma punctatum, (Ehrenb.) Cuv. \& Val. v. p. 302; Faun. Japon, Poiss. p. 60; Rüpp. Atl. Fische, p. 126 lam. 32 f. 2, et $N$. $W$. Fische, p. 1 25; Quoy. \& Gain. Voy. Astrol. Poiss. lam. i 2 f. 2, p. 699; Cuv. Régne Anim. Ill. Poiss. lam. 30 f. 3; Bleeker, Verh. Batav. Genaotsch. xxiii. Scicn, p. 25; Gthr. loc. cit. p. 323.

Holocentrus radjabon, Lacêp. iv. p. 3o7; Rüpp. Atl. p. 127. Hab. Luzón, Cavite, Santa Cruz; China.

2. D. emetum, T. et Schl. N. v.

(M. S. T.)

Tem. \& Schleg. Fam. Japon. Poiss. p. 61. lam. 26. f. 1. Richardson, Ichth. China, p. 226; Gthr. Cat. B. M. A. p. 325 (1859). Hab. Luzón, Manila; China.

22. D. chœtodonoides Lacép. N. v.

(M S. T.

Plectorhyunchus chœtodonoides, Lacèp. iii. p. 135. ii. lam. 13. f. 2.

Lutjanus chœtodonoides, Lacép id. pp. 195, 243.

Choetodon plectorhynchus, Shaw. Zool. id. p. 356. lam. 49.

Diagramma plectorhynchus, Cuv. \& Val. v. p. 294; Bleek. Verh. Batav. Genootsch. xxiii. Scran. p. 24.

D. chœtonoídes, Gthr. l. cit. p. 326.

Hab. Cebú; Batavia.

23. D. pictum, Thumb. N. v.

(M S. T.)

Seba, iii. 26. 32 .

Perca picta, Thunb. Nua. Handl. xiii, .p. I 4 I lam. 5.

Grammistes pictus, $B l$. Schn. 190. 
Intjanus pictus, Lacep. v. pp. $5^{87} 7,688$.

Jiagramma pictum Cuv. \& Val. r. p. In 5 ; Fatur. Japon. Poiss. p. 62; Richardson, Ichth. China, p. 227; Gthr. l. c. p. 327. Plectorhynchus baldeatus, Caut. Catal p. 78 .

$H a b$, C. de Samar, Cebú; China, Amboina.

24 D. balteatum, var. B. C. $V . \mathrm{N}$ v.

Diagramma balteatum, (Kuhl. \& v. Hass.) Cur. \& Val. r. I'. 316; Gthr. Cat. B. M, z. 328 (1859).

Hab. Paragua; Borneo.

25. D albovittatum, Rüpp N. v.

Rüppell, N. W. Fisch, p, 125. lam. 31. f. 2; Bleek. Act. Sixc. Sic. Indv-Nederl. i. Mam. en Makass. p. 46: Gthr. l. c. p. 3,3.. Hab. Cebú; Manado.

26. D. reticulatum, Gthr. N. v.

$(\mathrm{M} S \mathrm{~S} \text { - })^{2}$

I). reticulatum, Gthr. Cat. B. M. 1. p. 334 (I859).

Hab. Luzón, Manila; China.

5. Gén, LOBOTES, Cuv.

27. L. auctorum, Gtkr. N. v.

(M. S. T.)

Holocentrus surinamensis, Bl, lam. 243; Bl. Shn. p. 3 ғ6.

Bodianus triunus, Mitch, Trans. Lit. \& Phil. Soc. New. York, i. p. 4 I 8 lam. 3. f. Io.

Sobotes surinamensis, Cuv. \& Val. v. p. 3r9; Dekay, New. York. Fauna, Fishes, p. 88. lam. 18. f. 49; Holbr. Ichth. S. Carol. p. I 59. lam. 23. f. 2.

s. erate, Cuw. \& Val. v. p. 3.2; Bleek Verh. Bat. Genootsch, xxii. p. 4. et xxiii. Scian. p. 26; Cant. Catal. p. So; Cu: Régne, Anim. Ill. Poiss. lam. 3r. f. I.

S. farkharii, Cuv. \& Val. v. p. 324.

S. somnolentus, Citv. \& Val. v p. 324.

S. incurvus, Richards. Ichth. Chin. p. 237.

$\mathrm{I}$. auctorum, Gthr. l. c. p. $33^{8}$.

Hab. Luzón, Manila; China, Molucas.

$$
6 \text { Gén GERREs, Cur. }
$$

28. G. punctatus, $C U . \mathrm{N}$ v.

Cuv. \& Val. vi. p. $480 ;$ Gthr. Cat. B. M. 1. 1. $346 \quad 1859$. Hảb. Luzón, Manila, Cebú; China.

29. G. japonicus. $B / k$. N. v.

Rleeker Japon, p. 404, et Verh. Bat. Gen. xxvi. Japon, lam. 5 f. 2. Sthr. Cat. B. M. s). 351. (1859).

Hab. Luzón, Cavite, Sta. Cruz; China, Japón. 
Bleeker, Riuv. P. $482 ;$ Gthr. (at. B. M. 1. 1. 352, (1859). Hab. Luzón, Manila.

\section{Gén. SCOI.OPSIS, (îHR.}

3I. S. ciliatus, (Lacep) N. v.

(M. S. T )

Holocentrus ciliatus, Lacep. iv. pp. 333, $37 \mathrm{r}$.

Lycogenis argyrosoma, Küht. \& var. Hassets.

Scolopsides lycogenis, Cuv. \& Val. v. p. 346 lam. 127.

Sc. ciliatus, Gthr. l. c. 1. p. 355.

Hab. Luzón, Manila, C. de Cebú; Amboina, Java.

32. S japonicus, (Bloch.) N. v.

Anthias japonicus, Bloch. lam. 325. f. 2; Bl. Schn. p. 3(n7.

I, utjanus japonicus, Lacép. iv. p. 31 .

Kurete, Russell, lam. 106.

Scolopsis kate, Cuv. \& Val. v. p. 329.

Sc. kurite, Rüpp. Atl. Fische, p. 9 lam. 2. f. 3; Cuv. \& Val. v. p. 33r.

Scolopsides ruppelii, Cuv. \& Val. v. p. 332; Richardson, Ichth. China, p. 236.

Sc. japonicus, Gthr. l. c. p. 354 .

Hab. Luzón, Cavite; China.

33. S. bimaculatus, liüpp. N. v.

(M. S. T.).

Scolopsis bimaculatus, Rüpp. Atl. Fische, p. 8 lam. 2. f. 2 . et N. W. Fische, p. I26; Cuv. \& Val. v. p. 340; Gthr. l. c. 357.

Scolopsides tæniatus, (Ehrenb. Cuv. \& Val. l. c.

S. inermis, Rich. Ichth. China, p. 236 (no Schleg.)

Hab: Luzón, Manila; China.

34. S. cancellatus, $C$. V.

:Scolopsis lineatus, Quoy. \& Gaim, Voy. de l'Astral, lam. $60 \mathrm{f}$. 3 (verybad).

Scolopsides cancellatus, Cuv. \& Val. v. p. 35I; Bleek. Verh.

Batav. Genootsch. xxiii. Scian. p. 28; Gthr. l. c. 1. p. 36r. Hab. Marianas; Sumatra.

35 S. bleekeri, Blk.

Scolopsides lineatus, Bleeker, Solor, p. 37. (no Rüpp.); Gthr. Cat. B. $M$. 1. p. 36 I (1859).

Hab. C. de Cebú. 
8. Gén. heterognathodon, BlK.

36 H. nemurus $B / k$.

Bleeker, Célebes, iii. p. 754; Gthr. l. c. 1. p. $3^{65}$.
Hab. Cebú; Batavia.

9 Gén. SYNAGRIS, GTHR.

37. S. japonicus, (Bioch) N. v.

(M. S. T.)

Sparus japonicus, Bloch, t. 277. f. I. Dentex blochii, Bleeker, Natuwk Tydschr. India 1851, p. i76,
s. ja:onicus, Gthr. l. c. p. 378 .

Hab. Luzón, Manila; Batavia.

38. S. sinensis, (La.ép. N. v.

(M. S. T.)

Sparus sinensis, Lacép. iv. p. 46. Deutex setigerus, Cuv. \& Val. v. p. 253; Faun. Japon Poiss,
p. 73 lam. 37.f. I.

Sy. sinensis, Gthr. l.c. 1. p. 379.

Hab. Luzón, Manila; China.

1o. Gén. Dentex, Cuv.

39. D nematophorus, Blkr, N. v.

(M.S. T)

Dentex nematophorus, Bleeker, Sumatra, iii. p. 5\%).

Synagris nematophorus, Gthr. l. c. 1. p. 379.

Hab. Luzón, B. de Manila; Sumatra.

11. Gén. Pentapus, Cuv.

40. P. paradiseus, Gthr. N. v.

Gïnther, Cat. B. M. S. 1. 1859 , p. 883 lam. xxiv, f. A.

Hab. Marianas; Sumatra.

2 Gén. CaEsio, Cuv.

$4 \mathrm{C} \infty$ maculatus, $C . V . \mathrm{N} . \mathrm{v}$.

$\left(\begin{array}{lll}M & \mathrm{~S} & \mathrm{~T}\end{array}\right)$

Cuv. \& Val. vi. p. 439; Gthr. Cat. B. M. 1. p. 39 I (1859).

Hab. Cebú; Hong-kong China.

42. Co tile, $C . V . \mathrm{N}$.

Cuv. \& Val. vi. p. 428; Less. Voy. Cog. Zoot. Poiss. p. 181. Cuv. Regne Anim. Ill. Poiss. lam. 36, f. 2; Gthr. Cat. B. M. 1. p. 393 (1859).

Hab. Carolinas. 
Fam. MÚLIDOS.

I. Gén. UPENEOIDES, Bi.kr.

I U. vittatus, (Forck) N, v.

(M S. T)

Mullus vittatus, Forskal Faun. Arab. p. 3r; L. Gm. Syst. Nat.

i. p. 134 I, Bl. Schn. p. 79; Lacép iii. pp. 382, 401, lam. I4, f. I, Shaw, Zool. iv. p. 616 lam. 89.

Russell, lam. I 58 .

Mullus baudi, Shaw. Zool. iv. p. 515.

Upenoides vittatus, Gthr. l. cit. r. p. 397.

Hab. Luzón, B. de Manila, Batangas, Nasugbu; Amboina, Ceilan.

2. U. tragula, (Richaid), N. v

(M. S. T.)

Upeneus trayula, Richardson Ichth. China, p. 220.

l'penoides variegatus, Bleeker, Perc. p. $6+$ et Act. Soc. Nederl. ii. Amboina, p. 48.

U. tragula, Gthr. l. cit. r. p. 398.

Hab. Luzón, B. de Manila; China, Amboina.

3. U subvittatus, (Teml et $S c h$ ) N. v. (M. S. T)

Mulłus subvittatus, Tem. et Schleg. Faun. Japon. Poiss. p. 3(). Upeneus subvittatus, Richards Ichth. China, p. 219; Gthr. Cat. B. M. 1. p. 397 (1859).

Hab. Luzón, B. de Manila; China, Japon.

4. U. sulpbureus, C.V. N. v. Saramulete.

(M. S. T.)

Valent. iii. p. 504 fig. 505; Renard, i. 43, 216.

Upeneus sulphureus, Cuv. \& Val. iii. p. 450.

? Hypeneus vittatus, var., Cant. Catal. p. 35.

Upeneoides sulphureus, Bleeker, Act. Soc. Nederl. ii. Amboina, p. $45 ;$ Gthr. l. cit. 1. p. 398.

Hab. Luzón, B. de Manila; China, Mar Rojo.

5. U. moluccensis, Blkr. N. v.

(M. S. T)

Bleeker, Amboina, vi. p. 409; Gthr. loc. cit. 1. p. 399.

Hab. Luzón, Manila; Ambøina.

6 Upenoides, esp. N. v.

Hab. Luzón, B. de Manila.

2. Gén. MULluS, LiN.

7. Mullus, esp. N. v.

(M. S. T.)

Hab. Luzón, Manila. 
3. Gén. MULLOIIES, BlKR.

8. M. flavolineatus, Lacép. N. v.

(M. S. ' i.)

Mullus flavolineatus, Lacép. iii. p. 406.

M. aureovittatus, Shaw, Zool. iv. p. 418 .

l'peneus flavolineatus, Cuv. \& Val. iii. p. 456 ; Rüpp. $N . W$. Fische, p. ro1 t. 26, f. 1; Jenyns, Zool. Bengle. Fischer p. 24. : Hypeneus flavolineatus, var., Cant. Catal. p. $3^{6 .}$

Mulloides flavolineatus, Bleek. Ceram, ii. p. 697; Gthr. l. cit. p. 4(')

Hah. Luzón, Manila; Ch'na, Madagascar.

4. Gén. UpeneUs, Bikr.

9. U. indicus, (Shaze.) N. v.

(M. S. T)

Russell. ii. p. 42 lam. 157 .

Mullus indicus, Shaw; Zool. iv. pt. 2. p. 6I4.

Ipeneus russellii, Cuv. \& Val. iii. p. 465 ; Richards Ichth. China p. 220 ; Bleeker, Perc. p. 62.

1. indicus, Gthr. l. cit. p. 406.

? U. waigiensis, Cuv. \& Val. iii. p. 466.

Hab. Luzón, Camarines Sur, Pasacao; China.

10. U pleurospilos, Bleeker.

Bleeker, Natuurk. Tydschr. Nederl. Ind. iv. p. I Io, et Verhand Batav. Genootsch xxvi. Japon, p. 69; Gthr. l. cit. p. fo7. Hab. Cebú; Amboina.

I1. U. malabaricus, C. B. N. v.

(M. S. T.)

Cuv. \& Val. iii. p. 467; Gthr. C. B. M. 1. p. 407 (1859).

Hab. Luzón, Cavite, Santa Cruz, Cebú; Malabar.

12. T. barberinus, Lacép. N. v

(M. S. T )

Mullus barberinus, Lacép. iii. p. 406, lam. i 3. f. 3.

lipeneus barberinus, Cuv. \&. Val. iii. p. 46z; Rüpp. $N$. W. Fissche, p. IоI; Gthr. l. cit. p. fo5.

Hab. Luzón, B. de Manila, Cebú; Molucas.

13. U. trifasciatus (Lacép) N. v.

(MI. S. T.)

Iilullus bifasciatus, Lacép. iii. p. 49+, lam. 14. f. 2.

M. trifasciatus, Lacép. iii. p. to4, lam. I 5. f. I.

II. multifaciatus, Quoy \& Gaim Voy. Freyc. pl. 59. f. I.

Upeneus bifasciatus, Cuv. \& Val. iii. p. 468.

U. trifasciatus, Cuv. \& Val. iii. p. 468; Jeuyns, Zool. Beagle, Fishes p. 25; Bleek. Banda, i. p. 237; Gthr. l. ,it. p. 407; Vilan Creac. 5. p. 263. f. I 50; Wood. Nat. Host. 3. p. 238. fig.

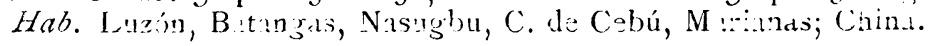


14. U. atherinoides. $C . V$.

L. atherinoides, Cuv. et Val. vii. p. 526 ; Gthr. C. B. M. 1. p. fot (Is 59 ).

Hab. Narianas, Guam.

25 U. chrysopleuron, T. et Sch. N. v.

(M Y. T)

Mullus chrysopleuron, Temm. \& Schleg. Fann. Japon. Poiss. P. 29, lam. 12. f. I.

Lpeneus biaculeatus, Gray, Rich Ichth. China, p. sig.

lpeneoides chrysopleuron, Bleek. Verhaud. Bataí. Genootsch. xxr. Japan, p. ro.

ipeneus chrysopleuron, Bleek. l. c. xxri. Japan, p. 7(); Githr. l. cit. p. 4 Io.

Hab. Samar, Villareal; China.

\section{Fam. ESPÁRIDOS.}

I. Gén. CANTharus Cuv.

I. C. grandoculis, C. V.

Cuv. \& Vat. vi. p. 34r; Cuv. Regne Anim. Ill. Poiss. lam. 35 f. 3; Gthr. C. B. M. 1. p. 417 (1859).

Hab. Marianas.

2. C. cœruleus, $C$. $V$.

Cuv. \& Val. vi. p. 343 ; Gthr. Cat. B. M. 1. p. 417 (1859). Hab. Marianas, Guam.

3. maculatus, $C . V . \mathrm{N} . \mathrm{v}$.

Cuv. \& Val. vi. p. 343 ; Gthr. Cat. B. M. 1. p. $4 \mathrm{I} 7$ (I859). Hab. Luzón, Manila; Oc. Indico.

4. C. lineolatus, C. V. N. v.

Cuv. \& Val. vi. p. 344; Gthr. Cat. B. M. 1. p. 418 (1859). Hab. Luzón, Cavite, Naic; Oceano Indico.

2 Gén. Girella, Gray.

5. G punctita, Gray. N. v.

Girella punctata, Gray, Ill. Ind. Zool. lam. $9^{8}$ f. 3, 4; Gthr. l. c. p. 427.

Melanichthys, Temm. \& Schleg. Faun. Japon, Poiss. p. 75 lam. 39.

Crenidens punctatus, Richrrdson Ichth. China, p. 242.

C. melanichthys, Richardson; Ichth. China, p. 242. Hab. Luzón, Cavite, Sta. Cruz; China, Japon. 


\section{Fam. PAGRÍNIDOS.}

I. Gén. Letrinus, Cuv.

1. L. richardsonii. Gthr. N. v

Lethrinus hamatopterus, Richardson, Zool. Sulph. Fishes, p. Itt, lam. 6t f. I. 3; Bleek. Verhand. Batav. Genootsch. Japon, p. 91. L. richardsonii, Gthr. C. B. M. 1, p. 456 (1859).

Hab. Luzón, Manila; Hong-kong, China.

2. L. nemacanthus, Blkr. N. v.

Bleeker Japon, p. 403, Verhand. Batav. Genootsch. xxvi. Japon, p. 9i, lam. 6; Gthr. Cat. B. M. 1, p. 456 (1859).

Hab. Cebú; Japon.

3. L. harak, Forsk. N, v.

Sciana harak, Forsk. p. 52.

Lethrinus harak, Rüpp. $N$. W. Fis he. p. i16 lam. 29 f. 3; Bleck. Verhand. Batav. Genootsch. xxiii. Spar. p. I5; Gthr. l. cit. 1. p. $45^{8}$.

? Lethrinus erythrurus, Cuv. \& Val. vi. p. 293.

?Lethrinus rhodopterus, Bleek, Singapore, i. p. 65. Hab. Luzon, Manila, Batangas, Nasugbú; Mar Rojo.

4. L ramak, Forsk. N. v.

(M. S. T.)

Sciana ramak, Forsk. p. 52.

Lethrinus ehrenbergii, Cuv. \& Val. vi. p. 312.

L. ramak, Rüpp. N. W. Fische, p. I 17 ; lam. 28 fig. 3 ; Gthr. l. c. I. p. 459.

Hab. Luzón, Manila, Nasugbú, Cebú; Mar Rojo, Ceilán.

5. I leutjanus, Lacep. N. v.

(M. S. T, )

? Bodianus leutjanus, Lacép. iv. p. $28 \mathrm{I}$.

¿Lethrinus leutjanus, Cuv. \& Val. vi. p. $3(09$; Gthr.l.c. г. p. +6r.

Lethrinus leutjanus, Bleek. Verhand. Bat. Genoostch. xxiii. Spar. p. I4.

Hab. Luzon, B. de Manila; Sumatra.

6. I. mahsenoides, $C . V . \mathrm{N}$, v.

(M. S. T )

? Lethrinus mahsenoides, Cuv. \& Val. vi. p. 286; Gthr. l. c. I. p. 464 .

Lethrinus mahsenoides, Bleek. Ver. Bat. Genootseh. xxiii. Spar. p. 15 .

Hab. Luzon, B. de Manila; Arboina. 
2. (ièn. SllH(lk()H)ON, Riur.

\section{7. $\mathbf{E p h}$. heterodon, iik.}

Pagrus heterodon, Bleeker, Gilolo, p. 54.

spharodon heterodon (ithr. l. cit. 1. p. to. $^{\circ}$.

Hab. Cebú; Ceilín.

3 Gèn. Pligus, Clv.

S. P. unicolor, $Q$ it $G$ N. v.

(M. S. T.)

Chrysophrys unicolor, Quoy \& Gain. Voy. Uratt. p. 299.

Pagrus unicolor, Cuo. \& Val. vi. p. 162; Kichardson, Ichth. China, p. $2+2$; Gthr. l. cit. 1. p. 468.

P. guttulatus, Cuo. \& Val. vi. p. IOo; Less. Voy. Coq. Zool. ii. p. 188 .

Hab. Luzon, Manila, Navotas;.Australia, Hong-kong, China.

9. P. cardinalis, Lacíp. N. v.

(M. S. T.)

Sparus cardinalis, Lacép. iv. p. 141 .

Chrysophrys cardinalis, Cuv. \& Val. vi. p. 130; Faun. Japon. Poiss. p. 69; pl. 33; Richarson, Ichth. Chima, p. $2+1$.

Pagrus cardinalis, Gthr. l. cit. i. p. 470 .

Hab. Luzon, Manila, Novotas; China.

1o. P. major, T. et Sch. N. v.

(M. S. T.)

Chrysophrys major, Temm. \& Schleg. Faun. Japon Poiss. 1. 71 lam. 35 .

Pagrus major, Gthr. l. cit. 1. p. 470.

Hab. Luzón, Manila, Malabon, China, Australia.

I P. spinifer, Forsk. N. v.

Sparus spinifer, Forsk p. 32; L. Gm. i. p. I273; Bl. Sch». p. 28 I Russell. ii. p. I. lam. Iо I.

Pagrus spinifer, Cuv. \& Val. vi. p. ${ }^{56} ;$ Räpp. N. W. Fische, P. I I 4 ; Gthr. l. cit. 1. p. 472.

P. longifilis, Cuv. \& Val. vi. r 59; Bleek. Celeb. iii. p. $75^{6}$. Hab. Cebú; Chiıa.

4. Gén CHRYSOPHRYS, Cuv.

12. Ch. aries, T. et Sch. N. v.

(M. S T.)

Temm. \& Schleg. Faun. Japon. Poiss. p. 68. lam. 31; Bleek. Verhand. Batav. Genootsch xxvi. p. 87; Gethr. l. cit. p. 489. Hab. Luzón, Cavite, Sta. Cruz; China, Java. 
12. Ch. hasta, $B l . \mathrm{N}$. v.

Sparus hasta, Bl. Schn. p. 27.5 .

Coius datnia, Buch. Ham. Fisch. Gatg. p. 88 lam. 9. f. 29.

Chrysophrys longispinis, Cuv. \& Val. vi. p. 116; Bleeker, Verhand.

Batav. Genootsch xxv. Nalez Faun. Beng. \& Hind. p. 93.

Ch. berda, Cuv. \& Val vi. p. I 3; Richardson, Ichth. Chinn, p. 240.

(h. langispinis, Faun. Japon. Poiss. p. 68 pl. 32 Richardson Ichth. China p. 240.

Ch. schlegelii, Bleeker, Japon. p. 400, et Verhand Batav. Cienootsch. xxvi. p. 86.

Ch. calamara, Cant, Catal. p. 48 (no $C$. \& $V$.)

Ch. xanthopoda, Richardson, Ichth. China, P. 241 .

Ch. auripes, Richardson, l. c. 1 .

Ch. hasta, Gthr. l. cit. 1. p. 490.

Hab. Luzón, Manila, Navotas; Ceilan, China.

14. Ch. calamara, Russell N. v. Dorada.

(M. S. T )

Calamara, Russell, i. lam. 92.

Chrysophrys calamara, Cuv. \& Val. vi. p. ir 7 ; Bleeker, Verhand.

Batav. Genootsch. xxiii. Spar. p. ro; Gthr. l. cit. 1. p. 493.

Ch. berda, Bleek. Topogr. Batav. et Ichth. Madura.

Hab. Luzón, Manila, Batangas, Nasugbú; Archip. Indico.

5. Gén. PIMEleptertẹs, Cuv.

15. P. tahmel, Forsk. N. v.

(M. S, 'T)

Scixna tahmel, Forsk. p. 53 .

Pimelepterus attipinis, Cuv. \& Val. vii. p. 270; Bleek. Banka, ii. p. 727 .

P. tahmel, Rupp. N. W. Fische, p. 35 lam. ro. f. 4; Githr. l. cit. 1. p. 499.

Hab. Luzón, B. de Manila; Mar Rojo, N. a Guinea.

\section{Fam. QUETODÓNTIDOS.}

I. Gén. Chatodon, Artidi

I. Ch setifer, $B l \mathrm{~N}$. v.

(M. S. T.)

Renard, i. p. 29. f. I98, ii. lam. 31. f. 145; Valent. iii. p. 385. f. I I6; Seba, iii. t. 25 f. I I.

Chatodon setifer, Bl. t. 426. f. I. Bl. Schn. p. 225; Cuv. \& Val. vii. p. 76; Guérin, Inconogr. Poiss. lam. 22. f. I; Less. Voy. Coq. Zool. ii. p. I75; Poiss. lam. 29. f. 2; Richards. Ichth. China. p. 246; Cuv. Régne Anim. Ill. Poiss. lam. 38. f. 1; Jenyns. Zool. Beagle, Fishes, p. 6r; Gthr. Cat B. M. 2. 1). 6 (1860).

Pomacentrus filamentosus, Lacép. iv. pp. 506, 511. 
Chætodon sebanus, Cuv. \& Val. vii. p. p. 74 .

Ch. auriga, zar. Rüpp. N. W. Fische, p. 28.

Ch. lunaris, Gronov. Syst. ed. Gray, p. 70.

Ch. auriga, Bleek. Célebes, iv. p. 164 .

Hab. Luzón, Cavite, Sta. Cruz; Amboina, China.

2. Ch ephippium, Cus N.v.

Chætodon ephippium, Cuv. \& Val. vii. p. 80. lam. 174; Less. Voy. Coq. Zool. ii. p. I74; Poiss. lam. 29. f. I; Bleek. Amboina, vi. p. 336 ; et Floris, p. 325; Gthr. l. c. 2. p. 7.

Ch. garnotii, Less. l. c.

Hab. Luzón, Currimao, Ilocos; Molucas, Polinesia.

3. Ch nesogallicius, Cuv N. v.

(M. S.. T.)

Renard, Poiss. Mol. i. lam. 5. f. 37.

Chætodon nesograllicus, Cuv. \& Val. vii. p. 63; Bleek. Banda. i. p. 240 .

Hab. Luzón, Manila, Navotas; Amboina.

4. Ch. ocellatus, Bluch. N. v.

(M. S. T)

Bloch, iii. p. 105. lam. 21 . f. 2; Bl. Schn. p. 223; Cuv, \& Val. vii. p. 75. (no Bleck. Natuurk. Tydschr. Nederl. Ind. vi. p. 21 2); Gthr. Cat. B. M. 2. p. Io (1890).

Hab. Luzón, Manila, Malabon; Indias Orientales.

5. Ch modestus, $T_{c / n ! m} . \mathrm{N}$ v.

Chætodon modestus, Temm. \& Schleg. Faun. Japon. Poiss. p. 80. lam. 4I. f. 2: Gthr. Cat. B. M. 2. p. 10.

Ch. ocellatus, Gronov. Syst. ed. Gray, p. 68.

Hab. Luzón, Manila; China.

6. Ch. unimaculatus, Bloch N. v

(M S. T.)

Bloch, t. 201. f. 1; Bl. Schn. p. 221; Cuv. \& Val. vii. p. 72; Cuv. Régne. Anim. Ill. Poiss. lam. 39. f. 3. Bleek. Banda, i. p. 24I; Gthr. Cat. B. M. 2. p. I I (1860).

Hab. Luzón, Manila, Cavite, Sta. Cruz.

7. Ch meyeri, $B l$. N. v.

(M. S. T.)

Valent iii. p. 455. f. 347; Renard, i. 25. 135.

Chætodon meyeri, Bl. Schu. p. 223; Cuv. \& Val, vii. p, r9; Cuv. Régne. Anim. Ill. Poiss, lam. 37. f. 2; Bleek. Act. Soc. Nederl. i. Amboina, p. 37: Gthr. l. c. 2. p. I3.

Holacanthus flavo-niger, Lacêp. iv. pp. 529, 538. lam. r3. f. 2. $H a b$. Luzón, Cavite, Naic; Amboina, Célebes.

8. Ch. ornatissimus, Cuv N. v.

Chætodon ornatissimus, (Solander). Cuv. \& Val. vii. 22; Less. 
Voy. Coq. Zool. ii. p. 179. lam. 3o. f. I Bleek. Act. Suc. Nedcrl. ii. Amboina, p. 55; Gthr. l. c. 2. p. 15.

Ch. ornatus, Gray, Zool Miscell. p. 33, et in Griff. Anim. Kingd. x. p. 323. lam. 20.

Hab. Carolinas.

9 Ch. reticulatus, $C . U$. N. v.

Cuv. \& Vat. vii. p. 32. lam. I7 ; Less. Voy. Coq. Zool. Poiss. p. I78. lam. 3o. f. 2; Gthr. Cat. B. M. 2. p. 22 (I860). Hab. Marianas.

10. Ch. kleinii, Bloch. N. v.

Klein, Miss. iv. lam. ro. f. 2. 255.

Chætonon kleinii, Bloch, lam. 2I8. f. 2; Bl. Schn. p. 225.

Ch. virescens, Cuv. \& Val, vii. p. 3o; Bleek. Verh. Batav. Genootsch. xiii. Chatodont. p. 18; Gthr. l. c. 2. p. 22.

Ch. flavescens, Benn. Proc. Comm. Zool. Soc. i. p. 61. Hab. Luzón, Manila; Java, Amboina.

11. Ch. fasciatus, Forsk. N. v.

(M S. 'T.)

Chætodon fasciatus, Forsk. p. 59; Gm. p. 1 266; Cuv. et. Lal. vii. p. 6I. Gthr. l. cit. 2. p. 24.

C. flavus, Bl. Schn. p. 225; Rüpp. Atlas, Fische, p. 40, lam. 9. t. I.

Hab. Luzón, Manila, Malabon; China.

12 Ch lunula, Cuv,

Pomacentrus lunula, Lacép. iv. pp. 507, 510, 513.

Chætodon lunula, Cuz. \& Val. vii p. 59. lam. I73; Bleek. Gilolo, p. 57 ; Gthr. l. cit. 2. p. 25.

Hab. Marianas.

13. Ch vagabundus, Linn. N. v.

(M. S. ' ' )

Renard. i. 23. 126.

Chætodon vagabundus, Linn. Mus. Ad. Fried. ii. p. 7I, et Syst. i, p. $465 ;$ L. Gm. p. $1251 ; B l$ lam. 204. f. 2 ; Bl. Schn. p. 222; Cuv. \& Val. iii. p. 50; Bleek. Verhand: Batav. Genootsch. xxiii. Chaetod. p. I8, (no Benn.); Gthr. l cit. 2. p. 25.

Hab. Luzón, Batangas, Nasugbú, Cavite, Naic, Cebú.

14. Ch. ocellicauda, $C V$ N. v.

(M. $S$ T.)

Cuv. \& Val. vii. p. 69; Gthr. l. cut. 2. p. 29.

Hab. Luzón, Cavi e; Oceano Indico.

15. Ch. lineolatus, Cuv. $\mathrm{N}$ v.

(M. S. T.)

Chætodon lineolatus, Cuv. \& Val, vii. p. 40; Bleek. Florio, p. 323 . 
C. lumatus, (Ehreub.) Cuv. \& Val. vii. p. 57; Rüpp. $N . U$. Fische, p. 3o. t. 9. f. 3; Gthr. l. ci. 2. p. 3o.

?Chetodon semilarvatus, (Ehrenb.) Cuv. \& Val. vii. p. 39 Young. Hab. C. de Mindoro; Mar Rojo.

16. Ch. miliaris, $Q$ et $G \mathrm{~N}$ v.

Quoy. \& Gaim. Voy. Freyc. Zool. p. 380. lam. 62. f. 6. (?) Ciuv. \& Val. vii. p. 26; Voy. Bonite. Poiss. p. 163 lam. 2. f. 2.; Gthr. l. cit. 2. p. 3o.

Hab. C. de Mindoro; Islas Saudwich.

17. Ch tau.nigrum, C. $V$.

Cuv. \& Val. vii, p. $3^{8 ;}$ Gth. l. cit. 2. p. 32. $H a b$. Marianas, Guam.

18. Ch. citrinellus, (Brouss). N. v.

(M. S T )

Renard, i. lam. 8. f. 59.

Chætodon citrinellus, (Brouss.) Cuv. \& Val. vii, p. 27; Regne Anim. Ill. Pois. pl. 39. f. I; Bleek. Sumatra, iii, p. 5or; Githr. l. cit. 2. p. 33 .

Hab. Luzón, Batangas, Nasugbú, Cavite, Naia.

19. Ch. sp

Hab. C. de Cebú.

2o. Ch. chrysozonus, (Kuhl \& v Hass.)

Chatodon chrysozonus, (Kuhl. \& v. Hass) Cuv. \& Val. vii. p. 82; Cuv. Régne Anim. Ill. Poiss. lam. 38. f. 2. Bleek. Verhand. Batav. Genootsch. xxiii. Chotodont. p. 17; Gthr. l. cit. 2. p. 34.

C. labiatus, (Kuhl. \& v. Hass.) Cuv. \& Val. vii. p. 83 .

C. melanopus, Cuv. \& Val. vii. p. 84 .

C. guttatus, Gronov. Syst. ed Gray. p. $7 \mathrm{I}$.

Haib. Luzón, Manila; China, Molucas.

21. Ch. sp.

Hab. C. de Cebú.

22 Ch. oligacanthus, Blk. N. v.

(M. S. T)

Bleeker. Verhund. Batav. Genwtsch. xxiii. Chatodant. p. 6; Gthr. l. cit. 2. p. 34 .

Hab. C. de Samar y de Cebú.

23. Ch. sp.

Hab. Cebú. 
24. Ch. strigatus, Langsd, $\mathrm{N}$ v.

(M. S. T )

('hrtodon strigatus, (Langsd.) Cuv. \& Val. vii, p. 25. lam. I20; Faun. Japon. Poiss. p. 8o. lam. 4r. f. I. Bleek. Verhand. Batav. Genwtsch. xxvi. Japon. p. 94; Gthr. l. cit. 2. p. 34. Hab. Luzón, Cavite; China.

\section{Ch. sp.}

Hab، C. de Cebú.

26. Ch. polylepis, Bleeizer. N. v.

(M. S. T.)

Bleeker, Act. Soc. Indo. Nederl. ii. Amboina, p. 54: Gthr. l. cit. 2. p. 35 .

Hab. Luzón, Manila; Amboina.

2. Gén. Chelmo, Cuv.

27. Ch. rostratus, $C u v . \mathrm{N}$ v.

Seba, iii. lam. 25 f. 17.

Chœetodon rostratus, L. Mus. Ad. Fried. i. p. 6r. lam. 33. f. 2; L. Gm. p. г 244; Bl. lam. 202. f. I; Bl. Schn. p. 221; Sharw, Zool. iv. p. 337, lam. 47; Gronov. Syst. ed. Gray, p. 73.

Jaculator schlosser, Phil. Trans. 1764 , p. 39, lam. 9.

Choetodon enceladus, Shaw. Nat. Misc. p. 2, lam. 67.

Chelmon rostratus, Cuv.-Régne. Anim.; Benn. Life of Raffles, p. 689; Cuv. \& Val. vii. p. 87; Cant. Catal. p. I58; Cuv. Régne Anim. Ill. Poiss. lam. 40. f. I; Bleek. Verhand. Batav. Genootsch. xxiii. Chatod. p. 20; Gthr. l. cit. 2. p. 36; Wood. Nat. Hist. 3. p. 243. fig.

Hab. Luzón, Manila, Navotas; Célebes, Australia.

3 Gén. HENIOChuS, C. V.

28. H. macrolepidotus, Cuv. N. v.

(M. S. T.)

Renard, i. 3, 13, 31, 168, ii. 14. 66; Ruysch. i. n." 3; Valent. iii. figs. I8, 201, 524, 372, 409; Seba. iii. 25, 8.

Chotodon macrolepidotus, Artedi, Species, p. 94; L. Syst. i. p. 464 ; L. Gm. p. I 248; Bl. lam. 200. f. I; Lacép. ix. p. 455, lam. I I. f. 3 et lam. I 2. f. I; Bl. Schn. p. 32 I; Gthr. l. cit. 2. p. 39 .

Ch. acuminatus, L. Mus. Ad. Fried. lam. 33. f. 3.

Ch. bifasciatus, Shaw, Zool. iv. p. 342; Gronov, Syst. ed Gray, p. 75 .

Heni .chus acuminatus, Cuv. \& Val. vii. p. 98.

H. permutatus, (Bennett) Cuv. \& Val. vii. p. 99.

H. macrolepidotus, Cuv. \& Val. vii. p. 93; Faun. Japon. Potss. p. 82, lam. 44 f. I Ricards. Ichth. Chin. p. 246; Bleeker, Verh. Bat. Gen. xxiii. Chatod p. 2I. 


\section{$-t^{3} y-$}

I)iphreutes macrolepidotus, Cant. Catal. p. 159.

Hab. C. de Cebú; Amboina, Ceilan.

22. H chrysostoma, $C, V$ N. v.

(M. S. T.)

? Heniochus chrysostomus, (Parkins.) Cuv. \& Val. vii. p. y9;

Cirv. Régne Anim. Ill. Poiss. lam. to. f. 2.

H. (hrysostomus, (Solander) Voy. Beech. Zool. p. 62, lam. iъ. f. I; Gthr. l. cit. 2. p. +r.

H. melanistion, Bleeker, Banda, iii. p. 98 .

Hab. Luzon, Manila; Amboina.

4. Gén. holacanthuUs, lacḱr.

3o. H. bicolor, Cuv N. v.

(il s. T)

Renard, i. lam. 19. f. ro6.

Chetodon bicolor, Bl. t. 206. f. I; Bl Schn. p. 218.

Holacanthus bicolor, Cuv. \& Val. vii. p. I68; Bleek. Solor. $\mu$. 77; Gthr. l. c. 2. p. 50.

Hab. Luzón, Manila; Indias Orientales.

3i. H. Xanthometopon, Bleckcr. N v.

(M. S. T)

Bleeker, Sumatra, ii. p. 258 ; Gthr. l. cit. 2. p. 5 I.

Hab. Luzón, Cavite; Sumatra.

32. H. semicirculatus, Bl. N. v.

(M. S. T )

Holacanthus semicirculatus, Cuv. \& Val. vii. p. I91, lam. 183; Bleek. Amboina, vi. p. 414 (no Banka, p. 452); Voy. Coqu. Zool. Poiss. p. I73, lam. 3o. f. 3; Gthr. l. cit. 2. p. 53.

Chotodon microlepis, Bleeker, Sumatra, ii. p. 257.

Hab. C. de Mindoro; Polinesia.

5. Gén. SCATOPhagus, C. V.

33. Sc. argus, Cuv. N. v. Quitang.

(I. S. T)

Piscis stercorarius, Willughby. $A p p$. p. 2, lam. 2. f. 2.

Renard, ii. 50, 2 I ; Valent. iii. p. 403. f. I80; Ruysch, Pis-Amb. p. 33. lam. 17. f. 6; Klein, Miss. iii. p. 36. n. ${ }^{\circ} 4$; Russell, i. lam. 78 .

Stront-risch, Nienhof. Braz. Land-und Zee-Reise, p. 269, lam. 268.

Chotodon argus, L. Gm. p. I248; Bl. lam. 204. f. ı; Bl. Schn. p. $232 ;$ Shaw, Zool. iv. p. 331.

C. pairatalis, Buch. Ham. pp. I22, 372, lam. I6. f. 41 .

Ephippus argus, Cuv. Régne. Anim.

Chetodon atromaculatus, Benn. Ceyl. Fisc. p. 18.

Scatophayus argus, Cuv. \& Val. vij. p. I 30 ; Richards. Ichth. Chin. p. 245; Bleek. Verh. Batav. Genootsch. xxiii. Chatod. p. 34; Gthr. loc. cit. 2. p. 58.

Cacoduxus argus, Cant. Catal. p. 163.

Scatophagus macronotus, Bleek. Topogr. Batav. 
Sargus maeulatus, Gronov. Syst. ed Gray, p. 65.

Hab. Luzón, B. de Manila, Nasugbú, Cebú, Visayas; China.

34. Sc. srnatus. $C . V$ N. V. Qnitang.

(M. S. T.)

Cuv. \& Val. vii. p. 142; ?Richards. Ichth. Chin. p. 245; Gthr. loc. cit. 2. p. 59.

Hab. Luzón, B. de Manila, C. de Cebú; Amboina.

6 Gén. DREPANE, C V.

35. D punctata, Cur. N. v Quitang.

(M. S. T.)

Chatolon punctatus, L. Gm. p. 1243; Shaw. Zool. ir. p. 365 .

C. longimanus, Bl. Schn. p. $23 \mathrm{I}$.

C. falcatus, Lacép is. pp. $45^{2}, 470$.

Russell, láminas $79,80,8 \mathrm{r}$.

Ephippus junctatus, Cur. Regne Anim.

Fi. longimanus, Cur. Regne Anim.

D epane punctata, Cuv. \& Val. vii. p. 132, lam. I79; Richards. Ichth. Chin. p. 245, pt. Ann. \& Mag. Nat. Hist. x. 18+2, p. $28 ;$ Githr. l. cit. 2, p. 62.

D. longimana, Cuv. \& Val. vii. p. 133; Richards. Ichth. Chin. p. 246; Bleek. Verh. Batav. Genootsch. xxiii. Chatod. p. 28. Harpochirus punctatus, Cant. Catal. p. 162.

H. longimanus, Cant. Catal. p. 163 .

Hab. Luzón, B. de Manila, Mindoro; China.

\section{Fam. TOXÓTIDOS.}

I. Gén. Toxotes, Cuv.

I. T. jaculatar, $C_{t \imath z^{\prime}}, \mathrm{N}$ v.

(M. S. T)

Sciana jaculatrix, Pall. Spicil. viii p. 41, et Phil. Trans. Vol. lvi. 1766 , p. 186 , lam. viii. f. 6.

Scarus schlosseri, L. Gm. p. I282; Lacep. iv. pp. 5, 17; Shaw', Zool. iv. p. 398.

Labrus jaculatrix, Lacép iii. pp. 425, 464; Shaw. Zool. iv. p. 485. lam. 68.

Cojus chatareus, Buch. Ham. pp. 20I, 370, lam. I4, f. 34 .

Toxotes jaculator, Cuv. \& Val. vii. p. 314 , lam. 192 Cant. Catal. p. 176; Agass. Poiss. Foss. iv. p. 262, lam. H. Cuv. Régne Anim. Ill. Poiss. lam. 44, f. 3; Bleeker, Verh. Batav. Genootsch. xxiii. Chatod. p. 31 ; Gthr. l. cit. 2, p. 67.

Hab. Luzón, Batangas, Nasuglou, Cavite, Sta. Cruz, C. de Cebú; Polinesia, Molucas. 


\section{Fam. CIRRÍTINOS.}

I Gén. CIRRHITICHTHYS. BLEk.

, C. maculatus, Laćp. N. v.

(M S. 'T.)

Cirrhites maculatus, Lacep. v. p. 3; Cur. \& Val. iii. p. 69.

Labrus marmoratus, Lacép. iii. p. 492, lam. 5, f. 3.

Cirrhites maculosus, Bennet, Zool. Journ. 1s29, p. 38; Gthr. l. cit. 2. p. 74.

Hab Lızón, Cavite, Sta. Cruz; Polinesia.

2. C aureus. Temm. et Schl. $\mathrm{N}$ v.

(M. S. T.)

Cirrhites aureus, Temm. \& Schleg. Faun. Japon. Poiss. p. 15, lam. 7. f. 2. Gthr. loc. cit. 2, p. 75 .

Hab. Luzón, Manila, Navotas; China, Japon.

2. Gén. Chilodaltylus, Cuv.

3 Ch. zonatus, Cuv. N. v

Labre du Japon, Krussenst. Ryise. All. lam. 63. f. ז.

Chilodactylus z matus, Cuv. \& Val. v. p. 365 lam. I 29; Faun. Japon. Poiss. p. 64 lam. 29; Richards. Ichth. China, p. 339, Proc. Zool. Soc. 1850. p. 66, et Ann. \& Mag. Nat. Hist. 1851, vii. p. 282 ; Bleek. Verhand. Batav. Genootsch. xxvi. Japan, p. 83 ; Gthr. l. cit. 2, p. 82 .

Hab. Luzón Cavite, Sta. Cruz; China.

Fam. TRÍGLIDOS.

r. ¿én SEbASTES, C V.

I. s. pachycephalus. Tem et Sihlegr. N. v.

(M. S T.)

Temm. \& Schleg. Faun. Japon. Poiss. p. 47 lam. 20. f. 3; Richards. Ichthyol. Chin. p. 214; Gthr. l. cit. 2, p. 97.

Hab. Luzón, Cavite, Sta. Crız; China.

2. §. marmoratus, Cuv N. v.

$\left(\begin{array}{lll}\mathrm{M} & \mathrm{S} & \mathrm{T}\end{array}\right)$

Sebastes marmoratus, Cuv. \& Val. iv. p. 345; Faun. Japon. Poiss. p. 46, lam. 21, f. I, 2; Gthr. l. cit. 2, p. I04.

Holocentrus albo-fasciatus, Lacép. iv. p. 372.

Sebastes albo-fasciatus, Cuv. \& Val. iv. p. 344.

S. sinensis, M'Clell. Calc. Journ. Nat. Hist. iv. p. 397, lam. 2 I

f. 3. (cfr. Richards. Ichth. Chin. p 215).

Hab. Luzón, Manila; China. 
3. S. strongensis, $C \quad V . \mathrm{N} . \mathrm{v}$.

Scorpæna strongia, Cuv. \& Val. iv. p. 323; Quoy. \& Gaim. Voy. Astrol. Poiss. p. 688, lam. 11, f. 2; Less. Voy. Dupery. Zool. Poiss. p. 2 I 3.

Sc. cyanostigma, Bleeker, Burn. p. Ioo.

Sebastes strongensis, Gthr. l. cit. 2, p. 106.

Hab. Zamboanga, Mindanao, Marianas.

4. S minutus, $C \quad V$.

Cuv. \& Val. iv. p. 348; Gthr. l. cit. 2, p. 106.

Hab. Marianas.

5 S. polylepis, Bleek. N. v.

$\left(\begin{array}{lll}M & \mathrm{~S} & \mathrm{~T} \\ \mathrm{~T}\end{array}\right)$

Scorpæna polylepis, Bleek. Natuurk. Tydschr. Nederl. Indie, 1851 , ii. p. 173 .

Sebastes polylepis, Gthr. .l. cit. 2, p. 107.

Hab. Cebú; Sumatra.

2. Gén. SCORPANA, ARtedi.

6. Sc. guamensis, Q. et $G$.

Quoy, et Gaim. Voy. Freyc. p. 326; Gthr. l. cit. 2, p. 107. Hab. Marianas, Guam.

7. 8c. picta, C. V. $\mathrm{N}$ v.

(M. S. '1.)

Scorpæna picta, (Kuhl \& van Hass.) Cuv. \& Val. iv. p. 321 ;

Cantor, Catal. p. 4\%; Gthr. l. cit. 2, p. I14.

Hab. C. de Cebú; Célebes.

8. Sc. pol prion, Blkr. N v.

(M. $\mathrm{S}$ T)

Bleeker Sclerop. p. 21 ; et Verhand. Batav. Genestsch. xxii. Bali, p. 5, and ibid. Sclerop. p. 7; Gthr. l. cit. 2, p. II 5.

Hab. Luzon, Batangas, Nasugbú, C. de Mindoro; Borneo.

9 Sc haplodactylus. Bleek. N. v.

Scorpæna bandanensis, Bleeker, Banda, i. p. 237.

Sc. aplodactylus, Ceram. ii. p. 698; Gthr. l. cit. 2, p. 117.

Hab. Cebú; Ceram.

ı. Ec. diabolus, Cuv. N. v.

(M. S. 'T.)

Ikan satan, Renard, ii. pl. 8 f. 35; Valent. iii. p. 399, f. 170; Duhamel, Pêches, ii. sect. 5 p. 92 lam. 3. f. i.

Scorpana diabolus, Cuv. \& Vol. iv. p. 312; Richreds. Ichth. Voy". 
Sulph. p. 76 lam. 40; Bleeker, Amboina \& Ceram, p. 266; Gthr. l. cit. 2. p. 117 .

Hab. Cebú; China, Amboina.

I1. Sc gibbosa, $B l$ N. v.

(M S. T.)

Bl. Schn. p. 192. lam. 44; Trosch. Arch. Naturgesch. vi. Band. 1. p. 274; Bleek. Act. Soc. Nederl. i. Amboina, p. 31; Gthr.

l. cit. 2, p. 118 .

Hab. Luzón, Cavite, Sta. Cruz; Amboina, China.

12. Sc. cirrhosa. Cutı. N v.

(M. S. T.)

Perca cirrhosa, Chunb. Nya Handl. Stockh. xiv. 1793, p. 199. lam. 7. f. 2 .

Scorpæna cirrhosa, Cuv. \& Val. iv. p. 318 ; Faun. Japon, Poiss. p. 42. lam. I7. f. 2. 3; Bleeker, Verhaud. Batav. Geuwtsch. xxvi. p. 79. Gthr. l. cit. 2. p. 120.

S. barbata, Rï̈pp. $N$. W. Fische. p. 105. lam. 27, t. I.

?Scorpæna leonina, Richards. Ichth. Chin. p. 216.

Scorpana oxycephalus, Bleek. Sclerop. p. 20. \& Verh. Batav. Genwlsch. xxii. Sclerop. p. 7.

Hab. Luzón, Cavite, Sta. Cruz; China.

13. Sc. laniaria, C. V. N. v.

Cuv. \& Val. iv. p. $324 ;$ Gthr. l. cit. 2. p. I 21.

Hab. Marianas, Guam.

3. Gén. PTERols, Cuv.

14. Pt. volitans, Cuv. N v Bantol.

Seba, iii. 28. 1, (bad). Renard, Poiss. i. 6, 41, i. 43, 215, ii. I 5, 72; Valentyn, Ind. ii. p. 413 f. 2ro. et p. 415. f. 213; Lacep. ii. t. I7. f. 3; Russell, lam. I33.

Amboynese visch, Nieuh. Bras, Lant-en Zee-Reize, p. 268.

Gasterosteus volitans, L. i. p. 49r.

Perca, sp., Gronov. Zoophye. n." 294.

Scorpæna volitans, L. Gm. i. p. 1217;Btoch, t. 184; Bl. Schn. p. 193; Gronov. Syst. ed. Gray, p. I19; Bennett, Fisches, of Ceylon, lam. 1.

Scorpana i. ahe, Lacép. iii. p. 278.

Pterois volitans, Cuv. \& Val. iv p. $35^{2}$, lam. 88 ; Bleeker. Berhand. Batav. Genwtsch. xxii, Sclerop. p. $8 ;$ Rïpp. N. W. Fische, p. IO7; Gthr. loc. cit. 2. p. I 22.

Hab. Cagayan de Misamis, Mindanao, Paragua; China.

15. Pt. antennata, Cuv. N. v.

(M. S T)

Pseudopterus sp., Klein, Miss. v. p. 76. n. $^{\circ}$ 1. lam. 4. f. 6.

Scorpæna antennata, Bl. t. $185 ; B l$. Schn. p. 193.

Pterois antennata, Cuv. \& Val. iv. p. $36 \mathrm{r}$; Less. Voy. Co.q. Zool. - Poiss. p. 212 ; Bleek. Solor, p. 72; Gthr.il. cit. 2. p. 124. 
P. geniserra, Cuv. \& Val. iv. p. $365 ;$ Belangzr, Voy. Ind. Orient. p. 353. lam I. f. 2.

Pterois russellii, Benn. Proc. Comm. Zool. Ssc. i. p. 128. Hab. Luzón, Cavite, Sta. Cruz; China, Amboina.

16. Pt. miles, Cant N. v.

Scorpæna miles, Bennett, Fishes of Ceylon, lam. 9.

Pterois muricata, Cuv \& Val. iv. p. 363 ; Rüpp. N. W. Fishe, p. 107.

P. miles. Cant. Catal. p. 42; Gthr. l. cit. 2. p. 125.

Hab. Luzón, Cavite; Oceano Indico.

17. Pt. zebra, Cuv. N. v.

(M S. T.)

Renard, i. lam. 6. f. 4 r.

Pterois zebra, Cuv. \& Val. iv. p. 367; Régne. Anim. Ill. Poiss. lam. 24. f. I. Bleek. Amb. \& Cer. p. 265; Qusy \& Gaim. Voy. Uran. Zool. p. 329, et Voy Astrol. Poiss. p. 692. lam. II. f. 6. Gthr. loc. cit. 2. p. 126.

Hab. Cebú, Samar, Villareal; China.

\section{Gén CEntropogon, Gtur.}

I8 C. robustus, Gtir. N. v.

(M. S. T.)

Gthr. loc. cit. 2. p. 128.

Hab. Cebú; Australia.

19. C. fuscovirens, $Q$. et $G . \mathrm{N}$. v.

? Apistus fuscovirens, Quoy \& Gaim. Voy. Astrol. Poiss. p. 695.

lam. II. f. 5; Cuv. \& Val. iv. p. 409; Gthr. l. cit. 2. p. I30.

A. fuscovirens, Bleeker Amboina \& Ceram. p. 269.

Hab. Marianas; Amboina.

\section{Gén Tetraroge, Gririr.}

20 T. longispinis, $(C$ et $V$.$) N. v.$

Apistus longispinis, Cuv. \& Val. iv. p. to8; Quoy \& Gaim. Voy. Astrol. Poiss. p. 594. lain. II. f. 4.

Tetraroge longispinis, Gthr. loc. cit. 2. p. 134.

Apistes multicolor, Richards. Voy. Samar. Fishes, p. 3. lam. 4. f. 3,4 .

Hab. Luzón. Cavite, Sta. Cruz, Samar; China, Borneo.

21. T amblycephalus, Bleek N v.

(M. S. T)

Apistus amblycephalus, Bleeker, Sclerop. p. 27 ; Gthr. l. cit. 2. p. I33.

Hab. Luzón, Manila; Padang. 
$22 \mathrm{~T}$. crista-galli, Githr. $\mathrm{N}$ v.

(M. S. T.)

Apistus tanianctus, Richrrds. Voy. Samarang, Fishes p. 5 lam. +. f. I, 2. ( 10 Cuv. \& Val.)

Tetrarogi crista-galli, Gthr. loc. cit. 2. p. I3t.

Hab. Luzón, Cavite, Sta. Cruz.

23. T barbata, $C . V . \mathrm{N}$ v

(M. S. T.)

Apistus barbatus, Cuv. \& V'al. iv. p. +iz; Bleeker, Act. Soc. Nederl. i. Amboina, p. $3^{6 .}$

A. melas, Bleeker, Natuurk. Tydschr. Nederl. Ind. i. p. 26. Hab. C. de Cebú; Molucas.

24. T. tæniknotus $C \quad V . \mathrm{N} \quad$ v.

'Tanianotus latovittatus, Lacep. iv. lam. 3. f. 2.

Apistus tænianotus, Cuv. \& Val. iv. p. 4o4; [no Richards. Voy.

Samarang, Fshes lan. 4. f. I, 2;] Bleeker, Amboina, ii. p. 557.

Tetraroge tænianotus, Gthr. l. cit. 2. p. i 36.

Hab. Cebú; Japon.

6. Gén PROSOPODASYS, Cantur.

25 Pr. leucogaster, (Richard). N. v.

(M. S. ' ' )

Apistus leucoyast:r, Richards. Voy. Samar. Zool. Fishes, lam.

5 f. I, 2; Bleeker, Ambo:na, iii. p. 2 I.

Pr. leucogaster, Gthr. loc. cit. 2. p. I 4 I.

Hab. Luzòn, Cavite, Sta. Cruz; Amboina, China.

26. Г'r cottoides, $C V$. N. v.

(M. S. T)

Perca cottoides, L. Mus. Ad. Firied. ii. p. 84 .

Apistus cottuides, Cuv. \& Val. iv. P. 4Io; Richards. Voy. Samar Fishes, p. 4 lam. 3. f. 6, 7; Bleek. Batavia p. 459.

Prosopodasys cottoides, Gthr. loc. cit. 2. p. 142.

Hab. Luzón, Manila; Borneo, China.

7. Gén APLOACTIS, Tem. ext Schleg.

27. A. asp?ra. Richards, N, v.

(M. S. T)

Aploactis, Temm. \& Schleg. Faun. Japon. Poiss. p. 5i. lam. 22. f. 3 .

A. aspera, Richards, Voy. Sulph. Fishes, p. 72; Gthr. l. cit. 2. p. 142

Hab. Luzón, Cavite, Sta. Cruz; China. 
8. Gén. trichopleura, kiaup.

28. Tr. mollis, (Rickard.) N. v.

(M. S. T.)

Sthenopus mollis, Richards. Voy. Samar. Fishes, p. Io. lam. 2.

f. 6,7 .

'Ir. mollis, Gthr. loc. cit. 2. p. 143.

Hab. Luzón, Manila; China.

8 Gén. SyNANCidium, Mült.

29. sy. horridum, (Lin). N. v.

(II. S. T.)

Renard, i. 39. f, r 99.

Scorpana horrida, L. Syst. i. p. 453; Bloch, t 183 ; Lacép. iii. p. 26 I, ii. t. I7. f. 2.

Perca sp., Gronov. Zoophyl. n. ${ }^{0}$ 292. lain. I I. 1, lam. 12. f. 1. la r. I3. f. I.

Synanceia horrida, Bl. Schn, p. 194; Cuv. \& Val. iv. p. H+(;

Bleek, Verhand. Batav. Genootsch. xxii. Sclerop. p. 9.

Scorpæna monstrosa, Gronov. Syst. ed. Gray, p. i 7 .

Synanceia trachynis, Richards. Ann. \& Mag. Nat. Hist. 1842 , ix. p. 385 .

Synancidium horridum, Gthr. l. cit. 2. p. I +4 .

Hab. Luzón, Cavite, Sta. Cruz; China, Australia.

9 Gén SYNANCEIA, C. V.

30 sy. verrucosa, Bloch. N. v.

(M. s. T)

Synanceia verrucosa, Bloch. Schn. Syst. p. 195. t. $45 ;$ Rüppell, $N$.

U. Fische. v. Io9; Gthr. l. c. 2. p. 146.

Scorpæna brachio, Lacép. iii. pp. 259, 272. lam. 12. f. 1.

S. brachio, Shaw, Zool. iv. pt. 2. p. 274.

Synanceia sanyuinolenta, Ehrenb. Pasc. t. 2.

Sy. brachio, Cuv. Régne Anim.; Cuv. \& Val. iv. 447; Bleeker,

Verhand. Batav. Genootsch. xxii. Sclerop. p. 9; Cuv. Régne. Anim. Ill. Paiss. lam. 25. f. 3; Agass. Oss. Foss. iv. p. 200. lam. L.

Hab. Luzón, Manila; Polinesia.

ro. Gén. PELOR, C V.

21. P cuvieri. Gr $\mathrm{N}, \mathrm{v}$.

Pelor cuvieri, Gray, Ill. Ind. Zool. ii. lam. 20. f. 2; Richards.

Ichthyol. Sulph. p. 72. lam. 39. f. 4, et Ichthyol. China, p.

212 ; Bleeker Sumatra, ii. p. 252; Gthr. l. cit. 2. p. 150.

? P. sinense, Cuv. \& Val. iv. p. 468.

Hab. Mindana, Zamboanga; China, Sumatra. 
32. P. japonicum, $C . V . \mathrm{N} . \mathrm{v}$.

(M. S. T.)

Pelor japonicum, Cuv. \& Val. iv. p. 437 ; Foun. Japon. Poiss. p. +4. lam. 18. f. 2: Gthr. l. cit. 2. p. 151.

P. tigrinum, Richards. Ichthyol. Chin. p 212.

Hab. Luzón, Carite, Sta. Cruz; China, Japón.

ri. Gén. pudabrus, Richariss.

33 P. cottoides, Kich. N. v

(M. S. 'I'.)

Richards. 1. c. p. 13. lain. 1. f. 1, 6; Gthr. l. cit. 2. 152.

Hab. Luzón, Cavite; China.

12 Gén CENTRIDERMICHTHYS, RICHARDS.

34. C fasciatus, Hiekel. N. v.

(M. S. T.)

'Trachidermus fasciatus, Heckel. Ann. Wien. Mus. ii. 2840 , p. I6o. lam. 9. f. I, 2 .

Centridermichthys ansatus, Richards. Voy. Sulph. Fishes, p. 74. lam. 5t. f. 6-io.

Cottus uncinatus, Temm. \& Schleg. Fann. Japon. Poiss. p. $3^{\text {x. }}$ (no Reinh).

(.) fasciatus, Gthr. l. cit. 2. p. I69.

Hab. Luzón, Manila; China.

13. Gén PLATYCEphalús, Bi.

35 Pl insidiatur, $B l . \mathrm{N}$ v. Sumoc.

(M. S T.)

Cottus insidiator, Forsk. p. 25; L. Gm. i. p. 1213; Shaw. Zool. iv. p. 260 .

Callionymus indicus, L. Gm. i. p. 1153 .

Cottus spatula, $B l$. lam. 424 .

Platycephalus insidiator, Bl. Schn. p. 59; Cuv. \& Val. iv. p. 227; Rïpp. N. W. Fische, p. Iо2; Bleek. Verh. Bat. Gen. xxii. Sclerop. p. 6; Faun. Japon. Poiss. p. 39. lam. I5. f. I; Cant. Catal. p. 37. Gthr. loc. cit. 2. 177.

Pl. spatula, Bl. Schn. p. 59 .

Batrachus indicus, Bl. Schn. p. 43.

Calliomorus indicus, Lacep. ii. p. 343 .

Cottus madagascariensis, Lacep. iii. p. 248 . lam. I I. f. I, 2; Schaw, l. c. p. $26 \mathrm{I}$. lam. 26.

Russell, lam. 46 .

Colliomorus chacca, Buch. Ham. pp. 133, 373.

Platycephalus endractensis, Qusy. \& Grim. Voy. Freyc. Zool. p. 353 ; Cuv. \& Val. iv: p. 240.

Pl. chacca, Gray, Ind. Zool. ii. lam. f. 2. Hab. Luzón, B. de Monila; C ina, Amboina. 
36. P1. japonicus, Tiles. N. v.

(M. S. T.)

Tiles in Krusenst. Reise. lam. 59. f. 1; Cuv. \& Val. iv. p. 256; Faun. Jaton. Poiss. p. 40 lam. i6. f. 3; Bleeker, Verhand. Batav. Genootsch xxv. p. 28. Hab. Luzón, Cavite, Santa Cruz; China.

37. Pl. isacanthas, $C \quad V$. N. v.

Cuv. \& Val. iv. p. 246; Cuv. Régne Arim. Ill. Poiss. lam. 22. t. 3 ; Less. Voy. Coq. Zool. Poiss. p. 214; Gthr. l. cit. 2. p. 183. Hab. Luzón, B. de Manila; Buru.

38. Pl. tentaculatus, Rüpp N. v.

?? Platycephalus longiceps, Cuv. \& Val. iv. p. 255.

Pl. tentaculatus, Rïpp. N.W. Fische, p. 104. lam. 26. f. 2; Gthr. loc. cit. 2. p. I 84 .

Hab. Cebú; Mar Rojo.

39. Pl. neglectus, Troschel. N. v.

(M S. T.)

Troschel in Wiegm. Arch. 1840 , p. 272; Gthr. l. cit. 2. p. 187. Hab. Luzón, Manila; China.

4o. Pl. fasciatus, Gthr. ‘ُubi?

Hab. Luzón, B. de Manila.

41. Pl. pristiger, C.V. N. v.

(M. S. T.)

Cuv. \& Val. iv. p. 260; Quoy. \& Gaim. Voy. Astrol. Poiss. iii.

p. 685 , lam. ı. f. 5. Bleek. Ternate viii. p. 205; Gthr. l. cit.

2. p. 189 .

Hab. Luzón, B. de Manila; Célebes, Java.

42. Pl. asper, C. V. N. v.

(M. S. T.)

Cuv. \& Val. iv. p. 257, lam. 82; Faun. Japon. Poiss. p. 4o, lam. 16. t. 4,5 ; Gthr. loc. cit. 2. p. I90.

Hab. Luzón, Manila; China.

14. Gén. HOPLICHThYS, C. V.

43. H. longsdorffii, $C$. $V$.

Cuv. \& Val. iv. p. 264, lam. 8ı; Temm. \& Schleg. Faun. Japon.

Poiss. p. 156, lam. 79. f. 2; Gthr. l. cit. 2. p. I91.

Hab. Luzón, Manila; China, Japon. 
15 Gèn. TRIGla, Cuv.

44. Tr. kumu, Le'ss ct Gain. N. v.

(M. S T.)

Trigla kumu, Less. \& Garn. Coqu. Polss. lam. r9; Cuv. \& Val. iv. p. 5o; Jenyns, Zool. Beagle, Fishes, p. 27; Faun. Japon. Poiss. p. 37, lam. I4 A. f. 3; Owen, Osteol. Catal. i. p. 5!; Bleek. Verhand. Batav. Genootsch. xxvi. Japaw, p. 7t; Githr. l. cit. 2. p. 204.

I'rigla spinosa, M'Clellanl, Calc. Journ. Nat. Hist. iv. p. 39', lam. 22 f. 2.

Hab. Luzón, Mindoro; China, Australia.

16 Gén. DACtyi.opterus, Lacép.

45. D orientalis, $C_{u v} \mathrm{~N}$. v.

(M. S. T)

Renard, i. lain. ro. f. 65; Valent. iii. f. 35 ; R:tssell. lam. 161.

Dactylopterus orientalis, Cuv. \& Val. iv. p. 134, lam. 76; Richardson, Ichth. Chin. p. $218 ;$ Bleek. Amb. \& Cer. p. 264. Temm. \& Schleg. Faun. Japon. Poiss. p. 37, lain. I5 A; Gthr. l. cit. 2. p. 222.

D. orientalis et japonicus, Bleeker, Japan, p. 395, et Verh. Bątav. Genootsch. xxvi. Japan, p. 72.

Hab. Lủzón, Mindoro; China, Amboina.

\section{Fam. TR.LQUÍNIDOS.}

1. Gén. URANOSCOPUS, Cuv
I. U bicinctus, Temt. et Sichl. N. v.
(M. S T.)

Temm. \& Schleg. Faun. Japon. Pouss. p. 26, lan. 10, B.; Bleek. Act. Soc. Nederl. ii. Amboinx, p. 41; Gthr. l. cit. 2. p. 228. Hab. Luzón, Cavite; China, Amboina.

2. U. asper, Tem. et Schl. N v.

(M. $\mathbf{S}$ T.)

Temm. \& Schl. Faun. Japon. Poiss. p. 26, lam. 9. f. 1. Richards. Ichth. Chin. p. 21 i; Gthr. loc. cit. 2. p. 228.

Hab. Luzón, Manila; China.

2. Gén. PERCIS, Br.

2. P. cylindrica, Block. N. v.

(M. S. T.)

Seba, iii. 27, I6.

Sciana cylindrica, Bloch, lam. 299. f. I; Lacep. iv. p. 314.

Bodianus sebæ, Bl. Schn. p. 335 .

Percis cylindrica, Cuv. \& Val. iii. p. 267; Bleek. Bandx, i. p. 235 (no Rüpp, Gthr. l. cit. 2. p. 239.

Hab. Cebú, Luzón, Cavite; China, Amboina. 
3. Gén. Sili,AGO, C. V.

4. S. sihama, Forsk. N. v.

(M. S. T )

Atherina sihama, Forsk. p. 70.

Sciæna malabarica, Bl. Schn. p. 8r. t. 19.

Platycephalus sihamus, Bl. Schn. p. 60.

Russell, t. I 13 .

Sillago sihama, Rüpp. Atl. Fische, p. 9 lam. 3. f. I; Gthr. l. cit. 2, p. 243 .

S. amta, Cuv. \& Val. iii. p. 400; Bleeker Perc. lam. 6r.

S. erythcea, Cuv. \&. Val iii. p. 409.

S. malabarica, Cant. Catal. p. 2 i; Bleek. Bali, iii p. I57.

Hab. Luzón, Manila; China, Amboina.

5. 8. maculata, Quoy et Gaín.

Sillago maculata, Quoy. \& Gain. Voy. Freyc. Zool. p. 26 I lam. 53 f. 2; Cuv. \& Val. iii. p. 4II; Bleeker. Perc. p. 62 et Babi, iii. p. I6I; Gthr. l. cit. 2, p. 245.

s bassensis, Cur. \& Val, iii. p. 412*; Quoy \& Gaim. Voy. Astrol. Poiss. p. 672 lam. I f. 2.

Hab. Marianas; Australia.

4. Gén. LatiluUs, C. V.

6. L. argentatus, C. $V$ N. v.

(M. S. T.)

Coryphæna sina, Bl. Schn. p. 296.

? Coryphæna sinensis, Lacép. iii. pp. I76 209.

Iratilus argentatus, Cuv. \& Val. v. p. 369, ix. p. 495; Faun. Japon, Poiss. p. 63 lam. 28 f. 2; Bleek. Verh. Batav. Genootsch. xxvi. p. 85 ; Gthr. l. cit. 2, p. 252.

Hab. Luzon, Cavite, Santa Cruz; China.

5. Gén. PSEUdOCHROMIS, Rüpp.

7. P. adustus, Müll et Tros. N. v.

(M. S. T)

Müll. \& Trosch. Hor. Ichthyal. iii. p. 23 lam. 4 fig. 3; Gthr. l. cit. 2, p. 258.

Hab, Luzon, Cavite, Samar.

Fam. ESCIÊNIDOS.

1. Gén. UMBRINA, Cuv.

I U. russellii, Cuv N. v.

(M. S. T.)

Russell, lam. in 8 .

Umbrina russelli, Cuv. \& Val. v. p. 178; ? Richards. Ichth. Chin. p. 226; Cant. Catal. p. 7 I; Gthr. l. cit. 2, p. 278. 
Vmbrina kuhlii, Cus. \& Val. v. p. I79; Rleek. I'erhand. Batar'. Genootseh. xxiii. Scizen. p. 19.

Hab. Luzon, Manila; China.

2. Gén. SCIENA, Crvv.

2. Sc. crocea, Richards. N. v

(M. S. T)

Richardson, Ichth. Chin. p. 224; Gthr. l. cit. 2, p. 284.

Hab. Luzón, Cavite; China.

3. Sc diacenthus Lacip $\mathrm{N}$.

(M S. T.)

Lutjanus diacanthus, Lacép. iv. pp. 195, 244.

Russell, ii. lam. I I $5,116$.

Bola chaptis, Buch. Hamm. p. 77 lam. 1o f. 25:

Johnius cataleus, Cuv. Règne Anim.; Cuv. \& Val. v. p. 128.

J. chaptis, Cuv. \& Val. v. p. I 30.

Corvina catalea, Belanger, Voy. Ind. Orient. Zool. p. 360; Richards. Ichth. Chin. p. 226; Bleek. Verhand. Batav. Gen. xxiii. Scion. p. i 8.

Johnius platycephalus, (Kuhl. \& Van Hasselt.) Cuv. \& Val. v. p. 132 .

Sciæna maculata, Gray. Ind. Zool. ii. lam. 83 f. I.

Johniı s diacanthus, Cant. Catal. p. 67; Bleek. Java, iv p. 326.

J. valenciennii, Eydoux \& Souleyet, Vov, Bonite, Zool. i. p. 159 . pl. I f. 2.

Sciæna diacanthus, Gthr. l. cit 2, p. 290

Hab. Luzón, Manila; China, Malaya.

4. Sc. $\sin \mathbf{a}, C \quad V . \mathrm{N}$ v.

$\left(\begin{array}{lll}M & S & T\end{array}\right)$

Johnius sina, Cuv. \& Val. v. p. 122.

Corvina sina, Belang. Voy. Inaes Orient. Zool. p. 359; Faun. Japon. Poiss. p. 58 lam. 24 f. 2; Bleek. Verhand. Batav. Genootsch xxvi. p. 82.

Sciæna sina, Gthr. l. cit. 2, p. 292.

Hab. Luzón, Manila; China, Malaya.

5. Se. dussumieri, $C . V$. N. v.

$\left(\begin{array}{lll}M & \text { S. } & \text { T.) }\end{array}\right.$

Corvina dussumieri, Cû. \& Val. v. p. Ir9; Règne Anim. Ill. Poiss. pl. 28 f. 2.

Johnius dussumieri, Cant. Catal. p. 64.

Sciana dussumieri, Gthr. l. cit. 2, p. 292.

Hab. Luzón, Cavite, Santa Cruz; China, Malabar.

6 Sc. borneensis, (Bleeker.) N. v.

(M. S. T )

Otolithus borneensis, Bleeker, Borneo, ii. p. 268; Gthr. l. cit. . 2, p. 294.

Hab. Samar; Borneo. 
3. Gén. CORvina, Cuv.

7. C. miles, Cuv.

(M $\mathbf{S}$ T.)

Holocentrus soldado, Lacép. iv. pp. 344, 373.

Russell. lam. i 7 .

Corvina miles, Cuv. \& Val. v. p. 94, ix. p. 479; Bleek. Verhand. Batav. Genwthsch. xxiii. Scion. p. 17; Gthr. l. cit. 2, p. 3\%). C. soldado, Cant. Catal. p. 70.

Hab. Luzón, B. de Manila; Ceilan.

8. C coitor, Cuv. N. v.

$\left(\begin{array}{lll}M & \mathrm{~S} & \mathrm{~T}\end{array}\right)$

Bola coitor, Buch. Ham. pp. 75 \& 368. lam. 27. f. 24.

Corvina coitor, Cuv. \& Val. v. p. I 6 ; Gthr. l. cit. 2, p. 301.

C. nalla-katchelec, Richards., Ichth. Chin. p. 226.

Hab. Luzón, Cavite, Sta. Cruz; China.

9. C belangerii, $C, V . \mathrm{N}$ v.

( $\mathrm{M}$ S $\mathrm{T}$.)

Corvina belangeri, Cuv. \& Val. v. p. 1 20, cop, in Belanger, p. 358 ; Gthr. l. cit. 2, p. 303 .

?C. kuhlii, Cuv. \& Val. v. p. ıгі; Bleeker, Verhand, Batav. Genwtsch. XXIII. Scian.

Iohnius belangeri, Cant. Catal. p. 65.

Corvina sampitensis, Bleeker, Borneo, vi. 421 .

Hab. Luzón, Cavite, Sta. Cruz; Borneo.

ro. C sémiluctuosa, $C \quad V$. N. v.

(M. S. T.)

Cuv. \& Vall. v. p. 97. lam. 106; Gthr. l. cit. 2. p. 304.

Hab. Luzon, Manila; China, Pondichery.

I1. C. olvida, C. V. N. v.

(M. S. T.)

Cuv. \& Val. v. p. 93. cop. in Belanger. Voy. Ind. Orient. Zool. p. 355. Gthr. l. cit. 2. p. 304.

Hab. Luzón, Manila; China.

4. Gén. OTOLIT..US, Cuv.

12. O. tridentifer, Richards. N. v.

(M. S. T.)

Richards. Ichth. China, p. 225; Gthr. l. cit. 2. p. 305.

Hab. Luzón, Manila; China.

13 O. ruber, $C u v \mathrm{~N}$.

(M. S. T)

Johnius ruber, $B l$. Schn. p. 75. lam. i 7 .

Otolithus ruber, Cuv. \& Val. v. p. 60. lam. 102; Cant. Catal.

p. 59; Gthr. loc. cit. 2. p. 309.

Hab. Luzón, Manila; Malabar. 
14. O. leuciscus, Gthr. N. v. Curvina.

Hab. Luzón, B. de Mánila.

15. O argenteus, (Kuhl. \& $v . H: z s s) \mathrm{N}$ v.

(M. S. T.)

(Otolithus argenteus, (Kühl. \& v. Hass. Cwv. \& Vall. v. p. 62; Richards. Ichth. China, p. 225; Bleek. Verhand. Batav. Genootsch. xxiii. p. I5; Gthr. l. cit. 2. p. 3 10.

? Sciæna japonica, Faun. Japon. p. $5^{8}$ lam. 24 f. I.

Hab. Luzon, Cavite; China, Java.

5. Gén. COllicthys, Githr.

16. C. lucida, Richards. N. v.

(M. S. T.)

Sciæna lucida, Richards. Ichth. Voy. Sulph. p. 87. lam. 44. f. 3,4 .

Collichthys lucida, Gthr. l. cit. 2. p. 312.

Hab. Luzon, Manila; China.

17. C. bi urita Cantor. N. v.

(M. S. T )

Otolithus biauritus, Cantor, Catal. Malay. Fishes, p. 57; Bleek. Act. Soc. Sc. Indo-Nederl iii. Borneo, p. 3.

C. biaurita, Gthr. l. cit. 2. p. 315.

Hab. Luzon, Manila; Borneo.

\section{Fam. POLINÉMIDOS.}

I Gén. POlynemus, Lin.

I. P. paradiseus, $C . V . \mathrm{N}$ v.

(M. S T )

Edwards, Av. p. 208. t 208; Russell, ii. f. 185.

Polynemus paradiseus, L. Syst. Nat. i. p. 140r. [no Bloch $]$; Gthr. l. cit. 2. p. 320.

Polynemus, n. ${ }^{\circ}$ 398, Gronov. Zoophyl.

P. risua aureus?, toposui?, Buch. Ham. Gang. Fishes, p. 228.

P. longifilis, Cuv. \& Val. iii. 365, vi. p. 512; Bleeks, Borneo, ii. p. 268 , et Borneo, vi. p. 4 r 8 .

Hab. Luzon, Cavite, Sta. Cruz; Siam.

2. P. multifilis, Schleg. N. v.

(M. S. T.)

Polynemus multifilis, Schleg. Faun. Japon. Poiss. p. 29. et Bydrage tot de dierkunde, 1851, p. 12, cum tab; Gthr. l. cit. 2. p. 320 .

P. quatuordecimfilis, Pel. Bydrage tot de diercunde, 1851 , p. 9.

P. polydactylus, Bleeker, Borneo, vi. p, 417.

Hah. Samar, Borongan; Borneo. 
3. P. multiradiatus, Gthr. N. v.

Githr. loc. cit. 2. p. 324.

Hab. Luzon, Manila; China.

4. P. lineatus, Gthr. N. v.

(M. S. T.)

Gthr. loc. cit. 2, p. 327.

Hab. Luzón, Manila; Amboina.

5 P. pleb`jus, Gm. N. v.

(M. S. T.)

Polynemus plebejus, Gm. L. Syst. p. 1401; Bloch, t. $400 ; B /$.

Schn. p. I7; Shaw. Zool. v. lam. 1 25; Cuv. \& Val. iii. p. $3^{80(\text {; }}$ (no Fauna Japon. lam. II. f. I); ?Bleeker, Perc. p. 58 ; Richards. Ann. \& Mag. Nat. Hist. 1842, ix. p. 210 ; Canlur, Catal. p. 27; Glhr. l. cit. 2. p. 329.

P. lineatus, Lacép. v. la:n. I 3, f. 2.

Bruce, Voyage, t. 4I.

Polynemus niloticus, Shaw. Zool. v. p. I5 I.

'Irigla asiatica, Forst. Descript. Anim. cur. Lichtenst. p. $23^{6}$. Hab. Luzón, Currimao, Ilocos; Amboina.

6. P. tetradactylus, Shaze. N. v.

(M. S T ;

Russell, lam. 183, Renard, Poiss. f. I 47.

Polynemus tetradactylus, Shaze, Zool. v. p. 155; Cuv. \& Val. iii. p. 375, vii. p. 5I4; M'Clell. Journ. As. Soc. viii. I\$39, p. 206; Richards. Ichth. China, p. 218; Bleeker, Perc. p. 57; Cuv. Régne Anim. Ill. Poiss. lam. I 9, f. I; Cantor. Catal. 1). 25; Gthr. l. cit. 2, p. 329.

P. teria, Bueh. Ham. pp. 224, 38I; Gray, Ind. Zool. i. lam. 92 f. 2 .

P. salliah et quadrifilis, Cantir Journ. As. Soc. v. p.' I66. Hab. Luzón, Manila; China, Borneo.

\section{Fam. ESFIRÉNIDOS.}

1. Gén. SPhYRæNa, Artedi.

I. S. jello, Cuv.

Jellow, Russell, lam. i 74 .

Sphyræna Jello, Cuv. \& Val. vi. p. 349; Bélanger, Voy. Zool. p. 346, lam. I, f. I, Rüppsll, N. Wirb. Fische, p. 98; Bleeker, Perc. p. 56; Cantor, Catal. p. 24; Bleeker, Batjan. p. 369, et Sphyran. p. 12; Gthr. l. cit. 2. p. 337.

Hab. Cebú; China, Amboina.

2. Sp. commersonii, C. V. N v. Batalai.

(M. S. T.)

Renard, Poiss. i. t. 4o f. 202 ; Valen'yn; Ind. iii. p. 369 , f. 70. 
Siphyrana commersonii, Cuv. \& Val. iii. p, 352; Bleeker, Perc. p. 5.5. Java, p. +25, et Sphyran. p. 15; Githr. l. cat. 2, p. $33^{\mathrm{x}}$. Has. Luzún, B. de Manila, Cebú; Java.

3. Sp. obtusata, Cuv. $\mathrm{N}$ v. $\quad$ (M. S. T)

Renard, Poiss. i. fo, 202; Valentyn, Ind. iii. p. 369, n. ${ }^{70 .}$

: Siphyrana chinensis, Lacép v. p. 321, t. 10. f. 2.

S. obtusata, Ciuv. \& Val. vl. p. 350; Bleeker, Perc. p. 56, Sphyrau.

p. 17, et Batjan, p. $36+$; Cant. Catal. p. 24 Finun. Japon.

p. 33, lam. 1 3, f. 2. Gthr. l. cit. 2, p. 339.

Hab. Luzón, Manila; Amboina.

4. Sp esp. N. v.

( $\mathbf{A}$ S. ' ' $)$

Hab. Luzón, Cavite, Sta. Cruz.

\section{Fam. TRIQUIÚRIDOS.}

1. Gén. TRICHIURUS, LiN.

I. T. savala, Cuv. N. v. Pez espada.

(M S. T.)

'Trichiurus savala, Cuv. Régne Anim.; Cuv. \& Val. viii. p. 25t, lam. 224; Cant. Ann. \& Mag. Nat. Hist. ix. p. 15, et Catal. p. I I 5; Cuv. Régne Anim. Ill. Po ss. lam. 68; Bleeker, Makr. Verh. Bat. Gen. xxiv. p. 4I; Gthr. loc. cit. 2 , p. 347.

'I. armatus, Gray, Zool. Misc. p. 9, et Ill. Ind. Zool. lain. f. I; Richards. Ichth. China, p, 268.

Hab. Luzón, B. de Manila, Cavite; China.

2 T. muticus? Gr. N. v.

(M. S. '1.)

Trichiurus muticus, Gray, Zool. Misc. p. ro; Griff. Anim. Kingd. Fisches, p. 349, la.n. 6. f. 2; G:hr. l. cit. 2, 348.

I. intermedius, Gray; Zool. Misc. p. Io; Richxrds. Ichth. Chinx. p. 268.

Hab. Luzón, Cavite, Sta. Cruz; China.

3 Tr. haumela, Forsk. N. v Balila.

(M. S. T.)

Willughby, App. lam. 3. f. 3 .

Clupea haumela, Forrsk. p. 72 ; L. Gm. p. 1408.

'Trichiurus lepturus, Lacép. ii. lam. 7, f. I, (no Cuv.)

Russel, lam. 4r.

Trichiurus ahumela, Bl. Schn. p. 518; Cuv. \& Val. viii. p. 249; Rüpp. N. W. Fische, p 41; Cant. Catal. p. I13; Bleek. Makr. Verh. Bat. Gen. xxiv. p. 41; Gthr. l. cat. 2, p. 348.

Hab. Luzón, B. de Manila, Cavite, Sta. Cruz; Oseano Indico.

4. $\operatorname{Tr}$ esp. $\mathrm{N}$ v.

(M. S. T)

Hab. Luzón, Cavite, Sta. Cruz. 
2. Gén, THYRSITES, C. V.

5. Th is. $\times$ v

(M. S T)

Hab. Luzon, Cavite, Sta. Cruz.

Fam. ESCÓMBRIIOS.

I. Gén, SCOMBER, Cuv.

sc microlepidotus, Krïpell. $\mathbf{N}$ v. (M. S. T.)

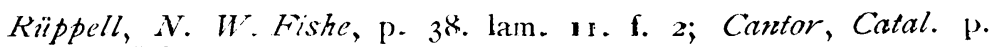
105; Gthr. l. cit. 2. p. 361 .

Hab. Luzon, Cavite, Sta. Cruz, Batangas, Nasugbú; China.

2. Gẹ́n. PELAMIYS, C. V.

2. I'. orientalis, 'Tim. et Schless. N. v.

(M S. T.)

Tomm. \& Schleg. Fani. Japon. Poiss. p. 94, Iam. 52; Gthr. l. cit. 2. 1). 368 .

Hab. Luzon, Cavite, Santa Cruz; Japon.

3. Gén. Cyibium, Cuv.

3. Cy. guttatum, Cuv N. v.

(M. S. T.)

Valent. iii. f. 505.

Scomber guttatus, Bl. Schn. p. 23 lam. 5 .

Russell, ii. p. 26, lam. 137.

Scomber leopardus, Shaw. Zool. iv. p. 591.

Cybium guttatum, Ciro. \& Val. viii. p. I73; Richards. Ichth. Chin. 1. 208; Cant. Catal. p. 11; Bleeker, Verh. Bat. Gemotsch. xxiv. Makr. p. 38; Gthr. Cat. B. M. 2. p. 37 I.

(y. kuhlii, Cuv. \& Val. viii. p. 178.

Hab. Luzón, Manila; China.

4. Gén. eCheneis, drtedi.

4. F, albescens, T.N. v.

(M S. T)

Temm. \& Schleg. Faun. Japon. Poiss. p. 272, lam. s 20. f. 3; Gthr. l. cit. 2. p. 377 .

Hab. Luzón, Cavite; China.

5. E. brachyptera, Lowe.

Remora, Catesby, Hist. Natur. South. Carol. ii. p. 26. lam. 26. Echeneis Catesby?, Loze, Trans. Zool. Soc. ii. p. I9r

E. brachyptera, Losce, Proc. Zool. Soc. 1839 , p. 89. et 7 ransc. 


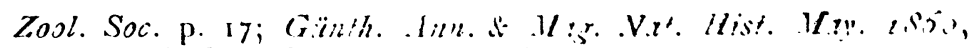
p. 399; id. Cat. B. .H. 2. p. 37's.

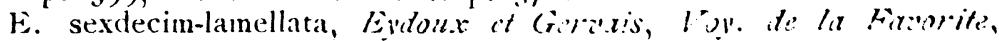
v. Zool. p. 77, lam. 3i.

?E. quatuorlecim-lamellatus, Storer, Report Fishes. Massidin. p. 155.

E. pallida, Temm. \& Schleg. Faruiz. Japon. Poiss. p. ż, lam. 20. f. 2,3 .

E. nieuhofii, Ble k. Sumatrit, ii P. 279.

Hab. Paragua, P. Princesa; C'hina.

.6 E. naucrates. I. N. v.

II. S. T.

Echeneis s. Remora, Aldratandi, iii. cap. 22. p. 335; Joustom, Thaumatogr. i. :it. 1. cap. 2. alt. t. lain. t. f. 3. lam. 25. $t$. 8; Marcgr. Iter. Bros. p. rso; ? Gotiorff. Liunstlammer, lam. 25. f. 2; Willughby, p. irg. lam. G. 8. f. 2; Ray, Syn. p. 7ו; Ruysch, Tha atr. Univ. p. 7. lam. t. f. 3, lam. 34 f. s; Seha, iii. p. roz. lan. 33 f. 2 ; Duturtre, Hist. Gén. des Antilles, ii. p. 299. fig. opp. p. $222 ;$ Brown, Hist. Jamaica, 1. +4.3.

E. naucrates, L. Syst. i. p. $+4^{6}$; Hasselg. Iter l'alest. p. $32 t$ Schoepff. Schritf. Gesellsch. Vaturf. Frennde. Berlin, rii'. . p. I45; Bl. ii. г3 . lam. 171; Bl. Schn. p. 239; Lacép. iii. lain. It6, r62. lam 9. f. 2; Shazo. Zool. iv. p. 209, la.n. 3i; Cur. Régne Anim; Storer, Rep. Fishes. Massach. p. I53; Mitch. Trans. Lit. \& Phil. Soc. Netu-York; i. p. 377; Richards. Faun. Bor. Amer. ii. p. 266, et Ann. \& Mag. N.t. Hs st. xi $1 \aleph^{2}+3$, p. 498; Cantor, Catal, 1). 199; Fitun Japon Poiss. p. 270, lam. I 20 , f. I; Richards. Ichthyol. China, p. 203; Guichen'Explor. Algér. Poiss. p. I I ; W bb. \& Berthel. Iles. Canar. Poiss. p. 87; Giunth. Ann. \& Mag. Nat. Hist. May. 1860, 1. 395; Gtrh. l. cit. 2. p. $38+$.

E. sp. Gronov. Zoophy! p. 75. n. ${ }^{\circ}$ 252 ct Mus. Ichtyhol. i. 1. ı 3. n. ${ }^{\circ} 34$; Klein, Miss. Pisc. iv. p. 5 1. n. 2.

Pegador, Parra p. 94, lam. 66. f.

Ala Mottah, Russell, i. p. 39, pl. 49.

Echeneis albicauda, Mitch. Ann. Mouth. Mag. ii. p. 244.

E. lunata, Bancroft. Proc. Comm. Zool. Soc. i. p. 134, et Zool. Journ. v. p. 4I I, lam. I 8 .

E. australis, Griff. Anim. King. Pisc. lam. opp. p. 504; Bennett. Whaling Voyage, ii. p. 273 .

E. vittata, Lowe, Proc. Zool. Soc. 1839, p 89, $185^{\circ}$, p. 252, et Trans. Zool. iii. p. 17.

Echeneis vittata, Rüpp. N. W. Fische, p. 82.

li. albicanda, pt., Dekry, New. York. Faun. Fishes, p. zo7. lam. 54. f. 177 .

E. fusca, Gronoy. Syst. ed. Gray, p. 92.

Skeleton, Agase. Recherches. Poiss. Foss. iv. tab. G. Hab. Luzón, B. de Manila; China. 
5. Gén. Stromateus, Artedi.

7. str argenteus, $B l$. N. v.

(M. S T.

Stromateus argenteus, $B l$. xii. p. 92 . lam. $421 ;$ Saw. Zool. iv. p. I Io; Cuv. \& Val. ix. p. 393; Cant. Catal. p. 142; Richards. Ichth. Chin. p. 272; Gthr. l. cit. 2. p. 400 .

Russell, pp. 31, 35. lam. 42.

Stromateus candidus, Cuv. \& Val. ix. p $39 \mathrm{r}$; Bélanger, Voy. ['. 369. lam. 2. f. 2 .

Sitr. securifer, Cuv. \& Val. ix. p. 394. lam. 273 Cant. Amn. \& Mag. Nat. Hist. ix. p. 15 ; Richards. Ichth. Chin. p. $2 \dot{7}$.

:St. punctatissimus, Schleg. Fuun. Japon. Po:ss. p. 1 21 . lam. 65. Hab. Luzón, Cavite; China.

8 str. niger, Bloch. N. v.

(M. S. T.)

Renard. i. lam: 5. f. 38; Russell, i. p. 32. lam. 44.

Stromateus niger, Bloch, xii: p. 93, lam. 422; Bl: Schn. p. 492. tab. 93; Shaw, Zool. iv. p. 111 ; Cuv. \& Val. ix. p. $385 ;$ Cant. Ann. \& Mag. Nat. Hist. ix. p. 15, et Catal. p. I39; Richards. Ichth. Chin. p. 272; Bleeker, Markr. p. 370, et Verh. Bat. Gen. xxiv. Markr. p. 77; Gthr. l. cit. 2. p. 40I. Str. paru, Bl. v. p. 75;Bl. Schn. p. 49r; Lacép. ii. p. 3I9; Shaw, Zool. iv. p. ro8.

Str. fiatola, $B l$. lam. 160 .

Apolectus stromateus, Cuv. \& $\mathrm{Kal}$. viii. p. 439. lam. 238; Cant. Catal. p. 123 .

? Seserinus vachellii, Richards. 'chth Chin. p. 273. Hab. Luzon, Cavite, Sta. Cruz; China.

\section{Gén BRAMA, Risso}

9. B. dussumieri, $C . V . \mathrm{N}$ v.

(M. S 'T )

Cuv. \& Val. vii. p. 294; Gthr. l. cit. 2. p. 409.

Hab. Luzón, Cavite, Samar; China.

7. Gén MENE, I,ACFr.

so M. maculata, Cuv N. v.

(M. S T)

Zeus maculatus, $B l$. Schn. p. 95. lam. 22.

Mene anna carolina, Lacep. v. pp. $47 \mathrm{p}, 480$. lam. I4. f. 2.

Russell, i. p. 47. lam. 60.

Mene maculata, Cuv. \&. Val. x. p. 104. lam. 285 ; Faun. Japon. Poss. p. 1 27. lam. 67. f. 3; Richards. Ichth. Chin. p. 276; Cant. Catal. p. I 54; Bleek. Verh. Bat. Gen. xxiv. Makr, p. 86; Cuv. Régne. Anim. Ill. Poiss. lam. 62. f. 2.

thab. Luzón, B. de Manila, Cebú; Península Malayana. 


\section{Fam. CARÁNGIDOS.}

I Gén. CARANX, C. V.

1. C. restus. Richards. N. v.

Ichth. China. p. 274; Gthr. l. cit. 2. P. 422.

Hab. Luzon, Cavite, Sta. Cruz; China.

2. C. margarita. Richards N. v

Richards, Ichth. Chin. p. 276.

Hab. Luzón, Cavite; China.

3 C. $\operatorname{sp} \mathrm{N}$.

(M. S T.)

Hab. Luzon, B. de Manila.

4. C. rottleri. Rụ̈p. N. v. Atun, Sábalo.

(M. S. T.)

? Scrmber cordyln, L. Syst. Nat. i. p. $4^{8} 3$; L. Gm. p. ч $36 z$; Bt. Schn. p. 23.

S. rottleri, Bl. x. p. 40. lam. 346; Bl. Schn. p. 25; Shaw. Zool. iv. p. 598 .

Russell, ii. p. 33. lam. I 43 .

? Scomber guara, Lacep. ii. p. 6o4.

Caranx rottleri, Rüpp. Atl. Fische, p. I02, et $N$. W. Fische, p. 48 ; Cuv. \& Val. ix. 29; Rüpp. N. W. Fische, pp. 48, 52; Richards. Ichth. China p. 273; Cant. Catal. p. 124; Gthr. l. cit. 2. p. 424.

Megalaspis rottleri, Bleeker. Verh. Bat. Gen. xxiv. Makr. p. 49. Hab. Luzon, Manila; China.

5. C. maruadsi, $T_{e} m m \mathrm{~N}$ v.

(M S. T.)

Caranx sanctæ Helenæ, Cuv. \&. Val. ix. p. 37.

C. maruadsi, Temm. \& Schleg. Faun. Japon Poiss. p. iog. lam. 58. f. 2; Gthr. l. cit. 2. p. 428.

Decapterus maruadsi, Bleeker, Verh. Bat. Gen. xxvi. Japan, p. IoO.

Hab. Luzón, Manila; China.

6.

C. lioglossus, Gthr: $\mathrm{N}$ v.

$(\mathrm{M} \quad \mathrm{S} \quad \mathrm{T})$

Leioglossus carangoides, Bleeker, Makr. p. 367, et Verhand. Batav. Genoolsch. xxiv Makr. p. 70 .

Caranx lioglossus, Gthr. loc. cit. 2. p. 432 .

Hab. Luzón, B. de Manila; China.

7. C. calla, $V . C$. N. v.

(M. S. T.)

Cuv. \& Val. ix. p. 49; Gthr. l. cit. 2. p. 433.

Hab. Luzón, B. de Manila; China. 
8. C. malabaricus, Cuv. N. v.

II. S T;

Scomber malabaricus, $B l$. Schn. p. 31 .

Russell, p. 37. lam. i 50 .

Caranx caruleo-pinnatus, Rüpp. Atl. Fische, p. ıоo, et $N$. II. Fische, p. 47. 1nn. I3. f. 2. (no Cuv.)

C. malabaricus, Cuv. of Val. ix. p. 121; Richards. Ichth. Chin. p. 275 ; Cant. Cotal. p. 128 ; Gthe l. cit. 2. p. $43^{6}$.

Caranroides talamparah, Bleeker, Verh. Bat. Géir. xxiv. Mark. p. 64 .

Hab. Luzón, Cavite, Sta. Cruz; China.

(. C leptolepis, Cur. $\mathrm{N}$ v

(M S. T)

Caranx leptolepis et mertensii, Cuv. \& Val. ix. pp. 63, 64.

C. leptolepis, Gant. Catal. p. I 27; Gthr. l. cit. 2. p 440.

Leptaspis leptolepis, Bleek. Verh. Bat. Gen. xxir. Makr. p. 7 I. Selaroides leptolepis, Bleek. l. c. p. 87.

Hab. Luzón, Manila; Archipiélago Indico.

10. C. speciosus, Lacép. N. v.

(M. S. T)

Scomber speciosus, Forsk.p. 54; L. Gm. p. I332; Shaw. Zool. iv. p. 603 .

Caranx speciosus, Lacép. iii. p. 72. lam. I. f. I; Gthr. l. cit. 2. p. $44+$.

Russell, ii. p. 36. lan. I 49 .

C. speciosus, Cuv. \& Val. ix. p. I 30 ; Cant. Catal. p. I 33 ; Peters. Wiegm. Arch. 1855 , p. 245 (no Rïpp.)

C. petaurista, Geoffr. Descr. Eg. pl 23. f. I. (no Rïpp).

Guathanodon speciosus, Bleek. Verh. Bat. Gen. xxiv. Makr. p. 72 .

Caranx poloosoo, Richards. Voy. Ereb. \& Terr. Ichth. lam. 58. f. 4,5 .

Hab. Luzón, B. de Manila; Madras, Borneo.

11. C. melampigus, Cuv. N. v.

(M. S. T.)

:Russell, ii. lam. I 45

Caranx melampygus, Cuv. \& Val. ix. p. iा6; Bleek. Gilolo, p. $5^{\text {}}$. Hab. Luzon, Manila, Nasugbú, Batangas; Polin sia.

I2. C. jarra, Cuv. N. v.

(M. S. T.)

Iarradanre parah, Rusell, ii. p. 35. lam. I47.

Caranx jarra, Cuv. \& Val. ix. p. ro9; Bleek. Verh. Bat. Gen. xxiv. Makr. p. 58; Gthr. l. cit. 2. p. 446.

Hab. Luzón, Cavite, Sta. Cruz, Nasugbú, Batangas; Amboina.

13. C. Sippos, Lin. N, v.

(M. S. T.)

Guara tereba, Marcgr p. 172; Seba, iii. 27. 3. 
Scomber hippos, L. Syst. Vat. i. p. $+9+$ ( 110 Mitch); Bl. Schn. p. 28; Forst. Descr. Anim. cur. Licht. p. 199.

C. Kleinii, Bl. lam. 347. f. 2; Bl. Schn. p. 3o.

Russell, ii. lam. its.

Caranx fallax, Cuv. of Val. ix. p. 95; Casteln. Anim. nouv. ou rares, Poiss. p. 22; Guichen. Poiss. in Ramon de la Sagra, Tist. Cuba, p. I1 1 .

(.) sem, Cav. \& Val. ix. p. io5.

C. forsteri, Cuv. \& Val. ix. p. Io7; Cant. Catal. p. I27; Bleek. Verhand. Batav. Genootsch. xxil. Makr. p. 57; et Natunrk. Tydschr. Nederl. Ind. 1852, iii. p. $16+$.

C. sexfaciatus, Quoy, of Gaim. Voy. Freyc. p. $35^{8}$ lam. 05. f. t; Cuv. \& Val. ix. p. ino.

?Caranx peronii, Cuv. \& Val. ix. p. 112.

C. lessonii, Cuv. \& Val. ix. p. II (no Bleek.); Less. Voy. Coq. Zool. Poiss. p. 11 5. lam. 31. f. 1.

C. belangerii, Cur. \& Val. ix. p. 116 .

C. hippos, Gthr, loc. cit. 2. p. +49 .

Caranx defensor, Dekay, New-York. Fauna, Fishes, p. 120, lam. 24. f. 72 ; Holbr. Ichth. S. Caral. p. 85, lam. 12. f. 1.

C. flavo-coruleus, Sclheg. Fann. Japon. p. 110 lam. 59 f. 2.

(. parapistes, Richards. Ichth. Voy. Ereb. \& Terr. p. 136 lam. 58 f. 6,7 .

Hab. Luzon, Bat ngas, Laguna de Taal, Cebú.

I 4 . atropus. Cantor. $\mathrm{N}$ v.

(M. S. T )

Brama atropus, Bl. Schn. p. 98, t. 23.

Russell, ii. p. 38 lam. I 52 .

Caranx nigripes, Cuv. \& Val. ix. p. I22; Richards. Ichth. Chin. p. 275; Cantor, Catal. p. 129.

Olistus atropus, Cuv. \& Val. iv. p. $1+1$.

Carangoides atropus, Bleek. Makr. p. 366; et Verh. Bat. Gen. xxiv. Makr. p. 66.

Caranx atropus, Cantor, Catal. p. I $30 ;$ Gthr. l. cit. 2, p. $45^{\circ}$. Hab. Manila, Luzon; China.

15. C. armatus, Cuv N. v.

(M. S. T.)

Sciana armata, Forsk. p. 53; Gm. L. p. 1306.

Russell, lam. ${ }_{15} \mathrm{I}$ p. 38.

(itula plumbea, Quoy \& Gaim. Voy. Freyc. Zool. Poiss. p. 361.

C. ciliaria, Rüpp. Atl. Fische. p. 102 lam. 25 f. 8 .

( armata, Rüpp. Atl. Fische, p roz, et $N$. W. Fische, p. $5^{\circ}$.

Caranx citula, Cuv. \& Val. ix. p. 126.

C. armatus, Cuv. \& Val. ix. p. I27; Cantor, Catal. p. I3I; Gthr. loc. cit. 2, p. 453 .

C. ciliaris, Cuv. \& Val. ix. p. 129 Faun. Japon. Poiss. p. I 12; Richards. Ichth. Chin. p. 276.

C. cirrhosus, (Ehrenb.) Cuv. \& Val. lam. 250.

Olistus malabaricus, Cuv. \& Val. ix. p. I 37, lam. 251; Cuv. Regne. Anim. Ill. Poiss. lam. 58. f. 1.

C. rüpellii, Cuv. \& Val. ix. p. Itt. 
Carangoides citula, Bleeker, Verh. Bat. Gen. xxiv. Makr. p. 65. Hab. Luzón, B. de Manila; China.

16. C. sansun, Firsk N. v.

Scomber sansun, Fiorsk. p. 56 .

Russell, ii. p. 33 pl. 144 .

('aranx sansun, Rüpp. Atl. Fische, p. ror; lam. N.W. Fische. p. 48 lain. i 3 f. 3; Gthr. l. cit. 2, p. 447.

Hab. Cebú; Mauricio.

17 C. sp. $\mathrm{N}$ v.

Hab. Luzon, Cavite, Sta. Cruz, Nasugbú, Batangas.

18. ('. gallus, $\operatorname{Lin} \mathrm{N}$ v.

$\left(\begin{array}{lll}M & \mathrm{~S} & \mathrm{~T}\end{array}\right)$

Meer-hæn, Nieuhof, Zee-en. Lint-Reize, i. p. 270, fig.

Valent. iii. p. 465 f. 376 ; Renard, ii. 26 1 28 ; Reysch., Theartr. Anım. i. 9, 7; Seba, iii. 26 34; Willughby, Append. lam 7, f, 1 .

Zeus, sp. n. ${ }^{\circ}$ 2, Arted, Genera, p. 50 (no synon).

7. gallus, Syst. i. p. 454 ; Bloch, lam. 192 f. I; Bl. Schn. p. 94; Gthr. l. cit. 2, p. 455 .

\%. sp., Gronov. Mus. i. n. ${ }^{\circ}$ 108.

Russell, i. p. 45, lam. 57, p. 46, lam. 58.

Gallus virescens, Lacép. iv. pp 583,584 .

Gallichthys major, Cuv. \& Val. ix. p. 168 lam. 254; Richarts. Ichth. Chin. p. 27I; Cantor, Catal. p. I 36.

(i. cherola, Cuv. \& Val. ix. p. I75.

Scyris indica, Rüpp. Atl. Fische, p. r 28, lam. 33, f. I; CuJ. \& Val. ix. p. 145 lam. 252; Rüpp. N.W. Fische, p. 51; Richards. Ichth. Chin. p. 276; Cantor, Catal. 1. I 34 ; Régne. Anim. Ill. Poiss. lam. 58 f. 2.

Carangoides gallichthys, Verh. Bat. Gen. xxiv. Makr. p. 68. Hab. Luzon, Cavite, Santa Cruz; China.

2. Gén SERIOLA, C. V

19 S. dumerilii. Risso. N. v.

(M. S. T)

('aranx dumerilii, Risso, Ichth. Nice, p. 175.

Seriola dumerilii, Risso, Eur. Mérid. iii. p. +24; Cuv. \& Val. ix. p. 2or lam. 258; Cuvv. Réone Anim. Ill. Poiss. lam. 56 f. I; Guichen. Explor. Algér. Poiss. p. 62; Webb. \& Berthel. Iles. Canar. Poiss. p. 57; Gthr. loc. cit. 2, 1. 462.

S. purpurascens, S. auro-vittata, et S. quinque-radiata, Richards. Ichth. Chin. p. 27 I (no Schleg.)

S. purpurascens, Schleg. Faun. Japon. Po:s. p. I I 3 lam. 6I; Bleek. Verhand. Batav. Genootsch. xxเi. Japan, p. 103.

Hab. Luzon, Manila, Cavite, Santa Cruz; China. 


\section{Gén CHORINEMUS, $C$ v.}

20. Ch. lysan. Cini, N, v.

(M. $\mathbf{s}$ T)

'Dampier, l'oy'. Nezi-Hollaild, i. lam. 3. f. 5 p. 162.

Scomber lysan, Forsk. n." 67. lam. 54.

S. forsteri, Bl. Schn. p. 26.

Scombersides commersonianus, Lacép. ii. lam. 20. f. 3.

Russell, ii. p. 31. lam. I+I.

Scomber madagascariensis, Saw. Zosl. iv. p. 590. Lam. 85; Belinelt, Life of Raffles, p. 689.

Lichia lysan, Rüpp. Atl. Fische, p. 91.

Chorinenus commersonianus, Cuv. \& Val. viii. p. 370; Bleek. Verh. Bat. Gen. xxiv. Makr. p. 44.

(h. lysan, Curv. \& Val. viii. p. 387; Rüppell, N. W. Fïsche, p. tt; Cantor, Catal. p. I I8; Gthr. l. cit. 2. 47 I.

?Chorinemus farkharii, Cav. \& Val. viii. p. 388.

('horinemus aculeatus, Cuv. \& Val. viii. p. $3^{8}+$ (no Bloch.)

(h. forsteri, Richards. Ann. \& Mag. Nat. Hist. $18+3$, xi p. 24. Hab. Luzon, B. de Manila, Cavite, Sta. Cruz; China.

2I. Ch. toloo, Cuv. N. v.

(M. S. T.)

T'oloo parah, Russell. ii. p. 29. lam. I 37 .

Lichia toloo-parah, Rüpp Atl. Fische, p. 9r.

Chorinemus toloo, Cuv. \& Val. viii. p. 377; ?Bleek. Verh. Batav. Gen. xxir. Makr. p. 45.

Hab. Luzon, Manila; Indias Orientales.

22 Ch. saucti-Petri, Cuv N v.

(M. S. T.)

? Coningo-visch, Nieuhof, Bras. Zee-en Lant-Reize, p. 269.

Renard, Poiss. Mol. i. 15. 86; Valent. iii. p. 351. f. г г.

Scomber, sp., Grouov. Zoophyl. n. ${ }^{0} 305$.

Chorinemus sancti Petri, Cuv. \& Val. viii. p. 379. lam. 236; Bleek. Verh. Bat. Gen. xxiv. p. 45; Peters, Wiegm. Arch. 1855, p. 245. Gthr. loc. cit. 2. p. 473.

Ch. modetta, Cuv. \& Val. viii. p. $382 ;$ Rüpp. N. W. Fìsche, p. 45 .

Ch. mauritianus, Cav. \& Val. viii. p. 382.

('h. tol, Cantor, Catal. p. rig (no Cuv.)

Thynnus moluccensis, Gronov. Syst. ed Gray. p. I 2 I. Hab. Luzón, Manila, Cebú; China, Amboina.

$23 \mathrm{Ch}$. orientalis, Temm, et Schl. N. v.

(M. S. T.)

Chorinemus orientalis, Temm. \& Schleg. Faun. Japon. Poiss. p. 106. lam. 57. f. I.

? Scomber aculeatus, Bloch, lam. 336 .

Hab. Luzón, Manila, Cavite; Japon. 
4. Gén. ILICHFA, Cuv.

24. I glauca, $B l$. N. v.

Prima glauci species, Randel. p. 252; Willughby, 1. 297.

Lampuge, Bellon. p. 155 .

Scomber n." 5, Artedi, Gentra, p. 52.

tc. glaucus, L. Syst. Nat. i. p. 494; Bl. Schrs. p. 33; Shaw. Zool. iv. p. 593 .

(iasterosteus glaucus, (Forst.) Bl. Schn. p. 539; Forst. Descript. Anim. cur. Lichtents. p. 5 .

(aranx gliucus, Lacép. iii. pp. 58, 66.

Centronotus glaycos, Risso, Ichth. Nice, p. 194.

Lichia glaucus, Risso, Eur. Mérid. iii. p. 429; Cuv. \& Val. viii. p. 358. lam. 234; Webb. \& Berthel. Ites. Canar. p. 56. lam. I 3. f. I; Jarrell, Brit. Fishes, edit. 3. ii. p. 232; Gthr. l. cit. 2. p. 477 .

Hab. Luzon, B. de Manila; Senegal.

\section{Gến TRACHYNOTUS, C. V.}

25. Tr. ovatus, Lin. N. v.

Gasterosteus ovatus, L. Syst. Nat. i. p. 49 .

Centronotus ovalis, Lacép. iii. pp. 309, 3 I6.

Valent. iii. p. 386 , f. I 8 , Renard, i. $27,45 \mathrm{k}$.

Scomber falcatus, Forsk. p. 57.

Coesiomorus blochii, Lacép. iii. p. 95. lam. 3, f. 2.

Trachinotus falcatus, Lacép. iii. p. 79; Rüpp. Atl. Fisch. p. 89; Cuv. \& Val. viii. p. 43 o.

Mookalee-parah, Russel, ii, p. 39, lam. 154.

'Trachinotus mookalee, Cuv. \& Val. viii. p. 523; Cantor, Catal. p. 1 20; Bleeker, Verhand. Batav. Genootsch. xxiv. Makr. p. $4^{8}$.

'T. blochii, Cuv. \& Val. viii. p. 425.

'I. affinis, Cuv. \& Val. viii. p. 428 .

'T. falciger, Cuv. \& Val. viii. p. 428.

'I. drepanis, Cuv. \& Val. viii p. 429.

'T. ovatus, Gthr. l. cit. 2. p. $48 \mathrm{I}$.

'I. auratus, Richards. Ichth. Chin. p. 270. Hab. Cebú; China.

\section{Gén. PSETTUS, C V.}

26. Ps. argenteus, Lir, N. v.

Chætodon argenteus, L. Aman. Acad. iv. p. 249; Bl. Schn. p. 230. Scomber rhombeus, Forsk. p. $5^{8}$. Centrogaster rhombeus, L. Gm. p. I $33^{8}$.

Acanthopus argenteus, Lacép. iv. pp. 558, 559.

Centropodus rhomboidalis, Lacép. iii. Pp. 303-305.

Russell, lam. 59. 
Psettus rhombeus, Cuĩ. \& Val. vii. p. 245; Cur. Rigne Anim. Ill. Poiss. lam. 42, f. 2; Bleek. Verh. Bativ. Genootsch. xxiii. Chetod. p. 29; Peters, Wiegm. Arch. 185.5, p. 247.

Monodactilus rhombeus, Cant. Catal. p. 172.

Psettus argenteus, Richards. loy. Ereb. \& Terr. Fiches, p. 57. lam. 35, f. 1-3; Gthl. l. cit. 2, p. $4^{87}$.

Hab Luzon, Nasugbú, Batangas, Pasacao, Camarines, Currimao, Ilocos; Australia.

27. Psetus. sp. N. v.

Hab. Luzón, B. de Manila.

7. Gén. Platax, C. V.

28. Pl vespertilio, Cuv. N. v.

Valent. n." 62; Renard, lain. 24, f. 129; Seba. iii. 25, 15.

Chætodon vespertilio, Bl. lam. I99 f. 2; L. Gm. p. i $857 ; B l$. Schm. p. 228; Shaw. Zool. iv. p. 344; Bennet, Ceylon Fishes, pl. 5 .

? Chætodon pinnatus, L. Mas. Ad. Frid. lam. 33, f. 6; L. Gm. p. I $24 \mathrm{I}$.

Plat:ix vespertilio, Cuv. Régne Anim.; Rüpp. Atl. p. I43, et N. W. Fische, p. 33; Cantor, Catal. p. 166; Peters in Wiegm. Arch. 1885 , p. 247; Gthr. l. cit. 2, p. 489.

P. gaimardi, Cuv. \& Val. vii p. 216.

P. guttulatus, Cuv. \& Val. vii. p. 227 , lam. 186.

P. albipunctatus, Rïpp. Atl. Fische, p. 69, t. I8, f. 4.

P. blochii, Cuv. \& Val. vii. p. 222 ; Bleeker, Verh. Bat. Gen. xxiii. Chetod. p. 27.

P. ehrenb rgii, Cuv. \& Val. vii. p. $22 \mathrm{I} ; R \ddot{p} p p . N$. W. Fische, p. 33 ; Richards. l. c. p. 245; Cuv. Régne Anim. Ill. Poiss. lam. 42, f. 1 .

Hab. Cebú; China.

29. Pl. teira, Cuv, N. v. Mayang.

(M. S. 'T.)

Chatodon teira, Forsk. p. 6o, lam. 22, Bl. lam. 199, f. I; $L$. Gm. p. 1265; Bl. Schn. p 22 I; Shawe. Zool. iv. p. 345. lam. 48 . Russell, lam. 87.

Fux teira, Cuv. Régne, Anım.; Rüpp. Atl. Fische, p. 68, et N. W. Fische, pp. 33, 37; Cuv \& Val. vii. p. 226; Cantor, Catal. p. I68; Bleck. Verh. Bat. Gen. xxiii. Chatod. p. 28; Peters. Wiegm. Arch. 1855, p. 247; Gthr. l cit. 2, p. 492.

P. leschen ildi, Cu. \& Val. vii. 1). 223.

P. vespertilio, Temm. \& Schleg. Faun. Japon. Poiss. v. 83, lam. 43. Hab. Luzón, Manila, C. de Cebú; China.

Hab. Luzon, B. de Manila. 


\section{Gén. PEMPHERIS, C. V.}

31. P. otaitensis, $C . V . \mathrm{N}, \mathrm{v}$.

P. oualensis, $C$. et $V$. vii. p. 299; Less. Voy. Coq. Zool. Poiss. p. 168; Bleeker, Banda. i. p. 422.

P. otaitensis, $C$. et $V$. vii. p. 304, lam. 191; Less. l. cit. P. I67; Gthr, l. cit. 2. p. 508 .

Hab. Cebú; Amboina.

9 Gén ẼUULA, Cuv.

32. E. fasciata, Lacíp. N. v.

(M. S. T )

Karah, Russell, i. P. 51, lam. 66.

Fiquula filigera, longispinis, carah, et fasciata, Cuv. \& Val. x. pi). 92-97, lam, 284.

F.. filigera, Cuv. Mem. Mus. i. p. 402, lam. 23. f. I; Cantor, Catal. p. I50 Bleeker Timor, i. p. 165 , et Verh. Bat. Gen. xxis. Makr. p. 79.

E, setigera, Agass, Recherch. Poss, Foss. v. p. 24. lam. B.

E. fasciata, Gthr. loc. cit. 2. p. 498.

Hab. Luzón, B, de Manila; Mar Rojo

33. E edentula. (Bloch). N. v.

(M. S. T.)

Scomber flavescens, \&c., Seba, iii. p. 75. lam. 27. f. 4 .

Sc. edentulus, Bloch, lam. 428 Bl. Schn. p. 36 .

Leiognathus argenteus, Lacepp. iv. pp. 448, 449 .

Koray-karah, Russell, i. p. 5o, lam. 63.

Equula ensifera, Cuv. \& Val. x p, 66; Bleek. Verh. Bat. Gen. xxiv, Makr, p. 8o.

E. coma, Cuv. \& Val. x. p. 76.

? E. serrulifera, Richards. Voy. Ereb. \& Terr. Ichthyol. p. 137, lam. 59. f, I 2-I4.

E. edentula, Gthr. l. cit. 2, p. 498.

Hab. Luzón, B. de Manila; China, Amboina.

34. E caballa, (Forsk.) N. v.

(M. S. 'T')

Scomber equula, Forsk. p. 58; Bl. Schn. p. 36; Shaze. Zool. iv. p. 596 .

Centrogaster equula, L. Gm. p. 1337.

Casio equulus, Lacêp. iii. pp. $85,90$.

Russell, pl. 62.

Equula caballa, Cuv. \& Val. x. p. 73; Rïppell, N. W. Fische, p. 51; Cantor Catal. p. I46; Gthr. l. cit. 2. p. 499.

Hab. Luzón, Cavite, Sta, Cruz, Nasugbú, Batangas; Mar Rojo.

35 E. dussumieri, $C . V . N$. v.

I.M. S. T.)

Cuv. \& Val. x. p. 77, lam. 283; Cuv. Régne Anim. Ill. Poiss. lam. 62. f. 1. Gthr. l. cit. 2. p. 500 .

Hab. Luzón, B. de Manila, C. de Cébú; Borneo. 
36. E nuchalis, Sihlor. N. v.

Schleg. Fann. Japon. Poiss. p. 126, lam. 67. f. 1; Gthr. l. cit. 2. p. 500 .

Hab. Luzón, Manila; China.

37. E splendens Car N. v

(M S. T.)

Goomorah karah, Russcll, i. 1. $t^{8}$, lam. 6r.

Equula splendens, Cuv. Régrne Auim.; Cantor, Catal. p. 149; Gthr. l. cit. 2. p. 501 .

E. gomorah, Cuz' \& Val.x. p. 8o; Rüppell, N. W. Piscke, p. 5I; Bicek. Vorh. Bat. Gen. vxiv. Makr. p. 82.

E. Caballa, Bleek. Oos-Java (no Cuv. \& Val.)

Hab. Luzón, B. de Manilia; Mer Rojo.

38. E oblonga, Cuv. N. v.

Scomber equula, var. Forsk. p. 58.

Equula oblonga, Cuv. \& Val. x. p. 85; Bleek. Verh. Bat. Genl. xxiv. Makr. p. 84 ; Githr. l. cjt. 2.

E. berbis, Cuv. \& Val. x. p. 85 .

Hab. C. de Samar; Java, Mar Rojo.

39. H leuciscus, Glhr.

Gthr. l. cit. 2. p. 303 .

Hab. Ce'.ú; Amboina.

40. H. insidiatrix, Bl. N. v. Sapi-sapi.

M. S. T)

Zeus insidiator, Bl. lam. 192. f. 2, 3; L. Gm. p. 1221; Bl. Schn. p. 95; Shaw, Zool. iv. p. 284. lam. 4r; Lacép. iv. pp. 572, 574. Equula insidiatrix, Cuv. \& Val. x p. 98; Cant. Catal. p. i5 ; Bleek. Verh. Bat. Gen. xxiv. Makr. p. 84; Gthr. l. cit. 2. p. 504 .

Hab. Luzón, Manila, Cavite, Sta. Cruz; China, Sumatra.

41. E interrupta, $C \quad V$. N. v.

(M. S. T.)

Chanda ruconius, Buch. Ham. Gang. Fisches. pp. ı06, 371, lam. I2. f. 35

Equula in:errupta, Cuv. \& Val.x. p. ıо2; Bleek. Verh. Bat. Gen. xxil. Makr. p. 85 ; Gthr. l. cit. 2. p. 504.

E. ruconius, Cuv. \&. Val. x. p. 73.

Hab. Luzón, Cavite, Sta. Cruz; China, Amboina.

9 Gén. GAZZA, Rüpr.

42. G. minuta, Bleek. N. v.

(M. S. T)

Scomber minutus, Bl. lam. 429. f. 2; Bl. Schn. p. 36 .

Equula minuta, Cuv. \& Val. x. p. 88 ; Gthr. l. cit. 2. p. 566. 
E. dentex, Cuv. \& Val. x. p. 91; Peters. Wiegm. Arch. 185.5, p. 247.

Gazza minuta, Bleek. Sumatra, ii. p. 25, et Verh. Bat. Gen. xxiv. Makr. p. 85 .

Hab. Luzon, B. de Manila, Cavite; Amboina.

43 G. argentaria, Forst. N. v.

Zeus argentarius, (Forst.) Bl. Schn. p. 96; Descript. Anim. cur. Lichtenst. p. 288.

G argentaria, Gthr. l. cit. 2. p. 506.

Gazza tapeinosoma, Bleeker, Sumatra, ii. p. Hab. Cebú; Sumatra.

44. G. equuløformis, Rüpp. $\mathrm{N}$ v.

(M. s. T.)

Rüppell, N.W. Fische, p. 4. lam. I. f. 3; Bleeker, Sumatra, ii. p. 26I; Cantor. Catal. p. I53; Gthr. l. cit. 2. p. 506.

Hab. Luzón, Cavite, Sta. Cruz, Nasugbú, Batangas; Mar Rojo.

Io Gén. PEMPHERIS, C. V.

45. P. mangula, Cnv. N. v.

(M. S. 'T.)

Mangula kutti, Russell, ii. lam. i 4.

Pempheris mangula; Cuv. \& Val. vii. p. 304; Bleek. Verhand. Batav. Genootsch. xxiii. Chatod. p. 30; Gthr, l. cit. 2. p. 509.

P. vanicolensis, Cuv. \& Val. vii. p. 305.

P. nesogallica, Cuv. \& Val. vii. p. 306.

Hab. Luzón, Cavite, Sta. Cruz; Amboina.

46. P. esp. N. v.

(M. S T.)

Hab. Luzon, Cdvite.

\section{Fam. XfFIDOS.}

I Gén. Histiophorus, C. V.

I. H gladius, N. v.

(II. S. T )

Nieuhoff, Embassy to China p. 237; Mahcgr. p. 171; Willughby, App. lam. 5. f. 9; Valent. iii. 509. f. I25; Renard, i. t. 34 . f. I 82 , ii. t. 54. f. 233.

Scomber gladius, Brouss. Mém. Acad. Sc. 1786 , p. 454. lam. io; $B l$. lam. 345 .

Xiphias velifer, $B l$. Schn. p. 93.

Istiophorus gladius, Lacep. iii. pp. 374, 375; Gthr. l. cit. 2. p. 5 I 3 .

Xiphias ensis, Lacep. ii. p. 296.

Notistium, Hermann. Observ. Zool. p. 305.

Xiphias platypterus, Shaw. Zool. iv. p. Ior.

Histiophorus indicus, Cuv. \& Val. viii. p. 293. lam. 229; Cuv. Regn? Anim. Ill. Poiss. lam. 53. f. I. 
H. americanus, Cuv. \& Val. viii. p. 303; Guchen. Poiss. In Ramon de la Sagra, Hist. Cuba. p. I05.

Hab. Luzon, Manila, Batanes: Oceano Indico.

\section{Fam. GÓBIDOS.}

I. Gén. GobiUs, A rTedi.

I. G. guamensis, Cuv. \& Vial.

C. et V. xii. p. I03; Gthr. Cat. B. M. 3. p. 5 .

Hab. Marianas, Guam.

2. G. baliurus, Cuv.

Gobius baliurus, Cuv. \& Val. xii. p. 6r; Bleek. Verhand. Batav. Genootsch. xxii. Blenn. en Gob. p. 31; Gthr. l. cit. 3, p. 18. Hab. Cebú; Amboina, Célebes.

3 G puntangoides, Blcek.

Gobius puntang, Bleek. Natuurk. Tydschr. Nederl. Ind. iii. p. 692 (n." ii. p. 486.)

G. puntangoides, Bleek. Ceram, iii. p. 242; Gthr. l. cat. 3 , p. 19.

Hab. Cebú; Amboina.

4. G lentiginosus, Richards.

Richards. Ichth. Voy. Ereb. \& Terr. p. 3. lam. I figs. 5, 6; Gthr. l. cit. 3, p. 20.

Hab. Cebú; N. Zelandia.

5. G. ornatus, Rüpp.

Gobius ornatus, Rüpp. Atl. Fische, p. 135, et N.W. Fische, p. 137; Gthr. l. cit. 3, p. 21.

G. ventalis, (Ehrenb.) Cuv. \& Val. xii. p. I 3 .

(x. interstinctus, Richards. Voy. Ereb. \& Teerr. Fisches, p. 3 lam. 5 figs. $3-6$; Bleek. Amb. \& Ceram, p. 275.

G. periophthalmoides, Bleek. Natuurk. Tydschr. Nederl. Ind. 1851, i. p. 249.

Hab. C. de Samar; Amboina, Molucas.

6. G. giuris, Buch. N. v.

(M.S. T.)

Korah-motta, Russell, lam. 50.

Koku, Russell, lam. $5 \mathrm{I}$.

Bullee korah, Russell, lam. 53.

Gobius giuris, Buch. Ham. Fish. Gang. p. 51 lam. 33 fig. 15; Cuv. \& Val. xii. p. 72 ; Bleek. Verh. Bat. Gen. xii., Blenn-iñ Gob. p. 24; Gthr. l. cit. 3, p. 22.

G. kokius, Cuv. \& Val. xii, p. .68; Jerdon in Madr. Journ. xr. 
p. 148; Bleek. l. c., Cant. C.al. p. $180 ;$ Jacq. Voy. Ind. Poiss. lam. 14, fig. 3 .

G. russelii, Cuv. \& Val. xii. p. 75 .

(i. catebus, Cuv. \& Val. xii. p. 76

G. kora, Cuv. \& Val. xii. p. 77.

G. celebicus, Cuv. \& Val. xii. p. 7 ; Bleek. Banten, p. 3 гъ.

G. kurpah, Sykes in Trans. Zool. Soc. ii. p. 352, lam. 6I fig. I.

G. platycephalus, Peters. Monatsber. Berl. Acad. 1852, p. 68I,

G. phaiosoma, Bleek. Verhand. Ba!av. Genootsch. xxii., Blenn. en Gob. p. 30; et Natuurk. Tydschr. Nederl. Ind. 1851. i. fig. 5 .

Gobius fusiformis, Bleeker, Verhand. Batav. Genootsch. l. c.

G. fasciato-punctatus, Richards. Voy. Sulph. Ichth. p. I45, lam. 62 figs. I 3,14 .

Hab. Luzón, B. de Manila, Laguna de Bay, C. de Cebú y Samar; China.

7. G. borneensis, B'eeker. N. v.

(M S. T.)

Bleeker, Borneo, i. p. Io; Gthr. l. cit. 3, p. 33.

Hab. Luzon, Cavite, Santa Cruz; Borneo.

8. G. echinocephalus, Rüpp. N. v.

(M S. T)

Gobius echinocephalus, Rïpp. Atl. Fische, p. г $3^{6}$, et $N . W$. F.sche, pp. I37 \& 138; Cuv. \& Val. xii. p. I $3+$; Gthr. l. cit. 3 , p. 34 .

Hab. Luzon, Manila, Cavite; China.

9. G. ophthalmotœnia, Bleeker.

Bleeker kokos-Eiland. p. 46; Gthr. l. cit. 3, p. 37. Hab. Cebú; China.

10. G. chlorostigmatoides, Bieker N. v.

$(\mathrm{M} \quad \mathrm{S} . \mathrm{T})$

Bleeker. Verhand. Batav. Genootsch. xxii. Blenn. en Gob. p. 26; Gthr. l. cit. 3, p. 39.

Hab. Luzón, Manila; Java, Borneo.

II. G. cyanoclavis, Cantor.

Cant. Catal. Mal. Fishes, p. $185 ;$ Gthr. l. cit. 3, p. to. Hab. Luzón, Manila; Pinang.

12. G. caninus, $C \cdot V \cdot \mathrm{N}, \mathrm{v}$.

(II S T )

Gobius caninus, Cuv. \& Val. xii. p. 86; Bleek. Verhand. Batav. Genootsch. xxii., Blenn. en Gob. p. 27 ; Gthr. l. cit. 3. p. 38 .

G. grandinosus, Valenc. in Voy. Bonite, Poiss. p. 177. lam. 5 fig 4 .

Hab. Luzon, Cavite, Sta. .Cruz; China. 
13. G. brevirostris, Githr. N. v.

(M. S. T.)

Gthr. l. cit. 3. p. 4 I.

Hab. Luzon, Manila; China.

14. G xanthosoma, Blecker. N. v.

(M S. T.)

Gobius xanthosoma, Bleeker, Ceram, ii. p. 703; Gthr. i. cit. 3. p. +2 .

Gobiodon xanthosoma, Bleeker, Enumer. Spec. p. 120 :

Hab. Luzon, Laguna de Bay; Célebes.

I 5 G. spectábilis, Gthr. N. V.

Gthr. l. cit. 3. p. 45.

Hab. Cebú; India.

16. G. schlegelii, Gthr. N. v.

(M. S. T.)

Gthr. l. cit. 3. p. $4^{6 .}$

Hab. Luzon, B. de Manila, Angat, Bulacan.

I 7. G.- microlepis, Bleek. N. v:

(M. S. T.)

Gobius acutipinnis, var., Cantor, Catal. p. 184.

G. microlepis, Bleek. Verhand. Batav. Genootsch. xxii., Blenn. en Gob. p. 35, et Java, ii. p. 436; Gthr. l. cit. 3. p. 49.

()xyurichthys microlepis, Bleek. Enum. Spec. p. 120.

Hab. Luzon, Cavite, Sta Cruz; China.

I8. G. grammepomus, Bleek. N. v.

(M. S. T.)

Gobius grammepomus, Bleek. Verhand. Batav. Genootsch. xxii., Blenn. en Gob. p. 34, et Batjan, ii. p. 200;.Gthr. l. cit. 3 . p. 63.

G. melanocephalus, Bleek. Verhand. Batav. Genootsch. l. c. p. 33 .

G. personatus, Bleek. l. c. p. 34, et Natuurk Tydschr. Nederl. Ind. 1851 , i. fig. 4

Hab. Samar; Borneo, Java.

19. G. bynoensis, Richards. N. v.

(M. S. T.)

Gobius bynoensis, Richards. Ichtyol. Ereb. \& Terr. p. I. lam." I figs. I, 2; Gthr. l. cit. 3. p. 70.

G. stethophthalmus, Bleek. Natuurk. Tydschr, Nederl. Ind. i. p. 249 fig. 7 , et xv. p. 236.

$H a b$. Luzon, R. Pasig, Manila; Australia.

20. G. sp. N. v.

(M. S. 'T.)

Hab. Samar. 
21. G. knuttelli, Bleek. N. v.

(M. S. T.)

? Gobius filifer, Cuv. \& Val. xii. p. 106.

Gobius knuttelli, Bleek. Act. Soc. Sci. Indo-Nederl. iii., Japon, p. 16 lam. 1. fig. 2; Gthr. 1. cit. 3. p. 73.

Hab. Luzon, Cavite, Sta Cruz; China.

22. G. lachrymosus, Ptıs. N. v.

Hab. Luzon, B. de Manila.

23. G. $\mathrm{sp} \mathrm{N.v}$

Hab. Luzon, Manila, Rio Munang, Antipolo.

2. Gén. APOCRYPTES, C. V.

24 A. serperater. Kichards. N. v.

(M. S. T.)

Richards. Ichthyol. China p. 206; Gthr. l. cit. 3. p. 82.

Hab. Luzon, Carite, Sta. Cruz; China.

25. A. nexipinnis, Cantor, N. v.

Cantor, Catal. p. i 88 ; Gthr. l. cit. 3. p. 83.

Hab. Luzon, Cavite, Sta. Cruz; Penang.

3. Gén. GOBIODON, Blefk.

26. G. ceramensis, Bleeker. N. v.

(M. S. T )

Gobius ceramensis, Bleeker, Ceram, ii. p. 703; Gthr. l. cit. 3. p. 88. Hab. Lazón, Cavite, Sta. Cruz; China, Borneo.

27. G. histrio, $C . V . N$. v.

Gobius histrio, (Kuhl \& v. Hass.) Cuv. \& Val. xii. p. I32. lam. 347; Bleek. Natuurk. Tydschr. Nederl. Ind. 1851, i. fig. 6; Gthr. l. cit. 3. p. 88.

Hab. Mindanao, Zamboanga; I. Amigos.

4 Gén TRI ÆNOPHORICHTYS, Gill.

28. Tr. trigonocephalus, Gill. N. v.

(M. S. T )

Gill. l. c. p. 18: Gthr. l. cit. 7. p. 89.

Hab. Luzón, Cavite, Sta. Cruz; China.

5. Gén SICYDIUM, Cur.

29. s. cyanocephalum, Cuv N. v.

Sicydium cynocephalum, Cuo. \& Val. xii. p. 177. lam. 352; Bleek. 


$$
-523-
$$

Batjan, ii. p. 201, et .V.tullk. Ty.tschr. Neterl. Ind. 1.550, p. 156: Gthr. l. cit. 3. p. 94.

S. lagocephalum, Bleek. Verhand. Batav. Gonoosch. xxii., Rlenn. en Gib. p. 39 (no l'al.)

Gobius hasseltii, Bleek. Natuurk. Tydschr. Nederl. Ind. i. p. 250. fig. 8 .

Hab. Luzón, Manila, Rio Lanateng, Morong; Indias Orientales.

\section{Gén PERIOPHTH:ILMUS, BL.}

\section{P koelreuteri Bl. N. v.}

(M. S T )

Valent. iii. p. 391. fig. 1fo; Renand, i. 16 65; Seba, iii. 29. 17; Kebreater in Nov. Coinm Petrop. viii. p. +21 .

? Gobius bariarus, L. Gm. i. p. 1201; B3l. Schn. p. 70.

G. kolreuteri, Pull. Spicil. viii p. 8. tab. 2. fig. 1.

Periophthalmus kuelreuteri, Bl. Schn. p. 65; Gthr. l. ctt. 3. p. 97. Hab. Cebú, Luzon, Manila, Malabon, Bulacan; Ceilan,

3r. P. schlosseri, Pall. N. v.

Gobius schlosseri, Pall. Spicil. viii. lam. r. fig. I-t; L. Gm.. i. p. I 201 ; Lxcep. ii. p 573; Shxw, Zool. iv. p. 246 .

Periophthalmus schlosseri, Bl. Sihn. p. 6i; Cuv. Regne Anim.; Cuv. \& Val. xii. p. 192; Bleeker, Verhand. Batav. Genootsch. xxii., Blenn. en Gob. p. 39; Cant. Catal. p. r91; Cuv. Regme Anim. Ill. Polss. lam. 81. fig. I; Gthr. l. cit. 3. p. I(x).

Gobius tredecim-radiatus, Buch. Ham. Fishes of the Ganges, 1). $4^{\text {r. }}$.

G. septem-radiatus, Buch. Ham. l. c. p. 46 .

G. novem-radiatus, Buch. Ham. l. c. p. 47. lam. 2. fig. 14.

Periophthalmus tredecin-radiatus, Cuv. \& Val. xii. p. 189.

P. septem-radiatus, Cuv. \& Val. xii. p. 196.

P. novem-radiatus, Cuv. \& Val. xii. p. 196.

P. freycineti, Cuv. \& Val. xii. p. I97; Quoy \& Gaim. Voy. Freyc. Poiss. p. I 57.

? P. borneensis, Bleek. Born. i. p. Ix.

Hab. C. de Sanar; Indias Orientales.

32. P. var. freycineti, $C$ it $V \quad \mathrm{~N} \mathrm{v}$

Cuv. et Val. xii. p. 197; Gthr. l. cit. 3. p. 100.

Hab. Filipinas.

7. Gén. ELeOTRIS, Cuv.

33. E. ophiocephalus ( ihl \& $\%$. Hasi.)

Eleotris ophiocephalus, (Kuhl.\& v. Hass.) Cuv. \& Val. xii. p. 339; Bleek. Verhand. Batav. Genootsch. xxii., Blenn. en Gob. lam. 22; Cantor, Catal. p. 196; Gthr. l. cit. 3. p. 107.

E. porocephalus, Cuv. \& Val. xii. p. 237 (no Cant. or Bleek.)

E. margaritacea, Cuv. \& Val. xii. p. 240.

Hab. Luzón, Manila, Rio Munang, Antipolo; $\underset{*}{\text { Borneo. }}$ 
34. E. hødtii, Bleek. N. v.

(M. S. T.)

Bleek. Amboina, v. p. 496; Gthr. l. cit. 3. p. 110.

Hạb. Luzón, Laguna de Bay; Amboina.

35. E. obscura, Schleg. N. v.

Schleg. Faun. Japon Poiss. p. 149. lam. 77. frgs. 1-3; Gthr. $l$. cit. S. p. II 5 .

Hab. Luzón, Manila; China.

36 E. oxycuphala, Schleg. N. v.

(M. S. T.)

Eloeotris oxycephala, Schleg. Faun. Japon. Poiss. p. 150. lam. 77 figs. 4, 5; Gthr. loc. cit. 3. p. I I 5 .

E. cantherius, Richards. Ichth. China, p. 209.

Hab. Luzón, Cavite; China.

37 E. butis, (Buch) N v.

Cheilodipterus butis, Buch. Ham. pp. 57, 367; Gray, \& Hardze. Itl. Ind. Zool. ii. lam. 93. f. 3.

Eloeotris humeralis, Cuv. \& Val. xii. p. 246; Bleek. Verhand. Batav. Genootsch. xxii., Blenn. en Gob. pp. 5, 2.2.

E. butis, Cant. Catal. p. I96; Gthr. l. cit. 3. p. 116.

? E. melanopterus, Bleek. Ceram, ii. p. 706.

Hab. Filipinas; Borneo, China.

38. E. melanostigma. Bleeker.

(M. S. T)

Bleeker, Verhand. Genootsch. xxii. Blenn. en Gob. p. 23; Gthr. l. cit. 3. p. 117 .

Hab. Luzón, Rio Pasig, Laguna de Bay; Java, Borneo.

39. E. caperata, Cantor. N. v.

(M. S. 'T)

Eleotris caperatus, Cant. Catal. p. 197; Gthr. l. cit. 3. p. i 7 .

E. koilomatodon, Bleek. Verhand. Batav. Genootsch. xxii., Blen:l. en Gob. p. 21 .

Hab. Luzón, Cavite, Sanța Cruz; China, Java.

40. E cyprinoides,? C. V. N. v.

(M. S T.)

Cuv. \& Val. xii. p. 248; Bleek. Sumatra, ii. p. 277.

Hab. Luzón, Cavite, Sanța Cruz, Manila; Sumatra.

4I. E. marmorata, Bleeker. N. v.

(M S. T.)

Bleeker, Borneo, vi. p. 424; Gthr. l. cit. 3. p. I23.

Hab. Luzón, Rio Pasig, Manila; Sumatra.

42 E. sinensis, Lacép. $\mathrm{N}$ v.

(M. S. 1.)

Bostrychus sinensis, Lacép. iii. p. 141, lam. I4. fig. 2. 
Gobius, C:rr. \& Val. xii. p. 94.

Philypnus occllicauda, Richards. Voy. Sulph. Fishes, pp. 58, 149), lam. 56 figs. I 5,16 .

Ph. sinensis, Richards. Ichth Chin. p. zia.

$\mathrm{Ph}$. ophicephalus, Bleek. in Verhand. Batav. Genootsch. xxii., Blenn. en Gob. p. 2o.

Bostrichthys sinensis, Gill, Proc. Acad. Nat. Sc. Philad. 1860, p. 125.

Eleotris sinensis, Gthr. l. cit. 3. p. I 27.

Hab. Luzón, Cavite, Sta. Cruz; Chinit, Java.

43. E. muralis, (Qu,y \& Gaim.) N. v.

(M. S. T.)

Eleotris moralis, (Quoy \& Gaim.) Cuv. \& Val. xii. p. 253, lam. 357 ; Bleek. Amboina \& Ceram, p. 276; Gthr. l. cit. 3. p. 130. Valenciennea muralis, Bleek. Boera, p. 4 I 2.

Eleotriodes muralis, Bleik. Goram, p. 2 г2.

Hab. Luzón, Cavite, Samar, Borongan; Archipiélago Indico.

\section{Gén AMBLYOPlS, C V.}

45. A. brachygaster, Gthr. N. v.

(M. S. T.)

Gthr. l. cit. 3. p. 134 .

Hab. Luzón, B. de Manila; Indias Orientales.

46 A. brachysoma, Blieker. N. v.

(M S. T )

Bleeker, Sumatra, iii. p. 5IO; Gthr. l. cit. 3. p. 136.

Hab. Luzón, B. de Manila, Bulacan, Angat; Priaman.

9. Gén. Platyptera, C. V.

47. Pl aspro, (v. Hass) $\mathrm{N}$ v.

Platyptera aspro (v. Hass.) Cuv. \& Val. xii. p. 32 I, lam. 360 ; Bleek. Celeb. viii. p 3 го; Gthr. l. cit. 3. p. г 38.

Pl. flavescens, Valenc. in Cur. Regne Anim. Ill. Poiss. lam. 83. fig. I.

Hab. Luón, B. de Manila.

io. Gén. CALlionymus, Lin.

48. C. longicaudatus, $T i m$. et Schl. N. v.

(M S. T.)

Callionymus japonicus, Houtt. Verh. Holl. Maatsch. Wet. Haarl. Xx. P. 3I I (no Val)

C. longicaudatus Temm. \& Schleg. Fishes, p. 60, lam. 78. figs. I, 2 lam. 79. A. fig. I; Bleek. Act. Soc. Sc. Indo-Nederl. iii. Japon, p. I 7 Gthr. loc. cit. 3. p. I 48.

C. reevesii, Richards. Voy. Sulph. Fishes, p. 6o, lam. 36. fig. I-3 (no fig. 4); et Ichth. Chin. p. 2 II; Bleek. Ceram, iii. p. $2+4$ Bleek. Nalez. Ichth. Jap. p. 44.

C. variegatus, Schleg. Faun. Japon. Poiss. p. I53. 
C. bəlcheri, Richards. Voy. Sulph. Fishes, p. 62, lam. figs. I \& 2. Hab. Luzón, Cavite, Santa Cruz; China, Amboina.

49. C schaapii, Blkr.

Blecker, Banka, p. 453 ; Gthr. l. cit. 3. p. I $4^{8 .}$ $H a b$. C. de Samar; Banka.

59. C. simplicicornis, C. $V$.

Cuv. \& Val. xii. p. $303 ;$ Gthr. l. cit. 3. p. $1+3$.

Hab. Marianas, Guam.

50. C. opercularis, $C, V$. N. v.

Cuv. et Val. xii. p. 305; Gthr. l. cit. 3. p. $15 \mathrm{I}$.

Hab. C. de Mindoro.

I1. Gén VULSUs, Gthr.

3r. V. dactylophus, $C . \quad V$. N. v.

Callionymus dactylopus, (Benn.) Cuv. \& Val. xii. p. 3io; Bleek. Amboina, ii. p. 559.

Dactylopus bennetti, Gill, l. c.

Vulsus dactylopu;, Gthr. l. cat. 3 p. $5^{2}$.

Hab. Cebú; Célebes.

Fam. BATRÁQUIDOS.

I. Gén. Batrachus, C. V.

1. B. $S p$, N. v.

(M. S. T)

Hab. Samar, Borongan.

Fam. ANTENÁRIDOS.

1. Gén, LOPHIUミ, ARTedr,

I. L. setigerus, $W_{a} h l$ N. v.

'M. S T.)

Lophius setigerus, Wahl. Skariut. of Naturh. iv. p. 214 , lam. 3 figs. 5, 6; Cuv. \& Val. xii. p. $3^{8} 3$; Schleg. Faun. Japon. Poiss. p. I58, lam. 80; Richards. Ichth. Chin. p. 203; Bleek. Verhand. Batav. Genootsch. xxv., Japon, p. $4^{6}$; Gthr. loc. cit. 3 p. 180.

L. viviparus, $B l$. Sch. p. I 42 tab. 32 .

$H a b$. Luzón, Manila; China. 


\section{Gén. ANTENNARiUS, Cummers.}

2. A. murmoratus, Gthr. N. v.

(M. S. T.)

Seba, i. $7 t$, t.

Cochinchina lophius, Shaw., Nat. Misc. vol. xxiii. lam. 1012.

Skeleton: Cuv. Mem. Mus. iii. pl. is, fig. +. (Chironectes lavigatius).

An. marmoratus, Gthr. l. cit. 3. p. 185.

Hab. Luzón, Manila; China, Australia.

3 A. tridens, (Schle $\left.e^{\prime}\right)$. N. v.

(M. S. T.)

Chironectes tridens, Schleg. Faun Japon. Poiss. p. 1 59, lam. 81 tigs. 2, 3 et 5; Bleek. Verhand. Batav. Genootsch. xxv. Japon, p. 47 .

Ant. tridens, Gthr. l. cit. 3. p. r9r. Hab. Luzón, Cavite, Sta. Cruz; China.

4 A. urophthalmus Bleek N.v. •

(M. S. T.)

Chironectes caudimaculatus, Richards. Voy. Ereb. \& Trr. Fishes, p. 1 25. 60. figs. 8, 9 (no Rüpp.)

Antennarius urophthalmus, Bleek. Nanumk. Tydschr. Nederl. Ind. ii.-p. 488, et xv. p. 237; Gthr. l. cit. 3. p. 192. Hab. Luzón, Manila.

5. A sp.

Hab. Mindanao, Zamboanga.

6. A notophthalmus, Bleek

Bleek. Natuurk. Tydschr. Nederl. Ind. v. I853, 'p. 54t; Gthr. l. cit. 3. p. 196. Hab. Visayas; Archipiélago Indico.

3. Gén, halieutea, C. V.

7. H. stellata, Cuv. N. v.

(M. S. T.)

Lophius stellatus, Wahl,"Skr. Naturh. Kjobenh. iv. p. 2It. lam. figs. 3,$4 ; B l$. Sch. p. I 42 .

L. faujus, Lacép. i. p. I 18 .

L. muricatus, Shaw. Zool. v. p. 382. lam. 162 .

Tiles. Krusenst. Reise, lam. 61. figs. 3, 4,

Halieutaa stellata, Cuv. \& Val. xii. p. 456. lam. 366; Faun. Japon. Poiss. p. I6o lam. 72; Bleek. Amb. \& Cer. p. 279; Gthr. l. cit. 3. p. 203 .

Hab. Luzón, Manila; China. 
Fam. BLÉNIDOS.

I. Gén. BLENNiÙ, Artedi.

I. Bl. sinensis, Gthr. N. v.

(M. S. T.)

Gthr. l. cit. 3. p. 212.

Hab. Luzón, Manila; China.

2. Gén PETROSCIRTES, Rüp.

2. P. tæniatus, (Q. et $G$.)

Aspidontus taniatus, Qusy \& Gaim. Voy. Astrol. Poiss. 1. $71 \%$. lam. i 9. fig. 4 .

Petroscirtes paradiseus, Bleeker, Amboina, v. p: 495.

Hab. Marianas, Guam; Amboina.

3. P. anema, Bleek'r. N:v.

Bleek. Amboina \& Ceram, p. 273; Gthr. l. cit. 3. p. 236. Hab. Cebú; China.

4. P. sp. N. v.

(M. S. 'T.)

Hab. C. de Samar.

5. P. grammistes, $G . \quad V . \mathrm{N}$. v.

Blennechis grammistes, Cuv. \& Val. xi. p. $2 \dot{8}_{5}$; Gthr. l. cit. 3. p. 236 .

Hab. C. de Samar, Cebú.

3. Gén. CLINUS, C. V.

6 Clinus, esp. $\mathrm{N}$ v.

(M, S. T.)

Hab. Luzón, Manila.

4. Gén. Salarias, cuv.

7. S. fasciatus, Cuv N. v

Blennius gattorugine, Forsk. Descr. Anim. p. 23.

B. fasciatus, $B l$. ii. p. I I I, lam. 162, fig. I; Bl. Schn. p. I68. Salarias quadripinnis, Rüpp. Atl. Fische, p. I 1 2, lam. 28 fig. 2 ; Cuv. \& Val. xi. p. 318; Bleek. Verhand. Batav. Genootsch. xxii., Blenx. en Gob. p. I9.

S. fasciatus, Cuv. \& Val. xi. p. 324.

S. priamensis, Bleek. Sumatra, ii. p. 268.

Hab. Luzón, Cavite, Sta. Cruz, Batangas, Nasugbú, Cebú; Borneo. 
8. S. 8p. N. v.

(M. 3. T.)

Hab. Cebú.

9 is ceramensis, Blecker.

Bleeker, Ceram, ii. p. 7or; Gthr. l. cit. 3, p. 246.

Hab. C. de Samar y Cebú; Célebes.

I0. A. sebø. Cuv. N. v.

(M. S. 'T.)

2. Seba, iii. 3o, 5 .

? Salarias sebx, Cuv. \& Val. xi. p. 323.

Salarias sebx, Bleek. Ternate, vii. p. 373 ; Gthr. l. cit. 3, p. 246.

Hab. Luzon, Cavite, Santa Cruz; Amboina.

I1. S. variolosus, $C \quad V$.

Cuv. \& Val. xi. p. 317; Gthr. l. cit. 3, p. 247.

Hab. Marianas, Guam.

12. S. sumatranus, Bleeker. N. v.

(M. S. T.)

Bleeker Bieen. en Gob. p. 256; Gthr. l. cit. 3, p. 252.

Hab. Luzon, Batangas, Nasugbú, Cavite, Santa Cruz; Archipiélago Indico.

13. S. quadricornis. G. $V$.

Cuv. \& Val. xi. p. 329, lam. 329; ?Jenyns, Zool. Beagle Fishes, p. 87 ; Gthr. b. c. 3, p. 255 .

Hab. Luzon, B. de Manila, C. de Cebú.

14. S. bellus, Gthr. N. v.

(M. S. T.)

Gthr. Cat. B. M. 3, p. 256.

Hab. Luzon, Cavite; China.

\section{Fam. TEÚTIDIDOS.}

I. Gén. TEUTHIS, Lin.

I. T, javus, $L:$ N. v。

(M. S. T.)

Teuthis javs, L. Syst. i. p. 507, L. Gm. i. p. 1362; Cant. Catal. p. 207; Gthr. l. cit. 3, p. 315.

Hepatus, Gronov. Zooph. p. I 3 n. ${ }^{\circ} 35^{2}$ lam. 8 fig. 4 ; Russell, ii. lam. Ioz.

Amphacanthus javus, Curv. \& Val. x. p. I1 $8 ;$ Bleek. Verhand. Batav. Gennotsch. xxiii. Teuth. p. 9; Schleg. \& Müll. Verhand. Nat. Gesch. Overz. Bezitt. p. Io. 
A. russelli, Bleek. Topogr. Batav.

Hab. Luzón, Manila, Cebú; Ceilán.

2. T vermiculats, $C . V$. N. v.

(M. S. T.)

Amphacanthus vermiculatus, (Kuhl \& v. H.sss.) Cusv. \& Val. x. p. 126; Mïll. \& Schleg. Verh. Overz. Bez. Vissch p. 11 lam. 3 fig. 2; Bleek Verhand. Batav. Genoorsch. xxiii. Teuth. p. I1. 'T. viomiculata, Gthr. l. cit. 3, p. 317.

Hab. Luzón, Batangas, Nasugbú, Cavite, Santa Cruz; Amboina.

3. T. margaritifera, C. $V$.

Amphacanthus margaritíferus, Cuv. \& Val.x. p. 145; Bleek.Java, iv. p. 334.

l'euthis margaritifera, Gthr. l. cit. 3, p: 317.

Hab. C. de Cebu; Archipiélago Indico.

4 T, albopinnctata, (Schleg) N v.

(M. S. T.)

Amphacanthus albopunctatus, Schleg. Faun.Japon. Poiss. p. 128. A. margaritiferus, Richard, Ichth. Chin. p. 243 (no Civo. \& Val.)

A. fuscescens, Richards. Ichth. Chin. p. 243 (no Auct.)

A. dorsalis, Bleek. Verhand. Batav. Genootsch, xiii., Teuth. p. 9, et Java, iv. p. 332; (no (uv. \& Val.)

'leuthis brevirostris, Gronov. Syst. ed. Gray, p. 142.

I. albopunctatus, Gthr. l. ct. 3, p. 318.

Hab. Luzón, Manila, Batangas, Nasugbú; China.

5. T. hexagonata, Bleek.

Amphacanthus hexagonatus, Bleek. Kokos, p. 4 r.

T. exagonata, Gthr. l. cit. 3, p. 320.

$H a b$. C. de Cebú; Java.

6 T lur: da, Rïpp.

Amphacanthus duridus, (Ehrenb.) Rïpp. Atl. Fische, p. 45; Cuv. \& Val. x. p. I50.

1. lurida, Gthr. l. cit. 3, p. $32 \mathrm{r}$.

Hab. Cebú; Mar Rojo.

7. T. nebulosa Q. et G. N. N

(M. S. T )

?Amphacanthus punctatus, Bl. Schn. p. 2ro; Civ. \& Val. x. p. 146; Forst. Deser. Anim. ed Licht. p. 286.

A. nebulosus, Quoy, \& Gaim. Voy. Uran. Zool. p. 369; Cuv. \& Val. x. p. 164; Schleg. \& Müll. l. c. p. 12.

A. maculosus, Quoy. \& Gaim. l. c. p. 370.

A. gymnopareius, Richards, Ann. \& Mag. Nat. Hist. 1843, xi. p. 174 .

A nebulosus, Gthr. l. cit. 3. p. $32 \mathrm{r}$.

Hab. Luzón, Manila, C. de Cebú; Australia. 
8 T argentes, $Q$. et $G$. N. v.

(M.S. T.)

Amphacanthus argenteus, Quor \& Gaim. Voy. Uran. Zool. p. 368. pl. 62. fig. 3 ; Cuv. \& Vial. x. p. 161.

A. argentea, Gihr. l. cit. 3. p. 322.

Hab. Luzón, Batangas, Nasugbú, Marianas.

9 T. virgata, $C V$ N. v.

(M. S. T)

Amphacanthus virgatus, Cuv. \& Val. x. p. r33; Mrül. \& Schleg. Verh. Overz. Bez. Vis ch. p. 14. lam. 3. fig. 1; Bleek. Merh. Batav. Genootsch. xxiii. Teuth. p. Ix.

T. virgata, Gthr. l. cit. 3. p. 323

Hab. Luzón, Manila, Cavite, Sta. Cruz; China.

10. T. vulpina. Sinieg N. v.

(M. S ' ' )

Valent. iii. p. 354. fig. 23, et p. 387. fig. 124*; Renard, i. 4. 29.

Amphacanthus vulpinus, Schleg. Mïll. Beschr. Zeevissch. in Verh. Nat. Gesch. Nederl. overz. Beçitt. Vissch. p. 1 2, et Bydrag. tot. de Dierkunde, v. I 852 , p. 38. fig. I; Bleek. Ternate, p. 135.

Teuthis tubulosat, Granov. Syst. ed. Gray, p. 142.

'T. vulpina Gthr. l. cit. 3. p. 324.

Hab. Luzón, Cavite, Sta. Cruz; Amboina.

11. T. sp N. v

(M S. T.)

Hab. Luzón, Manila, Batangas, Nasugbú.

Fum. ACRONÚRIDOS.

I. Gén. ACANThurus, Bl, Schn.

I. A triostegus. $B l . \mathrm{N} . \mathrm{v}$.

(M. $\mathrm{S}$ T )

Seba, iii. 25. 4; Russell, i. lam. 86.

Chatodon triostegus, L Syist. i. p. 463.

Acanthurus triostegus, Bl. Schn. p. 215; Cuv. \& Val. x.. p. 197; Blsek. Verhand. Batav. Gensotsch. xxiii., Teuth. p. I 3 ; Jeny.s, Voy. Beagls. Fische, p. 75; Gthr.

Harpurus fasciatus, Forst. Descr. Anim. ed. Licht. p. 2 I6.

Acanthurus zebra, Lacep. iv. p. 546. lam. 6. fig. 3 .

Chotodon zebri, Lacép. iii. lam. 25. fig. 3.

C. couagga, Lacep. iv. p. 727 .

Acanthurus hirudo, Benn. Ceyl. Fishes, p. ri. lam. Ir.

'Teuthis australis, Gray, in King's Survey of the Coasts of Austr.

ii. p. 435 .

Acanthurus subarmatus, Benn. Whating. Voy. ii. p. 278. Hab. Luzón, Manila, Ilocos, Currimao, C. de Samar. 
2. A. matoides, Cuv.

Acanthurus nigricans, pt., $B l$. Schn. p. 211.

?A. fuliginosus, Less. Voy. Cop. Zool. p. 149. lam. 27. fig. 2.

?A. rasi, Cuv. \& Val. x. p. 203.

A. matoides, Cuv. \& Val. x. p. 294; Bleek.. Verhand. Batav. Genootsch. xxiii., Tenth. p. I2; Gthr. l. cit. 3. p. 33 I.

A annularis, Cuv. \& Val. x. p. 209.

A. blochii, Cuv. \& Val. x. p. 209.

? A. xantopterus, Cuv. \& Val. x. p. 215; Cuv. Regne. Anim. Ill. Poiss. lam. $7 \mathrm{I}$. fig. 2.

A. xanthopterus, Cant. Catal. p. 209. lam. 4.

Skeleton: Agass. Poiss. Foss. iv. p. 206. lam. F. (A. xanthopterus).

Hab. Cebú, Marianas.

3. A. bipunctatus, Gthr. N. v.

Gthr. l. cit. 3. p. 331 .

Hab. Luzón, Cavite; China.

4. A. nigros, $C . V$.

Acanthurus nigroris, Cuv. \& Val. x. p. 208; Gthr. l. cit. 3. p. 332 .

Hab. C. de Samar; N. Hibridas.

5. A. lineatus, $B l . \mathrm{N}$ v.

Valent. iii. p. 360 fig. 47 , p. 424. fig. 257; Renard, i. 7.54 , i. 13. 80; Seba, iii. 25. I (?26. 37).

Chatodon, n. ${ }^{\circ}$ I Artedi, Spec. p. 89.

C. lineatus, L. Syst. Nat. i 3 th. edit. i. p. 1246.

Acanthurus lineatu , $B l$. Sch. p. 2r4. lam. 49; Lacép. iv. pp. 547,$549 ;$ Cuv. \& Val. x. p. 223; Bleek. Sumatra, ii. p. 26.3; Gthr. l. cit. 3. p. 333 .

A. vittatus, Benn. Fish. Ceyl. p. 2. lam. 2.

Hab. Luzon, Cavite, Sta. Cruz; Amboina.

6. A. striatus, $Q$. et $G$.

Quoy \& Gaim. Voy. Uran. Zo:l. p. 37.3, Poiss. lam. 63. fig. 3 Cuv. \& Val. x. p. 229; Gthr. l. cit. 3. p. 3.34 .

Hab. Marianas; Borneo.

7. A. achilles? Shaze. N. v.

(M. S T.)

Shaw. Zool. iv. p. 383; ?Cuv. \& Val. x. p. 218; Gthr. l. cit. 3. p. 340 .

Hab. Luzon, Cavite, Sta. Cruz; China.

8. A. ctenodon, C. V. N. v.

Acanthurus ctenodon, Cuv.\& Val. x. p. 24I. lam. 289, Gthr.l. cit. 3. p. $34^{2}$. 
A. strigosus, Bleek. Natuurk. Tydschr. Nederl. Ind. iv. p. 16q, \& vi. p. 102.

Hab. Carolinas, Amboina.

9. A. sp. N. v.

(M. S T.)

Hab. Luzon, Batangas, Nasugbú, Samar.

2 Gén. ACRONURUS, C. V.

10. A. argentous, $Q$ et $G$.

Q. et Gam. Voy. Uran. Zool. p. 372, lam. 63; f. 2; Cuv. et Val. x. p. 239; Gthr. l. cit. 3. p. 346.

Hab. Marianas.

3. Gén. NASEUS, Commirs.

II. N. olivaceus, Cuv. N. v.

(M. S. T.)

Chr todon olivaceus, Solander, M. S. Brit. Mus.

Naseus olivaceus, Cuv. \& Val. x. p. 288; Gthr. l. cit. 3. p. 352. Hab. Luzon, Manila; Otahiti.

\section{Gén KERIS, C. V.}

12. K. amboinensis, Bleeker. N. v.

Bleeker, Amboina \& Ceram, p. 272 Gthr. l. cit. 3. p. $35^{6}$.

$\mathrm{Hab}$ C. de Mindoro, y Cebú; Amboina.

\section{Fam. HOPLEGNÁTIDOS.}

1. Gén. hoplegnathus, Gthr.

1. H. punctatus. Richards. N. v.

(M S. T.)

Chatodon olivaceus, Salander, M. S. Brit. Mus.

Naseus olivaceus, Cuv. \& Val. x. p. 288.

H. punctatus, Gthr. l. cit. 3. p. $35^{8}$.

Hab. Luzon, Cavite; China.

\section{Fam. NÁNDIDOS.}

I. Gén. PLESIOPS, Cuv.

1. P1 cæruleo-lineatus, Rüpp, N v.

(M. S. T.)

Plesiops creruleo-lineatus, Rüpp. N. W. Fische, p.' 5. lam. 2. tig. 5; Bleek. Amboina, iii. p. I16; Gthr. l. cit. 3. p. 363. Pl. melas, Bleek. Verhand. Batav. Genootsch. xxii., Bali, p. 9. Hab. Luzon, Manila; Australia. 
2. PI. corallicola, Blick, $\mathrm{N}$ v

(M. S. T.)

Plesiops corallicola, (K. \& v. H.) Bleek. Sumatra, ii. p. 28o; Gthr. l. cit. 3. p. 364 .

Hab. Luzon, Cavite; Amboina.

\section{Fam. LABIRÍNTIDOS.}

I Gén, ANABAS, Cuv.

I. A, scandens, Cuv. N, v. Martinico.

Willughby, App. p. 4. lam. 4. fig. 4.

Perca scandens, Daldorff, Trans. Linn. Soc. iii. p. 62.

Anthias testudineus, $B l$. t. 322

Amphiprion testudineus, et scansor, Bl. Schn. pp. 204, 570 .

Lutjanus testudo et scandens, Lacep. iv. pp. 235, 239.

Sparus testudineus, Shaw, iv. p. $47 \mathrm{I}$.

Cojus cobojius, Buch. Ham. pp. 98, 370. lam. I3. fig. 33; Taylor in Breuster's Edinb. Journ Sc. 1831, v. pp. 34, 36.

Anabas testudineus, Cuv. Régne Anim.

A. scandens, Cuv. \& Val. vii. p. 333. lam. 93 \& 205; Cant. Ann. Nat. Hist. ix. 1842 , p. 28, et Calal. p. $82 ;$ Cuv. Régne. Anim. Ill. Poiss. p. 74. fig. I; Richards. Ichth China. p. 250; Jerdon in Madr. Journ. xv. p. 144; Bleck. Verh. Bat. Genootsch. xxiii. p. 8 lam. et Java, iv. p. 329; Gthr. l. cit. 3. p. 375 .

A. spinosus, Gray, Ind. Zool. ii. lam. 89. fig. I.

A. tripoliatus, Kaup. in Wiegm. Arch. 1850, p. 124. lam. 6. fig. $A$.

Hab. Luzón, Manila, Rio Pasig; Laguna de Bay.

2. $\Lambda$ macrocephalos, Bleck, N. v Liualo.

(M. S. T.)

Anabas scandens, Bleek. Verh. Bat. Gen. xxiii., Doolh. K. p. 8. A. inacrocephalos, Bleek. Java, ii. p. 430; Gihr. l. cit. 3. p. 376. Hab. Luzón, Manila; Borneo, Java.

3. A. oligolepis, Bleeker. N. v.

? Anabas oligolepis, Bleeker, Borneo viii. p. 161; Gthr. l. c1t. 3 . p. 376 .

Hab. Luzón, Cavite, Santa Cruz; China.

2 Gén. POlyaCan thus, c. V.

4. P. opercularis, Richards. N. v,

(M. S. T.)

Labrus opercularis, L. Aman. Acad. iv. p. 428 ; Bl. Schn. p. 245.

Chœtodon chinensis, Bl. lam. 218. fig. I; Lacép. iv. pp. 461, 496; Bl. Schn. p. 234. 
Polyacanthus chinensis, Cuv. \& Val. vii. p. 357; Ruchards. Ichth. Chin. p. 250.

Macropodus ocellatus, Cant. Ann. \& Mag. Nat. Hist. ix. 1842; p. 474.

Polyacanthus (?) opercularis, Richards. l. c.; Gthr. l. cit. 3. p. 379. Polyacanthus (?) paludosus, Richards. l.c. Hab. Luzón, Manila, Cavite; China.

3. Gén. MACROPUS, 'ithk.

\section{M. viridi-auratus,P Lacép, N. v.}

Macropodus viridi-auratus, Lacép. iii. p. 417, lam. I6. fig. I; Cuv. \&. Val. vii. p. 373; Cuv. Regne Anim. Ill. Poiss. lam. $7+$ tig. 2; Gthr. l. cit. 3. p. 382.

M. venustus, Cuv. \& Val. vii. p. 375, lam. 197. Hab. Luzón, Cavite; China.

\section{Gén. OSPHRONEMUS, Lacep.}

6. O. ulfax, (Commers.) N. v.

(M. S. T.)

Usphronemus gourami, Lacép. iii. p. 117 , lam. 3. fig. 2.

Trichopodus mentum, Lacép. iii. p. 125 , lam. 3. fig. 3.

'Trichopus goramy, Skaw, Zool. iv, p 388, lam. 55.

T. satyrus; Shaw, Zool. iv. p. 391, lam. 35.

(Osphronemus olfax, (Commers.) Hardw. Zool. Journ. iv. p. 309. lam. 36. suppl.; Cuv. \& Val. vii. p. 377; Richards. Ichth. Chin. p. 25I; Bleek. Verh. Bat. Genootsch. xxiii. p. Io; Cant. Catal. p. 88.

O. gourami, Cuv. \& Val. vii. p. 377, lam. 198; Cuv. Regne Anim. Iil. Poiss. lam. 73. fig. I.

O. satyrus, Bleek. Topogr. Batav.

Superbranchial organ, Cuv. \& Val. lam. 206 Peters. in Mïll. Arch. 1853 , lam. i 3. fig. A.

Osp, olfax, Gthr. l. cit. 3. p. 383 .

Hab. Samar; Borneo, Java.

7. O. trichoptcrus, Pall. N. v.

(M. S.. T.)

Sparus, Kalreater, Nov. Comm. Petrop. ix. p. 452, lam. 9. fig. 1. Labrus trichopterus, Pall. Spicil. viii. p. 45; L. Gm. p. I286; Bloch, lam. 295. fig. 2.

Trichogaster trichopterus, Bl. Schn. p. 165 .

'Irichopus trichopterus, Lacép. iii. p. I 29; Cuv. \& Val. vii. p. 388, lam. I99; Bleek. Verhand. Batav. Genostsch. xxiii. p. Io; Cantor, Catal. p. 89, lam. 2. fig. 5.

T. pallasii, Shaw, Zool. p. 392.

'T. Kerii, Bleek. Sumatra, i. p. 577.

Os. 'trichopterus, Gthr. l. cit. 3. p. 384.

Hab. Samar; Borneo. 
8. O. striatus, Bleck. N. v.

(M. S. T.)

? Osphronemus vittatus, (Kuhl \& v. Hass.) ('uv. \& Val. vii. p. 387. 'Trichopus striatus, Bleek Verhand. Batav. Genoosch. xxiii.; Labr. p. I . et Natuurk. Tydschr. Nederl. Ind. 1850, i. p. I06; Gthr. l. cit. 3. p. 386.

Hab. Luzón, Cavite; Java, Borneo.

\section{Fam. LUCIOCEFÁLIDOS.}

I Gén. LuCiocephalus, Bleek.

I. L. pulcher,? Bleek. N. v.

(M. S. T)

Diplopterus pulcher, Gray, Ill. Ind. Zool. i. lam. fig. I.

Luciocephalus pulcher, Bleek. Boruoo, ii. p. 274, et Blitong, p. 99; Gthr. l. cit. 3. p. 390.

Hab. Luzón, Manila; China.

\section{Fam. ATERÍNIDOS.}

I. Gén. Atherina, Artedi.

I. A. bleekeri, Gthr. N. v.

(M. S T.)

Atherina japonica, Bleek. Verhand. Batav. Genootsch, xxv. Japan, p. 4o. fig. 2 (no Houtt:); Gthr. l. cit. 3. p. 398.

Hab: Luzon, Manila; China.

2. A. lineata, Gthr.

Hab. Cebú.

3. A. sp N. v.

Hab. Cebú.

\section{Fam. MUGÍlLIDOS.}

1. Gén. MUGil, Artéd.

1. M cephalotus, Cuv $\mathrm{N}$.

Renard, i. lam. 2. fig. 10; Valent. p. $45^{8}$. n..$^{\circ} 35^{6 .}$

Mugil oür, Forsk. p. xiv. n. ${ }^{\circ}$ Io9. var. 7 ; Rüpp. N. W. Fische, p. I $3 \mathrm{I}$.

M. cephalotus, Cuv. \& Val. xi. p. 1 10 (no synon.); Cant. Ann. \& Mag. Nat. Hist. ix. I842, p. 484 (no Catal. Mal. Fishes); rEyd. \& Soul. Voy. Bonite, Zool. i. p. 175. lam. 4. fig. 4; Gthr. l. cit. 3. p. +19. 
M. japonicus, Schleg. Faun. Japon. Poiss. p. 134. lam. 72. fig. 1; Richards. Ichth. Chin. p. 247; Bleek: Verhand: Batav. Genootsch. xxv., Japon, p. $4 \mathrm{r}$.

M. macrolepidotus, Richards. Ichth: (hin. p. 249 (no anct.) Hab. Luzón, Cavite; China.

2 M. hœmatochilus. Schleg. N. V.

(M 3. T.)

Mugil homatocheilus, Schieg. Faun. Japon. Hoiss. p. i 35 . lam. 71. fig. 2; Bleek. Verhand: Batav. Genootsch. xxvi., Japon, p. IO7; Gthr. l. cit. 3. p. +22.

M. xanthurus, Richards. Ichthyol. Chin: p. 248 :

Hab. Luzón, Manila; China.

3 M. subviridis, $C . \quad \dot{V} . \mathrm{N}$. v.

Cuv. \& Val. xi. p. 115: Gthr. i. cit. 3. p. 423:

Hab. Manila; Madras.

4. M. sundanersis, Bleek. N. v. Talilon.

(M. S. T )

Mugil sundanensis, Bleek. Sumatra, ii. p. 265; Atl. Mugil. lam. I. fig. 1; Act. Sac: Sc. Indo-Nederl. vi., Borneo, xiii. p. 45. M. brachysoma, Bleek. Java, iii. p. 399.

Hab..Luzón; Rio Pasig, Laguna de Bay, Archipiélago Indico.

5 M. meyeri, Gthr N. v.

Hab، Luzón, Laguna de Bay.

6 M. sp. N. v. Taliton.

Hab. Luzón, Rio Pasig, Manila.

7. M. planiceps, Cuv. N. v.

(M. S. T)

Mugil planiceps, Cuv. \& Val. xi. p. 122; Bleek. Verhand. Batav. Genootsch. xxv., Bengal. en Hindost. p. ior. lam. I. fig. 5; Gthr. l. cit. 3. p. 428.

? M. dussumieri, Cuv. \& Val. xi. p. 147.

M. cephalotus, Cant. Catal. p. 95 .

M. dussumieri, Bleek. Java, iv. p. 339, no Journ. Ind. Archipel. ii., Ichth. Sumbawa, p. 637.

Hab. Luzón, Manila, Navotas; China.

8. M. kelaartii, Gthr. $\mathrm{N}$ v.

Günther. Cat. B. M. 3. p. 429.

Hab. Luzón, Manila, Navotas; Ceilan.

9. M. affinis, Gthr. N. v.

(M. S. T.)

Gthr. l. cit. \&. p. 433 .

Hab. Luzón, Manila; China. 
10. If cæruleo maculatus, Lacep. N. v.

? Mugil caruleo-maculatus, Lacep. v. pp. 385, 389; Cav. \& Val. xi. p. 128.

il. cxruleo-maculatus, Bleek. Rionzu, p. $4^{8} 4$, et Act. Soc. Sc. Indo-Nederl viii., Sumatra, ix. p. 5: Gthr. l. cit. 3. p. ++5 . Hab. Luzón, Manila; Mauricio

11. M. troschélii. Blecker., $\mathrm{N}$ v.

Bleeker, Natuurk. Tijschr. Nederl. Ind. xvi. p. 277, et Act. Soc. Sc. Indo-Nederl. vi, Sumatra, viii. p. 8o; Gthr. l. cit. 3. p. +48. Hab. Luzón, Manila, Navotas; Java, S'umatra.

12. M. borneensis Blieker. N. v.

(M. S. T.)

Mugil borneensis, Bleeker, Natumrk. 7ijdschr. Nederl. Ind. ii. 1851 , p. 2or, et Soc. Sc. Indo-Nederl. vi., Borneo, xiii. p. 55; Gthr. l. cit. 5. p. 448

M. adustus, Bleek. Natuurk. Tijdschr. Nederl. Ind. v. 1853 p. 503. Hab. Luzón, Cavite, Santa Cruz; Archipiélago Inclico.

13. MI. melinopterus, $C . V . \mathrm{N}$ v.

(M. S. T.)

Cuo. \& Val. xi. p. 146. pl. 314: Gthr. l. cit. 3. p. 452. Hab. Luzón, Cavite; Vanicolo.

Fam. OFIOCEFÁLIDOS.

I. Gén. OPHiocephalus, Bloch

1. O. rhodotœnia, Bleeker. $\mathrm{N}$ v Dalag.

(M. S. T)

Bleeker, Borneo, v. p. 425 ; Gthr. l. cit. 3. p. +72.

Hab. Samar; Borneo.

2. Oph melanopterus, Bleeker N. v. Dalag.

(M. S. T.)

Bleeker, Borneo ix. p. 420; Gthr. l. cit. 3. p. 473 .

Hab. Luzón, Bulacan; Borneo.

3. Oph striatus, $B l . \mathrm{N}$, D Dalag.

Ophiocepalus striatus, Bl. lam. 359; Bl. Schn. p 238; Shaw, Zool. iv. p. 530; Cuv. \& Val. vii. p. $4 \mathrm{I} 7$, lams. 202 \& 206; Jerdon, Madr. Journ. xv. p. 146; Cant. Catal. p. 92; cuv. Regne Anim. Ill. Poiss. lam. 75. fig. 2; Bleek. Verh. Bat. Gen. xxiii. Labyr. p. I 3; Gthr. loc. cit. 3, p. 474 .

Ophicephalus wrahl, Lacep. iii. p. 552; Buch. Ham. pp. 6o, 367 lam. 31 ; Russell, lam. I62.

Ophicephalus chena, buch. Hain. pp. 62, $3^{67}$. 
? Ophicephalus planiceps, Cuv. \& Val. vii. p. 424 .

Hab. Davao, Mindanao; Java, China.

4. Oph nigricans $C . V . N . v$.

(M. S. 'T.)

Cur. \& Val. vii. p. 431 ; Gthr. l. cit. 3, p. 477. Hab. Luzón, Maniia; Archipiélago Indico.

5. Oph. argus,? Cintor N. v.

(M. S T.)

Cant. Ann. \& Mag. Nat. Hist. 1842, ix. p. $48_{4}$; Gths. L. cit. 3. p. +80 .

Hab. Luzon, Laguna; China.

6. C ph. maculatus $C_{u v} . \mathrm{N}$.

(M. S T)

Bostrychus maculatus, Lacép. iii pp. 140, 143.

Ophicephalus maculatus, Cuv. \& Val. vii. p. 437; Richards. Ichth.

Chin. p. 251; Gthr. l. cit. 3, p. 480 .

Hab. Luzon, Bataan, Orion; China.

7 Oph vagus, Plis. N. v. Dalag.

(M S. T.)

Hab. Luzón, Laguna de Bay; Borneo, Sumatra.

\section{Fam. TRICONÓTIDOS.}

I. Gén. TRICHONOTUS, C. V.

I Tr. setigerus, $B l, \mathrm{~N}$ v.

(M. S. T.)

Trichonotus setigerus, Bl. Schn. p. 179 lam. 39; Cur' \& Val. xii. p. 316; Bleek. Celeb. v. p. 25I; Gthr. l. cit. 3, p. $4^{4}+$. T. polyophthalmus, Bleek. Ceram. iii. p. 243.

Hab. Samar, Borongan; Célebes.

\section{Fam. CEPÓLIDOS.}

I. Gén CEPOLA, LiN.

I C. abbreviata.? C. $V . \mathrm{N}$ v.

(M. S. 'T)

Caz. \& Vat. x. p. 4o3; Cant. Catal. p. i78; Bleek. Act. Soc.

Sc. Indo-Nederl. iii. Sumatra, p. $38 ;$ Gthr. l. cit. 3, p. 488. Hab. Luzon, Cavite, Santa Cruz; China. 


\section{Fam. CENTRÍSCIDOS.}

I. Gén. CENTRISCḷs, Lin.

1. C. gracilis, Lowe. N. v.

(M. S. T)

1.xwe Proc. Zool. Soc. 1839, p. 86, et Trans. Zool. Soc. iii. p. I2; Gthr. l. cit. 3. p. 521 .

$H a b$. Luzón, Manila, Navotas; China.

2. Gén. AMPHISEle, KLlis.

2. A scutata, Cuv. N. v.

(M $\quad \mathrm{S} \quad \mathrm{T}$.)

Valent. Amb. iii. p. 420 . figs. 243 \& 254; Seða, ĭii. p. ro7. lam. 34. fig. 5; Ruysch. Coll. Amb. lam. 3. n. ${ }^{\circ} 7$.

Centriscus scutatus, L. Syst. Nat, i. p. $4{ }_{5} ;$; $B l$. i. p. 57. lam. 1 23. fig. 2; Bl. Schn. p. In 3; Lacép. ii. p. 88, i. lam. 19. fig. 2; Shaw, Zool. v. p. 458. lam I8I.

Centriscus, sp., Grenev. Poophyl. n. ${ }^{\circ}$ 396. lam. 7. fig. 3.

Amphisile scutata, Cuv. Regne, Anim.; Guér. Iconogr. Poiss. lam. 45. fig. 3 ; Githr. L. cit. 3. p. 525 .

(entriscus scutatus, Grenov. Syst. ed. Gray, p. $13^{8}$.

Hab. Luzón, Cạite, Sța. Crụz; Chinạ.

3 A. strigata, Gthr.

Amphisile scutata, Bleek. Banda, i. p. 245 ; Gthr. l. ctt. 5. p. 528. Hab. Luzón, B. de Manila Cebú; Java.

Fam. FISTULȦRIDOS.

I. Gén FIstulaRia, I.in.

1. F. serrata, Cuv. N. v

(M. S. T)

Pike Fish Conger, Dampier, Voy. New. Holt. in Coll. Voy. iii. lam. p. 184 .

Fistularia tabaccaria, White, Voy. Bot. Cay, p. 296. fig. 2.

F. tabaccaria, var., $B l$. viii. p. г 30 . lam. $38 \mathrm{z}$. figs. 2,$3 ; \mathrm{Bl}$.

Schn. p. 114. Russell, ii. p. 58 . lam. 173.

Fistularia inmaculata, Cuv. Réone, Anim.; Richards. Ichth. Chin. p. 247; Faun. Japon. Poiss. p. 320; Bleek. Amb. \& Cer. p. 281 , et Verhand. Acad. Wetensch. Amsterd. i. Japan, p. I I.:

F. serrata, Cuv. Regne, Anim.; Gthr. l. cit. '3. p. 533.

F. comersonii, Rüpp. N. W. Fische, p. 142; Peters, in Wiegm. Arch. 1855 , p. 258.

Cannorhynchus immaculotus, Cant. Catal. p. 211.

Skeleton: Rosenth. Ichthyol. Taf. ix. figst 8-12 (Pistularia tabacaria).

Hab. Luzón, Cavite, Sta. Cruz; Amboina, China. 


\section{Fam. MASTACEMBÉLIDOS.}

I. Gén. RHYNCHOBDELla, C. V.

I. Rhy. aculeata, $B t$. N. v.

(M S. T )

Pentophthalmus, Willughb. App. Ham. I0. 428.

Mastacembelus, $s p$. Gronov. Zoophil. p. 133.

Enchelyopus, sp.; Klein, Pisc. Miss. iv. p. 58. n. ${ }^{\circ} 8$.

Ophidium aculeatum, Bl. lam, 159. f. 2.

Rhynchobdella orientalis, Bl. Schn. p. 478 .

R. aral, Bl. Schn. p. 479. lam. 89.

Nacrognathus aculeatus, Lacép. ii. p. $284 ;$ Buch. Hom. Fish. Gang. p. 29.

Ophidium rostratum, Shaze, Zool. iv: p. 73 .

Macrognathus ocellatus, Buch. Ham. l. c. p. 29.

Rhynchobdella aral, Cub. \& Val. viii. lam. 239.

R. ocellata, Cuv. \& Val. viii. p. 445; Bleek. Verh. Batav. Ginootsch. xxiii., Notac. p. 5 .

Mastacembelus pentophthalmus, Gronov. Syst. ed. Gray, p. 17 : Ph. aculeata, Gthr. l. cit. 3. p. 550.

Hab. Luzón, Cavite; Borneo, Molucas.

2 Gén. MASTACEMBEILSS, C V.

2. M. unicolor, C. V. N v.

(M. S. 'T)

Mastacembelus unicolor, (Kuhl. \& v. Hass). Cuv. \&. viii. p. 453; Bleek. Verhand. Batav. Genootsch. xxiii. Notac. p. 5; Gthr. l. cit. 3. p. 542 .

Hab. Luzón, Cavite; Java.

\section{Orden 2. ${ }^{\circ}$ FARINGOGNATOS.}

Fam. POMACÉNTRIDOS.

I. Gén. AMPhipkion, BL. Sch.

I. A bifasciatus. $B l, \mathrm{~N}, \mathrm{x}$

Percis, sp., Kölreut. N. Comm. Petrop. x. p. 340. lam. 8. fig. 4. ? Sciana sp., Gronov. Mus. Ich'h. p. 38.

Coracinus sp., Gronov. Zoophyl. n. ${ }^{\circ} 22$.

A'thias bitasciatus, $B l$. lam. 3i6. fig. 2.

Amphiprion bifasciatus, Bl. Schn. p. 204; Cuv. \& Val. v, p. 392 ; Schleg. Overz. Amphipr. \&c. in. Verh. Ned. Overz. Bezitt. p. 18; Bleek. Amb. \& Cer. p. 282; Gthr. Cat. B. M. 1852 4. p. 3 .

Holocentrus bifasciatus, $B l$. Schn. p. 567 . 
Lutjanus jourdin, Lacep., iv. pp. 191, 235.

Ampniprio laticlavius, Cuv. \& Val. y p. 296. lam. I32; tig. I.

Coracinus vittatus, Gronov. Syst. ed. Gray, p. 57. Hab. Cebú; Amboina.

2 A. intermedius, Sihleg. N. v.

Sciaena, var., Gronov. Mus. Ichthyol. p. 39.

Amphiprion intermedius, Schleg. l. c. p. 18 ; Gthr. l. cit. 7 . p. 4.

A. trifasciatus, Bleek. Celeb. iii. p. 767 (no (nv. \& Vai.) Hab. Luzón, Manila, Cebú; Molucas.

3 A. sp. N. v.

Hab. Luzón, Manila.

4. A. sebæ, Bleek. N. v.

Seba, iii. p. 70. lam. 26. fig. 24.

Amphiprion sebx, Bleek. Batav. p 478; Gthr. l. cul 4. 1. t. Hab. Santa Cruz, Luzón, Cavite; Sumatra.

5. A. clarkii, Cwv. N. v.

Athias clarkii, Benn. Fish. Ceyl. lam. 29.

A «phiprion clarkii, Cuv. \& Val. ix. p. 504; Peters nn Wiegn. Arch. 1855 , p. 265; Gthr. l. cit. 4. p. 5.

A japonicus, Sch!eg. Faun. Japon. Poiss. p. 66.

A. chrysargurus, Richards. Ichth. Chin p. $25+$.

A. xanthurus, Bleek. Amboin., ii. p. 560 (n) ( $u$. \& $V$ al).

A.nphiprion polymnus, Stendachner, Verhandl. Zool. Bot. Geselloch. Wien, 1861 , p. 79.

Hab. Luzón, Manila, Navotas; Amboina, Cbina.

6. A. percula, Cuv N. v.

(M. S. T)

Tetragonopterus, n. ${ }^{0}$ 5. Klein. Pisc. Miss. iv. p. 38. lam. I I. fig. 8.

Seba, iii. p. 69. lam. fig. 20.

Perca, sp., Tyson. in Philos, Trans. 1. xi. p. 2+7. lam. 7. fig. 8. Anthias polynna var., Bl. lam. 316; fig. 3 .

Lutjanus polymnus, var., Lacep. iv. p. 224.

L. Purcula, Lacep. iv. pp. 239, 248.

Amphiprion percula, Cuv. \& Val. v. p. 397; Bleek. Amb. \& Cer. p. 287; Sichleg. Overz. Amphipr. \&c in Verhand. Nat. Gesch. Nederl. Overz. Bezitt. p. I9; Steindachner, Verhand!. Zool. Bot. Gesellsch. Wien, i 86ı, p. 78 ; Gthr. l. cit. 4. p. 6.

A. tunicatus, Cuv. \& Val. v. p. 399. lam. i 32 . fig. 2; Less. Voy. Coqu. Zool. Poiss. p. 192. lain. 25. fig. 3.

A. ocellaris, Cuv. \& Val. v. p. 399.

A. melanurus, Cuv. \& Val. v. p. 400 .

Hab. Luzón, Cavite, Sta. Cruz; China, Amboina. 
7. A. boholensis, $O$ Cart. $N$ v.

(M. S. T.)

A. boholensis, O. Cart. Besch. Paryn Wiirzburg. 1873. Hab. B.hol, Cebú.

2 Gén PREMNAS, Cuv.

S F. biaculeatus. Bleek. N. v.

(M. S T )

Premnas biaculeatus, Bleek. Banda, iii. p. Io5; Gthr. Cat. B. M. 4. p Io.

Renard, Poiss. Mel. i. lam. 22. fig. 122.

Percis, sp., Kolreut. in Nov. Comm. Pctrop. x. p. 346. lam. 8. fig. 5 .

Chætodon biaculeatus, Bl. lam. 219. fig. 2.

Lutjanus trifasciatus, Bl. Schr. p. 568.

Holocentrus biaculeatus, Lacép. iv. pp. 528, 537 .

Holocentrus sonneratii, Lacép. iv. pp $34+391$.

Scorpæna aculeata, Lacép. iii. pp. 258, 268.

Preninas trifasciatus, Cuv. \& Val. v. p. 405; Cuv. Regme Anim. Ill. Poises. lain. 32. fig. 2; Schleg. Overz. Amphipr, Jc. in Verhand. Nat. Gesch. Nederl. Overz Bezitt. p. 20. lam. 6. fig. 6; Bleek. Verhand. Batav. Genootsch. xxi. Labr. Cten. p. y. Hab. Luzón, Cavite, Sta. Cruz; Ambuina.

3. Gén. DASCYLLUS, Cuv.

9. D aruanus, Cuz.

Valent. Amb. iii. p. 501. fig. 489 ; Renard, i. fig. 165; Seba, iii. 26.23.

Chatodon arcuatus, L. Mus. Ad. Fried. lam. 33. fig. 8; Shaz', Zool. iv. p. 34 I.

Ch. aruanus, L. Syst. Nat. i. p. $464 ; B l$. iii. p. 62. lam. igs. fig. 2; Bl. Schu. p. 220 ; Shaze. Zovl. iv. p. 348 .

Tetragonopterus, n. ${ }^{\circ}$ 6, Klein, Pisc. Miss. iv. p. $3^{8}$. lam. Ir. fig. 9 .

Chatodon abu dafur, Forsk. p. I 5 .

Lutjanus aruanus, Lacép. iv. p. 720 .

Pomacentrus aruanus, Rïpp. Atl. Fische, p. 39.

Dascyllus aruanus, Cuv. \& Val v. p. 434 ; Bleek. Banda, i. p. $24^{6}$. Banda, iii. p. Io8.

Cha todon araneus, Benn. Ceyl. Fish. lam. I 7 .

Titradrachmum arcuatum, Cant. Mal. Fish. p. 24 I. Hab. Cébú, Amboina.

Io. D. xanthosoma, Bleck N. v.

Dascyllus xanthosoma, Bleek, Banda, i. p. 247; Gthr. l. cit. 4 . p. I 2.

D. marginatus, var., Steindachner, Verhandl. Zool. Bot. Gesellsch. Wien, 1861 , p. 77 .

Hab. Luzón, Manila; China. 
I D. reticulatus, Bich.

Heliases reticulatus, Richards. Ichth. Clivin. p. 25t. Oberl, Beschret. p. 97 ; Gthr. l. cit. 7. p. 14.

$H a b$. Bohsl, Cebú; China.

4. Gén. pomachntrus, c v.

12. P fasciatus, $C$. $V$.

Cuv. \& Val. v. p. 426. lam. 134; Schleg. Overz. Amphipr. \& in. Verhand. Nat. Gesch, Nederi. Overz. Bezitt. p. 20. lain. 4. fig. I; Bleek. Batav. p. 482; Güer. Iconogr. Regne Anim. lam. I9. fig. 7 ; Gthr. l. cit. 4. p. 19.

Hab. C. de Cebú; Archipiélago Indico.

13. P. ovoides, $O$ Cart. N. v.

(M. S $\left.\mathrm{T}_{i}\right)$

P. ovoides, O. Cart. Beschrei, n. Phorgugognethen. p. 98. 1873. Hab. Luzón, Cavite, Sta. Cruz; Bohol.

r. P. trimaculatus, $C . V$. N. v.

(M. S. T)

Cuv. \& Val. v. p. 320; Schleg Overz. Amphipr. \& in Verhand. Nad. Gesch. Neterl. Overz. Bezitt. p. 20. lam. 4. fiig, 2 ; Bleek. Batav. p. 481 (no Rüpp.) Gthr. l. cit.

Hab. Luzón, Sta. Cruz, Cavite; China.

15 P. punctato-lineatus, O. Cart N. v.

Beschrei. nener Phorgugog nethen. p. 98. Hab. Bchol.

16. P. tæniurus, Bleek.

Bleek, Act. Soc. Sc. Indo-Nederl. i. Amboina, p. 51 ; Gthr. $l$. cit. 4. P, 22.

Hab. Bobol.

17. P. tæniurus, var. Cart.

Cart. Beschrei. Faryng, p. ioo. I873.

Hab. Cebú, Ubay, Bohol.

18 P. sinsiang, Bleek. N. v.

(M. S. T.)

Bleek. in Natutek. Tydschr. Nederl. Ind. 1856, xi. p. 90; Gthr. l. cit. 4. p, 22.

Hab. Samar, Borongan; Batavia.

19 P. sp. Cartier. N. v.

Hab. Cebú. 
20 P. pavo, Lacep.

(hotodon paro, Bl. t. 198. f. 1; Bl. Schn. p. 228.

Po naeentrus pavo, Lacép. iv. p. 508; Rïpp. Atl. Fische, p. \$7: Cuv. \& Va!. v. p. +1 3; Cuv. Regne. Anim. Ill. Poiss. lam. 32 fig. 3. Bleek. Banda i. p. 247; Peters in Wiegan. Archiz': 355, 1. $265 ;$ Gthr. l. cit. 4. p. 23.

Holocentrus diacanthus, Lacép. iv. pp. 338., 373.

Hab. Cebú; Amboina.

21. P. pavo, var. O. Cart. N. r.

Beschrei. Pharyng, p. 99-1873.

Hab. Cebú.

22. P. chrysopocitus, Kuhl. N. v.

Pomacentrus chrysopœcitus, (Kuhl. \& var. Hass.) Schleg. Overz: Amphipr. \&c. in Verhand. Nederl. Overz. Bezitt. p. 21. lam. 5. fig. 3; Bleek. Amboina \& Cieram, p. 284: Gthr. l. cit. 4. p. 21.

P. notostigmus, Richards. Voy. Sulph. Ichth. p. 89. lam. 44. figs. I \& 2 .

Hab. Cebú; Archipiélago Indico.

23. P. chrysurus, Cuv. N. v.

(M. S. T.)

Chrtodon chrysurus, Broussonet.

Pomacentrus chrysurus, Cuv. \& Val. v. p. 423: Gthr. l. cit. 4. p. 29. Hab. C. de Cebú; Amboina.

24. P. cyanospilus, Bleek. N. v.

(M s. T.)

Pomacentrus cyanospilus, Bleek. Ceram. ii. p. 709; Gthr. l. cit. 4. p. 30 .

P. prosoptænioidos, Bleek. Amb. \& Ceram, p. 286.

Hab. Luzón, B. de Manila, C. de Mindoro, Cebú; Amboina.

25. P. scolopseus, $Q$. et $G$. N. v.

Pomacentrus scolopseus, Quoy et Gaim. Voy. Uram. Zool. p. 398.

P. tæniops; Cuv. et Val. v. p. 423; Less. Voy. Coq. Zool. ii. p. I90. lam. 28 f. 2 ; Bleek. Sumatra, iii. p. . 51 2.

P. emano, Less. l. cit. p. r9o.

P. scolopsis, Gthr. l. c. 4. p. 28.

Hab. Cebú; Polinesia.

5. Gén. GLYPHIDODON, GTHr.

26. G cælestinus.

Renard, i. lam. 33. n. ${ }^{\circ}$ i76; Valent. n. ${ }^{\circ} 75$.

Sparus, sp., Gronov. Zoophyl. n. ${ }^{\circ} 22$. 
Chxtodon saxatilis, Forsk. p. 62; Bl. lam. 206. fig. 2. Labrus sexfaciatus, Lacép. iii p. 477. lam. 19. fig. 2. Rahti potah, Russell, i. p. 67. lam. 86.

Chatodon tyrwihtti, Benn. Fsh. of. Ceylon, lam. 25.

Glyphis don saxatilis, Rüpp. Atl. Fische. p. $35^{\text {t }}$ et $N$. W. I. Fische, p. 126 .

(i. rahti, Cuv. \& Val. v. p. 456, ix. p. 507; Cant. Mal. Fish. p. 2+2; Schleg. Oiverz. Amphipr. \&c. in Verh. Ned. Overz. Bezitt. p. 22; Bleek. Amb. \& Cer. p. 287; Richards. Ichth. Chin. p. 253.

G. cxlestinus, (Soland.) Cuv. \& Val. v. p. 464, ix. p. 508; Richards. l. c.; Bleek. in Verhand. Batav. Genootsch. xxi. Labr. Cten. p. I 5.; Guer.-Ménev. Iconogr. iii. lam. I9. fig. 8; Gthr. l. cit. 4. p. 38.

(i. tyrwhitti, Richards. l. c.

G. quadrifasciatus, Bleek. Labr. Cten. p. I7.

G. waigiensis, Bleek. Labr. Cten. p. I 3, et Batav. p. 484.

sparus fasciatus, Gronov. Syst. ed Gray', p. 60.

Hab. Luzón, Batangas, Nasugbú, C. de Cebú; China.

27. G. mutabilis. O. Cartier N. v.

(). Cart. Beschrei. Pharyng. p. soo. 1873 .

Hab. Cebú.

28 G septemfasciatus C. V. N. v

Giv. \& Val. v. p. 463; Bleek. Sumatra, i. p. $582 ;$ Gthr. l. cit. t. p. to.

Hab. Luzón, Batangas, Nasugbú; China.

29. G. tænioruptus O. Cart. N. v

o. Cart. loc. cit. p. sor.

Hab. Brhol.

3o. G affinis, Gthr. N. v.

Günthr. Cat. B. M. 4. 1862 , p. 41.

Hab. Luzón, Cavite, Sta. Cruz; China.

31. G. cingulatus, Kur. var. C:rit. N. v.

O. Cartier loc. cit. p. ror.

Hab. Ubay, Bohol.

32. G. sordidus, (Forsk.) N. v.

(M. S. T.)

Chatodon sordidus, Forsk. p. 62. n. ${ }^{\circ} 87$ Bl. Schn. p. 230.

Pomacanthus sordidus, Lacép. iv. p. 5 19; Russell, lam. 85 .

Glyphisodon sordidus, Rüpp. Atl. Fische, p. 3t. lam. 8. fig. I; Cuv. \& Val. v. p. 466; Bleek. Verhand. Batav. Genootsch. xxi. Labr. Cten. p. I6; Gihr. l. cit. 4. p. $+\mathrm{I}$. 
G. gigas, Lienard, Dix. Rapp. Soc. Nat. Maur. p. 35.

Hab. Luzon, Manila, Navotas; China.

33. G albofasciatus, $H$. et $\mathcal{F} . \mathrm{N}$. v.

(N. S. T.)

Hambr \& Jacq. Voy. Pole Ind. Poiss. p. 49, lain. 5. fig. 4; Gthr. l. cit. 4. p. 43 .

Hab. Samar, Borongan; Borneo.

34. G bonang, Blecker. N. v.

Bleeker, Sumatra, i. p. $5^{8}{ }_{2}$; Gthr. l. cit. f. p. 45. Hab. Luzón, B. de Manila; Java.

35. G. lachrymatus, $Q$ et $G$

Quoy \& Gaim. Voy. Uran. Zool. p. 388, lam. 62. fig. i; Cuo. \& Val. v. p. 478 ; Bleek. Ternate, v. p. 303 ; Githr. l. cit. p. 49. Hab. Guam, Marianas.

36. G florulentus, Gthr. N. v.

(M. S \%)

Günther. l. cit. 4. p. 49.

Hab. Luzón, B. Manila; Océano Indico.

37. G. antjerius, Bleeker

Glyphisodon antjerius, Bleek. Kokos, iv. pi $45+$; Gthr. l. cit. 4. p. 5o,

G. antjerius, (Kuhl. \& v. Hass:) Cuv. \& Val. v. p. $48 \mathrm{r}$.

G. biocellatus, Cuv. \& Val. v. p. 482; Quoy \& Gaim. Voy. Uran. Zool. p. 389; Schleg. Overz. Amphipr. \&s. in Verhand. Nat. Gesch. Nederl. Overz. Bezitt. p. 24. lam. 6. fig. 4 ; Bleek. Sum. ii. p. 286; Less. Voy. Coqu. Zool. Poiss. p. I 88.

G. zonatus, Cuv. \& Val. v. p. 483 ; Schleg. l. c.; Peters. in Wiegm. Arch. I 855, p. 266.

G. punctulatus, Cuv. \& Val. v. p. 484 .

G. andjerinus, Schleg. l. c. p. 24. lam. 6. fig. 3.

G. rossii, Bleek. Kokos, p. 48.

Hab. C. de Cebú; Borneo.

38. G plagiometopon, Bleeker. N. v.

Bleeker, Singapore, p. 67. Gthr. l. cit. 4. p. $5 \mathrm{I}$.

Hab. Luzón, Manila; Java.

39. G. unimaculatus, C. U. N. v.

(M S. T.)

Glyphisodon unimaculatus, Cuv. \& Val. v. p. 478; Schleg. Overs. Amphipr. \&c. in Verhand. Nat. Gesch. Nederl. Overz. Bezitt. p. 23. lam. 6. fig. I; Bleek. Sumatra, ii. p. 284; Gthr. l. cit. 4: P. $5 \mathrm{I}$. 
G. balinensis, Bleek. Verhand. Batav. Genootseh. xxii. Bali, p. 9. Hab. Borongan, Samar; Borneo.

40 G assimilis, Gthr. N. v.

Glyphisodon uniocellatus, Bleek. Amboin, iii. p. 19 (no Quoy \& Gaim).

Glyphidodon assimilis, Gthr. l. cit. 4. p. $5^{2}$. Hab. Luzón, Manila.

41. G dispar: Gthr. N. v.

Glyphidodon dispar, Günther. l. cit. 4. p.

Hab. Luzon, Batangas, Nasugbu; Amboina.

42. G. bankjeri, Rịchards. N. v.

(M. S. 'T.)

Glyphisodon bankieri, Richards. Ichth. Chin. p. 253; Gthr. loc. cit. 4. p. 54 .

G. nemurus, Bleeker, Booroo, ii. p. 73 .

Hab. Luzon, Santa Cruz, Cavite; Amboina.

43. G. modestus, Schleg. N. v.

(M. S. T.)

Schleg. Overz. Amphipr. \&c. in Verhan. Nat. Gesch. Overz. Bezitt. p. 23. tav. 6 fig. 2; Bleek. Sumatra, ii. p. 285. Hab. Luzón, Manila, Navotas; Amboina, China.

6. Gén. HELIATES, Githr.

44. H. cinerascens, $C . V . \mathrm{N} . \mathrm{v}$.

Cuv. \& Val. v. p. 495; Schleg. Overz. Amphipr. in Verhand. Nat. Gesch. Niderl. Overz. Bezitt. p. 24. lam. 6. fig. 5; Bleek. Labr. Ctea. p. 31; Gthr. l. cit. 4. p. 61.

Hab. C. de Cebú; Molucas.

45. H frenatus, $C$. $V$.

Cuv. \& Val. v. p. 498; Gthr. t. cit. 4. 62.

Hab. Marianas; Guam.

Fam. LÁBRIDOS.

I. Gén. CHAROPS, Rüpp.

1. Chæ. maerodon, Lacép.

Labrụs macrodantus, Lacép. iii. pp. 451, 522; Cuv. \& Val. xiii. p. 98 .

Chærops meleagris, Rüpp. l. c.

Cossyphus macrodon, Bleck. Verand. Batav. Genootsch. xxii. Labr. p. 10. 
? Labrus chlorudus, Gronov. Syst. ed. Gray, p. 80.

Ch. macrodon, Gthr. l. cit. 4. p. 94. Hab. C. de Cebú, Luzón, Batangas, Nasugbú; Australia.

2. Ch. meander, Cartier.

O. Cart. Beschrei; Pharyng. Icc. cit. p. 102. $H a b$. Cebú.

3. Chæ. ommopterus, Richarils. N. r.

?Cossyphus schonleinii, (Agass.) Cuv. \& Val. xiii. p. 143.

C. ommopterus, Richards. Ichth. Chin. p. 257.

C. sconleinii, Bleek. Celebes, v. p. 252.

Ch. ommopterus, Gthr. l. cit. 4. p. 94.

Hab. Luzón, Sta. Cruz, Cavite; China.

4. Ch. unimaculatus, Cart. N. v.

(M. S. T.)

Cart. loc. cit. p. 102.

Hab. Luzón, Cavite, Sta. Cruz.

5 Chæ. leucozona, Bleek.

Crenilabrus leucona, Bleek. Biliton, iv. p. 238.

Charops leucona, Gthr. l. cit. 4, p. 95.

$H a b$. Cebú; Biliton.

6. Chæ. anchorago. Bl. Schn.

Sparus anchorago, Bl. v. p. ro8. lam. 276; Bl. Schn. p. 276. Cossyphus anchorago, Trosch. in Wiegm. Arch. 1840, p. 279.

Ch. anchorago, Gthr. l. cit. 4. p. 95.

$H a b$. Cebú; Amboina.

7. Chæ. japonicus, (C.V.) N. v.

(M. S. T.)

Labrus japonicus, Cuv. \& Val. xiii. p. 99; Schleg. Faun. Japon. Poiss. p. 163. lam. 85.

Cossyphus japonicus, Bleek. Verhand. Batav. Genootsch. xxv. Nalez. Jap. p. 16. et xxvi. Nalez. Jap. p. II4.

Ch. japonicus, Gthr. l. cit. 4. p. 96.

Hab. Luzón, Cavite, Sta. Cruz; China.

8. Ch. oligacanthus, Bleek. N. v.

Crenilabrus oligacanthus, Bleek. Riouv, p. 489, et Singapore, p. 68.

Ch. oligacanthus, Gthr. l. cit. 4. p. 96.

Hab. Cebú; Archipiélago Indico. 
2. Gén. LABRichthys, Bleek.

9. L. rubiginosa, (Sihleg) N. v.

(M. S. T.)

Labrus rubiginosus, Schleg. Faun. Japon. Poiss. p. 168, lam. 86. fig. I.

Labrichthys rubiginosa, Gthr. l. cit. 4. p. II 4 .

Hab. Luzón, Cavite, Santa Cruz.

10. I (?) unilineata, Guichen.

Cossyphus unilineatus, Guichen. in Guer.-Menev. Rev. Zool. 1847 , p. 284.

Labrichthys unilineatus, Gthr. l. cit. 4. p. I 8.

Hab. Marianas, Guam.

3. Gén. LABRoides, BleEk.

I I. L. paradiseus, Bleck.

Valent. iii. p. 348 fig. 8, et p. 407 fig. I93; Renard, Poiss. Mol. i. lam. 24 fig. I 3 I.

Labroides paradiseus, Bleek. Banda, i. p. 249, et Atl. Ichth. p. lam. 44 fig. 2 ; Gthr. l. cit. 4, p. I I 9.

Hab. C. de Cebú; Archipiélago Indico.

4 Gén. Duymaria, Bleek.

12. D. aurigaria, Bleek N. v.

(M. S. T.)

Crenilabrus aurigarius, Richards. Voy. Sulph. Fishes, p. 90 lam. 45 , figs. I \& 2 .

C. rubellio, Richards. l. c. p. 93 lam. 45 fig. 3 .

Duymœria aurigaria, Bleek. Act. Sac. Sc. Indo-Nederl. i. Amboina, p. 53; Gthr. l. cit. 4, p. I 21.

D. rubellio, Bleck. l. c.

Hab. Luzon, Cavite, Sta. Cruz; China.

\section{Gén. Cheilinus, Cuv.}

13 Ch. festivus, C. $V$.

Cuv. \& Val. xiv. p. 124; Gthr. l. cit. 4, p. 125. Hab. Marianas.

14. Ch rostratus, Cartier.

Cartier. loc. cit. p. IOz.

Hab. Cebú.

15. Ch fasciatus, Rüpp.

Renard, Poiss. Mol. lam. 26 n. ${ }^{\circ}$ 132; Valent. iii. nos. 74 \& I I 3. 
Sparus fasciatus, $B l$. v. p. 18 , lam. $257 ; B l$. Schn. p. 269; Lacép. ix. pp. 39, I 27.

Labrus enneacanthus, Lacép. iii. pp. 433,480.

Cheilinus fasciatus, pt., Rüpp. Atl. Fische, p. 23.

C. fasciatus, Cuv. \& Val. xiv. p. 92; Rüpp. N. W. Fische, p. I8; Bleek. Verhand. Batai'. Genootsch. xxii. Labr. p. 3r; et Atl. Ichth. p. 67, lam. 26 fig. 2; Gthr. l. cit. 4, p. 129.

Hab. C. de Cebú; Amboina.

16. Ch. martensii, $C . V$.

Cuv. et Val. xiv, p. 102; Gthr. l. cit. 4, p. 125.

Hab. Guım, Marianas.

19. Ch. godeffroyi, Gthr.

Gthr. l. cit. 4. p. 126.

Hab. Cebú.

6. Gén, ANAMPSES, Cuv.

18. A meleggris, $C \quad V$.

Ruysch, Thes. i. p. 42. lam. 7. fiig. I, a.

Anampses meleagrides, Cuv. \& Val. xiv. p. г 2 ; Bleek. Act. Soc. Sc. Indo-Nederl. i. Amboina, p. 57, et Atl. Ichth. p. 102. lam. 24. fig. I.

A. meleagris, Gthr. l. cit. 4. p. 135.

Hab. C. de Césú; Amboina.

7. Gén. Stethojulis, Gthr.

19. St. strigiventer, Günth. N. v.

Julis strigiventer, Benn. Proc. Zool. Soc. 1832, p. 184; Cuv. \& Val. xiii. p. 468; Bleek. Banda, i. p. 25r.

Stethojulis strigiventer, Günth. Ann. \& Mag. Nat. Hist. 1861, viii. p. 386 ; Bleek. Atl. Ichth. p. I35. lam. 43. fig. I; Gthr. l. cit. 4. p. I 40 .

Hab. C. de Cebú; Ambrina.

2o. St esp. N. v.

(M. S. T.)

Hab. Luzón, Cavite, Sta. Cruz, Batangas, Nasugbú.

21. Pt. trilineata, Günth. N, v.

Seba, iii. 3I. 5; Valent. fig. 54.

Labrus trilineatus, $p t$., Bl. Schn. p. 253.

Julis sebanus, Cuv. \& Val. xiii. p. 474 .

J. casturi, Bleek. Celebes, iii. p. 768.

Stethojulis trilineata, Günth. Ann. \& Mag. Nat. Hist. 1861 , 
viii. p. 386; Bleek. Atl. Icth. p. I 3 I. lam 43. fig. 3; Gthr. l. cit. 4. p. 140.

Hab. Luzón, Manila; Amboina.

22. St. albovittata, Günth. N. v.

Gallenay castouri, Renard, fig. I 33 .

Labrus alboviitatus, (Bonnat.) Lacép. iii. pp. 443, 509 .

Julis balteatus, Quoy \& Gain. Voy. Uran. p. 267. lam. 56. fig. I; Cuv. \& Val. xiii. p. 475; Bleek. Banda, i. p. 253.

Stethojulis albovittata, Günth. Ann. \& Mag Nat. Hist. 1861 , viii. p. 386 ; Bleek. Atl. Ichth. p. I32. lam. 44. fig. 5; Gthr. l. cit. 4. p. $14 \mathrm{I}$.

Hab. C. de Cebú; Amboina.

23. St. kalosoma,? Bleck. N. v.

Bleek. Amboina \& Ceram, p. 289, et Atl. Ichth. p. i 34 . lam. 43. fig. 4 ; Gthr. loc. cit. 4. p. I 42.

Hab. Luzon, B. de Manila; Amboina.

\section{Gén. PLATYGLOSSUS, Kiein}

24 Pl. dussumieri, C. V. N. v.

(M. S. T.)

?Seba, iii. lam. 31. fig. 2 .

Sahnee moia, Russell, ii. fig. I 20.

?Labrus nigrescens, Bl. Schn. p. 263.

Julis dussumieri, Cuv. \& Val. xiii. p. 478, lam. 387. Cant. Catal. p. 236 .

J. exomatus, Richards. Ichth. Ciin. p. 258.

J. notophthalmus, Bleek. Verhand. Batav. Genootsch. xxii. Labr. Cyel. p. 20.

Halichæres nigrescens, Bleek. Syn. Labr. p. 14, et Atl. Ichth. p. I 8 . lam. 37. fig. 4 .

Platyglossus dussumieri, Gthr. l. cit. 4. p. I 44.

Hab. Luzon, Cavite, Sta. Cruz; China.

25. P. esp. N. v.

(M. S. T.)

Hab. Luzon, Manila, Samar.

26. P1. pseudogramma, Cartier. N. v.

Cartier: loc. cit. p. I04.

Hab. Ubay, Bohol.

27. P. esp. N. v.

Hab. Samar, Cebú.

28. Pl schwarzii, Bleek. N. v.

Julis schwarzii, Bleek. Verhand. Batav. Genootsch. xxii. Ball, p. 7 . 


$$
-553 \cdots
$$

J. dieschismenacanthus, Bleek. Banda. Neira, p. 645.

J. dieschismenacanthoides, Bleek. Amboina, ii. p. 121.

Halichares schwarzii, Bleek. Atl. Ichth. p. I17. lam.. 39. fig. 3.

Pl tyglossus schwarzii, Gthr. l. cit. 4. p. 149. Hab. Cebú; Amboina.

29 Pl. reticulatus, Cartier. N. v

O. Cartier: loc cit. p. 104.

Hab. Cebú.

3o. Pl. miniatus, C. V. N. v.

(M. S. 'T )

Julis miniatus, (Kuhl. \& v. Hass.) Cuv. \& Val. xiii. p. 460 ; Bleek. Verhand. Batav. Genootsch. xxii. Bali, p. 8, et Act. Soc. Sc. Indo-Nederl. i. Amboina, pt 60.

J. miniatus, pt., Bleek. Timor, p. 177.

Halichæres miniatus, Bleek. Atl. Ichlh. p. I14. lam. 41. fig. 5. Platyglossus miniatus, G/hr. l. cit. 4. p. 150.

Hab. Luzon, Cavite, Sta. Cruz; China.

3I P1. alternans, Cartier. N. v.

Cartier: loc. cit. p. ro4.

Hab. Cebú; Panglao.

32 Pl. guttatus, $B l$. N. v.

(M. S. T.)

Prochilus, sp., Klein, Miss. Pisc. v. lam. 12. fig. 5 .

Labrus guttatus, Bl. lam. 287. fig. 2; Bl. Schn. p. 247.

? Labrus argus, Bl. Schn. p. 263.

Julis argus, Zool. Journ. iii. p. 577. lam. I3. fig. 7 .

J. polyophthalmus, Bleek. Banka, p. $73 \mathrm{r}$.

Halichæres guttatus, Bleek. Synon. Labr. p. 13, et Atl. Ichth. p. I 24. lam. 35. fig. I.

Platyglossus guttatus, Gthr. l. cit. 4. p. 155 .

Hab. Luzón, Manila, C. de Samar, Cebú; Sumatra.

33. Pl. ubayensis, Cartier. N. v.

Cartier: loc, cit. p. I04.

Hab. Ubay, Bohol.

34. PI. binotopsis, Bleek N. v.

Halichæres binotopsis, Bleek. in Verhand. Batav. Genootsch. xxii. Bali, p. 7 ; Timor, p. 170, et Atl. Ichth. p. 122. lam. 4o. fig. 4 .

Platyglosus binotopsis, Gthr. l. cit. 4. p. 153.

Hab. Cebú; Archipiélago Indico.

35. P1. tenuispinis, Gthr. N. v.

(M. $\mathrm{S}$ T.)

Gïnther, l. cit. 4. p. $16 \mathrm{r}$.

Hab. Luzón, Cavite, Sta. Cruz; China. 


\section{P1. scapularis, Benn.}

Julis scapularis, Benn. Proc. Comm. Zool. $183^{1}$, p. 167.

J. corruleuvittatus, Rüpp. N.W. Fische, p. It lain. 4. fig. I (var).

J. elegans, (Kuhl \& v. Hass.) Cuv. \& Val. xiii. p. 467; Bleek.

Natwer. Eydschr. Nederl. Ind. iii. p. 289, ii. lam. 3.

J. leschenaultii, Cuv. \& Val. xiii. p. 4.53.

J. phaiotonia, Bleek. Bata, p. 322.

Güntheria coruleo-vittata, Bleek. Proc. Zool. Soc. 1861, p. 413;

Versl. Akad. Wet Amsterd. xiii. p. 291, et Atl. Ichth. p.

137. lam. 32. fig. 2.

Hab. Cebú; Amboina.

37. Pl pocilopterus, Schleg N. v.

Julis poeciiopterus, Schleg. Faun. Japon. Poiss. p. 169. lam. 86 bis, fig. I; Richards. Ickth. Chin. p. 260.

J. thersites, Richards. l. $c$.

Platyglossus poecilopterus, Gthr. l. cit. 4. p. 166.

Hab. Luzon, Manila, Navotas; China.

9. Gén. pSeudojulis, Bleek.

38. Ps. girardi, Bleek N. v.

Julis girardi, Bleek. Bali, iii p. 168 .

Pseudojulis girardi, Bleek. Proc. Zool. Soc. 1861, p. 412, et Atl. Ichth. p. I 27. lam. 33. fig. 3. Gthr. l. cit. 4. p. 168.

Hab. Manila, Luzón; C. de Boleling.

1o. Gén. NOVACUla, C. V.

39. R. tæniurus, Lacép. N. v.

Labrus fuscus, Lacép. iii. p. 437.

Sparus hemisphærium, Lacép. iv. pp. 53, 60.

S. brachio, Lacép. iv. pp. 54, I60.

Labrus tæniurus, Lacép. iii. pp. 448, 518. lam. 29. fig. I.

Xyrichthys tæniurus, Cuv.\&. Val. xiv. p. 54. lam. 363. (no Bleek.)

Novacula tæniurus, Gthr. l. cit. 4. p. I 72.

Hab. C. de Cebú; Mauricio.

40. N. pentadactyla, Ciøv. N. v.

Valent. figs. 67, 1 23, 262, Renard, i. fig. 84 , ii. figs. 6. I1 2; Ruysch, lam. 20. fig. 8.

Blennius, s, p., Ankarkrona, Act. Holm. i. I740 p. 451. lam. 3 . tig. 2

Coryphana pentadactyla, L. Syst. i. p. $447 ; B l$. lam. 173; Bl. Schn. p. 294.

Himipteronotus quinque-maculatus, Lacep. iii. p. 2 I 5.

Novacula pentadactyla, Cuv. \& Val. xiv. p. 69. lam. 395; Bleek. Cieieb. i. p. 222; Gthr. l. cit. 4. p. 177. 
N. sexmaculata, Cav. \& Val. xiv. p. 72.

Hemipteronotus pentadactylus, Bleek. Atl. Ichth. p. lam. 30. fig. 4. Hab. Luzón, Lobo, Batangas; China.

41. N. punctulata, C. I. N. v.

Cuv. \& Val. xiv. p. 73; Gthr. l. cit. 4. p. 177.

Hab. Luzon, Cavite, Sta. Cruz; China.

II. Gén JULIS, Gthr.

42 J. lunaris, Cuv. N. v.

(M. S. T)

Seba, iii. lam. 31. fig. 7; Valent. fig. 178; Renard, ii, fig. 39.

Labrus lunaris, L. Syst. Nat. i. p. 474; Lacep. iii. pp. 427,467; Gronou. Syst. ed. Gray. p. 82.

Labrus, n. ${ }^{\circ}$ Io, Gronov. Mus. Ichthol. ii. lam. 6. fig. 2; Zonphyl. p. 71, n. $2+2$.

Scarus gallus, Forsk. Descr. Anim. p. 26.

Labrus ceylonicus, (Forst). Penn. Ind. Zool. p. 56. lam. 16; Bl. Schn. p. 246; Lacép. iii. pp. 427, 472.

L. viridis, Bl. v. p. i 29. lam. 282; Bl. Schn. p. 243; Lacép. iii. pp. 454, 222.

L. gallus, L. Gm. i. p. 1284;Bl. Schn. p. 245 .

Osphronemus gallus, Lacép. iii. p. I 22.

Julis hardwickii, Gray. Ind. Zool. Pisc. lam. 9. fig. 1.

J. porphyrocephala, Benn. Proc. Comm. Zool. Soc. ii p. $8_{3}$.

J. duperrei, Quoy. \& Gaim. Voy. Wan. Zool. p. 268. lam. 56. fig. 2; Cuv. \& Val. xiii. p. 421.

J lunaris, Cuv. \& Val. xiii. p. 4c9; Bleek. Verhand. Batav. Genoo sch. xxii. Labr. p. 28, Synon. Labr. p. 6, et Atl. Ichth. p. 90. lam. 33. fig. 5; Gthr. l. cit. 4. p. 186.

J. viridis, Cuv. \& Val. xiii. p. 420.

J. meniscus; Cuv. \& Val. xiii. p. 4r5.

J. trimaculatus, Rüpp N.W. Fische, p. 13. (no Q. \& G.)

J. mertensii, Cuv. \& Val. xiii. p. $42 \mathrm{r}$.

J. celebicus, Bleek. Celebes. p. $3^{\mathrm{1}} 3$.

J. lutescens, (Solander), Zool. Beech. Voy. Fisches, p. 65 lam. 19. fig. 2.

Hab. Luzón, Manila, Cebú; China.

43 J. umbrostigma, Rüpp. N. v.

(M. S. 'T)

Julis umbrostigma, Rüpp. N.W. Fisch, p. ı1. laın. 3. fig. 2; Bleek. Atl. Ichth. p. 92; lain 34. fig. 2; Gthr. l. cit. 4. p. 185 . J. souleyetii, Cuv. \& Val. xiii. p. 457; Eyd. \& Soul. Voy. Bonite, Zool. i. p. 19o, Poiss. lism. 6. fig. 2; Bleek. Kokos, iii. p. 176. Hab. Luzon, Cavite, Sta. Cruz; China.

44. J. trilobata, Lacép. $\mathrm{N}$ v.

(M. S. T)

Labrus trilobatus, Lacép. iii. pp. 454, 526. (no Saw.)

L. fuscus, Lacép. iii. p. 437.

Julis bicatenatus, Benn. Proc. Coinm. Zool. Soc. i. 1831, p. 167. 
J. quadricolor, Less. Voy. Coqu. Zool. ii. p. 139. lam. 35. fig. I; Cuv. \& Val. xiii. p. 443; Bleek Nat. Tydschr. Nederl. Ind. xix. p. 339, et Atl. Ichth. p. 93. lam. 34. fig. 3.

J. semicoruleus, Rüpp. N.W. Fische, p. io. lain. 3. fig. 1; Cur. \& Val. xiii. p. 442 .

Scarus georgii, Benn. Fish. Ceyl. lam. 24.

Julis trilobatus, Cuv. \& Val xiii, p. 437 ; Gthr. l. cit. 4. p. 187.

J. formosus, Cuv. \& Val. xiii. p. 439 .

J. aruginosus, Cuv. \& Val. xiii. p. 441 .

J. cyanogaster, Cuv. \& Val. xiii. p. 444.

J. erythrogaster, Cuv. \& Val. xiii. p. 447.

Scarus (?) quinquevittatus, Richards. Voy. Blossom, Zool. p. 66. lam. 19. fig. 3 .

Hab. Luzón, Manila, Navotas; China.

45. J. dorsalis, Quoy. et Gaim. N. v.

(M. S. 'T.)

Renard, Poiss. Mol. i. lam. 28. fig. I55, ii. fig. 68; Valent. fig. 53 .

Sparus hardwickii, Benn. Fish. of Ceyl. pl. i 2 (no Julus hardwicki, Gray).

Julis dorsalis, Quoy. \& Gaim. Voy. Astrol. Zool. iii. Poiss. p. 713. lam. 15. fig. 5; Cuv. \& Val. xiii. p. 449; Bleek. Amboina, ii. p. 564, et Atl. Ichth. p. 94. lam. 34. fig. 4. Gthr. l. cit. 4, p. 190 .

J. semifasciatus, Cuv. \& Val. xiii. p. 448.

J. urostigma, Bleek. Sumatra, ii. p. 287.

Hab. Luzón, Manila, Navotas, Cavite, Santa Cruz, Samar, Villareal; China.

46. J. truncatus, Cartier.

Cartier, loc, cit. p. 105.

$H a b$. Cebú.

47. J. schwanefeldii, Bleeker.

Bleeker, Sumatra. ii. p. 288, et Atl. Ichth. p. 95. lam. 33. figr. 7; Gthr. l. cit. 4. 191.

Hab. Luzón, Batangas Nasugbú, Cebú; Sumatra.

12 Gén. GOMPHOSUS, Lacép.

48. G tricolor, Quoy et Gaim. N. v.

(M S. 'T)

? Renard, ii. lam. 22. fig. Io9.

Gomphosus tricolor, Quoy. \& Gaim. Voy. Uran. Zool. p. 280 . lam. 55. fig. 2; Bleek. Act. Soc. Sc. Indo-Nederl. i. Manado \& Makars. p. 54, et Atl. Ichth. -p. 85. lam. 21. fig. 6; Githr. l. cit. 4. p. 193 .

G. cepedianus, Cur. \& Val. xiv. p. 18. lam. 390. Hab. Luzón, Cavite, Santa Cruz; China. 
49. G. varius, Lacip.

Gomphosus varius, Lacép. iii. p. 104. lam. 5. fig. 2; Gtkr. l. cit. 4. p. 193.

G. pectoralis, Quoy. \& Gaim. Voy. Vram. Zool. p. 282.

G. fuscus, Cuv. et Val. xiv. p. 25.

Hab. Marianas; Mauricio.

13. Gén. Cheillo, lacér.

50 ch inermis, Bleek. N. v.

(M. S T.)

Valent. figs. 203. \& 516; Renard, i. lam. 34. fig. $18 \mathbf{1}$.

Labrus inermis, Forsk. p. 34; Bl. Schn. p. 262.

L. hassek, Lacép. iii. p. 5I 3 .

Cheilio auratus, (Commers,) Lacép. iv. p. 433; Quoy. \& Gxim.

Voy. Uran. Zool. p. 274. lam. 54. fig. 2; Cuv. \& Val. xiii. p. 34I; Bleek. Celebes, i. p. 22 I.

(h. fuscus, (Commers.) Lacép. iv. p, 433. Cuv. \& Val. xiii. p. 349 .

Labrus fusiformis, Rüpp. $N . W$ Fische, p. 7. lam, I fig. 4 .

Cheilio cyanochloris, Cuv. \& Val. xili. p. 346. lam. 382.

Ch. forskalii, Cuv. \& Val. xiii. p. 349.

Ch. hemichrisos, Cuv. \& Val xiii. p. 351; Bleek. Banda, i. p. 252.

Ch. viridis, Cuv. \& Val. xiii. p. 352 .

? Cheiliu microstoma, Cuv. \& Val. xiii. p. 353.

Cheilio ramosus, Jenyns, Zool. Beagle, Fisches, p. 102.

Ch. b:color, Bianc. Spec. Zool. Mosamb. p. 254. lam. 5.

Ch. inermis, Bleek. Atl. Ichth. p. 82. lam. 31 fig. 4; Gthr. l. cit. 4. p. 194.

Hab. Luzón, B. de Manila, C. de Cebú; Amboina.

14. Gén Cheillinus, Gill.

5 I. Ch. rostratus, Cartier.

Hab. Cebú.

14 Gén. PSEUdodaX, Blee $\mathrm{K}$.

52 Ps. moluccensis, (C. V.)

Renard, i. lam. 2 n. ${ }^{\circ}$ i $;$ Valent. p. 359 fig.

Odax moluccanus, Cuv. \& Val. xiv. p. 305 lam. 408 Cuv. Regne. Anım. Ill. Poiss. lam. 91 fig. 3; Bleek. Act. Soc. Sc. IndoNederl. i. Manado. en Makoss. p. 64; Kner, Pitagsber. Wien. Acad. 1860 , xl. p. 56, la.n. 2 fig. 18.

Pseudodax moluccanus, Bleek. Atl. Ichth. p. 80, lam. 22 fig. 2, lam. I 8 fig. 5.

Hab. Bohol, C-bú; Cèlebes. 


\section{Gén SCARICHTHYS, BleEk.}

16. Sc. auritus Bleek. N. v.

(M. S. T.)

Scarus auritus, (Kuhl. \& van Hass.) Cuv. \& Val. xiv. p. 2 I8. S. vaigiensis, pt., Quoy \& Gaim. Voy. Freyc. Zool. p. 288; Cuv. \& Val. xiv. p. 214.

S. novius, Cuv. \& Val. xiv, p. 253; Bleek. Celebes, iii. p. 760 . Callyodon chlorolepis, Richards. Voy, Sulph. Ichthyol. p. 137, lam. 64 figs. 4-7.

Scarichthys auritus, Bleek. Atl. Ichth. p. I 5 lam. I fig. 3. Hab. Luzón, Manila; China.

\section{Gén CALlyodon, C. V.}

54. C. carolinus, Cuv.

Callyodon carolinus, Cuv. \& Val. xiv. p. 291 .

Callyodon carolinus, Bleek. Natuurk. Tydschr. Nederl. Ind. 1859 , xx. p. 444, et Atl. Ichth. p. 14 lam. 2 fig. 4; Gthr. l. cit. 4, p. 214 .

Hab. Carolinas.

55. C. brachysoma, Bleeker.

Bleeker, Atl. Ichth. p. I4 lam. 2 fig. 3; Gthr. l. cit. t, 1. 215. Hab. Zumárraga, Samar; Ternate.

56. C. spinidens Q. et $G$.

Scarus spinidens, Quoy \& Gaim. Voy. Uran. Zool. p. 289.

S. vaigiensis, pt., Quoy \& Gaim. l. c. p. 288; Cuv. \& Val. xiv. p. $2 \mathrm{I} 4$.

Callyodon waigiensis, Cuv. \& Val. xiv. p. 296.

C. hypselosoma, Bleek. Amboina, vi. p. 425 .

C. spinedens, Bleek. Atl. Ichth. p. I3 lam. 2, fig. 2; Gthr. l. cit. 4, p. 2 I 5 .

Hab. C. de Samar; Amboina.

57 C. $8 p$ N. v.

Hab. Luzón, Manila.

17. Gén PSEUdOSCARUS, GrHr.

${ }_{5} 8$ Ps spilurus. $C . V$.

Scarus spilurus, Cav. \& Val. xiv. p. 279.

Hab. Carolinas.

59. Ps pulchellus, Bleek. N v.

(M S. 'T.)

Scarus pulchellus, Rüppell, $N$. W. Fische, p. 25. lam. 8. fig. 
3, et ierz. Fische Senckenl. Mus. p; 21; Cwo. \& Val. xir. p. 266; Bleek, Java, ii. p. 4to.

Pseudoscarus pulchellus; Bleek. Atl. Ichth. p. 34. lam. 10. fig. 3; Gthr. l. cit. 4. p. 219.

Hab. Luzón, Cavite, Sta. Cruz; China.

6o. P8. margaritus, Curticr.

Cartier. loc. cit. p. 105.

Hab. Cebú.

6I Ps. esp N. v.

(M. S T.)

Hab. Luzón, Pasacao, C marines Sur, Cavite, Sta. Cruz.

62. Ps chrysopoma, Bleek.

Scarus quoyi, (part.) Cuv. \& Val. xiv. p. 273.

S. chisopomus, Bleek. Verhand. Batav. Gen. xxii. Labr. Cylc. p. 53; Gthr. l. cit. 4. p. $22 \mathrm{I}$.

Pseudoscarus viridis, Bleek. (no Bloch.) Atl. Ichth. p. 45. lam. 17. fig. 2.

Hab. Cebú; Indias Orientales.

63. Ps sp Curtier.

Cartier, loc. cit. p. 105.

Hab. Cebú.

64. Ps. pyrrhostethus. Bleek. N v.

(M. S. T.)

Scarus, psittacus, Forsk. p. 29; Shaw, Zool. iv. p. 397; Rüpp. Atl. Fische, p. 77. lam. 20. fig. 1; Cuv. \& Val. xiv. p. 226. Cant. Catal. p. 239; (no Curyphana psittacus, L.)

Scarus pyrrhostethus, Richards. Jchth. Chin. p. 262.

S. harid, Cuv. \& Val. xiv. p. 247. (part.) lam. 404; Cuv. Regne Anim. Ill. Poiss. lam. 91, fig. i; Bleek. Verhand. Batav. Genootsch. xxii. Labr. Cycl. p. 48. (no Forsk.)

S. haridoides, Bleek. Enumer. p. 104.

Pseudoscarus pyrrhostethus, Bleek: Atl. Ichth. p. 42. lam. 9, fig. I; Gthr. l. cit. $4 \mathrm{t}$ p. 223 .

Hab. Luzón, Cavite, Sta. Cruz; China.

65. Ps æruginosus, $C . V$. N. v.

(M S. T.)

:Scarus æruginosus, Cuv. \& Val. xiv. p. 257.

S. lacerta, Cuv. \& Val. xiv. p. 217.

S. aruginosus, Bleek. Verhand. Batav. Genootsch. xxii. Labr. Cycl. p. 58. Gthr. l. cit. 4. p. 222.

Hab. Luzón, Cavite, Sta. Cruz, Batangas, Nasugbú; Archipiélago Indico.

66. Ps. (?) ocellatus, C. V.

Scarus ocellatus, Cuv. \& Val. xiv. p. 278. 
Ps. ocellatus, Gth. l. cit. 4. p. 233 . Hab. Carolinas.

67. Ps. celebicus. Bleck. N. v. (M. S. T.)

Scarus celebicus, Bleek Celebes., v. p. 253.

Pscudoscarus celebicus, Bleek. Atl. Ichth. p. 26. lam. 15. tig. 2; Gthr. l. cit. 4. p. 237.

Hab. Luzón, Cavite, Sta. (ruz; China.

6. Ps. muricatus, Bliek.

Scarus muricatus, Cuv. \& Val. xiv. p. 208. lam. 402; Bleek. Verhand. Batav. Genootsch. xxii. Labr. p. 46.

Pseudo carus muricatus, Blesk. Atl. Ichth. p. 26. lam. 7. fig. 3; Gthr. t. cit. 4. p. 238.

Hab. C. de Mindoro; Java.

69. Ps. balinensis Blkr.

Scarus balinensis, Bleek. Verhand, Batav. Genootsch. xxii. Bal', p. 8.

Pseudoscarus balinensis, Bleek. Atl. Ichth. p. 39. lam. i6. fig. 3; Gthr. l. cit. 4. p. 228.

Hab. Luzón, Pasacao, Camarines, Cebú; Banda.

\section{Fam. GÉRRIDOS.}

1 Gén GerRes, Cuv.

I. C. phílippinus, Gthr.

Günther, l. cit. 4. p. 258.

Hab. Filipinas.

2. G. kapas, Bleeker. N. v.

$\left(\begin{array}{lll}\mathrm{M} & \mathrm{S} & \mathrm{T}\end{array}\right)$

Bleeker, Rionv., p. $482 ;$ Gthr. l. cit. 4 p. 259.

Hab. Luzón, Manila, C. de Saınar; Archipiélago Indico.

3. G. punctatus, $C, V$. N. v.

(M. S. 'T )

Cuv. \& Val.'vi. p. 480 ; Gthr. l. cit 4. p. 260.

Hab. Luzon, Cavite, Sta. Cruz; China.

4. G. japonicus Bleeker. $\mathrm{N}$ v.

(M. S T)

Bleeker, Japan, p. 404, et Verh. Bat. Gin. xxvi. Jaban, p. 93. lam. 5. fig. 2. Gthr. l. cit. 4. p. 260.

Hab. Luzón, Sta. Cruz, Cavite; China, Emuy.

5. P. oyena, Cuv. N. v

Labrus oyena, Forste. p. $35 ; B l$. Schn. p. 245 ; Lxep iii. p. $4^{6} 3$. 
Sparus erythrurus Bl. lain. 261.

Cichla erythrura, Bl. Schn. p. 336.

Sparus britanus, Lacép. iv. pp. 132, 134 .

Labrus longirostris, Lacép. iii. p. 467 . lam. 19. fig. I.

Sinaris oyena, Rüpp. Atl. p. 11. lam. 3. fig. 2.

lierres oye la, Cuv. \& V'a!. vi. p. 472;? Jenyns, Zool. B!zgle, Fisches, p. 59; Bleek. Verhand. Batav. Genootsch. xxiii. Mania. p. 12; Gthr. l. cit. 4. p. 261.

(i. oblongus, Bleek. Topogr. Batav. (no Cuv.)

:Gerres equul, Temm. \& Schleg. Faun. Japon. Hoiss. p. 70. lam. 4. fig. I. Bleek. Verhand. Batav. Genootsch. xxvi. p. 92. Hab. Cebú; Mar Rojo.

\section{Orden $33^{\circ}$ ANACANTINOS.}

\section{Fam. GÁDIDOS.}

I. Gén. BREGMACEROS, Thomps.

I. B. macclellandii, Thomps. N. v.

Bregmacerọs mecclellandii, Thomps. l. c. fig.; Gthr. l. cit. f. p. 368 .

Calloptillum mirum, Richards. l. c. 95. lam. 46. figs. 4. 7.

Hab. Luzón, Cavite, Sta. Cruz, Samar, Villareal; China.

\section{Fam. OríDIDOS.}

I. Gén. ENCheliophis, Mülr.

I. E. vermiculıris, Müll N. v.

Müll. l. c. p. I54. lam. 5. figs. 4. \& 5; Gthr. l. cit. 4. p. $3^{8}+$. Hab. Filipinas.

2. Gén. CONGrogadus, Gthr.

2 C. subducens, Richards. N. v.

Machærium subducens, Richards. Ann. \& Mag. Nat. Hist. 1843, xii. p. I75. lam. 6, et Voy. Ereb. \& Terr. Fish. p. 72. lam. 44. figs. $I-6$.

M. reticulatum, Bleek. Banka. ii. p. 734 .

Congrogadus subducens, Gthr. l. cit. 4. p. 388. Hab. Cebú; Australia. 


\section{Fam. PLEURONÉCTIDOS.}

1. Gén PSETtOdes, BenN.

1. Ps. erumei, Sckn. N. v. Daba.

(I. S. T )

Pleuronectes erumei, Bl. Schn. p. I 5 \%.

Adalah, Russell, i. p. 54. lam. 69.

Nooree nalakah, Russell, i. p. 6o. lan. 77 .

Hippoglossus erumei, Cuv. Regne Anim.; Rüpp. Atl.' Fische, p. 121, et N.W. Fische, p. 84; Cant. Catal, P 216; Bleek. Verh. Batav. Genootsch. xxiv. Pleuron. p. I3.

H. nalaka, Cuv. Régne Anim

H. dentex, Richards Voy. Sulph. Fish. p. 102. lam. 47.

H. orthorhynchus, Richards. Ichth. Chin. p. 278.

? Psettodes belcheri, Benn. Proc. Comm. Zool. Soc. 1831, p. 147. Ps. crumei, Gthr l. cit. 4. p. 402.

Hab C. de Mindoro, Luzon, Manila, Cavite, Santa Cruz; China.

2 Gén. Tephritis, Githr.

2 T. sinensis, Lacép. N. v

(M. S. T.)

Pleuronectes sinensis, Lacép. iv. pp. 595, 658. lam. 14. fig. I Platessa chinensis, Gray, Ind. Zool, lam. fig. 1; Richards. Ichth. Chin. p. 277.

Tephritis sinensis, Gthr. l. cit. 4. p. 506. Hab. Luzon, Cavite, Sta. Cruz; China.

3 Gén. SAMARIS̀; Gray.

3. S. cristatus, Gray. $\mathrm{N}$ v.

(M. S. T.)

Gray, l. c. p. 5; Gthr. l. cit. 5. p. 420.

Hab. Luzón, Cavite, Santa Cruz; China.

4 Gén. PSEUdorhombus, Bleek.

4 Ps. russellii, (Gray.) N. v.

(M. S T )

Platessa russellii, Gray, Ill. Ind. Zool. lam. fig. 2; Cant. Catal. Mal. Fish. p. 214.

Rhombus lentiginosus, Richards. Ann. \& Mag. Nat. Hist. xi. 1843 , p. 495; Bleek. Verhand. Batav. Genootsch. xxiv. Pleuron. p. 15 .

Platessa chrysoptera, Richards. Ichth. Chin. p. 278.

Pl. balteata, Richards. $l$. c.

? Rhombus polyspilus, Bleek. Batav. p. 504 .

Rh. oligodon, Bleek. Verhand. Batav. Genootsch. xxvi. Nieuwe Nelez \&c. p. I21; Natuurk. Tydchr. Nederl. Ind. vi. p. 419; Act. Soc. Sc. Indo-Nederl. v. Japan, v. tab. 3. fig. 2.

Pseudorhombus russelii, Gthr. l. cit. 4. p. 424. Hab. Luzón, Manila; China. 
5. P8. pentophthalmus,? Gihr. N. v.

(M. S. T.)

Gthr. l. cit. 4. p. 428 .

Hab. Luzón, Manila, Narotas; China.

6 Ps. olivaceus, Sikhg. N. v.

(M. S. T.)

Hipoglossus olivaceus, Schleg. Faun. Japon. Poiss. p. 184. lam. 94. Rhombus wolftii, Bleck. Jafan, p. 421, t. Ait. Soc. Sc. IntcNederl. v. Japan. v. lam. 2. fig. 3.

Ps. olivaceus, Gthr. l. cit. 4. p. +29 .

Hab. Luzón, C.vite, Santa Cruz; China.

5. Gén. RHUMBOIDICHTHYs, Bt.kek.

7. Rh. pavo, Bleck. N v

(M S T $)$

?? Rombus macropterus, Gaim. Voy. Uran. Zool. p. 2.36; Poiss. lam. 50 .

Rh. pavo, Bleek. Kokos, iii. p. 177.

Rhomboidichthys pavo, Gthr. l. cit. 4. p. 435.

Hab. Luzón, Manila, Navotas; China.

8. $\mathrm{Rh}$ assimilis, Gihı. N. v.

(M. S T)

Gthr. l. cit. 4. p. 437.

Hab. Luzón, Manila, Navotas; China.

9. Rh. grandisquamma, Schley. N v.

(M. S. T.)

Rhombus grandisquamma, Schleg. Faun. Japon. Po ss. p. I8.3. lam. 12. f. $3 \& 4$.

Rhomboidichthys gr. ndisquama, Gthr. l. cit. 4. p. 537.

Hab. Luzón, Cavite, Santa Cruz; China.

\section{Gién. SOlEA, Cuv.}

10. S ovata, Richards N v. Sunoc, o Rodaballo.

(M S T)

? Solea ovata, Richards. Ichth. Ochin. p. 279 (no Cant); Gthr.

l. cit. 4. p. 472.

Hab. Luzón, Manila, Navotas; China.

\section{Gén PARDAChIRUS, Githr.}

II. P. pavorimus Lacep. $\mathrm{N}$ v.

Achirus pavonimus, Lacép. iv. pp. 658, 66r; Cant. catal. p. 225; Bleek. V'erhand. Batav. Genootsch. xxiv. Pleuron. p. 18. Pleuronectes pavoninus, Sharv. Zool. iv. p. 310.

Pardachirus pavoninus, Gthr. l. c. 4. p. 479. Hab. Cebú; Singapore. 
8. Gén. SyNAPtura, Cantor.

12. Sy. foliacea, Richards. N. v.

(N. S. T.)

Solea foliacea, Richards. Ichth. Chin. p. 279.

S. ovalis, Richards. l. c.

Sinaptura foliacea, Gthr. l. cit. 4. p. $48 \mathrm{r}$.

Hab. Luzon, Manila, Navotas; Chin..

13. Sy. zebra, Cant. N. v.

(M. S. T)

Pleuronectes zebra, Bl. Aust. Fische, iii. p. 27, lam. 117 Bl. Schn. p. 151; Lacep. iv. p. 597; Shazw. Zool. iv. p. 305. lam. 4 .

Sole' zebra, Cuv. Regne Anim., Bleek. Verhand. Bavat. Genootsch. xxiv. Pleuron. p. 16.

S. zebrina, Schleg Faun. Japon. Poiss. p. 86, lam. 95, fig. I.

S. ommatura, Richards Ichth. Chin. p. 279.

Synaptura zebra, Cart. Catal. Mal. Fish. p. 224; Gthr. l. cit. 4. p. 484.

Pleuronectes fasciatus, Gronov. Syst. ed. Gray, p. 91.

Hab. Luzon, Cavite, Santa Cruz, Samar; China.

14. Sy. quagga (Kaup.) N. v.

(M. S. T.)

Esopia quagga, Kaup in Wiegm. Arch. 18,58, p. 98.

Hab. Mindoro, Calapan; China.

9. Gén. CYNOGLOSSUS, Ham. Buch.

15. Cy. auro-limbata? Richards. N. v.

(M. S. T.)

Plagiusa auro-limbata, Richards Ichth. Chin. p. 289. China.

Cynoglussus auro-limbatus, Gthr. l. cit. 4. p. 492.

Hab. Luzon, Cavite, Santa Cruz; China.

16. Cy. abbreviatus, Gray. N. v.

(M. S. T.)

Plagusia abbreviata, Gray, Ill. Ind. Zool.

Arelia abbreviata, Kaup, l. c.

Cynoglossus abbreviatus, Gthr. l. cit. 4. p. 494.

Hab. Luzon, Manila, Navotas; China.

17. Cy. trigrammus, Gthr. N. v.

(M. S. 'T.)

Gthr. l. cit. 4. p. 494.

Hab. Luzon, Cavite, Santa Cruz; China.

18. Cy. melampetalus, Richarts. $\mathrm{N}$ v,

(M. S. 's.)

Plaguiusa melampetala, Richards. Ichth. Chin. p. 281.

Cynoglossus melampetalus, Gthr. l. cat. t. p. 406.

Hab. Luzón, Samar, Borongan; China. 
19. Cy. quadrilineatus, Blicker. N. v.

(M. S. T.)

Achirus bilineatus, Lacép. iv. pp. 659, 663.

Plagusia bilinea a, Räpp. Atl. Fische, p. 123.

Pi. quadrilineata, Bleek. Verhand. Batui'. Genootsch. xxiv. Plenron. p. 2t, or Natuurk. Thutschr. Naterl. Ind. i. p. 412.

Arelia quadrilineata, Kaup. in Viegm. Arch. 18.58, p. so7.

Cyoglossus quadrilineatus, Gthr. 1. cit. 4. p. 497.

Hab Luzón, Manila; Amboina.

20. Cy. oxyrhynchas $B_{i \imath k}$ N. v.

(M. S. T)

Plagusia oxyrhnchos, Bleek. Verhand. Batav. Genovtsch. xxiv. Plestron p. 26, or Natuurk. Tydschr. Nedterl. Ind. i. p. $4 \mathrm{I} 6$.

Arelia oxyrhynchos, Bleek. Enum. p. 185 .

Cynoglossus oxylumchus Gthr. l. oit. 4. p. 499:

Hab. Luzón, Cavite, Sta. Cruz; Amboina.

2I Cy puncticeps, Richaris. N. v.

(M. S. T)

Piagiusa punticeps, Richards. Ichth. Chin p. 280.

Plagusia javanica, Bleek. V'orhand. Batav. Genootsch. xxiv. Pleztron. p. 26, or Nat.nrk Tydschr. Nedsrl. Ind. i. p. 414.

Hab. Luzón, Manila; China.

22. Cy. brachyrynchus, (B!)

Plagusia brachyrynchus, Bleek. Verhand. Batar. Gen. xxiv. Ple:ron. p. 24, or. Natuurk Tydschr. Nederl. Ind. i. p. $t^{14}$

Arelia brachyrychus, B!sek. En:tın. p. I84.

Cynoglossus brachyrynchus, Gthr. l. cit. 4. p. 499.

Hab. Luzón, Manila.

\section{Orden. FISÓSTOMOS.}

\section{Fam. SILÚRIDOS.}

I. Gén Clarias, Gronov.

1. cl. macrocephalus, Gthy. $\mathrm{N}$ v Hito.

(M. S. T.)

Hab. Luzón, Manila, Laguna de Bay; Siam.

2. Cl. fuscus, La:ép N. v. Hito.

(M. S. T.)

Macropteronotus fuscus, Lacep. v. p. 88. lam. 2. f. 2.

Clarias pulicaris, Richards. Voy. Sulph. Fish. p. 135 lam. 62. figs. $5 \& 6$.

C. fuscus, Gthr. Cat. B. M. 5. p. 18. 1864.

Hab. Luzón, Manila, Navotas; China. 
3. Cl. melanoderna, Bleik.

(AI. S. T)

Clarias melanoderma, Bleek. Verh. Bat. Gen. xxi. Silur. Batai'. p. 54; Prodr. Silur. p. 341; et Atl, Ichth. Silur. p. 102. lam. 97. fig. 2; Gthr. l. cit. 5. p. 12.

C. melasoma (melanosoma), Bleek. Nat. Tydschr. Ned. Ind. iii. p. 427.

Hab. Luzon, Camarines Sur, Lagruna de Batos, Laguna de Bay; Java, Borneo.

4. Cl. liacanthus, Bikr. N. v.

(M. S. 'T.)

Bleeker, Nat. Tydschr. Nederl. Ind. ii. p. 430; Prodr. Silur. p. 347 ; et Atl. Ichth. Silur. p. Iot. lam. 98. fig. I. Gthr. Cat. Fische, p. 20: Gthr. l. cit. 5. p. 20.

Hab. Paragua; Java, Borneo.

5. Cl nieuhofii, Cuv et V. N. v.

Bontael, Nieuh. Lant-en Zee-reibe, p. 271.

Lampreta indica, W'ill Ichth. App. p. 4. lam. 6. f. 2

(larias nieuhoffii, Cuv. \& Val. xv. p. 386; Hyrtl. Denkschr. Acad. Wiss. Wien, 1859, xvi. p. I7; Bleek. Prodr. Silur. p. $35 \mathrm{I}$, et Atl. Ichth. Silur. p. 105. tab. 99. f. I; Gthr. l. cit. 5. p. 20.

C1. pentapterus, Bleek. Nat. Tydschr. Nederl. Ind. ii. p. 206. Hab. Filipinas; Archipiélago Índico.

\section{Gén HETEROdRANCHUS, Genfe.}

6. $\mathrm{H}$ tapcinopterus, Bicek. $\mathrm{N}$ v.

(M. S. T)

Bleek. Nat. Tydshr. Nederl. Ind. iii. p. 732; Prodr. Silur. p. 337; et Atl. Ichth. S.lur. p. 1or. lam. 97. fig. I.

Hab. Samar; Borneo.

\section{Gén. Illotosts, Lacér.}

7. Pl. anguillaris, Lacép. N. v.

(M. S T.)

Valent. Amb. iii. p. 502. fig. 496; Renard. Poiss. Mol. i. lam. 3 fig. II; Russell. ii. p. 5I. lam. I66.

silurus arab, Forsk. Descript. Anim. p. xvi. n. ${ }^{0} 36$.

Platystacus anguillaris, Bloch, viii. p. 6r. lam. 363. fig. I; $B l$. Schn. p. 373. lam. 74; Shaw. Zool. v. p. 30. lam. 99.

Plotosus anguillaris, Lacép. v. p. г 3o. lam. 3. f. 2; Rüpp. $N$. $W$. Fische, p. 76; Cant. Mal. Fish. p. 264; Peters, in Wiegm. Arch. xxi. p. 267; Bleek. Siaur. p. 314 ; Gthi. l. cit. 5. p. 24.

Plotosius ikapor, Less. Voy. Coqiı. Zool. ii. p. I32. lam. 31. f. 3. Plotosus marginatus, Benneti. in Life of Raffles, 691.

Pl. lineatus, Civ, \& Val. xv. p. 4I2; Richards. Fish. Chin. p. 286; Schleg. Faun, Jap. Poiss. p. 228. lam. 104. fig. 3; Bleek. in Verh. Bat. Gen. xxi., Silur. Batar. pp. 4, I7, 57. 
Pl. castaneus, Cur. \& Iral. Xr. p. \$2I.

Pl. castancoides, Bleek. Nat. Tydsckr. Nederl. Ind. ii. p. 490.

Pl. arab, Bleek. Atl. Ichth Silus. p. 98. Iam. 95. f. 2. Hab. I uzón, B. de Manila, Nisugbú, Batangits, C. de Cebú, ( . de Samar; Bcrneo, China.

S. Pl. canius. Hom. N. v.

Plotosus carius, Ham. Buch. Fish. Gang. P It2. lam. I 5. f. tt; Cur'. \& Val. xv. p. +25; Bleek. Verh. Bat Gen. xxv., Velez. Beng. p. 125; Prodr. Silur. p 318, et Atl. Ichth. Silur. p 99. lam. 90. fig. 2. Kner, Sitzgsber. Wien. Acad. xvii p. I 57 ; Gtin. l. cit. 5. P. 25.

Pl. unicolor, (Kuhl. \& v. Hass.) C'uv. \& Val. xv. p. t26; Bleek. lerh. Bat. Gen. xx., Silur. Batav. p. $5^{8 .}$

Pl. horridus, viviparus, multiradiatus, Blcek. l. c. p. 59, 60.

? Pl. casius, Hyrtl, Acad. Wiss. Wien. 1859, xvi. 1. 17., Hab. Cebú; Archipiélago Indico.

4. Cén. SILURUS, Artedi

9. S. asotus, $L$. N. v.

$(\mathrm{M} S \mathrm{~S}, \mathrm{~T}$.

Silurus asotus, L. Syst. Nat. i. p. 501; Cl. Schn. p. 378; Batsil. in Nouv. Mém. Soc. Nat. Mosc. 1855, x. p. 240. lam. 3. f. 4. (no C. \& V.); Gthr. l. cit. 5. p. 33 .

S. punctatus, Cant. Ann. \& Mag. Nat. Hist. 1842 , ix. 1. 485. S. xan hosteus, Richards. Voy. Sulph. Fish. p. 133. lam. 56. figs. I 2, I 4; Ichth. Chin. p. $28 \mathrm{I}$.

S. sinensis, Richards. Ichth. 'Chin. p. 28r. (no M'Clell.)

S. japonicus, Schleh. Faun. Japon. Poiss. p. 226. lam. Io4. f. I; Richard. Ichth. Chin. p. $28 \mathrm{I}$.

Hab. Samar, Calbayoc; China.

\section{Gén. SILURI HTHYS Bi.EeK}

o. S. phaiosoma, Bleck. $\mathrm{N}$ v.

( $\left.\begin{array}{lll}\mathrm{M} & \mathrm{S} & \mathrm{T}\end{array}\right)$

Bleeker. Prodr. Silur. p. 269, et Atl, Ichth. Silnr. p. 81. lam. 87. fig. 2. Gthr. l. cit. 5. p. 35.

Hab. Samar, Borongan; Borneo.

6 Gén. WALLAGO, BLElK

I I. W. leerii, N. v.

(M. S. 'T.)

Bleek. Natuurk. Tydschr. Nederl. Ind. ii. p. 427, et Atl. Ichth. Silur. p. 80. lam. 85. Gthr. l. cit. 5. p. 37.

Hab. Samar, Borongan; Sumatra, Borneo.

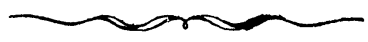




\section{Gén BEI,ODONTICH IHYS, BLELK.}

\section{B. macrochir Bieek N. v.}

Wallago dinema, Bleek. Nat. Tydschr. Nederl. Ind. ii. p. 202. Belodontichthys macrochir, Bleek. Prodr. Silur. p. 266. et Atl. Ichth Silur. p. 89. lam. 86. fig. 2: Gthr. l. cit. 5. p. 37. Hab. Samar, Borongan; Borneo.

\section{Gén. CRYPTOPTERUS, Bi.en.}

13. Cr limpok, N. v

(M. S. '..)

Silurus limpok, Bleek, Nat. Tydschr. Nederl. Ind. iii. p. $5^{83}$. Krytopterus limpok, Bleek. Prodr. Silur. p. p. 286, et Atl. Ichth. Silur. p. 87. lan. 91. fig. 2; Githr. l. cit. 5. p. 39. Hab. Samar, Borongan; Sumatra, Borneo.

14. Cr. schilbeides, ? Becker. N. v.

(M. S. T)

Henusilurus schilbeide:, Bleek. Prodr. Silur. p. 297, et Atl. Ichth. Silur. p. 94. lam. 90. fig. 4; Gthr. l. cit. 5. p. 41. Hab. Samar, Borongan; Sumatra, Borneo.

15. Cr. bicirrhis, $c: V \cdot$ N. v.

(M. S. T.)

Silurus bicirrhis, Cuv. \& Val. xiv. p. 367. lam. 4I I; Bleek. Vern. Bat. Gen. xxi., Silur. Batav. p. 20, et Nat. Sydschr. Nederl. Ind. i. p. $27 \mathrm{I}$.

Kryptopterichthys bicirrhis, Bleek. Prodr. Silur. p. 292, et Atl. Ichth. Silur. p. 88 lam. 90. fig. I; Gthr. l. cit. 5. p. 4I. Hab. Samar, Borongan; Java, Borneo.

16. Cr lais, Blcek. N. v.

(M. S. T.)

Silurus laïs, Bleek. Nat. Tydschr. Nederl. Ind. ii. p. 428.

Kryptopterichthys lais, Bleek. Prodr. Silur. p. 291, et Atl. Ichth. Silur. p. 88 lam. 9o. fig. 2; Gthr. l. cit. 5. p. 42.

Hab. Samar, Borongan; Borneo.

17. Cr. micropus, Bleek. N. v.

(M. S. T.)

Silurus crytopterus, Ble:k. Nat. Tydschr. Nederl. Int. i. p. 2jo. Kryptopterus micropus, Bleek. Prodr. Silur. p. 84, et Atl. Ichth. Silur. p. 86. lam. 89. fig. 3. G!ht. l. cit. 5. p. 42.

Hab. Samar, Rorongan; Sanatra.

18. Cr. micronema ? Bleek. $\mathrm{N}$ v.

(M. S. ' ')

Silurus micronemus, Bleek. Verh. But. Gen. xxi., Silur. Batay. p. 18.

S. phalacronotus, Bleek. Nat. Tydschr. Vederl. Ind. ii. p. +2). 
Nicronema typus, Bleek. Prodr. Silur. p. 300, et Atl. Ichth.

Silur. p. 91, lam. 92, fig. 2, lam. 93, fig. 1 .

Phalacronotus micruropterus, Bleek. Prodr. Silur. p. 207.

ilicronema phalacionotus, Bleek. Bnumer. p. 139.

Cryptopterus micronema, Gthr. l. cit. $5, \mathrm{p} .43$.

Hab. Samar, Borongan; Java, Borneo.

19. Cr. hexapterus, ? Bleek. N. v.

(M. S. 'T.)

Silurus hexapterus, Bleek. Nat. Tydschr. Nederl. Ind. ii. p. 203. Nicronema hexapterus, Bleek. Prodr. Silur. p. 301, et Atl. Ichth.

Silur. p. 91, lam. 92 f. I.

(ir. hexapterus, Gthr. l. wit. 5, p. 45.

Hab. Luzón, Manila, Navotas; Java.

\section{Gén. Callichrous, Gthr.}

2o. C. bimaculatus, Bleeḱ.

Witvisch, Nieuhof, Zee-en. Lant-reize, p. 276.

? Silurus bimaculatus, $B l$. viii. p. 24 lam. $364 ; B l$. Schn. p. 377; M'Clell. Calc. Journ. Nat. Hist. iv, p. 401.

()mpok siluroides, Lacép. v. p. 5\%, vi. lam. I f. 2.

Silurus bimaculatus, Cuv. \& Val. xiv. p. 360 .

Pseudosilurus bimaculatus, Bleek. Prodr. Silur. p. 277.

Phadacronotus siluroides, Bleek. Prodr. Silur. p. 304.

Callichrous bimaculatus, Bleek. Atl. Ichth. Silur. p. 84 lam. 87 fig. 3; Gthr. l. cit. 5. p. 45.

Hab. Samar, Borongan; Java, Borneo.

Io. Gán. LAIS, Bleek.

2 I. L. hexanema, Bleek. $\mathrm{N}$ v.

(M. S T )

Pangasius hexanema, Bleek. Nat. Tydschr. Nederl. Ind. iii. p. 589 . Lais hexanema, Bleek. Prodr. Silur. p. i71, et Atl. Ichth. Silur. p. 72 lam. 75 fig. 2 ; Gthr. l. cit. 5 ; p. 57.

Hab. Luzon, Manila, Navotas; Java.

$$
\text { 11. Gén. Pangasius, C. V. }
$$

22. P. macronema, Bleeker. N. v.

(M. S. T.)

Bleeker, Nat. Tydschr. Nederl. Ind. i. p. I1; Prodr. Silur. p. 183; et Atl. Ichth. Silur. p. 74, lam. 75 fig. 3; Gthr. l. cit. 5, p. 62 .

Hab. Lużón, Cavite, Santa Cruz; Borneo.

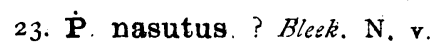

(M. S. T.)

Pseudopangasius nasutus, Bleek. Versh. Kon. Akad. Wet. xv. p. 72, et Atl. Ichth. Silur. p. 76 lam. 29, fig. i.

Pangasius nasutus, Gthr. l. cit. 5, p. 63 .

Hab. Luzón, Manila, Navotas; Borneo. 
12 Gén. PSEUDOBaGRU'; Beek.

24. Ps. vachellii, Richards. $\mathrm{N}$ v.

? Bagrus (?) bouderius, Richerds. Ichth. Chin. 1:. 283.

Bagrus (?) vachellii, Richards. l. c.p. 2 it.

Pseudobagrus vachellii, Gthr. l. cit. 5, p. 85.

Hab. Luzón, Manila, Navotis; China.

25. Ps fulvi-draco, P Richards. $\mathrm{N}$ v.

(M S. T)

Pimelodus (?) fulvi-draco, Richards. Ichth. Chin. p. 286.

? Silurus calvarius, Basıl. in Nono. Mém. Natur. Mosc. 1855, x. p. $2+1$ lam. 9 fig. I.

Pseudobagrus fulvi-draco, Gthr. l. cit. 5, 1. 14.

Hab. Luzon, Manila, Navotas; China.

13. Gén. RITA, Brlikk

26. R. manillensis, $C, V . \mathrm{N}, \mathbf{v}$

(M. S. T.)

Arius manillensis, Cuv. \& Val. xr. 93; Gthr. l. cit. 5, p. 94. Hab. Luzón, Manila, Narotas.

14. Gén. ARIUS, G's Hk.

27. A tonggol, Bleek.

Arius tonggol, Bleek. Verh. Bat. Gen. xxi. Silur. Bat. $\mu .4^{8}$. Arius crossocheilos, Bleck. l. c. p. $t^{6}$.

Ariodes tonggol, Bleek. Prodr. Silur. p. 83, et Atl. Ichth. Silur. p. 39. lam. 57 .

Arius tonggol. Gthr. . cit. 5. p. ${ }_{1} 64$. Hab. Luzón, Laguna de Bay; Java.

28. A. sagor, Ham. Buch. N. v.

Pimelodus sagor, Ham. Buch. p. i69.

Bagrus sondaicus, Cuv. \& Val. xiv. p. H+4; Cant. Mal. Fish. p. 255 .

B. javensis, Cuv. \& Val. xiv. p. 445.

B. sagor, Cuv. \& Val. xiv'. p. 446 .

Hexanematichthys sundaicus, Bleek. Prodr. Silur p. I27, et Atl. Ichth. Silur. p. 26. lam. 62.

Arius sagor, Gthr. l. cit. 5. p. I41.

Hab. Balabac, Samar; Borneo.

29. A. pidada, Bleek. N. v.

(M. S. T.)

Arius pidada, Bleek. Verh. Bat. Gen. xxi. Silur. Bat. p. 43, et Prodr. Silur. p. 74; Gthr. l. cit. 5. p. 167. 
A. viviparus, Bleik. l. c. p. 45.

Pseudarius pidada, blick. Atl. Ichth. Silur. p. 37. lam. 50). Hab. Luzón, Mamila, Narotas; Jaria.

3o. A. cælatus, C. $V^{\prime} N$. v

Arius calatus, Cini. \& Val. xiv. p. 66; Bleek. Atl. Ichth. Silur.

p. 35 lam. 53: Gthr. I. cit. 5. p. 15 ?.

A. aquibarbis, cue. of Val. xir. p. 68 .

A. granosus, Cuo. of Fal. xir. p. (o).

A. catatoides, Bleek. Verh. Bat. Gen. xxi. Silur. Batan. p. 32.

A. microgastropterygius, bleck l. c. p. 3.3 .

A. clypeaster, Blick. l. c. p. 3t.

A. clypeastro des, Bleek. l. c. p. 34.

A. chondropterygitis, Bleck. l. c. p. 35 .

A. melanopterygius, Bleek. l. c. xxii. Madur. p. 10

(ephaloçassis calatus, B'e k. Prodr. Silur. p. Iru. Hab. Samar, Joló, Burneo; Java, Sumatra.

31. A. falcarius, Richards. N. v

?'Tachisurus chinensis, Lacép. v. p. I5 I. lam. 5. fig. 2.

? Pimelodus tachisurus, Ciuv. \& Val. xv. p. 163.

Arius falcarius, $R$ chards. Voy. Sulph. Fish. p. I34. lam. 62. figs. 7-9; Gthr. l. cit. 5. p. 168.

Bagrus crinalis, Richards. Ichth. Chin. p. 282.

Pimelodus mong, Richards. Ichth. Chin. p. 286.

Arius schlegelii, Bleek. Nederl. Tydschr. Dierk. 1863,1 , 131. Hab. Luzon, haguna de Bay; China.

32. A. microcephalus, Blkr. N. v.

(M. S. T.

Arius microcephalus, Bleck. Nat. Tydschr. Nederl. Int. ix. p. 423, et Prodr. Silur. p. 77; Gthr. l. cit. 5. p. I70.

Pseudarius microcephalus, Bleek. Atl. Ichth. Sillur. p. 37. lam. 5I. f. I.

Hab. Luzón, Manila, Navotas; Borneo.

15. Gén. Hemipimelodus, Blelk.

33. H. borneensis, Bleek. N. v.

(M, S T.

Pimelodus borneensis, Bleek. Nat. Tydschr. Nederl. Ind. ii. p. 43 .

Hemipimelodus borneensis, Bleek. Prodr. Silur. p. 238 , et Atl. Ichth. Silur. p. +3. lam. 82. fig. 3; Gthr. l. cit. 5. p. 176. Hab. Samar, Borongan; Sumatra.

34. H. macroeephalus, \&leek. N. v.

(M S T.

Bleeker, Prodr. Silur. p. 239, et Atl. Ichth. Silur. p. 43. lam. 82. fig. 2; Gthr. l. cit. 5. p. 176.

Hab. Luzón, Cavite, Sta. Cruz. 
35. H. manillensis, $C$. \& $V$. N. v. Candole.

(M. S T.

Cuv. \& Val. xv. p. 192; Gthr. l. cit. 5. p. 176.

Hab. Manila, Luzón.

16. Gén. KETENGUS.s, BleEk.

$3^{6}$ K. typus, Bleek N. v.

(M. S. T.)

Ketengus typus, Bleek. Verh. Bat. Genootsch. xxi. Silur. p. \%; Natuurk. Tydschr. Nederl. Ind. i. p. 27 I; et Atl. Ichth. Silur. p. 44. lam. 82. fig. I. Gthr. l. cit. 5. p. 177.

Pimelodus pectinidens, Cant. Mal. Fisch. p. $26 \mathrm{I}$.

Hab. Samar, Borongan; Borneo.

\section{Fam. ESCOPÉLIDOS.}

I. Gén. SAƯRIDA, C. V.

I. S. tumbil. Cuv. $\mathrm{N}$ v.

(M. S T.)

Roover, Valent. fig. I 3 I; Renard, i. fig. 149.

Lacertus perígrinus, Rondel. De Pisc. xv. cap. 9. p. 428.

Padi mottah, Russell, lan. 172 .

Salmo tombil, Bloch. ix. p. i 2 . lam. 430; Bl. Schn. p. 405.

Saurus badimottah, Cuv. Régne Anim.; Rüppell. Nene. Wirtellh. Fische, p. 77; Cant. Mal. Fish. p. 270.

Saurida tombil, Cuv. \& Val. xxii. p. 500; Bleek. Verh. Batar.

Genootsch. xxiv. Chiv. p. 2o: G'hr. l. cit. 5. p. 399.

Hab. Luzón, B. de Manila, Navotas; Oceano Indico.

2. \$. argy ophanes. Richards. N. v.

(M. S. T)

Saurus argyrophanes, Richards. Ichth. Chin. p. 302.

Aulopus elongatus, Schleg. Faun. Japon. Fisc. p. 233. lam. I05. fig. 2 .

Saurida argyrophanes, Gthr. l. cit. 5. p. foo.

Hab. Luzón, B. de Manila, Navotas; China.

3. S. $\mathrm{sp} \cdot \mathrm{N}$ v.

(M. S. T )

Hab. Luzón, Rio Pasig, Manila, Navotas.

2. Gén. harpodon, iesueur.

4. H. nehereus., Hum-Buch. N. v.

(M. S. T.)

Bummaloh, or Bombay Duck.

Russell, ii. p. 55. lam. i 7 I.

Osmerus nehereus, Ham. Buck. Fish. Gang. p. 209.

Salmo (Harpadon) microps, Lesueur, Jouru. Acad. Nat. Sc. Plin lad. 1825, v. p. 48 . lam. 3. 
Saurus ophiodon, Cuv. Régne Anum.; Cuv. \& Val. xxii. p. 490. S. nehareus, Richards. Ichth. Chin. p. 301 ; Cant. Mal. v. Rept. p. 273 .

Harpodon nehereus, Gthr. l. cit. 5. p. 402.

Hab. Luzón, Manila, Navotas; China.

\section{Fam. SALMONIDOS.}

Gèn SALaNX, Cun:

I S. chinensis. $\left(O s b_{c} c k\right) \mathrm{N}$ v.

(M. S. T.)

Albula chinensis, Osbeck Reise in China, Rostock 1765, p. 309;

Gthr. Cat. B. Mus. 6. 1866, p. 205.

Hab. Luzón, Manila, Navotas; China.

\section{Fam. ESCOMBRESÓCIDOS.}

I. Gén. BELONE, Cuv.

1. B melanotus, Blk.

Belone melanotus, Bleeker, Natuurk. Tydscir. Ned. Ind. i. p.

94, or Verh. Genoctsch. xxiv. Sroek. Vissch. p. 14; Gthr. l. cit. 6 . p. $23^{8}$.

Mastacembelus crocodilus, Bleek. Ned. Tydschr. Dierk. iii.* (no syn.)

Hab. Cebú; Archipiélago Indico.

2. B. schismatorhynehus, Blecker. N. v.

(M. S T.)

Belone gracilis, Schleg. Faun. Japon. Poiss. p. 246. lam. ıо. f. I; Bleek. Verh. Bat. Gen. xxvi. Nieuwe Nalez. Japan. p. i if (no Lowe).

Belone schismatorhynchus, Bleeker, Nat. Tydschr. Ned. Ind. i. p. 95, or Verh. Genootsch xxiv. Sreok. Vissch. p. 15; Gthr. l. cit. 6. p. 239.

Mastacembelus gracilis, Bleek. Ned. Tydschr. Dierk. iii. Hab. Luzón, Manila, Batangas, Nasugbú; Mar Rojo.

3. B. annulata, $C . V$.

Russell, lam. I75.

?. Belona indica, Lesueur, Journ. Acad. Nat. Sc. Phılad. ii. I821, p. 331 .

Belone annulata, Cuv. \& Val. xviii. p. 447. lam. 55o; Bleek., Cant. Mal Fish. p. 244; Day. Fish. Malabar, p. 165; Gthr. l. cit. 6. p. 240.

Belone gigantea, Schleg. Faun. Japon. Poiss. p. 245; Bleek. Act. Soc. Sc. Indo-Nederl. iii. Japan, p. 21.

? B. melanurus, Bleek. Verhand. Batav. Genootsch. xxii. Mrdur. p. II. 
? B. cylindrica, Bleek., Verhand. Batav. Gensotsch. xxir. Snoek. p. 13.

B. brachyrinchus, Bleek. Nat. Tydschr. Ned. Ind. vi. p. 6r. Mastacembelus choram, Bleek. Nederl. Tydschr. Dierk. iii.

M. brachyrhynchus, Bleek. l. c.

Hab. Luzón, Batangas, Nasugbú; China.

4. B. caudimaculata, Cuv. N. v.

(M. S. T.)

Belone caudimaculata, Cuv. Régne Anim.; Cuv. \& Val. xviii. p. 452 ; Gthr. l. cit. 6. p. 245.

Hab. Luzón, Manila, R. Pasig; Amboina.

5. B. strongylurus, (Bleek.) N. v.

(M. S. T.)

Russell, ii. lam. I 76 .

Belone caudimaculata, Cant. Catal. p. 246; Bleek. Verhand. Batav. Genootsch. xxiv. Snoek. p. I 2 (no (uv.).

Mastacembelus strongylurus, (v. Hass.), Bleek. Ned. Tydschr. Dierk. iii. (no synon).

Be.one trongylurus, Gthr. l. cit. 6. p. 246. Hab. Luzón, Cavite, Santa Cruz; China.

6. B. anastomella, $C . V$. N. v.

Belune anastomella, Cuv. \& Val. xviii. p. 446; Gthr. l. cit. 6. p. 249.

Hab. Luzón, Manila, Navotas; China.

7. B. canciloides? B'eek. N. v.

(M. S, T.)

Belone canciloides, Bleek. Nat. Tydschr. Ned. Ind. v. p. 45 t Gthr. l. cit. 6. p. 253.

Mast cembelus canciloides, Bleek. Ned. Tydschr. Dlerk. iii. Hab. Samar, Borongan; Borneo.

\section{Gén. HEMIRHamphus, Cuv.}

8. H. viparus, Peters.

Peters, Monotsber. Akad. Wiss. Berl. i 865, p. I32; Gthr. l. cit. 6. p. 259.

Hab. Samar.

9. H. esp. N. v.

Hab. Luzún, Manila.

10. H. intermedius, Cantor. N. v.

(M. S. T.)

Hemirhamphus intermedius, Cant. Ann. \& Mag. Nat. Hist. 1842, ix. p. 485; Richards. Ichth. Chin. p. 264; Gthr. l. cit. 6. p. 260 . 
Hemirhamphus melanochir, Cuv. \& Val. xix. p. tl. Hab. Luzón, Manila, Cavite, Santa Cruz; Chine.

11. H. georgii, $C$ V.

Hemirhamphus georgii, Cuv. \& Val. xix. p. 37, lam. 335; Cant. Cat. p. 248 ; Gthrr. l. cit. 6. p. $26+$.

Hab. C. de Cebú; Océano Indico.

12 H. cantoris, Blecker. N. v.

(N. S. T)

Hemirhamphus longirostris, Bydr. Topogr. Bat (no Cuv.)

H. georgii, Bleek. Verh. Bat. Gen. xxiv. Snoek. 19 (no Cuv. if $(a l$.

H. cantori, Bleeker, Ned. Tydschr. Dierk. iii. p. 145: Gthr. l. cit. 6. p. $26+$.

Hab. Luzón, Cavite, Sta. Cruz; China.

13. H. melanurus, $C V . \mathrm{N}$ v.

(M. S T.)

Hemirhamphus melanorus, Cuv. \& Val. xix. p. $+2 ;$ Gthr. l. cit. 6. p. 264 .

Hab. Luzón, Cavite, Santa Cruz; China.

14. H. sinensis, ? Gthr. N v.

(M. S. T.)

Hemirhamphus sinensis, Gthr. l. cit. 6. p. 265. Hab. Luzón, Manila, Navotas; China.

15. H. quoyi, C. V.

Cuv. \& Val. xix. p. 26; Blek. Nat. Tydschr. Nederl. et ii. p. 491, cop. in Verh. Bat. Gen. xxiv. Snoek. p. 26, et Ned. Tydschr. Dierk. iii. p. I 53; Gthr. l. cit. 6. p. 267.

Hab. Luzón, Batangas, Nasugbu; N. ${ }^{a}$. Guinea.

16. H. gernaerti, C. V. N. v.

Cuv. \& Val. xix. p. 43; Gthr. l. cit. 6. p. 268.

Hab. Luzon, Manila, Navotas; China.

17. H. commersoriii, Cuv.

Valent fig. 318; Renard, ii. lam. 5. fig. 2.

Acus, sp., Willughby, Hist. Pisc. lam. p. 8. f. 3 .

Far, Forsk. Descr. Anim. p. 67.

Esox espadon, var., Lacép. v. lam. 7. f. 3.

Hemirhamphus commersonii, Cuv. Regnề Attim,; Cav. \& Vat. tix.

p. 28; Bleek. Verh. Bat. Genootsch. xxit. Snoek. p. 17; Gthr.

l. cit. 6. p. $27 \mathrm{I}$.

H. far, Rüpp. N. W. Fische, p. 74.

Hab. Luzón, Batangas, Nasugbú; Amboina. 
I8 H. dispar, C. V. N. v.

(M. S. T.)

Hemirhamphus dispar, Cuv. \& Val. xix. p. 58, lam. $45^{8}$; Bleek. Nat. Tydschr. Ned. Ind. vi. p. 498; Gthr. l. cit. 6. p. 274.

Zenarchopterus dispar, Bleek. Nederl. Tydschr. Dierk. iii. p. ${ }_{16} 4$. Hab. Luzón, Manila, Navotas; Indias Orientales.

19. H fluviatilis Bleeker. N. v.

Hemirhamphus fluviatilis, Bleeker, Nat. Tydschr. Ned. Ind. i. p. 95; cop. in Verh. Bat. Genootsch. xxiv. Snoek. p. 16; Peters. Monatsb; Gthr. l. cit. 6. p. 275.

Dermogenys pasillus, (v. Hass.) Bleek. Ned. Tydschr. Dierk. iii. p. 165 .

Hab. Samar, Borongan; Java.

20. H. sumatranu;, Bleeker.

Hemirhamphus sumatranus, Bleeker, Nat. Tydschr. Ned. Ind. v. p. 526 ; Gthr. l. cit. 6. p. 275 .

Dermogenys sumatranus, Bleek. Nederl. Tydschx. iii. p. 167.

Hab. Luzón, R. Lonaten, Morong; Sumatra.

3 Gén. EXocłtus, ARtedi.

21, Ex. apus, Cuv N. v.

(M. S. 'T )

Exocztus apus, Cuv. \& Val. xix. p. 107.

Hab. Luzón, Manila, Navotas; China.

22. Ex. monocirrhus, Richards. N. v.

$(\mathrm{M} \quad \mathrm{s} \quad \mathrm{T})$

Exocætus monocirrhus, Richards. Ichth. Chin. p. 265.

fiab. Luzón, Cavite, Sta. Cruz; China.

23. Ex. brachipterus, Solander. N. v.

(M. S. T.)

Exocætus brachipterus, Solander, M. S.; Richards. Ichth. Chin. p. 265; Gthr. l. cit. 6. p. 28 o.

Hab. Samar, Borongan; China.

24. Dx. monto, C. V. N. v. Botador.

(M. S. '5, )

Exocætus mento, Cuv. \& Val. xix. p. 124; Bloek. Verh. Bat.

Genootsch. xxiv. Snoek. p. $21 ;$ Gthr. 6. cit. 6. p. 281.

Parexocatus mento, Bleek. Nederl. Tydschr. Dierk. iii. pl. I 26. Hab. Luzón, Manila, C. de Samar; Indias.

25. Fx. volitans, Lin. N. v. Botador.

Hirundo, Salv. fol. I 85 , lam. 67.

Exocatus, n." I, Arledi, Genera, p. 8, et Spec. p. 35. 
Exucætus volitans, L. Aman. Acad. i. p. 320, Syst. Nat. i. p. 520. Cui'. \& l'al. xix. p. 83, lain. 559; Gthr. l. cit. 6. p. 29.3. Exocatus, Gronov. Zoophyl. p. I16.

Juhamel, Péches, ii. p. 480, lam. 22, fig. 2.

Exocatus exiliens, Bloch, lam. 397.

Exocatus quadriremis, Gronov. Syst ed. Gray, p. 14.5. Hab. Luzón, Manila, C. de Cebú y Samar.

26. Ex. Oligolepis. Blecker.

Exxatus unicolor, Bleek. Verh. Bat. Gen. xxiv. Snoek. Visch. p. 21. (no Cur. \& Val.).

li. oligolepis, Bleek. Ned. Tydschr. Dierk. iii. p. rog. Gthr. I. cit. o. p. 296.

Hab. Luzón, Manila; Archipiélagro Indico.

27 Ex evolans, Lin. N. v. Lauin

(M. S. T.)

Dutertre, Hist. Ant. ii. p. 21 2; Rochefort, Hist. Ant. i. p. 372 ; Catesby. Carol. lam. 8

Exocextus evolans, L. Syst. Nat. i. p. 52 i ; Bl. lam. 398; Bl. Schn. p. 430, lam. 84; Cuv. \& Val. xix. p. 1 38 ; Gronov. Syst. edt. Gray. p. I 45; Bleek. Cape of Good Hope, p. 28; Yarrell. Brit. Fish. 3r. edit. i. p. 474; Gthr. l. cit. 6. p. 282.

Exocætus, sp., Gronov. Zoophyl. n. ${ }^{\circ} 358$.

Jixocætus volitans, Lacep. v. p. 4or, lam. 12. f. 2: Penn. Brit. Zool. p. 44I, lam. 78; Donov. Brit. Fish. ii. p. 31; Brown, Philos. Trans lxviii. p. 790, pl. I 2; Yarrell. Brit. Fish. and. edit. i. p. 453; Benn. Whal. Voy. ii. p. 284.

Flying Fish White, Voy. Neru. Sauth. Wales, lam. 52 fig. 2.

Exocetus splendens, Clark. Abel, Journ. Chin. $1818, \mathrm{p} .4$.

Ii. volans, (Solander) Richards. Ichth. Chin. p. 264.

Hab. Luzón, Cavite, Sta. Cruz; China.

28. Ex. arcticeps, Gthr. N. v.

(M. S. T.)

Gthr. l. cit. 6. p. 289.

Hab. Luzón, Cavite, Sta. Cruz; China.

29. Eix. pæcilopterus? C. V. N. v.

(M. S. T.)

Valent. fig. ${ }_{1} 6_{5} ;$ Ruysch, lam. 7. n. ${ }^{\circ}$ i 8.

Exocatus pæcilopterus, Cuv. \& Val. xix. p. in 2, lam. 561; Gthr. l. cit. 6. p. 291.

Hab. Luzón, Cavite, Santa Cruz.

30. Ex. esp. N. v.

Hab. Luzón, Manila, Cebú, Samar.

3r. Ex. spilopterus, $C . V$.

Exocætus spilopterus, Cuv. \& Val. xix. p. I $3 ;$ Gthr. l. cit. 6. p. 292.

E. spilopterus, Bleek. Ned. Tydschr. Dierk. iii. p. I 6.

Hab. Carolinas. 
32. Ex. oxycephalus, Bleeirer: $N$ r

(M. S. T)

Bleeker, Nat. Tydschr. Net. Ind. 1859, iii. p. 77r, et Nederl. Tydschr. iii. p. 124; Gthr. l. cit. 6. p. 294.

Hab. Luzón, Manila, Navotas; Formosa.

33. Ex. brachycephalus, Gikr. $\mathrm{N}$ v

(M S. T )

Gthr. l. cit. 6. p. 297 .

Hab. Luzón, Cavite, Sta. Cruz; Chna.

\section{Fam. CIPRÍNIDOS.}

1 Gén CYPRINUS NiLs.

1. C. fossicola, ? Richards N. v.

(M. S. T )

Cyprinus (?) fossicola, Gray. Richards. Ichthyol. Chin. p. 291. Gthr. Cat. B. M. 7, 1868 , p. 28.

Hab. Luzón, Cavite, Sta. Cruz; China.

2. Gén. Cirrhina, Cuv.

2. C. chinensis, Gthr. N. v.

Gthr. l. cit. 7, p. 36.

Hab. Luzón, Cavite, Sta. Cruz; China.

3. Cén. DANGila, C V.

3. D. ocellata, Heckel. N. r

(M. S. T.)

Cyrene ocellata, Heckel, in Russegger's Reisen, i. p. 1025.

Dangila microlepis, Bleek. Nat. Tydschr. Ned. Ind. iii. p. 595.

D. ocellata, Bleek. l. c. xiv. p. 475 ; Prodr. Cypr. p. 194; or Atl. Ichthyol. Cypr. p. 43, lam. 16 fig. 3; Gthr. l. cit. 7, p. 37 .

Hab. Samar, Borongan; Borneo.

4. D cuvieri, Cuv. N. v.

(M. S T.)

Dangila cuvieri, Cuv. \& Val. xvi. p. 230, lam. 470; Gthr. l. cit. 7, p. $3^{8}$.

D. leptocheila, Cuv. \& Val. xvi. p. 234; Bleek. Prodr. Cypr. p. I98; or Atl. Ichthyol. Cypr. p. 45, lam. I7 fig. I.

Hab. Luzón, Cavite, Sta. Cruz; Java.

5. D. sumatrana, Blecker.

? Cyrene philippinia, Heckel, in Russegger's Reisen, i. p. 1025. Dangila sumatrana, Bleek. Nat. Tydschr. Ned. Ind. iii. p. 596; 
or Prodr. Cypr. p. 2(x); or All. Ichthol. Cypr. p. 46, lam. 15 fig. 2; Gthr. l. cit. 7, p. 39.

Hab. Filipinas; Sumatra.

6. D. festiva. Bleck. N. v.

Cyrene festiva, Heckel, in Russegger's Rcisen, i. p. 1025.

Dangila festiva, Bleek. in Act. Soc. Sc. Indo-Neerl. ii. Borneo, x. p. 16; or Prodr. Cypr. p. 202; or Atl. Ichthyol. Cypr. p. 47, lam. I 5 fig. 4; Githr. l. cit. 7, p. 39.

Hab. Samar, Villareal; Borneo.

\section{Gén OSTEOChILUS, Grir.}

7. O melanopleurus, Blecksr. N. v. Apahap.

(M S. T.)

Rohita melanopleura, Bleek. Nat. Tydschr. Ned. Ind. iii. p. 430; or Prodr. Cypr. p. 163; or. Atl. Ichthyol. Cypr. p. 62. lam. I.3. Osteochilus melanopleurus, Gthr. l. cit. 7, p. 4 o.

Hab. Samar, Borongan; Borneo.

8. O. hasseltii, Cuv. N r.

Rohita hasseltii, Cuv. \& Val. xvi. p. 274; Bleek. Nat. Tydschr. Ned. Ind. v. p 450; or Prodr. Cypr. p. 17I; or Atl. Ichthol. Cypr. p. 65, lam I4, fig. I.

R. leiorhynchos, Bleek. Verh. Bat. Gen. xxiii. Midd. Oost-Java, p. 19.

Osteochilus hasseltii, Gthr. l. cit. 7, p. 4I.

Rohita artedii, Bleek. Nat. Tydschr. Ned. Ind. i. p. 434. Hab. Luzón, Cavite, Sta. Cruz; Sumatra.

9. O. vittatus, C. V. N. v. Apahap.

(M. S T )

Rohita vittata, Cuv. \& Val. xvi. p. 267; Bleek. Nat. 7ydschr. Ned. Ind. v. p. 45I; or Prodr. Cypr. p. I78; or Atl. Ichthyol. Cypr. p. 68, lam. I 2. fig. 2.

R. erythrura, Cuv. \& Val. xvi. p. 268; Bleek. Nat. Tydschr. Ned. Ind. v. p. 452.

R. enneaporus, Bleek. l. c. iii. p. 596; or Prodr. Cypr. p. 184 ; or Atl. Ichthyol. Cypr. p. 70, lam. II. fig. 2.

Osteochilus vittatus, Gthr. l. cit. 7. p. 44. Hab. Luzón, Manila, Malabon; Borneo.

\section{Fam. CLUPÉIDOS.}

I. Gén. ENGraulis, Cuv.

1. E. tri, Bleek. $\mathrm{N}$ v.

Engraulis tri, Bleek. Verh. Bat. Gen. xxiv. Haring, p. 40; 0 Nat. Tydschr. Ned. Ind. iii. p. 436; Gthr. loc. cit. 7. p. 389. Hab. Luzón, Manila; Borneo. 
2. E. japonica, Houtt. N. v.

Atherina japonica, Houttuyn. Verh. Holl. Maatsch. Wet. Haarl. $\mathrm{xx} .2,1781$, p. 340 .

Eingraulis commersonianus, Richards. Ichth. Chin. p. 308. (no Lacép).

Engraulis japonica, Gthr. l. cit. 7. p. 390.

Hab, Luzón, Cavite, Sta. Cruz; China.

3. E. heterolobus, Rüppell. N. v.

Rüppell. $N$. W. Fische, p. 79, lam. 21. fig. 4; Bleek. Ned. Tydschr. Dierk. iii. p. 305; Gthr. l. cit. 7. p. 392.

Hab. C. de Samar; Mar Rojo.

4. E rbinorhynchus, Bleek. N. v.

Engraulis kammalensis, Blee. Verh. Bat. Gen. xxii. Madiera, p. I 3 .

E. rhinorhynchus, Bleek. l. c. xxiv. Haring, p. 4I; o Nat. Tydschr. Ned. Ind. iv. p. 432 ; Gthr. l. cit. 7. p. 394. Hab. Cebú; Borneo.

5. E. compressus, Girard N. v.

(M S. T.)

Girard, U. S. Pac. R. R. Exped. Fish. p. 336; Gthr. l. cit. 7. p. 395 .

Hab. Luzón, Manila; California.

6. E hamiltonii. Gray. N. v.

$\left(\begin{array}{lll}M & S & T\end{array}\right)$

'Ihrissa hamiltonii, Gray. Ind. Zool. c. fig.

'Ihryssa mystax, Richards. Ichthyol. Chin. p. 309.

Engraulis mystax, Cantor, Mal. Fish. p. 307.

E. grayi, Bleek. Verh. Bat. Gen. xxiv. Haring, p. 41; et Nat. Tydschr. Ned. Ind. ii. p. 492; Kner, Novara Fish. 1. 33.3; Gthr. l. cit. 7. p. 39j.

Hab. Luzón, Cavite, Sta. Cruz; China.

7 E. mystacoides, Bleek. N. v.

' M. S. T.)

Engraulis hamiltonii, Cuy. \& Val. xxi. p. 66 (no Gray); Kner, Novara, Fisch. p. 334.

Eingraulis mystax, Cuv. \& Val. xxi. p. 67 (no Bl. Schn).

Thyssa porava, Bleek. Verh. Bat. Gen. xxii. Modura, p. I 4 (no Ham. Buch.)

Engraulis mystacoides, Bleek. l. c. xxiv. Haring. p. 42. Cithr. l. cit. 7. p. 496.

E. mystax, Bleek. l. c. p. 43. (no Bl. Schn.).

Stolephorus (Thryssa) valenciennensii, Bleek. Ned. Tydschr. Dierk. iii. 1866 , p. 306 .

Hab. Luzón, Cavite, Sta. Cruz; China. 
Hab. Luzón, B. de Manila.

9. E setirostris, Brouss. N. v.

(M. S. T.)

Clupea setirostris, Brouss. Ichth. i. lam. 11. I. Gm. p. 1407; Bl. Schn. p. 428; Russel, ii. p. Ro, lam. 201.

C. mystacina, Forster, Descript. Anim. ed. Lichtensteln, p. 29.5.

Engraulis setirostris, Cui. \& Val. xxi. p. bo; Bleek. Werh. Bat. Gen. xxiv. Haring. p. 44. Gthr. l. cit. 7. p. 397.

Tryssa macrognathus, Bleck. l. c. xxii. Madura, p. 14. Hab. Luzón, Manila; Oceano Indico.

Io. F. breviceps? Cantor N. v.

Engraulis breviceps, Cant. Mal. Fish. p. 3 6; Gthr. l. cit. 7 . p. 401 .

E. pfeifferi, Bleek. Nat. Tydschr. Ned. Ind. iii. p. 433.

Hab. Luzón, Cavite, Sta. Cruz; Borneo.

\section{Gén Collia, Gray.}

I1. C clupeoides, (Lacép.) N. v.

(M. S. T)

Clupea mystus, L. Aman. Acad. iv. p. 252. fig. 12 Osbeck, Iter, p. 256.

Mystus clupeoides, Lacèp. v. P. 467 .

Chætomus playfairii, M'Clell. Calc. Journ. Nat. Hist. iv. I843, p. 405, lam. 24. fig. 3 .

Coilia playfairii, Richards. Voy. Sulph. Fish. p. roo, lam. 54. figs. 3-4; Cuv. \& Val. xxi. p. 86.

C. clupeoides, Gthr. l. cit. 7. p. 404.

Engraulis mystus, Cuv. \& Val. xxi. p. 73 .

Osteoglossum prionostoma; Basil. Now. Mém. Soc. Nat. Mosc. x. I 855 , p. 244.

Hab. Luzòn, Cavite, Sta. Cruz; China.

I2. C. grayi, Richards. N. v.

Richards. Voy. Sulph. Fish. p. 99, lam. 54. figs. I \& 2; Kner, Novara, Fische, p. 335; Gthr. l. cit. 7 . p. 4'5.

Hab. Luzón, Cavite, Sta. Cruz; China.

3. Gén. Chatoessus, C. V.

13. Ch. nasus, Cuv.

Clupea nasus, Bl. lam. 429; Russell, ii. lam. 197.

Chatoëssus altus, Gray, Ind. Zool. c. fig.; Day, Fish. Malab. p. 243 . 
C. nasus Cuv. \& Val. xxi. p. ıо4; Bleek. Nat. Tydschr. Ned. Ind. ii. p. 223; Gthr. l. cit. 7. p. 407.

Hab. Luzón, Manila; Java.

14 Ch. punctatus, Sihleg. N. v.

Chatoësus punctatus, Schleg. Falın. Japon. Poiss. p. 24 I, lam. ro9. fig. I; Cuv. \& Val. xxi. p. I07; Bleek. Verh. Bat. Gen. xxv. Japan, p. 50; Kner, Novara, Fisch. p. 336. Gthr. l. cit. 7. p. 408 .

Chatoëssus aquosus, Richards. Ichthyol. Chin. p. 307, Cuv. \& Val. xxi. p. 109 .

?Ch. punctatus, Kner, Novara Fisch. p. $33^{6}$.

Hab. Luzón, Cavite, Sta. Cruz; China,

15. Ch. chacunda, Cuv. N. v. Cabasi.

(M. S. T.)

Clupanodon chacunda, Ham. Buch. Fish. Gang. p. 246.

Chatoëssus chacunda, Cuv. \& Val. xxi. p. I I; Cant. Mal. Fish. p. 31 ; Bleek. Verh. Bat. Gen. xxiv. Haring, p. 47;. Gthr. l. cit. 7. p. $4 \mathrm{II}$.

Anodontostoma hasseltii, Bleek. Verh. Bat. Gen. xxii. Madura, p. 15 .

Chatoëssus selangkat, Bleek. l. c. xxiv. p. 47; or Nat. Tydschr. Ned. Ind. iii. p. $45^{8}$.

Hab. Luzón, Manila; China.

4 Gén Clupest, Cuv.

16. Clupea muuritiana, B:nn. $\mathrm{N}$ v.

(M. S. T)

Benn, Proc. Zool. Soc. i. p. 32; Mauritius; Gthr. l. cit. 7 . p. 4 r 3 .

Hab. Luzón, Sta. Cruz, Cavite; Maur icio.

17. C. isenglema, Rich.

Hab. Luzón, B. de Manila.

18. C. argyrotænia, Bleeker. N. v.

(M. S. T.)

Alausa champil, Cant. Mal. Fisch. p. $3 \mathrm{O} 2$ (no Gray).

Rogenia argyrotænia, Bleek. Verh. Bat. Gen. xxiv. Haring, p. 26; o Nat. Tydschr. Ned. Ind. iii. p. 457.

Clupea argyrotxnia, Gthr. l. cit. 7. p. 423.

Hab. Luzón, Cavite, Santa Cruz; Java.

19. C. fimbriata, N. v. Tamban, Tungsuy 6 sardina.

Spratella fimbriata, Cuv. \& Val. xx. p. 359, lam.600; Day, Fish. Malab. p. 233.

Kowala lauta, Cant. Mal. Fish. p. 270.

Clupea fimbriata, Gthr. l. cit. 7. p. 427.

Hab. Luzún, Manila; Océano Indico. 
20. C. nymphøa, Richrrds. $\mathrm{N}$ v.

(M. S. T)

Clupea nymphxa, Richards. Ichthyol. Chin. p. 3(1); Gthr. l. cit. 7. p. 428 .

Hab. Luzón, Manila; China.

2r. C. sp. $\mathrm{N}$ v

(M S. T )

Hab. Luzón, B. de Manila.

22. C. reevesii, Richards N. v. Sardina.

(M. S. T)

Alosa reevesii, Richards. Ichth. Chin. p. 305. -

A. palasah, Richards. l. c. p. 306.

? Alausa reveesii, Cuv. \& Val. xx. p. 437 (L. lat. 3o!).

Clupea reveesii, Gthr. l. cit. 7. p. 446 .

Hab. Luzón, Cavite, Sta. Cruz; China.

23 C kowal küppell N. v.

$\left(\begin{array}{lll}M & \mathrm{~S} & \mathrm{~T}\end{array}\right)$

Kowal, Russel, lam. I 86.

Clupea kowal, Rüppell, Neue Wirbelth. Fische, p. 79; Gthr. l. cit. 7 . p. $45^{\circ}$.

? Kowala thoracata, Cuv. \& Val. xx. p. 363.

K. thoracata, Cant. Mal. Fish. p. 269.

Clupalosa bulan, Bleek. Verh. Bat. Gen. xxii. Madura, p. I 2, et xxiv. Haring, p. 30.

Hab. Luzón, Cavite, Santa Cruz; China.

24. C zunasi. (Bleek) N. v.

$\left(\begin{array}{lll}M & \text { S. T. }\end{array}\right.$

Clupea kowal, Schleg. Faun. Japan. Poiss. p. 235, lain. 7. fig. I (no Rüpp).

Harengula zunasi, Bleek. Verh. Bat. Gen. xxvi. Japan, p. I 7 . Clupea zunasi, Gthr. l. cit. 7. p: 45I.

Hab. Luzón, Manila; Japon.

5. Gén. Pellona, C V.

25. P elongata, P Bennet. N. v.

(M S. 'T.)

Alosa elongata, Bennett, Mém. Raffes, p. 691.

Clupea affinis, Gray, Ind. Zool. c. fig.

Ilisha abnormis, (Gray,) Richards. Ichth. Chin. p. 306.

Clupea melastoma, Schleg, Faun. Japon. Poiss. p. 237, lam. 108 fig. I (no Bl.)

Pellona viıbella, Cuv. \& Val. xx. p. 3 r 7 .

P. grayana, Cuv. \& Val. xx. p. 315; Bleek. Verh. Bat. Gen. xxiv. Haring, p. 25.

P. affinis, Cant. Mal. Fish. p. 29r.

P. schlegelii, Bleek. Verh. Bat. Gen. xxvi. Japan, p. 118.

P. elongata, Gthr. l. cit. 7, p. 456.

Hab. Luzón, Cavite, Sta. Cruz; China. 
6. Gén. DUSSUmieria, C. V.

26. D. elopsoides, Blcek. N. v

(M. S. T.)

Dussumieria elopsoides, Bleek. Verh. Bat. Gen. xxii. Madura, p. I 2; xxiv. Chiroc. p. I 2; or Nat. Tydschr. Ned. Ind. i. p. 421 . D. hasseltii, Bleek. Verh. Bat. Gen. xxiv. Chiroc. p. I3; or Nat. Tydschr. Ned. Ind. i. p. 422.

Hab. Luzón, Manila; China.

7. Gén. ELOPS, Liv.

27. E. saưrus, Lin. N. v.

(M. S. T.)

Sloane, Jamaica, ii. p. 284, lam. 250, fig. I.

? Harengus minor, Catesby, ii. p. 24.

Elops saurus, L. Syst. Nat. i. p. 5i 8; Bloch., lam. 393 fig. 1; Bl. Schn. p. 430 lam. 82; Deka', New-York, Fauna, Fish. p. 267, lam. 4I f. I31; Cuv. \& Val. xix. p. 365; Cant. Mal. Fish. p. 287; Bleek. Verh. Bat. Gen. xxiv. Haring, p. 1+; Gronov. Syst. ed. Gray, p. i68; Kner, Novara, Fisch. p. 338; Gthr. i. cit. 7, p. 470 .

Argentina carolina, L. Syst. Nat. i. p. 5r9.

Argentina machnata, Forsk. Descr. An. p. 68.

Mugilomorus annæ carolinæ, Lacép. v. p. 398.

Russell; lam. i 79 .

lilops inermis, Mitchill. Lit. \& Phil. Trans. New.York, i. p. $4+5$. Elops machnata, Rüpp. N. W. Fische, p. 80; Richards. Voy. Ereb. \& Terr. Fish. p. 59, lan. 36 figs. 3-5; et Ichth. Chm. p. 3I I; Schleg. Faun. Japon. Poiss. p. 24I, lam. iog fig. 2; Günth. Fish. Zanz. p. I 21 .

Elaps purpurascens, Richards. Ichth. Chin. p. 3II.

E. capensis, Smith. Ill. Zool. S. Afr. Pisc. lam. 7. Hab. Luzón, B. de Manila; China.

\section{Gén. MEGalops, Lacep.}

28. M. cyprinoides, Bleek. N. v. Buambuan.

(M. S. T.)

Clupea cyprinoides, Brouss. Dec. Ichlhyol. i. lam. 9 (no synon.); Bonnat. Encycl. Méth. Ichth. p. I87, lam. 75. fig. 3it; (cop. Brouss.); Lacép. v. lam. I3. fig. 3; Forster, Descr. Anim. ed. Licht. p. 296.

Megalops filamentosus, Lacep. v. pp. 289, 290. lam. i 3. fig. 3; - Bleek. Ned. Tydschr. Dierk. iii. i 866, p. 286.

Kundinga, Russell, ii. p. 81, lam. 203.

Cyprinodon cundinga, Ham. Buch. Fish. Gang. pp. 254, $3^{8} 3$.

Megalops setipinnis, Richards. Ann. Nat. Hist. xi. p. 493; et Ichth. Chin. p. 3 Io.

M. curtifilis, Richirds. Ichth. Chin. p. 310.

M. indicus, Cuv. \& Val. xix. p. '388, lam. 542; Bleek. Verh. Bat. Gen. xxiv. Chiroc. p. I5; Kner, Novara, Fisch. p. 339.

Elops cunliaga, Caut. Mal. Fish. p. 289. 
Meyalops macrophthalmus, Bleek. $l$. $c$.

M. macropterus, Bleek. Ned. Tydschr. Dierk. iii. 1866, p. 284.

M. kundinga, Bleek. l. c. p. 288.

iA. cyprinoides, Bleek. l. c. p. 290; Gthr. l. cit. 7. p. +7 r.

il. oligolepis, Bleek. l. c. p. 292.

Elops apelike, Day, Fish. Malab p. 228.

$H a b$. Luzón, B. de Manila; Amboina.

\section{Gén. Chanos Lacel.}

29 Ch. salmoneus, Bl. Schn. N. v. Bang̃os

(M. S. T.)

Renard, Poiss. d'dmb. fol. 34. n." 184.

Mugil chanos, Forsk. Descr. An. p. 74; Bl. Schn. p. 16.

IM. salmoneus, (Forst.) Bl. Shcn. p. 121; Forst. Descr. Anim. ed. Lichtenstein, p. 299.

Chanos arabicus, Lacep. v. p. 39'; Cuv. \& Val. xix. p. 187.

Palah bontah, Russell, ii. lam. 207.

'Toolelvo, Russell, ii. lam. 208.

Cyprinus (leuciscus) palah, Cuv. Kégne Anim.

1: tolo, Cuv. Régne. Anim.

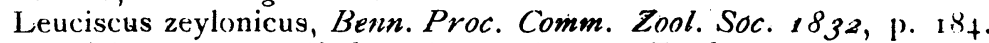
Lutodeira chanos, (kishl.) Rüpp. N. W. Fische, p. I8, lain. 5. fig. I; Günthr. in Fish. Zanz. p. I 20.

Leuciscus (Ptycholepis) salmoneus, Gray, in Dicffenbach, Travels, in New. Zealand, ii. p. 2 I8.

Butirinus maderdspatensis, Jerdon, Madr. Journ. Lit. \& Sc. xv. 1). 344 .

Lutodeira salmonea, Richards. Voy. Ereb. \& Terr. Ichthol. p. 58 , lam. 36 ; fig. I.

Chanos mento, Cuv. \& Val. xix. p. r94.

C. chloropterus, Cuv. \& Val. xix. p. 195; Kner, Novxra, Fisch. p. $34 \mathrm{I}$ :

C.. nuchalis, Cuv. \& Val. xix. p. igf.

C. orientalis, Cuv. \& Val. xix. p. I97; Kner, l. c.

C. cyprinella, Cuv. \& Val. xix. p. $19^{8}$.

C. salmoneus, Cuv. \& Val. xix. p. 201; Gthr. Cat. B. Mus. 7. p. 473 .

C. pala, Cant. Mal. Fish. p. 278; Day, Fish. Malab. p. 224.

C. tolo, Cant. l. c. p. 283.

C. indicus, Bleek. Verh. Bat. Gen. xxiv. Chiroc. p. I . Hab. Luzón, Estanques de Malabon, Manila.

\section{Fam. QUUROCÉNTRIDOS.}

I. Gén. Chirocentrus, Cuv.

1. Ch dorab, Forsk. N. v.

Clupea dor b, Fursk. Descr. An. p. 72 ; Lacep. v. p. 425; Rus. sell, ii. lam. I99.

C. dentex, Bl. Schn. p. 428 .

Esux chirocentrus, Lacepp. v. p. 296. 
Chirocentrus dorab, Cuv. Rigne Anim.; R̈̈̈pp. N. IV. Fasche, p. 81; Richards. Ichthyol. Chin. p. 311; Cuv. \& Val. xix. p. 159. lam. 565; Bleck. Verh. Bat. Gen. xxii. Maduera, p. 6; Cant. Mal. Fish. p. 277; Day, Fish. Malab. p. 223; Gthr. l. cit. 7, p. 475 .

('h. hypselosoma, Bleek. Verh. Bat. Gen. xxiv. Charoc. p. 25; o Nat. Tydschr. Ned. Int. iii. p. $7 \mathbf{1}$.

Hab. Luzón, Cavite, Santa Cruz; China.

\section{Fam. SIMBRÁNQUIDOS.}

I. Gén. Symbranchus, Bloch.

I. \&. bengalensis, $M^{\prime}$ Clell. N. v.

(M. S. T.)

Ophisternon bengalensis, M'Clell. Calc. Journ. Nat. Hist. v. pp. 197, 200, lam. 11. figs. I \& 2; Kaup, Apod. p. 12 1, lam. 15. fig. 76 .

Ophisternon hepaticus, $M^{\prime}$ Clell. l. c. p. 198, figs. 3 \& 4.

Symbranchus immaculatus, Mïller, Abhandl. Ak. Wiss. Berlin, 1839, p. 245; Cantor, Mal. Fish. p. 537; Bleek. Verh. Bat. Gen. xxr. Mur. p. 57; o Nat. Ty'ds. Net. Ind. iii. Borneo, vi. $4.43^{8}$.

S. gutturalis, Richards. Voy. Ereb. \& Terr. Fish. p. 49, lam. 30 figs. $14-17$.

'Ietrabranchus microphthalmus, Bleek. Nat. Tyds. Ned. Ind. ii. P. 69.

Symbranchus bengalensis, Bleek. Atl. Ichth. Mur. p. I19, lam. $4^{8}$. fig. I; Gthr. Cat. B. M. 8. 1870 , p. i6.

Hab. Luzón, Manila, Navotas; Archipiélayo Indico.

\section{Fam. MURÉNIDOS.}

I. Gén. ANGullla, Cuv.

\section{A. mauritiana, Benn:tt.}

Anguilla mauritiana, Bennet, Proc. Comm. Zool. Soc. 1831, p. I 28; Gthr. l. cit. 8. p. 25.

A. labrosa, Richards. Voy. Ereb. \& Terr. Ichth. p. II3.

Murena maculata, Bleek. Ned. Tydschr. Dierk. i. p. 237, o Atl. Ichthol. Muran. p. 9, lam. s. fig. 2 (no $H$. B.)

M. 'manillensis, Bleek. Atl. Ichthyol. Muran. p. Io, lam. 44. f. 2; o Ned. Tydschr. Mierk. 1864 , p. 31.

Anguilla johanna, Günth. in Fish. Zanz. p. I 24.

Muræna marmorata, Kner, Novara, Fisch. p. 369.

Hab. Luzón, Laguna de Bay; Amboina.

2. A. fidjiensis, Gthr.

A. fidjiensis, Gthr. l. cit. 8. p. 26. Hab. Luzon, Manila. 
3. A. amboineusis, I'trs N. v.

$(\mathbf{M} S \mathbf{T}$.)

Peters, Monatsber. Ak. W'iss. Berl. 1 Sron, 1. 523; Gthr. I. cit. 8. p. 34 .

Hab. Luzon, Manila; Amboina.

4. $\lambda$ esp. $\mathrm{N} . \mathrm{v}$

(A. S. T)

Hab. Luzón, Laguna; Stit. Rosil.

2 Gén L'ROCONGEK, Kill.

5. U. lepturas. Kiaup. N. v.

(II. S T)

Congrus lepturus, Richards. Voy. Sulph. Fish. p. 106, lam. 5(). figs. I-6, et Voy. Ereb. \& Terr. Fish. p. ıng; Bleek. Act. Soc. Sc. Indo-Neerl. iii. Sicmatra, vi. p. +5.

Uroconger lepterus, Kaup, Apod. p. r ro; Bleek. Atl. Ichth. Mur. p. 29, lam. 5. fig. I; Kner, Novara, Fisch. 1. 37.3; Gthr. I. cit. \&. p. 44 .

Hab. Luzón, B. de Manila; China.

3. Gén MURANeS()X, M Crefl.

6. M. talabon, Bleck. N. v.

Tala Bon, Russell, i. p. 27, lam. $3^{8 .}$

Conger (Murxna) talabon, Cuv. Rerne An.; Cant. Mal. Fish. p. 3I 2; Bleek Nat. Tyds. Ned. Ind. iii. p. 78 (parte), et v. p. 456 ; et Verh. Bat. Gen. xxv. Mur. p. 18.

Muranesox lanceolata, M'Clell. Calc. Journ. Nat. Hist. iv. p. 4 (og.

M. exodon, $M^{\prime}$ Clell. l. c.

M. serradentata, M'Clell. l. c., et v. p. $2 \mathrm{I}$ (o.

M. exodentata, $M^{\prime}$ Clell. v. p. I80, lam. 8. fig. 4, et p. 210.

M. pristis, Kaup, Apod. p. I 6 .

M. talabon, Bleek. Atl. Ichth. Mur. p. 22, lam. 8. fig. 2; Kner, Novara, Fisch. p. 372 ; Gthr. l. cit. 8. p. 45.

Hab. Luzon, Cavite, Santa Cruz; Indias-Orientales.

7. MI cinereus, (Firsk) N. v.

(M. S T)

Muræna cinerea, Forsk. Descr. An. pp x. et 22.

M. arabica, Bl. Schn. p. 488 .

M. bagio, Ham. Buch pp. 24, 364 .

Ophisurus rostratus, Quoy \& Gaim. Voy. Uran. Zool. 1. 242, lam. 5i. fig. I.

Conger longirostris, Benn. in Life of Raffles, p. 692.

C. oxyrhinchus, Eydoux \& Soul. Voy. Bon. i. p. 203, lam. 9. fig. 2.

Murænesox tricuspidata, M'Clell. Calc. Journ. Nat. Hist. iv. p. 409, lam. 24. fig. I.

M. hamiltonii, M'Clell. l. c.

Congrus tricuspidatus, Richards. Voy. Sulph. Fish. p. I05, lam. 
51. fig. 2 Ichth. Chin. p. 312; or Voy. Ereb. \& Terr. Fish. p. 110 .

Conger hamo, Schleg. Faun. Japon. Poiss. p. 262, lam. 11 t. fig. 2; Richards, Voy. Ereb. \& Terr. Fish. p. I1 1.

C. bagio, Cant. Mal. Fish. p. 316; Bleek. Nat. Tyds. Ned. iii. p. 777 ; o Verh. Bat. Gen. xxv. Mur. p. 22

Congrus protervus, Richards. Voy. Ereb. \& Terr Fish. 1. I11.

C, angustidens, Richards. l. c.

C. breviscuspis, Richards. l. c. p 1 ir.

Conger singapurensis, Bleek. Verh. Bat. Gen. Mur. xxv. p. 2 I.

Murenæsox bagio, Peters, Wiegm. Arch. 1855, p. 270; Kaup, Apod. p. 116, lam. 14, fig. 73; Bleek. Ati. Ichth. Mur. 1. 24, lam. 26. fig. 2; Kner, Novara, Fisch. p. 373.

M. cinereus, Gthr. l. cit. 8. p. 46.

M. singapurensis, Bleek. l. c. p. 25, lain. 7. fig. 2; Kner, l. c. p. 373 .

Hab. Luzón, B. de Manila, R. Munang, Mindoro; China.

4. Gén. MUR ENICHTHYS, BLELK.

8. M. macropterus, Bleek. N. v.

Sleek. Act. Soc. Sc. Iudo-Nederl. ii. Amboina, viii. p. gr; o Atl. Ichth. Mur. p. 31, lam. 7. tig 3; Gthr. l. cit. 8. 1. 52. Hab. P. Princesa, Paragua; Amboina.

9. M. schultzii, Bleek N. v.

Bleek. Nat. Tyds. Ned. Ind. xiii. p. 366; or Atl. Ichth. Mur. p. 33, lam. 4. fig. 3. Gthr. l. cit. 8. p. 52.

Hab. Luzón, B. de Manila; Java.

ı. M. sp. N. v.

Ha.b. Luzón, B. de Manila.

\section{Gén OPHICHTHYS, GTHR.}

I1. O adspersus, Gthr. N. v.

(M. S T

Gthr. l. cit. 8. p. 57.

Hab. Luzón, Manila; China.

12. O dicellurus, Richards. N. v.

(M S. T.

Ophisurus dicellurus, Richardson, Voy. Sulph. Ichthyol. p. Iof, lam. 48. figs. 2-4; Voy. Ereb. \& Terr. Fish. p. 105.

Ophichthys dicellurus, Gthr. l. cit. 8. p. 59.

Hab. Luzón, Cavite, Sta. Cruz; China.

13. O. palleas, Richards. N. v.

(M. S. T.)

Ophisurus harancha, Richards. Ichth. Chin. p. 33 3 (mo H. B.) (). pallens, Richards. Voy. Ereb. \& Terr. Fish. p. 1оп. 
Pisnodonophis pallens, Kaup. Apod. p. 17.

Ophichthys pallens, Gthr. l. cit. \&. p. 6I.

Hab. Luzón, Cavite, Sta. Cruz; China.

14. O. broekmeyeri. Bleck. N. v.

(M S. T )

Ophisurus broekmeyeri, Bleek. Act. Soc. Sc. Indo-Nederl. i. Manado p. 7 I.

O. celebicus, Bleek. l. c. p. 7o.

Ophichthys amboinensis, Bleek, Ned. Tyds=hr. Dierk. ii. p. 45; o. Atl. Ichthyol. Muran. p. 54, lam. 45. fig. 1.

O. broekmeyeri, Bleek. Atl. Ichthyol. Muran. p. 53. lam. 15. fig. I.

O. celebicus, Bleek. l. c. p. 54, lain. i 5. fig. 3; Gthr. l. cit. \&. p. 6r.

Hab. Luzón, Cavite, Sta. Cruz; Amboina.

15 O cirrochilus, Bleek. N. v.

(M. S. T)

Ophisurus cirrhocheilus, Bleek. Act. Soc. Sc. Indo-Nederl. ii. Amboina, viii. p. 89 .

Brachysomophis cirrhochilus, Bleek. Atl. Ichthjol. Muran. p. 38', lain. 9.

Ophichthys cirrochilus, Gthr. 'l. cit. 8. p. 65.

Hab. Luzón, Cavite, Sta. Cruz; Formosa.

16. O. cephalozona, Bleek. var. G hr. N v.

(M. S. T)

Centrurophis spadiceus, Kaup, Apol. fig. I (no descript., n) Richards).

Murænopsis marginatus, Bleek. Ned. Tydschr. Dierk. i. p. 179) (no Ptrs.)

Ophichthys cephalozona, Bleek. Atl. Ichthyol. Muran. p. 49, lam. I 2. fig. 2; Kiner, Novara, Fisch. p. 377; Gthr. t. cit. 8. p. 60. Hab. Luzón, Manila, Cebú; Japon.

7. O. apicalis, Bennett N. v.

(M. S. 'T)

Ophisurus apicalis, Bennett, in Life of Raffles, p. 692.

O. s adiceus, Richards. Ichthyol. Chin. p. 313; Voy. Ereb. \& Ferr. Ichthyol. p. Iо3.

O. compar, Richards. Voy. Ereb. \& Terr. Ichthyol. p. 105.

O. bangko, Bleek. Verh. Bat. Gen. xxv. Muran. p. 67.

Centrurophis spadiceus, Kanp, Apod. p. 2 (no fig.)

C. bangko, Kaup, Apod. p. 3 .

Coecilophis compar, Kaup, Apod. p. 6.

Ophisurus diepenhorsti, Bleek. Act. Soc. Sc. Indo-Nederl. viii. Sumatra, viii p. 85 .

Ophichthys banko, Bleek. Atl. Ichthyol. Muran. p. 5I, lam. I4, fig. I .

O. diepenhorsti, Bleek. Atl. Ichth. Muran. p. 52, lam. i 5. fig. 4.

O. apicalis, Gthr. l. cit. 8. p. 70 .

Hab. Luzón, B. de Manila; China. 
18. O. chinensis, Kaup. N. v.

(M. S. T.)

Cirrhimuræna chinensis, Kaup, Apod. p. 27.

Ophisurus polyodon, Bleek. Act. Soc. Indo-Nederl. viii. Sumatra, viii. p. 86.

Cirrhimuræna polyodon, Bleek. Atl. Ichth;ol. Murcen. p. 4, lam. 8. fig. I.

()phichtys chinensis, Gthr. l. cit. 8. p. 75 .

Hab. Luzón, B. de Manila; China.

19. O. boro, (Ham-Buch).

Ophisurus boro, Mam. Buch. Fish. Gang. pp. 20, 363; Gray, Ill. Ind. Zool.; M'Clell. Calc. Journ. Nat. Hist. v. p. 2 in; . Richards. Ichth. Chin. p. 313, et Voy. Ereb. \& Terr. Fish. p. 99; Bleek. Verh. Bat. Gen. xxv. Beng. p. 156; Cant. Mal. Fish. p. 322, lam. 5. fig. 2.

O. harancha, Ham. Buch. l. c. pp. 2. 363 ; Gray, l. c.; M'Clell. l. c. lam. I2. fig. 4 .

O. caudatus, $M^{\prime}$ Clell. l. c. p. 185 , lam. I 2. fig. 3.

Conger microstoma, Eyd. \& Soul. Voy. Bonite, i. p. 2(15, Poiss. lam. 9. fig. 3.

Ophisurus potamophilus, Bleek. Nat. Tydschr. Ned. Ind. v. p. 458, o Verh. Bat. Gen. xxv. Muran. p. 68.

Pisoodonophis boro, Kaup, Apod. p. I7; Bleek. Atl. Ichth. Mu. ran. p. 62, lam. 20. fig. 3; Day, Fish. Malab. p. It8.

P. potamophilus, Kaup, Apod. p. 20; Bleek. l. c. p. 63, lam. 28. fig. 2.

Ophichtys boro, Gthr. l. c. 8. p. 77 .

$H a b$. Cebú; Indias Orientales.

20. O. cancrivorus, Richards. N. v.

(M. S. T)

? Conger flavipinnatus, Bennett, Proc. Comm. Zool. Soc. 1831 , p. 168.

Ophisurus cancrivorus, Richards. Voy. Ereb. \& Terr. Fish. p. 97 , lam. 5o. figs. 6-9.

O. sinensis, Richards. l. c. p. 98.

(). baccidens, Cant. Mal. Fish. p. 320, 1im. 5. fig. I.

(). schaapi, Bleek. Nat. Tydschr. Ned. Ind. iii. p. 735; o Veril. Bat. Gen. xxv. Murcen. p. 53.

(). brachysoma, Bleek. l.cc. $1^{\circ}$, p. $776 ; 0$ l. c. $2^{0}$, p. 55.

Pisoodonophis cancrivorus, Kaup, Apod. p. I 5 , fig. 9 (cop. de Ri.hards.); Kner, Novara, Fish. p. 379.

P. brachysoma, Kaup, Apod. p. i9; Bleek. Atl. Ichth. Muran. p. 6o, lam. 18. fig. 2.

P. schaapi, Kaup, l. c.; Bleek. l. c. lam. i 7. fig. I.

P. moluccensis, Bleck. Atl. Ichthyol. Muran. 1. 72 , lam. 49 (no. 45) fig, 2.

Ophicthys cancrivorus, Gthr. l. c. 8. p. 78.

Hab. Luzón, B. de Manila; Amboina. 
21. O. culubrinus, Boddacrt.

Murana culubrina, Boddaert, in Pallas's Nene .Vord. Beytr. ii. 1781 , p. 56, lam. 2. fig. 3 .

11. annulata, Ahl, De Murcena et Ophichtho, 789 , p. 8 lam. 1. fig. 1 .

M. fasciata, Ahl, l. c. p. y.

Gymnothorax annulatus, $B l$. Schn. p. 257.

G. fasciatus, Bl. Schn. p. 529.

G. culubrinus, Bl. Schn. p. 529 .

Ophisurus fasciatus, Lacép. iv. p. 686; Richards. Ereb. \& Terr. Fish. p. IoO; Bleek. Atl. Ichthyol. Muran. p. 64, lam. 21. fig. I; Kner, Navara, Finsch. p. 379.

(). alternans, Quoy \& Gaim Voy. Urun. i. p. 243 ; lam. +5. f.g. 2. (). colubrinus, Richards. l. c. p. i(x); Bleek. Nat. Tydschr. Ned. Ind. xi. p. ios.

Ophithorax colubrina, M'Clell. Calc. Journ. Nat. Hist. v. p. 212 (no synon.)

Pisoodonophis fasciatus, Kaup, Apod. p. 23.

()phichtys colubrinus, Gthr. l. cit. 8. p. 81 .

Hab. Cebú; Borneo.

22. O. quadratus, Richards $\mathrm{N}$ v.

(M S. T.)

Sphagebranchus quadratus, Richards. Voy. Sulph. Fish. p. 115, lam. 528 -1 5 .

Ophicthys quadratis, Gthr. l. cit. \&, p. 8g. $H a b$. Luzón, Manila; China.

\section{Gén. MORINGUA, Gray.}

\section{M. lumbricoidea, Richards}

? Moringua linearis, Gray, Zool. Misc. p. 9; et Ill. Ind. Zool. c. fiy, Moringua lumbricoidea, Richards. Voy. Sulph. Ichth. p. 113, lam. 56 figs. 7-1 I; Kaup, Apod. p. I07; Gthr. l. cit. 8, p. 91 . M. microchir, Bleek. Nat. Tyds. Ned. Ind. iv. p. I 24; o Verh. Bat. Gen. xxv. Mur. p. 66; or Atl. Ichth. Mur. p. ı6, lam. + fig. 2 .

Hab. Luzón, Manila; Amboina, China.

24. M. abbreviata, Bleek.

A phthalmichthys abbreviatus, Bleek. Ncd. Tyds. Dierk. i p. I63; Atl. Ichth. Mur. p. i 7, lam. I fig. 'I.

Moringua abbreviata, Gthr. l. cit. 8, p. 92.

Hab. Cebú; Archipièlago Indico.

7. Gén. MURENA, Githr.

25. M. punctato-fasciata, Bleeker

Murona catenata, Bleck. Act. Soc. Indo-Neerl. i. Amboma, p. 66 (ni) synon.) 
M. punctatofasciata, Gthr. l. cit. 8, p. 106.

Gymnothorax punctato-fasciatus, Bleek. Atl. Ichth. Mur. p. 99, lam. 31 fig. 4 .

Hab. Luzón, B. de Manila, Cebú; Amboina.

26. M. tessellata, Richarils. N. v.

(M. S. T.)

? Gymnothorax favagineus, $B l$. Schn. p. 525. lam. IO5.

? Murœna favaginea, Cuv. Régne. Anim.

'Thorodontis reticulata, $M$ 'Clell. Galc. Journ. Nat. Hist. v. 1845, pp. 188, 216, lam. 7. fig. I (no Bl. Schn).

Murcena tessellata, Richcrds. Ichth. Sulph. p. Io9, lam. 55 figs. 5-8, et Ereb. \& Terr. Fish. p. 88; Bleek. Nat. Tydschr. Ned. Ind v. p. 530, o Verh. Bat. Gen. xxv. Muran. p. 74; Gthr. l. cit. 8, p. 106.

M. isingleena, Richards. Ichth. Sulph. p. Io8, lam. $4^{8}$ fig. I; et Ereb. \& Terr. Fish. p. 86.

IA. python, Kaup, Apod. p. 68, fig. $5^{2}$.

II. favarinea, Kaup, Apod. p. 68, fig. 53.

'Thyrs jidea tessellata, Kaup. Apod. p: 76 .

T. isingleena, Kaup, Apod. p. 75, fig. 57.

Gymnothorax isingleena, Bleek. Atl. Ichth. Mur. p. 92, lam. 37 fig. I.

G. tessellatus, Bleek. l. c. p. 93, lam: 27, fig. 3, et lam. 28 fig. I.

Hab. Luzón, Cavite, Santa Cruz; China.

27. M. fimbriata, Bennett. N. v

(M. S. T.)

Murcena fimbriata, Bennett. Proc. Comm. Zool. Soc. i. irzi, p. 168 .

M. bullata, Richards. Ereb. \& Terr. Ichth. p. 86 (cop. de Kaup, Apod. p. 81, fig. 60); Gthr. l. cit. 8, p. 1o8.

M. isingleena, Bleek. Nat. Tyds. Ned. Ind. ix. p. 277 (no Richards.)

M. isingleenoides, Bleek. Verh. Bat. Gen. xxv. Mur. p. +8.

Gymnothorax isingleenoides, Bleek. Atl. Ichth. Mur. p. 9r, lain. 35 , fig. I, lam. 36 , fig. $I$.

Hab. Luzón, Cavite, Santa Cruz; Amboina.

28 M. melanospila, Bleek.

Muroena melanospilus, Bleek. Nat. Tydschr. Ned. Ind. ix. p. 279.

Gymnothorax melanospilus, Bleek. Atl. Ichth. Mur. p. 9), lam. 42 fig. I.

M. melanospila, Gthr. l. cit. 8, p. Iog.

Hab. Luzón; Sumatra.

29. M. macassariensis, P Bletk N. v.

(II. S. T.)

Muroena thyrsoidea, Richards. Voy. Sulph. Fish. lain. t9 fig: I (no descript).

Gymnothorax makassariensis, Bleek. Ned. Tydschr. Dierk. i. p. 168; or Atl. Ichth. Mur. 1. 104, lan. 37 fis. 3. 
?? Murœna elegantíssima, Kaup, Apod. p. 67.

Thyrsoidea macrops, Kaup, Apod. p. 74.

M. macassariensis, Gthr. l. cit. 8, p. 111 .

Hab. Luzón, Manila, Malabon, Bulacan; China.

29 M. thyrsoidea, Richu'ds. N. v.

(M. S. 'T)

Muroena thyrsoidea, Voy. Sulph. Ichth. p. 111 (no fig.), et Voy. Ereb. \& Terr. p. 9o; Cant. Mal. Fish. p. 330, lam, 5 f. 5; Gthr. l. cit. 8 , p. I I 3 .

M. grisseobadia, Richards. Voy. Ereb. \& Terr. Fish. p. 89.

M. prosopeion, Bleek. Nat. Tyds. Ned. Ind. iv. p. z(x); ó Verh. Bat. Gen xxv. Mur. p. 73.

Thyrsoidea gi iseobadia, Kaup, Apod. p. 74, (cop. Richards).

T. arenata, Kaup, Apod. p. 85 (cop. Richards.)

Gymnothorax prosoption, Bleek. Atl. Ichth. Mur. p. 88, pl. 39. fig. 3 .

$H a b$. Luzón, Cavite, Sta. Cruz; China.

3o. M. picta, $A h l . \mathrm{N}$ v.

(M. S r.

? Conger indicus maculosus, Willughby, Apennd. p. 24, lam. G. Io. fig. I.

Muræna picta, Ahl. De Mur. et Ophichth. in Thumb. Dissert. iii. p. 6, lam. 2, fig. 2; Gïnth. in Fish. Zanz. p. 126; id. Cat. B. $M .8$. p. 116 .

Gymnothorax pictus, Bl. Schn. p. 529; Bleek. Atl. Ichth. Muran. p. 87, lam. 26. figs. 3, 4, lam. 28. fig. 3, lam. 29. fig. 1, lam. 45. fig. 3; Kner, Novara, Fisch. p. 384.

Murænophis pantherina, Lacép. v. pp. 628, 641, 643.

Muræna variegata, Quoy \& Gaım. Voy. Uran. Zool. p. 246, lam. 52. fig. 1 .

M. lita, Richards. Voy. Ereb, \& Terr. Fish. p. 84 ; Bleek. Nat. Tydschr. Ned. Ind. iii. p. 294, et x. p. 383, $o$ Verh. Bat, Gen. xxv. Muran. p. 47.

M. siderea, Richards. l. c. p. 85 ; lam. 48 . figs. I-5.

M. pfeifferi, Bleek. Nat. Tyds. Ned. Ind. v. p. I73; ó Verh. Bat. Gen. xxv. Muran. p. 72.

Sidera pfeifferi, Kaup. Apod. p. $70^{\circ}$.

S. pantherina, Kaup. Apod. p. 7 I.

Gymnothorax panterinus, Bleek. Ned. Tydschr. Dierk. i. p. I52. Hab. Luzón, Cavite, Sta. Cruz; Amboina.

31. M. nubila, Richards. N. v.

(M. S. T.)

Muræna nubila, Richards. Voy. Ereb. \& Terr. p. 81, lam. 46. figs. 6-i 4 (cop. de Kaup. Apod. p. 57, fig. 48); Gthr. l. cit. p. I I 7 . M. similis, Richards. l. c. p. 83 (cop. de Kaup. Apod. p. 63).

M. kidako, Schleg. Faun. Japon. Poiss. p. 266, lam. I I 7.

Gymnothurax sagenodeta, Bleek. Atl. Ichth. Mur. p. ioo, lam. to. fig. 4 (no synon.)

G. chilospilus, Bleek. Ned. Tydschr. Dierk. ii. p. 52; ó Atl. Ichth. Mur. p. IO3, lam. 44. fig. I

Hab. Luzón, Cavite, Sta. Cruz; Amboina, Formosa. 
32. M. tenebrosa, Richards. N. v.

Richards. Voy. Ereb. \& Terr. Fish. 1. 84; Gthr. l. cit. 8. p. 119. Hab. Marianas.

33. M macrurus, Bleek N. v

Murana macrurus, Bleek. Nat. Tyds. Ned. Ind. ii p. 324 ; Gthr. l. cit. 8. p. 127 .

Thyrsoidea longissima, Kaup. Apod. p. 82 .

T. macrurus, Bleek. Atl. Ichth. Mur. P. II I, lam. 22. fig. 2; Kner, Novara, Fisch. p. 386.

Hab. Luzón, Manila, Navotas; Ceilan.

34. M. zebra, Bleek. N. v.

(M. S. T.)

? Seba, ii. p. 72, lam. 7o. fig. 3.

Gymnomuræna zebra, Shaw, Zool. Misc. p. ror; Richards. Voy. Ereb. \&. Terr. Fish. p. 95 (cop. de Kaup, Apod. p. IOt, tig. 70$)$.

Gymnothorax zebra, $B l$ Sch. p. 528 .

Gymnomuræna doliata, Lacep. v. pp. 648, 649, lam. i 9. fig. 4 . Muræna molendinaris, Bennett, Proc. Zool. Soc. i. I 833, p. 32. M. zebra, Bleek. Nat. Tyds. Ned. Ind. xiii. p. 8o; et Act. Soc. Sc. Indo-Neerl. ii. Amboina, viii. p. 93: Gthr. l. cit. 8. p. I 28. Gymnomuræna fasciata, Kaup, Apod. p. Io3, fig. 69.

Echidna zebra, Bleck. Atl. Ichthyol. Atur. p. 81, lam. 27. fig. 1. Hab. Luzón, Cavite, Santa Cruz, Batangas, Nasugbú; Sumatra.

35. M. polyzona, Richards. N. v.

(M. S. T.)

Muræna polyzona, Richards. Voy. Sulph. Zool. p. i I 2, lam. 55. figs. I I-14, et Voy. Ereb. \& Terr. Fish. p. 95; Bleek. Act. Soc. Sc. Indo.Neerl. Manado, p. 73; Gthr. l. cit. 8. p. I 29. M. dizona, Bleek. Nat. Tyds. Ned. Ind. xxii. p. 260.

Pocelophis polyzonus, Kaup, Apod. p. IoI (cop. de Richardson); Kner, Novara, Fische, p. 382.

Echidna polyzona, Bleek. Ati. Ichth. Mur. p. 81, lam. 24. fig. 3. Hab. Luzón, Manila; Archipièlago Indico.

36. M. nebulosa, Ahl. N. v.

Seba, ii. lam. 69. figrs. I, I 7 .

Muræna nebulosa, Ahl, D. Mur. et Ophichth. p. 5, lam. 1. fig. 2; Gthr. l. cit. 8. p. I 30.

Gymnothorax nebulosus Bl. Sch. p. 528 .

G. echidna, Bl. Schn. p. 526.

Echidna variegata, Forst. Descr. An. ed. Licht. p. I8ı; Bleek. itl. Ichth. Mur. p. 80, lam. 24. fig. 2.

Murana ophis, Rüpp. Atlas, Fische. p. i16, lam. 29. fig. 2; Richards. Voy. Ereh. \& Terr. Fish. p. 93.

'Thurodontis ophis, M'Cleli. Calc. Journ. Nat. Hist. v. p. 2 i 7. 
Muræna variegata, Richars. Voy. Ereb. if Terr. Fish. p. 94, lam. 47, figs. I1-16; Bleek. L'at. Tyds. Ned. Ind. iii. p. 295;

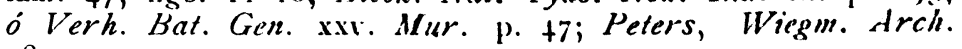
I 855, p. 270 .

Pacilophis variegata, Kaup, Apod. p. 98, lam. 13. fig. 67; Kn'r, Nov:ra Fisch. p. $38 \mathrm{~s}$.

Hab. C. de Cebú; China.

\section{Gén. Grinomur.ina, Br.eEk.}

6 G. marmorata, Rivi.

Gymnomurana marmorata, Lacep. v. pp. 648, 650; Bleek. All. Ichth. Mur. p. I1 3, lam. 31. fig. 3; Gth. l. cit. 8. p. 133.

Ichthyophis pantherinus, Less. Voy. Coq. Poiss. ii. p. 131, $d t l$. lam. I 3; Bleek. Versl. Ak Wet. Natuork. xv. p. 467.

Muræna micropterus, Bleck. Nat. Tyds. Ned. Ind. iii. p. 29s; i Verh. Bat. Gen. xxv. Mur. p. 50 (joven).

Uropterigius xanthopterus, Bleek、Nat. Tyds. Ned. Ind. xix. p. 35('; (joven).

Gymnomuræna macrocephalus, Bleek. Ned. Tyds. Dierk. ii. p. 54; or. Atl. Ichth. Mur. p. II 4 , lam. 2 r. fig. 2 (joven).

Hab. Mindanao, Zamboanga.

37. G. esp. N. v.

(M. S. T.)

Hab. Zamboanga, Mindanao.

\section{Fam. PEGÁSIDOS. \\ I. Gén PEgasus, Lin.}

I. P. volans, Linn. N. v.

(M. S. T.)

Pegasus volans, L. Syst. Nat. i. p. 418 ; Gthr. l. cit. 8. p. 148 .

P. laternarius, Cuv. Régne An.; Kaup, Lophobr. p. 3, lam. I, fig. 4 .

Hab. Luzón, Manila; China.

2. P. natans, Linn. N. v.

(M. S. T.)

Pegasus natans, L. Syst. Nat. i. p. 418 ; Bl. Ausl. Fish. i. p. 53, lam. I 21. figs. 2. \& 3; Richards, Voy. Sulph. Fish. p. I18, lam. 50. figs. 5-7; Gthr. l. cit. 8. p. 148. Wood. Nat. Hist. 3. p. 340, fig.

Cataphractus, sp., Gronov. Zoophyl. $n .^{\circ} 357$, lam. II. figs. $2 \& 3$. Pegasus spatula, Lacép. ii. p. 85 .

P. volans, Richards. Voy Sulph. Fish. lam. 50. figs. 8-10.

P. pristis, Bleek. Nat. Tyds. Ned. Ind: iii. p. 6o6; ó Verh. Bat. Gen. xxv. Trosk. p. 28.

Cataphractus anceps, Gronov. Syst. ed. Gray, p. 144. Hah. Luzón, Santa Cruz, Cavite, Samar. 
3. P. lancifer, Kaup. N. v.

(M. S. T.)

Pegasus natans, Kaup. Lophobr. p. 4, lam. I. fig. 2.

P. lancifer, Kaup. Wiegm. Arch. 1868, p. I17; Gthr. l. cit. 8. p. 149.

Hab. Luzòn, Santa Cruz, Cavite; Tasmania.

\section{Orden $5 \cdot^{\circ}$ LOFOBRANQUIOS.}

Fam. SOLENOSTÓMIDOS.

I. Gén. SOlEnOSTOMA, Lacép.

I. S cyanopterum, Bleek. N. v.

(M. S. T.)

Solenostomus paradoxus, Richards. Icht. Chin. p. 203; Bleek. Nat. Tyds, Ned, Ind. iii. p. 308; Kaup. Lophobr. p. 2.

Solenostoma cyanopterum, Bleek. l. c. vi. p. 506; Gunth. in Fish. Zanz. p. 137 , lam. 20 . figs. 2 \& 3 ; id. l. cit 8. p. $15 \mathrm{I}$.

Hab. Luzón, Cavite, Sta. Cruz; China.

2. S. brachyurum, Bleek. N. v.

(M. S. T )

Bleek. Nat. Tyds. Ned. Ind viii. p. 433; Gthr. l. cit. 8. p. 132. Hab Luzón, Cavite, Sta. Cruz; Amboina.

\section{Fam. SINGNÁTIDOS.}

1. Gén. SYNGNATHUS, ARTEdi.

1. S schlegelii, Kaup. N, v.

'M. S T)

Syngnathus tenuirostris, Schleg. Faun. Japon. Poiss. p. 273, lam 120 fig. 5 .

S. schlegelii, Kaup. Lophobr. p. 46; Gthr. l. cit. 8 p. 160. Hab. Luzón, Cavite, Sta. Cruz; China.

2. S. zanzibarensis, Gthr. N. v.

(M. S. T.)

Gunther, in Fish, Zanz. p. 140, lam. 20. fig. 5; ed. Cat. R. $M$ 8. p. 168 .

Hab. Luzón, Manila; China.

3 S. spicifer, Rüppell. N. v.

Syngnatbus spicifer, Rüppell, N.W. Fische p. 143, lam. 33 fig. 4 ; Kaup, Lophobr. p. 34; Day, Fish. Malab. p. 264; Gthr. l. cit. 8. p. I72. 
S. djarong, Bleek. Verh. Bat. Gert. xxv. Trosk. p. 22; et Nat. Tyds. Ned. Ind. vii. p. 325.

S. gastrotonia, Bleek. l. c. $1^{\circ}$, et l. c. $2^{\circ}$, iii p. 713.

S. helfrichii, Bleek. l. c. $2^{\circ}$, ix. p. 428.

S. argyrostictus, Kaup, Lophobr. p. 33 .

S. biserialis, Kaup, Lophobr. p. 33 .

Hab. Luzón, Manila, Navotas; China, Borneo.

4. S. conspicillatus, fenyns. $\mathrm{N}$ v.

(M. S. T.)

Valentyn, fig. 81; Ruysch, Amboina, lam. 6. fig. 18; ? Renard, i. lam. 4. fig. 30

Syngnathus fasciatus, Gray, Ind. Zool. c. fig. (no Risso).

?S. perlatus, Binn. in Beechey's Voy. Fish. p 68, lam. 21. fig. I.

S. conspicillatus, Jenyns, Voy. Beagle, Fish. p. 147, lam. 27. fig. 4 ; Gthr. loc cit. 8. p. 174.

S. hæmatopterus, Bleek. Nat. Tyds. Ned. Ind. ii. p. 258; 0 Verh. Bat. Gen. xxv. Trosk. p. 20.

Corythoichthys fasciatus, Kaup, Lophobr. p. 25; Kner, Novara, Fisch. p. 39r.

Hab. Luzón, Manila, Cebú, P. Princesa, Paragua; Amboina.

\section{Gén ICHTHYOCAMPHUS, Kaup.}

5. I. belcheri, Kaup. N. v.

(M. S. T.)

Ichthyocampus belcheri, Kaup, Lophobr. p. 30; Gthr. l. cit. 8. p. 177.

Hab. Luzón, Manila, Navotas; China.

3. Gén. DORYIHTHYS, KaUP.

6. D. boaja, Bleek. N. v.

Syngnathus boaja, Bleek. Nat. Tyds. Ned. Ind. i. p. 16; o Verh. Bat. Gen. xxv. Trosk p. I 4.

Doryichthys spinosus, Kaup, Lophobr. p. 57.

D. boaja, Gthr. l. cit. 8. p. 180.

Hab. Luzón, Cavite, Sta Cruz; China.

7. D. caudatus, (Plrs).

Microphis caudatus, Peters. Monatsber. Ak. Wiss. Berlin, 1868 , p. 278 .

Doryichtys caudatus, Gthr. l. cit. 8. p. 182 .

Hab. Samar; Java.

8. D. pl -urostictus, (Ptrs).

Microphis pleurostictus, Peters, Monatsber. Ak. Wiss. Berlin, 1868 , p. 278 .

Doryich hys pleurostictus, Gthr. l. cit. 8. p. 185.

Hab. Luzón. 


\section{Gén. GaSterotokeus, Kaup.}

\section{G. biaculeatus, Kaup.}

Valent. Amb. iii. p. 500, n. ${ }^{\circ} 48 \mathrm{r}$; Renard, fig. 73.

Syngnathus biaculeatus, Bl. Ausl. Fisch. iv. p. io, lam. 121. figss. I \& 2; Bl. Schn. p. 515, lam. I07; Cant. Mal. Fish. P 3īi. S. tetragonus, L. Gm. i. p. I 433 ; Lxcép. ii. p. 42

Syngnathoides blochii, Bleek. Nat. Tyds. Ned. Ind. ii. p. 259.

Solegnathus blochii, Bleek Verh. Bat. Gen. xxv. Trosk. 1. 24. Gasterotokeus biaculeatus, Kaup. Lophobr. p. i9; Gthr. l. cit. 8. p. 194 .

Hab. C. de Samar; China.

5. Gén. Solenognathus, Kaup.

1o. S. hardwickii, Kaup. $\mathrm{N}$.

(M S. T )

Syngnathus, hardwickii, Gray, Ind. Zool. $c$ fig.

Solegnatus polyprion, Bleek. Verh. Bat. Gen. xxv. Trosk. p. 25. Solenognathus hardwickii, Kaup. Lophobr. p. 2o; Gthr. l. cit. 8 . p. I 95 .

Hab. Luzón, Cavite, Sta. Cruz; China.

\section{Gén hippocampus, Kaup.}

I. H. longirsstris, Sckleg. N. v.

(M. S. T.)

? Willughby, lam. J. 25. fig, 4 .

Hippocampus longirostris, Cuv. Régne An.

Hippocampus longirostris, Schleg. Faun. Japon. Poiss. p. 273; Gthr. l. cit. 8. p. 201 .

Hab. Samar, Borongan; China.

12. H. guttulatus, Cuv. N. v.

(M. S. T.)

Hippocampus kuda, Bleek. Nat. Tyds. Ned. Ind. iii. p. 82; Verh. Bat. Gen. xxv. Trosk. p. 26.

H. moluccensis, Bleek. Nat. Tyds. Ned. Ind. iii. p. 305.

H. tœniopterus, Bleek. l. c. p. 306.

H. polytænia, Bleek. l. c. vi. p. 338 .

H. melanospilus, Bleek. l. c. p. 505 .

H. comes, Kaup, Lophobr. p. ıо; Day, Fish. Malabar, p. 262, (no Cant.)

H. punctulatus, Kaup, Lophobr. p. I4.

H. punctulatus, guttulatus et monckei, Günth. Fish. Zanz p. I39; Id. Cat. B. M. 8. p. 202.

Hab. Luzón, B. de Manila, Cebú; Amboina.

13. $\mathrm{H}$ trimaculatus, Leach $\mathrm{N}$ v.

Hippocampus trimaculatus, Leach, Zool. Misc. p. ı 4 ; Gthr. l. cit. 8. p. 204. 
H. mannulus, Cant. Mal. Rept. p. 388, pl. I1. fig. 1; Kaup, Lophobr. p. It.

? H. chinensis, Basilezsky. Now. Men. Nat. Mesc. x. 1855, p. $2+9$.

? H. kampylotrachelos Blcek. Na'. Tyds. Ned. Ind. vii. p. 107.

? H. manadensis, Bleek. Act. Soc. Sc. Indo-Necrl. i. Manado, p. 79. Hab. Luzón, Cavite, Sta. Cruz; China.

14 H. comes, Cantor. N. v.

(M. S. T.)

Cantor, Mal. Fesh. p. 389. lam. 11. p. 2; Gthr. l. cit. 8. p. 204 .

Hab. Luzón, Cavite, Sta. Cruz.

\section{Orden 6..$^{\circ}$ PLECTOGiNATOS.}

\section{Fam. TRIACÁNTIDOS. \\ I. Gén. TRiacanthus Cuv.}

I. T. brevirostris, Schleg. N. v.

(M. S. T.)

Hoornvisch Nieuhof, Lant-en Zee-R. p. 272.

Piscis eornutus Willughby, Append. p. 5, lam. io fig. 2.

Balistes, sp., Gronov. Mus. i. p. 52 n. ${ }^{\circ}$ I 5 ; Zoophyl. p. 53, n." I94; Russell, i. p. I4, lam. 2 r.

Balistes biaculeatus, Benn. Fish. Ceyl. lam. I5 (no Bl).

Triacanthus brevirostris, Schleg. Faun. Japon. Poiss. p. 294, lam. I 29, fig. 2; Hollard, Ann. Sc. Nat. 1854, i. p. 45, lam. 2, fig. I; Bleek. Atl. Ichth. Balist. lam. 17, fig. 3; Gthr. l. c.t. $8, \mathrm{p} .209$.

'T. biaculeatus, Bleek. Verh. Bat. Gen. xxii. Madur. p. 6 (no Cuv.)

'T. rholopterus, Bleek. l. c. xxiv. Balist. p. 25, lam. 4. fig. 8.

I. russellii, Bleek. $l . c$.

'T. nieuhofi, Bleek, l. c. p. 26, lam. 4. fig. 9, or Nat. Tyds. Ind. iii. p. 459, or Atl. Ichth. v. p. 92, lam. 3, fig. 3.

T. brachysoma, Bleek. Nat. Tyds. Ned. Ind. iv. p. г 28.

Balistes bipes, Gronov. Syst. ed. Gray, p. 37. Hab. Luzon, Cavite, Sta. Cruz; China, Formosa.

2. T. biaculeatus, Cuv. $\mathrm{N}$ v.

Balistes biaculeatus, $B l$. lam. 148. fig. 2 .

'Triacanthus biaculeatus, Cuv. Regine, An.; Cant. Mal. Fish. p. 360; ? Day, Fish. Malabar, p. 260; Gthr. l. cit. 8. p. 2 10.

'T. oxycephalus, Bleek. Verh. Bat. Gen. xxiv. Balist. p. 27, lam. 5. fig. ro, o Nat. Tyds. Ned. Ind. ii. p. 496, o Atl, Ichth. v. p. 9o, Balist. lam. 6. fig. 3 .

'T. blochii, Bleek. Nat. Tyds. Ned. Ind. iii. p. 81, o Atl. Ichth. v. p. 89, Balist. lam. 3. fig. I; Kner, Novara, Fische, p. 394.

T. angustifrons, Hollard, Ann. Sc. Nat. 1854, i. p. 45, lam.

2. fig. 2 . 
T. macrurus, Blcek. Atl. Ichth. v. p. 91, Balist. lam. 8. fig. 3. Hab. Luzon, B de Manila, C. de Cebú; Australia.

3. T strigilifer, Cant. N. v.

(M. S. T.)

Triacanthus strigilifer, Cant. Mal. Fish. p. 363, lam. 9; Bleek. Act. Soc. Sc. Indo-Neerl. ii. Amboina, viii. p. 97; o Atl. Ichth. v. p. 89, Balist. lam. 15. fig. 3; Gthr. l. cit. 8. p. 2 I I. 'T. longirostris, Hollard, Ann. Sc. Nat. 1854, i. p. 46, lam. 2. fig. 3 .

Hab. Luzón, Cavite, Sta. Cruz; China.

\section{Fam. BALISTIDOS.}

1. Gén. BALISTES, Cuv.

I. B. stellatus, Lacép. N. v.

(M. S. T)

Willughby, Append. p. I, lam. I, fig. I; Valent. fig. 202 ; Renard, ii. lam. 33. fir. 153; Ruysch, Anb. lam. 2. fig. 8.

Balistes stellatus, Lacép. i. p. 330, lam. r 5, fig. I; Rüpp. Atl. Fish. p. 31; Blee). Verh. Bat. Gen. xxi. Balist. p. I 3; Bleek. Verh. Bat. Gen. xxiv. Balist. p. I 3; Gthr. l. cit. 8. p. 2 1 2.

Balistes stellaris, Bl. Schn. p. 476; Hollard, Ann. Sc. Nat. 1854, i. p. 320 .

B. vachellii, Richards. Voy. Sulph. Fisch. p. I 29.

B. phaleratus, Richards. in Stokes, Discov. in Austral. i. p. 484 , lam I. figs. 4-5.

Leiurus stellatus, Bleek. Atl. Ichth. Balist. p. I05, lam. I; Kner, Novara, Fisch. p. 398.

Hab. Luzón, Cavite, Santa Cruz; China, Amboina.

2. B. maculatus, Gm. N. v.

(M. S. T.)

Guaperva longa, Willughby, Append. p. 21, lam. I 20.

Sabaco, Parra, p. i7, lam. io.

Balistes maculatus, Gm. L. i. p. I468; Bl. ii. p. 25, lam. I5I; Bl. Schn. p. 464; Lacép. i. p. 36r; Holl.urd, Ann. Sc. Nat. i. 1854 . p. I58; Gthr. l. cit. 8. p 213 .

B. suffamen, Mitch. Lit. \& Phil. Trans. N. York, i. p. 467 , lam. 6. fig. 2.

B. oculatus, Gray, Ill. Ind. Zool.

B. willughbeii, Benn. Proc. Comm. Zool. Soc. i. p. I68; et in Beechey's Voy. Zool. p. 68, lam. 21. fig. 2.

? B. angulosus, Quoy \& Gaim. Voy. Uran. Zool. p. zio; Hollard, l. c. p. 57 .

B azureus, Less. Voy. Coq. Zool. ii p. izi, lam. io. fig. 2.

? B. rotundatus, Prbc. Bull. Soc. Philom. 1822 , p. I 30.

B. conspicillum, Cant. Mal. Fish. p. 344 (no syn).

B. longus, Gronov. Syst. ed. Gray, p. 37 .

B. senticosus, Richards. Voy. Samar. Fish. p. 23, lam. 9. figs. 5-8; Bleek. Nat. T. N. Ind. v. p. 93.

B. longissimus, Hollard. Ann. Sc. Nat. i. p. 6o, lam. 3. fig. 3. 
B. brevissimus, Hollard. Arts. Sc. Nat. 1854 , i. p. 56, lam. 3. fig. I.

B. (Canth:dermis) oculatus, Bleek. Atl. Ichth. Balist. p. 121. lam. 4. fig. 2.

B. (Canthidermis) maculatus, Bleek. l. c. p. 122, fig. 4; Kner, Novara, Fisch. p. 401 .

B. sobaco, Poey, Mem. Cub. ii. p. 324.

B. macrops, Poey, l. c. p. 326.

Hab. Samar, Borongan; China.

3. B. vidua, Richards. N. v

(M. S. T.)

Renard, i. lam. 17. fig. 96.

Balistes vidua, Richards. Voy. Sulph. Fish. p. 128, lam. 59. figs. 9, io; Bleek. Nat. Tyds. Ned. Ind. iii. p. 565; Hollard, Ann. Sc. Nat. 1854 , i. p. $319 ;$ Gthr. l. cit. 8. p. 216.

Melichthys vidua, Bleek. Atl. Ichth. v. p. 109, lam. 217. fig. 2. Hab. Luzón, Cavite, Santa Cruz; China.

4. B. mitis, Benn. N. v.

Baliste bridé, Lacep. i. pp. 335, 38t, lam. 15. fig. 3.

Balistes amboinensis, Gray, Ill. Ind. Zool. c. fig.

B. mitis, Benn. Proc. Comm. Zool. Soc. i. p. 169; Gthr. l. cit. 8. p. $2 \mathrm{I} 8$.

B. frenatus, Richards. Voy. Sulph. Fish. p. 129, lam. 6o. fig. 1; Hollard, Ann. Sc. Nat. $4^{\circ}$ serie. i. p. 322, lam. 5. fig. 3; Bleek. Act. Soc. Sc. Indo-Neerl. i. Manado, p. 74.

B. hihpe, Richards. l. c. p. r 27, lam. 6o. fig. 2.

B. schmittii, Bleek. Verh. Bat. Gen. xxiv. Balist. p. 37.

B. (Balistapus) frenatus, Bleek. Atl. Ichth. Balist. p. I 14 , lam. 223. fig. 2; Kner, Novara, Fisch. p. 400.

Hab. Luzón, Manila; China.

\section{B. bursa, Bl. N. v.}

Valent. figs. $377 \& 403$; Renard, i. lam. 1. fig. 7, ii. lam. 26. fig. I 27; Ruysch, lam. 19. figs. 5 \& 6 .

Baliste bourse, Lacép. i. pp. 335.

B. bursa, Bl. Schn. p. 476; Bleek. Nat. Tyds. Ned. Ind. v. p. 350; Hollard, Ann. Sc. Nat. 1854 , i. p. 328; Gthr. l.cit. 8. p. 219.

Balistes (Balistapus) bursa, Bleek. Atl. Ichth. Balist. p. i I6, lam. 9. fig. 3.

Hab. Cebú: Oceano Pacífico.

6. B. aculeatus, $L$. N. v.

Seba, iii. lam. 24. fig. I5; Valent. figs. 22 \& 422 ; Renard, i. t. 28. f. I 54 , ii. t. 28 . f. I 3 ; Ruysch, t. 2. f. 2, t. 19. f. 8. Balistes, sp., Gronov. Zoophyl. n. ${ }^{\circ}{ }_{1} 88,0$ Mus. Ichth. i. p. 53. n. ${ }^{\circ}$ I 7 .

Capriscus, sp., Klein, Pisc. Miss. iii. p. 25. n. ${ }^{\circ} 7$, lam. 3. fig. Io.

Balistes aculeatus, L. Syst. Nat. i. p. $405 ; B l$. ii. p. I9, lam. I 49; Bl. Schn. p. $46_{5} ;$ Lxcep. i. p. $3^{67}$, lam. I7. fig. I; 
Bennett, in Beechsy's Voy. Zool. p. 69, lam. 22. fig. 2; Tenyns, Zool. Beagle, Fish. p. I55; Bleck. Verh. Bat. Gen. xxiv. Balist. p. I5; Hollard. Ann. Sc. Nat. i. 1854, p. 333. Gthr. l. cit. 8. p. 223.

B. ornatissimus, Less. Voy. Coq. Zool. Poiss. i. p. Ir9, lam. I0. fig. I .

B. armatus, Cuv. R. An: Ill. Poiss. lam. ir 2. fig. 2.

B. striatus, Gronoz. Syst. ed. Gray, p. 32.

B. (Balistapus) aculeatus, Bleek. Atl. Ichth. Balist. p. $120,1 \mathrm{~m}$. 2. fig. 3 .

Hab. Luzón, B. de Manila; China.

7. B. verrucosus, L Syst. N. $\overline{\mathbf{v}}$

Valent. fig. I73; Renard, ii. Iam. 34, fig. 157; Ruysch, Amb. lam. 14, fig. I4; Seba, iii. t. 24, f. 17.

? Balistes verrucosus, L. Sy'st. Nat. i. p. 405.

Baliste pralin, Lacép. i. p. 363 .

Balistes viridis, $B l$. Sch $n$. p. 476 .

B. verrucosus, $B l$. Schn. p. $476 ;$ Gthr. l. cit. 8, p. 225.

B. unimaculatus, Share, Zool. v. pt. 2, p. 4 Io.

R. praslinensis, Quoy \& Gaim. Voy. Urak. Zool. p. 205, lam. 46, fig. 1 .

B. praslinoides, Less. Voy. Coq. Zoot. ii. p. i i7, lam. 9, fig. 3.

B. melanopleura, Bleek. Verh. Bat. Gen. xxiv. Balist. p. 14.

13. praslensis, Hollard, Ann. Sc. Nat. 1854 , i. p. 332 .

B. (Balistapus) verrucosus, Bleek. Att. Ichth. Balist. p. I 20, lam 2, fig. 2; Kner, Novara, Fisch, p. 399.

Hab. Luzón, Batangas, Nasugbú, Mindoro, Cebú; China.

\section{B. rectangulus, $B l$}

Baliste echarpe, Lacép. i. pp. 333, 352, lam. 16 tig. I.

Balistes rectangulis, Bl. Schn. p. 465; Günth. in Fish. Zanz. p. I 34 ; id., Cat. B. M. 8 , p. 225.

B. medinilla, Quoy \& Gaim. Voy. Uran. Poiss. lam. 46, fig. 2.

B. erythropteron, Less Voy. Coq. Zool. ii. p. i 23, lain. io fig. 3.

B. cinctus, Ann. Sc. Nat. 1854 , i. p. 335 .

13. (Balistapus) cinctus, Bleek. Ned. Tyds. Dierk. iii. p. 24, et Atl. Ichth. v. p. I I9, lam. 228 fig. I.

Hab. Luzon, Batangas, Nasuģbú; Polinesia.

o. B. undulatus, Mongo Park. N v Pacol.

(M S T.)

Valent. figs. $78 \& 3.33$; Renard, i. lam. 43, f. 217 , ii. lam. 2, f 7, lam. 25, fig. I23; Ruysch, lam. 7, f. 4 .

Balistes undulatus, Mungo Park, Trans. Linn. Syc. iii. p. 37; Lacép. iv. p. 682; Gthr. l. cit. 8, p. 226.

B. lineatus, Bl. Schn. p. 466, lasn. 87; Bleck. Verh. Bat. Gen. xxiv. Balist. p. 14; Hollard, Ann. Sc. Nat. i. 1854, p. 337.

B. aculeatus, $\beta$ viridis, Benn. Fish. Ceylon, lam. ro.

B. capistratus, Files. Mem. Ac. Sc. St. Petersh. vii. p. зor, lam. 9. 
B. Lamourouxii, Quoy \& Gaim. Loy. Uran. Zool. p. 208, lam. ti fig. 1.

B. sexquilineatus, Bent. in Beechey, Voy, p. bo, lam. 2 fig. 3.

B. porcatus, Cironoi. Syst. e'd Gray, p. 32 .

R. (Balistapusilineatus, Bleck Ned. Tyds. Dierk. iii. 1805, p. 24 ; Atl. Iihth. $\checkmark$ p. Iik, lam. 229 fis. 2; Kiner, Notara, Fisch. p. $f(x)$.

Hab. Luzón, Carite, Santa Cruz, Celoú; China.

2. Gén. MONACANIHUS, Cuv.

10. M. chinensis ('ui) N. v

(MI. s. T.)

Valent. fig. 4 (6).

ballistes cuinensis, Bl. ii. p. 29, lam. 52. fig. 1; Bl. Schn. p. $46 x$.

B. sinensis, Gim. L. i. p. 1470 .

B. punctatus, Marion de Procé, Bull. Philom. 1822, p. 130.

Monacanthus chinensis, Cuv. Régne. An.; Bleek. Ned. Tyds. Dierk. iii. p. 26, $\dot{o}$ Atl. Ichth. v. p. 125, lam. 22 f. 2; Githr. I. cit. 8, p. 236.

M. greográjhicus, Cuv. Régne An.; Cant. Mal. Fish. p. 348 ; Bleek. Verh. Bat. Gen. xxiv. Balist. p. 17.

Monacanthus cantoris, Bleek. 6. c.; o Nat. Tyds. Ned. Ind. iii. p. . 80.

Balistes granulosus, Gronov. Syst. et Gray, p. 34.

Hab. Samar, Luzon, Cavite, Santa Cruz; China.

I . M. ep. N. v.

(M S. T.)

Hab. Luzón, Nasugbú, Batangras.

12. M. tomentosus, Cuv.

Balistes, sp., Gronov. Mus. Ichth. i. p. 53, n. ${ }^{0144 .} \dot{o}$ Zoophyl. n." I9I, lam. 6, fig. 5; Seba, iii. lain. $2+$ fig. I 8 .

Balistes toinentusus, L. Syst. Nat. i. p. 405; Gronov. Syst. et Gray, p. 54; Wood. Nat. Hist. 3, p. 354, fig.

Baliste velu, Lacép. i. pp. 333, 359 .

Monacanthus tomentusus, Cuv. Régne. An.; Bleek, Verh. Bat. Gen. xxiv. Balist. p. 19, et Atl. Ichth. v. p. 127, lain. 220, fig. I law. 229 i; Gthr. loc. cit. 8, p. $23^{8 .}$

M. hajam, Bleek. l. c. I. ${ }^{\circ}$, p. I8, lain. I fig. I, et l. c. 2." p. 126, lain. 230 figs. 1 \& 3 .

M. trichurus, Bieek. Nat. Tyds. Ned Ind. iv. p. 125 ,

il. helleri, Stendachner, Sitzgsber. Ak. Wiss. Wien, $1867,1 \mathrm{v}$. p. 7 1 2, la:s. 3. fig. 3 .

1tab. C. de Mindoro, Luzón, y Samar, Cebú; China.

13. M sulcatus, Hollard, $\mathrm{N}$ v

(M. S. T.)

Monacanthus sulcatus, Hollard, Ann. Sc. Nat. 1854, ii. p. 363, lam. 14. fis. 3; Gihr. l. cit. 8. p. 239. 
M. isogramma, Bleek. Nat. Tyds. Ned. Ind. xiii. 1857, p. 367 ; Atl. Ichth. Balist. p. I 28, lam. 222. fig. I.

Hab. Luzon, Cavite, Santa Cruz; China.

14. M. setifer. Bennelt. N v.

M nacanthus setifer, Bennett, Proc. Comm. Zool. Soc. 1830 , p. I 2 ; Hollard, Ann. Soc. Nat. 1854 , ii. p. 342, lam. I2. fign. 4; Gthr. l. cit. 8. p. 239.

M. cirrifer, Schleg. Faun. Japon. Poiss. p. 290, lam. I 30. fig. i; Bleek. Act. Soc. Sc. Indo Neerl. iii. Japan, iv. p. 3 I.

M. varius, Ranzani, Nov. Comm. Ac. Sc. Inst. Bonon. 1842, v. p. 6, lam. 2 .

M. broccus, Storer, Proc. Bost. Soc. Nat. Hist. 1842, p. $8+$ (no Mitch.)

M. filamentosus, Valenc. in Webb. \& Berthel. Iles Canar. Poiss. p. 95, lam. I7. fig. I.

M. gallinula, Valenc. $l . c$.

M. signifer, Storer, Mem. Am. Ac. ii. 1846, p. 497, et viii. p. 426 (lam, 35 fig. I).

M. auriga, Lowe, Proc. Zool. Soc. 1850 , p. 253 .

M. setifer, Dekay, New. York. Faun. Fisch. p. 337, lam. 59. fig. 194 .

M. massachusettensis, Storer, Fish. Mass. p. 174, et Mem. An. Ac. viii. p. 425, (lam. 34. fig. 4); Dekay, l. c. p. 336, lam. 57. fig. I 87 .

M. komuki, Bleek. l. c. lam. 3. fig. 3, et Verh. Ak. Wet. i. Japan, p. 13, c. fig.

M. auratus, Castelnau, Potss. d'Afr. Austr. p. 77.

? M. oppositus, Poey, Mem. Cuv. ii. p. 331 .

Hab. Luzon, B. de Manila; China.

15. M. monoceros, Osbeck. N. v.

(M. S. 'T.)

Acara muca, Willughby, lam. E. 2. fig. 2; Valent. fig. 3ro; Renard, i. lam. 4. fig. 24, ii. lam. 4. fig. 16.

Balistes, sp. Genov. Zoophyl. n. ${ }^{\circ}$ 8, lam. 3. fig. 11.

B. monoceros, Osbeck, ft. p. I Iо; L. Syst. Nat. i. p. 404; Bl. lain. I47; Bl. Schn. p. 462 .

Capriscus, sp., Klein, Pisc. Miss. iii. p. 25. n. ${ }^{\circ} 8$, lam. 3. fig. I I .

Lija barbuda, Parra, p. 48, lam. 22. fig. 2.

Balistes kleinii, Gm. L. p. I 472 .

B. monoceros, var. unicolor, Bl. Schn. p. 463.

B. serraticornis, Fremunv: Naw. Bull. Sc. Soc. Phulom. n. ${ }^{\circ} 67$, p. 249, lam. 4. fig. I.

Aleuteres berardi, Less. Voy. Coq. Zool. p. io7, lam. 7; Richards. Voy. Sulph. Ichth. p. I32, lam. 6r. fig. I.

Alutera cinerea, Schleg. Faun. Japon. Poiss. p. 292, lam. I3I. fig. I.

? Balister unicornu, Basılewsky, Now. Mem. Soc. Nat. Mosc. x. 1855, p. 263 .

Alutarius macracanthus, Bleek. Verh. Bat. Gen. xxiv. Balist. p. 22, lam. 3. fig, 6.

A. amphacanthoides, Bleek. l. c. p. 23, lam. 2. fig. 5 . 
A. obliteratus, Cant. Mal. Fisk. p. 358 .

Balistes linguatula, Gronov. Syst. ed. Gray, p. 35.

Aluterus anginosus, Hollard, Ann. Sc. Nat. 1855 , iv. p. 1.

Alutera güntheriana, Poey, Proc. Ac. Nat. Se. Phil.ad. $186_{3}$,

Aleuteres m noceros, B'e?k. Ned. 7yds. Dierk. iii. 1865, p. 28; Atl. Ichth. v. p. 140, lain. 226. fig. 2.

Monocanthus monoceros, Gthr. l. cit. 8. 25 x.

Hab. Samar, Luzón, Cavite, Santa Cruz; China.

\section{Fam. OSTRACIÓNTIDOS.}

\section{Gén OSTRacrón, ARtedf.}

1. O. gibbosus, Lin. N. v. Tagb6.

(M. S. 'T.)

Ostracion alter, Aldrov. iv. p. $5^{61}$ (cop. de Jonston, lam. 25. firs. 6, y de Willughby; lam. J. 9. fig. 1).

Ostracion, sp., Artedi, Synon. p. 83. n." 2, et Genera, p. 55. n. ${ }^{\circ} 2$.

Ostracion gibbusus, L. Syst. Nat. i. p. 409; Gthr. l. cit. 8. p. 25 \%. Valent. pp. 395, 425, figs. 159, 262; Rluysch, p. 9; lam. 5. fig :. 5, 9; Renard, ii. lam. 6. fig. 24, lam. 9. fig. 46:

Crayracion, sp., Klcin, Pisc. Miss. iii. p. 20. n. ${ }^{\circ}{ }_{17}$.

Ostracion turritus, Forsk. Descr. Anr. p. 75. n. ${ }^{\circ}$ I13; Bl. Aus'. Fisch. i. p. I1 3, lam. 136; Bl. Schn. p. 5(0; Lacep. i p. 4in; Bleek. Verh. Bat. Gen. xxiv. Balist. p. 31 , et Act. Scc. Sc. Indo-Neerl. vii. Japan, vi. p. I3; Hollard, Ann. Sc. Nat. 1857, vii. p. 156 .

Lactophrys camelinus, Dekay, New Fork Faun. Fish. p. 341, lam. 58. fig. 190 .

Ostracion (Tetrosomus) turritus, Bleek. Atl. Ichth. Ostrac. p. 31, lam. 3. fig. 3 .

Hab. Luzón, Cavite, Santa Cruz; Archipiélago Indico.

2. O. concatenatus $B l . N$. v.

(M. S T)

Ostracion concatenatus, Bl. lam. 131 (adult.); Bl. Schn. p. 49'; Lacép. i, p. 454; Hollard, Ann. Sc. Nat. 1857, vii. p. I55; Gthr. l. cit. 8, p. 259.

O. stellifer, Bl. Schn. p. 499 , lam. 98 (joven).

O. bicuspis, Blumenbach, Abbild. lam. 58; Smith, lll. Zool. S. Afr. Pisc. lam. 18.

Hab. Luzón, Cavite, Santa Cruz; China.

3. O. cubicus, Lin. N. v.

Ostracion prior, Aldrov. ii. c. 19, p. 560; Jonston, p. 125, lam. 25. fig. 7 .

Willughby, Append. p. 20, lam. J. 10 et J. 12; Valent. p. 386, fig. 120; Seba, iii. lam. 24. fig. II.

Ostracion, sp.; Artedi, Synon. p. 85, n. ${ }^{\circ} 8$; et p. $84.1 .^{\circ} 6$; Genera. pp. 55, 56. nos. I \& 4; Gronov. Mus. i. p. $5+\mathrm{n}{ }^{\circ}$ I 9 ; Zoophyl. p 54. n. 173 . 
O. tetrigonus, L. Mus. Ad. Fred. p. 59; Bleek. Atl. Ichth. Ostrac. p. 39, latm. I. fir. 2, et lam. 3, fig. 2; Gïnth. in Fish. Zanz. p. I 29; Day, Fish. Malab. p. 254.

O. cuberculat is, L. Syst. Nat. i. p. 4\%).

O. cubicus, L. l. c. p. tro; Bloci, Ausl. Finsh. i. p. II 5 , lin. 137; L.tess. i. p. 45r, lan. 22., fig. I; R.̈̈p. Atlus, Finsch. p. 3; Bleek. Ver $\therefore$ B.xt. Goil. Xxiv. Batist. p. 35, lan. 7. fig. it; Lefebr. Voy Po'ss. p. 238 , la.n. 8; Holl.zr., Ann. Sc. Nit. N.xt. 1857 , vii. p. i62; Gith l. c.t. 8. p. 260.

Abu senduk, Forsk. Discr. Air p. 17. $n^{\prime \prime} .48$.

() itracion deux-tubercules, Lirép. i. p. 459.

O. bituberculatus, Bl. Schn. 50 r.

O. cyanurus, Rï̈sp. Atl. Fische, p. 4, lam. I. fig. 2; Hollard, Ann. Sc. Nat. 1857 , vii. p. 167.

O. argus, Rüpp. l c. fig. I.

?. inaculatus, Quoy et Givin. Voy. Uran., Zool. p. 2 I8.

O. inmacuiatus, Schleg. Fanti. Japon. Poiss, p. 206; Bleek. Nat. Ichth. Japan, p. 55 ; Brevoort, Not. Jap. F.sh. p. $28_{+}$.

O. tesserula, Bleek. Nat. Tyds. Ned. Ind. iii. p. Z(5). Hab. Cebú, Parıgua; A.rbuina.

\section{4. $O$ diaphanus, $B l$.}

Ostracion, sp., Gronov. Zoophyl. p. 45. n.. 176 .

(). diaphanus, Bl Schn. p. Zor; Bleek. Act. Soc. Sc. Indo-Neerl. iii. Japan; ir. p. 38; Hollard. Ann. Sc. Nit. 1857, vii. 1. 157; Gthr. l. c. 8 . p. 264.

O. brevicornis, Schleg. Faun. Japon. Poiss. p. 297, lam. 13\%. fig. 3 .

O. undecim-aculeatus, Sinith, Ill. Zool. S. Afr. lam. 17; Casleln. Mem. Poiss. Atr. Austr. p. 78.

O. pentacornis, Bennett, Whaling Voyage, p. 266.

L:tophrys diaphanus, Kaup, Arch. Ntrgesch. 185.5. p. 217.

Ostracion (acantinostracion) cornutus, Bleek. Atl. Iinth. Ostric. p. 33, lam. 2. fig. 2, et lam. 4. fig. 3. (no L., vide Peters, Monatsber. Ak. Wiss. Berl. 1868 , p. 461).

Hab. Zamboanga, Mindanao; Japon.

5 O. cornutus Lin N. v. Pez toro.

$\left(\begin{array}{lll}\mathrm{M} & \mathrm{S} & \mathrm{T}\end{array}\right)$

Willughby, Atpend. p. 20. n. ${ }^{\circ}$ 12, lam. J. 13. fig. I; Va'ent. pp. $35^{8}, 45 \mathrm{I}, 455,+6 \mathrm{I}$, figs. $36,333,344,367$; Ruysch, pp. 9, I0, 17, lim. j. firs. 8. \& 20 ; lan. 9. fig. 8; Rentrd, i. lam. 39, fig. 197, ii. lam. 8. fig. 38, lam. s3. fig. 60, lam. 27. fig. I 35; “eba, iii. lam. 24. flys. 8, 9, \& 13 .

Crayracion n." 26, Klin, Pisc. Mss. iii. p. 22.

Ostracion, sp., Gronov. Mus. i. p. 54. n." I 8 ; Zoophyl. p. 45. n. ${ }^{\circ}$ I 75 .

O. cornutus, L. Syst. Na!. i. p. fog; B!och, i. p 105, lam. 133; Bl. Schn. p. 5co; L.tcés. i. p. 470; Shaze, Zool. v. p. 223, lain. 170; Schles. Fatil. Japon. Pciss. p. 299, lan. 131. fig. 4; Bleek. Verh. Bat. Gen. xxv. Ba!ist p. 32 ; Holl.ird, Ann. S N.t. 1857 , vi. p. I58; P.tors, Monutsh. Ak. Wiss. Berl. 1868 ; 
p. $f(x) ; G t h r$. l. cit. 8. p. $265 ;$ Wood. Nat. Hist. s. p. 345. figs.

O. arcus, Bl. Schn. p. 502.

(). cornutum, Cant. Ma!. Fsh. p. 365.

() ra!entini, Blcek. Journ. In.t. Archipel. $18 \& 8$.

(). (ac inthosiracion) arcus, Bleek. All. Ichth. Ostr. p. 35, lam. 2. fig. 3, lam. 4. fig. 4 .

Hab. C. de Cebú, Davao, Mindanao, Joló; China.

\section{Fain. TETROLÓNTIDOS.}

\section{Gén Xenoplerus, Bibrun.}

I. X. naritus,; (Richarls) $\mathbf{N}$ v.

(M S. T.)

Tetraodon naritus, $R$ chards. Voy. Sammr. Fish. p. I8, lan. 8; Cant. Mal. Fish. p. 383. lan. Io; Ble:k. Verh. Bat. Gen. xxiv. Blootk. p. 21, o Nat. Ty'dschr. Ne.t. Ind. iii. p. 439.

'Tetrodon granlispina, Van. der Hoeven, Handb. Dierk. and ednt. ii. p. 275 .

Chonerhinus naritus, Bleek. Atl. Ichth. Gymnod. p. 77, lam. 7. fig 2.

Xenopterus naritus, Gthr. l. cit. 8. p. $27 \mathrm{I}$.

Hab. Samar, Borongan; Borneo.

2 X modestus, (Bleek) $\mathrm{N}$ v.

(M S T.)

'Tetraodon modestus, Bleek. Nat. Tyds. Ned. Ind. i. p. 16, et iii. p. 440, o Verh. Bat. Gen. xxiv. Blootk. pp. 19, 21.

Chonerhinus modestus, Bleek. Atl. Ichth. Gymnád. p. 78, lam. 9. fig. 8.

Xencpterus modestus, Gthr. l. cit. 8. p. 27 I.

Hab. Luzón, Cavite, Sia. Cruz; Burneo.

2. Gén. TETRODON, LiN.

3. T. lunaris Bl. Schn. N. v.

(M. S 'I')

Tetrodon lunaris, Bl. Schn. p. 505; Schleg. Fiann. Japon. Poiss. p. 277, lam. I22. fig. 1; Rï̈pp. $N$. W. Fisck. p. 59; Cant. Mal. Fish. p. 378 ; Bleek. Verh. Bat. Gen. xxiv. Blootk. p. I 2 , et Atl. Ichth. Gymnod. Russell, i. p. 20. n. ${ }^{\circ} 29 ;$ Gthr. l. cit. 8. p. 274 .

'T. tepa, Ham. Buch. pp. Io, 362.

'T. leiopleura, Gray, Ind. Zool.

Physogaster lunaris, Müll. Abiandl. Ak. Wiss. Berl. 1839. p. $25^{2}$.

(iastrophysus lunaris, Mïll. Wiegm. Arch. ix. p. $330^{\circ}$

P'romecocephalus lunaris, Bibr. Rev. Zool. 1855 , p.. 279.

'Tetrodon spadiceus, Richurts. Voy. Swlph. Ichth. p. I23, lam. 
58. figs. 4 \& 5; Bleek. Atl. Ichth. Gymnod. p. 64, lam. 3. fig. I.

Hab. Luzón, B. de Manila, C. de Mindoro; China.

4. T. var spadiceus (Rich.) N. v.

Hab. Luzón, Cavite, Sta. Cruz; China.

5. T. sceleratus, (Forst.) N. v.

'Tetrodon sceleratus, (Forst.) Gm. L. i. p. 1444; Bl. Schn. p. 506; Lacép. i. pp. 476, 508; Gthr. l. cit. 8. p. 275.

'I. argenteus, Lacép. Ann. Mus. d'Hist Nat. iv. I804, p. 2 I I, p. 58. fig. 2; Schleg. Faun. Japon. Poiss. p. 275, lam. I21. fig. 2; Bleek. Nat. Tyds Ned. Ind. iii. p. 737, et Atl. Ichth. Gymnod. p. 6+. lam. 5. fig. I.

'T. argyropleura, Bennett, Proc. Comm. Zool. Soc. ii. 1832, p. 184 .

'I. argentatus, Blyth, in Kelaart's Prodr. Faun. Zeyl. i. Apped. p. 49.

Promecocephalus argentatus, Bibron, Rev. Zool. 1855, p. 279. Tetraodon bicolor, Brevoort, Not. Japan, Fish. p. 283. Hab. Luzon, Manila; Formosa.

6. T. hypselogenion, Bleek. N. v.

'Tetrodon honckenii, Rüpp. Atl. Fisch. p. 65, lam. 17. fig. 2 (no Bl.)

'I'. hypselogeneion, Bleek. Nat. Tyds. Ned. Ind. iii. p. 300, o Verh. Bat. Gen. xxiv. Blootk. p. 24, or Atl. Ichth. Gymnod. p. 6I, lam. 9. fig. 5.

$H a b$. Luzón, Manila; Australia.

7. T. oblongus, $B l$. N. v.

(M. S. T)

Tetraodon oblongus, $B l$. Ausl. Fisch. ii. p. 6, lam. 146. fig. I; Lacép. i. pp. 476, 502; Bl. Schn. p. 504; Cant. Mal. Fish. p. 380 ; Bleek. Verh. Bat. Gen. xxiv. Blootk. p. 12, et Atl. Ichth. Gymnod. p. 62, lam. 4. fig. 4; Gthr. l. cit. 8. p. 278.

Physogaster oblongus, Muller, Abhandl. Ac. Wiss. Berl. 1839, p. $25^{2}$.

'I'etraodon alboplumbeus, Richards. Voy. Sulph. Ichthyol. p. I21, lam. 58. figs. 6 \& 7; et Ickth. Chin. p. r 99; Bleek. l. c. p. 62 , lain. I. fig. 1 .

'T. precilonotus, Schleg. Faun. Japon. Poiss. p. 279, lam. I 24. fig. 2.

'T. patoca, Bleek. Verh. Bat. Gen. xxiv. Blootk. p. I . (no H. B.) Gastrophysus alboplumbeus, Bleek. Nat. Tyds. Ned. Ind. vii. p. 104 .

'Tetraodon niveatus, Brevoort, Notes on Japan. Fish. p. 284.

'T. hartlaubii, Bianconi, Mem. Accad. Bologn. vi. p. 146, la'n. 2, fig. I. 
Gastrophysus microphthalmus, Blyth, Journ. As. Soc. Besig. xxix. $1852, \mathrm{p} .174$.

Hab. Luzún, Cavite, Sta. Cruz; China.

8. T oblongus, var. albo-plumbous Kich. N. T. (M S. T.)

Hab. Luzón, B. de Manila; China.

9. T. Ocellatus, Osb.ck. N. v.

(M s. '.)

Tetrodon (Dioton) ocellatus, Osbeck. It. p. 226, $\dot{0}$ Eisql. edit. i. p. 364, ii. p. 33I; L. Syst. Nat. i. p. 411. Bl. lam. 145; Lacép. i. Pp. 475, 497; Bl. Schn. p. 504; Richards. Voy'. Siulph Fish. p. I 20, lain. 57. figs. I \& 2 ; Gthr. loc. cit. \&. p. 279.

'Tetrodon fasciatus, M'Clell. Calc. Journ. Nat. Hist. iv. p. 412 , lain. 2 r. fig. 2.

'T. sp., Benn. in Beechey's Voy. Zool. p. 50.

'T. bimaculatus, Richards. l. c. p. I I9, lam. 57. figs. 7-9.

Hab. Luzón, Cavite, Sta. Cruz, China.

Io T. rubripos, Schley. N v.

$\left(\begin{array}{lll}\mathrm{M} & \mathrm{S} & \mathrm{T}\end{array}\right)$

'Tetraodon rubripes, Schleg. Faun. Japon. Poiss. p. 283. fig. I. Gthr. loc. cit. 8. p. 279.

'l. xanthopterus, Schleg. l. c. p. 284, lam. t25. fig. I.

Gastrophysus rubripes, Bleek. Act. Soc. Sc. Indo-Neerl. viii. Japan, vi. p. 68.

G. xanthopterus, Bleek. l. c. p. 69.

Hab. Luzon, Manila; China.

I I. T. patoca, Ham. $\mathrm{N}$ v.

(M S. T.)

Kappa, Russell, i. p. I8, fig. 25.

'Tetrodon patoca, Ham. Buch. pp. 7, 363, lam. 18, fig. 2; Bibron, Rev. Zool. 1855 , p. 280; Gthr. l. cit. 8, p. 288.

'Tetrodon dissutidens, Cant. Mal. Fish. p. 382.

'. kappa, Bleek. Verh. Bat. Gen. xxiv. Blootk. p. I6, xxv. Beng. p. i6o, o N.tt. Ty.ts. Ned. Int. iii. p. 3or.

Leiodon patoca, Blcek. All. Ichth. Gymnod. p. 76, lam. 6, fig. 2. Hab. Luzón, Manila, C. de Samar; China.

12. T. inmaculatus, $B !$ var. virgata, Rich. N. v.

(M. S. T.)

Tetrodon manilensis, Procé, Bull. Philom 1822, p. 130.

'T. virgatus, R chards. Voy. Ereb. \& Terr. Fish. p. 62, lam. 39 fios. 8 \& 9, et Voy. Herald, Zool. p. 163 , lam. 28 figs 6-8; Bleek. Verh. Bat. Gen. xxiv. Blootk. p. 24, o Nat. Tyds. Ned. Ind. iii. p. 229.

H locanthus pilosus, Gronov. Syst. et Gray, p. 28.

Dibolomycter lonsicaudus, Bibron, Giér. Rev. Zool. 1855, p. 279.

Crayracion manillensis, Bleek. Atl. Ichth. Gymnod. p. 69, lam. 4, fig. 2. Hab. Luzón, Cavite, Santa Cruz, Manila; Amboina. 
13. T. nigropuntatus, $B l$.

'letrodon nigropunctatus, Bl. Schn. p. 507; Gthr. l. cit. 8, p. 293. 'Tetraodon trichoderma, Bleek. Nat. Tyds. Ned. Ind. v. p. 532.

'T. trichodermatoides, Bleek. l. c. vi. p. 336.

- Irothron melanorhynchus, Bleeek. $l$. $c$. ix. $p$ in.

Crayracion nigropunctatus; Bleek. Atl. Ichth. Gymnod. p. 74, lam.

2 fig. 4.

Hab. Cebú; Sumatra.

14 T. mappa, Less.

Tetraodon mappa; Less. Voy. Coq. Poiss. p. 102, lam. 5; Gthr. l. cit. 8. p. 293 .

'T. reticulatus, Bleek. Verh. Bat. Gen. xxii. Madura, p. 16, et xxiv. Blootk. p. 18 .

'Tetraodon calamaroides Bleek. l. c. xxiv. p. 16, o Nat. Tyds. Ned. Ind i. p. 96 (adult.)

'T. meleagris, Bleek. N.tt. Tyds. Ned. Ind. v. p. 9i. (no synon).

Crayracion mappa, Bleek. Atl. Ichth. Gymnod. p. 72, lam. 6, fig. 3 (adult.)

C. meleagris, Bleek: l. c. fig. I.

Crayracion mappa, Bleek. Atl. Ichth. Gymnod. p. 72, lam. 6, fig. 3 (adult.)

(.) meleagris, Bleek. l. c. fig. 1.

Hab. Cebú; Archipiélago Indico.

15. T. reticularis, $B l$.

'Tetrodon testudineus, (no L.) Bl. Aust. Fisch. i. p. 123, lam. I 39; Lacép. i. p. 477 ; Bl. Schn. p. 502; Shaw, Zool. v. p. 444, lam. 178; Cant. Mal. Fish. p. 376; Bleek. Verh. Bat. Gen. xxiv Blootk. p. 14 o Nat. Tyds. Ned. Ind. iii. p. 78.

'T. reticularis, Bl. Schn. p. $506 ;$ Gthr. l. cat. 8. p. 296.

Arothron testudineus, Bleck. Atl. Ichth. Gymnod. p. 7 r, lam. 8. fig. 3; Day, Fish. Malabar, p. 257.

Hab. Cebú; Célebes.

16. T. hispidus, Lin var. semistriatus, Rïpp. N. v.

?Tetrodon hispidus, L. Syst. Nat. i. p. 411 (no synon.)

'T. hispidus, Bl. Ausl. Fisch. i. p. 130, lam. 142; Bl. Schn. p. 504 .

'T. hispidus, Lacép. i. p. 487 , lam. 24. fig. I; Richards. Voy. Samar. Fish. p. 17, lam. 9. figs. 3 \& 4; Gthr. l. cit. 8. p. 297.

'Tetraodon perspicillaris, Rüpp. Atl. Fische, p. 63.

'I. semistriatus, Rüpp. $N$. $W$. Fische. p. 58, lam. 16. fig. 3.

'T. implutus, Genyns, Voy. Beagle, Fish. p. 152.

T. stellatus, Eyd. \& Soul. Voy. Bonite, Poiss. p. 212, lam. 10. fig. 2. (no Lacep).

'1. laterna, Richards. Voy. Sulphur, Zool. p. 124, lam. 6r. fig. 2, et Ichth. Chin. p. 199; Bleek Verh. Bat. Gen. xxiv. Blootk. 
p. 23, ó Nat. Tyds. Ned. Ind. iii. p. 299; Grinth. Fisch. Zanz. p. 131.

Crayracion implutus, Bleek. Atl. Ichth. Gymnod. p. 71.

T. laterna, Bleek. l. c. lam. 1. fig. 3.

Hab. Luzón Sta. Cruz, Cavite, Samar; Amboina.

17. T. meleagris, Laich.

Tetrodon melegris, Lacepp. i. pp. 476, 505; Bl. Schs. p. 507; Richards Voy. Sulphutr, Fish. p. 122, lam. 57. figs. 1-3; Gthr. loc. cit. 8. p. 299.

?'Tetrodon lacrymatus, (Ciuv.j Quoy \& Gaim. Voy. Uran. Poiss. p. 204 .

Hab. Marianas.

18. T fluviatilis, Ham. N. v.

(M. S T )

Tetrodon fluviatilis, Ham. Buch. Fish. Gang. p. 6, lam. 3o. fiy. I; Gthr. l. cit. 8. p. 299.

T. nigroviridis, Proce Bull. Philom. 1822, p. 130.

T. simulans, Cantor, Mal. Fish. p. 374.

'T. potamophilus, Bleek. Verh. Bat. Gen. xxii. Madura, p. if, or xxiv. Blootk. p. 17.

Arothron dorsovittatus, Blyth, Journ. As. Soc. Beng. xxix. 1861, p. 173 .

Criayracion fluviatilis, Bleek. Atl. Ichth. Gymnod. p. 68, lam. 6. fig. 4; Day, Fish. Malab. p. 256.

Hab. Luzón, Manili, Laguna de Bay; Ceilan.

19. T. papua, B.eek. $\mathrm{N}$ v

(M. S. T.)

Valent. Amb. p. 353, fig. 21, et p. 412, fig. 498; Ruysch, Amb. p 9, jam. 5. fig. 10; Renard, i. lam. 39. fig. 200, ii. lam. 25. fig. 124 .

Tetraodon margaritatus, (no Rïpp.) Bleek. Ver. Bat. Gen. xxiv. Blootk. p. 25, o Nirt. Tyds. Ned. Ind. iii p. 302.

T. papua, Bleek. Journ. Ind. Arch. ii. p. 638, et Verh. Bat. Gen. xxiv. Blootk. p. 13; Gthr. l. cit 8. p. 301.

Tropidichtys margaritatus, Bleek. Nat. Tyds. Ned. Ind. vi. p. 5 ().

Canthogaster margaritatus, Bleek. Atl. Ichth. Gymnod. p. $8 \mathrm{I}$.

Psilonotus margaritatus, Bleek. l. c. lam. 9. fig. 4. tiab. Luzon, B. de Manila, Cebú; Amboina.

2o. T. striolatus, Quoy \& Grim N. v.

(M. S T.)

Valentyn, p. 422, fig. 249; Renard, i. lam. 25. fig. I 38.

Tetraodon striolatus, Quoy \& Gaim. Voy. Uran. Zool. p. 203; Gthr. l. cit. 3, p. 304.

T. compressus, Proce, Bull. Soc. Philom. 1822, p. 130.

Tropidichthys striolatus, Bleek. Nat. Ty'ds. Ned. Ind. vi. p. 503.

Psilonotus striolatus, Bleek. Atl. Ichth. Gymmod. lam. 9. fig. そ.

Canthogaster striolatus, Bleek. l. c. p. 82.

Hab. Luzón, Manila, Navotas; Amboina. 


\section{Gén DIODON, GTrhk.}

Histrix piscis, Clusius, Exot, vi. c. 23 ; Jonston, Hist. Nat. Pisc. lam. 45. fig. 4; Willughby, Hist. Pisc. lam. p. 5.

Reversus indicus, Aldrov. Pisc. iii. p. I1 3, lam. 15. fig. 12; Jonston, l. c. lan. 3. fig. I.

Valent. Amb. p. 458, fig. 357; Renard, i. p 9, lam. 5. fig. 32. Ostracion, sp., Arted, Gen. p. 60. n." i9; Synon. p. 86. n." 2 I; Gronov. Mus. ii. p. 40 n. ${ }^{1}{ }_{1} 81$, et Zoophil. p. 47. n. ${ }^{\circ}{ }_{1} 81$. Seba, iii. p. 58, lam. 23. figs. I \& 2, et p. 62, lam. 24. fig. 10. Crayracion, sp., Klein, Pisc. Miss. iii. p. 20. nos. $13 \& 14$.

Erizo, Parra; p. 60, lam. 29. fig. I.

Jiodun hystrix, L. Syst. Nat. i. p. 413; Rris. Rarnev. Rev. Zool. 1846 , p. 141: Gthr. l. cit. 8. p. 306; Wood. Nat. Hist. 3. p. 337. fig.

D. atinga, Bl. lam. 125; Schn. p. $511 ;$ Lacep. ii. pp. 1 \& 3 ; i. lam. 25. fig. 3; Kaup, Wiegm. Arch. 1855, p. 227 (no L.)

D. plumieri, Lacep. ii. pp. 2 \& ro, i. lam. 3. fig. 3 .

D. brachiatus, Bl. Sichn. p. 5 I 3 .

1). punctatus, Cuv l. c. p. 132; Bleek. Verh. Bat. Gen. xxiv. Blootk. p. 19.

Holocanthus hystrix, Gronov. Syst. ed. Gray, p. 27.

Paradiodon hystrix, Bleek. Atl. Ichth. Gymnod p. 56, lam. 3 fig. I. Hab. Luzón, B. de Manila; Océano Indico.

22. D. maculatus, (Gthr) N. v.

(M S. T)

Diodon tachete, Lacep. ii. p. I 3 .

D. novemualuiatus, Cizv. l. c. p. 136, c. fig.; Blrek. Nat. Tyds. Ned. Ind. iii. p. 567 .

D. sexmaculatus, Cuv. l. c. p. 1 36 , c. fig.; Kaüp, l. c. p. 229.

D. multimaculatus, $C u v$ l. c. p. I 36 , c fig; Kaup, l. c. p. 227.

D. quadrimaculatus, Cuv. l. c. p. 1 37 , c. fig.; Bleek. Act. Sor. Sc. Indo-Neerl. ii. Amboina, viii. p. 94.

D. spinusissinus, .Kuup, l. c. p. 228 (no (uv.).

Paradiodon novemmactilatus, Bleek. Atl. Ichth. Gymnod. p. 5i, lam. 2. fig. 3.

P. quadrimaculatus, Bleek. l. c. p. 58 , lam. 8. fig. 2.

Diodon maculatus, Gthr. l. cit. 8. p. 307. Hab. Luzón, Cavite, Sta. Cruz; China.

\section{Gén. CHILOMYCTERLS, GThr.}

\section{Ch. orbicularis. $B l$. N. v.}

Diodon orbicularis, Bl. lam. I 27; Lacep. ii. p. 16; Bennett, Whaling. Voy. ii. p. 264; Barneville, Rev. Zool. 1846, p 141; Blee.i. Nat. Tyds. Ned. Ind. v. p. 92, o Act. Soc. Sc. Indo-Neerl. ii. Amborna, viii. p. o Atl. Iihthyol. Gymnod. p. 55, lain. 1. fig. 4

D. caruleus, Quoy \& Gaim. Zool. Uran Poiss. p. 201, lam. 65. fig. 5 . 
D. triedricus, Cant. Mal. Fish. p. $37 \mathrm{I}$ (110 Cuv.)

Chil.mycterus orbicularis, Gthr. loc. cit. 8. p. 312.

Hab. Luzón, Paragua; Océano Indico.

\section{Orden: CONDROSTEOS.}

\section{Fam. ACIPENSÉRIDOS.}

\section{Gén. ACIPENSER, AkTteds}

A sinensis. Gray N. v.

(M. S. T.)

Acipenser sinensis, Gray, Ill. Ind. Zool. c. fig., \& Proc. Zco!. Soc. 1834 , p. 122. Gthr. l. cit. 8. p. 338.

??Acipenser mantschuricus, Basil. Nouv. Mem. Soc. Nat. Mosc. x. 1855, p. 250 .

Hab. Luzón, Cavite, Sta. Cruz; China.

\section{Orden: PLAGIÓSTOMOS.}

\section{Fam. I. CARCÁRIDOS.}

t. Gén. Carcharias, Cuv.

1. C. laticaudus, Müll N, v. Patin:

(M. S. T.)

Carcharias (Scoliodon) laticaudus, Mïll. \& Henle, p. 28, lam. 8; Dumeril, Elasmobr. p. 343.; Gthr. l. cit. 8. p. $35^{8}$.

C. macrorhynchus, Blee, Verh. Bat. Gen xxiv. Plag.ost. p. 3 1, lam. I. fig. I; Duméril, $\iota . c$.

Hab. Luzón, Cavite, Sta. Cruz; China.

2. C acutus Rüpp. N. v. Pating.

(M. S. T.)

Carcharias acutus, Rüpp. N. W. Fische, p. 65, lan. 18. fig. 4; Gthr. l. cit. 8. p. 358 .

Squalus russellii, Gray, Ill. Ind. Zool.

Ca charias (Scoliodon) acutus, Mïll. \& Henle, p. 29; Cant. Ma!. F.sh. p. 399; Bleek. Verh. Bat. Gen xxiv. Plag. p. 30; Ditmeril, Elasmobr. p. 345

Hab. Luzón, Manila; China.

3. C. hemiodon. Mâll. N v. Pating ó Tiburon. Ijo.

(it. S. T )

Carcharias (Hypoprion) hemiodon, Müll. \& Henle, p. 35, lam.' 19. fig. 2 Dumeril, Elasmobr. p. 35I; Gth. l. cit. 8. p. $3^{52}$.

Hypoprionodon hemiodon, Gill, Ann. Syc. Nat. Hist. New. York, vii. p. 4ng.

Hab. Luzón, B. de Manila; Célebes. 
Carcharias, (Pricnodon) borneensis, Bleek. Act. Soc. Sc. Indo Neerl. v. Borneo, xii. p. 8; Gthr. l. cit. 8. p. $37 \mathrm{I}$.

Hab. Samar, Borongan; Borneo.

2. Gén. Galeus, Cuv.

5 G canis. Kiontel. N. $\mathbf{v}$

(M. S T T.)

Galeus canis, Rondel. De Pisc. p. 377.

Canis galeus; Salvian. p. 130, fig. 4r; Willughby, Hist. Pisc. p. 5 I lam. $B$ 6. fig $\mathrm{I}$.

Squalus sp., Artedi, Gen. p. 68. n. ${ }^{\circ}$ 9; Synon. p. 97.

S. galeus, L. Sy'st. Nat. i. p. 319; Brïnn. Pisc. Massil. p. 9; Bl. p. I 8 ; Bl. Schn. p. 128; Risso, Ichth. Nice, p. 32; Turt. Brit. Faun. p. I 2 , Genyns, Man. p. 501; Nilss. Skand. Fann. Fisk. p. 714; Couch, Fish, Brit. Isl. i. p. 45, lam. 9.

Milandre, Duham. Pesch. ii pl. 22. figs. I \& 2; Lacép. i p. 237.

Tope, Penn Brit Pool. iii, p. 98, ó edit. 1812 , iii. p. It (no lam. I8).

Carcharias galeus, Risso, Eur. Mérzd. iii. p. I21.

Galeus vulgaris, Flem. Brit. An. p. 165; Yarrell, Brit. Fish. and. edit. ii. p. 509 o 3rd. edit. ii. p. 491; Parnell, Wer. ner. Mem. vii. p. 4I4; Kröyer. Danm. Fisk. iii. p. 834.

G. canis, Bonap. Faun. Ital. Pe'sce; Müll. \& Henle. p. 57; Gaimard, Voy, Isl. et Groenl. Poiss. lam. 21, Wright \& Ekstr. Skand. Fisk. p. 185 , lam. 45; Dumeril, Elasmobr. p. 390; Bocage \& Capello, Peix. Plagisst. p. i $8 ;$ Gtr. l. cit. \&, p. 379:

G. communis, Owen, Osteol. Cat.ul. i. p. 92.

Hab. Luzón, Cavite, Santa Cruz; Océano Indico.

3. Gén. ZYGAXNA, Cuv.

6. Z. mallous, Shciv. N. v.

(M. S. T.)

Libella, Bellon. de Aquat. p. 6r; Salvian, p. 128, lam. 40; Aldrov. p. 408; Jonston, lam. 7. fig. 8; Willughby, lam. B 1.

Zygæna, Rondel. p. 389; Gesner, De Aquat. p. 1050.

Squalus, sp., Artedi, Gen. p. 44. n. ${ }^{\circ} 7$; Synon. p. 96. n. ${ }^{\circ} 7$; Gronov. Mus. Ichth. i. pp. 63, I 39; Zooohyl. p. 146.

Zygxne, Dutertre, Hist. Nat. ii. p 207; Duhamel, Pesches, ii. sect. ix. pl. $2 \mathrm{r}$. figs. 3-8.

Squalus zygæna, L. Syst. Nat. p. 399; Bl. lam. I17; Bl. Schn. p. I 31 ; Lacép. i. p. 257; Briinn. Pisc. Mass. p. 4; Forsk. Descript. Anim. p. xviii; Mitch. Trans. List. \& Phil. Soc. New. York, i. p. 284; Gronov. Syst. ed. Gray, p. 6.

Cestr. cion, $s p$. n. ${ }^{\circ}$ I, Klein, Pisc. $M s s$. iii. p. I 3 .

Cornuda, Parra, lam. 32.

Koma sorra, Russell, lam. I 2.

Squalus malleus, Risso, Ichth. Nioe, p. 34.

Zygrna malleus, Shaw, Nat. Misc. lam. 267; Val. Mem. Mus. ix. I832, p. 223, lam. I , fig. I; Risso, Eur. Mérid. iii. p. I25; 
Cant. Quart. Med. Jour. Calcutta, 1837, fig. 1; Sçhlig. Faun. Japon. Poiss. p. 306, lam. 138; Jenyns, Man. p. 507; Yarrell, Brit. Fisch. and edlit. ii. p. 504, o 3rd eilit. ii. p. 486 ; Dekay, New. Iork Faun. Fish. p. 362; la.n. 68. fig. 804; Storer, Proc. Bost. Soc. Nat. Hist. i. p. 36; Bleek Verh. Bat. Gen. xxil. Plag. p. 48 , lam. 3. fig. 8; Couch, Fish. Brit. Isl. i. p. 7o, lam. 16 .

Z. lewini, Gritf. An. Kingiton, x. p. 640, lam. 50.

Sphyrna zygana, Mïll. \& Henle, p. 5 I; Bonap. Faun. Ital. Pisce; Cant. Fish. Brit. Isl. i. p. 7o. lam. 16.

Cestracion zygæna, Gill. Ann. Lyc. Nat. Hist. New. York, vii. p to3; Duméril, Elasmobr. p. 382; Day, Fish. Malab. p. 270.

C. leeuwenii, Dumeril, l. c. p. $3^{83}$.

Hab. Luzón, B. de Manila, Laguna de Bay; China.

7. Z tiburo, Valenc. N. v.

(M. S. T.)

'Tiburonis species minor, Pison. Hist. Nat. Bras. 181; Willughby, p. 55 , lam. B 9. n. ${ }^{\circ} 3$.

Cestracion, n. ${ }^{\circ}$, Klein, Miss. iii. p. 13, lam. 2. figs. $3 \& 4$.

Syualus tiburo, L. Syst. p. 399; Bl. Sck. p. 131; Gronov. Syst. ed. Gráy, p. 6.

Hear-headed Shark, Shaw, Gen. Zool. v. 2. p. 355, lam. I5t, et Nat. Misc. lain. 229.

Sphyrna tiburo, Müll \& Henle, p. 53 ; Gthr. l. cit. 8. p. 382. Zygand tiburo, Valenc. Mem. Mns. ix. p. 226, lam 12. fig. 2. ZZ. subarcuata, Storer, Proc. Bost. Soc. Nat. Hist. 1848, p. 70. Cestracion tiburo, Duméril, Elasmobr.

Hab. Luzón, Manila, Nayotas; China.

4. Gén. TRLENODON, GThr.

8. T. obesus, Küipp. N. V.

(M $\quad$ S $\quad \mathrm{l}$.)

Carcharias obesus, Rüpp. N. W. Fish. p. 64, lam. i8. fig. 2 . 'Trianodon obesus, Müll. \& Henle; p. 55, lam. 20; Dumerib, Elasmobr. p. $386 ;$ Gthr. l. cit. 8. p. 383 .

Hab. Luzón, B. de Manila; Océano Indico.

\section{Fam. RINODÓNTIDOS.}

I. Gén, RINODON, Smith.

I. Rh. typicus, Smith. N. v

(M S. T.)

Smith, Ill. Zool. S. Afr. Fish. lam. 26; Müll. \& Henle, p. 77, lam. 35. fig. 2 Duméril, Elas,nobr. p. 428; Gthr. l. cit. 8.' p. 396 .

Hab. Luzon, Manila; Seychelles. 
Fam. ESCÍLIDOS.

I Gén. SCylliun, M. ET H

I. Scy. marmoratum, Benn N. v.

(M. S. T)

Scyllium marmoratum, Benn. Life of Raffles, p. 693; Gthr. i. cat. 8. p. 400 .

S'cy. maculatum, Gray, Ind. Zool.; Richards Ichth. Chin. p. 193; Müller \& Henle, p. 5, lam. 7; Can!. Mal. Fish. p. 391; Bleek. Verh. Bat. Gen. xxiv. Pl.rgost. p. i6; Duméril, Elismobr. p. 319 (no Bl. Schn.)

Hab. Luzón, Manila; Sumatra.

2. scy maculitum. (Bl. Sch.) $\mathrm{N}$ v.

(M. $\mathrm{S}$ T.)

Squalus maculatus, Bl. Schn. p. I $3^{\circ}$ (no auct.)

?. S. cuvier, Peron \& Les. Journ. Ac. Nat. Sc. Phil. ii. p. 35 I. Scyllium maculatum, Gthr. l. cit. 8. p. 401 .

Hab. Luzon, Cavite; Australia.

3. Scy. b̈̈rgeri, Müller, $\mathrm{N} v$

(M. $\mathrm{S}$ T.)

Scyllium bürgeri, Müller. \& Henl, p. 8, lam. 2; Schleg. Fann.

Japon. Poiss. p. 301; Bleek. Act. Soc. Sc. Int.-Neerl. i. Amboyna, p. 69.

Halælurus burgeri, Gill, Ann. Syc. Nat. Hist. New. York. vii. p. 414.

Scylliu.n bürgeri, Gthr. l. cit. 8. p. 404.

$H a b$. Luzón, Currimao, Ilocos; Ainboina.

\section{Gén STEgostomA, $\mathrm{M}$ ET $\mathrm{H}$}

4 \&. tigrinum. (Gm.)

Seba, iii. p. 105, lam. 34. fig. I.

Squalus, sp., Gronov. Mus. Ichth. i. p. 62, et Zoophyl. p. 3r. n. ${ }^{\circ}$ i 47.

Squalus tigrinus, Gm. L. i. p. 249; Fiorst. Zjol. Int. p. 2t, lam. 13. fig. 2.

s. Inngicaudus, Gin. L. i. p. 1496.

S. fasciatus, Bl. la.n. I $3 ; B l$. Schn, p. 1 30 .

Zebra shark, Shaw, Nat. Misc. lam. 434.

Pollee maku.n, Russill, Fish. Coron. i. lam. I8.

Scyllium heptagonuin, Rïpp. $N$. W. Fisch. p. 6i, lam. i7. fig. I.

Stegostoma fasciatum, Mïller \& Henle, p. 25, lam. 17; Can'. Mal. Fish. p. 395; Blesk. Verh. Bat Gen. xxiv. Plag. p. 23; Dumérel. Elasmobr. p. 336; Gïnth. in Fish. Zanz. p. 140.

St. tigrinum, Gthr. loc. cit. 8. p. 409.

St. carinatum, Blyth. Journ. As. Soc. Beng. xvi. 1847, p. 725 , lam. 25 b. fig. 1 . 
sंualus cirrosus, Gromov. Sy'st. ed. Gray, p. 6. H.zb Parayud, P. Princesa; Ceilan.

3. Gén. Chitoscrlalici, M. Er H.

5. Ch indicum. (Gm.) N. r

siuntus, sp., Gronse Mus. Ichth. i. p. n." 133; Zoophyl. n." 150. Squalus indicus, Gm. L. i. p. $1503 ; B l$. Schn. p. 137.

Sqquale dentelé, Lxcép. i. p. 281, lam. I r. fig. 1.

Squalus gronorianus, Lacép. i. p. 280.

Sq. tuberculatus, Bl. Schn. p. 137 .

Russell, p. 10, lam. 16 (Bokee Sorrah et Ra Sorrah.

Scyllium plagiosum, Bennett, Life of Raffles, p. 694.

Sc. ornatuin, Gray, Ill. Ind. Zool. c. fis.

Chiloscyllium plagiosum, Mïller. \& Henle, p. 17; Cant. Mal.

Fisch. p. 352; Bleek. Verh. Bat. Gen. xxiv. Plagiost. p. 17;

Dumeril Elasmobr. p. 328; Day, Fish. Malabar, p. 267.

Ch. indicum, Gthr. l. cit. 8. p. 411 .

Ch. griseum, Mïll. \& Henle, p. r9, lam. 4 (no buena).

Ch tuberculatum, Mïll. \& Henle, p. I9; Bleek. l. c. p. 20; Dumérll, l. c. p. 331; Kner, Novara, Fisch. pp. 412, 413.

Squalus caudatus, Gronov. Syst. ed. Gray, p. 8.

Scyllium hasseltii, Bleek. l. c. p. I9.

Sc. phymatodes, Bleek. l. c. p. 21 ; Dumeril, l. c. p. 331.

Hemiscillum malayanum, Bleek. Nat. Tyds. Ned. Ind. vii. p. 376 (no Less.)

Chiloscyllium margaritiferum, Bleek. Ned. Tyds. Dierk. i. p. 243; Dumeril, l. c. p. 329 (joven).

Synchismus tuberculatus, Gill, Ann. Syc. Nat. Hist. New. York, 1861, I. 408.

Hab. Luzón, Sta. Cruz, Cavite; China.

\section{Fam. RÍNIDOS.}

1 Gén. RHINA, KLein.

I. Rh. squatina Dumeril. N. v.

(M. S. T.)

Pivn, Aristot. Hist. An. ii. c. I 5 ; v. c. 5 , Iо, I I; ix. c. 37.

Squatina, Bellon De Aquat. p. 78 ; Rondel. p. 367; Gesner, Dz Aquat. p. 899; Aldrov. p. 472; Sxlvian. p. 152, fig. 53; Willughby, p. 79, lam. D. 3; Jonston. p. 23, lam. I I. fig. 7.

Squalus, sp. Artedi, Synon. p. 95. n. ${ }^{\circ} 6 ;$ Gen. p 67. n. ${ }^{\circ} 6$; Gronov. Zoophyl. p. $15 \mathrm{I}$.

Rhina, Klein, Pisc. Miss. iii. p. 14. sp. 1, lan. 2: figs. $5 \& 6$. Angel-fish, Borlase Cornwall, p. 265. lam. 26. fig. 5; Penn:ınt, Brit. Zool. iii. p. 74, lam. 12, o edit. 1812 , iii. p. 130, lam. 15.

Squalus squatina, L. Syst. Nat. i. p. $39^{8}$; Brïnn. Ichth. Mass. p. 5; Bl. Ausl. Fisch. i. p 25, lam. I16; Bl. Schn. p. I37; Donov. Brit. Fish. i. pl. I7; Turton, Faun. p. 114.

Angelo, Comide. Pec. Galic. p. 129.

Squaine, Duhamel, Pesch. ix. lam. I4. figs. 1-4. 
Squale ange, Lacep. i. p. 293, lam. 1z. fig. 1 .

Angel-shark, Shav, Nat. Misc. xxi. lam. 906.

Squatina lavis, Cür. Regne Arrim.

Sq. aculeata, Cuv. Regne Anim.

Siq. vulyaris, Risso, Ichth. Nice, p. 45; Fleming, Brit. Ais. p. r6g; Müll. \& Hente Plagiost. p. 99, lan. 35. fig. 4; Kröy. Danm. Fisk. iii. p. 935; Schleg. Jaun. Japone. Pisc. p. 305, lam. I 36 .

Sq. angelus, Dumeril, Zool. Anal. p. roz; Blaino. Jaun. Jr. p. 53, litm. 13. figs. I \& 2; Risso, Eur. Merid. iii. p. 139; Jeny'ns, Manual, p. 507; Bonap. Jaun. Ital. Pesce; Yarrell, Brit. Fish. 2nd. edit. ii. p. 539, o 3rd. edit. ii. p. 536; Parnell, Werwer. Mem. vii. p. 42:1; Gromov. Syst. ed Gray, p. 14.

Squatina lewis, Couch, Limn. Trans. xiv p. qo; Jenyns, Manual, p. 508 .

S. dumerilii, Lesseur, Journ. Ac. Nat. Sc. Philad. i. p. 225, lım. 10; Dekay, New-York, Fanen. Fish. p. 363, lam. 62 fig. 2033 Leidy, Proc. Ac. Nat. Sc. Phibad. 1847, p. 247.

S. fimbriati, Mïll. \& Henle, pp. so1, 192, lam. 35.

S. oculata, Borsap. Faun. Ital. Pesc.

S. japonica, Bleek Act. Soc. Sc. Indo-Neerl. iii. Japan, iv. p. 4 o. Monk-fish, Couch, Fish. Brit. Isl. i. p. 37, lam. 17.

Rhina squatina, Duméril, Elasmobr. p. $464 ;$ Gthr. l. cit. 8 , p. 43 ().

R. aculeata, Dumeril, l. c. p. 465.

R. dumerilii, Duméril, l. c. p. 467.

R. californica, Ayres, Proc. Calif. Ac. Nat. Sc. Philad. 1859, p. 29,1860 , fig. 7 .

Hab. Luzón, Manila, Navotas; Japon.

\section{Suborden. BATOIDEOS.}

\section{Fam. PRÍSTIDOS.}

1. Gén. PRISTiS, Larh.

I. P. pectinatus, Latham. N. v.

(M. S. T.)

Pristis pectinatus, Latham, Trans. Linn. Soc. 1794, ii. p. 278, lam. 26, fig. 2; Bl. Schn. p. 351, lam. 70, fig. 1; Risso, Ichth. Nice, p. 22; et Eur. Merid. iii. p. 141; Blainv. Faun. Fr. p. 51; Müll. \& Henle, p. 109; Blyth, Journ. As. Soc. Beng. 1860 , xxix. p. 36; Owen, Odontogr. lam. 8, fig. 1; Dltméril, Elasmobr. p. 475 ; Gthr. l. cit. 8, p. 437 .

Squalus scie, Lacép. i. p. 286 , lam. 8.

Pristis megalodon, Duméril, Elasmobr. p. 476 , lam. 9, fig. 4.

P. acutirostris, Duméril, l. c. p. 479.

P. occa, Duméril; l. c. p. 479.

P. leptodon, Dumeril, l..c. p. 480 .

P. brevirostris, Duméril, l. c. p. 480 .

Hab. Luzón, B. de Manila, Laguna de Bay. 


\section{Fam. RINOBÁTIDOS.}

\section{i. Gien. RaY NChObatLS, Girk.}

I. Rh. ancylostomus, $B l$ Sckn. N. v.

LiEndormie (Seychelles).

Rhina ancylostomus, Bl. Schn. p. 352, lam. 7.2; Gray, IIl. Ind. Zool.; Agass. Ponss. Foss. ii. p. 82, lam. H. figs. 3 y 4 Richards, Ichth. Chit. p. 195; Mill. \& Hexle, p. Ino; Ozen, Odontogr. lam. 23; Cantor, Mal. Fish. p. 409; Bleek. Verh. Bat. Gen. xxiv. Plag. p. 56.

Rhanphobatis aucylostomus, Gill, Anu. Lyc. Nat. Hist. Nex:York, 1861 , vii. p. 408.

Rhynchobatus ancylostomus, Gthr. $l$. cit. 8, p. + th.

Hab. Luzón, Cavite, Santa Cruz; China.

2. Rh. djeddensis. Cant N. v.

(M. S T)

Rhinobatus, Duhawel, Pesches, ii. lam. 15, figs. I 2.

Raja djiddensis, Forsk. Descr. Anien, p. 18.

Raie bokhat, Lacep. i. p. 139 .

Raie rhinobate, Lacep. i. p. 145, lam. 6 fig. 3.

Rhinobatus laevis, Bl. Sckn, p. 354, lam. 71; Schleg. Fann. Japon. Poiss. p. 3o6, lam. I39.

R. djidsensis; Bl. Schae. p. 356.

Walawah tenkere, Russell, i. p. 6, lam. Io.

Rhinobatus djeddensis, Rüpp. Atl. Fisch. p. 5t, lam. 14 fig. I; Benn. in Life of Raffles, p. 693; Gthr. l. cit. 8, p. 441.

R. duhameli, Blaimv. Faun. Fr. p. 48.

Rhyachobatus lavis, Hül. \& Henle, p. In; Bleek. Verh. Bat. Gen. xxiv. Plag. p. 58 ; Duméril, Ela mobr. p. 483.

R. djeddensis, Caxt. Mal. Fish. p. 412; Day, Fish. Malab. p. 273.

Hab. Samar, Luzón, Sta. Cruz, Cavite; Sumatra.

2. Gén RHINOBATUS, GTHR.

3 Rh, halavi, Rüpp. N. v.

(M. S. T)

Raja halavi, Forsk. Deser. An. p. I 9 .

Rhinobatus halavi, Rüpp. Atl. Fisch. p. 55, Iam. 4, fig. 2; Mrïll. \& Henle, p. 20; Guichen. Explor. Alger. Poiss. p. 129; Duméril, Elasmobr. p. 496; Gthr. L. cit. 8, p. 442.

Hab. Luzón, La Unión; China.

4. Rh. schlegelii, ( $M$ et $H$ ) N. v.

(M S T.)

Rhinobatus schlegelii, Müll. \& Henle, p. 123, lam. 42; Richards. Ichth. China, p. 195; Schleg. Faun. Japon. Poiss. p. 207; Bleek. Act. Soc. Sc. Indo-Neerl. iii. Japan, iv. p. 41; Gthr. loc. c. 8. p. 445 . 


$$
-620-
$$

Rh. hynnicephalus, Richards. Iclith. Chin. p. 195.

Hab. Luzón, Manila, Navotas; China.

\title{
Fam. TORPEDÍNIDOS.
}

\author{
I. Gén. TEMERA, Gray.
}

1. T. hardwickii, Gray,

Gray, l. c., et Ill. Ind. Zool. c. fig.; Müll. \& Henle, p. 131, lam. 59. fig. 2; Cant. Mal. Fish. p. 420 , lam. 12; Bleek. Nat. Tyds. Ned. Ind. xx. p. 451; Gthr. loc. cit. 8. p. 455.

Hab. Filipinas; Indias Orientales.

\section{Fam. RÁYIDOS.}

$$
\text { 1. Gén. RAJA, Uv. }
$$

I. R. kenojei, $M$. et $H$. N. v. Pagrui.

(M. S. T.)

Müll. \& Henle, p. I49, lam. 47 (no buena); Rickards. Ichth. Chin.

p. 197; Schleg. Faun Japon. Poiss. p. 308; Bleek. Act. So:

Sc. Indo-Neerl. ii. Japan, iv. p. 42 (part.), et viii. Japan, vi.

p. 65; Dumeril, Elasmobr. p. 556; Gthr. l. cit. 8. p. 46r.

$H a b$. Luzón, Manila, Samar, Borongan, Paragua, P. Princesa.

2 Gén. Platyrhina, M. et $\mathrm{H}$.

2. Pl sinensis. Müll. N. v.

(M. S. T.)

Raja chinensis, Lacép. i. pp. 34, I 57, lam. 2. fig. 2. Rhina sinensis, Bl. Schn. p. 352 .

Platyrhina sinensis, Müll. \& Henle, p. 125, lam. 43; Dumerl; Elasmobr. p. 576 ; Gthr. l. cit. 8. p. 47 I.

Hab. Luzon, Cavite, Sta. Cruz; China.

\section{Fam. TRIGÓNIDOS.}

\section{TRYGON, Adauson.}

1. T. gerrardi, Gray. N. v.

(M. S. T )

Trygon gerrardi, Gray, Chondropt. p. I16; Gthr. l. cit. 8. p 474. T. macrurus, Bleek. Verh Bat. Gen. xxiv. Plag. p. $74 ; 0$ Nat. Tyds. Ned. Ind. iii. p. 607.

Hab. Luzón, Cavite, Sta. Cruz; Japon.

2. T. bennetii, $M$. et $H$. N. v.

Trygon bennetii, Müll. \& Henle, p. 16o, lam. 52; Dumeril, Elasmobr. p. 595 ; Gthr. l. cit. 8. p. 480 . 
T. carnca, Richards. Ichth. Chin. p. 197.

Hab. Luzón, Manila; China.

2. 'én. PTEROPLATEA, N. ET H.

3. Pt. hirundo, Laide. $\mathrm{N}$ v.

(M. S. T.)

Pteroplatea hirundo, Lozve, Proc. Zool. Soc. I8f.3, 4. 94; Githr. $l$ cit. 8. p. 487.

P. japonica, Schleg. Finun. Japon. Poiss. p. 3oy, lam. 141; Bleek. Act. Soc. Sc. Indo-Neerl. ii. Japan, iv. p. +5 .

? Pt. :narmorata, Cooper, Proc. Calif. Acad. Nat. Sc. iii. p. 112 , fig. 25.

$H a b$. Luzón, Manila; China.

\section{Fam. MILIOBÁTIDOS.}

I. Gén. Myliobatis, Cuv.

I. M. nieubofii. Cur' N v.

Zee-vleermuis, Nieuh f, Lant en Zee-Reize, ii. p. 278, fig.; Willughby, App. p. 6, lam. 1o. fig. 3; Russell, Fish. Coroit. lam. Io.

Raja nieuhofii, Bl. Schn. p. 364 .

R. fasciata, Shaw, Gen. Zool. v. 2. p 286 , lam. $1+3$.

Myliobatis nieuhofii, Cuv. Regne. An.; Müll. \& Henle. p. i77;

Cantor, Mal. Fish. p. 432; Bleek. Verh. Bat. Gen. xxiv. Plag.

p. 85 ; Duméril, Elasmobr. p. 638 ; Gihr. l. cit. 8 p.

Hab. Luzon, Cavite; Océano Indico.

2. M. milvus, Müll. N. v.

(M. S. T.)

Myliobatis milvus, Müll. \& Henle, p. I78; Bleek. Verh. Bat. Gen. xxiv. Plag. p. 87; Dumeril, Elasm br. p. 638.

M. vultur, Mïll. \& Henle, p. 179; Dumeril, l. c. p. 640.

M. oculeus, Richards. Ichth. Chin. p. 198.

Hab. Luzón, Manila; China.

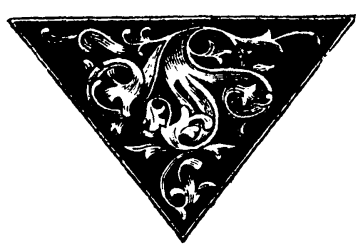





\section{APÉNDICE.}

Pag. 2. 9. M. philippinensis, Gïnther, P. Z. S. 1879, p. 74 .

Pag. 4. N. tardigradus, Everet. Mamm. of the Bornean Islands, 1893 p. 494.

Hab. Bongao, 'lawi-tawi.

Pag. 4. T. spectrum, Everett. P. Z. S. 1893 , p. 494.

Pag. 16. G. philippiaensis, Gthr. P. Z. S. 1879 , p. 74.

Hab..... Basilan.

Pag. i 7. 'T. javanica, Everett. P. Z. S. 1893, p. 495.

Pag. 21. 14. Sciurus nigripes, Thos; Everett. $P . Z .1893$, p. 495.

Hab. Paragua.

Pag. 15. Sciurus mindanensis, Steere List B. et M. Steere Exped. 1890, p. 29; Sc. caggsi Meyer.

Hab. Mindanao, Basilan.

Pag. 16. Sc. coccinus, Thomas. Ann. et Mag. Nat. H. (6) vol. I I. p. 407 ( I 888); Sc. philippinensis, Steere List $B$. et $M$. Steere Exped. p. 59.

Hab..... Mindanao, Basilan.

Pag. 17. Sc. samarensis, Steere, List B. et M. Steere Exped. p. 30 .

Pag. 24. A. pumilla, Everett, P. Z. S. 1893, p. 495. Hab..... Calamianes, Culion.

Pag. 25. M. marchei, Hent. esp. 2.; Everet. P. Z. S. 1893, p. 495 .

Hab..... Calamianes.

Pag. 27. A. binturong, Raffl: Everret. P. Z. S. 1879. p. 75. $H d b . .$. .. Mindoro, Panay, Negros, Mindanao, Basilan, Paragud.

Pag. 28. V. tangalunga, Gray: Everet. P. Z. S. 1863 , p. 495. Hab.... Siquijor, Panay, Mindoro, Calamianes, Paragua; Mindanao.

Pag. 31 linea 3: Blanford, P. Z. S. 1887, p. 631. Hab..... Cebú, Toledo.

Pag. 33. linea i. " B. mindorensis, Heude: Nehring Sitzzungsberitcht. der. Gesellschaft naturforschender Freunde zu Berlin. n. ${ }^{\circ}$ 6, I89o, p. IoI; Probubalus mundorensis, Steere, List B. et $M$. Steere Exped.

Pag. 37. S. celebensis, var. philippinensis, Nehring.

Hab. Luzón, Mindoro, Baco, Sibuyan, Masbate, Panay, Negros, Samar, Borongan, Mindanao, Basilan, Joló, 'Tawi-tawi.

Pag. 8. S. ahaenobarbus, Hent; Everett. P. Z. S. 1893 , p. 596. Hab. Paragua, Calamianes. 
Pag. 52. Fam. NESTÓRIDAS.

Gén. NESTOR.

N. productus, Gunld. $\sigma^{\prime} \mathrm{N} \mathrm{v}$

Wilson's parrakeet, Lath. Gen. Hist. ii. p. 170 (1822).

llyctolophus productus, Gould, P. Z. S. 1836 , p. I9.

Nestor productus, Goull, Syn. Austr. B. et adi. Isl. pt. i. lam.f. I (133-?); $B$ urrot, Perr. la.n. 59 ( 1 837-39); Guu!d, B. Aistr. v. lam. 6 (part. v., 18+1); G. R. Gr. Gen. B. ii. p. +26, n." 2 (1845); Rchns V̈g. NEuh)ll. n. ${ }^{\circ}{ }_{1} 87$ (1950); Bp. C川sp. i. p. 8 (1850); Rcins. Journ. f. Orn. 1853, p. 10; Bp. Rev. et Mag. de Zoo!. 1854 , p. 155, n." 252; Souancé, Rev. et M.r. de Zool. 1855, p. 224 ; Sclat. Pr. Linn. Soc. 1858, p. 132; G. R. Gr. List. Psitt. Brit. Mus. p. Iow (1859; Sclat. Journ. Orn. 1860 , p. 34, Pelz. Verh. $k$ Ak. Wissench. Wien, xli. Pp. 322-325, la ...-fi. 2, 34,5 (1860); Schleg Journ. f. Orn. 1861 p. 377; G. R. Gr. Ibis, 1852 , p. 230 Schleg. Nerent. p. 84 (1854); i.t. Mics. P.-B. Psitt. p. 157 (1854); Guubl, Hanth. B. Austr. ii. p. $55^{\circ}$ (1865); Finsc.t. Journ. f. Orn. 1867 , p. 322 ; Finsch. Papag. ii. p 888 (г868); Nwt. Ibis, 1859, p. 323; Selys-Longch. Ibis, 1870 , p. 450 (Mus. de Florencia); G. R. Gr. Hantl-l t. p. 150, n. ${ }^{\circ} 825^{8}$ (1870), R)wley, P. Z. S. 1874 , p. 497, Gieb. Tes. Orn. ii. p. 701 (1875); Ibis, 1877 , p. I 29 (spec. in Turati's Mius..); Rchnw. Vogelbild. t. xriii. f. 6 (1878-83); id. Journ. f. Orn. 1881, p. 2 I (Consp. Psitt. p. 21); Salvad. Ib: 1881 , p. 609; Salv. Cat. Strickl. Coll. p. 463 (1882); id. Cat. B. M. 20. 1894, p. 9; Lxyard, Ibis, 1884, p. I 23, Wag!, Bull. B. New. Zeal. and ed. i. p. 164 (1888); Ibis, 1888, p. 650; Rainsay, Tab. List. p. 38 (1888).

Centrurus productus, $B p$. Nalumannia, 1856 , Consp. Psitt. n. ${ }^{0} 265$. Hab. Filipinas?

\section{Pag 52. Cacatua hæmaturopygia.}

Cacatua minor, Briss, Orn. iv. p. 2 г 2, n. $^{0}$ I I, lam. xxii. f. ( 1760$)$. Gerini, Orn i. p. 96, t. I 19; Schleg, Dierent. p. 81 (1864);

Psittacus hamaturopygius, Cass. Proc. Ac. Nat. Sc. Philad. 1864, p. 239, n. ${ }^{\circ} 5 \mathrm{I}$.

Red-vented cockatoo, Brozon, Ill. p. Io. t. (1776); Lath. Syn. i. p. 258 (178I); id. Gen. Hist. ii. p. 204 (1822).

Psittacus philippinarum, Lath. Ind. Orn. i. p. I08, n. 79 (1790); Bechst. Kurz? Ueburs. p. 88 (1 8 I I); Shaw. Gen. Zool. viii. p. 48I (I8I1); Kuhl, Consp. Psitt. p. 85 (1820); Desm. Dict. Sc. Nat. xxxix. p. 1 I 4 (1826); Vo:gt, Cuv. Uebers. p. 337 (1831); Temm. Tabl. Méth. p. 56 (1839).

Cacatua philippinarum, Vieill. N. D. xvii. p. IO (I8I7); id. Enc. Méth. iii. p. I4I3, la.n. 227. f. I (1823), Wagl. Mon. Britt. p. 692 (1832 ; Hartl. Syst. Verz. Ges. Mus. p. 89 (1844); G. R. Gr. Gen. B. ii. p. +25, n. 2 (18+5); Bp. Rev. et Hag. te Zool. 1854 , p. I55, n. ${ }^{\circ} 25$; id. Nartinannia, $185^{5}$, 
Consp. Psitt. n. ${ }^{\circ} 27^{0}$; Cass. Un. St. Expl. Exp. p. 233 (1858); G. R. Gir. List. iPsitt. Brit. Mus. p. 92 (18.59); Sibla. P.Z. S. 1850 , p. 225; Sihleg. Jaur3. N.at. Art. Migstru. 1851, p. I 83 ; id. Jour. f. Orn. 1661 , p. 378 Sclat. P. Z. S. 1 S6, p. Zif, 1864, p. 188; I'all. P. Z. S. 1864, pp. 279293 ; Sch! g. Mus. P.-B. Psittici, p. Itt (18jt); id. Ned. Tijdschr. Derk. iii. p. 319 (1865); M.trt. To:trn. f. Orn. 1856, p. 21; Sclat P. Z. S. 1857 , p. 2צt; W'ald. \& Lay.ard, Ibis, 1873 , p. 95; Sch!eg. Mis. P. B3. Psitt.xci, R.ju=, p. 07 (187t); G.urrod, P. Z. S. 1876, p. 692 1877 , p. 793; Tiweed:t. Orn. Works, p. II6 (1881); Sclal. List. Vert. Ali. 8th. ed. p. 311 (1883); id. P. Z. S. $19 S 5$, p. 251 ; Tristr. Cat. Coll. B. P. $72(1889)$.

Plyctolophus philippinarum, Sleph. Gen. Zool. xiv. p. IOS (1826); Selby, Nat. Libr., Parrots, p. I 31 (1836).

Kakac oe philippinarum, Bourjot, Perr. lam. 81 (18.37-38).

Eolophus philippinarum, Sou.znce, Rev. et Mag. de Zoo!. 1856, p. $22+$; B3. Compt. R:nt. xliv. p. 537 (1857).

Pliclolophus philippinarum, Finsch, Papag. i. p. 310 (1861); Gulliver, P. Z. S. 1875, p. 490; Ciebel, Thes. Orn. iii. 1'. $230(1877$ ).

Cacatua hæmaturopygia, G. R. Gr. Hand-list, ii. p. 169, n." $838+($ 1870); Wald. \& Layard, Ibls, 1872, p. 96; Wald. Tr. Zool. Soc. ix. pp. 132, 250 (1875; Sharpe, Tr. Linn. Soc. (2) Zool. i. p. 31 2 (1876); Tweedd. P. Z. S. 1877, pp. 755, $756,817,1878$, pp. 107, 281, 340, 379, 937; Sharpe, P. $Z$. S. 1879 , p. 313; Tweedd. Orn. Works, pp. 116, 299, 410, 529, $43^{\circ}, 5+4,566,587575,602,628$ (1881); Wardl. Rams. Trecedd. Orn. Works, p. 655 n. ${ }^{\circ}$ I (188r); Salvin, Cat. Strickl. Co!l. p. 453, n. ${ }^{2216}$ (1882); Kutter, Journ. f. Orn. 1882, p. 176, 1883 , p. 293; Sharpe, Ibis, 1884 , p. 316; Guillem. I'. Z. S. 1885 , pp. 250, 251; Sharpe, Ibis, 1888 , p. 192; Blas. Ornis. ir. p. 305 (1889); Everett. Journ. St. Br. R. As. Soc. 1889 , 1. 176; Wh ted. Ib.s, 1890, p. 4I ; Steere, List, p. 5 (1890); Salv Cat. B. M. 2o. p. 130.

Camptolo, shus philippinarum, Suntev. Meth. nat. Av. disp. Tennt. p. 69 (1872).

Plissolophus philippinaram, Rchnze. Journ. f. Orn. $1 \mathcal{S} 1$, p. 25. (Consp. Psitt. p. 25); id. Vegelbild. lam. xxxii. f. 2 (1878-83). Hab.... Calamianes, Culion, Panay, 'Tablas, Siquijor, 'Iawitawi.

\section{Pág. 53. Tanygnat’us luzonensis.}

Psittacus lucionensis, Lxth. Ind. Orn. i. p. I 20, n. ${ }^{\circ}$ i6 r (1790). Psittacus mi 1or, sive Psittacula lusionensis, Gerini, Orn. ii. p. 21, t. I 38 (i 769 ).

Psittacus margin itus, Gm. S. N. i. p. 324, n. ${ }^{\circ} 7$ I (1788); Lath. Irs.t. Orn. i. p. 95, n." 42 (1790); Shxtw. Gen. Zool. viii. (2) p. 420 (191 I); Bechst. Lexth. U:b. p. 73 (1812); Vieill. N. D. xxv. p. 35) (1817); Kuhl, Consp. Psitt. p. 37 (1820); Vieill. Ent. Wéth. iii. p. 1399 , lan. 223 . f. 2 (1823); Voigt, Cuv. Uebers. p. 734 (1831); Cass. Pr. Acad. Nat. Sc. Philad. 1854 , p. 240. Varied-winged parrot, Lith. S,n. i. p. 233 (179r); id. Gin. Hist. ii. p. 155 (1822). 
Lace-ringed parrot, Lath. Syn. i. p. $24+(1781)$; id. Gen. Hist. ii. p. $157(1822)$.

Manilla green parrot, Lath. Syn. i. p. 296 (г781); id. Gen. Hist. ii. p. $239(1822)$.

P'sittacus olivaceus, Gm. S. N. i. p. 326, n." 76 (iz88) (ex Buffon); Lath. Ind. Orn. i. p. 97, n. ${ }^{\circ}$ 43. (1750); B.chist. Kurze Ueb. 1. 73 (1812); Vieill. Enc. Meth. iii. p. ${ }^{8} 3(1823)$.

Arara marginata, Less. Tr. d'Orn. p. I 87 ( $183 \mathrm{I}$ ).

'Tanygnathus marginatus, II agl. Mon. Psitt. p. 678 (1832); Bp. Consp. Av. i. p. 5 (1850); id. Rev. et Mrg. de Zool. 1854 , l. 153, n." I 56, Sounnee, Rev. et Mizg. de ZJol. 18.55 , p. 209; Kp. Nanmannia, 1856 , Consp. Psitt. n. ${ }^{\circ} 154$; Sclat. P. Z. S. 18.57 , p. 225 ; G. R. Gr. P. Z. S. 1858 , p. 195; id. Cat. 13. Nezw. Guin. 1. Go (1859); Rosenb. Journ. f. Orn. 186 4, p. I I 5; Sousa, Mus. Nac. Lish. Psitlace, p. I6 (1869); Saliad. Orn. Pap. é Mol. i p i 35 (1880).

T. gala, G. R. Gr. Gen. B. ii. p. 420 , n. ${ }^{2}$ (18+6.)

'T. macrorhynchos, part., Blyth, Cat. B. Mus. A. S. B. p. 3 $(1849)$.

'T. pileatus, Cass. Un. St. Expl. Exp., Birds, p. 230 (1858).

'I. lucionensis, G. R. Gr. List. Psitt. Brit. Mus. p. 67 (1859); Sclat. P. Z. S. 1860 , p. 225 G. R. Gr. P. Z. S. 1851 , Finsch, Ned. Tijdschr. Dierk. i. Berigt. p xviii. (1863i; Wall. P. Z. S. 1864, pp. 285,294 ; G. B. Gr. Hand-list, ii. p. 158 n." 8247 (1870); Sitnd. Meth. nat. Av. disp. Tent. p. 69 (1872); Treeedd. P. Z. S. 1877, PP. 436, 588; Sharpe, P. Z. S. 1879 , 1. 133; Treeedd. Voy. Chall., Birds, p. 9 (1881); Kutter, Journ. f. Orn. 1882, p. I76 I883, p. 294; Blass. Ornis, iv. pp. 305, 559 (1888); Tristr. Cat. Coll. B. p. 76 (1889); Salo. Cat. B. M. $20,1897, \mathrm{p} .424$.

Eclectus luci nensis, Scleg. Mus. P.-B. Psittaci, 1). 48 (1864); id. op. cit. Revue. p. 26 (1874).

E. mülleri, part., Scleg. Mus. P.-B. Psittaci, p. 48, n. ${ }^{\circ} 7$ (1864).

Loriculus (Tanygnathus) luzoniensis, v. Mart Journ. f. Oor. I $860, \mathrm{p} .23$.

Eclectus luconensis, Finsch, Papag. ii. p. 362 (I868); Gieb. Thes. Orn. ii. p. 75 (1875).

'1. luconensis, Sclat. P. Z. S. 1871 , p. 479 ; id. Ibis, 1872 , p. 324; Wa'd. Trans. Zool. Soc. ix. 1). I33, 249 (1875); IVardl. Rams. Trieedd. Orn. Works, p. 655 (1881); Rchnw. Journ. f. Orn. 1881, p. 244 (Consp. I'sitt. p. 132); Meyer, Sitzb. r. Abh. Ges. Isis, 1834 , Abh. i. p. 6; Sharpe, Ib.s, 1884 , p. 3 I6; Stceire, List. p. 6 (189ri).

'T. luzonensis, Brilg;. Abh. Brem. v. p. 38 (1876); Koch, Verz. Vogelb. aus Celetes und Sanghir, Febr. 1876, p. I; Sala'ad. Orn. Mus. Civ. Gen. ix. p. 33 (1876); Treedi. P. Z. S. 1877 , pp. 755,756817 i 878, p. 28 I p. 340 p. $6+2$ p. 9371879 , p. 68; Trwedd. Ora. Works, pl). 301, 410, 460, 462, 529, $530,544,537,595,607,628,646$ (1881); Rchuro. Vogelbild. Nachtr. 62(1883); Sclat. L st Vert. An. 8 th. ed. p. 325 (1883); Sharpe, Ibis, 1838, p. 194; Whiteh. Ib.s, $1890, \mathrm{p} .4 \mathrm{I}$.

'T. luzoniensis, Sharpe, Tr. Inm. Soc. (2) Zool. i. p. 3т2 (18;6); M:yer, Ibs, 1879, 1. 48; Guillein. P. Z. S. (1885); 1. 252; 
p. 418 Everitt, Ib:s, 1888 , p. 282 ; id. Journ. Str. Br. R. As. Soc. 1889 , P, I 76 .

T. su natranus, Tristr. (no Raffl.) Cat. Coll. B. p. 76 (1889).

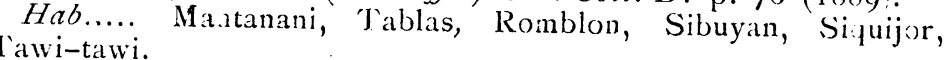

Pag 54. T. everetti.

'Tanygnathus sumatranus, G. R. Gr. (no Raff.) List Psit.'. Brit. Mus. p. 68; 1859 ; id. Hand-list, ii. p. I58, n. ${ }^{\circ} 8248$ (1870). Loriculus (Tanygnathus) sumatranus, v. Mart. Journ. f. Orn. 1885 , p. 22 (part.).

'Tanygnathus mülleri, part., Wald. Tr. Zool. Soc. viii. p. 3r (1872).

'T. everetti, Treedit. Anil. \& Mag. N. H. (4) xx. p. 53.3 (1877); id. P. Z. S. 1877 , p. 818; Sharpe, P. Z. S. 1879, p. 3r4; Treedd. Orn. Works, pp. 544, 55 ( $\left.188_{1}\right) ;$ Wardl. Rams. Trieedd. Orn. Works, pp. 655, 670 (1881); Rchunc. Journ. f. Orit. 1881, p. 246 (Consp. Psilt. p. 134); id. Vogelbild text of pl. xxvii. f. o $(1878-83)$; Steere, List, p. 6 (1890); Salv. Cat. B. M. 20, p. 432, 1894 .

Hab..... Panay, Concepción.

\section{Pag 54. T. burbidgei.}

'Tanygnathus burbidgei, Rchnew. u. Schal. Journ. f. Orn. 1879, P. 427; Wardl. Rams. Trueedd. Orn. Works, p. 655 (1881); id. Ibis, 1884 , p. $470 ;$ Salo. Cat. B. M. 20, 1894, p. 432 . 'T. albirostris, part., Rchnw. f. Orn. 1881 , 1. 246; id. Consp. Psitt. p. 134 (1881).

Hab..... Tawi-tawi.

\section{Pag. 57 C. furmutats, (Scop.)}

Peruche á collier, Buff. Hist. Nat. Ois. vi. p. iz3 ( (779).

Collared parrakeet, Lath. Syn. i. p. 317 (178I) (ex Sonnerat); id. Gen. Hist. ii. p. 270 (1822).

Ps. torquatus, Lath Ind. Orn. 1. p. I 33, p. I 54 (1790); Bechst. Kurze Uebers. p. 54 (1811); Shauv, Gen. Zool. viii. 2, p. 55 (1811); Vielll. N. D. xxv. p. 374 (1817); Kuhl, Consp. Psitt. p. 66 (1820); Vienll. Enc. Meth. iii. p. I405 (1823; Voigt. Cui. Uebers, p. 747 (1431); Pucher. Rev. et Mag..de' Zool. 1853, 1. I63.

P. streptephorus, Desm. Dict. Sc. Nat. xxxix. p. 88 (1826); Bp. Compt. Rend. xliv. p. 538 (I 857 ).

Psittacula torquata, Steph. Gen. Zool. xiv. 2, p. I44 (1826); Wagl. Mon. Psitt. p. 630 (1832); Lear, Parr. pl. 40 (1832).

Psittacula loxia, Less. Tr. d'Orn. p. 204 (173I); Bourj. Perr. lam. 94 (1837-38); Less. Comp!. de Buff. ix. p. 209 (1837); id. op. cit. and ed. p. $607(1838)$; G. R. Gr. Gen. B. ii. p. 423 , n." 22 (1846); id. List. Psitt. Brit. Mus. p. 90 (1 859).

P. squammato-torquata, Bourj. Perr. lam. 97 (1837-38) (ex Leart).

P. lunulata, G. R. Gr. Gen. B. ii. p. 423, n." $22(1846)$; id. List Psitt. Brit. M1us. p. 9ा (1869); Schlog. Mus. P.-B. Psittaci, 
p. 72 (1864); Finsch, Papag. ii. P. 616 (1868); Schleg. op. cit. Rei. p. 31 (1873); Gieb. Thes. Orn. iii. p. 328 (1877).

Cyclopsitta loxia, Bp. Rev. et Mag. de Zool. 1854, p. 1.54, n." Hartl. Journ. f. Orn. i 854, B. p. Lxiii; Souancé, Res' et Mag. de Zool. 1856, p. 217 ; id. Naumannia, 18.56 , Consp. Psit.. n." 229; G. R. Gr. Hand-list; ii. p. 164, n. ${ }^{\circ} 8376$ (1870).

Psittinus torquatus, Kp: Nantrannia, 1856, Consp. Psitt p. 159.

Loriculus (Cyclopsitta) loxia, v. Mart. Journ. f. Crn. 1866, p. 2 I, n. ${ }^{\circ}$ I 17 .

Loriculus (Cyclopsilta) lunulatus, v. Mart. ibid. n. ${ }^{0}$ I 8.

Psittacula luxias, Gieb. Thes. Orn. iii. p. 328 (18.77).

Cyclopsitta lunulata, G. R. Gr. Hand list, ii. p. 163, n. ${ }^{0} 8377$ (1870); Wald. Tr. Zool. Soc. ix. pp. 333, 249 (1075); Sharpe, Trans. Linn. Soc. (2) Zool. i. p. 3 г (1876); Trieedd. P. Z. S. 1887 , pp. 689, 818, г878, p. 9.37; id. Orn. Works, pp. $3^{\circ 1}$ 4 I0 514 (I88I); IVarld. Ram say, Taucedd. Orn. Works, App. p. 655 (part.) (1881); Meger, Vogel-Skeletten, i. pl. xxi. (1879-88); Tristr. Cat. Coll. B. p. 78 (1889); Steere, List. p. 6 (1890).

Cyclopsittacus lunulatus, Rchnw. Jonm. $f$. Orn. 1881 , p. i 36 (Consp. Psitt. p. I 36.)

Cyclopsittacus loxias, Rehnw. Journ.f. Orn. 1881 , p. I 37 (Consp. Psitt. p. 73); id. Vogelbild, Nachtr. I8 (1883).

Bolbopsittacus lunulatus, Salv. Cat. B. M. 20, 1894, p. 504. hab..... Manila, Monta.ban, S. Mateo, Bataan, Orion.

\section{Pag 55. Cyclopsitta intermedia, (Sala)}

Bolbopsittacus intermędius, Salv, Cat. B. M. 20, 1894, p. 505 lan. xiii.

Hab. Luzón, Mindanao, Samar, Borongan.

\section{Pag. 55 Cy. mindanensis.}

Cyclopsitta lunulata, (part.), Treeedd. Tr. Zool. Soc. ix. pp. i34, 249 (1875); id. Orn. Works, pp. 301, 410 (1881).

Cyclopsitta lunulata, Tzueedd. (nec Scop.) P. Z. S. 1878, p. 818 1878, p. 937 id. Orn. Works, pp. 545628 (1881); Wardl. Rams. Tweedd. Orn. Works, App. p. 655 (1881).

Cyclopsitta mindanensis, Steere, List B. \& M. Steere. Exped. to the Philipp. p. 6 (1890).

Bolbopsittacus mindanensis, Salv. Cat. B. M. 20, 1894, p. 505, lam. XIII.

Hab..... Surigao, Panaon, Butuan.

\section{Pag. 55. Prioniturus discurus.}

Psittacus discurus, Viell. Gal. des Ois. i. p. 7, lam. 26; id. Enc. Meth. iii. p. I 369 (1823).

Psittacus discosurus, Wagl. Mon. Psitt. p. 524 (I 832).

Psittacus spatuliger, fonz., Bourj. Perx. lam. 53 bis (1837-38).

Prioniturus discosurus, Bp. Consp. Av. i. p. 6 (1850).

Urodiscus spatuliger, Bp. Rev. et Mag. de Zool. 1854 , p. I55, n.. 226; G. R. Gr. Hand-list, ii. p. I 4 I, n. ${ }^{\circ} 8048$ (1870).

Urodiscus spatulifer, $B p$. Naumania, 1856 , Consp. Psitt. n. ${ }^{\circ} 233$. 
Prioniturus discurus, G. R. Gr. List Psitt. Brit. Mus. p. 18 (18.59; Philppine Isl.) Sclat. P. Z.S 1860, p. 224; Gould, 13. of $A s$ a, vi. lam. 15 (pt. xiv., 1862 ); v. Mart. Journ $f$. Urn. $1 \delta 66$, p. 22; Il ald. Tr. Zocl. Soc. ix. pp. 132, 249, (part) (1875); Sharpe, Tr. Linn. Soc. (2) Zool. i. p. 312 (1876); Triecdd. P. Z. S. $13^{2} 77$, pp. 538, 756, 817, 18,8, pp. 379, 937, 1879, p. 68; Trieedd. I'oy. Chall., B.ids, p. 9 (1881); idt. Orn. Ilorks, pp. 3co (part.), 410, 401; 462, 5.50, 54t, $602,6286+6$ (1881); Wardl-Rams.; Tiee dd. Orm. Worls, p. 655 ( 881$)$; Schw Vogelb lit. t. xxvii. f. 3 (18;-8.83); id. Juidrn. ff. Orn. $188^{\prime} 1$, p. I5t (Consp. Psitt. 1). 1 +2$)$; Tristr. Cat. Coll. B. p. 75 (1889); Steere, List, p. 6 (1890; Salv. Cat. B. MI. $20,1894, \mathrm{P} 5 \mathrm{I} 7$.

Prioniturus spatuliger, G. R. Gr. List Psitt. Brit. Mfus. p. 18 (1859); Gould, B. of Asia, vi. lam. I4 (pt. xiv., 1862); Wald. P. Z. S. 1864 . 1 294; Mart. Journ. f. Orn. 1856, p. 22.

Prioniturus, sp., Wal.'. P. Z. S. 1864 , p. $28+$.

Pionias discurus, Finsch, Papag. ii. P. 401 (1868); Gieb. Thes. Orn. iii. p. Igo (1875).

Urodiscus discurus, G. R. Gr. Hand-list, ii. p. I4I, n. ${ }^{n} 80+7$ $($ I $8-0)$.

Hlab..... Tablas, Sibuyan.

\section{Pag. 55. P. cyaniceps}

Prioniturus platena*, Blass. Braunscha'eigische Anzeiger, n. ${ }^{\circ} 37$, Febr. 12 (1888), p. 335; id. Ornis, iv. p. 305 (1888); id. Ibis, $1888, \mathrm{pp} .372,374$.

Prioniturns cyaniceps, Sharpe, Ibis, April. 1888 , p. I9t (Palawan); Whiteh. Ibis, 1800 ; p. 42; Everett, Journ. Str. Br. R. As. Soc. 1880 ; p. 176; Steere, List. p. 6 (1890); Salv. Cat. B. M. 20, 1894, p. 419.

Hab... Calamianes, Culion, Paragua, P. Princesa.

l'ag. 55. Fr. suluensis.

Prioniturus discurus, Sharpe (nec Vieill.), P. Z. S. 1879, p. 313 Gullen. P. Z.S. 1885 , p. 251.

Prioniturus discurus, var. Sulïensis, Blas. Journ. f. Orn. 1800 , p. 140.

P. suluensis, Salv. Cat. B. M. 20, 1894, p. 419. Hab. Joló.

Pagg. 56 P. verticalis, Sharpe.

Ibis, 1894 , p. 248 , lam. vi. f. I. 2. Hab. 'Tawi-tawi.

Pag 56. P. luzonensis.

Prioniturus discurus, (part.), 7weedd. Tr. Zool. Soc. ix. p. 3 I 2 (1875) id. (no Vieill.) P. Z. S. 1877 , p. 688. id. Orn. Works, p. 300 (part.) et p. 5 I4 (no Vieill.) (1887).

Prioniturus luconensis, Steere, List. B. \& M. Steere, Steere Exped. to the Philipp. p. 6 (1890).

Hab..... Manila, Montalban. 
Pag. 56. P mindorensis, St.

Prioniturns mindorensis, Steere, List: B. \& M. Steere, List to the Pinilipp. p. 6 (1890).

Hab..... Calapan.

\section{Palg 56. Loriculus philippinensis.}

Psittacula philippensis, Bris. Orn. iv. p. 392, lam. xxx. f. i (1;601); Stegh. G.⿲丿 Zool. xiv. 2. p. Itt (1825); Mycn, Nov. det. C. L.-C. Nat. Cuv. xvi Suppl. prim. p. 94 (1834); Bourj. Perr. latn. 8) (I837-38); Licht. Nomeincl. Aí. p. 7 I (I854).

Psittacus philippensis, Voigt, Cuo. Uebers, p 747 (1831); Kuhl, Consp. Psitt. p. 64 (1820).

? Petite perruche de l'isle de Lazon quatriéne espéce, Sonner. Vov. á la Nazu. Guin. p. 77, lam. 40 1776).

?La Perruche á ailes noires, Buff. Hist. Nat. Ois. vi. p. 174 (i 779 ) (ex Sonnerat).

Philippine parakeet, Lath. Syn. i. p. 3 І г (г 78 г); id. Gen. Hist. ii. p. 265 (0) (1822).

Luzonian parrakeet, Lath. Syn. i. p. 3 r8 (r73r) (ex Sonnerat); id. Gen. Hist. ii. p. 27 I (1822).

P. galgulus, var. $\beta$ Gm. S. N. i. p. 349 , n. $4^{5}$ (г 788$)$ (ex Brisson); Lath. Ind. Onr. i. p. I3 1, n. I48 (1788); Shat, Gin. Zool. viii. 2, p. $55^{\circ}$ (I 8 I I ).

?P. minor, Gm. S. N. i. p. 35I, n. I 35 ( 7833 ) (ex Sonnerat); Lath. Int. Orn. i. p. I33, n. I55 (i790); Vieill. Eins. Meth. iii. p. I 404 ( 1823$)$.

P. coulacissi, Vieill Enc. Meth iii. p. 1405 (1823).

Psittacula coulaci, part., Less. Tr. d' Orn. p. 202 (1831).

P. rubrifrons, Lear, Parots, lam. 4 I (I832); Bourj. Perr. lam. 87 ( $1837-38)$ ex Lear).

P. ru irifrons, Vig. P. Z. S. 1831, p. 97.

Psittacula rubrifrons, Siv. Class. B3. ii. p. 303 (1836).

Psittacula melanoptera, G. R. Gr. Gen. B. ii. p. 423, n. ${ }^{\circ}$ ig $(18+6)$.

Loriculus rubrifrons, $B p$. Rev. et $M a g$. de Zool. 1854, p. I55, n. ${ }^{\circ} 242$; Hartl. Journ. $f$. Orn. 1854, p. 1xiii.

L. philippensis, Surtucé. Rev. et Mag. de Zool. 1856 , p. 221 ; Schleg. Mus. P.-B. Psitiaci, p. Izo (1864), v. Mart. Journ. f. Orn, 1566 , p. 21; op. cit. Revute, p. 59 (1874); Wald. Tr. Zool. Soc. ix. Pp. I35, 249 (1875), Tzeeedd. I'. Z. S. 1877, p. 820; id. Orn. Works, pp. 299, 303, 304, 410, 547 (I881); Wardl. Rams. Tzueedd. Orn. Works, p. 655 (I 881); Zristr. Cat. Coll. B. p. 76 (1899); Steere, List. p. 6 (189.); Salv. Cat. B. M. 20. 1894, p. 524

Licmetulus philippensis, B. Nammania, 1856, Consp. Psitt. n. ${ }^{\circ} 254$.

Loriculus coulacissi, G. R. Gr. List. Psitt. Brit. Mus. p. 56 (1859); Wall. P. Z. S. 1854 , p. 288; Gieb. Thss. Orn. ii. p. 493 (1875), Meyer, Row!. Orn. Misc. ii. p. 232, 11. 6 (I877); id. Vogel-Skelett. t. ii. (i 879 ).

?L. melanopterus, Wall. P. Z.S. 1864 , pp. 288, 294. 
Coryllis culacissi, Finsch, Pafag. ii. p. 705 (1868).

Licmetulis culacissi, G. R. Gr. Hant-list, ii. p. 852, n. ${ }^{0} 8181$ $(18-0)$.

Coryllis philippensis, Rchna. Journ. f. Orn. $1 \delta \delta 1$, p. 228 (Consp. Psitt. P. II 6 ); id. Iagclh:ld Nichtr. 47 (1883).

\section{Pág 56. L. mindorensis}

L. mindercrsis, stcre, List. B. ct $M$. Stccre Exfed. to tho

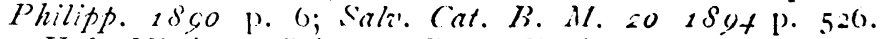
Hab. Mincioro, Calapan, Baco, Naujan.

\section{Pág 56. L. Eiquujoren£is}

L. siquijorcnsis, Stcere, List. B. \& MI. Stecre. Exped. Philipp. 1890 p. 6; Salv. Cat. B. M. 201897, p. 527.

\section{Pág. 56. L. indicus}

I.. indicus, (Bris).

The smallest green et red Inclian paroquet, Edaw. i. lam. 6 (17+5); Seligm. Samml. Ausländs. Vög. i. pl. xi. f. I (1749).

Psittacula indica, Briss. Orn. iv. p. 390 (1760); Red et Green Indian. Parrot, Lath. Syn. i. 31 I (1781); id. Gen. Hist. ii. p. $264(1822)$.

Psittacus indicus, Gm. $S . \quad N$. i. p. 349 n. ${ }^{\circ} 28$ (no n." 58 ) (1788) (ex Brisson); Bechs. Kitrze Uebers. p. Iot (181 ); Kithl. Consp. Psitt. p. 65 (8 no \&) (1820); Vorgt, Cuv. Uebers. 1). 747 (1831).

P. asiaticus, Lath. Ind. Orn. i. p. I 30. nn. ${ }^{0}$ I 6 (1790); Shaz's. Gen. Zool. viii, 2, p. 553 (181 I); Vieill, Enc. Meth. iii. p. I $407(1823)$.

P. indica, part., Steph. Gen. Zool. xiv, 2, p. I1t $(8126) ; G . R$. Gr. Gen. B. ii. p. $423, \mathrm{n}^{\circ}$ I 7 ( 1846 ).

P. coulaci, part., Less. Tr. d'Orn. p. 202 (\&) (1831).

P. minor, part., Mon. Psitt. p. 628 (8) (r832).

P. indicus vel minor, Bourj. Perr. text, p. 89 (1837-38).

Loriculus philippensis, Blyth. (no Briss.), Cat. B. Mus. A. S. B. p. IO, n. ${ }^{\circ} 46$ (i 849$)$.

L. asiaticus, Blyth, op. cit. p. 312 (1849); id. Journ. A. S. B. xviii. p. 801 (1849), xix. p. 236 (1850); Kelaar, Prodr. Fann. (eyl. p. 127 (1852); Layard, Ann. \& Mag. N. H. (2) xiii. p. 261 ( 854$)$; Hartl. Journ. f. Orn. 1854 , p. 1 58 ; Hsrsf. \& Moore, Cat. B. Mus. E. 1. Comp. ii. p. 628 (1856) Wald. P. Z. S. 1866 , p. 539; Garrod, P. Z. S. 1873 , p. 634, 1874, p. 587 ; Trucedd. Orn. Warkfi, p. I7 (1881); Sclat. List. Vert. An. 8th. ed. p. 326 (1883).

L. indicus, Bp. Rev. et Mag. de Zool. 1854 , p. I 55, n. ${ }^{\circ} 240$; Souancé, Rev. et Mag. de Zool. 1856 , p. 219 , n. ${ }^{\circ}$ i73, p. 220 (5 no ९), p. 22 г Bp. Naumannia, 1.856 , Consp. Psitt. n. 247; Cass. Narr. Exp. under Comm. Perry p. 242 (1856); Journ. f. Orn. $185^{8}$, p. 449; G. R. Gr. List. Psitt. Brit. Mus. P. 55 (1859'; Schleg. Mus. P.-B. Psittaci, P. I32 (1864); 
Pelz. Nöara Reise, Vög. p. 99 (1865); G. R. Gr. Hand-list, ii. p. 152, n. ${ }^{\circ} 8173$ (1870; Holdsu. P. Z. S. 1872 , pp. 485,425 ; Logoe, Ibis, 1874, 15; Hune, Str. Feath. ii. p. 186 (1874); Schleg. Mus. P. B. Psittaci, Revus, p. 6o (187t; Leoge, vir. Feath. iii. p. $365(1875) ; G e b$. Thes. Orn. ii. p. 493 (1873); Meyer. Row!. Oru. Misc. ii. p. 232, n. ${ }^{\circ}$ (1877); T.ieedd. P. Z. S. 1877 , p. 820; Hilme, Str. Feath. vii. p. 368 (1878), viii. p 87, n. ${ }^{\circ}$ I 53 bis (1879); Legse, B. of Ceyl. pp. 13, г2 12, lan. iv. inferior (ad.), lam. vii. superior fis. (joven) (1880); Tzueed. Orn. Works, pp. 546, 457 (1881); Tristr. Cat. Col. B. p. 76 (1830); Salv. Cat. B. M. 20. 1894 p. 525.

?. L. puniculus (sinens's), Bp. Rev. et Mag. de Zool. 1854 , p. I55, n. 241 ; Souancé, Rvv. et Mag. de Zool. 1856 , pp. 219, 22 ; Bp. Naumannia, 1855 , Consp. Psitt. n. ${ }^{\circ}$ 250; G. R. Gr. List. Ps t.. Brit. Mus. p. 55 (1859)*; Sclat. Ibis, 1850, p. $36 \mathrm{I}$ (nota'; Swinh. Ibis, 1861 , p. 4 I I 1862, p. 305; G. R. Gr. Handlist, ii. p. 1 52, n. 8174 (1870); Swing.P. Z. S. 1871 . p. 391 .

L. coulaci, Blyth, Ibis, 1853, p. 7, 1867, p. 226.

L. edwardsi, Blyth, I6 s, 1867 , p. 294, 31 ; Wald. Ibis, 1857 , p. 45 ; Nevull. Journ. A. S. B. (Cey!. Br.) 1870-71, p. 32; lewsedd. Orn. IVorks, p. 4I (1881).

Cory.lis inlica, Fins=h. Pxp.xg. ii. p. 714 (1853) R:hnu. Jo:urn.f. Orn. 1881 , p. 227 (Conss. Psitt. p. II 5); id. Vugelbild. N.uchtr. $46(188.3)$

Hab. Minlanao; Ceilán.

Pag. 56. L chr, sonotus.

Loriculus regulus, G. R. Gr. (n) Souancé) List Psitl. Brit. Mus. p. 56 (1859); Wall. P. Z. S. 1864 , p. 288; Finsch, Ibis, 1874 , p. 208; Wald. Trans. Zool. Soc. ix. p. I 37 (1875); Tiveedd. Orn. Works, p. 304 (1891).

Coryllis regulas, (pirt.), Finsch, Papag. ii. p. 7 I I (r868); id. Ibis, 1872, p. 208.

Loriculus culacissi, Sclat. (noc Viell.) P. Z. S. 1871, p. 479; id. Rev. Lyst. of Ver!. p. 212 (1872).

L. chrysonotus, Sclat. Ibis, 1872, p. 324 , lam. xi. * id. P. Z. S. 1872 , p. 790; Wiald. P. Z. S. ${ }_{1} 873$, p. 525; G.trrod, P. Z. S. 1873, p. 634,1874, p. $587 ;$ Gieb. Tizes Orn. ii. p. 497 (1875); Wald. Trans. Zool. Soc. ix. pp. I37, 249 (1875); Sharpe, Tr. Linn. Soc. (2) Zool. i. p. 3 1 3 (1876); Meyer, Row!. Orn. Misc. ii. p. 232, n. 8 (1877); Twsedl. P. Z. S. 1877 , pp. 539757820 ; id. Orn. Works, pp. 299, 304, 410, 463, 530 547 (1881); Wardl. Rams. Tweedd. Orn. Works, p. 655 (1881); Trueedd. Voy. Chall., Birds, p. Io (1881); Sclat. List Vert. Au. 8th. ed. p. 325 (1833); Steere, List etc. p. 6 ( 1890$)$.

Coryllis occipitalis (part.), Finsch, Ibis, 1874, S. 209t; Rchnw. Journ.f. Orn. 1881 , p. 228 (Consp. Psitt.p. 116); id. Vogrlbild. Nachtr. 47 (i 883 ).

C. chrysonotus, Finsch. Ibis, 1874, p. 208.

Loriculus oc ipitalis, Wald. Tr. Zool. Sxc. ix. pp. I 35 (not.z), 249 (1875); Meyer, Rizw!. Orn. Misc. ii. p. 232, n. 9 (1877); Tweedd. Orn. Works, pp. 303, 5 IO (1881); Wardl. Rams. Tweedd. Orn. Works, p. (1881). 
Coryllis chrysonota, Rchnw. Jo:trn. f. Orn. 1881, p. 229 (Cunsp. Psitt. p. I I 7 ); id. Vogelbild. Nachtr. 48 (1883). Hab... Mindanao.

\section{Pag. 56. Loriculus vernalis (Sparr.)}

Psittacus vernalis, Sparrm. Mus. Carls. t. 29 (1787); Lath. Ind. Orr. i. P. 130, n. ${ }^{1}+7$ (1790); Rechst. Kurze U:b. p. 105 (18Ir); Vicill. V. D. xखv. p. 3751877 ; id. Ens. Meth. iii. p. If07 (182.3); Voigt, Ciuv. Uebers. p. 7t7 (1831).

Vernal parrakeet, Lath. Syn. Suppl. ii. p. 95, n. 26 (1802); id. Gen. Hist. ii. p. $265(1822)$.

Psittacus indicus, fam., Kuhl, Consp. Psitt. p. 65 (1820).

Psittacula gralgulus, farm. Wagl. Mon. Psitt. p. $626\left(18_{32}\right)$.

Ps. minor, feem., Wagl. op. cit. p. 529 (1832).

Psittaculus galgulus, Selby, Nat. L:br., Pivrots, p. 167 (1836).

Ps. pendulus, Pears. Journ. A. S B. x. p. $65+(18+1)$.

Ps. vernalis, Blyth, Journ. A. S. B. x. p. 921 (18+t); Jerd. Madras Journ. xi. p. 209 (18fo).

Psittacula pyrhopyga v. vernalis, Hods. Gr.zy's Zool. Misc. p. 85 $(18+4)$.

Ps. vernalis, Blyth, Cat. B. Mus. A. S. B. p. 10, n. ${ }^{\circ}$ to $^{6}$ (part.) (18+9); id Journ. A. S. B. xviii. p. 801 (1849), xix. p. 337 (1850); Bp. Rev. et Mag. de Zool. 1854, p I55, n. ${ }^{\circ}$ 239; ? Souance, Rev. et Mag. de Zool. 18.56, p. 2r9; Bp. Natumatnia, 1855 , Consp. Psitt. n. ${ }^{2}+6$; Horsf. \& Moore, Cat. B. Mus. E. I. Comp. ii. p. 627 (1858); G. R. Gr. List Psitt. Brit. Mus. p. 5t (1859); Jerd. B. of Ind. i. p. $26_{5}$ (1862); Gray, Cat. Hodgs. Coll. B. Mus. and ed. p. 62, n." 45 (186.3); Blyth, Ibis, 1853, p 6 (part.); id. App. Zool. of the Antam. Isl. in Mouxt's Arch. Antim. Isl. p. 355 (1863); Wald. P. Z. S. 1866, p. 538; Blyth, Ibis, 1867 , p. 295; Beav. Ibis, 1857 , p. 323 1859, p. 412; Eilzes, Ibis, 1870, p. 527 G. R. Gr. Hzut.lis!, ii. p. 152, n. 8172 (1870); Ball, Journ. A. S. B. xli. p. 279 (1872); Wald. Ibis, 1873 , p. 299; Bull, Str. Feath. i. p. 62 (1873); Hume, Nests \& Eggs, p. I19 $(1873)$; Wald. Ibis, 1878 , p. 298; Hune, Str. Featí. ii. pp. 57 i85, 47 I (1874), iii. p. 57 (1875); Blyth. \& Wald. B. of Burve. p. 69 (1875); Gieb. Thes. Orn. ii. p. $499(\mathbf{1} 876)$; Hume, ibit. p. 393 (1876), v. p. 25 (1877); Fuirb. ibid. p. 395 (1877); Meyer, Rowel. Orn. Misc. ii. pp. 23I, 234, n. ${ }^{\circ}$ (i877); Hume \& Davis. Str. Feath. vi. pp. I20, 500 (1878); Hume, Str. Fexth. viii. p. 87, n. ${ }^{\circ} 53$ p. 153 (1879); Vidal, Str. Fexth. x. p. 52 (. 830$)$; Bing,h. ib.d. p. I6r (1890); Buth. ib d. p. $3^{3}+$ (1330); Ottes, Str. Futh. x. p. r97 (1932); Salo. Cat. B. Strickl. Coll. p. 459 (1882); Oates, B. of Brit. Burm. ii. p. I $+^{6}(1833)$; Silvat. Ann. Mus. Civ. $G$ n. (2) v. p. 560 (i 888 ), viii. p. 376 (1889); Tristr. Citt. Coll. B. p. 25 (1889); Mitrr. Ayjf. of Brit. Int. ii. p. 463 (1 89:); Oxtes, Nests \& Eggs. Ind. B. iii. p. 92 (1890).

- Loriculus indicus, (p.urt.), Sonancé, Rev. et M.yg. de Zool. 1856, $(A, f(x))$.

L. apicalis, (p.rrt.), Sounnce, ibid. (B. fam).

Coryllis verialis, Finsch, Papag. ii. p. 721 (1859); Rchmw. Vo- 
gelbild. t. xi. f. $4(1878-83)$; id. Journ. f. Orn. 1881, p. 226. (Consp. Psitt. p. r 14); Miill. Journ. f. Orn. 1882, p. 428.

Hab. Península Malaya, Filipinas.

\section{Pag. 57. L. hartlaubi.}

? Psittacus melanopterus, Scop. Del. Flor. et Fiaun. Insubr. ii. p. 86, n. $^{\circ} 23(\sigma)$ ( 786$)$ (ex Sonnerat).

: Loriculus indicus, A, Sonan'é, Rev. et Mag. de Zool. 1856, p. 220 (parte).

L. apicalis, Sonancé, 'Revo. et Mag. de Zool. 1856 , pp. 220, 22 I;

- Bp. Nanmannia, 1856 , Consp. Psitt. n. ${ }^{\circ} 49 ;$ Wald. P. Z. S. 1864 , pp. 288, 294; v. Mart. Journ. f. Orn. 1866, p. 21; $G$. R. Gr. Hant-list, ii. p. 15r, n. ${ }^{\circ}$ 8176 (1870); Legge, $B$. of Cicyl. p. 18 i (1880).

? L. sp., Souanié, l. c. p. 221.

L. cyanxmus, Bp. Naumannia, 1856 , Consp. Psitt. Brit. Mus. p. 55 v. Mart. Journ. f. Orn. 1866 , p. 21 ; G. R. Gr. Handlist, ii. p. 152, n. 8 I 75 ( 1870$)$.

L. melanopterus, G. R. Gr. List. Piitt. B. M. p. 55; v. Mart. Journ. f. Orn. 1866 , p. $2 \mathrm{1}$; P. R. Gr. Hand-list, ii. p. 152, I 870, n. $^{\circ} 8175$.

Corillis hartlaubi, (part.), Finsch, Papag. ii. p. 7r r (o) (I868).

Loriculus hartlaubi, Wald. P. Z. S. 1873, p. 256; id. Tr. Zool. Soc. xx. pp. г31, 249 (г875); Meyer, Rowl. Orn. Misc. ii. p. 233, n. ${ }^{\circ}$ IO (1877); Tried. PP. $Z$. S. 1877 , p. 8I9, Iam. Ixxxii. f. I. $\sigma, 2$ o 1878, pp. 107, 340 (?) 709,937 (1879), p. 68; id. Orn. Works, pp. 299, 303, 304, 410, 545, 546, 547, 566, 655, 621, 628, 646 (1881); Wardl. Rams. Tiveedd. Orn. Works, p. 655 (1881); ? Kutter, Journ. f. Orn. $188_{3}$, p. 294; Steere, List. p. 6 ( 1890$)$.

Coryllis apicalis, Rchnze. Journ. f. Orn. 1881, p. 229 Consp. Psitt. p. I I 7 ); 2d. Vogelbild. Nachtr. 49 (1883).

Loriculus bonapartei (part.), Guillem. P.Z. S. 1885, p. 252.

Loriculus worcesteri, Steere, List B. \& M. Stecre Exped. to the Philipp. p. 6 (1890).

Hab... Samar, Borongan, Paric, Leyte, Amparo, Zamboanga, Tawi-tawi.

\section{Pag. 57. I. regulus,}

Loriculus regulus, Sclat. Ibis, 1872, p. 324;id. P. Z. S. 1872 , p. 7oo; Wald. P. Z. S. 1873, P. 526 ; Gieb. Thes. Orn. ii. p. 499 (parte) (1875); Wald. Tr. Zool. Soc. viii. p. I35 (1875); Sharpe, Trans. Linn. Soc. Zool. i. p. 313 (1876); Meyer, Rowl. Orn. Misc. ii. p. 232, n. 7 (1877); Rowl. \& Meyer, ibid. p. 238 ; lam. $5^{8}$ (1877); Tiveedd. P.Z. S. $1377, \mathrm{pp} .539,820$, I 878 , p. 281 ; id. Orn. Works, pp. 303, 410, 587 (1881); Ward!. Rams. Tweedd. Orn. Works, p. 655 ( $188 \mathrm{I}$ ); Steve, List. p. 7 (1890); Sali. Cat. B. M. 20, 1894 , p. 523 .

Licmatulus regulus, Bp. Nammania, ${ }_{1} \delta_{5} \sigma$, Consp. Psitt. n. ${ }^{0} 25$.

Loriculus (Licmetulus) regulus, v. Mart. Journ. f. Orm. 1866, p. 21.

Curyllis regulus, Finsch, Ibis, 1874 , p. 208†; Rchnw. Journ. $f$. 
Orn 1881, p. 223 (Consp. Psitt. p. 816); at. L'ogibilat. Nacht. $47(1883)$.

Loriculus uccipitalis (part.), W'ald. Tr. Zoo!. Soc. ix. pp. 13.5 (nota), $2+9(1 S 75)$; Mejer, Ro:u!. Or;i. Miso. ii. p. $232 ;$ n. $^{0}$ y (1877); Tivedd. Orn. Works. p. 655 (1831).

Loriculus panayensis, Tivedtl. $P$. $Z$. S. 1877, pl. 530, 538; id. Orn. Works, p. 402, (1881) Wardl. Rams. ibid. pp. 055,667 (I881); Tweedd. Voy. Chall., Bwds, p. 9, lam. I, f. I, 2 (I881 i;

Coryllis panayensis, Rchure. Jouru. f. Or-n. 13931 , p. 227 (Consp. Psltt. p. I1 5); id. Vegelvild. Nirchtr. $\$ 5$ (1883).

Hab... Negros, Valencia, Tablas, Roinblon, Sibuyan.

Pag. 57. Fclectus roratus, (S. Miill).

Purper roode lori, Vosmaer, Mon. p. 10, t. 7 (1769).

Lory de la Nouvelle Guinée, D'Aubent. Pl. Enl. 683.

Psittacus roratus, P. L. S. Mïll. S. N. Suppl p. 77 (1776); Cass. Pr. Ac. Sc. Philad. $186+$, p. 2fo, n. ${ }^{0}$.'

Le grand lori, Buff. Hist. Nat. Ois. vi. p. I 35 (1879).

Grand lory, Lath. Syn. i. p. $275(178 \mathrm{r})$; id. Gen. Hist. ii. p. $230(1822)$.

Psittacus ceclanensis, (sic), Bodd. Titbl. Pl. Einl. p. 42 (1783).

P. grandis, Gm. S. N. i. p. 335, n. ${ }^{\circ}$ I)2 (1 788$)$; Lath. In.t. Orn. i. p. 116 (1790); Sa?u, Gen. Zool. viii. p, 133, lain. 8in (1811); Bechst. Kurze Uebers. p. I 4 (1811); Kuhl, Consp. Psitt. p. 30 (1820); Vieill. Ens. Méth. p. I.378 (1823); Voigt, Cuv. Uebers. p. $73+$ (1831); Hahn, Orn. Atl., Pasag. p. 88, t. 68 (1837); Teinm. Tab!. Méth. p. 59 (1839); S. Mïll. Verh. Land-en Volkenk. p.). 107, Io8 (parte) (1 $839-44)$; Thienem. Foutpflanz. d. ges. Vög. p. 76 (1852); Dehne, Naumanma, 1854, p. 39; Finsch, Neu-Giuin. p. 157 (parte) (1865).

Le Perroquet grand lory, Levaill. Perr. lanı. 126, 127 (var.), i 28 (var.) (1805).

Lorius grandis, Steph. Gen. Zool. xiv. p. 131 (1826); Hartl. Syst. Verz. Ges. Mus, p. 87 (1844).

Mascarinus puniceus, Less. (no Gm.) Tr. d'Orn. p. 188 (1831).

Eclectus grandis, Walo. Mon. Psitt. p. 143; id. Rev. et Mag. de Zool. 1854, p. $155, \mathrm{n}^{\circ}$ 236; id. Naumannia, 1856, Consp. Psitt. n. ${ }^{\circ} 2+4$; Sounnce, Rev. et Mag. de Zool. 1856, p. 218 ; Horsf. \& Moore, Cat. B. Mus. E. I. Comp. ii. p. 62t (1858); G. R. Gr. P. Z. S. 1860 , p. 357 ; Berust. Nat. Tijitschr. Ned. Ind. xxvii. p. 295 (1864); Wall. P. Z. S. 1864 , p. 286; Schleg. LWus. P-B. Psittaci, p. 38 (1864); Finsch, Papag. ii. p. 340 (1868); Mcyer, Zool. Gart. 1874 , p. 161; Schleg. Mus. P. B. Psittaci, Revile, p. I 3 (1874); Gieb. Thes. Orn. ii. p. 74 (1875); Satvad. Ann. Mus. Civ. Gen. vii. p. 757, n. ${ }^{\circ} 6(1875)$; Kosenb. Reist. naar Geclvinkb. p. 6 (1873); Forbes, Ibis; 1877 , p. 282; Salvad. Ibis, 1877 , p. 475; Rosenb. Zool. Gart. 1878 , p. 3+5; Meyer, Zeitschr. $f$. wissensch. Zool. xxxvii. p. 156 y siguientes (1882). E. ceylonensis, G. R. Gr. Gen. B. ii. p. $4 \mathrm{I} \&, \mathrm{n}^{\circ} 2$ (18+5).

Polychlorus magnus, Sclat. P. Z. S. 1860, pp. 226, 227 (parte.) Eclectus polychlorus var., G. R. Gr. P. Z. S. 1860, p. 358.

Psittacodis magnis, Rosenb. Journ.f. Orin. 1862, p. 62 ; id. Nat. Tijdschr. Ned. Ind. xxx. pp. 141, 142 (1863). 
E. polychlorus, Bernst. (no Scop) Nat. Tijdschr. Ned. Ind. xxvii. p. 294 (1864); Wall. P. Z. S. 1864, p. 286 (parte); Schleg. Mus. P.-B. Psittaci, p. 40 (parte) (1864); Finsch. Papag. ii. p. 333 (parte) (1868); Miger, Zool. Gart. 1874, p. 16 I (parte); Schleg. Mus. P.-B. Psittaci, Revue, p. 14 (parte) (1874); Rosenb Rcitt. uaar Geelvinkb. p. 6 (1875); id. Zool. Gart. 1878 , p. 345 (parte); Musschenbr. in Bernst. Dagbsek, p. 256 (parte) (1883).

Psittacus polychlorus part., Finsch, Neu-Guin. p. 157 (1867).

Eclectus roratus, G. R. Gr. Hand-list, ii. p. 157, n. ${ }^{\circ} 8240(1870)$; Salvad. P. Z. S. 1878 , p. 78 ; id. Orn. Pap. e Mol. i. p. 206 (1880); id. Cat. B. M. 20. 1894, p. 393; Rchuw. Vogelbild. t. xxvii. f. 6 (o) (1878-83); id. Journ. f. Orn. 1881, pp. 252, 397 (Consp. Psitt. pp. 140, 229); Salvad. Voy. Chall., Birds, p. 59 (1881); Sclat. P. Z. S. $188^{2} 3$, p. 196; Meyer, Zeitschr. $f$. ses. Orn. i. p. 274, lam. xvi. (1884); Guillem. P. Z. S. 1885, p. 563; Saload. Mem. R. Ac. Sc. Tor. ser. 2, xl. p. 165 (1889); Meyer; Ibis, 1890, p. 26, pl. i.

Muscarinus (sic) polychlorus, part., G. R. Gr. Hand-list, ii. p. $15^{8}$, n. $8243(1870)$.

Eclectus cardin. lis, Silvat. (nec Bodd.) Aun. Mus. Civ. Gen, vii. p. 757, n. ${ }^{\circ}$ (nec n." 5) (1875).

\section{Varictas.}

Perroquet violet, Fermin. Descr. gen. de Surinam, ii. p. 175, ( 769 ).

Violet lory, Lath. Syn. i. p. 220 (1 $\left.7_{1}\right)$; $d$. Gen. Hist. ii. p. 135 (1822).

Psittacus janthinus, Gm. S. N. i. p. 319, n. ${ }^{\circ}$ 6r (1;88).

Psittacus guebiensis, var. $\beta$ Lath. Ind. Orn. i. p. 90. Hab. Filipinas.

\section{Pag. 57. E. pectoralis, (L.S. Mï̈ll)}

Green and red parrot from China, Edw. Glean. v. lam. 231 (1758); Seligm. Ausl. Vög. vii. pl. I 4 (1770).

Psitt..cus sinensis, Briss. Orn, iv. p. 291 (1760) Gim. S. N. i. p. 337, n. ${ }^{0} \operatorname{sog}(1788)$; Lath. Ind, Orin. i. p. 117 (1790); Becht. Kurze Lebers. p. 94 (1814); Kithl. Consp. Psitt. p. 84 (1820); Vieill. Enc. Mêth. p. I369 (1823); Liss. Voy. (o1., Zool i. p. $342(1826)$ ii. p. 627 (1828); Voigt, Ci.v. Uebers. p. 743 (1831); Temm. Tabl. Méth. p. 59 (1839).

Perroquet de la Chine, $D^{*}$ Aubent. Pl. Enl 514.

Grand Perroquet verd de la Nouvelle Guinée, Sonner. Voy a la Now. Guin. p. 174, lam. "1o8 (1776).

Psittacus pectoralis, P. L. S. Miiıl. S. N. Suppl. p. 78, n. $^{\circ} 5^{8}$ ( 1776$)$; Cass. Pr. Ac. Nat. Ss. Philad. 1864, p. 240.

P. aur.ntius, P. L. S. Mäll. op. cit. p. 80, n. ${ }^{\circ} 64$; Cass. l. c.

Perroquet vert, Buff..Hist. Nat. Ois. vi. p. 116 (1779).

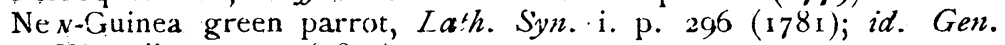
Hist. ii. p. 233 (1822).

Psittacus polychlorus, Scop. Del. Flor. et Faun. Insubr. p. 87, 
n. 27 (1786) (ex Sonnerat); Finsch, Nen-Guin. 'p $15^{8}$ (parte) (1865); Rosenb. Reist. naar Geelvinkb. p. 83 (1875).

P. magnus, Gm. S. N. i. p. 344, n. ${ }^{\circ} 22$ (1788) (ex Sonnerat); Bechst. Kurze Uebers. p. I(x) (1811); Vieill Buc. Meitk. p. 1366 (1823); S. Mïll. Verh. Laum-den Volkenk. pp. 22, 167 (is $39-1844$ ).

P. viridis, Lath. Ind. Orn. i. p. 125, n. 123 (1750! (ex Gmelin). Le perroquet à flancs rouges, Lovaill. Perr. l:un. 132 (1805):

Psittacus lateralis, Shave, Gen. Zool. viii. p. 490 (1811).

Green y red chinese parrot, Lath. Gen. H.st. ii. p. 233 (1822).

Psittacus grandis, Less. (no Gm.) Voy. (icq., Zsol. i. p. 342 (1826); S. Müll. Verh. Land-en, Volkenk. p. $22(1835-18+4)$. P. puniceus (part.), Less. Voy. Co7., Zool. i. 2, p. 627 (1828).

Mascarinus prasinus, Less. Tr. d'Orn. p. I88 (1831).

Eclectus linn:ei, Wagl. Mon. Psitt. p. 57 I, t. xxi. (1832); G. R. Gr. Gen. B. ii. p 418, n. ${ }^{\circ}$ ( $18+5 ; B p$. Nannainnia, 1855 Consp. Psitt. $12 .^{\circ}$ 24.3; Souancé, Rev. et Mag. de Zios. 1955 , p. 219; Wall. Ann. \& Mag. N. H. (2) xx. p. 473 (1857);G. R. Gr. P. Z. S. $1 \delta_{5} \delta$, pp. 182, 194, 1859, p. 15 ; it. Cat. B. Neze Guin. pp. 40, 59 (1859); id. Lisl Psitl. Brit. Mus. p. 65 (1859); Wall. Ib s, 1861 , p. 286; Cass. Pr. Ac. Sc. Phlizd. 1862 , p. 319 ; Sclat. Ibis, 1853, p. 229; Finsch, Ned. Tijdschr. Dierk. i. Berigten, p. xvii. (1863); Wall. P. Z. S. 1864 , p. 286; Bernst. Nat. Tijdschr. Ind. xivii. p. 297 (1864); Schleg.. Mus. P.-B. Psittaci, p. 38 (1861); Rosin). Rois n.x.ur Zuidoostereil. pp. 48, 80 (1867); Finsch, Papag. ii. p. $34^{6}$ (1868); G. R. Gr. Hand-list, ii. p. 157, n. ${ }^{\circ} 839$ (1870); id. Ann. \& Mag. N. H. (4) v. F. 329 (1870); Meyer Z jol g. Gart. 1874 , p. 16r; Schleg. Mus. P.-B. Psittaci, Revue, p. 11 (1874); Rosenb. Reist. naar Geelvinhb. pp. 36, 55, II 3 (1875; id. Zנرlog. Gart. 1878 , p. 345 .

Psittacodis magnus, Wagl. Mon. Psitt. p. 575 (1832); Bp. Consp. i. p. 4 (1850); id. P. Z. S. 1850 , p. 26; idt. Rev. et Mag. de Zool. 1854 , p. 155, n. ${ }^{\circ} 232$; ilt. Naumanniti, $185^{5}$, Co.ts?. Psitt. n. ${ }^{\circ} 239 ;$ Soulance, Rev. et Mag. de Zool. 1856, p. 218 ; Wall. Ann. \& Mag. N. H. (2) xx. p. 473 (1852); Rosenb. Journ. f. Orn 1852 , pp. 62, 64, 65, 68; id. Nat. Tijdschr. Ned Int. xxv. pp. I $43,144,145,148,223, n^{\circ} 22(1863)$.

Psi tacus li inæi, Hahn, Orn. Atlas, Papag. t. 77 (1836); Finsch, Neu-Guin. p. 157 ( 1865 ).

Psittacus grandis, var., S. Müll. Verh. Land-en Volkznk. pp. I07, 108 (1839-1844).

Eclectus polychlorus, G. R. Gr. Gen. B. ii. p. 428, n. ${ }^{4}$ (i843); id. Cat. B. New Guin. pp. 4o, 59 (1859); id. C.t.. B. Trop. Isl. p. 34 (1859); id. List Psiti. Bris. Mus. p. 6' (1859;; Cass. Pr. Ac. Sc. Phlad. 1852, p. 319; Sclat. Ibis, 1853 , p. 229; Rosenb. Nat. Tijdschr. Ind. xxv. p. 226, n. ${ }^{\circ}$ I (1863); id. Journ. 'f. Orn. 1864 , p. I14, n." 21; Wall. $P$. Z. S. 1864 , p. 286 (parte); Schleg. Mus. P.-B. Psittuci, p. 4 I (parte) (I 864); id. Ned. Tijdschr. Dierk. iii, p. 332 (1865); Rosenb, Reis. naar Zuidosstere:l. pp. 48, 80 (1867); Finsch, Papag. ii. p. 48, Gesellsch. Wien, 1874 , p. 179; 1d. Zosl. Gart. 1874 , p. 161; Schleg. Mus. P.-B. Psittaci, Rovue, pp. 14. I5 (1874); Gieb. Thes. Orn. ii. p. 76 (1875); Meyer, M:tth. z3o!. 
Mus. Dresd. i. p. I I (1875); Sitzb. Isis Dresd. 1875, p. 77; Salvad. Ann. Mus. Civ. Gen. vii. p. 756 (1875); Salvad. e D'Alb. Ann. Mus. Civ. Gen. vii. p. 8 I I (1875); Rosenb. Reist. naar Geelvinks. pe. 36, 55, I I8 (1875); Sizloud. Ann. Mus. Civ. Gen. ix. p. I I (1875); Ramsay, Pr. Linn. Suc. N. S. W. i. p. $39+$ (1876), Cis. u. Rchnu. Journ. f. Orn. 1's; , p. 324; Salvad. Ann. Mus. Cov. Gen. x. p. 31, n." 49, p. I 2 I (1877); Sclat. P. Z. S. 1877 , p. I05; Meyer, P. Z. S. 1877 , p. 800, lam. 79; Forbes, Ibis, 1877 , p. 282 (parte); Salvad. Ibis, 1877 , p. $475 ;$ id. P. Z. S. 1878 , p. 92; Sctat. P. Z. S. 1878. pp. 289, 290; Sharpe, Journ. Linn. Soc., Zool. xiii. p.: 49I, n. ${ }^{1} 5$ (1878); Meyer, Orn. Centralblatt. 1878 , Pp. I19, I37; Gould. B. New Gitin. pt. viii. lam. 4 (1878). Rosenb. Zool. Gart. 1878 . p. 345 (parte); Meyer, Zool. Galt. 1878: p. 377; Rosenb. Malay. Archip p. 37 I (1878-79); Ramsay, Pr. Linn. Soc. N. S. W. iii. p. 253 (1878-79); iv. p. 69 (1879); Finsch, P. Z. S. 1879 , p. I2; D'Alb. e Salvad Ann. Mus. Civ. Gen. xiv. p. 36 (1879); Salvad. Ann. Mus.. Civ. Gen. xiv. p. 639 (1879); Rchnw. Vogelbil. t. xi. ff. 3,4 (1878-83); Salvad. Voy. Chall., Birds, p. 75 (1881) Meyer, Zeitschr. f. wissensch. Zool. xxxvii. p. I 46 (I882); Ramsay, Pr. Linn. Soc. N. S. W. viii. p. 33 (г882); Tristr. Ibis, 1882 , p. I +3; Muss:chenbr. Dagboeh, p. 256 (parte) (1883); Finsch, Vög. d. Sïdsee, p. I7 (1884); Mever, Zeitschr.f.ges. Orn, i. p. 373 (1834); Grant, P. Z. S. 1887 , p. 329, 1888, p. I89; Meyer, VogelSkelett. t. x., xliv. (1878-89).

Eclectus puniceus, Bp.P.Z.S. I 879 , p. It2 id. Rev. et Mag. do Zool. 1854, p. $155, \mathrm{n}^{\circ}{ }^{2} 35$; Rosenb. Nat. Tijdschr. Ned. Ind. xxv. pp. 143, 145, 226, n. ${ }^{\circ}$ 20 (1863); id. Journ. f. Orn. 1864 , p. I I 4, n. $^{\circ} 20$.

Eos puniceus, Licht. Nomencl. Av. p. 7 I (185t).

Polychlorus magnus, Sclat. P. Z. S. 1857, p. .26, 1860, p. 227 Marie, Act. Soc. ,Linn. Bord. xxvii. p. 327 (1870) Oust. Bull, Soc. Philom. Paris, 1879, p. 218.

Polychlorus grandis, Sclat. (nJ Gm.) Journ. Pr. Linn. Soc. ii. p. 165, n. ${ }^{\circ}$ II 3 (I $85^{8}$.

Eclectus polychlorus, var. aruensis, G. R. Gr. P. Z. S. 1858 , p. 183 ; id. Cat B. Neze Guin. pp. 40, 59 (1859); Bernst. Nat. Tijdschr. Ned. Ind. xxvii p. 297 (186+).

Psittacodis polychloros, G. R. Gr. P. Z. S. 1858 , p. 194.

Mascarinus polychiorus, Horsf. \& Moore, Cat. B. Mus. E. I. Comp. ii. p. $625(1858)$; Finsch, Ned. Tijdschr. Dierk. i. Berigten, p. xvi. (1863).

Eclectus ceylonensis, G. R. Gr. (no Bodd.) Cat. B. Trop. Ist. p. $34(1859)$.

Eclectus cardinalis, Wall. (no Bodd.) Ibis, 1859 , p. 210 ; Sclat. P. Z. S. 1860, p. 227; Rosenb. Journ. f. Orn. 1862 , pp. 63, 64, 65; id. Malay. Archip. p. 37 (1878-79); De Vis, Ann. Rep. Brit. New Guin. p. $5^{8}$ (1890); 2d. Colonial Papers, n." I03, p. 108 (r890); id. Ibis. 1891 , p. 29.

Psittacodis magna, Rosenb. Journ. f. Orn. 1864 , p. IIt, n. ${ }^{\circ} 22$.

Muscaris (sic) polychlorus, G. R. Gr. Hand-list, ii. p. I 58 , n." $82+3(1870)$. 
M. (sic) intermedius, part., G. R. Gr. Hand-list, ii. p. 158 ; n. ${ }^{\circ}$ $82+5(18 ; 0)$.

Eclectus (sic) intermedius, G. R. Gr. (no Bp.) Ann. \& Mag. A. H. (4) r. p. 32) (1870):

Lorius sinensis, Gullin. P. Z. S. 1875, p. 489 .

Eclectus pectoralis, Salvad. Orn. Pap. e Mol. i. p. 197. (1880); Rchnzi. Journ. f. Orn. 1881 , p. 252 (Consp. Psitt. p. 140); at Vogelbild. Bericht. (1883); Sclat. P. Z. S. 1883, p 196; Meyer, Sitz. u. Abh." Gesellsch. Isis, $18 \delta^{\circ}$, Abh. i. p. 16; Nehrk. Journ. f. Orn. 1895, p. 3 1; Guillem. P. Z. S. 189.5 , p. 620: Satiat. Mem. R. Ac. Sc. Tor. ser. 2, xl p. 165 (1889); id. Cat. B. M. 20. 1894 , Tristr. Cat. Coll. B. p. 76 ( 1889 ).

? Eclectus grandis, Berust. (no Gm.) I)agboek, p. It4 (1883).

Ec. intermedius, (part.), Musschenbr. in Bernst. Dagboek, p. 256 ( 1883 ).

Hab. Filipinas.

Pag. 5 . Lorias lory, (Lin).

?Psittacus orientalis exquisitu Loeri dictus, Seb.t, Thes. i. p. 37, f. 4 (I734).

First black-capped lory, Eowe. Birds, iv, lam. i70 (i751).

?Ara moluccensis raria, Bris. Orn. iv. p. 197 (i76o) (ex Seba).

Lorius philippensis, Briss. Orn. iv. p. 225, lam. xxiii. f. 2 (175o); Hartl. Syst. Verz. Ges. Mus. 85 (18+4); Horsf. \& Moore, Citt. B. Mus. E. I. Consp. ii. p. 629 (1856).

Psittacus lory, Linn. S. N. i. p. 145 (1766); Gm. S. N. i. p. 335 , n. ${ }^{\circ} 27$ (г788); Lxth. Int. Orn. ii. p. I 55 , n. ${ }^{\circ} 99$ (г 790); Shaze, Nat. Misc. lam. 633 (1863?); id. Gen Zool. viii. p. 534 (1812); Becnst. Kurze Uebers. F. 92 (1811); Kuhl, Consp. Psitt. p. 4r (1820); Vell. Enc. Meth. p. г 380 (1823); Less. Voy. Coq. i. p. $628(1828)$; Vo:gt, Cui. Uebers. p 7+5 (885r); Temm. Tabl. Meth. p. 6o (I839); S. Mïll. Verh. Land-en Volkenk。 p. I 07 ( $1839-18+4)$.

Lory des Philippines, D'Aub. Pl. Enl. 168.

L. tricolor, Buff. Hist. Nat. Ois. vi. p. I32 (г779).

?Grand perruche á bandean noir, Buff. Hist. Nat. Ois. vi. p. i 58 ( I 779$)$.

!Black-crowned parrot, Lath. Syn. i. p. 2г3 (г78г); id. Gen. Hist. ii. p. I 25 (1 822 ).

Black-cilped lory, Lath. Syn. i. p. 273, n. ${ }^{0} 78$ (r78r).

Psittacus atricapillus, Gm. S. N. i. p. 317 (1788); Lath. Ind. Orn. i. p. 88, n." I8 (1790); Bechst Kurze Uebers. p. 63 (1881); Vieill. Enc. Meth. p. I 387 (1823).

Lory á scapulaire blew, Levaill, Perr. lams. 123, 124 (1805).

Psittacus solanus, Shaw', Gers. Zool..viii. p. 459 (1812(.

Lorius tricolor, Steph. Gen. Zool. xiv. p. I32 (1826); Less. Voy. Coq., Zool. i. p. 628 (1828); id. Tr. d'Orn. p. I92 (1831); G. R. Gr. Gen. B. ii. p. $416, \mathrm{n}^{\circ} 2$ (1845); Bp. Rev. et Mag. de Zool. 1854 , p. 156 sp. 282; id. Naumannia, 1856 , Consp. Britt. Psitt, sp. 295; Scuancé, Rev. ct Mag. de Zool. 1856, p. 225; Sclat. Journ. Pr. Linn. Soc. ii. p. $16_{5}$, sp. $108(1158) ; G . R$. Gr. P.Z. S. 1858 , p. 794 (parte); id. Cat. B. New. Guin, 
pp. 3959 (parte) (1859); id. P. Z. S. 1839 , p. 158; Sclat. P. Z. S. 1860 , p. 227; Wall. Ibis, 1861 , p. 311; G. R. Gr. P. Z. S. 1861 , p. 436; Rosenb. Journ.f. Orn. 1862 , pp. 63. 64; id. Nat. Tijdschr. Ned. Ind. xxv. pp. 142, 143, 147, 225, sp. 17 (180́3); Bernst. Nat. Tojdschr, Ned. xxvi. p. 297 (1854); Rosenb. Journ. f. Orn. 1864 , p. 114 , sp. 17; Schleg Mus. P.B. Psttaci, p. I 8 (185 ); Finsch. Nev. Gitin. p. I57 (1855); Schleg. Mus. P.-B. Psittaci, Revue, p. 54 (1874); Garrod, P. Z. S. 1874 , p. 587 ; Sclat. 1'. Z. S. 9874 , p. 684, pl. xvi. f. 11 ; Sclat. Vert. An. 8a-ed. p. 318 (1883).

Domicella lory, Wagl. Mon. Psitt. p. 568 (1832); Wall. Ann. \& Mag. N. H. (3) iii. p. I 47 (1859).

Psittacus lorius, Weinl. Journ. f. Orn. 1854 ; B. p. lxxv.

Lorius lory, Wall. Ann. \& Mag. N. H. (3) iii. p. I +8 (1859); id. P. Z. S. 1864 , p. 288; Salvad. Ann. Mus. Civ Gen. vii. p. 759 (1875), x. p. 32 , n. ${ }^{\circ} 56$, p. I22, (1877); id. Orn. Pap. d Mo! i. p. 223 (1830; id. Ca!. B. M. 20. 1897 p. 33; Ne'rk. Journ.f. Orn. 1885 , p. 31; Guillem. P. Z. S. 1885, p. 6r 2; Salvad. Ibis, 1886 , p. I54; id. Men. R. Ac. Sc. Tor. sar. 2, xl. p. 167 (1889); Myor, Vogel-Skelett. t. 1xix. (1878-89).

L. cyanauchen, Rosenb. (n) Mïll.), Nat. Tijdsuhr. Ned. In.t. xxi. pp. 143, 225, sp. I8 (1863; id. Journ. f. Orn. 1874, p. I14, sp. I8; Bernst. Detgboek, pp. 82 I26, I5 I : 1833).

Donicella lory, Finsch. P.ip.rg. ii. p. 769 (1868); Meyer, Sitzb. k. Ak. Wissensch. Wien, lxx. pp. 233, 238 (parte), (1874); id. Sitzb. Isis, Dresd. 1875 , p. 78 (parte); id. Ibis. 1875, p. 147; Rchno Vogelbild. t. xi. f. 2 (1878-83).

Lorius lori, G. R. Gr. Hant-list, ii. p. I53, sp. 8397 (187)); Garrod, P. Z. S. 1874 , p. 587 ; Salv. Cat. B. Sirickl. Coll. p. 455 (1882); Tristr. Cat. Coll. B. p. 73 (1839).

Domicella tr color, Rchna. Vogelbild. t. xi. f. 2 (1878-83); id. Journ. f. Orn. 1881, p. 272 (Consp. Ps.tt. p. 1oS).

Eos (!) tricdor, Sclat. List. Vert. An. 8th. ed. p. 318 (1883. $H a b$. Filipinas?

\section{Gén. PALEORNIS.}

Pag. 58. P. torquita (Briss.) ơ N. v.

(M. S. T)

'The long-tailed green parrot, Eduv. Glean. iv. pl. I75 (175I);

(West Indies); Lath. Gen. Syn. i. p. 230 (w.thout vitr. A)

1781 ; id. Gen. Hist. ii. p. I 53 (1822).

Psittaca, Briss. Orn. iv. p. 319, (1 760$)$.

Ps. torquata, Briss. Orn. iv. p. 323 , n. 55 (1760).

Psittacus alexandri (part.), Linn. S. N. i. p. I41 ( 1766).

? Psittacus rufirostris, Linn. S. $N$. i. p. I43, n. i 8 ( ?) (1765.)

Gm. S. N. i. p. 323, n. ${ }^{\circ} 8$ (i788); Lah. Ind. Orn. i.

p. 95, n. 39 (1790); Bechs. Lath. Uebers. p. 73. (I8I1);

Kuhl, Consp̈. Psitt. p. 26 (1820); Vieill. Ens. Méth. iii. p.

I 4)1 (1823); Veig', Cuv. Ues. p. 729 (1831).

? Ps. krameri, Scop. Annus. I. Hist. N.t. p. 31 ( 1769).

La Perruche, D'Aubent. Pl. Enl. 550 ( $)$.

La Perruche á collier, D'Aubent. Pl. Enl. 55 I (o). 
La perruche á collier couleur de rose, Buff. Hist. Nat. Ois. vi. p. 152 (1779).

Le Sincialo, Buff. Hist. Nat. Ois. ri. p. 265.

Alexandrine parrakeet, var. $A$, Rose-ringed Parrake't, Lath. Syn. i. p. 235 ( 1781$).$

Psittacus torquatus, Bodd. Tabl. Pl. Enl. p. 32 (I783); Kiahl. Consp. Psitt. p. 3o (1820); Voigt, Cuv. Ueb. p. 727 (1831); Sundev. Physiogr. Sallsk. Tidskr. 1837, p. 173; Thienem. Furtpft. d. ges. Vög. p. 73 (1846).

P. alexandri, var. B. Gm. S. N. i. p. $32 x$ (1788); Lath. Ind. Orn. i. p. 98 (1790).

P. manillensis, Bechst. Stubenvög. p. 6r 2 (1794); id. Lath. Ueh. p. 75, n. 50 (1811); Hahn, Orn. Atl., Papag. p. 17, t. 6 (o) (I 834$)$.

Alexandrine parraqueet, Lath. Syn. Suppl. ii. p. 86 (1802).

La perruche á collier rose, Levaill. Perr. p. 42 (o'), lam. 23 (joven) (1801).

La perruche sincialo, Levaill. Perr. p. 42 (Q) (1801).

La perruche souffre, Levaill. Perr. lam. 43 (var.) (I801).

Sulphur parraqueet, Shaw, Gen. Zool viii. 2, p. 428 (181 I); Lear, Parr. lam. $33(1832)$.

Psittacus s ncialo, Shaw, Gen. Zool. viii. 2, p. 44r (1811).

Rose-ringed parrakeet, Lath. Gen. Hist. ii. p. I60 (1822).

Palæornis torquatus, Vig. Zool. Journ. ii. p: 50 (part.) (I825); Steph. Gen. Zoob. xiv. 2, p. 126 (1826); Frankl. P. Z. S. 1831 , p. 120; Sykes, P. Z. S. 1831 , p. 120; Sykes, P. Z. S. $1832 ;$ p. 96 (Dukhum); M. Clell. P. Z. S. 1839 , p. 165; Irzuin, J. A. S. B. viii. p. I0J7 (1839) (Oxus); Jerd. M.tdr. Journ. xi. p. 207 (1840); Blyth, Ann. \& Mag. N. H. xii. p. 90 (1840); Eiver, P. Z. S. 1842 , p. 92; Strickl. P. Z. S. 2842, p. I67; Hodgs; Gray's Misc. p. 85 (1844); Gray, Cat. Hogds. Coll. B. Mus. p. II $3(18+6)$; G. R. Gr. Gen. B. ii. p. 409, n. 3 (parte) (1846); Blyth, Cat. B. Mus. A. S. B. p. 4 (18+9); id J. A. S. B. xix. p. 23I (1850); Kelaart, Prodr. Faun. Ceyl. p. I 27 (1852); Layard, Ann. \& Mag. N. H. (2) xiii. p. 262 (1854); Burg. P. Z. S. 1854, p. 256; Theobald, J. A. S. B. xxiii. p. 595 (1854); Hartl. Journ. f. Orn. 1854 , p. I $58 ; B p$. Rev. et Mag. de Zool. 1854, p. I 52, n. ${ }^{\circ}{ }^{\text {I }} 3^{8}$; Chesnoy, Jour. of the Euphr. Exped. i. pp. 443537 ; Sonancé, Rev. et Mag. de Zool. 1856 , p. 057; Bp. Naumannia, 1856 , Consp. Psitt. n." I 43; Horsf. \& Moore, Cat. B. Mus. E. I. Comp. ii. p. 61 1 (1856.58); Philipps, P. Z. S. 1857, p. 99; Adams. P. Z. S. 1858 , p. 490, 1859 , p. 173 Morre, P. Z. S. 1859 , p. $453 ;$ G. R. Gr. List Psitt. Brit. Mus. p. I9 (1859); Irby, Ibis, 1861 , p. 218 ; lerd. B. of Ind. i. p. 257 (1 862); Blyth, Ibis, 1863 , p. 2; Finsch, Ned. Tijdschr. Dierk. i. Berigt. p. ix. (1863); Schlog. Mus. P.-B. Psittaci, p. 80 (parte) (1864); Park. P. Z. S. 1865 , p. 238 ; Beav. ibid. p. 691; id. Ibis, 1865 , p. 409, 1857 , p. 320; Layard, B. of S. Afr. p. 320 (nota) (1867); Finsch, Papag. ii. p. 17 (parte) (1868); Hume, Ibis, 1869, p. 4; Sousa, Mus. Nuc Lisb. Psittaci, p. It (1869); Blyth, Ibis, 1870 , p. 162; G. R. Gr. Hand-list, ii. p. 142, n." 855 (1870); Anters. P. Z. S. 1871, p. 679, 1872 , p. 80; Holdsw. P. Z. S. 1872 , p. 425; Elwes, P. Z. S. 
1873 , p. 661; Hume, Str. Feath. i. p. 170 (1873); 1d. Nests \& Eggs, p. I16 (1873); Lloy.t, Ibis, 1873, p. 407; Hume, Str. Feath. ii. p. 13 (1874); Ball, ibid. p. 389 (Chota Nagpur); Legge, Ibis, 1874 , p. I 4 ; Wald. Ibis, 1874 , p. 282; Schleg. Mus. P.-B. Psittaci, Revule, p. 35 (part.) (1874); Gulliz. P. Z. S. 1875, p. 489; Bly't', B. of Buurm. P. 55 (1875) Legge, Ibis, 1875 , p. 282; Hume, Str. Feath. iii. p. 56 (1875); Butl. Str. Feath, iii. p. 457 (Mount. Aboo et N. Guzerat) (1875); Gieb. Thes. Orn. iii. p. I I (part.) (1877); Hume. \& Davis. Str. Feath. vi. p. I 8 (1878); Hume, Str. Feath. viii. pp. 5I, 86. n." I 48 (1879); Scully; Str. Feath. viii. p. 240 (1879); Schmidt. P. Z. S. 1880 p. 309; Legge, B. of Ceyl. p. I7 I (1880); Rchnzw. Vogelbild. t. v. f. 7 (1878-83); id. Journ. f. Orn. 1888, p. 235 (Consp. Psitt. p. 124); Salv. Cat. B. Strickl. Coll. p. 457 (part.) (1882); Oates, Str. Feath. x. p. I89 (1882); 2d. B. Brit. Burm. ii. p. I41 (1883); Sclat. List Vert. An. 8th ed. p. 321 (1883); Szunh. \& Barn. Ibis, 1885, p. 65; Park. Ibis, 1886, p. 183; Sundev. Ibis, i886, p. 454; Hume, Str. Feath. xi. p. 5t (1888); Tristr. Cat. Coll. ${ }_{B}$ p. 75 (1889); Murr. Avif. of Brit. Ind. ii. p. 467 (1890); Oates Nests \& Eggs. Ind. B. iii. p. 85 (1890); Salv. Cat. B. M. 20. 1894 , p. 443 .

Conurus rufirostris, Less. Tr. d'Orn. p. 213 (1831); Le Maout, Hist. Nat. Ois. p. 97 (1853).

C. Torcunatus, Less. Tr. a'Orn. p. 215 (1831).

Palaarnis cubicularis, Wagl. Mon. Psitt. p. p. 507 (1832).

Psittacus torquatus luteo varians, Bourj. Perr. lam. $2\left(1837-3^{8}\right)$.

Palaornus bitorquatus, Blyth (no Kuhl), Cat. B. Mits. A. S. B. p. $4(1849)$.

P. latyardi, Blyth, Cat. B. Mus. A. S. B. P 34 (1849); Bp. Rev. et. Mag. de Zool. 1854 , p. I52, n. ${ }^{3}$ it2; G. R. Gr. List Psitt. Brit. Mus. p. 20 (1859); Sousa, Mus. Nac. lisb. Psittaci, p. 15 (1869); G. R. Gr. Hand-list, ii. p. I43, n." $8060 \quad(1870)$.

P. frenatus, (part.), Licht. Nomencl. Av. p. 7 I (185t).

P. torquata, Beav. Ibis, 1869 , p. 4ro; Luyard, Ibis, 1869 , p. 450 (var) Garrod, P. Z. S. 1873 , p. 634, 1874, p. 587; Parker, Ibis, 1883 , p. 193 .

Hab. Ceilan, Luzón.

Pag 58. Gén. EOS, Wagler.

E. rubiginosa, $B p$.

Chalcopsitta rubiginosa, Bp. Consp. An. i. p. 3 (1850); id. Compt. Rend. xxx. p. 134 (1850); id.P. Z. S. 1850 , p. 26 (no), lam. xvi.); id. Rev. Mag. Zool. 1854 , p. I56, n. 292 ; id. Naumannia, 1856 , Consp. Psitt. n. 306 ; Sclat. P. Z. S. $1860, \mathrm{p}$. 227; Wal.'. Ibir, 1861 , p. 29?; G. R.Gr.P.Z. S. 1861 , p. 436; Rosenb. Journ. f. Orn. 1362, p. 63; id. Nat. Tijdschr. Ned. Ind. xxv. p. I43 (1853); Wall. P. Z. S. 1864 , pp. 289, 294; Pclz. Novara Reise, Vög. p. 99 (i 865$)$ ) G. R. Gr. Hand-list, p. I53, 11." 8193 (1870); Tristr. Cat. Coll. B. p. 73 (1889). 


$$
-6+3-
$$

Eos rubiginosa, G. R. Gr. List Psitt. Brit. Mïs. p. 53 (1859; id Cit. B. New Giuin. Pp. to, 59 (1859); Rosens. Journ. $f$. Orn. (1864), p. I It; Salvad. Orn. Pap. é Mol. i. p. 267 (1880); id. Cat. B. M. 20. $18^{2} 97$, p. 29.

Lorius rubiginosus, Schleg. M/us. P.-B. Psittaci, p. I 29 (1864); Finsch, New-Guin. p. I 57 (1865); Schleg. Mus. P.-B. Psittaci, Revue, p. 58 (1874); Giel. Thes. Orn. ii. p. 502 (1875).

Dumicella rubiginusa, Finsch, Papag. ii. p. 78 I (1868); id. P. $Z$. S. 1869 , pp. 127 I 28,1872, p 88.

'Trichoglossus rubiginosus, Finsch, Journ. Mus. Godeffr. Heft. xii. P). I 7, I 8 (1876); id. P. Z. S. 1877, p. 778 ; id. Journ. f. Orn. 1880, p. $284 ;$ id. Ibis, $1 \& \delta 1$, pp. IIo, III, IIt; Rchnzi. Journ.f. Orn. 1881 , p. 162 (Consp. Psitt. p. 98); id. Vogelbild. t. xxx. f. $6(1878-83)$.

Hab. Carolinas, Ponapé.

Pág. 66. Accipiter manillensis, Miyin.

Wardlaw Ramsay, Ibis, 1884, p. 330 .

Hab..... Guimarás.

Pág 71. Spilornis holospilus, $V i_{S}$.

Hab.... Masbate; Romblon, 'Tablas, Sibayan, Joló, 'Tawi-tawi.

Pág. 72 S. panayensis.

Steere, List. B. etc. M. Steere, Expect. i 890, p. 7 (es sinonimia de la especie i I) S. holospiclus, Vig.

Hab... Masbate, Tablas, Romblon, Sibuyan, Joló, 'Tawi-tawi.

Pág. 72. Butastur indicus, ( $\mathrm{Gm}$.)

Hab... Calamianes, Masbate, Joló, Tawi-tawi.

Pág. 74. Cuncuma leucogaster, (Gm.)

Hab... Mindoro, Naujan, Calamianes, Culión, Masbate, Tablas, Romblon, Sibuyan, Siquijor, Basilan, 'Tawi-tawi.

Pág. 75 Haliastur intermedius, Gurn.

$H a b \ldots$ Tablas, Romblon, Masbate, Sibuyan, Calamianes, $\mathrm{Cu}-$ lión, Basilan, Tawi-tawi.

Pág. 77. Elanus hypoleucus, Gould.

Hab... Calamianes, Culión, Tawi-tawi.

Pág. 77. Pernis ptilonorhynchus, (Tem.)

Hab... Cebú, Sibuyan. 


\section{Pág. 81. Hypotriorchis severus, (Horsf.)}

Hab... Cebú, Romblon, Siquijor, Calamianes, Sibuyan, Tawitawi.

Pág 88. Ninox lugubris, Tiek.

Hab... Masbate, Joló.

Pág. 88. N. philippin€nsis.

Hab... Masbate.

Pág. 89. N. Epilonotus Fr. et Worc.

Prelim. Not. Menage Exped. Philipp. 1894, p. 8.

Hab. Cebú, Sibuyan, "I’ablas, Mindoro, Baco.

Pág. 89. N. japonica, $T$. et $S$.

Sharpe, P. Z. S. 1879 , p. 325.

Hab. Cebú.

Pág. 94. Corone philippina.

Hab... Romblon, Tablas, Sibuyan, Tawi-tawi.

Pág. 98, Oriolus achorhynchus, Vig.

Hab... Romblon, Masbate, Sibuyan, Tablas, Calamianes, Tawitawi.

Pág. 99. O. samarensis,

Steere, List. B. et M. Steere Exped. 1890, p. 17; id. Ibis, 1891, p. $31 \mathrm{I}$.

O. cinereogenys, Fr. et Worc.

Prelim. Not. Menag. Exped. Philipp. 1894, p. 16. Hab. 'Tawi-tawi.

O. nigrostriatus, Fr. et Worc.

Prelim. Not. Menage Exped. Philipp. 1894 , p. 16. Hab. Negros, Masbate.

Pág. Ioo Buchanga palawanensis, Whiteh.

Whilehead, Ibis, 1890 , p. 47.

Hab. Calamianes, Paragua. 
Pág. 101. Chibia menagei, Fr. et Horc.

Prelim. Not. Menage Exped. Philtpp. 1894 p. 15.

Hab. Sibuyan.

Ch palawanersis, (Tw.)

Sharpe, Ibis, 1884 , p. 318 .

Hab. Paragua, Calamianes.

Ch. borneensis, Sharpe.

Sharpe, P. Z. S. 1879, p. 246.

Hab. 'Tawi-tawi.

Fàg. id. Artamides sumatrensis, (S. Mïll.)

Hab... Calamianes, Culion.

Pág. 102 A. mindorensis.

Steere List, B. et M. Steere Exped. Philipp. 1890, p. 14. Hab... Tablas.

Pag. 102. A. guillemardi, (Salv).

Salvad. Ibis, 1886 , p. I 54 .

Hab. Tawi-tawi.

Pag. 103. Edoliisoma everetti, Sharpe.

Sharpe, Ibis, 1894, p. 254.

Hab. Joló, 'Tawi-tawi.

Pag. 104. Pericrocotus leytensis, Steere.

List B. et $M$. Steere Exped. p. I 5 (1890).

Hab... Samar, Borongan, Paric.

Pug. 105. Lalage terat, (Boaic).

Hab... Calamianes, Tablas, Romblon, Sibuyan, Siquijor.

Pag. 10 5. I. minor, (Steere).

Pseudolalage minor, Steere, List Bel. u. Steere, Exped. Philipp. $1890, \mathrm{p}, 15$.

Hab... Samar. 
Pág. 106. Gén. CRYi'TOLOPHA, Swainson.

1. C Olivacea, Mossely.

Mossely, Ibis, 1891 , p. 46 .

Hab. Mindanao, Joló, 'lawi-tawi.

2. C flavigularis, Fr. et Worc.

Prelim. Not. Menage Exped. Philipp. 1894, p. 23. Hab. Cebú.

\section{Gén MUSCiCapula, Salv.}

M. mindanensis, Blas.

Blas. J. f. O. 1890, p. 147 .

Hab. Basilan.

M. samarensis, Gr. et Wore.

Prelim. Not. Menage Exped. Phlipp. 1894, p. 26.

Hab. Samar.

Pag. 107. Muscicapa griseisticta, (Swinh).

Hab. . Mindoro, Baco, Siquijor, Masbate, Calamianes, Tawitawi.

Pag 108 P. caprata.

Hab... Masbate, Siquijor.

Pag. I 10. Hypothymis azurea, (Bodd).

Hab... Tablas, Romblon, Sibuyan, Masbate, Siquijor, Calamianes, Tawi-tawi.

Pag. 111. Cyanomyas cælestis, Tiv.

Hab... Sibuyan, (Men. Exped.)

Pag. 1 I 1 . C. belenæ, Steere.

List B. et M. Steere Exped. Philipp. 1890 , p. 16; Ibis, 1891 , p. 3 II.

Pag. II . Rhipidura sauli, Fr. et Worc.

Prelim. Not. Menage Exped. Philipp. 1894, p. 26.

Hab. Tablas. 
Pag. 112. Rh. nigritorquis. Vis,rs.

Hab... Samar, Borongan, Min!loro, Calamianes, Tablas, Romblon, Sibuyan, Masbate, Siquijor, 'Tawi tawi.

Pag. 112. Zeocephus rifu3, $B_{.}$.

Hab... Tablas, Romblon, Sibuyan, Cabú.

z. cinnamomeus, Shaps.

Hab... Tawi-tawi.

Pag. 113 Z. cyanescens, Sh.

Hab... P. Princesa, Calamianes, Culion.

Rhinomyas albigularis, fir. et Worc.

Prelim. Not. Menage Exped. Philipp. 1894, p. 27.

Hab. Negros, Guimarás.

Rh. ozularis, Gr. it Worc.

Prelim. Not. Menage Exped. 1894, p. 28.

Hab. Joló, 'Tawi-tawi.

Cu'icicapa penayensis, Sharpe.

Haß... Romblon, Iablas, Sibuyan, Guimarás, Masbate, Siquijor, 'Tawi-tawi.

Pag I15. Siphia philippinensis, Sharpe.

Hab... Tablas, Siquijor, Romblon, Sibuyan, Guimarás, Masbate, 'Tawi-tawi.

\section{S. lemprieri}

Sharpe, Ib:s, 1894, p. 319.

Hab... Calamianes.

Pag. 116. Phylloscopus borealis.

Hab... Samar, Borongan; Calamianes, Tablas, Sibuyan, Joló, Tawi-tawi.

Pag. 118. Acrocepharus orientalis, Tim.

Hab... Mindoro, Baco. 
Pag. 121. Geocichla cinerea, Fr. et Worc.

Prelim. No'. Menage Exped. Philipp. 1894, p. 23.

Hab. Mind ro, Naujan.

Pag. 128. Erithacus calliope

Hab... Masbate.

Pag 129. Monticola solitaria, (Bris.)

Haß... 'Tablas, Masbate, Romblon, Sibuyan, Siquijor, Joló, 'Tawi-tawi.

Pag. 133 Iole haynaldi, (Blis.)

Sharpe, Ibis, 1894 , p. 253.

Hab. 'Tawi-tawi.

Ioie cinereiceps. Fr. et Worc.

Prel. Not. Menage Exped. Phalipp. 1894, p. 25.

Hab. Tablas, Romblon.

Iole monticola, Fr. et Worc.

Prel. Not. Menage Exped. Philipp. 1894, p. 25. Hab. Cebú.

Pág 135. Pycnonotus goiavier.

Hab... Masbate, Tablas, Panay.

Pag. 136. P. cinereifrons.

Hab... Calamianes.

Pág. I36. Irena tweaddt.li.

Hab... Calamianes, Çulion.

Pag. I38. Copsychus mindanensis.

Hab... Mindoro, Naujan, Tablas, Sibuyan, Masbate, Siquijor, Tawi-tawi.

Pag 139. Cittocincla cebuensis, Steere.

Steere, List. B. et M. Exped. Philipp. 1894, p. 20; Id. Ibus, 1891, p. 3 I 4 .

Hab. Cebú. 
C. superciliaris, Fr. et Worc.

Prel. Not. Menage Exped. Philipp. 1894, p. 23.

Hab. Masbate.

Pig. 140. Megalurus palustris.

Hab... Masbate.

M. ruficeps.

Hab... Calamianes, Culion.

l'ág. I4I. Orthotomus castaneiceps.

Hab... Masbate.

l’ág. 142. O. ruficeps.

Hab... Calamianes, Culion.

Hag. 145. Cisticola exilis, Vig.

Hab... Samar, Borongan, Tablas, Sibuyan, P.ınay, Cebú, Siquijor, Masbate, Mindors, Baco, Calamianes, Culion.

Pag. 147, Mixornis plateni, Blas.

Blas. J. F. O. 1890, Pp. 145,147 .

Hab. Samar, Paric.

Ptilocichla minuta, Fr. et Worc.

Prelim. Not. Menage Exped. Philipp. 1894, p. 21.

Hab. Samar, Borongan, Calbayog.

Pág. 149. Parus elegans.

Hab... Mindoro, Baco, Tawi-tawi.

Pag. 150. Pachycephala winchelli, (Fr. et Worc).

Hyloterpe winchelli, Franck et Worcest. Prelim. Not. Menage Exped. Philipp. 1894 , p. 21.

Hab. Panay, Negros, Masbate.

P. major, (Fr. et Worc).

Hylcterpe major, Franck et Worc. loc, cit. p. 22.

Hab. Cebú, 'Tablas, Siquijor: 
P. mindorensis, Fr. et Worc, oo N. v.

Hyloterpe mindorensis, Gr. et Worc. loc. cat. p. 22.

Hab. Mindoro, Calapan, Naujan.

P. homeyeri, Blas.

Blas. J. f. O. 1890, p. 143 .

Hab. Mindanao, 'Tawi-tawi.

Pág. 151. Lanius nigriceps.

Hab... Masbate, Calamianes, Culion.

\section{I. lucionensis.}

Hab... Calamianes, Cu ion, Tablas, Romblon, Sibuyan, Siquijor, Joló, Tawi-tawi.

Pég. 154. Climacteris mystacalis.

Hab... Negros, Masbate.

P’ág. 156. Sitta oenochlemyø.

Hab... Samar, Borongan, Parauas.

l'ag. 158. Ethopyga arolasi, Fr. et Worc.

P;el. Not. Menage Exped. Philipp. 1894, p. 17. Hab. Joló, 'Tawi-tawi.

Ae. bonita, Fr. at Worc.

Prelim. Not. Menage Exped. Philipp. p. 17 (1894).

Hab. Negros, Cebú, Masbate.

Ae. minuta, Fr. ct Worc. oo N. v.

Prelim. Not. Menage, Exped. Philipp. 1894 , p. I8.

Hab. Mindoro, Naujan.

Pág. 157. Ae. magrifica.

Hab... Panay, Tablas, Sibuyan.

Pág. 158. $\Lambda$ e. shelleyi.

Hab... Calamianes, Culion. 


\section{Cinuyris sperata.}

Hab.... Calamianes, Culion, Tablas, Romblon, Sibuyan, Panay, Siquijor, Cebú.

Pag. 159 C. juliæ.

Hab..... Tawi-tawi.

Pag. 160. C. guimaraensis.

Steere, List B. et M. Exped. 1890, p. 22 ; it. Ihis, 1894, p. 305. Hab... Panay.

C. jugularis.

Hab... Tawi-tawi, Tablas, Romblon, Sibuyan, Masbate.

Pag. ${ }^{6} 63$ Anthothreptes, chlorogaster.

Hab... Tablas, Sibuyan, Ro.nblon, Panay, Tawi-tawi.

Pag. 166. 14. bis. Zosterops cinerea, Kittl.

Hab. Marianas.

15. bis. $\mathrm{Z}$ siquijorensis, Fr, ct Worc.

Prel. Not. Menage Exped. Philipf. 1897, p. 2 I. Hab. Siquijor.

Pag. 166. 7, basilanica.

Steere, List. B. et M. Exped. 1890, p. 91 ; id. Ib.s, 1891, p. 314. -Z. everetti.

Hab... Tawi-tawi.

Pag. 167. Dicæum rubriventer.

Hab... Samar, Borongan, Masbate.

Pag. 167. D. palidior, Fr. et Worc.

Prelim. Not. Menage Exped. Philipp. 1894, p. 19. Hab. Cebú.

D. sibuyanica, Fr. et Worc.

Prclim. Note Menage Exped. Philipp. 1894, p. 19. Hab. Sibuyan. 


\section{$-652-$}

D. intermedia, Fr. et Worc.

Prel. Not. Menage Exped. Philipp. 1894, p. I9. $H a b$. Rombion, Tablas.

D. assimilis, Fr. et Wore.

Prel. Not. Menage Exped. Philipp. 1894, p. 19. $H a b$, Joló, 'Táwi-tawi.

D. mindanense.

Hab... Basilan, Joló, Tawi-tawi.

Pag. 169. D. pygmœum.

Hab... Samar, Borongan, Siquijor, Masbate, Sibuyan.

Pag. 170. Prionochilus æruginosus, Fr. et Worc.

Prel. Not. Menage Exped. Philipp. 1894, p. 20. $H a b$. Cebú, Mindanao.

P. bicolor, Fr. et Worc.

Prel. Not. Menage Exfed. Philipp. 1894, p. 20. Hab. Mindanao, Zamboanga.

P. modestus, Hume.

Hume, Str. F. p. 289, (1875).

Hab. Paragua.

Pag. 174. Hirundo gatturalis.

Hab... Mindoro, Baco, Basilan, Siquijor, Joló.

P’ag. 775. H. javanica.

Hab... Panay, Guimarás, Luzón, Laguna, Los Baños, Masbate, Siquijor, Calamianes, Tawi-tawi.

H. striolata.

Hab... Masbate.

Pag. I8o. Motacilla melanope

Hab... Romblon, Sibuyan, Masbate, 'Tablas, Joló, 'Tawi-tawi. 
Pag i $\$ 7$. Anthus ruphulus.

Hab... Calamianes, Culion, Tablas, Romblon, Sibuyan, Mashate.

Pag 190. A. gustavi.

Hab. Masbate, Romblon, Sibuyan, Tawi-tawi.

Pag. 197. Artamus leucogaster.

Hab... Tablas, Romblon, Sibuyan, Masbate, Calamianes, 'Tawi-tawi.

Púg. 200 Sarcops calvus.

Haß... Tablas, Romblon, Sibuyan, Tawi-tawi, Luzón, Laguna, Calamba.

Pas 202. Mainatus polawanensis.

Hab... Calamianes, Culion.

Pag. 205 Calornis panayensis.

Hab... Luzon, Laguna, Calamba, Samar, Borongan, Paranas, Calamianes, Culion, 'Tablas, Romblon, Sibuyan, Tawi-tawi.

Pag. 206 Munia oryzivora.

Hab... Manila, Pasig, Laguna, Calamba, Panay, Samar, Paric.

Pag 207. M. jagorii.

Hab... 'Tablas, Romblon, Sibuyan, Siquijor, Calamianes, Basilan, 'Tawi-tawi.

Pag. 208 Uroloncha everetti.

Hab... Calamianes, Tablas, Romblon, Sibuyan, Tawi-tawi.

Pag 216. Pitta erytrogastra.

Hab... Tablas, Sibuyan, Romblon, Panay, Masbate, Cebú, Siquijor, 'Tawi-tawi.

Pag. $2 \times 8$ P. atricapilla.

Hab:.. Tablas, Cebú, Calamianes, Tawi-tawi.

Pag 223 Colocalia fuciphaga.

Hab. Luzón, Pangasinan, Sual. 
Pag. 224. C. troglodytes.

Hab... Samar, Borongan, Cebú, Mindoro, Siquijor, Masbate, Sibuyan, Romblon.

Pag. 225. C. marginata.

Hab... Masbate.

Pag. 225. C. francica, (Gm)

La petite Hiro:adelle noire á croupion gris, Buff. Hist. Nat. Ols. vi. p. 696 (1779); ibid. fol. vii. p. 345 (1783).

Grey-rumped swallow, Lath. Gen. Syn. ii. p. 582 (1783).

Hirundo francica, Gm. Syst. Nat. ii. p. Ior 7 (1788); Lath. Ind. Orn. p. 580 (1 790); Steph. Gen. Zool. x. p. I I 5. (1817); Griff. An. Kingd. vii. p. 67 (1829).

Hirundo francisca, Vieill. Noze. Dict. xiv. p. 525 (18 I7).

Hirundo francia, Less. 7 r. Orn. p. 270 (1831).

Collocaila francica, Gray. List. Spec. B. Brit. Mus. p. 2 I (1848); E. Newton, Ibls, 1860 , p. 201; Cab. \& Heine, Mus. Hein. iii. p. 82 (1 863); Hartl. Orn. Bentr. Fiun. Madag. p. 26 (1861); Coquerel, Alb. Reunion, p. 21, fig. I (1855); Schleg. P. Z. S. 1866 , p. $42 \mathrm{I}$; Gray, Amn. \& Mag. Nat. His'. 1856 , xvii. p. I 22; E. Newton, Ibis, 1861,271 ; ibid. 1877 , p. 359; Schleg. \& Pollen, Faun. Madag. ii. p. 67, (1868); Gray, Hand-l. i. p. 65 (1869); Walden, Ibis, 1874 , p. I 32 (part.); i.t. ib.d. I876, p. $35^{6}$ (part.); Hartl. Voy. Madag. p. 59 (1877); Milne-Edw. \& Grandid. Madagascar, p. 198, pls.-lxxv. (1879); Salo. Cat. Strickl. Coll. p. 372 (1882); Hart. Cat. B. M. 16,1892, p. 503.

Macropteryx spodiopygia, Peale, U. S. Expl. Exp. 1848, p. 170, pl. 49 i 848 , Hartl. Wiegm. Arch. 1852 , p. I 2.

Collocalia spodiopygia, Cass. U. S. Expl. Exp. 1858 , p. I $8+$, lam. xii. fig. 3 (1858); Gray; Ann. \& Mag. Nat. Hist. $188^{2} 6$, xvii. p. i22; id. Hand-l. i. p. 65 (1869); Hartl. \& Finsch, Orn. Centr.-Polynes, p. 48 (1867); Finsch. \& Hartl. P. Z S. 1871, p. 23; Whitmee, Ibis, 1875, p. 437; Lxyard, Ibïs, 1876 , p. 391; Kleinschm. Journ. Mus. Godeffr. xii. p. 866 (1875); Cab. * Reichen. J. F. O. 1876, p. 324; Finsch, P. Z. S. 1876 , p. $19 ;$ id. P. Z. S. 1877 , p. 723 ; Ramsay. Pr. Linn. Soc. $N$. S. W. iii. p. 265 (1878); S.zlvad. Ibs, 1879, p. 323; id. Atti. R. Ac. Sc. Tor. xv. p. 347 (1880); id. Orn. Papuasia, i. p. 546 (1880); Scl. Voy. Challeng. pp. 34, 44 (1880).

Cypselus francicus, Schlg. \& Pollen, Ois. Mad. ii. p. 67 (1868).

Cypselus terræ regina, Ramsay, P. Z.S. I874, p. 6о1.

Collocalia terræ reginx, Gould, B. N. Guinea, iv. pl. 38 (I875); Ramsay, Proc. Linn. Soc. N.S. W. ii. p. 178 (1877); Sharpe, Journ. Limn. Soc. N. S. W. iii. p. 265 (1878); Salvad. Ibis, 1879, p. 323.

Collocalia infuscata*, Salvad. Atti. R. Ac. Sc. Tor. xu. p. $34^{8}$ (1880); id. Orn. Papuasia, i. p. 447 (1880); id. Atti. R. Ac. Sc. Tor. xvii. p. $45^{\circ}$ (1882); id. Agg. Orn. Pap. i. p. 64 (1889). Hab. Pànı, Calamianes; Australia. 


\section{Pag. 226. Macropteryx comata.}

Hab... Samar, 'Tablas, Masbate, Joló, 'Tawi-tawi.

Pag. 227. I bis. Caprimulgus jotgka, Time it Schleg.

Caprimulgus jotaka, Temm. \& Scileg. Fantn. Jap., Aves, p. 37, lam. 12 (1847); Bp. Consp. i. p. 60 (1850); Schrenck, Vög. Amurland, ii. p. 253 (185i); Sivinx, Ib:s, 1851 , p. 327; id. P. Z. S. 1863 , p. 263 ; id. Ibis, 1870 , p. 89; id. P. Z. S. 1871, p. 344 ; id. Ibis, 1874 , p. 434 ; id. Ibis, 1875 , p. $33 \mathrm{I}$; Radde, Rus. Sib:r., Vög. p. 133 (1853); Whitely, Ibis, 1837 , p. 195; Gray, Hand-l. i. p. 57 (1869); Swinh. P. Z. S. 1871 ; p. 344; Tircz. J. f. O. 1872 , p. 351; 2d. Bull. Sos. Zool, Fr. 1876, p. 132; Prjevalski, Rowol. Orn. Misc. ii. p. 157 (1877); Dav. \& Oust. Ois. Chine, P. 67 (1877); Blak. \& Pryer, Ibis, 1878 , p. 251; Huine \& D.tvison, Stray, F. vi. p. $5^{6}$ (1878); Scully, Stray F. viii. p. 235; 1879); Jo:Ly, Proc. U. S. Nat. Mus. vi. p. 310 (188.3); Oates, B. Burm. ii. p. 21 (1883); id. ed. Hume's Nest. \& Eggs. Ind. B. iii. p. 45 (1890); 'Tristr. Ibis, 1885, p. 194; Styan, Ibis, 1887, p. 228, Hume, Stray F. xi. p. 38 (1888); Seeb. P. Japan. Emp. p. 178 (1890); Hartert, Ibis, 1892, p. 283 .

Caprimulgus indicus, Pelz. Reis. Nov. Vög. p. 34 (1865); Plyth, B. Burma, p. 83 (1875); Hume, Stray F. v. p. 17 (1877); p. 3 18 Hume \& Davis. Stray, F. vi. p. 56 (1878).

C. dytîscivorus, Swing. Ibis, 1860 , p. 130 .

C. melanopogon, Salvad. Ann. Mus. Civ. Gen. vii. p. 918 (1875); x. p. 31 1 (1877); id. Orn. Papuas, i. p. 530 (1880).

Caprimulgus innominatus, Hume, Stray F. 1875, p. 318, nota Hab. Paragua, P. Princesa; Borneo, China.

\section{C. macrurus.}

Hab... Sibuyan.

Pag. 228. Isyncornis mindanensis.

Hab... Basilan.

Pag. 230. Batrachostomus menagei. Fr. et Wirc.

Prclim. Not. Menage Exped. Philipp. 1894, p. 11. Hab. Filipinas.

Pag. 231. Eurystomus orientalis.

Hab... Tablas, Sibuyan, Romblon, Calamianes, Tawi-tawi.

Pag. 232. İierops bico'or.

Hab... Tablas, Masbate, Calamianes. 
Pag. 236. Pelargapsis gouldi.

Hab... Calamianes, Culion.

P. gigantea.

Hab... Cebú, Negros, Tablas, Sibuyan, Tawi-tawi.

Alcedo ispida.

Hab... Tablas, Romblon, Sibuyan, Panay, Tawi-tawi.

Pag. 242. Ceyx rufidorsa.

Hab... Calamianes, Tawi-tawi.

Pag. 243. Ceyx nigrirostris, Fr. et Worc.

Prel. Not. Menage Exped. Philipp. 1894, p. I3.

Hab. Panay, Negros, Cebú.

Pag. 245. Halcyon coromandus.

Hab... Masbate, Sibuyan, Tawi-tawi.

249. H. winchelli.

Hab... Tawi-tawi, Siquijor, Cebú, Tablas, Romblon, Sibuyan. lag. 250. H. chlzris.

Hab... Calamianes, Culion, 'Tawi-tawi, 'Tablas, Sibuyan, Masbate, Siquijor.

Pag. 258. Harpactes ardens. N. v.'Sinaguilala, Adac.

Hab... Laguna, Calamba.

Pag. 262. Iyngipicus menagei, fr. et Worc.

Prelim. Not. Menage Exped. Philipp. 1894, p. 262.

Hab. Sibuyan.

Pag 269. Thriponax philippinensis.

Steere, List. B. et M. Steere Exped. 1890, p. 8; 2d. Ibis, 1891 p. 315 .

Hab... Panay. 
Pag. 271. Xautholæma intermedia.

Hab... Tablas, Masbate.

Pag. 27 . Coccystes coromandus.

Hab... Paragua P. Princesa, Siquijor.

l'ag 274. Surniculus velutinus.

Hab... Samar, Borongan, Joló, 'Tawi-tawi.

Pag. 276 Cuculus canorus.

Hab... Siquijor.

Pag. 280. Cacomantis merulinus.

Hab... 'Tablas, Calamianes, 'Tawi-tawi.

Pag. 284. Eudynamis mindanensis.

Hab... Cebú, Siquijor, Masbate, Panay, Sibuyan, Romblon, Tablas, 'Tawi-tawi.

Pag. 289. Centropus viridis.

Hab... Siquijor, Tíablas, Romblon, Sibuyan.

C. javanicus.

Hab... Mindanao, 'Tawi-tawi, Siquijor, Cebú.

Pag 291 C. steerii, Fr. ct Worc. \& $\&$ N. v. Talahiban.

Prel. Not. Menage Exped. Philipp. 1894, p. I4.

Hab. Mindoro, Baco, Calapan.

Pag. 292. Dryococcy $x$ harringtonii.

Hab... Calamianes.

Pag. 294 Osmotreron axillaris.

Hab... Siquijor, 'Tawi-tawi.

Pag. : 95 O. v:rnans.

Hab... Negros, Masbate.

Pag. 298. Phabotreron occipitalis.

Hab... Samar, Mindoro, Calapan. 
Ph. nigrorum.

Hab... l'ablas, Masbate, Sibuyan.

Pag. 299. Leucotreron occipitalis.

Hab... Samar, Paranas, Mindoro, Naujan.

Pag. 300. I. leclanderi.

Hab... 'Tablas, Calamianes, Culion.

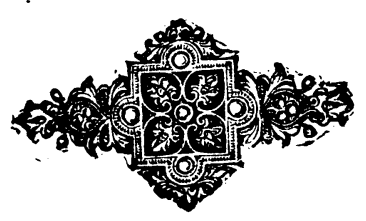




\section{INDICE.}

Tipo $10^{\circ}$ VERTEBRADOS. Clase $I^{\circ}$ MAMIFEROS. Subclase I. ${ }^{\mathrm{a}}$ MONODELFOS.

\section{Ordeu 1.० CUADRUMANOS.}

Fam. I. ${ }^{a}$ SIMIDOS.

Subfam. 1. ${ }^{a}$ SIMINOS.

I. Gén. Simia, Linn.

satyrus, Lin. var. bi-

color.

Subfam. 2." HILOBATINOS.

Gén. Hylobates, Illig. . . » funereus, Is. Geoff. . 》

Fam. 2. ${ }^{\mathrm{a}}$ CERCOPITÉCIDOS.

Subfam. SEMNOPITECINOß.

Gén. Semnopitecus, Cuv. . " entellus, $F$. Cav. . . 》 dussumieri, Desm. . . 2 leucoprymnus, Desm. . " albipes, I. Geoff. . . "

Gén. Nasalis, Geoff. . . nasica, Schreb. . . "

Gén. Cynamolgus, Reich. . 3 cynamolgus, Schr.. . 2 philippinensis, $I$. Geoff. var. albina. 2 et 623 palpebrosus, $I$. Geoff: 3 fur, Slack. var. . . " var. cumingii, Gray. ."

Gén. Macacus, Lacep. . . " speciosus, $F$. Cuv.. . » maurus, $F$. Cuv. . . 》 rhesus, Desm. . . ” sinicus, Lin.. . . . $\frac{\text { Pág. }}{3}$ nemestrinus, Dum.. . nemestrimus, var. philippinina. 4

Gén. Cynopithecus, Less. . " athiops, Zim. . . . 》

Fan. CÉBIDOS.

Gén. Cebus, Erxl. . . » chrysopus, $F$. Cuv. . »

Orden $2 .{ }^{\circ}$ PROsIMIOS.

Fam. LEMÚRIDOS.

Subfam, NICIICEBiNOS.

Gén. Nycticebus, Geoff . " tardigradus, (Lin.) 4 et 623

Fam. TÁRSIDOS.

Gén. Tarsius, Storr.. . . » spectrum, Pall. 4 et 623

Orden 3.० QUIRÓPTEROS.

\section{Suborden \\ MEGAQUIRÓPTEROS.}

Fam. TEROPÓDIDOS.

Gén. Pteropus, Bris. d 5 phœocephalus, Oldfield. " breviceps, Oldfield. . » leucopterus, Tem. . 》 edulis, Geoff. . . , " hypomelanus, Tem. . " keraudrenii, $Q$. et $G$.. 6 insularis, $H$. et $G$. var. jubatus, Esch. . . . " philippinensis. . . ” 
Gén. Cynonycteris, Ptrs. $\quad \frac{\text { Páf. }}{6} \mid$ *muricula, Hodg. . . $\frac{\text { Pág. }}{1.3}$

Gén. Kerivoula, Gray. . . " pellucida, Wat.. . . " marginatus, Geoff.. .
latidens, Dobs... . Gén. Miniopterus, Bp. . .

Subgen. Ptenochirus, Plrs. jagorii, Ptrs. . . .

Gén. Macroglosus, $F$. Cuv. " minimus, Geoff.. • . 》

\section{Suborden: \\ MICROQUIRÓPTEROS.}

\section{Fam. RINOLÓFIDOS.}

Subfam. RINOI.OFINOS.

Gén. Rhinolophus, Geoff. luctus, Tem.. . . philippinensis, Wat. . rufus, Ptrs.

schreibersii, Nat. . . > vrr. pusillus, Dobs. . 》 tristis, Wat.. . . . "

Fam. EMBALONÉRIDOS.

\section{Subfam. EMBALONLRINOS}

Gén. Emballonura, Tem. . " semicaudata, Pexle. . » monticola, Temm... .

Gén. Taphozous, Grof.. • I 5 melonopogon, Tem. . 》

Gén. Nyctinsmus Geoff. - " plicatus, Buch. . »

Subfam. FILORRRNINOS.

Gén. Phyllorhina, $B p$. . . 》 diadema, Geoff. . . . » pygmax, Wat.. . . 9 Jarvata, Horsf. . . . 》 obscura, Ptrs. . . 》 coronata, Ptrs. . . . " bicolor, Temm. . . 》

Fam. NICTÉRIDOS.

Gén. Megaderma, Geoff. . Io spasma, (Lin).. . . »

Fam. VESPERTILIÓNIDOS.

Gén. Vesperugo, Keys. . . 》 pachypus, Temm. . »

* noctula, Schr. . . . »

* maurus, Blas. . . . 11 abramus, Temm. . . 》 var. irretitus, Cantor. 》

* tylopus, Dobs. . . . I 2

Gín. Scotophilus, Lach. ." temminckii, Horsf. . 》

Gén. Vespertilio, Keys. . . 》 macrotarsus, tWat. . 》

* chínensis, Jobs. . ."

* formosus, Hodg. . . 》 capaccini, BP. . . 23

\section{Orden 4. INSECTIVROA.}

Subord. MESODONTOS.

\section{Tribu: IDERMÓPTEROS.}

Fam. GALEOPITÉCIDOS.

Sulfam. GALEOPITECINOS.

Gén. Galeopithecus, Pall . I6 volans, (Shaw). . . " rufus, Geoff. var.. . " variegatus, Geoff. var. » marmoratus, Tem. var. 》 philippinensis, Wat. var. 16 et 623

\section{Subord. INSECTIVOROS} (VERDADEROS.)

Fam. 'TUPÁYIDOS. Subfam. TUPAINOS.

Gen. Tupaia, Raffl. . . I ferru rinea, Raffl. . " javanica, Horst. i 7 et 623

Fam. ERINACÉIDOS. Gén. Erinaceus, L. . . . 17 
europœus, $L i n . . \quad . \quad \frac{P a g .}{17}$

Fam. SORÍCIDOS.

Gén. Crocidura, Wagler. cœrulescens, Shaze. murina, (Lin.). luzoniensis, Plrs. albina, $B / y / h$ edwardsiana, Trt. - aranea, Sclireb.

Gén. Mygale, $G, C u v$. pyrenaica, E. Geoff. .

Fam. CENTÉTIDOS.

Gén. Centetes, Illig. . . I9 ecaudatus, Schreb.

\section{Orden ROEHORES.}

Fam. ESCIÚRIDOS.

Subfam. ESCIĹRINOS.

Gén. Pteromys, G. Ciiv. . " petaurista, Pell. philippensis, Elliot. inornatus, Mäll, var.

Gén. Sciuropterus, F. Curv. melanotis, Gray. . pulverulentus, Gthr.

Gén. Fesciurus. Lat. affinis, Rafit.

Gén. Heterosciurus, Tr. prevostii, Desm. philippinensis, Wat. steerii, Gthr. lateralis, Var. . "

Gén. Nannosciurus, Trt. . » exilis, Müll. et Schl. . "

Gén. Funanbulus, Less. . . 21 vittatus, Raff... . .

Gén. Sciurus, Lin. . . . 》 vulgaris, Lin. . . . 》 samarensis, Steere. 2 r et 623 mindanensis, Steere. $2 \mathrm{I}$ coccinus, Thomas. 2 I et 623 nigripes Thos. . . .623

Fam. MIÓXIDOS.

Gén. Eliomys, Wagnner.. . 2 I nitela, Pall..

\section{Fam. MÚRIDOS.}

Subfam. FLEOMINOS.

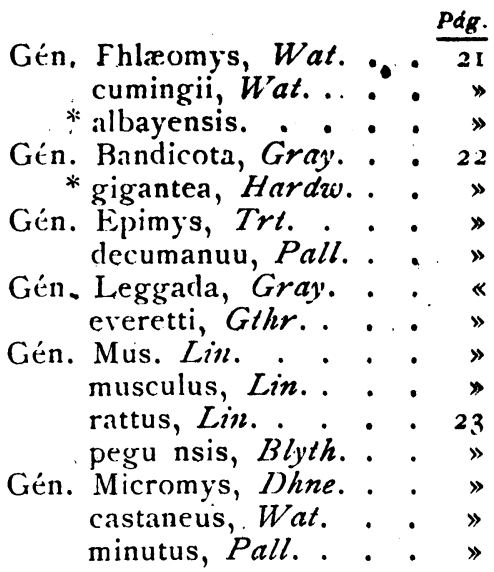

Fam. HISTRÍCIDOS.

Gén. Acanthion, F. Cuv. . 24 pumila, Gthr. 24 et 623

Fam. CÁVIDOS.

Gén. Cávia, Pall. . . . . 24 cobaya, Marcgu. . . ”

Fam. LEPÓRIDOS.

Gén. Lepus, Lin. . . . . " variabilis, Pall. . . 》

Gén. Oryctolagus, Lillj. . » cunículus, Lin. . . . "

\section{Orilen 6.० CARNÍVOROS.}

Subord. FISÍPEDOS.

Fam. ÚRSIDOS.

Gén. Ursus, Lin.. . . . » arctus, Lin. var. pyre-

naicus. 》

Fam. MUSTÉLIDOS.

Gén. Meles, Storr. . . 25 taxus, Bodd. . . . 》 Gén. Maydaus, F. Cuv.. . " 
meliceps, $F$. Cuv. 25 et $\frac{\text { Pág. }}{623}$

G'én. Mustela, Nils. . . 25 erminea, Lin. . . . " vulgaris, Briss. - "

Gén. Lutra, Erxl. • • 26 vulgaris, Erxl. . "

* sumatrana, Gray. . . »

Fam. CÁNIDOS.

Gén. Canis, Lin. . . - " japonicus, Tem. - \ molossus, Lin. . . . 》 terræ novæ. . . . 》 * pollokensis. . . . . »

Gén. Vulpes, Briss. . . " vulgaris, Briss. . . "

Fam. VIVÉRRIDOS.

Subfam. ARCTICTINOS.

Gén. Artictis, Tem. . . 27 binturung, Raff. 27 et 623

Gén. Paradoxurus, $F$. Cuv. 27 philippensis, Cam. . 》

* dubius, Gray. . . . »

* nigrifrons, Gray. . : »

* crossii, Gray. . . . $\mathbf{2} 8$

Gén. Viverra, Lin. . . . 》 tangalunga, Gray. 28 et 623 zibetha, Lin. . . . 29

Gén Viverricula, Hodg. . » * malacensis, Cantor. . 》

Gén. Herpestes, Illig. . . 》 auropunctatus, Hogids. »

Fam. FÉLIDOS.

Gén. Cynailurus, Wagler. . 30 tigris, Lin. . . . . » macroscelis, Gray. . »

Gén. Felis, Lin. . . . . 》 viverrina, Bennt. . . 》 javanensis, Horsf. 30 et 623 domésticus, Briss. . $3 \mathrm{r}$ angorensis, Briss. . . 》

\section{Orden $7,^{\circ}$ PINNÍPIDOS.}

Fam: TRIQUÉQUIDOS. Gén. Trichechus, Gray.

\author{
rosmarus, Schre. (col- \\ millos). $3 \mathrm{r}$
}

\section{Orden 8. SIRENIOS.}

Fam. HALTCÓRIDOS.

Gén. Halicore, Illig. . • 》 dugong, Lin. • • »

\section{Orden $90^{\circ}$ CET ICEOS.}

Fam. BALÉNIDOS.

Gén. Balæna, Lin. . . »

* australis, Desm. (bar-

Fam. BALENOPTÉRIDOS.

Gén. Balænoptera, Gray. . 32

* swinhoei, Gray (verte.

* mindorensis. bra). 》

Fam. CATODÓNTIDOS.

Gén. Catodon, Gray. . . . 》

* macrocephalus, Gray. »

Fam. DELFÍNIDOS.

Gén. Steno, Gray. . . . 》

* chinensis, Osbeck. . . »

Gén, Delphinus, Gray. . . "

Orden 10. ARTIDÁCTILOS.

Subord. CAVICORNIUS.

Fam. BÓVIDOS.

Gén. Bos, Lin. . . . . ” taurus, Gray. . . . 》

Gén. Bubalus, H. Smith. . 》 carabao, . . . . . 》 mainitensis, $P$. Heude. 》 mintorensis, $P$. Heude.

33 et 623 
Fam. ANTILÓPIDOS.

Gén. Rupicapra, Gray. . $\frac{}{33}$ tragus, Gray.

\section{Fam. CÁPRIDOS.}

Gén. Hircus, Gray.. . . ” agagrus, Gray. . • »

Fam. ÓVIDOS.

Gén. Ovis, Gray . • • 》 aries, Gray . . • »

Fam. ÁlCIDOS.

\section{Subord. CARREOLOS.}

Gén. Alces, Gray. • • 》 malkis, Gray. . . . "

\section{Fam. CÉRVIDOS.}

Gén. Strongyloceros, Gray. canadensis, Gray. . . 》

Gén. Pseudaxis, Gray. . . " tunquinensis : . . 》 soloensis, Heude. • . 34

Gén. Sikelaphus, $H$. . 》 soloensis, $H$. . . • 》

Gén. Ussa, $H$. . . . : 》 gorrichanus, $H$. • \ barandanus, $H$. . 》 crasşicornis, $H$. . . " francianus, $H$. • • • 》 nublanus, $H$. . . \ tuasoninus, $H$. . . ' 》 spatharius, $H . \quad$. $\quad$. ramosianus, $H$. . . . 》 ambrosianus, $H$. . . 》 macarianus, $H$. . . 》 elorzanus, $H$. $\quad$ • $\quad$ • 35 garcianus, $H$. . . 》 guidoteanus, $H$. . . 》 rosarianus, $H$. . . . nigricans, $H$. . . • maraisianus. $H$. - . 》 dailliardianus, $H$. : " marzaninus, $H$. . . " roxasianus, $H . \quad$. . . longicuspis, $H$. . . 》 microdontus, $H$.

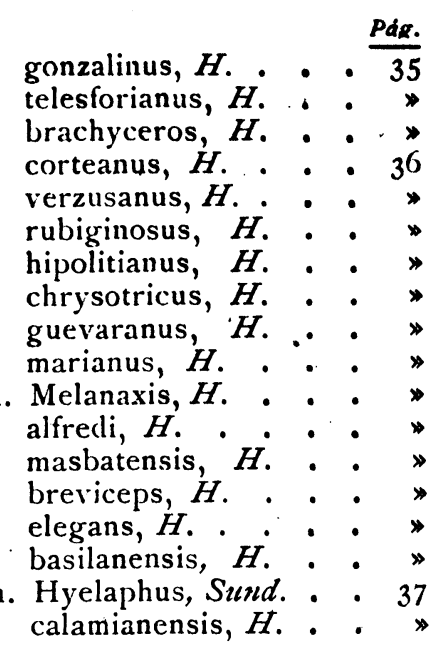

Fam. TRAGÚLIDOS.

Gén. Tragulus . . . . javanicus, Pall. . "

* fulviventer, Gray. . . "

* pygmaus, Gray. . . »

Fam. SÚIDOS.

Gén. Sus, Lin. . . . " scrofa, Lin. . . . . " papuensis, Less. . " effrenus, $H$. . . . . . frenatus, $H$. . . . . » microtis, $H$. . . . " cebrifons, $H$. . . . » celebensis, var. philippi nensis. 623 ahaenobarbus; Hent. . 623 minutus, $H$. • • • $~ 38$ arietinus, $H$. . . " bucculutus, $H$. . . . " calamianensis, $H$. . . » Gén. Babirusa, Gray. • " alfurus, Less. • • »

\section{Ord. $110^{\circ}$ PERISODÁCTILOS.}

\section{Fam. ÉQUIDOS.}

Gén. Equus, Lin. - . » caballus, Lin. • • • "

Gén. Asinus, Lin. . : " vulgaris, Lin. • • • » 
Fam. TAPÍRIDOS.

Gén. Tapirus, Lin. . . . $\frac{P_{a ́ g}}{3^{8}}$ indicus, Desm. . . ”

Orden 12.० PROBOS IDIOS.

Fam.. ELEFÁNTIDOS.

Gén. Elephas, Lin. . . . 39 maximus, L. (molar.) "

\section{Orden 13. DESIENTADOS.} Fam. MANÍDIDOS.

Gén. Pholidotus, Briis. . . » javanicus, Desm. . . ” dalmanii, Saud. . . " culionensis. . . . . "

Fam. DASIPÓDIDOS.

Gén. Tatusia, F. C̀uv. . . 4o novencincta, Lin.. . "

Sub-clase 2. ${ }^{\text {a }}$ DIDELFOS.

Orden. 14. MARSUPIALES.

Fàm: PERAMÉLIDOS.

Gén. Perameles, Geoff. . . " nasuta, Geof. . . . "

Fam. DASIÚRIDOS.

Gén. Dasyurus, Geoff. . . + I viverrinus, Shatw. . "

Fain. DIDÉLFIDOS.

Gén. Didelphis, Lin. . . 42 marsupialis, $L: n$. . . »

Fam. MACROPÓDIDOS.

Gén." Macropus, Shaw. . . 44 antilópinus, Gould. . " giganteus, Shazv. . 45
Fam. FALANGÍSTIDOS.

Gén. Trichosurus, Less. $\frac{\text { pág. }}{47}$ vul inus, (Myer). . "

Fam. FASCOLÁRCTIDOS.

Gén. Phascolarctos, Bl. . +8 cincreus, Schr. . . . 》

Sub-clase $3 .^{\mathrm{a}}$ ORNITODELFOS.

Orden $15 .^{\circ}$ MONOTREIIOS. Fam. EQUÍDNIDOS.

Gén. Echidna, G. Cuv. • 49 aculeata, Shazw. . . "

Fam. ORNITORÍNQUIDOS.

Gén. Ornithorhynchus, Blum. $5^{\circ}$ anatinus, Shaw. . . "

\section{Clase 2." AVES.}

Orden 1.० PRENSOR.IS.

Fam. CACATÚIDAS.

Gén. Cacatua, Vieill. .. . 52 hximaturopygia, ( $S$.

Mïll. 52 et 624

pusilla, var. . . 52

rosea, $V$. . . . . .

sulphurea, Gm. . 》

nasicd, Tem. .... . 》

moluccensis, $G m$. . .53

Gén. Microglossus, Geoff. . 》 aterrinus, Gm. . . . 》

Fam. NESTÓRIDAS.

Gén. Nestor. . . . . : $62+$ productus, Gould. . . 》

- Fàm. SITÁCIDAS.

Gén. Tanygnathus, Wagler. 53 
luzonensis, Briss. 53 et $\frac{\text { Pir. }}{625}$ viridis, var. . . . . 53 cyanescens, var. . . 54 tor fuatus, var. . '. . everetti, Tw. . 54 et 627 burbidgei, Sharpe. 54 et 627 macrorhynchus, Wagl. 54

Gén. 'Triclaria, Wagl. . . cyanogaster, Shaw. . »

Gén. Platycercus, Vig. . * * eximius, Shaw. . *

Gén. Cyclopsitta, $H$. et $J$. lunulata, Scop. . 54 et 627 squammotorquata, var. 55 intermedia, (Salv.). $\quad 628$ mindanensis, (Steere.)

55 et 628

Grén. Prioniturus, Wigl. . 55 discurus, (Vieill.) 55 et 628 cyaniceps, Sharpe, var. 55 et 629

cyaneus. . . . . 55 suluensis, Blas. . 629 luzonensis, Steere. 56 et 629 mindorensis, Steere.

56 et 630

verticalis, Sharpe.. . 629

Gén. Loriculus, Blyth. . . $5^{6}$ philippinensis; (Briss.) 56 et 630

siquijorensis, Steere. 56 et $63 \mathrm{I}$

mindorensis, Steere. 56 et $63 \mathrm{I}$

worcesteri, Steere. . 56 occipitalis, (Finsch.) . " chrysonotus, $\mathrm{Scl}$. 56 et $63^{2}$ indicus, $(\mathrm{Gm}$.). . . $63 \mathrm{I}$ hartlaubi, (Finsch.) 57 et 634

regulus, Sow. , 57 et 634 galgulus, (Lin.) . . " vernalis, (Sparr.). . 633

Gén. Ecclectus, Wagler. . 57 grandis, (Gm.). : . " polychlorus, (Scop.) $.5^{8}$ roratus, S. Miull. . . 635 pectoralis, S. Müll. . 633

Gén. Melopsittacus; Gould . ${ }_{58}^{8}$ undulatus, Share...

Fam. TRICOGLÓSIDAS. domicella, Line. . $\frac{P \text { Pder }^{\circ}}{5^{\circ}}$ garrulus, (Lin.) . . lory; (L'11.). . . .634

Gén. Chalcopsittacus, Salv. 58 ater, (Scop).

Gén. Coriphilus, Wagl placens, (Teem)

Gén. Charmosyna, Wagl. . 54 papuensis, (Gem.). .

Gén. Calyptorhynchus, Saly. " macrorhynchus, Gould *

Gén. Tricoglossus, Wig. . » hommtopus, Gm. . . .

Gén. Paleornis, Vig. . .6fr, torquata, Briss. . . 640

Gén. Eo;, Wagl. . . 6+t rubiginosa, $(B p$.$) . . 641$

\section{Orilen 2. RIPACES.}

Sub orden: HALCONES.

\section{Fam. I. VUL'Túridas.}

subfam. VULTURINAS.

Gén. Gyps, Lav . . . . 59 hispaniolensis, Sharp.

Fam. FALCÓNIDAS.

Subfam. ACCIPITRINAS.

Gén. Circus, Lacéb . . 60 spilonotus, Kaup . . " melanoleucos, Forst . "

* cruginosus, Lin . . " cinerescens, . . 6r philippinensis? Steere. striatus, . . 》

Gén. Astur, Lacép . . . \ palumbarius, Lin. . trivirgatus, Tem. . . 62 soloensis, Lath. . . 63

* cuculoides, Tem. . . . poliopsides, $\quad . \quad 64$

Gén. Accipiter, Briss. . . i nisus, (Lin.) . . . virgatus, $(R e i n+\infty . . .65$ stevensonii, Gurmey. 6: maculosus. . . . : 》 m inillensis, Meyen. .643

Subfam. BUTEONINAS.

Géa. Buteo, Cu». . . . 66 
vulgaris, Leach. . . Pág. * plumipes, Hodgs. . . 67

Subfim. AQUILINAS.

Gén. Neopus, Hodgs. malayensis, (Tem.)

Gén. Spizaetus, Vieill.

* Kieneri, Gerv. .

* lanceolatus, $B p$.

* alboniger, (Blyth:). limnaetus, Horsf. ph lippinensis, Sharpe.

* caligatus, (Raffl.).

Gén. Spilornis, Gray.

* cheela, Lath.

* bacha, (Daud.). pallidus, Wald. . holospilu, Vigors. $7 \mathrm{I}$ ảnayensis, Steere. 72 et 643 et 643

Gen. Butastur, Hodgs. . . 72 * liventer, (Tem.) - . " indicus, ( $\mathrm{Gm}$.). 72 et 643

Gén. Haliztus, Sav. . . . 73 albicillus, (Lin.) . .

* pelagicus, (Pall.). . 74

Gén. Cuncuma, (Hogds.) leucogaster, (Gm.). 74

Gén. Haliastur, Selby. . . 75 indus, (Bod:.). internedius, Gapk. 75

* girrenera, (Vieilh.) . 76

Gén. Milvus, Cuv. . . . " ictinus, Sav.. . . . 》

Gén. Elanus, Sav. • • 77 hypoleucus, Gould. 77

Gén. Pernis, Curv. . . 77 ptilonorhynchus, ( $\mathrm{Tem}$.)

77 et 643

Subfam. FAICOCNINAS.

Gén. Baza, Hodgs. maynirostris, leucopais, Sharpe. .636

Gén. Micruhierax, Sharpe. 78

* fringillarius, (Horsf). " eryihrogenys, Vigors. "

* latifrows, Sharpe.. 79
Gén. Falco, Lin. ... . . communis, Gm.. . . 》

* peregrinator, Sund. . "

* meḱnogenys, Gould. . 80

Gén. Hypotriorchis, Boie. . $8 \mathbf{I}$ s verus, (Horsf.) $8 \mathrm{I}$ et 644 Gén. Cerchneis, Boie. . . 82

* tinnuncula, Lin. . . .

\section{Subord. 2.० PANDIONES.}

\section{Fam. PANDIÓNIDAS.}

Gén. Pandion, Saw. . . »

* haliatus, Lin. . . . . . »

* leucocephalus, Gould. 83

Gén. Polioatus, Kaup. .. . "

* ichthyatus, (Horsf.). 84

Subord. $3 .^{\circ}$ NOCTURNAS.

Fam. BUBÓNIDAS.

Subfam. BLBONINAS.

Gen. Bubo, ckv. . . . » ignarus, Forster. .. . " philippinensis, Schl. . 85 gurneyi, Tw. . . . "

Gén. Scops, Savig. . . 86 megalotis, Gray. • . 》 lempiji, (Horsf.) . . " rufescens, Horsf. . . everetti, Tw. . . $\quad 87$ asio, Lin. . . . . "

Gén. Carine, Kaup. . • 》 noctua, (Scop.). • \

Gén. Ninox, Hodos. . . 88 lugubris, 7ich. 88 et 644 paraguanensis. . . . 88 philippinensis, $B p .88$

* scutulata, Raff.. . . 89 spilocephalus, (Steere). " spilonotus, Fir. et Worc. 644 japonica, T. et S.. . »

Subfam. SIRNINAS.

Gén. Syrnium, Sau. . . 
* leptogrammicum (Tem). $\frac{P \dot{q} g .}{89}$

* sinense, (Lath). . . 90

Fam. ESTRÍGIDAS.

Gén. Strix, Lin. flammea, Lin. . . . 》 candida, Tick. . . 92

Gén. Phodilus, Geoff. • . 93

* badius, (Horsf). . •. ”

\section{Orden: 3. PidskOs.}

Grupo: coraOnOREOS.

\section{Fam. córVIDOS.}

Gén. Colrus, Kaup. . . " monedula, (Lin). . . 》

Gén. Corone, Kizup. . . . 94 philippina, $(B p) .9+$ et $6+4$ pusilla, (Trv.).... . 94

Gén. Cissa, Boie. .. . . " chinensis, (Bodd). . 》

Gén. Xanthoura, $B p$. . 95 cyanocapilla, (Cab.). »

Fan. FARADISÉIDOS.

Subfam. EPIMAQUINOS.

Gén. Seleucides, Less. . . » niger, (Shaw). . . . "

Subfam. PARADISEINOS.

Gén. Paradisea, Lin. . • 96 apoda, Lin. . . . . sanguinea, Shaze. . "

Gén. Semioptera, Gray. • 97 wallacii, Gray. . . . 》

Gén. Lophorhina, Vieill. superba, (Penn). . "

Gén. Phonygama, Less. . . 98 keraudreni, Less. . "

Fam. ORIÓLIDOS.

Gén. Oriolus, Lın. . . • 》 philippinensis, Gray. . ». unicator. unicalor. achrorchynchus, Vigors. 98 et $6+4$

steerii, Sharpe. . . 9) assimilis, $T w$. . . . xanthonotus, Horesf. . \ suluensis, Sharp. . . " palawanensis, . Tw. . " samarensis, Steere: 99 et $6+7$ cinereogenys, Fr. et Worc. 6tt nigrostriatus, Fr. $e^{\prime}$

Worc. "

Fam. DICRÚRID́s:.

Gén. Dicrurus, Mieill. . .97 balicassius, Lin. . . ” mirabilis, $W a l '$. . . I0) striatus, Tw. . . . ” palawanensis, Tw. . \

Gén. Buchanga, Hodgs. . . " cineracea, (Horsf.) . " palawanensis, Whitek. $6+4$

Gén. Chibia, Hodzs. . . 100 pectoralis, (Wal!.) . " inenagei, Fr. et Worc. $6+5$ palawauensis, (Trv.). 》 borneen is, Sharpe. . "

\section{Grupo: cilitollongos.}

Fan. CAMPEFÁGIDOs.

Gén. Artamirles, Hartl. . IOI sumatrensis, (S. Mäll.)

pollens, salv. et 64.5 striatus, (Bodd.) . . " mindorensis, Steere. 102 et 645 mindanensis, Stcere. . I02 panayensis, Steere. . 》 guillemardi, (Salv.). . 645

Gén. Edoliisoma, Jacq. . . 102 corulescens, Blyth. . " everetti, Sharpe . 102 et 645 mindanensis, Tw. . . I 93 panayensis, Steere. . " Gén. Pericrocotus, Boie. igneus, Blyth. . • 
* miniatus, Tem. . . $\frac{\text { Rág. }}{103}$

* cinereus, Lafresn. . 104 marchesa, Guill. . . * leytensis, Steere. 104 et 645

Gén. Lalage,'Boie. . . . 104 melanoleuca, Blyth.' . terat, Bood. . 104 et 645

* aurea, (Tem). . . . 105 insperata, Finsch. . . » monacha, $(H$. et Fins.

ch.). . "

* leucopygialis, (Gray.) 105 minor, Steere. 105 et 645

Fam. MUSCICÁPIDOS.

Gín. Alseonax, Cab. . . 106 latirostris, Raff. . . 106

Gén. Muscicapa, Lin. . . 106 caruleocephala; Scop. 106 luzoniensis, Gm. . . 107 macrura, Scop.. . . Jo7 philippinensis, $G m$. $\quad 107$ manillensis, Gm. . . 107 griseisticta, (Swnh.) I07 et 646

Gén. Muscicapula, Blyth. . 646 mindanensis, Blas. . » samarensfs, . Fr. et Worc.

Gén. Cryptolopha, Swaison. olivacea, Mosseley. . » flavigularis, Fr. et Worc.

Gén. Pratincola, Koch. . . 108 ceprata, Lin. so8 et 646 pampilonensis, . .

Gén. Gerygone, Gould. . . simplex, Cab. . . . 408

* flaveola, Cab. . , . 109

Gén. Metabolus, $B p .$. . . 》 rugensis, $H$. et $J$. . "

Gén. Xanthopygia, Blyth, . " narcissina, Tem. . . "

* cyanomelana, Tem. . "

Gén. Hypothymis, Boie. . . 110 azurea,(Bodd.). I ro et 646 occipitalis, (Vigors.). I 10 superciliaris, $S h$. . . I I samarensis, Steere, . "

Gén. Cyanomyas, Sharp. ." calestis, Tw. I I et 646 helena, Steere, 》
Gén. Rhipidura, Vigors. . $\frac{P g^{\prime}}{111}$ kubarii, Finsch. . . 》 lepida, H. et Finsch. . " versicolor, H. et Finsch. $\gg$ sauli, Fr. et Worc. . 646 cyaniceps, Cass. . . 112 albiventris, Sharp. . " nigritorquis, Vigors.

112 et 647

Gén. Zeocephus, $B p$. . . . I1 2 rufus, $B p$. . 112 et 647 cinnamomeus, Sharp.

I 2 et 647

cyanescens, Sh. 11 3 et 647

Gén. Philentoma, Eyton. . "

* velatum, Tem.. . . "

Gén. Rhinomyas, Sharp. . " ruficauda, Sharp.. . " albigularis, Fr. et Worc. 647 samarensis, Steere. . " ocularis, Fr. et Worc. 1 I 3 et 647

Gén. Culicicapa, Swinh. . " panayensis, Sharp. II 3 et 647

Gén. Myiagra, Vigors. . . " pluto, Finsch. . . . 》 oceanica, Jacq. et Puch. II 4 erythrops, Hart et Finsch.

Gén. Monarcha, vig. et Horsf. " godeffroyi, Hartl. . " Gén. Stoparola, Blyth. . . 》 panayensis, Sharp . "

Gén. Siphia, Hodgs. . . . » elegans, Tem. . . " banyumats, Horsf.. . " philippinensis, Sharpe. I 5 et 647

lemprieri, Steere. II 5 et $\gg$

\section{Fam. TÚRDIDOS.}

\section{Subfam. SILVINAS.}

Gén. Phylloscopus, Boie. - I15 borealis, (Blas). i 5 et 647 lugubris, Blyth. . . I16 * superciliosus, Gm. . . " 
Gén. Acrocephalus, Naum.. $\frac{\text { Pag. }}{18}$ orientalis, Tem. i 6 et 647

Gén. Locustella, Kaup.

* fasciolata, Gray. . . * ochotensis, Middl. . . I 9

Gén. Lusciniola, Gray. . . • fuscata, Blyth.. . .

\section{Subfam TÚRDINOS.}

Gén. Geocichla, Kuhl. . . I 20 varia, Pall. . . . . "

* interpres, Kuhl. . . I $2 \mathrm{I}$ cinerea, Fr. et Worc. 648

Gén. 'Iurdus, Lin. . . . I 2 I músicus, Lin. . . . » viscivorus, Lin. . . 122 mustelinus, $G m$. . 123 pallasi, Cab. . . . . 123 leucauchen, Sclat. . I 24 grayii, $B p$. . . . . 》

Gén. Merula, Leach. . . merula, Lin. . . . torquata, Lin. .. . . . 125 obscura, $G m$. . . . 126 chrysolaus, Tem. . . I 27

Gén. Erithacus, Cuv. ${ }^{2} 8^{\circ}$ et 648

Gén. Monticrla, Boie. . . I 28 affinis, Blyth. . . . " solitaria, Briss. 128 et 648

Gén. Sialia, Sw. . . . . $\quad 129$ sialis, (Lin). . . . ”

Gén. Ruticilla, Brehm. . . 130 phœnicurus, Lin. . .

\section{Fam. 'TIMÉLIDOS.}

Subfam BR.AQUIPODINOS.

Gén. Aegithina, Vieill. . . viridis, $(B p$.$) . . I 3 \mathbf{I}$

Gén. Chloropsis, Jard. . . . $3_{2}$ palawanensis, Sharp. . » flavipennis, $T z$. . . » nigricollis, (Vicill.) . »

Gén. Ixocincla, Blyth. . . » crassirostris, E. Newt. "

Gén. Iole, Blyth. • • • olivacea, Blyth. . . » everetti, $T w$. . . I 33 r. figularis, Sharpe. . » philippinensis, Montb. ». guimarasensis, Steere. haynaldi, (Blas). . $\frac{\text { Pie. }}{648}$ cinereiceps, Fr. et Worc. monticola, Fr. et Worc. mindorensis, Steere. . I34 siquijorensis, Gm. . . .

Gén. Poliolophus, Sharpe. . • urostictus, Shalv. . . » basilanicus, Steere. -

Gén. Micropus, Swain. . . * melanocephalus, Gm. . \

Gén. Criniger, Tem. . I 35 frater, Sharpe. . . . palawanensis, Tw. . \

Gén. Pycnonotus, Boie. . goiavier. (Scop.) I 35 et 648

* sinensis, Gm. cinereifrons, $T w$. 136 et 648

Subfam. IRÉNINOS.

Gén. Irena, Horsf. . . . 136 cyanogastra, Vigors. . melanochlamys, Sharpe.

* turcosa Horsf. 136 et 648 ella, Steere. . . . . » tweeddallii. Sharpe. 136 et 648 paraguanensis, . . .

Subfam. TROGLODINOS.

Gén. Campylorhynchus, Spix. 173 zonatus, Less.

Gén. Thryothorus, Vieill. . » maculipectus, Lafr. : "

Gén. Henicorhin ', Scl. . " leucosticta, Licht. . . »

Subfam. TIMELINOS.

Gén. 'Thamnobia, Swa. • I 38 * fulicata, (Lin). . . "

Gén. Copsychus, Wagl. " mindanensis, $(\mathrm{Gm}$.) i 38 seychellarum, $A$. Newt. cebuensis, Steere. . . I 39

Gén. Cittocincla, Sclat. . .. nigra, Sharpe. . . . cebuensis, Steere. . . 648 luzoniensis, Kittl. . . \ 
superciliaris, Fr. et $\frac{\text { Pdg. }}{\text { Worc. } 649}$

annæ, $H$. et F. . . 139

Gén. Bebrornis, Sharp. . " seychellensis, Oust. . "

Gén. Megalurus, Horsf. . 140 palustris', Horsf. 1 40 et 649 ruficeps, Tw.

Gén. Sutoria, Nichol. sepium, Raff.

Gén. Orthotomus, Horsf. . I $4 \mathrm{I}$ frontalis, Sharp:. . . " cinereiceps, Sharp. . » nigriceps, $T w$.... . » castaneiceps, Wald. 14 I et 649 derbianus, Moore. . . " ruficeps; Less. I 4 I et 649 cineraceus, Blyth. . . " panayensis, Steere. : 142 sa arensis, Steere. . "

Gén. Cisticola, Kaup. . . "

* cisticola, Vieill. . . I 42

* exilis, Vig. et Horsf. 144 et 649

Gén. Cinclosoma, Vigors. . I 45 punctatum, (Lath.) . "

Gén. 'Tatare, Less. . . I 46 syrinx, Seeb. . . . " luscinia, $Q$. et $G$. . . »

Gén. 'Turdinus, Blyth. . . " rufifrons, Tw. . . . •»

Gén. Dasycrotopha, Tiw. . ” speciosa, Tw. . . . »

Gén. Mixornis, Hodgs. . . " woodi, Sharpe. . . . 》 cagayanensis, Guill. . . » capitalis, T $w$. . . . I 47 nigro-capitatus, Steere. plateni, Blas. . . . $6+9$

Gén. Macronus, Jard. . . I47 striaticeps, Sharpe. . 》 mindanensis, Steere. . 》 kettlewelli, Guill. . . "

Gén Ptilocichla, Sharpe. . " falcata, Sharpe... . " basilanica, Steere. . . ” mindanensis, Steere. . " minuta, Fr. et Worc. 649

Gén. Anurops, Sharpe. . I 48 cinereiceps, (Tzk.) . "

Gén. Dendrobiastes, Sharpe. " basilanica, Sharpe."
Fam. PÁRIDOS.

Subfam PARINOS.

Gén. Parus, Lin. . . . $\frac{\text { Pág. }}{"}$ cæruleus, Lin. . . . ” amabilis, Sharpe. . . 149 elegans, Less. 149 et 649

Subfam RECULINOS.

Gén. Regulus, Koch. . . I 49 ignicapillus, Brehm. 》

Fam. LÁNIDOS.

Subfam GIMNORRINOS.

Gén. Gymnorhina, Gray. . I50 tibicen, (Lath.). . "

Subfam. P:QUICEFAL!NOS.

Gén. Pachycephala, Vig. et Horsf. 》

philippinensis, Wald. . $15^{\circ}$ homeyeri, Blas. I 50 et 650 winchelli, Fr. et Worc. 649 major, Fr. et Worc. . 》 mindurensis, Fir. et Worc. 650

\section{Subfam LANINOS.}

Gén. Lanius, Lin. . : . 15r schach. Lin. . . . . " nigriceps, Frankl. I 5 I et $65^{\circ}$ cephalomelas, $B p$. . I 52 superciliosus, Lath. . " lucionensis, $\operatorname{Lin}$. 152 et $65^{\circ}$

Fam. VIREÓNIDOS.

Gén. Vireo, Vieill . . 153 gilvus, Vieill. . . . " tlavoviridis, Cass. . .

Gén. Hylophilus, Tèm. . " decurtatus, (Bp.). . "

Gén. Vireolanius, Du Bus. $15+$ pulchellus, Scl... . " 


\section{Grupo CERTIOHORFOS.}

Fam. CÉRTIDOS.

Subfam CERIINOS.

Gén. Certhia, Lin. . . . $\frac{P a_{\alpha}}{»}$ mex:cuna, Glog. . . »

Gén. Climacteris, Temm. * * mystacalis, (Tem.) 154

Subfam. Srinos.

Géz. Sitta, $L$ n. . . . I 55 carolinensis, Wils. . " frontalis, Size. . . . " anochlamys, Sharpe. $15^{6}$ et $65^{\circ}$

\section{Grupo 4. CINNIRIMORFOS.}

Fam. NEC'TARÍNIDOS.

Gén. Chalcostetha, Cab.. . " insignis, (Jerd.) . . 156

Gén. Æthopyga, Cab. . . 157

* temmincki; (S: Mïll.) 》

* eximia, (Horsf.) - . ” magnifica, Sharpe i 57 et $65^{\circ}$

arolasi, Fr. et Worc. bonita, Fr. et Worc. . " minuta, Fr. et Worc. " shelleyi, Sharpe $15^{8}$ et ." bella, Tz⿺. . . . I 58 pulcherrima, Sharpe. . *

Gén. Cinnyris, Cuv. . . . " sperata, (Lin:) 158 et 651 juliæ, (Trueed.) I 59 et 》 aurora, (Tw.) . . . 》 flagrans, (Oust.) . . » guimaraensis, Steere. I6o et $\gg$ jugularis, (Lin.) " et . * pectoralis, (Horsf.) . I6 1

Gén. Arachnothera, Tem. ..»

* longirostris, (Lath.)."

* flainmifera, Tw. . . . 162 dilutior, Sharpe. . ."

Gén. A-thothreptes, Gadow. 163 chlorogaster, Gadow. 163 et 651 griseigularis, Tiveedd. 163

Fain. MELIFÁGIDOS.

Subfain. MIZOMELINOS.

Gén. Mizomela, Vig. et rubratra (Less.) Horsf. 163 c. cheriresina, Gray... . "

Subfam ZOSTEROPINOS.

Zosterops, Vig. et Horsf. 163

everetti, Tw. . . . 167

* palpebrosus, (Tem.). " meyeni, $B P$. . . . . 165 semperi, $H$. et Finsch. . nigrocum, T $u^{\prime} . . . . "$ hypolais, Hartl. et Finsch. 165 oleagina, $H$. et $F$. . " conspicillata, (Kittl.). " semiflava, Neziton. $:$ I 66 modesta, Nezwton. . . " finschii, (Hartl.) : . " ponapensis, Finsch. . " basilanica, Steere. 166 et $65 \mathrm{I}$ cinirea, (Kittl.). . . 65 siquijorensis, Fr. et

Worc. "

Subfan. MELIFAGINOS

Gén. Puilemon, Vie:llot. . 166 philippinensis, Steere."

\section{Grupo FRINGILIFORMES.}

\section{Fam. DICÉIDOS:}

Gén. Dicoum, Cuv. . . . 166 retrocinctum, Gould. . " hæmatostictum, Shar- • pe. ${ }^{1} 67$ rubriventer, Less. 167 et $65 \mathrm{i}$ h.ypoleucum, Sharpe.." mindanense, $T w$. 167 et $65^{2}$ 
* trigonosticma; (Scop.). $\frac{P a ́ g .}{10 \gamma}$ dorsale, Sharpe. xanthopygium, $T w$.

cinereigulare, $T w$. . . 169

besti, Steere.

p y g mæu m, (Kittl.)

169 et $65^{2}$

everetti, Tw.

palidior, Fr. et Worc. 65I

sibuyanica, Fr. et

Worc.

intermedius, Fr.et

Worc. 652

assimilis, Fr. et Worc.

Gén. Prionochilus, Strickl. samarensis, Steere. .

* percussus, Tem. . . quadricolor, $T w . \quad$. 170 olivaceus, $T w$. . . \$

* xanthopygius, Salv. . " joannes, Steere. . " aruginosus, Fr et

Worc. $65^{2}$

bicolor, Fr. et W'orc.

modestus, Hume. .

Fam. HIRUNDÍNIDOS.

Gén. Chelidon, Boie. . I 170

* urbica, (Lin.) . . . ”

* dasypus. $B p$. . . . I7 1

Gén. Cotile, Boie. . . 172

* sinensis, Gray.. . . 172

Gén. Hirundo, $L n$. . . 173 rustica, Lin . . . . ” gutturalis, Scop. 174 et 652 javanica, Sparr. I75 et 》 striolata, (Bore.) i 76 et »

Fam. MNIOTÍLTIDOS.

Gén. Mniotilta, V'ieill. • . 177 varia, (Lin.)

Gén. Dendraca, Sharpe. . " pennsylvanica, (Lin). " cærulescens, Gm. . . »

Gén. Geothlypis, Cab. . . ” philadelphia, Wils. . "

Gén. Setophaga, Sw. . 178 ruticilla, (Lm.). . ”

Fam. MOTACÍLIDOS.

Gén. Motacilla, Lint. . 178
* alba, Lin. . . . . $\frac{\text { Pág. }}{178}$

* ocularis, Swinh. . . I 8 : melanope, (Pall.) 180

* flava, Lin. . . . 183

* borealis, (Suntev.) . 185

Gén. Anthus, Bechts. . . 186

* richardi, Vieill. . . rufulus, Vieill. 187 et 653

* cervinus, Pall. . . 188 gustavi, Swinh. r9o et 653 undatus. . . . . Igo

* maculatus, Hodgs. . . . "

Fam. CERÉBIDOS.

Subfam. CEREBINOS.

Gén. Chlorophanes, Reich. I9I spiza, LIn.

Fam. TANÁGRIDOS.

Gén. Calliste, Boie. . . . Igr larvata, Du Bus. . . "

Gén. Pyranga, Vieill. , " rubra, (Linn.). . ."

Gén. Phænicothraupis, Cab. 192 rubicoides, Lafr. . . "

Gén. Saltator. Vieill. . . " magnoides, Lafr. . . »

Gén. Pitylus, Cuv. . . . ” poliogaster, Du Bus. 192

Fam. IC'TÉRIDOS.

Gén. Dolichonyx, Sw. . . 192 oryzivorus, Lin. . . ”

Gén. Molothrus, Sw. . . ” pecoris, Gm. . . . 》 aneus, Wagl. . . . 193

Gén. Agelaus, Vieill. . . ” phæniceus, Lin. . . "

Fam. FRINGÍlidoś.

Gén. Volatinia, Reich. . . 193 jacarini, (Lin). . . 》

Gén. Carduelis, Briss. . . ” carduelis, (Lin). . . »

Gén. Passer, Briss. . . . 194 * montanus, (Lin). . . »

* flaveolus, Blyth. . : Ig6 

Gén Poacetes, Baird.... $\frac{\text { Pág. }}{195}$

(i)n. Serinus, koch. canaria, (Lin)

Gén. Loxia, Lin. curvitostra, Lir

\section{Gufo: ESTLRNIFORMIES.}

Fam. ARTÁMIDOS.

Gén. Artamus, Vieill. leucogaster, Valenc. 197 et 653

Fam. ESTÚRNIDOS.

Gén. Sturnia, Less. . . . violacea, (Boid). . . 198

Gén. Acridotheres, Vie ll. . I 99 crist.ttellu;, (Gm). . 》

Gén. Sarcops, Waldt. . . 200 colvus, Lin. 200 et 653

Gún. Mainatus, Less. . . " religiosus, (Lin). . . " javanicus, Osbek. . . $20 \mathrm{I}$ palawanensis, Sharp. 202 et 653

Gén. Mino, Less. . . . . 》 dumon:i, Less. . . . 》

Gén. Aplunis, Gould. . . » pelzelni, Finsch. . . " khittlitzi, Fusch. . . "

Gén. Calornis, Gray. . . 203 * metallica, Tie.. . . 》

* chalybad, (Horsf). . 204 panayensis, $(S c o p)$. 2.)5

Gen Streptociti, $B p$. . . » torquata, (Temm). . »

Gén Enodes, Tem. . . 206 * erytrophrys, (Tem). . »

Fam. PLOCÉIDOS.

Gén. Munia, Hodg. . . . 206 oryzivora, (Lin). 206 et 653 jagorii, Cab. 207 et " minuta, Meyer. . . . 207

* nisoria, (Tem). . . 208 cabanisi, Sharp. . . ”

Gén. Uroloncha, Cab. . . » everetti, (Tiv). 208 et $\frac{p_{\text {oir. }}}{653}$

* leucorastra, B'yth. .

Gén. Erythrurd, Sparr. (2)

* prasina, (Sparr.). . . trichroa, Kitll. . . .

Gén. Ploceus, Cuv. . . . 211 baya, Blyth.

* atrigula, Hodgs. . . $21 \mathrm{I}$

Gén. Ploceella, Oates . . javan nsis, (Less). . »

Fam. ALÁUDIDUS.

Gén. Melanocoryphi, Boie. 212

* calandra, Lir.. . . *

Gén. Alauda, Lin. . . . »

* gulgula, Frankl. . . "

Gén. Mirafra, Horsf. . . 2r 3

* javanica, Horsf. . . » philippinensis, Wardl. »

Fam. 'TIRÁNIDOS.

Gén. 'Todirostrum, Less. . 2 I 3 schistaceips, Scl. . .

Gén. Legatus, Sch. . . . albicollis, (Vieill). . »

Gén. Myodinastes, $B p$. . 214 luteiventris, $B p$. . .

Gén. Megarhynchus, Tumb. » pitangua, Lin.. . . »

Gén. Myobius, Gray. . . " sulphureipygius, Scl.: »

Gén, Myarchus, Cab. . . » lawrencii, Gir. . . .

Gén. 'Iyrannus, Cuv. . . 214 pipiri, Vie.ll. . . . »

Fam. PÍPRIDOS.

Gén. Chiroxiphia, Bab. . . 214 caudata, Shaze. . . ."

Gén. Chiromachæris, Cab. 215 candxi, Parz. . . . "

Fam. CoTíngIDOS.

Gén. Pachyrhamphus, Gray. 215 major, Sclat. . . . "

Gén. Lipaugus, Boie. . . " holerythrus, Scl. . . "

Gén. Rupicola, Briss. . ” peruviana, (Lath). ... 
Gén. Xipholena, Glog. . . $\frac{\text { Pág. }}{215}$ pompadora, (Lin.). . »

Fam. PÍTIDOS.

Gén Pitta, Vifall . . 216

* cyanoptera, Tem. . . erythrogastra, Tem. 216 et 653 propinqua, Sharpe 217 et 653

kochi, Brug. . . . "

* makloti, Tem. . . . " atricapilla, Bris. . . 118

* muelleri, $B p$. . . . » steerii, Sharp. . . . 219 luzonica. . . . . . » guttata. . . . . . " maxima, Mïll et Schleg. "

* nympha, Tem. et Schl, 》

Fam. EURILÉMIDOS.

Gén. Sarcophanops, Sharre. 220 steerii, Sharpe. . " samaremsis, Steere. . "

Gien. Eurylæmus, Sclat. .." * jaranicus, Horsf . . ”

Fam. DENDROCOLÁP ITIDOS.

Gén. Synallaxis, Vieill. . 220 erythrothorax, Scl. . "

Gén. Automolus, Reich. . 221 cervinigularis, Scl. . "

Gén. Dendrornls, Eyton. . " eburneirostris, (Less). * erythropygia, Scl. $.22 \mathrm{I}$

Fam. FORMICÁRIDOS.

Gén. 'Thamnophilus, Vieill. $22 \mathrm{I}$ doliatus, (Lin). . . "

Gén. Rhamphocanus, Vieill. " rufiventris, Bp... . »

Gén. Cercomacra, Scl. . . " tyrannina, Scl. . . . »

\section{Orilen 4. PICIRIO:.}

Suborden UPUPOS.

Fam. UPÚPIDOS.

Gén. Llupa, Lin. . . . 222 epops, (Lin). . . . $\frac{\text { Pag. }}{222}$

Suborden: TROQUILOS.

Fam. TROQUÍLIDOS.

Gán. Eulampis, Bore. . . 222 jugularis, (Lin). . . ”

Gén. Florisuga, $B p$. . . » inellivora, (Lin.) . . »

Gén. Amazilia, Reich. . . " riefferi, (Bourc.). . »

\section{Suborden: CORACIOS.}

Fam. CIPSÉLIDOS.

Subfam. CHETURiNos.

Gén. Chœtura, Steph. . . 223

* gigantea, (Tem.). . »

Collocalia, Gray . . "

lowi, (Sharfe.). . . 》

fuciphaga, (Thumb.)

22.3 it 653

troglodytes, Gray 224

marginata, Salv. 225 et "

* linchi, H. et Moore . »

francica, $(\mathrm{Gm}$.). . .654

Subfam. MACROPTERIGINOS.

Gén. Macropteryx, Swe ins. 226 comatus, (Tem.) 226 et 655

Fam. CAPRIMÚLGIDOS.

Subfam. CAPRIMULGINOS.

Gén. Caprimulgus, Lin . 226 europaus, Lin . . . " macrurus, Horsf. 227 et 655 affinis, Horsf. . . . " manillensis, Gray . 228 griseatus, Gray. . . ” phalona, Hart . . . » jotaka, Tem. et Schleg. 655

Gén. Lyncornis, Gould . . 228 macrotis, Vig. . . . » mindanensis, Treedd. 228 et 655 


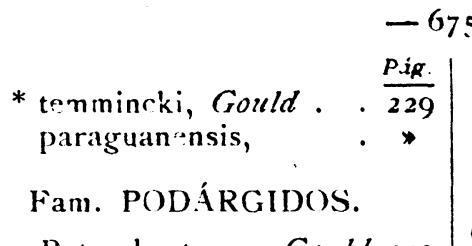

Gén. Batrachostomus, Gould. 229

* auritus, Gr. . . . » septimus $T w$. . . " javanensis, Horsf. . 230 menagei, Fr. et Worc. 655

\section{Fam. CORÁCIDOS.}

Gén. Eurystomus, Vieill - 230 orientalis, (Lin.) 23 oet 6.55

* calonyx ? Hodgs. . . $23 \mathrm{r}$

Fan. MEROPÓDIDOS.

Gén. Merops, $L$ in. . . 232 bicolor, Bodd 232 et 656

* sumatranus, Raffl. . " apiaster, Lin. . . . 233 philippinus, Lin. . . "

\section{Suborden: ALCIONES.}

Fam. ALCEDínidos.

Subfam. ALCEDININOS.

Gén. Pelargopsis, Gloger. . 235 melanorhyncha, (Ten.) 》 leucocephala, (Gm.) . 》 gouldi, Sharpe 236 et 656 gigantea, Wald. 236 et mindorensis, . . 236

Gén. Alcedo, Lin. . . . " ispida, Lin. . 236 et 656 meninting, Horfs . . 240

Subfam. DACELONINOS.

Gén. Ceyx, Lacép . . 24I

* tridactyla, (Pall) . . " rufidorsa, Sharpe 242 et 656 melanura, Kaup. . . 》 b. silanica, Steere. . mindanensis, Steere. . 243 lepida, Tem. . . . " malamaui, Steere . . » bournsi, Stcere. . . » nigrirostris, Fr. et Worc. $65^{6}$ cyanipectus, L.zfr . . $\frac{p_{\text {igg }}}{2 t t}$ steeri, Sh.urps. . . argeniata, To... . fluminicola, $T w$. . . Gén. Dacelo, Leach . . . » gigas, (Bodt.). . . "

Gén. Halcyon. Szu iing . 245 coromandus, (Lath.) 245 et $65^{6}$ s.nyrnensis, $(L ; n) . \quad \cdot 24^{6}$ gularis, (Kiuhl.) . . 248 pile itus, (Bodt.) . . " albicilius, (Cuv.) . . 349 winchelli, Sharpe. 249 et $65^{6}$ cinnamominus, Szexitus. $25^{\prime \prime}$ mediocris, Sharp?. . " chloris, (Bodx). $25^{\circ}$ et 6.50 humii, Sharpe. . 252 variegatus, (J.et Puch.) 》 lindsayi, (Vig.). . . » moseleyi, Sharpe . 253

Gén. Tanysiptera, Vigors ." dea, (Lin.). . . . "

Fam. MOMÓTIDOS.

Gén. Hylomanes, Licht . 253 momotula, Licht. . . ”

Suborden: BUCEROTES.

Fam. BUCERÓTIDOS.

Gén. Hydrocorax, Briss. $\quad 253$ hydrocorax, (Lin.). . " mindanensis, Tweedd . 254 semigaleatus, (Tweed.) 》

Gén. Anthracoceros, Reich 255 montani, Oust . . . »

Gén. Gymnolœmus, Ogilv. . » marchii, (Oust.). . . »

Gén. Penelopides, Reich. . » panini,(Bodd.) . . . $\gg$ manillœ, (Bodd.) . . 256 mindorensis, Steere. . 》 aftinis, Tw.. . . . 》 basilanica, Sterre. . » samarensis, Steere. $\quad 257$

Gén. Cranorrhinus, $C$. et Heine. 257 cassidix, Tem. . . " 
leucocephalus, (Vieill.) $\frac{\text { Pág. }}{357} \mid$ Gén. Hemilophus, Sw. . . $\frac{\text { Pág. }}{267}$ waldeni, Sharpe. . 258

Gín. Anorrhinus, Reich. . " galeritus, (Tem.) . . »

* pulverulentus, (Tem.) "

Gén. 'Thriponax, Cab. et. Heine. 268

Suborden: TROGONES.

Fam. TROGónIDOS.

Gén. Harpactes, Sw.xins. . 258 ardens, (Tem.) 258 et 656

\section{Suborden: TREPADORES.}

\section{Fam. PÍCIDOS.}

Subfarn PÍCINOS.

Gén. Gecinus, (Boie.) . 257 viridis, (Lin.) . . . 259 puniceus, (Horsf.). . »

Gén. Chloronerpes, Swa. . 260 yucatanensis, (Cabot.). 》

Gén. Chrysophlegma, Gould. " mentale, (Tem.). javensis, (Horsf.). . " pectoralis, Tzw . . 260 hargitti, Sharpe. . . " philippinensis, Steere. 269 et $65^{6}$ mindorensis, Steere. - 269

Fam. CAPITÓNIDOS.

Gén. Xantholæma, $B p$. . . $260^{\circ}$ h: $m$ a tocephal a, $(S$. Miill.). 269 et 657

* rosea, (Dum.) . . . 27 I intermedia, (Shell.) $27 \mathrm{I}$ et 657

Gén. Chotorhea, Bp. . . 271 versicolor, Raffl. . .

Fam. RAMFÁSTIDOS.

Gén. Rhamphastos, Lin. . 27 I dicolorus, Lin. . . . »

Fam. GALBÚlidos.

Gén. Melanerpes, Swa . . 26I pucherani, (Malh.). »

Gén. Dendrocopus, Koch. . " mixtus, (Bodd.). . . »

Gén. Sphyropicus, Bair. $\quad 262$ varius, $(\operatorname{Lin}.) . .$. . 》

Gén. Yyngipicus, $B p$. . . » maculatus, $(S c o p$.$) . . »$ fulvifasciatus, Harg. . 》 basilanicus, Steere. . 262 menagei, Fr. et . 263

Gén. Tiga, Kaup . • 》 everetti, Thw, · · 》 javanensis, (Ljung.) . »

Gén. Chrysocolaptes, Blyth. 264 erytrocephalus, Sharp. homatribon, (Wagl:). lucidus, Scop. . . $\cdot 265$ rufopunctatus, Harg . 266 xanthocephalus, Wald, »

Gén. Microstictus Harg. . » fuliginosus, Tw. . . " funebris, (Valen.). . »

Gén. Galbula, Briss. . . . 272 melanogenia, Scl. . .

Suborden: COCCIGEOS.

Fam. CUCÚLIDOS.

Subfam. CUCULINOS.

Coccystes, Glog. . . 272 coromandus, (Lin.) 272

Gén. Surniculus, Less. . . 273 lugubris, (Horsf.). . " velutinus, Sharpe. 274

Gén. Hierococcyx, S. Müll. $27+$ sparverioides, (Vig.). " fugax, (Horsf.). . . 275

Gén. Cuculus, Lian. . . canorus, Lin. 276 et 657 intermedius, Vahl. .278

* poliocephalus, Lath. . 279

Gén. Cacomantis, S. Mïll. 280 merulinus, ( $S c o p$.) 280 
Gén. Chalcoccyx, Cab. . . $\frac{P_{a g} \text {. }}{2 \gamma_{1}}$

* xanthorhy chus, (Horsf.) 282

* basalis, (Hor.sf.) .. . »

* lucidus, (Gm.) : . 283 malayanus, (Raffi.) . »

Gén. Eudrnamis, Vig. . . 284 mindanensis, (Limn.). $28+$ et 657

* honorata, (Lin.). . . 286 rufiventer, (Less.). . "

Gén. Coccyzus, Vieill. erithrophthalmus, Wils. 286

Gén. Scythrops, Lath. nova-hollandiae, Lath 287

Subfam. CENTROPODINOS.

Gén. Centropus, Ill. . . . 287 mindorensis, (Steere.) " sinensis, (Steph.). . » viridis, ( $S c 0 p$ ) 289 et 657 javanicus, (1)um.) 289 et melanops, (Les.) . . 290 unirufus, $C a b$. $A$.

Heine. 291

var. albina. . . . . " steerii, Fr. et Worc. 657

Subfam. FENICOFAINOS.

Gén. Piaya, Less. . . . . 291 cayana, (Lin.). . . " melanogastra, (Vieill.) *

Gén. Rhopodytes, Cab. et Hein. 29I tristis, (Less.). . . 291

Gén. Rhinortha, Vig. . . 292 chlorophoea, (Raffl.)."

Gén. Dryococcyx, Sharpe..» harringtoni, Sharpe.. " et 657

Gén. Dasylophus, Swa. . 292 superciliosus, (Cuv.)."

Gén. Lepidogrammus, Reich. » cumingi, Frasser. . . »

Subfam. CROTỌFAGINOS.

Gén. Crotophaga, Lin. . . 292 sulcirostris, Sw. . . 293
Orden: ' PALOMAS.

Fam. 'TRERÓNIDAS.

Gén. Treron, Vieill. . . $\frac{\text { Fág. }}{29.3}$ nipalensis, (Hodgs.) . "

Gén. Os:notrerin, $B p$. . . " axillaris, Gray. 29+ et 657 vernans, (Lin.) 295 et 》

Gén. Phabotreron, Bp. . 297 amethystina, $B p$. . . 》 cinereiceps, Fran et Worc. 297

brunneiceps, Fr. et IVorc. 297

maculipectus, Fir. et frontalis, Fr. et leucotis, (Tem.). . . " occipitafis, Tw. 298 et 657 nigrorum, Sharpe 29?

brevirostris, Tw. . $\begin{array}{r}\text {. } 298 \\ \hline\end{array}$

Fam. PTILÓPIDAS.

Gén. Leucotreron, Bp. . . 299 occipitalis, Gr. 299 et $65^{8}$ marchei, Oust. . . . 3ou leclanderi, $B p$. 300 et $65^{8}$ roseicollis, Gr. . . . 3(ख) gularis, $Q$. et $G$. . . 301 sp... . . . . . 3(2) jambu, (Gm.). . . . "

Gén. Ptilopus, Strickl. . . 3)3 ponapensis, Finsch. ." hernsheimi, Finssh. . 3०3 roseicapillus, (Less.). 》

Gén. Lamprotreron, $B p$. . 304

* temminckii, Des Murs. »

Gén. Spilotreron, Salv. . . » bangueyensis, Meyer. 》

Gén. Alectrænas, Gray.. . 3c5 pulcherrima, (Scop.). "

Fam. CARPOFÁGIDAS.

Gén. Globicera, $B p$. . . 306 oćeánica, (Less.) . . "

Gén. Carpophaga, Selby. . » nuchalis, Cab. . . . " ænea, (Lin.) . . 307 
chalybura, $B p . . \cdot \cdot \frac{\text { Pág. }}{308} \mid$ luzonica, $(S c o p.) \cdot \frac{\text { Pág. }}{326}$ palawanensis, Blas. . perspicillata, (Tem.) . 309 pickeringi, Crss. . . "

Gén. Ptilocalpa, $B p$. . . 310 griseipectus, $B p$. . . carola, $(B p$.$) . . . . "$

Gén. Zonophaps, Salv. . . " poliocephalus, Gray. . »

Gén. Myristicivora, Rchub. . 3 I I bicolor, (Scop.). . . "

\section{Fam. COLÚMSIDAS.}

Gén. Columba, Lın. . . . 313 livia, Briss. . . . " griseigularis, Wald. . cinerea, Scop. . . . »

Fam. MACROPÍGIDAS.

Gén. 'Turacæna, $B p$. . . 3I4 * modesta? . . . . . "

Gén. Macropygia, Siw. . . » tenuirostris, Gr. . . »

Fam. PERISTÉRIDAS.

Subfam. TURTURIDAS.

Gén. Homopelia, Salv. . . 315 rostrata, (Bp.). . . " picturata, (Tem.) . . 316

Gén. Streptopelia, $B p$. . . 317 dussumieri, (Tem.) . " humilis, (Tem.). . . 318

Gén. Spilopelia, Sundev. 319

* chinensis, (Scop.). .

* tigrina, (Tem.). . . »

Subfam. GEOPELINAS.

Gén. Geopelia, Sw. . . . $32 \mathrm{I}$ striafa, (Lin.) . . . $\rightarrow$

Subfam. FABINAS.

Gén. Chalcophaps, Gould. · 323 indica, (Lin). . . . "

Gén. Ocyphaps, Gould. . $\quad 326$ lophoses, (Tem.) . .

Subfam. GEOTRIGONINAS.

Gén. Phlogænas, Rchnb. 326 luzonica, var. albina. 327 samarensis, . . . " mindorensis, . . . " crinigera, (H. et Jacq.) 328 platena, Blas. . . yapensis, H. et Funsh. 》 Kub iryi, Finsch. . . " menagei, Fr ct Worc. 327 pampusan, $Q$. et $G$. . "

Subfam CALENADINAS.

Gén. Calæenas, Gray. . $33^{\circ}$ nicobarica, (Lin). . ”

\section{Fam. GÚRIDAS}

Gén. Goura, Sleph. . . $\cdot 33^{2}$ coronata, (Lin.) . . " victoria, Fraser. . . 》

\section{Orden: GALLINAS.}

\section{Fam. FASIÁNIDAS.}

Gén. Caccabis, Kaup. . 332 rufa, $(L \cdot n$.$) . . . . "$

Gén. Perdix, Briss. . . . ” perdix, (Lin). . . . "

Gén. Arboricola, Hodgs. $\quad 333$ * gingica, $(G m$.$) . . . 》$

Gén. Rollulus, Bonn. . . " roulroul, (Scop.) . . »

Gén. Coturnix, Moehring. · 334 coturnix, (Lin.). . 》

Gén. Excalfuctoria, Bp. · 334 chinensis, (Lin.) . . 》 lineata, Scop. . . 335

Gén. Lophura, Fleming. .336 rufa, Raffl . . . . ”

Gén. Tragopan, Cuv. . . " satyra, (Lin) . . . 》 temmincki, Gray. 337

Gén. Gennæus, Wagl. . . " nyctemerus, $($ Lin) $)$. ”

Gén. Phasianus, Lin. . . ” colchicus, Lin. . . . • » torquatus, Gm. . . . »

Gén. Chrysolophus, Gray. . ” pictus, (Lin.) . . . ” 
Gén. Gallus, Lin. . $\cdot \frac{P \text { dag. }}{337}$ stramineicollis, Sharpe.

gallus, Lin.

varius? Shaw.

domesticus, (Lin.)

d. patavinus, (Lim.)

d. cochinchinensis.

sunnerati, (Tem.).

Gén. Polypleciron, $T_{\mathrm{t}} m$. .

emphanes, Scl. . .

napoleonis, Less. . . $34^{\circ}$

Gén. Argusianus, Raf. . 34 I argus, Lin. . . . . "

Gén. Pavo, Lin. . . . . » cristatus, Lin. . . . * muticus, Lin. . . . "

Gén. Meleagris, Lin. . . * gallo-pavo, Lin. . . "

Gén. Numida, Lin. . . . » meleagris, Lin. . . . » id. vir. alba, . . . »

Gén. Lophortyx, $B p$. . $34^{2}$ californicus, Shaw.

Fam. MEGAPÓDIDAS.

Gén. Megapodius, $Q$. et cumingi, Dilliv. Gaim. $34^{2}$ laferousii, (Tem). . . »

\section{Orden: HEMIPODIOS.}

Fam. 'TURNÍCIDOS.

Gén. Jurnix, Bonnat. • 343 pugnax, (Tem.) . . 343 fascia a, (Tem.) . . " ocellat', $(S c o p.) \cdot 34 t$

\section{Orden: CORREDORAS.}

Fam. CASÚARIDAS.

Gén. Casuarius, Lin. • 344 emeu, Lath . . . . '” bicarunculatus, Sclat. 》

\section{Orden: ZANCUDAS.}

Fam. OTÍDIDAS.

Gén. Otis, Lin. . . $3+4$ tarda, L. . . . . . $\frac{P \text { sig. }}{3 t t}$

Fam. EDICNÉMIDAS.

Gén. Ortioramphus, Salv. 345

* magnirostris, Geoff. .

Fam. CHARÁDRIDAS.

Gén. Squaterola, $C u$. . $3+5$ helvetica. (Briss.) . . "

* grisea, (Briss.) . . "

* varia, (Briss.) . . . $3 t^{6}$

Gén. Belenopierus, Reich." cayennensis, $G m$. . . "

Gén. Charadrius, Lin. . . » fulvus, $G m$. . . . . "

* pluvialis, Lin. . . . 347

Gén. Aegialites, Boie. . ."

* peronii, (Tem.). . . " dubius, $(S c o p$.$) . . . "$ mongolicus, (Pall.) . $3 t^{8}$ geoffro,i, (V'agl.) . 349

* cantianus, (Lxth.) . "

Fam. GLAREÓLIDAS.

Gén. Glareola, Less. . . 349 orientalis, Leach. . . "

* isabella, Vieill. . . . 35()

Fam. HEMATOPÓDIDAS.

)

Gén. Strepsilas, Illig. . $\cdot 35^{\circ}$

* interpres, (Lin.) . . 350.

Gén. Hamatopus, Linn. . . 35I

* osculans, Swinh. . ."

Fam. ESCOLOPÁCIDAS.

Gén. Himantopus, Briss. . 35 $\mathrm{I}$

* leucocephalus, Gould. " autummalis, Has. . .

* minor, Nat. . . . .

Gén. Recurvirostra, Lin. $\quad 35^{2}$

* avocetta, Lin. . . . »

Gén. Calidris, Illiger. . . »

* arenaria, Lin. . . . »

Gẻn. Limicola, Koch. . . »

* platyrhincha, (Tem.) . »

Gén. Pelidna, Cuv. . . . "

* subarquata, (Gulden.) "

* alpina, (Lin.) . . 353 
* acuminata, (Horsf.) $\frac{P \text { s } g}{353}$

Gún. Actodromas, Kaup. . ”

* albescens, (Tem.) . . "

* teinminckii, (Leisl.) . $35+$

* salina, (Pall.) . . . "

Gén. 'Tringa, Lin. . . . ”

* cassirostris, Tem. et

* canutus? (Lath.) . $\quad 355$

Gén. Tringoidles, $B p$. . . " hypoleucus, (Lin.). . "

Gén. 'Totunus, Bechst. . . glareola, (Lin.) . . 355 ochropus, Lin. . . $\quad 355$ calidris, $\left(G_{\mathrm{m}}\right.$.) . . . "

* stagnalis, Bechst. . . " glottis, $(L i n$.$) . . 357$ inc a lus, $(\mathrm{Gm}$. ) . . . 》

Gén. Terekia, $B p$. . $.35^{8}$ cinerea, (Gulden). . "

Gén. L:mo ia, Briss. . . . 359

* m lanuroides, Gould. . "

* buveri, Nuxum. . . . 》

* agocephala, Lin . . 360 brevipes, Gray. . . "

Gén. Pseudoscolopax, Blyth. »

* semipalinatus, (Jerd). »

Gén. Numenius, Briss. . 36r arquatus, (Lin). . . "

* lineatus, Steere. . . »

* major, Tem. Schleg. . »

* australis, Gould. . ."

* phropus, (Lin.). . . I"

* minutus, Gould. . . $3^{52}$ luzoniensis, $(\mathrm{Gm}) . \quad 3^{62}$

Gén. Gallinago, Steph. . . ” * megala, Swinh. . . »

* scolopacina, Bp. . . "

Gén. Pavoncella, Leach. · 363 pugnax, (Lin). . . 》

Gén. Spilura, $B p$. . . . ” stentra, (Kuhl). . . 》

Gén. Rhynenixa, Cuv. . . » bengalensis, (Lin). . 》

Gén Scolopax, Lin. . . . $3^{6}+$ rusticola, (Lin). . . ”

Fam. RÁlIDAS.

Gén. Hypotænidia, Rchb. $\cdot 3^{6} 4$ striata, (Lin). . . . ” torquata, (Briss). . . " philippinensis, (Briss). $3^{5} 5$
Gén. Anaurornis, Reich. $\frac{\text { Pág. }}{305}$ olivacea, (Meyer). . "

Gén. Rallina, Rchb . . . ” fasciata, (R.rffl). . . " fusca, $(L ; n n)$. . . . 》

* rufigenys, Wall. . . 365

G’́n Ortygonetra, Lin. . cinerea, (Vieill). . . 355

Gén. Porzana, $V$. . . . 367 erythrothorax, Steere. " palykuli, Steere. . . ”

* bailloni, Vieill. . . "

Gén. Gallicrex, Blyth. . ” cristata, (Lxth). . . "

Gén. Erythra, R=h3. . . . 358 phuticura, (Penn). . 》

Gén. Gallinula, Briss. . . ” orientalis, Horsf. . . " sp. . . . . . . 》

Gén. Porpiyrio, Briss. . 357 * pulverulentus, Te:n. . indicus, Forsf. . . . »

Gén. Fulica, Lin. . . . ” atra, Lnn. . . . ”

Fam. PÁRRIDAŚ.

Gén. Hydrophasianus, IV zol. 369 chirurgus, (Scop). . ”

\section{Fam. GRÚIDAS.}

Gén. Antigone, Relch. . . 370 * toryuat.l, (Wie.ll). . "

Fam. A RDÉIDAS.

Gén. Ardea, Lin. . . $37^{\circ}$ * sumitrana, Raffl. . " cinerea, Lin. . . . 37 I parpurea, Lin. . . . ”

Gén. Diniegretta, Blyth. . ” sicra. (Gin). . . . 》

Gén. Herodias, Bone. . 372 * torra, (Buch-Hamilt). 》 internedia, (Hasself). 373 garzetta, (Lin). . . 374

Gén. G.ırzetta, $B p$. . . . 》 * nigripes, (Tem.) . . 》 Gén. Babslcus, $B p . \quad$. . . 》 coromandus, (Botd). . »

Gén. Arlesla, Bose. . . 375 
* specinsa, (Horsf). . $\frac{\text { Pig. }}{375}$

Gín. Butorides, Blyth. . javanica, (Hors/). 375

* macrohyncha, (Gould).

Cién. Ardeirala, Verr. . 376 flaricollis, (Lath). . "

Gén. Ardetta, G. R. Gray. » sinensis, (Gm.) . . » paraguanensis. . . 377 cinnamoinea, $(G m)$. . 》 sp. . . . . . . »

Botaurus, Steph. . . . . » limnophylax, (Gen.). . 》 stellaris, Lin. . . . »

Gén. Buphus, Boie. . . 378 * malaccensis, $(G m)$. . ”

Gén. Zebrilus, $B p$. . . . "

* radiolatus, Bp. . . . »

Gén. Gorsachius, Pıeg. . " *. 1.elanolophus, (Raffl). $z$

Gén. Nycticorax, steph. 379 griseus, Lin. . . . " manillensis, Vig. . . "

Fam. CICÓNIDAS.

Gén. Leptoptilos, Less. . · 379 dubius, $(G m) . \cdot$. . "

Gén. Melanopelargus, Rchb. » episcopus, (Bodd). . »

Fam. 'TANTÁLIDAS.

Gén. Falcinellus, Bechst. $\quad 380$ * igneus, $(G m)$. . . . ”

\section{Fam. PIAATALÉIDAS.}

Gén. Platalea, Lin. . . . $3^{8_{1}}$ leucurodia, Lin.

Gén. Leucorodius, Reich. . » luzoniensis, $(S \operatorname{cop})$. . »

\section{Orden: NADADORAS}

Fan. ANÁTIDAS.

Gén. Querquedula, Steth. . 》 multicilor, $(S c o p)$. . 》

* circia, $(\operatorname{Lin}) . . .0$

Gén. Anas, lín. .. . . $3^{x_{2}}$ luzonice, firas. . . .

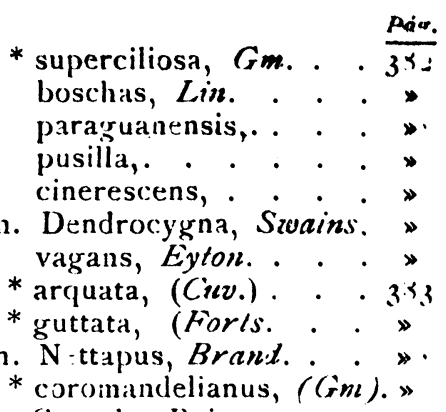

Gén. Siratula, Boie.

* clypeata, Lin. . . . »

Gén. Anser, Lin. . . 3rit

* segetuin, Gm. . . . \$ cinereus, Meyer. . .

Gén. Olor, Wagler. . . . " musicus, (Lin.). . . 》

Gén. Aix, Boie. . . . " galericulata, Lin. . ."

Gén. D.tfila, Leach. . . . »

* acuta, Lin. . . . . "

Gén. Fulix, Siundco. . . ."

* crist.ıta, Lin. . . . »

Fam. PEL.ECÁNIDAS.

Gén. Pelecanus, Linn. . . $38_{5}$ roseus, Gm. . . . . " javanicus, Horsf. . . "

Gén. Fregata, Ill. . . "

* aquila, (Linn.) . . . ”

* minor, $(G \mathrm{G})$. . . . $3 \% 6$

Gén. Gráculus, Linn. . "

* carbo, Linn. . . . "

Gén. Microcarbo, BD. . . "

* sulcirostris, (Brandt). 》

* pygmæus, (Pall.) . . $3 \% 7$

Gén. Sula, Briss. . . . 898

* cyanops, Sund. . . . piscatrix, $(\operatorname{Linn})$. . . " fiber, Linn. . . . $3^{39}$

\section{Fam. PLÓTIDAS.}

Gén, Plotus, Linn. . · . ” melanogaster, (Penu.). »

Fam. FETÓNIDAS.

Gén. Phaeton, Linn.. . . 390 rubricauda, Bodi. . . "

* candidus, (Briss.) . . 》 s6 
Fam. LÁRIDAS.

Gén. Larus, Linn. . . . $\frac{P a ́ g}{390}$

* fritzei, (Bruch.) . . ridibundus, Lin. . . 391 hardyi, $B p$. . . . . "

Fam. ESTÉRNIDAS.

Gén. Gelochelidon, Brehm. 》

* anglica, (Mont).

Gén. Hydrochelidon, Boie. . * nigra, $(\operatorname{Linn})$. . . " hybrida, Pall. . . $39^{2}$

Gén. Onychophrion, Wagl. fuliginosus, $(G m$.). anaethetus, (Scop.) • 393 sumatranus, (Raffl) ."

Gén. Gygis, Wagl. . . 394 alba, (Sparrm.) . . "

Gén. Sterna, Linn. . . . "

Subgén. Pelecanopus, Wagl. cristatus, Steph. .

* medius, Horsf. . . . 395

Subgen. Sterna, Lin. . 596 melanogastra, Tem. . "

* sinensis, Gm.

Gén. Sternula, Boie. . . . " minuta, (Linn.). . . ”

Gén. Anous Leach. . . " stodidus, (Linn.) . . 》 melanogenys, Gr. . 397

\section{Fam. PODIcÍPIDAS.}

Gén. Sylveocyclus, $B p$. . . » philippinensis, (Bon.). "

* var.. . . . . . . 》

Gén. Podiceps, Lath. . . 398

* australis, Gould. . . "

Gén. Pedeaithyia, Kaup. . » rubricollis, Lath. . . ”

\section{Fam. COLÍMBIDAS.}

Gén. Colymbus, Lin. . * arcticus, Lin. . . . ”

Fam. PROCELÁRIDAS.

Gén. Diomedea, Lin. . . exulans, Lin. . . . "

Gén. Puffinus, Briss. . . \ leucomelas, (Tem) $\cdot \frac{\text { Paig. }}{390}$

Fam. ÁlCidAS.

Gén. Cheniscus, Moehr. . " cirrhatus, $(G m$.$) . . »$

\section{Clase 3.' REP TILES.}

\section{Orlen 1.० QUELONIOS.}

\section{Suborden: ATECOS.}

Fam. ESFÁRGIDOS.

Gén. Dermochelys, Blainv. 397

* coriacea? (Lin). . . 399

Suborden: TECÓFOROS.

Fam. PLATISTÉRNIDOS.

Gén. Platysternum, Gray.. ”

* megacephalum, Gray. "

Fam. TESTUdínIDOS.

Gén. Callagur, Gray. . . 400

* picta, Gray. . . "

Gén. Ocadia, Gray. . . .

* sinensis, (Gray). . . ”

Gén. Damonia, Gray. . . »

* reevesii, Gray.. . . "

Gén. Bellia, Gray. . . . 40I

* crassicollis, (Gray). . ”

Gén. Nicoria, Gray.. . . »

* spengleri, Gray. . . "

Gén. Cyclemys, Bell. . . "

* trifasciata, (Gray.). . » amboinensis, Daud. . 402

* flavomarginata, (Gray) »

* platynota, (Gray.). . »

Fam. QUELÓNIDOS.

Gén. Chelone, Strauch. . " mydas, (Lin.). . . " imbricata, Strauch. . 404

Gén. Thalassochelys, Fitzing. . . . . " caretta, (Lin.). . . 》 
Fam. TRIONÍCIDOS.

Gén. Trionyx, Gray. . . $\frac{P a g}{405}$

* subplanus, Geoffr. . "

* sinensis, Wiegm. . . 406

Gén. Pelochelys, Gray. . . I I cantoris, Gray.. . . 406

Gén. Chitra, (Gray.) . . . 407

* indica, Gray. . . . "

\section{Oriten 2.0 EMIIDOS.lURIOS.}

Fam. CROCODÍlIDOS.

Gén. Crocodilus, Cuv.. . » porosus, Schneid.. . "

*. palustris, Less. . . . 408

Or.len 3. SAURIOS.

Subord. I. ${ }^{\circ}$ LAGARTOS.

Fam. GECÓNIDOS.

Gén. Gymnodáctylus, Spix. 》

* marmoratus, D. \& B. » philippinicus, Steind . »

* consobrinus, Peters. . "

* platurus, White. . . 409

Gén. Hemidáctylus, Cuv. . ” frenatus, Gray. . . "

* maculatus, Gray. . . " garnotii, Dum. Brib. 4 Io platyurus, Lach. . . "

Gén. Gehyra, Gray. . . » mutilata, Wiegze. . . 》

Gén. Perochirus, Blgr.. . 4II ateles, Dum. . . . 》 depresus, Fisch. . . " scutellatus, Fisch... . " var. articulatus, Fisch. "

Gén. Spathoscalobates, Gthr. "
Gthr. . .

Gén. Lepidodacty tus, lugubris, D. B. . . " labialis, Ptrs. . . . 41 2

Gén. Luperosaurus, Gray. » cumingii, Gray. . . "

Gén. Gecko, Laur. . I I I verticillatus, Laur. $\frac{P d g}{t^{12}}$

* vittatus, Gray.. . . 413 vir. bivittatus. . .

monarchus, Gray. . .

Fam. AGÁMIDOS.

Gén. Draco, Lork. . . . volans, Gray. . . . 》

reticulatus, Gthr. . . 414

id. v. cy a nopterus, Ptrs. guentheri, Blgr. . . * everetti, Blgr. . . . * ornatus, Gün'lir. . . . " spilopterus, D. \& $B$. »

* spilonotus, Gthr. . . * bimaculatus, Gthr. . " dussumieri, D. \& B. 415 quinquefasciatus, Gray. 》

Gén. Gonyocephalus, Kaup. » sophioe, (Gray). . . ” semperi, Plrs . . . 》 interruptus, Blgr. . . »

Gén. Calotes, Cuv. . . . » cristatellus, (Kuhl.). ” marmoratus, Gray. . 416

* oph:omachus, Gray. ."

Gén Lisphura, Gray. . . » amboinensis, Gray. . »

Fam. VARÁNIDOS.

Gén. Varanus, Merr. . . 417 grayi, (Gthr.). . . " rudicollis, Gray. . . 》 salvator, Laur. . . . » nuchalis, Gthr. . . . » cumingii, Mart. . . . 418

Fam. LACÉRTIDOS.

Gén. Lacerta, Lin. . . . ” viridis, (Laur.). . . »

Fam. ESCÍNCIDOS.

Gén. Egernia, Gray.. . . » cunninghami, Gray. . »

Gén. Mabuia, Fitz. . . " multicarinata, Gray. . " multifasciata, Kuhl. . ”

Gén. Lygosoma, Gray. . . 419 fasciatum, Gray. . . » 
jagorii, Ptrs. : . . $\frac{P d g}{42 \mathrm{I}}$

variegatum, Ptrs. . .

acutum, Ptrs. . . . »

rumingii, Gray . . . »

chalcides, (Lin.). . . 》

quadrivittatum, Ptrs. . 420

smaragdignum, Less. .

olivaceum, Gr. . . . »

semperi, Ptrs. . . . »

pulchellum, $G r$. . $42 \mathrm{I}$

vulcanium, Civ. . . »

atrocostatum, Less. . 》

nigrum, H. et Jacq. . "

Gén. 'Tropidophorus, $D . B$. ” leucospilus, (Pts.). . » grayi, Gthr. . . .

Gén. Brachymeles, $D$. $B$. . 》 schandenbergii, (Fucher.). 422 gracilis, Fisch.. . . " bicolor, Gray. . . . " bonitæ, D. B. . . . »

\section{Suborden: RIPTOGLOSOS.}

Fam. CAMALEÓNTIDOS.

Gén. Chamæleon, Laur. . " vulgaris, Daud. . . ”

\section{Orden 4. OFIDIOS.}

Fam. TIFLÓPIDOS.

Gén. 'Typhlops, D. B. . . 423 braminus, (Dand.). . " philippinus, (Mïll.). » jagorii, Ptrs. . . . » petersi, Steind. . . » ruficaudus, (Gray.). . » sp. . , . . , . . »

Gén. Onychocephalus, $D . B$. " cumingii, (Gray.). . 》 olivaceus, Gray. . . 424

Fam. UROPÉLTIDOS.

Gén. Uropeltis, D. B. . . 》 philippinus, (Cuv.!. . "

Gén, Rhinophis, Cuv. . . » philippinus, Boie. . . ”
Fam. CALAMÁRIDOS.

Gén. Calamaria, Boie. . . $\frac{P_{a ́ g}}{4^{24}}$

* bitorques, Pts. . . . $\gg$ gervasii, $D . B$. . . ” grayi, Gthr. . . " lumbricoidea, Boie. 425 philippinica, Steind. . " vermiformis, $D . B$. . "

* temminckii, D. et B. . 》

Gén. Oxycalamus, Gthr. . " oxycephalus, Gthr. ."

Gén. 'Typhlogeophis, Gthr. » brevis, Gthr. . . . 》

Gén. Geophis, Wagl. . . 》 schadenbergi, Fisch. ." brevirostris, Ptrs. . . 》 modestus, (D..B.). . 426

Gén. Rabdion, $B . B$. . " torquatum, B.B... ”

Gén. Aspidura, Walger. . 》

* brachyorhos, Gth. . "

Fam, OLIGODÓNTIDOS.

Gén. Oligodon, Bore. . ” modestus, Gthr. . . ” notospilus, Gthr. . . 》

* sublineatus, Gthr. . ."

Gén. Adlabes, D. B. . $\quad 4^{27}$

* collaris, (Gray.) : . "

Gén. Simotes, D. B. . . ” ancoralis, Jan. . . ” aphanospilus, Cope. . ” phornochalinus, Cope.. 》 purpurascens, (Scleg.). »

* russelli, Jan. . . . »

Fam. $4 \cdot^{a}$ COLÚBRIDOS.

Subfam. COLUbRiNos.

Gén. Elaphis, Aldr. . . 428 subradiatus, (Sclig.) . 》

Gén. Coryphodon, D. B. . 》

* korros, (Reinw.) . . "

* hexanotus, (Cantor). . 》

* mucosus, (Lin.). . . »

* fuscus? Gther. . . »

Gén. Compsosoma, D. B. . " melanurum, (Schleg.). " var. erythrura, D. B. 429

Cén. Spilutes, Wagl.. . . » 
samarensis, Ptrs. . $\cdot \frac{P_{a r r}}{429} \mid$ xanthozonius, var. Boie. $\frac{\text { Paig. }}{435}$

Fam. DRIADÍNIDOS.

Gén. Passerita, Gray. . . »

* mycterizans, (Lin). . »

Gén. Zaocys, Cope. . . . 》

luzonensis, Gthr. . . »

Fam. NA'TRÍCIDOS.

Gén. Tropidonotus, Kuhl. . 》 aur:culatus, Gthr. . . » chrisargus, Boie. . . » dendrophiops, Gthr. . 》 lineatus, Ptrs. .. . » spilogaster, Boie. . . ” sp. . . . . . . . 》 sp. . . . . . . . » stolatus, (Lin.). . . 》 aff. dorsalis, Gthr. . $43 \mathrm{I}$ aff. hypomelas, Ghtr. " schistosus, (Daud.) . 》

Fam. HOMALÓPSIDOS.

Gén. Cerberus, Cuv... . . 》 rhynchops, (Schneid.j. "

Gén. Campylodon, D.B. $\cdot 432$ prevostianum, $D . B$. . "

Fam. PSAMMÓFIDOS.

Gén. Psammodynastes, Glhu. 》 pulverulentus, (Boie.). »

Fam. DENDRÓFIDOS.

Gén. Gonyosoma, Wagl. . » oxycephalum, Boie. . »

* frenatum, (Gray.). . "

Gén. Dendrophis, Boie. . 433 pictus, $G$ ml.) . . . " punctalatus, (Gray.) . 》 terrificus, Pts. . . . »

Gén. Chrysopeleà, Boie. . ” ornata, (Shaw.) . . 》 rubes ens, (Gray.) • 434

Gén. Lepthophis, Bell. . . " vertebralis, D. B. . . ”

Fam. DRIÓFIDOS.

Gén. Tragops, Wagl. . . " prasinus, (Reinw.). . " var. Ixta, Cap. . . ”
Fam. DIPSÁDIDOS.

Gén. Dypsas, Boie. . . . \ angulata, $P$ ts. . . . » cynodon, Cuv. . . . 》 sp. . . . . . 436 dendrophila. Wagl. . drapiezi, (Boie.) . . " guiraonis, Stetnd. . . ”

* fusca, (Gray.). . . » philippina, $P t s$. . . »

Fam. LICODÓN'Tidos.

Lycodon, Boie . . . 》 aulicus, (Lin.) . . . 4.37

* capucinus, Boie var. P. Russel. » bairdi, Stend. . . . 》 modestum, . . 》 tesselatus, Jan . . . 》 cucullatum, . . "

Gén. Odontomus, D.B.. . » mülleri, (D.B.) . . ”

Gén. Cyclocorus, D.B. $\cdot 43^{8}$ lineatus, (Reinh.). . 》

Fam. ESCITÁLIDOS.

Gén. Hologerrhum, Gthr. .. » philippinum, Gthr. . "

Fam. AMBLICEFÁLIDOS.

Gén. Amblycephalus, Kuhl. » boa, Boie. . . . . »

Fam. BÓIDOS.

Gén. Piesigaster, Seoane. . 》 boettgeri, Seoane. . »

Fam. PITÓNIDOS.

Gén. Python, Daud. . . 439 reticulatus, (Schneid.). 》

Fam. ACROCÓRDIDOS. Gín. Chersidrus, Cuv. . . » 
granulatus, (Schneid.). $\frac{P_{a ́ g}}{439}$

Fam. ELÁPIDOS.

Gén. Naja, Laur . . . . ” tripudians, Merr. . . » sp.

var. samarensis, Pts. . 440 s putatrix, (Reinw.)

Gén. Ophiophagus, Gthr. . " elaps, (Schleg.). . . " fasciatus, (Pts.). . . "

Gén. Hemibungarus, Ptrs. ." calligaster, (Wiegm.) . 》 collaris, (Schleg.). . " gemmiannulis, Ptrs. . 44 I

Gén. Callophis, Gray. . . » bilineatus, Ptrs . . . »

Gén. Adeniophis, Ptrs. . . » philippinus, (Gthr.) . "

Gén. Elaps, Schueid. . »

* intestinalis, (Seba.). . "

* gracilis, (Gray.). . . ”

Fam. HIDRÓFIDOS.

Gén. Platurus, Latr . . . 442 laticaudatus, (Lin.). . " var. colubrirra, Shneid. 》

Gén. Hydrophis, Daced. . » abreviatus, Jan . . . brevis, Jan. . . . . »

* nigrocinctus, $D . B$. . » fasciatus, Schneid . . " loreata. (Gray.). . . » semperi, Garm . . 443 spiralis, (Shaw.) . . " westermanni, Jan. . . ”

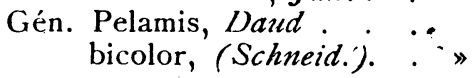

Fam. CROTÁlidOS.

Gén. Trimeresurus, Gthr. . " erythrurus, (Cantor.). 》 flavomaculatus, (Gray). 444 hombroni, (Ginsh)..." philippinensis, (Gray). » sp. schadembergi, Fisch . " wagleri, Schleg.
* hypnale, $D$. et $B \cdot \frac{\text { Pág. }}{445}$

* viridis, $D$. et $B$. . . "

\section{Clase $4 \cdot^{\circ}$}

ANFIBIOS, (Batracios.)

\section{Orilen 1. ANUROS.}

\section{Subord. FANEROGLOSOS.}

Fam. RÁNIDOS.

Gén. Oxyglossus, Tschudı. ” lovis, Gtír . . . . ”

* lima, Gthr. . . . . »

Gén. Rana, Lin. . . . . » mindanensis, (Giv). . "

* corrugata, Ptrs. . . $44^{6}$

* kuhlii, Gïnth . . . " macrodon, Gthr. . . » tigrina, Gthr. . . 》

* gracilis, Wiegm. . . 447

* guentheri, Boul. : . »

* macrodactyla, Gthr. . " erythrœa, Gthr. . . » chalconata, Gthr . . 》

* jerboa, Gihr. . . . 448

* luctuosa, (Ptrs.) . . » natatrix, Gthr . . . 》 similis, Gthr. . . . " sp. . . . . . 》 everetti, Boul. . . . "

* glandulosa, Boul . . "

Gén. Rhacophorus, Knhl. . 》 hecticus, Ptrs . . . » surdus, Ptrs . . . . 449 maculatus, Githr . . " var. quadrilineata, Wiegm. 》 appendiculatus, Gthr. . pardalis, Gthr. . . . »

Gén. Ixalus, $D$. et $B$. . . » acutirostris, Peters. . "

* pictus, Peters. . . . 45' bimaculatus, Ptrs. . 》

Gén. Cornufer, Tschudi. . » gürtheri, (Boul). . . 》 meyeri, Gthr. . . . " jagorii, Plrs. . . . 》 corrugatus, Dum. . . » 
Fam. ENGISTOMÁTIDOS.

Gén. Mycrohyla, Tschude. . $\frac{P \dot{d i g}}{45^{\circ}}$ achatina, Gthr. . . .

Gén. Callula, Gr. . . . . 45I pict ı, Gthr. . . . . * conjuncta, Ptrs. . . baleata, Mïll. . . .

Fam. BUFÓNIDOS.

Gén. Nectophryne, Buch. . » guentheri, (Boul.). . " sundana, Ptrs. . . . »

Gén. Bufo, Laur . . . . » brevipes, Ptrs. . . . 》 melanostictus, Gthr. . » biporcatus, Gthr. . . ” panayensis, Seoan . . ”

Fam. HÍlidos.

Gén. Hyla, Laur

* chinensis, Gthr. $\cdot 45^{2}$

Fam. HILEDACTÍlIDOS.

Gén. Hyledactylus, Tschudi. » * pictus, Bibr. . . . 453

Fam PELOBÁtidos.

Gén. Megalophrys, Kuhl. . 》 montana, Gthr . . . »

\section{Orden: UROIELOS.}

Fam. SALAMÁNDRIDOS.

Gén. Molge, Merv. . . . 》 * sinens's, Co:l. . . . »

\section{Orde : ÁPODOs.}

Fam. CECÍlIDOS.

Gén. Ichthophis, Fitz. . $\quad .454$

* munochrous, Ptrs.

\section{Clase 5.a PECES.}

Sub-clase:

1. TELEOSTEOS.

Orden: IC INTOPTERIGIOS.

Fam. BERÍCIDOS.

Gén. Monocentris, Schnsid. $\frac{P \div \text {. }}{45 t}$

* juponicus, Houtt. . . 》

Gén. Myripristis, Cız. . . 》

* pralinius, Cuv. . . . »

* murdjan, Forsk. . . 455

* japonicus, Cuv. . . . "

Gén. Holocentru.n, Artedi. * rubrum, (Forsk.) . . » punctatissimum, Cuv. » stercus-muscarum, $C . V$. " spiniferu:n, C. $V . \quad .456$ binotituin, $Q$ et $G$

* spinosissimuin, Tem et Sch. 》

* diadena, Lucep. . . »

* melanopterum, Blkr. » cornutum, Blkr. . . 》

* violaceum, Blkr. . . 》 sammara, (Forsk.) . "

Gén. R'hynchichthys, C. $V .457$

* pelamides? C. V. . . "

Fam. PÉRCIDOS.

Gén. Percichthys, Girard. . "

* ciliata, Gthr. . . . 》

Gén. Lates, Cuv. . . . . 》

* calcarifer, (Flo॰k.) . "

Gén. Cnidon, $M$. et Trosch. "

* chinensis, Müll. . . "

Gén. Psammoperca, Rich. . "

* waigienensis, $C$. et $V$. 》

Gén. Percalabrax, Tem. $\cdot 4^{3}$

* japonicus, $C$. et $V$. . "

Gén. Etelis, $C . V$. . . . » carbunculus, $C . V$. . "

Fam. SERRÁNIDOS.

Gén. Aprion, $C . V$. . $\quad \cdot 45^{8}$

virescens, $C . V . \quad$. . "

Gén. Anthias, Blk. . . . 》 
${ }^{*}$ cichlops, Blk. .j . . $\frac{\text { Pág. }}{450}$

* schelegelii, Gthr.

Gén. Anyperodon, Gthr. · 459

* leucogrammicus, Githr. "

Gén. Serranus, Cuv. . . . "

* brunneus, Glhr. . ."

* albofuscus, Gthr. . . ” oceanicus, $C . V$. . . "

*louti, Forsk. . . . "

* trimaculatus, C. $V$. . "

* flavimarginatus, Rüpp. 460

* diacanthus, Ciıv. . . "

* stigmapomus, Rich. . * tigrinus, $C$. $V$. . . » zananella, Blk. . . . "

* micropion, Blk. . . 》

* cyagnostigma, C. $V$. »

* aurantius, $C: V$. . . 461

* guttatus, Bl. . . . 》

* urodelus, C. V. . . "

* lumbatus, $C . V$. . . 》 suillus, $C: V$. . . . 》

* altivelioides, Blh.. . 》

* marginalis, $C . V$. . . "

* seba, Blk. . . . . 462

* celebicus, Blk. . . . " sp. . . . . . . . 》 hexagonatus, Gthr. ." var. hexagonata, Forst. " var. merra, C. $V$. . "

* punctatissimus, Gthr. 》

* gilberti, Richard. . . "

* awoara, Schleg. . . 463

* chlorostigma, Gthr. . "

* altivelis, C. V. . . "

* formosus, (Shaw). ."

* lineatus, C. V. . . . "

Gén. Plectropoma, Cuv. . "

* oligacanthus, Blkr. .

* susuki, C. V. . . . "

Gén. Grammistes, Cuv. . . 464 orientalis, Bl. . . . "

Gén. Diploprion, $C$. $V$. . ”

* bifasciatum, $C . V$. . "

Gén. Myriodon, Briss. . . 》

* waigiensis, G. A. I. "

Gén. Genyoroge, Cantor. . "

* bengalensis, Bloch. . "

* macolor, (C:V.) . . 465

* seba (C.V.) . . . " rosea, $(C: V$.$) . . : "$ bottonensis, (C.V.) . * riruluta, (C. V.) . $\frac{p^{\prime} \dot{c} .}{465}$

Gén. M esoprion, Ciwv. . . gembra, $C . V$. . . . " bohar, Forsk. . . . " johnii," Bloch. . . . 466

* fuscescens, $C . V$. . . .

* quinquilineatus, $C . V$. 》 fulviflamma, (Forsk.) " annularis, $C . V$. . $\cdot 4^{66}$

* malabaricus, $B l$. . . " decussatus, C: $V$. . "

* vitta, Q. et $G$. . . . . "

Fam. PRIACÁNTIDOS.

Gén. Priacanthus, $C$. $V$. . " * japonicus, $C . V$. . . " carolinus, $C . V$. . . »

* holocentrum, Blkr. . 468

* tayenus, Richard. . »

Fam. APOGÓNIDOS.

Gén. Ambassis, $C . V$. . . ”

* rubustus, Gthr. . . " interruptus, Blk. . . " safgha, Forsk. . . . " urotænia, Blk. . . . "

* apogonoides, Blk. . . "

* dussumieri, $C . V$. . . "

* buruensis, Blk. . . ‘ 469

* wolffü. Blk. . . . . "

* batjanensis. Blk. . . "

Gén. Apogon. Lacep. . . taniatus, $C . V$. . . 》

* hyalosoma, Blkr. . . "

* nigripinnis, C. $V$. . . "

* precilopterus, C.V. . "

* inultitzniata, $C . V$. . " monochrous. Bleek. . 470 bif sciatus, Rïppell. . "

* annularis. Rpp. . . " quadrifasciatus, $V a l$. . " frenatus, $\mathrm{Val}$. . . . " fasciatus, Whitc. . . " guamensis. Val. . ." cyanotienia, Blesk. . $47 \mathrm{I}$ fuscus, $Q$. et $G$. . . 》

Gén. Apogonichthys, Blkr. " * glaga, Blkr. . . . ”

* carinatus, $C . V$. . . "

* polystigma, Blk. . . "

Gén. Chilodipterus, Lacep. 》 
quinquelineatus, $C . V . \frac{P_{a} g .}{47 \mathrm{I}} \mid *$ nemurus, $B l k . \quad \cdot \frac{p_{i}}{4 j_{i}}$

Fam. GRÍSTIDOS.

Gén. Synagris, Gthr. . .

* japonicus, (Bloch.). . »

* sinensis, (Lacép.). . »

Gín. Dules, $C . V$. . . . " tæniurus, $C . V$. . . guamensis, $C . V . \quad . \quad 472$

\section{Fam. PRISTIPOMÁTIDOS.}

Gén. Therapon, Cuv. . . » theraps, Cuv. . . . * servus, Block. . . . » elipticus, Rich . . . » trivittatus, (Buch.). . » oxyrhynchus, . . » quadrilineatus, $($ Bloch $) .473$ argenteus, $C . V$. . . "

Gén. Pristipoma, Cuv. . . » nigrum, $C$. $V$. . . . » hasta, (Bloch.) . . . 》 leucurum, $C$. et $V$. . » argyreum, Bleek. . . » maculatum, (Bl.). . $\quad 474$ japonicum, $C \cdot V_{t} \cdot 474$

- Gén. Ha palogenys, $R i-$ nigripinnis, $(T e: n)$. Schl.) 》 mucronatus, (Eyd. et Soul.) 》

Gén. Diagramma, Cuv. . . " gilbosum, H. et Jacqq. » affine, Gthr . . . . 》 lineatum, Lin. . . . 475 pardalis, $V C$. . . . . punctatum, $C . V$. . . »

* cinctum, T. et Schl. . 475

* chætodonoides, Lacép. . " pictum, Thumb . . . »

* bolteatum, var. $b$. C.

* reticulatum, Gthr.

Gén Lobotes, Cuv.

* auctorum, Gthr. . . »

Gén. Scolopsis, Gthr. : . 477 ciliatus, (Lacép.) . . ”

* japonicus, (Bloch.). . »

* bimaculatus, Rïpp. . »

* cancellatus, $C$. $V$. . $\gg$ bleekeri, Blk. . . . ”

Gén. Heterognathodon, Blk. 478

Gén. Dentex, Cuv.

* nematophorus, Blkr. . Gén. Pentapus, Cuv. . . . »

* paradiseus, Gthr. . . »

Gén. Coesso, Cuv. . . . . ”

* maculatus, $C . V$. . . " tile, $C . V . \quad . \quad . \quad .{ }^{\circ}$

Fam. MÚLIDUS.

Gén. Upeneoides, Blkr. . +7') vittatus, (Forck.). . tragula, (Richard.) . »

* subvittatus, (Tem. et Sch.). » sulphureus, $C$. $V$. . . » moluccensis, Blkr. . . » esp. . . . . . . 》

Gén. Mullus, Lin. . . . esp. . . . . . .

Gén. Mulloides, Blkr. . $\cdot 4^{\text {xo }}$

* flavolineatus, Lacép. . "

Gén. Upeneus, Blkr. . . " indicus, (Shaze.) . . »

* pleurospilos, Bleek. . » malabaricus, $C$. B. . » barberinus, Lacep. . . " trifasciatus, (Lacep.) . »

* atherinoides, $C$. V. . $4^{8 \mathrm{r}}$

* chrysopleuron, $T$. et Sch. »

\section{Fam. ESPÁRIDOS.}

Gén. Cantharus, Cuv. . : ” grandoculis, C. V. . 》 coruleus, $C . V$. . . " maculatus, $C . V$. . . » lineolatus, $C . V$. . . » Gén. Girella, Gray. . . . ” * punctata, Gray. . . . »

Fam. PAGRÍNIDOS.

Gén. Letrinus, Cuv. . . $\quad \cdot 4^{82}$

* richardsonii, Gthr. . »

* nemacanthus, Blkr. . » harak, Forsk. . . . "

* ramak, Forsk. . . . » 


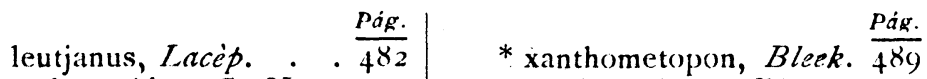

mahsenoides, $C . V$. . 》

Gién. Spharodon, Rüpp. $\cdot 4^{8} 3$

* heterodon, Blk. . . "

Gén. Pagrus, Cuv. . . . 》

* unicolor, $Q$. et $G$. . . 》

* cardiralis, Lacép. . . ”

* major, T. et Sch. . . "

* spinifer, Forsk . . . "

Gín. Chrysophrys, Cuv . . "

* aries, T. et Sch. . . »

* hasta, Bl. . . . . . 484 calamara, Russell . . "

Giér. Pimelepterus. Cuv. . " tahmel, Forsk. ... . »

\section{Ham. QUE'TODÓNTIDOS.}

Gén. Chotodon, Artedi. . "

* setifer, Bl. . . . . "

* ephippium, Cav. . . 485

* nesogallicus, Cuv . . " ocellaius, Bloch. . . "

* modestus, Tenmm. . . " unimaculatus, Bloch. . "

* meyeri, Bl. . . . . "

* ornatíssimus, Cuo... . »

* reticulatus, C. V. . . 486

* kleinii, Bloch. . . . " tasciatus, Forsk. . . " lunula, Cuv . . . . 》 ragabundus, Linn. . . 》

* ocellicauda, C. B. . . 》 lineolatus, $C u v$. . . » miliaris, $Q$. et $G$. . . 》

tau-nigrum, $C . V . . .487$

* citrinellus, (Brouss.) . " sp. . . . . . . . "

* chrysozonus, (Kuhl \& Hass.) 》 sp. . . . . . . . " oligacanthus, Bik . . " sp. . . . . . . . "

* strigatus, Langsd . . 488 * sp. i · · · "

Gién. Chelmo, Cuv. . . . rostratus, Cuv. . . . »

Gén. Heniochus, $C$. $V$. . - 》 macrolepidotus, Cuv. . "

* chrysostoma, C. V. . 489

Gín. Holacanthus, Lacép. . "

* bicolor Cuv. . . . »

Gén. Scatopliagus, $C . V$. . 》 argus, Cuv. . . . . " ornatus, $C . V . \quad$. $\quad .490$

Gén. Drepane, $C \cdot V$. . . " punctata, Curv. . . . "

Fam. TOXÓTIDOS.

Gén. Toxotes, Cuv. . . . ” jaculator, Cuv. . . . "

\section{Fam. CIRRÍTIDOS.}

Gén. Cirrhitichthys, Bleek . 49r maculatus, Lacép. . . 》

* aureus, Temm . et Schl. 》

Gén. Chilodactylus, Catv. . "

* zonatus, Caro. . . . »

Fam. 'TRÍGLIDOS.

Gén. Sebastes, C. $V$. . . 》

* pachycephalus, Tem.et Schleg. 》

* marmoratus, Carv. . . "

*. strongensis, $C$. $V$.. $\quad 492$ minutus, $C$. $V$.. . . »

* polylepis, Bleek. . . "

Gén. Scorpœna, Artedi. . " guamensis, $Q$. et $G$. . " picta, C. $V . . \quad . \quad . \quad »$ polyprion, Blkr. . "

* haplodactylus, Bleek. . ».

* diabolus, Cuv. . . . "

* gibbosa, Bl.. . . : 493

* cirrhosa, Cav. . . . »

* laniaria, C.V. . . . "

Gén. Pterois, Cù. . . . » volitans, Cuv. . . . »

* antennata, Cuv. . . . »

* miles, Cant. . . . 494 zebra, Cuv. . . . . »

Gén. Centropogon, Gthr. . "

* robustus, Gthr. . . "

* fuscovirens, $Q$. et $G$. "

Gén. I'etraroge, Gthr. . . "

* longispinis, (C.et V.) " amblycephalus, Bleek. » crista-galli, Gthr. . . 495 barbata, $c . V .$. . . 
* toenianotus, $C . V . . \quad \cdot \frac{P i g .}{495}$

Gén. Prosopodasis, Cantor.

* leucogaster, (Richard.)

* cottoides, C. V.

Gén. Aploactis, Tem. et Sch-

* aspera, Richards. leg. "

Gén. 'Trichopleura, Kaup. . 496

* mollis, (Richard.). . "

Gén. Synancidium, Mïll. . 》

* horridum, (Lin) . . "

Gén. Synanceia, Bloch.. . 》 verrucosa, Bloch.. . »

Gén. Pelor, $C$. $V$. . . " curieri, Gr.. . . . »

* japonicum, C. V. . . 497

Gén. Podabrus, Richards. . "

* cottoides, Rich. . . "

Gén. Centridermichthys, $R z-$ chards. 》

Gén. Platycephalus Bl." insidiator, Bl. . . " "

* japonicus, Tiles. . . 498

* isacanthus, C: V...."

* tentaculatus, Rüpp.. . "

* neglectus, Troschel. . »

* fasciatus, Gthr. . . " pristiger, $C . V$. . . "

* asper, $C . V$. . . . "

Gén. Hoplichthys, $C . V$. . 》

* longsdorffii, C. V. . »

Gén. 'Trigla, Cuv.. . . . "

* kumu, Less. et Garn. 499

Gén. Dactylopterus, Lacép.

* orientalis, Cuv.. . . "

\section{Fam. TRAQUÍNIDOS.}

Gén. Uranoscopus, Cuv . : . » bicinctus, Tem. et $S c h l$. asper, Tem. et Schl.

Gén. Percis, Bl. . . . . " cylindrica, Block. . "

Gén. Sillago, C. $V$. . . 500 sihama, Forsk. ... . maculata, Quoy et Gaim. "

Gén. Latilus, $C . V . .$. . » * argentatus, $C . V .$. . »

Gén. Pseudochromis, Rïpp. 》 * adustus; Mull. et Tros.
Fam. ESCIÉNIDOS.

Gén. Umbrina, Cuv. . . . $\frac{P \text { ág. }}{500}$ russellii, Cuv. . . .

Gén. Sciona, Cuv. . . . 501

* crocea, R.chards. . "

* diacanthus, Lacép.. . " sina, C. V. . . . . »

* dussuanieri, $C$. $V$. . "

* burneensis, (Bleeker.).

Gén. Corvina, Cuv. . . 502 miles, Cuv. . . . ."

* coitor, Cur.. . . . "

* belangerii, $C . V$. . . "

* semiluctuosa, $C$. V. . »

* olvila, $C . V$. . . . "

Gén. Otoliius, Cub. . . . »

* tridentifer, Richards. . ruber, Cus. . . . . " leuciscus, Gthr. . . 51)3

* argenteus, (Kuhl. \& v. Hass.) 》

Gén. Collicthys, Gthr. . .

* lucida, Richards. . . "

* biaurita, Cantor. . . »

Fam. POLINEMIDOS.

Gén. Polynemus, Lin. . . " paradiseus, $C . V$. . . »

* multifilis, Schleg. . . "

* multiradiatus, Gthr. . 504 líneatus, Gthr. . . . " plebejus, Gm. . . . » tetradactylus, Shazv. . »

Fam. ESFIRÉNIDOSS.

Gén. Sphyrona, Artedi. . 》 jello, Cuv. . . . . 》 commers unii, $C . V$. . " obtusata, Cuv. . . 505 $\operatorname{esp} \cdot . \cdot$. . . . ”

Fam. 'TRIQUIÚRIDOS.

Gén. Trichiurus, Lin. . . ” savala, Cuv. . . . . 》 muticus? $G r$. . . " haumela, Forsk. . . " esp. . . • $\cdot$. . "

Gén. Thyrsites, $\left.\dot{C} \cdot \dot{V} . \cdot 5^{(}\right)$ $\operatorname{esp}$. . . . . » 
Fam. ESCÓMBRIDOS.

Gén. Scomber, Cuv. . . . $\frac{P_{a g}}{506}$ microlepidotus, Rüpp.

Cién. Pelamys, $C$. $V$. orientalis, Tem. et Sch-

Cién. Cybium, Cuv.

guttatum, Cuv. . . . 》

jén. Echeneis, Artedi. . . "

* albescens, $T$. . . . " brachyptera, Loz'e. . " naucrates, $L$. . . . 507

Gén. Stromateus, Artedi. . 508

* argenteus, $B l$.

* niger, Bloch. . .

Gién. Brama, Risso. . . "

* dussumieri, $C . V .$. . 》

Gén. Mene, Lacép. . . . " maculata, Cuv. . . . "

Fam. CARÁNGIDOS.

Gén. Caranx, C. $V$. margarita, Richards. . » sp. . rottleri $\dot{R} \ddot{p} \dot{p} \cdot \cdot \cdot \cdot$ maruad i, Temm. . . » lioglossus, Gthr. . . " calla, $C . V$. . . . " malabaricus, Cuv. . . 510 leptolepis, Cur . . . " speciosus, Lacép. . . ” melampigus, Cuv. . . " jarra, Cuv. . . . . » hippos, Lin. . . " atropus, Cantor. . $5 \mathrm{II}$ armatus, Cuv. . . ." sansun, Forsk.. . 5 12 sp. . . . . . " gallus. Lin... . "

Gén. Seriola, $C . V$. . . * dumerilii, Risso, . . "

Gén. Chorịnemus, $C \cdot V \cdot 5^{1} 3$ lysan, Cavv. . . . "

* toloo, Cav. . . . . " sancti-Petri, Cuv. . . » orientalis, Tem. et Scht. "

Gén. Lichia, Cuv, . . 514 glauca, $B l$. . . . . "

Gén. Trachynotus, $C \cdot \dot{V} \cdot \overrightarrow{.} \gg$ opatus, Lin, . . . .
Gén. Psettus, C. $V . \quad . \quad \frac{\text { Pág. }}{51+}$ argenteus, Lin. . " sp. . . . . . . 515

Gén. Platax, $C . V$. . . . "

* vespertilio, Cuv. . . " teira, Cuv. . . . . » sp. . . . . . . 》

Gén. Pempheris, $C . V$. ${ }_{5}{ }^{6}$ * otaitensis, $C . V$. . . ”

* mangula, Cuv. . . . 518 esp . . . . . . 》

Gén. Equula, Cuv. . . . 》 fasciata, Lacép. . . edentula, (Bloch). . 》 caballa, (Forsk). . . " dussumieri, $C$. $V$. . " nuchalis, Schleg. . . $5^{17}$ splendens, Cuv. . . " oblonga, Cuv. . . . "

* leuciscus, Gthr. . . " insidiatrix, Bl. . . . "

* interrupta, $C . V$. . . 》

Gén. Gazza, Rüpp. . . . 》 minuta, Bleek. . . 》 argentaria, Forst. . . ” equulæformis, Rüpp. . 》

Fam. XÍFIDOS.

Gén. Histiophorus, $C . V$. . " * gladius . . . . . . "

Fam. GÓBIDOS.

Gén. Gobius, Artedi. . . 519 guamensis, Cuv.\& Val. ”

* baliurus, Cuv. . . . »

* puntangoides, Bleek. . »

* lentiginosus, Richards. " ornatus, Rüpp. giuris, Buch . . . » borneensis, Bleeker. . 520 echinocephalus, Rüpp. " ophthalmotænia, Bleek. » chlorostigmatoides. .

Bleek. " " cyanoclayis, Cantor. . " caninus, $C . V . . .$. . brevirostris, Gthr. . $52 \mathrm{I}$ xanthosoma, Bleek. . spectabilis, Gthr. . . " schlegelii, Gthr. . . » microlepis, Bleek. . . ” 
P.ig.

grammepomus, Bleek. $\overline{520}$ bynoensís, Richards.

sp. . Bleek. $\cdot 522$ lachrymosus, Ptrs. . " sp. . . . . . . 》

Gén. Apocriptes, C. $V$. . » serperaster, Richards, " nexipinnis, Cantor. . "

* madurensis, Blhr.. . »

Gen. Gobiodon, Bleek. . . "

* ceramensis, Blecker. . " histrio, C. V. . . . "

Gén. 'Irianophoriehtys, Gill.

* trigonocephalus, Gill.

Gén. Sicydium, Cuv. . . » cyanocephalum, Cuv. . »

Gén. Periophthalmus, $B l$. $\quad 523$ koelreuteri, $B l$. . . " schlosseri, Pall. . . 》 $v .7 r$. freycineti, $C$. et $V$. 》

Gén. Eleotris, Cù. . . • " ophiocephalus, (Kuhl. \& v. Hass. 》 hædtii, Bleek. . . . 524 obscura, Schleg. . ."

* oxycephala, Schleg. . » butis, (Buch). . . . 》 melanostigma. Bleek. . "

* caperata, Cantor. . " cyprinoides? $C$. $V$. . " marmorata, Bleek. . "

* sinensis, Lacép. . . ” muralis, (Quoy \& Gaim). 525

Gén. Amblyopus, $C . V$. ." brachygaster, Gthr. . ” brachysoma, Bleek. ."

Gén. Platyptera, C. V. . . "

- aspro, (v. Hass). . . "

Gén. Callionymus, Lin. . . "

* longicaudatus, Tem. et Schl. 》 schaapii, Blkr. . . . 526 simplicicornis, C. V. ." opercularis, $C . V$. . »

Gén. Vulsus, Gthr. . . . "

* dactylophus, C. $\ddot{V}$. . "

Fam. BATRÁQUIDOS.

Gén. Batrachus, C. V. . . " $\mathrm{sp}$
Fam. ANTENÁRIDOS.

Gén. Lophius, Artedi. $\quad \cdot \frac{P a ́ g .}{526}$

* setigerus. .

Gén. Antennarius Commers. 527 marmoratus, Gthr. .

* tridens, (Schleg). . . \

* urophthalmus, Bleek. . " sp. . . . . . . . » notophthalmus, Bleek. 》

Gén. Halieutæa, $C . V$. . . »

* stellata, Cuv. . . . »

Fam. BLÉNIDOS.

Gén. Blennius, Artedi. . 528

* sinensis, Gthr. . . .

Gén. Petroscirtes, Rüpp. . » tæniatus, (Q. et $G$.) . 》

* anema, Bleek. . . . » sp. . . . . . . . »

* grammistes, $G \cdot \dot{V} \cdot \dot{ }$.

Gén. Clinus, $C . V$. . . . " esp . . . . . . . 》

Gén. Salarias, Cuv. . . . 》 fasciatus, Cuv. . . . »

sp. .. . . . . . 529

* ceramensis, Bleek. . . "

* sebæ, Cuv. . . . . »

* variolosus, C. V. . . » sumatranus, Bleek. . "

* quadricornis, G. 'V. . »

* bellus, Gthr. . . . »

Fam. TEUTÍDIDOS.

Gén. Teuthis, Lin. . . . 529 javus, L. N. .. . . " ver.s.iculata, $C . V . \cdot 53^{\circ}$ margarifera, $C$. $V$. . " albopunctata, (Schleg). 》 hexagonata, Bleek. ."

* lurida, Rüpp. . . . " nebulosa, $Q$. et $(\dot{r}$. . »

* argentea, $Q$. et $G$. $.53 \mathrm{I}$ virgata, $C . V$. . . . " vulpina, Schleg. , . 》 sp. . . . . . . »

Fam. ACRONÚRIDOS.

Gén. Acanthurus, Bl. Schn. 》 triostegus, $B l$. . . . » 
matoides, Cuv. . . . $\frac{P_{a ́ g .}}{532}$

bipunctatus, Gthr. . .

nigros, $C . V$. . . .

lineatus, $B l$.. . . . »

striatus, $Q$. et $G$. . .

achilles? Shaw. . . .

ctenodon, $C . V$. . . "

sp. . . . . . . . "

Gén. Acronurus, $\dot{C} \cdot \dot{V} . \cdot 533$ argenteus, $Q$. et $G$. . "

Gén. Naseus, Commers . . " olivaceus, Cinv.. . . »

Gén. Keris, $C . V$. . . . " amboinensis; Bleek. . "

Fam. HOPLEGNÁTIDOS.

Gén. Hoplegnathus, Gthr. . " punctatus, Richards. ."

\section{Fam. NÁNDIDOS.}

Gén. Plesiops, Cuv... . " cœruleo lineatus, Rüpp. "

* corallicola, Bleek. . 534

Fam. LABIRÍN'TIDOS.

Gén. Anabas, Cuv. scandens, Cur. macrocephalus, Bleek.

* oliucele

Gén. Polyacanthus, $C . \dot{V} . \dot{.}$ "

* opercularis, Richards. 534

Gén. Macropus, Gthr. . . 535

* viridi-auratus? Lacép. ."

Gén. Osphronemus, Lacép. " olfax, (Commers.) . . " trichopterus, Pall. . . " striatus, Bleek. . . 536

Fam. LUCIOCEFÁLIDÓS.

Gén. Luciocephalus, Bleek. » * pulcher? Bleek. . . . »

Fam. A'TERÍNIDOS.

Gén. Attherina, Artedi. . 》

* bleekeri, Gthr. . . . "

* lineata, Gthr. . . . " sp.
Fam. MUGílidos.

Gén. Mugil, Artedi. . . $\frac{P a \dot{g} .}{»}$

* cephalotus, Cuv. . . "

* hæmatochilus, Schleg. 537 subviridis, $C . V$. . . " sundanensis, Bleek. ." meyeri, Gthr. . . . " sp. . . . . . . . " planiceps, Cuv.. . . " kelaartii, Gthr... . 》

* affinis, Gthr. . . . » cœruleo-maculatus, $\mathrm{La}$ troschelii, Bleek. . ." cép 538

* borneensis, Bleek. . . " melinopterus, $C . V$. . »

Fam. OFIOCEFÁLIDOS.

Gén. Ophiocephalus, Bloch. 》

* rhodotænia, Bleek. . »

* melanopterus, Bleek. . » striatus, Bl.. . . . " nigricans, $C: V . \quad .539$

* argus? Cantor. . . . "

* maculatus, Curv. . . . » ragus, Ptrs. . . "

Fam. 'TRICONÓTIDOS.

Gén. 'Trichonotus, $C . V$. . * setigerus, Bl. . . . »

Fam. CEPÓLIDOS.

Gén. Cepola, Lin . . . . »

* abbreriata? $C . \dot{V} \cdot \dot{-} "$

\section{Fam. CEN'TRÍSCIDOS}

Gén. Centriscus, Lin. . 540 * gracilis, Low'e. . . "

Gén. Amphisele, Klein. . . ” scutata, Cuv. . . . . » strigata, Gthr.. . . »

Fam. FISTULÁRILOS.

Gén. Fistularia, Lin.. . . ” * serrata, Cuv. .. . » 
[ Fam. MAS'TACEMBÉIDIDOS.

Gén. Rhynchobdella, $C . V \cdot \frac{\text { Pig. }}{5+1}$ * aculeata, $\mathrm{Bl}$.

Gién. Mastacembelus, $C \cdot \dot{V}$. ” * unicolor, $C . V$. . . »

\section{ord. 2. FARINGOGNATOS.}

\section{Fam. POMACÉNTRIDOS.}

Gién. Amphiprion, Bl. Sch. ” bifasciatus, Bl. . . " intermedius, Schleg. . $5 t^{2}$ sp. . . . . . . » * sela. Blee... . . " * clirkii, Cuv.. . . . "

* percula, Cav. . . . 》 boholensis, O. Cart. . $5+3$

Gén. Premnas, Cuıv.. . . 》 biaculeatus, Bleek. . "

Gén. Dascyllus, Cuv. . . " aruanus, Cuv. . . . *

* xant!: osoma, Bleek. . " reticulatus, Bich . . $54 t$

Gén. Pomacentrus, $C . V$. " fasciatus, $C . V$. . . ovoides, O. Cart. . ."

* trimaculatus, $C . \quad V$. . " punctato-lineatus, $O$. Cart.. » tæniurus, Bleek. . . " teniurus, var. Cart. $\quad 544$

* simsiang, Bleek. . . " sp. Cartier. . . " paro, Lacép. . . 545 paro, var O. Cart. . "

* chrysopacilus, Kuhl. » chrysurus, Ciıv. . . " cyanospilus, Bleek. . "

* scolopsis, $Q$. et $G$. . "

Gén. Gryphidodon, Glhr. . " coelestinus. . . . 》 mutabil:s $O$. Cartier.. 546 septemfasciatus, $C . V$. " tænicruptus, $O$. Cart. "

* affinis, Gthr. . . . " cingulatus, Kur. var. Cant.. . 》

* sordidus, (Fiors?.). . "

* albofasciatus, $H$. et $J \cdot 547$ bonang, B!esk. lachrymatus, $Q$. et $G . \overline{5+7}$

florulentus, Gthr. . .

* antjerius, Bleek. . . * playiometopon, Bleek. »

* unimaculatus, $C$. V. . " assimilis, Gthr. . $54^{9}$ dispa:", Gihr.. . . ."

* bankieri, Richards. . "

* molestus, Schleg.. . *

Gén. Heliates, Gthr.. . . 》 cinerascens, $C: V$. . "

* frenatus, $C . V$. . . . »

Fam. LÁBRIDOS.

Gén. Chærops, Rïp.. . . 》 macroilon, Lacép. . ."

meanler, Cartier.. . 5+3

* ommopterus, Richards. " unimaculatus. Cart. . "

* leucozona, Bleek. . . "

* anchorago, Bl. Schn." jiponicus, (C. V.) . "

* oligacanthus, Bleek. .."

Gén. Labrichthys, Bleek. 55 s

* rubiginosa, (S'ih'eo.) ." unilineata, Guichen. . "

Gén. Labroides, Bleek. • paradiseus, Bleek. . ..»

Gén. Duynariา, Bleek. . . "

* aurigaria, Bleek. . . "

Gén. Cheilinus, Cuv. . . »

* festivus, $C . V$. . . . . . rostratus, Cartier. . . »

* fasciatus. Rïpp. . . »

* martensii $C . V . \quad$. 55 .

* gudeffroyi, Gthr. . . "

Gén. Anampses, Cuv. . . " meleagris, $C . V$. . . "

Gén. Stethojulis, Gthr. . . \ strigiventer, Günth. . " 'sp. . . . . . . " triliaeata, Gïnth. . . " albovittata, Gïnth. . " kalosoma? Bleek. . . "

Gén. Platyglossus, Klein. $\quad 55^{2}$

* clussumieri, $C . V$. . . " esp. . . . . . . " pseudogramna, Cartier." esp. . . . . . . 》

* schwarzii, Bleek. . ." reticulatus, Cartier. $\quad 553$ 
* miniatus, $C . V . \quad . \quad \frac{P a ́ g}{553}$ alternans, Cartier. ."

* guttatus, Bl. . . . » ubayensis, Carter. .

* binotopsis, Bleek. ... . 》

* tenuispinis, Gthr. . . "

* scapularis, Benn... . 554

* pacilopterus, Schleg. "

Gén. Pseudojulis, Bleek. . » girardi, Bleck. . . . »

Gén. Novacula, $C . V . .$. . » taniurus, Lacep. .. . » pentadactyla, Cuv. . . »

* punctulata, $C$. $V$. .555

Gén. Julis, Gthr. . . " lunaris, Cuv. .... . 》

* umbrostigma, Rüpp. . »

* trilobata, Lacép. . . » dorsalis, Quoy. et Gain. $55^{6}$ truncatus, Carti.r. . 》

* schwanefeldii, Bleek. . 》

Gén. Gomphosus, Lacép. . "

* tricolor, Quoy et Gaim. 》

* varius, Lacép. . . . 557

Gén. Cheilio, Lacép. . . 》 i rermis, Bleek. . . . »

Gén. Cheillinus, Gill. . . » rostratus, Cartier. . . "

Gén. Pseudodax, Bleek. . " moluccensis, $C$. $V$. . 》

Gén. Scarichthys, Bleek. $\cdot 55^{8}$ auritus, Bleck.

Gén. Callyolon, C. $V$. . . » (earolinus, Cuv. . . $55^{8}$ brachysoma, Bleek. ." spinidens, $Q$. et $G$. . » sp. . . . . . . . »

Gén. Pseudoscarus, Gthr. "

* pulchellus, Bleek. . . " margaritus, Cartier. . 559 esp. . . . . . . 》

* chrysopoma, Bleek. . » sp. Cartier. . . .

* phyrrhostethus, Bleek. » aruginosus, $C$. $V$. . " ocellatus, $C . V$. . . »

* celebicus, Bleek. . . 560 muricatus, Bleek. . . ”

* balinensis, Blkr. . . "

Fam. GÉRRIDOS.

Gén. Gerres, Cuvv. 560 et 477 phallippinus, Gthr.» et $\frac{P a g .}{477}$ kapas, Bleek. " et " punct itus, $C . V$. » et $\gg$ * japonicus, Bleek. 》 et » * oyena, Cuv. . . » et»

\section{Ordèn $3 .^{\circ}$ ANIC.INTINos.}

Fam. GÁDIDOS.

Gén. Bregmaceros, Thomps. 56 $\mathrm{I}$ macclellandii, Thomps. "

\section{Fam. OFÍDIDOS.}

Gén. Encheliophis, Mïll. . » verinicularis, Mïll. . »

Gén. Congrogadus, Gthr. . " "

* subducens, Richards. »

Fa!n. PLEURONÉCTIDOS.

Gén. Psettodes, Benn. . . 552 erumei, Schn. . , . 》

Gón. Tephritis, Gthr. . . "

* sinensis, Lacép. . . "

Gén. Samaris, Gray.. . . "

* cristatus, Gray. . . 》

Gén. Pseudorhombus, Blıek. " russellii, (Gray.) . . 》

* pentophthalinus? Gthr. $5^{6} 3$

* olivaceus, Schleg. . . "

Gén. Rhomboidichthys, Bleek. $5^{6} 3$

* paro, Bleek. . . . »

* assimilis, Gthr. . .

* grandisquamma, Schleg. »

Gén. Sollea, Cuvv. . . . » ovata, Richards. . . "

Gén. Pardachirus, Gthr. . ”

* pavoninus, Lacép. . »

Gén. Synaptura, Cantor. . 56t

* foliacea, Richards. . »

* zebra, Cant. . . . » quagga, (Kaup.) . . "

Gén. Cynoglossus, Ham.

Buch. »

* auro-limbatus Richards. "

* abbreviatus, Gray. . »

* trigrammus, Gthr. . »

* melampetalus, Richards." 
quadrilineatus, Bleek. $\frac{P_{a ́ g}}{565}$

* oxyrhynchos, Bleek.

* puncticeps, Richards. brachyrynchus, (Bl.)

\section{Orden: FISÓSTOMUS.}

\section{Fan. SILÚRIDOS.}

Gén. Clarias, Gronov. . . ” macrocephalus, Gthr.

* fuscus, Lacép. . . . . "

* melanoderma, Bleek. . 566

* liacanthus, Blkr. . . nieuhofii, Cuv. et $V$. »

Gén. Heterobranchus, Geofe. »

* tapeinopterus, Bleek. . "

Gién. Plotosus, Lacép. anguillaris, Lacép. . » canius, Ham. . . . 567

Gen. Silurus, Artedi. . .

* asotus, L. . . . . "

Gén. Silurichthys, Bleek. . »

* phaiosoma, Bleek. . .

Gién. Wallago, Bleek. . . .»

* leerii, Bleek. . ... »

Gén. Belodontichthys, Bleek. 568

* macrochir, Bleek. . . »

Gén. Cryptopterus, Bleek. . *

* limpok, Bleek. . . . 》

* schilbeides? Bleek. . *

* bicirrhis, C. $V$. . .

* lais, Bleek. . . . . »

* micropus, Bleek. . . ”

* micronema? Bleek. . \

* hexapterus? Bleek. . . 569

Gén. Callichrous, Gthr... .

* bimaculatus, Bleek. . ”

Gén. Laiis, Bleek. . . . • »

* hexanema, Bleek. . . ”

Gén. Pangasius, $C . V$. . ..»

* macronema, Bleek. . \$

* nasutus,? Bleek. . . »

Gén. Pseudobagrus, Bleck. 570

* vachellii, Richards.

* fulvi-draco? Richards.. "

Gén. Rita, Bleek. . . . . » manillensis, $C . \quad V$. . »

Gén. Arius, Gthr. . . »

* tonggol, Bleek. . . »

* sagor. Ham. Buch. . pidada, Bleek. . . . \
* colatus, $C . V . . \ldots \frac{P_{\text {dig. }} \text {. }}{570}$ falcarius, Richards. .

* microcephalus, Bleek. . *

Gén. Hemipimelodus, Bleek. 57 I

* borneensis, Bleek. . *

* macrocephalus, Bleek. * manillensis, $C$. \& V. $57^{2}$

Gén. Ketengus, Bleek. . .

* typus, Bleek. . . . .

\section{Fam. ESCOPÉLIDOS.}

Gén. Saurida, C. V.. . . * tumbil, Cuv..

a rgyroph anes, $R i$ chards. *

Gén. Harpodon, Lesueur. * * nehereus, Ham-Buch. *

Fam. SALMÓNIDOS.

Gén. Salanx, Cuv. . . 573

* chinensis, (Osbeck.).

Fam. ESCOMBRESÓCIDOS.

Gén. Belone, Cuv . . . . ”

* melanotus, Blk. . . *

schismatorhynchus,

Bleek. *

annulata, C. V. . . . »

caudimaculata, Cuv. .574

* strongylurus, Bleek. . "

* anastomella, $C . V$. . »

* canciloides? Bleek. . .

Gén. Hemirhamphus, Cuv. . » viviparus, Peters. . . * esp. . . . . . . »

* intermedius, Cantor. . * georgii, C. V... . 575

* cantoris, Bleek. . .

* melanurus, $C$. V. . . »

* sinensis,? Cthr. . . quoyi, $C: V$. . . . *

* gernaerti, $C . V$. . . * commersonii, Cuv. . » dispar, C. V. . . .576 fluviatilis, Bleek. . . sumatranus, Bleck. . »

Gén. Exoccetus, Artedi. . *

* apus, Cuv. . . . . * 
* monucirrhus, Ri- ${ }_{\text {chards. }}^{\text {Pág. }}$

* brachipterus, Solander. " mento, $C$. $V$.

* volitans, Lim.

evolans, Lan. . . . "

* arcticeps, Gthk. . . 》

* parcilopserus,? C. $V .$. " esp. . . . . . . 》 spilopterus, C. $V$. . 》

*.oxycephalus, Bleek. .578

* brachycephalus, Gthr. "

\section{Fam. CIPRÍNIDOS.}

Gín. Cyprinus, Nils.

* fossicola ! Richards. . 》

Gén. Cirrhina, Cuv. . . \

* chinensis, Gthr. . \

Gén. Dangila, $C$. $V$. . . 》

* ocellata, Heckel. . . »

* curieri, Cav. . . . » sumatrana, Bleek. . »

* festiva, Bleek. . . . 579

Gén. Osteochilus, Gthr. . »

* melapopleuras, Blcek. 》

* hasseltii, Cuv. . . . 》

* vittatus, $C$. $V$. . . »

Fam. CLUPÉIDOS.

Gén. Engrauliş Cuथ. . . 》 tri, Blamk, . . . "

* japonica, Houtt. . . 580 heterolobus, R̈̈pp. ."

* rhinorhynchus, Bleek. » ampressus, Girard. . »

* hamittonii. Gray. . . 》

* mystacoides, Bleek. . 》 esp. . . . . . $58 \mathrm{I}$ setirostois, Brouss. . "

* brevicepas? Cantor. - 》

Gén. Coilia, Graj.. . . »

* clupecuides, (Lacép.) . »

* grayi, Rachards. . . 》

Gén. Chatoessus, $C$. $V_{*}$. . 》 nasus, Carv... . . 》

* punctatus, Selhleg. . . 582 chacunda. Cuv.. . . "

Gén. Clupea, CHv. . . . »

* mauritiana, Benn. . »

* istenglema, Rich. . . 》 argyrutania, Bteck. $\quad \frac{p_{i j s}}{5^{\gamma / 2}}$ fimbriata, $C . \quad V$. . . 》

* nymphaea, Richards. . 5r 3 sp.

* revesii, Richards. "

* koval, Rüppell. . . " zunasi, (Bleek.) . ."

Gén. Peilona, $C$. $V$. . . . "

* clongata? Bennel. . . 584

Gén. Dussumieria, Bleck. . 》 elopsoides, Bleek. . ”

Gién. Elops, Lin.. . . . ” saurus, Lin.. . . . 》

Gén. Megahos, Lacép. . . » cyprinoides, Bleek. . "

Gén. Chanos, Lacep. . . . $5^{8} 5$ salmoneus. Bl. Schn. »

Fam. QUIROKENTRIDOS.

Gén. Chirocentrus, Cuv. 585

* dorab, Forsk. . . "

Fam. SIMBRÁNQUIDOS.

Gén. Symbranchus, Bloch. . ${ }^{866}$ bengaleusis, M'Clell. ."

Fam. MURFNIDOS.

Gén. Anguilla, Cnv. . . . $5^{86}$ mauritiana, Bessnett. . " fidjiensis, Gthr. . ”

* amboinensis, Retrs. . " (sp). . . . . 587

Gxín. Uroconger, Kaup. . "

* lepturus, Kaup. . . »

Gín. Murancxos, M'Clell. »

* talabon, Bleek. . . . » cinereus, (Forsk.) . "

Gén. Murænichthys Bleek. $5^{88}$ macropterus; Bleek. . » schultzii, Bleek. . . 》 sp. - . . . . . 》

Gén. Ophichthys, Gthr. . »

* adspersus, Gthr. . . 》

* dicellurus, Richards. . 》

* pallens, Richards. . 》

* broekmeyeri, Bleek. . 589

* cirrochilus, Bleek. . " cephalozona, Bleek. var. Gihr. 》 apiculis, Bennelt. . . » 
chinensis, Kaup. . . $\frac{P(g g}{590}$ boro, (Ham-Buch.) cancrivorus, Richards.

* culubrinus, Bodd.

* yuadratus, Richards.

Gén. Moringua, Gray.

* lumbricoidea, Richards

* abbreviata, Ble k.

Gén. Murena, Gthr. punctato - fasciata. Blcek. 》

* tessellata, Richards. $\quad 592$

* fimbriata, Bennett. . " melanospila, Bleek. . "

* macassariensis, ? Bleek.

* thyrsoidea, Richards. 593

* pict 1 , Ahl. . . . "

* nubila, Richards. . . . "

* tenebrosa, Ri hards. 594

* macrurus, Bleck. . ." zebra, Bleek. . . . ” polyzona, Richards. ." nebulosa, $A h l$. . . . »

Gén. Gymnomurana, Bleek. 595 marmorata, Bleek.. ." esp. . . . . . "

\section{Fam. PEGÁSIDOS.}

Gén. Pegasus, Lin. . . ” * volins, Linn. . . . "

* natans, Linn. . . . "

* lancifer, Kaup. . . . 596

\section{Orilen 5." LofOBra neUIOS.}

Fam. SOLENOSTÓMIDOS.

Gén. Solenostema, Lacep. . "

* cyanopterum, Bleek. . "

* brachyurum, Bleek. . 》

Fam. SYNGiNÁTIDOS.

Gín. Syngnatus, Artedi. . "

* schlegelii, Kaup. . . "

* zanzibarensis, Gthr. . " spicifer, Rïppell. . . ” conspicillatus, Jenyns. 597

rin. Ichthyocampus, Kaup. "

* belcheri, Kaup. . . ”

Gín. Doryichthys, Kaup. . "

* buaja, Bleek. . . . " caurlatus. (P/rs.) . . $\overline{597}$

pteumstictus, Ptr.s. .

Gín. Gasterntokeus, Kaup. biaculeatus, Kants. . 597

Gén. Solenognathus, Karep. " * hardwickii, Kane.. . *

Gén. Hippocampus, Kinup. .

* longirostris, Schleg. . » guttulatus, Crevo. .

* trimaculatus, leach. . *

comes, Cantor.. . . 599

Orden 6. PLECTOGNiTOS.

Fam. 'TRIACÁN'TIDOS.

Gén. 'Triacanthus, Cuv.. . "

* brevirostris, Sclheg. . "

biaculeatus, Cuv:. . "

strigilifer, Cant. . . "

Fam. BALÍS'TIDOS.

Gén. Balistes, Cuv. . . . foo

* stellatus, Lacép. . . *

* maculatus, Gra. . . "

* vilua, Richards. . . 6or

* mitis, Benn. . . . "

* bursa, Bl. . . . . " aculeatus, $L . . .$. " verrucosus, L. Syst. . б02 rectangulus, $B l$. . . " undulatus, Afungo Park. "

Gén. Monacanthus, Civv. .603

* chinensis, Cuv. . . » sp. . . . . . . » tomentosus, Cur. . . 》

* sulcatus, Hollard. . " setifer, Bennet. : . 604

* monoceros, Osbeck. . "

Fam. OSTRACIÓN'TIDOS.

Gén. Ostración, Artedi. . 605 gibbosus, Lin. . . . . ”

* concatenatus, $B l$. . . »

cubicus, Lin. . . . "

diaphanus, $B l$. . . . 606

cornutus, Lin. . . ”

Fam. TE'TRODÓNTIDOS.

Gén. Xenopterus, Bibrona . 607 
* naritus, (Richards.) . $\frac{\text { Pag. }}{607}$

* modestus, (Bleek.) . "

Gén. Tetrodon, Lin. . . " lunaris, Bl. Schn. . " var. spadiceus, (Rich.) 608 sceleratus, (Forst.)." hypselogenion, Bleek. 》

* oblongus, $\mathrm{Bl}$. . . . " oblongus, var. alboplumbeus Rich. 609

* ocellatus, Osbeck. . »

* rubripes, Schleg. . . » patoca, Ham. . . . » inmaculatus, $B l$. var. virgata, Rich, »

* nigropunctatus, $B l$. . 610

* mappa, Less. . . . ” reticularis, Bl... . »

* hispidus, Lin, var. semistriatus, Rïpp,

* meleagris, Lacép. . . 6i fluviatilis, Ham, . . papua, Bleek. . . . " striolatus, Quoy, \& Gaim. 》

Gén. Diadon, Gthr. . . . ” hystrix, Lin .. . . . " * maculatus, (Gthr). . »

Gén. Chilomycterus, Gthr. . 》 orbicularis, Bl.. , , 》

\section{Orden: CONOROSTEOS.}

Fan, ACIPENSÉRIDOS.

Gén. Acipenser, Artedi. . Gr 3 * sinensisis, Gray. . . . ”

\section{Orden: PLilgIOSTOMOS.}

Fam. I. CARCÁRIDOS.

Gén, Carcharias, Cuv.

* lațicaudus, Müll.

* acutus, Rüpp, . . . 》 hemiodon, Mïll. . . "

* borneensis, Bleek. . . 6it

Gén. Galeus, Cuf: . . . "

* canis, Rondel. . . . »

Gén. Zygæna, Cuv. . . . »

* malleus, Share. . . . »
* tiburo, Valenc. . . . . $\frac{\text { Pár. }}{615}$

Gén. Irianodon, Gthr. . " obesus, Rïpp. . . . "

Fam, RINODÓN'TIDOS.

Gén. Rinodon, Smith. . . "

* typicus, Smith . . . "

Fam. ESCÍLIDOS.

Gén. Scyllum, M. et $H$. . 616

* marmoratum, Benn. . "

* maculatum, (Bl. Sch.) 》

* burgeri, Mïller. . . "

Gén. Stegastoma, $M$. et $H$. 》 tigrinum, $(G m$.$) . . 》$

Gén. Chilloscyllum, M. et H. 617 indicum, (Gm.) . . "

Fam. RínIDOS.

Gén. Rhina, Klein. . . . \

* squatina, Dumeril. . "

Subord, BATOIDEOS.

Fam, PRÍS'IIDOS.

Gén, Pristis, Lath. . . . 618

* pectinatus, Latham. . 》

Fam. RINOBÁtIDOS.

Gén. Rhynchobatus, Gthr. 619

* ancylostomus Bl, Schne. "

* djedlchnsis, Cant. . . "

Gén. Rhinobatus, Gthr. . "

* halavi, Rüpp... . . »

* schlegelii, (M. et H.) "

Fam. TORPEDÍNIDOS.

Gén. Temera, Gray. . .620 hardwickii, Gray. . ”

Fam. RAYIDOS,

Gén. Raja, Cuv. . . . . 》 * kenojei, $M$. et $H$. . 》 


$$
-701-
$$

Gén. Platyrhina, M. et H. $\frac{\text { Pag. }}{620} \mid$ Gén. Pteroplatea, M. et H. $\frac{\text { Pag. }}{621}$

* sinensis, Müll. . . ”

Fam. TRIG(ONIDOS.

Gén. 'Trygon, Adanson. . "

* gerrardi, Gray. . . ”

* bennetti, $M$. et $H$. . "
* hirundo, Lozve. . . "

Fam. MILIOBATIDOS.

Gin. Myliobatis, Cuv. . . "

* nieuhofii, Cuv. . . . "

* milvus, Mïll. . . . » 






, 
UNIVERSITY OF MICHIGAN

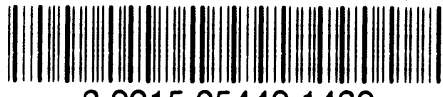

उ 9015054491439

Museums 
